\title{
SCIENTIFIC PROGRAM OF 37TH WORLD CONGRESS OF ENDOUROLOGY PROGRAM BOOK
}

BSP1-01 Comparison of recurrence rate between "en-bloc" resection of bladder tumor and conventional technique for non-muscle invasive bladder cancer

M Bangash, H Ather, N Khan, S Mohammad

Aga Khan University Hospital, Karachi

BSP1-02 Ex-vivo Multiphoton microscopy imaging of prostate biopsy slides as a new tool for prostate cancer prognosis

P. Treacy, I. Pavlova, U. Falagario, E. Wajswol, N. Tzavaras, R. Brody, R. Cabal, J. BravoCordero, P. Wiklund, M. Durand, A. Tewari

BSP1-03 Urinary N-GAL secretion predicts postoperative change in renal function after robotic-assisted partial nephrectomy for localized kidney cancer M. Takenaka, K. Fukaya, K. Takahara, N. Fukami, M. Ichino, M. Kusaka, R. Shiroki

\section{BSP1-04 Withdrawn}

BSP1-05 Transurethral Resection of a Bladder Tumor (TURBT), for the treatment of Non-Muscle Invasive Bladder Cancer (NMIBC), results in changes to selective inflammatory and angiogenic biomarkers

P. Ella-Tongwiis, I.S. Shergill, S.F. Hughes

North Wales Clinical Research Centre

BSP1-06 Correlation between serum testosterone and prostate-specific antigen (PSA) in ordinary males

J. Lee, J. Choi

BSP1-07 An upfront multiparametric MRI in the prostate cancer diagnostic pathway avoids prostate biopsy in $56 \%$ of patients. Can PSA density be used to further reduce Likert 3 biopsies?

T. Birks, J. Marenco, A. Roy, D. Cohen, P. Singh Royal Free Hospital, London

BSP1-08 Uro-TC and ureteroscopy: useful tools in the diagnosis of upper urinary urothelial carcinoma

F. Lara Pérez, S. Martín Martín, J. Torrecilla García-Ripoll, J. Diaz Romero, M. Bedate Núñez, F. Natal Alvarez, J. Soto Rodríguez, A. Ruano Mayo, A. Zamora Horcajada, C. de Castro Olmedo, R. Calvo González, L. Pesquera Ortega, J. Calleja Escudero, E. Valsero Herguedas, M. Pascual Samaniego, D. Rivero Martínez, A. Alonso Villalba, J. Díez Rodríguez, C. Conde Redondo, J Cortiñas González Hospital Clinico Universitario de Valladolid
BSP1-09 Prostate specific antigen kinetics predict prostate cancer progression following holmium laser enucleation of the prostate

K.M. Rose, A. Nevo, K.S. Faraj, A. Navaratnam, K.A. Arora, S.M. Cheney, M.R. Humphreys Mayo Clinic

BSP1-10 Photodynamic Therapy in Combination with EGFR Targeted Photosensitizers Enhances the Long-Term Cure of Bladder Cancer in Animal Models

F.A. Durrani, J. Cacaccio, R.R. Cheruku, N.A. Aldhaam, K.A. Guru, R. Pandey

Roswell Park Comprehensive Cancer Center

BSP1-11 The EGFR, and FABP7 gene mutations had discriminating power to differentiate metastatic overall survival in liver metastasis from metastatic renal cell carcinoma after targeted therapy

W. Park, D. Hong, J. Park, J. Chung, S. Kim, S. Kim

BSP1-12 Closed loop audit of muscle sampling in trans-urethral resection of bladder tumor

R. Georgiou, R. Varma, A. Chetwood, A. Ali

BSP1-13 Withdrawn

BSP1-14 Possible role of 5-alpha reductase inhibitors in non-invasive bladder urothelial neoplasm: multicentric retrospective study

A.A. Pastore, C. De Nunzio, M. Balzarro,

Y. Al Salhi, A. Fuschi, A. Martoccia, N. Amigoni,

A. Tubaro, W. Artibani, A. Carbone

Sapienza University of Rome, Faculty of

Pharmacy and Medicine

BSP1-15 MiR-193a-3p inhibits aldosteronoma growth by down-regulating CYP11B2 expression

Z. Guoxi, Z. Xiaofeng, L. Quanliang,

X. Tianpeng, H. Zhihua

BSP1-16 Feasibility of the Epicheck test in upper urinary tract tumor

R. Boissier, A. Territo, A. Gallioli, F. Regis,

D. Vanacore, J. Palou, A. Breda

Fundació Puigvert, Autonomous University

of Barcelona, Barcelona. Spain.

BSP1-17 Connective tissue transcriptomic signature and worst clinicopathological outcomes in prostate cancer patients

P. Treacy, A. Martini, P. Ratnani, S. Nair,

U. Falagario, A. Horowitz, M. Durand, A. Tewari 
BSP1-18 Preoperative neutrophil/lymphocyte ratio (NLR) as a prognosticator of recurrence and progression in upper tract urothelial carcinoma (UTUC) patients treated conservatively:

a retrospective evaluation

M. Antonucci, L. Defidio, N. Russo,

M. De Dominicis, A. Patel

BSP1-19 Epidermal Growth Factor Receptor (EGFR) Targeted Multifunctional Photosensitizers for Bladder Cancer Imaging (Fluorescence and PET) and Photodynamic Therapy (PDT)

R.R. Cheruku, J. Cacaccio, F.A. Durrani,

W.A. Tabaczynski, R. Watson, A. Marko,

M. El-Khouly, S. Fukuzumi, R. Kumar, J. Missert,

R. Yao, M. Sajjad, D. Chandra, A.A. Hussein,

K.A. Guru, R. Pandey

Roswell Park Comprehensive Cancer Center
BSP1-20 Single cell multiplex proteomic imaging on nephrectomy and renal tumor biopsy papillary renal cell carcinoma pairs

K.G. Sands, J. Hsieh, D. Cao, R. Figenshau,

A. Ramachandran, L. Yang, A. Sivaraman,

E. Kim, R. Venkatesh

Washington University School of Medicine,

St. Louis, United States

BSP1-21 Aquaporin 3 low expression in cancer tissue of urothelial carcinoma patients

V. Pavlov, K. Danilko, L.M. Kutliyarov,

A. Sivkov, R. Khairullina, E. Shut

Bashkir State Medical University

BSP1-22 Knockdown of COPS3 Inhibited the progress of Prostate Cancer through reducing phosphorylated p38 MAPK expression and impaired the Epithelial-Mesenchymal Transition process

Y. Hong, Z. Zhu, Q. Xu, X. Huang, F. Zhang,

L. An

Peking University People's Hospital
BSP2-01 Endoscopic early realignment or delay urethral reconstruction for traumatic urethral total disruption: a single center experience T Chang China Medical University Hospital

BSP2-02 Efficacy and safety of low power (50W) HoLEP in the management of LUTS/BPH:

Wrexham Maelor initial experience

O Obi-Njoku, C Bell, SL Moore, M Abdimalik, SF Hughes, I Shergill

Member Royal College of Surgeons England

BSP2-03 Is there a role for minimally invasive pyeloplasty in children less than 20kg?

S Palaniappan, A Ganpule, A Singh, R Sabnis, M Desai

Muljibhai Patel Urological Hospital

BSP2-04 Magnetic Stimulation Therapy for Urinary Incontinence: a Meta-analysis Q He, K Xiao, D Luo, H Li, K Wang Institute of Laboratory of Reconstructive Urology, West China Hospital, Sichuan University, Chengdu, Sichuan, P.R.C

BSP2-05 Effect of Holmium Laser Enucleation of the Prostate (HoLEP) on Prostate-Specific Antigen (PSA) Kinetics for Prostate Cancer Patients Actively Managed by Observation A Ibrahim, A Zakaria, M Wissing, W Kassouf, M Aube, S Tanguay, S Carrier, M Elhilali, A Aprikian McGill University
BSP2-06 Is Detrusor Overactivity a predictor factor of Posterior Tibial Nerve Stimulation outcome in the treatment of Overactive Bladder?

W Alamari, B Malallah, A Alabbad, M Algahwari, M Aldokhi, O Sarhan, R Almousa

BSP2-07 Management of recurrent stricture after previous ureteral reconstruction:

Robot-assisted laparoscopic surgery versus open surgery

W Qing, S Wang

Tongji Hospital, Tongji Medical College, Huazhong University of Science and Technology

BSP2-08 Autological fat stromal-vascular fraction injection for male stressed urinary incontinence treatment

V Pavlov, S Maksimova

Bashkir State Medical University

BSP2-09 Epidemiologic Characteristics and Risk Factors in Patients with Ketamine-associated Lower Urinary Tract Symptoms Accompanied by Urinary Tract Infection:

A Cross-sectional Study

W Liu, W Wu, Y Wei, R Zhang, J Wu

Fujian Provincial Hospital

BSP2-10 Intravesical injections of platelet-rich plasma is effective and safe in treatment of interstitial cystitis refractory to conventional treatment-A prospective clinical trial

C Yesildal, A Albayrak, A Yavuzsan, M Ilgi,

N Turkmen, S Kirecci

Sisli Etfal Training and Research Hospital 
BSP2-11 How international is the International Prostate Symptom Score - a literature review of validated translations of the IPSS

M Yao, J Green, SS Folkard

BSP2-12 Diagnosis and management of false urethral anastomosis in cases of posterior urethral trauma S Jain, R Goel, D Bansal India Institute of Medical Sciences, New Delhi

BSP2-13 Posterior Tibial Never Stimulation (PTNS) in the treatment of voiding dysfunction. Single center experience

B. Malallah, W Alamari, A Alabbad,

M Algahwari, O Sarhan, R Almousa

BSP2-14 Ketamine-induced lower urinary tract symptoms: a study of International Prostate Symptom Score and Quality of Life

Y Wei, J Wu

Fujian Provincial Hospital

BSP2-15 Outcomes of robotic ureteral reimplantation in tertiary care centers:

a bi-institutional case series

SS Komaravolu, DD Eun, D Kim, PM Youssef, JC Deolankar, PD Kothari, Z Lee, M Billah, H Koster, M Ahmed, GG Lovallo, R Munver, MD Stifelman

Hackensack Meridian Department of Urology

BSP2-16 Comparison of perioperative and mid-term outcomes between thulium laser vaporization and vapoenucleation:

a propensity score analysis

S Nazzani, G Motta, O Blezien, P Acquati,

R Stubinski, C Yameogo, S Maruccia, S Casellato,

L Carmignani
BSP2-17 The Effect of Platelet-Rich Plasma Injection on Post-internal Urethrotomy Stricture Recurrence: A Prospective Study A Yavuzsan, A Albayrak, C Yesildal, M Ilgi, N Turkmen, S Kirecci

Health Sciences University, Sisli Hamidiye Etfal

Training and Research Hospital

Urology Department

BSP2-18 Beta-adrenoreceptor regulates the secretion of inflammatory cytokines by autophagy in human bladder smooth muscle cells under pathological hydrostatic pressure

G Chen, K Xiao, K Wang, H Li

Institute of Laboratory of Reconstructive Urology, West China Hospital, Sichuan University, Chengdu, Sichuan, P.R.C

BSP2-19 Hemorrhagic Cystitis due to Cytomegalovirus in a patient with AIDS

W Padayachee, S Sadhwani, S Doherty,

E Van Der Berg, A Botha

University of Kwa-Zulu Natal, Grey's Hospital

BSP2-20 Utilizing da Vinci ${ }^{\circledR}$ Robotic Surgical System for Ureteroneocystostomy

MW Salkini, D Riggs, B Jackson

West Virginia University

BSP2-21 Evaluation of spongiofibrosis of urethral stricture disease

A Farganov, V Pavlov, A Izmailov,

R Kazihinurov, R Safiullin

BSP2-22 Genito-Urinary Tuberculosis Revisited - 10

Years' Experience Of a Single Centre

P Mylarappa, K Dokania, A Kulkarni

Ramaiah Medical College and Hospital
MP01-01 Impact of a National Comprehensive Cancer Network Compliant Multidisciplinary Consortium on Treatment Decisions in Patients with Localized Prostate Cancer

AA Hussein, AS Elsayed, NA Aldhaam, Z Lone, S Hall, S Kamal, K Carroci, E Kauffman, B Sullivan, Q Li, T Schwaab, M Kuettel, KA Guru Roswell Park Comprehensive Cancer Center

MP01-02 Cryotherapy for prostate cancer: oncological and functional outcomes

A Territo, D Vanacore, R Boissier, F Sanguedolce, F Regis, A Gallioli, J Gaya, J palou, A Breda Fundació Puigvert. Autonomous University of Barcelona. Barcelona. Spain.

MP01-03 Study on the difference between clinical stage and pathological stage of $\mathrm{T} 1$ renal cell carcinoma patients

M Li, C Li, G Bao, Z Gao
MP01-04 T1-substaging after Thulium fiber laser en bloc resection of bladder tumors

A Dymov, L Severgina, D Kislyakov, D Enikeev, N Sorokin, A Proskura, L Rapoport

Institute for Urology and Reproductive Health, Sechenov University

MP01-05 Is post voiding residual volume related to toxicity of intravesical bacillus Calmette-guelin for urothelial non-muscle invasive bladder cancer?

K Tochigi, K Hattori, N Sassa, M Kato,

T Yamamoto, M Gotoh

Nagoya University Hospital

MP01-06 Percutaneous nephrostomy in obstructing pelvic malignancy: does it facilitate further oncological treatment and what is the associated morbidity? SS Folkard, S Banerjee, J Reason, E Psallidas, J Green 
MP01-07 Neoadjuvant Chemotherapy Before Radical Prostatectomy For Locally Advanced Prostate Cancer: A Systematic Review And Meta-Analysis

T Lin, X Yang, Q Wei

West China Hospital, Sichuan University

MP01-08 Experience of laser treatment for contralateral recurrence of upper urinary tract transitional cell carcinoma after radical resection M Li, C Li, Bao, Z Gao

MP01-09 The effective observation of the non muscle-invasive bladder cancer with the transurethral thulium laser radical resection of bladder tumor

Z Xin, C Hanmin, X Kunlin, Z Xiaofeng, X Rihai, Z Guoxi

MP01-10 Patient-Reported Outcomes Via Online Questionnaires: Post-Radical Prostatectomy Quality Improvement and Outcome Assessment KJ See, J Tran, LM Huynh, TE Ahlering UC Irvine Health

MP01-11 Preoperative prognostic value of trigone involvement of muscle invasive bladder cancer

K Tochigi, N Sassa, S Inoue, Y Funahashi, M Kato, T Yamamoto, M Gotoh

Department of Urology, Nagoya University Graduate School of Medicine

MP01-12 Evidence for the Integration of Total and Free Testosterone in Management of Prostate Cancer

M Towe, LM Huynh, F el Khatib, MM Osman, F Yafi, TE Ahlering

UC Irvine Health

MP01-13 Perioperative and functional outcomes of RARP in Elderly Men> $=80$

M Shahait, RW Dobbs, J Kim, N Eldred, V Patel, DI Lee

University of Pennsylvania

MP01-14 Creation of the Harmonized Arabic version of the Expanded Prostate Index Composite for Clinical Practice (AREPIC-PC)

G Barayan, M Awad, M Plante, R Grunert

University of Vermont/ Umm AlQura

University
MP01-15 Thulium Laser en-bloc resection of bladder Tumor (THUEB-BT): ESUT study to compare laser and electrical en-bloc transurethral resection of bladder tumor

G Bozzini, U Besana, G Pini, AS Gozen, E Liatsikos, C Buizza, M Sighinolfi, B Rocco ASST Valle Olona - Busto Arsizio VA

MP01-16 Active surveillance - Is it feasible for intermediate risk localized prostate cancer? S Mukherjee, I Promponas, J Abbaraju, S Madaan Dartford and Gravesham NHS Trust

MP01-17 Nurse led one stop hematuria clinic: Outcomes from 1581 patients

MA Hamdoon, MO Breish, D Whiting, A Elliott, D Pearce, S Madaan

Dartford and Gravesham NHS Trust

MP01-18 The Role of Urine and Washing Cytology during Primary Transurethral Resection of Bladder Tumors (TURBT) T Ozimek, MC Hupe, JP Struck, M Hennig, N Gilbert, J Cordes, AS Merseburger, MW Kramer Universitätsklinikum Schleswig-Holstein (Campus Lübeck)

MP01-19 Do all patients with suspicious prostate cancer need Multiparametric Magnetic Resonance Imaging before prostate biopsy?

S Anacleto, J Alberto, E Dias, B Caló, P Mota, N Morais, J Torres, P Passos, A Cordeiro, U Falagario, E Lima

MP01-20 Predictors of Failure of Active Surveillance in Patients with Clinically Localized Prostate Cancer NA Aldhaam, AS Elsayed, AA Hussein, W Ji, G James, o Abdel Razzak, I Saad, M Abdelhakim, E Kauffman, J Mohler, KA Guru

Roswell Park Comprehensive Cancer Center

MP01-21 The clinical differences between patients of pheochromocytoma with hypertension and normotension

Z Liu, L Zhou, H Li, K Wang, Y Zhu

Sichuan University

MP01-22 Evaluation of the actual tumor volume evaluated from surgical specimens following radical prostatectomy and its clinical impact

on the prognosis of patients with prostate cancer O Kwon, H Lee

Hallym University Kangnam Sacred Heart Hospital
MP02-01 Avoiding Unnecessary MRI and Biopsies: NPV and PPV of MRI according to PSA Density, 4Kscore and Risk Calculators

U Falagario, A Martini, E Wajswol, P Treacy,

P Ratnani, R weil, i Jambor, SC Lewis,

L Cormio, G Carrieri, AR Rastinehad, P Wiklund,

A Tewari
MP02-02 Clinical Safety and Diagnostic Efficacy of Multiparametric Magnetic Resonance Imaging and Ultrasound (mpMRI-US) Fusion Targeted and systematic Transperineal Prostate Biopsy: The preliminary Experience of First 100 Cases

S Mehmood, A Alwehaibi, KI Alothman King Faisal Specialist Hospital \& Research Center Riyadh 
MP02-03 Contemporary Practice Patterns in Treatment of Malignant Extrinsic Ureteral Obstruction in the Nationwide Inpatient Sample

C Haas, C Sayegh, J Pak, O Shah, E Hyams

Columbia University

MP02-04 Prevalence of risk factors in patients presenting for hematuria evaluation - A prospective patient reported data analysis \& outcomes

G Kanda Swamy, B James, B Rai, M Jefferies,

P Bose

Swansea Bay University Health Board

MP02-05 Effect of Travel Distance to Treatment Facility on Presenting Stage and Overall Survival in Kidney Cancer: an NCDB Analysis

A Kaldany, KE Okhawere, PC Yong, KK Badani Icahn School of Medicine at Mount Sinai

MP02-06 Magnetic Resonance Imaging-Targeted 3D Transrectal Ultrasound Guided Fusion Biopsy of Prostate Cancer Suspected Patients: Results of 292 patients S Yilmaz, H Özdemir, E Gazel, E Kaya, S Yalcin, M Ezer, A Öner, M Yörubulut, L Tunc Ankara Acibadem Hospital

MP02-07 Transurethral resection in one piece (TURBO) for bladder tumor assisted by photodynamic diagnostic cystoscopy with 5-ALA

Y Yamauchi

Nagoya Daini Red Cross Hospital

MP02-08 Risk factor and survival of female-predominant upper urinary tract urothelial carcinoma in Taiwan

$\mathrm{Y} \mathrm{Wu}, \mathrm{H}$ Luo

Department of Urology, Kaohsiung Chang Gung Memorial Hospital, Kaohsiung, Taiwan

MP02-09 Operator Variant Outcomes in Prostate Cancer Detection Rates: Doctor versus Nurse Operator MM Quraishi, M Sheikh

Department of Urology. Kent \& Canterbury Hospital. East Kent Hospitals University NHS Foundation Trust

MP02-10 The number of metabolic features as a significant prognostic factor in patients with metastatic renal cell carcinoma

O Kwon, H Lee, J Yu

Sanggye Paik Hospital/Inje University

MP02-11 Impact of Gonadotropin-releasing Hormone Agonist on Cardiovascular Disease and Diabetes Mellitus: A Nationwide Population-based Cohort Study

W Bang, M Shim, C Oh, Y Lee, J Cho

Hallym Sacred Heart Hospital
MP02-12 The utility and safety of diagnostic ureteroscopy in the diagnosis of upper tract urothelial carcinoma (UTUC): the experience of a high volume center

T Birks, N Pindoria, C Cumby, A Goyal, D Allen,

R Kucheria, P Singh, L Ajayi, G Ellis

Royal Free Hospital, London

MP02-13 Outcomes of imperative conservative treatment of upper tract urothelial cancer S PROIETTI, M Marchioni, B Eisner, ME Rodriguez Socarras, G Saitta, C D' Orta, P Bellinzoni, L Schips, F Gaboardi, G Giusti Department of Urology, Ospedale San Raffaele-Turro, Milan, Italy

MP02-14 Iatrogenic Kaposi Sarcoma of the Bladder in the Post-Renal Transplant Patient

P Mistry, M Edelson, I Efthimiou, V Vasudevan, C Tabib, D Mikhail, Z Okeke Arthur Smith Institute for Urology

MP02-15 Comparison of Intra- and Post- Operative Outcomes of Straight Laparoscopic, Hand-Assisted Laparoscopic, and Robotic-Assisted Laparoscopic Partial Nephrectomies for Small Renal Masses at a Single Institution

M Apoj, L Wang, W Shaun, M Katz, D Wang

Boston Medical Center Department of Urology

MP02-16 Angiomyolipoma and its follow up in a tertiary referral center

B Vissamsetti, t Bhambra, S Biyani, A Khan FRCS

MP02-17 Comparison of partial nephrectomy and percutaneous radiofrequency ablation for small renal mass in the manner of preoperative aspects and dimensions used for an anatomical (PADUA) classification W Kuo, C Yu, J Lin

Kaohsiung Veteran General Hospital

MP02-18 Transurethral laser ablation of low-grade bladder: a service evaluation, financial implications and importance of a comprehensive business case

AJ Ridgway, K Charitopoulos West Middlesex University Hospital

MP02-19 Is there a difference in long term renal functional outcome between benign and malignant renal tumors managed with robotic partial nephrectomy?

BD Dayal, KE Okhawere, AA Elbakry, A Beksac, K Meilika, J Sfakianos, R Mehrazin, R Abaza, DD Eun, A Bhandari, A Hemal, J Porter,

KK Badani

Icahn School of Medicine at Mount Sinai 
MP02-20 Study of the Prostatic Urethral Lift in the Real-World Setting

NJ Barber, G Eure, S Gange, PJ Walter, A Khan,

C Chabert, T Mueller, P Cozzi, M Patel,

S Freedman, P Chin, S Ochs, A Hirsh, M Trotter,

D Grier

Frimley Park Hospital

MP02-21 Upper tract Ultrasound scan is an adequate screening modality for patients presenting with Visible Hematuria - retrospective cohort study of a prospective database

G Kanda Swamy, B Rai, R David, N Gill, P Bose

Swansea Bay University Health Board
MP02-22 Outcome of Adjuvant Versus Salvage Androgen

Deprivation Therapy Following Radical

Prostatectomy for Prostate Cancer Patients with Adverse Pathologic Feature(s)

or with Positive Regional Lymph

Node(s) Metastasis

P Boonyapanichskul, V Woranisarakul, S Leewansangtong, C Nualyong, S Srinualnad, T Taweemonkongsap
MP03-01 Surgeons musculoskeletal problems following endourologic prostatectomy: an ergonomic survey study of the endourology society members M Omar, M Sultan, E El Sherif, M Abdallah, M Monga

Menoufia University

MP03-02 High Incidence of Urinary Tract Infections after Greenlight Laser Vaporization of Prostate: A Risk Factor Analysis of 665 Patients

J Motzer, J Roth, A Widmer, H Seifert,

C Wetterauer

Department of Urology, University Hospital

Basel

MP03-03 Retrospective Analysis of Urethral Stenosis Following Transurethral Prostate Resection: Preventing Effect of the Using Low Molecular Weight Heparin on Development of Urethral Stenosis

M Ilgi, A Albayrak, C Yesildal, A Yavuzsan, N Turkmen, S Kirecci

MP03-04 Energy impact on voiding symptoms after thulium enucleation of prostate:

A large multi institutional analysis

S Nazzani, G Motta, O Blezien, F Molinari, P Acquati, R Stubinski, S Casellato, C Yameogo, S Maruccia, L Carmignani

MP03-05 The New 4D and Standard Prostatic Urethral Lifting (UroLift) Techniques:

Day Case, Effective, No Sexual Dysfunction M Ravindraanandan, W Mahmalji, Y Phan Hereford County Hospital

MP03-06 Vaporesection of the prostate with Tm : YAG laser : The Oyster technique P Kallidonis, D Kotsiris, C Adamou, P Ntasiotis, K Pagonis, I Kyriazis, E Liatsikos, A Al Aown Armed Forces Hospital Southern Region

MP03-07 Two-Year Outcomes after Aquablation Compared to TURP:

Efficacy and Ejaculatory

Improvements Sustained

PJ Gilling, T Bach, C Roehrborn

Asklepios Hospital Harburg
MP03-08 Functional outcomes post Holmium Laser Enucleation of the Prostate (HoLEP) for patients with huge prostates $(>150 \mathrm{cc})$

A Oluwole-Ojo, M Hadjipavlou, E Di Benedetto, A Sujenthiran, R Catterwell, B Namdarian, O Elhage, R Popert, B Challacombe NHS

MP03-09 Prostatic Urethral Lift in Subjects with Acute Urinary Retention (PULSAR)

M Rochester, NJ Barber, T Page, R Kavia, $\mathrm{N}$ Thiruchelvam, O Kayes

Norfolk and Norwich University Hospital

MP03-10 Does targeted bladder neck cautery prevent bleeding events? Learning after 700+ Aquablation procedures using different hemostasis methods in prostates $20 \mathrm{ml}$ to $280 \mathrm{ml}$ in size

D Elterman, T Bach, E Rijo Mora, V Misrai, P Anderson, K Zorn, N Bhojani, B Chughtai Asklepios Hospital Harburg

MP03-11 Early clinical outcomes of transurethral enucleation with bipolar compared with transurethral resection in saline for Benign Prostatic Hyperplasia treatment

Y Endo, J Akatsuka, K Takahashi, N Hodotsuka, H Mikami, K Obayashi, S Nomura, M Yanagi, H Takeda, T Hayashi, Y Toyama, T Hamasaki, H Shimizu, Y Kondo Nippon Medical School

MP03-12 Thulium laser enucleation (thULEP) versus Holmium laser enucleation of the prostate: a two institution trial to compare intra and early postoperative outcomes.

G Bozzini, U Besana, J Roche, P Broggini, G Pini, C Buizza, M Sighinolfi, B Rocco ASST Valle Olona - Busto Arsizio VA

MP03-13 Safety and efficacy of Holmium laser enucleation of the prostate (HoLEP) in patients with previous transperineal biopsy:

outcomes from a multi-center case-control study C Bell, SL Moore, O Obi-Njoku, A Gill, SF Hughes, A Saleemi, G Ellis, F Khan, IS Shergill North Wales Clinical Research Centre 
MP03-14 Efficacy and safety of prostate artery embolization for benign prostatic hyperplasia in patients with catheter-dependent urinary retention and lower urinary tract symptoms M Fadel, R Das, B Hawthorn, S Katmawi-Sabbagh St George's Hospital

MP03-15 Withdrawn

MP03-16 Safety of holmium laser enucleation of prostate (holep) in patients on antiplatelet or anticoagulation medications

K Ramaswami, P Bhirud, R Raveendran, H Pothiyedath

Metromed Institute Of Advanced Urology

And Renal Translplant

MP03-17 Surgical outcome of huge benign prostatic hyperplasia treated by Holmium laser enucleation of the prostate using en bloc enucleation technique with lateral median approach

Y Shibata, S Arai, A Kanayama, D Oka, A Otsu, M Aoki, H Nakayama, Y Miyazawa, T Syuto, M Nomura, Y Sekine, H Koike, H Matsui, K Suzuki

MP03-18 En-Bloc HoLEP vs En-Bloc GreenLEP: comparing intra and post-operative outcomes for En-bloc enucleation technique using two kinds of laser to treat patients affected by benign prostatic enlargement (BPE)

G Saitta, ME Rodriguez Socarras, E Rijo, J Reinoso Elbers, J Fernandez Del Alamo, L Llanes

Gonzalez, J Gomez Rivas, F Gómez Sancha

Department of Urology, Ospedale San Raffaele-

Turro, Milan, Italy
MP03-19 Improved Efficiency of Holmium Laser Enucleation of the Prostate Utilizing a Modulated Pulse Platform

BB Whiles, A Martin, A Brevik, K Glavin,

KL Thurmon

University of Kansas Medical Center

MP03-20 ProstaCare:

Water electrolysis system for the treatment of benign prostate hyperplasia

WJ Chua, Y Lim, PJ Gilling, HY Tiong, A Sim, $\mathrm{H}$ Ho

National University Hospital, Department of Urology

MP03-21 Online Interest in Minimally Invasive Outpatient Surgical Treatment for BPH Using Google Trends

S Merheb, D Wang, S Wason

Boston University School of Medicine

MP03-22 Does the complexity affect the outcome of Top-Down Holmium Laser Enucleation of prostate (Top-Down HoLEP)?

A Hodhod, FA Oquendo Aponte, W Shahrour, A Kotb, O Prowse, H Elmansy

Thunder Bay Regional Health Science Centre
MP04-01 Mutational analysis of AGXT gene in Chinese patients with primary hyperoxaluria type 1

W Wang, J Li, Y Tian

Beijing Friendship Hospital, Capital

Medical University

MP04-02 Dual energy CT value in urolithiasis S El Rai

Al Zahra Hospital Sharjah

MP04-03 Inter- and Intra-observer Agreement in Measuring Urolithiasis Density on Non-Enhanced Computed Tomography GW Disselhorst, JJ Reimerink, RK van der Vijgh, R Sandkuyl, PC Kauer, R Reinhard, M Tjeenk Willink, S Jensch, EP van Haarst

OLVG Hospital, Amsterdam

MP04-04 Genome Wide Polygenic Score and Urinary Tract Stone Diagnosis in a Multiethnic Cohort I Paranjpe, M Paranjpe, S Jaladanki, A Kapoor, L Chan, C He, S Coca, R Do, M Gupta, G Nadkarni, A Zampini

MP04-05 Withdrawn
MP04-06 In vitro comparison of ablation rates between SuperPulsed Thulium Fiber Laser and Ho:YAG Laser for endocorporeal lithotripsy F Panthier, S Doizi, L Berthe, O Traxer Service d'Urologie, Hopital Tenon, APHP

MP04-07 High concentration of calcium promotes the expression of Matrix Metallopeptidase-9 through reaction oxygen species dependent NFкB signaling pathway in nephrolithiasis formation Y Wu, Y Lu, H Hu, b Qin, S Wang, Q Wang Tongji Hospital

MP04-08 Comparison of Teflon Durability Between Guidewires in a Benchtop PCNL Model M Hajiha, A Amasyali, J Groegler, J Smith, R Moghisi, A Goyne, M Shah, M Alsyouf, P Stokes, D Baldwin, J Maldonado, D Baldwin

MP04-09 Comparison of the fissures and fragments produced with $150 \mu \mathrm{m}$ and $272 \mu \mathrm{m}$ laser fibers with Superpulsed Thulium Fiber Laser: an in vitro study

F Panthier, S Doizi, L Berthe, O Traxer Service d'Urologie, Hospital Tenon, APHP 
MP04-10 The long-term effect of short periods of unilateral ureteric obstruction on the renal function: Does it deteriorate despite the initial apparent full recovery?

F Hammad, L Lubbad, S Al-Salam, J Yasin

United Arab Emirates University and

Tawam Hospital

MP04-11 Are There Clinical and Metabolic Characteristics that Correlate with the Formation of Calcium Oxalate Monohydrate or Dihydrate Stones?

N Stern, J Bjazevic, L Nott, H Razvi

Western University

MP04-12 Wielding a Stone Analysis to its Full Potential JE Brinkman, T Large, CU Nottingham, J Williams, A Krambeck

MP04-13 Development of a simple and practical nomogram for predicting stone-free rate after flexible ureteroscopy or percutaneous nephrolithotomy for solitary medium sized renal stones in adults

L Bevilacqua, R Milandri, S Ciarlariello, C Del Prete, C Di Pietro, F fidanza, A Elsherbiny, A Eissa, M Sighinolfi, S Puliatti, G Bianchi, B Rocco, S Micali Department of Surgical, Medical and Dental Morphological Sciences related to Transplant, Oncology and Regenerative Medicine - Section of Urology, University of Modena and Reggio Emilia, Modena, Italy

MP04-14 Novel technique for residual stones removal: second-look flexible nephroscopy in gas (CO2) medium

D Korolev, L Rapoport, D Tsarichenko, D Enikeev, M Enikeev, P Glybochko Institute for Urology and Reproductive Health, Sechenov University

MP04-15 What factors drive staghorn versus non-staghorn pattern growth in patients with metabolic stones? JS Winoker, JN Bamberger, RA Chandhoke, A Kapoor, H Kwun, W Atallah, M Gupta The James Buchanan Brady Urological Institute, Johns Hopkins University School of Medicine
MP04-16 The Effects of a High Sodium Diet on Lithogenesis in an Experimental Rat Model of Renal Calcium Oxalate Stone Formation Y Hong, Q Xu, X Huang, L An, Z Zhang Peking University People's Hospital

MP04-17 Defining the Relationship between Urinary Infection and Calcium Urolithiasis: A Potential Novel Role or Osteopontin and Zinc Transport

J Bjazevic, K Al, J Gorla, H Razvi, J Burton

Western University

MP04-18 Association of Obesity with Increased

Endogenous Oxalate Synthesis

K Wood, C Boyd, R Holmes, J Knight, B Gower, D Assimos

University of Alabama at Birmingham

MP04-19 Clinical Risk Factors that Predict the Known Lithogenic Metabolic Diagnoses in Patients with Kidney Stones

BO Manzo, JD Cabrera, JE Torres, FC Vicentini, E Emiliani, B Eisner, H Sánchez

Hospital Regional de Alta Especialidad del Bajío

MP04-20 Indwelling ureteral stents induce ureteral aperistalsis, injury and fibrosis

K Scotland, L Wang, BH Chew, C Seow, D Lange University of British Columbia

MP04-21 Critical role of short chain fatty acids in the prevention of renal calcium oxalate stones K Wang, Y Liu, L Cheng, Q Jiang, L Zhou, Y Li, Q Sun, $\mathrm{H} \mathrm{Li}$

Institute of Laboratory of Reconstructive Urology, West China Hospital, Sichuan University,

Chengdu, Sichuan, P.R.C

MP04-22 The Impact of Laser Pulse Type on Temperature Changes During Ureteroscopic Laser Activation B Winship, RS Terry, D Wollin, K Boydston, E Carlos, P Whelan, D Ho, P Zhong, G Preminger, M Lipkin

Duke Urology
MP05-01 Integral monitoring system for patients with ureteral stents

C Solano, A Chica, E Castellanos, A Contreras,

C Duarte, J Restrepo, J Meneses, P Villegas,

N Fernandez, J Aristizabal, N Tarazona,

A Vasquez

\section{MP05-02 Withdrawn}

MP05-03 Patient Reported Outcome Measures (PROMs) in patients undergoing flexible ureteroscopy and shockwave lithotripsy for renal stones B Starmer, S Tolofari, J Broome, H Garrod, K Agarwal, M Iskander, S Javed, P Kelly, A Samsudin, H Lazarowicz, R Calvert
MP05-04 US versus CT urography in the assessment of urinary tract stone size - can we trust it? H Tran, H Pan, H Yao, P McCahy Peninsula Health

MP05-05 Renal Forniceal Rupture in the Setting of Obstructing Ureteral Stones (FROU): An Analysis of Stone Characterization and Urologic Intervention Pattern S Setia, P Massie, M Epsten, A Sharma, L Fogg, AK Chow Rush University Medical Center 
MP05-06 Immediate and early postoperative complications after ureteroscopy and Ho:YAG laser lithotripsy for urinary stones: real-life findings from a single center series.

L Villa, G Fallara, M Menean, A Briganti,

A Salonia, F Montorsi

Division of Experimental Oncology/Unit of Urology; URI; IRCCS Ospedale San Raffaele, Milan, Italy

MP05-07 Outcomes of patients who have undergone percutaneous bladder stone removal using a laparoscopic entrapment bag B Gallante, JN Bamberger, RA Chandhoke, W Atallah, E Kaplan-Marans, M Gupta, AM Zampini

Mount Sinai

MP05-08 Utilizing da Vinci ${ }^{\circledR}$ Robotic Surgical System to Treat Challenging Urinary Stone C Crigger, MW Salkini

MP05-09 Does long-term relative renal function improve after intervention for chronic ureteric obstruction? - a case review of 268 adults Z Low, V Arumuham, S Allen, L Davis, J Bomanji, R Smith Institute of Urology, University College London Hospitals NHS Foundation Trust

MP05-10 To TURP or Not to TURP: Does it Matter When It Comes to Men with Concomitant BPE and Bladder Calculi? JN Bamberger, J Chong, A Katims, C Brown, B Gallante, A Kapoor, H Kwun, J Thai, W Atallah, RA Chandhoke, M Gupta, AM Zampini Mount Sinai

MP05-11 Stage II percutaneous nephrolithotomy combined retrograde intrarenal surgery under lateral lithotomy position for residual renal stones after percutaneous nephrolithotomy $\mathrm{X}$ Lin Department of Urology Hong Kong University Shenzhen Hospital

MP05-12 Outcome of Mini PCNL for large renal stones in 72 patients with spinal deformities DA Rajyaguru

MP05-13 Early Surgical Intervention for Symptomatic Renal and Ureteral Stones Reduces Narcotic Requirement Relative to Medical Expulsive Therapy C Valadon, CU Nottingham, T Large, A Krambeck University of Louisville School of Medicine

MP05-14 The Impact of the Number of Lifetime Stone Events on Quality of Life:

Results From the North American Stone Quality of Life Consortium

S Tapiero, L Limfueco, K Osann, Z Okhunov, RM Patel, JA Antonelli, NM Streeper,

KL Penniston, SY Nakada, S Sivalingam,

DP Viprakasit, T Chi, VG Bird, VM Pais, RL Sur, $\mathrm{S}$ Andonian, NE Canvasser, BH Chew,

J Landman, RV Clayman

University of California, Irvine
MP05-15 Justifying CT scans for sub $5 \mathrm{~mm}$ renal stones identified on ultrasound scans.

MM Quraishi, B Osman, OA Ramadan, I Ahmed, E Latif, J Dorrell, A Simoes, G Papadopoulos, J Hale, N Shrotri

Department of Urology. Kent \& Canterbury Hospital. East Kent Hospitals University NHS Foundation Trust

MP05-16 Endourological management (PCNL, URS, SWL) of stones in solitary kidney - A systematic review from European Association of Urologists (EAU) Young Academic Urologists (YAU) and Uro-Technology (ESUT) groups

T Reeves, A Pietropaolo, T Tailly, M Oezsoy,

P Kallidonis, OM Aboumarzouk, A Skolarikos,

E Liatsikos, BK Somani

MP05-17 Simultaneous ureteroscopic and percutaneous nephro-lithotripsy for management of combined ureteric and renal stones in the Galdakao modified supine Valdivia position: Safety and outcomes in a single-center experience

A Ali, L Abbas, T El-Leithy

Theodor Bilharz Research Institute

MP05-18 Correlation between peripheral blood inflammatory indexes and Systemic Inflammatory Response Syndrome in patients received Percutaneous Nephrolithotomy

L An, Q Xu, X Huang

Peking University Applied Lithotripsy

Institute/Peking University People's Hospital

Urology and Lithotripsy Center

MP05-19 Risk factors for resistance to preservation treatment of calculus pyelonephritis

N Hodotsuka, T Hayashi, Y Endo, T Ueda, J Akatsuka, H Takeda, S Osawa, G Kimura, Y Kondo

Nippon Medical School Hospital, department of Urology

MP05-20 The impact of phone counseling on urinary stone prevention

A Nevo, A Navaratnam, MT Keddis,

MR Humphreys

Mayo Clinic

MP05-21 Value of urinary pH in assessment of high-risk stone patients: Outcomes from a dedicated metabolic stone clinic

M Ramachandra, M Saraogi, A Pietropaolo,

BK Somani, P Cook

University Hospital Southampton

MP05-22 How much energy is needed to ablate one cubic millimeter of stone volume during flexible ureteroscopy using a low-power laser device? Results from a proof-of-principle clinical study

E Ventimiglia, S Doizi, F Panthier, O Traxer

Division of Experimental Oncology/Unit of

Urology; URI; IRCCS Ospedale San Raffaele 
V01-01 Step by step ThuLEP (Thulium laser enucleation of prostate): an Indian multicentric experience J Jagtap, S Veer, G Agrawal, K Jadhav, P Rao, S Mistry

V01-02 Endoscopic management of bladder mesh erosion following previous incontinence surgery A Pietropaolo, BK Somani, M Ramachandra

V01-03 New Advancements of Moses for HoLEP: Transforming Enucleation into a Day Case CU Nottingham, T Large, C Stephens, A Krambeck

V01-04 Aquablation of the prostate: a guide through a novel robotic procedure for $\mathrm{BPH}$ A Hajj, J El-Asmar, N Abdallah, G Bustros, M Bulbul

V01-05 Moses Laser Enucleation of the Prostate: Early Experience MW Pickhardt, T Large, CU Nottingham, A Krambeck

V01-06 Staying Out of Incontinence Zone: Our technique of Ultra Low Power Precision HoLEP S Prabhakar, Y KK
V01-07 Thulium Laser Enucleation of Prostate In A Patient Who Has 170 cc Prostate Volume HC Aybal, TB Aydogan, E Gazel, E Kaya, $S$ Yalcin, S Yilmaz, L Tunc

V01-08 Different Approaches For Robotic Simple Prostatectomy For Benign Prostatic Hyperplasia BD Dayal, K Meilika, A Beksac, KE Okhawere, AA Elbakry, KK Badani

V01-09 ProTouch Laser Enucleation of the Prostate: A retrospective analysis of the first 77 cases M Emmerling, S Wason, W Pate Boston Medical Center Department of Urology

V01-10 HoLEP with Moses technology: MoLEP Y Hussein M.I., S Corti, F Ceresoli, R Milesi, I Vavassori

V01-11 4D UroLift ${ }^{\circledR}$ approach to Anatomically challenging Benign Prostatic Hyperplasia with middle lobe KL Ng, S Giona, NJ Barber

V01-12 Colposacropexy laparoscopic: Technique and functional Results C Conde Redondo, F Castroviejo Royo, A Alonso Villalba, S Martin Martin, J Torrecilla, J Diez, J Cortiñas González
V02-01 Indocyanine green (ICG) assisted video endoscopic inguinal lymphadenectomy (VEIL) for penile carcinoma C Gurbani, K Chen, A Sim

V02-02 Conservative treatment of a large UTUC in solitary kidney

L Defidio, M De Dominicis, M Antonucci, N Russo

V02-03 Ultrasound Visibility of Instruments in Zero-exposure Retrograde Intrarenal Surgery

D Takahashi, Y Kawanishi, K Izumi, M Yamanama, T Tsujioka, K Seto, Y Atagi, K Hirota, H Bando

V02-04 Endoscopic Management of Giant Ureteral Fibroepithelial Polyp With Laser Ablation B Onal, Y 1lki, e bulbul, B Ercili, S Citgez

V02-05 Laser Endopyelotomy: where are we in 2019? G Celentano, H Abboudi, F Schoofs, S Choong

V02-06 Ureteroscopic management of parapelvical renal cysts.

A Martov, N Baykov, D Ergakov

V02-07 Transperineal prostate biopsy under local anesthetic in the outpatient setting with a reusable needle guide

A Patel, T Poloskey, C Rogers
V02-08 Percutaneous Ablation of Upper Renal Urothelial Carcinoma with Thulium laser

A Breda, L Mosquera Seoane, D Vanacore, A Territo, F Regis, F Sanguedolce, O Mayordomo Ferrer, A Gallioli, A Mercadé, R Boissier, J Palou

V02-09 Robot-assisted Retroperitoneal Lymph Node Dissection in Post-chemotherapy Residual Mass Adherent to Left Kidney Vessels O Aytac, A Garayev, F Atuğ

V02-10 MRI-based 3D printing of prostate for clinical-radiological-pathological correlation T Chan, C Kan, K Lai, L Lam, T Chung, Y Stone, $\mathrm{N}$ Chia, W Au

V02-11 Office-Based, Ultrasound-Guided Renal Mass Biopsy: Technique and Results

FA Jefferson, Z Okhunov, J Sung, C Cottone, RM Patel, J Landman

V02-12 Robotized Laparoscopy demonstration in various Urological scenarios

K Ramaswami, P Bhirud, R Raveendran, H Pothiyedath

V02-13 Application of ureteroscopy combined with laparoscopy in the treatment of ureteral septal injury after cervical cancer operation J Huang, X Zhan, G Hu, H Zhang, Y Xu 
V03-01 Intrafascial radical prostatectomy by robotic laparoscopic assisted surgery with complete Neurovascular bundle and Urethra preservation

F Adawi, M Alhamad, A Alkhalili, A Demey, $\mathrm{X}$ Taccoen, $\mathrm{P}$ Loock, F Ozone

V03-02 Robotic-Assisted Radical Prostatectomy with minimal apical dissection and lateral prostatic fascia preservation: an evolution of our conventional technique.

M Covas Moschovas, O Fikret, S Bhat, T Rogers, V Patel

V03-03 Less is More: Modified Robotic Prostatectomy Techniques to Decrease Operative Time and Improve Outcomes

NH Patel, M Iorga, M Stern, M Zhang, R Yau, M Esposito, G Lovallo, M Ahmed

V03-04 An analysis of the trans-umbilical approach of reduced port surgery in laparoscopic radical prostatectomy

K Araki, K Ohtsuka, K Hou, T Suyama, S Kojima, Y Naya, H Masuda, K Mikami

V03-05 Modified Dissection Technique of the Urethra provided Improved Early Urinary Continence after Robot-Assisted Radical Prostatectomy R Munver, M Degen, JF Tsui, B Desroches

V03-06 Robotic assisted radical prostatectomy in a patient with complete ureteral duplicity with ectopic prostate drainage

LM Calazans, NJ Barretto, N Barretto, T Mourão, R Oliveira
V03-07 Salvage robot-assisted laparoscopic prostatectomy: tips and tricks for challenges due to different primary treatments

O Fikret, M Covas Moschovas, S Bhat, T Rogers, V Patel

V03-08 Transvesical Robot Assisted Radical Prostatectomy G Wang, X Zhou, B Fu, C Zhang

V03-09 Surgical Factors Improving Continence in Robotic Assisted Laparoscopic Radical Prostatectomy, Real Surgical Experience In Ramathibodi Hospital, A Case Report

W Ratanapornsompong, P Sungkum,

S Pacharatakul, C Leenanupan,

W Kongcharoensombat

V03-10 Athermal and Clip-free Nerve-Sparing Technique in Robotic Radical Prostatectomy: Feasibility Study of Autologous Fibrin Glue as Hemostatic Agent

O Aytac, A Garayev, F Atuğ

V03-11 Design and Validation of a full-immersion robot assisted radical prostatectomy simulation using three-dimensional printing and hydrogel molding technology

A Ghazi, M Witthaus, R Melnyk, G Wu, TP Frye, H Rashid, JV Joseph

V03-12 Maximum conservation in laparoscopic prostatectomy step by step

C Conde Redondo, F Castroviejo Royo,

A Alonso Villalba, J Torrecilla, S Martín Martín, F Lara Pérez, R Cortiñas Gonzalez
MP06-01 Construction and Assessment of an innovative indigenous TURP Simulator

A Rawandale-Patil, LG Patni, PA Rawandale,

A Hindustanwala, A Gade, S Kaushik

Tejnaksh Hospital's Institute of Urology

MP06-02 Effective Utilization of Legos to Create a Ureteroscopic Trainer for Urology Residents SL Best, KA Richards, BJ Lewis, DR Gralnek, CJ Kozler, TG Leffler, BL Miller, DH Williams, SY Nakada, BV Le

University of Wisconsin School of Medicine \& Public Health

MP06-03 Immersive Virtual Reality for Percutaneous Nephrolithotomy Training

E Parkhomenko, S Resad, D Wang, S Wason Boston Medical Center

MP06-04 A vascularized peritumor area model based on quantitative three-dimensional reconstruction facilitates precise partial nephrectomy: FACS scoring criteria and four defining steps for surgery planning

W Xiong, H Liu, Y Du, J Yang, P Guo, J Chen, J Lyu, X Huang, K Dou, Q Ran
MP06-05 Machine Learning using a Multi-task

Convolutional Neural Networks Can Accurately Provide Robotic Skills Assessment

JC Gahan, RL Steinberg, A Garbens, X Qu,

BA Johnson, E Larson

University of Texas Southwestern

MP06-06 Training in urolithiasis procedures using simulation-based models:

A comparative evaluation

U Baig, A Aydin, A Al-Jabir, N Raison, T Abe, J Ramón Cansino Alcaide, Y Farahat, K Sarica, P Dasgupta, K Ahmed

MRC Centre for Transplantation, King's College London, Guy's Campus, King's Health Partners,

London, United Kingdom

MP06-07 Can video-based learning support

the improvement of surgery safety by reducing surgeon error? An experimental study

S Anacleto, V Fernandes, P Mota, N Carvalho,

M Costa, J Torres, N Morais, P Passos,

R Rodrigues, E Dias, E Lima, J Correia-Pinto 
MP06-08 Development and Validation of a Novel Skills Training Model for PCNL G Bozzini, U Besana, G Pini, E Liatsikos, AS Gozen, C Buizza, M Sighinolfi, B Rocco ASST Valle Olona - Busto Arsizio VA

MP06-09 Utility and feasibility of video-based assessment of residents performing ureteroscopy and laser lithotripsy

Y Wang, K Dore, D Russell, J Hoogenes, B Shayegan, NC Wong, ED Matsumoto McMaster University

MP06-10 Early Exposure to Robotic Surgery is Improving Urology Resident Robotic Skills

T Rogers, S Bhat, M Covas Moschovas, F Onol, T Larson, V Patel, D Thiel

Global Robotic Institute

MP06-11 Evaluation of the Non-Technical Skills for Urological Surgeons (NTS) Curriculum and Assessment Scale

CM Griffin, A Aydin, O Brunckhorst, C McIlhenny, J Brewin, N Raison, A Al-Jabir, T Brouwers, J Palou Redorta, M Khan, P Dasgupta, K Ahmed

King's College London

MP06-12 Construction and Assessment of an Innovative Indigenous All-Endo-Uro-Sim - a One Stop

Solution For Endourology Training A Rawandale-Patil, LG Patni, A Gade, S Kaushik, PA Rawandale, A Hindustanwala Tejnaksh Hospital's Institute of Urology

MP06-13 Evaluation of The Learning Curve of the European Training In Basic Laparoscopic Skills (E-BLUS) Validated Training Model:

A Prospective Cohort Study

MS Amin, N Abbud, A Aydin,

B Van Cleynenbreugel, D Venenziano, BK Somani, AS Gozen, J Palou Redorta, M Khan, P Dasgupta, J Makanjuola, K Ahmed MRC Centre for Transplantation, King's College London, London, UK

MP06-14 Assessment of Construct validity of virtual reality TURP simulator

E Lim, A Sim, H Ho, w Lau, K Chen

Singapore General Hospital
MP06-15 Objective Mental Workload Evaluation Using Functional Brain Network Metrics and Artificial Neural Network

S Besharat Shafiei, AA Hussein, NA Aldhaam, KA Guru

Roswell Park Comprehensive Cancer Center

MP06-16 Trial of A Near-Miss Resident Conference J Ahn, B Dalkin, H Wessells, M Sorensen University of California San Francisco

MP06-17 Evaluation of the Microsoft HoloLens as a Training Tool in Urological Laparoscopic Procedures - a Randomized Control Trial N Abbud, A Aydin, MS Amin, C Scott, D Venenziano, B Van Cleynenbreugel, BK Somani, AS Gozen, J Palou Redorta, M Khan, P Dasgupta, K Ahmed MRC Centre for Transplantation, King's College London, London, UK

MP06-18 Development and testing of an online motor imagery training tool

M Goble, N Raison, J Chong, M Khan, P Dasgupta, K Ahmed

King's College London Medical School

MP06-19 Intrinsic Streaming Platform for Remote Live Surgical Mentoring and Review - Advanced Mentoring in a High Volume Robotic Urological Training Centre J MacKinlay, E Latif, MM Quraishi, B Osman, M Thomas, EH Streeter, BA Eddy, SS Kommu Department of Urology. Kent \& Canterbury Hospital. East Kent Hospitals University NHS Foundation Trust

MP06-20 Evaluation of effective urological ward rounds S Murali-Krishnan, J Krishnan, N Osman, $\mathrm{R}$ Inman Sheffield Teaching Hospitals

MP06-21 Withdrawn

MP06-22 Residency Training for Robotic Partial Nephrectomy with a Porcine Model K Du, N Badhiwala, S Monda, A Sivaraman, E Kim, R Venkatesh, J Vetter, A DeClue, R Figenshau Washington University in St. Louis
MP07-01 Withdrawn

MP07-02 Mini versus standard percutaneous nephrolithotomy for treatment of pediatric renal stones: Is smaller enough?

Sn Mahmood, BO Mohammed, HM Toffeq,

SS Fakhraldin

Univesrsity of Sulaimania, College of Medicine, Department of Surgery
MP07-03 Percutaneous Nephrolithotomy with intraoperative CT-guided evaluation of stone free rate - a feasibility study X Zhu, A Kusters, H Langenhuijsen, F d'Ancona Radboudumc 
MP07-04 Supine Percutaneous Nephrolithotomy in Horseshoe Kidneys? Results of a Multicentric Study

FC Vicentini, E Mazzucchi, M Gokce, M Sofer, Y Tanidir, T Sener, P Melo, B Eisner, T Batter, T Chi, M Armas-Phan, C Scoffone, C Cracco, B Manzo, O Angerri Feu, E Emiliani, O Maugeri, K Stern, C Batagello, M Monga

Brigadeiro Hospital

MP07-05 Practice of Percutaneous Nephrolithotripsy and Outcomes at the Philippine General Hospital

J Flor, A Cabungcal, M Lapitan

University of the Philippines- Philippine

General Hospital

MP07-06 Partial tubeless with single step dilatation percutaneous nephrolithotomy with a novel technique of zero degree $\mathrm{c}$ arm angel puncture without a single blood transfusion: 1000 cases from single center in rural INDIA.

H Tanwar, Y Kaje, A Pardeshi

MP07-07 Withdrawn

MP07-08 Infra-costal Puncture for Upper Pole Calyceal Access for Percutaneous Stone Extraction in Both Prone and Supine Positions

H Burns, N Smyth, S Nalagatla

University Hospital Monklands

MP07-09 Trends of PCNL in Saudi Arabia

WK Kamal, A Alhazmy, M Alharthi, A Al-Solumany

Consultant Urology and Endourology King Fahd Hospital Jeddah

MP07-10 Stone Related Preoperative factors affecting postoperative PCNL Stone Free Rate (SFR)

G Bozzini, G Pini, U Besana, S Proietti, G Giusti, C Buizza, M Sighinolfi, B Rocco

ASST Valle Olona - Busto Arsizio VA

MP07-11 The Outcome of Ultrasound-Guided

Percutaneous Nephrolithotomy in the Treatment of Horseshoe Kidney with Calculi:

A Decade's Experience

Y Hong, Q Xu, X Huang, L Xiong, F Zhang, L An, $\mathrm{K}$ Ma

Peking University People's Hospital

MP07-12 Outcome of Ultrasound-Guided Percutaneous Nephrolithotomy in the Treatment of Medullary Sponge Kidney with Symptomatic Stones: ten years' experience

Y Hong, Q Xu, X Huang, L Xiong, L An, H Ye

Peking University People's Hospital

MP07-13 Percutaneous Nephrolithotomy:

Is Monoplanar better than Biplanar Access?

D Irani, M Hosseini, A Eslahi, A Haghpanah

Shiraz Nephrology Urology Research Center
MP07-14 Which one is the easiest and quickest scoring system to predict percutaneous nephrolithotomy outcomes by Junior surgeons?

N Iqbal, T Bhatty, S Akhter, J Rahim

Pakistan Kidney and Liver Institute and

Research Center

MP07-15 Supine mini-percutaneous nephrolithotomy using a novel access sheath with negative pressure aspiration system: A prospective evaluation from a Swedish tertiary center

M Popiolek, D Al-Rammahi, P Georgouleas, M Wagenius, S Andersson, O Andrén

Department of Urology, Univeristy

Hospital Örebro

MP07-16 Ideal Candidates for the Tract Dilation

Monitored by Ultrasound

in Percutaneous Nephrolithotomy

S Wang, X Zhang, Y Tang, B Xiao, J Li

University of Maryland School of Medicine

\section{MP07-17 Withdrawn}

MP07-18 Mini-Percutaneous Nephrolithotomy versus Retrograde Intrarenal Surgery for the treatment of 10-20mm lower pole renal stones. A Systematic Review and Meta-Analysis

JD Cabrera, B0 Manzo, JE Torres, FC Vicentini, H Sánchez, E Rojas, E Lozada

Hospital Regional de Alta Especialidad del Bajío

MP07-19 Preoperative factors associated with perioperative complications in Percutaneous Nephrolithotomy

V Grajales, D Miller, A Mohapatra, A Delmonte, J Bigley, T Averch, M Semins

UPMC

MP07-20 Serum Cystatin C, 24 Urine Creatinine Clearance, and Renal scan (DTPA) as a markers of renal function, pre and postoperative percutaneous nephrolithotomy (PCNL)

C Martinez-Arroyo, G Veliz-Cabrera,

P Cortes-Raygoza, M Cantellano-Orozco,

G Fernandez-Noyola, G Morales-Montor,

C Pacheco-Gahbler

Hospital General Dr. Manuel Gea Gonzalez

MP07-21 Outcomes of Percutaneous Nephrolithotomy with or without Nephrostomy Tube:

A Comparative Study

M Ali, P Saha, S Chowdhury, M Rahman,

N Kamal

Dhaka Medical College Hospital

MP07-22 Percutaneous Nephrolithotomy for Renal Stones in Chronic Kidney Disease and Poorly Functioning Kidney:

A Prospective Study

M Abdulrahman, M Gadelmoula, EO ElGanainy, YM Abdelsalam

Assiut University 
MP08-01 Could RezumTM water vapor ablation therapy for benign prostate enlargement be an option for patients with urinary retention? The first UK center experience

S Sarkar, M Johnston, T Farmer, T Gehring,

G Rajkumar, T Nedas, A Emara, R Hindley

MP08-02 A Novel Technique of Morcellation Using a Pneumovesicum After Holmium Laser Enucleation of the Prostate in Complicated Situations: Our Initial Experience and Tips $\mathrm{H}$ Choi

Korea University Ansan Hospital, Korea

University College of Medicine

MP08-03 A matched comparison between $120 \mathrm{~W}$ Moses and $100 \mathrm{~W}$ standard holmium laser enucleation of the prostate in treating symptomatic benign prostate hyperplasia

M Meskawi, J Alamiri, ME Rivera

MP08-04 Prostatic Urethral Lift Study:

Experiences in Northern Ireland (PULSE NI)

J Morrow, D Curry, F Farag, R Fiala, A Pahuja NHS

MP08-05 Postoperative Storage Symptoms of Holmium Laser Enucleation versus GreenLight Vaporization of the Prostate for Symptomatic Benign Prostatic Hyperplasia

A Ibrahim, M Elkoushy, M Aube, S Carrier McGill University

MP08-06 Utility of Post-Operative Day (POD) Zero Labs in Bipolar Trans-urethral Resection of Prostate (TURP)

B Patel, S Ekbal

MP08-07 Evaluation of the New Holmium Laser Xpeeda ${ }^{\mathrm{TM}}$ Side-firing Fiber versus GreenLight XPS 180W Vapoenucleation of the Prostate for Benign Prostatic Obstruction: An Early Clinical Experience

A Ibrahim, M Aube, H Elmansy, S Carrier McGill University

MP08-08 A good bye to High Power Settings! A single center experience on Ultra Low Power HoLEP S Prabhakar, Y KK

MP08-09 Thuvep of large prostates: a prospective single-center study

F Germinale, L Tosco, A Giacobbe, D Collura, M Kurti, G Muto, E Berdondini Humanitas Gradenigo, Turin, Italy

MP08-10 Withdrawn

MP08-11 WATER vs WATER II: Aquablation Therapy for Benign Prostatic Hyperplasia

A Kasraeian, N Bhojani, D Nguyen

Kasraeian Urology
MP08-12 A Comparison of Outcomes After Holmium Laser Enucleation of the Prostate in Patients with Chronic Retention, a History of Acute Retention, and No Prior Retention A Martin, BB Whiles, A Brevik, J Thompson, KL Thurmon

University of Kansas Medical Center

MP08-13 Is RezumTM Water Vapour ablation therapy a suitable option for men with larger prostate glands?

S Sarkar, M Johnston, T Farmer, T Gehring, G Rajkumar, T Nedas, A Emara, R Hindley

MP08-14 Aquablation for Benign Prostatic Hyperplasia: Early Outcomes from a Tertiary Care Center in the Middle East

N Abdallah, A Mailhac, M Bulbul, H Tamim, R Jaafar, A Hajj

American University of Beirut Medical Center

MP08-15 Ablation Efficiency Utilizing a Modulate Pulse Platform for Holmium Laser Ablation of the Prostate

BB Whiles, A Martin, A Brevik, KL Thurmon University of Kansas Medical Center

MP08-16 HoLEP as a day case procedure N Raison, H Godbole, N Kale, MK Bhattacharyya North Middlesex University Hospital

MP08-17 Outcomes of transurethral resection and holmium laser enucleation in more than $60 \mathrm{~g}$ of prostate: A prospective randomized study

RJ SINHA, V SINGH, S MEHROTRA, S MEHDI, S PANDEY, R Sinha King Georges Medical University, Lucknow, India

MP08-18 Telementored bipolar enucleation of the prostate:

A magic way to teach surgery M Amato, R Milandri, S Ciarlariello, G Guarino, M Sighinolfi, A Meneghini, B Rocco, S Micali Department of Surgical, Medical and Dental Morphological Sciences related to Transplant, Oncology and Regenerative Medicine - Section of Urology, University of Modena and Reggio Emilia, Modena, Italy

MP08-19 Antegrade Ejaculation Preserving Technique with Green Light XPS

PN Contreras, N Bonanno, L Blas, $\mathrm{N}$ villasante, H Ríos Pita, C Ameri Hospital Alemán 
MP08-20 Clinical Comparison of Holmium Laser Enucleation of the Prostate and Bipolar Transurethral Enucleation of the Prostate: A short term follow-up

A Tuncel, C Aykanat, E Gazel, S Akdemir, T Oksay, Y Aslan, M Balci, O Guzel, M Arslan, L Tunc

University of Health Sciences, Ankara Numune

Research and Training Hospital
MP08-21 Preliminary data on prospectic randomized trial on the comparison of holmium laser enucleation of the prostate (HOLEP) vs. prostate enucleation with Moses technology (MOLEP)

Y Hussein M.I., F Petrelli, S Corti, R Milesi,

F Ceresoli, I Vavassori

ASST Bergamo Ovest

MP08-22 Single Surgeon Experience with Procept Aquabeam Aquablation of Prostate: First 40 Cases

A Kasraeian

Kasraeian Urology
MP09-01 A novel one-stop LUTS clinic model from a tertiary referral university hospital in the United Kingdom

U Walters, T Latimer, S Dean, M Morgan,

V Jeram, T El-Husseiny

Charing Cross Hospital, Imperial College

Healthcare NHS Tust

MP09-02 Analysis of the Factors Influencing Holmium Laser Enucleation of the Prostate Training BA Penev, A Henderson, E Peneva, M Cynk Maidstone and Tunbridge Wells NHS Trust

MP09-03 The incidence of Urethral complications with using 26-French versus 28 -French resectoscope sheaths in Holmium Laser Enucleation of the Prostate (HoLEP) KH Thai, JC Smith, J Stutz, J Sung, M El Tayeb Baylor Scott \& White

MP09-04 Prostatic amyloidosis- What is the clinical significance?

A Nevo, ML Stanton, A Navaratnam, MR Humphreys

Mayo Clinic

MP09-05 Urethra and ejaculation preserving robot-assisted simple prostatectomy: near infrared fluorescence imaging-guided Madigan technique

G Simone, L Misuraca, U Anceschi, F Minisola, M Ferriero, S Guaglianone, G Tuderti,

M Gallucci, R Mastroianni

MP09-06 Risk factors for febrile complications after holmium laser enucleation of the prostate Y Kyono, F Endo, M Sakurai, M Sano, K Komatsu, K Narimoto, M Shimbo, K Ohwaki, K Hattori

MP09-07 Outcome of Unilateral bladder neck incision in comparison with Bilateral incision incase of Primary bladder neck obstruction

M Alam

Bangabandhu Sheikh Mujib Medical

University (BSMMU)
MP09-08 Is TURP safe even in super-aged patients over 85 years of age?

J Kang, S Shin, J Cho, K Moon, D Cho, J Choi, m Kim, J Park, T Yoo

Eulji Medical center

MP09-09 A randomized controlled study to compare the efficacy of thulium laser $200 \mathrm{~W}$ enucleation versus Greenlight laser $180 \mathrm{~W}$ vaporesection of the prostate for the treatment of benign prostatic obstruction: Midterm report

F Hsu, C Chou, H Chang

Taipei City Hospital

MP09-10 Correlation of Frailty Scores and Stress Incontinence post Holmium laser enucleation of the prostate for Benign Prostatic Hyperplasia J Smith, J Stutz, J Sung, H Hashim, M El Tayeb Baylor Scott \& White

MP09-11 Robot-assisted Total Prostatectomy:

A New Technique for the Management of Benign Prostatic Hyperplasia

R Pathak, A Hemal

Wake Forest University

MP09-12 Ejaculation Sparing Thulium Laser Enucleation of the Prostate (ES-ThuLEP): Outcomes on a large cohort.

G Bozzini, U Besana, A Calori, A Govorov, G Pini, C Buizza, M Sighinolfi, B Rocco ASST Valle Olona - Busto Arsizio VA

MP09-13 Initial experience with intraoperative fluid warming during Holmium Laser Enucleation of the Prostate (HoLEP).

T Large, CU Nottingham, A Krambeck

MP09-14 Safety of Holmium Laser Enucleation of the Prostate in the Outpatient Setting

D Agarwal, K Hebert, ME Rivera

Indiana University

MP09-15 Withdrawn

MP09-16 Convective water vapor thermal prostate ablation: experience at a tertiary center G Greear, T DiPina, K Kader, T Hsieh, J Buckley, J Woo, S Bechis

UC San Diego 
MP09-17 Perioperative Outcomes of Bladder Neck Sparing

Robot-assisted Simple Prostatectomy

M Shahait, K Patel, J Kim, DI Lee

University of Pennsylvania

MP09-18 Randomized Comparison of Moses Enucleation of the Prostate to Standard Holmium Enucleation of the Prostate (HoLEP)

T Large, CU Nottingham, J Williams,

A Krambeck

MP09-19 Long-Term Functional Outcomes of Robot-Assisted Simple Prostatectomy in Patients with >3 Year Follow-Up

Z Lee, A Keehn, AM Asghar, D Strauss, M Lee, DD Eun

Temple University
MP09-20 Predicting outcomes of robotic assisted simple prostatectomy in patients with large prostate volumes

RL Steinberg, H Trivedi, BA Johnson, A Garbens, C Roehrborn, JC Gahan

MP09-21 HoLEP learning curve - is there a plateau after 50 cases?

A Coscione, S Kampantais, D Cohen, K Gordon, P Acher

Southend University Hospital NHS Trust

MP09-22 Comparison of Different Types of Catheters for Intermittent Self-Catheterization: A Systematic Review and Network Meta-analysis D Ye, Y Chen, Z Jian, H Li, K Wang West China Hospital
MP10-01 Cascade of Allium ureteral stent for ureteral stenosis after radiotherapy for gynecologic cancer

W Qi

Peking university People's Hospital

MP10-02 The First UK experience of Super-Mini Percutaneous Nephrolithotomy

H Abboudi, G Celentano, S Bilal, G Kazantis, F Schoofs, V Arumuham, S Choong University College London Hospital

MP10-03 Use of analgesia used for shockwave lithotripsy (SWL): Twitter survey from EULIS/ESUT

A Pietropaolo, K Sarica, A Skolarikos, E Liatsikos, BK Somani

MP10-04 Initial Experience with Swiss Lithoclast Trilogy Demonstrates Reliable and Rapid Lithotripsy during Percutaneous Nephrolithotomy CU Nottingham, T Large, K Cobb, RL Sur, NE Canvasser, C Stoughton, A Krambeck Indiana University School of Medicine

MP10-05 Comparison of Small Core Diameter Optical Laser Fibers Used for Holmium:YAG and Super Pulse Thulium Laser Lithotripsy BE Knudsen, WR Molina, BH Chew The Ohio State University

MP10-06 Comparison of Stent-Related Symptoms Between Conventional Metal Stent and Allium Ureteral Stent

W Qi

Peking University People Hospital

MP10-07 Evaluation of a single use flexible cystoscope: a multi-institutional international study K Scotland, ER Tawfiek, A Chiura, BH Chew, D Bagley

University of British Columbia
MP10-08 Initial human experience with a novel stone aspiration device used during ureteroscopic lithotripsy for renal stones

S Agrawal, A Singh, RL Sur, B Eisner, G Haleblian, G Preminger, A Ganpule, R Sabnis, M DESAI

Muljibhai Patel Urological Hospital, Nadiad

Gujarat, India

MP10-09 Comparing dusting and fragmenting efficiency using the new Super Pulse Thulium Fiber Laser vs a 120W Holmium:YAG laser

BH Chew, BE Knudsen, WR Molina

University of British Columbia

MP10-10 To investigate the clinical efficacy of transurethral ureteroscopic laser lithotripsy in the treatment of ureteral calculi under ultrasound guided paravertebral nerve block anesthesia

Y Wu, Y Lu, H Hu, S Wang, Q Wang

Tongji Hospital

MP10-11 Integrated, Real-Time Digital Stone

Measurement in Ureteroscopic Stone

Procedures:

A Workflow Feasibility Study

K Koo, T Aro, S Lim, D Petrisor, D Stoianovici, B Matlaga

Johns Hopkins University School of Medicine

MP10-12 Comparison of Mini PCNL v/s Retrograde intrarenal surgery (RIRS) v/s Extracorporeal shockwave lithotripsy (ESWL) for the treatment of renal stones up to 2.5 centimeters

P. Pattnaik

Bombay Hospital PG Institute

MP10-13 Rapid, Efficient Crowdsourcing Using Social Media for the Surgical Management of Nephrolithiasis

K Koo, T Aro, B Matlaga

Johns Hopkins University School of Medicine 
MP10-14 Operator-assisted vs self-achieved basketing during ureteroscopy:

results from an in-vitro preference study

E Ventimiglia, S Doizi, T Besombes, O Traxer

Division of Experimental Oncology/Unit of

Urology; URI; IRCCS Ospedale San Raffaele

MP10-15 Two-step precise method with transurethral flexible ureteroscopy for treatment of parapelvic cyst combined with renal calculi

G Jingyang, Y Wenzeng, Z Yanqiao, S Xiaoqiang,

A Feng, G Deqiang

Affiliated Hospital of Hebei University

MP10-16 Super Pulse Thulium Fiber Laser Compared to 120W Holmium:YAG Laser:

Impact on Retropulsion and Laser Fiber

Burn Back

BE Knudsen, BH Chew, WR Molina

The Ohio State University

MP10-17 RIRS for lower pole small renal stones:

Novel SuperPulse Thulium (Tm) fiber laser lithotripsy. First clinical results.

D Korolev, R Klimov, D Enikeev, D Tsarichenko,

M Enikeev, A Dymov, S Ali, G Akopyan,

V Lekarev, L Rapoport

Institute for Urology and Reproductive Health,

Sechenov University
MP10-18 Ultrasound guided paravertebral nerve block anesthesia and SVOF accurate puncture for percutaneous nephrolithotomy — an innovative ambulatory surgery mode

Y Wu, Y Lu, J Zhang, S Wang, Q Wang

Tongji Hospital

MP10-19 Evaluation of safety and efficacy of combined Extracorporeal shockwave lithotripsy and retrograde intrarenal surgery for the treatment of large lower calyceal calculi PK Pattnaik, SK Pattnaik, MP Pattnaik S S Urological \& Research Insitute, Bombay Hospital PG Institute

MP10-20 Ureteroscopic Doppler Ultrasonography: Where is the Least Vascular Renal Access Site for Percutaneous Nephrolithotomy?

RM Patel, V Cooper, L Limfueco, S Tapiero, M O'Leary, D Regan, J Landman, RV Clayman University of California, Irvine

MP10-21 Selective Utilization of the LithoVue Single-Use Digital Flexible Ureteroscope for the Management of Upper Urinary Tract Stones Results in Shorter Operative Times and Cost Savings

R Munver, J Tsui, B Desroches, M Degen, M Ahmed, G Lovallo, MD Stifelman Hackensack University Medical Center
V04-01 Ultrasound-guided retrograde intrarenal surgery is safe and useful

T Tsujioka, Y Kawanishi, D Takahashi, K Hirota,

Y Atagi, K Seto, K Izumi, M Yamanama

V04-02 Challenging case of RIRS in Horse Shoe Kidney A Puri

V04-03 Moving away from Percutaneous Nephrolithotomy; Retrograde intrarenal surgery in complex stone scenarios

Y KK, S Prabhakar

V04-04 Single-use digital flexible ureteroscopy: Pelvic kidney with pyelic lithiasis

O Angerri, L Sabiote, J Balañà, E Emiliani,

A Kanashiro, F Sánchez-Martín, F Millán

Rodriguez

V04-05 Endoscopic Removal of Foreign Body within the Ureter

S Setia, L Deane

V04-06 Withdrawn

V04-07 Blue spritz technique - a simple way to identify the elusive ostium of a calyceal diverticulum W Low, N Mohd Zam
V04-08 The Cost of Ureteroscopy:

Can Single-Use Flexible Ureteroscopes Advance Past Reusable?

C Tabib, V Vasudevan, D Hoenig, Z Okeke, AD Smith

V04-09 Impacted stone or Basket AZ Osman, AZ Osman NMC Royal Hospital, Dubai, UAE

V04-10 Piecemeal retrograde removal of encrusted and encased stuck ureteral stent: Video tips and tricks A Pietropaolo, BK Somani

V04-11 Establishment of internal circulation system in rigid ureteroscopy X Zhan, g Hu, J Huang, H Zhang, Y Xu

V04-12 The Complete Endoscopic Laser Management Of A Ureterocoele With An Associated Obstructive Calculus M Ravindraanandan, W Mahmalji, Y Phan Hereford County Hospital

V04-13 Tips and tricks: Limiting radiation exposure during retrograde intrarenal surgery T Chi, T Chi 
V05-01 Laparoscopy assisted PCNL for Staghorn calculus in Pelvic kidney using Stone Morcellator A Novel Technique PK Pattnaik, SK Pattnaik, DP Pattnaik

V05-02 Laparoscopic ureterocalicostomy for complicated upper urinary tract obstruction without hydronephrosis after treatment of staghorn calculi: A case report.

K Mikami, K Ohtsuka, K Hou, T Suyama, H Masuda, S Kojima, Y Naya, K Araki

V05-03 'Is this man pregnant ??' - An unusual presentation of a renal cyst

O Andura, D Marghawal, C Wülfing

V05-04 A prospective evaluation of outcomes of transperitoneal 3-D laparoscopic adrenalectomy in adrenal masses

A Kumar, S Yadav, S Kumar, K Saurav,

Y Prashant, V Tyagi, V Prasad

V05-05 Sling Technique for Ureteropelvic Junction Repair - An Autologous Gonadal Vein Sling

For Transposing the Crossing Vessel

J Goodman, T Chi, M Stoller, T Chi

V05-06 Technical Tips in Ureterocalicostomy in Failed Pyeloplasty

S Nachimuthu, R Manickam, S Kallappan
V05-07 Laparoscopic pyeloplasty and pyelolithotomy in a pelvic kidney MA Hamdoon, D Whiting, MO Breish, S Madaan, S Sriprasad

V05-08 Combined Antegrade Intrarenal Surgery, Laparoscopy and Retrograde Intrarenal Surgery for absolute clearance of large volume renal stone A versatile approach

JM Ramanathan, A Konay

V05-09 Laparoscopic Ileal Ureter In Failed Laparoscopic Ureterocalicostomy R Manickam, S Nachimuthu, S Kallappan

V05-10 Two cases of laparoscopic surgery for the local recurrence of pheochromocytoma in patients with multiple endocrine neoplasia

K Tomita, T Yoshida, S Kageyama, K Johnin, M Narita, A Kawauchi

V05-11 Appendiceal interposition for $3 \mathrm{~cm}$ mid-ureteric stricture

A Alaqqad, W Hassen

V05-12 Successful laparoscopic resection of a paraganglioma immediately behind the inferior vena cava and bilateral renal veins. T Hakariya, Y Shida, Y Miyata, H Sakai
V06-01 Robotic Assisted Laparoscopic Lymphatic Sparing Varicocelectomy In An Adolescent Male J Jensen, A Rensing, D Frimberger, B Meenakshi-Sundaram

V06-02 Safe Laparoscopic Technique Port Placement In Pediatric Patient

B Onal, D Abdullahoglu, Y Ilki, E Bulbul, B Ercili, S Citgez

V06-03 Patient-Specific Surgical Simulation Using 3D Printed Hydrogel Models for Living Donor \& Recipient Kidney Transplant Surgery A Ghazi, R Melnyk, E Belfast, T Campbell, G Wu, R Kashyap, JV Joseph

V06-04 Solo-Surgeon Pure Laparoscopic Living Donor Nephrectomy Using Camera Holder D An, D You, Y Kim, J Aum, K Kim, J Han

V06-05 Robot-assisted Bilateral Orchidopexy: Persistent Mullerian Syndrome MI Al Khalaf, AM Alenizi, TA Al Saad

V06-06 Four Renal Artery Kidney Donor and Transplant technique

V Agarwal
V06-07 Pneumovesicoscopic Bilateral periureteric diverticulum excision with Ureteric Reimplantation in 6years old child A Aljneibi

V06-08 Laparoscopic retroperitoneal living donor nephrectomy with simultaneous cholecystectomy D Perlin, I Alexandrov, V Zipunnikov, I Dymkov, M Kretov

V06-09 Combined antegrade and retrograde endoscopic approach for management of a forgotten stent in the pediatric patient

JT Barnard, AJ Hajiran, CE Morley,

O AL-Omar

V06-10 25 Year Old Male with Voiding Dysfunction in the Setting of Prostatic Utricle Cyst

K Parikh, J Moore, R Pak

V06-11 Robot-assisted Simple Nephrectomy for Management of Persistent Urinary Incontinence Secondary to an Ectopic and Atrophic Right Kidney and Ureter M Lee, Z Lee, AM Asghar, MG Packer, DD Eun 
MP11-01 Implementation of a Single-Use Digital Flexible Ureteroscope Results in a Decrease in Reusable Digital Ureteroscope Repair Costs, and Increase in Reusable Digital Ureteroscope Life, and Prevention of Operative Case Cancellation R Munver, J Tsui, B Desroches, M Degen, G Lovallo, M Ahmed, MD Stifelman Hackensack University Medical Center

MP11-02 Tissue incision, excision and ablation using Super Pulse Thulium Fiber (SPFL) LASER: Comparison with High Power 120W

Holmium-YAG LASER (Ho:YAG) BH Chew, BE Knudsen, WR Molina University of British Columbia

\section{MP11-03 Withdrawn}

MP11-04 Holmium laser versus thulium laser versus Thulium Fiber Laser enucleation of the prostate: a single center study

PK Pattnaik, SK Pattnaik, DP Pattnaik S S Urological \& Research Institute, Bombay Hospital PG Institute

MP11-05 Digital application developed for evaluation of functional results following assisted robotic radical prostatectomy

L Faria, S Reis, K Leite, J da Cruz, R Maia, R Pimenta, MM Xavier, D Ilias, G Xavier, F Fakhouri, M Srougi, C Passerotti, G Magalhaes, M Belkovsky

Hospital Alemão Oswaldo Cruz

MP11-06 Hemostatic properties of Super Pulse Thulium Fiber (SPTF) LASER: Comparison with High Power 120W Holmium-YAG LASER (Ho:YAG) in an ex-vivo Porcine Kidney Model

WR Molina, BH Chew, BE Knudsen University of Kansas Medical Center

MP11-07 Initial Evaluation Of A Novel Modulated Radiofrequency-Based Bladder Denervation Device Z Okhunov, FA Jefferson, R Mao, RM Patel, $\mathrm{S}$ Tapiero, T Lee, G Ghoniem, G Li, J Landman University of California, Irvine

MP11-08 Immersive Virtual Reality (iVR) Renal Models as an Educational and Preoperative Planning Tool for Laparoscopic Donor Nephrectomy:

Initial Experience

FA Jefferson, E Parkhomenko, M O'Leary,

J Sung, L Limfueco, S Tapiero, RM Patel, H Ichii, D Dafoe, J Landman

University of California, Irvine

MP11-09 Comparing the utilization trends and 90-day outcomes for open, laparoscopic, and robot-assisted radical nephrectomy cases in New York State between 2009-2014.

KH Bilal, M Finkelstein, E Kaplan-Marans, M Palese

Mount Sinai Medical System
MP11-10 Concomitant treatment of ureteropelvic junction obstruction and complicated renal calculi with laparoscopic pyeloplasy and $19.5 \mathrm{~F}$ rigid nephroscopy

L An, L Xiong, X Huang

Peking University Applied Lithotripsy

Institute/Peking University People's Hospital

Urology and Lithotripsy Center

MP11-11 Microwave assisted, off-clamp no renorrhaphy laparoscopic partial nephrectomy in a porcine model - A feasibility study

AK Chow, A Sivaraman, M Talcottt, S Greco,

S Arafenian, R Venkatesh

Washington University School of Medicine,

St. Louis, USA

MP11-12 Initial experience with Robotized Laparoscopy at single center

K Ramaswami, P Bhirud, R Raveendran,

$\mathrm{H}$ Pothiyedath

Metromed Institute of Advanced Urology

and Renal Translplant

MP11-13 Non-Ischemic or Ischemic Laparoscopic Partial Nephrectomy Using a Newly Developed Hybrid Pencil Type Energy Device in a Porcine Model.

K Nakagawa, S Irie, H Nishimatsu, J Inokuchi, M Eto, M Tanaka

Tokyo Dental College Ichikawa General Hospital

MP11-14 In Vivo Porcine Evaluation of a Novel Self-Contained Bladder Irrigation System (MultiphzeTM)

P Jiang, RM Patel, S Tapiero, J Landman,

RV Clayman

UC Irvine Urology

MP11-15 A pilot study of the diagnostic accuracy using fluorescence confocal microscopy for urothelial carcinoma of the bladder during transurethral resection

B Becker, E von Rohden, A Gross, B Feyerabend, D Scholz, C Netsch

Departement of Urology, Asklepios Hospital

Barmbek, Hamburg, Germany

MP11-16 Evaluation of The Robotic Camera Holder on The Learning Curve for Basic Laparoscopic Skill: A Randomised Control Trial

MS Amin, N Abbud, A Aydin, B Van

Cleynenbreugel, D Venenziano, BK Somani,

AS Gözen, JP Redorta, M Khan, P Dasgupta,

J Makanjuola, K Ahmed

MRC Centre for Transplantation, King's College

London, London, UK 
MP11-17 Thulium-Holmium:YAG laser in conservative treatment of upper tract urothelial cancer: 13 years-experience from a tertiary national referral center

L Defidio, M Antonucci, M De Dominicis,

N Russo, G Fuchs, A Patel

MP11-18 Matched Comparison of Laparoscopic Vs Robotic Assisted Nephro-Ureterectomy and Bladder Cuff Excision

S Nalagatla, I Ibrahim, NO Umez-Eronini, OM Aboumarzouk

Queen Elizabeth University Hospital

MP11-19 Multi-Porous Open-Ended Catheter Technique for Intraureteral Indocyanine Green (ICG) Injection During Surgery

E Kaplan-Marans, J Fulla, M Palese

Cleveland Clinic
MP11-20 On-clamp versus purely off-clamp robotic partial nephrectomy in solitary kidneys: comparison of chronic kidney disease progression at two highvolume centers

U Anceschi, G Tuderti, A Brassetti, M Ferriero, S Guaglianone, R Mastroianni, R Flammia, M Gallucci, R Bertolo, F Minisola, M Costantini, J koauk, G Simone

"Sapienza" University

MP11-21 Robot assisted Supine Extraperitoneal Approach for midline retroperitoneal masses GK Pooleri, A LAddha

Amrita Institute of Medical Sciences

MP11-22 Decreased Risk of Upper Extremity Neuropraxia During Robotic Assisted Laparoscopic Prostatectomy:

A Novel Technique M Watson, B Koch, R Xu, G Heath, A Singh
MP12-01 Effect of Perioperative Continuation of Antithrombotic Therapy on Outcome of Robot-Assisted Radical Prostatectomy : A Prospective Clinical Trial at Single-Institution M Kubota, T Matsuoka, S Murata, I Suzuki, N Makita, Y Tohi, Y Sugino, K Inoue, M Kawakita

Kobe City Medical Center General Hospital

MP12-02 Institutional Learning Curves for Robotic Radical Prostatectomy using CUSUM Analysis F Endo, M Shimbo, K Ohwaki, K Hattori St. Luke's International Hospital

MP12-03 Predictors of Length of Hospital Stay in Robotic Assisted Radical Prostatectomy:

a Retrospective Cohort Study

MS Labban, J Zarka, NF Abou Heidar, A Mailhac, H Tamim, A Hajj

American University of Beirut Medical Center

MP12-04 The Value of Nerve Sparing during RARP in Men with Low SHIM score

M Shahait, J Kim, A Malhotra, RW Dobbs, DI Lee

University of Pennsylvania

MP12-05 The effect of USPSTF recommendation on post-operative outcomes in Robot-assisted laparoscopic prostatectomy (RALP) based on analysis of a single surgeon series.

S Bhat, O Fikret, M Moschovas, T Rogers, C Jenson, B Rocco, V Patel

Global robotics institute, Celebration, FL

MP12-06 Evaluation of lymphoceles after pelvic lymph node dissection in robot-assisted radical prostatectomy

Y Muro, H Iijima, C Nakamura, T Fujimoto, R Yabusaki, Y Shiraishi, M Imamura,

K Yoshimura

Department of Urology, Shizuoka

General Hospital
MP12-07 Impact of Median Lobes on Urinary Function after Robotic Radical Prostatectomy

OE Martinez, R Abaza

OhioHealth Dublin Methodist Hospital

MP12-08 Long-term Oncologic Outcomes After Limited Pelvic Lymph Node Dissection for Intermediate and High Risk Prostate Cancer AS Elsayed, NA Aldhaam, AA Hussein, G James, O Abdel-Razzak, I Saad, M Abdelhakim, KA Guru

Roswell Park Comprehensive Cancer Center

MP12-09 Impact of membranous urethral length on the urinary continence outcomes of patients after robotic-assisted radical prostatectomy Y Murakami, K Tabata, H Tsumura, T Hirayama, K Matsumoto, M Iwamura

MP12-10 Nerves at the posterior of the prostate base contribute to erectile function:

An intraoperative electrical stimulation assessment Y Kaiho, J Ito, Y Kohada, J Mikami, G Anan, T Kudo, M Sato Department of Urology, Tohoku Medical and Pharmaceutical University

MP12-11 Use Of Transversus Abdominis Plane Block To Decrease Pain Scores And Narcotic Use Following Robot Assisted Laparoscopic Prostatectomy T Rogers, F Onol, S Bhat, M Covas Moschovas, C Jenson, S Roof, N Gallo, B Gallo, M Sandri, V Patel Global Robotic Institute

MP12-12 Transvesical Robot Assisted Radical Prostatectomy G Wang, X Zhou, B Fu, C Zhang The First Affiliated Hospital of Nanchang University 
MP12-13 Can tumor volume percentage in radical prostatectomy predict risk of biochemical recurrence in prostate cancer? Determining a cut-off point and composite risk factors approach

A Alenezi, C Eden, M Ismail

Mubarak Hospital and Sabah Al Ahmad

Urology Centre

MP12-14 Acute kidney injury and its impact on renal prognosis after robot-assisted laparoscopic radical prostatectomy

S Kanda, S Narita, H Sato, T Inoue, T Nara, K Numakura, M Saito, T Habuchi

Akita University Graduate School of Medicine

Department of Urology

MP12-15 Comparison of clinical outcomes of laparoscopic and robotic radical prostatectomy

H Nakanishi, K Ohba, S Gohno, K Kawada,

T Matsuda, K Mitsunari, Y Shida, T Matsuo,

T Hakariya, Y Miyata, H Sakai

MP12-16 Preservation of umbilical ligaments and Retzius space reconstruction improves early urinary incontinence after robot-assisted laparoscopic radical prostatectomy

H Hospital, K Shiga, K Miyoshi, S Naito

Harasanshin Hospital

MP12-17 Predictive value of serum testosterone levels as early PSA recurrence after Robot-assisted laparoscopic radical prostatectomy in patients with higher Body Mass Index

S Koga, Y Horiguchi, A Ogata, N Sakamoto,

F Tsukuda

Department of Urology, Edogawa Hospital
MP12-18 Comparison of biochemical recurrence between open and robot-assisted radical prostatectomy in patients with positive surgical margins T Ohashi, T Majima, R Ishida, Y Matsukawa, T Nishikimi, M Kato, T Tsuzuki, M Gotoh,

H Kobayashi

MP12-19 Comparison of outcomes in salvage robotic-assisted laparoscopic prostatectomy for post-primary radiation vs. ablation therapies

O Fikret, S Bhat, T Rogers, M Covas Moschovas, H Ganapathi, S Roof, V Patel

Global Robotic Institute

MP12-20 The Therapeutic Outcomes of Robot-assisted Radical Prostatectomy (RARP) Following Neoadjuvant Chemohormonal Therapy for High-risk Prostate Cancer

T Yoneyama, H Yamamoto, A Imai,

S Hatakeyama, Y Hashimoto, C Ohyama

Hirosaki University Graduate School of Medicine

MP12-21 Effect of Puboprostatic Ligaments Preservation during Robotic-Assisted Laparoscopic Radical Prostatectomy on Early Continence:

Randomized Controlled Trial

W Ratanapornsompong, P Sungkum,

S Pacharatakul, C Leenanupan,

W Kongcharoensombat

Ramathibodi Hospital

MP12-22 Salvage Pelvic Lymph Node Dissection (sPLND) for Nodal Recurrence of Prostate Cancer after Radical Prostatectomy

V Berge, O Hopland, F Ottosson, S Fosså,

E Hernes, K Hole, A Svindland, J Hoff,

B Brennhovd, L Eri

Oslo University Hospital, Oslo University
MP13-01 Robotic partial nephrectomy versus radical nephrectomy in elderly patients with large renal mass: a multi-institutional propensity score match paired analysis

A Veccia, A Antonelli, A Mottrie, A Minervini, I Derweesh, C Lau, G Simone, J Kaouk, K Jacobsohn, DD Eun, B Challacombe, S Perdonà, J Porter, C Zang, U Capitanio, M Aron, C Simeone, L Hampton, F Porpiglia, R Autorino Virginia Commonwealth University Health System, Richmond, VA

MP13-02 Retroperitoneoscopic partial nephrectomy Trifecta outcome analysis in a single-institution series of 700 consecutive cases

C Wülfing, D Marghawal, O Andura

Asklepios Klinik Altona

MP13-03 Diagonal renorrhaphy technique in robot-assisted partial nephrectomy for endophytic mid-pole renal tumor of size $\mathbf{>} \mathbf{4 m}$ G Wang, X Liu, X Zhou, B Fu, L Chen, Y Li Department of Urology, The First Affiliated Hospital of Nanchang University; Jiangxi Institute of Urology
MP13-04 Is Robot-assisted Radical Nephroureterectomy with Bladder Cuff Excision Superior to Pure Laparoscopic Approach in The Treatment of Upper Urinary Tract Urothelial Carcinoma? Y Huang, E Huang, H Chung, M Tai, T Huang, T Wei, Y Fan, C Lin, T Lin, J Kuo, S Lu, Y Chang, A Lin, W Huang

Taipei Veterans General Hospital

MP13-05 The Incidence and Pattern of Renal Cell Carcinoma Recurrence after Robotic Partial Nephrectomy

MW Salkini, A Lamoushi

West Virginia University

MP13-06 Laparoscopic transperitoneal living donor nephrectomy early experience at a single center in Saudi Arabia

H El-Tholoth, T Alzahrani, H Al Akrash, R Zahid,

A Al Zahrani

Urology Department, Prince Sultan Military

Medical City

MP13-07 Treatment outcomes of large angiomyolipoma according to treatment method

T Kwon, S Park, S Park, K Moon, S Cheon

Ulsan University Hospital 
MP13-08 Discharge protocol for asymptomatic patients post pyeloplasty with satisfactory MAG 3 scans at annual review

N Kale, S Sangaralingam, Y Wagh, S Huq,

Z Billoo, H Godbole

Department of Urology, North

Middlesex Hospital

MP13-09 Outcomes and predictors of benign histology in patients undergoing robotic partial nephrectomy for renal masses: a multicenter study

A Veccia, A Antonelli, I Derweesh, A Mottrie, A Minervini, M Aron, G Simone, U Capitanio, C Simeone, DD Eun, S Perdonà, J Porter, C Sundaram, C Zang, R Uzzo, B Challacombe, L Hampton, J Kaouk, F Porpiglia, R Autorino Virginia Commonwealth University Health System, Richmond, VA

MP13-10 Initial Experience with Robotic Assisted Left Renal Vein Extravascular Stent Placement in the Treatment of Nutcracker Phenomenon RL Steinberg, A Garbens, BA Johnson, JA Cadeddu

MP13-11 The role of intra-operative mitomycin C intravesical administration during robotic assisted nephroureterectomy C Huang

MP13-12 Long-Term Stone Recurrence after Zero-Fragment Laparoscopic Pyelolithotomy compared with Percutaneous Nephrolithotomy H Pakmanesh, R Sharifian-Amiri, S Mohammad-Salehi Kerman University of Medical Sciences

MP13-13 The "N" component of R.E.N.A.L. nephrometry scores correlates with postoperative renal function after robotic-assisted partial nephrectomy.

R Shimizu, E Moriyasu, N Sakamoto, T Hoshino, A Yamamoto, R Nishikawa, S Teraoka,

Y Kimura, H Iwamoto, P Tsounapi, S Morizane, K Hikita, M Honda, A Takenaka

Tottori University Faculty of Medicine, Yonago, Japan

MP13-14 Comparative Analysis of Donor And Allograft Outcomes Based on Laterality of Kidney Donation: Proof of Equivalence of The Safety and Efficacy of Laparoscopic Left vs. Right Donor Nephrectomy

MA Dinno Francis, EL Gerial Jr., JA Abraham, DT Rubio

National Kidney and Transplant Institute
MP13-15 Laparoscopic pyelolithotomy in a cross-fused renal ectopia guided by 3 Dimensions imagine reconstruction

L Ye, M Hu, J Wu, Q Zu, Z Yang, J Wu

MP13-16 Laparoscopic Off-Clamp Partial Nephrectomy for Complex RENAL nephrometry score Tumors; its effects on peri-operative renal function

A Thwaini, M Evans, W Elbaroni, Z Aslam,

K Randhawa

Craigavon Area Hospital

MP13-17 Necessity and feasibility of 4th arm

for Robot-assisted partial nephrectomy by da Vinci Si

S Kira, T Mitsui, N Sawada, H Nakagomi, T Ihara, N Takahashi, M Takeda Interdisciplinary Graduate School of Medicine and Engineering University of Yamanashi

MP13-18 Three versus four arm robotic partial nephrectomy:

Is it worth the hassle?

J El-Asmar, R Sebaaly, A Mailhac, M Bulbul, R Khauli, H Tamim, A Hajj

American University of Beirut Medical Center

MP13-19 Withdrawn

MP13-20 Effect of Perioperative Continuation of Antithrombotic Therapy on Outcome of Robot-Assisted Partial Nephrectomy : A Prospective Clinical Trial at Single-Institution M Kubota, T Matsuoka, Y Tohi, S Murata, N Makita, I Suzuki, Y Sugino, K Inoue, M Kawakita

Kobe City Medical Center General Hospital

MP13-21 Laparoscopic donor nephrectomy:

Strategies to reduce vascular complications and improve outcome

Y Saifee, S Bhatia, C Chamania, J Kriplani, P Salgia, A Sepaha

Choithram Hospital \& Research Centre

MP13-22 Prospective development of a prediction model for postoperative renal function evaluation after unilateral nephrectomies using preoperative risk factors and measured glomerular filtration rate with 51Cr-EDTA

HS Andrade, V Srougi, MA Arap, AI Mitre, CP Camargo, H Zargar, J Kaouk, WC Nahas, M Srougi, RJ Duarte

Clinicas Hospital - University of Sao Paulo 
MP14-01 Can Preoperative Renal Mass Biopsy Change Clinical Practice And Reduce

Surgical Intervention? Z Okhunov, MA Gorin, FA Jefferson, M Allaf, P Pierorazio, J Sung, RM Patel, LM Huynh, S Tapiero, LR Kavoussi, RV Clayman, J Landman University of California, Irvine

MP14-02 Prostate Artery Embolization for Lower Urinary Tract Symptoms - Prostatic Specific Antigen (PSA) Response Patterns

I Ahmed, MM Quraishi, E Latif, B Osman, BA Eddy, EH Streeter, N Das, SS Kommu Kent \& Canterbury Hospital. East Kent Hospitals NHS Foundation Trust

MP14-03 Withdrawn

MP14-04 Pre-Biopsy Multiparametric MRI (mpMRI) In The Prediction of the Pathological Stage at Robotic Assisted Radical Prostatectomy Results From a Series of 1,421 mpMRIs MM Quraishi, M Stanowski, B Osman, N Lobo, I Morrison, E Latif, EH Streeter, SS Kommu, BA Eddy Department of Urology. Kent \& Canterbury Hospital. East Kent Hospitals University NHS Foundation Trust

MP14-05 Utility of prostate-specific antigen density in patients with inconclusive or negative multiparametric magnetic resonance imaging.

L Rico, PN Contreras, L Blas, G Vitagliano, H Ríos Pita, C Ameri

Hospital Alemán

MP14-06 Effectiveness of Lead Aprons in Shielding the Surgeon during Percutaneous Nephrolithotomy

J Smith, M Hajiha, A Amasyali, Z Campwala, H You, M Alsyouf, J Ewald, A Krause,

D Baldwin, R Moghisi, D Baldwin

MP14-07 Diagnostic Accuracy of Computed Tomography Urography (CTU) in Upper Tract Urothelial Transitional Cell Carcinoma (TCC)

S Nalagatla, O Babawale, I Ibrahim, S Prattley, R Lockyer, NO Umez-Eronini, BK Somani, OM Aboumarzouk

Queen Elizabeth University Hospital

MP14-08 Can Patients With Negative Multiparametric MRI Avoid Prostate Biopsy? Results From 1000 Pre-Biopsy Multiparametric MRIs

N Lobo, M Stanowski, MM Quraishi, E Latif, I Morrison, M Eraibey, SS Kommu, EH Streeter, BA Eddy

Kent \& Canterbury Hospital. East Kent Hospitals University NHS Foundation Trust

MP14-09 Factors influencing fluoroscopy use during Ureteroscopy at a residency training program SP Hager, S Desai, M Amster, N May Main Line Health, Mercy Health Network
MP14-10 Do we protect ourselves? Review of radiological protection during endourological procedures with fluoroscopy

JM López, A Sierra, P Luque, A Alcaraz

Clínic Hospital Barcelona - University of

Barcelona, Urology department

Barcelona/Spain, Spain

MP14-11 Multiparametric Magnetic Resonance Imaging is a Cost Effective Method in the Evaluation of Renal Masses for Clear Cell Renal Cell Carcinoma

RL Steinberg, BA Johnson, R Ghandour, R Rasmussen, A Garbens, Y Xi, T Yokoo, A Diaz de leon, I Pedrosa, JA Cadeddu University of Texas Southwestern

MP14-12 Is characterization of renal stone composition by using fast kilovoltge switching dual-energy computed tomography can detect $100 \%$ of uric ucid stone?

U Romsaithong, K Jongjitaree, P Korpraphong,

V Woranisarakul, T Taweemonkongsap,

C Nuanyong, E Chotikawanich

Siriraj Hospital

MP14-13 First French experience of prostate targeted biopsies with URONAV system

V Bailly, P Henry, C Darcq, M Hintzy

Centre d'Urologie, clinique Saint Vincent, Besançon - FRANCE

MP14-14 "The Dirty Prostate" - Background Peripheral Zone Apparent Diffusion Coefficient Does Not Appear to Differ Between False and True Negatives on Prostatic mpMRI

E Wajswol, Y Kuo, H Koster, A Bogomol,

K Basralian, MD Stifelman, M Naik

Hackensack Meridian Department of Urology

MP14-15 Final pathology of radical prostatectomy specimens in patients with negative pre-biopsy multiparametric MRI

M Stanowski, N Lobo, EH Streeter, I Morrison, BA Eddy

Frimley Park Hospital

MP14-16 Phase II Multi-Center Trial: MR/US Fusion Guided Ultra-Focal Gold Nanoshell Directed Laser Ablation of Prostate Tumors: Reporting 3-Month Outcomes and Safety Data E Wajswol, AK George, S Diamond, M Davenport, V Tamisetti, JS Winoker, SK Doppalapudi, BA Taouli, SC Lewis, JA Schwartz, S Canfield, AR Rastinehad Hackensack Meridian Department of Urology

MP14-17 Cancer detection rate at MPMRI-TRUS Fusion guided Prostate Biopsy: the PI-RADS version 2 and the role of the radiologist

G Bozzini, U Besana, A Calori, P Broggini, G Pini, C Buizza, M Sighinolfi, B Rocco

ASST Valle Olona - Busto Arsizio VA

MP14-18 Low dose CT KUB in urolithiasis S EL RAI

Al Zahra hospital Sharjah 
MP14-19 Periprocedural and Diagnostic outcomes of Transrectal versus Transperineal US/MRI guided Fusion Prostate Biopsy: Multi-institutional Propensity Score Matched Pair Analysis M Ferriero, M Oderda, V Forte, A Peltier, P Kumar, P Mozer, A Giacobbe, R Mastroianni, G Tuderti, U Anceschi, A Brassetti, J Roche, M Ciccariello, S Guaglianone, T Piechaud, M Rouprèt, P Gontero, G Muto, M Gallucci, G Simone

"Regina Elena" National Cancer Institute

MP14-20 Insertion of a Bioprotect balloon spacer between prostate and rectum prior to photon or proton beam radiotherapy in the treatment of carcinoma of prostate: early experience from a single UK centre

JA Thomas, A Warlow, J Snell, J Clorely, R Davies, JF Lester

Rutherford Centre South Wales
MP14-21 Radiation dose and usage of CTKUB over a 5-year period (2014-2018): Observed trends in a University Hospital

A Pietropaolo, A Ho, T Bryant, I Wilson, F Wall, R Geraghty, BK Somani

MP14-22 Comparison of a Transperineal Versus Transrectal Targeted Biopsy with Magnetic-Resonance/Ultrasound Fusion Guidance for Suspicious Lesions on Prostate Magnetic Resonance Imaging E Wajswol, JS Winoker, H Anastos, SK Doppalapudi, J Sfakianos, P Treacy, C Knauer, SC Lewis, BA Taouli, AR Rastinehad Hackensack Meridian Department of Urology
MP15-01 Withdrawn

MP15-02 Is laparoscopic radical cystectomy really suitable for the elderly?

G Kaneko, S Hattori, S Shirotake, K Kanao,

S Hara, M Oyama

Saitama Medical University International

Medical Center

MP15-03 Complete Hybrid Transvaginal Natural Orifice Transluminal Endoscopic Nephroureterectomy Z Xiaofeng, z guoxi, X Tianpeng, Y Yuanhu, $\mathrm{X}$ Rihai, W Gengqing

MP15-04 Long-term outcome of laparoscopic vesicouterine fistula repair: Experience from a tertiary referral centre

S Mehrotra, S Mehdi, V Singh, S Mehrotra, S Mehdi, S Pandey, R Sinha, V Singh Department of Urology, King Georges Medical university, Lucknow, India

MP15-05 Laparoscopic partial cystectomy for different benign bladder pathologies

AA ALSUNBUL, H El-Tholoth, T Alzahrani, A Al Zahrani

Urology Department, Prince Sultan Military Medical City

MP15-06 Robotic Cystectomy in the Over Eighties Study (RCOES) - A United Kingdom Multicenter Study E Latif, J Noel, V Kusuma, D Moschonas, S Woodhams, M Swinn, P Pavlakis, H Mostafid, B Ayers, R Nair, M Perry, EH Streeter, HR Patel, R Issa, R Thurairaja, N Vasdev, K Patil, PD Rimington, S Khan, SS Kommu Kent \& Canterbury Hospital. East Kent Hospitals NHS Foundation Trust
MP15-07 Comparison of synchronous bilateral transperitoneal and posterior retroperitoneal laparoscopic adrenalectomy in high volume adrenal centers

A Tuncel, J Langenhuijsen, A Erkan,

T Mikhaylikov, M Arslan, Y Aslan, D Berker, Y Ozgok, E Gallyamov, AS Gozen University of Health Sciences, Ankara Numune Research and Training Hospital

MP15-08 Suprapubic-assisted laparoendoscopic single-site surgery (SA-LESS) for secondary ureteral stricture

Z Guoxi, Z Xiaofeng, Y Yuanhu, X Rihai, W Gengqing, W Xiaoning

MP15-09 Impact of a Complex Pelvic Operative Field on Robot-Assisted Radical Cystectomy Morbidity AS Elsayed, NA Aldhaam, B Hull, A Nagra, A Siam, M Durrani, Z Jing, AA Hussein, KA Guru Roswell Park Comprehensive Cancer Center

MP15-10 The mid-long-term follow up after transvaginal natural orifice transluminal endoscopic surgery in urology

Z Xiaofeng, Z Guoxi, Y Yuanhu, X Rihai,

W Gengqing, W Xiaoning

MP15-11 Withdrawn

MP15-12 Single-Port Robotic Surgery Allows Same-Day Discharge in Majority of Cases

R Abaza, OE Martinez OhioHealth Dublin Methodist Hospital

MP15-13 Extraperitoneal Single Port Robotic Prostatectomy: Initial Experience with Same Day Discharge RJ Yau, GV Sawczyn, RJ Valero Carrion, M Eltemamy, J Kaouk Cleveland Clinic Foundation 
MP15-14 RARC and U-configuration ileal neobladder with totally intracorporeal procedure after neoadjuvant chemotherapy in patients with muscle-invasive bladder cancer

C Ohyama, Y Hashimoto, T Yoneyama,

S Hatakeyama, A Imai

Hirosaki University Graduate School of Medicine

MP15-15 Global assessment of outcomes of Robot-assisted radical cystectomy with orthotopic neobladder: Trifecta vs Pentafecta

A Brassetti, U Anceschi, G Tuderti,

S Guaglianone, M Ferriero, F Minisola,

R Mastroianni, R Flammia, M Gallucci, G Simone

"Regina Elena" National Cancer Institute

MP15-16 Perioperative efficacy and long-term survival benefit of Robotic-assisted radical cystectomy in elderly patients: a nationwide multi-institutional study in Japan

H Iwamoto, S Morizane, T Koie, R Shiroki, M Kawakita, T Gondo, K Matsumoto, T Habuchi, A Takenaka, H Kanayama

Tottori University Hospital

MP15-17 Laparoscopic urethrectomy following laparoscopic radical cystectomy T Yoshida Fukuoka Wajiro Hospirtal, Japan

MP15-18 Sex sparing vs standard Robot Assisted Radical Cystectomy with Intracorporeal Padua Ileal Neobladder in female: perioperative, oncologic and functional outcomes

G Tuderti, R Flammia, R Mastroianni, S Guaglianone, F Minisola, U Anceschi, L Misuraca, A Brassetti, M Ferriero,

M Gallucci, G Simone

"Sapienza" University
MP15-19 Innovation and development of laparoendoscopic nephroureterectomy:

one-center experience

Z Xiaofeng, Z Guoxi, Y Yuanhu, X Rihai,

W Gengqing, W Xiaoning

MP15-20 Robotic-Assisted Radical Cystectomy: a multi-centric Brazilian experience and learning curve

M Covas Moschovas, D Chade, MA Arap,

Á Sarkis, W Nahas, L Tanure, G Ebaid, A Fazoli, G Guglielmetti, C Moreira, M Cordeiro,

P Carvalho, R Coelho

Global Robotic Institute

MP15-21 Robotic Revision of Uretero-Iliac Anastomotic Stricture: Technique and Outcome

R Madi

Augusta University Health at the Medical College of Georgia

MP15-22 Laparoscopic adrenalectomy

for pheochromocytoma

in the hemodialysis patient

S Tatarano, H Hayami, M Yonemori, H Enokida,

M Nakagawa, H Yoshino

Department of Urology, Graduate School of

Medical and Dental Sciences,

Kagoshima University

MP15-23 Pathological Staging of Bladder Cancer, What Lies Beneath? Results from the International Robotic Cystectomy Consortium NA Aldhaam, AS Elsayed, C Wijburg, L Richstone, A Wagner, J Palou, M Stoeckle, M Menon, F Gaboardi, T Maatman, A Hemal, B Yuh, Z Jing, M Roupret, P May, J Kaouk, P Wiklund, J Peabody, KA Guru

Roswell Park Comprehensive Cancer Center
V07-01 Rezum Steam Ablation of the Prostate: A New Wave of Day Case, Effective Prostate Surgery with no Sexual Dysfunction M Ravindraanandan, W Mahmalji, Y Phan Hereford County Hospital

V07-02 The New 4D and Standard Prostatic Urethral Lifting (UroLift) Techniques:

Day Case, Effective, No Sexual Dysfunction M Ravindraanandan, W Mahmalji, Y Phan Hereford County Hospital

V07-03 En Bloc MoLEP (Moses HoLEP) With Early Apical Release F Gómez Sancha, ME Rodriguez Socarras, G Saitta, J Reinoso Elbers, J Fernandez Del Alamo, L Llanes Gonzalez, J Gomez Rivas

V07-04 Top-Down Green Light Laser enucleation of the prostate (GreenLEP): Technical Aspects H Elmansy, A Hodhod, FA Oquendo Aponte, W Shahrour
V07-05 Ablation in Holmium Laser Enucleation of the Prostate: A Novel Method to Avoid Termination of Difficult Morcellation BB Whiles, K Glavin, KL Thurmon

V07-06 Top Down technique of Holmium laser enucleation of prostate

K Ramaswami, P Bhirud, R RAVEENDRAN, H Pothiyedath

V07-07 Holmium Laser Enucleation of the Prostate after Failed Urolift: Surgical Considerations for Morcellation

K Parikh, C Dora

V07-08 Thulium Laser enucleation of the prostate: Top or down technique adopting during the early learning curve? M Omar, M El Shazly

V07-09 En-bloc Laser Enucleation of Prostate with Multi-pulse laser

T Chang, J Tseng 
V07-10 Non-anatomical thulium laser enucleation of prostate for elderly BPH patients with oversize prostate volum

Y Jing, Y Zhu, Y Ruan, F Zhao, B Han, S Xia
V07-11 Various techniques of Holmium Laser Enucleation of Prostate

K Ramaswami, P Bhirud, H Pothiyedath

V07-12 Improving the safety profile of morcellation technique during Hoelp

H Pothiyedath, K Ramaswami, P Bhirud
V08-01 Simultaneous Robot-assisted Radical Cystectomy and Nephroureterectomy for Concomitant Bladder and Upper Urinary Tract Urothelial Carcinoma in Patient with Solitary Kidney

A Garayev, O Aytac, F Atuğ

V08-02 Robot-Assisted Radical Cystectomy with Intracorporeal Ileal Conduit in a Patient with Situs Inversus Totalis

F Munshi, C Polotti, SE Elsamra

V08-03 Evolution in technique of robotic intracorporeal continent catheterizable pouch after cystectomy

R Matulewicz, G Chesnut, A Goh

V08-04 Minimally Invasive management of Robot-Assisted Radical Cystectomy Related complications: 15 Years of Experience

NA Aldhaam, AS Elsayed, M Durrani, Z Jing, AA Hussein, KA Guru

V08-05 A Novel Technique of Intracorperal Ileal Neobladder After Robot Assisted Radical Cystectomy: Modified Anatolian Pouch

B Onal, C Demirdag, B Selcuk, B Ercili, S Citgez, A Erözenci

Istanbul University- Cerrahpaşa

V08-06 Treatment of Multipl Diseases in One Session by Robot Assisted Laparoscopic Method:

A Case Report

B Onal, C Demirdag, B Selcuk, B Ercili, U Aferin, M Ertem

V08-07 Robot-assisted radical cystectomy with intracorporeal urinary diversion

V Pavlov, R Safiullin, M Urmantsev, A Deneyko
V08-08 Robotic-assisted anterior pelvic exenteration with intracorporeal urinary diversion

V Pavlov, R Safiullin, M Urmantsev, A Deneyko, R Abdrahimov

V08-09 Robotic intracorporeal Studer pouch reconstruction with internal $\mathbf{J J}$ stenting following robotic radical cystectomy:

Balbay's technique

MD Balbay, AE Canda, MC Kiremit, E Koseoglu

V08-10 Robotic Nerve Sparing Radical Cystectomy: Maintaining Oncologic Efficacy while Improving Quality of Life

M Billah, S Ahsanuddin, G Lovallo, M Stern, M Ahmed

V08-11 Sex-sparing vs standard Robot Assisted Radical Cystectomy with Intracorporeal Padua Ileal Neobladder in female: step-by-step surgical technique, perioperative, oncologic and functional outcomes

G Tuderti, R Mastroianni, R Flammia, S Guaglianone, F Minisola, U Anceschi, L Misuraca, A Brassetti, M Ferriero, M Gallucci, G Simone

V08-12 Tips For Optimizing the Outcome of Robotic Segmental Ureterectomy for Distal Ureteral Urothelial Carcinoma

KE Okhawere, BD Dayal, AA Elbakry, K Meilika, A Beksac, KK Badani
V09-01 Robotic assisted, single-docking nephroureterctomy and bladder cuff excision with regional lymph node dissection W Li, L Tsai, G Chen, C Huang, C Chang, C Yang, $\mathrm{H} \mathrm{Wu}$

V09-02 Single stage transperitoneal robotic nephroureterectomy in patient with ventriculoperitoneal shunt A Veccia, A Tracey, R Autorino
V09-03 Robotic Uretero-Vesical Reimplantation For Ureteral Stricture After Kidney Transplant A Beksac, AA Elbakry, KE Okhawere, BD Dayal, K Meilika, KK Badani

V09-04 Robot assisted supine extra peritoneal (RASE) approach for RPLND: Our experience GK Pooleri, A LAddha

V09-05 Retroperitoneal Approach Single-Site Robot-Assisted Partial Nephrectomy in High Complexity Renal Mass: Step-by-step technique using da-Vinci Xi system

Y Yoon, J Na, D Kim, K Rha, W Han, H Lee 
V09-06 Embolizing left renal artery before Robotic assisted radical left nephrectomy and renal vein thrombectomy to treat left renal cancer with renal vein thrombosis J Wu, M Chen, L Zhang

V09-07 Single-site Robotic-Assisted Pyeloplasty Using the Da Vinci SP Robotic System

RL Steinberg, A Garbens, BA Johnson, JA Cadeddu

V09-08 Perineal Single-Port Approach for Robotic Radical Prostatectomy Using The New SP ${ }^{\circledR}$ da Vinci Surgical System: An option for selected patients GV Sawczyn, RJ Valero Carrion, J GaristoRisco, RJ Yau, J Kaouk
V09-09 Bladder Cuff Excision: An Integral Component of Radical Nephroureterectomy R Pathak, A Hemal

V09-10 Single-port Robotic Partial Nephrectomy Using The New SP ${ }^{\circledR}$ da Vinci Surgical System RJ Valero, GV Sawczyn, J GaristoRisco, RJ Yau, J Kaouk

V09-11 Robotic Single Port Partial Nephrectomy: First Experience Using a Magnetic Retraction Device J Fulla, E Kaplan-Marans, M Palese

V09-12 Bilateral Pyeloplasty performed with the da Vinci Single Port Robotic System: A new approach to a classic case

M Zhang, NH Patel, G Lovallo, M Ahmed
MP16-01 Super-Mini Laser Cystolitholapaxy:

A useful approach in those with challenging retrograde access

H Abboudi, G Celentano, K Adasonla, F Schoofs, S Choong

University College London Hospitals

MP16-02 Laparoscopic nephroureterectomy is safe and established procedure, but it needs to be paid attention to intravesical recurrence

S Shimura, K Matsumoto, M Ikeda, D Ishii, K Tabata, Y Taoka, T Utsunomiya, D Matsuda, N Okuno, A Irie, M Iwamura

Kitasato University

MP16-03 The Endockscope:

A Disruptive Endoscopic Technology

S Lu, R Yoon, C Cottone, FA Jefferson, J Sung, Z Okhunov, S Tapiero, RM Patel, J Landman, RV Clayman

University of California, Irvine

MP16-04 Impact of Photodynamic Diagnosis and Narrow Band Imaging on Detection Rate of Urothelial carcinoma in Transurethral Resection of Non-Muscle Invasive Bladder Cancer

N Makita, S Murata, I Suzuki, M Kubota, Y Tohi, Y Sugino, K Inoue, M Kawakita

Kobe City Medical Center General Hospital

MP16-05 Narrow Band Imaging reduces persistence of cancer in patients with pT1 high grade bladder cancer

R Giulianelli, B Gentile, G Mirabile, L Albanesi, L Mavilla, G Rizzo, P Tariciotti, A Lopes Mendes, P Aloisi, M Vermiglio, R Lombardo

MP16-06 Role of endoscopic management in synthetic sling/mesh erosion following previous incontinence surgery: A systematic review S Sulaiman, BK Somani, A Pietropaolo, T Hughes University Hospital Southampton
MP16-07 Factors impact on intravesical recurrence of endoscopic management for high-grade upper urinary tract urothelial cell carcinoma followed by radical nephroureterectomy with bladder cuff excision

A Sawangchareon, E Chotikawanich

Division of Urology, Department of Surgery,

Faculty of Medicine Siriraj Hospital,

Mahidol University

MP16-08 Endoscopic Bladder Suture after ureteral and bladder cuff excision during laparoscopic Nephroureterectomy

CA Oliveira, AA Barros, R Reis, J Correia-Pinto, E Dias, E Lima

Life and Health Sciences Research Institute, School of Medicine, University of Minho, Braga, Portugal. ICVS/3B's, PT Government Associate Laboratory, Braga / Guimarães, Portugal. Department of Urology, Hospital de Braga, Braga, Portugal

MP16-09 Short term outcome of mini-percutaneous Holmium laser endopyelotomy for treating secondary ureteropelvic junction obstruction O Elgebaly, H Rashad, A Balah, A Zahran Urology Department, Alexandria University

MP16-10 Is single-use flexible endoscope Uscope Pusen performant enough for Endoscopic Stones Recognition?

V Estrade, I Jour, P Meria, J Ferriere, H Bensadoun, J Bernhard, G Capon, F Bladou, G Robert, O Traxer Hospital Pellegrin

MP16-11 En-bloc resection of bladder tumors (ERBT) for pathological staging: the value of lateral margins analysis R Milandri, S Ciarlariello, C Del Prete, L Sarchi, A Eissa, S Puliatti, M Rizzo, M Sighinolfi, G Bianchi, B Rocco, S Micali Department of Surgical, Medical and Dental Morphological Sciences related to Transplant, Oncology and Regenerative Medicine - Section of Urology, University of Modena and Reggio Emilia, Modena, Italy 
MP16-12 Ureteral stent failures and complications in patients with malignant ureteral obstruction NA Pickersgill, K Du, R Figenshau, A Desai, J Vetter, R Venkatesh

Barnes Jewish Hospital/Washington University School of Medicine

MP16-13 A Systematic Review of Transurethral Resection of Ejaculatory Ducts for the Management of Obstructive Azoospermia:

Outcomes and Predictors of Success

A Mekhaimar, M Goble, O Brunckhorst, HM Alnajjar, D Ralph, A Muneer, K Ahmed King's College London

MP16-14 Evaluation of a novel 3-D printed portable cystoscopy device: The "GoScope"

M Landin, A Aydin, B Smith, F Kum, M Khan, P Dasgupta, K Ahmed

King's College London

MP16-15 Withdrawn

MP16-16 Evaluation Of Single-Use Flexible Ureteroscopes Available In The Saudi Market:

In Vitro Comparison Between Four Disposable Ureteroscope Clinically Oriented Approach WK Kamal, M Abuzenada, A Alhazmy, A Al-Solumany, M Alharthi, Y Alobairi Consultant Urology and Endourology King Fahd Hospital Jeddah

MP16-17 Impact of refractive errors on Da Vinci SI robotic system

MB Tuna, AE Kılavuzoğlu, TS Doğanca, Ö Argun, I Tufek, o ozisik, C Obek, A Kural Acibadem Maslak Hospital
MP16-18 Comparison of Multi-tract minimally invasive percutaneous nephrolithotomy and Endoscopic Combined Intrarenal Surgery for Staghorn Renal Calculi: a single institution experience $\mathrm{K} \mathrm{Xu}, \mathrm{Z} \mathrm{Li}$

MP16-19 Sequential Layered Muscle Inclusive Relook TURBT (SeL-MIRT) In High Grade Bladder Cancer - Results from a High Volume United Kingdom Centre

MM Quraishi, B Osman, A Simoes, I Ahmed, E Latif, M Jones, J Hale, OA Ramadan, A Rajagopalan, B Varga, N Shrotri, M Thomas, P Keirandish, BA Eddy, R Krishnan, EH Streeter, SS Kommu, G Papadopoulos

Kent \& Canterbury Hospital. East Kent Hospitals University NHS Foundation Trust

MP16-20 Correspondence between contrast enhanced computed tomography and ureteroscopy in the management of upper tract urothelial carcinoma

A Gallioli, A Territo, F Sanguedolce, F Regis, R Boissier, J Gaya, M Martinez, P Gavrilov, D Vanacore, J Palou, A Breda Fundació Puigvert. Autonomous University of Barcelona. Barcelona. Spain

\section{MP16-22 Withdrawn}

MP16-22 Treatment of iatrogenic ureteric lesions after abdomino-pelvic surgery with metallic stents S Martín Martín, J Torrecilla García-Ripoll, F Lara Pérez, j Diaz Romero, M Bedate Núñez, J Soto Rodríguez, A Ruano Mayo, A Zamora Horcajada, F Natal Alvarez, J Cortiñas González HOSPITAL CLINICO UNIVERSITARIO DE VALLADOLID
MP17-01 Impact of Modified Bladder Neck Suspension on Early Recovery of Continence after Robot Assisted Radical Prostatectomy

H Moon, J Yang, Y Park, W Bae, H Cho, U Ha, S Hong, S Kim, J Lee

Seoul St. Mary's Hospital

MP17-02 The Impact of Body Mass Index (BMI) on Pelvic Lymph Node Yield in High Risk Prostate Cancer

K Parikh, R Bateh, R Pak

Mayo Clinic Florida

MP17-03 Combined Anterior and Posterior Reconstruction during Robotic Assisted Radical Prostatectomy - Efficacy and Early Continence Rates

M Stanowski, N Lobo, N Petrides, MM Quraishi, B Osman, E Latif, SS Kommu, EH Streeter, BA Eddy

Kent \& Canterbury Hospital. East Kent Hospitals University NHS Foundation Trust
MP17-04 Impact of prior Transurethral Resection Of The Prostate on the outcomes of Robotic-Assisted Laparoscopic Prostatectomy: A Systematic Review M Belkovsky, G Magalhaes, J da Cruz, D Ilias, MM Xavier, AL Sousa, CM Silva, F Fakhouri, R Maia, M Srougi, C Passerotti Faculdade de Medicina da Universidade de São Paulo

MP17-05 Does prostate size influence the outcomes of Robotic-Assisted Laparoscopic Radical Prostatectomy (RALP)? MM Xavier, G Magalhaes, M Belkovsky, CM Silva, AL Sousa, J da Cruz, R Maia, S Reis, C Passerotti Hospital Alemão Oswaldo Cruz

MP17-06 Outcomes of Salvage Robotic Pelvic Lymphadenectomy for Recurrent Prostate Cancer Detected on Fluciclovine Positron Emission Tomography (PET) Scan K Parikh, A Shumate, R Pak 
MP17-07 Lessons Learnt From 1010 Consecutive Robotic Assisted Radical Prostatectomies (RARP) MM Quraishi, B Osman, N Petrides, N Lobo, m stanowsk, SS Kommu, B Eddy

Kent Sussex and Surrey

MP17-08 10-Year Oncological Outcomes of Prostate Cancer after Robotic-assisted Laparoscopic Radical Prostatectomy : Single Center Experience

N Yodkhunnatham, V Woranisarakul, $\mathrm{T}$ Taweemonkongsap, T Hansomwong, S Leewansangtong, S Srinualnad

MP17-09 Comparison of Retzius sparing vs standard robot-assisted radical prostatectomy for prostate cancer

A Tahra, U Sen, A Inkaya, E Kucuk, U Boylu University of Health Sciences, Umraniye Teaching Hospital, Urology

MP17-10 Robot-Assisted Simple Prostatectomy: Does Gland Size Affect Outcomes? AM Asghar, Z Lee, D Strauss, A Keehn, DD Eun Temple University

MP17-11 Robotic Salvage Radical Prostatectomy. An efficient yet underutilized treatment for prostate cancer recurrence

R Madi

Augusta University Health at the Medical College of Georgia

MP17-12 Modified simple prostatectomy: an approach to address large volume BPH and associated prostate cancers.

M Covas Moschovas, S Bhat, O Fikret, T Rogers, V Patel

Global Robotic Institute

MP17-13 Comparison between robot-assisted radical prostatectomy and minimum incision endoscopic radical prostatectomy for high-risk prostate cancer following neoadjuvant therapy A Imai, H Yamamoto, S Hatakeyama, T Yoneyama, Y Hashimoto, C Ohyama Hirosaki University Graduate School of Medicine

MP17-14 Early comparison of the Single Port robotic-assisted radical prostatectomy vs its multi-port predecessor

S Crivellaro, WR Halgrimson, YJ Lee, RW Dobbs

University of Illinois at Chicago
MP17-15 Retzius-sparing Robotic-assisted Radical Prostatectomy versus Open Retropubic Radical Prostatectomy: A Prospective Comparative Study with 19-month Follow-up A Abdel Raheem, M Alenzi, K Chang, I Alowidah, A El-Bahnasy, Y Choi, K Rha Urology Department, King Saud Medical City

MP17-16 The Impact of Low Free Testosterone on Prostate Cancer: High Risk Disease, Biochemical Recurrence, and Testosterone Replacement After Radical Prostatectomy

LM Huynh, M Towe, KJ See, J Tran, F el Khatib, $\mathrm{H} \mathrm{Su}, \mathrm{F}$ Yafi, TE Ahlering

UC Irvine Health

MP17-17 Clinical study on Extraperitoneal approach in Robot-Assisted Radical Prostatectomy Compared to Transperitoneal approach S Murata, R Yamaguchi, H Kokubun, N Makita, I Suzuki, R Suzuki, A Fukunaga, Y Abe, M Kubota, Y Tohi, N Tsutsumi, K Inoue, M Kawakita Kobe City Medical Center General Hospital

MP17-18 Management of urethro-vesical anastomotic disruption: Strategies for managing delayed healing of urethro-vesical anastomosis post robotic radical prostatectomy

LM Alzweri, EJ Shaw, R Thomas

Tulane University School of Medicine

MP17-19 Single Port radical prostatectomy versus Xi Multi-Port radical prostatectomy: a human factor analysis follow up study SM Talamini, WR Halgrimson, RW Dobbs, S Crivellaro

University of Illinois at Chicago

MP17-20 Diagnostic and staging performance of mpMRI-US fusion prostate biopsy: prospective analysis on consecutive radical prostatectomy specimens from a multicentre series

M Ferriero, R Flammia, G Tuderti, R Mastroianni, U Anceschi, A Brassetti, M Oderda, P Gontero,

A Giacobbe, G Muto, G Malossini, M Puglisi,

A Peltier, K Pradeep, T Piechaud, J Descotes,

R Papalia, S Guaglianone, M Gallucci, G Simone

"Regina Elena" National Cancer Institute

MP17-21 Urinary and sexual function outcomes at 12 months post-radical prostatectomy: robotic-assisted versus laparoscopic approach C Moore, J Hoogenes, J Saini, A Molawi, B Shayegan, ED Matsumoto McMaster University
MP18-01 Laparoscopic Donor Nephrectomy Without Drainage Does Not Increase Postoperative Morbidity D An, D You, J Han, K Kim, J Aum, Y Kim
MP18-02 Local Regional Recurrence of Renal Cell Carcinoma after Robotic Partial Nephrectomy PM Youssef, JC Deolankar, PD Kothari, SS Komaravolu, D Kim, N Diab, H Koster, M Ahmed, R Munver, G Lovallo, MD Stifelman 
MP18-03 The Impact of Postoperative AKI after Robotic PN on Patient Outcomes and Predictors to Ascertain Risk

O Omidele, M Finkelstein, M Palese

Icahn School of Medicine

MP18-04 Retroperitoneoscopic or laparoscopic? which is the best for posterior tumors partial nephrectomy?

$\mathrm{J}$ Correia, C Tavares, P Principe, R Versos, R Borges, M Silva-Ramos

Department of Urology, Centro Hospitalar Universitário do Porto; Hospital Lusíadas Porto

MP18-05 The Effect of Adhesive Perirenal Fat on Operative Outcomes KE Okhawere, AA Elbakry, BD Dayal, A Beksac, K Meilika, J Daza, J Sfakianos, DD Eun, A Hemal, R Abaza, A Bhandari, R Mehrazin, J Porter, KK Badani Icahn School of Medicine at Mount Sinai

MP18-06 The effect of retroperitoneal approach at robotic partial nephrectomy for post-operative VAS pain score: Match-Paired Case-Control Study

B Kim, T Shin, W Jung, J Ha, c Park, C Kim Department of Urology, Keimyung University School of Medicine, Dongsan Hospital

MP18-07 Surgical treatment of local retroperitoneal recurrence after retroperitoneal laparoscopic radical nephrectomy for renal cell carcinoma H Kim, H Choe, D Lee, S Lee St. Vincent's Hospital

MP18-08 Learning Curve In Robotic Assisted Kidney Transplantation: Results From The ERUS RAKT Working Group A Territo, A Gallioli, R Boissier, R Campi, D Vanacore, A Alcaraz, M Musquera, K Decaestecker, V Tugcu, S Serni, A Breda Fundació Puigvert. Autonomous University of Barcelona. Barcelona. Spain

MP18-09 Robot Assisted Renal Allograft Nephrectomy H Abdul-Muhsin, S McAdams, A Syal, R Nunez-Nateras, A Navaratnam, KM Rose, K Farraj, E Castle Mayo Clinic, Arizona

MP18-10 Outcomes of Laparoscopic Donor Nephrectomy in presence of double renal arteries R Mohsin, B Ali, A Shahzad, M Hussain Sindh Institute of Urology and Transplantation

MP18-11 Parenchymal Volume Loss Affects Postoperative Contralateral Kidney Volume Following Partial Nephrectomy

A Beksac, KE Okhawere, AA Elbakry, BD Dayal, J Daza, K Meilika, KK Badani Icahn School of Medicine at Mount Sinai
MP18-12 Enhanced Recovery After Surgery (ERAS) Programme for Robotic Assisted Partial Nephrectomy (RAPN) - Outcomes in a High Volume Robotic Urology Centre MM Quraishi, B Osman, E Latif, N Lobo, M Jones, SS Kommu, BA Eddy, M Thomas, $S$ Matthew

Kent \& Canterbury Hospital. East Kent Hospitals University NHS Foundation Trust

MP18-13 Operative and functional outcomes for selective clamping in robotic partial nephrectomy for patients with a solitary kidney KK Badani, PD Kothari, JC Deolankar, PM Youssef, D Kim, SS Komaravolu, M Billah, KE Okhawere, H Koster, R Munver, M Ahmed, G Lovallo, MD Stifelman Hackensack University Medical Center

MP18-14 Oncological Outcomes of distal ureter management followed by Laparoscopic/Robotic nephroureterectomy for Upper Tract Urothelial Carcinoma W Kuo, J Lin, C Yu Kaohsiung Veteran General Hospital

MP18-15 Zero Ischemia Robot-Assisted Partial Nephrectomy: Trifecta Outcomes MB Tuna, TS Doğanca, I Tufek, Ö Argun, C Obek, A Kural Acibadem Maslak Hospital

MP18-16 Long-term oncologic outcomes after robotic assisted partial nephrectomy for renal cell carcinoma

K Du, J Vetter, A Paradis, R Venkatesh, R Figenshau Washington University in St. Louis

MP18-17 Robotic Partial Nephrectomy Outcomes in Highly Complex Kidney Cancers: Are We Pushing the Boundaries Too Far? D Kim, NH Patel, PM Youssef, JC Deolankar, PD Kothari, SS Komaravolu, M Billah, H Koster, Y Kuo, M Ahmed, G Lovallo, R Munver, MD Stifelman

Hackensack Meridian Department of Urology

MP18-18 Are There Any Functional Benefits From Ischemia Sparing Techniques among Patients with Chronic Kidney Disease? A Beksac, KE Okhawere, D Rosen, D Paulucci, AA Elbakry, BD Dayal, J Sfakianos, R Abaza, PD Kothari, DD Eun, A Bhandari, A Hemal, J Porter, MD Stifelman, KK Badani Icahn School of Medicine at Mount Sinai

MP18-19 Robot assisted radical nephrectomy and inferior vena cava thrombectomy: surgical technique, perioperative and mid-term oncologic outcomes U Anceschi, G Tuderti, A Brassetti, M Ferriero, F Minisola, M Costantini, S Guaglianone, R Flammia, R Mastroianni, M Gallucci, G Simone "Regina Elena" National Cancer Institute 
MP18-20 Evaluation of postoperative parenchymal reduction with or without renorrhaphy using computed tomography volumetry in robotic-assisted partial nephrectomy

R Sagehashi, T Inoue, S Nakajima, S Kanda,

T Nara, K Numakura, M Saito, S Narita, S Satoh,

T Habuchi

Akita University School of Medicine
MP18-21 Head to head impact of MIC vs a novel TRIFECTA score on oncologic and functional outcomes after robotic assisted partial nephrectomy: results of a multicentric series

U Anceschi, R Bertolo, A Brassetti, G Tuderti,

U Capitanio, M Ferriero, A Larcher, A Veccia,

A Mottrie, A Antonelli, A Minervini,

P Dell'Oglio, I Derweesh, F Porpiglia,

F Montorsi, J koauk, R Autorino, M Gallucci,

G Simone, R Mastroianni

MP18-22 Fate of Residual Ureteral Stump in Patients Undergoing Incomplete Robot-assisted Radical Nephroureterectomy for High-risk Upper Tract Urothelial Carcinoma

R Pathak, A Hemal

Wake Forest University
MP19-01 Evaluation of a routine clinical prostate multiparametric MRI and Decipher Score for prediction of Biochemical Recurrence in patients who underwent robotic-assisted laparoscopic prostatectomy

P Ratnani, U Falagario, I Jambor, A Martini, S Sobotka, A Beksac, P Wiklund, S Nair, A Tewari, T Bashorun, D Phillip, R Weil Icahn School of Medicine

MP19-02 Outcomes of radical prostatectomy in transplanted- kidney patients who have localized prostate cancer

W Kongcharoensombat, W Kongchareonsombat Mahidol University

MP19-03 A Multicentre United Kingdom Study of Robot Assisted Ureteric Reimplantation E Latif, J Chong, J Pain, M Wright, EH Streeter, RA Persad, PD Rimington, SS Kommu, BA Eddy Kent \& Canterbury Hospital. East Kent Hospitals NHS Foundation Trust

MP19-04 Decreased Short-Term Complications in Minimally-Invasive Approaches to Nephrectomy in the Elderly PC Yong, A Kaldany, KK Badani Icahn School of Medicine at Mount Sinai

MP19-05 Open Retrieval Post Retroperitoneoscopic Radical Nephrectomy does not increase risk of port site recurrence

N Kale, Y Wagh, H Godbole, F Harris

Department of Urology, North

Middlesex Hospital

MP19-06 Withdrawn

MP19-07 The Role of the Prostatic Vasculature as a Landmark for Bladder Neck Sparing during Robot-assisted Radical Prostatectomy

M Shahait, A Sandberg, DI Lee

University of Pennsylvania
MP19-08 Do Dialysis and Transplant Patients have Worse Surgical Outcomes with Laparoscopic Nephrectomy Compared to Normal Controls? K Parikh, A Kahn, D Thiel Mayo Clinic Florida

MP19-09 The use of multiple video assessment methods to determine the influence of surgical skill on potency and continency in patients after robot-assisted radical prostatectomy

A Beulens, W Brinkman, R Meijer, E Koldewijn, $\mathrm{J}$ van Basten, $\mathrm{H}$ van der Poel, J van Merriënboer,

C Bangma, C Wagner

Catharina Hospital Eindhoven

MP19-10 Face and Content Validity of a novel chicken model for Laparoscopic Neobladder Reconstruction

A Singh, Z Kareem, GR TAK, A Ganpule, R Sabnis, M DESAI

Muljibhai Patel Urological Hospital

MP19-11 Laparoscopy has better conditional survival than open radical nephroureterectomy in patients with stage pT3 advanced UUTC: a retrospective multicenter conditional survival analysis

M Song, B Hong, S Kang, B Jeong, J Ku, H Seo, S KIM

MP19-12 Indocyanine Green Angiography for Use in Robotic Spermatic Cord Denervations

NV Halstead, SP Catarinicchia, GL Lloyd

MP19-13 Factors associated with Increasing trend in benign pathology at partial nephrectomy for cT1 tumors in the era of advanced renal imaging

AK Chow, A Sivaraman, R Figenshau, J Ippolito, J Vetter, R Venkatesh

Washington University School of Medicine, St. Louis, USA 
MP19-14 The Efficiency and Safety of Different Analgesic Drugs for patients with Postoperative Pain in Department of Urology: A Randomized Controlled Trial

D Ye, L Ma, L Liu, B Liao, H Li, K Wang

West China Hospital

MP19-15 Robot-Assisted Nephrouretercomy A Case Series.

S Nalagatla, I Ibrahim, J Hendry,

OM Aboumarzouk

Queen Elizabeth University Hospital

MP19-16 Drains Are No Longer Necessary

in Urologic Surgery

R Abaza, OE Martinez

OhioHealth Dublin Methodist Hospital

MP19-17 Withdrawn

MP19-18 Enigma of the crossing vessel in pelviureteric junction obstruction

S Sangaralingam, H Godbole, C Carr, H SafarAly,

N Kale

Department of Urology, North

Middlesex Hospital
MP19-19 Aim: To assess the feasibility of Robotic assisted kidney transplantation in Graft with multiple vessels (GMV).

K Thekke Adiyat, V Raveendran

Aster Medcity

MP19-20 Prospective comparative study on robot-assisted vs open kidney transplantation: trend to less perioperative inflammatory response and similar functional results

A Territo, J Subiela, G Theil, L Gausa, F Regis, R Boissier, D Vanacore, M Nasreldin, P Fornara, A Gallioli, L Guirado, A Breda

Fundació Puigvert. Autonomous University of Barcelona. Barcelona. Spain

MP19-21 Initial Outcomes of Minimally Invasive versus Open Inguinal Lymph Node Dissection SS Pandya, NJ Farber, R Patel, KD Radadia, J Sterling, A Tabakin, RE Weiss, IY Kim, TL Jang, EA Singer, SE Elsamra

MP19-22 Risk factor for erectile dysfunction after robot-assisted radical prostatectomy Y Nakano, J Furukawa, K Harada, K Shigemura, T Ishimura, N Hinata, M Fujisawa
MP20-01 Trends of urolithiasis management in the Chilean private health system from 2008 to 2018 GM Astroza, VI Giraudo Urology Department, Pontificia Universidad Catolica de Chile

MP20-02 Incorrect imaging for hematuria workup across specialties is leading to poor value of healthcare

B Adamic, J Aizen, C Labbate, S Park

MP20-03 Post-operative narcotics prescribing patterns following endoscopic procedures: How much is too much?

C Tabib, K Cwach, P Mistry, M Schwartz Arthur Smith Institute for Urology

MP20-04 Clinical, fiscal and environmental benefits of a specialist led virtual ureteric colic clinic: a report of a prospective study

MJ Connor, S Miah, M Edison, J Brittain,

M Kondjin-Smith, C Gan, M Hanna, R Dasgupta, T El-Husseiny

Imperial College Healthcare NHS Trust, Charing Cross Hospital

MP20-05 High Fidelity Registry Demonstrates Differences in Stone Presentation Between China and the United States:

Results from ReSKU

J Ahn, T Chi, T Chi, J Li, W Hu, M Stoller, Y Tang

University of California San Francisco
MP20-06 Is the introduction of single use flexible ureteroscopes capable of preventing reusable scopes breakages? Results from a high-volume center

E Ventimiglia, S Doizi, L Villa, O Traxer Division of Experimental Oncology/Unit of Urology; URI; IRCCS Ospedale San Raffaele

MP20-07 New Persistent Opioid Use after Outpatient Ureteroscopy for Upper Tract Stone Treatment C Tam, CA Dauw, K Ghani, V Gunaseelan, T Kim, D Leavitt, J Raisky, JM Hollingsworth University of Michigan

MP20-08 Streamlining Costs of Robot-Assisted Radical Prostatectomy: Can We Optimize the Financial Burden? AA Hussein, AM Toenniessen, NA Aldhaam, AS Elsayed, Z Lone, P May, S Tauber, K Sniadecki, R Grady, KA Guru Roswell Park Comprehensive Cancer Center

MP20-09 Management of Rare PCNL Complications is a Fellowship Required?

D Mikhail, L Griffiths, Z Kozel, C Tabib, A Rai, D Hoenig, Z Okeke, AD Smith

MP20-10 Over-investigation of Non-visible Hematuria Optimizing Current Platforms to Reduce Excessive Investigations MM Quraishi, A Simoes, B Osman, J Dorrell, G Papadopoulos, M Thomas, P Kheirandish, R Krishnan, BA Eddy, EH Streeter, SS Kommu, N Shrotri

Kent \& Canterbury Hospital. East Kent Hospitals University NHS Foundation Trust 
MP20-11 Time Trends in Metabolic Diagnoses of Recurrent Stone Formers

S Dianatnejad, K Piranviseh, S Tapiero, P Jiang,

$\mathrm{R}$ Youssef Yaacoub

University of California, Irvine

MP20-12 Withdrawn

MP20-13 Kidney stones in black women in the United States: Data from the Black Women's Health Study M D'Amico, S Wason, L Rosenberg, Y Cozier Boston Medical Center Department of Urology

MP20-14 Utilization and 5-Year treatment free rates for Radical Nephrectomy and Partial Nephrectomy for the diagnosis of Renal Cell Carcinoma between 2000-2014 in New York State KH Bilal, M Finkelstein, M Palese Mount Sinai Medical System

MP20-15 The Impact of "Freeing Up" The On-Call Consultant From Elective Activity on Emergency Urological Admissions, Length of Hospital Stay and Re-Admission Rates

A Simoes, N Shrotri, P Keirandish, J Hale, D Bogard, G Papadopoulos, M Thomas, BA Eddy, EH Streeter, R Krishnan, SS Kommu

Kent \& Canterbury Hospital

MP20-16 A pattern of increased presentation of patients with kidney stones during Abu Dhabi's summer months

Z Almallah, D Delaney, A Alaqqad, A Burham Cleveland Abu Dhabi

MP20-17 A Systematic Review of the Effects of Intraoperative Stress on Surgical Performance E Good, N Raison, M Khan, P Dasgupta, $\mathrm{K}$ Ahmed King's College, London
MP20-18 The Cost of Gross Hematuria following Robotic-Assisted Laparoscopic Partial Nephrectomy

O Kott, DW Sobel, B Golijanin, C Mueller-

Leonhard, O Alalao, C Tucci, G Pareek,

D Golijanin

Brown University

MP20-19 Fate of normal hematuria investigations patients in the immediate to long term - outcomes and clinical implications

G Kanda Swamy, R David, B Rai, B James,

K Thomas, P Bose

Swansea Bay University Health Board

MP20-20 The Impact of Video Visits on Follow-Up Care for Robot Assisted Radical Prostatectomy Patients J Kim, c GU, E Kim, M Shahait, RW Dobbs, DI Lee

University of Pennsylvania

MP20-21 Cystoscopic follow-up of low-risk bladder Transitional Cell Carcinoma -Are the National Institute of Health and Care Excellence guidelines from the United Kingdom appropriate in suggesting discharge after 12 months of follow up?

M Alsawi, H Burns, R Sohail Khan, J De Souza Department of Urology -University

Hospital Monklands

MP20-22 Recommendations for Opioid Prescribing after Endourological Surgery:

An Expert Panel Consensus

K Koo, F Faisal, N Gupta, A Meyer, H Patel, P Pierorazio, B Matlaga

Johns Hopkins University School of Medicine
V10-01 3D Retroperitoneoscopic nephrectomy. A patient friendly procedure A Soomro, M Azad, S Saulat, S Qadri, A Ayub, M Ejaz

V10-02 Retroperitoneoscopic Partial Nephrectomy The technique step by step C Wülfing, D Marghawal, O Andura

V10-03 "Unlock” accidents of polymeric Hem-O-Lok clips during laparoscopic kidney surgeries T Abe, A Sazawa, D Iwami, T Osawa, R Matsumoto, H Kikuchi, S Murai, N Shinohara

V10-04 Laparoscopic resection of retrohepatic suprarenal mass D Tan, A Sim Soon Phang

V10-05 Robotized Laparoscopy In Stone Disease R Raveendran, K Ramaswami, P Bhirud, $\mathrm{H}$ Pothiyedath

V10-06 Laparoscopic Ureterolithotomy for large distal ureteric stone JM López, R Martos, A Sierra, M danna, P Luque, A Alcaraz
V10-07 Laparoscopic Management of Vascular Injuries C Vaddi, S PM, R Paidakula, P Gopi, M B, S Ganesan

V10-08 Ureteral Obstruction Due To Crossing Vessel At The Level Of The Pelvis

HV Patel, BJ Lichtbroun, SE Elsamra Rutgers Robert Wood Johnson Medical School

V10-09 Laparoscopic Transposition of Cecal Appendix in Ureteral Injury Repair CA Gonzalez-Santana, D Soriano-Morillo, MA Fernandez-Camilo, JP Mejía-Rosario, I Peralta-Bernal, M Joa-Cabrera, G Castillo, M Pichardo-Garcia

V10-10 Withdrawn

V10-11 Total Laparoscopic Retroperitoneal tumor excision: challenging but possible J Jagtap, S Veer, G Agrawal, P Rao, S Mistry 
V11-01 Bladder Neck Sparing Robot-assisted Simple Prostatectomy M Shahait, DI Lee

V11-02 Robotic-Assisted Excision of Retroperitoneal Schwannoma A AlMulhim, A AlGosaibi, AM Alenizi

V11-03 Testicular vein syndrome:

First described case of robotic assisted treatment JB de Oliveira, L Welter, VR Jr, CP dos Santos, HO Santos, GF Canuto, TB Santana, LM Calazans, NJ Barretto

V11-04 Robotic-Assisted Laparoscopic Excision Of Calyceal Diverticulum And Removal Of Calyceal Calculi Y Phan, M Ismail, O Karim

V11-05 Simplified transvescical robot-assisted simple prostatectomy: technical nuances A Veccia, A Balthazar, L Hampton, R Autorino

V11-06 Robotic Fascial Sparing Rectus Abdominis Harvest LC Zhao, ML Schulster, ML Schulster, GW Dy, NM Vranis, JP Levine, M Jun
V11-07 Robotic Bladder Neck Reconstruction Techniques for Retropubic and Trans-Vesical Approaches A Beksac, AA Elbakry, KE Okhawere, BD Dayal, K Meilika, R Purohit, KK Badani

V11-08 Robot-assisted transvesical simple prostatectomy with concurrent bladder stone removal.

O Aytac, A GARAYEV, F Atuğ

V11-09 Robotic assisted Ureteric Mitrofanoff conduit with Augmentation Cystoplasty and Malone Antegrade Continence Enema procedure S Harinatha, M Keshavamurthy, S Tabrez, P Kumar, B Neelagar

V11-10 Robot-Assisted Radical Prostatectomy of a $500 \mathrm{~g}$ Gland AM Asghar, Z Lee, DD Eun

V11-11 Combined robot assisted laparoscopic nephrectomy and ureterocystoplasty C Wetterauer, A Feicke, S Moehr, T Horn, H Seifert

V11-12 Robotic assisted repair of traumatic intraperitoneal bladder rupture M Stanowski, MM Quraishi, E Latif, SS Kommu, BA Eddy
V12-01 Laparoscopic pyelolithotomy in a cross-fused renal ectopia guided by 3 Dimensions imagine reconstruction

$$
\text { L Ye, J Wu, Y Wei }
$$

V12-02 3-D Transperitoneal Lap anterior exenteration in females with muscle invasive bladder carcinoma :A prospective evaluation

A Kumar, S Yadav, S Kumar, Y Prashant, K Saurav, V Tyagi, V Prasad

V12-03 Modified Ileal Conduit Intracorporeally Accomplished following Laparoscopic Radical Cystectomy Y Niu, H Wei

V12-04 Laparoscopic Excision Of Infected Urachal Cyst Following Laparoscopy Assisted Vaginal Hysterectomy C Vaddi, S PM, R Paidakula, P Gopi, M B, S Ganesan

V12-05 Radical cystectomy, urethrectomy and ileal substitution in woman C CONDE REDONDO, F Castroviejo Royo, F Lara Pérez, J Torrecilla, S Martín Martín, A Alonso Villalba, R Cortiñas Gonzalez

V12-06 Suprapubic cystoscopy guided Heygrove dilator placement in anastomotic urethroplasty for PFUI S Nachimuthu, R Manickam, S Kallappan
V12-07 Laparoscopic treatment of uterovesical fistula after caesarian delivery

V Agarwal

V12-08 Laparoscopic urachal remnant excision DG Sucupira, HS Andrade, MA Arap, V Srougi, G Eufrasio da Silva, A Mitre, RJ Duarte, M Srougi

V12-09 Recurrent Epididymo-orchitis: Uncommon Presentation of Zinner Syndrome F Alhajeri, S Salem, A Harraz, S Alkhudir, K Alali

V12-10 Technical Tips In Laparoscopic Excision Of Giant Bladder Diverticulum S Nachimuthu, R Manickam, S Kallappan

V12-11 A new transurethral approach to repair a Vesicovaginal Fistula

E Hernandez-Mendez, G Morales-Montor, C Martinez-Arroyo, P Cortes-Raygoza, I Calvo-Vazquez, M Cantellano-Orozco, G Fernandez-Noyola, C Pacheco-Gahbler

V12-12 Laparoscopic VVF Repair Using Barbed Suture And 3D Camera

R Manickam, S Nachimuthu, S Kallappan

V12-13 Rare cause of persistent hematuria: Diverticulum formation after conservative management of extraperitoneal bladder injury associated with pelvic bone fracture Gr Choudhary, P Gupta, L Pallagani 
MP21-01 Introduction of a nurse led stent removal service using the single use ISIRIS in a tertiary referral stone unit and the effect on patient waiting times J Collie, S Shah, J Durrant, S Al-Hayek,

K Saeb-Parsy, O Wiseman

Cambridge University Hospitals NHS Trust

MP21-02 The Importance of Stone Retrieval In The Modern Management Of Urolithiasis: The UroPro Stone catcher vs Traditional Sieving A Harbias, M Ravindraanandan, W Mahmalji Hereford County Hospital

MP21-03 Ureteral Access Sheath-Related Injuries VS Post-Operative INFECTIVE conditions: is sheath insertion always needed? G Bozzini, U Besana, A Calori, S PROIETTI, G Pini, C Buizza, M Sighinolfi, B Rocco ASST Valle Olona - Busto Arsizio VA

MP21-04 Ureteral stenting after routine ureteroscopy: is earlier stent removal feasible?

S Hill, A Boyko, S Merheb, M Biebel, M Katz, R Babayan, S Wason, D Wang

Boston Medical Center Department of Urology

MP21-05 Benchtop Comparison of 13 Different Guidewires in 3 Different Impacted Stone Models A Amasyali, J Groegler, M Shah, M Hajiha, J Maldonado, M Alsyouf, P Stokes, D Baldwin Loma Linda University Health

MP21-06 Retrospective study of the effect of preoperative indwelling double $\mathbf{J}$ tube on ureteroscopic lithotripsy M Li, G Bao, C Li, Z Gao

MP21-07 Prospective Randomized Comparison of Standard Hand Pump Infuser Irrigation and an Automated Irrigation Pump for Operating Room Efficiency During Percutaneous Nephrolithotomy and Ureteroscopy

FA Jefferson, J Sung, L Limfueco, S Lu, C Cottone, S Tapiero, RM Patel, P Ghamarian, R Youssef Yaacoub, RV Clayman, J Landman University of California, Irvine

MP21-08 The Safety of Ureteral Stenting with The Use of Potassium Citrate for Management of Renal Uric Acid Stones

M El Shazly, N Alenezi, F Zanaty, E El Sherif, A Badawy, M Elgharabawy

MP21-09 The Japanese linguistic validation of the Ureteral Stent Symptoms Questionnaire (USSQ).

J Matsuzaki, T Matsuda, A Yamaguchi, S Mugiya, K Wada, J Miyazaki, S Yamamoto

Department of Urology, Ohguchi Higashi General Hospital, The section meeting for ureteral stent in Japanese Society of Endourology

MP21-10 Ureteric stent with extraction string - strategies to reduce complications

S Mukherjee, C Popanes, A Raza

Dartford and Gravesham NHS Trust
MP21-11 The Utility of Instruments and Disposables during Endourologic Practice among Egyptian Urologists

M Omar, K Sayedahmed, YA Noureldin

Menoufia University

MP21-12 Randomized clinical trial on urinary pH monitoring and nutraceutical intervention in the prevention of ureteral stent incrustation.

J Fernández-Concha Schwalb, C Torrecilla Ortiz, J Ramón Cansino Alcaide, J Antonio Mainez, J H. Amón Sesmero, S Costas Ochoa, O Angerri Feu, E Emiliani, M Arrabal Martin, M A. Arrabal Polo, A García Romero, M Carlos Reina Ruiz,

J Francisco Sánchez García, A Budía Alba,

D Pérez-Fuentes, F Grases Freixedas,

A Costa-Bauzá, J Cuñé Castellana

La Fe University and Polytechnic Hospital

MP21-13 Impact of Ureteral Access Sheath on Renal Stone Treatment: Prospective Comparative Non-Randomized Outcomes over a 7-year Period

A Lima, T Reeves, R Geraghty, A Pietropaolo,

L Whitehurst, BK Somani, T Hughes

University Hospital Southampton

MP21-14 PCNL Tract Dilation Efficiency - Balloon vs. Sequential Dilators?

D Mikhail, V Vasudevan, A Rai, A Perez-Orozco, S Patwa, D Hoenig, AD Smith, Z Okeke

MP21-15 Does ureteral balloon dilatation for ureteral access sheath (UAS) placement during retrograde intrarenal surgery (RIRS) increase risk of de novo ureteral strictures? : a retrospective comparative study

J Choi, S Jeon, M Baek, H Jeon, H Sung,

M Kang, D Han

Samsung Medical Center, Sungkyunkwan

University School of Medicine, Seoul, Korea

MP21-16 Holmium Laser versus thulium fiber laser for treatment of urinary calculus disease PK Pattnaik, SK Pattnaik, MP Pattnaik S S Urological \& Research Institute, BOMBAY HOSPITAL PG INSTITUTE

MP21-17 JJ stent attached to a string - reasons for low uptake by urologists despite benefits to patients and improved utilization of hospital resources

S Mukherjee, C Popanes, A Raza

Dartford and Gravesham NHS Trust

MP21-18 Impact of the Ureteral Stent Diameter on Ureteral Stent-Related Symptoms

M Taguchi, K Yoshida, M Sugi, H Kinoshita,

T Matsuda

Department of Urology, Osaka Saiseikai

Izuo Hospital 
MP21-19 Temperature rise during Laser Lithotripsy: Comparison of Super Pulse Thulium Fiber LASER (SPTF) vs High Power 120W

Holmium-YAG LASER (Ho:YAG)

WR Molina, BH Chew, BE Knudsen

University of Kansas Medical Center

MP21-20 In Vitro Assessment of the Olympus URF-P7 and V3 Flexible Ureteroscopes and Comparison to Prior Generation Scopes

RS Terry, B Winship, K Boydston, P Whelan,

E Carlos, G Preminger, M Lipkin

Duke Urology
MP21-21 Analysis of ureteral stents encrustation and biofilm formation after 3-week dwell time: Results of a Prospective Randomized Multicentre Clinical Comparative Study of Silicone Hydrocoated (Imajin ${ }^{\circledR}$ hydro) versus Percuflex ${ }^{\mathrm{TM}}$ Plus

O Wiseman, J Letendre, J Cloutier, M Daudon, F Kleinklauss, S Doizi, O Traxer Cambridge University Hospitals NHS Trust

MP21-22 Comparison of Conventional 30, 120 Watt, and 120 Watt Moses Laser in a Benchtop Cystolitholapaxy Model

M Hajiha, A Amasyali, J Groegler, Z Campwala, J Smith, A Goyne, D Baldwin, R Moghisi, M Shah, M Alsyouf, P Stokes, R Belay, D Baldwin
MP22-01 Urinary metabolic abnormalities in patients with pure vs. mixed uric acid stones RA Chandhoke, B Gallante, JN Bamberger, W Atallah, M Gupta, AM Zampini Mount Sinai

MP22-02 Buyer Beware: Evidence-Based Evaluation of Dietary Supplements for Nephrolithiasis K Koo, T Aro, B Matlaga Johns Hopkins University School of Medicine

MP22-03 Risk Factors for the Development of Multiple-Drug Resistant Bacteria in Patients With Urinary Stones S Wang, Y Zhang, X Zhang, Y Tang, B Xiao, $\mathrm{J} \mathrm{Li}$

University of Maryland School of Medicine

MP22-04 Impact of Demographic Factors and Systemic Disease on Urinary Stone Risk Parameters Amongst Stone Formers K Wood, C Boyd, B Gower, W Poore, D Assimos University of Alabama at Birmingham

MP22-05 Renal Stone Disease in Patients with Primary Hyperparathyroidism - Findings in a Tertiary Referral Stone Centre MM Quraishi, T Olaomi, I Ahmed, SS Kommu, J Davis

Kent \& Canterbury Hospital. East Kent Hospitals University NHS Foundation Trust

MP22-06 Association between visceral adipose tissue loss post-bariatric surgery and 24-hour urinary profile changes

M Uy, R DiLena, J Hoogenes, B Al-Harbi, A Woodward, S Gmora, B Shayegan, ED

Matsumoto

McMaster University

MP22-07 Screening for Primary Hyperparathyroidism in a Tertiary Stone Clinic, a Useful Endeavor K Wood, C Boyd, D Assimos University of Alabama at Birmingham

MP22-08 Withdrawn
MP22-09 Does Severity of Disease Impact Efficacy of Pharmacotherapy Versus Conservative Therapy in Reducing Stone Recurrence? BA Johnson, RL Steinberg, SL Best, SY Nakada, T Marien, C Tracy, N Miller, M Pearle, Y Lotan, JA Antonelli

University of Texas Southwestern

MP22-10 Are we following NICE Guidelines for metabolic stone screening?

M Ravindraanandan, P Jayawardena

Hereford County Hospital

MP22-11 24-hour Creatinine Excretion is a Poor Indicator of Adequate 24-hour Urine Collection in Obese Stone Formers

BA Johnson, RL Steinberg, SL Best, SY Nakada, C Tracy, N Miller, T Marien, M Pearle, JA Antonelli, Y Lotan

University of Texas Southwestern

MP22-12 Healthcare utilization of alpha-blockers for medical expulsive therapy in patients with ureteral stones - a United States national claims database study

SP Argade, A Desai, J Vetter, M Keller, M Olsen, A Paradis, A Parker, R Venkatesh

Barnes Jewish Hospital/Washington University School of Medicine

MP22-13 Use of sipIT Intervention to Reduce Common Perceived Barriers to Increasing Fluid Intake Among Adult Patients with Kidney Stones NM Streeper, D Brunke-Reese, E Thomaz, A Sanders, D Conroy

Penn State Health

MP22-14 Comparison of 24-hour Urinary Sodium and Potassium Excretion in U.S. Stone Formers vs. Non-Stone Formers from 2010-17

KA Healy, O Shah, G Li, J Asplin Columbia University Medical Center

MP22-15 Withdrawn 
MP22-16 Factors That Contribute to Inadequate 24-hour Urine Collections in Patients with Nephrolithiasis: a Multi-center Analysis

BA Johnson, RL Steinberg, SL Best, SY Nakada, C Tracy, N Miller, T Marien, M Pearle, JA Antonelli, Y Lotan

University of Texas Southwestern

MP22-17 Post-operative radiographic and 24-hour urine profile changes in bariatric patients with a history of nephrolithiasis: risk factors for recurrent stone formation associated with Roux-en Y gastric bypass versus sleeve gastrectomy

M Uy, R DiLena, J Hoogenes, B Al-Harbi, S Gmora, B Shayegan, ED Matsumoto

McMaster University

MP22-18 Metabolic evaluation of adults with Urolithiasis: guidelines vs practice

L Saunders, E Maher, F Hinds, N Thakare,

SH Moochhala, R Kucheria, D Allen, L Ajayi,

A Goyal

Royal Free Hospital

MP22-19 Magnesium Intake and the Risk of Incident Kidney Stone in Women Health Initiative (WHI)

O Kott, C Chen, X Wen, B Nicholas, M Huang,

J Li, J Wactawski-Wende, L Snetselaar, S Liu,

J Tang

Nephrology, Alpert Warren School of Medicine,

Brown University
MP22-20 Radiological vs. Urological Determination of Ureteral Stone Density. Monocentric Retrospective Study

JR Wießmeyer, J Ring, J Landmesser, T Ozimek, MW Kramer, AS Merseburger

Universitätsklinikum Schleswig-Holstein

(Campus Lübeck)

MP22-21 Entering to 2.0 cystinuric management: medical digital solution to monitor and control urinary pH

O Angerri Feu, F Rousaud Baron, S Gracia Garcia,

E Emiliani, A Kanashiro, F Sánchez-Martín,

F Millán Rodriguez, J Palou Redorta,

J Cuñé Castellana

Fundacio Puigvert

MP22-22 Adherence to pharmacologic treatment and its improvement in the twenty-four urine parameters in patients with kidney stones

B0 Manzo, E Emiliani, H Sánchez, JD Cabrera,

JE Torres, E Lozada

Hospital Regional de Alta Especialidad del Bajío
MP23-01 The use of immunization and probiotic therapy in the prevention of recurrent nephrolithiasis after puncture nephrolithotripsy A Pushkarev, V Pavlov Bashkir State Medical University

MP23-02 The Comparison of Flexible Ureterorenoscopy and Percutaneous Nephrolithotomy in 20 - 40 mm in Kidney Stones

A Albayrak, M Allahverdiyev, C Yesildal, A Yavuzsan, M Ilgi, S Kirecci

MP23-03 Management of Large Proximal Ureteral Calculi: A Comparative Study Between Simultaneous Supine Percutaneous Nephrolithotomy with Retrograde Ureterolithotripsy (ssPCNL) and Semi-rigid Ureteroscopic Lithotripsy

Y Chen, H Chen, Y Juan, W Wu, C Li, T Huang

Kaohsiung Medical University Hospital,

Kaohsiung Medical University,

Kaohsiung, Taiwan

MP23-04 The effectiveness and safety of four tract dilation methods of percutaneous nephrolithotomy: a meta-analysis Y Wu, Y Lu, Y Xun, S Wang, Q Wang Tongji Hospital, Tongji Medical College, Huazhong University of Science and Technology
MP23-05 Life threatening severe bleeding requiring angioembolization after percutaneous nephrolithotomy

H Kim, S Lee, H Choe, K Lee, J Yoo, D Lee

St. Vincent's Hospital

MP23-06 Endoscopic Combined IntraRenal Surgery(ECIRS) in treating complex high burden stone

Y Tsao, T Huang

Kaohsiung Medical University Hospital

MP23-07 Multi Institutional Experience with Single Access Percutaneous Nephrolithotomy

for Staghorn Calculi

T Large, C Forbes, K Scotland, D Agarwal,

H Jarvis, R Yan, CU Nottingham, ME Rivera,

BH Chew, A Krambeck

MP23-08 Ultrasound-guided micro-percutaneous nephrolithotomy in the treatment of pediatric nephrolithiasis

C Ning, W Wang, J Li

Beijing Friendship Hospital, Capital

Medical University

MP23-09

Predisposing Factors Affecting Blood Loss During Percutaneous Nephrolithotomy (PCNL)

JN Bamberger, B Gallante, RA Chandhoke, A Kapoor, H Kwun, W Atallah, M Gupta, AM Zampini

Mount Sinai 
MP23-10 The Experience of handling Channel Loss during the Procedure of Nephrostomy in Percutaneous Nephrolithotripsy: Report of 17 Cases

X Lin, L Guangyan, L Bingfeng

Department of Urology Hong Kong University

Shenzhen Hospital

MP23-11 Microperc-The Next Generation Of PCNL A Soomro, M Murtaza

MP23-12 Outcomes of non-lower pole puncture for supine PCNL: a multi-center study

C Mejia Arbalaez, M Gokce, B Manzo, M Gonzalez, S Proietti, L Ajayi, J Torres Garcia, A Yaghoubian, G Giusti, P Daels, C Cristallo, G Saitta, T Birks, B Eisner Massachusetts General Hospital

MP23-13 Non-papillary mini-percutaneous nephrolithotomy: Early experience

P Kallidonis, A Vagionis, C Adamou, P Ntasiotis, K Pagonis, E Liatsikos

University of Patras

MP23-14 Factors Increasing the Likelihood of SIRS in Patients Undergoing Percutaneous Nephrolithotomy

AR Omorogbe, JN Bamberger, E Rosenbluth, H Kwun, A Kapoor, M Gupta, AM Zampini Mount Sinai

MP23-15 The Effect of Continuing Aspirin on Postoperative Outcomes in Percutaneous Nephrolithotomy

E Rosenbluth, JN Bamberger, AR Omorogbe, B Gallante, RA Chandhoke, H Kwun, A Kapoor, M Gupta, AM Zampini

Mount Sinai
MP23-16 Percutaneous nephrolithotomy in the transplant kidney - a prospective 20 year multicenter experience

T Birks, C Ferreiro, J Withington, M Moghul, C Torrecilla, F Vigués, O Angerri Feu, A Breda, L Ajayi, E Emiliani

Royal Free Hospital, London

MP23-17 Surgical outcomes of percutaneous nephrolithotomy in 3402 Patients and results of stone analysis in $\mathbf{1 5 5 9}$ patients

M Hussain, S Askari, A Hashmi, M Lal, M Jabeen, A Khalique

Sindh Institute of Urology and Transplantation

MP23-18 PCNL: A safe alternative to guideline recommendations for stones less than $1 \mathrm{~cm}$

T Chi, M Armas-Phan, S Wiener, W Hu, B Xiao, B Su, M Stoller, T Chi, J Li

University of California, San Francisco

MP23-19 ULTRA-mini PERC under local anesthesia: a novel approach in select cases

C Chalouhy, M Moukarzel, S Slaba, A Tabche,

E Hanna, N Harake, T Smayra

MP23-20 Treatment of kidney stones by micro-NLPC in patients with urinary diversion. R Boissier, T Negre, F Michel, G Karsenty, E Lechevallier Aix-Marseille University, Marseille, France; Department of Urology and Renal Transplantation, APHM, Conception University Hospital, Marseille, France

MP23-21 Withdrawn

MP23-22 It is Time For Supine PCNL Now:

A Case Control Study

PK Pandey, S Shukla

Hind Institute of Medical Sciences
MP24-01 Opiate Prescribing Patterns and Risk Factors for Prolonged Opiate Use in Patients with Acute Nephrolithiasis

B Cotta, S Bechis

UC San Diego

MP24-02 Calcium Phosphate Stones Predominate Among Patients with Recurrent Nephrolithiasis Requiring Surgical Intervention A Arnouk, A Vig, MD White Albany Medical College

MP24-03 Comparison of Mini PCNL v/s Retrograde intrarenal surgery (RIRS) v/s Extracorporeal shockwave lithotripsy (ESWL) for the treatment of renal stones up to 2.5 centimeters PK Pattnaik, SK Pattnaik, MP Pattnaik S S Urological \& Research Institute, Bombay Hospital PG Institute
MP24-04 The Risk of Post-Operative Sepsis following Ureteroscopy or Percutaneous Nephrolithotomy: An Analysis of NSQIP Data

J Bjazevic, H Razvi

Western University

MP24-05 Nephrolithiasis and Diabetes:

Risk factors predicting stone disease V Talanki, K Ieong, A Zlatopolsky, S Eng, J Miller, Y Sheynkin, D Schulsinger Stony Brook University Hospital

MP24-06 How should the follow-up be done after stone surgery? CT scan vs Ultrasonography A Rivero, J Montero, J Adot, J Hernández, A Robles, E Gutierrez

MP24-07 Can the qSOFA Score Predict the Need for ICU Admission and Outcomes in Patients with Obstructed Infected Ureteral Stones? M Alsyouf, P Stokes, S Cho, Z Gilbert, A Amasyali, M Hajiha, M Shah, J Maldonado, J Groegler, D Baldwin Loma Linda University Health 
MP24-08 Utility of the POPVESL score for risk stratification of intrarenal vascular complications following percutaneous nephrolithotomy

P Shadpour, R Maghsoudi, N Yousefzadeh

Kandevani, M Etemadian, N Abian

Hasheminejad Kidney Center (HKC), Iran

University of Medical Sciences (IUMS),

Tehran, Iran

MP24-09 Evaluation of $40 \mathrm{cmH} 20$ vs $100 \mathrm{~cm} \mathrm{H} 2 \mathrm{O}$ gravity irrigation system in fURS

T Tefik, B Aydin, R Aydın, S Turan, F Ozcan, I Nane

Istanbul University, Istanbul Faculty of Medicine, Department of Urology

MP24-10 Bilateral Simultaneous Procedures for Bilateral Urolithiasis: The Future? Evidence from a Systematic Review

R Geraghty, A Pietropaolo, B Rai, P Jones, BK Somani

MP24-11 Initial clinical experience of LithoVue ${ }^{\mathrm{TM}}$ in patients of Urolithiasis:

An Indian perspective

J Jagtap, L Sinha, S Veer, G Agrawal, P Rao, S Mistry

Gleneagles Global Hospitals, Mumbai

MP24-12 Body Mass Index impacts Percutaneous Nephrolithotomy and Retrograde Intra-renal Surgery results

J Torres, P Pereira, N Morais, S Anacleto, R Rodrigues, P Passos, P Mota, R Leão, E Lima

MP24-13 Emergent urologic consultation for renal colic - what factors lead to admission C Tabib, W Wu, P Samson, C Hartman Arthur Smith Institute for Urology

MP24-14 Identification of Stone Composition Based Upon Gross Appearance A Ng, V Vasudevan, C Hartman, D Hoenig, Z Okeke, AD Smith, C Tabib
MP24-15 Implementation of a Hospital-wide Protocol Reduces Time to Nephrostomy Tube and Length of Stay in Patients with Stone Related Obstructive Pyelonephritis

C Haas, C Sayegh, J Pak, E Sebesta, E Hyams, O Shah

Columbia University

MP24-16 Prognostic performance of sepsis-3 and SIRS criteria for patients with urolithiasis-associated sepsis transferred to the ICU following surgical interventions: a single center study

Y Huang, J Ding, B Shi

MP24-17 Creation and implementation of the DIRECT Pathway (Delayed Imaging to Reduce Excessive Computed Tomography) for the evaluation of patients with suspected renal colic

K Sternberg, DW Sobel, A Greenspun, N Moring, D Bak, R Bidad, P Weimersheimer

Brown University

MP24-18 Bladder Lithiasis - Percutaneous Suprapubic or Transurethral Cystolithotomy:

A Retrospective Study

S Anacleto, V Fernandes, J Torres, N Morais,

P Passos, R Rodrigues, P Mota, E Dias, E Lima

MP24-19 Does Preoperative Turbid Urine Predict for Infectious Complication Following PCNL?

D Mikhail, E Lynch, A Rai, S Mehta, A Greico,

D Hoenig, Z Okeke, AD Smith

\section{MP24-20 Withdrawn}

MP24-21 Evaluation of Bone Mineral Density in Kidney Stone Formers with Computed Tomography:

A Single-Center Case-Control Study

B Timuroglu, B Dolek, I Sari, N Gul, O Ozkan, T Sener, Y Tanidir

Marmara University School of Medicine,

Department of Urology

MP24-22 Do all patients with obstructing ureteric calculi and urinary nitrites need emergency decompression?

T Marsden, A Pai, O Blach, A Alanbuki, A Symes

Brighton and Sussex University Hospitals
MP25-01 Can a disposable ureteroscope push up the 20-mm threshold?

R Takazawa, Y Waseda, S Yoshida,

M Kobayashi, T Tsujii

Tokyo Metropolitan Ohtsuka Hospital

MP25-02 Comparing Emergency and Elective Semi-Rigid Ureteroscopic Lithotripsy for Patients with Ureteric Calculi

SM Nazim, M Bangash, N Khan

The Aga Khan University Hospital,

Karachi Pakistan
MP25-03 Awake Flexible Ureteroscopy May Improve Patient-Urologist Communication and Stone Free Rate: A Single Surgeon Experience J Feghali

KMC Hospital, Department of Urology, American University of Beirut, Jounieh-Lebanon; SZUMC, Department of Urology, Lebanese University, North-Lebanon; NINI Hospital, Department of Urology, Beirut Arab University, North-Lebanon

MP25-04 Dedicated Day Case Ureteroscopy List: standardising the pathway and improving outcomes

C Bastianpilliai, S Mathew, A Golash, H Fernando University Hospitals North Midlands 
MP25-05 Defining thermally safe laser lithotripsy power and irrigation parameters: in vitro model A Aldoukhi, K Black, T Hall, K Ghani, AD Maxwell, B MacConaghy, WW Roberts Department of Urology, Univeristy of Michigan

MP25-06 Safety of Live Retrograde Intrarenal Surgery; Results from Society of Urological Surgery in Turkey-Stone Group (SUST-SG) Courses O ÖZMAN, S ÇITGEZ, C Basatac, H AKGÜL, C YAZICI, Y Tanidir, H AKPINAR, B Onal Gayrettepe Florence Nightingale Hospital

MP25-07 Assessment of the clinical and economic relevance of the use of single-use digital flexible ureteroscopes:

A systematic review

C McMartin, J Cloutier, S L'Espérance, R Drolet, A Nourissat, $M$ Rhainds

CHU de Québec - Université Laval

MP25-08 The electron-microscopic characteristics of stones - new tool in the retrospective evaluation of laser settings applied in individualized lithiasis' treatment BF Geavlete, G Balan, DA Georgescu, PA Geavlete Saint John Emergency Clinical Hospital

MP25-09 Compared endoscopic and microscopic recognition of urinary stones: preliminary concordance study G Verzotti, M Daudon, P Meria, C Almeras, O Traxer, V Estrade Hospital Pellegrin

MP25-10 New proposed "NASA" score for measurement of stone free rate in flexible ureteroscopy P Cortes-Raygoza, C Martinez-Arroyo, M Ortega-Gonzalez, E Hernandez-Mendez, I Calvo-Vazquez, G Fernandez-Noyola, M Cantellano-Orozco, G Morales-Montor, C Pacheco-Gahbler Hospital General Dr. Manuel Gea Gonzalez

MP25-11 Large Ureteric Stones:

No To Knife-The Laser Edge In Stone Age! SM Mahmud

Lifecare Hospital (Abu Dhabi Campus)

MP25-12 Application of Soton ureteroscopy in the treatment of long Segment of Ureteral Stone Street

W Xiaoning, Z Xiaofeng, Y Yuanhu, X Rihai, Z Guoxi, W Gengqing
MP25-13 “VaporTunnel” Ureteroscopic Holmium laser lithotripsy: intraoperative and early postoperative outcomes G Bozzini, U Besana, A Calori, S PROIETTI, G Giusti, C Buizza, M Sighinolfi, B Rocco ASST Valle Olona - Busto Arsizio VA

MP25-14 Comparison of early vs delayed ureteroscopy following obstructive pyelonephritis treated with urinary diversion

S Lagacé, J Cloutier, I Fugaru, D Simonyan, N Laflamme

Université Laval

MP25-15 Withdrawn

MP25-16 Clinical effects of Poly- Flexible Ureteroscopy combined with ultrasound guided all seeing needle Microperc to treatment of Lower Pole Renal Calculi larger than $2 \mathrm{~cm}$ with infundibulopelvic angle less than 60 degrees

G Jingyang, Y Wenzeng, Z Yanqiao Affiliated Hospital of Hebei University

MP25-17 A single center prospective randomized controlled trial of two single use flexible ureteroscopes - LithoVue (Boston Scientific, USA) and Pusen PU3022a (Zhuhai Pusen, China) A Patil, S Agrawal, A Singh, A Ganpule, R Sabnis, M DESAI Muljibhai Patel Urological Hospital, Nadiad, Gujarat, India

MP25-18 Beware of Laser, Ureteral Stenosis after Ureteroscopy F Pauchard, N Calvo, D Gonzalez, C Ondarza, F Kramer, C Mülchi Hospital Carlos Van Buren

MP25-19 Withdrawn

MP25-20 Flexible ureteroscopy and holmium laser lithotripsy for ureteral stones in renal transplant recipients with ureteroureterostomy type anastomosis V Ulker, O Celik, Y Ilbey Health Sciences University Tepecik Training and Research Hospital

MP25-21 Different morbidity using different flexible ureteroscopes - is it a reality?

PA Geavlete, R Multescu, DA Georgescu, M Stan, C Bucur, BF Geavlete Saint John Emergency Clinical Hospital

MP25-22 A Prospective Comparative Study Between Flexible and Semi-rigid Ureteroscopic Lithotripsy in the Management of Single Large Proximal Ureter Stone H Chen, Y Chen, Y Juan, W Wu, C Li, T Huang Kaohsiung Medical University Hospital, Kaohsiung Medical University, Kaohsiung, Taiwan 
V13-01 Magnetic-assisted Robotic Radical Prostatectomy Using the Da Vinci SP Robotic System RL Steinberg, A Garbens, BA Johnson, JA Cadeddu

V13-02 Robotic, Single Port, Trans-vesical Bladder Diverticulectomy K Fareed, J GaristoRisco, J Kaouk

V13-03 Circumferential Periumbilical Incision for Single Port Robot-Assisted Surgery JR Slawin, GW Dy, R Bluebond-Langner, LC Zhao NYU Langone Health

V13-04 Single Port Robotic Y-V Plasty of Bladder Neck Contracture K Fareed, J GaristoRisco, H Wood, J Kaouk

V13-05 Robotic Assisted Laparoscopic Posterior Urethroplasty Using the SP Robot M Jun, W Liu, GW Dy, X Meng, LC Zhao NYU Langone Health

V13-06 Pure single-site robot-assisted pyeloplasty with the da Vinci SP ${ }^{\circledR}$ system: Initial experience J Heo, S Kang, D Koh, J Na, Y Lee, W Han, Y Choi, W Jang
V13-07 Pure Transvesical Single-Port Robotic Simple Prostatectomy Using The New SP ${ }^{\circledR}$ da Vinci Surgical System GV Sawczyn, RJ Valero Carrion, J GaristoRisco, RJ Yau, J Kaouk

V13-08 Initial Report of da Vinci Single Port Robot-Assisted Orchiectomy for an Undescended Inguinal Testis R Munver, JF Tsui, K Basralian, B Desroches

V13-09 Single Port Robotic Assisted Laparoscopic Radical Prostatectomy M Stern, M Billah, S Ahsanuddin, G Lovallo, M Ahmed

V13-10 Single Port Robotic Pyeloplasty: Similar Outcomes, Excellent Cosmesis M Billah, D Kim, PM Youssef, PD Kothari, JC Deolankar, SS Komaravolu, MD Stifelman

V13-11 Robotic, Single Port, Trans-peritoneal Simple Prostatectomy K Fareed, J GaristoRisco, J Kaouk

V13-12 Same Day Discharge Extra-peritoneal Single-Port Robotic Radical Prostatectomy: New Device, New Approach, New Paradigm

RJ Valero Carrion, GV Sawczyn, J GaristoRisco, RJ Yau, J Kaouk
V14-01 Microwave Assisted Clampless Laparoscopic and Robotic Partial Nephrectomy

B Pham, A Parker, R Venkatesh

V14-02 Robotic assisted partial nephrectomy for endophytic renal tumor T Lin, C Ou, Y Lin

V14-03 Management of Major Complication After Robot Assisted Partial Nephrectomy B Onal, M Simsekoglu, U Aferin, B Ercili, A Erözenci

V14-04 Sutureless technique in robot-assisted partial nephrectomy M Ishida, Y Miyazaki, Y Konnai, K Shojo, H kawasaki, S Watanabe, H Kobayashi, M Kosugi

V14-05 Robot-assisted Partial Nephrectomy in Patient with solitary Kidney and multiple renal masses O Aytac, A Garayev, F Atug

V14-06 Off-clamp Robot-assisted Partial Nephrectomy (RPN) for cT2 renal cancers

G Simone, U Anceschi, A Brassetti, M Costantini, M Ferriero, R Flammia, S Guaglianone, R Mastroianni, F Minisola, G Tuderti, M Gallucci
V14-07 The Utility of 3D Virtual Reality Models In Preoperative Planning For Robotic Partial Nephrectomy BD Dayal, AA Elbakry, KE Okhawere, K Meilika, A Beksac, KK Badani

V14-08 Robotic Partial Nephrectomy after Previous Open Ipsilateral Partial and Contralateral Robotic Partial Nephrectomy S Aly, JF Tsui, R Munver, B Desroches

V14-09 Renorrhaphy during robotic partial nephrectomy: technique evolution A Patel, T Poloskey, C Rogers

V14-10 Robotic-assisted laparoscopy Partial nephrectomy for intra parenchymal renal tumor assisted by endoscopic ultrasound in a patient known case of multi cystic kidney disease.

A Alkhalili, F Adawi, M Alhamad

V14-11 Robotic-assisted laparoscopic partial nephrectomy for renal cell carcinoma in horseshoe kidney: a hybrid technique with conventional laparoscopic surgery

Y Muto, K Numakura, T Nara, S Kanda, M Huang, M Saito, S Narita, T Inoue, S Satoh, T Habuchi

V14-12 Robotic zero ischemic partial nephrectomy with quantitative three dimensional reconstruction and triple-screen real-time display (Tilepro ${ }^{\circledR}$ )

W XIONG, P GUO 
V15-01 Robot-assisted ureteral stricture resection and primary anastomosis in a solitary kidney: illustration of an optimized technique utilizing indocyanine green and reconstructive surgical principles

PM Gurung, G Wu

V15-02 Left sided Robot assisted radical Nephrectomy for $\mathrm{RCC}$ with thrombus in the renal vein reaching up to IVC

S Schakaki

V15-03 Robotic arm assisted ureteroureterostomy $\mathrm{K}$ Lin, $\mathrm{C}$ Ou, K Lin

V15-04 Robotic Assisted Laparoscopic Bilateral Ureterolysis: A Novel Approach M Billah, SS Komaravolu, JC Deolankar, D Kim, PD Kothari, PM Youssef, MD Stifelman

V15-05 Robotic pyeloplasty in anomalous kidney : point of technique

A Singh, S Agrawal, A Ganpule, R Sabnis, M DESAI

V15-06 Combined robotic-assisted laparoscopic and endoscopic surgery for a ureteropelvic junction obstruction caused by aberrant vessel with removal of kidney stones

F Adawi, M Alhamad, A Alkhalili
V15-07 Robot-assisted Synchronous Bilateral Nephrectomy for Autosomal Dominant Polycystic Kidney Disease PM Gurung, TP Frye, H Rashid, JV Joseph, G Wu

V15-08 Robotic Stone Removal in the Challenging Patient C Crigger, MW Salkini, A Pearce

V15-09 Diagonal renorrhaphy technique in robot-assisted partial nephrectomy for endophytic mid-pole renal tumor of size $>4 \mathrm{~cm}$

G Wang, X Liu, X Zhou, B Fu, L Chen, Y Li

V15-10 Magnetic-assisted laparoscopic and robotic renal surgery:

Applications of a new technology

J Fulla, E Kaplan-Marans, M Palese

V15-11 ICG-Near Infrared guided robot-assisted pyeloplasty in patient with retrocaval ureter A Veccia, A Tracey, R Autorino

V15-12 Robotic Inferior Vena Cava Thrombectomy Using A Novel Intracaval Balloon Occlusion Technique

A Alahmari, K Algarni, T Alamoudi, A Abdelsalam, T Kaid, A Kaki, E Younes, R Jamjoom, R Azhar
MP26-01 Safety and Efficacy of Stone Dusting Utilizing a 30-Watt Laser system Versus a High Power (100/120W) System

O Alalao, O Kott, DW Sobel, C Tucci,

G Pareek

Brown University

MP26-02 Analysis of ureteral stent's encrustations: comparison between silicone and Percuflex ${ }^{\mathrm{TM}}$ L Bevilacqua, M Amato, G Guarino, C Del Prete, R Milandri, M Sighinolfi, S Puliatti, B Rocco, S Micali

Department of Surgical, Medical and Dental Morphological Sciences related to Transplant, Oncology and Regenerative Medicine - Section of Urology, University of Modena and Reggio Emilia, Modena, Italy

MP26-03 Is the irrigation method important for maintaining safe irrigation temperatures during flexible ureteroscopy when a high power Holmium:YAG laser is used? Evidence from an experimental study in porcine model

E Farsari, P Ntasiotis, C Adamou, A Vagionis,

A Gerakaris, K Gkialas, GA Callas, E Liatsikos,

P Kallidonis

University of Patras
MP26-04 Effectiveness of using thinner Double J stents following flexible ureterorenoscopy for stone fragment expulsion

E Fernandes, S Kanekar, H Maienkar Vision Hospital, Healthway Hospital

MP26-05 Buried upper end of the double coil stent following perforation of the collecting system during percutaneous nephrolithotomy F Hammad United Arab Emirates University and Tawam Hospital

MP26-06 Stent Syndrome. Does the diameter matter? Preliminary results of a prospective trial PN Contreras, N Bonanno, L Rico, M Ringa, F Frascheri, C Ameri Hospital Alemán

MP26-07 FECal (forgotten, encrusted, calcified) grading system for forgotten ureteral stents. Is it a reliable system? OE FUGITA, J AMARO, F MARCHANT, N BERNARDO, M BAPTISTUSSI, A LUNA, M GONZALEZ, J CAMPOS, A CARDENAZ-ORTIZ FACULDADE DE MEDICINA DE BOTUCATU - UNESP 
MP26-08 A Novel Cost Effective Patient Engagement Based Educational Tool for Informed Stent Insertion Consent

I Ahmed, OA Ramadan, B Osman, MM Quraishi, J Hale, P Keirandish, N Shrotri, R Krishnan,

SS Kommu

Kent \& Canterbury Hospital. East Kent Hospitals NHS Foundation Trust

MP26-09 How to place a loop type ureteral stent safely?: From an early experience of a loop-type ureteral stent

H Byun, J Choi, D Han, H Ryoo, J Chung

MP26-10 Evaluation of the initial efficacy

and safety in endoscopic combined intrarenal surgery (ECIRS)

Y Miyazawa, T Miyao, Y Fukuma, M Hatori,

N Otake, T Sekihara, K Suzuki

MP26-11 A Clinical Tracking Program for Ureteral Stents Integrated Into EMR

F Ghali, D Papagiannopoulos, R Sur, S Bechis

UC San Diego

MP26-12 Comparison of three different endoscopic treatment in the treatment of large bladder stone over $3 \mathrm{~cm}$

J Choi, Y Kim, J Chang, Y Ko, P Song, K Moon, H Jung

Yeungnam University Medical Center

MP26-13 Withdrawn

MP26-14 Analysis of Disposable Equipment Utilization on the Cost of Ureteroscopy:

Impact of Technique and Training

K Parikh, R Pak

Mayo Clinic Florida

MP26-15 Comparison of Holmium Lithotripsy pulse duration on stone dusting

W Kuo, C Yu, J Lin, J Tsai

Kaohsiung Veteran General Hospital

MP26-16 Impact on renal function and risk factors associated with the calcification of ureteral stents in a south hospital at México

KA Mendoza, A Ávila, E Cruz, MA Osorio,

FE Navarrete, A Esqueda

Hospital Regional de Alta Especialidad de la

Península de Yucatán
MP26-17 Reusable Versus Single-use Cystoscopes for removal of DJ Stent:

A Prospective Randomized Comparison and Cost Analysis

S Bin Hamri, MA Alkhamees, M Alzughabi,

A Alothman, I Alshohayeb, A Almujallid,

M Alsadhan, A Alasker, Y Ghazwani

Assistant Professor, Urology Department, College

of Medicine, Majmaah University

MP26-18 Should the use of stent-on-strings become common practice during supine miniature percutaneous renal stone extraction?

H Burns, S Nalagatla

University Hospital Monklands

MP26-19 The risk factors and complications of forgotten double-J stents:

a single center experience

T Lin, W Lin, M Chen, S Jhang, T Yang, J Hsu

Mackay Memorial Hospital

MP26-20 Suctioning the dust out in mini PCNL, does it change the outcome?

A Singh, N Reddy, A Ganpule, R Sabnis,

M Desai

Muljibhai Patel Urological Hospital

MP26-21 Thulium:YAG vs Holmium:YAG laser effect on soft tissue: evidence from an ex vivo experimental study

ME Rodriguez Socarras, S PROIETTI,

R Luciano', F Scarfo', G Saitta, F Gaboardi, G Giusti

Instituto de Cirugía Urológica Avanzada (ICUA), Madrid, Spain

MP26-22 Initial In Vivo Experience with a Novel Single Probe Dual Energy Lithotriptor

MM Dunne, JE Abbott, JG Davalos

University of Maryland Medical Center
MP27-01 Stone composition in children:

A single center study in Pakistan.

N Iqbal, A Hasan, M Mughal, T Bhatty, S Akhter,

J Rahim

Pakistan Kidney and Liver Institute and

Research Center

MP27-02 In vitro evaluation of a novel pediatric flexible cystoscope - A practical comparison of endoscopes for pediatric lower urinary tract evaluation

H Truong, J Rosado, J Hagerty, SG Hubosky,

D Bagley
MP27-03 Does Early Extracorporeal Shock Wave Lithotripsy (ESWL) Failure Predict Overall Treatment Failure - Results from A Tertiary Referral Stone Centre

E Latif, MM Quraishi, J Dorrell, J Hale, S Subramaniam, P Keirandish, OA Ramadan, B Osman, G Papadopoulos, I Ahmed, A Simoes, SS Kommu, R Krishnan, N Shrotri

Kent \& Canterbury Hospital. East Kent Hospitals NHS Foundation Trust 
MP27-04 Endoscopic hybridization in the treatment of complex upper urinary calculus in children L Zhong, J Sun

Shanghai Jiaotong University School of Medicine

MP27-05 The use of a virtual reality device $\left(H_{y p n o V R}{ }^{\circledR}\right)$ during external shock wave lithotripsy

for treatment of urinary stones:

initial results from a feasibility study

E Ventimiglia, S Doizi, E Ayani, G Raynal,

O Traxer

Division of Experimental Oncology/Unit of

Urology; URI; IRCCS Ospedale San Raffaele

MP27-06 Withdrawn

MP27-07 Role of dusting and pop-dusting using high power (100w) laser in treatment of pediatric stones

T Reeves, S Griffin, A Pietropaolo, S Chugh,

BK Somani

Portsmouth Hospitals NHS Trust

MP27-08 Expulsive therapy using tamsulosin after shock wave lithotripsy of renal and ureteral stones: randomized prospective study

N Mouad

Clinique Achifaa Oujda

MP27-09 Role of stone size, stone density, and skin-to-stone distance by non- contrast computed tomography abdomen in predicting success of shock-wave lithotripsy for kidney stones.

A Hamid, A Shah, N Choh

SKIMS, SRINAGAR, KASHMIR, INDIA

MP27-10 Ultrasound Guided ESWL for VUJ Stones RS Bashetty, SB Alur, JB Alur, S Raju Matoshree Kidney Stone Center

MP27-11 Outcome of anterograde ureterography for ureteral stone detection in extracoporeal shock wave lithotripsy

J You, Y Shin, Y Kim

Department of Urology, Chonbuk National

University Medical School and Research Institute

of Clinical Medicine of Chonbuk National

University-Biomedical Research Institute of

Chonbuk National University Hospital,

Jeonju, Korea

MP27-12 Using Algorithm-Based Tools to Improve

Shockwave Lithotripsy Outcomes

BD Hamilton, R Seltzer, D Klett, SY Nakada,

G Gerber

University of Utah
MP27-13 Analysis of infrared spectrum stone composition of children in Gannan in Jiangxi Province:

52 cases report and clinical analysis

X Ruiquan, Y Yuanhu, Z Xiaofeng, Z Guoxi,

$\mathrm{X}$ Rihai, W Gengqing

\section{MP27-14 Withdrawn}

MP27-15 Safety and efficacy of flexible ureteroscopy and laser stone fragmentation for pediatric renal stones: A systematic review of the literature A Barr, S Chugh, A Pietropaolo, P Jones, OM Aboumarzouk, BK Somani

Portsmouth Hospitals NHS Trust

MP27-16 The prediction of treatment success and complications of shockwave lithotripsy using artificial intelligence

R Seltzer, BD Hamilton, D Klett, Z Chen, SY Nakada, G Gerber

MP27-17 Pediatric urolithiasis: overview of treatment and outcomes from a single series of 50 patients R Milandri, L Bevilacqua, C Di Pietro, P Ceccarelli, E Morini, A Elsherbiny, A Eissa, S Puliatti, M Sighinolfi, G Bianchi, S Micali, B Rocco Department of Surgical, Medical and Dental Morphological Sciences related to Transplant, Oncology and Regenerative Medicine - Section of Urology, University of Modena and Reggio Emilia, Modena, Italy

MP27-18 Prospective analysis of the predictors of success after ESWL

F Quadrini, A Boni, P Guiggi, M Del Zingaro,

E Mearini

University of Perugia

MP27-19 Spectrum of pediatric urinary stone composition in North-western India

R Saran, K Mirdha, S Saran

MP27-20 Totally Ultrasound Guided ESWL Monotherapy for Impacted Uretero-Vesical junction (UVJ) calculi.

PK Pattnaik, SK Pattnaik, MP Pattnaik S S Urological \& Research Institute, Bombay Hospital PG Institute
MP28-01 Comparison of S.T.O.N.E nephrolithometry and Guy's scoring systems for predicting stone clearance and complications after percutaneous nephrolithotmy SM Nazim, N Khan, M Farhan, B Salam, H Ather The Aga Khan University Hospital, Karachi Pakistan
MP28-02 Comparison of Quality of Life between standard tubeless PCNL and Mini Tubeless PCNL by using WISCONSIN Stone QOL Questionnaire N Iqbal, A Hasan, A Mughal, M Nazir, S Akhter, J Rahim

Pakistan Kidney and Liver Institute and Research Center 
MP28-03 Efficacy and safety of Ureteroscopic Lithotomy and Percutaneous Nephrolithotomy for Upper Ureteral Calculi

Q Xu, Y Hong, X Huang

Urology and Lithotripsy Center, Peking

University People's Hospital

MP28-04 Effect of percutaneous nephrolithotomy over the renal replacement therapy-free time on patients with chronic kidney disease KDIGO IV and $\mathrm{V}$

KA Mendoza, A Ávila, E Cruz, M Basulto, A Esqueda, A Romero, J Flores, H Rendon, S Canul, T Diaz

Hospital Regional de Alta Especialidad de la Península de Yucatán

MP28-05 Ambulatory Percutaneous Nephrolithotomy Performed in a Free-standing Surgery Center: Outcomes of the First 550 Cases MM Dunne, AI Kim, MV Silva, JE Abbott, JG Davalos

University of Maryland Medical Center

MP28-06 Bacteriology and Antibiotic Sensitivity Pattern of Isolates in Patients Who Underwent Percutaneous Nephrolithotripsy at the Philippine General Hospital

J Flor, M Lapitan

University of the Philippines- Philippine

General Hospital

MP28-07 Comparison of Cost-Effectiveness and Postoperative Outcomes following Integration of a Stiff Shaft Glidewire into Percutaneous Nephrolithotripsy

C Valadon, CU Nottingham, T Large,

A Krambeck

University of Louisville School of Medicine

MP28-08 Analysis of the clinical efficacy and safety of PCNL in patients with anatomical variations: A single center retrospective study

DT Rubio, JA Abraham

National Kidney and Transplant Institute

MP28-09 Investigating the Roll of Smaller Tract Percutaneous Nephrolithotomy in the Fully Anticoagulated Patient

MM Dunne, JE Abbott, JG Davalos

University of Maryland Medical Center

MP28-10 Prospective Outcomes of Single Stage Tubeless mini-PCNL for Renal Stones $\geq \mathbf{2 0 m m}$

S Khadgi, M Darrad, AR EL-Nahas, A Al-Terki Urology Department, Al-Amiri Hospital, Kuwait

MP28-11 Evaluation of Efficacy and Safety Profile of Bilateral Simultaneous Endoscopic Stone Surgery in Kidney Stones

Y Tanidir, T Sener, G Ozgur

Marmara University School of Medicine,

Department of Urology
MP28-12 A control research between super-mini percutaneous nephrolithotomy(SMP) and minimally invasive percutaneous nephrolitripsy(MPCNL)

Z Guoxi, W Xiaoning, Z Xiaofeng, Y Yuanhu, $X$ Rihai, W Gengqing

MP28-13 Feasibility of Percutaneous Nephrolithotomy (PCNL) under Spinal Anesthesia for Staghorn Kidney Stones:

Safety and Efficacy

JA Kalathia, S Agarwal, C Saurabh

Fortune Urology Clinic

MP28-14 A Nomogram to Predict Significant Estimated Glomerular Filtration Rate Reduction After Percutaneous Nephrolithotomy.

U Falagario, A Mangiatordi, M Auciello,

A Martini, P Treacy, JN Bamberger, M Gupta,

G Carrieri, L Cormio

MP28-15 Does skeletal muscle quality impact the outcomes of percutaneous nephrolithotomy?

Y Wu, Y Lu, Y Xun, H Hu, S Wang, Q Wang

Tongji Hospital

MP28-16 Novel use of betadine and antibiotic irrigation for renal pelvis sterilization during PCNL a pilot study

D Mikhail, E Lynch, A Rai, T Benjamin, M Mekhail, AD Smith, D Hoenig, Z Okeke

MP28-17 Withdrawn

MP28-18 Comparative analysis of microbial contamination of stone and urine in recurrent nephrolithiasis

A Pushkarev, V Pavlov

Bashkir State Medical University

MP28-19 Withdrawn

MP28-20 Analysis of Risk Factors for Pulmonary Complications in Patients undergoing Upper Pole Access Percutaneous Nephrolithotomy:

A Single Center Experience

ME Amponin, JA Abraham

National Kidney and Transplant Institute /

Philippine Urological Association

MP28-21 ECIRS in treatment of Large Upper Ureteric Stones

DK Mishra, MS Agrawal

Global Rainbow Healthcare, Agra, India

MP28-22 Can CT-based measurements of renal parenchyma, perirenal fat and subcutaneous fat predict the complication rate of Percutaneous Nephrolithotomy? A single center retrospective study

J Torres, C Gonçalves, N Morais, S Anacleto, R Rodrigues, P Passos, P Mota, R Leão, E Lima 
MP29-01 Impact of an Enhanced Recovery After Surgery Protocol on Patient Symptoms in the Immediate and Intermediate Post-operative Period After Ureteroscopy for Stones

BA Johnson, J Tse, A Akhtar, J Crivelli, RL Steinberg, J Sasaki, JA Antonelli, M Pearle University of Texas Southwestern

MP29-02 FRAIL: Feasibility of Retrograde IntrArenal Surgery In eLderly - Preliminary Outcomes S Biligere, C Heng, V Gauhar $\mathrm{Ng}$ Teng Fong General Hospital

MP29-03 Novel Biocompatible Adhesive to remove stone dust: Usability Trial in a Kidney Model

TJ Hausmann, C Netsch, B Becker, A Gross, CM Rosenbaum

Asklepios Clinic Barmbek, Hamburg, Germany

MP29-04 Efficacy and Safety of Semi-rigid Ureteroscopic Lithotripsy for Proximal Ureteral Stone Larger than $10 \mathrm{~mm}$

M Bangash, SM Nazim, S Jamil, M Ghani, S Naeem

The Aga Khan University Hospital, Karachi Pakistan

MP29-05 What is the Current Role of Single Use Ureteroscopes? A Snapshot World Survey M Ravindraanandan, W Mahmalji, Y Phan Hereford County Hospital

MP29-06 The Impact of Endourological Experience on Flexible Ureteroscopy (fURS) Outcomes. Retrospective Multifactorial Analysis. T Ozimek, MW Kramer, MC Hupe, JM Laturnus, JP Struck, M Hennig, AS Merseburger, J Cordes Universitätsklinikum Schleswig-Holstein (Campus Lübeck)

MP29-07 The role of antegrade irrigation via percutaneous nephrostomy on surgical outcomes in semi-rigid ureteroscopy among patients with upper ureteral stones

W Jung, H Byun, D Lee

Keimyung University School of Medicine, Dongsan Medical Center

MP29-08 Frequency and predictors of access failure in patients undergoing Ureterorenoscopy for upper urinary tract calculi

A Rafi, H Ather, W Aziz

Aga Khan University Hospital

MP29-09 Fluoroless Ureteroscopy for ureteric stones: feasibility, outcomes and assessment of factors necessitating fluoroscopy.

R Gupta, G Garg

MP29-10 Outcomes and complications of ureteroscopy and ureterorenoscopy for stones management using Holmium laser in a tertiary stone center for ureteric and renal stones

MA Hamdoon, MO Breish, M Smith, D Whiting, D Browne, H Marsh, F Anjum, S Sriprasad

Dartford and Gravesham NHS Trust
MP29-11 The efficacy of the disposable flexible ureterorenoscopy (Lithovue $^{\circledR}$ ) for renal stone smaller than $2 \mathrm{~cm}$

I Seo, J Lee, S Park, T Oh

Wonkwang University School of Medicine \& Hospital

MP29-12 Developing a Free Three-Dimensional Tool for Kidney Stones' Surgical Planification: "Calculator"

F Panthier, S Doizi, L Berthe, O Traxer Service d' Urologie, Hospital Tenon, APHP

MP29-13 Durability of fiber optic reusable flexible Ureterorenoscopes :Retrospective analysis after 313 consecutive cases of Retrograde Intra Renal Surgery; the lesson learned

Y KK, S Prabhakar Renai Medicity Multi Super Specialty Hospital, Kochi, Kerala, India

MP29-14 Donor and post-transplant ureteroscopy for stone disease in patients with renal transplant Evidence from a systematic review T Reeves, A Pietropaolo, BK Somani, H Ho

MP29-15 Reporting outcomes of a successful treatment in Ureteroscopy and Retrograde intrarenal surgery for stones:

The STONE-FECTA.-A Single center experience

N Iqbal, A Hasan, M Mughal, S Akhter, J Rahim Pakistan Kidney and Liver Institute and Research Center

MP29-16 Intramuscular Ketorolac Administered at Time of Stent Removal Decreases Pain-related Return Visits in a Randomized Control Trial

BA Johnson, I Sorokin, RL Steinberg, JA Antonelli, M Pearle

University of Texas Southwestern

MP29-17 Intrarenal arteriovenous malformation following flexible ureterorenoscopy and holmium laser stone fragmentation:

report of a case

A Bashar, F Hammad

Salford Royal NHS Foundation Trust

MP29-18 S.T.O.N.E nephrolithometry scoring system vs. Guy's scores for predicting stone-free status post sheathless retrograde intrarenal surgery:

Which is better?

SN Mahmood, AA Saeed, HM Toffeq, SS Fakhraldin University of Sulaimania, College of Medicine, Department of Surgery 
MP29-19 Does the working channel position have a role on the efficacy of Ho:YAG laser lithotripsy? Results from a laboratory test

L Villa, E Ventimiglia, S Doizi, S PROIETTI,

G Giusti, A Briganti, A Salonia, F Montorsi,

O Traxer

Division of Experimental Oncology/Unit of Urology; URI; IRCCS Ospedale San Raffaele, Milan, Italy

MP29-20 S.O.L.V.E. nephrolithometry: a novel scoring system for predicting the stone-free rate of flexible ureteroscopy S Luo, D Hu, W Tang

Department of Urology, The First Affiliated

Hospital of Chongqing Medical University
MP29-21 Treatment of isolated small renal stone leads to resolution of symptoms and should be routinely offered to patients:

Outcomes (of stone $\leq 7 \mathrm{~mm}$ and $8-10 \mathrm{~mm}$ )

from a University Hospital

A Pietropaolo, R Geraghty, BK Somani

MP29-22 Retrograde ureteroscopy versus antegrade mini percutaneous flexible ureteroscopy for treating impacted proximal ureteric stones $\geq 10 \mathrm{~mm}$

O Elgebaly, H Abdeldaiem, F Idris, A Zahran

Urology Department, Alexandria University
MP30-01 Does Previous History with Urosepsis Predict the Likelihood of Urosepsis Post-Ureteroscopy

OA Ramadan, J Dorrell, SS Kommu, J Hale, P Keirandish, R Krishnan, N Shrotri

Kent \& Canterbury Hospital. East Kent Hospitals NHS Foundation Trust

MP30-02 Efficacy and outcomes of ureteroscopy and Holmium laser on calculus anuria by ureteric stones: A single center experience

JA Kalathia, C Saurabh, S Agarwal, R Agarwal

Fortune Urology Clinic

MP30-03 Is the use of a ureteral access sheath necessary for maintaining safe irrigation temperatures during flexible ureteroscopy when a high power Holmium:YAG laser is used? Clues from an in-vivo experimental study

C Adamou, E Farsari, P Ntasiotis, A Vagionis, A Gerakaris, K Gkialas, GA Callas, K Pagonis, E Liatsikos, P Kallidonis

University of Patras

MP30-04 Pulse Modulation with Moses Technology Improves Popcorn Laser Lithotripsy

K Black, A Aldoukhi, J Teichman, WW Roberts, T Hall, K Ghani

Department of Urology, Univeristy of Michigan

MP30-05 RIRS with SuperPulse Thulium fiber laser for kidney stones larger than $\mathbf{2}$ cm

A Dymov, D Tsarichenko, G Akopyan,

D Chinenov, S Ali, R Klimov, V Lekarev,

D Korolev, L Rapoport

Institute for Urology and Reproductive Health, Sechenov University

MP30-06 Evaluation of Risk Factors for Urinary Tract Infection After Flexible Uretero-Renoscopy: A Retrospective Study Including 604 Interventions

R Boissier, M Baboudjian, B Gondran-Tellier, G Karsenty, E Lechevallier

Aix-Marseille University, Marseille, France; Department of Urology and Renal

Transplantation, APHM, Conception University Hospital, Marseille, France
MP30-07 Negative Ureteroscopies For Ureteric Stones M Moghul, O Elhalawaty, L Da Silva, A Almushatat, P Patki, Z Ali

Barts Health NHS Trust

MP30-08 Worldwide survey of Flexible Ureteroscopy (FURSL) Practice: A survey from EAU sections of Young Academic Urologists (YAU) and Uro-Technology (ESUT)

A Pietropaolo, A Skolarikos, E Liatsikos, N Rukin, E Niewad, T Sener, P Kallidonis, M Oezsoy, E Emiliani, MA Maestro, T Tailly, M talso, OM Aboumarzouk, S Proietti,

G Giusti, BK Somani

Fundacion Puigvert. Universidad Autonoma de Barcelona, Spain

MP30-09 Fluoroscopy-free ureterorenoscopy for kidney stones treatment: technique description by an endourology expert

G Verzotti, G Capon, F Bladou, J Bernhard, H Bensadoun, G Robert, J Ferriere, V Estrade Hospital Pellegrin

MP30-10 Predictors and results of negative ureteroscopy for treatment of consecutive ureteric stones done as a primary procedure: Prospective outcomes from a university hospital

S Prattley, P Rice, A Pietropaulo, H Ching Ho, BK Somani

MP30-11 Factors Influencing the Cost of Ureteroscopy M Alsyouf, P Stokes, D Hur, S Kown, A Amasyali, M Hajiha, M Shah, R Belay, J Maldonado, D Baldwin

MP30-12 Removing Stones from the Lower Pole during Retrograde Intrarenal Surgery (RIRS)Improved Stone Free Rates or a Waste of Time? A Prospective Randomized Study B Gallante, JN Bamberger, RA Chandhoke, W Atallah, M Gupta, AM Zampini Mount Sinai 
MP30-13 Incidence of Hydronephrosis and Ureteric Stricture with the Use of Ureteral Access Sheaths in the Treatment of Nephrolithiasis

LF Reynolds, D Ghiculete, AM Althunayan,

JY Lee, J Honey, M Ordon, KT Pace

St. Michael's Hospital

MP30-14 Pain Intensity and Interference after

Ureteroscopy for Nephrolithiasis

G Lin, A Fombona, B Friel, H Stambakio, GE Tasian, JB Ziemba

Perelman School of Medicine at the University of Pennsylvania

MP30-15 Use of a Coagulum during Ureteroscopic Stone Removal Adds No Additional Case Time or Complications

C Valadon, CU Nottingham, T Large,

A Krambeck

University of Louisville School of Medicine

MP30-16 Fire the Laser: Lithotripsy activation patterns for ureteroscopic stone dusting

A Aldoukhi, K Black, T Hall, JM Hollingsworth, SN Ambani, CA Dauw, WW Roberts, K Ghani

Department of Urology, University of Michigan

MP30-17 Predictors of urosepsis after ureteroscopy (URS): Prospective results from 900 consecutive patients S Chugh, A Pietropaolo, BK Somani Portsmouth Hospitals NHS Trust
MP30-18 RIRS for renal pelvic stones 1.5 to $2.0 \mathrm{~cm}$ using semi-rigid uretero-renoscopy

H Qureshi, G Sultan, M Hussain, T Gazdar,

A Hashmi, A Rizvi

Sindh Institute of Urology and Transplantation

MP30-19 Infectious pathogens in urine, blood and ureteral stents in ureterolithotripsy

TM Brasil, MQ Fortes, BN Lana, GB Araújo, RF Ferraz, CM Celjar, DC Perdigão, AT Dias, JP Pedrosa, HS Almeida, RM Medeiros, AA Carvalho, B Marroig Hospital Quinta D’Or

MP30-20 Effect of ureteric stenting on female sexual function- A prospective cohort study Z Kazmi, D Umer, H Ather

The Aga Khan University Hospital

MP30-21 Ureteric stenting versus non-stenting following uncomplicated ureteroscopic lithotripsy: A Prospective randomized trial S Al Demour, A Alrabadi, A AlSharif, M Ababneh, M Melhem, H Mansi, S Aljamal, M Abufaraj The University of Jordan/ Jordan University Hospital/ Urology Department

MP30-22 Peri-Calculus Ureteral Thickness (P-CUT) on CT Predicts Stone Impaction at Time of Surgery: A Prospective Analysis RA Chandhoke, B Gallante, JN Bamberger, W Atallah, M Gupta, AM Zampini Mount Sinai
V16-01 Complete retroperitoneal Laparoscopic radical nephroureterectomy for the treatment of upper urinary tract urothelial carcinoma

J Wu, B Xu, M Chen, L Zhang

V16-02 Pure intraperitoneal laparoscopic nephroureterectomy with bladder cuff resection for upper urinary tract urothelial carcinoma G Kaneko, S Hattori, S Shirotake, K Kanao, S Hara, M Oyama

V16-03 A prospective evaluation of impact of adherent perirenal fat on perioperative outcomes of 3-D laparoscopic transperitoneal partial nephrectomy for clinical $\mathrm{T} 1$ renal tumors

A Kumar, S Yadav, S Kumar, K Saurav,

Y Prashant, V Tyagi, V Prasad

V16-04 Solitary kidney and thirteen tumors (13): laparoscopic radical nephroureterectomy, bench ex-vivo nephron-sparing surgery and autotransplantation

La Zanettini, La Zanettini, Ac Zanettini,

Aa Paulmichl, Ov Eye, Mv Rizzon,

Ie Litvin, Ra Guelfii, O Santos,

F Ribeiro, La Zanettini
V16-05 Retroperitoneal Laparoscopic Radical Nephrectomy Technique for Advanced Left T3a Renal Cancer

C Yogeswaran, J Roberts, S Hugo, K Ekwueme

V16-06 Treatment of complex renal tumors, laparoscopic is still available?

C Conde Redondo, F Castroviejo Royo, S Martín Martín, J Torrecilla, F Lara Pérez, A Alonso Villalba, R Cortiñas Gonzalez

V16-07 Off-Clamp Laparoscopic Standard Partial Nephrectomy using PlasmaJet Surgical System R Papalia, a Civitella, E Luperto, P Tuzzolo, F Prata, R Scarpa

V16-08 Renal cell carcinoma in left kidney with tumor caval thrombus: full laparoscopic management

D Perlin, I Alexandrov, I Dymkov, A Shmanev, V Zipunnikov

V16-09 Pure laparoscopic nephroureterectomy with bladder cuff excision and lymph node dissection

J Fang, L Tsai, G Chen

V16-10 Spill Free Technique of Laparoscopic Nephroureterectomy In Multiple Lower Ureteral TCC

S Nachimuthu, R Manickam, S Kallappan 
V16-11 Partial nephrectomy by robotic-assisted laparoscopy for intra parenchymal renal tumor assisted by endoscopic ultrasound in a patient presents a multi-cystic kidney

F Adawi, X Taccoen, A Demey, F Ozone, P Loock
V16-12 Laparoscopic Partial Nephrectomy and Adrenalectomy for Incidentally Detected Left Renal Hilar Mass with Left Adrenal Adenoma. R T.P., D Sarma, SK Pratihar
V17-01 Holmium Laser En-Bloc Resection of Bladder Tumors: The Real Deal M Nobbs, C Chemalsé

V17-02 A case of bladder cancer involving ureteral orifice, which was completely resected by photodynamic diagnosis assisted transurethral resection using oral but not intravesical administered 5-aminolevulinic acid.

K Watanabe, S Shirotake, Y Umezawa, T Takahashi, T Yamanaka, N Santo, T Okabe, G Kaneko, K Sano, M Yasuda, K Kanao, M Oyama, K Nishimoto

V17-03 Memokath 051 migrated to bladder: a solution

J Torrecilla García-Ripoll, F Lara Pérez, S Martín Martín, M Bedate Núñez, J Soto Rodríguez, A Ruano Mayo, A Zamora Horcajada, F Natal Alvarez, M Pina Pallín, J Díaz Romero, J Cortiñas González

V17-04 Current status of thulium: fiber laser in the bladder cancer management A Martov, D Ergakov, N Baykov
V17-05 MPM imaging, a new intraoperative tool for TURB diagnosis

P Treacy, M Durand, I Pavlova, J Sfakianos, R Mehrazin, U Falagario, E Wajswol, F Barthe, Y Ahallal, N Tzavaras, D Chevallier, A Tewari, P Wiklund

V17-06 Holmium Laser Enucleation of a Bladder Tumor: Early Experience

T Large, J Chavali, A Krambeck

V17-07 Aggressive Bladder Tumor- Can intravesical morcellation help?

A Puri

V17-08 En-bloc TURB:

all over the bladder

R Giulianelli, B Gentile, G Mirabile, P Tariciotti, L Albanesi, L Mavilla, G Tema, P Aloisi, A Lopes Mendes, G Rizzo, R Lombardo

V17-09 Transurethral resection of a large bladder hemangioma: a case report

A Martov, D Ergakov, N Baykov

V17-10 Endoscopic Bladder Suture after ureteral and bladder cuff excision during laparoscopic Nephroureterectomy CA Oliveira, AA Barros, R Reis, E Dias, J Correia-Pinto, E Lima
V18-01 Efficient Use of Low Watt Lasers: Good Things Come in Small Packages - Pt. 2. Pulse Length BF Schwartz, DT Lybbert

V18-02 Surgical treatment of matrix stone. Soft stone, hard surgery JM López, A Sierra, P Luque, T Ajami, A Alcaraz

V18-03 Traditional Prone for PCNL: Fundamental Versatility D Mikhail, C Tabib, AD Smith, D Hoenig, Z Okeke

V18-04 Percutaneous Nephrolithotomy for Erosion of Calcified Embolization Coils into the Renal Collecting System

J Alamiri, J Joseph, C Fleming, ME Rivera
V18-05 Efficient Use of Low Watt Lasers: Good Things Come in Small Packages - Pt. 1. Energy and Frequency BF Schwartz, DT Lybbert

V18-06 Innovative technique Bullseye fluoroscopic renal access in supine PCNL K Bhatti

V18-07 Mini Mini- Endoscopic Combined Intrarenal Surgery with LithoVue disposable ureteroscope for severely encrusted double-j ureteral stent A Mangiatordi, U Falagario, M Auciello, G Carrieri, L Cormio

V18-08 Supine Percutaneous Nephrolithotomy (PCNL) in Horseshoe Kidney MO Breish, D Whiting, O Abdalla, S Sriprasad, F Anjum 
-P50-

V18-09 RIRS in Cross Cohen's ureteric re-implantation : A difficult situation but safe and feasible KP Parikh, R Soni, AK Parikh

V18-10 SIMULTANEOUS BILATERAL ENDOSCOPIC SURGERY (SBES): a new technique for the treatment of bilateral renal stones S PROIETTI, ME Rodriguez Socarras, B Eisner, G Saitta, P Bellinzoni, F Gaboardi, G Giusti
V18-11 Endoscopic cystolithotripsy and Mini-PCNL with ballistic and ultrasonic energy for bladder and transplanted kidney stones

JM López, A Sierra, T Ajami, P Luque, L Peri, M Musquera, A Alcaraz

V18-12 ECIRS in Kidney with Complex Anatomy F Pauchard, M Escobar

V18-13 Miniaturized PCNL for renal stone larger than $3 \mathrm{~cm}$ in Galdakao-modified supine Valdivia position

C Liu, T Lin, H Huang 


\section{BASIC SCIENCE POSTER 1: BASIC SCIENCE: ONCOLOGY}

\section{BSP1-01 Comparison of recurrence rate between "en-bloc" resection of bladder tumor and conven- tional technique for non-muscle invasive bladder cancer}

\author{
M. Bangash, H. Ather, N. Khan, S. Mohammad \\ Aga Khan University Hospital, Karachi
}

Introduction \& Objective: Conventional piecemeal Transurethral Resection of Bladder (TURBT) using a wire loop diathermy is the accepted standard of care for bladder cancer. However, this technique violates the basic principle of oncological surgery, i.e. dissection through normal tissue with en bloc removal of the tumor as a single specimen. We conducted to compare the quality of the specimen and report the recurrence rate at first cystoscopy at 3 months duration in both the en-bloc and conventional resection technique.

Methods: After informed consent, we included patients undergoing resection for newly diagnosed bladder tumor with solitary or multiple lesions, none more than $4 \mathrm{~cm}$. All patients received single instillation of chemotherapy (SICA) within 6 hours of resection as per standard. Patients with CIS, prior TURBT, and muscle invasive tumor on histopathology were excluded. En-bloc TURBT was performed using Collins knife attached to diathermy, whereas conventional TURBT was performed using routine resection loop attached to diathermy. Standard bladder diagram was made on operating notes from which the data regarding size, site, and number were retrieved.

Results: A total of 41 en-bloc tumor resections were compared with 41 conventional resections during the study duration. Eight were excluded ( 5 muscle invasive, 3 other histopathologies). Mean operative time was 31.59 minutes (+/-11.44) in en-bloc resections compared to 56.27 minutes (+/-52.21) in conventional resections $(\mathrm{p}=0.005)$. Mean tumor diameter was $2.52 \mathrm{~cm} \mathrm{(+/-0.971)} \mathrm{in} \mathrm{en-bloc} \mathrm{and} 2.74 \mathrm{~cm}(+/-1.266)$ in conventional group (0.370). In terms of specimen quality, $14(34 \%)$ patients among the en-bloc group had detrusor muscle at the base of the primary tumor but only $7(17 \%)$ patients in the conventional group had detrusor muscle in the base of the primary tumor on histopathological examination $(p=0.128)$. Among en-bloc patients, 4 patients had Recurrence at 3 months cystoscopy compared to 9 patients in the conventional group $(\mathrm{p}=0.226)$. No patient had bladder perforation requiring prolonged catheterization ( $>5$ days), holding single instillation chemotherapy or significant bleeding requiring intervention in both the groups.

Conclusions: En-bloc resection is a feasible and safe technique which follows oncological principles. En-bloc resection can potentially improve histopathological quality of specimen and recurrence of bladder tumors at 3 months. In future, it will be interesting to see the effect of en-bloc TURBT on 1-year recurrence.
BSP1-02 Ex-vivo Multiphoton microscopy imaging of prostate biopsy slides as a new tool for prostate cancer prognosis

P. Treacy, I. Pavlova, U. Falagario, E. Wajswol, N. Tzavaras, R. Brody, R. Cabal, J. Bravo-Cordero, P. Wiklund,

M. Durand, A. Tewari

Introduction \& Objective: Multiphoton microscopy (MPM) allows quantitative analysis of collagen surrounding cancerous cells through second harmonic generation (SHG) signal (image 1). Decipher test is a genomic prognosis prediction score. Measure the percentage of collagen fibers in Multiphoton Microscopy (MPM) images of cancerous prostate biopsy pathology slides and correlate them with the Gleason Group Grade (GGG) and Decipher Score. Methods: MPM imaging of cancerous prostate biopsy slides was done on patients who underwent radical prostatectomy. 5 to 10 images $\left(0.5^{*} 0.5 \mathrm{~mm}\right)$ per slide were acquired, and mean collagen percentage was measured dividing SHG by composite area pixels, using ImageJ software (Image 2). Spearman correlation between Decipher test and collagen percentage was done.

Results: 31 patients were selected, 22 patients had Decipher test. Mean collagen percentage was higher as the GGG increased
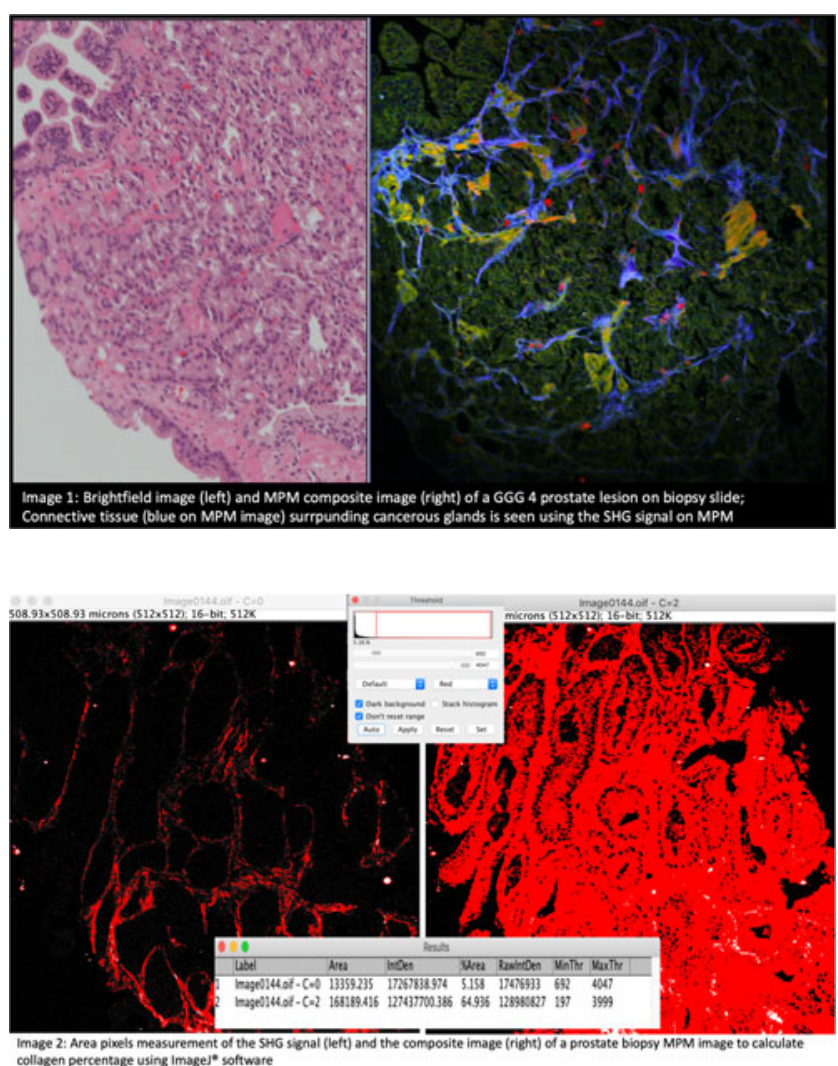


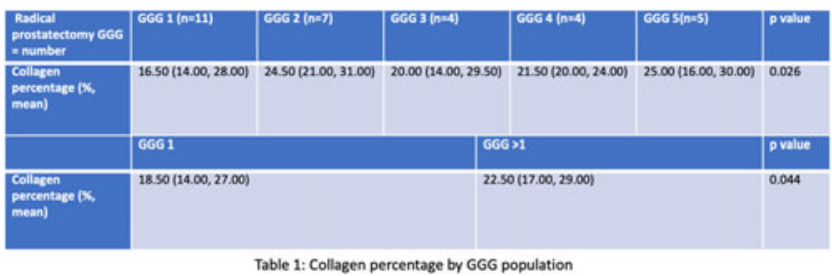

Table 1: Collagen percentage by GGG population

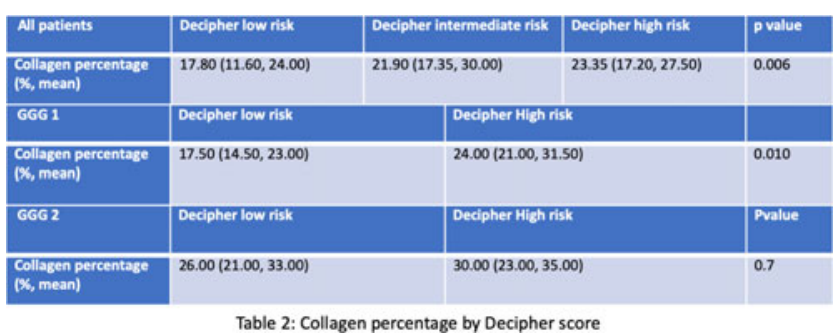

$(\mathrm{p}=0.026$, table 1$)$. Patients with a higher Decipher score had a higher collagen percentage $(p=0.006$, table 2$)$ and GGG 1 population with high Decipher score had an increase in collagen percentage (24\% (21-31.50 vs $17.5 \%(14.5-23), \mathrm{p}=0.01)$. Spearman correlation between Decipher score and collagen percentage was significant in GGG 1-2 population (Spearman's rho $=0.3204, \mathrm{p}=0.0009)($ graph 1$)$.

Conclusions: Collagen percentage increases with the GGG and correlates with Decipher score. MPM imaging of prostate biopsy slides can be an interesting complementary tool in the therapeutic management of GGG 1 and 2 population.

\section{BSP1-03 Urinary N-GAL secretion predicts postop- erative change in renal function after robotic-assisted partial nephrectomy for localized kidney cancer}

M. Takenaka, K. Fukaya, K. Takahara, N. Fukami, M. Ichino, M. Kusaka, R. Shiroki

Introduction \& Objective: Robotic-assisted partial nephrectomy for localized kidney cancer was demonstrated to provide shorter ischemic time and less perioperative complications compared with laparoscopic PN. On the other hand, RAPN still needs warm ischemic time (WIT), which possibly leads to a temporary acute renal injury (AKI) by vascular reperfusion. The serum creatinine level needs time to rise after a renal damage, which means not appropriate for a prediction of AKI. Development of the sensitive biomarker which can predict AKI after PN from an early stage is expected.

Methods: This study included 40 cases of localized kidney cancer treated with RAPN. Tumor size and RENAL NS ranged from 12 to $67 \mathrm{~mm}$ (mean 32) in diameter, from 5 to 9 (median; 7), respectively. Urinary stent catheter was placed before surgery and urine was collected in 1, 3, 12, 24 hours after declamping the renal artery. The data of urinary biomarkers were analyzed whether they have any predictive value for postoperative kidney function or not.

Results: On RAPN procedure, there was no positive surgical margin or open conversion cases noted. WIT ranged from 8 to 47 minutes, 16.5 in median. WIT showed significant negative correlation with eGFR change in one month postoperatively $(\mathrm{p}<0.0001)$. Among urinary biomarkers, NGAL in one hour post reperfusion showed correlation with WIT $(\mathrm{p}=0.0015)$. In addition, neutrophil gelatinase-associated lipocalin (NGAL) in twelve-hour post reperfusion was recognized to have negative correlation with the eGFR change in one month $(\mathrm{p}<0.0001)$, and a significant cutoff level for worse eGFR change was estimated to 100 Î1/4g/g, Cr. Conclusions: Urinary NGAL concentration of diseased side at 12-hour post reperfusion was demonstrated to be a usefulness biomarker to predict the postoperative kidney function after RAPN for localized kidney cancer.

\section{BSP1-04 Withdrawn}

BSP1-05 Transurethral Resection of a Bladder Tumor (TURBT), for the treatment of Non-Muscle Invasive Bladder Cancer (NMIBC), results in changes to selective inflammatory and angiogenic biomarkers

\section{P. Ella-Tongwiis, I.S. Shergill, S.F. Hughes}

North Wales Clinical Research Centre

Introduction \& Objective: Non-muscle invasive bladder cancer (NMIBC) is associated with significant morbidity and mortality, due to high recurrence rates (50-60\%) and the potential for disease progression. Currently, only histology and imaging techniques are routinely used for determining recurrence and disease progression. The discovery of a serum-based biomarker, or panel of biomarkers, could identify patients who are at increased risk of disease recurrence and progression. Sustained increases in serum inflammatory and angiogenic biomarkers following TURBT may be associated with NMIBC recurrence or progression. The main aim of this pilot study was to evaluate changes in inflammatory and angiogenic biomarkers in patients who underwent Transurethral Resection of a Bladder Tumor (TURBT) for the treatment of NMIBC.

Methods: 40 patients ( 7 females, 33 males, average age $=72.9$ years), were prospectively recruited after written informed consent (NHS ethical approval REC: 14/WA/0033). Venous blood samples were taken before TURBT surgery and postoperatively at 1, 3 and 6 months. Selective biomarkers to assess inflammation were measured using commercially available ELISA and BioPlex multiplex assay kits.

Results: Significant increases in serum CD31 $(\mathrm{p}=0.003)$ and Stem Cell Factor $(\mathrm{SCF})(\mathrm{p}=0.005)$ concentration were observed in $\mathrm{BC}$ patients up to 6 months post-operative. There were also significant decreases in Macrophage Inflammatory Protein -2 (MIP-2) $(\mathrm{p}=0.001)$, Platelet Derived Growth Factor (PDGF) $(\mathrm{p}=0.028)$, Matrix Metalloproteinase-9 (MMP-9) $(\mathrm{p}=0.001)$ and Vascular Endothelial Growth Factor C (VEGF-C) serum concentration (Table 1).

\begin{tabular}{|c|c|c|c|c|c|c|}
\hline & CD31 (pg/ml) & SCF (pg/ml) & MIP-2 (pg/ml) & PDGF (pg/ml) & MMP-9 (pg/ml) & VEGF-C (pg/ml) \\
\hline $\begin{array}{c}\text { Easeline (pre- } \\
\text { operative) }\end{array}$ & $9004(7546-12247)$ & $516(430-617)$ & $347(308-499)$ & $1714(1128-2632)$ & $4141(3058-11322)$ & $2711( \pm 971)$ \\
\hline $\begin{array}{c}\text { 1 month post- } \\
\text { operative }\end{array}$ & $12254(8570-14862)$ & $598(396-652)$ & $389(303-536)$ & $1594(783-2119)$ & $2700(1242-3515)$ & $2821( \pm 988)$ \\
\hline $\begin{array}{c}3 \text { months post- } \\
\text { operative }\end{array}$ & $10717(9845-17196)$ & $539(508-713)$ & $285(235-530)$ & $1331(702-1496)$ & $3121(1168-4801)$ & $2406( \pm 795)$, \\
\hline $\begin{array}{c}\text { 6 months post- } \\
\text { operative }\end{array}$ & $11160(10052-14692)$ & $546(480-671)$ & $206(189-320)$ & $1431(903-1932)$ & $2257(1515-3503)$ & $2421( \pm 785)$ \\
\hline pvalue & $p=0.003$ & $p=0.032$ & $p=0.001$ & $p=0.012$ & $p=0.002$ & $p=0.04$ \\
\hline Statistical test & Friedman & Friedman & Friedman & Friedman & Friedman & ANOVA \\
\hline
\end{tabular}

Table 1.0: Changes in various serum biomarkers following TURBT, for the treatment of NMIBC. Doto analysed via Friedman or ANOVA are presented as median \pm lar or mean $\pm S O$ respectively $1 " s$ po. 05 following 
Conclusions: Findings from this study may provide an alternative approach in the monitoring and management of patients with BC. Sustained changes in various markers of inflammation, angiogenesis and tumor growth, such as MIP-2, MMP-9, PDGF, SCF, VEGF-C and CD31, may be utilized as a potential biomarker panel for monitoring patients following surgical treatment of BC. These biomarkers may be used in addition to, or in combination with, currently used scoring systems to predict cancer recurrence and progression. However, verification and validation of these biomarkers are needed using larger cohorts.

BSP1-06 Correlation between serum testosterone and prostate-specific antigen (PSA) in ordinary males

\section{J. Lee, J. Choi}

Introduction \& Objective: Prostate specific antigen (PSA) dose a major role in diagnosis of prostate cancer. In many cases additional considerations such as PSA density, PSA velocity or free PSA are necessary because PSA is not specific for prostate cancer. There are few studies about relations between serum testosterone and PSA. We investigated the association of serum testosterone and PSA.

Methods: We retrospectively reviewed 9318 male patients who visited our urology clinic for benign prostatic hyperplasia or regular health check-up. 582 patients were excluded because of PSA level $>4.0 \mathrm{ng} / \mathrm{ml}$, current usage of 5a-reductase inhibitors, pyuria and lack of data. Total 8736 patients were enrolled for analysis. We evaluated the relation between serum testosterone and PSA with adjustments of age, prostate size, plasma volume and metabolic syndrome.

Results: Mean age of enrolled patients were 50.2 \pm 5.6 years. PSA showed positive relationship with serum testosterone. After fully adjusting for age, total prostate volume, plasma volume and metabolic syndrome, the geometric mean PSA linearly and significantly increased as a quartile testosterone increased $(\mathrm{P}=0.047)$. Additionally, there was significant difference in adjusted mean of PSA between 1st quartile and 4th quartile group of testosterone $(\mathrm{P}=0.006)$ and between 2nd quartile and 4th quartile group of testosterone $(\mathrm{P}=0.006)$. There was significant difference in adjusted mean of PSA between a group with hypogonadism (serum testosterone $<3.5 \mathrm{ng} / \mathrm{mL}$ ) and between a group without hypogonadism $(\mathrm{P}=0.002)$.

Conclusions: The present study suggests that PSA may have positive correlation with serum testosterone. Careful considerations about serum testosterone are recommended for the interpretation of PSA results.

BSP1-07 An upfront multiparametric MRI in the prostate cancer diagnostic pathway avoids prostate biopsy in $56 \%$ of patients. Can PSA density be used to further reduce Likert 3 biopsies?

T. Birks, J. Marenco, A. Roy, D. Cohen, P. Singh

Royal Free Hospital, London

Introduction \& Objective: Multiparametric MRI (mpMRI) based triage reduces the number of men undergoing prostate biopsy. The PRECISION trial demonstrated reduced insignificant prostate cancer detection from $22 \%$ to $9 \%$ and increased significant cancer detection from 26 to $38 \%$. Here we report

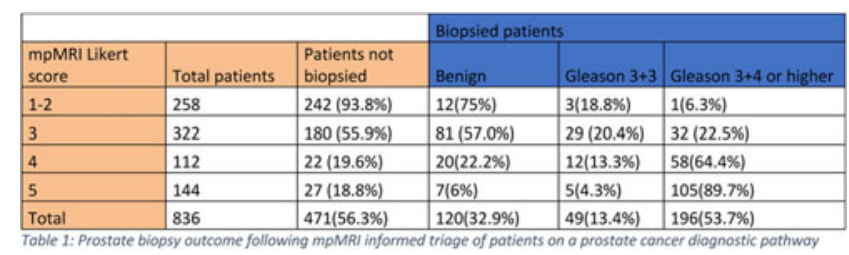

\begin{tabular}{|c|c|c|c|c|c|}
\hline $\begin{array}{l}\text { PSA density } \\
\text { threshold in } \\
\text { patients with a } \\
\text { Likert } 3 \text { mpMRI } \\
\left(\mathrm{ng} / \mathrm{ml}^{2}\right)\end{array}$ & $\begin{array}{l}\text { Number of men } \\
(n=322=100 \%)\end{array}$ & Biopsied & $\begin{array}{l}\text { Benign } \\
\text { ( } \% \text { of } \\
\text { biopsied } \\
\text { men) }\end{array}$ & $\begin{array}{l}\text { Gleason } 3+3 \\
\text { ( } \% \text { of } \\
\text { biopsied } \\
\text { men) }\end{array}$ & $\begin{array}{l}\text { Gleason } 3+4 \\
\text { or higher }(\% \\
\text { of biopsied } \\
\text { men) }\end{array}$ \\
\hline PSAd $<0.12$ & 125 & $39(12 \%)$ & $27(69 \%)$ & $8(6 \%)$ & $4(10 \%)$ \\
\hline PSAd $>0.12$ & 197 & $103(32 \%)$ & $54(52 \%)$ & $21(20 \%)$ & $28(27 \%)$ \\
\hline PSAd $<0.15$ & 174 & $66(21 \%)$ & $41(62 \%)$ & 14 (21\%) & $11(17 \%)$ \\
\hline PSAd $>0.15$ & 148 & $76(24 \%)$ & 39 (51\%) & $15(20 \%)$ & $22(29 \%)$ \\
\hline
\end{tabular}

1-year data of mpMRI-first prostate cancer diagnosis pathway and consider if PSA density may help triage Likert 3 MRI.

Methods: Prospective data was analyzed from 836 biopsy naïve men undergoing mpMRI (1.5T) referred to us on suspicion of prostate cancer. MRI suspicion of significant prostate cancer was Likert scored 1-5 (very unlikely, unlikely, indeterminate, likely or very likely). Patients were counselled regarding biopsy or surveillance based on MRI and clinical risk. Biopsy was systematic or systematic plus fusion when a target was present. Biopsy results following Likert 3 MRI were analyzed to model risk stratifying these men using two different PSA density thresholds, 0.12 or $0.15 \mathrm{ng} / \mathrm{ml}^{2}$.

Results: Median age was 66 years (range 33-93). Median PSA was $7.1 \mathrm{ng} / \mathrm{ml}$ (0.3-2096). Median prostate volume was 44ml (9-444). 471 men (56\%) avoided a prostate biopsy. Of the 365 men biopsied, 49 (13\%) showed insignificant prostate cancer (Gleason 6). 196 (54\%) had significant prostate cancer (Gleason $3+4$ or higher). Rate of significant prostate cancer increased with LIKERT score (table 1). $78 \%$ of biopsies in men with Likert 3 MRIs were either benign or Gleason 6 (table 2). If a threshold of $0.12 \mathrm{ng} / \mathrm{ml}^{2}$ had been applied 39 fewer men would have proceeded to biopsy but 4 significant cases of cancer would have been missed. A threshold of $0.15 \mathrm{ng} / \mathrm{ml}^{2}$ would have prevented 66 men undergoing biopsy but missed 11 significant cases of cancer. A similar significant cancer detection rate of $27-29 \%$ was found at both thresholds.

Conclusions: This single institution dataset shows upfront MRI, with clinical consultation, prevents $56 \%$ of men referred proceeding to prostate biopsy. A threshold of $0.12 \mathrm{ng} / \mathrm{ml}^{2}$ may be the optimal threshold to triage Likert 3 MRIs to biopsy without significantly increasing the risk of missing significant prostate cancer.

\section{BSP1-08 Uro-TC and ureteroscopy: useful tools in the diagnosis of upper urinary urothelial carcinoma}

F. Lara Pérez, S. Martín Martín, J. Torrecilla García-Ripoll, J. Diaz Romero, M. Bedate Núñez, F. Natal Alvarez,

J. Soto Rodríguez, A. Ruano Mayo, A. Zamora Horcajada, C. de Castro Olmedo, R. Calvo González, L. Pesquera Ortega, J. Calleja Escudero, E. Valsero Herguedas,

M. Pascual Samaniego, D. Rivero Martínez, A. Alonso Villalba, J. Díez Rodríguez, C. Conde Redondo, J Cortiñas González

Hospital Clinico Universitario de Valladolid 
Introduction \& Objective: Although the Uro-CT is the goldstandard test for the diagnosis of UTUC, the ureteroscopy may be of value in case of uncertain diagnosis and provides information for therapeutic management. The goal of our study was to determine the correlation between the Uro-CT and the ureteroscopy in the diagnosis of the Upper Urinary Tract Urothelial Carcinoma (UTUC).

Methods: Retrospective and descriptive study including all patients with a UTUC suspicion that underwent Uro-CT from January 2013 to March 2019. The Uro-CT images were analyzed by 2 independent radiologists, that classified the likeliness of UTUC of as high suspicion (HS) or low suspicion (LS). The Uro$\mathrm{CT}$ result was then compared with the result of the ureteroscopy. Demographic and pathological anatomy results of the selective urinary cytology during the ureteroscopy and biopsy samples from during the intervention were analyzed.

Results: 80 patients were included, $88.75 \%(\mathrm{n}=71)$ were males with a median age of 66.7 years [43-85]. 53.85\% $(n=43)$ had a lesion diagnosed by Uro-CT classified as HS by both radiologists, while $38.8 \%(n=31)$ had a lesion classified as LS by both radiologists. The inter-observer correlation coefficient $(\mathrm{k})$ was $0.847(\mathrm{p}<0.001)$. There was no correlation of the results between radiologist in $7.6 \%$ of cases $(n=?$ ? $)$. From the 43 patients in the HS group, in $79.1 \%(n=34)$ the ureteroscopy biopsy result confirmed the diagnosis. $4.6 \%(n=2)$ had a severe ureteral stenosis that prevented a successful catheterization. $8.75 \%(n=7)$ cases were false positives. On the other hand, from the 31 patients in the LS group, $32.4 \%(\mathrm{n}=12)$ had a suspicious image in the ureteroscopy and in $21.6 \%(n=8)$ the malignancy was confirmed by the biopsy, with a moderate $\mathrm{k}$ coefficient $(0.488$; $\mathrm{p}<0.001)$. Thirty-five patients $(43.75 \%)$ underwent biopsy, $28.75 \%(n=23)$ were in the HS group and in all cases the biopsy confirmed the diagnosis of UTUC. For patients in the LS group $(32.4 \%, \mathrm{n}=12)$ positive results for UTUC were obtained in 8 cases. The $\mathrm{k}$ coefficient was $0.301(\mathrm{p}<0.05)$.

Conclusions: The concordance between the two radiologists is high for the diagnosis of UTUC. One fifth of patients with a low risk image in the Uro-CT had a positive biopsy result for UTUC, whilst in a not negligible number of patients $(67.5 \%)$ classified as high risk the biopsy did not confirmed the initial suspicion. Our experience suggests that the diagnosis and management of patients with suspicion of UTUC must be individualized and perhaps in low risk cases a second Uro-CT before the ureteroscopy might be of help.

\section{BSP1-09 Prostate specific antigen kinetics predict prostate cancer progression following holmium laser enucleation of the prostate}

K.M. Rose, A. Nevo, K.S. Faraj, A. Navaratnam, K.A. Arora, S.M. Cheney, M.R. Humphreys

Mayo Clinic

Introduction \& Objective: Introduction and Objective: Holmium laser enucleation of the prostate (HoLEP) is an effective outlet procedure and has a higher incidental prostate cancer (PrCA) rate than traditional BPH procedures. Outcome data regarding the clinical progression of patients with prostate cancer following HoLEP is lacking. We aim to describe the clinical course as well as disease progression of PrCA following HoLEP. Methods: A retrospective review of HoLEP surgeries at our institution by a single surgeon was performed. We included patients with prostate cancer diagnosed preoperatively or incidentally at the time of HoLEP. Clinical progression was defined as the need for additional therapies following HoLEP. We excluded patients undergoing HoLEP prior to planned definitive therapy for prostate cancer in the sub-analysis for clinical progression. Results: 1,288 HoLEP cases were performed by a single surgeon, 152 of whom had PrCA. $133(10.4 \%)$ were incidentally diagnosed. Following HoLEP, 23/149 (15.4\%) patients met criteria for clinical progression at a median of 20 months. The clinical progression group demonstrated a significantly higher preoperative prostate specific antigen (PSA), preoperative PSA density (PSAD), first postoperative PSA, postoperative PSAD, and PSA velocity. After HoLEP, twelve $(7.8 \%)$ patients underwent radiation therapy, $18(11.8 \%)$, underwent androgen deprivation, $6(4 \%)$ underwent robot assisted radical prostatectomy, and $1(0.6 \%)$ underwent high intensity focused ultrasound of the prostate. Patients were more likely to demonstrate clinical progression with PSA velocity $>0.75 \mathrm{ng} / \mathrm{dL} /$ year (OR 19.3, 95\% CI 4.9-75.8, $\mathrm{p}<0.01$ ) and a PSA doubling time (PSADT) $<36$ months (OR 10.2, 95\% CI 1.3-81.6, p<0.01). When predicting clinical progression following HoLEP, the sensitivity of PSADT $<36$ months was $94 \%$, the negative predictive values for a PSA velocity $<0.75 \mathrm{ng} / \mathrm{dL} /$ year was $95 \%$, and PSADT $>36$ months was $97 \%$. The estimated overall survival for all patients at 5 and 10 years was $87.4 \%$ and $58.7 \%$, respectively. There was no significant difference in estimated overall survival in those that did vs. did not clinically progress (89.2 vs. 97.3 months, $\mathrm{p}=0.72$ ).

Conclusions: Following HoLEP, patients with PrCA should be surveyed closely for clinical progression. We identified PSA kinetics to be critical in assessing the risk of clinical progression following HoLEP. While PSA surveillance is an important determinant in the decision making regarding additional therapies, there is also a need to identify factors that guide patients to watchful waiting, without exposing them to additional testing and biopsies involved in active surveillance.

\section{BSP1-10 Photodynamic Therapy in Combination with EGFR Targeted Photosensitizers Enhances the Long- Term Cure of Bladder Cancer in Animal Models}

F.A. Durrani, J. Cacaccio, R.R. Cheruku, N.A. Aldhaam, K.A. Guru, R. Pandey

\section{Roswell Park Comprehensive Cancer Center}

Introduction \& Objective: Photodynamic therapy (PDT) treating bladder cancer, mice bearing UMUC3 (human transitional cell carcinoma) and T24 (human urinary bladder) tumors using photosensitizers (PS) with and without conjugating with an erlotinib moiety. PS with high tumor-selectivity over Photofrin, a current clinical standard, should show enhanced long-term survival of bladder cancer patients with reduced skin phototoxicity, a major problem associated with Photofrin-PDT.

Methods: IVIS/Spectrum: A noninvasive in vivo, fluorescence imaging. The SCID mice bearing either UMUC3 or T24 tumors were injected with the PS $\mathbf{1}$, and the corresponding erlotinib conjugates 3, 5 (Pluronic). The PSs were injected (i.v.) in tumored mice, imaged at time intervals, uptake and pharmacokinetics of the PS in tumor, liver and skin was measured.

Results: In vitro PDT efficacy: To compare the cytotoxic potential of PS 1 and erlotinib analogs $3 \& 5$, an MTT assay was conducted, and to assess the EGFR target specificity, UMUC3 cells with overexpression of EGFR and T24 cells with low expression of EGFR were used. Interestingly, PS 1 (a non-erlotinib analog) showed similar photosensitizing activity in both the cell lines with low (T24) and high (UMUC3) EGFR expression. In vivo Efficacy Studies: To determine a comparative in vivo PDT 
efficacy of PS 1 and the corresponding erlotinib analogs $3 \& 5$, the PS were injected (iv) at a dose of $0.47 \mathrm{mmol} / \mathrm{kg}$ in SCID mice bearing UMUC 3 tumors. The tumors were then exposed with a laser light $\left(665 \mathrm{~nm}, 135 \mathrm{~J} / \mathrm{cm}^{2}, 75 \mathrm{~mW} / \mathrm{cm}^{2}\right)$ at $24 \mathrm{~h}$ post injection. PS 5 in which the erlotinib moiety attached at position-17 of the PS showed improved long-term cure $(\mathrm{CR}) 5 / 11=55 \%$ ) than PS 3 in which the erlotinib moiety attached at position-3 of the PS $(0 / 5=0 \%)$ and PS 1 with no erlotinib moiety only (CR) 1 / $5=20 \%$ on day 60 .

Conclusions: The PS with or without erlotinib showed higher in vivo uptake (determined by fluorescence), in UMUC3 (EGFR positive) and T24 (low) bladder cancer tumors with significant differences in the two tumor types. The PS formulated in Pluronic showed higher uptake in both UMUC3 and T24 tumor and significant difference in their pharmacokinetic profiles The PDT efficacy of PS with and without an erlotinib moiety in mice bearing UMUC3 tumors showed enhanced long-term tumor cure based on the conjugation of erlotinib at specific position. Before initiating this approach to human Phase I clinical trial, further studies are underway.

BSP1-11 The EGFR, and FABP7 gene mutations had discriminating power to differentiate metastatic overall survival in liver metastasis from metastatic renal cell carcinoma after targeted therapy

\section{W. Park, D. Hong, J. Park, J. Chung, S. Kim, S. Kim}

Introduction \& Objective: This study aimed to find out any significant mutated genes to predict the metastasis to either liver, bone or other sites in tissues from primary and metastatic renal

Figure 1. The (A) spreading metastatic pattern and (B) Kaplan Meier overall survival curves according to metastatic sites among 32 patients with $\mathrm{mRCC}$
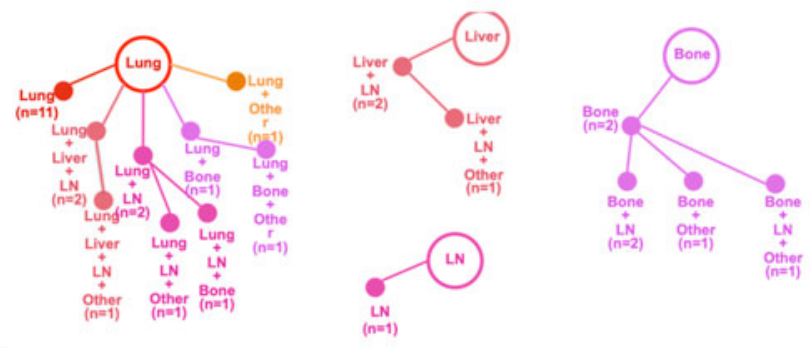

A.

B.

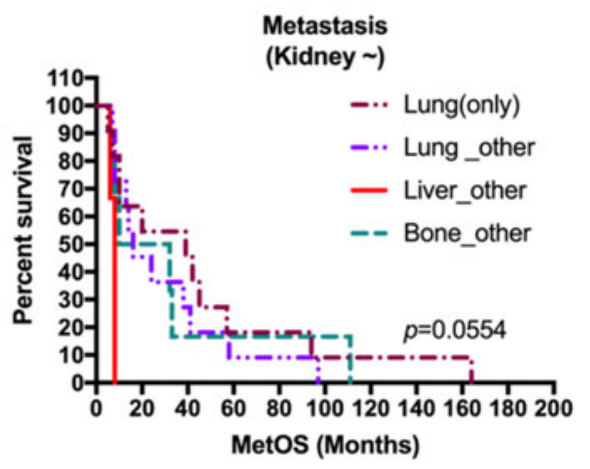

cell carcinoma after treated with systemic first-line targeted therapy in patients with metastatic renal cell carcinoma (mRCC). Methods: Between 2005 and 2017, 32 paired primary kidney and metastatic tumor tissues were obtained among $56 \mathrm{mRCC}$ patients and the targeted gene sequencing (TGS) using the 88 targeted genes from the National Cancer Center were performed. The metastatic OS was used for the statistical outcome and defined as the time interval between the diagnosis of metastasis and death. Any significantly mutated genes with metastatic organs were analyzed to find out any significant mutated gene predictive for metastatic OS (log rank p-value <0.05).

Results: There were 6 liver metastasis, 9 bones, and 17 other lesions such as brain, lung, lymph node and others in 32 patients. The 32-paired tissues were analyzed that liver metastasis was the worst OS-paired metastasis than other metastatic lesions $(p=0.05)$. Among the 88 TGS, EGFR $(\log$ rank test $p=0.0081$, HR: 14.3), and FABP7 (log rank test $p=0.0229$, HR: 5.38) were significantly differentiate the metastatic OS among different metastatic lesions. We then applied the combinational mutation number from two candidate genes in the liver metastatic samples that presence of mutated EGFR2 and FABP7 gene (log rank test $p=0.0058$, HR: 5.11 had significantly worse OS than those in other metastatic lesions.

Conclusions: The study showed EGFR, and FABP7 gene mutations as candidate genes that can significantly discriminate the metastatic OS in liver metastatic RCC in mRCC.

BSP1-12 Closed loop audit of muscle sampling in trans-urethral resection of bladder tumor

\author{
R. Georgiou, R. Varma, A. Chetwood, A. Ali
}

Introduction \& Objective: Our experience with presence of detrusor muscle (DM) following transurethral resection of bladder tumor (TURBT).

Objectives: To evaluate the presence of DM in primary TURBT specimens as a quality improvement exercise in Frimley Park Hospital, UK. In addition, to determine the clinical efficacy of the en-bloc resection technique in all intermediate and high-risk non-muscle-invasive bladder cancer cases.

Methods: Retrospective electronic record histopathology reports from a single urology center, servicing a group of urologists, were obtained for TURBT procedures performed between January 2014 and May 2018.

Results: A total of 242 cases were performed under the care of 7 consultant urologists. Analysis revealed a significant improvement in the quality of resections from $39 \%$ to $84 \%$ after the introduction of the following interventions: (1) analysis of current practice, (2) introduction of European Association of Urology (EAU) guidelines by separating samples and sending a second specimen pot marked as bladder tumor base to look for DM, (3) dedicated urologists with an interest in bladder cancer performing TURBTs using the en-bloc resection technique in appropriate cases.

Conclusions: Through our closed loop audits we have demonstrated a considerable improvement in the quality of resections. In the future we aim to analyze the effect of these measures on cancer recurrence and progression. Our simple interventions have improved the rate of DM yield in order to accurately stage the tumor.

\section{BSP1-13 Withdrawn}


BSP1-14 Possible role of 5-alpha reductase inhibitors in non-invasive bladder urothelial neoplasm: multicentric retrospective study

A.A. Pastore, C. De Nunzio, M. Balzarro, Y. Al Salhi, A. Fuschi, A. Martoccia, N. Amigoni, A. Tubaro, W. Artibani, A. Carbone

Sapienza University of Rome, Faculty of Pharmacy and Medicine, Dept. of Urology

Introduction \& Objective: About $75 \%$ of urothelial carcinoma of the bladder are non-invasive (non-muscle-invasive bladder cancer, NMIBC), therefore limited to mucosa (Ta or CIS) or submucous (T1). Several studies have shown that androgens are implicated in bladder carcinogenesis: the increase in androgen expression and androgen receptors have a positive effect on oncogenic expression, which can lead to an increase of those specific proteins that promote the proliferation, invasiveness and motility of neoplastic cells. The aim of the study was to evaluate whether 5-alpha reductase inhibitors (5-ARI) have a role in NMIBC.

Methods: The retrospective analysis was conducted on 293 patients diagnosed with NMIBC who underwent transurethral resection of the bladder (TURB), from 2013 to 2018. The study was conducted evaluating demographic characteristic of each patient, intraoperative and postoperative parameters. We analyzed the number of resections, number of total recurrences, time of recurrences, and histopathology details. The population was divided into two main groups: treated and untreated whit 5-ARIs. Patients were treated with dutasteride for lower urinary tract symptoms due to prostatic hyperplasia (mean treatment time 16.8 months) The patients were followed according to EAU follow-up guidelines for NIMBC. Mean follow-up time was 29.5 months (range 24-50 months).

Results: No significant differences were observed among the different groups at baseline. The group treated with 5-ARIs presented a lower rate of recurrences $(14.78 \%)$ than the untreated group $(37.11 \%)$. Regarding the mean number of recurrences statistically significant difference was observed between the untreated (1.944 \pm SD 0.141) and the treated group (1.235 \pm SD 0.206; p-value: 0.0066), respectively. Furthermore, evaluating the recurrences, the 5-ARIs group showed a lower T stage when compared to the untreated group ( $p$-value $=0.037$ ). No significant differences were obtained regarding the grade of the recurrences $(p$-value $=0.756$ ).

Conclusions: Long-term treatment with 5-alpha-reductase inhibitors might play a role in reducing the risk of tumor recurrence and $\mathrm{pT}$ and extension of the lesions. Starting from this experience, a long-term, randomized prospective study could definitively assess the possible role of this widely used drugs in NMIBC.

\section{BSP1-15 MiR-193a-3p inhibits aldosteronoma growth by down-regulating CYP11B2 expression}

Z. Guoxi, Z. Xiaofeng, L. Quanliang, X. Tianpeng, H. Zhihua

Introduction \& Objective: To explore the correlation and possible mechanism between mir-193a-3p and adrenal aldosterone tumor.

Methods: A total RNA of 15 patients with adrenal aldosterone tumor were collected after operation from aldosterone tumor and adjacent normal adrenal gland. The expression of mir-140-3p, mir-193a-3p, mir-22-3p and CYP11B2 mRNA were detected by quantitative reverse transcription PCR (qrt-pcr). Cultured adrenocortical cell line H295R and transfected with mir-193a-3p. Cell proliferation was detected by colorimetry and apoptosis and cell cycle progression were analyzed by flow cytometry. The levels of aldosterone in the supernatant were detected by ELISA. Results: By detecting three miRNAs, miR-193a-3p expression was significantly decreased and CYP11B2 mRNA expression was significantly increased in APA compared with adjacent normal adrenal tissue. The miR-193a-3p mimic was transfected into the human adrenocortical cell line H295R, and it was found that increased expression of miR-193a-3p inhibited cell proliferation and aldosterone secretion, induced G1 arrest and promoted apoptosis of H295R cells. In addition, by luciferase gene assay, overexpression of miR-193a-3p in H295R cells significantly reduced the luciferase activity of the wild-type CYP11B2 3'-UTR construct and found that it can pass miR-193a- Reversal of mutation of the $3 p$ binding site. In addition, miR-193a-3p overexpression down-regulated the expression of CYP11B2 mRNA and protein. Finally, overexpressed CYP11B2 attenuated the effect of miR-193a-3p on H295R cells.

Conclusions: Our study suggests that miR-193a-3p may be involved in down-regulating the expression of CYP11B2 and inhibiting APA growth. This also explains that down-regulation of miR-193a-3p promotes cell growth and inhibits apoptosis during APA formation.

\section{BSP1-16 Feasibility of the Epicheck test in upper urinary tract tumor.}

R. Boissier, A. Territo, A. Gallioli, F. Regis, D. Vanacore, J. Palou, A. Breda

Department of Urology. Fundació Puigvert. Autonomous University of Barcelona. Barcelona. Spain

Introduction \& Objective: Several studies reported the low specificity and sensitivity of urine cytology for both diagnosis and surveillance of UTUC and the increased risk of bladder recurrence when a diagnostic ureteroscopy is performed prior to nephroureterectomy. Bladder EpiCheck ${ }^{\mathrm{TM}}$ (EpiCheck) is a urine test developed by Nucleix Ltd for monitoring the recurrence of bladder cancer based on analysis of 15 informative DNA methylation biomarkers that demonstrated a high NPV 95\% in bladder cancer and even $99 \%$ for high grade. The objective was to assess the diagnostic accuracy of Epicheck in UTUC.

Methods: Single center prospective not comparative study including all patients who underwent a ureteroscopy for UTUC since June 1st, 2018 in our center. All patient had a preoperative CT urography with a suspicion of uni or bilateral UTUC. As first step of diagnosis ureteroscopy, a cystoscopy was performed to exclude a synchronous bladder tumor. Bladder urine was collected and ureteral urine was collected with a monoJ. Cytology and Epicheck were performed on each bladder and ureteral urine samples. A diagnostic ureteroscopy was performed in each patient to identify UTUC and biopsy were performed in case of tumor.

Primary outcome was to calculate sensitivity, specificity, negative predictive value of voided and selective. Epicheck. Secondary outcome was the accuracy of Epicheck in identifying high grade UTUC.

Results: A total of 25 patients were included. $3(12 \%)$ patients had a history of cystectomy. $4(16 \%)$ had a suspicion of bilateral UTUC. Mean age was 70 years and $83 \%$ were male. The diagnosis of UTUC was confirmed by ureteroscopy and biopsy in 15 ureters (52\%), with 7 high grade, 6 low grade and 2 undetermined grade. Sensitivity, specificity, negative of bladder and ureteral Epicheck 
were: $55 \%, 55 \%, 55 \%, 55 \%$ and $67 \%, 85 \%, 83 \%, 69 \%$ for the diagnosis of all UTUC ; $100 \%, 75 \%, 71 \%, 100 \%$ and $89 \%, 88 \%$, $89 \% 88 \%$ in the diagnosis of high grade UTUC. Diagnostic performances of Bladder Epicheck was similar in bladder vs ureteral urine with a high sensitivity and specificity for high grade UTUC. Conclusions: These preliminary results shew the feasibility and the high diagnostic performance of Bladder Epicheck in UTUC. If further results confirm theses preliminary results, Bladder Epicheck could change the diagnostic, treatment and follow-up strategies of UTUC. In case of radiological suspicion of UTUC, Epicheck could avoid unnecessary ureteroscopy to confirm the diagnosis or recurrence of UTUC and avoid an attempt of unadaptated endoscopic conservative treatment in case of high grade UTUC.

BSP1-17 Connective tissue transcriptomic signature and worst clinicopathological outcomes in prostate cancer patients

P. Treacy, A. Martini, P. Ratnani, S. Nair, U. Falagario, A. Horowitz, M. Durand, A. Tewari

Introduction \& Objective: Understand the correlation between genomic features of connective tissue and worst clinicopathological outcomes (extra-capsular extension (ECE), lymph node invasion $(\mathrm{LN}+)$, early biochemical recurrence (BCR) on radical prostatectomy specimens, and their impact on progression-free survival.

Methods: 652 patients who underwent radical prostatectomy and had a Decipher diagnostic test were included. Single Channel Array-Normalized expression of coding genes and transcript results were analyzed. Relationship between transcript results and clinical features such as ECE, LN+ and early BCR (Biochemical Recur-
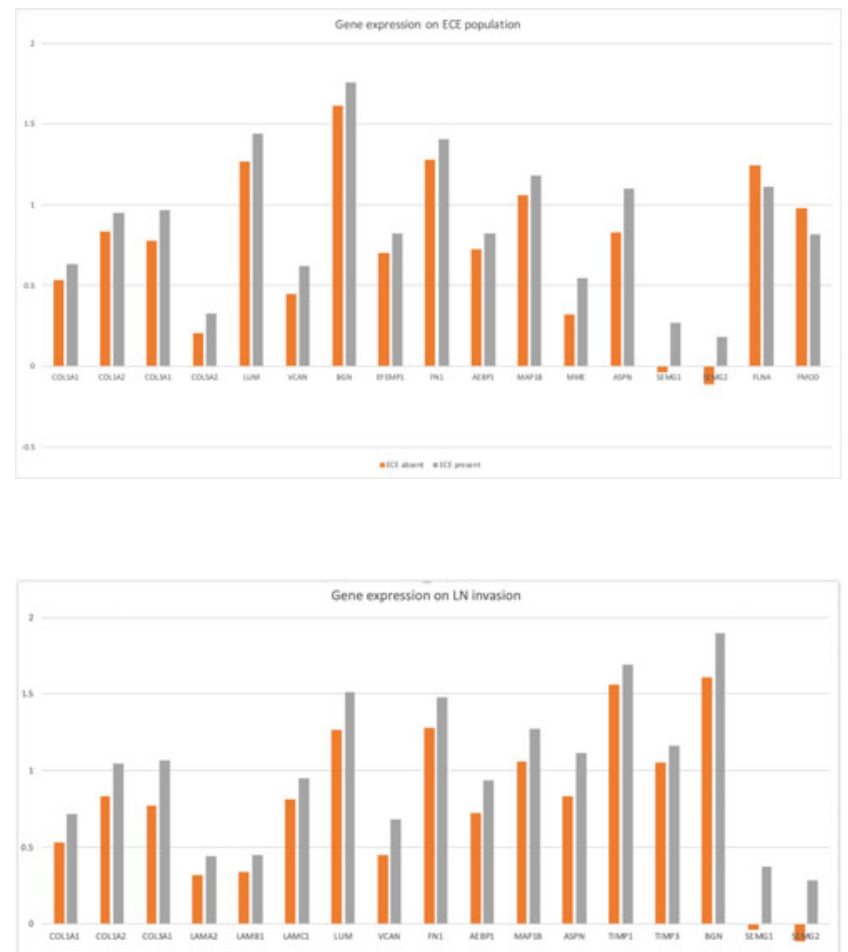

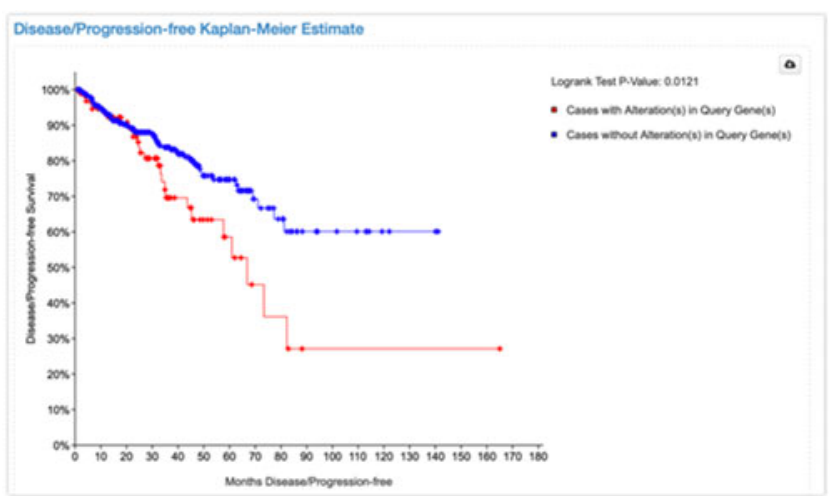

rence) was investigated with multiple t test and Benjamin Hochberg correction. The Cancer Genome Atlas (TCGA) was used to evaluate genes prognostic role on progression-free survival (PFS).

Results: 214 patients had ECE, 33 patients had LN+, and 136 had an early BCR. Multiple $t$ test on microarray data revealed significant differential gene expression on word clinical outcomes: 1433 for ECE (501 unregulated), 1114 for LN+ (407 unregulated), and 7539 for early BCR (1538 unregulated). COL1A1 and COL1A2 (collagen type I), COL3A1 (collagen type III), ASPN (asporin), VCAN (versican), FN1(Fibronectin), LUM (lumican), BGN (biglycan), MAP1B (microtubule associated protein 1B) and AEBP1 were over expressed in patients with ECE and LN+. In early BCR, only COL1A1 (0.5316vs $0.006)$ and ASPN (0.899 vs 0.82) were over expressed $(\mathrm{p}<0.001)$ On TCGA population, overexpression of these genes showed a worst 5-year PFS rate $(52 \%$ vs. $74 \%(\mathrm{p}=0,0121))$.

Conclusions: RNA overexpression of connective tissue genes is correlated to worse clinical features, such as ECE, LN invasion, and early BCR. TCGAp cohort analysis showed a worse PFS when overexpression of the connective tissue genes.

BSP1-18 Preoperative neutrophil/lymphocyte ratio (NLR) as a prognosticator of recurrence and progression in upper tract urothelial carcinoma (UTUC) patients treated conservatively: a retrospective evaluation

M. Antonucci, L. Defidio, N. Russo, M. De Dominicis, A. Patel

Introduction \& Objective: To evaluate the potential prognostic value of NLR during follow up (NLR - an expression of the involvement of systemic inflammation in neoplastic growth + progression) in conservatively treated UTUC patients.

Methods: Between 2005 and 2017110 patients who underwent uretero-renoscopy and RIRS (retrograde intra renal surgery) UTUC treatment with endoscopic follow-up as per EAU guideline recommendations, in a single tertiary referral national center, were retrospectively evaluated. Patients dropped out after radical treatment. NLR was calculated at baseline before $1^{\text {st }}$ treatment episode. The patients were stratified into two groups according NLR value (cut-off point 3). Upper tract disease free survival (uDFS), bladder disease free survival (bDFS) and progression free survival (PFS), were evaluated by age adjusted multivariate Cox regression. Kaplan-Meier plot was drawn and log rank test was performed.

Results: Overall 110 patients were enrolled with a mean follow up of 28.4 months (SD 27 - range 12 to 144), median age of 73 years (IQR 67/79), and mean NLR of 3.09 (SD 2.04). Group 1 


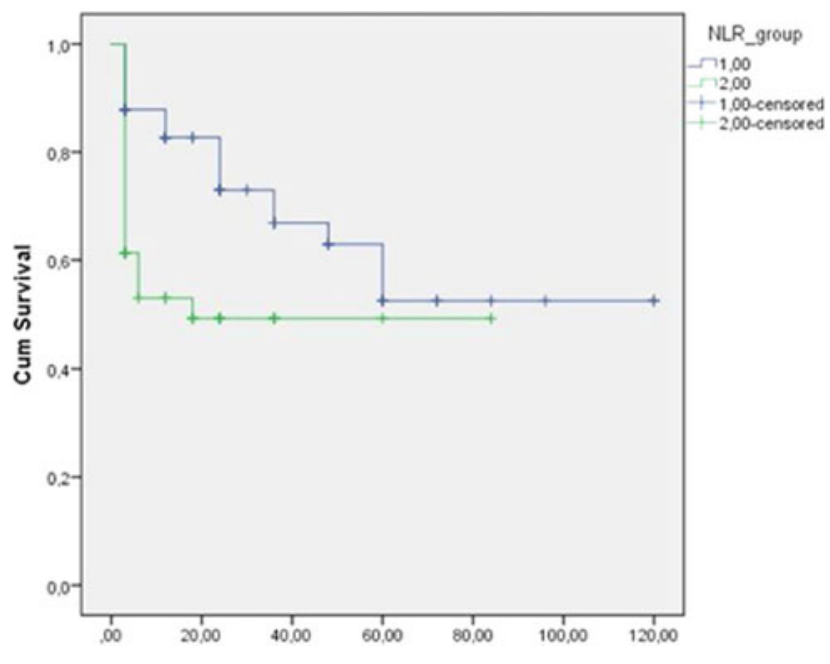

consisted of 66 patients ( $60 \%$ ) with baseline NLR $<3$ and Group 2 of 44 patients $(40 \%)$ with NLR $>3$. On multivariate Cox regression, which included age, grade, size and single/multiple tumor(s), baseline NLR $>3$ significantly predicted for shorter PFS $(\mathrm{HR}=0.47 ; \mathrm{p}=0.019 ; 95 \% \mathrm{CI}=0.25-0.89)$ but not for $\mathrm{uDFS}$ $(\mathrm{p}=0.26)$ or bDFS $(\mathrm{p}=0.52)$. Figure 1 Kaplan Meier plot graphically showing the PFS difference between the two Groups - Mean PFS in Group 1=76 mo. (SD 7) vs. Group 2=43 mo. (SD 6.3). Log rank test 0.007.

Conclusions: Baseline NLR evaluation before $1^{\text {st }}$ UTUC conservative treatment may provide valuable prognostic information for disease progression risk and an opportunity for early risk stratification. It may also prevent under-treatment in those at high progression risk and an individualized surveillance strategy where extirpative surgery is initially declined, not safely possible for any reason, or where an imperative for renal preservation exists. Baseline NLR variation did not significantly impact upper tract or bladder recurrence in this cohort. Prospective multicenter studies with larger study populations are needed to validate the prognostic role of NLR.

\section{BSP1-19 Epidermal Growth Factor Receptor (EGFR) Targeted Multifunctional Photosensitizers for Bladder Cancer Imaging (Fluorescence and PET) and Photo- dynamic Therapy (PDT)}

R.R. Cheruku, J. Cacaccio, F.A. Durrani, W.A. Tabaczynski, R. Watson, A. Marko, M. El-Khouly, S. Fukuzumi, R. Kumar, J. Missert, R. Yao, M. Sajjad, D. Chandra, A.A. Hussein, K.A. Guru, R. Pandey

\section{Roswell Park Comprehensive Cancer Center}

Introduction \& Objective: This report describes the advantages of a multifunctional 124I-labeled [methyl- 3-(1'-m-iodobenzyloxy) ethyl-3-devinyl-pyropheo phorbide-a (PETONCO) over the 18Ffluorodeoxy glucose (18F-FDG) for imaging bladder cancer by positron emission tomography (PET). As a non-radioactive iodinated analog, it can be used for fluorescence-guided PDT (photodynamic therapy) of mice bearing bladder cancer.

Methods: In our attempt to develop EGFR targeted multifunctional photosensitizer (PS), an erlotinib moiety known for targeting EFGR was conjugated either at position-3 or at position-17 of the iodinated PS following the well-established Sonogashira cross coupling methodology.

Results: The comparative in vitro efficacy (cell uptake, intracellular localization, and photosensitizing efficacy and EGFR target specificity) of the iodinated PS with and without an erlotinib moiety was investigated in UMUC-3 (EGFR positive) and T24 (EGFR low) cell lines. Among the compounds investigated, the PS containing the erlotinib moiety at position-17 of the PS showed higher tumor-uptake, improved tumor contrast and long-term tumor cure by PDT in SCID mice bearing UMUC3 tumors.

Conclusions: The comparative in vitro efficacy (cell uptake, intracellular localization, and photosensitizing efficacy and EGFR target specificity) of the iodinated PS with and without an erlotinib moiety were investigated in UMUC-3 (EGFR positive) and T24 (EGFR low) cell lines. Among the compounds investigated, the PS containing the erlotinib moiety at position-17 of the PS showed improved PET imaging and PDT efficacy. The position-17 erlotinib conjugate also showed higher in vivo uptake (determined by fluorescence), PET-imaging ability and PDT efficacy in SCID mice bearing UMUC3 tumors.

BSP1-20 Single cell multiplex proteomic imaging on nephrectomy and renal tumor biopsy papillary renal cell carcinoma pairs

K.G. Sands, J. Hsieh, D. Cao, R. Figenshau, A. Ramachandran, L. Yang, A. Sivaraman, E. Kim, R. Venkatesh

Washington University School of Medicine, St. Louis, USA

Introduction \& Objective: The scant translational progress made in non-clear cell renal cell carcinoma (nccRCC) concurs with its lagging in clinical improvement. Papillary renal cell carcinoma (pRCC) represents most nccRCC. Besides cancer genomics, key tumor features including the heterogeneity of cancer-immune interactions in pRCC have not been thoroughly interrogated. To address this unmet need, we developed a multiplex immunofluorescence (MxIF) platform and examined a small pilot cohort whose paired primary nephrectomy and biopsy tumor samples were available for single cell proteomic imaging (SCPI).

Methods: A search of our single institutional renal biopsy database identified 32 pRCC cases among which 6 have sufficient primary nephrectomy and biopsy materials for SCPI study using the GE CellDive MxIF system. The pathological features of paired samples were examined by a designated GU pathologist and subjected to the MxIF-based single cell tumor-immune interaction profiling. An established custom RCC antigen panel consisting of 13 immune, 3 epigenetics, 3 segmentation, 2 signaling, and 1 proliferation markers were developed and applied to this cohort. 22 images were taken on the same single slide per tumor sample and interrogated at single cell resolution for immune subtyping (Figure 1).

Results: The size of the pRCC tumors at biopsy was $3.97+$ /$0.77 \mathrm{~cm}$. When comparing matched nephrectomy and biopsy tumor pairs, nuclear grades were consistent in all cases, whereas
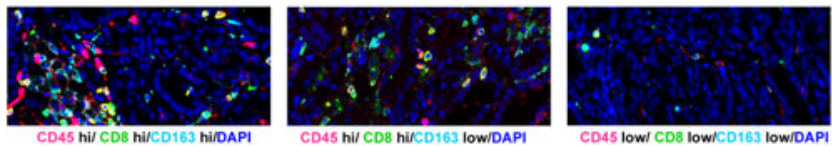
tumor immune subtypes exhibited an unexpectedly high discordance. Based on membranous positive staining (hi defined as $>5 \%$; low as $<2.5 \%$ ) of CD45 (pan-immune), CD8 (cytotoxic $\mathrm{T}$ ), and CD163 (M2 macrophage) in the nephrectomy tumors, three distinct Immune profiles of pRCC was identified, i.e. CD45hiCD8hiCD163hi; CD45hiCD8hiCD163low; and CD45lowCD8lowCD163low.

Conclusions: SCPI of pRCC identified three distinct tumor immune subtypes, uncovering distinct tumor-immune interaction patterns. This pilot study is limited by small sample size. Further analyses and future studies on a large cohort $(>100)$ of pRCC are warranted to confirm tumor-immune heterogeneity and provide needed clinical insights.

\section{BSP1-21 Aquaporin 3 low expression in cancer tissue of urothelial carcinoma patients}

V. Pavlov, K. Danilko, L.M. Kutliyarov, A. Sivkov,

R. Khairullina, E. Shut

Bashkir State Medical University

Introduction \& Objective: Aquaporins (AQPs) are well known water channel proteins. Increasing evidence strongly suggest that AQP3 plays a pivotal role in cancer progression and metastasis. AQP3 is not a specific marker for benign or malignant epithelial neoplasms and changes in its expression can be multidirectional in the tissues of various forms of cancer. But the role of this protein in urothelial carcinoma of the bladder has been investigated only in one patient cohort. The objective of this study was to investigate the pattern of expression of AQP3 in neoplastic and benign tissues of bladder.

Methods: Fresh tissue samples of the 39 patients with urothelial carcinoma following transurethral resection or radical cystectomy were collected. Gene expression profile of AQP3 mRNA transcript in 33 tumor tissues samples or 17 visually normal tissue samples were determined using real-time PCR (RT-PCR). The differences in mRNA expression between neoplastic and benign bladder tissues was assessed using Mann-Whitney U test and GraphPad Prism v.6.0 software.

Results: $\Delta \Delta \mathrm{Ct}$ method was used to compere the mRNA expression levels in cancer and benign bladder tissues of 39 urothelial carcinoma patients using RT-PCR. MRNA expression of AQP3 gene was significantly lower in tumor tissues than in benign tissues (Mann-Whitney $\mathrm{U}=173.0, \mathrm{P}=0.0274$ ).

Conclusions: AQP3 is expressed significantly lower in bladder cancer tissue than in normal tissue. This finding can be used to improve the early diagnostic of urothelial carcinoma.

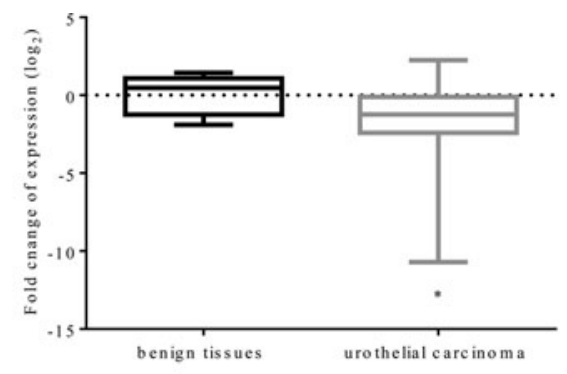

Fig. Relative mRNA expression level of AQP3 in urothelial carcinoma and benign
BSP1-22 Knockdown of COPS3 Inhibited the progress of Prostate Cancer through reducing phosphorylated p38 MAPK expression and impaired the Epithelial-Mesenchymal Transition process

\author{
Y. Hong, Z. Zhu, Q. Xu, X. Huang, F. Zhang, L. An
}

Peking University People's Hospital

Introduction \& Objective: The amplification of gene COPS3 was closely related to the development of osteosarcoma and hepatocellular carcinoma. However, the effects of COPS3 on prostate cancer are poorly understood.

Methods: In this study, the protein expression of COPS3 in prostate cancer tissues, adjacent normal tissues and bone metastasis tissues of prostate cancer were analyzed by immunohistochemistry. Furthermore, the cell proliferation, colony formation, migration and invasion assay were performed in DU145 and PC-3 cells after knockdown COPS3 by si-RNAs.
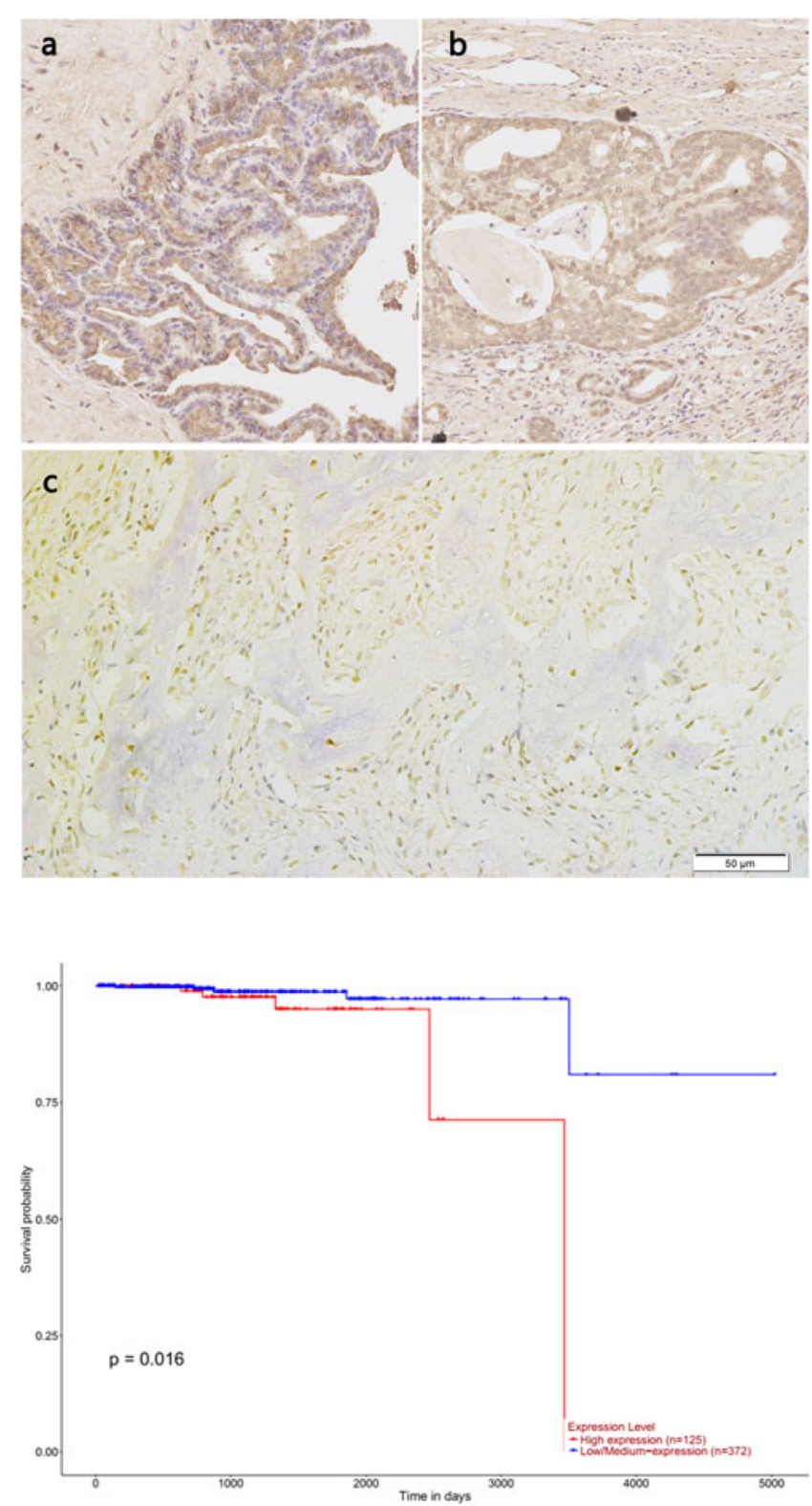
a

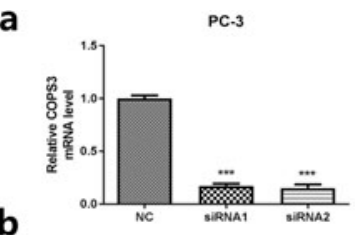

b

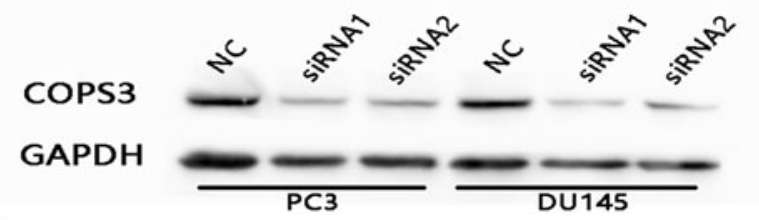

C

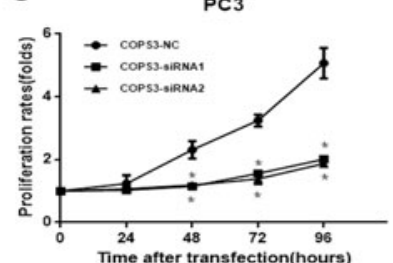

d

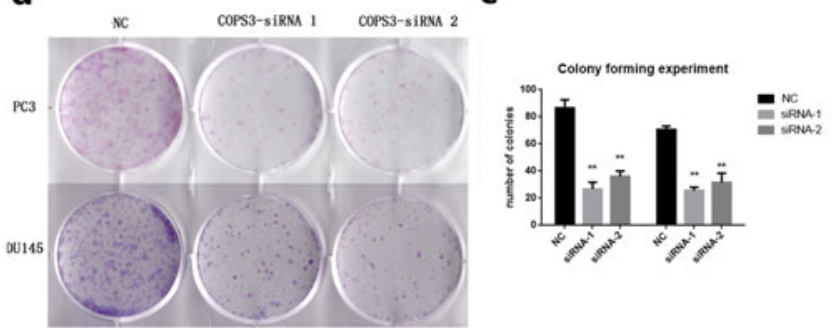

Furthermore, we performed western blotting to explore the potential mechanisms underlying it.

Results: This study found that the overall survival of the COPS3 high expression group was significantly shorter than the low expression group. This report discovered that the protein expression of COPS3 in prostate cancer tissues was higher than that in the matched non-tumor prostate tissues. In addition, tissues from bone metastasis of prostate cancer had a high percentage of over-expressing COPS3. After knockdown of COPS3 gene in DU145 and PC3 cells, two classic human prostate cancer cell lines which had a high level of COPS3, the abilities of migration, invasion and proliferation were inhibited. Finally, protein levels of phosphorylated P38 MAPK and N-cadherin was significantly decreased after knockdown the expression of COPS3, and the protein levels of E-cadherin was significantly increased.

Conclusions: In conclusion, COPS 3 may be closely related to the progress of prostate cancer. Knockdown of COPS3 inhibited the progress of $\mathrm{PCa}$ through reducing the levels of phosphorylated P38 MAPK and impaired the Epithelial-Mesenchymal Transition (EMT) process. a

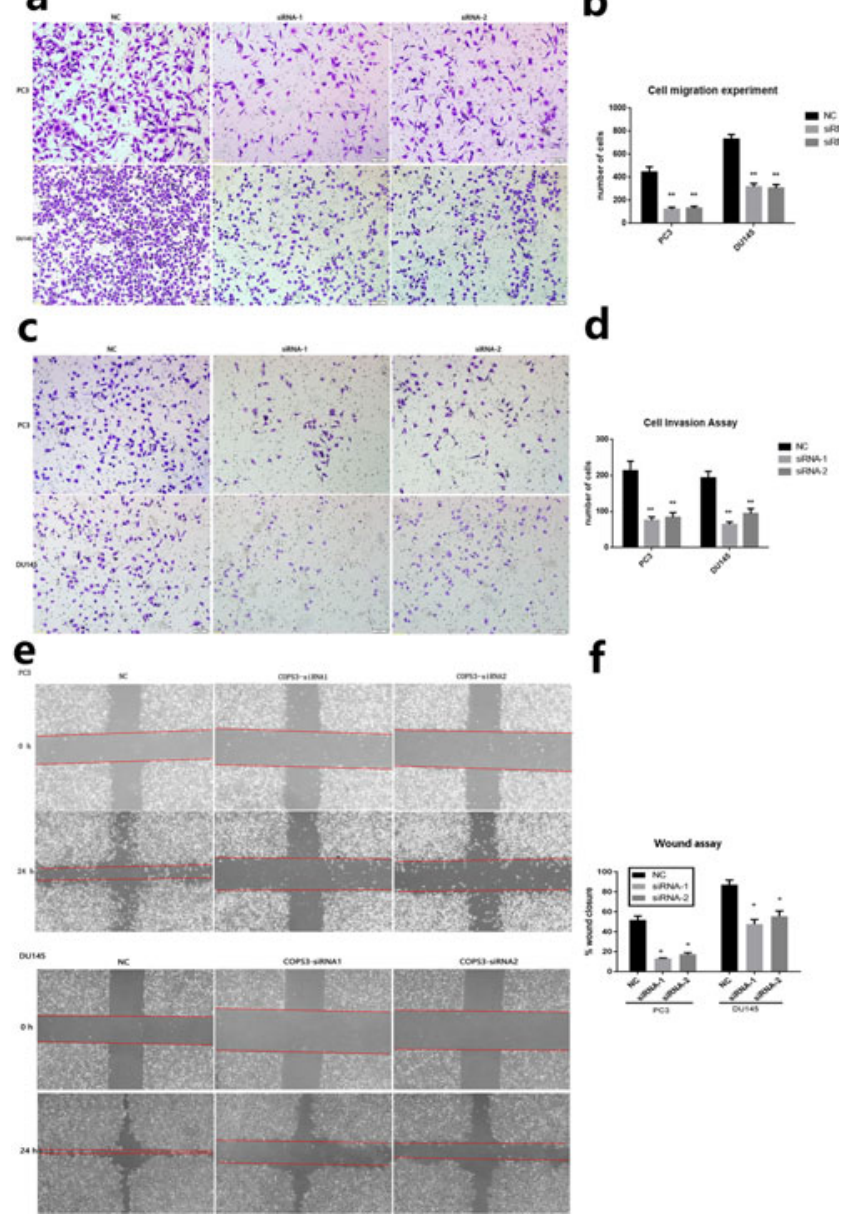

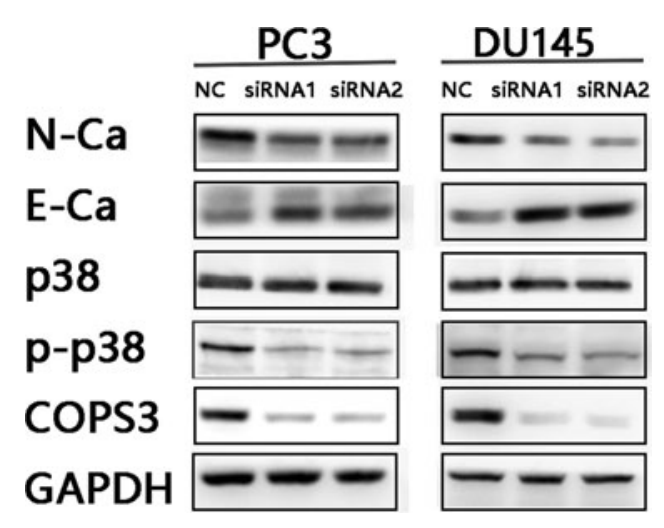




\section{BASIC SCIENCE POSTER 2: BASIC SCIENCE: BPH/LUTS \& RECONSTRUCTION}

\begin{abstract}
BSP2-01 Endoscopic early realignment or delay urethral reconstruction for traumatic urethral total disruption: a single center experience
\end{abstract}

\section{T Chang}

China Medical University Hospital

Introduction \& Objective: The optimal management strategy for urethral injuries remains controversial. Suprapubic cystostomy followed by delayed urethral reconstruction had been view as standard therapy in guidelines but it was also associated with higher complication rate. Early endoscopic realignment for urethral injuries provides early definitive procedure with low complications. In this study, we report our experience of management urethral total disruption.

Methods: Between January 2009 and April 2019, all patients with traumatic urethral total disruptions were reviewed. Both patients of anterior and posterior urethra total disruption were included. Patients were managed by endoscopic urethral realignment or suprapubic cystostomy followed by delay urethroplasty. Preoperative retrograde urethrography, or abdominopelvic CT were performed to evaluate the urethral defect length, the prostate position, and the extent of the pelvic hematoma, presence of pubic bone fracture, severity of dislocation and presence of other organ damage. Early realignment was defined by placement of urethra foley within 14 days. Failure of realignment was defined by urethral foley balloon not within the bladder. Retrograde urethrography or urethroscopy were performed 1-3 month after realignment or urethroplasty.

Results: 25 patients in the age group 16-75 (mean 41) were treated. 9 patients suffered from anterior urethra total disruption, and 15 patients suffered from posterior urethra total disruption. Presence of pubic bone fracture, femoral bone fracture and ISS was significant higher in posterior urethral disruption group. Early realignment was performed in 5(55.5\%) and $8(53 \%)$ patients each group. Suprapubic cystostomy followed by delay reconstruction as initial management was performed in 4(44.4\%) and 7(46.7\%) patients each group. The rate of urethra stricture that required further dilation or DVIU was similar in both early and delay reconstruction group. But early realignment was associated with shorter operative time, shorter hospital stay, less blood loss for both anterior and posterior urethra disruption. We also proposed an algorithm for the management of urethra total disruption in

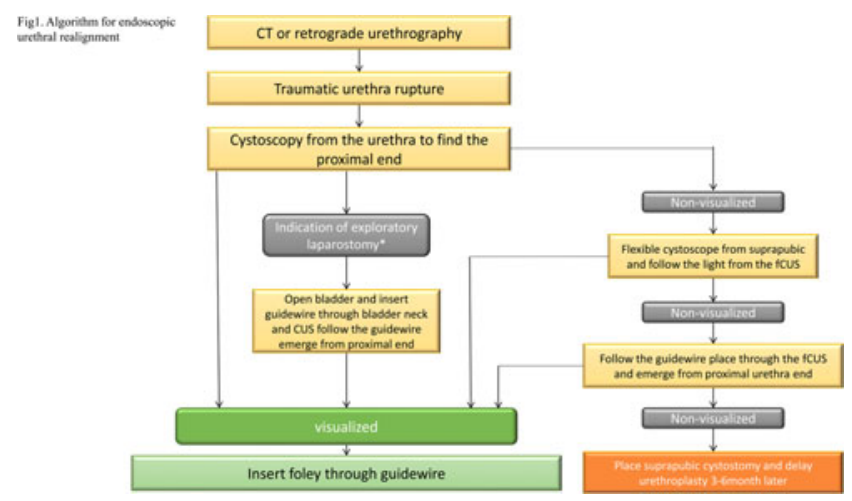

\begin{tabular}{|c|c|c|}
\hline & anterior urethra injury & posterior urethra injury \\
\hline No. & 9 & 15 \\
\hline age & 47 & 34 \\
\hline pubic bone fracture & 4 & 14 \\
\hline unstable pelvic fx & 3 & 4 \\
\hline other associated injury & 3 & 13 \\
\hline femur fx & 2 & 7 \\
\hline bladder rupture & 1 & 3 \\
\hline rectal injury & 0 & 2 \\
\hline brain injury & 1 & 0 \\
\hline ISS score(mean) & 15 & 25.1 \\
\hline$<9(\mathrm{No} / \%)$ & 0 & 0 \\
\hline 9 to $15(\mathrm{No} / \%)$ & $6(66.6 \%)$ & $2(13 \%)$ \\
\hline$>=16(\mathrm{No} / \%)$ & $3(33.3 \%)$ & $13(86.7 \%)$ \\
\hline \multicolumn{3}{|l|}{ method of surgery } \\
\hline \begin{tabular}{|c|} 
early realigment \\
\end{tabular} & $5(55.5 \%)$ & $8(53 \%)$ \\
\hline $\begin{array}{l}\text { suprapubic + delay } \\
\text { reconstruction }\end{array}$ & $4(44.4 \%)$ & $7(46.7 \%)$ \\
\hline
\end{tabular}

Table2. Outcome of posterior urethra injury

\begin{tabular}{|c|c|c|}
\hline & early realignment( $\mathrm{N}=8)$ & $\begin{array}{l}\text { suprapubic + delay } \\
\text { reconstruction( }(\mathrm{N}-7)\end{array}$ \\
\hline operative time mean (min) & 120 & 435 \\
\hline blood loss & 90 & 258 \\
\hline \multicolumn{3}{|l|}{ method to identified proximal end } \\
\hline direct & 1 & . \\
\hline endoscopic light & 1 & - \\
\hline Guidewire from suprapubic flexible cystoscope & 4 & $\cdot$ \\
\hline open bladder & 2 & - \\
\hline stricture & $7(87.5 \%)$ & $7(87.5)$ \\
\hline DVIU (times) & $1(0-4)$ & $1.16(0-3)$ \\
\hline failure & $2^{*}$ & $2 * *$ \\
\hline
\end{tabular}

"one patient was found Foley catheter ballon dislocation was noted about $4 \mathrm{~cm}$ away from bladder neck in cystography about 3 month after alignment and endoscopic optical incision to the proximal end was performed and urethra foley was final inserted.

The other patient had severe urethra stricture after early realignment and reconstructive urethroplasty was performed

which early realignment as initial approach and delay urethral reconstruction as salvage management if early realignment fail.

Conclusions: Compared with delay reconstruction, endoscopic early realignment of urethral rupture is less invasive and a safer procedure, without additional injuries. It has shorter operative time, shorter hospital stay, less blood loss, and delay urethral reconstruction may serve as salvage management if early realignment fail.

BSP2-02 Efficacy and safety of low power (50W) HoLEP in the management of LUTS/BPH: Wrexham Maelor initial experience

O Obi-Njoku, C Bell, SL Moore, M Abdimalik, SF Hughes, I Shergill

Member Royal College of Surgeons England

Introduction \& Objective: We evaluated the efficacy and safety of Low Power (50W) HoLEP in the management of LUTS/BPH. Methods: A review of the first 100 consecutive cases in a prospectively maintained database between January 2016 and August 2018 (31 months). Primary outcome measure was efficacy, 
Table 1:

Secondary

outcome measure

\begin{tabular}{|c|c|}
\hline Parameter & Median (Range) \\
\hline $\begin{array}{c}\text { On-table operative time } \\
\text { (mins) }\end{array}$ & $90.1(10-210)$ \\
\hline $\begin{array}{c}\text { Prostate weight enucleated } \\
(\mathrm{g})\end{array}$ & $39(2-150)$ \\
\hline $\begin{array}{c}\text { Enucleation time } \\
\text { (mins) }\end{array}$ & $0.7(0.1-4.5)$ \\
\hline $\begin{array}{c}\text { Enucleation efficiency } \\
\text { (g/min) }\end{array}$ & $7(0.5-40)$ \\
\hline $\begin{array}{c}\text { Morcellation time } \\
\text { (mins) }\end{array}$ & $4.7(0.3-59.5)$ \\
\hline $\begin{array}{c}\text { Morcellation efficiency } \\
\text { (g/min) }\end{array}$ & \\
\hline
\end{tabular}

defined as successful voiding in catheterized patients and improvement in IPSS/QOL in non-catheterized patients. Secondary outcome measures were safety (Clavien-Dindo Classification), and the factors shown in Table 1.

Results: The median age of patients in this study was 73 years (48-89), with a median prostate volume of 70cc (20-190). Efficacy was $97 \%$ of patients with retention (57/59) and 95\% (39/ $41)$ of those with LUTS. Average pre-and-post-operative IPSS/QOL score was $22.7 / 5$ and 6/1. Overall it was safe with minimal complications (less than $10 \%$ ), all cases managed conservatively (Clavien-Dindo Grade 1) with no further intervention and were mostly temporary stress urinary incontinence. Results of other secondary outcome measures are listed; Table 1. Conclusions: In Wrexham Maelor hospital, Low Power (50W) HoLEP is efficacious and safe with an overall success rate of $96 \%$ based on the available data in this study.

\section{BSP2-03 Is there a role for minimally invasive pye- loplasty in children less than $20 \mathrm{~kg}$ ?}

\section{S Palaniappan, A Ganpule, A Singh, R Sabnis, M Desai}

Muljibhai Patel Urological Hospital

Introduction \& Objective: Our objective is to assess if there is difference in outcomes between open, laparoscopic and robotic pyeloplasty in children under $20 \mathrm{~kg}$.

Methods: A retrospective analysis of all open, laparoscopic and robotic pyeloplasties done for children of weight under $20 \mathrm{~kg}$ was carried out. Indwelling stents was the standard of care. However in smaller children, a ureteric catheter was placed as splint across the anastomosis with a ureteric catheter. Patient demographics, duration of surgery, length of hospital stay, post-operative complications as well as the need for re-intervention were collected. Success was defined as resolution of symptoms or absence of hydronephrosis during the follow-up.

Results: A total of 157 pyeloplasty was performed in children of weight less than $20 \mathrm{~kg}$. Half of them were open pyeloplasty $(\mathrm{n}=78)$ procedures while laparoscopic pyeloplasty numbered 52 of them and robotic pyeloplasty made up 27 in number. Over the mean follow-up of 16.3 months, the success rates were comparable (98.7\% vs $96.2 \%$ vs $96.3 \%$ ) in the OP, LP and RP groups respectively. Mean operative time was significantly longer by 35 mins in minimally invasive pyeloplasty when comparing with open pyeloplasty. Being a more demanding procedure, laparos- copy requires advanced suturing skills while robotic surgery is very challenging in the small child as there are no specialized equipment currently available. Hospitalization was significantly shorter in the minimally invasive groups (4 days) compared to the open pyeloplasty group (7days). Post-operative complications were not significantly different.

Conclusions: Open pyeloplasty remains the gold standard in children less than $20 \mathrm{~kg}$. However laparoscopic and robotic pyeloplasty had equivalent outcomes to open surgery with a shorter hospital stay. We conclude that in this large series of small children, the minimally invasive approach is technically challenging but a valid approach in high volume centers.

\section{BSP2-04 Magnetic Stimulation Therapy for Urinary Incontinence: a Meta-analysis}

\author{
Q He, K Xiao, D Luo, H Li, K Wang
}

Department of Urology, Institute of Urology (Laboratory of Reconstructive Urology), West China Hospital, Sichuan University, Chengdu, Sichuan, P.R.C

Introduction \& Objective: Magnetic stimulation (MS) treatment as a novel approach for urinary incontinence (UI), the applicability of it is still unclear. This systematic review and metaanalysis was conducted to evaluate the effects of MS treatment on UI.

Methods: Literature searching was performed in Embase, PubMed and Cochrane Library (up to August 26th, 2018), and all English-language randomized control trials (RCTs) were screened whether or not meeting the inclusion criteria. Manual searching the reference lists was also executed among retrieved studies. The efficacy of MS treatment was assessed between active stimulation and sham stimulation.

Results: Eleven studies involving 612 patients were included in this review. According to the results of the meta-analysis, MS therapy relieved UI symptoms evaluated using the International Consultation on Incontinence Questionnaire-Short Form (ICIQSF) score (MD $-3.03,95 \%$ CI -3.27 to -2.79 ). In addition, the frequency of UI in the MS treatment group was also alleviated compared with sham group (MD -1.42, 95\% CI -2.15 to -0.69). Finally, MS treatment improved the quality of life of patients with UI (SMD -1.00, $95 \%$ CI -1.24 to -0.76 ).

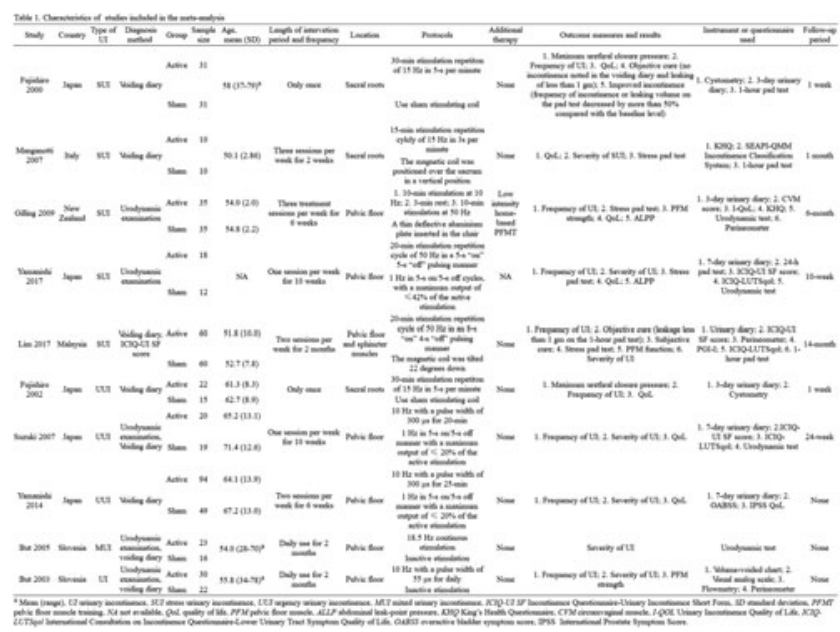



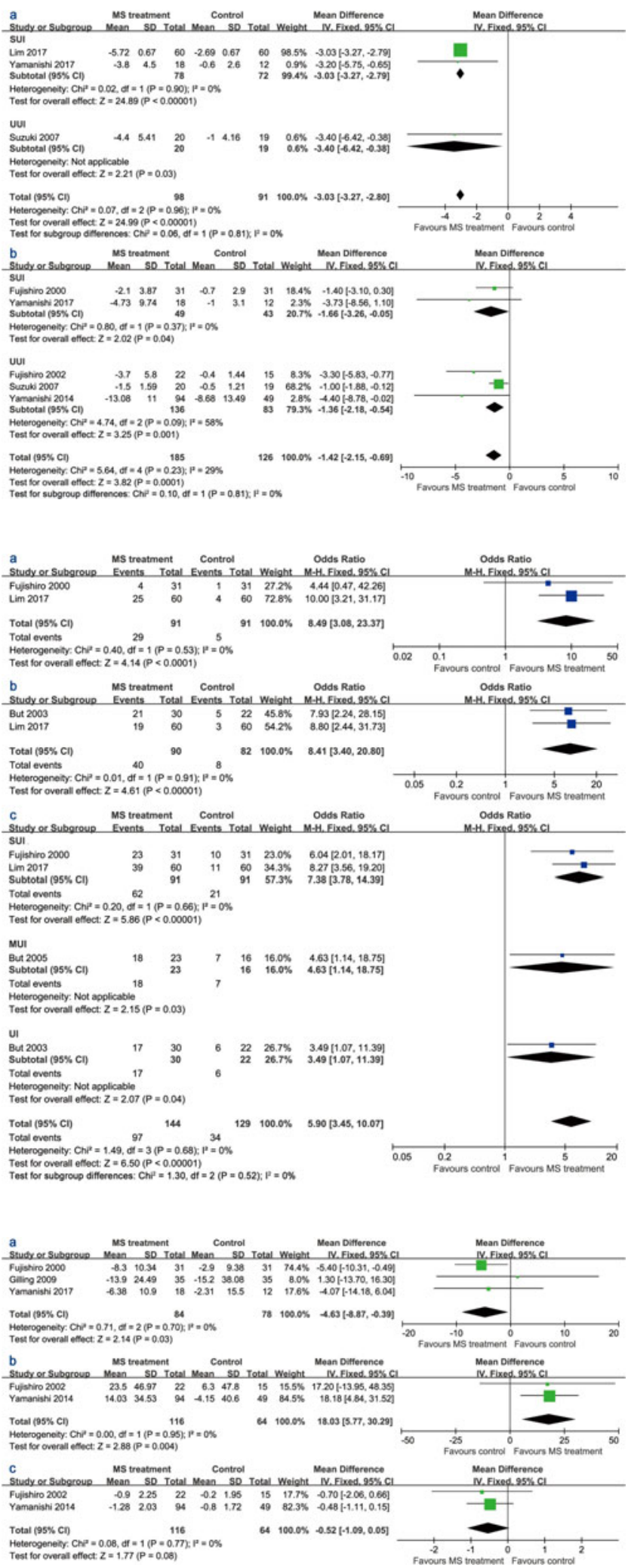

Conclusions: Our meta-analysis preliminarily indicates that MS treatment is an effective therapeutic modality for patients with UI. Nevertheless, additional large, high quality RCTs with a longer follow-up period that use consistent stimulation methods and analyze comparable outcomes are required to validate the efficacy.

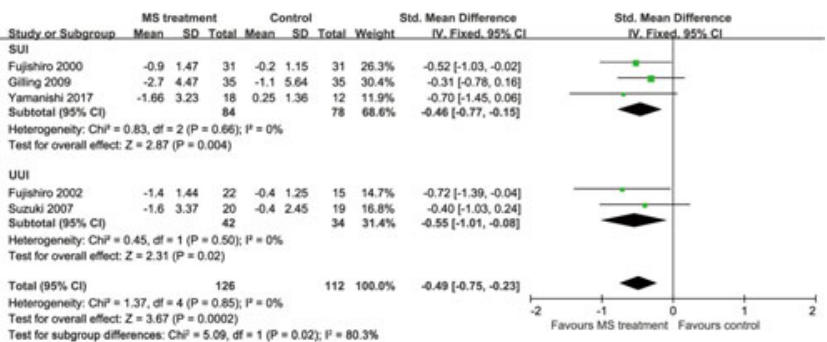

BSP2-05 Effect of Holmium Laser Enucleation of the Prostate (HoLEP) on Prostate-Specific Antigen (PSA) Kinetics for Prostate Cancer Patients Actively Managed by Observation

A Ibrahim, A Zakaria, M Wissing, W Kassouf, M Aube, S Tanguay, S Carrier, M Elhilali, A Aprikian

\section{McGill University}

Introduction \& Objective: Prostate-specific antigen (PSA) has been used as a surrogate predictor for prostate volume. When prostate cells are destroyed, the serum PSA level decreases proportionally to the amount of prostatic tissue removed. However, the effect of Holmium Laser Enucleation of the prostate (HoLEP) on prostate-specific antigen (PSA) dynamics in patients with localized prostate cancer ( $\mathrm{PCa}$ ) previously managed by observation, is not well known. Our aim was to evaluate the effect of HoLEP on PSA Kinetics for prostate cancer patients actively managed by observation.

Methods: A prospectively maintained database for patients undergoing HoLEP was reviewed. Patients with PCa diagnosis between 1998 and 2016 managed by active surveillance (AS) or watchful waiting (WW) were included for analysis. We excluded patients with incidental PCa diagnosis at the time of HoLEP or PCa treatment before HoLEP. Cohort demographics, clinical, laboratory and pathological data were collected at baseline, pre-HoLEP and post-HoLEP. PSA velocity was defined as the difference between PSA measurement immediately post-HoLEP and the last obtained measurement (or the last measurement before a new treatment was initiated) divided by the time between these two measurements. Linear regression models were used for analysis. Results: Of the 1555 HoLEP cases, 92 (5.9\%) patients were identified to have prostate cancer and only $22(1.4 \%)$ patients met the study inclusion criteria (AS and WW), the median age at the time of surgery was $72.5(69-79)$ years and the median postHoLEP follow up time was 7.3 years. The cohort had a pre-HoLEP median PSA of $8.0 \mathrm{ng} / \mathrm{dL}$ (5.6-16.8) and median prostate volume of $64.5 \mathrm{~mL}$ (47.6-111.4). The absolute median decline in the first PSA measurement post-HoLEP which was $-4.7 \mathrm{ng} / \mathrm{ml}$ (-2.3-9.7) with a relative decrease of $47.4 \%$. The median lowest PSA reached post-HoLEP was $1.8 \mathrm{ng} / \mathrm{ml}(0.7-5.4)$ during a median period of 5.2 months (3.7-52.6). Over the study period, the cohort median PSA velocity was $0.73 \mathrm{ng} / \mathrm{ml} /$ year $(0.36$ for the AS-group and 3.58 for the WW-group). On subgroup analysis, higher baseline primary Gleason score and a lower percentage of cancer in post-HoLEP specimen were found to significantly increase relative PSA changes post-HoLEP $(\mathrm{P}=0.004$ and 0.022 , respectively. $)$

Conclusions: Active Surveillance prostate cancer patients undergoing HoLEP had a very slow PSA velocity after HoLEP. This could be attributed to higher resection velocity (efficiency) after HoLEP. Further prospective studies are definitely warranted to compare HoLEP with other minimally invasive procedures and to determine the effect of efficient resection on postoperative PSA nadirs. 
BSP2-06 Is Detrusor Overactivity a predictor factor of Posterior Tibial Nerve Stimulation outcome in the treatment of Overactive Bladder?

W Alamari, B Malallah, A Alabbad, M Algahwari, M Aldokhi, O Sarhan, R Almousa

Introduction \& Objective: Posterior Tibial Nerve Stimulation (PTNS) is a well-known treatment for overactive bladder (OAB) not responding to conventional treatment. Herein, our aim is to assess the significance of Urodynamic proven Detrusor Overactivity (DO) on the outcome of PTNS in patients with OAB syndrome.

Methods: Single center, retrospective study, reviewed the medical charts of all adult patients with $\mathrm{OAB}$ with or without detrusor Overactivity (DO) who underwent Posterior Tibial Nerve Stimulation (PTNS) in our center between January 2012 until December 2018. Hospital Institutional review board was obtained before starting the study. Patients' demographic data, diagnosis, voiding diary pre and post Posterior Tibial Nerve Stimulation (PTNS) treatment, and outcome collected. All patients had baseline investigations (urine analysis, serum Creatinine, Urodynamic study, Renal Ultrasound). Each Patient had to fill a voiding diary and quality of life questionnaire at the beginning of therapy (Week 0) and after completion of the initial weekly therapy (week 12). The success of treatment was defined as $50 \%$ or more improvement of voiding dysfunction symptoms in voiding diary. PTNS was continued for 24 sessions in patients who showed $50 \%$ improvement or more of symptoms after 12 sessions. Patients who were considered as success completed another twice/month sessions for three months then once/month sessions for another 6 months (total of 12 Months therapy).

Results: Forty-nine patients (35 female and 14 male) with a mean age of 43 years (range 18-77) were included. Two patients were excluded from the study because no urodynamic study was done. Twenty-nine patients $(59.2 \%)$ had no DO and 20 patients (40.8\%) had DO. PTNS treatment showed an overall success rate of $61.5 \%$. In OAB patients with no DO, 15 patients $(51.7 \%)$ had improvement, while 16 OAB patients with DO (80\%) had Improvement. There is a statistically significant difference between the outcomes in both groups $(\mathrm{p}<0.05)$.

Conclusions: In patients with $\mathrm{OAB}$, a urodynamic proven Detrusor Over activity (DO) predicts posterior Tibial Nerve Stimulation outcome. Success rate is more frequently encountered after PTNS treatment in patients in whom urodynamic evaluation showed DO compared to those with negative DO on urodynamic (Urgency frequency syndrome).

BSP2-07 Management of recurrent stricture after previous ureteral reconstruction: Robot-assisted laparoscopic surgery versus open surgery

W Qing, S Wang

Department of Urology, Tongji Hospital, Tongji Medical College, Huazhong University of Science and Technology

Introduction \& Objective: To report our experience in treating recurrent benign ureteral strictures and demonstrate whether robot-assisted procedures for redo ureteral reconstruction are as effective as open procedures while remaining less invasive.

Methods: We retrospectively assessed 41 patients (22 robotassisted surgeries and 19 open surgeries) who underwent consecutive robot-assisted and open procedures for redo ureteral reconstruction from January 2014 to January 2018 at our institution. Perioperative outcomes, including demographics, operative

\begin{tabular}{ccc} 
Table 1.Descriptive characteristics for patients. & & \\
\hline Item & Robot ( $\mathbf{n = 2 2 )}$ & Open (n=19) \\
\hline Symptoms, $\mathbf{n}(\%)$ & & \\
Flank pain & $14(63.64)$ & $9(47.37)$ \\
Fever & $1(4.55)$ & $2(10.53)$ \\
Haematuria & 0 & $1(5.27)$ \\
Asymptomatic & $7(31.82)$ & $7(36.86)$ \\
Interval, yr, median (range) & $8(1-25)$ & $8(0.5-31)$ \\
History, $\mathbf{n}$ & & \\
Open pyeloplasty & 9 & 6 \\
Open uretero-ureterostomy & 1 & 5 \\
Laparoscopic pyeloplasty & 5 & 4 \\
Laparoscopic uretero-ureterostomy & 1 & 2 \\
Open pyeloplasty + nephrostomy & 1 & 1 \\
Open uretero-ureterostomy + balloon dilation & 1 & 0 \\
Open pyeloplasty + endopyelotomy & 1 & 0 \\
Open pyeloplasty + double J stent & 2 & 0 \\
Laparoscopic pyeloplasty + nephrostomy & 1 & 0 \\
Laparoscopic pyeloplasty + balloon dilation + & & 1 \\
endopyelotomy & 0 & \\
\hline
\end{tabular}

\begin{tabular}{|c|c|c|c|}
\hline Item & Robot (n=22) & Open $(n=19)$ & $P$ value \\
\hline Mean age, yr (median) & $37.82 \pm 18.56(36)$ & $36.16 \pm 15.54(39)$ & 0.760 \\
\hline Mean BMI, $\mathrm{kg} / \mathrm{m}^{2}$ (median) & $22.75 \pm 3.65(22.48)$ & $22.79 \pm 2.94(22.86)$ & 0.969 \\
\hline Gender, n (\%) & & & 0.737 \\
\hline Male & $16(72.73)$ & $12(63.14)$ & \\
\hline Female & $6(27.27)$ & $7(36.86)$ & \\
\hline Side of disease, $\mathbf{n}(\%)$ & & & $>0.999$ \\
\hline Left & $14(63.64)$ & $12(63.16)$ & \\
\hline Right & $8(36.36)$ & $7(36.84)$ & \\
\hline ASA, n (\%) & & & 0.742 \\
\hline I & $5(22.73)$ & $4(21.05)$ & \\
\hline II & $15(68.18)$ & $14(73.68)$ & \\
\hline III & $2(9.09)$ & $1(5.27)$ & \\
\hline
\end{tabular}

BMI: Body mass inder.

ASA: American Society of Anesthesiologists Score.

Table 3.Postoperative characteristics of patients.

\begin{tabular}{|c|c|c|c|}
\hline Item & Robot $(n=22)$ & Open $(n=19)$ & $P$ value \\
\hline Procedure, n (\%) & & & 0.145 \\
\hline Pyeloplasty & $19(86.36)$ & $12(63.16)$ & \\
\hline Uretero-ureterostomy & $3(13.63)$ & $7(36.84)$ & \\
\hline Mean operative time, min & $124.55 \pm 48.45$ & $185.11 \pm 49.71$ & $<0.0001$ \\
\hline Mean blood loss, ml & $100.00 \pm 18.43$ & $182.60 \pm 23.89$ & 0.008 \\
\hline \multicolumn{4}{|c|}{ Postoperative complications, n (\%) } \\
\hline Grade II ${ }^{*}$ & $2(9.09)$ & $7(36.84)$ & 0.057 \\
\hline Grade IIIa & 0 & $1(5.26)$ & 0.463 \\
\hline Grade IIIlb & $\mathbf{0}$ & $1(5.26)$ & 0.463 \\
\hline Median VAS (range) & $2.5(1-5)$ & $2(1-3)$ & 0.053 \\
\hline Mean hospital stay, d & $7.50 \pm 2.24$ & $12.21 \pm 6.16$ & 0.002 \\
\hline Cost, $\boldsymbol{v}$ & $61161.77 \pm 8567.67$ & $38470.79 \pm 9764.00$ & $<0.0001$ \\
\hline
\end{tabular}

VAS: Visual analogue score.

\begin{tabular}{cccc} 
Table 4. Follow-up & \multicolumn{3}{l}{} \\
\hline Item & Robot $(\mathbf{n}=\mathbf{2 1})$ & Open $(\mathbf{n}=\mathbf{1 7})$ & P value \\
\hline Median follow up, month (range) & $21(11-41)$ & $39(10-53)$ & 0.001 \\
Success, $\mathbf{n}$ (\%) & $18(85.71 \%)$ & $14(82.35 \%)$ & 0.775 \\
Outcomes, $\mathbf{n}$ & & & \\
Decreased hydronephrosis & 14 & 12 & - \\
Stable hydronephrosis + symptom resolution & 4 & 2 & - \\
Stable hydronephrosis + unrelieved symptoms & 3 & 1 & - \\
Increased hydronephrosis & 0 & 2 & - \\
\hline
\end{tabular}

time, estimated blood loss, complications, hospital stays, pain scores, success rates and cost, were compared between two groups. Results: There was no significant intergroup difference in terms of age, gender composition and American Society of Anesthesiologists (ASA) scores. Compared with the open group, robot-assisted group showed shorter operative time $(124.55 \mathrm{~min}$ vs. $185.11 \mathrm{~min}$, $\mathrm{p}<0.0001)$, less estimated blood loss $(100.00 \mathrm{~mL}$ vs $182.60 \mathrm{~mL}$, 
$\mathrm{p}=0.008)$, shorter hospital stays $(7.50 \mathrm{~d}$ vs $12.21 \mathrm{~d}, \mathrm{p}=0.002)$ and higher cost $(61161.77 ¥$ vs $39470.79 ¥, p<0.0001)$. Complication rates and pain scores were similar between two groups. Median follow-up periods were 21 and 39 months for robot-assisted and open group respectively. Success rates in the robot-assisted (85.71\%) and the open group (82.35\%) were not significantly different.

Conclusions: A robot-assisted procedure for recurrent stricture after previous ureteral reconstruction is as effective as an open procedure and is associated with shorter operative time, less estimated blood loss and shorter hospital stays.

BSP2-08 Autological fat stromal-vascular fraction injection for male stressed urinary incontinence treatment

\section{Pavlov, S Maksimova \\ Bashkir State Medical University}

Introduction \& Objective: Stress urinary incontinence (SUI) after radical prostatectomy is a major problem that affects $9-16 \%$ of patients. Post-prostatectomy incontinence results from surgical damage to the urethral sphincter as well as to surrounding nerves and supportive tissue. Treatment of urinary incontinence can be conservative including drug therapy, behavioral therapy, physiotherapy, pelvic floor exercises or surgical including sling operations or periurethral injection therapy with bulking agents. Whereas more invasive approaches like sling operations are more effective but have higher morbidity, while the injection of allogeneic bulking agents is minimally invasive but has poor long-term efficacy. Some studies showed the autologous stromal vascular fraction (SVF) from adipose tissue exhibited promising long-term efficacy.

Methods: Here we report our experience of SVF transplantation into the periurethral region as a new method of stress urinary incontinence treatment. Seventeen patients with symptoms of stress urinary incontinence (grade 1-2) after radical prostatectomy or transurethral resection of prostate were treated by injections of SVF into the bladder sphincter. The first stage is tumescent lipoaspiration. After this, the stromal-vascular fraction is separated using special double syringes and centrifugation. After excretion of SVF, transurethral introduction to the zone of the external sphincter of the bladder is performed. Part of the SVF is sent to the laboratory for further study of the cellular composition using flow cytofluorimetry. Successful differentiation of the obtained cells into bone, muscle, cartilage tissue demonstrates the presence of multipotent mesenchymal stem cells.

Results: Our study showed that injection of the SVF into the bladder sphincter area is a safe treatment option for SUI. The effectiveness of the method was 50\% according to the results of the Pad test, ISIQ-SF and QoL. In the group with a positive effect, the Pad test value decreased on average from 2-3 to 0-1, the score on the ISIQ-SF questionnaire from 7-11 to 1-4 and the QoL test from 4-6 to 1-2.

Conclusions: Further studies will develop new therapeutic strategies with finding the optimal dosage, the number and localization of injections are needed to improve its long-term efficacy.

BSP2-09 Epidemiologic Characteristics and Risk Factors in Patients with Ketamine-associated Lower Urinary Tract Symptoms Accompanied by Urinary Tract Infection: A Cross-sectional Study

W Liu, W Wu, Y Wei, R Zhang, J Wu

Fujian Provincial Hospital
Introduction \& Objective: Young adults with longstanding ketamine abuse present with lower urinary tract symptoms (LUTS), which may be accompanied by urinary tract infection (UTI). However, the morbidity and risk factors for ketamine-associated LUTS accompanied by UTI (KALAUTI) are still unknown. To ascertain these, we surveyed patients with a history of ketamine abuse and LUTS at the time of their initial presentation.

Methods: One hundred untreated patients with ketamineassociated LUTS were initially surveyed at three medical institutions. The patients' basic demographic and clinical information, KALAUTI status, and possible risk factors were obtained via a questionnaire and analyzed.

Results: Eighty-one patients were finally enrolled. Eight patients $(9.88 \%)$ had a definitive diagnosis of KALAUTI and $16(19.75 \%)$ had suspected KALAUTI. The diagnosis of KALAUTI was ruled out in the remaining 57 patients $(70.37 \%)$. Patients with upper urinary tract involvement, longer duration of drug use, or more severe LUTS $(\mathrm{P}>.05)$, were more prone to KALAUTI. Frequent urine culture and a higher voiding symptom score (VSS) were risk factors for KALAUTI $(\mathrm{P}<.05)$, increasing the risk of KALAUTI by 44.241-fold and 1.923-fold, respectively.

Conclusions: The study indicates that frequent urine culture and severe VSS are risk factors for KALAUTI. The possibility of UTI should be considered in ketamine abusers with LUTS in the clinical setting.

BSP2-10 Intravesical injections of platelet-rich plasma is effective and safe in treatment of interstitial cystitis refractory to conventional treatment-A prospective clinical trial

C Yesildal, A Albayrak, A Yavuzsan, M Ilgi,

N Turkmen, S Kirecci

Sisli Etfal Training and Research Hospital

Introduction \& Objective: Current treatments for interstitial cystitis are usually unsuccessful in achieving long-term irritable symptom improvement. Therefore, in this study we aim to investigate the clinical efficacy of platelet-rich plasma (PRP) intravesical injections on interstitial cystitis patients, who refractory to conventional therapies.

Methods: 30 patients, who applied to our clinic between 20152019, received one intravesical injections of $10 \mathrm{~mL}$ autologous PRP extracted from $50 \mathrm{~mL}$ of whole blood. The primary endpoint was Global Response Assessment (GRA) at the first month after the PRP injection. Secondary endpoints included changes in O'Leary-Sant symptom score (OSS), daily frequency, nocturia, post-void residual volume (PVR) from baseline to first month after the PRP injection.

Results: All 30 patients (all of them are women) completed the first injections and first month follow-up visits. GRA improved after the PRP injection and the success rate was $85 \%$. OSS also decreased (mean 32 to $20 \mathrm{p}<0.05$ ) (Table 1). The PVR did not change. Frequency, and nocturia were decreased after the PRP injection. Urinary tract infections or voiding difficulties were not seen.

\begin{tabular}{|l|l|l|l|l|}
\hline & Pre-Op (Mean) & Post-Op (Mean) & $p$ & \\
\hline GRA & 2 & 5 & $p<0.05$ & \\
\hline OSS & 32 & 20 & $p<0.05$ & \\
\hline PVR & $<50$ & $<50$ & $p>0.05$ & \\
\hline
\end{tabular}

Table 1: Results of the primary and secondary end-points 
Conclusions: The study demonstrated that intravesical injections of autologous PRP can provide interstitial cystitis symptom improvement. Autologous PRP injection is safe and effective in selected patients.

\section{BSP2-11 How international is the International Prostate Symptom Score - a literature review of val- idated translations of the IPSS}

M Yao, J Green, SS Folkard

Introduction \& Objective: The ubiquitous IPSS was validated by the AUA with $210 \mathrm{BPH}$ and 108 control patients and is accepted by the WHO. Our London hospital covers an area where over 120 languages are used. However, we receive complaints and requests from patients asking for the IPSS in their native language. We thus set out to investigate which validated translations exist and assess how international the IPSS truly is.

Methods: Literature search revealed 14,000 articles. Amongst 33 relevant articles, 19 were validated translation studies. Statistical validation criteria for consistency and reliability was assessed. Total language population was used to estimate global access.

Results: 24 translations of the IPSS have been validated. Studies used different techniques of translation methodology, clinical correlation, and statistical analysis to validate translations. These 24 translations have a potential global reach of over 4.1 billion people across all continents of the world.

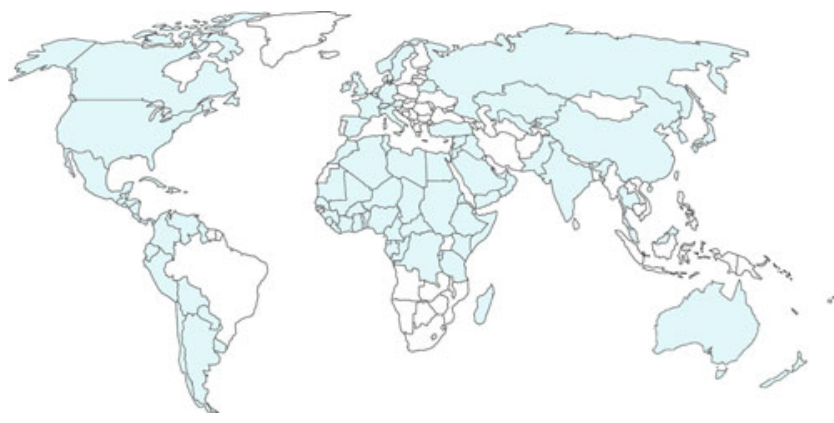

\begin{tabular}{|c|c|c|c|c|c|c|}
\hline \multirow[b]{2}{*}{ Nethor, yeer } & \multirow[b]{2}{*}{ Langungen } & \multirow{2}{*}{ vationesed } & \multirow{2}{*}{ 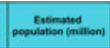 } & \multirow{2}{*}{ 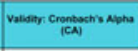 } & \multicolumn{2}{|c|}{ Roliebiatry } \\
\hline & & & & & 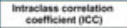 & ones \\
\hline Bery et a, 1902 & Englath & Yos & 11224 & 0.80 & 0.92 & \\
\hline Quek et $\alpha, 2005$ & Wandarin Chinese in & rese & 9178 & 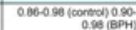 & 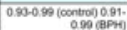 & \\
\hline Bada et al, 1997 & Spamian & Yes & 5443 & & & tranaluson methosologyr \\
\hline Hammad ee all: 2010 & Arabic & Yes & 315.4 & $0.85\{0.78 .0889\}$ & 088 & \\
\hline Bada et at, 1997 & French & Yes & 2798 & & & transiascon methosodiogy \\
\hline 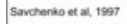 & Pussian & res & 258.2 & & & not aratabite \\
\hline Salman et at, 2018 & unow & Yos & 170.2 & 0.72 & 0.75092 & \\
\hline Homma of at, 2003 & Japanesese & Yes & 122.4 & 0.83 & & Wolphtos kappas = 0.62 \\
\hline Remantalib of at, 2012 & Marater (nsa) & Yes & s.1 & $0.890 .089 .0 .89)$ & 0.94 & \\
\hline Chol et ax, 1996 & Korean & res & $\pi 2$ & 0.99 & & 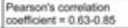 \\
\hline Bozive et $\alpha, 2002$ & Twivish & res & 749 & & & dinical transation \\
\hline Bada ex act 1990 & spoesian" & Yes & 46.6 & 0.78 & 0.92 & \\
\hline Baley et al. 2000 & 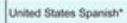 & Yes & 30 & & & tranalusen methosology \\
\hline Quek et $x, 2002$ & Malasy in Macagyia & Yes & 31.6 & 0.53 (contsol) 0.88 (turs) & 0.51 & \\
\hline Avilov of a, 2012 & Uetoke & Yos & 205 & 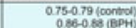 & 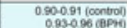 & \\
\hline Lemma ex al 2004 & Amtharic (Enicopis) & Yes & 256 & 0.99 & 0.95 & \\
\hline Bada ef al $19 \mathrm{~m}$ & butch & Yes & 23.1 & & & transulason methosology \\
\hline Ouek et $x, 2001$ & Englath in Malaysia" & Yes & 156 & 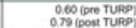 & 0.59 & \\
\hline Bada et ax. 1997 & Smosin & Yes & 128 & & & tranalason methosoloby \\
\hline Onoi et a. 2014 & Hong Kong Clinesese & Yos & 75 & 0.71 & 0.70080 & \\
\hline Bada et al, 1997 & Canasian French" & Yes & 72 & & & transilabon methosolobyry \\
\hline Bada ef al, 1997 & Danish & res & 56 & & & 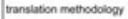 \\
\hline Bada et ax. 1997 & Flemish & Yes & 8.5 & & & transistoson methodiciogy \\
\hline Bada ex al, 1997 & Nonwogian & res & 8.3 & & & transistocon methosologyr \\
\hline & sects & Total & 4113.2 & & & \\
\hline
\end{tabular}

Conclusions: The IPSS is currently available in 138 languages, spanning 51 countries, but not all translations have been validated. Over $54 \%$ of global population can currently access a validated translation of the questionnaire. Translation of languages used in developing countries requires further validation, and problems arise in utilizing the IPSS in illiterate populations. However, the IPSS is still one of the most validated symptom scoring systems in the world and is truly deserving of its international title.

BSP2-12 Diagnosis and management of false urethral anastomosis in cases of posterior urethral trauma

\section{S Jain, R Goel, D Bansal}

India Institute of Medical Sciences, New Delhi

Introduction \& Objective: Failed urethroplasty due to false urethral tract anastomosis is a dreadful complication. Despite advancement in the field of urethral trauma management, we continue to see such patients.

Methods: Here we describe a case of false urethral tract anastomosis, aiming to bring to notice this very real complication of treatment for pelvic fracture urethral injury. The patient presented with total urinary incontinence following open perineal urethroplasty done for management of posterior urethral trauma. Retrograde urethrogram and micturating cysto-urethrogram (RGU/MCU) showed an apparently normal urethra with a posteriorly oriented false tract.

Results: Endoscopic evaluation showed anastomosis of the anterior urethra with the false tract with the true bladder neck and posterior urethra lying posteriorly. The patient underwent dismemberment of the anastomosis with redo progressive perineal
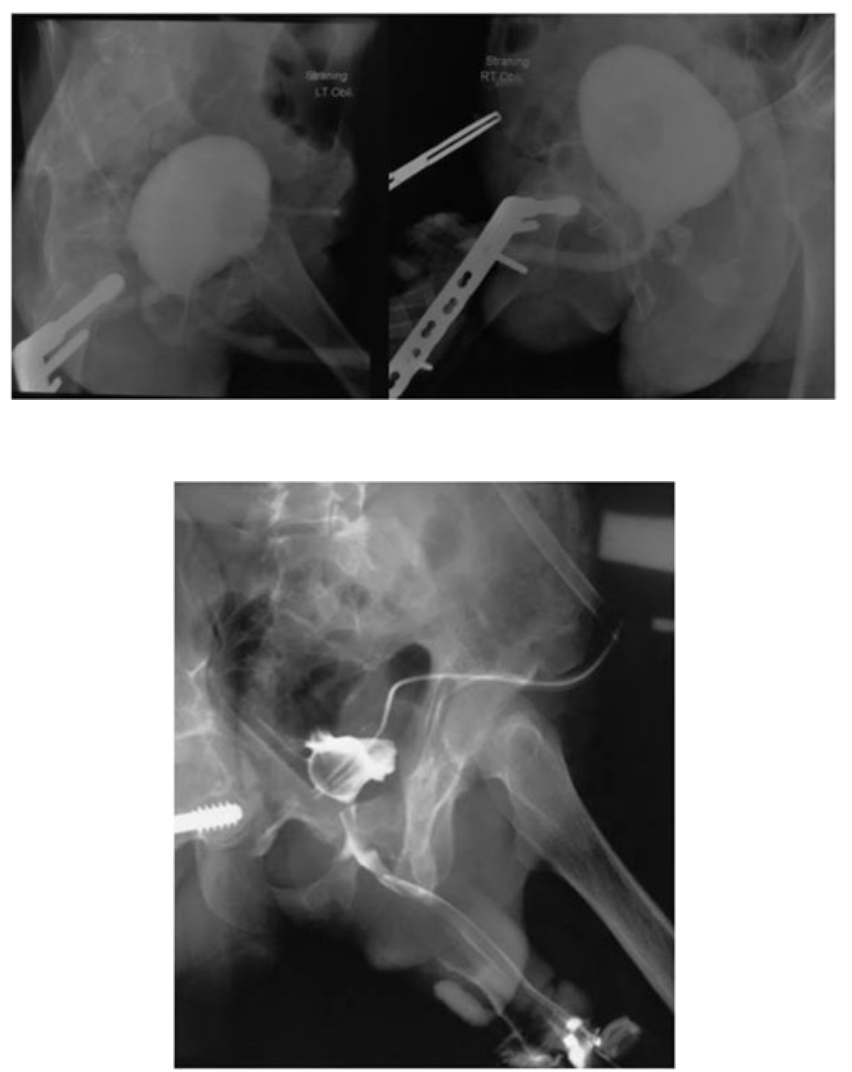
urethroplasty. After per urethral catheter removal, the patient was continent and voided with good urinary stream.

Conclusions: The case presented describe the importance of recognizing this devastating complication. Surgeon experience and meticulous endoscopic evaluation are the keys to success. Careful dissection of the posterior urethra with removal of all fibrous tissue during urethroplasty is essential to identify the true posterior urethra. Use of flexible cystoscopy for evaluation may give additional help in identification of such false tracts at the time of urethroplasty.

\section{BSP2-13 Posterior Tibial Never Stimulation (PTNS) in the treatment of voiding dysfunction. Single center experience}

B. Malallah, W Alamari, A Alabbad, M Algahwari, O Sarhan, R Almousa

Introduction \& Objective: Posterior Tibial Neuromodulation was described more than 20 years ago as a minimally invasive treatment for patients with Over active bladder syndrome. Posterior Tibial Neuromodulation has been tested at various centers and has been proven as effective as the usually prescribed anticholinergic medications. More recently, it has been successfully used to treat other voiding dysfunction problems like NonObstructive urinary retention and / or chronic Pelvic pain syndrome. Our aim is to assess the efficacy of Posterior Tibial Nerve Stimulation (PTNS) in treatment of patients with voiding dysfunction (over active bladder, idiopathic non obstructed urinary retention, or chronic pelvic pain syndrome) who are unresponsive to medical therapy.

Methods: Single center, retrospective study, reviewed the medical charts of adult patients with voiding dysfunction who underwent Posterior Tibial Nerve Stimulation (PTNS) in our center between January 2012 till December 2018. IRB obtained. Patients' demographic data, diagnosis, voiding diary pre and post PTNS treatment, and outcome collected. All patients had baseline investigations (urine analysis, serum Creatinine, Urodynamic study, Renal Ultrasound). Each Patient had to fill a voiding diary and quality of life questionnaire at the beginning of therapy (Week 0) and after completion of the initial weekly therapy (week 12). The success of treatment was defined as 50\% or more improvement of voiding dysfunction symptoms in voiding diary. PTNS was continued for total of 24 sessions in patients who showed 50\% improvement or more of symptoms after 12 sessions. Patients who were considered as success completed another twice/month sessions for three months then once/month sessions for another 6 months.

Results: 108 medical charts were reviewed. 70 patients (41 female, 29 male) were included in our study. 38 patients were excluded due to missing data or incomplete file. Age ranged between 18-77 years (mean 42). Patients' diagnoses were overactive bladder in 51 (73\%), idiopathic on obstructed urinary retention in $14(20 \%)$, and chronic pelvic pain syndrome in 5 patients $(7 \%) .61 .2 \%$ of overall 1 patients had $50 \%$ or more improvement of symptoms. Success rate was $62.2 \%, 76.9 \%$ and $40 \%$ in patients with Overactive bladder, idiopathic on obstructed urinary retention, and with chronic pelvic syndrome, respectively.

Conclusions: PTNS is a safe and an effective treatment modality in treating patients with voiding dysfunction disorders who failed conservative treatment. PTNS treatment showed a higher success rate in patients with Idiopathic non-obstructive urinary retention compared with other disorders.
BSP2-14 Ketamine-induced lower urinary tract symptoms: a study of International Prostate Symptom Score and Quality of Life

Y Wei, J Wu

Fujian Provincial Hospital

Introduction \& Objective: Ketamine associated cystitis was first reported in 2007. Although many articles have been published concerning the effects of ketamine on lower urinary tract, not much focus had been put on the Quality of Life (QoL). This study was to assess lower urinary tract symptoms (LUTS) and QoL in ketamine abusers.

Methods: One hundred ketamine abusers with LUTS were included in the study. All patients were interviewed by one of the three trained investigators through face-to-face interview. Their LUTS were evaluated by the survey instrument consisting of International Prostate Symptom Score (IPSS) and 7-points Likert-type QoL scale.

Results: Sixty-six of them had moderate symptoms and 16 suffered from heavy symptoms. Eighty-one percent patients reported storage symptoms compared to $41 \%$ with voiding symptoms. Mono-ketamine abusers (MKA) had higher scores in IPSS, QoL, storage and voiding symptoms than polydrug abusers (PDA). Multiple linear regression analysis demonstrated that lower educational background, MKA, and longer duration of ketamine abuse were found to be predicting factors on IPSS and QoL.

Conclusions: LUTS in ketamine abusers are mostly moderate to severe, and their quality of life were wrecked. Mono-ketamine abusers have more severe LUTS and lower quality of life than poly-drug users. Lower educational level, mono-ketamine abuser and longer duration of abuse are independent predictors of severity of LUTS and QoL.

\section{BSP2-15 Outcomes of robotic ureteral reimplantation in tertiary care centers: a bi-institutional case series}

SS Komaravolu, DD Eun, D Kim, PM Youssef, JC Deolankar, PD Kothari, Z Lee, M Billah, H Koster, M Ahmed, GG Lovallo, R Munver, MD Stifelman

\section{Hackensack Meridian Department of Urology}

Introduction \& Objective: Robotic ureteral reimplantation is an area of growing interest and some controversy. We set out to review and report our bi-institutional experience at two tertiary care centers of ureteral reimplantation in adults, specifically extravesical, psoas hitch, Boari flap, and side-to-side techniques.

Methods: Data from our IRB-approved retrospective and prospective databases from October 2008 to October 2018 was queried to identify all ureteral reimplantations. Demographic, perioperative, hospital stay, and success rate data was summarized.

Results: 69 patients were included and all procedures were completed robotically. Demographic data, peri-operative data, and post-operative data are presented in Tables 1, 2, and 3 respectively. Of interest $78.3 \%$ (54/69) of patients had previous abdominopelvic surgery, $18.8 \%$ radiation, and $24.6 \%$ previously attempted repairs. $55.0 \%$ of patients required mobility procedures, $20.3 \%$ adjunctive vascularity procedures, and $58.0 \%$ utilized intraoperative ICG. Only $10.3 \%$ had $>2$ Clavien complications. At a mean follow up of $13.1 \pm 3.1$ months, $95.6 \%$ of 
Table 1. Demographic data.

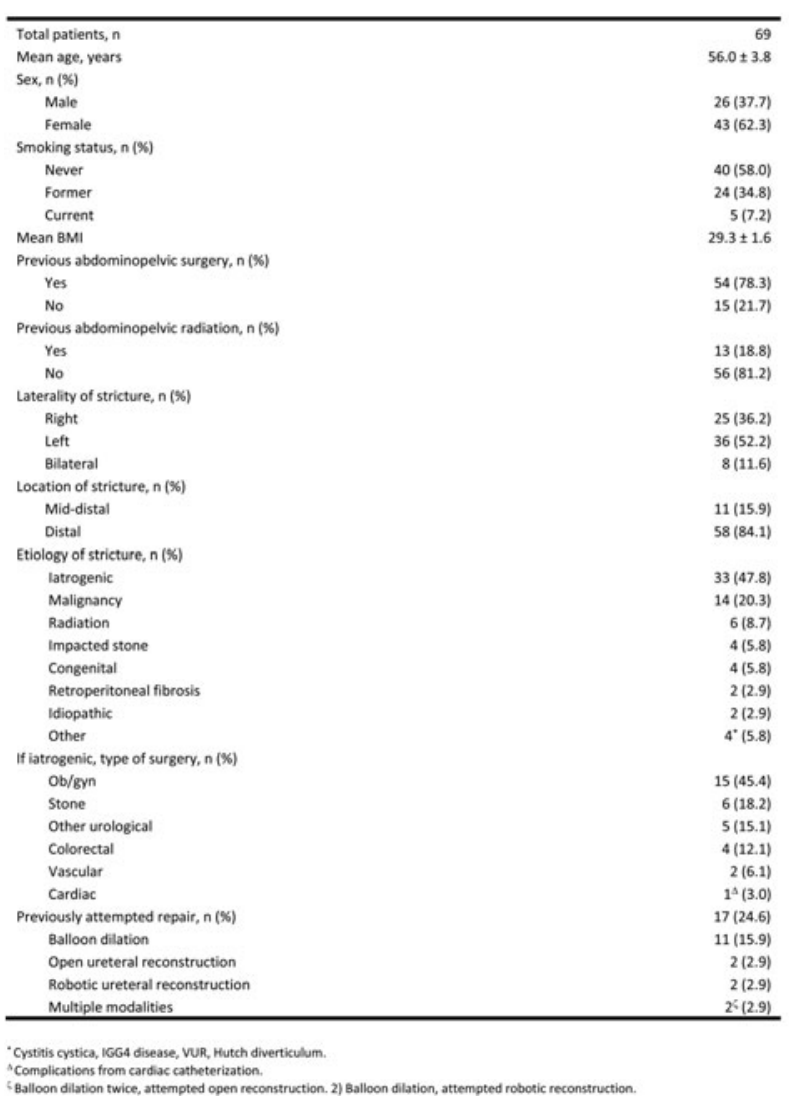

Table 2. Peri-operative data, $\mathrm{n}=69$.

\begin{tabular}{lr}
\hline Main procedure, $n$ (\%) & \\
$\quad$ Ureteral re-implantation & $62^{*}(90.0)$ \\
$\quad$ Side-to-side bladder re-implant & $8^{*}(11.6)$ \\
Adjunctive mobility procedure, $n$ (\%) & $38(55.0)$ \\
Psoas hitch & $27(39.1)$ \\
$\quad$ Psoas hitch and boari flap & $11(15.9)$ \\
Adjunctive vascularity procedure, $n(\%)$ & $14(20.3)$ \\
$\quad$ Omental wrap & $10(14.5)$ \\
Peritoneal wrap & $2(2.9)$ \\
$\quad$ Amniox wrap & $2(2.9)$ \\
Intraoperative ICG, $n$ (\%) & $40(58.0)$ \\
$\quad$ Intraureteral & $16(23.2)$ \\
Intravenous & $24(34.8)$ \\
Mean length of stricture, cm & $3.08 \pm 0.48$ \\
Mean operative time, min & $200.2 \pm 21.2$ \\
Mean estimated blood loss, cc & $100.5 \pm 22.6$ \\
Intraoperative complications, $n(\%)$ & \\
Ureterotomy & $1(1.4)$ \\
None & $68(98.6)$ \\
Mean time from diagnosis to surgery, months & $11.2 \pm 3.5$ \\
Drainage on day of surgery (pre-op), $n$ (\%) & \\
\hline
\end{tabular}

- One patient with bilateral strictures received both.

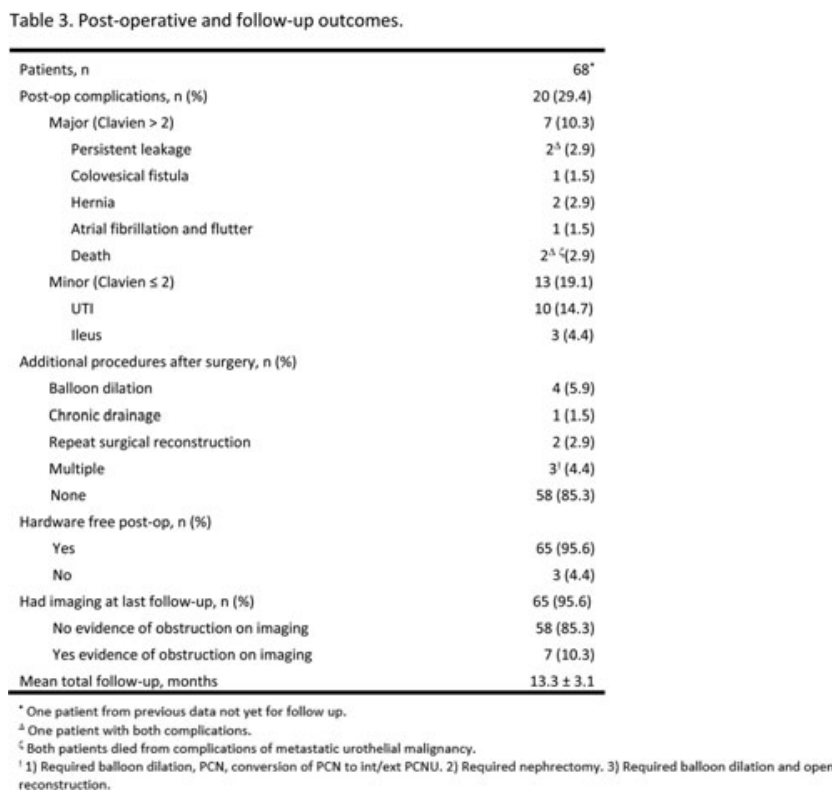

patients were hardware-free and $89.2 \%$ of patients who were scanned were obstruction-free.

Conclusions: In the largest robotic ureteral reimplantation series published to date we report excellent stent-free and low complication rates despite many patients with complex histories of previous repairs, or abdominopelvic radiation or surgery.

\section{BSP2-16 Comparison of perioperative and mid-term outcomes between thulium laser vaporization and vapoenucleation: a propensity score analysis}

S Nazzani, G Motta, O Blezien, P Acquati, R Stubinski, C Yameogo, S Maruccia, S Casellato, L Carmignani

Introduction \& Objective: Thulium laser vapoenucleation (ThuVep) is considered equivalent to transurethral resection of prostate for treatment of benign prostatic obstruction. Conversely, thulium laser vaporization (Thuvap) is not yet considered comparable to the gold standard according to European Association of Urology Guidelines. We compared early and late outcomes between ThuVep and ThuVap.

Methods: Thulium laser vapoenucleation (ThuVep) is considered equivalent to transurethral resection of prostate for treatment of benign prostatic obstruction. Conversely, thulium laser vaporization (Thuvap) is not yet considered comparable to the gold standard according to European Association of Urology Guidelines. We compared early and late outcomes between ThuVep and ThuVap.

Results: Between 2012 and 2018, we identified 692 patients treated with either ThuVep[442 (63.9\%)] or ThuVap[250 (36.1\%)]. Median follow-up was 44 months. Median International Prostatic Symptoms Score drop was 16. ThuVap patients had median higher preoperative maximum flow ( $8 \mathrm{vs} 8.6 \mathrm{ml} / \mathrm{sec}$; $\mathrm{p}=0.04)$ and were more frequently in anticoagulant or antiplatelet therapy (29.5 vs 20.3; $\mathrm{p}=0.002)$. In MLRMs testing for surgical technique predictors anticoagulant or antiplatelet therapy achieved independent predictor status for higher ThuVap rates (OR: 1.49; $\mathrm{p}=0.001)$. However, no difference was found in early (OR: $0.90 ; p=0.08)$ and late (OR: $0.85 ; \mathrm{p}=0.2)$ complication rates between Thuvap and Thuvep. Finally, Thuvap did not achieved independent predictor status for lower IPSS drop. Results were confirmed after IPTW adjustment. 
Conclusions: Based on our results no statistically significant difference exist between ThuVap and ThuVep in early and late complication rates. Moreover, IPSS drop was similar in the two groups.

BSP2-17 The Effect of Platelet-Rich Plasma Injection on Post-internal Urethrotomy Stricture Recurrence: A Prospective Study

A Yavuzsan, A Albayrak, C Yesildal, M Ilgi, N Turkmen, S Kirecci

Health Sciences University, Sisli Hamidiye Etfal Training and Research Hospital Urology Department

Introduction \& Objective: Local injection of platelet-rich plasma (PRP) works by delivering growth factors to the injection site. Thus, enhance recovery process starts. In this study, we aim to investigate the effect of local submucosal PRP injections, applied along with urethrotomy, on the post-internal urethrotomy stricture recurrence rate.

Methods: 30 male patients with symptomatic bulbar urethral stricture (diagnosed by retrograde urethrography) enrolled to the study. Internal urethrotomy with submucosal autologous plateletrich plasma injection applied to the patients. Functional bladder capacity (FBC), maximum and average flow rate, voided volume, post-void residual volume (PVR) of the patients were evaluated from baseline to 1, 3 and 6 months after the PRP injection. We planned to follow our patient at 3 months intervals for 5 years after internal urethrotomy or until urethral stricture recurrence.

Results: For now, sixth month follow ups were done and recurrence rates were 0 . We are planning to follow our patient at 3 months intervals for 5 years after internal urethrotomy or until urethral stricture recurrence.

Conclusions: In this study, submucosal PRP injection at the site of internal urethrotomy, decreased the rate of stricture recurrence at 6 months after the intervention. However, we need more time to evaluate this protective effect duration.

BSP2-18 Beta-adrenoreceptor regulates the secretion of inflammatory cytokines by autophagy in human bladder smooth muscle cells under pathological hydrostatic pressure

G Chen, K Xiao, K Wang, H Li

Department of Urology, Institute of Urology (Laboratory of Reconstructive Urology), West China Hospital, Sichuan University, Chengdu, Sichuan, P.R.C

Introduction \& Objective: Accumulating evidence demonstrates that bladder tissue remodeling of partial bladder outlet obstruction (PBOO) is from inflammation to fibrosis, the molecular mechanism remains unclear. To further explore the pathological development of $\mathrm{PBOO}$, we investigated expression of inflammatory cytokines of human bladder smooth muscle cells (HBSMCs) under pathological hydrostatic pressure treated with beta-adrenoreceptor (ADRB) and/or autophagy intervention agents.

Methods: The expression of inflammatory factors, autophagyrelated markers and $\mathrm{ADRB}$ subtypes were detected by wholegene microarray in PBOO rat model. To simulate pathological hydrostatic pressure in vitro, we exposed HBSMCs to hydrostatic pressure apparatus with $200 \mathrm{~cm} \mathrm{H} 2 \mathrm{O}$ pressure for $0.5 \mathrm{~h}, 2 \mathrm{~h}, 6 \mathrm{~h}$, $24 \mathrm{~h}$, then examined the autophagy, the expression of ADRB subtypes and inflammatory cytokines; Investigate the effect of ADRBon the secretion of inflammatory cytokines and autophagy levels under pathological hydrostatic pressure; Further intervene autophagy to explore ADRB's effect on inflammation.

Results: The expression of CCL2, CCL5 and IL6 in PBOO model rats gradually increased to the highest level at 1, 3 and 6 weeks, and the increasing trend began to weaken at 12 weeks ; the expression of ADRB2 and ADRB3 decreased at 3 weeks; The autophagy level variation trend of HBSMCs under $200 \mathrm{~cm} \mathrm{H} 2 \mathrm{O}$ hydrostatic pressure in vitro was similar to inflammatory cytokines; The secretion of CCL2, CCL5 and IL6increased markedly, while the expression of ADRB2 and ADRB3 decreased significantly;Bycontrast, ADRB2 agonists suppressed the secretion of inflammatory cytokines and the level of autophagy under hydrostatic pressure; Autophagy agonists inhibited the anti-inflammatory effect of ADRB2 agonists;

Conclusions: ADRB2 agonists down-regulate the secretion of inflammatory cytokines via reducing autophagy level under pathological hydrostatic pressure, which provides appropriate treatment time points for inflammation and a new insight for into efficient treatment of PBOO.

\section{BSP2-19 Hemorrhagic Cystitis due to Cytomegalo- virus in a patient with AIDS}

W Padayachee, S Sadhwani, S Doherty, E Van Der Berg, A Botha

University of Kwa-Zulu Natal, Grey's Hospital

Introduction \& Objective: Hemorrhagic Cystitis due to $\mathbf{C y}$ tomegalovirus in a patient with AIDS Winston $\mathrm{P} \mathrm{R} \mathrm{Pa}$ dayachee $^{1}$, Sanjay Sadhwani ${ }^{2}$, Sean W Doherty ${ }^{1}$, Eunice Van Der Berg ${ }^{3}$, Adam R Botha ${ }^{3}{ }^{1}$ Department of Urology, University of Witwatersrand, Johannesburg, South Africa ${ }^{2}$ Department of Urology, Chris Hani Baragwanath Academic Hospital, Johannesburg, South Africa. ${ }^{3}$ Department of Anatomical Pathology, University of Witwatersrand, Johannesburg, South Africa. Symptomatic cytomegalovirus infection is common in immunocompromised individuals and may produce a diverse spectrum of disease. Whilst gastrointestinal, ocular and neurologic manifestations are well described in the setting of AIDS, urinary tract manifestations are rare and are reported predominantly following stem cell transplants. We present an unusual case of CMV associated hemorrhagic cystitis in an individual infected with HIV. Methods: A 33-year-old HIV positive female presented with acute onset hematuria and suprapubic pain. She had defaulted on her anti-retroviral regimen 3 years earlier, due to drug

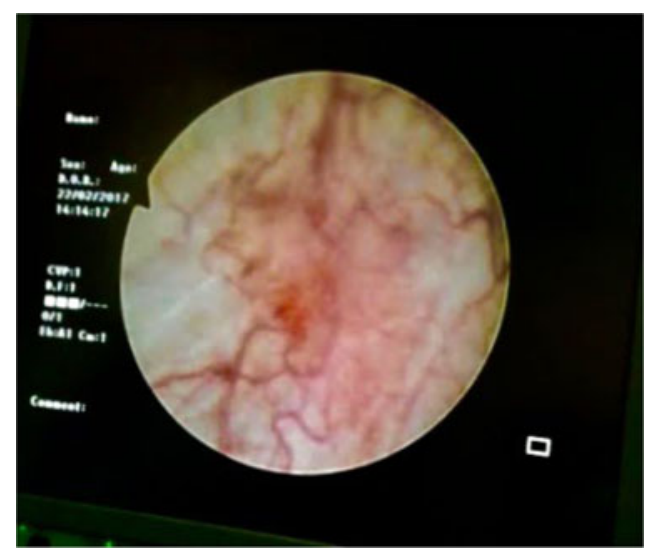



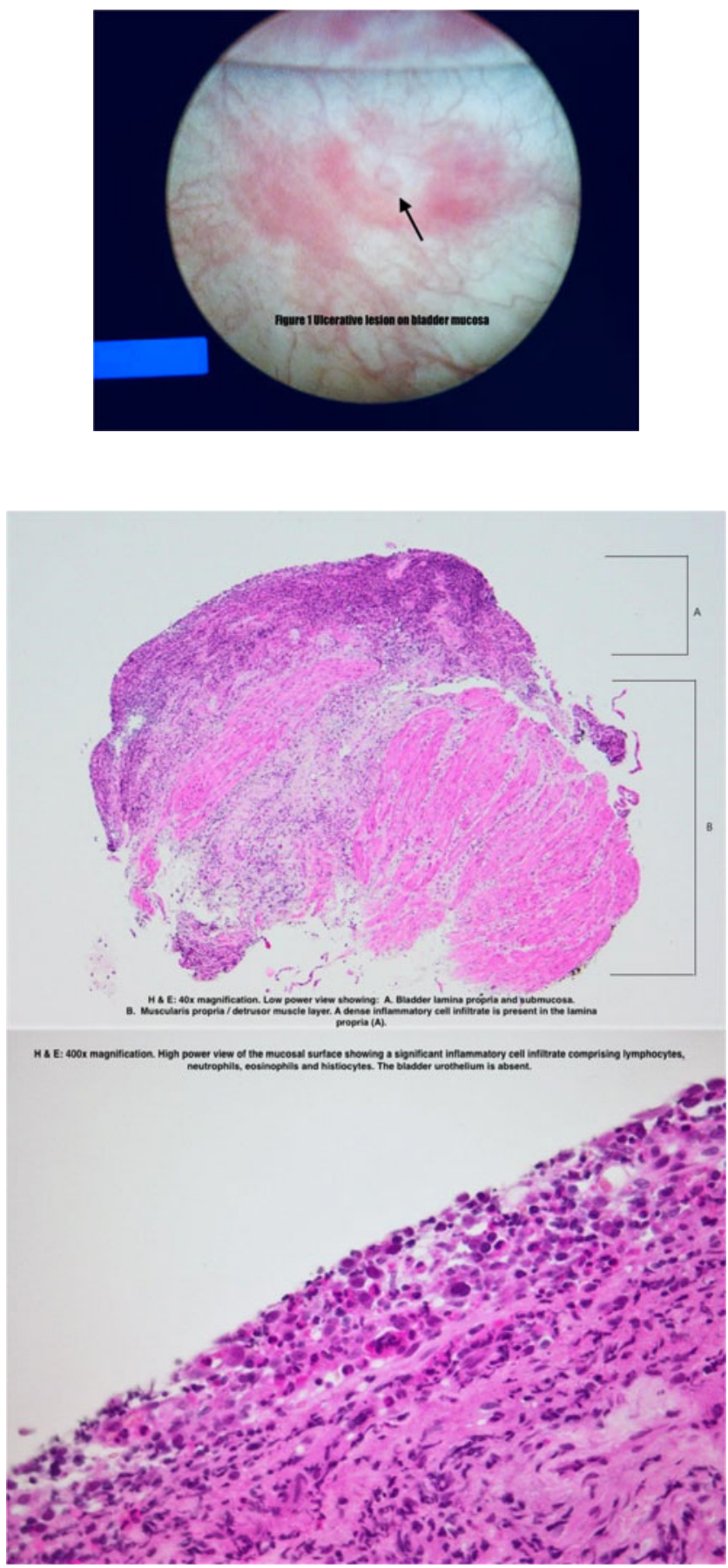

intolerance. Her current absolute CD 4 count was 20 cells $/ \mu \mathrm{L}$ and her HIV viral load was $2,110,000$ copies $/ \mathrm{mL}$. This case report/poster will describe the clinical features, endoscopic presentation and Histological findings of a rare case of CMV hemorrhagic cystitis in a patient with AIDS. Visual images of the cystoscopic evaluation showing an ulcer. Furthermore, detailed and labelled images of the histology slides will be presented, along with hematological viral studies performed. The patient was referred for re-initiation of anti-retroviral treatment, with a reduction in the HIV viral load to 2,130 copies/mL after six weeks of treatment. Post treatment images showed resolution of bladder changes.

Results: The hematological, visual and histological findings will be presented on a poster format. Images attached.
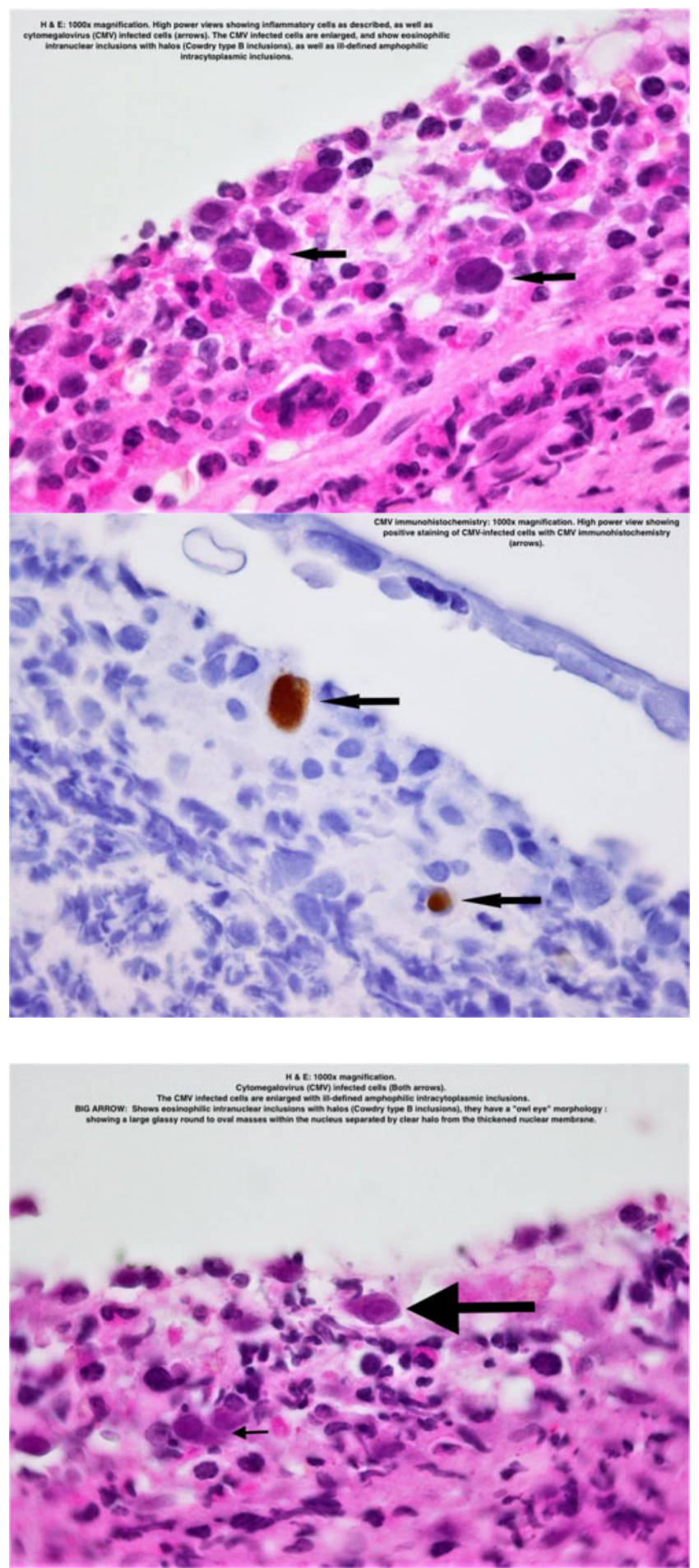

Conclusions: This case appears to be the first of its kind reported in South Africa. We believe that it highlights the importance of considering CMV as a potential infectious etiology in HIV positive patients with hematuria. Bladder biopsy may play an important role in the evaluation of undiagnosed lower urinary tract symptoms or hemorrhagic cystitis in AIDS patients. Multisystem disease may not always be present and cystoscopic features are not pathognomic, therefore a low threshold for biopsy should be maintained. The case also emphasizes the role that antiviral therapy may play in the resolution of symptoms and the prevention of complications in these patients. 


\section{BSP2-20 Utilizing da Vinci ${ }^{\circledR}$ Robotic Surgical System for Ureteroneocystostomy}

MW Salkini, D Riggs, B Jackson

West Virginia University

Introduction \& Objective: Ureteroneocystostomy, occasionally with creation of Boari flap, is the best option to substitute for the loss of the distal ureter in both benign and malignant conditions. The procedure is usually performed through a large midline or Gibson incision. Utilizing the robotic da Vinci ${ }^{\circledR}$ surgical system made it feasible to achieve the objectives of the procedure with minimally invasive approach. Our aim is to report our experience with robotic assisted ureteral substitution with and without creation of Boari flap.

Methods: Between September 2009 and March 2019, we used the de $\mathrm{Vinci}^{\circledR}$ robotic system was used to reconstruct 34 distal ureters in 29 patients (5 patient needed bilateral ureteral reimplantation). 15 benign and 19 malignant conditions needed ureteral substitution. The distal ureter was excised with bladder cuff in cases of TCC involving the distal ureter. The proximal ureteral end was spatulated and re implanted to the bladder either directly or after developing Boari flap to enable tension free, leak proof anastomosis. Negative margin was insured in all the malignant cases.

Results: The average patient age was 67 years (ranging from 2779). We had 15 male and 19 female patients. All cases were completed robotically except one that needed to be converted to open due to adhesion. The patients were followed for an average of 35 months (ranging from 3 months to 72 months). All the ureters (\%100) with benign strictures were patent after reimplantation during the time of follow up. However, 4 ureters (\%21) who had lower ureteral TCC developed ureterovesical anastomosis stricture. All strictures were due to recurrence of
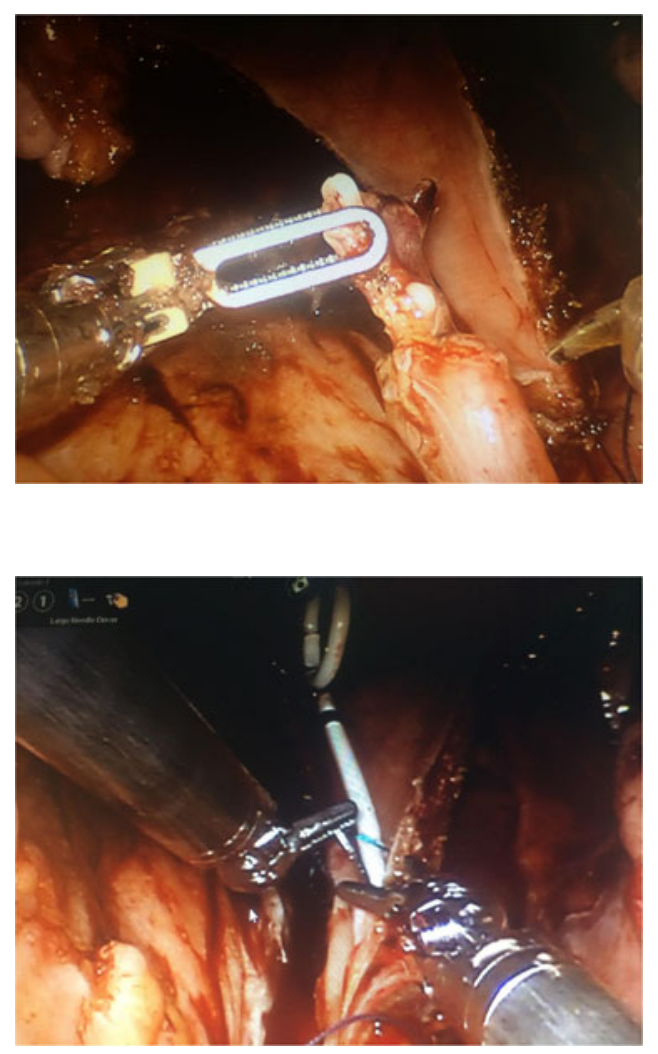

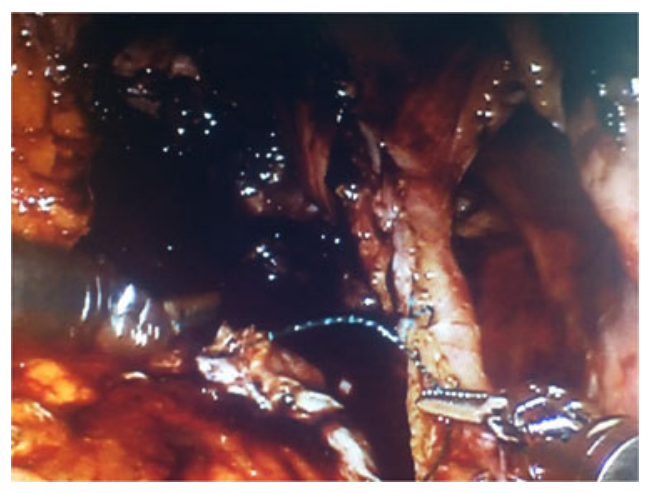

high grade TCC on the initial and final pathology after nephroureterectomy with excision of the flap. None of the 4 patient who had hx of radiation developed stricture.

Conclusions: Robotic reconstruction of the lower ureter with Boari flap is feasible and has an acceptable oncologic outcome. High grade TCC of the lower ureter is predictor of recurrence at the anastomosis. Longer follow up is needed especially in cases of malignancy.

\section{BSP2-21 Evaluation of spongiofibrosis of urethral stricture disease}

A Farganov, V Pavlov, A Izmailov, R Kazihinurov, R Safiullin

Introduction \& Objective: A urethral stricture is the pathological narrowing of any part of the urethra surrounded by the corpus spongiosum and as a result - development of spongiofibrosis. Preoperative evaluation of spongiofibrosis and right planning of surgical intervention in compliance with the basic surgical principles allows to obtain good results. The result is determined by the histological differentiation of the urethra and spongy tissue in the surgical area. An accurate evaluation of the resection zones of the urethra's damage tissue, evaluation of tissue viability in the area of the future anastomosis during augmentation and anastomotic surgical techniques are an important aspects when we performing urethroplasty.

Methods: In the period from September 2015 to April 2018, 36 patients (median age was $48 \pm 9.5$ years) with a urethral stricture which had different localization and etiology before surgical treatment, in addition to standard diagnostic studies, was performed an optical coherence tomography (OCT) of the urethra and surrounding tissue. The main purpose is to determine the length and depth of spongiofibrosis, which, in our opinion, is also an important detail for the surgical treatment planning. Patients with urethral obliteration were not include in examination group. The data performed by contrast urethrography and OCT were compared with each other. The results were correlated during the surgical treatment with an express biopsy of the resection margin for histological evaluation of urethral tissue.

Results: The average length of the urethral stricture according to urethrography was $3.12 \pm 0.8 \mathrm{~cm}$, by OCT was $4.15 \pm 0.4 \mathrm{~cm}$ and according to an intraoperative express biopsy the average length of the urethral stricture was $4.2 \pm 0.9 \mathrm{~cm}$. The results performed by OCT and express biopsy were comparable. The intraoperative visual data and data performed by urethrography were not coincide with the histological and OCT data.

Conclusions: Optical coherence tomography (OCT) showed highly informative in evaluating of spongiofibrosis in patients with a urethral stricture and can be recommended as a reliable diagnostic method for the planning further treatment tactics. 
BSP2-22 Genito-Urinary Tuberculosis Revisited - 10 Years' Experience Of a Single Centre

P Mylarappa, K Dokania, A Kulkarni

Ramaiah Medical College and Hospital

Introduction: Tuberculosis had been declared by the World Health Organization (WHO) as 'public health emergency' in 1993. Extra pulmonary tuberculosis (E.P.T.B.) comprises 20$25 \%$ total burden of the disease in which genitourinary tuberculosis (G.U.T.B.) is $4 \%$. Timely diagnosis and treatment will prevent the sequelae of this disease.

Objective: To know the various clinical presentations, diagnostic modalities and management of Genito urinary tuberculosis.

Methods: During a 10-year-period from January 2007 to December 2016, 117 retrospective cases of Genito urinary tuberculosis were admitted in our center. They were analyzed for clinical presentation, diagnostic modalities and management outcomes.

Results: Young patients mainly in their third decade of life were commonly affected with higher incidence in female patients. In our study, the most common presentation was irritative voiding symptoms $(66.47 \%)$ followed by hematuria (47.60\%). Although it can affect the entire genito-urinary system, kidney was the most affected organ (64.9\%) following ureter (27.35\%), urinary bladder $(17.09 \%)$, prostate $(3.4 \%)$ and epididymis $(5.19 \%)$. Among the different diagnostic modalities in this study, the diagnostic positivity rate was $21.6 \%$ for the urine AFB test, $35.4 \%$ for the urine $M$. tuberculosis culture test and $67.7 \%$ for PCR. Chest X-ray was positive in $25.6 \%$ (30). ESR was raised in $62.5 \%$ and Mantoux test was positive in $61.2 \%$ patients.

Conclusions: A high index of suspicion and a wide range of investigations may be required to achieve a complete diagnosis of genitourinary tuberculosis. Though short course chemotherapy with fourdrug-regimen for six month-duration is the mainstay of treatment, surgical interventions were required in $60 \%$ of cases in our study.

\section{MODERATED POSTER SESSION 01: CLINICAL ONCOLOGY: OUTCOMES \& COMPLICATIONS (I)}

\begin{abstract}
MP01-01 Impact of a National Comprehensive Cancer Network Compliant Multidisciplinary Consortium on Treatment Decisions in Patients with Localized Prostate Cancer
\end{abstract}

AA Hussein, AS Elsayed, NA Aldhaam, Z Lone, S Hall, S Kamal, K Carroci, E Kauffman, B Sullivan, Q Li, T Schwaab, M Kuettel, KA Guru

Roswell Park Comprehensive Cancer Center

Introduction \& Objective: National Comprehensive Cancer Network (NCCN) guideline-based treatment provides a benchmark of quality patient care. The impact of compliance with guidelines on the treatment of prostate cancer patients has not been defined.

Methods: We retrospectively reviewed a prospectively maintained Prostate Cancer Database from 2014 through 2017. All patients were presented to the biweekly Localized Prostate Cancer Conference (LPCC) that includes urologists, radiation oncologists, genitourinary pathologist, and patient advocates. All patients received a formal letter with the consensus recommendation. Data were analyzed for concordance among the LPCC recommendations, community recommendations, and the treatment received. Chi-square tests assessed equality of proportions, with correction for continuity. All tests were two-sided with significance defined as p-value $<0.05$.

Results: Community-based institutions have not complied with NCCN recommended staging studies in $10 \%$ of patients. Community-based and LPCC recommendations concurred in $68 \%$ of patients. LPCC's recommendations matched treatment received $76 \%$ versus $64 \%$ for community-based recommenda- tions, for patients treated at LPCC institution. Community-based recommendations offered radiation therapy more frequently than LPCC $(22 \%$ vs $4 \%, \mathrm{p}<0.001)$.

Conclusions: NCCN-based recommendations were more concordant with patient's treatment decisions than to communitybased recommendations. To our knowledge, this is the first study that compared NCCN vs community-based recommendations and its impact on decision making.

MP01-02 Cryotherapy for prostate cancer: oncological and functional outcomes

A Territo, D Vanacore, R Boissier, F Sanguedolce, F Regis, A Gallioli, J Gaya, J palou, A Breda

Department of Urology. Fundació Puigvert. Autonomous University of Barcelona. Barcelona. Spain

Introduction \& Objective: Cryoablation, has been developed to reduce morbidity and improve functional outcome without compromising oncological results in selected patients with prostate cancer ( $\mathrm{PCa}$ ) The aim of our study is to evaluate oncological, functional and peri-post-operative results of hemi (HA) vs whole gland (WA) cryoablation of the prostate as first line treatment in localized prostate cancer.

Methods: We retrospectively evaluated patients who underwent cryotherapy as first line treatment for localized low-intermediate risk (D'Amico classification) PCa from 2010 to 2018 in the Puigvert's Foundation, Barcelona, Spain. Pre-operative work up 
included multiparametric magnetic resonance (MRI) and prostate biopsy. Hemi-ablation was proposed in case of biopsy and prostate MRI proven unilateral prostate cancer. Recurrence was suspected in case of rising PSA $>$ PSA nadir $+2 \mathrm{ng} / \mathrm{mL}$. Each patient suspected of recurrence underwent local evaluation with prostate MRI and systemic + targeted biopsy. The primary endpoint was defined as any second line treatment (systemic or local treatment) and was evaluated by second line treatment free survival according to the Kaplan-Meier test and compared in a Cox regression model. Secondary outcomes were complications, urinary incontinence and sexual dysfunction.

Results: Overall, 66 patients were included. In WG $(n=40)$ vs. HB group median patients age was 74 [42-81]vs 76 [71-80] years $(\mathrm{p}=0,08)$. and Median Prostate size (ml) was 39 [20-90] vs 56 [23$120]$ in WG and HG group, respectively $(\mathrm{p}<0,01)$. Low and intermediate D'Amico risk group were respectively $15 \%$ and $85 \%$ vs $23 \%$ and $77 \%(\mathrm{p}=0,75)$. Median follow up was $41[1,5-99,00]$ vs 27 [0.9-93] months $(\mathrm{p}=0,03)$. Overall, $31(46 \%)$ patients needed a MRI or biopsy for suspicious recurrence at a median delay of 36 months after cryotherapy. Overall, retreatment has been recorded in $9(13,6 \%)$ cases. Of these $5(12,5 \%)$ and $4(15,3)$ patients were in WG and HB group respectively. All of them had an intermediate risk except one patient of HA arm. Early incontinence was $60 \%$ vs $72 \%$ in WG vs HA $(\mathrm{p}=0,26)$ but 1 -year continency was $83 \%$ vs $83 \%(p=0,99) .80 \%$ vs $57 \%$ of patients had no sexual function preoperatively. Postoperative de novo impotency was $75 \%$ vs $46 \%(\mathrm{p}=0,33)$. Adverse event occurred in $48 \%$ and $23 \%$ of patients in WG and Hemi ablation group $(\mathrm{p}=0,04)$.

Conclusions: In well selected patients with low to intermediate $\mathrm{PCa}$ risk, WG and $\mathrm{HB}$ cryoablation is an alternative treatment to the radical prostatectomy and radiation therapy, showing good medium- term oncological outcome in both group (hemi and whole gland ablation). Further studies with greater sample and longer follow up are necessary to confirm our preliminary results.

\section{MP01-03 Study on the difference between clinical stage and pathological stage of $\mathrm{T} 1$ renal cell carci- noma patients}

\section{Li, C Li, G Bao, Z Gao}

Introduction \& Objective: To compare the difference between cTNM and pTNM in patients with stage T1 renal cancer, assess the occurrence of overly-staged and under-differentiated stage in clinical stage, and then to provide the basis for developing the best treatment plan for kidney cancer patients.

Methods: According to the pTNM, 158 patients of T1 stage renal cell carcinoma were counted from January 1, 2016 to January 1, 2018 in the department of urology of First Hospital of Chifeng college. Preoperative imaging data and postoperative pathological data were collected for retrospective study. Comparing the data about patients' clinical T stage, pathological types, preoperative imaging methods, and the distance of tumor collection system. And identify the difference between the cTNM and pTNM of patients. Results: The postoperative pTNM of 158 patients with renal cell carcinoma, T1a: 81 cases, T1b: 77 cases; the preoperative cTNM, T1a: 50 cases, T1b: 99 cases, T2a: 9 cases. The average maximum diameter of preoperative tumors was $48.11 \pm 13.09 \mathrm{~mm}$ from imaging, and the postoperative pathological average maximum diameter was $44.21 \pm 12.34 \mathrm{~mm}$. The difference of the two groups was statistically significant $(\mathrm{P}<0.001)$. Compared with pTNM, there were 44 cases with cTNM $>$ pTNM, accounting for $27.8 \%$, and 4 cases with cTNM $<$ pTNM, accounting for $2.5 \%$. According to the stratified analysis of pTNM, we found 35 patients of pT1a with cTNM $>$ pTNM, $\mathrm{P}<0.001$, there was a significant difference;
9 patients of pT1b with cTNM $>$ pTNM, $p=0.004$, there was a significant difference. In patients with pTla, there were no cases with $\mathrm{cTNM}<\mathrm{pTNM}$, there were 4 patients of pT1b with $\mathrm{cTNM}<$ pTNM, $p=0.184$, there was no significant difference.

Conclusions: 1 : In the $\mathrm{T} 1$ renal cancer patients, there was a significant difference between the average maximum diameter of the $\mathrm{CT}$ and the pathological average maximum diameter, and the difference was about $3.91 \pm 6.13 \mathrm{~mm}$. About $27.8 \%$ of patients with cTNM had overly high stage compared with pTNM, and about $2.5 \%$ had too low stage. 2 : Patients with pT1a are more likely to be overly high cTNM stage, and about $22.2 \%$ of patients with pT1a tumors have high cTNM stage, about $5.7 \%$ of patients with pT1b tumors have high cTNM stage, which may affect the choices of some patients' best surgical program and prognosis. About the patients with T1 kidney cancer had too low cTNM stage, CT examination showed no significant difference.

\section{MP01-04 T1-substaging after Thulium fiber laser en bloc resection of bladder tumors}

A Dymov, L Severgina, D Kislyakov, D Enikeev, N Sorokin, A Proskura, L Rapoport

Institute for Urology and Reproductive Health, Sechenov University

Introduction \& Objective: The depth and extent of invasion into the lamina propria (T1 substaging) has been demonstrated to be of prognostic value in retrospective cohort studies. Its use is recommended by the most recent 2016 World Health Organization (WHO) classification. The optimal system to substage T1 remains to be defined. Probably the use of en bloc resection of bladder tumors when layer by layer is preserved would be beneficial for accurate $\mathrm{T} 1$ substaging. The aim of this study is to compare the quality of specimens after Thulium fiber laser en bloc resection of bladder tumors and conventional TURBT in terms of accurate T1 substaging. Methods: We prospectively analyzed specimens after Thulium fiber laser en bloc resection $(\mathrm{N}=71)$ and conventional TURBT $(\mathrm{N}=58)$ for NMIBC. En bloc resections were performed with thulium fiber laser «Fiberlase» (IRE Polus, Russia). We evaluated the possibility of T1 substaging in 24 cases in laser group and 18 cases in cTURBT group. All these procedures were performed by one experienced surgeon and all pathomorphological investigations were done by one uromorphologist.

Results: T1 substaging was carried out in all 24 cases $(100 \%)$ after laser en bloc resection while conventional TURBT provided accurate T1 substaging in 8 cases $(42 \%), p<0,001$. In laser en bloc resection group T1a was detected in 17 cases $(71 \%)$ and T1b in 7 cases (29\%). In cTURBT group, T1a was detected in 5 cases $(63 \%)$ and T1b in 3 cases $(37 \%)$.

Conclusions: According to our data specimens after Thulium fiber laser en bloc resection of bladder tumors are very well suitable for proper T1 substaging compare to conventional TURBT. Despite the fact that there is inconsistency in T1 substaging systems at present either $\mathrm{T} 1 \mathrm{a} / \mathrm{T} 1 \mathrm{~b}$ or $\mathrm{T} 1 \mathrm{~m} / \mathrm{T} 1 \mathrm{e}$ classifications should be used routinely as it may alter treatment strategy.

MP01-05 Is post voiding residual volume related to toxicity of intravesical bacillus Calmette-guelin for urothelial non-muscle invasive bladder cancer?

K Tochigi, K Hattori, N Sassa, M Kato, T Yamamoto, M Gotoh

Nagoya University Hospital 
Introduction \& Objective: Intravesical bacillus CalmetteGuerin (BCG) therapy is recommended for high-risk, nonmuscle invasive bladder cancer (NMIBC). Although it reduces the risk of intravesical recurrence and progression of NMIBC, $19 \%$ of patients cannot continue to receive the treatment due to severe side effects. Little is known about the relationship between post voiding residual volume and toxicity of intravesical BCG therapy. In this article, we investigated the relationship between post voiding residual volume after intravesical BCG instillation and the side effects of the treatment.

Methods: We retrospectively reviewed the medical records of 32 patients who were diagnosed with NMIBC by transurethral resection (TUR) and treated with intravesical BCG therapy in Nagoya University Hospital from July 2017 to August 2018. They received the instillation once weekly for 6-8 weeks after an interval of more than 4 weeks from the initial TUR or $2^{\text {nd }}$ TUR. They received full dose of BCG induction instillation with Tokyo-172 strain (Immunobladder intravesical 80mg) and kept it for 1 hour. After first urination, their post voiding residual volume was measured with BladderScan ${ }^{\circledR}$ BVI 6100 ultrasound device. If necessary, urethral catheterization was performed. We used ManWhitney $U$ test to evaluate the association of post voiding residual volume with toxicity. $\mathrm{P} \leq 0.05$ considered to indicate significance. Results: The median patients' age was 75 years old. Of the patients, $25(78 \%)$ were male and $7(22 \%)$ were female. Eleven received intravesical BCG therapy after first TUR and 21 did after TUR for recurrent tumors. The median post voiding residual volume was $15.7 \mathrm{ml}(0-55 \mathrm{ml})$. Twenty-two patients $(68.7 \%)$ experienced any grade toxicity and more than grade 2 toxicity was observed in 7 patients $(21.8 \%)$. Furthermore, 4 patients $(12.5 \%)$ could not continue the treatment. We found that post voiding residual volume tended to increase in the group with any grade toxicity compared with that without any grade toxicity $(19.25 \mathrm{ml}$ vs $6.25 \mathrm{ml}, \mathrm{p}=0.07)$. However, there was no significant difference between post voiding residual volume in the group without any grade toxicity and that in the group with more than grade 2 toxicity $(p=0.1)$ nor that in the group with stopping the treatment $(p=0.23)$. Conclusions: In this study, it was suggested that side effects with intravesical BCG therapy for NMIBC occurred more frequently accordance with the increase of post voiding residual volume.

\section{MP01-06 Percutaneous nephrostomy in obstructing pelvic malignancy: does it facilitate further oncologi- cal treatment and what is the associated morbidity?}

\section{SS Folkard, S Banerjee, J Reason, E Psallidas, J Green}

Introduction \& Objective: The optimal management of patients with ureteric obstruction in advanced malignancy is unclear. The treatment is associated with complications and frequent readmissions. How the patients' quality of life is affected by a nephrostomy and how many of these patients undergo further oncological treatment remains uncertain. The objective of this retrospective multicenter study was to look at the outcomes of patients who had percutaneous nephrostomy for malignancy.

Methods: We identified patients who had a nephrostomy inserted for ureteric obstruction due to malignancy at our institution from Jan 2015 to Dec 2018. We obtained data retrospectively from our electronic patient record system. Patients who had nephrostomy insertion for other causes such as ureteric calculi or injury were excluded from the study.

Results: 105 patients (55 male and 50 female) underwent nephrostomy insertion during this time interval. Average age was 68.8 (range $30-93)$ years. $51.42 \%$ patients $(n=54)$ had urological malignancies (bladder and or prostate cancer) and $40.97 \%$ $(n=43)$ had non-urological pelvic malignancies (colorectal, gynecological) and the rest $7.61 \%(n=8)$ other malignancies (testis, pancreatic, hematological). The median LOS was 14 days (range 1-104 days) post procedure and 39.04\% $(n=41)$ had at least one 30-day readmission to hospital. The average starting Creatinine levels was $348 \mathrm{mmol} / \mathrm{L}$ (range 49-1133) and the average creatinine at discharge was $170 \mathrm{mmol} / \mathrm{L}$ (range 44-651). Although the average change in the creatinine $(190 \mathrm{mmol} / \mathrm{L})$ is statistically significant $(\mathrm{p}<0.001)$ it did not seem to prolong life of the patients. Only $26(24.76 \%)$ patients were alive (all-cause mortality) at the end of the 4-year period with an average life expectancy of 139 days following nephrostomy. Interestingly after nephrostomy insertion only $30.47 \%(n=32)$ patients underwent further oncological treatment as the rest were too frail to undergo any chemotherapy.

Conclusions: In our series most, patients who had nephrostomy insertion for ureteric obstruction due to malignancy had no further oncological treatment following insertion. Percutaneous nephrostomy is a procedure not without associated morbidity and does not always prolong survival. Given the associated poor prognosis in cases of advanced malignancy, we advocate multidisciplinary approaches to decision-making in terms of nephrostomy insertion.

\section{MP01-07 Neoadjuvant Chemotherapy Before Radical Prostatectomy For Locally Advanced Prostate Can- cer: A Systematic Review And Meta-Analysis}

T Lin, X Yang, Q Wei

\section{West China Hospital, Sichuan University}

Introduction \& Objective: Radical prostatectomy (RP) only is not adequate for the locally advanced prostate cancer, instead a multimodal approach is implemented. Neoadjuvant or adjuvant therapies such as radiation therapy, androgen deprivation therapy (ADT) and chemotherapy are used to minimalize the risk of positive surgical margin and recurrence. Chemotherapy has been the standard of care for castration resistant prostate cancer since the development of the taxanes. In recent years, its application has been widened to metastatic hormone sensitive prostate cancer. The question remains whether neoadjuvant administration of cytotoxic agents with RP can improve perioperative outcome and long-term survival. The present study aims to synthesize current available evidences on the tolerance and efficacy of neoadjuvant chemotherapy (NAC) followed by RP, and to combine data from multiple studies using meta-analysis.

Methods: This study was conducted according to Preferred Reporting Items for Systematic Reviews and Meta-Analyses guidelines and registered on the international prospective register of systematic reviews (PROSPERO registration number: CRD42019123375). Authenticated databases including PubMed/Medline, Embase, Web of Science, Ovid, Web of Knowledge, and Cochrane Library were extensively searched. MeSH words and free words with the following searching strategy: "prostate cancer" AND "neoadjuvant" AND ("docetaxel" OR "taxane" OR "chemotherapy") were used in the literature search. The quality of selected studies was appraised using The Grading of Recommendations Assessment, Development and Evaluation (GRADE) approach.

Results: We included 20 studies with 746 participants for analysis. NAC was used independently or together with neoadjuvant ADT or tyrosine kinase inhibitors for dose optimization. The median Gleason Scores of the included patients ranged from 7 to 8. A significant proportion of locally advanced prostate cancer patients were able to tolerate NAC, with only $90(12.1 \%)$ 
discontinued treatment. Partial PSA response (more than $50 \%$ reduction) before RP was achieved in 38 of 110 patients received NAC only, while in 122 of 123 patients received NAC plus neoadjuvant ADT. In term of long-term survival, $35.7 \%$ to $73.3 \%$ patients were disease-free during follow-up (median 13.0 to 141.6 months), with a pooled disease-free survival rate of $52.9 \%$ (95\% CI $46.9 \%-59.7 \%)$.

Conclusions: The neoadjuvant administration of taxanesbased chemotherapy before RP is safe and effective for locally advanced prostate cancer patients. Future research should optimize the dosage and the combination regimens of NAC, as well as investigate which subsets of patients will benefit from NAC.

MP01-08 Experience of laser treatment for contralateral recurrence of upper urinary tract transitional cell carcinoma after radical resection

\section{Li, C Li, Bao, Z Gao}

Introduction \& Objective: Introduction: Some patients who have received nephroureterectomy for one side upper urinary tract transitional cell carcinoma (UUT-TCC) will have recurrent carcinoma in opposite upper urinary tract. It is dilemma for urologist to choose effective therapy. Laser therapy would be a choice.

Purpose: To investigate the effectiveness of laser therapy for recurrent UUT-TCC after opposite side nephroureterectomy. Materials and Methods: We retrospectively reviewed all 15 patients who had undergone opposite side nephroureterectomy received laser therapy for recurrent UUTT from January 2014 to December 2018. Clinical characteristics, operative results and functional outcomes were investigated. Mean follow-up were 19.6 months. End points of follow-up including: complete ureteral obstruction, or tumor locally advanced, or distant metastasis.

Results: clinical features :5 patients presented mild gross hematuria, the rest had no serious symptoms. There were multiple lesions in 7 cases and mild hydronephrosis in 9 cases. All patients had superficial lesions. Results: the average operation time was 3313 minutes, and the average number of operations per patient was 2.5 0.6. The mean recurrence time was 3.92 .3 months. All patients had no other complications except ureteral perforation (36\%). Functional Results: renal function was maintained in 8 patients $(53.3 \%)$. Five patients $(33.3 \%)$ had to undergo open surgery or nephrostomy because of complete ureteral obstruction, local tumor progression, or distant metastasis.

Conclusions: Laser therapy for patients in this situation is palliative. This procedure minimize invasion and is duplicable in spite of less efficient for cure and function preservation. However, for patients who have undergone opposite side nephroureterectomy, laser therapy could be a choice.

\section{MP01-09 The effective observation of the non muscle- invasive bladder cancer with the transurethral thu- lium laser radical resection of bladder tumor}

\section{Z Xin, C Hanmin, X Kunlin, Z Xiaofeng, X Rihai, Z Guoxi}

Introduction \& Objective: To evaluate the effect and security of the non muscle-invasive bladder cancer with the transurethral thulium laser radical resection of bladder tumor.

Methods: 24 cases of bladder tumor patients with transurethral thulium laser radical resection of bladder tumor from May 2017 to May 2018 were performed. According to the imaging examination, the preoperative clinical stage was Ta and T1N0M0, and cystoscopy was performed before the operation to determining the tumor location, number and size. Using the rickon thulium laser and setting the laser as continuous wave mode, with the maximum power of 50W. Excising the whole tumor in a area of $1.0 \mathrm{~cm}$ from the tumor base, and cutting it into the deep muscle layer or serous layer of the bladder. Postoperative intravesical instillation of pirarubicin or gemcitabine was treated for 1 year. Results: All operations were successfully completed. The operation time was $16 \mathrm{~min}$ ( 5 to $25 \mathrm{~min}$ ). No hemorrhage and obturator nerve reflex occurred during the operation. The catheter was retained for 1 to 2 days and the bladder was flushed for 1 day. The pathology was urothelial carcinoma, including 15 cases of low level, 9 cases of high level, the cases of postoperative pathological stage Ta was 11, the cases of T1 was 8 and the cases of T2 was 5. Follow-up time was 2 to 12 months, and 2 cases recurrence occurred.

Conclusions: The transurethral thulium laser radical resection of bladder tumor can quickly resect the whole piece of bladder tumor, which is ideal effective, high security, Meanwhile, the transurethral thulium laser radical resection of bladder tumor can provide pathological specimens sufficiently, which is the ideal method to treating the non muscle-invasive bladder cancer and worth of clinical popularization and application.

\section{MP01-10 Patient-Reported Outcomes Via Online Questionnaires: Post-Radical Prostatectomy Quality Improvement and Outcome Assessment}

KJ See, J Tran, LM Huynh, TE Ahlering

UC Irvine Health

Introduction \& Objective: Securing a reliable source for tracking patient-reported outcomes accurately and in a timely manner is an essential component for counseling post-radical prostatectomy (RP) patients. In 2011, we identified a simple means to predict time to post-RP continence recovery via padfree cards (PFC) and daily urinary pad logs (DUPL). Subsequently, in 2017, we presented an automated, HIPAA-compliant system using an electronic questionnaire to assess early urinary continence rates. The present study seeks to validate the accuracy, efficiency, and ease of use this system in tracking post-RP continence recovery.

Methods: 510 patients undergoing robot-assisted RP (RARP) were prospectively enrolled to one of three outcomes tracking systems: 1) a pre-addressed paper packet containing a DUPL and PFC 2) an automated, email questionnaire, or 3) both. An email survey was sent 30 days following catheter removal and reminders were automatically sent if no response was received within two days, up to 3 reminders. Pad-free dates of patients in group (3) were analyzed to ensure concordance between paperand electronic questionnaires. Two-tailed, Student t-tests and ANOVA were used to compare demographic characteristics, response rates, and continence rates.

Results: Demographic characteristics, stratified by each tracking system are presented in Table 1. At 30-days post-RP, response rates in paper versus electronic questionnaire systems were $80.7 \%(201 / 249)$ and $92.7 \%(229 / 250)$, respectively $(\mathrm{p}=0.0001)$. Pad-free rates in paper versus electronic questionnaires were not significantly different between groups: $64.2 \%$ (129/201) and $64.2 \%(147 / 229)$, respectively $(\mathrm{p}=1.00)$. At 1 -year post-RP, response rates in paper versus electronic questionnaires were $87.6 \%(218 / 249)$ and $97.3 \%(144 / 148)$, respectively $(\mathrm{p}=0.0013)$. Again, pad-free rates were not significantly different between groups: $91.7 \%(200 / 218)$ in paper and $94.4 \%(136 / 144)$ 
Table 1. Patient Demographic Table

\begin{tabular}{|c|c|c|c|c|c|}
\hline & $\begin{array}{l}\text { Paper } \\
\text { Questio } \\
N=249\end{array}$ & & $\begin{array}{l}\text { Electronic } \\
\text { Questionn } \\
\mathrm{N}=250\end{array}$ & & \\
\hline & Mean & SD & Mean & SD & P-Value \\
\hline Age, years & 63.48 & 7.32 & 63.76 & 7.81 & 0.6774 \\
\hline IIEF-5 score & 19.00 & 6.84 & 18.14 & 7.53 & 0.1796 \\
\hline Pre-PSA, ng/mL & 10.05 & 12.09 & 8.60 & 14.46 & 0.2212 \\
\hline AUA score & 9.88 & 7.35 & 9.05 & 7.37 & 0.2053 \\
\hline Bother & 2.01 & 1.53 & 1.74 & 1.47 & 0.0436 \\
\hline Prostate weight, g & 57.24 & 24.45 & 54.39 & 24.38 & 0.1899 \\
\hline Estimated blood loss, mL & 87.99 & 41.80 & 89.20 & 39.36 & 0.7377 \\
\hline Body mass index, $\mathrm{kg} / \mathrm{m} 2$ & 27.01 & 3.85 & 27.05 & 3.42 & 0.9018 \\
\hline & N & $\%$ & $\mathrm{~N}$ & $\%$ & P-Value \\
\hline Pathological stage & 253 & & 243 & & \\
\hline pT2 & 150 & $59.3 \%$ & 147 & $60.5 \%$ & 0.7854 \\
\hline рT3/pT4 & 103 & $40.7 \%$ & 96 & $39.5 \%$ & 0.3255 \\
\hline Pathologic GGG & 253 & & 241 & & \\
\hline 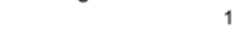 & 54 & $21.3 \%$ & 39 & $16.2 \%$ & 0.1475 \\
\hline 2 & 78 & $30.8 \%$ & 103 & $42.7 \%$ & 0.0061 \\
\hline 3 & 59 & $23.3 \%$ & 58 & $24.1 \%$ & 0.8346 \\
\hline 4 & 18 & $7.1 \%$ & 21 & $8.7 \%$ & 0.5099 \\
\hline 5 & 44 & $17.4 \%$ & 20 & $8.3 \%$ & 0.0026 \\
\hline $\begin{array}{l}\text { Seminal vesicle } \\
\text { invasion }\end{array}$ & 254 & & 243 & & \\
\hline Yes & 35 & $13.8 \%$ & 29 & $11.9 \%$ & 0.5276 \\
\hline No & 219 & $86.2 \%$ & 214 & $88.1 \%$ & 0.5276 \\
\hline Surgical margins & 254 & & 244 & & \\
\hline Positive & 58 & $22.83 \%$ & 67 & $27.46 \%$ & 0.234 \\
\hline Negative & 196 & $77.17 \%$ & 177 & $72.54 \%$ & 0.234 \\
\hline
\end{tabular}

electronic, respectively $(\mathrm{p}=0.339)$. In group $(3)$, dates of padfree continence recovery were concordant in $89.6 \%$ (43/48) of patient's \pm 5 days.

Conclusions: Not only did the electronic questionnaires system yield a significant increase in response rates at 30-days and 1year, it also proved to be an effective and reliable method in determining pad-free continence.

\section{MP01-11 Preoperative prognostic value of trigone involvement of muscle invasive bladder cancer}

K Tochigi, N Sassa, S Inoue, Y Funahashi, M Kato, T Yamamoto, M Gotoh

Department of Urology, Nagoya University Graduate School of Medicine

Introduction \& Objective: Radical cystectomy is golden standard treatment of muscle invasive bladder cancer (MIBC). Some reported association tumor location and tumor progression, but little is known. Trigone develops from the mesonephric ducts, it's different from other locations. In this article, we investigated preoperative prognostic value of trigone involvement of MIBC.

Methods: We retrospectively reviewed the medical records of 112 patients who were diagnosed with MIBC and treated with radical cystectomy in Nagoya University Hospital from January 2004 to May 2018. We excluded patients who have past history of upper tract urothelial cancer. The association between trigone involvement of MIBC and other factors with overall survival (OS), Cancer specific survival (CSS), and disease-free survival (DFS) was assessed. Kaplan-Meier estimated survival curves were generated for OS, CSS, and DFS. Cox proportional hazards analysis was performed to identify factors that predicted survival. $\mathrm{p} \leq 0.05$ considered to indicate significance.

Results: The median patients' age was 70 years old. Of the patients, $85(76 \%)$ were male. $40(36 \%)$ have trigone involvement of MIBC. 45 (40\%) received neo-adjuvant chemotherapy. The median follow-up was 40.5 months. 5 years OS, CSS, and DFS of all population are $75.2 \%, 80.2 \%$, and $65.3 \%$. The 5 -year OS, CSS, and DFS rate of trigone involvement patients was $71.1 \%, 79.6 \%$, and $58.2 \%$, which was not significantly different from that of no trigone involvement patients (OS: $70.9 \%$, $\mathrm{p}=0.911$, CSS: $73.8 \%, \mathrm{p}=0.596$, DFS: $68.6 \%, \mathrm{p}=0.285)$. On multivariate analysis, only advanced clinical $\mathrm{T}$ stage is associated with a decreased CSS (hazard ratio, 4.2; $\mathrm{p}=0.002$ ).

Conclusions: Trigone involvement of MIBC is not associated with OS, CSS, and DFS.

\section{MP01-12 Evidence for the Integration of Total and} Free Testosterone in Management of Prostate Cancer

M Towe, LM Huynh, F el Khatib, MM Osman,

F Yafi, TE Ahlering

UC Irvine Health

Introduction \& Objective: Within the context of prostate cancer (PC), there has been a historic fear of high serum testosterone, a hesitance towards testosterone supplementation, and a resultant lack of systematized testosterone screening. Herein, we seek to investigate the utility of serum total and calculated free testosterone (cFT) in PC management.

Methods: 830 patients underwent RP, with prospectively-drawn total testosterone (TT), sex hormone binding globulin (SHBG), and cFT. Impact of age was assessed with linear regression modeling. Correlation of low cFT with adverse oncologic characteristics [i.e. high-risk Gleason Grade Group (GGG) 9-10, extraprostatic extension (pT3/pT4) and seminal vesicle invasion (SVI)] was assessed with receiver-operator characteristic (ROC) curves.

Results: Levels of TT remained constant as men aged ( 0.09 per year; $R=0.02)$, while SHBG increased ( 0.87 per year; $R=0.32$ ), and $\mathrm{cFT}$ decreased $(0.08$ per year; $\mathrm{R}=-0.2)$. Low $\mathrm{cFT}$ independently predicted very high-risk GGG $(\mathrm{AUC}=0.435, \mathrm{p}=0.036)$, extraprostatic extension $(\mathrm{AUC}=0.557, \mathrm{p}=0.011)$, and SVI $(\mathrm{AUC}=0.396, \mathrm{p}=0.005)$ TT was not a predictor.

Figure 1: Trends of TT, cFT, and SHBG by age.

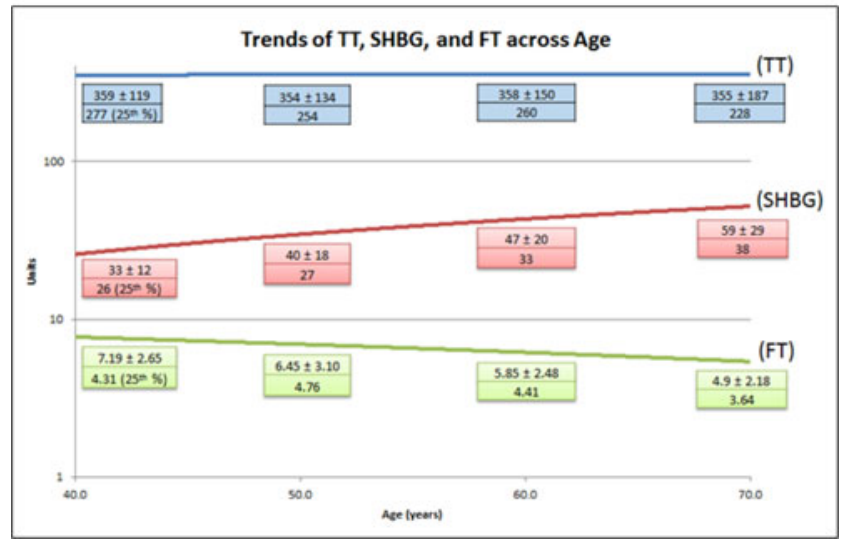




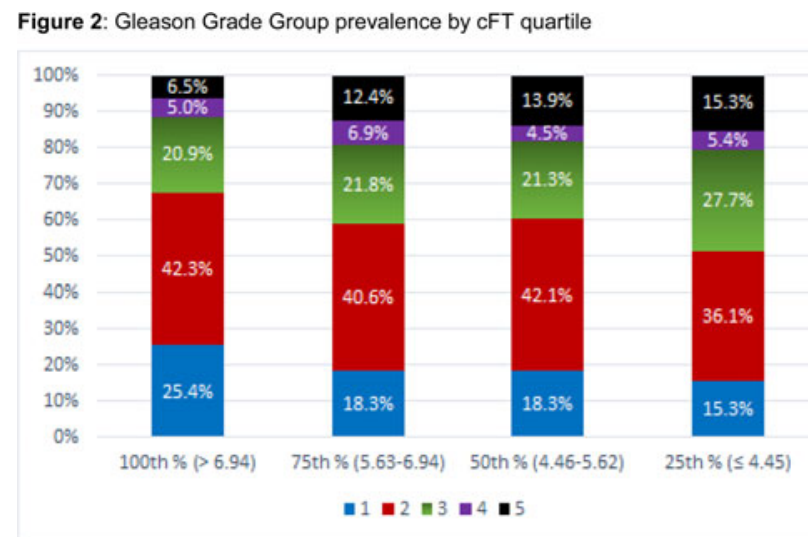

Figure 3: Receiver-operator characteristic curve of GGG 9-10, predicted by age preoperative PSA, and $\mathrm{CFT}(\mathrm{AUC}=0.698)$.

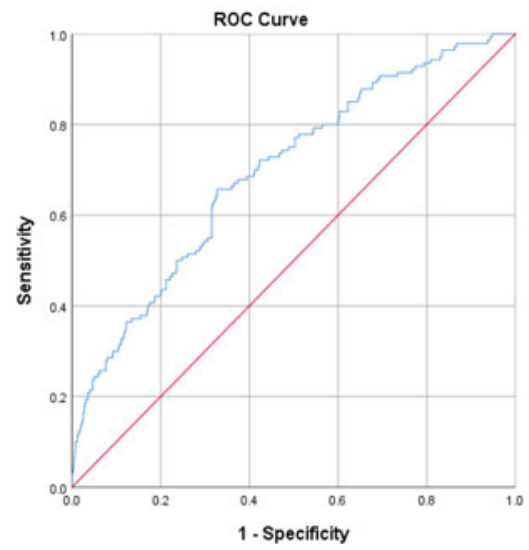

Conclusions: TT remained stable as men aged, while cFT decreased $2-3 \%$ per year. Low cFT was an independent predictor of more aggressive, high-risk disease and adverse pathological characteristics. These relationships stress the importance of $\mathrm{cFT}$ integration into prostate cancer management.

\section{MP01-13 Perioperative and functional outcomes of RARP in Elderly Men> $=80$}

M Shahait, RW Dobbs, J Kim, N Eldred, V Patel, DI Lee

University of Pennsylvania

Introduction \& Objective: The number of highly active octogenarian men with prostate cancer seeking definitive treatment is on the rise. The functional and oncological outcomes of RARP in this population are not well studied. Herein, this study aims to report perioperative, functional, and oncological outcomes of RARP in men $>=80$ years old

Methods: Between January 2010-2019, 39 patients with PCa underwent RARP by two surgeons (DIL \& VP). Clinical and pathological features, perioperative and postoperative complications were evaluated. The patient reported continence rate, and oncological outcomes were reported. Continence was defined as the use of zero or one safety pad per day.

Results: The median age was 81 (80-83), median ASA score and CCI was 2 (2-3), and 5 (5-6), respectively. The median preop- erative IPSS was $14(2.3-26.8)$, and median SHIM score was 3 (1-19); $87 \%$ of the patient had intermediate or high-risk PCa. The mean (SD) operative time was 122 (28) minutes, and the mean (SD) blood loss was 167(32) ml. All cases were completed robotically, no intra-operative complications were encountered, and the median length of stay was $1.26(0.85)$. Regarding, 30 and 90 day complication, nine patients had postoperative complications; 7 were Clavien-Dindo grade I-II, and two were Clavien-Dindo grade $\geq$ III. Post-RARP continence rate at 3 months, and 12 months were $60 \%$ and $79 \%$, respectively. Two patients had PSA persistence, and ten patients had a biochemical recurrence; of those, six received radiation therapy, and one patient received androgen deprivation therapy.

Conclusions: RARP is a feasible option to treat $\mathrm{PCa}$ in well selected elderly men $>80$. Majority of these men had significant LUTS and harbored high-risk disease, who might benefit from RARP by alleviating their symptoms and providing local control of their disease.

\section{MP01-14 Creation of the Harmonized Arabic version of the Expanded Prostate Index Composite for Clin- ical Practice (AREPIC-PC)}

\section{G Barayan, M Awad, M Plante, R Grunert}

University of Vermont/ Umm AlQura University

Introduction \& Objective: The Expanded Prostate Cancer Index Composite for Clinical Practice (EPIC-CP) is a validated Patient Reported Outcome (PRO) widely used for assessing the quality of life in prostate cancer patients. It specifically measures the urinary, bowel, sexual and vitality / hormonal health function of prostate cancer patients. It is used internationally and has been translated and, in turn, validated into at least 4 languages in addition to English. This effort represents the initial steps as defined by guidelines set forth by the Professional Society for the Health Economics and Outcomes Research (ISPOR) to create the an Arabic harmonized version of the EPIC-CP (AREPIC-CP).

Methods: Using the established protocol as defined by the ISPOR for translating patient care questionnaires, two native Arabic speaking physicians (two Urology residents from our program) separately translated an independent draft of the EPIC-CP into Arabic. This was then reviewed by our nonphysician certified lead Arabic translator to create a harmonized Arabic version. In turn, an additional non-physician certified translator then back-translated the harmonized version into English. The entire team then collectively reviewed and made changes to this English version to adjust for any cross-cultural differences. The non-physician lead translator then retranslated this finalized version into Arabic to create the final harmonized translation for clinical use with approval by the entire team.

Results: This harmonized version of the Arabic EPIC-CP is submitted herein. Again, the translation was reviewed extensively with consideration of cultural context. See figure 1.

Conclusions: The creation of this harmonized translation of the Arabic EPIC-CP (AREPIC-CP) was successfully performed following ISPOR guidelines. Although yet to be validated for clinical research, this approved harmonized version can be considered for use to help guide the clinician and prostate cancer patients to discuss quality of life both before and after prostate cancer therapies among Arabic speaking patients. We are 
currently working to validate this important PRO for clinical use and future research in Arabic speaking patients.

\section{MP01-15 Thulium Laser en-bloc resection of bladder Tumor (THUEB-BT): ESUT study to compare laser and electrical en-bloc transurethral resection of bladder tumor}

G Bozzini, U Besana, G Pini, AS Gozen, E Liatsikos, C Buizza, M Sighinolfi, B Rocco

ASST Valle Olona - Busto Arsizio VA

Introduction \& Objective: En bloc resection of bladder tumors (EBRBT) may improve staging quality and perioperative morbidity and influence tumor recurrence. We want to evaluate the safety, efficacy, and recurrence rates of electrical versus Thulium laser en bloc resection of bladder tumors (THUEBBT).

Methods: This European multicenter study included 362 patients at four tertiary centers in Lombardia. TUR-BT was performed with monopolar/bipolar current or Thulium laser energy. Staging quality measured by detrusor muscle involvement, tumor size, switch to conventional TURBT rate, early postoperative outcomes, hemoglobin loss and 18-month follow-up data were analyzed.

Results: Electrical EBRBT and THUEB-BT resection were used to treat 178 and 184 patients, respectively. Median tumor size was $1.9 \mathrm{~cm}$; largest tumor was $4.4 \mathrm{~cm}$. Detrusor muscle was present in $89.4 \%$. A switch to conventional TURBT was significantly more frequent in the electrical en-bloc group (23.7 vs. $3.7 \%, \mathrm{p}<0.05)$. Mean operation time, postoperative irrigation, catheterization time, and hospitalization were not different from a statistical point of view. Overall complication rate was low (Clavien $\geq 3,3.3 \%$ ). Hemoglobin was significantly lower after electrical ERBT $(p=0.0013)$. Patients were followed for a mean follow up of 20.3 months; 72 (19.8\%) had recurrences. In total, $72.2 \%$ recurrences occurred outside the ERBT resection field, mainly for THUEB-BT.

Conclusions: ERBT is safe and reliable regardless of the energy source and provides high-quality resections of tumors $>1 \mathrm{~cm}$. Recurrence rates did not differ between groups, and the majority of recurrences occurred outside the ERBT resection field, mainly in THUEB-BT group.

\section{MP01-16 Active surveillance - Is it feasible for in-} termediate risk localized prostate cancer?

S Mukherjee, I Promponas, J Abbaraju, S Madaan

Dartford and Gravesham NHS Trust

Introduction \& Objective: Active surveillance (AS) is a wellrecognized treatment option for low risk localized prostate cancer (PC). However, its role in intermediate risk localized PC is yet to be fully established. Here we compared the outcome of AS between low risk and intermediate risk PC patients.

Methods: Between November 2002 and April 2019, 318 men with localized PC underwent initial expectant management in our hospital based on local departmental protocols mainly adapted from NICE guideline and a prospective database was maintained (demographic details summarized in Table-1). Intervention was offered when there was progression in Gleason
Table-1: Demographic details

\begin{tabular}{|c|c|}
\hline Demographic details $(\mathrm{n}=318)$ & Number (percentage) \\
\hline $\begin{array}{l}\text { 1. PSA } \\
: \quad<10 \\
: 10-<20 \\
:>20 \\
\text { - unknown }\end{array}$ & $\begin{array}{c}282(88.68 \%) \\
32(10.06 \%) \\
2(0.63 \%) \\
2(0.63 \%)\end{array}$ \\
\hline $\begin{array}{l}\text { 2. Gleason score } \\
\begin{array}{l}\text { - } 3+3 \\
\text { - } 3+4 \\
\text { - } 4+3\end{array}\end{array}$ & $\begin{array}{c}275(86.48 \%) \\
42(13.21 \%) \\
1(0.32 \%)\end{array}$ \\
\hline $\begin{array}{l}\text { 3. Clinical tumour staging } \\
\text { - } \mathrm{Tla} \\
\text { - } \mathrm{T} 1 \mathrm{~b} \\
\text { - } \mathrm{Tlc} \\
\text { - T2a } \\
\text { - T2b }\end{array}$ & $\begin{array}{c}29(9.12 \%) \\
14(4.40 \% \\
208(65.41 \%) \\
64(20.13 \%) \\
3(0.94 \%)\end{array}$ \\
\hline $\begin{array}{l}\text { 4. D'Amico risk group } \\
\text { - Low risk } \\
\text { - Intermediate risk } \\
\text { - High risk }\end{array}$ & $\begin{array}{c}241(75.79 \%) \\
73(22.96 \%) \\
4(1.26 \%)\end{array}$ \\
\hline $\begin{array}{l}\text { 5. Type of initial biopsy } \\
\text { - TRUS } \\
\text { - Template } \\
\text { - TURP }\end{array}$ & $\begin{array}{c}209(65.72 \%) \\
65(20.44 \%) \\
44(13.84 \%)\end{array}$ \\
\hline $\begin{array}{l}\text { 6. Disease progression } \\
\text { - Total } \\
\text { - Histology progression } \\
\text { - Radiology progression } \\
\text { - PSA rise }\end{array}$ & $\begin{array}{c}102(32.08 \%) \\
64(20.13 \%) \\
19(5.97 \%) \\
19(5.97 \%)\end{array}$ \\
\hline $\begin{array}{l}\text { 7. Intervention done } \\
\text { - Total } \\
\text { - Radical prostatectomy } \\
\text { - External beam radiotherapy } \\
\text { - Brachytherapy }\end{array}$ & $\begin{array}{c}100(31.45 \%) \\
67(21.07 \%) \\
29(9.12 \%) \\
4(1.26 \%)\end{array}$ \\
\hline $\begin{array}{l}\text { 8. Indications for intervention } \\
\text { - Histology progression } \\
\text { - Radiology progression } \\
\text { - PSA rise } \\
\text { - Patient's preference }\end{array}$ & $\begin{array}{l}56(17.61 \%) \\
18(5.66 \%) \\
18(5.66 \%) \\
8(2.52 \%)\end{array}$ \\
\hline
\end{tabular}

score (GS), worsening of magnetic resonance imaging (MRI) finding, consecutive rise in prostate-specific antigen (PSA) level, or change in patient's preference.

Results: Out of 318 localized PC patients who underwent AS during this period, $241(75.79 \%)$ were low risk, $73(22.96 \%)$ were intermediate risk and $4(1.26 \%)$ were high risk as per D'Amico risk classification. The median (interquartile range) total follow-up time from the first biopsy was 61.50 (36.0-88.0) months. Out of 318 patients, 14 (4.40\%) died (11 from low risk group and 3 from intermediate risk group) and rest 304 are alive

Table-2: Comparison between low risk and intermediate risk prostate cancer patient

IQI - Interquartile range

\begin{tabular}{|c|c|c|c|}
\hline Demographic details & $\begin{array}{c}\text { Low risk } \\
(\mathrm{n}=241)\end{array}$ & $\begin{array}{c}\text { Intermediate risk } \\
(\mathrm{n}=73)\end{array}$ & $\mathrm{p}$ value \\
\hline 1. Age [(Median (IQR)] & $64.0(59.0-68.0)$ & $65.0(61.0-70.0)$ & 0.0640 \\
\hline $\begin{array}{l}\text { 2. Total follow up period in } \\
\text { months [(Median (IQR)] }\end{array}$ & $63.0(38.75-95.0)$ & $\begin{array}{l}57.00(30.75- \\
76.25\end{array}$ & 0.0441 \\
\hline $\begin{array}{l}\text { 3. Period of active } \\
\text { surveillance in months } \\
\text { [(Median (IQR)] }\end{array}$ & $41.0(19.75-72.0)$ & $\begin{array}{l}39.0(15.77- \\
51.79)\end{array}$ & 0.4804 \\
\hline $\begin{array}{l}\text { 4. Disease progression } \\
\text { [Number (percentage)] }\end{array}$ & $78(32.37 \%)$ & $24(32.88 \%)$ & 0.9349 \\
\hline $\begin{array}{l}\text { 5. Intervention needed } \\
\text { [Number (percentage)] }\end{array}$ & $80(33.20 \%)$ & $20(27.40 \%)$ & 0.3524 \\
\hline $\begin{array}{l}\text { 6. 5-years overall survival } \\
\text { rate (Percentage) }\end{array}$ & $94.90 \%$ & $97.60 \%$ & 0.9890 \\
\hline $\begin{array}{l}\text { 7. Probability of being } \\
\text { untreated at } 5 \text { years } \\
\text { (Percentage) }\end{array}$ & $63.29 \%$ & $66.95 \%$ & 0.8311 \\
\hline $\begin{array}{l}\text { 8. 5-years biochemical } \\
\text { recurrence free survival }\end{array}$ & $89.62 \%$ & $93.50 \%$ & 0.6731 \\
\hline
\end{tabular}


Figure-1: Kaplan-Meire overall survival curve. 1 (purple colour) indicates total patient population, 2 (green colour) indicates low risk group and 3 (orange colour) indicates intermediate risk group.

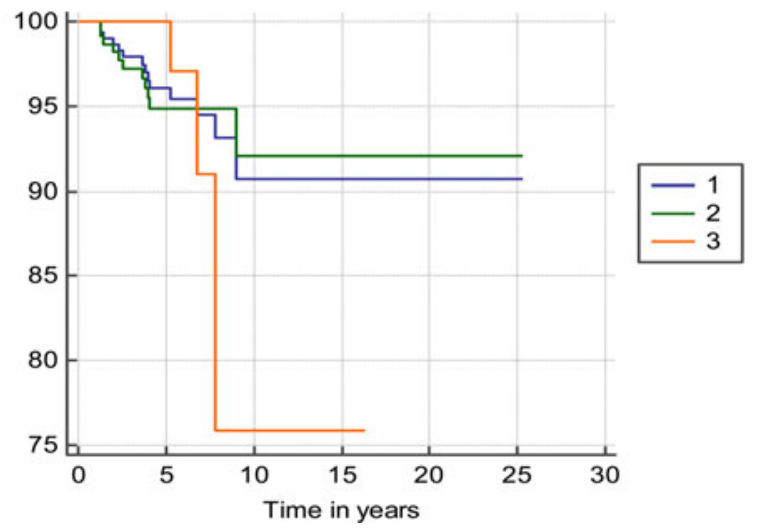

Figure-2: Time to intervention curve. 1 (purple colour) indicates total patient population, 2 (green colour) indicates low risk group and 3 (orange colour) indicates intermediate risk group.

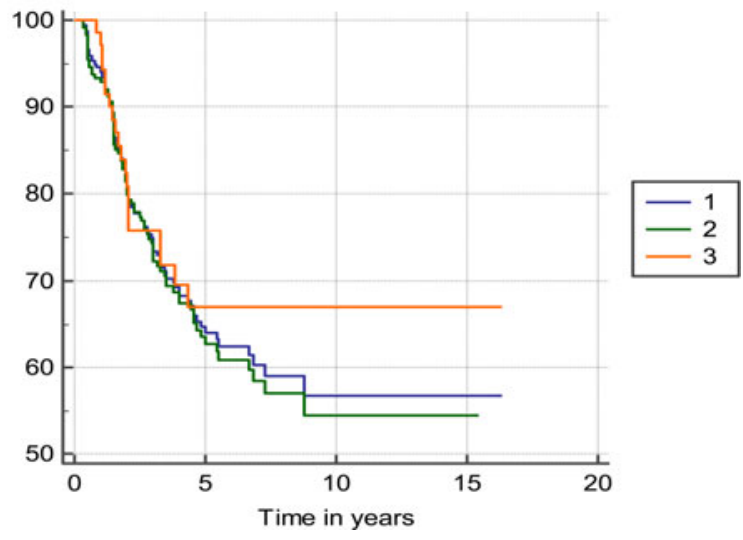

(censored rate, $95.60 \%$ ). No death was recorded from prostate cancer in the cohort. Overall, $102(32.08 \%)$ patients developed disease progression - $78(32.37 \%)$ belonged to low risk group and $24(32.88 \%)$ to intermediate risk group with no statistically significant difference between the two groups $(\mathrm{p}=0.9349)$. The 5-years overall survival rates (Fig-1) for the whole cohort was $96.02 \%$ which was nearly similar at $94.90 \%$ and $97.60 \%$ for low risk and intermediate risk group respectively without any statistically significant difference $(\mathrm{p}=0.9890)$. Similarly, no significant difference was observed in the estimated 5-years curatively intended treatment-free survival (Fig-2) between low risk $(63.29 \%)$ and intermediate risk $(66.95 \%)$ groups $(\mathrm{p}=0.8311)$. Lastly, there was no difference in 5-years biochemical recurrence free survival for treated patients between low and intermediate risk group. Table-2 illustrates the comparative data between these two groups.

Conclusions: The option of AS can be considered in carefully selected patients of intermediate risk localized prostate cancer, but the patients need to be informed that long term ( $>10$ years) follow up data are limited.

\section{MP01-17 Nurse led one stop hematuria clinic: Out- comes from 1581 patients}

MA Hamdoon, MO Breish, D Whiting, A Elliott, D Pearce, S Madaan

Dartford and Gravesham NHS Trust

Introduction \& Objective: The aim of this study is to assess the diagnostic approach \& outcomes from the referrals to the urology outpatients at a district general hospital with nurse led one stop hematuria clinic.

Methods: Analysis of prospective data was performed for all patients referred to hematuria clinic between October 2014 \& December 2017 (Figure 1).

Results: Total 1581 patients were seen. Of these 65\% (1020) were males and 35\% (561) were females; $75 \%$ of all referrals were visible hematuria. $73 \%$ of patients with visible hematuria

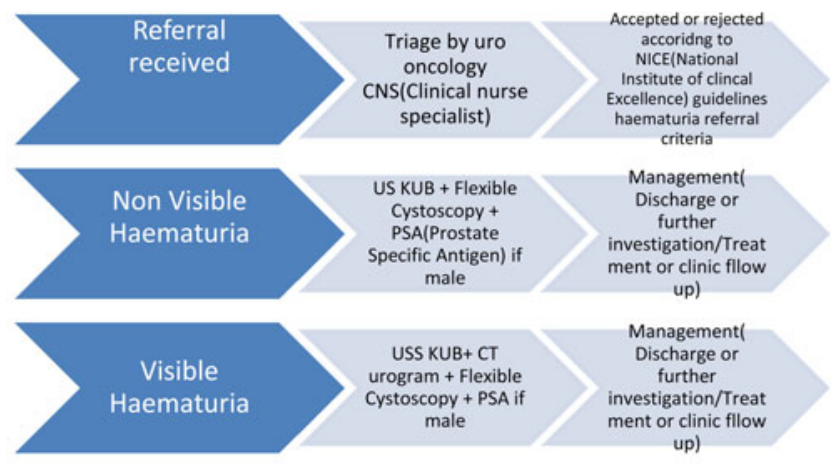

Fig 1: Haematuria clinic pathway

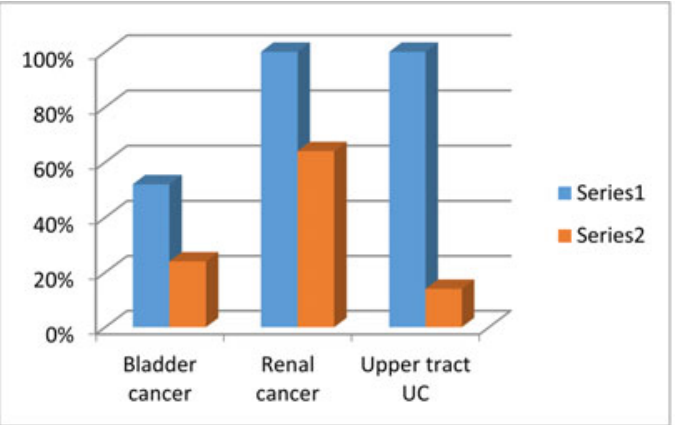

Figure 2: Sensitivity of CT urogram and US KUB in urological cancer detection

\begin{tabular}{|l|l|l|l|l|}
\hline & US KUB & CT Urogram & $\begin{array}{l}\text { Both US and CT } \\
\text { Urogram }\end{array}$ & $\begin{array}{l}\text { Total number } \\
\text { of cancer } \\
\text { cases }\end{array}$ \\
\hline Bladder Cancer & $48 \%$ & $60 \%$ & $67 \%$ & 152 \\
\hline Renal Cancer & $83 \%$ & $100 \%$ & $100 \%$ & 15 \\
\hline $\begin{array}{l}\text { Upper Tract } \\
\begin{array}{l}\text { Urothelial } \\
\text { Carcinoma (Upper } \\
\text { tract UC) }\end{array}\end{array} 0 \%$ & $86 \%$ & $86 \%$ & 14 \\
\hline
\end{tabular}

Fig 3: Urological malignancies diagnosis by US KUB and CT urogram 
and $72 \%$ of patients with non-visible hematuria had no cause found. The overall cancer detection rate was $13 \%$ [17\% with visible hematuria (200/1185) and 5\% with non-visible hematuria (21/396)]. (Figure 2) Of those with positive findings on investigations; bladder cancer was the commonest cause of both visible (25\%) and non-visible hematuria (27\%). Approximately $60 \%$ of patients harboring malignancy were either current or Ex-Smokers. CT (Computerized Tomography) urogram is superior to US KUB (Ultrasound Kidney Ureter and bladder) in detecting bladder cancer ( $60 \%$ Vs $48 \%)$, renal cancer $(100 \% \mathrm{Vs}$ $83 \%)$, and upper tract urothelial malignancies (86\% Vs $0 \%)$ (Figure 3).

Conclusions: Nurse led hematuria clinic is feasible with good outcomes and flexible cystoscopy can be performed safely by trained nurses and the nurse led hematuria clinic can be applied to other multiple diagnostic urological clinics following the appropriate training. The investigations showed no cause of hematuria in majority of patients while bladder cancer is the commonest cause of both types of hematuria. CT urogram remains the gold standard imaging for visible hematuria.

\section{MP01-18 The Role of Urine and Washing Cytology during Primary Transurethral Resection of Bladder Tumors (TURBT)}

T Ozimek, MC Hupe, JP Struck, M Hennig, N Gilbert, J Cordes, AS Merseburger, MW Kramer

Universitätsklinikum Schleswig-Holstein (Campus Lübeck)

Introduction \& Objective: Urine cytology (UC) is a recommended supplementary tool for diagnosis and follow-up of high-grade urothelial malignancies of lower and upper urinary tract. So far, no specific recommendations regarding the role of washing cytology (WC) was included in the guidelines. Clinical practice shows that UC and WC are often gathered not only during out-patient cystoscopies but also intraoperatively during TURBT. The goal of our study was to retrospectively analyze the relationship between the histology of resected tumors and intraoperative UC or WC findings.

Methods: Five hundred consecutive primary TURBT cases conducted between November 2010 and October 2015 at our department were retrospectively analyzed. Data of patients in which additional intraoperative UC and WC were gathered underwent further investigation for specificity and sensitivity of given cytological test. Presence of synchronous or metachronous urothelial cancer of upper urinary tract (UTUC) was additionally registered. Sensitivity of UC and WC were compared with McNamara Chi2 test.

Results: Urothelial cancer of the bladder at any stage had been histologically confirmed in 313 out of 500 investigated procedures $(62.6 \%) .151(30.2 \%)$ patients demonstrated benign lesions. $36(7.2 \%)$ patients had intravesical malignant lesion of non-urothelial origin. Any cytological samples were gathered in 210 of 313 urothelial cancer patients: UC was performed in 192 cases (positive UC in 99/192 - sensitivity of 51.6\%) and WC in 185 cases (positive WC in 107/185 - sensitivity of 57.8\%). Both types of samples were gathered in 167 cases, with positive UC or WC in 105 of 167 patients - sensitivity of $62.8 \%$. No statistical difference regarding sensitivity of UC vs. WC could be found $(p>0.05)$. We observed only one false positive result of urine cytology out of all cytological samples in 106 cytologic records out of 151 patients with benign histology. Positive cytologic results in patients with benign intravesical lesion indicating UTUC were not observed in any case.

Conclusions: Cytologic testing is an important diagnostic part in diagnosis and follow-up of urothelial cancer. Urine and washing cytology present similar sensitivity. Our results advocate however against intraoperative cytologic testing during TURBT. The clinical benefit from taking both types of samples at once is minimal.

\section{MP01-19 Do all patients with suspicious prostate cancer need Multiparametric Magnetic Resonance Imaging before prostate biopsy?}

S Anacleto, J Alberto, E Dias, B Caló, P Mota, N Morais, J Torres, P Passos, A Cordeiro, U Falagario, E Lima

Introduction \& Objective: Multiparametric magnetic resonance imaging (mpMRI) is a promising tool to diagnose prostate cancer but its cost is not negligible. In order to avoid unnecessary costs and minimize time to diagnosis, it is necessary to establish which patients benefit from doing mpMRI prior to prostate biopsy (PB). Our aim is to test if mpMRI still predicts prostate cancer ( $\mathrm{PCa}$ ) and clinically significant prostate cancer (csPCa) in patients with high clinical suspicion of cancer, defined as prostate specific antigen (PSA) $>10 \mathrm{ng} / \mathrm{ml}$, PSADensity (PSAD) $>0.15 \mathrm{ng} / \mathrm{ml} / \mathrm{cc}$ or suspicious digital rectal examination (DRE).

Methods: We retrospectively collected data on 594 patients who underwent transrectal ultrasound-guided PB at the Urology Department of Hospital de Braga from January 2017 to July 2018. From these, 206 patients who underwent mpMRI were selected. mpMRI results were classified using Prostate Imaging Reporting and Data System (PI-RADS) version 2. In primary analysis, ChiSquared and Fisher's-Exact Tests were used to evaluate the

Table 1. Association of PI-RADS with PCa and csPCa stratified for PSA $\leq 10 \mathrm{ng} / \mathrm{ml}$ and $>10 \mathrm{ng} / \mathrm{ml}, \mathrm{PSAD} \leq 0.15 \mathrm{ng} / \mathrm{m} / \mathrm{cc}$ and $>0.15 \mathrm{ng} / \mathrm{ml} / \mathrm{cc}$ and normal and suspicious DRE

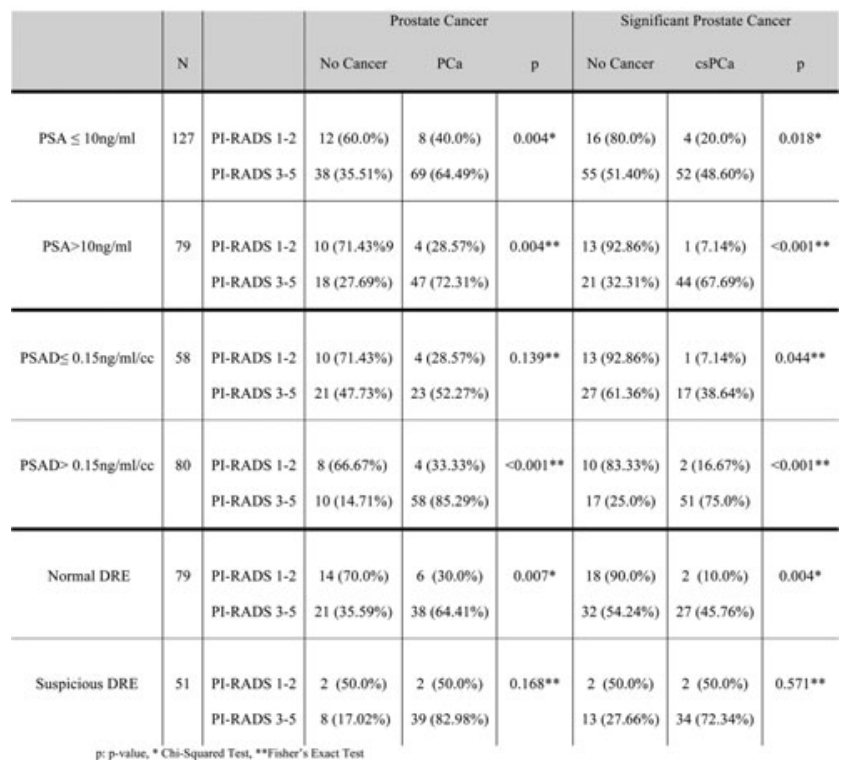


association of mpMRI with PCa and csPCa. Stratification of this model for low versus high clinical suspicion of cancer, defined by different PSA and PSAD cutoffs and DRE, was then performed. Results: In primary analysis and overall, mpMRI was predictive of PCa and csPCa $(\mathrm{p}<0.001)$. In stratified analysis, we found that in patients with high clinical suspicion of cancer (PSA $>10 \mathrm{ng} / \mathrm{ml}$ and PSAD $>0.15 \mathrm{ng} / \mathrm{ml} / \mathrm{cc}$ ), mpMRI was still significantly associated with $\mathrm{PCa}(\mathrm{p}=0.004$ and $\mathrm{p}<0.001$, respectively) and csPCa $(\mathrm{p}<0.001)$. In men with suspicious DRE, however, mpMRI was no longer predictive of PCa nor $\operatorname{csPCa}(\mathrm{p}=0.168$ and $\mathrm{p}=0.571$, respectively).

Conclusions: $\mathrm{mpMRI}$ is still useful in predicting csPCa in patients with PSA $>10 \mathrm{ng} / \mathrm{ml}$ and PSAD $>0,15 \mathrm{ng} / \mathrm{ml} / \mathrm{cc}$. In patients with suspicious DRE though, mpMRI might be no longer useful in the prediction of cancer, since almost all men will have csPCa.

MP01-20 Predictors of Failure of Active Surveillance in Patients with Clinically Localized Prostate Cancer

NA Aldhaam, AS Elsayed, AA Hussein, W Ji, G James, o Abdel Razzak, I Saad, M Abdelhakim, E Kauffman, J Mohler, KA Guru

\section{Roswell Park Comprehensive Cancer Center}

Introduction \& Objective: Active surveillance (AS) has gained acceptance as a method for reducing the over-treatment associated with PSA-based early detection without compromising

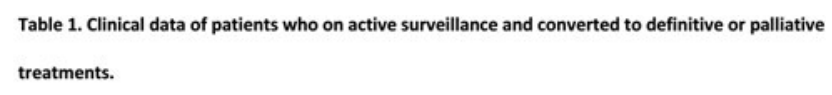

\begin{tabular}{|c|c|}
\hline Variable & value \\
\hline $\begin{array}{l}\text { Number of patients converted to } \\
\text { treatment, }(\%)\end{array}$ & $83(27)$ \\
\hline Age, mean (SD) & $62(6)$ \\
\hline Body mass index, mean (SD) & $30(5)$ \\
\hline Follow up (months), mean (SD) & \\
\hline $\begin{array}{l}\text { Core positive biopsy, median } \\
\text { (IQR) }\end{array}$ & $0.25(0.2)$ \\
\hline PSA doubling time, mean(SD) & $128(311)$ \\
\hline Reasons of conversion, $n(\%)$ : & \\
\hline Anxiety & $1(1)$ \\
\hline Increase in Grade & $19(23)$ \\
\hline Increase in Grade and Stage & $3(4)$ \\
\hline Increase in Grade and Volume & $26(31)$ \\
\hline Increase in PSA and Grade & $11(13)$ \\
\hline Increase in Volume & $6(7)$ \\
\hline Increase in Volume and PSA & $3(4)$ \\
\hline Increase in Volume and Stage & 2(2) \\
\hline Not Documented/Unknown & $1(5)$ \\
\hline \multicolumn{2}{|l|}{ Type of treatment, $n(\%)$ : } \\
\hline RARP & $64(78)$ \\
\hline Radiation & $12(15)$ \\
\hline hormonal & $6(7)$ \\
\hline
\end{tabular}

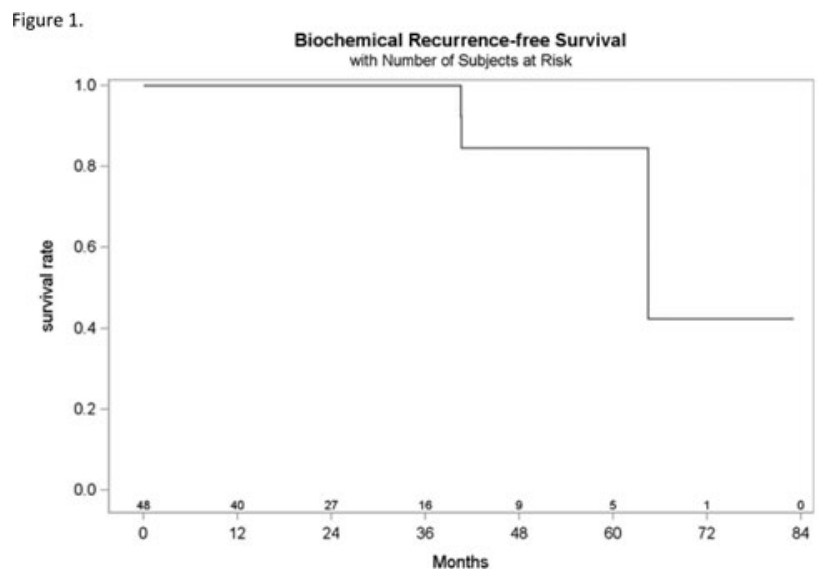

cancer-related outcomes. However, the possibility of progression from low- to high-grade cancer or patient anxiety may reduce retention in AS programs. We assessed the predictors of AS failure (conversion from AS to either definitive or palliative treatment).

Methods: A retrospective analysis of a prospectively populated and continuously updated database was conducted of all patients (pts) enrolled from 2009 to 2018 in the AS program at Roswell Park Comprehensive Cancer Center. Follow up included prostate-specific antigen (PSA) measurements every 6 months and surgeon-specific use of MRI and repeat prostate biopsies. Demographics, clinical and oncological outcomes were analyzed. Multivariate logistic regression models were used to assess for predictors of AS conversion. Kaplan-Meier curves were used for biochemical recurrence-free survival (BcRFS).

Results: 306 patients on AS had mean follow-up 24 months (range 21 - 37). 27\% of patients converted to treatment $(78 \%$ underwent robot-assisted radical prostatectomy (RARP), $15 \%$ underwent radiation and 7\% underwent androgen deprivation therapy). Median time from diagnosis to conversion was 2.1 years. The main reasons to convert from AS to treatment were increase in the Gleason score and the percentage of the volume of involved core biopsy on follow up biopsies $31 \%$ (Table 1). Variables that predicted AS failure were PSA doubling time $(\mathrm{P}=0.02)$ and percent positive core biopsies $(\mathrm{P}<0.01)$. No deaths from prostate cancer have occurred. The BcRFS (Figure 1) rate at 5 years was $85 \%$.

Conclusions: PSA doubling time and percent positive core biopsies were associated with conversion to the treatment of men on active surveillance.

MP01-21 The clinical differences between patients of pheochromocytoma with hypertension and normotension

Z Liu, L Zhou, H Li, K Wang, Y Zhu

Sichuan University

Introduction \& Objective: To explore the clinical differences between patients of pheochromocytoma with hypertension and normotension. Besides, we would like to determine the risk factors associated with development of hypertension. 
Methods: All patients in our hospital undergoing surgical removal of pheochromocytoma (PHEO) from 5th January 2009 to 19th July 2018 were retrospectively analyzed to determine those with and without hypertension. Preoperatively demographic data and clinical data on hypertension were recorded.

Results: Finally, 164 (31.3\%) patients were with normotension among 524 patients of PHEO undergoing surgery. Older patients and those with longer courses of PHEO were more likely to develop hypertension. Patients with hypertension were less likely to have extra-adrenal PHEO. Last but not least, the blood norepinephrine of patients with hypertension was higher. Besides, patients with hypertension had higher blood glucose and were more likely to develop diabetes.

Conclusions: $31.3 \%$ of PHEO patients might be with normotension. Older patients and those with longer courses of PHEO were more likely to present hypertension preoperatively. Patients with hypertension had higher the blood norepinephrine and blood glucose. They were also more likely to develop diabetes.

MP01-22 Evaluation of the actual tumor volume evaluated from surgical specimens following radical prostatectomy and its clinical impact on the prognosis of patients with prostate cancer

O Kwon, H Lee

Hallym University Kangnam Sacred Heart Hospital
Introduction \& Objective: The tumor volume (TV) of prostate cancer (PCa) was regarded as significant criterion factor to predict the prognosis of patients. We sought to evaluate the actual distribution of TV and relationship with PCa prognosis.

Methods: We measured the TV during the pathologic evaluation of surgical specimen following the radical prostatectomy in 2,316 patients with localized PCa. We tried to evaluate the clinical impact of TV in predicting the postoperative biochemical recurrence by using Kaplan-Meier analysis and Coxproportional model.

Results: The mean prostate volume of entire cohort was $36.5 \mathrm{cc}$ and mean TV was $5.9 \mathrm{cc}$. There were significant positive relationships between the TV and elevation of the D'Amico and National Comprehensive Cancer Network risk groups (all $\mathrm{p}<0.001$ ). The high TV showed significant worse pathologic outcomes compared with low TV group in terms of high rate of extra-capsular extension, seminal vesicle invasion, and positive surgical margin (all $\mathrm{p}<0.05$ ). The patients with high TV showed significant shorter biochemical recurrence-free survival than the low TV group $(\mathrm{p}<0.001)$. Finally, the TV was revealed to be an independent predictor of postoperative biochemical recurrence from multivariate Cox-proportional analyses as both categorical variable (HR 1.42, 95\% CI 1.13-1.78, $\mathrm{p}=0.003$ ] and continuous variable (HR 1.04, 95\% CI 1.04-1.05, $\mathrm{p}<0.001$ ).

Conclusions: The TV was revealed as an independent prognostic factor in predicting the postoperative biochemical recurrence. The patients with high number and longer tumor length in the biopsy cores were revealed to have a high possibility to have high tumor volume.

\section{MODERATED POSTER SESSION 02: CLINICAL ONCOLOGY: OUTCOMES \& COMPLICATIONS (II)}

\author{
MP02-01 Avoiding Unnecessary MRI and Biopsies: \\ NPV and PPV of MRI according to PSA Density, \\ 4Kscore and Risk Calculators \\ U Falagario, A Martini, E Wajswol, P Treacy, P Ratnani, \\ R weil, i Jambor, SC Lewis, L Cormio, G Carrieri, \\ AR Rastinehad, P Wiklund, A Tewari
}

Introduction \& Objective: The 2019 EAU Guidelines recommend obtaining mpMRI in case of clinical suspicion for $\mathrm{PCa}$ in biopsy naive patients and avoiding biopsy in patients with a negative mpMRI and low clinical suspicion. However, a consensus on the optimal biopsy strategy including biomarker and MRI is currently lacking.

Methods: After Institutional Review Board approval, we reviewed data of 266 biopsy naïve patients. PSA higher than $3 \mathrm{ng} / \mathrm{ml}$ or positive DRE was the trigger for a biopsy. If mpMRI was positive, 2 to 4 extra cores were taken from each lesion in addition to the 12-core standard sampling. In order to provide urologists with an outline for deciding when to perform MRI and biopsy we calculated NPV and PPV of mpMRI stratified by the patient's risk of csPC based on all currently available clinical tools, such as RCs (European Randomized Study of Screening for PC Risk Calculator [ERSPC-RC]) and biomarkers (4kscore and PSAd). Finally, we investigated different clinical scenario to assess a cost-effective strategy to avoid unnecessary biopsies and reduce the diagnosis of clinically insignificant cancers.

Results: The highest NPV for men with PIRADSv2.0 1-2 lesions were found in men with low and intermediate risk 4Kscore $(96.9 \%$ and $97.1 \%)$, PSAd $<0.10(98.7 \%)$ and ERSCP-RC $<2 \%(98.7 \%)$. Likewise, the PPV for men with PIRADSv2.0 3-5 lesions was lowest in patients with low $4 \mathrm{~K}$ risk score $(0 \%)$, PSAd $<0.10$ $(13.2 \%)$ and ERSCP-RC $<2 \%(12.3 \%)$ (Table 1) Three different sequences and combinations of clinical screening tools were 

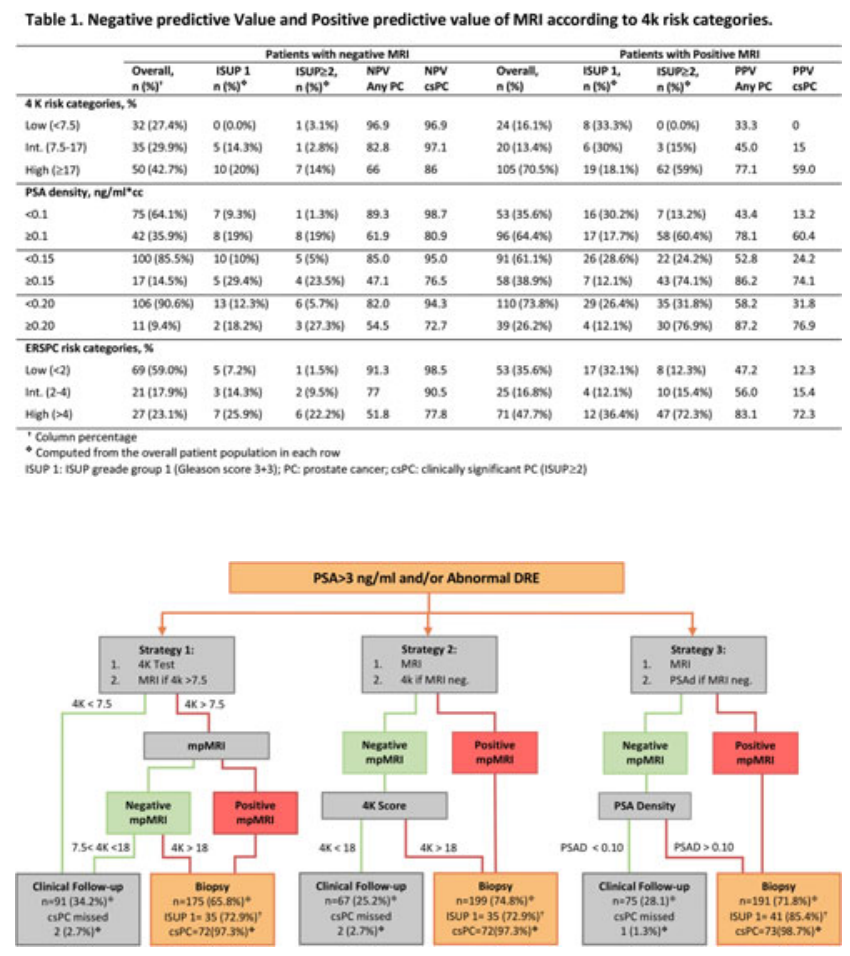

examined (Figure 1). Performing an initial 4Kscore followed by MRI if the 4Kscore was greater than $7.5 \%$ and a subsequent biopsy if the MRI was positive or 4 Kscore was $>18 \%$ resulted in $2.7 \%$ of csPCa missed and $34.2 \%$ biopsy avoidance (Strategy 1; Figure 1). Performing an initial MRI followed by biopsy in negative MRI if 4 Kscore was $>18 \%$ or PSAd was $>0.10$ resulted in a similar percentage of csPCa missed (2.7\% and $1.3 \%$ ) but slightly less biopsies avoided (25.2\% and $28.1 \%$ ) (Strategy 2 and 3; Figure1).

Conclusions: Physicians should consider clinical risk screening tools when ordering and interpreting mpMRI results to avoid unnecessary testing and diagnostic errors.

\section{MP02-02 Clinical Safety and Diagnostic Efficacy of Multiparametric Magnetic Resonance Imaging and Ultrasound (mpMRI-US) Fusion Targeted and sys- tematic Transperineal Prostate Biopsy: The prelimi- nary Experience of First 100 Cases}

\section{S Mehmood, A Alwehaibi, KI Alothman}

King Faisal Specialist Hospital \& Research Center Riyadh

\section{Introduction \& Objective:}

Background: Post biopsy urosepsis is one of the major concerns in terms of patient morbidity and medical cost. Transperineal targeted and systematic biopsy is reported to have less complications and higher detection rate of clinically significant prostate cancer.

Objectives: To determine the clinical safety and diagnostic efficacy of mpMRI-US fusion transperineal targeted and systematic prostate biopsy in our first 100 patients with elevated prostatic specific antigen (PSA).

Material and Methods: A prospective single institution study included men with elevated PSA $>3 \mathrm{ng} / \mathrm{ml}$ and previous negative biopsy from January 2018 to April 2019. All patients had mpMRI and suspicious lesions reported as Prostate Imaging Reporting and Data System (PIRADS) score version 2. Average twelve

\begin{tabular}{|c|c|}
\hline Variables & Values \\
\hline No. of patients & 100 \\
\hline Age (yr) median & 64.0 (range 47-84) \\
\hline Initial PSA (ng/ml) & $6.1(3.2-224)$ \\
\hline PSA $<4$ & 13 \\
\hline $4-10$ & 57 \\
\hline$>10$ & 30 \\
\hline BMI $\left(\mathrm{kg} / \mathrm{m}^{2}\right)$ & $27.3(19.0-37.0)$ \\
\hline Prostate volume(ml) & $53.0(15.0-147.0)$ \\
\hline $\begin{array}{l}\text { mpMRI to biopsy } \\
\text { Time (months) }\end{array}$ & $4.1 \pm 3.6$ \\
\hline Prior Biopsy (n, \%) & $12(12)$ \\
\hline $\begin{array}{l}\text { Cancer detection } \\
\text { rate }(\%)\end{array}$ & $45(45 \%)$ \\
\hline Biopsy cores & \\
\hline Targeted cores & $2(1-5)$ \\
\hline Systematic cores & $12(10-18)$ \\
\hline
\end{tabular}

PSA $=$ prostatic specific Antigen, $B M I=$ Body mass index, mpMRI= Multiparametric Magnetic Resonance Imaging

\begin{tabular}{|c|c|c|c|c|c|c|c|}
\hline \multirow{3}{*}{$\begin{array}{l}\text { PI-RADS } \\
\text { Score }\end{array}$} & \multirow{3}{*}{$\begin{array}{l}\text { No. of } \\
\text { patients }\end{array}$} & \multicolumn{3}{|c|}{ Targeted Biopsy } & \multicolumn{3}{|c|}{ Systemic Biopsy } \\
\hline & & \multirow[t]{2}{*}{ Negative } & \multicolumn{2}{|c|}{ Positive } & \multirow[t]{2}{*}{ Negative } & \multicolumn{2}{|l|}{ Positive } \\
\hline & & & $\begin{array}{l}\text { Total No. } \\
(\%)\end{array}$ & $G S>7(\%)$ & & $\begin{array}{l}\text { Total } \\
\text { no.(\%) }\end{array}$ & $G S>7(\%)$ \\
\hline PI-RADS 5 & 36 & $11(33.55)$ & $25(69.44)$ & $23(63.88)$ & $22(61.11)$ & 14 (38.88) & $10(27.77)$ \\
\hline PI-RADS 4 & 27 & $16(59.25)$ & $11(40.74)$ & 09(33.33) & $22(81.48)$ & $05(18.51)$ & $02(7.40)$ \\
\hline PI-RADS 3 & 17 & 15 (88.23) & $02(11.76)$ & 0 & $14(82.35)$ & 03(17.64) & $01(5.88)$ \\
\hline PI-RADS 1-2 & 20 & $20(100)$ & 0 & & $20(100)$ & 0 & \\
\hline
\end{tabular}

PI-RADS= Prostate imaging reporting and data system, $\operatorname{cSPSA}=$ clinically significant prostate cancer, GS= Gleason score

systematic and two targeted cores were biopsied in every patient. All patients underwent biopsy under general anesthesia and received single prophylactic dose of antibiotic and no postoperative antibiotics. PIRADS score were compared with cancer detection in targeted cores and systematic cores.

Results: First consecutive 100 patients having median age of 64.0 years with initial median PSA of $6.1 \mathrm{ng} / \mathrm{ml}$ (range 3.2-224) were included for mpMRI-US fusion transperineal targeted and template prostate biopsy. Overall cancer detection rate was $45 \%$ (targeted $36 \%$ and template $23 \%$ ) with clinically significant prostate cancer (csPCa) 34\% (targeted 32\% and template 13\%). Cancer detection rate including csPCa with MRI fusion targeted biopsy were higher 69.44\% in PI-RADS 5, 40.74\% in PI-RADS 4 and $11.76 \%$ in PI-RADS 3. $20 \%$ of patients who had PI-RADS score 1-2 and high PSA, all turned out benign. Urinary retention developed in 5 patients (5\%) post biopsy, requiring temporary catheterization. There was not a single case of urinary tract infection or urosepsis.

Conclusions: Transperineal mpMRI-US fusion prostate biopsy is highly safe and effective in diagnosing clinically significant prostate cancer. There is no risk sepsis or major complications. 
MP02-03 Contemporary Practice Patterns in Treatment of Malignant Extrinsic Ureteral Obstruction in the Nationwide Inpatient Sample

C Haas, C Sayegh, J Pak, O Shah, E Hyams

Columbia University

Introduction \& Objective: Malignant extrinsic ureteral obstruction (MEUO) presents a clinical challenge and generally has a poor prognosis. Strategies for decompression include retrograde ureteral stenting (RUS) and nephrostomy tube (NT) placement, however there is limited evidence on optimal approaches. The objective of this study was to investigate practice patterns in the use of RUS and NT for MEUO across malignancy types in a large national sample.

Methods: Using the National Inpatient Sample (2010 - 2015), we abstracted all adults with diagnoses of hydronephrosis and concurrent metastasis or lymphoma, excluding any record with a urinary tract stone or a procedure code suggesting intrinsic obstruction. Primary outcome was receipt of RUS or NT compared to no decompression. Weighted multinomial logistic regression assessed predictors of undergoing no decompression, RUS, or NT.

Results: There were an estimated 242,944 cases of MEUO, with genitourinary (GU) cancers representing the largest subgroup $(31.3 \%)$. Bladder and prostate cancer had the lowest ratio of RUS/NT among cancer groups at 0.81 and 0.61 , indicating a preference of NT in these cancers (Fig. 1). On multinomial logistic regression with no decompression as the referent, factors that significantly increased odds of NT included prostate (OR 1.5), bladder (OR 1.6), and cervical (1.6) cancer, teaching (1.4) and large (1.3) hospitals, chronic kidney disease (1.4), acute kidney injury (2.5), and pyelonephritis (2.0). Decreased odds of NT was observed in lymphoma (0.6), rural (0.5), and southern (0.9) hospitals. Factors that increased odds of undergoing RUS included ovarian (1.3) and colon (1.3) cancer, female gender (1.4), Midwest (1.4), and southern (1.2) hospitals. Medicaid insurance predicted lower odds of RUS (0.8) but higher odds of NT (1.2).

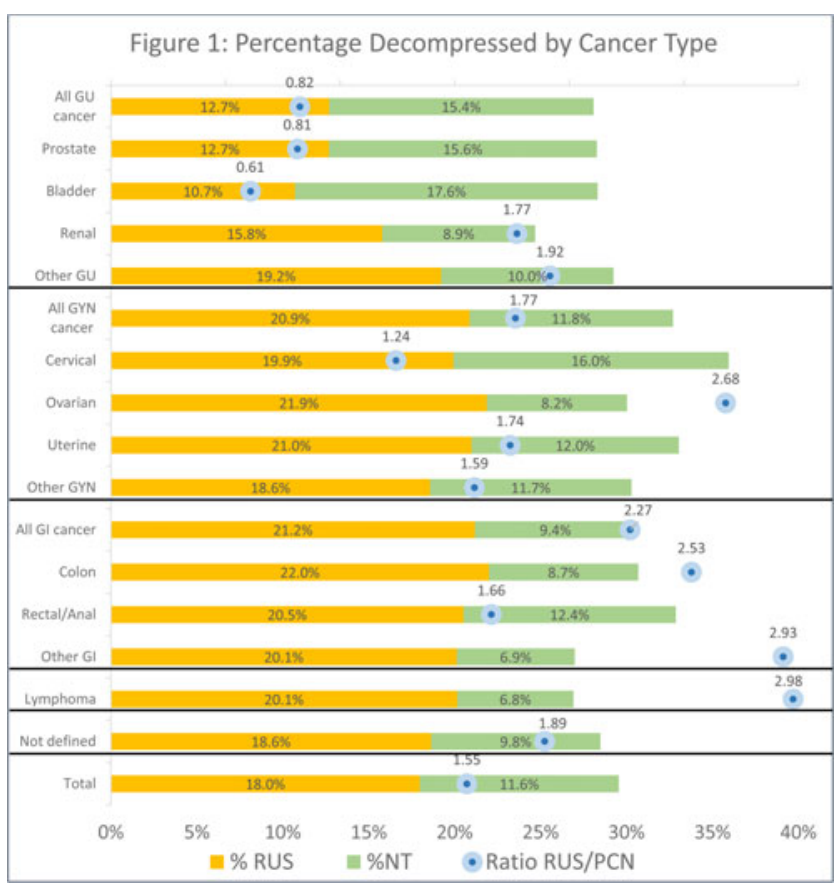

Conclusions: There is substantial variation in decompression approaches for MEUO among patient, hospital and disease types. A preference for NT was observed in larger coastal academic institutions for Medicaid patients with bladder, prostate, or cervical cancer, while RUS was preferred in female patients, and patients with lymphoma, ovarian, or colon cancer in the Midwest and South. Further investigation into best approaches for certain disease subtypes may help to standardize care and reduce potential disparities.

MP02-04 Prevalence of risk factors in patients presenting for hematuria evaluation - A prospective patient reported data analysis \& outcomes

\section{G KANDA SWAMY, B James, B Rai, M Jefferies, P Bose}

Swansea Bay University Health Board

Introduction \& Objective: The risk factors for Urological cancers are well known. With more awareness, many changes at different levels have been implemented to minimize exposure to risk factors. We aimed to look at the contemporary prevalence of risk factors for urothelial cancers in all patients attending hematuria clinic (HC).

Methods: A questionnaire was developed comprising of all risk factors for urothelial malignancy. We asked all patients attending $\mathrm{HC}$ to fill in the questionnaire. Data was collected between prospectively between Jan to Nov 2018, which included demographics, race, smoking history, diabetes, hypertension, body mass index (BMI), pioglitazone \& ketamine use, prior cancer, cyclophosphamide use, family history of cancers, exposure to dyes, paints, rubber, textiles, leather, high risk occupations, predisposing factors, genetic conditions and schistosomiasis exposure.

Results: During the ten-month study period, 583 patients were evaluated in HCs. Mean age was 64.8 years (range 18 - 99), with male: female ratio of $1.87: 1.88 \%$ were Caucasians, $15.5 \%$ smoked tobacco, $3.4 \%$ use e-cigarettes, $37.2 \%$ were Ex-smokers and $42.5 \%$ never smoked. Hypertension in $10.1 \%$, Diabetes in $6 \%$, and occupational exposure to potential carcinogens in $21.2 \%$ were noted. $\mathrm{BMI}>30$ (obese) was seen in $35.9 \%$ and another $35 \%$ were overweight (BMI 25-30). Cancer in family is seen in 50.6\% of HC patients. Multiple cancers were seen in $23 \%$ \& the other $77 \%$ had single cancer only. The top three cancers are lung (17.2\%), bowel $(16.2 \%)$ and breast $(15.9 \%)$. Incidence of prostate and bladder cancer in family are $8.1 \%$ and $3.7 \%$ respectively. Table below summarizes the comparison of prevalence of risk factors in the study cohort as compared against national standards and also highlights certain data that were not studied before.

\begin{tabular}{|c|c|c|}
\hline & Study cohort rate & $\begin{array}{c}\text { Welsh rate }\left({ }^{*} \text { regional }\right) \\
(16+\text { years })\end{array}$ \\
\hline Tobacco smoking & $15.5 \%$ & $20 \%^{*}$ \\
\hline $\mathrm{E}-\mathrm{cig}$ & $3.4 \%$ & $8 \% *$ \\
\hline $\mathrm{BMI}>25$ & $71 \%$ & $78 \% *$ \\
\hline Diabetes & $6 \%$ & $7.4 \%$ \\
\hline Hypertension & $10.1 \%$ & $20 \%$ \\
\hline Cancer in family & $50.6 \%$ & NA \\
\hline $\begin{array}{c}\text { Carcinogen } \\
\text { exposure }\end{array}$ & $21.2 \%$ & NA \\
\hline Travel to Africa & $8 \%$ & NA \\
\hline $\begin{array}{c}\text { Genetic } \\
\text { conditions }\end{array}$ & $0 \%$ & NA \\
\hline
\end{tabular}


Conclusions: Prevalence of smoking, BMI distribution, diabetes and hypertension are better than general population in Wales among patients presenting with hematuria. Prevalence of few other risk factors are reported for the first time in this study. Further studies are underway to study the relationships of these factors to Urological malignancy. These data in the meantime will provide valid insight into certain previously un known factors.

MP02-05 Effect of Travel Distance to Treatment Facility on Presenting Stage and Overall Survival in Kidney Cancer: an NCDB Analysis

A Kaldany, KE Okhawere, PC Yong, KK Badani

Icahn School of Medicine at Mount Sinai

Introduction \& Objective: Previous studies have demonstrated mixed findings on the relationship between travel distance to care and outcomes for many urologic malignancies, however this association has not yet been studied in renal cell carcinoma (RCC). We used data from the National Cancer Database (NCDB) to investigate the association between distance travelled for treatment and presenting oncologic stage as well as overall survival in patients with RCC in the United States.

Methods: Overall, 199,675 RCC patients meeting eligibility criteria were drawn from the NCDB from 2004 to 2015. A multilevel ordered logistics regression was used to evaluate the relationship between distance to care facility and clinical stage at presentation, controlling for demographic variables. KaplanMeier analysis was used to evaluate the overall mortality by strata of distance to care facility (short [ $<12.5$ miles], intermediate [12.5-49.9 miles], and long [49.9-249.9 miles]). The relationship between distance to care and overall mortality was evaluated using a multilevel parametric survival model, controlling for clinical and treatment variables. We also evaluated the interaction between distance to care and median income status on overall mortality.

Results: In total, $57.4 \%, 31.8 \%$, and $10.8 \%$ of patients traveled short, intermediate, and long distances, respectively. Patients who travelled intermediate (OR: $0.85 ; 95 \%$ CI: $0.83,0.87$ ) and long (OR: $0.75 ; 95 \%$ CI: $0.72,0.78)$ distances had significantly lower odds of being diagnosed with stage IV RCC compared to those who travelled short distances. In the bivariate analysis, mortality was significantly higher among those who travelled a short distance (34.64\% vs. $29.14 \%$ intermediate vs. $29.79 \%$ long;

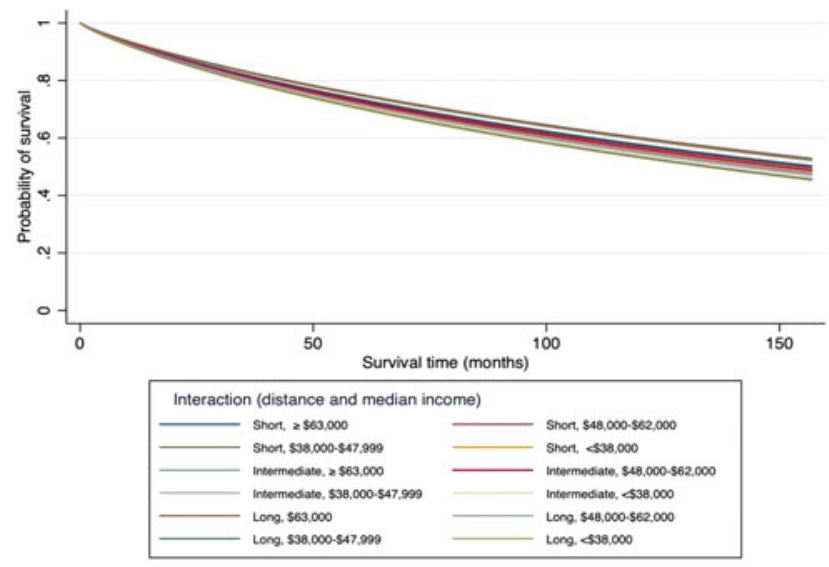

$\mathrm{p}<0.001)$. After controlling for all covariates in the multilevel model, long distance to care facility was significantly associated with overall mortality (HR: 1.04; 95\% CI: 1.02, 1.08). An inverse relationship between median income and mortality was seen for each stratum of distance travelled.

Conclusions: Our study demonstrates lower likelihood of stage IV presentation with increasing distance to treatment facility for RCC. We have also demonstrated an interaction between travel distance and income, namely that increasing distance to care and decreasing income negatively impact overall survival.

\section{MP02-06 Magnetic Resonance Imaging-Targeted 3D Transrectal Ultrasound Guided Fusion Biopsy of Prostate Cancer Suspected Patients: Results of 292 patients}

S Yilmaz, H Özdemir, E Gazel, E Kaya, S Yalcin, M Ezer, A Öner, M Yörubulut, L Tunc

Ankara Acibadem Hospital

Introduction \& Objective: Magnetic resonance imagingtargeted 3D transrectal ultrasound guided (MRI-3D TRUS) fusion biopsy is one of the newest diagnostic tool in patients with suspected prostate cancer. In this study, we aim to share the results of MRI-3D TRUS fusion biopsy patients.

Methods: The study included 292 patients who decided to undergo MRI-3D TRUS fusion biopsy because of suspected prostate cancer, From March 2016 to January 2019. Patients were scanned with 1.5 or 3.0 Tesla prostate multiparametric magnetic resonance imaging (mpMRI) prior to the procedure. The suspicious lesions obtained in the mpMRI were scored according to

\begin{tabular}{ll}
$\begin{array}{l}\text { Table 1: Lesion localizations and biopsy results of patients } \\
\text { who undergoing biopsy }\end{array}$ \\
\hline & $\begin{array}{l}\text { Quantity } \\
(\%)\end{array}$ \\
Lesions applied fusion biopsy & 332 \\
Peripheral zone & $236(69.8)$ \\
Central zone & $22(6.6)$ \\
Transitional zone & $72(21.6)$ \\
Anterior Fibromuscular Stroma & $2(0.6)$ \\
PIRADS 3 lesions applied fusion biopsy & 170 \\
Benign & $129(75.7)$ \\
Malignant & $41(12.3)$ \\
PIRADS 4 lesions applied fusion biopsy & 127 \\
Benign & $82(64.6)$ \\
Malignant & $45(35.4)$ \\
PIRADS 5 lesions applied fusion biopsy & 35 \\
Benign & $14(40)$ \\
Malignant & $21(60)$ \\
Patient with a positive biopsy pathology & 107 \\
TRUS-Bx positivity & 86 \\
MRI/TRUS Fusion Positivity & 74 \\
MRI/TRUS Fusion (+), TRUS Bx (-) & 21 \\
MRI/TRUS Fusion (-), TRUS Bx (+) & 33 \\
MRI/TRUS Fusion (+), TRUS Bx (+) & 53 \\
\hline & \\
\hline &
\end{tabular}


Table 2: Characterization of malignancy detected lesions by MRI / TRUS Fusion Biopsy

\begin{tabular}{lllll}
\hline & PI-RADS & PI-RADS & PI-RADS \\
ASAP & $\mathbf{3}$ & $\mathbf{4}$ & $\mathbf{5}$ & Total \\
LPIN & 9 & 3 & 1 & 13 \\
HPIN & 5 & - & - & 5 \\
Gleason 3+3 & 4 & 2 & 1 & 7 \\
Gleason 3+4 or 4+3 & 21 & 29 & 9 & 59 \\
Gleason 4+4 or 3+5 & - & 7 & 4 & 13 \\
Gleason 4+5 & - & 3 & 4 & 7 \\
Total & 41 & 45 & 2 & 3 \\
ASAP: Atypical Small & Acinar Proliferation, LPIN: Low Grade Prostatic \\
Intraepithelial Neoplasia, HPIN: High Grade Prostatic Intraepithelial Neoplasia
\end{tabular}

the PI-RADS score and scores of 3 and above were considered as suspicious for prostate cancer and the biopsy procedure was decided. MRI-3D TRUS fusion biopsy procedures were performed by a single radiologist (HÖ) who had at least 30 years of expertise in the field. Following the 12-core conventional biopsy with Tru-Cut ${ }^{\circledR} 18$-gauge needle routinely by transrectal route to the patients, three core fusion biopsies were performed from each suspicious lesion by transrectal route with GE Logiq ${ }^{\circledR}$ E9 device. Results: Between March 2016 and January 2019, 292 patients underwent MRI-3D TRUS fusion biopsy. The mean age of the patients was 61.9 (42-84). The mean tPSA value was $14.2 \mathrm{ng} / \mathrm{mL}$ (0.75-46.68) and the mean prostate size was $69.1 \mathrm{~g}(18-331)$. In total, fusion biopsy was performed on 332 mpMRI suspected lesions. The localization of the lesions and the degree of suspicious lesions are given in Table 1. Malignancy was reported in 107 (36.6\%) case. Pathological results are listed in Table 2.

Conclusions: The main issue of fusion biopsy is prostate cancer, which cannot be determined by conventional techniques but can be diagnosed by fusion biopsy. In our study, the number of patients with isolated fusion biopsy was 21 . This number constitutes $7.1 \%$ of the total number of patients and $19.6 \%$ of the patients with malignancy positivity. These ratios are of great importance in demonstrating the importance of MRI-3D TRUS fusion biopsy.

MP02-07 Transurethral resection in one piece (TURBO) for bladder tumor assisted by photodynamic diagnostic cystoscopy with 5-ALA

Y Yamauchi

Nagoya Daini Red Cross Hospital

Introduction \& Objective: Transurethral resection of bladder tumor (TURBT) is the gold standard strategy for removing bladder tumors. TURBT does not only remove all visible tumor but provides tumor specimens for pathological evaluation. We can get more accurate pathologic results by performing transurethral resection in one piece (TURBO) assisted by photodynamic diagnostic (PDD) cystoscopy with 5-ALA.

Methods: In this study, one surgeon have performed TURBO for bladder tumors on 25 patients at Nagoya Daini Red Cross Hospital between August 2016 and June 2019. PDD cystoscopy was performed at the beginning of TURBO to detect tumor surgical margin. Those with pathological findings other than urothelial carcinoma were excluded. Records of 23 patients were analyzed retrospectively. We have not performed intravesical instillation in the periods.
Results: The median age was 71 years old (48-84), with median follow up period of 18 months (4-28). There were 20 males and 3 females, 17 single and 6 multiple tumors. They were T2 in 1, T1 in 3 and Ta in 19. The tumor grade was high grade in 7 and low grade in 16. During the follow up period, recurrence occurred in two patient and progression in no patients. There were no complications including postoperative bleeding or bladder perforation. Pathologically, 18 patients of 23 have muscle layer. This rate of containing the bladder muscle tissue layer was comparable to previous reports of conventional TURBT. In addition, bottom of specimens by TURBO are less heat denaturation than TURBT. In $\mathrm{T} 1$ and Ta patients, surgical margins were all negative.

Conclusions: We considered TURBO as safety and utility procedure for visible bladder. And our study shows that PDD cystoscopy detects surgical margins accurately and help performing TURBO. But, this study had a short follow up span and only small experience, so that further studies are needed.

MP02-08 Risk factor and survival of femalepredominant upper urinary tract urothelial carcinoma in Taiwan

Y Wu, H Luo

Department of Urology, Kaohsiung Chang Gung Memorial Hospital, Kaohsiung, Taiwan

Introduction \& Objective: Upper urinary tract urothelial carcinoma (UTUC) is a relatively uncommon malignancy in Western countries, affecting more men than women. UTUC is more common in Taiwan and diagnosed more in women than in men. Previous studies regarding gender effect on cancer behavior of UTUC is controversial. Investigation in female predominance for UTUC in Taiwan may help improve UTUC management in Taiwan as well as understand gender effect on urothelial carcinoma.

Methods: Between January 2005 and December 2015, 828 patients with localized UTUC treated by radical nephroureterectomy were enrolled. Cox-regression analysis was performed to assess the independent roles of perioperative factors on local recurrence and systemic recurrence.

Results: Multivariate analysis revealed male gender is borderline associated with higher systemic recurrence $(p=0.096)$ and independently associated with higher bladder recurrence $(p=0.011)$, cancer-specific mortality $(p=0.014)$ even after taking smoking and other established prognostic factors into consideration. Bladder cancer history and multifocal disease are strong associated factors for bladder recurrence.

Conclusions: Compared with females, male UTUC patients in Taiwan are associated with more bladder recurrence and higher cancer-specific mortality and have a trend to have more systemic recurrence.

MP02-09 Operator Variant Outcomes in Prostate
Cancer Detection Rates: Doctor versus Nurse Operator MM Quraishi, M Sheikh

Department of Urology. Kent \& Canterbury Hospital. East Kent Hospitals University NHS Foundation Trust

Introduction \& Objective: Prostate Cancer is the commonest cancer in men in the UK, responsible for a major burden on national healthcare. A significant proportion of hospitals across the world still utilize transrectal ultrasound guided biopsy of the prostate (TRUS), with a view to train nurses to perform the 
procedure. Limited data exists on the outcomes comparing TRUS biopsy of the prostate between nurses and urologists. Our aim was therefore to assess if the background of the operated impacted on cancer detection rates.

Methods: A retrospective review was performed of the data that had been collected prospectively, documenting the age, digital rectal examination findings, the pre-biopsy PSA reading, the operator identity and the final histology on the specimen. Analysis was performed using a variety of relevant statistical tests using Stata Statistical Software: 2015, TX: StataCorp LP.

Results: A total of 392 consecutive patient's records were reviewed who underwent a TRUS Biopsy of the Prostate. A hundred and thirty-six $(35 \%)$ biopsies were performed by a urologist and $256(65 \%)$ were performed by a trained nurse. The nursing cohort had a cancer detection rate of $56 \%$, whilst the urologist picked-up malignancy in $57 \%$ of the biopsied cohort. No statistical difference was identified between the cancer detection rates in the two cohorts $(p=0.77)$. In order to ensure both cohorts had a similar group of patients, analysis were performed individually on age, PSA and DRE findings in both the nurse and urologist biopsied patients. Mean age was 65 and 66 years in the doctors and nurses' cohort of biopsied patient'. Median PSA was $6.5 \mathrm{ng} / \mathrm{ml}$ and $6.7 \mathrm{ng} / \mathrm{ml}$ for the doctors and nurses cohort of patients. No significant statistical difference was found in age or PSA, between the doctors and nurses' cohort of patients. Multivariate logistic regression analysis has demonstrated in our cohort that PSA (OR 1.07; $\mathrm{p}<0.001)$, age (OR 1.04; $\mathrm{p}=0.02]$ ) and $\mathrm{T}$ status on DRE findings (OR 1.62; $\mathrm{p}=0.04)$ are independent predictors of a diagnosis of prostate cancer. Multivariate linear regression analysis on our cohort has revealed that a urologist is significantly more likely $(\mathrm{p}=0.01)$ to pick up a higher grade of prostate cancer on TRUS Biopsy than compared to a nurse, when controlled for DRE findings, PSA level and patient age. However, this statistically significant difference to identify a higher-grade cancer appears to be absent in patients with PSA level less than 10 .

Conclusions: Our study reveals that trained nurses have the same cancer detection rates when performing TRUS biopsy as doctors. We are also able to appreciate higher-grade Gleason cancer pick up by doctors when confirmed predictors such as DRE findings, PSA and age are controlled.

MP02-10 The number of metabolic features as a significant prognostic factor in patients with metastatic renal cell carcinoma

O Kwon, H Lee, J Yu

Sanggye Paik Hospital/Inje University

Introduction \& Objective: The effect of metabolic characteristics on the prognosis of patients with metastatic renal cell carcinoma remains controversial. We investigated the associations between metabolic features of each individual and disease prognosis in patients with metastatic renal cell carcinoma.

Methods: Data of 1,584 patients with metastatic renal cell carcinoma from a multi-institutional database were retrospectively analyzed. The entire cohort was stratified into three subgroups according to how many patients had abnormal metabolic features (hypertension, diabetes mellitus, and low body mass index). The Kaplan-Meier and Cox proportional analyses were performed to investigate the associations between abnormal metabolic features and disease prognosis.

Results: There were 465 subjects without any metabolic features, 995 with one or two, and 124 with three. When the survival outcomes were compared according to the number of metabolic features, patients with higher numbers of metabolic features had significantly shorter overall and cancer-specific survival than those with fewer metabolic features (all $\mathrm{p}$ values $<0.05$ ). The multivariate Cox analysis showed that the number of metabolic features was an independent predictor for shorter cancer-specific and overall survival (all $\mathrm{p}$ values $<0.05$ ). When performing subgroup analysis according to the cellular type, significant results were only obtained among the clear cell subtype subgroup, with the association not being significant in the non-clear cell subtype cohort.

Conclusions: Patients with more metabolic features had significantly worse survival outcomes than those with fewer metabolic features. However, the association was only statistically 3 significant in patients with clear cell-type metastatic renal cell carcinoma.

\section{MP02-11 Impact of Gonadotropin-releasing Hormone Agonist on Cardiovascular Disease and Diabetes Mel- litus: A Nationwide Population-based Cohort Study}

W Bang, M Shim, C Oh, Y Lee, J Cho

Hallym Sacred Heart Hospital

Introduction \& Objective: Several studies have demonstrated the use of androgen deprivation therapy (ADT) increased the risk of cardiovascular disease and diabetes mellitus (DM) in patients with prostate cancer (Pca). We investigated to clarify whether use of gonadotropin-releasing hormone agonist (GnRHa) is associated with these diseases in the cohort based from the entire Korean population.

Methods: Using the National Insurance Service Database, we conducted an observational study of 579,377 men who sought treatment due to Pca between January 1, 2012 and December 31, 2016. After excluding patients with previously diagnosed of cardiovascular disease, DM and Chemotherapy, we extracted data of 2,053 patients who started $\mathrm{GnRHa}$ (GnRHa use) and 2,678 men who were newly diagnosed as Pca (Nonuse) from July 1, 2012 to December 31, 2012, with follow-up though December 31, 2016. Primary outcomes were development of cerebrovascular attack (CVA), ischemic heart disease (IHD), acute myocardial infarction (AMI), and DM. We also observed the association of ADT duration with the outcomes.

Results: Mean age of patients with GnRHa use and nonuse were $72.57 \pm 8.34$ and $66.77 \pm 9.00$, respectively $(\mathrm{p}<0.0001)$. GnRHa Users were older, reside more in the rural area, had lower socioeconomic status and had more comorbidities than Nonusers (all $\mathrm{p}<0.0500$ ). Although GnRHa Users demonstrated an increased incidence of only CVA $(p=0.0134)$ in the univariate analysis, GnRHa use was not associated with an increased risk of CVA, IHD, MI, and DM while age at diagnosis was strongly associated with all 4 diseases in the multivariate analysis. The cumulative duration of ADT was also not associated with the outcomes.

Conclusions: Our complete enumeration for Korean Pca population suggest that ADT was not associated with and increased risk of cardiovascular disease and DM. Differences in patient.

MP02-12 The utility and safety of diagnostic ureteroscopy in the diagnosis of upper tract urothelial carcinoma (UTUC): the experience of a high volume center

T Birks, N Pindoria, C Cumby, A Goyal, D Allen, R Kucheria, P Singh, L Ajayi, G Ellis

Royal Free Hospital, London

Introduction \& Objective: EAU guidelines recommend risk stratification of patients with newly diagnosed upper tract 


\begin{tabular}{|c|c|c|c|c|c|c|}
\hline \multirow[b]{3}{*}{ CT finding } & \multirow[b]{3}{*}{$\begin{array}{l}\text { Number of } \\
\text { diagnostic } \\
\text { cases }\end{array}$} & \multirow[b]{3}{*}{$\begin{array}{l}\text { Number of } \\
\text { UTuC } \\
\text { confirmed }\end{array}$} & \multicolumn{4}{|c|}{ Patients with confirmed UTUC } \\
\hline & & & \multicolumn{2}{|c|}{ Biopsy/cytology } & \multicolumn{2}{|c|}{ EAU risk stratification } \\
\hline & & & $\begin{array}{l}\text { High grade } \\
\text { UTUC }\end{array}$ & $\begin{array}{l}\text { Low grade } \\
\text { UTUC }\end{array}$ & $\begin{array}{l}\text { High risk } \\
\text { disease }\end{array}$ & $\begin{array}{l}\text { Low risk } \\
\text { disease }\end{array}$ \\
\hline Hydronephrosis & 14 & $5(36 \%)$ & 2 & 3 & 5 & 0 \\
\hline $\begin{array}{l}\text { Filling defect/ } \\
\text { lesion }\end{array}$ & 29 & $16(55 \%)$ & 11 & 5 & 11 & 5 \\
\hline $\begin{array}{l}\text { Urothelial } \\
\text { thickening }\end{array}$ & 9 & $2(22 \%)$ & 1 & 1 & $\begin{array}{c}2 \\
\text { (1 multifocal } \\
\text { low grade) }\end{array}$ & 0 \\
\hline Normal & 9 & 0 & 0 & 0 & 0 & 0 \\
\hline
\end{tabular}

urothelial carcinoma (UTUC) and offering kidney sparing surgery to patients with low risk disease. Ureteroscopy (URS) has a vital role in the diagnostic pathway, risk stratification and potential kidney sparing management of UTUC but must be balanced against the risk of increased bladder recurrence in those patients with confirmed UTUC. Centralization of kidney cancer services in North London, U.K. has brought a large volume of patients through our upper tract diagnostic pathway. Here we present our twelve-month cohort from 2018.

Methods: Patients coded as having had URS in 2018 at the Royal Free Hospital, London were included if the rationale for URS was suspicion of UTUC. Data was retrospectively collated from electronic case notes to include demographics and pre-operative imaging findings. Operative findings recorded included whether biopsies or ureteric washings were taken and whether the urothelium was visually suspicious. Cytology, biopsy and nephroureterectomy histology were all recorded.

Results: In 2018, 491 URS were performed, of which diagnostic URS for suspected UTUC was performed in 61 patients. 15 of these patients required a second diagnostic URS. Mean age was 67 years (range 33-88). Presenting complaints were hematuria $(\mathrm{n}=28)$, incidental finding $(\mathrm{n}=11)$, suspicious upper tract imaging whilst under surveillance for urothelial cancers of the bladder $(n=11)$, pain $(n=9)$ and urinary tract infections $(n=2)$. 23 cases of UTUC were diagnosed. Table 1 groups the outcomes by initial CT findings. Definitive management was nephroureterectomy in 15 cases $(65 \%)$ and $6(26 \%)$ are being treated endoscopically. 2 cases were either unfit or declined radical treatment.

Conclusions: Diagnosis of UTUC requires a multimodal approach to facilitate accurate diagnosis and risk stratification. Despite concerns about bladder seeding after diagnostic URS, its use to confirm a diagnosis of UTUC and for precise risk stratification is crucial. In our series 38 patients were reassured that there was no upper tract disease and there were no pT0 nephroureterectomies. A thorough diagnostic URS enabled us to identify 5 patients as having low risk disease who have gone on to have kidney-sparing endoscopic management. Following 61 diagnostic cases in 2018 we expect to accumulate high numbers of similar patients in years to come.

\section{MP02-13 Outcomes of imperative conservative treatment of upper tract urothelial cancer}

S Proietti, M Marchioni, B Eisner, ME Rodriguez Socarras, G Saitta, C D' Orta, P Bellinzoni, L Schips, F Gaboardi, G Giusti

Department of Urology, Ospedale San Raffaele-Turro, Milan, Italy

Introduction \& Objective: To date, few studies have focused on conservative treatment of upper tract urothelial cancer (UTUC) in patients with imperative indications. Herein we report our
Figure 1- UTUC recurrence-free survival in imperative indications for KKS

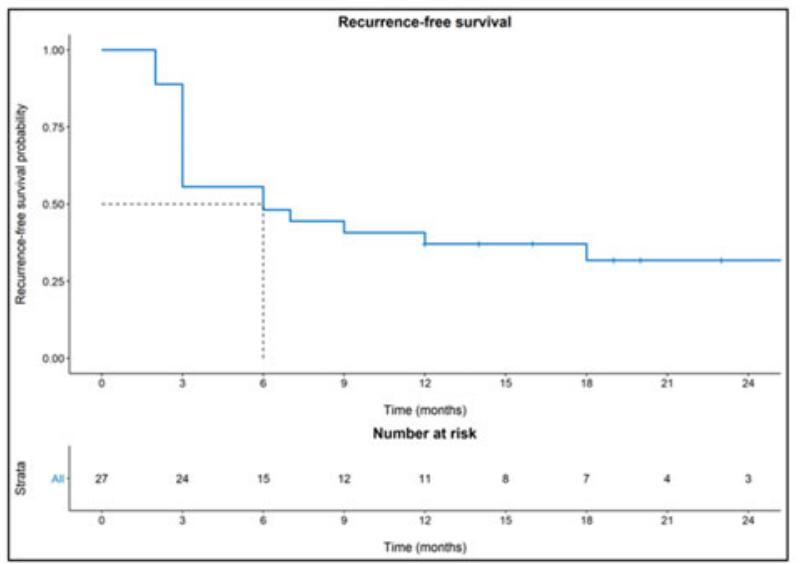

experience in this subset of patients affected by UTUC with the aim of highlighting the importance of kidney sparing surgery (KSS).

Methods: Retrospective data were collected for all patients who underwent KSS with imperative conditions at our institution from September 2013 to January 2019. Inclusion criteria were as follows: solitary kidney caused by absent or nonfunctioning contralateral kidney, bilateral UTUCs, severe chronic renal failure, both low and high risk UTUC disease. Peri/postoperative complications were reported according Clavien-Dindo classification system. Comorbidity was determined by using the ageadjusted Charlson comorbidity index (CCI). The primary endpoint of the study was to evaluate the overall survival (OS) and the secondary endpoints were the recurrence free-survival and Clavien-Dindo complications grade 1 or higher.

Results: A total of 29 patients, 22 males and 7 females, met inclusion criteria and were enrolled in the study. Age at diagnosis was 69 years (IQR 63-79); A total of 137 endoscopic procedures (135 FURS and 2 PCNL) were performed for a median of 4 per patient (IQR 3-6) during a median follow-up of 23 months (IQR 14-35). Twelve patients had high grade UTUC at the time of first diagnosis. The median recurrence-free survival was 6 (95\% CI: 3 - NA) months (Figure 1). The recurrence rate was higher in patients with high grade vs low grade tumors $(\mathrm{p}<0.05)$. A median of 2 endoscopic procedures per patient were positive for UTUC recurrence. The median overall mortality-free survival was not reached. During the follow-up 2 patients $(6.9 \%)$ died of extra-urological disease. The median CCI was 6 (IQR 4-8) and the median estimated 10-year survival was $2 \%$ (IQR $0-53 \%$ ). There was statistical difference in creatinine levels when comparing baseline to last follow-up $(\mathrm{p}=0.018)$. Clavien-Dindo complications are shown in Table 1.

Conclusions: KSS for UTUC in patients with imperative indication is acceptable in terms of local control and safety. allowing for renal function preservation. However, due to the high recurrence rate of UTUC, stringent endoscopic follow-up is mandatory.

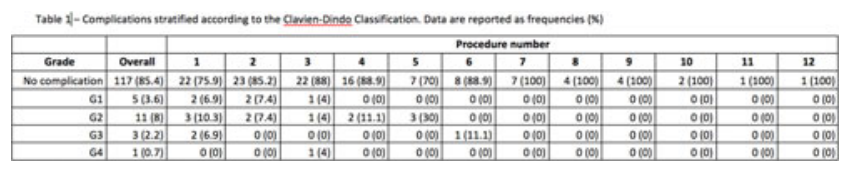


MP02-14 Iatrogenic Kaposi Sarcoma of the Bladder in the Post-Renal Transplant Patient

P Mistry, M Edelson, I Efthimiou, V Vasudevan, C Tabib, D Mikhail, Z Okeke

Arthur Smith Institute for Urology

Introduction \& Objective: Iatrogenic Kaposi sarcoma (KS), caused by Herpes Virus 8 (HHV8), is a common complication associated with immunosuppression following solid organ transplant. It commonly develops in mucocutaneous zones, however visceral involvement occurs in $40 \%$. Visceral $\mathrm{KS}$ usually occurs in the gastrointestinal tract or lung but may occur in any organ. Prevalence of KS after renal transplant is $0.45 \%$, with only 5 previous cases of bladder KS reported in the literature. We present a case of KS of the bladder after immunosuppression post-renal transplant successfully managed with transurethral resection and chemotherapy.

Methods: A 44-year-old female with history of IgA nephropathy received a live donor kidney transplantation. Maintenance immunosuppression was tacrolimus, mycophenolic acid and prednisone. She presented with severe abdominal pain after treatment for a multi-drug resistant E. coli urinary tract infection. CT scan revealed severe asymmetrical right-sided bladder wall thickening, pelvic and retroperitoneal lymphadenopathy (Fig. 1).

Results: Cystoscopy demonstrated diffuse bullous and erythematous changes over the right lateral wall and abutting the right ureteral orifice, extending to the floor of the bladder and posterior wall. Approximately $2.5 \mathrm{~cm}$ of tumor was resected. Pathology revealed HHV-8 positive KS, staining positive for SMA, CD-31 and CD-34 (Fig. 1). CT-guided retroperitoneal lymph node biopsy demonstrated a CD-34 and CD-31 positive spindle cell lesion. Tacrolimus and mycophenolic acid were stopped, followed by eight cycles of liposomal doxorubicin. Post-chemotherapy CT scan showed significantly decreased bladder wall thickening and interval improvement in retroperitoneal and pelvic lymph nodes. One-year follow-up was uneventful, and she remains on prednisone only for immunosuppression.

Conclusions: Iatrogenic Kaposi sarcoma commonly develops in the skin after solid organ transplant with rare instances of bladder involvement. Management ranges from immunosuppression withdrawal, radiation and chemotherapy. We demonstrated successful management of extensive KS with withdrawal of immunosuppression and liposomal doxorubicin.

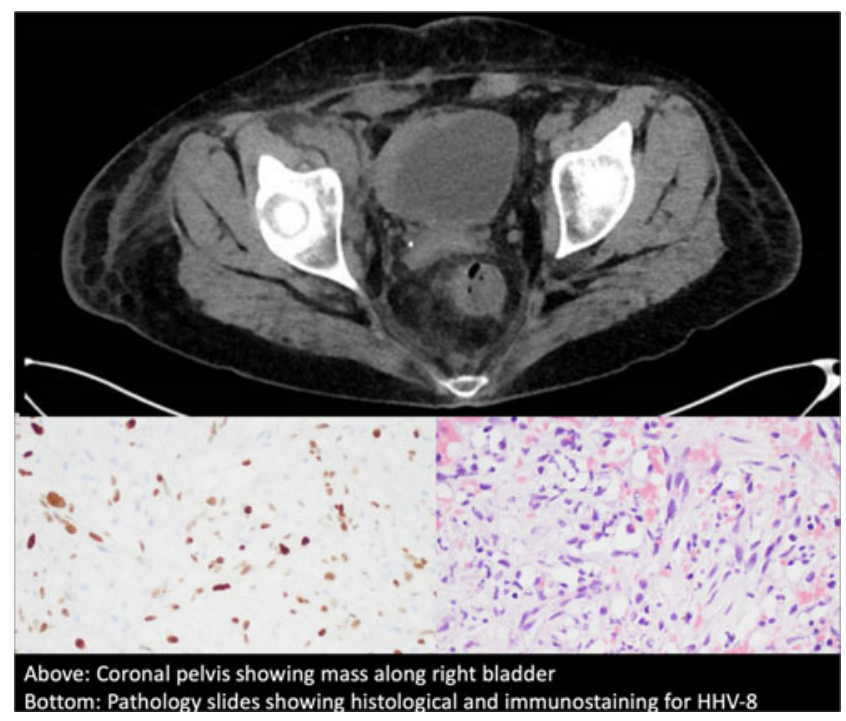

MP02-15 Comparison of Intra- and Post- Operative Outcomes of Straight Laparoscopic, Hand-Assisted Laparoscopic, and Robotic-Assisted Laparoscopic Partial Nephrectomies for Small Renal Masses at a Single Institution

\author{
M Apoj, L Wang, W Shaun, M Katz, D Wang \\ Boston Medical Center Department of Urology
}

Introduction \& Objective: Partial nephrectomy has become the standard operative procedure for the treatment of small renal masses. Due to a decrease in morbidity with similar long-term outcomes, the minimally invasive compared to open approach has become the preferred surgical technique. This surgery can be performed via Straight Laparoscopy (SL), Hand-Assisted laparoscopy (HA), or Robotic-Assisted laparoscopy (RA). We compared the intra- and post-operative outcomes of these techniques at our institution.

Methods: A single-institution retrospectively review of intraand post-operative outcomes (up to 30 days) in minimally invasive partial nephrectomy cases between 2012 and 2018 was performed. A linear regression model adjusting for age, gender, demographics, size of tumor on pathology, and RENAL Nephrometry score (RNS) was used to investigate differences in estimated blood loss (EBL), operative time (OT), length of stay (LOS), and warm ischemia time (WI). We used a chi square analysis to investigate differences in post-operative complications, demographics, and gender, and analysis of variances (ANOVA) to check for differences in positive margin rate, RNS, and age among the cohorts.

Results: A total of 164 cases were reviewed. 36, 65, and 63 surgeries were performed using the SL, HA, and RA technique, respectively. No differences in age, gender, intra-operative complications, and positive margin rates were found among the cohorts ( $p>0.05$ ). The HA technique had a higher RNS (mean of 1.38) compared to RA and SL (1.2 and 1.1, respectively) $(\mathrm{p}<0.05)$. There were no differences in EBL in the SL, HA, and RA techniques $(193,291$, and $259 \mathrm{~min}$, respectively) ( $\mathrm{p}>0.05)$. OT was longer for RA (229 min) compared to SL and HA (187 and $198 \mathrm{~min}$, respectively) $(\mathrm{p}<0.05)$. WI time for HA $(19.6 \mathrm{~min})$ was shorter than for SL and RA (21.3 and $25.3 \mathrm{~min}$, respectively) $(\mathrm{p}<0.05)$. LOS was longer for HA (3.1 days) when compared to SL and RA (2.4 and 2.3 days, respectively) $(\mathrm{p}<0.05)$. HA and SL had a higher overall incidence of Clavien-Dindo grade 3 and 4 post-operative complications compared to RA $(15.3 \%$ vs $13.8 \%$ vs $1.5 \%$, respectively) $(\mathrm{p}<0.05)$.

Conclusions: Our study demonstrates that at our institution, the RA approach is starting to overshadow traditional SL. The RA technique had a low incidence of post-operative complications, but the HA technique was used in more anatomically complex cases. The HA approach had a shorter WI and thus can be better suited for patients who need greater preservation of their renal function. More prospective studies are needed to validate our results.

\section{MP02-16 Angiomyolipoma and its follow up in a ter- tiary referral center}

B Vissamsetti, T Bhambra, S Biyani, A Khan

FRCS

Introduction \& Objective: Angiomyolipoma (AML) is a benign mesenchymal tumor, which can occur sporadically or as 
part of tuberous sclerosis complex. Most are diagnosed incidentally on imaging as they are asymptomatic in majority of the patients. Bleeding is the most severe complication and the incidence increases with the size of the tumor. There is no agreed protocol on follow up of these tumors. EAU guidelines suggest prophylactic treatment if they are $4-5 \mathrm{~cm}$ or more. To assess the follow up and management strategies of AML at our institute and their outcome.

Methods: Retrospective analysis of 100 patients who were diagnosed with AML on imaging.

Results: Out of the 100 patients diagnosed with AML M:F is $17: 83$ with a mean age of 51years. $62 \%$ of the AMLs are less than $10 \mathrm{~mm}$. Only $3 \%$ are greater than $30 \mathrm{~mm}$. None of these patients were admitted to the hospital with bleeding over the period of 5 years. $46 \%$ of these patients had further scans, of which around $50 \%$ are dedicated follow up scans. Majority of the follow up is by a single Ultrasound scan within 3-6 months. There is a $20 \%$ increase in size without any significant complications. 2 out of 100 patients under went prophylactic embolization.

Conclusions: Most of the AMLs diagnosed incidentally are small and asymptomatic. There is no standardized follow up at our center. They show slow growth with no significant complications. There no justification of routine follow-up of these benign asymptomatic tumors although a larger study would be beneficial to strengthen this justification.

\section{MP02-17 Comparison of partial nephrectomy and percutaneous radiofrequency ablation for small renal mass in the manner of preoperative aspects and di- mensions used for an anatomical (PADUA) classifi- cation}

W Kuo, C Yu, J Lin

Kaohsiung Veteran General Hospital

Introduction \& Objective: We present our experience with the clinical feature, efficacy and outcome of comparison of partial nephrectomy and radiofrequency ablation (RFA) for small renal tumors in the manner of Preoperative Aspects and Dimensions Used for an Anatomical (PADUA) Classification as a minimal invasive treatment in VGHKS.

Methods: A total of 78 patients in 15 years with small renal tumors were enrolled. We use Preoperative Aspects and Dimensions Used for an Anatomical (PADUA) Classification for renal tumor classification and compared PN (42 patients) and RFA (36 patients) with clinical outcome (local recurrence-free, metastases-free, and overall survival rates), RFA procedure (RFA needle, No. of treatment, Anesthesia, complication), renal function deterioration after procedure and pathology report. We also use clustered needle or switch mode control of RFA and D5W instillation for separating bowel loop.

Results: The median value of the PADUA score was 7.5 (IQR: $7 \hat{a} €$ "9), which is no significant difference between two groups. Of the 56 cT1 a patients, 32 underwent PN, 24 underwent RFA. In this cohort, local recurrence-free survival, metastases-free survival was similar among the two treatments $(p=0.53 / 0.46)$. Of the $22 \mathrm{cT} 1 \mathrm{~b}$ patients, 10 patients underwent $\mathrm{PN}$, and 12 patients were managed with RFA. In this cohort, local recurrence-free survival $(p=0.76)$ and metastases-free survival $(p=0.51)$ were similar between PN and RFA. In both the cT1a and cT1b groups, PN patients were significantly younger, with lower Charlson scores and had superior overall survival $(\mathrm{p}<0.001$ for all). In the RFA group, all patients have been biopsy before RFA and 22 been proved RCC. The number of RFA needle treatment were significantly correlated to PADUA score. 13 patients were under local anesthesia, the other were under IVGA by anesthesiologist. Only one patient presented mild pneumothorax after RFA without intervention treatment. Renal function deterioration after procedure did not reveal clinical significance. Tumor recurrence was recognized by $\mathrm{CT}$ scan in 3 patients during follow up, who repeated RFA treatment without recurrence.

Conclusions: For carefully selected patients, RFA represent a less invasive alternative associated with less morbidity and fewer complications and a promising treatment compared with partial nephrectomy. However, the long-term efficacy of these approaches remains to be established.

MP02-18 Transurethral laser ablation of low-grade bladder: a service evaluation, financial implications and importance of a comprehensive business case

\section{AJ Ridgway, K Charitopoulos \\ West Middlesex University Hospital}

Introduction \& Objective: Recurrent low-grade bladder TCC poses a challenge in a typically frail and elderly patient population. Trans-Urethral Laser Ablation (TULA) using a Diode laser is an alternative management method to TURBT that can be used under local anesthetic (LA) in the outpatient (OP) Setting. We present our experiences of TULA to date.

Methods: This retrospective study examined patient satisfaction, readmission rates and financial implications. 79 patients underwent TULA between March 2018 and February 2019 using the Leonardo Dual Mini Diode laser from Promed LTD under (LA) using Instillagel (2\% Lidocaine). Post treatment, 20 randomly selected patients were interviewed and gave satisfaction ratings regarding the procedure. We examined the 2018-19 NHS tariffs for procedures carried out in OP and theatres.

Results: 23 of the procedures were performed as OP and 56 in theatre as a day case procedure. One patient ended the procedure due to pain; one was booked for a TURBT due to tumor burden. There was one admission overnight due to bleeding and one overnight stay due to social reasons. Feedback from patients favored the OP setting. There was a financial gain for the Trust of $10 \%$ per patient from the OP compared to theatre tariff.

Conclusions: TULA has allowed patients to have an LA procedure instead of a TURBT. Benefits of this include: avoidance of GA which has implications given the frailty and co-morbidity often seen in these patients, better utilization of theatre and anesthetists' time and a reduction in overnight admissions. For procedures performed in $\mathrm{OP}$, the advantages include freeing up space on elective lists, time and resource efficiency and improved patient satisfaction. In the UK introduction of new initiatives has to be through a business case. In our department frequent changes of managers led to loss of continuity with the project therefore OP activity was not properly remunerated and job plans of urologists were difficult to adjust. Furthermore, the success of the project created more demand, with GPs from outside the catchment area referring in to the service. All these factors did not allow establishment of TULA in OP. TULA has transformed the patient experience and has been adopted as a technique by all Urologists working in our department. Nevertheless, our experience has provided valuable lessons in successfully introducing a novel modality; addressing the challenge of frequent managerial changes, careful job planning and agreed financial remuneration beforehand can all lead to effective implementation of innovative procedures. 
MP02-19 Is there a difference in long term renal functional outcome between benign and malignant renal tumors managed with robotic partial nephrectomy?

BD Dayal, KE Okhawere, AA Elbakry, A Beksac, K Meilika, J Sfakianos, R Mehrazin, R Abaza, DD Eun, A Bhandari, A Hemal, J Porter, KK Badani

Icahn School of Medicine at Mount Sinai

Introduction \& Objective: We sought to evaluate the effect of tumor pathology (benign vs. malignant) on renal functional outcome after robotic partial nephrectomy.

Methods: Data was obtained from our prospectively maintained multicenter database of patients who underwent robotic partial nephrectomy between January 2006 and January 2018. We included patients with at least 6 months post-operative estimated glomerular filtration rate (eGFR). Median follow up was 14 months (IQR: 7-26). Patients were divided according to the tumor pathology into benign $(n=145)$ and malignant $(n=729)$ groups. The associations between baseline demographic and clinical data, perioperative data, and renal functional outcome data were compared between the two groups using chi-square, Fisher's exact test and Kruskal Wallis test.

Results: The malignant group was more likely to have a younger median age at surgery ( 60 years vs. 63 years of age; $\mathrm{P}=0.026$ ), higher number of patients with diabetes mellitus $(22.5 \%$ vs $13.1 \% ; \mathrm{P}=0.01$ ), higher tumor complexity (R.E.N.A.L score 7 vs. $6 ; \mathrm{P}<0.001)$, and smaller tumor size $(2.4$ vs $2.8 \mathrm{~cm}$; $\mathrm{P}=0.007)$. There was no significant difference in the baseline eGFR (benign, 82.4 vs malignant, 79.6; $\mathrm{P}=0.721$ ) as well as last follow-up eGFR (benign, 75.7 vs malignant, 72.3; $\mathrm{P}=0.212$ ). The association between tumor pathology and the development of a post-operative De novo stage III CKD (benign, $13.79 \%$ vs malignant, $12.89 \% ; \mathrm{P}=0.769$ ) as well as delta eGFR (benign, -7.53 vs malignant, $-8.12 ; \mathrm{P}=0.212$ ) was also not significant.

Conclusions: Our findings suggest that renal function after robotic partial nephrectomy would be affected to the same extent regardless of tumor pathology. These results may indicate the need for a better strategy to identify small benign lesions that can

\begin{tabular}{|c|c|c|c|}
\hline & Benign & Malignant & P-value \\
\hline & $n(\%)$ & $n(\%)$ & \\
\hline $\mathbf{N}$ & $145(16.59)$ & $729(83.41)$ & \\
\hline Age median (IQR) & $63(55,69)$ & $60(51,67)$ & 0.026 \\
\hline \multicolumn{4}{|l|}{ Gender } \\
\hline Male & $64(44.14)$ & $442(60.63)$ & $<0.001$ \\
\hline Female & $81(55.86)$ & $287(39.37)$ & \\
\hline \multicolumn{4}{|l|}{ Race } \\
\hline White & $63(43.45)$ & $258(35.39)$ & 0.309 \\
\hline Black & $10(6.90)$ & $53(7.27)$ & \\
\hline Hispanics & $1(0.69)$ & $11(1.51)$ & \\
\hline Others & $0(0.00)$ & $10(1.37)$ & \\
\hline Unknown & $71(48.97)$ & $397(54.46)$ & \\
\hline Body Mass Index median (IQR) & $28.47(25.26,33.15)$ & $29.26(26.03,34.31)$ & 0.073 \\
\hline \multicolumn{4}{|l|}{ Hypertension } \\
\hline No & $81(55.86)$ & 387 (53.09) & 0.541 \\
\hline Yes & $64(44.14)$ & $342(46.91)$ & \\
\hline Diabetes & $19(13.10)$ & $164(22.50)$ & 0.011 \\
\hline \multicolumn{4}{|l|}{ Tumor Laterality } \\
\hline Right & $58(40.00)$ & $344(47.71)$ & 0.229 \\
\hline Left & $77(53.10)$ & $337(46.74)$ & \\
\hline Bilateral/multiple tumor & $10(6.90)$ & $40(5.55)$ & \\
\hline $\begin{array}{l}\text { Renal Score } \\
\text { Ischemia type* }\end{array}$ & \multicolumn{3}{|c|}{ Ischemia type* } \\
\hline Warm & $120(88.24)$ & $619(91.98)$ & 0.002 \\
\hline No Ischemia & $16(11.16)$ & $54(8.02)$ & \\
\hline $\begin{array}{l}\text { Ischemia time, mins median } \\
(I Q R)\end{array}$ & $14(9,18)$ & $15(11,20)$ & 0.058 \\
\hline Tumor size $(\mathrm{cm})$ median (IQR) & $2.8(2.0,4.0)$ & $2.4(1.8,3.5)$ & 0.007 \\
\hline Post-operative complication* & $24(16.67)$ & $83(11.43)$ & 0.434 \\
\hline
\end{tabular}

Table 2: Post-operative renal function between patients with malignant vs benign tumors after robotic partial nephrectomy

\begin{tabular}{llll}
\hline & Benign & Malignant & P-value \\
\hline $\begin{array}{l}\text { Baseline eGFR median } \\
\text { (IQR) }\end{array}$ & $82.44(66.79,98.79)$ & $79.62(64.92,96.92)$ & 0.721 \\
Last follow-up eGFR & $75.74(59.08,88.83)$ & $72.33(57.77,87.52)$ & 0.245 \\
De novo stage III CKD* & $20(13.79)$ & $94(12.89)$ & 0.769 \\
$\Delta$ DeGFR median (IQR) & $-7.53(-15.25,2.28)$ & $-8.12(-19.06,1.61)$ & 0.212 \\
\hline *De novo stage III CKD is estimated glomerular filtration rate $<60 \mathrm{ml} / \mathrm{min} / 1.73 \mathrm{~m}^{2}$
\end{tabular}

be managed conservatively with active surveillance to avoid unnecessary loss of renal function.

\section{MP02-20 Study of the Prostatic Urethral Lift in the Real-World Setting}

NJ Barber, G Eure, S Gange, PJ Walter, A Khan, C Chabert, T Mueller, P Cozzi, M Patel, S Freedman, P Chin, S Ochs, A Hirsh, M Trotter, D Grier

Frimley Park Hospital

Introduction \& Objective: Introduction: Prostatic Urethral Lift (PUL) has been shown to provide rapid, significant and durable improvement in symptoms and quality of life, including preservation of sexual function, for patients with BPH. The true test of clinical applicability, however, is how real-world experience compares to results from controlled studies. To determine whether PUL outcomes in the real-world support those from clinical studies, a retrospective study within a large, unconstrained, multicenter data set of PUL commercial cases was conducted.

Methods: Retrospective analysis of 1,413 consecutive PUL patients in North America and Australia was performed under IRB approval. Baseline demographics and symptom outcomes of real-world retrospective (RWR) subjects were compared to subjects in the randomized L.I.F.T. study. IPSS, QoL and Qmax were evaluated at $1,3,6,12 \& 24$-months post-procedure for all non-urinary retention subjects (Group A) and retention subjects (Group B). Within Group A, outcomes were further analyzed using paired t-tests and $95 \%$ mean confidence intervals for the following parameters: IPSS baseline $\geq 13$, age, prostate size, site of service, prostate cancer treatment, and diabetic status. Surgical interventions, adverse events and catheterization rates were summarized in detail.

Results: RWR subjects were older, had lower baseline IPSS and QoL and higher Qmax compared to those from the L.I.F.T. study. Following PUL, mean IPSS for Group A improved significantly from baseline by at least 8.1 points throughout follow up and $84 \%$ of subjects required no catheter. No significant differences were observed between Group A and B absolute symptom scores. Within Group A, subjects with an IPSS baseline $\geq 13$ behaved

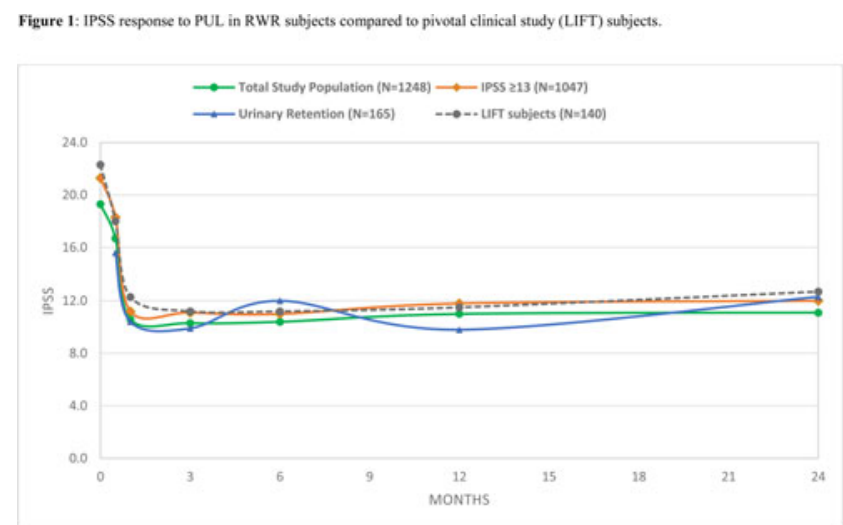


similarly to L.I.F.T (Figure 1 ). Age $(<50$ vs $\geq 50 \mathrm{yr}$ ), prostate volume ( $<30 \mathrm{cc} ; 30 \mathrm{cc}$ to $<80 \mathrm{cc} ; \geq 80 \mathrm{cc})$, site of service, prior cancer treatment and diabetic status did not significantly affect PUL effectiveness. Previous prostate cancer treatment did not elevate adverse events of high concern such as incontinence and infection. When completed in a clinic office, PUL resulted in less side effects and catheter placement compared to other sites of service.

Conclusions: This is the largest study of commercial PUL cases to assess effectiveness. PUL performs well in a real-world setting in terms of symptom relief, morbidity and patient experience for all examined patient cohorts, and confirms pivotal clinical study results.

MP02-21 Upper tract Ultrasound scan is an adequate screening modality for patients presenting with Visible Hematuria - retrospective cohort study of a prospective database

G Kanda Swamy, B Rai, R David, N Gill, P Bose

Swansea Bay University Health Board

Introduction \& Objective: To evaluate the clinical presentations and imaging modalities used to screen \& diagnose Upper Tract Urothelial Carcinoma (UTUC) to develop Hematuria screening models.

Methods: All cancer diagnosis in Wales are prospectively captured in Cancer Network Information System Cymru (CaNISC) database, after multidisciplinary team (MDT) input. We retrospectively reviewed all UTUC recorded in CaNISC between Jan 2013 - Dec 2017, registered through our MDT. We obtained data on demographics, initial presentation and imaging modalities used in diagnosis of UTUC.

Results: In the 5-year study period, 67 patients with UTUC were identified in the database. The male: female ratio was 1.68:1 and mean age was 72.9 years (range 49-90). Visible hematuria (VH) was the commonest presentation (55\%), followed by loin pain. Ultrasound (US) detection rate of UTUC was $94.5 \%$ in patients presenting with $\mathrm{VH}$, with hydronephrosis being the commonest abnormality. But abnormal US was noticed in only $38 \%$ of UTUC patients who did not present as VH.

Conclusions: UTUC diagnosis is a challenge. In patients with visible hematuria US is an adequate screening modality. Further studies including biomarker technology has to be evaluated for improving diagnosis of UTUC.

MP02-22 Outcome of Adjuvant Versus Salvage Androgen Deprivation Therapy Following Radical Prostatectomy for Prostate Cancer Patients with Adverse Pathologic Feature(s) or with Positive Regional Lymph Node(s) Metastasis

P Boonyapanichskul, V Woranisarakul, S Leewansangtong, C Nualyong, S Srinualnad, T Taweemonkongsap

Introduction \& Objective: Prostate cancer is currently the $4^{\text {th }}$ leading cancer for men in Thailand. The standard treatment for localized disease is radical prostatectomy. Despite of aggressive surgery, the risk for biochemical recurrence at 2 years after surgery is about $45 \%$. Androgen deprivation therapy (ADT) after radical prostatectomy has been recommended for the patients with regional lymph node metastasis or high-risk feature(s) in combination with radiotherapy. However, the duration and timing of ADT was not well studied. Some studies suggest early

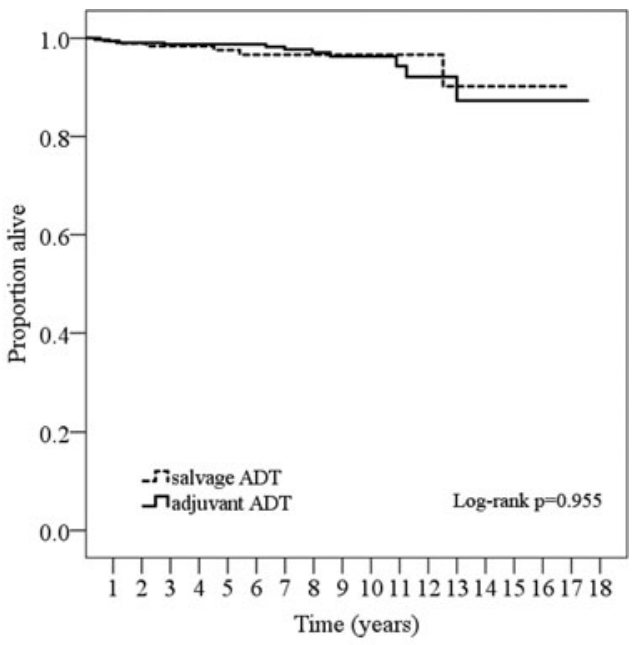

Figure 1: Kaplan-Mier curve for overall survival

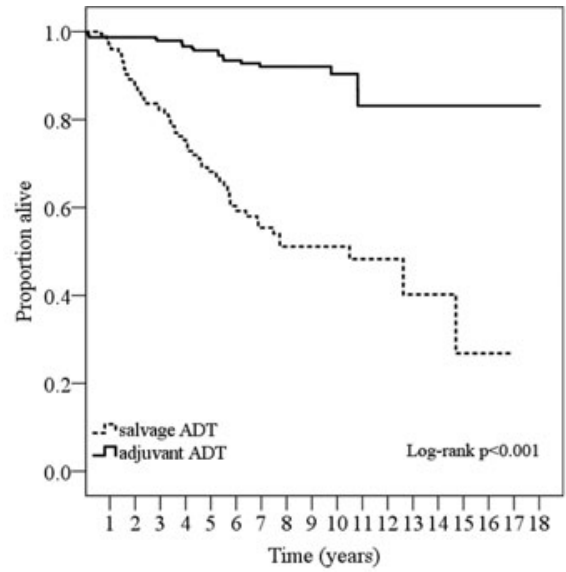

Figure 2: Kaplan-Meier curve for Biochemical-recurrence-free survival

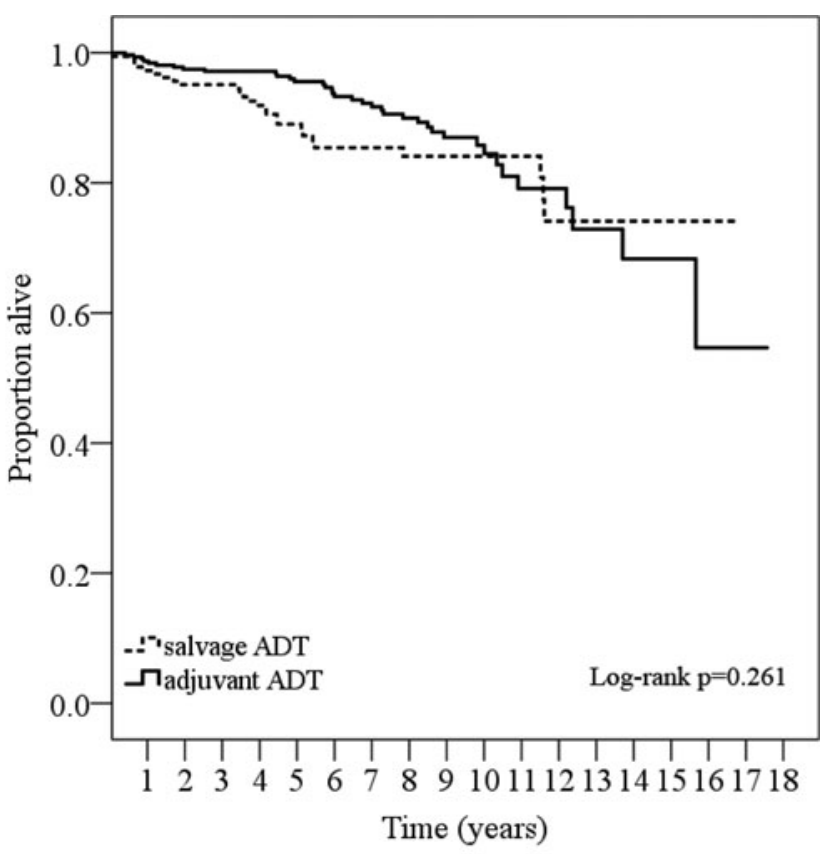

Figure 3: Kaplan-Meier curve for Metastasis-free survival 


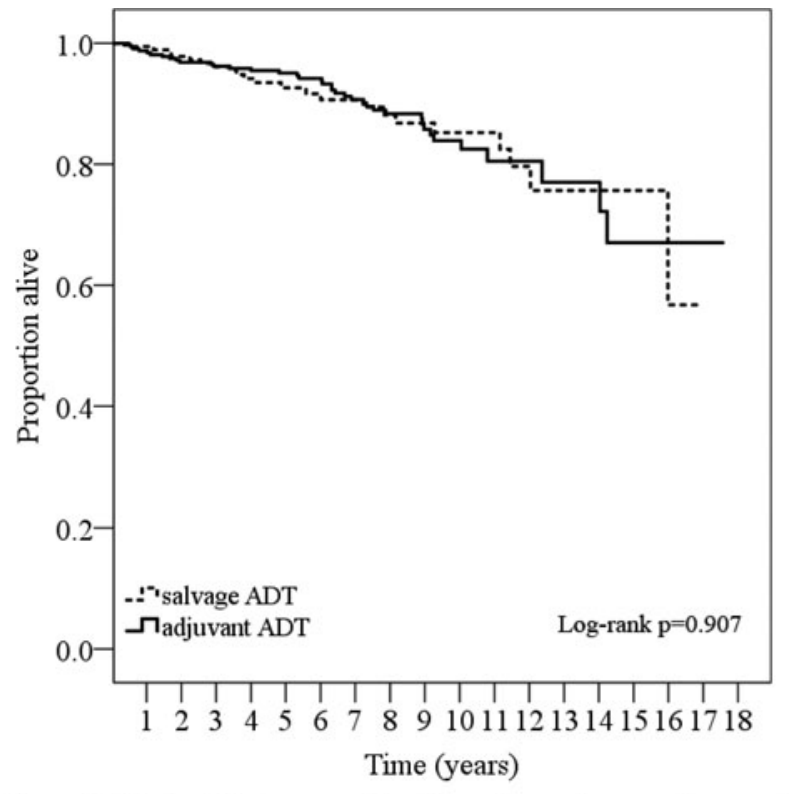

Figure 4: Kaplan-Meier curve for Castration-resistance-free survival

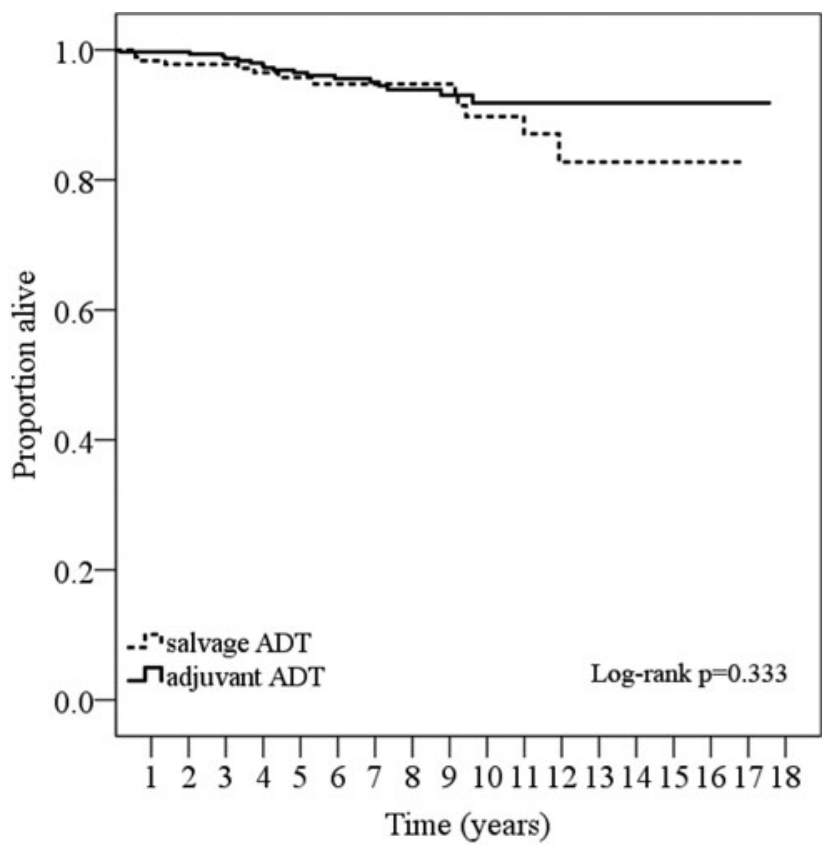

Figure 5: Kaplan-Mier curve for CVS-morbidity-free survival

ADT due to better survival benefit. Although an increased risk of developing cardiovascular events in early ADT group has also been reported. This study's aim is to evaluate and compare the overall survival of patients after radical prostatectomy with high risk feature(s) or regional lymph node metastasis who received adjuvant ADT and salvage ADT.

\begin{tabular}{|c|c|c|c|}
\hline & Adjuvant ADT $(\mathrm{n}=320)$ & Salvage ADT $(\mathrm{n}=184)$ & $p$-value \\
\hline Age (Mean, SD) & $68(8)$ & $67(7)$ & 0.737 \\
\hline ASA Classification & & & 0.716 \\
\hline 1 & $39(12.3 \%)$ & $17(9.2 \%)$ & \\
\hline 2 & $239(75.4 \%)$ & $145(78.8 \%)$ & \\
\hline 3 & $39(12.3 \%)$ & $22(12.0 \%)$ & \\
\hline Median BMI (Min, Max) & $24.3(11.3,37.2)$ & $24.2(17.5,36.7)$ & 0.709 \\
\hline $\begin{array}{l}\text { Median PSA ng/mL } \\
\text { (Min, Max) }\end{array}$ & $19.0(1,250)$ & $15.9(0,258)$ & 0.226 \\
\hline Gleason Grade group & & & 0.017 \\
\hline 1 & $15(4.8 \%)$ & $8(4.7 \%)$ & \\
\hline 2 & $68(21.7 \%)$ & $55(32.0 \%)$ & \\
\hline 3 & $50(16.0 \%)$ & $35(20.3 \%)$ & \\
\hline 4 & $68(21.7 \%)$ & $35(20.3 \%)$ & \\
\hline 5 & $112(35.8 \%)$ & $39(22.7 \%)$ & \\
\hline Type of operation * & & & 0.178 \\
\hline RRP & $77(24.1 \%)$ & $45(24.5 \%)$ & \\
\hline EERPE & $41(12.8 \%)$ & $26(14.1 \%)$ & \\
\hline LRP & $39(12.2 \%)$ & $34(18.5 \%)$ & \\
\hline RALRP & $163(50.9 \%)$ & $79(42.9 \%)$ & \\
\hline \multicolumn{4}{|l|}{ Pathological staging } \\
\hline -T staging & & & $<0.001$ \\
\hline $\mathrm{pT} 2$ & $45(14.1 \%)$ & $55(30.2 \%)$ & \\
\hline pT3 & $266(83.1 \%)$ & $126(69.2 \%)$ & \\
\hline pT4 & $1(0.3 \%)$ & $1(0.5 \%)$ & \\
\hline \multicolumn{4}{|l|}{ - Lymph node } \\
\hline $\mathrm{pN} 0$ & $273(88.1 \%)$ & $165(94.3 \%)$ & 0.026 \\
\hline $\mathrm{pNI}$ & $37(11.9 \%)$ & $10(5.7 \%)$ & \\
\hline \multicolumn{4}{|l|}{ - Margin } \\
\hline $\begin{array}{l}\text { Positive } \\
\end{array}$ & $280(87.5 \%)$ & $144(78.3 \%)$ & 0.006 \\
\hline Negative & $40(12.5 \%)$ & $40(21.7 \%)$ & \\
\hline \multicolumn{4}{|l|}{ Seminal vesicle invasion } \\
\hline Yes & $150(46.9 \%)$ & $39(21.2 \%)$ & $<0.001$ \\
\hline No & $170(53.1 \%)$ & $145(78.8 \%)$ & \\
\hline \multicolumn{4}{|l|}{ - Extraprostatic extension } \\
\hline Yes & $248(77.5 \%)$ & $121(66.1 \%)$ & 0.005 \\
\hline No & $72(22.5 \%)$ & $62(33.9 \%)$ & \\
\hline \multicolumn{4}{|l|}{-Detectable PSA after RP } \\
\hline Yes & $37(11.6 \%)$ & $33(18.1 \%)$ & 0.044 \\
\hline No & $281(88.4 \%)$ & $149(81.9 \%)$ & \\
\hline - Combination with RT & & & 0.962 \\
\hline Yes & $93(29.1 \%)$ & $135(29.2 \%)$ & \\
\hline No & $227(70.9 \%)$ & $327(70.8 \%)$ & \\
\hline
\end{tabular}

Methods: The authors have conducted a retrospective cohort study by reviewing the chart of eligible patients in Siriraj hospital who underwent radical prostatectomy from January 2000 to December 2016. We included patients with adverse pathological feature(s) or regional lymph node metastasis. The patient was classified into 2 groups, the adjuvant $\mathrm{ADT}$ group is the patient who received $\mathrm{ADT}$ within 3 months after surgery and the salvage ADT group is the patient who had delayed the ADT for more than 3 months after surgery or start ADT after presence of PSA recurrence.

Results: There was 504 eligible patients. At 15 years, the overall survival for adjuvant ADT and salvage ADT group was $87.3 \%$ and $90.2 \%(p=0.955)$. The recurrence free survival was $83.2 \%$ and $26.8 \%(\mathrm{p}<0.001)$. The metastasis-free survival at 15 years was $68.3 \%$ and $74.1 \%(\mathrm{p}=0.261)$. The CVS-morbidity-free survival was $91.9 \%$ and $82.8 \%$ respectively $(\mathrm{p}=0.333)$.

Conclusions: No difference in overall survival between adjuvant or salvage ADT group was demonstrated at 15 years after therapy. Adjuvant ADT group had significant better recurrence-free survival at 15 years. There were no statistical differences in metastasis and CVS-morbidity-free survival between the groups. 


\section{MODERATED POSTER SESSION 03: BPH/LUTS: ELECTROSURGERY, LASERS \& OTHER TECHNOLOGY (I)}

\begin{abstract}
MP03-01 Surgeons musculoskeletal problems following endourologic prostatectomy: an ergonomic survey study of the endourology society members
\end{abstract}

M Omar, M Sultan, E El Sherif, M Abdallah, M Monga

Menoufia University

Introduction \& Objective: Benign prostatic hyperplasia (BPH) is the commonest urologic disorders affecting older men, necessitating medical or surgical intervention. Limited data exist, regarding the affection of endourologist musculoskeletal system following these surgeries because of the required difficult posture, prolonged procedures, repetitive movements and the settings of an adjustable VDT (visual display terminal) workstation. The aim of our work was to survey the prevalence and possible causes of musculoskeletal disorders among endourologists performing TURP or laser prostatectomy (HoLEP or ThuLEP). [i] Reyes DA, Tang B, Cuschieri A. Minimal access surgery (MAS)-related surgeon morbidity syndromes. Surg Endosc. 2006;20(1):1-13.

Methods: An e-mail inviting all members of the Endourological Society for survey participation was sent to 3000 members worldwide. The questionnaire included different demographic and practice characteristics with concern regarding being performing either TURP or laser prostatectomy and the incidence and type of musculoskeletal incidents following these surgeries. All responses were collected by a commercially available

TABLE 1: Different characteristics of complaining and non-complaining urologists

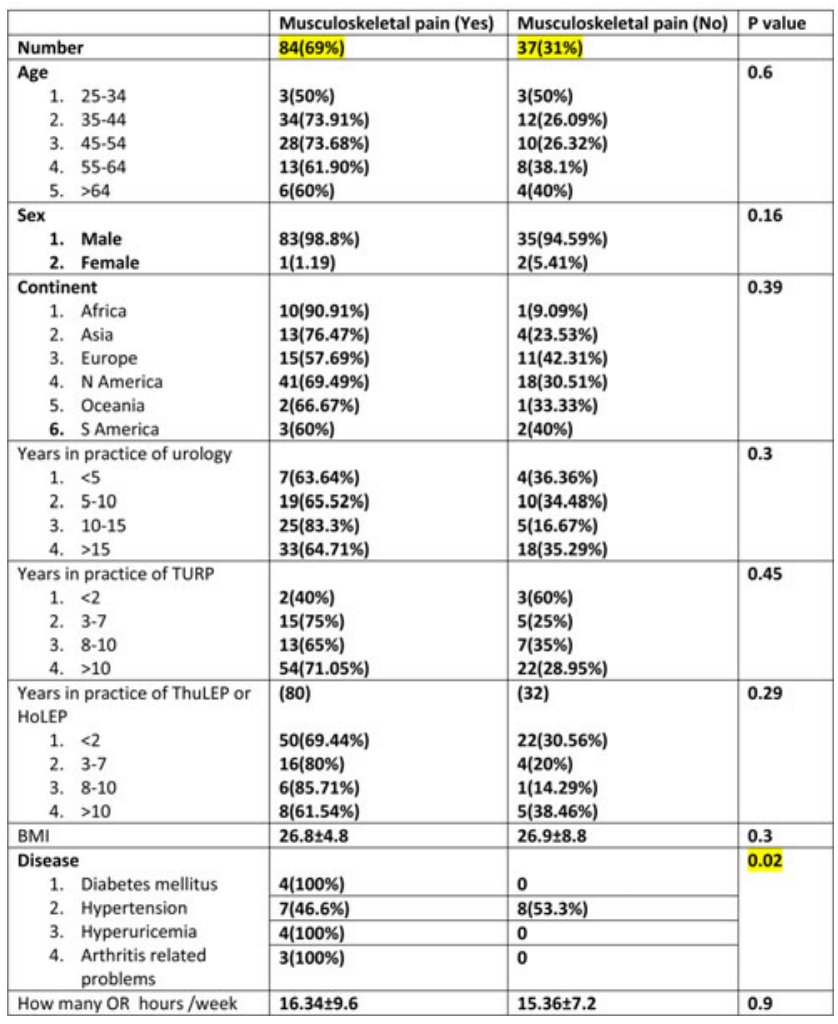

TABLE 2: Operative related differences between complaining and non-complaining urologists

\begin{tabular}{|c|c|c|c|}
\hline & Musculoskeletal pain (Yes) & Musculoskeletal pain (No) & P value \\
\hline Number & $84(69 \%)$ & $37(31 \%)$ & \\
\hline TURP surgeries per week & $2(1-3)$ & $2(1-3)$ & 0.6 \\
\hline ThuLEP or HoLEP per week & $1(0-2)$ & $0(0-1)$ & 0.2 \\
\hline Average prostate size (gram) & $75.8 \pm 33.2$ & $59.32 \pm 19.62$ & 0.004 \\
\hline Hour of exercise per week & $3.9 \pm 3.3$ & $4.6 \pm 4.1$ & 0.5 \\
\hline $\begin{array}{l}\text { Operating position } \\
\text { 1. Sitting } \\
\text { 2. Standing }\end{array}$ & $\begin{array}{l}67(72.8 \%) \\
17(58.62 \%)\end{array}$ & $\begin{array}{l}25(27.17 \%) \\
12(41.28 \%)\end{array}$ & 0.1 \\
\hline $\begin{array}{l}\text { Corrective eye wear } \\
\text { 1. Contact lens } \\
\text { 2. Eye glasses } \\
\text { 3. None }\end{array}$ & $\begin{array}{l}11(64.71 \%) \\
42(67.74 \%) \\
31(73.81 \%)\end{array}$ & $\begin{array}{l}6(35.29 \%) \\
20(32.26 \%) \\
11(26.19 \%)\end{array}$ & 0.7 \\
\hline $\begin{array}{l}\text { Dominant hand } \\
\text { 1. Left } \\
\text { 2. Right }\end{array}$ & $\begin{array}{l}8(80 \%) \\
76(68.47 \%)\end{array}$ & $\begin{array}{l}2(20 \%) \\
35(31.53 \%)\end{array}$ & 0.4 \\
\hline $\begin{array}{l}\text { Type of working element } \\
\text { 1. Active } \\
\text { 2. Passive } \\
\text { 3. Combined } \\
\text { 4. Iglesias } \\
\end{array}$ & $\begin{array}{l}38(69.09 \%) \\
17(80.95 \%) \\
6(66.67 \%) \\
23(63.89 \%)\end{array}$ & $\begin{array}{l}17(30.91 \%) \\
4(19.05 \%) \\
3(33.33 \%) \\
13(36.115) \\
\end{array}$ & 0.57 \\
\hline $\begin{array}{l}\text { Brand of working element } \\
\text { 1. Storz } \\
\text { 2. Wolf } \\
\text { 3. Olympus } \\
\text { 4. Others }\end{array}$ & $\begin{array}{l}51(68392 \%) \\
8(80 \%) \\
35(70 \%) \\
1(25 \%) \\
\end{array}$ & $\begin{array}{l}23(31.08 \%) \\
2(20 \%) \\
15(30 \%) \\
3(75 \%)\end{array}$ & 0.1 \\
\hline $\begin{array}{l}\text { Brand of the camera } \\
\text { 1. Storz } \\
\text { 2. Wolf } \\
\text { 3. Olympus } \\
\text { 4. Others }\end{array}$ & $\begin{array}{l}46(66.67 \%) \\
4(80 \%) \\
28(62.22 \%) \\
13(86.67 \%)\end{array}$ & $\begin{array}{l}23(33.33 \% \\
1(20 \%) \\
17(37.78 \%) \\
2(13.33 \%)\end{array}$ & 0.14 \\
\hline $\begin{array}{l}\text { Type of the monitor } \\
\text { 1. Flat screen } \\
\text { 2. Non-flat screen }\end{array}$ & $\begin{array}{l}82(69.49 \%) \\
2(66.67 \%)\end{array}$ & $\begin{array}{l}36(30.51 \%) \\
1(33.33 \%)\end{array}$ & 0.9 \\
\hline $\begin{array}{l}\text { Previous surgery in the } \\
\text { dominant hand }\end{array}$ & $11(68.75 \%)$ & $5(31.25 \%)$ & 0.9 \\
\hline
\end{tabular}

Internet-based survey host (www.surveymonkey.com) over a period of 6 weeks.

Results: Of 121 complete response, 84(69\%) endourologist complained of a musculoskeletal disorder following TURP or laser prostatectomy. The most frequent complaint was for neck $64 \%$, followed by back $57 \%$, shoulder $48 \%$, hand $40 \%$ and elbow $18 \%$. The average prostate volume turned to be the most compelling predictor for musculoskeletal disorder occurrence with 76 gram average prostate volume operated by the complaining group compared to 59-gram in the other group. Those with more severe symptoms were more linked to mean prostate size of 80 gram (60146) and constantly complained of shoulder, neck or back trouble compared to less severely complaining group.

Conclusions: High prevalence of musculoskeletal complaints among urologists performing endourologic prostatectomy and proportionally related to size of the prostate. Most of surgeons denied attributing their symptoms to specific brand of working element. Integration of ergonomic specialist inside the operation room for watching and correcting surgeon position during endourologic procedure may reduce the endourologist's exposure to these occupational hazards.

MP03-02 High Incidence of Urinary Tract Infections after Greenlight Laser Vaporization of Prostate: A Risk Factor Analysis of 665 Patients

J Motzer, J Roth, A Widmer, H Seifert, C Wetterauer

Department of Urology, University Hospital Basel 
Introduction \& Objective: Although Greenlight laser vaporization is a recognized alternative to transurethral resection in treating benign prostatic obstruction, there is limited data at present on the incidence and determinants of postoperative urinary tract infections. We assessed patients subjected to Greenlight laser vaporization, evaluating incidence and potential determinants of postoperative urinary tract infections.

Methods: Consecutive patients undergoing Greenlight laser vaporization between April 2010 and August 2018 were candidates for this retrospective cohort study. The primary outcome measure was microbiologically confirmed postoperative urinary tract infection. We fitted uni- and multivariable Cox models to identify potential risk factors.

Results: Among the 665 included patients, 20\% developed postoperative urinary tract infections. The overall incidence rate per 100 patient-days was 0.65 (95\% confidence interval [CI]: 0.55-0.77). Risk factors for postoperative urinary tract infections were end-stage renal failure (adjusted hazard ratio $[\mathrm{aHR}]=$ 14.10, 95\% CI: 2.08-64.58; $\mathrm{p}=0.001)$ and presence of at least one of the following factors in the 3 months preceding GLV: (i) placement of urinary catheter, (ii) bacteriuria, (iii) urinary tract infection, or (iv) antimicrobial treatment (composite aHR $=1.99$, 95\% CI: $1.22-3.24 ; \mathrm{p}<0.001$ ). There was no apparent association between choice or duration of antimicrobial prophylaxis and incident urinary tract infections.

Conclusions: Our analysis revealed a high incidence of urinary tract infections after Greenlight vaporization of the prostate and served to identify certain preoperative risk factors. Neither the choice of antimicrobial regimen nor its duration affected the incidence of urinary tract infections. Prolonged antimicrobials proved to be disproportionately high, warranting further scrutiny in randomized controlled trials.

MP03-03 Retrospective Analysis of Urethral Stenosis Following Transurethral Prostate Resection: Preventing Effect of the Using Low Molecular Weight Heparin on Development of Urethral Stenosis

M Ilgi, A Albayrak, C Yesildal, A Yavuzsan,

N Turkmen, S Kirecci

Introduction \& Objective: In this study, the protective effect of low molecular weight heparin (LMWH) treatment on urethral stricture after transurethral resection of the prostate (TURP) was investigated.

Methods: 551 patients who underwent TUR-P operation between May 2007-April 2013 were included in the study. 167 patients were receiving anticoagulant therapy because of increased thrombosis due to various diseases. Anticoagulant therapies of the patients were changed with LMWH injection on the peroperative days. Patients were separated into two groups; TURP $(n=384)$ group and LMWH+TURP $(n=167)$ group. The groups were compared in terms of age, location and length of the stricture, duration of resection, duration of postoperative catheterization, number of internal urethrotomies, recurrence time and necessity of urethroplasty.

Results: Mean ages of the TURP and LMWH+TURP groups were 55.4 and 58.6, respectively. Urethral stricture rates for TURP and LMWH+TURP groups were (24/384, 6.2\%) and (9/ $167,5.3 \%)$ and time to first stricture time for TURP and LMWH+TURP groups 8.7 (1-26) and 12.6 (1-33) months, respectively (Table 1). Recurrence rates for TURP and LMWH+TURP groups were $(10 / 24,42 \%)$ and $(3 / 9,33 \%)$, and mean time to recurrence for TURP and LMWH+TURP groups were 6.2 (1-18) and 13 (3-26) months, respectively (Table 2).

\begin{tabular}{|l|l|l|l|}
\hline & TUR-P & LMWH+TUR-P & $\mathrm{p}$ \\
\hline Patients & 384 & 167 & $\mathrm{p}>0.05$ \\
\hline Age (year) & $55,4 \pm 17,2$ & $58,6 \pm 19,4$ & $\mathrm{p}>0.05$ \\
\hline Pre-Op Qmax (mL/sn) & $6,1 \pm 5,3$ & $7,9 \pm 4,8$ & $\mathrm{P}<0.05$ \\
\hline Operation Time & $55,2(25-98)$ & $59,4(20-120)$ & $\mathrm{p}>0.05$ \\
\hline Urethral Catheterization time & $1,9 \pm 1,3(1-6)$ & $2,0 \pm 1(1-8)$ & $\mathrm{p}>0.05$ \\
\hline
\end{tabular}

Table 1: Comparison of preoperative and operative data between groups

\begin{tabular}{|l|l|l|l|}
\hline & TUR-P & LMWH+TUR-P & $\mathrm{p}$ \\
\hline $\begin{array}{l}\text { Postoperative stenosis } \\
\text { development }(\mathrm{n} / \%)\end{array}$ & $24(\% 6,1)$ & $9(\% 5,3)$ & $\mathrm{p}=0.053$ \\
\hline $\begin{array}{l}\text { Time to first stricture } \\
\text { (months) }\end{array}$ & $8,7(1-26)$ & $12,6(1-33)$ & $\mathrm{p}<0.05$ \\
\hline $\begin{array}{l}\text { First development of } \\
\text { recurrence stenosis (n) }\end{array}$ & $10 / 24(\% 42)$ & $3 / 9(\% 33)$ & $\mathrm{p}<0.05$ \\
\hline $\begin{array}{l}\text { Time to first } \\
\text { development of } \\
\text { recurrence stenosis }\end{array}$ & $6,2(1-18)$ & $13(3-26)$ & $\mathrm{p}<0.05$ \\
\hline $\begin{array}{l}\text { Second development of } \\
\text { recurrence stenosis (n) }\end{array}$ & $7 / 10(\% 70)$ & $2 / 3(\% 66)$ & $\mathrm{p}=0.055$ \\
\hline $\begin{array}{l}\text { Time to second } \\
\text { development of } \\
\text { recurrence stenosis }\end{array}$ & $5,7(1-12)$ & $9(5-14)$ & $\mathrm{p}<0.05$ \\
\hline $\begin{array}{l}\text { Third development of } \\
\text { recurrence stenosis }(\mathrm{n})\end{array}$ & $4 / 7(\% 57)$ & 0 & $\mathrm{p}<0.05$ \\
\hline $\begin{array}{l}\text { Urethroplasty } \\
\text { requirement }\end{array}$ & 3 & 0 & $\mathrm{p}<0.05$ \\
\hline
\end{tabular}

Table 2: Recurrence rates of urethral stricture between groups.

No patient needed more than 3 internal urethrotomy and urethroplasty in the LMWH+TURP group. However, in the TUR-P group 3 patients had severe recurrent urethral stricture and urethroplasty operation was performed.

Conclusions: Considering the rates of recurrence and urethroplasty, the incidence of severe urethral stricture development is less in those receiving anticoagulant therapy with LMWH. Heparins anticoagulant effect can make surgery complicated, however fibrinolytic activity is helpful for long-term urethral stricture complication.

MP03-04 Energy impact on voiding symptoms after thulium enucleation of prostate: A large multi institutional analysis

S Nazzani, G Motta, O Blezien, F Molinari, P Acquati, R Stubinski, S Casellato, C Yameogo, S Maruccia, L Carmignani

Introduction \& Objective: Impact of energy delivery during enucleation of prostate on storage symptoms is still unknown. We aimed to test how energy delivery influences postoperative storage symptoms in a large cohort of benign prostatic obstruction patients who underwent Thulium enucleation of prostate (ThuVep).

Methods: Within our multi-institutional database with prospective collected data we selected patients treated with ThuVep (Quanta System Cyber TM 200 W). Preoperative, 7 days and 30 days International Prostatic Symptoms Score (IPSS) questionnaires were administered. Storage symptoms were considered as frequency, urgency and nocturia according to IPSS questionnaire. Energy administered during the procedure was recorded and its ratio with trans rectal ultrasound prostate size was calculated. Finally, patients were stratified according to median energy delivered per gram of prostate. The statistical significance of differences in medians and proportions was tested with the Kruskal-Wallis and chi-square tests. 
Results: Between 2014 and 2018, we identified 228 patients treated with ThuVep. Median prostate volume was 70 [Interquartile Range: (IQR) 50-104 grams] and median energy delivered per gram was 3207 Joule (IQR: 1904 - 4956 Joule). Median operative time was 86 minutes. Low energy $(<3207$ Joule) patients were less frequently in antiplatelet (10.5\% vs $26 \% ; \mathrm{p}=0.01)$ and anticoagulant therapy $(2.6 \%$ vs $7.9 \%$; $\mathrm{p}=0.01)$. Preoperative urgency $(1.5 \mathrm{vs} 1.5 ; \mathrm{p}=1)$, frequency $(1.7$ vs $1.9 ; \mathrm{p}=0.7)$ and nocturia $(2.3$ vs $2.5 ; \mathrm{p}=0.3)$ were similar in the two cohorts. Similarly, no differences were found in term of preoperative indwelling catheter. Hemoglobin drop ( -0.9 vs -0.7 grams $/ \mathrm{dL} ; \mathrm{p}=0.03$ ) and catheterization time were lower (37 vs $30 ; p=0.05)$ in high energy patients. 7 and 30 days urgency and frequency were comparable in the two cohorts. Finally, 7 days nocturia was higher ( 1.8 vs $2.3 ; \mathrm{p}=0.1)$ in high energy patients, albeit in a non-significant fashion.

Conclusions: Based on our results no statistically significant difference exist in term of overall storage symptoms according to energy per gram delivered. Conversely, 7 days nocturia appears to be more frequent in high-energy patients. However, no statistically significant difference was found. Finally, high-energy patients had lower catheterization time and hemoglobin drop.

\section{MP03-05 The New 4D and Standard Prostatic Ure- thral Lifting (UroLift) Techniques: Day Case, Effec- tive, No Sexual Dysfunction}

M Ravindraanandan, W Mahmalji, Y Phan

Hereford County Hospital

Introduction \& Objective: Benign prostatic hypertrophy (BPH) associated with lower urinary tract symptoms (LUTS) and sexual dysfunction is common amongst the aging male population. Surgery for LUTS is one of the most common in the UK, with a rise in prevalence increasing financial pressure and burden on the healthcare system. UroLift is a minimally invasive surgery used to treat BPH with advantages compared to conventional techniques.

Methods: The UroLift procedure was performed within a single institution. Parameters measured included IPSS score, post-void residuals, QMAX, sexual dysfunction, theatre time and length of stay. Data was analyzed using a two-sample equal-variance t-test.

Results: We performed UroLift on 37 patients, average age 69 years (range 53-90). Table 1 summarizes primary outcomes achieved following the UroLift procedure, with significant improvements in IPSS $(\mathrm{p}<0.02)$, post-void residuals $(\mathrm{p}<0.04)$ and QMAX ( $\mathrm{p}<0.04)$. No patients suffered any sexual dysfunction side effects with quality of life scores improving by 1.8 $(\mathrm{p}<0.04)$. Hospital stay and theatre time were significantly reduced. Average length of stay was just 10.6 (6-18) hours and average theatre time just 18.7 (12-30) minutes.

Conclusions: UroLift is substantially shorter than other surgery for LUTS. Significant benefits are felt for both the patients, who are discharged home much sooner, and also the hospital, who can

Table 1 - primary outcomes from UroLift

\begin{tabular}{|c|c|c|c|c|c|}
\hline Characteristics & $\begin{array}{c}\text { Baseline } \\
\text { Mean }\end{array}$ & $\begin{array}{c}4 \text { months } \\
\text { post-op } \\
\text { mean }\end{array}$ & $\begin{array}{c}\text { Mean } \\
\text { Difference }\end{array}$ & P-value & $\mathrm{n}$ \\
\hline $\begin{array}{l}\text { International } \\
\text { Prostate Symptom } \\
\text { Score (IPSS) }\end{array}$ & 21.8 & 13.8 & 8 & 0.02 & 37 \\
\hline Quality of life & 4.6 & 28 & 1.8 & 0.04 & 37 \\
\hline $\begin{array}{l}\text { Post-void residual } \\
\text { volume (ml) }\end{array}$ & 229 & 138 & 91 & 0.04 & 37 \\
\hline QMAX (ml/s) & 7.7 & 12.1 & 4.4 & 0.04 & 37 \\
\hline
\end{tabular}

perform a higher number of procedures on patients in a similar time period. Patients also do not require an inpatient bed so should not be cancelled on the day of theatre.

\section{MP03-06 Vaporesection of the prostate with Tm: YAG laser: The Oyster technique}

P Kallidonis, D Kotsiris, C Adamou, P Ntasiotis, K Pagonis, I Kyriazis, E Liatsikos, A Al Aown

Armed Forces Hospital Southern Region

Introduction \& Objective: To evaluate clinical and functional parameters of an anatomical technique on Thulium (Tm:YAG) laser transurethral prostatectomy.

Methods: The study included 122 men diagnosed of benign prostatic Obstruction (BPO) with IPSS score $>15$, Q-max $<15 \mathrm{ml} / \mathrm{sec}$, prostate volume $>60 \mathrm{~mL}$ in absence of prostate cancer. The operation was performed using a 200W Tm:YAG laser (Revolix 200, Lisa Laser Products GmbH, Germany) and included partial enucleation of the prostatic lobes followed by vaporesection (Oyster technique). After six weeks, follow up with IPSS, Qmax, and PVR took place.

Results: The average duration of the operation was $53.62 \mathrm{~min}$ (range: 20-85) and the bladder irrigation was stopped on the first postoperative day to all patients. The urethral catheter was removed after a median of 1.55 days (range 1-5). The median duration of hospitalization was 3.03 (range: 1-7) days. Moreover, the median hemoglobin drop was found to be $0.96 \mathrm{~g} / \mathrm{dL}$ (range: 0.4 to 3.1 ). Six weeks postoperatively, there was significant improvement in all clinical and functional outcomes (Table 1). Conclusions: The Oyster technique seems a safe approach for Tm:YAG laser prostatectomy with significant improvement in the clinical and functional outcome parameters and with negligible hemoglobin drop. Randomized controlled trials would further strengthen the above results and confirm the efficacy of this technique.

Patients' characteristics and operative outcomes $(\mathrm{n}=122)$

Age (years old)

$69.049(54-84)$

Operative time ( $\mathrm{min})$

$53.62(20-85)$

Hemoglobin drop $(\mathrm{g} / \mathrm{dL})$

$0.96(-0.4$ to 3.1$)$

IPSS improvement

$-14.44(-6$ to -33$)$

Q-max improvement ( $\mathrm{mL} / \mathrm{sec})$

$7.11(-1.7$ to 19.4$)$

Post void residual $(\mathrm{mL})$

$73.52(0-250)$

Catheterization period (days)

Length of hospital stay (days) 
MP03-07 Two-Year Outcomes after Aquablation Compared to TURP: Efficacy and Ejaculatory Improvements Sustained

PJ Gilling, T Bach, C Roehrborn

Asklepios Hospital Harburg

Introduction \& Objective: To compare two-year safety and efficacy outcomes after Aquablation or transurethral resection of the prostate (TURP) for the treatment of lower urinary tract symptoms (LUTS) related to benign prostate hyperplasia (BPH). Methods: 181 patients with $\mathrm{BPH}$ were assigned at random (2:1 ratio) to either Aquablation or TURP. Patients and follow-up assessors were blinded to treatment. Assessments included International Prostate Symptom Score (IPSS), Male Sexual Health Questionnaire (MSHQ), International Index of Erectile Function (IIEF) and uroflow. The focus of analysis was two-year outcomes. Results: At two years, IPSS scores improved by 14.7 points in the Aquablation group and 14.9 points in TURP $(\mathrm{p}=.8304,95 \% \mathrm{CI}$ for difference -2.1 to 2.6 points). Two-year improvements in maximum flow rate (Qmax) were large in both groups at 11.2 and $8.6 \mathrm{cc} / \mathrm{sec}$ for Aquablation and TURP, respectively $(\mathrm{p}=.1880,95 \%$ CI for difference -1.3 to 6.4). Sexual function as assessed by MSHQ was stable in the Aquablation group and decreased slightly in the TURP group. At two years, PSA was reduced significantly in both groups by 0.7 and 1.2 points, respectively; the reduction was similar across groups $(\mathrm{p}=.1816)$. Surgical retreatment rates after 12 months for Aquablation were $1.7 \%$ and $0 \%$ for TURP. Over two years, surgical BPH retreatment rates were $4.3 \%$ and $1.5 \%(\mathrm{p}=.4219)$, respectively. Conclusions: Two-year efficacy outcomes after TURP and Aquablation were similar and the rate of surgical retreatment was low and similar to TURP.

MP03-08 Functional outcomes post Holmium Laser Enucleation of the Prostate (HoLEP) for patients with huge prostates $(>150 \mathrm{cc})$

A Oluwole-Ojo, M Hadjipavlou, E Di Benedetto, A Sujenthiran, R Catterwell, B Namdarian, O Elhage, R Popert, B Challacombe

NHS

Introduction \& Objective: Holmium laser enucleation of the prostate (HoLEP) is recommended for patients with benign prostatic hyperplasia with large prostates. The aim of the study was to assess the functional outcomes in men undergoing HoLEP with huge prostates $(>150 \mathrm{cc})$ and compare these to men with smaller prostates $(<150 \mathrm{cc})$.

Methods: We retrospectively evaluated all patients undergoing HoLEP in a UK single tertiary hospital between August 2017 and December 2018. Data on pre-operative and post-operative International Prostate Symptom Score (IPSS), peak urinary flow rate (Qmax), post void residual (PVR) and prostate specific antigen (PSA) was evaluated for patients with huge prostates and compared to those with smaller prostates.

Results: In total, HoLEP was performed on 196 patients with a preoperatively measured prostate size of $20-450 \mathrm{cc}$. Of these patients, 62 had a huge prostate $(>150 \mathrm{cc})$ with a mean volume (measured by MP-MRI) of 193cc (range: 150-450cc). The mean total operative time was 109.8 mins (range: $30-225$ mins) with a mean morcellated prostate volume of $126.5 \mathrm{~g}$ (range: $60-270 \mathrm{~g}$ ). With regard to functional outcomes, patients with huge prostates undergoing HoLEP had significant improvements in IPSS (from 21.4 \pm 9.3 to $7.9 \pm 6.0$ ),

\begin{tabular}{|c|c|c|c|}
\hline & Huge (>150mL) & Smaller $(<150 \mathrm{~mL})$ & $P$ value \\
\hline IPSS improvement & 9.14 & 11.76 & 0.3870 \\
\hline PVR improvement (mL) & 24.32 & 37.56 & 0.8617 \\
\hline PSA Improvement (ng/mL) & 7.83 & 6.94 & 0.5573 \\
\hline Qmax improvement (mL/s) & 29.34 & 12.91 & 0.0235 \\
\hline Incontinence (\%) & 6.4 & 6.7 & 0.9448 \\
\hline
\end{tabular}

Qmax (from $10.0 \pm 4.3 \mathrm{~mL} / \mathrm{s}$ to $34.9 \pm 17.5 \mathrm{~mL} / \mathrm{s}$ ), PVR (from $144 \pm 117 \mathrm{~mL}$ to $76 \pm 60 \mathrm{~mL}$ ) and PSA (from $13.2 \pm 11.1 \mathrm{ng} / \mathrm{mL}$ to $4.9 \pm 14.5 \mathrm{ng} / \mathrm{mL})$. Four patients $(6.5 \%)$ with huge prostates had significant stress urinary incontinence following surgery at 3-6 months follow-up. These were all managed non-operatively with intensive physiotherapy. All patients were catheter-free following surgery. Comparison of IPSS, PVR, PSA and continence rates between patients with huge and smaller prostates showed no statistically significant differences. Qmax showed a greater improvement in the huge prostate group, which was significant (Table 1).

Conclusions: Our study shows that HoLEP is a safe and effective operation for treatment of BPH in patients with huge prostates $(>150 \mathrm{cc})$ with excellent functional outcomes and a low complication rate. This should make open prostatectomy redundant in appropriately trained HoLEP centers.

\section{MP03-09 Prostatic Urethral Lift in Subjects with Acute Urinary Retention (PULSAR)}

M Rochester, NJ Barber, T Page, R Kavia, $\mathrm{N}$ Thiruchelvam, O Kayes

Norfolk and Norwich University Hospital

Introduction \& Objective: Patients with acute urinary retention (AUR) due to BPH who fail medical therapy are often limited to long-term catheterization or invasive surgery options. The objective of this study was to assess safety and feasibility of Prostatic Urethral Lift (PUL) in patients with AUR secondary to BPH.

Methods: Men age $\geq 50$ diagnosed with BPH with at least one failed TWOC while on alpha blocker and prostates $\leq 100 \mathrm{cc}$ were enrolled. PUL implants were placed to retract the prostatic lobes. Void trials were performed 3 days ( \pm 1 day) post-PUL. International Prostate Symptom Score (IPSS), quality of life (QoL), BPH Impact Index (BPHII), peak flow rate (Qmax), post void residual (PVR) and the Sexual Health Inventory for Men (SHIM) were assessed through 12 months and compared to the L.I.F.T. study. Patient satisfaction and return to normal were also assessed.

Results: 52 men (age $71.4 \pm 7.9$, prostates $55.2 \pm 22.8 \mathrm{cc}$ ) were enrolled across 6U.K. sites. They were catheterized for 132.4 mean days before PUL, and $40 \%$ subjects had LUTS $\geq 6$ years. An average of $4.8 \pm 1.4$ implants were placed per subject. Symptoms and QoL were significantly better than L.I.F.T. at 6 and 12 months (Table 1). 22 (42\%) subjects had a related adverse event and most events were mild-moderate, resolving by 2 weeks. 4 subjects had a serious adverse event (1 urinary tract infection, 3 hematuria) that resolved by 2 weeks. $58 \%$ subjects had a successful first TWOC at 3 days. $67 \%$ subjects became catheter-free by 1 month and $79 \%$ by 3 months. $67 \%$ subjects stopped alpha blockers pre-PUL and all remained off medication. Mean baseline SHIM (16.0 \pm 8.7$)$ was stable or improved (19.5 \pm 7.5$)$ at 3 months. $87.5 \%$ subjects returned to normal in 8.5 average days. 3 subjects had surgery (2 HoLEP, 1 TURP) and 2 had repeat PUL. 96.2\% subjects were "much or very much better" at 6 months and $88.5 \%$ would recommend PUL at 6 months. 


\begin{tabular}{|c|c|c|c|c|}
\hline $\begin{array}{r}\text { Measurement } \\
\text { (mean } \pm S D, \text { range) }\end{array}$ & $\begin{array}{l}\text { PULSAR } 6 \text { Months } \\
\qquad(n=26)\end{array}$ & $\begin{array}{l}\text { L.I.F.T. } 6 \text { Months } \\
(n=133)\end{array}$ & $\begin{array}{c}\text { PULSAR 12 } \\
\text { Months (n=6) }\end{array}$ & $\begin{array}{c}\text { L.I.F.T. } 12 \\
\text { Months ( } n=123)\end{array}$ \\
\hline \multirow[t]{2}{*}{ IPSS } & $5.8 \pm 4.1,[0-14]$ & $11.2 \pm 7.3,[0-31]$ & $5.5 \pm 6.2,[2-18]$ & $11.5 \pm 7.3,[0-23]$ \\
\hline & \multicolumn{2}{|c|}{$p=0.0003$} & \multicolumn{2}{|c|}{$p=0.0495$} \\
\hline \multirow[t]{2}{*}{ Qol } & $1.2 \pm 1.4,[0-5]$ & $2.2 \pm 1.6,[0-6]$ & $0.7 \pm 0.8,[0-2]$ & $2.3 \pm 1.6,[0-6]$ \\
\hline & \multicolumn{2}{|c|}{$p=0.0047$} & \multicolumn{2}{|c|}{$p=0.0202$} \\
\hline \multirow[t]{2}{*}{ BPH II } & $0.8 \pm 2.0,[0-8]$ & $2.7 \pm 2.8,[0-12]$ & $0.3 \pm 0.8,[0-2]$ & $2.8 \pm 2.9,[0-13]$ \\
\hline & \multicolumn{2}{|c|}{$p=0.0017$} & \multicolumn{2}{|c|}{$p=0.0355$} \\
\hline
\end{tabular}

Conclusions: PUL can safely and quickly restore voiding and significantly improve LUTS and QoL in BPH patients suffering from AUR.

\section{MP03-10 Does targeted bladder neck cautery prevent bleeding events? Learning after $700+$ Aquablation procedures using different hemostasis methods in prostates $20 \mathrm{ml}$ to $280 \mathrm{ml}$ in size}

D Elterman, T Bach, E Rijo Mora, V Misrai, P Anderson, K Zorn, N Bhojani, B Chughtai

Asklepios Hospital Harburg

Introduction \& Objective: Prostate tissue resection for patients with LUTS remains the most effective means to provide the largest improvement in symptomatic relief. Many studies have evaluated the bleeding complication profile post-operatively for TURP and report a range up to $7 \%$ of patients requiring a blood transfusion. Aquablation is a new surgical alternative for resective BPH surgery, but optimization of hemostasis has not been fully vetted.

Methods: The current commercial AquaBeam robot that performs Aquablation Therapy was first used in 2014. Since then numerous clinical studies have been conducted in various countries; Australia, Canada, Germany, India, Lebanon, New Zealand, United Kingdom, and the United States. All of the clinical data will be pooled with the early commercial procedures from France, Germany, and Spain. The hypothesis is to determine if athermal methods are as effective in preventing postoperative major bleeding events (defined as either a transfusion or take back to OR for fulguration) as the use of bladder neck cautery across various prostate volume sizes.

Results: Over 700 Aquablation procedures are included in this analysis. The categorical prostate volume sizes used for analysis were $<50 \mathrm{cc}, 50 \mathrm{cc}-100 \mathrm{cc}$, and $>100 \mathrm{cc}$. The major bleeding events in each prostate volume category was $5 \%, 8 \%$, and $10 \%$, respectively. The major bleeding events in the athermal group were $5 \%, 9 \%$, and $11 \%$, respectively across prostate size. Lastly, the major bleeding events in the bladder neck cautery group were $5 \%, 5 \%$, and $0 \%$, respectively.

Conclusions: While the athermal subgroup had comparable major bleeding rates as TURP, even in very large prostates $(>100 \mathrm{cc})$, a trend suggests that the optimal hemostasis technique may be bladder neck spot cautery, which resulted in a lower bleeding event rate, especially in prostates $>50 \mathrm{cc}$.

\section{MP03-11 Early clinical outcomes of transurethral enucleation with bipolar compared with transurethral resection in saline for Benign Prostatic Hyperplasia treatment}

Y Endo, J Akatsuka, K Takahashi, N Hodotsuka, H Mikami, K Obayashi, S Nomura, M Yanagi, H Takeda, T Hayashi, Y Toyama, T Hamasaki, H Shimizu, Y Kondo

Nippon Medical School
Introduction \& Objective: Transurethral resection of the prostate (TURP) has been the standard surgical treatment for benign prostatic hyperplasia (BPH). Nowadays, various minimally invasive alternatives including transurethral resection in saline of prostate (TURisP), Transurethral enucleation with bipolar (TUEB), holmium laser enucleation and green laser vaporization was developed. However, there are a few reports that compared these new methods directly. The present study provides early clinical results of TUEB from a retrospective review of a single-institution experience comparing the efficacy and safety of TUEB and TURisP for managing BPH.

Methods: A retrospective review of a single-institution experience of 86 consecutive TUEB or TURis between 2016 and 2017 at our related hospital was performed aiming to compare the efficacy and safety of TUEB and TURisP for managing BPH (64 patients in TUEB group and 24 in TURP group). Both groups were well matched for age, preoperative prostate volume, IPSS, QoL and uroflowmetry findings. Peri-operative data included operating time, resected tissue weight, changes in hemoglobin, and early complications. Postoperative outcomes included International Prostate Symptom Score (IPSS), Over Active Bladder Symptom Score (OABSS), Quality of Life score (QoL), uroflowmetry findings, change in serum prostate-specific antigen level and all late complications at 3 months after these operations in the two groups.

Results: Operating time on TUEB group was significantly longer than on TURis group. The average retrieved tissue weight in the TUEB group was larger than that in the TURis group (29.3 vs $19.7 \mathrm{~g} ; \mathrm{P}=0.048$ ). The change in hemoglobin at postoperative day 1 in two groups was comparable. (1.15 vs $1.07 \mathrm{~g} / \mathrm{dL}$; $\mathrm{P}=0.728$ ). Early complication was equally low with each procedure. IPSS at postoperative 3 months in the TUEB group was significantly less than in the TURis group (5.76 vs 10.44; $\mathrm{P}<0.027)$. Patients in the two arms had comparable improvements in postoperative QoL, maximum flow rate and average flow rate post-void residual urine volume. The rates of complications were equally low with each procedure.

Conclusions: Although TUEB needs much operating time compared with TURisP, we could retrieve more tissue result in better IPSS.

MP03-12 Thulium laser enucleation (thULEP) versus Holmium laser enucleation of the prostate: a two institution trial to compare intra and early postoperative outcomes

G Bozzini, U Besana, J Roche, P Broggini, G Pini, C Buizza, M Sighinolfi, B Rocco

\section{ASST Valle Olona - Busto Arsizio VA}

Introduction \& Objective: To compare clinical intra and early postoperative outcomes between Thulium laser enucleation of the prostate (ThuLEP) and Holmium laser enucleation of the prostate (HoLEP) to treat benign prostatic hyperplasia (BPH).

Methods: This prospective study enrolled consecutive patients with BPH to ThuLEP $(n=115)$ or HoLEP $(n=121)$. All patients were evaluated preoperatively with regards to blood loss, catheterization time, irrigation volume, hospital stay and operative time. At 3 months after surgery they were also evaluated by International Prostate Symptom Score (IPSS), maximum flow rate (Qmax), and postvoid residual urine volume (PVR).

Results: The patients in each study arm each showed no significant difference in preoperative parameters. Compared with 
HoLEP, ThuLEP had same operative time $(63.69 \pm 41.44$ vs $71.66 \pm 38.70$ minutes, $\mathrm{P}=.245$ ) but resulted in less hemoglobin decrease $(0.45$ vs $2.77 \mathrm{~g} / \mathrm{dL}, \mathrm{P}=.005)$. ThuLEP and HoLEP had same catheterization time (1.9 vs 2.0 days, $\mathrm{P}=.45)$, irrigation volume (29.4 vs $33.2 \mathrm{~L}, \mathrm{P}=.762)$, and hospital stay (2.2 vs 2.8 days, $\mathrm{P}=.216$ ). During the 3 months of follow-up, the procedures did not demonstrate a significant difference in Qmax, IPSS, PVR, and QOLS.

Conclusions: ThuLEP and HoLEP both relieve lower urinary tract symptoms equally, with high efficacy and safety. ThuLEP was statistically superior to HoLEP in blood loss. Catheterization time, irrigation volume, hospital stay and in operation time showed no differences. However, procedures did not differ significantly in Qmax, IPSS, PVR, and QOLS through 3 months of follow-up.

MP03-13 Safety and efficacy of Holmium laser enucleation of the prostate (HoLEP) in patients with previous transperineal biopsy: outcomes from a multicenter case-control study

C Bell, SL Moore, O Obi-Njoku, A Gill, SF Hughes, A Saleemi, G Ellis, F Khan, IS Shergill

North Wales Clinical Research Centre

Introduction \& Objective: We investigated the surgical feasibility, safety and effectiveness of 50W (low power) Holmium Laser Enucleation of the Prostate (HoLEP) in patients who have undergone previous template biopsy of the prostate (TPB).

Methods: Data encompassing pre-operative baseline characteristics, intra-operative measures and post-operative outcomes was collected for 109 patients undergoing HoLEP across two UK centers. Patients were stratified into two groups; group $1(n=24)$ had undergone previous TPB were compared with 'controls' (no previous TPB) in group $2(n=85)$. The primary outcome was successful HoLEP.

Results: 24 patients had undergone previous TPB; 8/24 (33\%) of patients had Gleason score $\geq 3+3$. Median time to HoLEP was 38.6 weeks. There were no statistically significant differences in either key baseline characteristics or mass of prostate enucleated between groups 1 and 2 . There was no statistically significant difference in enucleation or morcellation times parameters between the two groups other than enucleation efficiency in favor of group $1(\mathrm{p}=0.024)$. Functional outcomes improved significantly in both groups, but there were no sta-

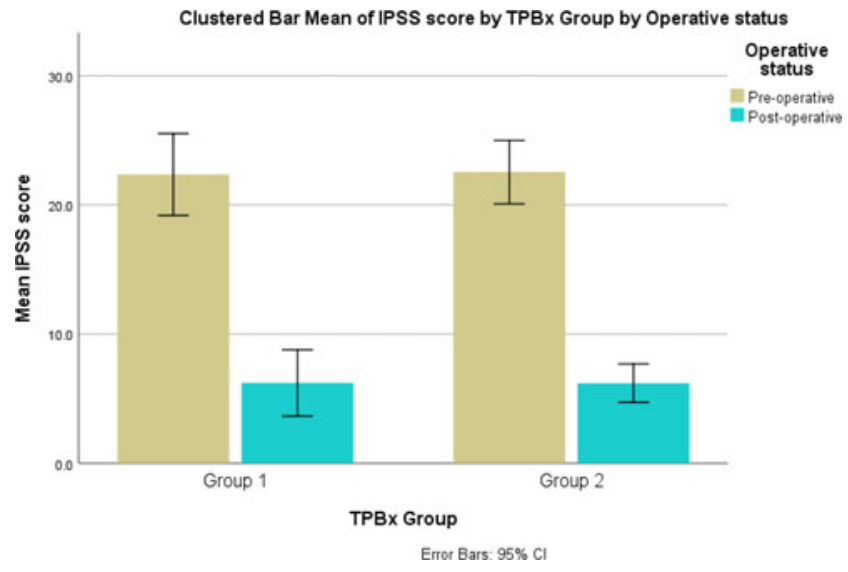

tistically significant differences between the final functional outcomes (figure).

Conclusions: In patients with a previous TPB, HoLEP is surgically feasible, safe and effective. TPB should not be considered a contraindication to HoLEP. Our work provides a strong foundation for undertaking more research in this area, although further work involving more patients are needed to validate reliability in a clinical setting.

MP03-14 Efficacy and safety of prostate artery embolization for benign prostatic hyperplasia in patients with catheter-dependent urinary retention and lower urinary tract symptoms

M Fadel, R Das, B Hawthorn, S Katmawi-Sabbagh

St George's Hospital

Introduction \& Objective: Prostatic artery embolization (PAE) has emerged as an alternative treatment for benign prostatic hyperplasia $(\mathrm{BPH})$. We report the clinical outcomes of PAE (up to 3 years) for the management of lower urinary tract symptoms (LUTS) and catheter-dependent urinary retention as a result of $\mathrm{BPH}$.

Methods: 90 patients (mean age 75.8 years) with BPH treated with PAE in a single institution were analyzed prospectively (mean follow-up 10.3 months). All patients were evaluated by prostate-specific antigen, transrectal ultrasound (TRUS) and computed-tomography angiogram. PAE was performed using $300-500 \mu \mathrm{m}$ embospheres, under sedation by unilateral/bilateral femoral approach. Clinical follow-up included prostatic volume (PV) using TRUS or magnetic resonance imaging, International Prostate Symptom Score (IPSS), quality of life (QoL), peak urinary flow (Qmax) and post-void residual volume (PVR) recorded at 1, 3, 6-12 months.

Results: $48 \%$ of patients presented with LUTS and $52 \%$ of patients had catheter-dependent urinary retention. Bilateral PAE was performed in $83 \%$ of cases. Sixty nine percent of patients were catheter-free at 1 month and $65 \%$ had complete resolution of symptoms at 6-12 months. PAE provided significant improvement in all measurements at 6-12 months. The mean prostate volume (pre-PAE vs post-PAE $99.3 \mathrm{ml}$ vs $77.2 \mathrm{ml}$ ) with a mean reduction of $22.3 \%$, mean IPSS (20.3 vs 8.4), mean QoL (4.6 vs 2.2), mean Qmax $(8.5 \mathrm{ml} / \mathrm{s}$ vs $13.7 \mathrm{ml} / \mathrm{s})$ and mean PVR $(165.8 \mathrm{ml}$ vs $103.5 \mathrm{ml})$ were significantly different $(p<0.01)$ with respect to baseline in patients with LUTS. The mean prostate volume (pre-PAE vs post-PAE $146.6 \mathrm{ml}$ vs $86.0 \mathrm{ml}$ ), with a mean reduction of $41.3 \%$, and mean QoL (4.7 vs 1.2 ) were also significantly different $(p<0.01)$ with respect to baseline in patients with catheter-dependent retention. The post-PAE mean findings in these patients for IPSS, Qmax and PVR were 6.2, 12.3ml/s and $158.7 \mathrm{ml}$ respectively. There were no major complications reported and the total reoperation rate was $6.7 \%$.

Conclusions: Clinical symptoms and uroflowmetry improved after PAE, with an overall mean 11-point IPSS improvement from baseline. We have included a larger group of catheterdependent patients compared to other previous studies and more than two-thirds of these patients became catheter-free. We believe that PAE is a safe and effective procedure which should be considered in medically refractory LUTS as well as catheterdependent urinary retention.

\section{MP03-15 Withdrawn}


MP03-16 Safety of holmium laser enucleation of prostate (holep) in patients on antiplatelet or anticoagulation medications

K Ramaswami, P Bhirud, R Raveendran, H Pothiyedath

Metromed Institute Of Advanced Urology And

Renal Translplant

Introduction \& Objective: Holmium Laser Enucleation of Prostate (HOLEP) has been proved to be as effective as transurethral resection of prostate (TURP) for the treatment of benign prostatic hyperplasia (BPH). Oral anticoagulation is considered a strict contraindication to TURP. In recent years, however, safe and effective surgical alternatives such as HOLEP have emerged. Methods: A prospective study was done including all patients who underwent HoLEP at our institution from December 2014 to April 2019. Follow up period raged from 1 month to 24 months. A pulsed high power 100 watts Holmium Laser with $26 \mathrm{~F}$ resectoscope sheath was used for enucleation. Thirty-six patients were taking Aspirin tablet, 9 were on clopidogrel table and 7 patients were on tablet warfarin who were substituted with un-

\begin{tabular}{|l|l|}
\hline \multicolumn{2}{|l|}{ Baseline Patient Characteristics } \\
\hline $\begin{array}{l}\text { Number of patients } \\
\text { 1. On Anticoagulant medications }\end{array}$ & 2190 \\
2. Withheld anticoagulants & 182 \\
3. Not withheld & 130 \\
\hline Age(yrs) & 52 \\
\hline Q Max(m//s) & $66.5 \pm 8.1$ \\
\hline IPSS & $8 \pm 3.1$ \\
\hline PVR (ml) & $24 \pm 5.6$ \\
\hline Prostate volume (cc) & $115 \pm 35$ \\
\hline Total Serum PSA (ng/ml) & $62 \pm 34$ \\
\hline Urinary retention & $4.6 \pm 3.5$ \\
\hline Bladder Calculi & 122 \\
\hline Bladder Diverticuli & 47 \\
\hline
\end{tabular}

\begin{tabular}{|l|l|}
\hline Intra- and perioperative data & \\
\hline Total operative time (min) & $80.8 \pm 39.7$ \\
\hline Laser enucleation time (min) & $54 \pm 20.9$ \\
\hline Morcellation time (min) & $18.3 \pm 9$ \\
\hline Morcellation efficiency (g / min) & $2.89 \pm 1.5$ \\
\hline Resected weight (g) & $40 \pm 27.5$ \\
\hline Hb drop (g/ dl) & $1.1 \pm 0.9$ \\
\hline Na drop (meq/ I) & $2 \pm 1.4$ \\
\hline Post operative irrigation & 8 \\
\hline Clot evacuation & 0 \\
\hline Blood transfusion & 0 \\
\hline Traction for 6 hours & 21 \\
\hline Catheter time (hrs) & $36 \pm 14.7$ \\
\hline Hospital stay (hrs) & $48 \pm 26$ \\
\hline
\end{tabular}

fractionated heparin perioperatively. These medications were not stopped prior to surgery.

Results: 130 patients out of 2190 patients were on antiplatelets. 52 patients proceeded with Holep without stopping antiplatelets, there was no intra-op or post -op bleeding, 7 patients had mild hematuria managed conservatively.

Conclusions: Holep Represents an effective and safe surgical intervention for BPH, even in patients taking oral anticoagulants. With Current increasing number of high-risk men who are on antithrombotic therapy requiring surgical intervention of $\mathrm{BPH}$, HOLEP is one of the best options.

MP03-17 Surgical outcome of huge benign prostatic hyperplasia treated by Holmium laser enucleation of the prostate using en bloc enucleation technique with lateral median approach

Y Shibata, S Arai, A Kanayama, D Oka, A Otsu, M Aoki, H Nakayama, Y Miyazawa, T Syuto, M Nomura, Y Sekine, H Koike, H Matsui, K Suzuki

Introduction \& Objective: Holmium laser enucleation of the prostate (HoLEP) has become an increasingly popular treatment for symptomatic benign prostatic hyperplasia (BPH). HoLEP allows complete removal of the tissue in the transition zone regardless of the prostate size. Despite the advantages of HoLEP, huge $\mathrm{BPH}$ requires considerable surgical technique for safe operation. In this study, we have examined the surgical outcome of patients with enucleated tissue weight of over 100 grams.

Methods: Nearly 450 patients underwent HoLEP in our institution since the introduction of this surgical method. The first ten patients were operated with an ordinary technique introduced by Gilling, et al in 1996, which enucleate the lobes separately starting with the median lobe. Afterwards, we have started the en bloc enucleation technique with lateral median approach to the median lobe which was applicable for large BPH. The procedure consists of following maneuvers: 1) Early encircling mucosal incision at apical adenoma, 2) Lateral approach to bladder neck at upper portion, 3) Lateral to median exfoliation of median lobe at bladder neck, 4) Retrograde exfoliation of hang down adenoma at 12 o'clock position, 5) En bloc downward morcellation of the enucleated adenoma. The surgical outcomes and complications were examined in 29 patients with over 100 grams of enucleated tissue weight.

Results: Mean age at surgery was 74.6 (range 61-88) and 21 $(72.4 \%)$ had a urinary catheter. Prior BPH treatment by antiandrogens or 5 alpha-reductase inhibitor was performed on 4 $(13.8 \%)$ patients. The mean estimated prostate volume and adenoma evaluated by trans-rectal ultrasound were $137.7 \mathrm{ml}$ (range 51.4-250.7) and $102.9 \mathrm{ml}$ (range 38-220.9), respectively. The mean operative time was160.9minutes (range 76-278) and the mean enucleation weight was 135.6 grams (range 101-275). The operation efficiency (enucleated weight/operation time) was $0.90 \mathrm{~g} / \mathrm{min}$ (range $0.51-1.55$ ). Three patients (10.3\%) required either homogeneous or autologous blood transfusion, respectively. The mean post-operative duration of catheterization and post-operative hospital stay period were 2.6 days and 4.7 days, respectively. One patient $(3.5 \%)$ required re-catheterization by hematuria and one re-operation (3.5\%) for removal of hard enucleated adenoma a week after first operation.

Conclusions: We feel that HoLEP represents a safe and effective treatment for patients with huge BPH. HoLEP require shorter length of hospital stay and fewer complications compared to open surgery. 
MP03-18 En-Bloc HoLEP vs En-Bloc GreenLEP: comparing intra and post-operative outcomes for Enbloc enucleation technique using two kinds of laser to treat patients affected by benign prostatic enlargement (BPE)

G Saitta, ME Rodriguez Socarras, E Rijo, J Reinoso Elbers, J Fernandez Del Alamo, L Llanes Gonzalez, J Gomez Rivas, F Gómez Sancha

Department of Urology, Ospedale San Raffaele-Turro, Milan, Italy

Introduction \& Objective: En-Bloc enucleation has been introduced recently as a safety and valid technique to treat patients affected by benign prostatic enlargement (BPE). The aim of this study is to compare intra and post-operative outcomes of EnBloc HoLEP vs En-Bloc GreenLEP.

Methods: A prospective analysis of 312 consecutive patients who underwent En-Bloc enucleation in our institution, between January 2013 - March 2017, was performed (137 in En-Bloc HoLEP group vs 175 in En-bloc GreenLEP group). All procedures were performed by a single surgeon with large experience in laser surgery for BPE. Patients were followed-up at 1, 3, 6, 12, 24 months and intra- and post-operative parameters as well as functional results were assessed: International Prostate Symptom Score (IPSS), International Index of Erectile Function (IIEF), maximum flow rate (Qmax), postvoid residual urine (PVR), prostate specific antigen (PSA), mean residual prostate volume (ml). Complications were recorded according to Clavien-Dindo classification. Analyzes were carried out with the statistical

Table 1: En-Bloc HoLEP vs En-Bloc GreenLEP; comparing intra and post-operative outcomes for En-bloc enucleation technique using two kinds of laser to treat patients affected by benign prostatic enlargement (BPE).

\begin{tabular}{|c|c|c|c|c|}
\hline & Time (months) & $\begin{array}{c}\text { HoLEP } \\
\text { (137 patients) }\end{array}$ & $\begin{array}{c}\text { GreenLEP } \\
\text { (175 patients) }\end{array}$ & p (overall) \\
\hline \multirow[t]{6}{*}{ IPSS (score) } & 0 & $24.2 \pm 7.9$ & $21.4 \pm 6.98$ & 0.687 \\
\hline & 1 & $10.6 \pm 5.86$ & $11.0 \pm 6.2$ & 0.69 \\
\hline & 3 & $7.88 \pm 5.68$ & $7.74 \pm 5.08$ & 0.881 \\
\hline & 6 & $5.22 \pm 4.55$ & $5.69 \pm 3.9$ & 0.66 \\
\hline & 12 & $6.4 \pm 5.09$ & $12.0 \pm 7.36$ & $\underline{0.001}$ \\
\hline & 24 & 5.0 & $4.31 \pm 3.03$ & $\cdot$ \\
\hline \multirow[t]{6}{*}{ MEF (score) } & 0 & - & - & - \\
\hline & 1 & $9.5 \pm 5.06$ & $17.9 \pm 8.87$ & $\underline{0.001}$ \\
\hline & 3 & $10.3 \pm 4.08$ & $21.5 \pm 7.01$ & $\leq 0.001$ \\
\hline & 6 & $10.0 \pm 9.54$ & $22.0 \pm 6.13$ & 0.158 \\
\hline & 12 & $11.0 \pm 4.24$ & $25.0 \pm 0.00$ & 0.134 \\
\hline & 24 & $-(-)$ & $23.0 \pm 5.49$ & - \\
\hline \multirow[t]{6}{*}{$Q \max (m / / s)$} & 0 & $7.55 \pm 4.25$ & $8.76 \pm 4.19$ & 0.044 \\
\hline & 1 & $21.5 \pm 10.2$ & $19.8 \pm 10.7$ & 0.235 \\
\hline & 3 & $24.8 \pm 12.2$ & $24.1 \pm 11.1$ & 0.735 \\
\hline & 6 & $24.0 \pm 9.23$ & $25.4 \pm 13.7$ & 0.527 \\
\hline & 12 & $27.8 \pm 12.0$ & $25.6 \pm 12.7$ & 0.717 \\
\hline & 24 & $23.0( \pm 12.1)$ & $28.8 \pm 15.1$ & 0.306 \\
\hline \multirow[t]{6}{*}{$P V R(m l)$} & 0 & $>75$ & $>125$ & 0.276 \\
\hline & 1 & $11.6 \pm 40.6$ & $9.86 \pm 50.7$ & 0.86 \\
\hline & 3 & $27.3 \pm 90.5$ & 0 & 0.341 \\
\hline & 6 & $25 \pm 7.3$ & $1.23 \pm 6.83$ & 0.427 \\
\hline & 12 & $7.5 \pm 15$ & $23 \pm 75.8$ & 0.422 \\
\hline & 24 & {$[0-30]$} & {$[0-30]$} & $\cdot$ \\
\hline \multirow[t]{6}{*}{ PSA $(n g / m l)$} & 0 & $3.6 \pm 1.7$ & $5.09 \pm 1.5$ & 0.724 \\
\hline & 1 & $1.34 \pm 2.16$ & $0.92 \pm 1.12$ & 0.249 \\
\hline & 3 & $0.75 \pm 0.63$ & $0.97 \pm 1.69$ & 0.288 \\
\hline & 6 & $1.25 \pm 1.54$ & $1.08 \pm 1.98$ & 0.628 \\
\hline & 12 & $1.06 \pm 1.14$ & $0.84 \pm 0.73$ & 0.381 \\
\hline & 24 & $1.35 \pm 1.06$ & $0.79 \pm 0.72$ & 0.225 \\
\hline \multirow[t]{6}{*}{ SUI $(n, \%$ rate $)$} & 0 & - & $\cdot$ & - \\
\hline & 1 & $7(5.8 \%)$ & $10(6.29 \%)$ & 0.915 \\
\hline & 3 & $2(1.5 \%)$ & $13(6.67 \%)$ & $\underline{0.032}$ \\
\hline & 6 & $1(0.7 \%)$ & $11(6.38 \%)$ & 0.104 \\
\hline & 12 & 0 & $2(2.38 \%)$ & 1.0 \\
\hline & 24 & 0 & 0 & - \\
\hline
\end{tabular}

$n=312$ patients; $* p<0.05$

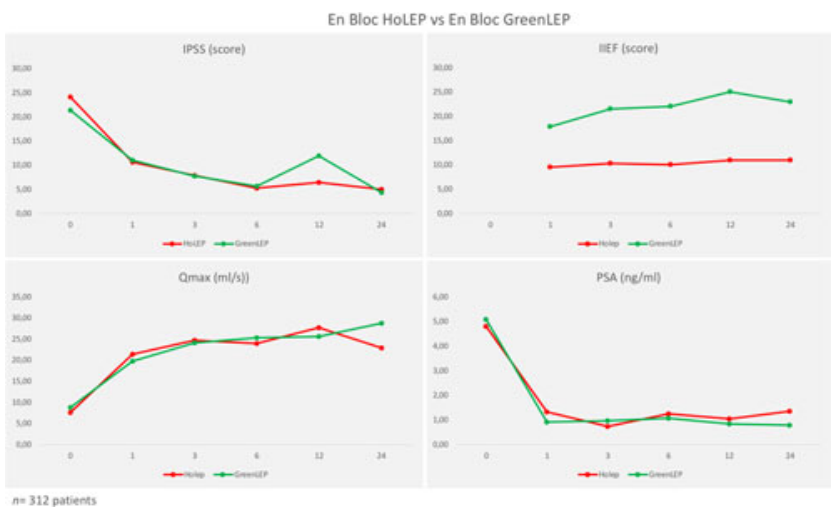

software R (version 3.3.2) using Chi-square, t-Student, ANOVA, Kruskall Wallis \& U-Mann-Whitney as statistical tests.

Results: Mean patients' age was $66 \pm 5,3$ years; pre-operative PSA was $3.6 \pm 1.7$ vs. $5.09 \pm 1.5 \mathrm{ng} / \mathrm{ml}(\mathrm{p}=0.724)$, prostate volume $80 \pm 41.5$ vs. $75 \pm 42,3 \mathrm{ml}(\mathrm{p}=0.07)$, surgical time was $52 \pm 23$ vs. $47.6 \pm 21.3 \mathrm{~min}(\mathrm{p}=0.081)$, enucleation time 25 vs. $31.5(\mathrm{p}=0.011)$, morcellation 6 vs. $6.9 \mathrm{~min}(\mathrm{p}=0.008)$, catheterization time and hospital stay were 1 vs 1.2 day $(\mathrm{p}=0.353)$ for En-Bloc GreenLEP and En-Bloc HoLEP, respectively. En-Bloc GreenLEP demonstrated a significant improved IIEF score at 1 and 3 months compared to En-Bloc HoLEP (all p < 0.05). En-Bloc HoLEP showed lower rates of SUI at 3 months and more reduction of IPSS score at 12 months compared to GreenLEP (all $\mathrm{p}<0.05$ ). Conclusions: Both En-Bloc HoLEP \& En-Bloc GreenLEP are feasible and safe to treat patients with benign prostatic enlargement (BPE). En-Bloc HoLEP shows lower rates of temporary urinary incontinence and reduction of IPSS, while IIEFs seems better in En-Bloc GreenLEP group.

\section{MP03-19 Improved Efficiency of Holmium Laser Enucleation of the Prostate Utilizing a Modulated Pulse Platform}

BB Whiles, A Martin, A Brevik, K Glavin, KL Thurmon

University of Kansas Medical Center

Introduction \& Objective: Holmium laser enucleation of the prostate (HoLEP) is as well-established treatment option for men with lower urinary tract symptoms attributed to benign prostatic hyperplasia. With multiple advancements in technology various holmium laser platforms have emerged. Moses ${ }^{\mathrm{TM}}$ is one laser platform which has shown promise for use in laser lithotripsy. This same technology can be used in HoLEP; however, to our knowledge, no studies to date have formally investigated the use of Moses technology in this specific patient population.

Methods: This is a retrospective cohort study of patients who underwent HoLEP (CPT 52649) at an academic medical center between $04 / 2018-10 / 2018$ by a single urologic surgeon. Intraoperative variables were compared between patients who underwent enucleation of the prostate with Pulse $120 \mathrm{H}$ laser fitted with a $550 \mu \mathrm{m}$ fiber with and without use of Moses Technology (Moses fiber and laser settings). Prostate size was determined by preoperative imaging, and enucleated tissue weight was determined by pathology. Intraoperative parameters such as enucleation time and laser energy usage were obtained from operating room documentation. Data analysis was completed in SAS Studio via Student's t-test since all were parametrically distributed.

Results: A total of 49 patients underwent HoLEP were included in our cohort, 31 patients without and 18 patients with the use of 
Table 1. Holmium laser enucleation of the prostate (HoLEP) without and with Moses

\begin{tabular}{|c|c|c|c|}
\hline & $\begin{array}{l}\text { Without Moses } \\
\qquad(n=31)\end{array}$ & $\begin{array}{l}\text { With Moses } \\
\quad(n=18)\end{array}$ & p-value \\
\hline $\begin{array}{c}\text { Mean Age } \\
\text { (Years) }\end{array}$ & 68.8 & 68.8 & 0.98 \\
\hline $\begin{array}{l}\text { Mean Prostate Size } \\
\text { (grams) }\end{array}$ & 119 & 152 & 0.03 \\
\hline $\begin{array}{l}\text { Mean Enucleation Time } \\
\text { (minutes) }\end{array}$ & 76.3 & 79.1 & 0.68 \\
\hline $\begin{array}{l}\text { Mean Enucleated Volume } \\
\text { (grams) }\end{array}$ & 68.3 & 100.7 & $<0.01$ \\
\hline $\begin{array}{l}\text { Enucleation Rate } \\
\text { (grams/min) }\end{array}$ & 0.92 & 1.32 & 0.01 \\
\hline $\begin{array}{l}\text { Percentage Enucleated } \\
(\%)\end{array}$ & 58.2 & 73.9 & 0.17 \\
\hline $\begin{array}{l}\text { Mean Laser Energy Usage } \\
(\mathrm{KJ})\end{array}$ & 167.3 & 181.6 & 0.48 \\
\hline $\begin{array}{l}\text { Energy Usage Rate } \\
(\mathrm{KJ} / \mathrm{min})\end{array}$ & 2.22 & 2.41 & 0.49 \\
\hline $\begin{array}{l}\text { Enucleated Tissue per KJ Energy } \\
\text { (grams/KJ) }\end{array}$ & 0.47 & 0.64 & 0.09 \\
\hline
\end{tabular}

Moses. Prostate size was larger in patients who underwent HoLEP after implementation of Moses (152g vs $119 \mathrm{~g}$; $\mathrm{p}=0.03)$. Enucleation time was similar (79.1 minutes vs 76.3 minutes; $\mathrm{p}=0.68)$; however, enucleated volume $(100.7 \mathrm{~g}$ vs $68.3 \mathrm{~g} ; \mathrm{p}<0.01)$ and enucleation rate $(1.32 \mathrm{~g} / \mathrm{min}$ vs $0.92 \mathrm{~g} / \mathrm{min} ; \mathrm{p}=0.01)$ were larger and faster with use of the Moses laser technology. Percentage of tissue enucleated was $73.9 \mathrm{~g}$ vs $58.2 \mathrm{~g}$ in those with and without use of Moses, although this was not statistically significant $(p=0.17)$. There were no differences in mean laser energy usage or energy usage rate between these two groups. Energy efficiency, defined by enucleated tissue per KJ of energy used, was $0.64 \mathrm{~g} / \mathrm{KJ}$ vs $0.47 \mathrm{~g} / \mathrm{KJ}$ with and without the use of Moses which approached by was not statistically significant $(\mathrm{p}=0.09)$.

Conclusions: Use of the Moses laser and laser fiber are associated with increased enucleation efficiency in patients undergoing HoLEP. Further studies are needed to further determine if intraoperative as well as postoperative outcomes differ when this technology is utilized.

\section{MP03-20 ProstaCare: Water electrolysis system for the treatment of benign prostate hyperplasia}

\section{WJ Chua, Y Lim, PJ Gilling, HY Tiong, A Sim, H Ho}

National University Hospital, Department of Urology

\section{Introduction \& Objective:}

Introduction: Established treatment of benign prostate hyperplasia $(\mathrm{BPH})$ with medical or surgical therapy provides symptom relief but have associated side effects. Newer technology offers treatment options with the aim to minimize these side effects. The ProstaCare water electrolysis system was developed to treat BPH by creating necrosis in the lateral lobes of the prostate around the urethra. Water electrolysis is achieved by passing direct current between a pair of electrodes in tissue. This creates hydroxyl ions at the cathode and hydrogen ions at the anode. The resulting acidic and alkaline regions cause cellular necrosis. The ions created are proportional to the charge passing through the electrodes and an ion concentration gradient ensures as they diffuse through the tissue. The controlled shape and size of the concentration gradient allow for the predictable creation of necrotic treatment zones.
Objective: We report our multi-center clinical experience with ProstaCare water electrolysis treatment of prostate for $\mathrm{BPH}$ and the outcomes with 12 months' follow-up.

Methods: A total of 49 patients with moderate to severe lower urinary tract symptoms (LUTS) were recruited in a single arm study. The ProstaCare treatment catheter device was inserted per urethra using lignocaine gel as local anesthesia in the outpatient clinic. The pre and post procedure assessments included International Prostate Symptom Score (IPSS), Quality of Life (QOL) score, and peak urinary flow rate. The duration the patient had a catheter was also recorded.

Results: Our patients were aged (years) median 66, range (51$86)$ with prostate volume $(\mathrm{ml})$ median 39 , range (27-54.9). They reported significant improvement in IPSS, QOL and peak urinary flow rate through 12 months after water electrolysis therapy. The mean improvement in IPSS of -9.6 was sustained from the 3 months through to the 12 months follow up $(p=0.004)$. The mean improvement in QOL of -2.1 at 3 months reduced to -1.5 at the 12 months follow up $(\mathrm{p}=<0.001)$. The mean peak urinary flow rate improvement of $+2.2 \mathrm{ml} / \mathrm{s}$ at 3 months $(\mathrm{p}<0.001)$ was not sustained and reduced to $+1.1 \mathrm{ml} / \mathrm{s}$ at 12 months $(\mathrm{p}=0.06)$. Treatment failure was reported in $\mathrm{n}=3(6 \%)$ patients. The duration of catheter insertion was (days) median 1 (range 0-6).

Conclusions: This study demonstrates that ProstaCare is a safe treatment for BPH with sustained symptomatic improvement at 12 months follow up. Water electrolysis treatment of prostate can be considered in selected patient group as an alternative to the use of pharmaceutical medication.

\section{MP03-21 Online Interest in Minimally Invasive Out- patient Surgical Treatment for BPH Using Google Trends}

\section{S Merheb, D Wang, S Wason}

Boston University School of Medicine

Introduction \& Objective: BPH affects about $70 \%$ of men (6069 years) in the US. Globally, 1.2 million men undergo surgery for BPH per year, Transurethral Resection of the Prostate (TURP) being the most common procedure. Currently, surgical management for BPH includes: TURP, Photoselective Vaporization of the Prostate (PVP), Holmium Laser Enucleation of the Prostate (HoLEP), Water Vapor Energy Ablation (WAVEREZÚM), and Prostatic Urethral Lift (Urolift). The online interest for these interventions is unknown. Google Trends is a feature of the Google Search Engine that provides access to internet search patterns. We sought to determine online interest in surgical treatment options for BPH by using Google Trends to evaluate US and Worldwide online search patterns for TURP, PVP, HoLEP, WAVE-REZÚM, and Urolift.

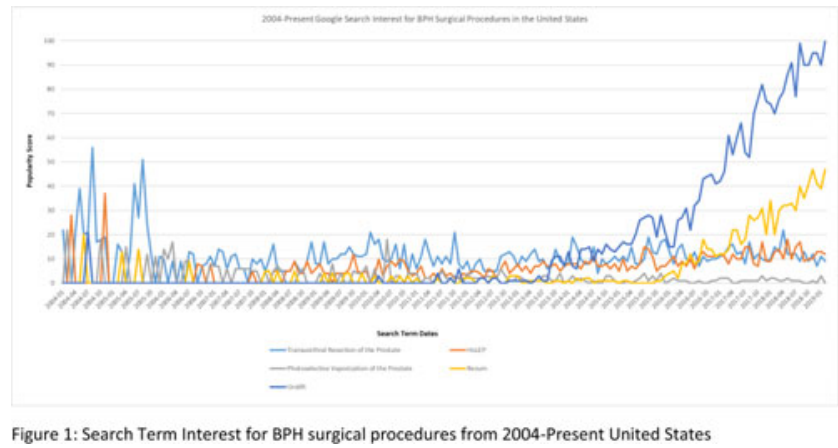




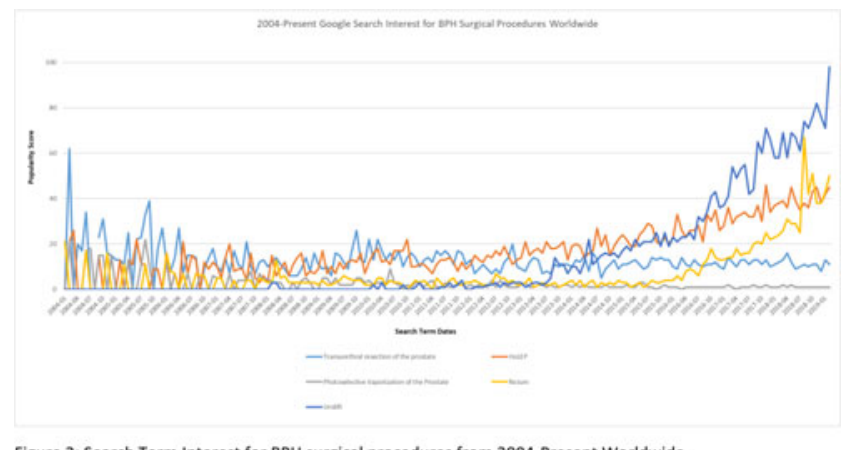

Figure 2: Search Term Interest for BPH surgical procedures from 2004-Present Worldwide

Methods: TURP, PVP, HoLEP, WAVE- REZÚM, and Urolift were selected from the 2018 AUA Guideline for the Surgical Management of Lower Urinary Tract Symptoms Attributed to BPH as search terms of interest within Google Trends. Google Trends normalizes the popularity of a search term on a $0-100$ scale. A score of 0 indicates a low relative search popularity and 100 indicates a relative peak in popularity for the term.

Results: Searches in the US and Worldwide exhibited downtrends in the popularity of TURP; meanwhile, there was an upward trend in the popularity of Urolift, and REZÚM. Within the US, Urolift surpassed TURP in August 2014, while REZÚM surpassed TURP in September 2016. Worldwide, Urolift surpassed TURP in May 2014, while REZÚM surpassed TURP in October 2016. In Worldwide searches, HoLEP surpassed TURP in 2013.

Conclusions: A steady increase in the search term popularity for Urolift and REZÚM was demonstrated in the US and Worldwide, both of which surpassed online interest for TURP. Additionally, search interest for HoLEP surpassed TURP worldwide. This trend reflects a shift in online interest towards minimally invasive surgery for BPH and a declining interest in the current gold standard, TURP.

\section{MP03-22 Does the complexity affect the outcome of Top-Down Holmium Laser Enucleation of prostate (Top-Down HoLEP)?}

A Hodhod, FA Oquendo Aponte, W Shahrour, A Kotb, O Prowse, H Elmansy

Thunder Bay Regional Health Science Centre

Introduction \& Objective: The Top-Down HoLEP approach was recently introduced to accelerate the learning curve, while promising outcomes and improved continence. In this study, we evaluated the outcome of Top-Down HoLEP for complex cases due to repeated transurethral resection of the prostate (TURP) and prostate cancer, in comparison to non-complex prostates.

Methods: We retrospectively reviewed the charts of prospectively collected patients who underwent Top-Down HoLEP, by a single urologist (H.E), between 2017 and 2018. We used a 100-W holmium: YAG laser (VersaPulse ${ }^{\circledR}$ PowerSuite, Lumenis) with a $550 \mu \mathrm{m}$ laser fiber and a $28 \mathrm{Fr}$ continuous flow resectoscope. Enucleated tissue was morcellated using a Karl Storz DrillCut ${ }^{\mathrm{TM}}$ Morcellator. We recorded the enucleation time, the morcellation

\begin{tabular}{|c|c|c|c|c|}
\hline \multicolumn{2}{|c|}{ Parameter } & Complex Group & Control Group & $P$ value \\
\hline \multicolumn{2}{|c|}{ Number of patients (\%) } & $27(45)$ & $33(55)$ & $\ldots$ \\
\hline \multicolumn{2}{|c|}{ Age at surgery mean (range) } & 74.5 years $(57-87.4)$ & 71.5 years $(54-88)$ & 0.051 \\
\hline \multicolumn{2}{|c|}{ Prostate Size mean (range) } & 144.7 grams $(70-237)$ & 127.6 grams $(74-266)$ & 0.31 \\
\hline \multicolumn{2}{|c|}{ Pre-IPSS mean (range) } & $24.3(17-31)$ & $19.6(10-34)$ & 0.15 \\
\hline \multicolumn{2}{|c|}{ Pre-PVR mean (range) } & $190.5 \mathrm{ml}(11-508)$ & $267.9 \mathrm{ml}(21-780)$ & 0.18 \\
\hline \multicolumn{2}{|c|}{ Pre-Qmax mean (range) } & $11.3 \mathrm{ml} / \mathrm{s}(8.3-14.2)$ & $8.1 \mathrm{ml} / \mathrm{s}(5.5-15)$ & 0.53 \\
\hline \multicolumn{2}{|c|}{ Pre-QoL mean (range) } & $5.1(3-6)$ & $4.5(2-6)$ & 0.96 \\
\hline \multicolumn{2}{|c|}{ Preoperative PSA mean (range) } & $8.1 \mathrm{ng} / \mathrm{ml}(2-17)$ & $9.2 \mathrm{ng} / \mathrm{ml}(1.2-27.1)$ & 0.55 \\
\hline \multicolumn{2}{|c|}{$\begin{array}{l}\text { Cause of complexity } \\
\text { Recurrent after BPH n (\%) } \\
\text { Prostate cancer n (\%) }\end{array}$} & $\begin{array}{r}24(88.9) \\
3(11.1)\end{array}$ & $\ldots$ & $\ldots$ \\
\hline \multicolumn{2}{|c|}{ Enucleation time mean (range) } & $102.1 \min (42-131)$ & $80.8 \min (45-128)$ & 0.04 \\
\hline \multicolumn{2}{|c|}{ Moreellation time mean (range) } & $18.1 \min (6-58)$ & 19 minutes $(4-45)$ & 0.96 \\
\hline \multicolumn{2}{|c|}{ Resected weight mean (range) } & 115.5 grams $(46-202)$ & 103.5 grams $(44-242)$ & 0.41 \\
\hline \multicolumn{2}{|c|}{ Energy mean (range) } & $\begin{array}{l}187.7 \text { Kilojoules ( } 93.6- \\
319 \text { - }\end{array}$ & $\begin{array}{c}172.7 \text { Kilojoules } \\
(92.3-257)\end{array}$ & 0.23 \\
\hline \multirow{2}{*}{$\begin{array}{l}\text { Catheter Stay } \\
\text { n (\%) }\end{array}$} & $<24$ hours & $26(96.3)$ & $31(93.9)$ & \multirow[t]{2}{*}{0.68} \\
\hline & $>24$ hours & $1(3.7)$ & $2(6.1)$ & \\
\hline \multirow{2}{*}{$\begin{array}{l}\text { Hospital Stay } \\
\text { n (\%) }\end{array}$} & $<24$ hours & $25(92.6)$ & $32(97)$ & \multirow{2}{*}{0.44} \\
\hline & $>24$ hours & $2(7.4)$ & $1(3)$ & \\
\hline
\end{tabular}

Table 1. Patient's characteristics and perioperative data.

time, and intraoperative and postoperative complications. All patients were followed-up postoperatively at 3, 6, and 12 months. The evaluation included the IPSS score, Quality of Life assessment (QoL), Qmax and the post-void residual (PVR).

Results: Sixty patients were enrolled in this study. The complex group consisted of 27 patients, while the control group included 33 patients. Patients' characteristics are shown in Table 1. The complexity of HoLEP was due to recurrent TURP (24 patients) and prostate cancer ( 3 patients). The mean enucleation time for the complex group was 102.1 minutes (42-131), and $80.8 \mathrm{~min}$ utes $(45-128)$ for the control group $(p=0.04)$. There was no significant difference between both groups in terms of morcellation time, resected weight, catheter duration, and hospital stay. One patient in the control group with a slight bladder mucosal injury. The postoperative outcomes regarding IPSS, QoL, Qmax, and PVR were comparable between both groups (Table 2). At one-year follow-up, 1 patient in the complex group had stress incontinence $(\mathrm{p}=0.26)$ and 1 patient in each group experienced urge incontinence $(\mathrm{p}=0.89)$.

Conclusions: Apart from a significantly longer enucleation time in the complex group, the safety and postoperative outcomes of Top-Down HoLEP were similar for both groups.

\begin{tabular}{|l|c|c|c|}
\hline \multicolumn{1}{|c|}{ Parameter } & Complex Group & Control Group & P value \\
\hline IPSS & & & \\
Mean (range) 3 months & $5.9(0-21)$ & $3.1(0-11)$ & 0.11 \\
Mean (range) 6 months & $3.9(0-14)$ & $3(0-12)$ & 0.55 \\
Mean (range) 12 months & $3.1(0-7)$ & $2.1(0-9)$ & 0.87 \\
\hline QoL & & & \\
Mean (range) 3 months & $0.63(0-5)$ & $0.7(0-4)$ & 0.77 \\
Mean (range) 6 months & $0.88(0-4)$ & $0.4(0-4)$ & 0.44 \\
Mean (range) 12 months & $0.67(0-3)$ & $0.7(0-4)$ & 0.68 \\
\hline PVR & & & \\
Mean (range) 3 months & $56.2 \mathrm{ml} \mathrm{(0-200)}$ & $48.7 \mathrm{ml}(0-109)$ & 0.94 \\
Mean (range) 12 months & $18.4 \mathrm{ml}(0-48)$ & $10.8 \mathrm{ml}(0-43)$ & 0.79 \\
\hline Qmax & & & \\
Mean (range) 3 months & $30.7 \mathrm{ml} / \mathrm{s} \mathrm{(12.3-48.7)}$ & $24.3 \mathrm{ml} / \mathrm{s} \mathrm{(12.5-48.1)}$ & 0.13 \\
Mean (range) 6 months & $27 \mathrm{ml} / \mathrm{s} \mathrm{(14.1-48)}$ & $25.5 \mathrm{ml} / \mathrm{s}(14.8-42)$ & 0.28 \\
$\quad$ Mean (range) 12 months & $23.6 \mathrm{ml} / \mathrm{s} \mathrm{(14.8-32.1)}$ & $25.8 \mathrm{ml} / \mathrm{s}(14-44.8)$ & 0.86 \\
\hline PSA & & & \\
$\quad$ Mean (range) 3 months & $0.99 \mathrm{ng} / \mathrm{ml}(0.29-5.1)$ & $1.1 \mathrm{ng} / \mathrm{ml}(0.16-9)$ & 0.56 \\
\hline Stress Incontinence (1y) n (\%) & $1(3.7)$ & 0 & 0.26 \\
\hline Urge incontinence (1y) n (\%) & $1(3.7)$ & $1(3)$ & 0.89 \\
\hline
\end{tabular}

Table 2. Top-Down postoperative outcome measures at 3,6, 12 months. 


\section{MODERATED POSTER SESSION 04: BASIC SCIENCE: STONES}

\begin{abstract}
MP04-01 Mutational analysis of AGXT gene in Chinese patients with primary hyperoxaluria type 1
\end{abstract}

W Wang, J Li, Y Tian

Beijing Friendship Hospital, Capital Medical University

Introduction \& Objective: Primary hyperoxaluria type 1 (PH1), a rare autosomal recessive disorder, is characterized by renal stones, nephrocalcinosis, and chronic kidney disease. PH1 is caused by defects in alanine glyoxylate aminotransferase (AGT, 392 amino-acid residues), which is encoded by the alanineglyoxylate and serine-pyruvate aminotransferase (AGXT) gene. The most common mutation, c.33dupC, accounted for about $12 \%$ of the total alleles in PH1 patients in the literatures. This study aimed to determine the clinical, and mutation spectrum of patients with PH1 from mainland China.

Methods: Seventy-five patients with early-onset nephrolithiasis were suspected of having PH. DNA was extracted from patient's white blood cell. The whole exome sequencing was performed. We searched for AGXT gene mutations in the patients and his parents. All coding regions, including exon, intron-exon boundaries, were analyzed using PCR followed by direct sequence analysis.

Results: Fifteen PH1 patients (two adults and thirteen children, age range: 1 to 34 years) were found. The adult patients had chronic kidney disease, while the pediatric patients retained the normal kidney function except one child needed renal dialysis. Seventeen mutations of the AGXT gene were detected. One adult male with late-onset PH1 is a compound heterozygote of the c.815_816insGA and c.1015delG mutations. These frame-shift mutations could result in the production of truncated AGT proteins. Five boys that are respectively homozygous for the c.815_816insGA mutation, c.614C $>$ T mutation, c.33dupC mutation, c. $484 \mathrm{G}>\mathrm{A}$ mutation and c.26dupC mutations. Other patients include an adult female and two boys who are heterozygous for c. $473 \mathrm{C}>\mathrm{T}$, c.909delG, c.28-c.29delCCinsA and c.815_816insGA mutations. And thus, the c.815_816insGA mutation accounts for $6 /$ 30 alleles in the present study; importantly, the position c. 815 represents the 5 '-end of the consecutive wild-type sequence of GAGAGAGA.

Conclusions: We describe a few novel mutations, including c.1015delG, c.679-682delAAGT, etc, and a common mutation, c.815_816insGA, of the AGXT gene among fifteen unrelated families with $\mathrm{PH} 1$. Moreover, we suggest that the short repeat of the GA dinucleotide may represent a mutation hotspot in the Chinese population.

\section{MP04-02 Dual energy CT value in urolithiasis}

S El Rai

Al Zahra Hospital Sharjah

Introduction \& Objective: Unenhanced CT KUB is the recommended gold standard imaging investigation for patients who present with acute renal colic due to its high level of safety and sensitivity. The positive diagnosis of urolithiasis is easy in CT KUB but it is not allowing useful differentiation between stones based on the use of CT attenuation number. Previous studies have shown an overlap of attenuation values when used for characterization of renal calculi, leading to a lower specificity. Such stone composition differentiation is very useful in daily practice as uric acid could be treated medically and to avoid extra-corporeal shock wave lithotripsy for uric acid and calcium oxalate monohydrate. Dual-energy computed tomography (DECT) using the 3rd generation CT utilizes the technique of simultaneous image acquisition of a body part at two different energy levels of the X-ray tubes and provide a stone's attenuation ratios between low-and high-energy. The main objective of this presentation is to spotlight the added-value of DECT in the daily clinical practice in urolithiasis.

Methods: Through a review of literature, we will discuss the DECT accuracy in vivo characterization of kidney stone composition and will show its remaining limitations. We will provide small study regarding consecutive KUB CT exams acquired in our hospital over 3 months on Siemens Somatom Force CT scan 384 slices in order to compare the amount of patient-incurred radiation dose, and to show the superiority and impact of DECT in case management changes and to spotlight some limitations of the DECT KUB.

Results: DECT can help differentiation via analysis of material composition. DECT appears to be superior to conventional CT in differentiating stone composition and is particularly accurate in differentiating non-uric acid from uric acid stones. Therefore, DECT allow defining those who might benefit from medical treatment at an early stage. There is no significant difference in the incurred patient radiation dose between the DECT than the conventional helical acquisition. Yet, the DECT has some limitation in obese patients related to its smaller field of view. Another drawback is in some mixed stones, which have a coating of another material.

Conclusions: Unenhanced CT KUB is the recommended gold standard imaging investigation in urolithiasis but suffers from lower specificity regarding its characterization of renal calculi. New emerging techniques could differentiate the material composition of stones: DECT, spectral CT and upcoming photoncounting detector. DE CT KUB stone characterization is an accurate and useful method for the chemical characterization of urinary stones and can change patient management.

\section{MP04-03 Inter- and Intra-observer Agreement in Measuring Urolithiasis Density on Non-Enhanced Computed Tomography}

GW Disselhorst, JJ Reimerink, RK van der Vijgh, R Sandkuyl, PC Kauer, R Reinhard, M Tjeenk Willink, S Jensch, EP van Haarst

OLVG Hospital, Amsterdam

Introduction \& Objective: Treatment choice for urolithiasis is partially based on measuring stone density in Hounsfield Units (HU) on Non-Enhanced Computed Tomography (NECT). Interobserver variability in these measurements could have treatment consequences. The aim of this study was to assess the observer 
agreement of measuring stone density and whether using a protocol leads to a better agreement.

Methods: We retrospectively included 155 consecutive NECTs of patients with stones $\geq 4 \mathrm{~mm}$. Five observers (two radiologists, one urologist and two residents) assessed all anonymized NECTs four times in randomized order.

HU was measured without instruction (rounds 1;2) and subsequently using two protocols (A; B, rounds 3 ; 4). Protocols comprised bone setting, using zoom and measuring $\mathrm{HU}$ without the penumbra, in either three (A) or one (B) axial plane. The inter- and intra-observer agreement were evaluated using the Intraclass Correlation Coefficient (ICC) and Kappa values (K).

Results: Inter-observer agreement on HU measurement without protocol was: $\mathrm{ICC}=0.84$ (CI-0.79-0.87), $\mathrm{K}=0.68$. Agreement diminished with protocol A to $\mathrm{ICC}=0.62$ (CI-0.37-0.76), $\mathrm{K}=0.40$ and improved with protocol $\mathrm{B}$ to $\mathrm{ICC}=0.90$ (CI-0.86$0.92), \mathrm{K}=0.75$. Intra-observer agreement without protocol was $\mathrm{ICC}=0.87, \mathrm{~K}=0.74$, with protocol A $\mathrm{ICC}=0.87, \mathrm{~K}=0.68$ and with protocol $\mathrm{B}$ ICC $=0.93, \mathrm{~K}=0.81$. The biggest improvement was seen for urologists' agreement from no protocol to protocol $\mathrm{B}$, ICC improved from 0.81 (CI-0.70-0.87) to 0.91 (CI-0.840.94 ); K from 0.66 to 0.78 .

Conclusions: Observer agreement of HU measurement of urolithiasis without protocol is already good but using zoom, bone setting and measuring in a representative plane is recommended. This protocol results in higher agreement, especially for urologists. Measuring in 3 axial planes does not increase agreement.

\section{MP04-04 Genome Wide Polygenic Score and Urinary Tract Stone Diagnosis in a Multiethnic Cohort}

I Paranjpe, M Paranjpe, S Jaladanki, A Kapoor, L Chan, C He, S Coca, R Do, M Gupta, G Nadkarni, A Zampini

Introduction \& Objective: Urinary tract stones are highly heritable and clinical risk factors only explain a portion of stone

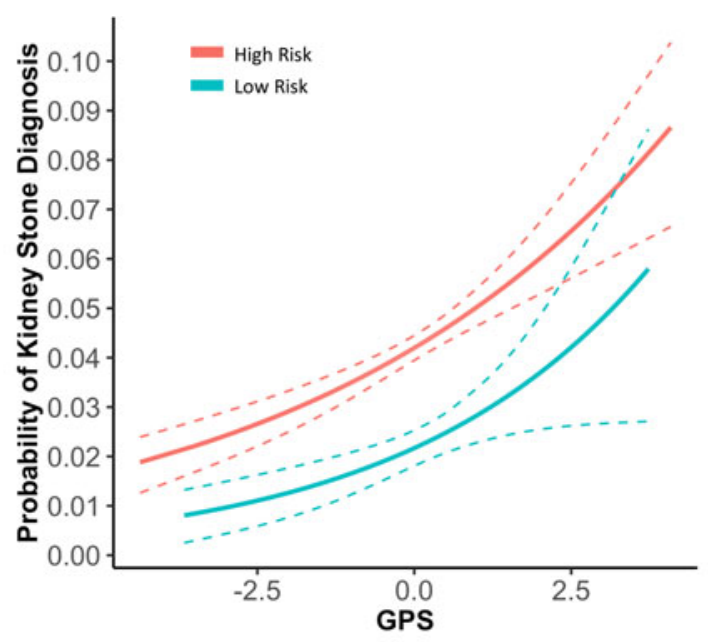

Figure 1 Association of GPS with Kidney Stone Diagnosis Stratified by Clinical Risk. The teal function represents probability of kidney stone diagnosis as a function of GPS for low risk individuals, defined as having $\mathrm{BMI}<25$, no hypertension, no type 2 diabetes, and no gout. The red function represents probability of kidney stone diagnosis as a function of GPS for high risk individuals. Kidney stone diagnosis probability was estimated using a logistic regression function with GPS as input. occurrence. Additionally, urinary tract stones may occur in the absence of risk factors. We aimed to develop a develop a polygenic score for association with urinary tract stones.

Methods: We used genome-wide association studies (GWAS) summary statistics for urinary tract stones from the UK Biobank $(n=361,141)$, to generate polygenic scores with increasing number of single nucleotide variants (SNVs). We then determined the association of the best performing polygenic score with urinary tract stone diagnosis in individuals with and without clinical risk factors (diabetes, hypertension, gout and obesity) in a biobanked cohort using imputed genotyping data (BioMe Biobank, 1208 cases, 30,233 controls) and calculated odds ratios (aOR) with $95 \%$ confidence intervals (CI) adjusting for age, sex and genetic ancestry.

Results: A genome wide polygenic score $\left(\mathrm{GPS}_{\text {stone }}\right)$ utilizing the most SNVs ( $\sim 7$ million) explained the highest variance and was chosen for further analysis. In individuals with at least one risk factor, every SD increase in GPS $_{\text {stone }}$ was associated with an increment of $14 \%$ in diagnosis, $(\mathrm{aOR} 1.14 ; 1.08-1.22 ; \mathrm{p}=4.3 \mathrm{x}$ $10^{-5}$ ) In individuals without no clinical risk factors, every SD

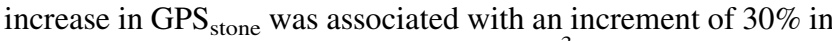
diagnosis, (aOR 1.3; $1.1-1.5 ; \mathrm{p}=4.7 \times 10^{-3}$ ). (Figure 1 ) In these individuals the top $10 \%$ had four-fold adjusted odds relative to the lowest 10\% (aOR 3.7; 95\% CI 1.7 - 9.3; p=0.003).

Conclusions: We identified a genome wide polygenic score that is associated with urinary tract stones overall and even in the absence of known clinical risk factors and identifies a subgroup with genetic risk comparable to a monogenic mutation.

\section{MP04-05 Withdrawn}

MP04-06 In vitro comparison of ablation rates between SuperPulsed Thulium Fiber Laser and Ho:YAG Laser for endocorporeal lithotripsy

\section{F Panthier, S Doizi, L Berthe, O Traxer}

Service d'Urologie, Hopital Tenon, APHP

Introduction \& Objective: Holmium: YAG (Ho:YAG) laser is currently the standard for lithotripsy. Recently, Superpulsed Thulium Fiber Laser (TFL) has been evaluated as an alternative for lithotripsy, using laser fibers with core-diameters (CDF) down to $50 \mu \mathrm{m}$ and additional available settings suitable for "dusting" technique. This in vitro study aimed to compare the efficiency of SuperPulsed Thulium Fiber Laser (TFL) and Holmium:YAG Laser based on ablation volumes.

Methods: A 50W TFL (IPG Photonics ${ }^{\circledR}$ ) and a 30W MH1 Ho:YAG laser $\left(\right.$ Rocamed $^{\circledR}$ ), were used with $150 \mu \mathrm{m}$ and $272 \mu \mathrm{m}$ CDF (Sureflex, Boston Scientific (C), comparing three TFL settings, "fine dusting" (FD:0.15J/100Hz); "dusting" (D:0.5J/30Hz); "fragmentation" (Fr:1J/15Hz) and two Ho:YAG settings: "dusting" (D:0.5J/30Hz); "fragmentation" (Fr:1J/15Hz). An experimental setup consisting in immerged cubes of calcium oxalate monohydrate (COM) or uric acid (UA) stone phantoms (Begostone Plus, Bego()) was used with a 20 seconds' laser activation time and an Archimed type 4 spiral trajectory jointly controlled, in contact mode with the stones using a micrometric screw. Experiments were repeated four times. Stones were dried and weighed before three-dimensional scanning (Micro-CT Quantum FX, Perkin Elmer()). Ablation rates were assessed on ablation volumes $\left(\mathrm{mm}^{3}\right)$ measured by 3D segmentation using 3DSlicer $(\mathrm{NIH} \odot)$. 
Figure 1: Ablation rates $\left(\mathrm{mm}^{3} / \mathrm{min}\right)$ beyond interface ( $\mathrm{COM}=$ calcium oxalate monohydrate; $\mathrm{UA}=$ uric acid), laser settings, laser source and fiber-core diameter.

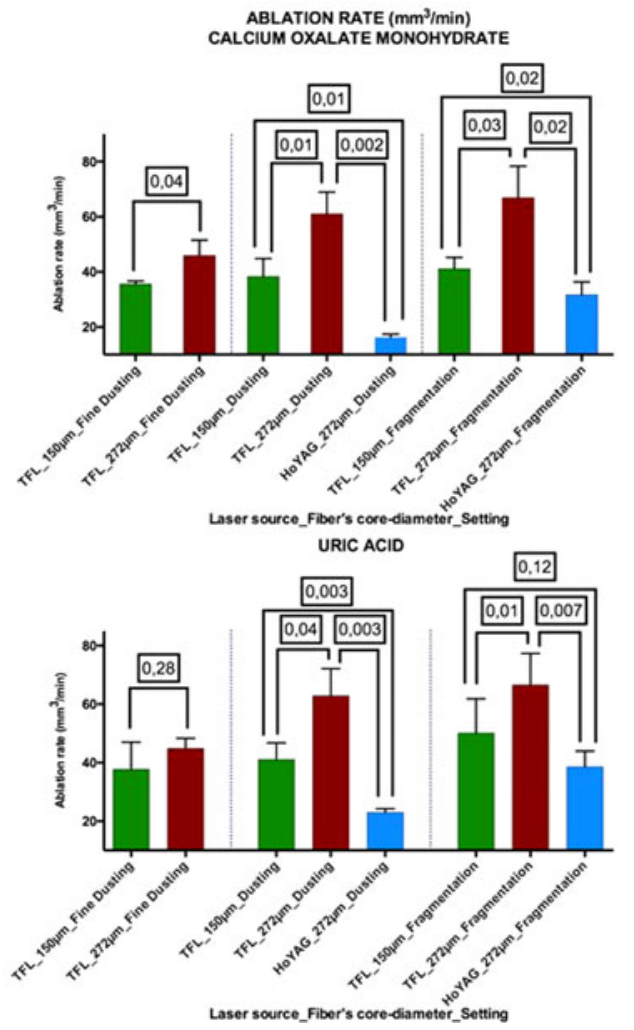

Results: In "dusting" and "fragmentation" with $272 \mathrm{CDF}$, ablation volumes were four and two fold higher with TFL than Ho:YAG against COM $(\mathrm{p}<0,05)$, and three and twofold higher against UA $(\mathrm{p}<0,05)$, respectively (Figure 1). Ablation rates for 150CDF-TFL were significantly higher than 272CDF-Ho:YAG, except in "fragmentation" against UA $(p=0,12) .272 \mathrm{CDF}$ was associated with significant higher ablation volumes than 150CDF with TFL, except in "fine dusting" against UA $(p=0,28)$. Ablation rate in "Fine dusting" mode with 150CDF-TFL and 272CDF-TFL was associated with a twofold and threefold higher ablation rate compared to $272 \mathrm{CDF}-\mathrm{Ho}$ :YAG in "dusting" mode. Conclusions: With equal settings and laser fibers, in vitro ablation volumes by TFL are two (fragmentation) to four (dusting) times higher compared to the Ho:YAG. Moreover, when using 150CDF with TFL, ablation volumes are still fifty percent higher to $\mathrm{Ho}$ :YAG with $272 \mathrm{CDF}$.

MP04-07 High concentration of calcium promotes the expression of Matrix Metallopeptidase-9 through reaction oxygen species dependent NFKB signaling pathway in nephrolithiasis formation

Y Wu, Y Lu, H Hu, b Qin, S Wang, Q Wang

Tongji Hospital

Introduction \& Objective: To address the effects of Matrix Metallopeptidase-9(MMP-9) on nephrolithiasis formation under high concentration of calcium and the mechanisms underlying such effects.
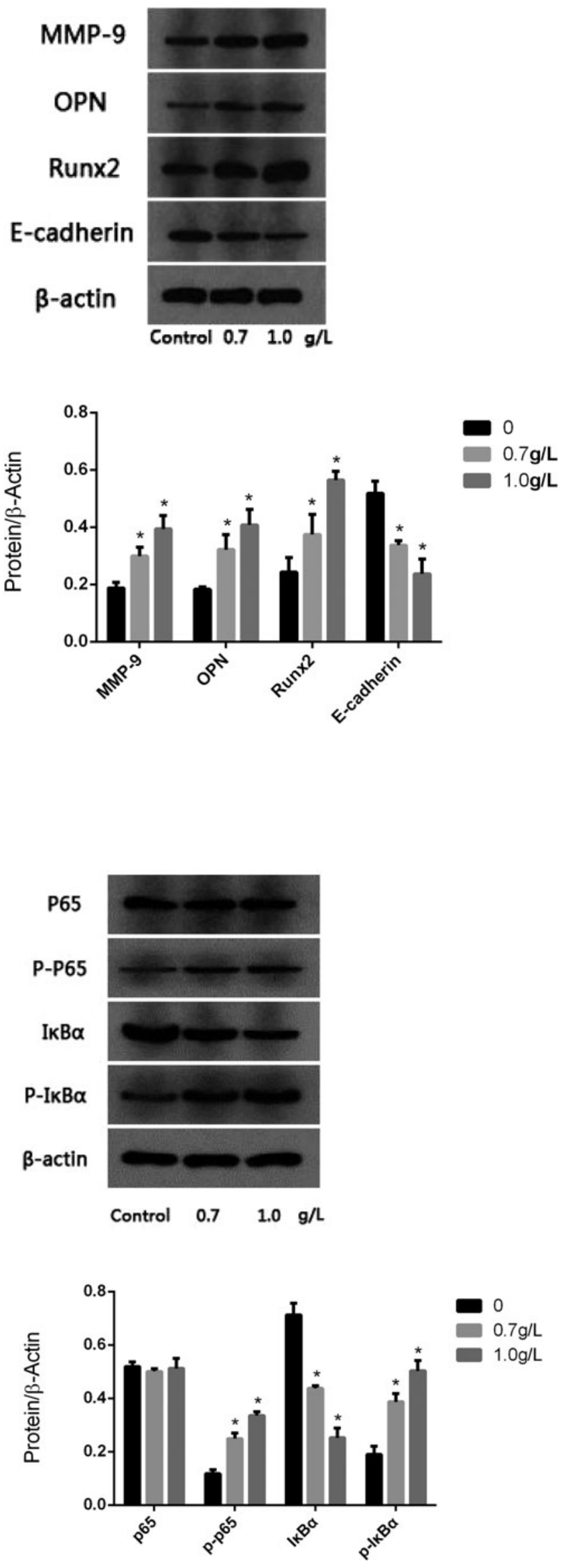
Methods: NRK-52E cells or pretreatment of NRK-52E cells with reaction oxygen species (ROS) scavenger or inhibitor of $\mathrm{NF} \kappa \mathrm{B}$ were treated with high concentration of calcium. The cell viability, apoptosis and intracellular ROS were detected by cell counting kit-8 and flow cytology, respectively. Real-timepolymerase chain and Western blotting were conducted to detect the expression of MMP-9, NF $\kappa \mathrm{B}$ signaling pathway protein, Runt-related transcription factor 2(Runx2), E-cadherin and osteopontin (OPN). Cells were transfected with plasmid-MMP-9 or siRNA-MMP-9 for up- or down-regulation of the expression of MMP-9, respectively. Alizarin red staining was conducted to detect the adherent and deposition of calcium salt.

Results: High concentration of calcium promoted intracellular ROS release, NF $\kappa$ B signaling pathway activation, MMP-9, OPN and Runx2 expression in NRK-52E cells, but inhibited Ecadherin expression. Pretreatment of NRK-52E cells with ROS scavenger or inhibitor of NF $\kappa$ B significantly suppressed MMP-9, OPN and Runx2 expression. Overexpression of endogenous MMP-9 increased the adherent and deposition of calcium salt to NRK-52E cells, while silence of MMP-9 showed reverse results. Conclusions: MMP-9 plays a stimulative role in the stone formation. High concentration of calcium promotes MMP-9 expression through ROS dependent $\mathrm{NF} \kappa \mathrm{B}$ signaling pathway and then promotes mineralization in NRK-52E cells.

\section{MP04-08 Comparison of Teflon Durability Between Guidewires in a Benchtop PCNL Model}

M Hajiha, A Amasyali, J Groegler, J Smith, R Moghisi, A Goyne, M Shah, M Alsyouf, P Stokes, D Baldwin,

J Maldonado, D Baldwin

Introduction \& Objective: Lithotripter use or repetitive nephroscope movements may damage the Teflon (Polytetrafluoroethylene) coating on wires during percutaneous nephrolithotomy (PCNL). Teflon fragments have previously been shown to be lithogenic in urine. The aim of this study was to evaluate the Teflon coating durability of various wires during a simulated PCNL.

Methods: A 3D printed PCNL model with $30 \mathrm{Fr}$ access sheath was developed to test the durability of 10 commonly used Teflon coated guidewires divided into 3 groups (standard, hybrid and Amplatz wires) (Table 1). Two sources of wire trauma were created including use of the ultrasonic lithotripter adjacent to the wire $(30 \mathrm{sec})$ and repeatedly inserting and removing a 26 Fr rigid nephroscope (50x). Ten trials were performed for each wire type using a new wire for each trial. Light microscopy was used to measure the length and degree of damage to the Teflon coating. Damage was graded as grade 1: minimal Teflon coating removed without exposure of metal core, grade $2: 2-25 \%$, grade $3: 25-$ $50 \%$ and grade $4:>50 \%$ of Teflon coating removed. Total wire damage score was calculated using the sum of grades multiplied by length of damage. ANOVA and Tukey's Post hoc with significance set at $\mathrm{P}<0.05$ was used to compare the 3 groups.

Results: The ultrasonic lithotripter induced damage was significantly different between the 3 groups, with hybrid wires having the lowest damage compared to standard and Amplatz wires (mean damage: 83.9 vs. 94.3 and 107.5, $\mathrm{p}=0.049$ ). The ultrasonic lithotripter induced damage was lowest for the Olympus PTFE wire (mean damage: 50.33) and highest for Cook Amplatz fixed core wire (mean damage: 147.40) compared to all other wires (Table 1). The rigid nephroscope induced damage was significantly lower for hybrid wires compared to standard and Amplatz wires (mean damage: 210.8 vs. 367.9 and 280.6, $\mathrm{p}<0.001)$. The rigid nephroscope induced damage was lowest
Table 1: wire damages for ultrasonic lithotripter and rigid nephroscope movements

\begin{tabular}{|c|c|c|c|c|c|}
\hline & & & \multicolumn{3}{|c|}{ Ultrasonic lithotripter induced damage } \\
\hline & & & Total damage score & $\begin{array}{l}\text { P value Olympus PTFE v. } \\
\text { other wires }\end{array}$ & $\begin{array}{l}\text { P value Cook Amplats } \\
\text { iv. other wires }\end{array}$ \\
\hline \multirow[t]{3}{*}{ Standard } & Olympus & PTFE & 50.33 & NA & 0.000 \\
\hline & Cook & Fixed core & 114.40 & 0.000 & 0.803 \\
\hline & Bard & Fised core & 119.50 & 0.000 & 0.938 \\
\hline \multirow[t]{4}{*}{ Hybrid } & $\begin{array}{c}\text { Boston } \\
\text { Scientific }\end{array}$ & Sensor & 91.15 & 0.001 & 0.025 \\
\hline & Olympus & Uttratrack & 66.80 & 0.340 & 0.000 \\
\hline & Cook & Motion & 96.30 & 0.000 & 0.05 \\
\hline & Bard & $\begin{array}{l}\text { Solo Plus } \\
\text { Hybrid }\end{array}$ & 81.40 & 0.009 & 0.002 \\
\hline \multirow[t]{5}{*}{ Amplatz } & Cook & $\begin{array}{l}\text { A Amplatz Fixed } \\
\text { eore }\end{array}$ & 147.40 & 0.000 & NA \\
\hline & Olympus & Amplatz & 69.80 & 0.198 & 0.000 \\
\hline & $\begin{array}{l}\text { Boston } \\
\text { Scientific }\end{array}$ & $\begin{array}{l}\text { Amplatu Super } \\
\text { Stiff }\end{array}$ & 105.40 & 0.000 & 0.345 \\
\hline & & & \multicolumn{3}{|c|}{$\begin{array}{l}\text { Rigid Nephroscope induced damage } \\
\end{array}$} \\
\hline & & & Total damage score & $\begin{array}{l}\text { Pvalue Olympas Uitratrack } \\
\text { va. other wires }\end{array}$ & $\begin{array}{l}\text { P value Bard fixed core } \\
\text { w. other wires }\end{array}$ \\
\hline \multirow[t]{3}{*}{ Standard } & Olympus & PTFE & 160.50 & 1.0 & 0.006 \\
\hline & Cook & Fixed core & 396.70 & 0.001 & 0.638 \\
\hline & Bard & Fixed core & 547.70 & 0.005 & NA \\
\hline \multirow[t]{4}{*}{ Hybrid } & $\begin{array}{c}\text { Boston } \\
\text { Sckentifie }\end{array}$ & Sensor & 186.10 & 0.937 & 0.010 \\
\hline & Olympus & Uttratrack & 151.50 & NA & 0.005 \\
\hline & Cook & Motion & 275.80 & 0.012 & 0.063 \\
\hline & Bard & $\begin{array}{c}\text { Solo Plus } \\
\text { Hybrid }\end{array}$ & 229.90 & 0.481 & 0.025 \\
\hline \multirow[t]{3}{*}{ Amplatz } & Cook & $\begin{array}{l}\text { Amplatz Fixed } \\
\text { eare }\end{array}$ & 359.50 & 0.001 & 0.354 \\
\hline & Olympus & Amplatz & 203.00 & 0.765 & 0.014 \\
\hline & $\begin{array}{l}\text { Boston } \\
\text { Scicatific } \\
\end{array}$ & $\begin{array}{l}\text { Amplatz Super } \\
\text { Stiff }\end{array}$ & 279.45 & 0.004 & 0.066 \\
\hline
\end{tabular}

NA: Not Available

for the Olympus Ultratrack wire (mean damage: 151.50) and highest for the Bard fixed core wire (mean damage: 547.70).

Conclusions: Independent of the brand, the Teflon coating of hybrid wires was the least likely to sustain damage compared to the standard or Amplatz wire types. Knowledge of the resilience of Teflon coated guidewires could allow surgeons to select wires which are less likely to results in a nidus for future stone formation.

MP04-09 Comparison of the fissures and fragments produced with $150 \mu \mathrm{m}$ and $272 \mu \mathrm{m}$ laser fibers with Superpulsed Thulium Fiber Laser: an in vitro study

F Panthier, S Doizi, L Berthe, O Traxer

Service d'Urologie, Hospital Tenon, APHP

Introduction \& Objective: Holmium: YAG (Ho:YAG) is currently the standard for lithotripsy. Recently, Superpulsed Thulium Fiber Laser (TFL) has been evaluated as an alternative for lithotripsy, using laser fibers with core-diameters (CDF) down to $50 \mu \mathrm{m}$ and additional available settings suitable for "dusting" technique. This in-vitro study compared stone fissures and fragments' size produced with $150 \mu \mathrm{m} \mathrm{CDF}$ or $272 \mu \mathrm{m} \mathrm{CDF}$ with different laser settings using TFL.

Methods: $150 \mu \mathrm{m} \mathrm{CDF}$ and $272 \mu \mathrm{m} \mathrm{CDF}$ were compared using three TFL settings, "fine dusting" (FD:0.15J/100Hz); "dusting" (D:0.5J/30Hz); "fragmentation" (Fr: $1 \mathrm{~J} / 15 \mathrm{~Hz})$. An experimental setup consisting in immerged $10 \mathrm{~mm}$ cubes of calcium oxalate monohydrate (COM) or uric acid (UA) stone phantoms was used with a 30 seconds' laser activation time and a spiral trajectory jointly controlled, in contact mode with the stones. Fragments were acquired through sieves. Fragments and stones were observed under optical microscopy to measure fragments 'size and fissure (WOF) mean widths.

Results: $150 \mu \mathrm{m}$ CDF produced significantly smaller WOF than $272 \mu \mathrm{m}$ CDF in all modes against UA (FD: $304.4 \mu \mathrm{m} \pm 45.04$ vs $410.2 \mu \mathrm{m} \pm 66.5, \mathrm{p}<0.001$; D: $503 \mu \mathrm{m} \pm 59.91$ vs $573.5 \mu \mathrm{m} \pm 38.09$, $\mathrm{p}<0.01$; Fr: $615.9 \mu \mathrm{m} \pm 59.18$ vs $689.2 \mu \mathrm{m} \pm 51.4, \mathrm{p}<0.01)$ and COM except in "fragmentation" (FD: $396.9 \mu \mathrm{m} \pm 68.27$ vs $540.8 \mu \mathrm{m} \pm 76.93, \mathrm{p}<0.0001 ; \mathrm{D}: 602.5 \mu \mathrm{m} \pm 35.42$ vs $656.3 \mu \mathrm{m} \pm 65.21$, $\mathrm{p}=0.04 ; \quad$ Fr: $971.3 \mu \mathrm{m} \pm 153.8$ vs $930.5 \mu \mathrm{m} \pm 67, \quad \mathrm{p}=0.39)$ (Figure 1). $150 \mu \mathrm{m}$ CDF produced smaller fragments' diameters 
Figure 1. Mean width of fissure after Thulium Fiber Laser emission on synthetic Uric Acid (UA) and Calcium-Oxalate Monohydrate Stones (COM).

\section{Mean Width of Fissures COM vs UA}
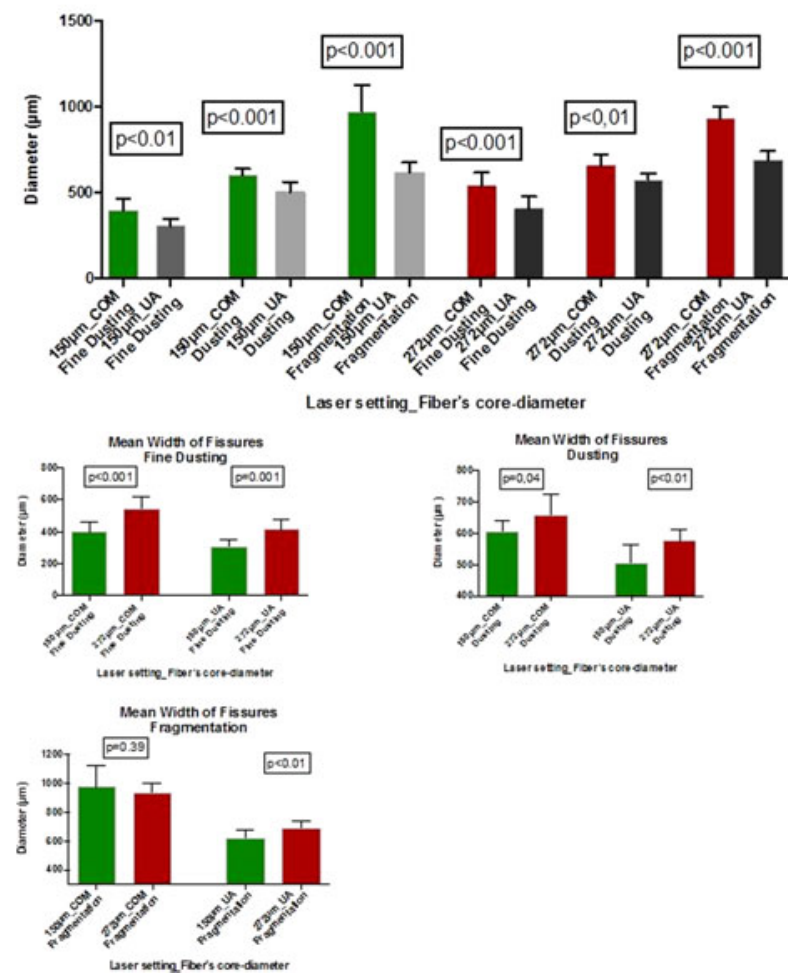

Figure 2. Fragment sizes after Thulium Fiber Laser emission on synthetic Uric Acid and Calcium-Oxalate Monohydrate Stones. Fine Dusting
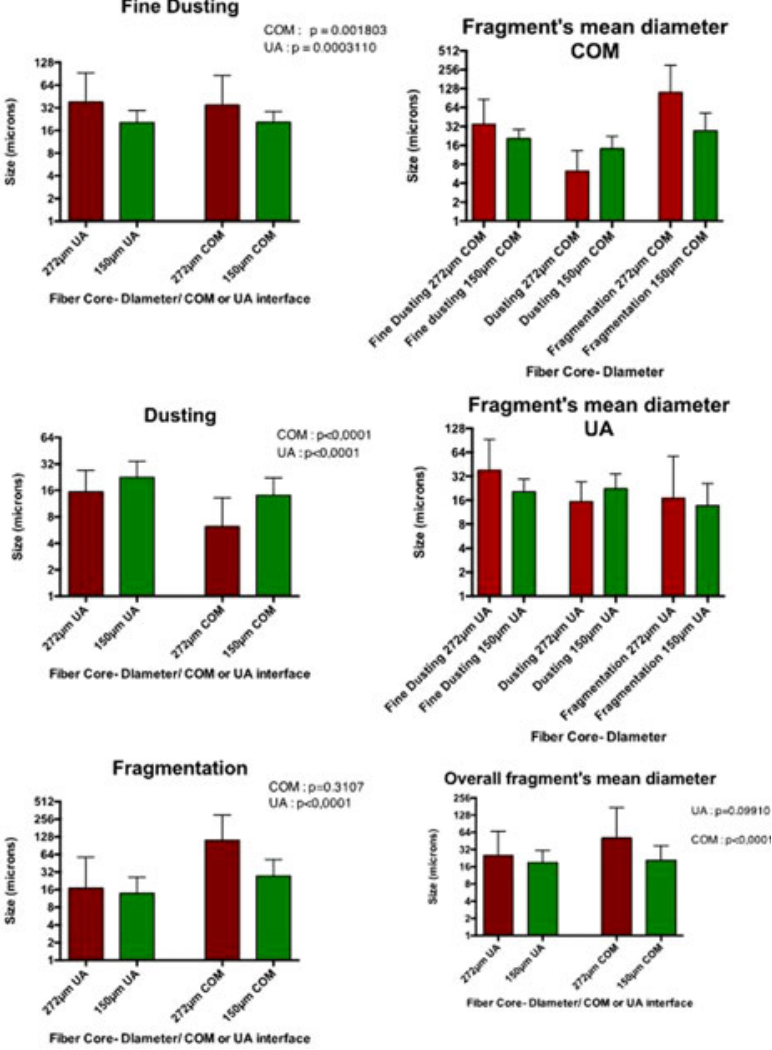

than $272 \mu \mathrm{m}$ CDF in all modes except "dusting" against UA (FD: $20.52 \mu \mathrm{m} \pm 9.001$ vs $38.41 \mu \mathrm{m} \pm 54.42, \mathrm{p}=0.0003 ; \mathrm{D}: 22.65 \mu \mathrm{m} \pm$ 11.95 vs $15.45 \mu \mathrm{m} \pm 11.69, \mathrm{p}<0.0001 ;$ Fr: $13.78 \mu \mathrm{m} \pm 12.3$ vs $17.01 \mu \mathrm{m} \pm 40.66, \mathrm{p}=0,3107)$ and COM (FD:20.66 $\mu \mathrm{m} \pm 8.099 \mathrm{vs}$ $34.91 \mu \mathrm{m} \pm 51.21, \quad \mathrm{p}=0.0018 ; \quad \mathrm{D}: 14.2 \mu \mathrm{m} \pm 8.108$ vs $6.28 \pm 7$, $\mathrm{p}<0.0001$; Fr: $27.56 \mu \mathrm{m} \pm 24.61$ vs $111.9 \mu \mathrm{m} \pm 189.8, \mathrm{p}<0.0001)$ (Figure 2).

Conclusions: These preliminary findings demonstrate that $150 \mu \mathrm{m}$ CDF produces smaller fragments (except in "dusting" mode) that meets the definition of "dusting" lithotripsy and smaller stone fissures than $272 \mu \mathrm{m} \mathrm{CDF}$.

MP04-10 The long-term effect of short periods of unilateral ureteric obstruction on the renal function: Does it deteriorate despite the initial apparent full recovery?

\section{F Hammad, L Lubbad, S Al-Salam, J Yasin}

United Arab Emirates University and Tawam Hospital

Introduction \& Objective: The recovery of renal functions following release of ureteric obstruction (UO) depends on several factors including duration and degree of UO. So, for instance, in the rat, release of 3-day unilateral UO was associated with a total recovery of hemodynamic and tubular renal functions by 14 days post-reversal of UO. However, this was associated with a significant increase in several pro-inflammatory cytokines and in tubular apoptosis, cellular proliferation and interstitial fibrosis which continued up to 28 days post-reversal despite the recovery in renal functions. The renal functions, however, have not been studied beyond 28 days post-reversal of obstruction and it is not yet known if the ongoing interstitial fibrosis which was shown to cause longterm deterioration in glomerular filtration rate (GFR), would result in an ongoing slow deterioration in the renal functions despite the apparent initial recovery post-reversal. Thus, the aim of this study was to investigate the renal functions in the long-term following the initial recovery of the hemodynamic functions post-reversal. Methods: Male Wistar rats underwent reversible complete unilateral left UO for 72 hours. The renal hemodynamic and tubular of the right non-obstructed (NOK) and left obstructed (OK) kidneys were measured using clearance techniques one month (Group-1), four months (Group-2) and 18 months (Group-3) following reversal of UO.

Results: In Group-1, all renal parameters in OK were similar to NOK. This include renal blood flow, GFR, urine volume, total urinary sodium and potassium excretion and fractional excretion of sodium and potassium $(6.39 \pm .050$ vs. $7.04 \pm 0.59,0.83 \pm 0.06$ vs. $0.93 \pm 0.09,17.1 \pm 1.8$ vs. $18.7 \pm 2.7,3.02 \pm 0.38$ vs. $3.86 \pm 0.58$, $1.17 \pm 0.11$ vs. $1.52 \pm 0.09,0.04 \pm 0.003$ vs. $0.04 \pm 0.003$ and $0.54 \pm 0.03$ vs. $0.68 \pm 0.05$, respectively, $\mathrm{P}>0.05$ for all). Up to 18 months, the functions of $\mathrm{OK}$ did not show any deterioration as shown by the similarity of the renal parameters between the $\mathrm{OK}$ and NOK in Group-2 and Group-3 (Table 1).

Table-1: The ratio of the left (OK) to right (NOK) kidney for each parameter in the three groups. The results are expressed as percentages (mean \pm SEM).

\begin{tabular}{lccccccc}
\hline & GFR & RBF & UV & UNsV & FE $_{\text {No }}$ & U.V & FE $_{\text {s }}$ \\
\hline Group-1 & $94 \pm 6$ & $95 \pm 6$ & $104 \pm 9$ & $90 \pm 10$ & $94 \pm 7$ & $80 \pm 8$ & $82 \pm 6$ \\
\hline Group-2 & $110 \pm 15$ & $110 \pm 11$ & $119 \pm 20$ & $118 \pm 28$ & $95 \pm 8$ & $100 \pm 17$ & $91 \pm 4$ \\
\hline Group-3 & $103 \pm 9$ & $114 \pm 15$ & $104 \pm 13$ & $110 \pm 17$ & $111 \pm 16$ & $93 \pm 8$ & $91 \pm 8$ \\
\hline
\end{tabular}

Table-1: The ratio of the left (OK) to right (NOK) kidney for each parameter in the three groups. The results are expressed as percentages (meantSEM). 
Conclusions: In this model of unilateral UO, both hemodynamic and tubular functions were maintained up to 18 months post reversal of UO with no sign of long-term deterioration in kidney functions. This might indicate a large reserve in kidney functions to withstand short periods of UO.

\section{MP04-11 Are There Clinical and Metabolic Char- acteristics that Correlate with the Formation of Cal- cium Oxalate Monohydrate or Dihydrate Stones?}

N Stern, J Bjazevic, L Nott, H Razvi

Western University

Introduction \& Objective: Calcium oxalate is the most predominant stone composition and can be found in two main chemical forms, calcium oxalate monohydrate (COM) and dihydrate (COD). COM stones are known to be denser and harder to fragment, thus impacting treatment success of shockwave lithotripsy. We aimed to identify clinical and biochemical characteristics which may predispose to the formation of COM or COD stones in a large contemporary cohort, to potentially allow for improved treatment planning and patient counselling. Methods: A retrospective analysis of a prospectively maintained metabolic stone clinical database was performed. All patients presenting from September 2001 to February 2017 with predominantly $(>50 \%) \mathrm{COM}$ or COD stones were analyzed including demographic data, serum biochemistry, and 24-hour urine abnormalities. Statistical analysis was performed using a Pearson's chi squared test and ANOVA.

Results: A total of 347 patients were identified, the majority $(85.9 \%)$ were primarily COM in composition, compared with only $14.1 \%$ patients with COD stones. Patients with COD stones were slightly younger compared with COM stone formers ( $48 \pm 17$ versus $54 \pm 13$ years, $\mathrm{p}=0.012$ ). There was no correlation between calcium oxalate stone type and patient gender, BMI, medical comorbidities (hypertension, cardiac disease, diabetes mellitus, prior stroke, gout, inflammatory bowel disease, or sarcoidosis), family history of stone disease, or prior history of stone treatment including shockwave lithotripsy or ureteroscopy. No association was found between stone composition and 24-hour urine findings including urinary $\mathrm{pH}$, sodium, calcium, oxalate, urate, phosphate and urea. Low urine volume was found in the majority of patients (66\%) with COD stones, representing a statistically significant proportion $(\mathrm{p}=0.006)$. In addition, no significant correlation was present between calcium oxalate stone type and serum biochemistry findings including serum calcium, parathyroid hormone, urate, and vitamin D levels.

Conclusions: Previous studies have shown some associations between 24-hour urine collection results and the propensity to form predominately COM or COD stones; however, these studies have been small and reported conflicting results. Our large contemporary series does not demonstrate a correlation between clinical and biochemical characteristics and the formation of specific calcium oxalate stone composition. Further research is required in order to better understand the conditions which predispose to either COM or COD stone formation.

\section{MP04-12 Wielding a Stone Analysis to its Full Potential}

JE Brinkman, T Large, CU Nottingham, J Williams, A Krambeck
Introduction \& Objective: Current AUA guidelines recommend a 24-hour urine collection be obtained in high-risk and recurrent stone formers. While 24-hour urine are valuable in guiding stone prevention they are not always available due to lack of compliance, the goal of our study was to determine if a stone analysis can be utilized to guide preventative lifestyle, dietary and medical interventions.

Methods: We retrospectively reviewed stone analyses from Beck Analysis laboratories database from January 1, 2002 - July 1,2018 . We identified 3,760 patients with pure COM $(\mathrm{COM}=$ $791)$, pure $\mathrm{COD}(\mathrm{COD}=210)$, pure brushite $(\mathrm{BRU}=436)$, pure hydroxyapatite $(\mathrm{HA}=1,391)$, or pure uric acid $(\mathrm{UA}=932)$ stones. Patients included for analysis had to have a paired 24hour urine study with their first stone event managed at our institution. Patients younger than 18, with mixed or discordant stone analyses, or already on medical therapy were excluded. Urinary characteristics for each group were characterized with averages and 95\% confidence intervals using statistical software. Results: After meeting all inclusion and exclusion criteria, 123 COM, 70 COD, 88 BRU, $174 \mathrm{HA}$, and 110 UA patients were analyzed (Figure 1). Patient with pure COD, HA and BRU stones were hypercalciuric ( $>150 \mathrm{~g} /$ day) and had higher urinary $\mathrm{pH}$ $(\mathrm{pH}>6.0)$. COM stone formers had a neutral $\mathrm{pH}(6.0+0.1)$ and hyperoxaluria $(45+4 \mathrm{~g} /$ day $)$. Lastly, UA stone formers had aciduria $(\mathrm{pH}<5.8)$, hypernatraturia $(209+16 \mathrm{mEq} /$ day $)$, and hyperuricosuria $(0.66+0.04 \mathrm{~g} /$ day $)$.

Conclusions: The presence of pure stone composition provides insight into certain metabolic derangements. Our results align with prior studies suggesting that a stone analysis represents an aggregate of crystal deposition over a prolonged period and representative of underlying urinary abnormalities. In the absence of a 24-hour urine study, the stone analysis can guide dietary, lifestyle, and medication interventions aimed at preventing future stone events.

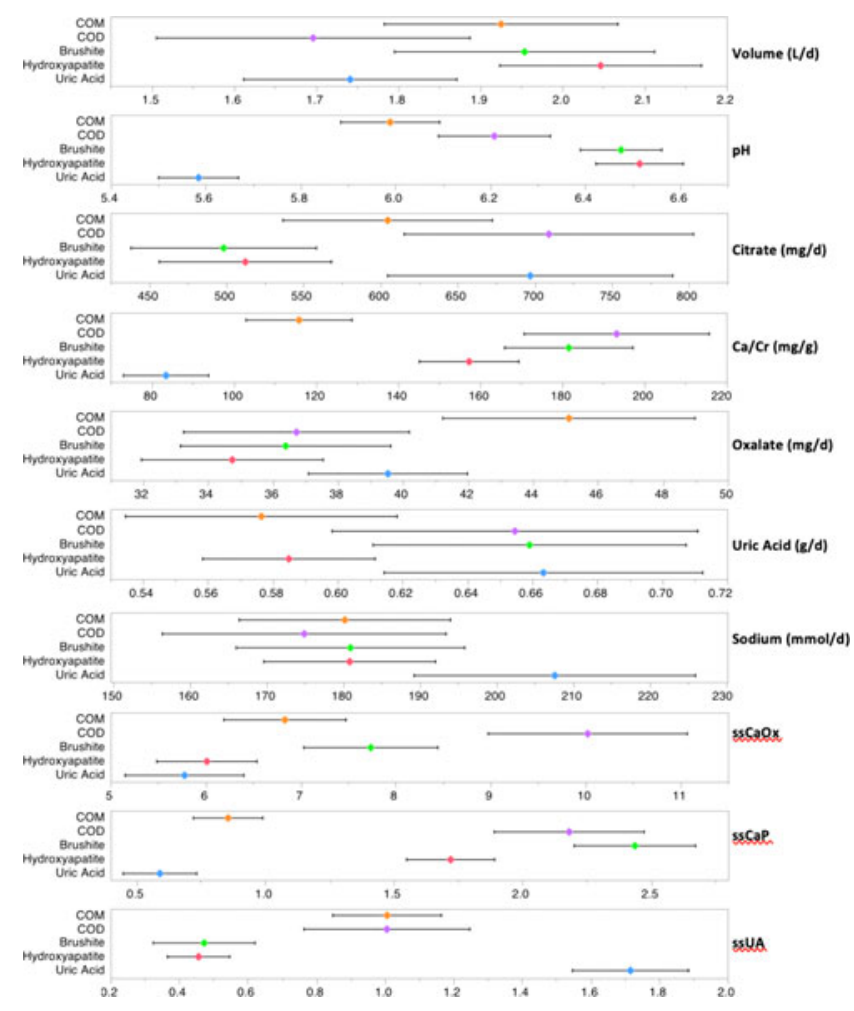


MP04-13 Development of a simple and practical nomogram for predicting stone-free rate after flexible ureteroscopy or percutaneous nephrolithotomy for solitary medium sized renal stones in adults

L Bevilacqua, R Milandri, S Ciarlariello, C Del Prete, C Di Pietro, F fidanza, A Elsherbiny, A Eissa, M Sighinolfi, S Puliatti, G Bianchi, B Rocco, S Micali

Department of Surgical, Medical and Dental Morphological Sciences related to Transplant, Oncology and Regenerative Medicine - Section of Urology, University of Modena and Reggio Emilia, Modena, Italy

Introduction \& Objective: to develop a preoperative nomogram for predicting stone-free rate (SFR) in case of performing flexible ureteroscopy (FURS) or percutaneous nephrolithotomy (PCNL) for patients with solitary medium sized (ranging from 1 to $2 \mathrm{~cm}$ ) renal stones in adults.

Methods: we retrospectively analyzed the data of 100 FURS and 100 PCNL procedures for removal of a solitary medium sized renal stone $(1-2 \mathrm{~cm})$ in adults starting from January 2015 to September 2018. All the patients were evaluated by ultrasound (1 month) and CT scan (6 months) after procedures to detect the stone free rate. The stone-free status was defined as absence of any gravel more than $2 \mathrm{~mm}$. Preoperative predictors, that were statistically significant $(\mathrm{P}<0.05)$ in the univariate logistic analysis, were included in the multivariate logistic regression analysis, which was performed to screen independent predictors for stone free status. Independent predictors $(\mathrm{P}<0.05)$ in the multivariate logistic regression analysis as well as clinically significant predictors were included in the nomogram construction. The nomogram was validated internally by a calibration method and from the area under the receiver operating characteristic (ROC) curve (AUC), the AUC indicate good concordance.

Results: multivariate analyses identified stone site $(\mathrm{p}=0.0146)$, maximum density using Hounsfield unit $(\mathrm{p}=0.0212)$, skin to stone distance $(\mathrm{p}=0.0375)$, associated urinary tract infection $(\mathrm{p}=$ $0.0002)$, comorbidities $(\mathrm{p}=0.0029)$ and age $(\mathrm{p}=0.0403)$ as independent predictive factors for the stone-free status after shock wave lithotripsy. Based on these parameters, we developed a nomogram to predict SFR after FURS and PCNL. Total nomogram score (maximum 11) was derived from summing individual scores of each variable; a high total score was predictive of successful outcome; a low total score was predictive of unsuccessful outcome. The area under the receiver operating characteristics for nomogram predictions was 0.72 for PCNL, 0.86 for FURS and 0.76 for both procedures.

Conclusions: this nomogram can be considered as feasible and reliable tools to predict SFR after FURS and PCNL treatment of solitary medium sized renal stones in adults.

MP04-14 Novel technique for residual stones removal: second-look flexible nephroscopy in gas (CO2) medium

D Korolev, L Rapoport, D Tsarichenko, D Enikeev, M Enikeev, P Glybochko

Institute for Urology and Reproductive Health, Sechenov University

Introduction \& Objective: The residual stones problem is still relevant, especially in group of patients who has undergone
PCNL for complex forms of stone disease. Flexible second-look nephroscopy in liquid irrigation medium significantly increases the cost of percutaneous surgery and also may increases complication rate associated with high intrarenal pressure especially for infected stones. To evaluate safety and efficacy of secondlook flexible nephroscopy in gas (CO2) medium for patients with residual stones after previous PCNL.

Methods: There were a total of 22 patients, mean age was $51 \pm 14$ years. 13 patients had staghorn stones and other 9 patients had large multiple stones with stone size $23.7 \pm 2.4(20-28) \mathrm{mm}$. Conventional PCNL was performed with standard technique using a 30 Fr Amplatz sheath and ultrasound lithotripsy. The mean residual stone size and stone density were $8.3 \pm 2.7(5-17) \mathrm{mm}$ and $1019 \pm 245$ (485-1483) HU respectively. Second-look flexible nephroscopy in gas (CO2) medium using existing nephrostomy tract was performed on postoperative day 3. Irrigation was applied as gas (CO2) medium which was pumped by laparoscopic insufflator. To assess the impact of gas (CO2) medium we control acidbase balance $(\mathrm{ABB})$ immediately before the operation, during it and 3 hours after its completion as well. Stone removal was performed by nitinol baskets. In one case we performed Holmium YAG Laser Lithotripsy for $17 \mathrm{~mm}$ residual stone after pre-filling pelviocaliceal system (PCS) with saline. In 2 cases we failed to blow PCS with gas (CO2) medium because of the latter high rigidity and we had to continue nephroscopy with saline.

Results: The mean second-look flexible nephroscopy time was $42.7 \pm 14.4 \mathrm{~min}$. No postoperative complications were experienced. No stone migration was evolved. Blood $\mathrm{pH}$ during the operation and 3 hours after did not go beyond the limits of acceptable values $(7,35-7,45)$ and remained normal in all patients. Stone-free rate was $86.3 \%$ at 1,5 -mo follow-up (low dose CT). Conclusions: Second-look flexible nephroscopy in gas (CO2) medium is an effective and safe method for residual kidney stones removing. Benefits of this technique are as follows: relative cheapness due to lower cost of gas (CO2), and low migration rate that reduces operating time. Low pelvic pressure allows to eliminate the risk of pyelovenous reflux and subsequent septic complications. Limitations of the technique are related with PCS rigidity, making difficult to adequately fill it with gas and also and inability to use laser energy in gas (CO2) medium.

\section{MP04-15 What factors drive staghorn versus non- staghorn pattern growth in patients with metabolic stones?}

JS Winoker, JN Bamberger, RA Chandhoke, A Kapoor, H Kwun, W Atallah, M Gupta

The James Buchanan Brady Urological Institute, Johns Hopkins University School of Medicine

Introduction \& Objective: It is unknown why some large metabolic calculi form as solitary or multiple separate, non-branching calyceal stones while others develop as staghorn stones forming a cast of the collecting system. We sought to compare these two groups of metabolic stone formers (SF) in an attempt to shed light on these disparate stone-forming phenomena.

Methods: From January 2017 to September 2018, 190 patients underwent percutaneous nephrolithotomy for stones $>2 \mathrm{~cm}$. We identified $86(45 \%)$ patients with a metabolic stone, defined as $\geq 80 \%$ calcium oxalate monohydrate or dihydrate and/or calcium phosphate. Exclusion criteria included stones composed of cystine, $>20 \%$ uric acid, or any infectious element (struvite or carbonate apatite). Metabolic staghorn and non-staghorn SF were compared with respect to medical comorbidity, 24-hour urine 


\begin{tabular}{|c|c|c|c|}
\hline Characteristic & $\begin{array}{l}\text { Staghorn } \\
(\mathrm{N}=25)\end{array}$ & $\begin{array}{c}\text { Non-Staghorn } \\
(\mathrm{N}=65)\end{array}$ & $P$-value \\
\hline \multicolumn{4}{|c|}{ Urinary Metabolic Abnormalities } \\
\hline Hypercalciuria & $8 / 18(44.4 \%)$ & $13 / 48(27.1 \%)$ & 0.112 \\
\hline Hyperoxaluria & $11 / 18(61.1 \%)$ & $16 / 48(33.3 \%)$ & 0.041 \\
\hline Hypocitraturia & $7 / 18(38.9 \%)$ & $22 / 48(45.8 \%)$ & 0.558 \\
\hline Hyperuricosuria & $3 / 18(16.7 \%)$ & $8 / 48(16.7 \%)$ & 0.815 \\
\hline Hypomagnesuria & $1 / 18(5.6 \%)$ & $3 / 48(6.3 \%)$ & 0.886 \\
\hline Hypernatriuria & $8 / 18(44.4 \%)$ & $24 / 48(50.0 \%)$ & 0.718 \\
\hline \multicolumn{4}{|c|}{ Mean 24-Hour Urine Parameters } \\
\hline Volume 24 (L/day) & $2.2 \pm 0.9$ & $2.0 \pm 0.9$ & 0.441 \\
\hline $\mathrm{SS} \mathrm{CaOx}$ & $7.5 \pm 2.6$ & $6.7 \pm 3.6$ & 0.737 \\
\hline Calcium 24 (mg/day) & $236.4 \pm 109.8$ & $203.4 \pm 94.7$ & 0.335 \\
\hline Oxalate 24 (mg/day) & $45.3 \pm 19.0$ & $35.8 \pm 12.8$ & 0.040 \\
\hline Citrate 24 (mg/day) & $579.8 \pm 305.4$ & $513.9 \pm 268.2$ & 0.408 \\
\hline SS CP & $0.9 \pm 0.8$ & $1.2 \pm 1.0$ & 0.299 \\
\hline $\mathrm{pH}$ & $5.8 \pm 0.6$ & $6.1 \pm 0.7$ & 0.128 \\
\hline SS UA & $1.2 \pm 1.2$ & $0.8 \pm 0.7$ & 0.220 \\
\hline UA 24 ( $g /$ day) & $0.6 \pm 0.2$ & $0.6 \pm 0.2$ & 0.552 \\
\hline Sodium 24 (mmol/day) & $171.3 \pm 89.1$ & $154.2 \pm 78.4$ & 0.768 \\
\hline Potassium 24 (mmol/day) & $63.0 \pm 21.0$ & $58.2 \pm 22.1$ & 0.867 \\
\hline Magnesium 24 (mg/day) & $117.3 \pm 42.0$ & $89.6 \pm 35.6$ & 0.070 \\
\hline Phosphorus 24 (g/day) & $0.9 \pm 0.4$ & $0.8 \pm 0.3$ & 0.264 \\
\hline Ammonium 24 (mmol/day) & $35.9 \pm 18.6$ & $34.1 \pm 19.1$ & 0.947 \\
\hline Chloride 24 (mmol/day) & $180.6 \pm 86.8$ & $160.8 \pm 76.4$ & 0.692 \\
\hline
\end{tabular}

parameters, stone and urine microbiology, stone compositions, and intraoperative findings. Statistical differences were assessed using chi-square analysis, Fisher's exact test, and student t-test. Results: In total, 25 (29\%) staghorn and 61 (71\%) non-staghorn SF were included for analysis. The groups were statistically similar in age, sex, body mass index, and medical comorbidity. Staghorn SF were more likely to have hyperoxaluria $(\mathrm{p}=0.041)$ and higher mean 24 -urine oxalate levels $(p=0.040)$. There were no other significant differences in 24-hour urine profiles, rates of metabolic abnormalities, stone compositions, stone or urine cultures, presence of collecting system obstruction, or pelvicalyceal anatomy.

Conclusions: Though potentially driven by urinary oxalate, whether a metabolic stone will form into a staghorn configuration or not does not appear significantly influenced by standard determinants of stone development, including metabolic profile, cultures, hydronephrosis, and pelvicalyceal anatomy, among others. Further work is needed to elucidate the physicochemical factors that govern the pathogenesis of this increasingly prevalent entity.

MP04-16 The Effects of a High Sodium Diet on Lithogenesis in an Experimental Rat Model of Renal Calcium Oxalate Stone Formation

Y Hong, Q Xu, X Huang, L An, Z Zhang

Peking University People's Hospital

Introduction \& Objective: The aim of this study was to investigate the effects of a high and low sodium diet on lithogenesis in a rat experimental model of calcium oxalate stone formation. Methods: Twenty male Wistar rats were randomly divided into the following four groups: group $\mathrm{A}-4 \% \mathrm{NaCl}+1 \%$ ethylene

glycol (EG); group B- $8 \% \mathrm{NaCl}+1 \%$ EG; group C- $8 \%$ $\mathrm{NaCl}+$ normal drinking-water; and group D- $1 \% \mathrm{EG}+$ normal diet. The rats were fed in metabolic cages, and their urine volume was recorded every day. All rats were sacrificed four weeks later, and blood samples were collected via cardiac puncture. Kidney samples were collected for Von Kossa staining to evaluate the formation of calcium-containing crystals. The final $24 \mathrm{~h}$ urine samples were also gathered for metabolic analysis.

Results: Von Kossa staining demonstrated that rats in both groups A and B had significantly more renal calcium crystals than those in group D. In addition, the final $24 \mathrm{~h}$ urinary volume of group B was significantly increased $(142.26 \pm 20.91 \mathrm{~mL})$ compared to group $\mathrm{A}(100.52 \pm 28.23 \mathrm{~mL})$, group $\mathrm{C}(107.35 \pm 14.23 \mathrm{~mL})$, and group $\mathrm{D}(40.78 \pm 8.71 \mathrm{~mL})(\mathrm{P}=0.004,0.012$, and 0.000 , respectively). Levels of urine sodium $(\mathrm{Na})$, potassium $(\mathrm{K})$, chlorine $(\mathrm{Cl})$, calcium $(\mathrm{Ca})$, and urea nitrogen of group $\mathrm{B}$ were significantly higher than group D. The levels of urine phosphorus, oxalate, creatinine and the urine specific gravity and urine $\mathrm{pH}$ between group $\mathrm{B}$ and group $\mathrm{D}$ were similar. The concentration of serum sodium of group $B(151.26 \pm 4.06 \mathrm{mmol} / \mathrm{L})$ was higher than that of group $\mathrm{D}(145.56 \pm 1.12 \mathrm{mmol} / \mathrm{L})(\mathrm{P}=0.002)$.

Conclusions: A high salt diet increased urine volume and resulted in urine metabolism changes and promoted stone formation in this rat calcium oxalate lithogenesis model. A lower salt diet may suppress the development of urinary stones.

\section{MP04-17 Defining the Relationship between Urinary Infection and Calcium Urolithiasis: A Potential Novel Role or Osteopontin and Zinc Transport}

\section{J Bjazevic, K Al, J Gorla, H Razvi, J Burton}

Western University

Introduction \& Objective: The formation of calcium-based stone disease may be impacted by urinary bacteria. Prior epidemiological studies have demonstrated a strong association between a history of culture-proven urinary tract infections and the development of stone disease. In addition, bacteria can be directly isolated from the majority of pure calcium-based calculi. However, the nature of the relationship between urinary bacteria and stone disease, as well as the potential mechanisms involved, have yet to be elucidated. Osteopontin (OPN) which is a known potent inhibitor of calcium oxalate $(\mathrm{CaOx})$ urolithiasis and zinc (Zn) which is involved as an early nidus for the mineralization process of urinary stones; have both also been shown to be involved in bacterial pathogenesis. We aimed to further investigate the potential effect of a non-urease producing bacteria, OPN, and $\mathrm{Zn}$ on the adherence of $\mathrm{CaOx}$ crystals to renal epithelial cells.

Methods: Crystal adherence assay was performed using MDCK renal epithelial cells grown to $90 \%$ confluence on cell culture plates. Cells were exposed to a non-urease producing strain of Escherichia coli UTI89 $\left(10^{3} \mathrm{CFU}\right)$ at $37 \mathrm{C}$ for 20 mins, and then incubated with $\mathrm{CaOx}$ crystal suspension $(0.5 \mathrm{mg} / \mathrm{mL})$ in artificial urine with or without the addition of OPN $(0.1 \mathrm{mg} / \mathrm{mL})$, or zinc chloride $(500 \mathrm{mg} / \mathrm{mL})$ for an additional 20 minutes at $37 \mathrm{C}$. Unattached crystals were washed free with culture media. Pixel intensity of adherent $\mathrm{CaOx}$ crystals under birefringent microscopy was quantified with MATLAB (2018).

Results: Exposure to UTI89 significantly increased $\mathrm{CaOx}$ crystal adherence compared with controls $(\mathrm{p}<0.001)$. Treatment with OPN and $\mathrm{Zn}$ had opposing effects. OPN significantly decreased crystal adherence and attenuated the effect of UTI89 ( $p<0.001$, figure 1). Whereas $\mathrm{Zn}$ increased adherence of $\mathrm{CaOx}$ crystals and 
A.
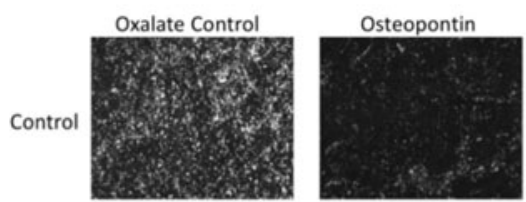

UT189

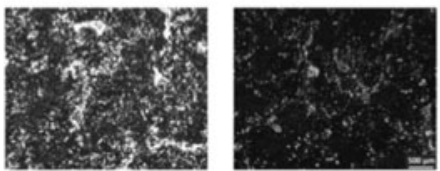

B.

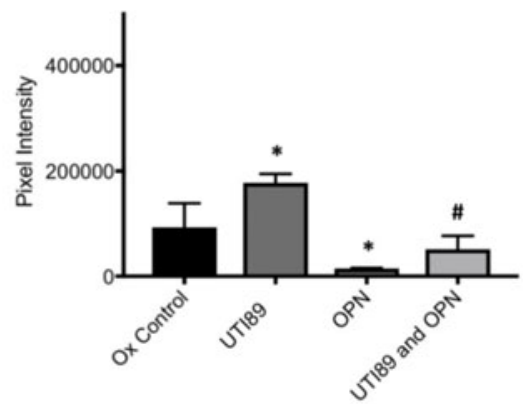

Figure 1: $\mathrm{CaOx}$ crystal adherence to MDCK renal epithelial cells following treatment with $E$. coli UTI89 $\left(10^{3} \mathrm{CFU}\right)$ or osteopontin $(0.1 \mu \mathrm{g} / \mathrm{mL})$ (A) Representative birefringence microscopy images of $\mathrm{CaOx}$ crystals adherent to MDCK cells under 100x magnification. (B) Measured pixe intensity of $\mathrm{CaOx}$ crystal adherence shown as mean $\pm \operatorname{SEM}\left(\mathrm{n}=15 /\right.$ group), ${ }^{*} \mathrm{p}<0.001$ compared with control, \# $\mathrm{p}<0.05$ compared with UTI89.

A.

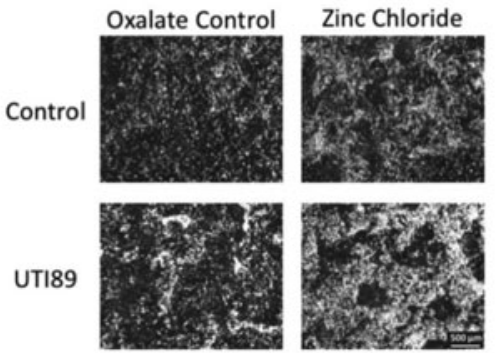

B.

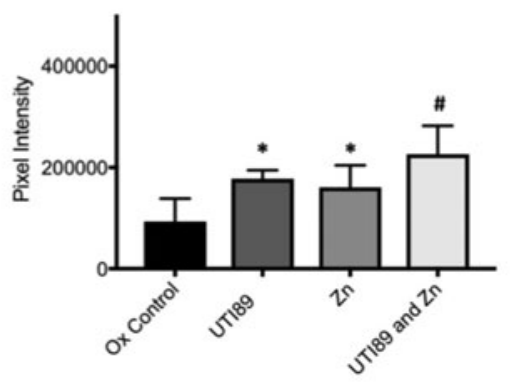

Figure 2: $\mathrm{CaOx}$ crystal adherence to MDCK renal epithelial cells following treatment with $E$. coli UTI89 $\left(10^{3} \mathrm{CFU}\right)$ or zinc chloride $(500 \mu \mathrm{g} / \mathrm{mL})$ (A) Representative birefringence

microscopy images of $\mathrm{CaOx}$ crystals adherent to MDCK cells under 100x magnification. (B) Measured pixel intensity of $\mathrm{CaOx}$ crystal adherence shown as mean $\pm \mathrm{SEM}$ ( $\mathrm{n}=15 /$ group), * $\mathrm{p}<0.001$ compared with control, \# $\mathrm{p}<0.05$ compared with UTI89. further enhanced the observed effect of UTI89 on crystal adherence $(\mathrm{p}<0.05$, figure 2$)$.

Conclusions: Our findings suggest that exposure to a non-urease producing strain of E. coli, as well as OPN and Zn, impact the adherence of $\mathrm{CaOx}$ crystals to renal epithelial cells. In addition, both OPN and $\mathrm{Zn}$ may play a novel role in the relationship between urinary pathogens and the development of calcium stone disease. Additional investigation is required to confirm these results in an in vivo model and further delineate the potential mechanisms involved.

\section{MP04-18 Association of Obesity with Increased En- dogenous Oxalate Synthesis}

K Wood, C Boyd, R Holmes, J Knight, B Gower, D Assimos

University of Alabama at Birmingham

Introduction \& Objective: Urinary oxalate levels are affected by both dietary and endogenous components. Prior studies have demonstrated the positive correlation between weight/body mass index (BMI) and urinary oxalate excretion. Our objective was to determine if this association is secondary to increased endogenous oxalate synthesis.

Methods: Healthy subjects, between 18 and 65 years old, with variable BMI were recruited. Subjects of various BMIs were recruited. Subjects consumed a low oxalate-controlled diet containing $16 \%$ protein, $30 \%$ fat, $54 \%$ carbohydrate, $1000 \mathrm{mg}$
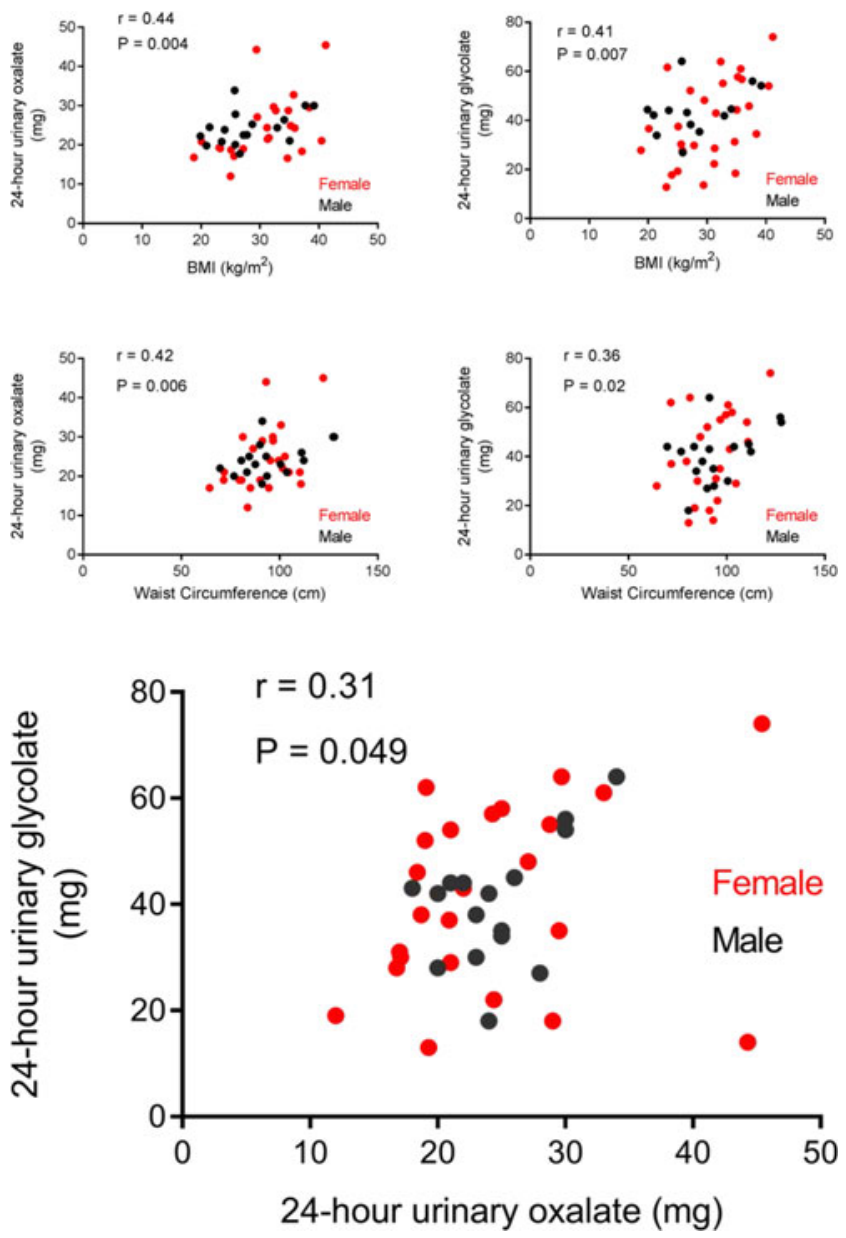
calcium, and $30 \mathrm{mg}$ oxalate which was devoid of vitamin $\mathrm{C}$ and calcium supplements. This diet limits the contribution of diet to the urinary oxalate pool. Subjects remained on this diet for 3 days. 24hour urine collections were performed on the last two days. Urinary oxalate was measured by ion chromatography coupled with mass spectroscopy. Statistical analysis included Chi-squared, correlation and linear regression analysis, and student t-test.

Results: There were 41 subjects recruited with various BMIs (19-42). Urinary oxalate excretion (mg/day) was positively correlated with BMI $(\mathrm{r}=0.44, \mathrm{p}=0.004)$ and waist circumference $(r=0.42, p=0.006)$. Similar correlations were seen with urinary glycolate excretion (mg/day) with BMI ( $r=0.41$, $\mathrm{p}=0.007)$ and waist circumference $(\mathrm{r}=0.36, \mathrm{p}=0.02)$. Urinary oxalate and glycolate excretion were positively correlated $(\mathrm{r}=0.31, \mathrm{p}=0.049)$.

Conclusions: These results demonstrate a positive correlation between urinary oxalate derived from endogenous oxalate synthesis and BMI as well as other measures of obesity. This also provides an explanation for the association between stone risk and obesity.

\section{MP04-19 Clinical Risk Factors that Predict the Known Lithogenic Metabolic Diagnoses in Patients with Kidney Stones}

BO Manzo, JD Cabrera, JE Torres, FC Vicentini, E Emiliani, B Eisner, H Sánchez

Hospital Regional de Alta Especialidad del Bajío

Introduction \& Objective: Risks factors that predispose to kidney stones are well known, but there is limited data about which factors are associated with lithogenic metabolic diagnoses in patients with kidney stones. This study aims to assess which clinical risk factors are associated with a specific urine lithogenic diagnosis in patients with kidney stones.

Methods: All patients with a diagnosis of kidney stones from a referral center were evaluated from February 2014 to April 2019. All patients with a complete metabolic (plasma and urine) evaluation were included. Exclusion criteria were patients with incomplete data or lack of metabolic evaluation. Statistical analysis was performed with Fisher's exact test, and Chi-square test and odds ratios were calculated considering a confidence interval of $95 \%$ as statistically significant.

Results: A total of 3,720 patients were evaluated, 2,606 had a diagnosis of urinary stones, 1,562 patients had a diagnosis of renal stones, and 156 patients were stone free after surgical treatment and had a complete metabolic evaluation. 148 (94.8\%) presented at least one metabolic alteration. Male gender was encountered as a risk factor for hyperuricemia, OR 3.2 (IC 95\% 1.3-7.9), and exhibiting various metabolic alterations, OR 3.0 (IC 95\% 1.2-7.6). Multiple calculi were found as risk factor for presenting hypercalcemia (OR 10.2, IC95\% 1.3-82.4), hypomagnesuria (OR 3.2, IC95\% 1.2-8.7), hyperoxaluria (OR 2.4, IC95\% 1.2-4.5), low urinary volume (OR 2.5, IC95\% 1.03-6.2) and multiple metabolic alterations (OR 5.6, IC95\% 2.1-14.8). Obesity, family history, occupation, and high urinary stone volume were not found as risk factors for any lithogenic metabolic diagnoses.

Conclusions: In patients with renal stones, the presence of multiple calculi is the leading risk factor for presenting various lithogenic metabolic alterations, like hypercalcemia, hypomagnesuria, hyperoxaluria, and low urine volume. These findings are significant because we can perform a directed metabolic assessment instead of extensive and expensive tests. It is necessary to evaluate these factors in patients without renal stones and assess if patients with multiple stones would benefit from empiric treatment.

\section{MP04-20 Indwelling ureteral stents induce ureteral aperistalsis, injury and fibrosis}

\author{
K Scotland, L Wang, BH Chew, C Seow, D Lange \\ University of British Columbia
}

Introduction \& Objective: Ureteral stenting causes symptoms of pain and discomfort in more than $80 \%$ of patients. We propose that pain, in part, may be due to dysfunctional ureteral peristalsis in response to the indwelling stent. Our previous work suggests a role for the Gli family of proteins and erythropoietin in regulating ureteral smooth muscle function. To study these in a more relevant animal model, we investigated the effects of Gli effector proteins, tamsulosin and erythropoietin on ureteral smooth muscle function in stented porcine ureters.

Methods: Pigs were stented for 2-14 days and degree of hydronephrosis, ureteral dilation, and rate of peristalsis were evaluated. Tissues were examined for inflammation by a blinded pathologist. RNA and protein level experiments evaluated expression of potential biomarkers. Ureteral physiology was evaluated by investigating ureteral contractile force.

Results: Stent placement triggered extensive ureteral dilation, aperistalsis and moderate hydronephrosis in 48 hours. Gli1 expression, collagen 4A2 (a marker of fibrosis), clusterin alpha (marker of kidney injury), and cox2 (marker of stretch-induced inflammation) expression increased in stented ureters compared to contralateral unstented ureters. Erythropoietin did not improve peristalsis or contraction force but did decrease non-purposeful spasms seen exclusively in stented ureters. Tamsulosin increased contractile force but did not affect rate of peristalsis in stented ureters as compared to control and contralateral non-stented ureters. Conclusions: Stent placement causes kidney injury and fibrosis, leading to activation of members of the Gli family likely to induce repair. Increased expression of cox 2 suggests that stentinduced ureteral dilation specifically causes stretch induced injury. Regulation of peristalsis in stented ureters is multifactorial. Prophylactic erythropoietin may regulate ureteral stabilization in stretch induced spasming following stent placement while tamsulosin increases contractile force, consistent with previous theories on alpha blocker function in ureteral obstruction. Continuing work will elucidate the role of these agents in coordinating ureteral contractions and combatting stent-induced injury.

\section{MP04-21 Critical role of short chain fatty acids in the prevention of renal calcium oxalate stones}

K Wang, Y Liu, L Cheng, Q Jiang, L Zhou, Y Li, Q Sun, H Li

Department of Urology, Institute of Urology (Laboratory of Reconstructive Urology), West China Hospital, Sichuan University, Chengdu, Sichuan, P.R.C

Introduction \& Objective: Calcium oxalate stone (60\%-90\%) is the most common type of nephrolithiasis, while the etiology is still unclear. Emerging evidence has linked the gut microbiome to nephrolithiasis. Some studies found that Oxalobacter formigenes, a member of the human gut microbiome, can degrade oxalate and thus decrease the level of oxalate in serum and urine. However, several prospective researches indicated that patients with calcium oxalate stones showed no significant change in urinary oxalate after oral administration of $\mathrm{O}$. formigenes. 
Therefore, the objectives of the study were to explore the new gut microbiome and metabolites which may affect the formation of calcium oxalate stones.

Methods: We performed microbiome analysis using $16 \mathrm{~S}$ ribosomal RNA (rRNA) amplicon sequencing on fecal samples from 153 Chinese individuals, including 20 with recurrent renal calcium oxalate stones (RS), 49 with occasional stones (OS) and 84 non-stones (NS) healthy people. The $\alpha$ and $\beta$ diversity analyses were performed. LEfSe analysis was also employed to filter out significant gut bacteria between patients with nephrolithiasis and healthy people. Multi-class linear discriminant analysis (mLDA) was used to build a predictive model of renal calcium oxalate stones. We also predict the metabolic function of gut microbiome with PICRUSt.

Results: RS and OS patients both exhibited higher fecal microbial diversity than controls. We also found significant associations between total community composition and disease status by assessing partial least squares discrimination analysis (PLS-DA). At phylum level, Firmicutes-Bacteroidetes ratio in RS group (3.595) was the highest, followed by OS (2.789) and NS group (2.646). At genus level, the abundance of Blautia, Anaerostipes, Coprococcus-2, Fusicatenibacter, Eubacteriumhallii-group, Fusobacterium, Ruminococcus-1 and Dorea, all producing short chain fatty acids (SCFAs) (like acetic acid), were significantly lower in stone patients' gut microbiome. By mLDA, we found 10 bacteria of great importance and then formed a predicting model based on them. The overall predictive accuracy of renal stone patients and healthy people are $86.9 \%$ after cross validation. The genes and genomes (KEGG) pathways of SCFAs metabolism were higher in NS patients $(492815 \pm 83945)$ than in KS patients $(473121 \pm 88053)(\mathrm{P}<0.001)$.

Conclusions: Our findings revealed association between gut microbiome alterations and renal calcium oxalate stones. SCFAs and their producers (like Blautia) may play an important role in the prevention of renal calcium oxalate stones.

MP04-22 The Impact of Laser Pulse Type on Temperature Changes During Ureteroscopic Laser Activation

B Winship, RS Terry, D Wollin, K Boydston, E Carlos, P Whelan, D Ho, P Zhong, G Preminger, M Lipkin

Duke Urology
Introduction \& Objective: Moses technology is a Holmium:YAG laser pulse delivery system shown to minimize stone retropulsion. This may allow laser lithotripsy at higher power. However, power and heat production are proportional and temperatures capable of tissue injury may be achieved during ureteroscopic lithotripsy. While prior in vitro studies have shown the importance of irrigation and laser activation time, the impact of laser pulse type on temperature has not been evaluated.

Methods: A flexible ureteroscope with $365 \mu \mathrm{m}$ laser fiber in the working channel was placed in a $36 \mathrm{~cm}, 11 / 13 \mathrm{Fr}$ ureteral access sheath inserted into a full $250 \mathrm{cc}$ bag of saline to simulate a normal caliber ureter, renal pelvis reservoir, and antegrade irrigant flow. A thermocouple was placed adjacent to the laser tip, and the laser was fired for $30 \mathrm{sec}$ at $0.6 \mathrm{~J} / 6 \mathrm{~Hz}, 0.8 \mathrm{~J} / 8 \mathrm{~Hz}, 1 \mathrm{~J} / 10 \mathrm{~Hz}$, $1 \mathrm{~J} / 20 \mathrm{~Hz}$, and $0.2 \mathrm{~J} / 70 \mathrm{~Hz}$ at constant irrigation pressure of $100 \mathrm{mmHg}$. We tested 4 runs per setting using short pulse (SP), long pulse (LP), Moses-contact (MC) and Moses-distance (MD) modes. The maximum change in temperature (dT) and time to reach 6 degrees above an adjusted baseline of $37^{\circ} \mathrm{C}$ were compared, and thermal dose was calculated in cumulative equivalent minutes at $43^{\circ} \mathrm{C}$ (CEM43). 120 minutes was used as a threshold for tissue injury.

Results: The dT at $0.6 \mathrm{~J} / 6 \mathrm{~Hz}$ was similar across all pulse types, and the thermal dose remained under the threshold for injury. At $0.8 \mathrm{~J} / 8 \mathrm{~Hz}$, dT for LP was higher than MC $(\mathrm{p}=0.041)$, though CEM43 did not reach the thermal injury threshold and all other pulse types were statistically similar to one another. At $1 \mathrm{~J} / 10 \mathrm{~Hz}$, dT was again higher for LP than MC as well as MD ( $p=0.024$, 0.045 ). Thermal dose approached but did not exceed the injury threshold at 116 minutes. No differences in dT were seen between pulse types at $0.2 \mathrm{~J} / 70 \mathrm{~Hz}$ or $1 \mathrm{~J} / 20 \mathrm{~Hz}$. At $0.2 \mathrm{~J} / 70 \mathrm{~Hz}$, all pulse types approached but only MC exceeded the thermal injury threshold. At $1 \mathrm{~J} / 20 \mathrm{~Hz}$, the thermal dose exceeded the injury threshold for all pulse types. Temperatures quickly reached 6 degrees above baseline in most $0.8 \mathrm{~J} / 8 \mathrm{~Hz}$ tests and all conditions over 10 watts of power; pulse length and type did not impact the speed that these temperatures were reached.

Conclusions: Laser pulse length and pulse type impact the production of heat in our ureteral model. Long pulse produced significantly greater maximum temperature changes from baseline at $0.8 \mathrm{~J} / 8 \mathrm{~Hz}$ and $1 \mathrm{~J} / 10 \mathrm{~Hz}$. Fortunately, thermal dose remained safe at these settings. Both long pulse and Moses technology have been shown to reduce stone retropulsion; the latter may confer this advantage with decreased heat production.

\section{MODERATED POSTER SESSION 05: CLINICAL STONES: OUTCOMES (I)}

\section{MP05-01 Integral monitoring system for patients with ureteral stents}

C Solano, A Chica, E Castellanos, A Contreras, C Duarte, J Restrepo, J Meneses, P Villegas, N Fernandez, J Aristizabal, $\mathrm{N}$ Tarazona, A Vasquez

Introduction \& Objective: Ureteral stents are used in urology in different types of treatments. One of the most feared complications is the forgetfulness or not timely withdrawal of them, this occurs in about $12 \%$ of cases, increasing costs, complications and reinterventions, becoming a public health problem. One of the reasons for forgetting is the lack of follow-up or inadequate communication with the patient. The objective of this study is to determine the impact of a integral monitoring system for management of patients with ureteral stents.

Methods: An integral monitoring system for patients with ureteral stent was designed, which is made up of: administrative staff, nurses and urologists from a single institution; through which the patient is educated and the recall date is generated by placing handcuffs on patients. The data were collected prospectively in an encrypted database between the months of January and May of 2019. On the day of stent removal, the handle 
is checked and removed from the patient. Finally, the information is recorded in the encrypted database and the variables of placement date, withdrawal date, indication of ureteral stent placement, if the stent presented threads are analyzed.

Results: A total of 161 patients were collected between January and May of 2019, within the indications for surgery, urolithiasis was $93 \%$, ureteral stricture $3 \%$, stenosis of the UPJ $2.4 \%$, diagnostic ureteroscopy $0.6 \%$, partial nephrectomy $0.6 \%$. In $20.4 \%$, the stent was left with threads. The stents were removed $80 \%$ on the established date, the withdrawal past the date, by $16 \%$, but with a median of days of withdrawal of 7 days. He was not retired at $1 \%$. There was loss of follow-up in $1 \%$, since it was not possible to communicate by telephone with the patients.

Conclusions: This integral monitoring system for patients with ureteral stents is a simple and safe alternative for the timely withdrawal of the same, reducing the incidence of forgotten ureteral stents in institutions with limited resources. More studies are needed to confirm these findings in other institutions.

\section{MP05-02 Withdrawn}

\section{MP05-03 Patient Reported Outcome Measures (PROMs) in patients undergoing flexible ureteroscopy and shockwave lithotripsy for renal stones}

B Starmer, S Tolofari, J Broome, H Garrod, K Agarwal, M Iskander, S Javed, P Kelly, A Samsudin,

H Lazarowicz, R Calvert

Introduction \& Objective: Patient reported outcome measures (PROMs) assess the quality of care delivered from a patient's perspective. There is a paucity of evidence reporting PROMs in treatment of urolithiasis. In this study, we aim to assess the impact of urological stone procedures on patient's perceptions of their morbidity.

Methods: Data was prospectively collected over 12 months using the Cambridge Renal Stone PROM questionnaire. Patients undergoing flexible ureterorenoscopy (fURS) or a first session of shockwave lithotripsy (SWL) for renal stones were asked to complete a pre-operative questionnaire. This questionnaire was repeated at 1, 6 and 12 weeks postoperatively.

Results: A total of 106 patients were collected over the 12-month period. 60 patients underwent SWL with 46 patients having fURS. Mean age was 51 years. Median stone size in the fURS cohort was $10.43 \mathrm{~mm}$. $86 \%$ of fURS patients were stented. Complete stone clearance was $77 \%$. Of the SWL cohort, median stone size was $6.53 \mathrm{~mm}$. Only $3 \%$ of patients had a ureteric stent

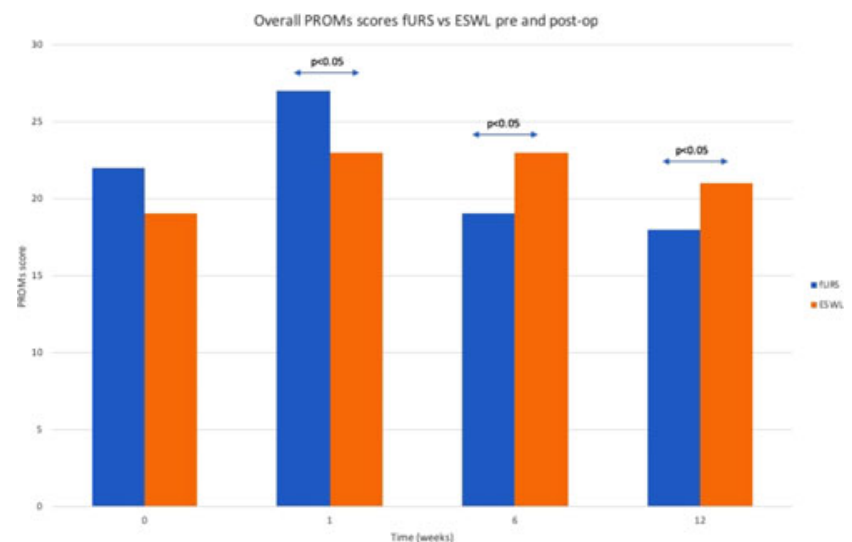

in situ. $74 \%$ of patients required 2 sessions. There were no significant differences in pre-op PROMs scores for both treatment groups. At one week, the fURS PROMs were significantly higher than ESWL $(p<0.01)$. However, at both six and twelve weeks SWL had a significantly higher PROMs scores $(\mathrm{p}<0.05)$.

Conclusions: The fURS cohort has a significantly higher PROMs score at week one, suggesting a higher initial morbidity. However, PROMs scores at six and twelve weeks post-operatively are significantly higher with ESWL. This maybe be due to factors including ureteric stent symptoms in the immediate post-operative period. Repeated sessions with SWL perhaps explain the higher PROMS score at $6 \& 12$ weeks, which may lead to continued symptoms or anxiety associated with repeated treatment.

\section{MP05-04 US versus CT urography in the assessment} of urinary tract stone size - can we trust it?

H Tran, H Pan, H Yao, P McCahy

Peninsula Health

Introduction \& Objective: Computed tomography (CT) urography is considered the gold standard imaging modality for the assessment of urolithiasis. However, it exposes patients to ionizing radiation. This is especially important in young patients, women of child bearing age and patients with recurrent urolithiasis. Some referring clinicians choose to order ultrasound imaging as an alternative imaging modality in the investigation of patients with suspected urolithiasis. This study aims to determine whether the ultrasound measurement of stone size is comparable to the gold standard measurement on CT urography.

Methods: From July 2010 to June 2016, 21,060 ultrasound scans of the renal tract performed at Monash Health were identified. Only patients with an ultrasound scan reported to have urolithiasis and a comparison CT scan performed within one month of the ultrasound were included. Patients were excluded if ultrasound did not report on stone size or if the stone was not visible on CT scan. Demographic data was extracted from electronic medical records. Statistical analyses were performed using SPSS.

Results: A total of 881 sets of USS and CT KUB were included in the study analysis. The median age was 51 and there was a male preponderance of 59\%. Ultrasound stone size was concordant with $\mathrm{CT}$ in $16 \%$ of stones, under-estimated stone size in $21 \%$, and overestimated stone size in $63 \%$. Furthermore, $10 \%$ of stones reported on the ultrasound were not visible on CT. Overall, ultrasound under or over-estimated stone size by $2.0 \mathrm{~mm}$. The median stone size of the study cohort on ultrasound was $8.97 \mathrm{~mm}$ compared with $7.66 \mathrm{~mm}$ measured on CT ( $\mathrm{p}=0.001$, Wilcoxon signed-ranked test $)$. Conclusions: Sonographic urolithiasis stone size is often inaccurate in comparison with CT scan. Ultrasound tends to overestimate the size of urolithiasis and over report the presence of stones and thus cannot be relied upon to guide the management of urolithiasis.

MP05-05 Renal Forniceal Rupture in the Setting of Obstructing Ureteral Stones (FROU): An Analysis of Stone Characterization and Urologic Intervention Pattern

S Setia, P Massie, M Epsten, A Sharma, L Fogg, AK Chow

Rush University Medical Center

Introduction \& Objective: Forniceal rupture due to obstructing ureterolithiasis (FROU) is a seldom encountered radiographic 
finding but the impact of its diagnosis and influence on stone management has not been well studied in the literature. The purpose of the study is to examine stone characteristics and intervention patterns of patients with radiographic evidence of FROU.

Methods: A retrospective analysis of all patients with radiographic evidence of FROU was matched with patients with obstructing ureteral stones without forniceal ruptures (noFROU). All patients presented to our Emergency Department from January 2015 until December 2018. Data analyzed included patient characteristics, clinical presentation, stone characteristics, and management pattern. Primary outcome was need for hospital admission and surgical intervention.

Results: Thirty-two patients with FROU (mean age =45) were compared to fifty patients with noFROU (mean age $=57$ ). Univariate analysis revealed that days of symptoms, history of diabetes mellitus, history of hypertension, degree of hydronephrosis, degree of perinephric stranding, and positive urine culture were associated with forniceal rupture $(p<0.05)$. Upon multivariate analysis, only degree of perinephric stranding remained significant $(\mathrm{p}<0.05)$. Average maximum axial stone diameter in the FROU group was $5.1 \mathrm{~mm}$ vs $4.7 \mathrm{~mm}$ in the noFROU group $(p=0.66, C I-1.2-1.9) .46 .8 \%$ of stones were located within the distal ureter in the FROU group vs $53.3 \%$ in the noFROU group $(\mathrm{p}=0.07)$. There was no difference in hospital admission (FROU 35.3\% vs noFROU 44\%, $\mathrm{p}=0.56$ ) and need for surgical intervention (FROU $50 \%$ vs noFROU $48 \%$, $\mathrm{p}=0.86$ ). There were no 30-day-complications in patients with FROU.

Conclusions: Ureteral stone location and size does not seem to impact the presence of FROU. FROU may be an alarming reported finding but its presence does not appear to impact clinical outcomes or affect urological management including admission or need for urologic intervention.

\section{MP05-06 Immediate and early postoperative compli- cations after ureteroscopy and Ho:YAG laser litho- tripsy for urinary stones: real-life findings from a single center series}

\author{
L Villa, G Fallara, M Menean, A Briganti, \\ A Salonia, F Montorsi \\ Division of Experimental Oncology/Unit of Urology; URI; \\ IRCCS Ospedale San Raffaele, Milan, Italy
}

Introduction \& Objective: The reported complications rate following ureterorenoscopy (URS) in patients treated for stones is low. We assessed the rate of and the predictors of immediate urosepsis and early postoperative complications at 30-day postURS in a homogenous series operated at a single institution.

Methods: Data from 212 patients treated with URS with holmium:YAG laser stone lithotripsy (March 2016 - June 2018) were analyzed. Any preoperative concomitant urinary tract infection (UTI) was treated before surgery in every patient; moreover, every patient received preoperative antibiotic prophylaxis. Rates of and type of early complications over the 30 postoperative days were investigated. Stone-free status was defined as a complete absence of any residual fragments at radiological investigations (ultrasound + abdomen X-Ray or CT scan) at 30 days. Descriptive statistics was applied to detail clinical features of all patients. Logistic regression analyses tested the impact of patients' characteristics (age, Charlson Comorbidity Index (CCI) score, UTI history, stone size) and intraoperative data (presence of DJ stent at surgery, operative time and the use of ureteral access sheath) in terms of rate of immediate sepsis (defined as for the 3rd International Consensus Definitions for Sepsis and Septic Shock) and emergency room (ER) visits at discharge.

Results: Overall, 26 (12.3\%), 7 (3.3\%), 4 (1.9\%) and $1(0.4 \%)$ patients developed postoperative fever, urosepsis, septic shock, and death, respectively. At 30-day postoperative assessment, 19 $(8.9 \%)$ patients accessed to the ER due to pain $(n=8 ; 3.8 \%)$ or fever $(\mathrm{N}=11 ; 5.1 \%)$. Of $19,9(4.2 \%)$ patients were hospitalized and $4(1.8 \%)$ patients required an endoscopic intervention. Stone-free rate at 30 -day was $60.5 \%$. A history of UTI and greater CCI scores were both univariably $(\mathrm{OR}=15.7 ; 95 \%$ $\mathrm{CI}=2.8-83.1$ and $\mathrm{OR}=1.7 ; 95 \% \mathrm{CI}=1.2-2.5$, respectively) and multivariably $(\mathrm{OR}=28.8 ; 95 \% \mathrm{CI}=2.6-326.2$ and $\mathrm{OR}=2.1 ; 95 \%$ $\mathrm{CI}=1.1-3.8)$ associated with a higher risk of immediate postoperative urosepsis. The stone-free status at 30 days post-URS was associated with a reduced risk of an ER visits (UVA: $O R=0,1 ; 95 \%$ $\mathrm{CI}=0.03-0.38$; MVA: $\mathrm{OR}=0.07 ; 95 \% \mathrm{CI}=0,02-0,32$ ).

Conclusions: The risk of having early complications after discharge is not negligible in patients submitted to URS and stone lithotripsy. An optimal surgical stone clearance seems to reduce the risk of subsequent ER visits. Our findings recommend a comprehensive counselling and an accurate monitoring of candidates to URS with either comorbidities or a previous UTI history.

\section{MP05-07 Outcomes of patients who have undergone percutaneous bladder stone removal using a laparo- scopic entrapment bag}

B Gallante, JN Bamberger, RA Chandhoke, W Atallah, E Kaplan-Marans, M Gupta, AM Zampini

\section{Mount Sinai}

Introduction \& Objective: Percutaneous management of large bladder stones with the use of a laparoscopic entrapment bag is a minimally invasive procedure that has advantages over both transurethral and open removal, which can be plagued by longer operative times, residual stones, bladder injury, and hematuria. After being introduced in 2008 , the procedure was refined in 2013 to reduce complications and recovery time. Urethral catheters are used instead of suprapubic catheters to reduce the risk for wound infection and leakage. A sheath is placed inside of the bag rather than outside to allow for continuous flow and quicker stone retrieval. The objective of this study is to examine and compare long term morbidity and complications of the two cohorts: 2008-2012 and 2013-2019.

Methods: 43 male patients underwent this procedure from 20082019. After percutaneous bladder access is achieved, the tract is dilated to $30 \mathrm{~F}$ and stones are captured in a laparoscopic entrapment bag. The opening of the bag is exteriorized and stone fragmentation and comminution are achieved using a nephroscope and pneumatic or ultrasonic lithotripters. After extracting the bag, either a 22F SP tube is inserted into the bladder, or the wound is closed and a $16 \mathrm{~F}$ urethral catheter is placed for drainage. Operative and follow up parameters were compared between the 2 cohorts.

Results: All patients were rendered stone free. Procedure time was longer in the SP tube group $(\mathrm{p}=.001)$. Length of stay was shorter in the catheter group $(\mathrm{p}=.001)$, with most patients leaving the hospital the day of the procedure. There were no complications of fluid extravasation, hematuria, or urethral trauma. The SP group had higher rates of leakage from the SP site ( $25 \%$ vs $0 \%$ ), prolonged need for drainage over 5 days ( $18.8 \%$ vs 


\begin{tabular}{|l|c|c|c|}
\hline \multicolumn{3}{|c|}{$\begin{array}{l}\text { Table 1: SP Tube vs Catheter in Patients who have undergone Percutaneous Bladder Stone } \\
\text { Removal }\end{array}$} \\
\hline $\begin{array}{c}\text { SP tube (2008- } \\
\text { 2012) }\end{array}$ & $\begin{array}{c}\text { Foley Catheter } \\
\text { (2013-2018) }\end{array}$ & p-value \\
\hline DEMOGRAPHICS & 16 & 27 & \\
\hline Number of Participants & 71 & 68 & 0.218 \\
\hline Mean Age & & & \\
\hline OPERATIVE DATA & 112.56 & 57.63 & $0.001^{*}$ \\
\hline Mean Procedure Time (mins) & 25.31 & 16.37 & 0.486 \\
\hline Mean EBL (mL) & 3.50 & 3.93 & 0.781 \\
\hline Mean Number of Stones & 6.22 & 4.70 & 0.278 \\
\hline Mean Stone Burden & & & \\
\hline FOLLOW UP DATA & 1.00 & 0.19 & $0.001^{*}$ \\
\hline Mean Length of Stay (days) & 139.64 & 68.45 & 0.296 \\
\hline Mean PVR & N/A & $3.12(1.6)$ & N/A \\
\hline Mean Number of Days with Catheter (SD) & 46 & 15 & $<.001^{*}$ \\
\hline Length of follow up (months) & \multicolumn{3}{l}{} \\
\hline Analysis conducted using ANOVA, * denotes significance & & & \\
\hline
\end{tabular}

$0 \%)$, and recurrent retention $(12.5 \%$ vs $7 \%)$. There was one complication in each group requiring an ER visit: acute retention in the SP group and a dislodged catheter in the catheter group. Mean follow up was longer in the SP group (46 vs 15 months). Conclusions: The use of a urethral catheter after percutaneous entrapment bag bladder stone removal leads to fewer complications and reduces the length of stay, making this an outpatient procedure. This is a safe and easy technique that can reduce morbidity during the management of large bladder stones.

\section{MP05-08 Utilizing da Vinci ${ }^{\circledR}$ Robotic Surgical System to Treat Challenging Urinary Stone}

\section{Crigger, MW Salkini}

Introduction \& Objective: A worldwide mounting in the incidence and prevalence of urolithiasis has been observed. The standard treatment of urologic stone disease (USD) has changed from open surgery to extracorporeal shock wave lithotripsy (ESWL), percutaneous nephrolithotomy (PCNL) or ureteroscopy depending on the size and location of the stone. Open stone surgery is reserved for challenging urinary stone (CUS) that is not amenable to the above-mentioned techniques. We utilized de Vinci robotic surgical system to treat CUS. We are sharing our experience in utilizing Da Vinci ${ }^{\circledR}$ robotic surgical system to treat patient with urolithiasis instead of open surgical approach.

\begin{tabular}{|c|c|c|}
\hline Indication of RSS & number & Percentage \\
\hline Morbid Obesity & 8 & $31 \%$ \\
\hline Need for other surgery & 8 & $31 \%$ \\
\hline Inability to Position & 2 & $8 \%$ \\
\hline Failed PCNL & 4 & $15 \%$ \\
\hline Large Diverticular Stone & 4 & $15 \%$ \\
\hline \multicolumn{2}{|c|}{ Variables } & values \\
\hline \multicolumn{2}{|c|}{ Mean BMI } & $37.4(21-64)$ \\
\hline \multicolumn{2}{|c|}{ Mean Age } & 52.9 \\
\hline \multicolumn{2}{|c|}{ Female/male } & $9 \mathrm{~F} / 12 \mathrm{M}$ \\
\hline \multicolumn{2}{|c|}{ Mean ASA score } & $2.65(1-4)$ \\
\hline \multicolumn{2}{|c|}{ Mean Stone Volume } & $19 \mathrm{~cm}^{3}(9-32)$ \\
\hline \multicolumn{2}{|c|}{ Operative Time } & 198 minutes $(120-240)$ \\
\hline \multicolumn{2}{|c|}{ Console Time } & 155 minutes $(75-180)$ \\
\hline \multicolumn{2}{|c|}{ Stone Free Rate } & $26(90 \%)$ \\
\hline \multicolumn{2}{|c|}{ LOS } & 2.0 days $(1-8)$ \\
\hline \multicolumn{2}{|c|}{ Complications } & $5(17 \%)$ \\
\hline
\end{tabular}
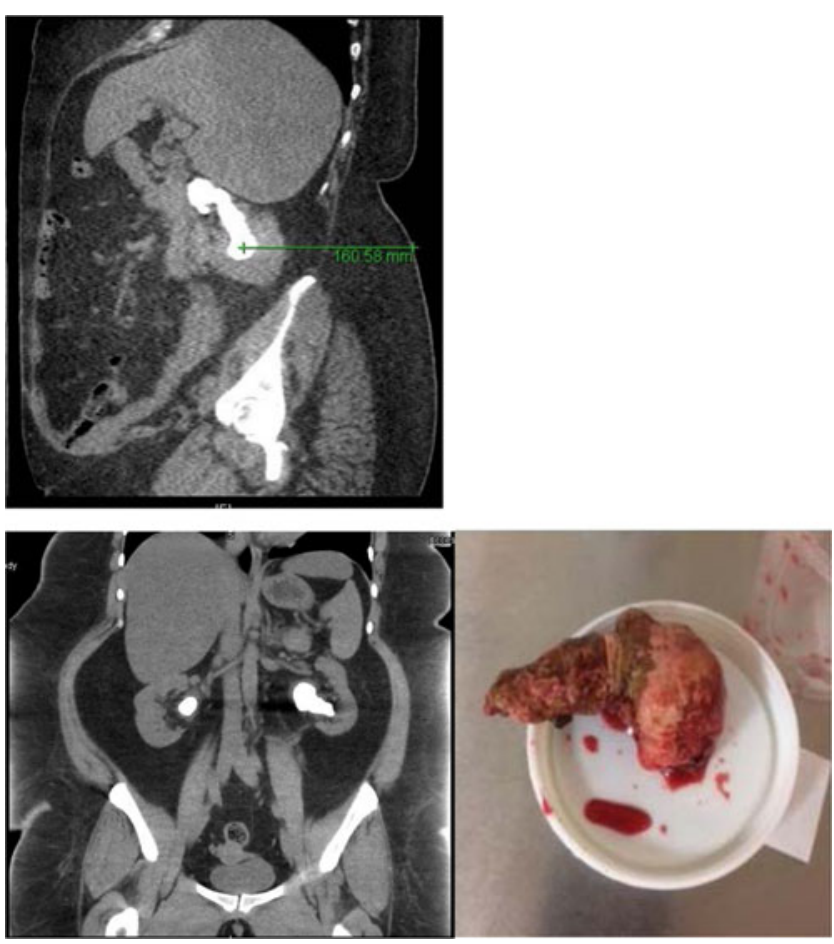

Methods: We reviewed prospectively collected data of 26 patients who underwent robotic assisted stone surgery (RSS) between January 2010 and March 2019 at our institute for USD involving 29 nephroureteral units.

Results: A total number 29 RSS were accomplished with no conversion to open. 3 patients had bilateral stone and needed to have RSS on each side separately. 13 RSS were performed on the right. The indications for RSS included: morbid obesity $(n=8$, mean BMI $56.4 \mathrm{~kg} / \mathrm{m} 2)$, need for concurrent renal surgery $(\mathrm{n}=8)$ severe contractures limiting positioning for retrograde endoscopic surgery or PCNL $(n=4)$, symptomatic calyceal diverticular stone with failed endoscopic approach $(n=4)$ and inability to position the patient $(n=20) .26$ nephroureteral unit $(90 \%)$ were rendered stone free on the first attempt with complication occurring after 5 surgeries $(17 \%)$.

Conclusions: RSS is viable options in the treatment of CUS with high success rate and low risk of complication. The need for open stone surgery was eliminated by RSS at our center. Multiple centers need to participate in RSS with bigger number of patients to create an established role for RSS.

\section{MP05-09 Does long-term relative renal function im- prove after intervention for chronic ureteric ob- struction? - a case review of 268 adults}

Z Low, V Arumuham, S Allen, L Davis, J Bomanji, R Smith

Institute of Urology, University College London Hospitals NHS Foundation Trust

Introduction \& Objective: Unilateral renal function often deteriorates with chronic ureteric obstruction. Our objectives were to determine the change in relative renal function (RRF) by Technetium-99m MAG3 renography after intervention for ureteric obstruction, and to identify any clinical/epidemiological factors which influence long-term outcomes.

Methods: We identified 268 patients who had MAG3 renography before and after intervention for unilateral ureteric 


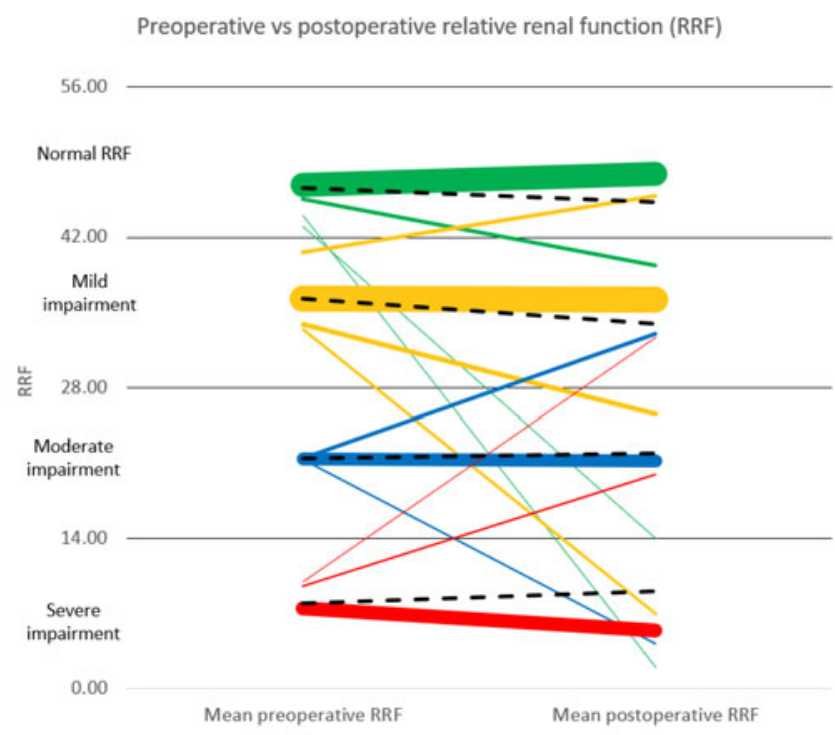

Figure 2 Mean preoperative vs postoperative RRF stratified into categaries; widths of lines are proportional to number of patients. Dotted lines show the overall trend within each cotegory. RRF, relative renal function

obstruction. Patients were divided into categories with normal preoperative RRF (43-57\%), mild (29-42\%), moderate (15-28\%) and severe $(<15 \%)$ impairment of preoperative RRF in the affected kidney. Patient demographics, type of obstructive uropathy and type of intervention employed were analyzed. Each group was assessed both for the absolute change in RRF and change in RRF category postoperatively.

Results: The mean patient age (SD) was 50.5 years (16.5), with $69.1 \%$ being female. Overall, the mean pre- and postoperative RRF did not differ significantly (32.54 [13.6]\% vs 31.84 [14.8]\%, $\mathrm{P}=0.181)$. The majority of patients remained in their preoperative RRF group: $82.3 \%$ of normal, $67.3 \%$ of mild, $65.3 \%$ of moderate and $76.9 \%$ of patients with severe RRF impairment. Patients with mildly impaired preoperative RRF showed a significant worsening postoperatively $(36.30 \%$ vs $33.96 \%, \mathrm{P}=0.007)$. The other three groups showed no significant change in RRF postoperatively. Logtransformed RRF similarly did not differ significantly before and after intervention ( 1.01 vs $1.02, \mathrm{P}=0.67)$. When patients were stratified by preoperative RRF, no group showed significant improvement in log-transformed RRF postoperatively. Multivariate logistic regression analysis showed receiving a nephrostomy (OR 10.52, 95\% CI 1.60-69.13) or endometrial ablation/surgery (OR 33.794, 95\% CI 0.81-6.23) were positive predictors of improvement in RRF category postoperatively.

Conclusions: Our results show that RRF does not improve significantly after intervention for ureteric obstruction. The aim should therefore be maintaining existing renal function and relieving symptoms, in which regard the pros and cons of nephrostomy versus stent drainage should be discussed.

\section{MP05-10 To TURP or Not to TURP: Does it Matter When It Comes to Men with Concomitant BPE and Bladder Calculi?}

JN Bamberger, J Chong, A Katims, C Brown, B Gallante, A Kapoor, H Kwun, J Thai, W Atallah, RA Chandhoke, M Gupta, AM Zampini

Mount Sinai

Introduction \& Objective: The objective of this study is to evaluate and compare the longitudinal bladder stone recurrence

\begin{tabular}{|c|c|c|c|}
\hline & $\begin{array}{l}\text { Concurrent TURP } \\
(n=18)\end{array}$ & $\begin{array}{l}\text { Medical Management } \\
(\mathbf{n}=22)\end{array}$ & $p$-value \\
\hline \multicolumn{4}{|l|}{ Demographics and Clinical: } \\
\hline Mean Age (years) & $74.00( \pm 6.60)$ & $67.95( \pm 9.68)$ & 0.0301 \\
\hline Mean BMI & $27.54( \pm 4.65)$ & $29.39( \pm 6.83)$ & 0.3325 \\
\hline Bladder Stone Recurrence* & $33.33 \% n=6$ & $27.27 \% n=6$ & 0.677 \\
\hline Time to Recurrence (months) & $50.43( \pm 33.60) n=6$ & $17.14( \pm 11.21) n=6$ & 0.044 \\
\hline Mean Follow-Up Time (months) & $17.39( \pm 9.43) n=17$ & $20.39( \pm 18.57)$ & 0.516 \\
\hline Prostate Size (grams) & $82.79( \pm 43.19) n=17$ & $71.85( \pm 31.83) n=11$ & 0.477 \\
\hline Intraprostatic Protrusion (mm) & $12.39( \pm 6.03) n=15$ & $19.00( \pm 2.65) n=3$ & 0.086 \\
\hline Max Flow Rate (mL/s) & $7.49( \pm 4.69) n=16$ & $7.09( \pm 3.58) n=14$ & 0.799 \\
\hline Preoperative PVR (cc) & $189.00( \pm 115.1) n=17$ & $145.15( \pm 163.60) n=20$ & 0.361 \\
\hline Postoperative PVR (cc) & $41.59( \pm 37.26) n=17$ & $50.88( \pm 46.79) n=8$ & 0.597 \\
\hline Change in PVR (cc) & 147.41 & 94.27 & $0.001,0.124$ \\
\hline Urinary $\mathrm{pH}$ & $5.97( \pm 0.69) n=17$ & $6.17( \pm 0.91) n=20$ & 0.459 \\
\hline Mean Stone Burden $(\mathrm{cm})$ & $3.59( \pm 2.09) n=11$ & $2.17( \pm 1.23) n=18$ & 0.029 \\
\hline Mean Stone Weight (grams) & $2.15( \pm 2.94) n=17$ & $1.57( \pm 2.40) n=8$ & 0.630 \\
\hline Proportion of Calcium Oxalate Stones" & $76.47 \% n=13$ & $36.36 \% n=8$ & 0.013 \\
\hline Proportion of Uric Acid Stones" & $31.25 \% \mathrm{n}=5$ & $40.90 \% n=9$ & 0.542 \\
\hline
\end{tabular}

rates in men who receive concurrent transurethral resection of the prostate (TURP) with bladder stone removal vs. those who received bladder stone removal only and were medically managed for their benign prostatic enlargement (BPE).

Methods: A retrospective analysis of men who had concurrent TURP with bladder stone removal $(n=18)$ was compared to men who had bladder stone removal but no outlet procedure for BPE $(n=22)$. Differences in clinical features, time to recurrence (TTR) and stone features were assessed to establish longitudinal bladder stone recurrence results.

Results: Men receiving concurrent TURP tended to be older. Overall mean follow-up time was 19.1 months. BMI, Prostatic size, intraprostatic protrusion, max flow rate, preoperative post void residual (PVR), urinary $\mathrm{pH}$ and stone weight were similar between groups. Eighteen patients received concurrent TURP and bladder stone removal procedures, of which there were 6 bladder stone recurrences (TTR: 50.4 months, range: 12-103 months). In the medical management group, $6 / 22$ patients developed recurrences despite maximal medical management (TTR: 17.1 months, range: 7-36 months). Change in PVR was higher after TURP compared to medical management $(\Delta 147.41$ vs $\Delta 94.27 \mathrm{cc}, \mathrm{p}<0.001, \mathrm{p}=0.124)$. TTR was greater in the TURP group (50.4 vs 17.1 months, $\mathrm{p}=0.044)$. Patients receiving concurrent TURP had greater stone burden $(3.59$ vs $2.17 \mathrm{~cm}$, $\mathrm{p}=0.029)$ and were more likely to have calcium oxalate predominate stones $(76.5 \%$ vs $36.4 \%, \mathrm{p}=0.0127)$ (Table 1$)$.

Conclusions: While prostatic reduction surgery was previously recommended with the diagnosis of bladder calculi, medical management can be equally effective in preventing recurrence. TURP is useful in reducing post-void residual and may lengthen time before bladder stone.

MP05-11 Stage II percutaneous nephrolithotomy combined retrograde intrarenal surgery under lateral lithotomy position for residual renal stones after percutaneous nephrolithotomy

$\mathrm{X}$ Lin

Department of Urology Hong Kong University Shenzhen Hospital

Introduction \& Objective: We sought to describe and evaluate the effectiveness and complications related to Stage II 
percutaneous nephrolithotomy (pcnl) combined retrograde intrarenal surgery (rirs) under lateral lithotomy position for residual renal stones after pcnl.

Methods: Retrospective record review of patients performed Stage II combined rirs under lateral lithotomy position for residual renal stones after pcnl between January 2016 to April 2019. We first place the patient lateral position under general anesthesia, and then use the Allen boot-type stirrups to fix the legs for lateral lithotomy position. One doctor use flexible ureteroscopy via the urethra and the other doctor use the rigid ureteroscopy through the original pcnl channel. We use the 200um holmium laser fiber to crush the stones and take out the stones through the original pcnl channel.

Results: 20 patients were performed the operation smoothing for 22 times. the stones of 5 patients have been cleaned. 15 patients were effectively reduced the stone load $40 \%$ to $90 \%$. There were no inoperative or postoperative complications.

Conclusions: Stage II percutaneous nephrolithotomy combined retrograde intrarenal surgery under lateral lithotomy position for residual renal stones after percutaneous nephrolithotomy is effective and low risk.

MP05-12 Outcome of Mini PCNL for large renal stones in $\mathbf{7 2}$ patients with spinal deformities

DA Rajyaguru

Introduction \& Objective: Patients with spinal deformity (SD) pose special challenge in stone management due to altered anatomy and compromised cardiorespiratory reserve. Retrograde intrarenal surgery (RIRS) though preferred method, is not viable options in remote areas of India where stone bulk is high and affordability is an issue. We have studied mini PCNL (mPNL) in 72 patients with SD to look for its safety and feasibility in large renal stones.

Methods: We have studied 72 patients with spinal deformities (between Sep2000 to Sep'18) with a stone $>1.5 \mathrm{~cm}$ (single or multiple). Spinal deformities were- varying amount (scoliosis, kyphosis, lordosis and kyphoscoliosis). Stone size was calculated with the help of CT IVP after confirming their operative fitness. Exclusion criteria were - children and elderly population, unfit for surgery. General anesthesia was used in all, after putting open end 5 Fr ureteric catheter in lithotomy, patient was tuned prone by appropriate padding. Fluoroscopy guide puncture was done and tract dilated over hydrophilic $0.35 \mathrm{f}$ guide wire and tract dilated upto $14 \mathrm{~F}, 16 \mathrm{~F}$ or $18 \mathrm{~F}$ dilators (mPCNL). Stone was fragmented to dust with the help of the $30 \mathrm{wt}$ holmium laser 365 fiber as wells as mini pored lithoclast. After the procedure, 5.0Fr double $\mathbf{J}$ stent and percutaneous drain was put. Post-operative $\mathrm{X}$ ray was done on 1st day after bowel preparation. Patient was discharged on 2nd day.

Results: Out of 72 patients, males were 55(76.3\%). Mean age of the patients was $45 \mathrm{yrs}$ (32-62 yrs). Stone position was pelvis 35 $48,61 \%$, Pelvic + calyx $-23(32.22 \%)$ cases, only calyx 14 cases (19.44\%). Four patients were complete staghorn. Mean operative Time was: 50-minute (20-120) min. Complete clearance was 66 $(91.66 \%)$, remaining required relook $\mathrm{mPCNL}$ for clearance. Minor complications in 9 patients $(12.5 \%$ ) (minor bleeding in 6 , culture positive infection in 3) occurred 11 (15.2\%), major complications in 9 patients $(12.5 \%)$ (bleeding required transfusion in 5 patients, persistent urine leak from surgery site in 2, hydrothorax in 1and colonic rupture in 1).

Conclusions: The mPCNL is safe effective in patients with spinal deformities and large renal stones. Stringent patient selection and pre-operative stone mapping is very important. Fi- nancial conflicts and Disclosure statement - None Source of funding - None

\section{MP05-13 Early Surgical Intervention for Sympto- matic Renal and Ureteral Stones Reduces Narcotic Requirement Relative to Medical Expulsive Therapy}

C Valadon, CU Nottingham, T Large, A Krambeck

University of Louisville School of Medicine

Introduction \& Objective: Given the substantial burdens of the current opioid crisis, clinicians are tasked with reducing excessive narcotic analgesia. The purpose of this study was to evaluate if medical expulsive therapy or initial surgical intervention resulted in less narcotic analgesia utilization in patients with acute renal colic due to stone disease.

Methods: We retrospectively evaluated patients at our institution who presented with acute renal colic due to a renal or ureteral stone. We excluded patients who required surgical intervention for acute kidney injury or infection. Patients are standardly offered medical expulsive therapy (MET) or surgical intervention with ureteral stenting or ureteroscopy (URS) at the time of diagnosis. Our standard practice following surgery is to provide no narcotics upon discharge except for pain refractory to non-narcotic analgesics. We compared rates of narcotic prescription over the entire treatment course for patients electing MET versus surgery at initial diagnosis. We secondarily analyzed rates surgical intervention among initial MET patients.

Results: We included 143 patients for analysis, with $78(55 \%)$ electing MET as initial treatment, 40 (28\%) stent, and $25(17 \%)$ URS. Ultimately, 46 (59\%) MET patients underwent URS at a median time of 18 days (IQR 8-33 days) from diagnosis. A significantly higher proportion of MET patients required a narcotic prescription (59\% vs $30 \%$ vs $32 \%$, respectively; $\mathrm{p}=0.006$ ) compared to patients electing initial stent or URS.

Conclusions: Patients electing initial treatment with MET for renal colic due to stone disease were more likely to require a narcotic prescription than patients electing initial surgical intervention.

\section{MP05-14 The Impact of the Number of Lifetime Stone Events on Quality of Life: Results From the North American Stone Quality of Life Consortium}

S Tapiero, L Limfueco, K Osann, Z Okhunov, RM Patel, JA Antonelli, NM Streeper, KL Penniston, SY Nakada, S Sivalingam, DP Viprakasit, T Chi, VG Bird, VM Pais, RL Sur, S Andonian, NE Canvasser, BH Chew,

J Landman, RV Clayman

University of California, Irvine

Introduction \& Objective: Urolithiasis is associated with a significant negative impact on patients' health-related quality of life (HRQOL). However, for many people urolithiasis is an acute one-time event and not a series of chronic recurring events. We sought to evaluate the impact of chronic stone recurrence on an individual's HRQOL using the validated Wisconsin Stone Quality of Life (WISQOL) in a multi-institutional study.

Methods: A total of 2,665 patients from 14 institutions in North America were prospectively enrolled and completed the WISQOL questionnaire between June 2014 and September 2018. The 28-question survey has a 1-5 point scale for each item (total score range 0-140) and is divided into 4 domains; D1 social 
Figure 1. Correlation between number of stone events and total WISQOL score

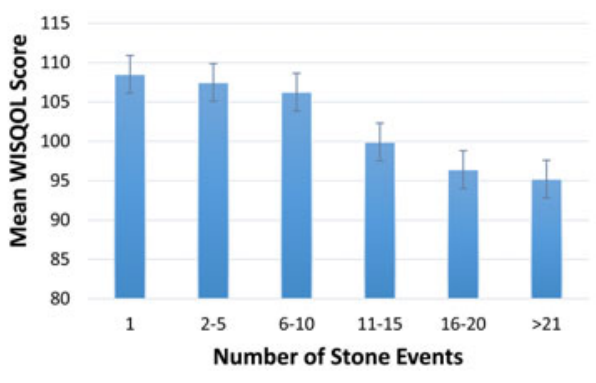

functioning, D2 emotional functioning, D3 stone-related impact, and D4 vitality. Demographic data, number of stone events, and WISQOL scores were recorded. A stone event was defined as renal colic, stone-related procedure, or emergency department visit. Regression analyses with both univariate and multivariate analyses were performed using general linear models to determine the impact of the number of stone events on quality of life. Results: Patients were 51\% males, had a mean age of 54.1 \pm 14.4 years, and had a mean of $7.4 \pm 16.4$ lifetime stone events. There were 606 patients $(23 \%)$ with a single stone event. The mean age at first stone event was $41.6 \pm 16.6$ years. Mean total score for all urolithiasis patients was $101.5 \pm 28.9$. On univariate analysis, WISQOL score decreased significantly with number of stone events, younger age, younger age at first stone event, higher $\mathrm{BMI}$, increasing number of comorbidities, female gender, and stone-related symptoms. On multivariate analysis, after adjusting for other covariates, the number of lifetime stone events was a significant predictor of lower WISQOL score $(p=0.008$, Figure 1). While there was no difference in quality of life among patients with 1 versus 2-5 stone events, WISQOL score declined significantly across all 4 domains beyond 5 stone events (D1: 31.1 vs. 30 , D2: 24.4 vs. 23.5 , D3: 28.2 vs. 27.3 , D4: 10 vs. 9.6 , and total score 102.5 vs. $98.8, \mathrm{p}<0.05$ ).

Conclusions: The cumulative number of lifetime stone events was associated with a lower quality of life. The negative impact on the quality of life was most pronounced after 5 stone events.

\section{MP05-15 Justifying CT scans for sub $5 \mathrm{~mm}$ renal stones identified on ultrasound scans}

MM Quraishi, B Osman, OA Ramadan, I Ahmed, E Latif, J Dorrell, A Simoes, G Papadopoulos, J Hale, N Shrotri

Department of Urology. Kent \& Canterbury Hospital. East Kent Hospitals University NHS Foundation Trust

Introduction \& Objective: The accuracy of ultrasound for renal stones varies significantly depending on the operator, machine, size and location of stone. It still remains the first line investigation for renal stones in many health care systems. Sub $5 \mathrm{~mm}$ renal stones have a very high likelihood of spontaneous passage based on evidence. We begin to question the logic of performing a CT Scan to follow up ultrasound scans for small renal stones. What benefit does a CT scan add to the ultrasound scan for sub $5 \mathrm{~mm}$ renal stones.

Methods: The stone patient database at our district general hospital was reviewed over a 12-month consecutive period to identify patients who underwent a CT scan following ultrasound findings of renal tract stone disease. One hundred and sixty-three patients were identified during the study period to have an ultrasound scan followed up with a CT scan. Medical records were reviewed to assess the findings of stone disease between the imaging modalities. Analysis was performed following database creation.

Results: Of the 163 patients identified to have stone disease on the USS, $50(31 \%)$ did not have any stone findings on the CT Scan, $25(15 \%)$ had stone/calcification findings adding to $4.6 \mathrm{~mm}$ which would have likely been managed conservatively even if led to ureteric colic. Eighty-eight $(54 \%)$ of patients who had ultrasound findings of stone had a stone burden of more than $5 \mathrm{~mm}$. Overall comparable findings of stone disease between USS and CT was seen in only $29 \%$. Of the 28 patients identified to have stone size less than $5 \mathrm{~mm}$, a CT scan confirmed comparable findings on 3 patients, over-reporting by the USS in 19 patients, and under-reporting in 3 patients. The CT scan found no stones in 15 of the 28 patients (54\%), identified to have sub $5 \mathrm{~mm}$ stones on USS. Overall analysis revealed that 19 of the 28 patients $(68 \%)$ either had no stones, or stones small enough to warrant conservative management.

Conclusions: Urology clinics tend to be overwhelmed with findings of small stones. Expectations often result in clinicians investigating small renal stones with CT scans, at the expense of radiation without additional benefit in the management of patients. We reveal that in over $2 / 3^{\text {rd }}$ of our cohort of patients with sub $5 \mathrm{~mm}$ stones, performing a CT scan would not have changed out stance from a conservative management. We may consider community follow up USS or virtual follow up clinics as means of minimizing unnecessary burden on the healthcare systems, and radiation to the patient for sub $5 \mathrm{~mm}$ stones.

MP05-16 Endourological management (PCNL, URS, SWL) of stones in solitary kidney - A systematic review from European Association of Urologists (EAU) Young Academic Urologists (YAU) and UroTechnology (ESUT) groups

T Reeves, A Pietropaolo, T Tailly, M Oezsoy, P Kallidonis, OM Aboumarzouk, A Skolarikos, E Liatsikos, BK Somani

Introduction \& Objective: Urolithiasis in the solitary kidney (SK) presents significant management dilemma as any insult to the kidney or its drainage can lead to significant morbidity. The treatment options includes shock wave lithotripsy (SWL), percutaneous nephrolithotomy (PCNL) and ureteroscopy (URS). Our aim was to conduct a systematic review of literature on all available endourological techniques reporting on stones in a solitary kidney.

Methods: We conducted a systematic review according to Cochrane and PRISMA checklist for all English-language articles from inception to December 2018. All studies with a minimum of 10 patients that reported on endourological management (SWL, PCNL or URS) were included. Data was extracted for patient and stone demographics, outcomes including adverse events and renal function.

Results: After an initial search of 553 papers, 27 were included for the final review (10 PCNL, 1 mini-PCNL, 9 URS, 1 SWL and 6 comparative studies). The choice of treatment seems to be based on stone size with PCNL, URS and SWL offered for mean stone sizes between $25-50 \mathrm{~mm}, 10-28 \mathrm{~mm}$ and $12-15 \mathrm{~mm}$ respectively. PCNL, URS and SWL were reported in 1445, 792 and 186 patients respectively with a final SFR of $67-97.7 \%, 43-100 \%$ and $73-80 \%$ and a complication rate of $26.4 \%, 15 \%$ and $16.7 \%$ across the three groups. The renal function deterioration was reported in 

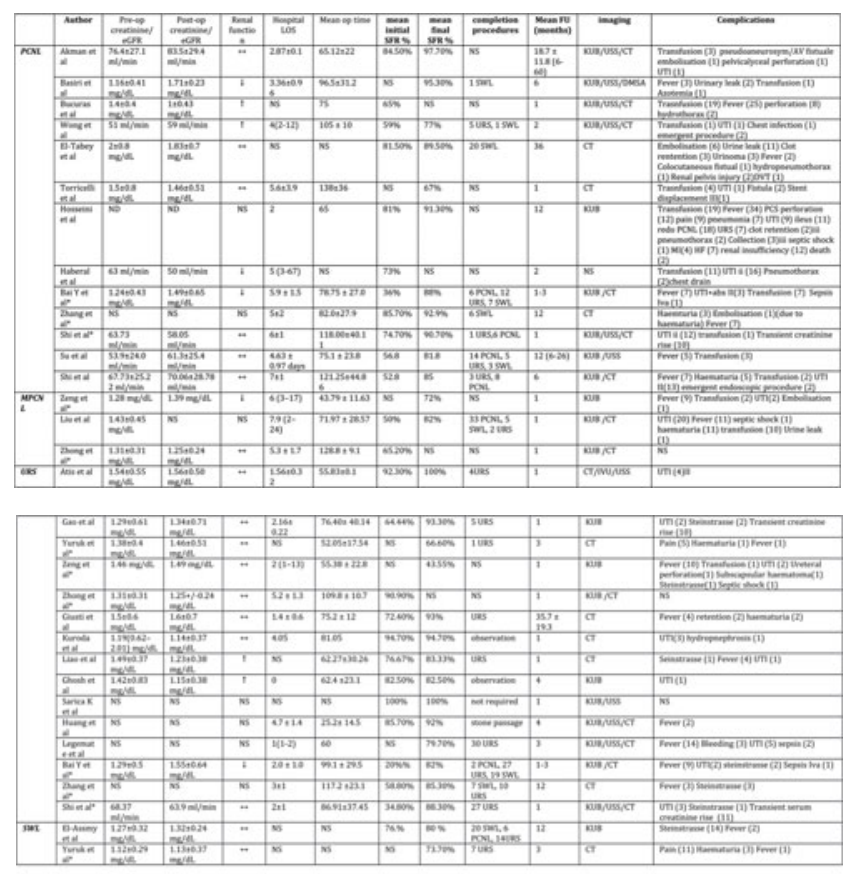

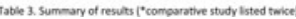

\begin{tabular}{|c|c|c|c|c|}
\hline Procedure & Type of complication & Frequency (n) & Clavien Grade & $\%$ \\
\hline \multirow{19}{*}{$\begin{array}{l}\text { PCNL } \\
(314 / 1264, \\
24.8 \%)\end{array}$} & Fever & 91 & \multirow{2}{*}{1} & \multirow{2}{*}{8} \\
\hline & Transient creatinine rise & 11 & & \\
\hline & Transfusion & 69 & \multirow{4}{*}{ "I } & \multirow{4}{*}{10.1} \\
\hline & UTI & 56 & & \\
\hline & Chest infection & 2 & & \\
\hline & DVT & 1 & & \\
\hline & Collection & 6 & \multirow{4}{*}{ III } & \multirow{10}{*}{5.6} \\
\hline & Clot rentention & 5 & & \\
\hline & Fistulae & 2 & & \\
\hline & Stent displacement & 1 & & \\
\hline & Emergent additional procedure & 4 & \multirow{5}{*}{ IIIa } & \\
\hline & Pelvicalyceal perforation & 23 & & \\
\hline & Embolisation & 9 & & \\
\hline & Pneumothorax & 5 & & \\
\hline & Hydrothorax & 3 & & \\
\hline & Urine leak & 13 & $\overline{\mathrm{IIIb}}$ & \\
\hline & Heart Failure & 7 & \multirow{3}{*}{ IV } & \multirow{3}{*}{1} \\
\hline & MI & 4 & & \\
\hline & Septic shock & 2 & & \\
\hline \multirow{7}{*}{$\begin{array}{l}\text { MPCNL (68/181, } \\
37.5 \%)\end{array}$} & Fever & 20 & \multirow{2}{*}{1} & \multirow{2}{*}{17} \\
\hline & Haematuria & 11 & & \\
\hline & UTI & 22 & \multirow{3}{*}{ II } & \multirow{3}{*}{19.3} \\
\hline & Transfusion & 12 & & \\
\hline & Urine leak & 1 & & \\
\hline & Embolisation & 1 & IIIla & 0.5 \\
\hline & Septic shock & 1 & IV & 0.5 \\
\hline \multirow{12}{*}{$\begin{array}{l}\text { URS (119/792, } \\
15 \%)\end{array}$} & Fever & 47 & \multirow{4}{*}{1} & \multirow{4}{*}{9.6} \\
\hline & Transient creatinine rise & 21 & & \\
\hline & Pain & 5 & & \\
\hline & Haematuria & 3 & & \\
\hline & UTI & 23 & \multirow{4}{*}{ II } & \multirow{4}{*}{3.4} \\
\hline & Retention & 2 & & \\
\hline & Transfusion & 1 & & \\
\hline & Subscapsular haematoma & 1 & & \\
\hline & Ureteral Perforation & 1 & \multirow{2}{*}{ III } & \multirow{3}{*}{1.5} \\
\hline & Hydronephrosis & 1 & & \\
\hline & Steinstrasse & 10 & IIIta & \\
\hline & Septic shock & 4 & IV & 0.5 \\
\hline \multirow{4}{*}{$\begin{array}{l}\text { SWL (31/186, } \\
16.7 \%)\end{array}$} & Pain & 11 & \multirow{3}{*}{1} & \\
\hline & Fever & 3 & & 9.1 \\
\hline & Haematuria & 3 & & \\
\hline & Steinstrasse & 14 & IIII & 7.5 \\
\hline
\end{tabular}

4/16 PCNL studies and 1/15 URS study, while it remained unaffected in the SWL study.

Conclusions: Our review shows a rise of endourological techniques in the management of stones in solitary kidney. Although PCNL was used for larger stones, it had a higher risk of major complications including blood transfusion. While, good stone free rate was obtained for patients irrespective of the treatment modality, the selected intervention needs to be balanced with the safety profile and the need for ancillary procedures.

\section{MP05-17 Simultaneous ureteroscopic and percutane- ous nephro-lithotripsy for management of combined ureteric and renal stones in the Galdakao - modified supine Valdivia position: Safety and outcomes in a single-center experience}

\section{A Ali, L Abbas, T El-Leithy}

Theodor Bilharz Research Institute

Introduction \& Objective: To evaluate the safety and efficacy of simultaneous ureteroscopic (URS) laser lithotripsy and percutaneous nephrolithotripsy (PCNL) for the management of combined ureteric and renal stones (larger than $20 \mathrm{~mm}$ ) in its longest diameter in the Galdakao - modified supine Valdivia (GMSV) position.

Methods: We performed simultaneous combined URS and PCNL for 20 patients with ipsilateral ureteric and renal calculi from November 2018 to March 2019. The study was performed in accordance with the declaration of Helsinki. The Institutional Review Board approved this study protocol. Twelve patients were male and 8 were female with age ranged from 21 to 74 years old. Patients were suffering from ureteric stones with mean stone size \pm SD $\{13.604 \pm 2.438\} \mathrm{mm}$ and from renal stones larger than $20 \mathrm{~mm}$ in its longest diameter with mean stone size \pm SD $\{26.65 \pm 4.70\} \mathrm{mm}$. Patients were positioned in (GMSV) position. Both ureteric and renal stones were targeted simultaneously by performing combined ureteroscopic laser lithotripsy for ureteral stones and Percutaneous nephrolithotripsy by pneumatic lithotriptor. Tract dilatation was 20 $\mathrm{F}$ in 8 patients and $30 \mathrm{~F}$ in 12 patients. In 7 patients a nephrostomy tube was left indwelling while 13 patients were tubeless. Double J stents were applied at the end of the procedure in all patients. The procedure outcome in terms of operative time, blood loss, hospital stay, complications using modified Clavien grading system, the need of auxiliary procedures, stone free rates after 4 weeks by using non contrast computed tomography (NCCT), were evaluated statistically. Our results were compared with those from recent large series of supine PCNL.

Results: The median (range) operative time was 95 (69-114) min. Regarding blood loss in terms of the $\{$ mean $\pm S D\}$ change in $\mathrm{Hb}$ level was $\{1.098 \pm 0.398\} \mathrm{g} / \mathrm{dl}$ with no need of blood transfusion in a single patient. The hospital stay ranged from 1 day to 4 days. The stone free rate was $85 \%$ after 4 weeks by using NCCT, Three patients had an auxiliary procedure in the form of shock wave lithotripsy because of residual stones more than $4 \mathrm{~mm}$ and less than $1 \mathrm{~cm}$. Regarding complications using modified Clavien grading system 5 patients developed complications (3 patients had grade I, 2 patients had grade II).

Conclusions: Simultaneous combined endourological surgery for targeting both ureteric and renal stones is technically feasible, safe and effective using the (GMSV) position with shorter operative time compared to staged URS followed by PCNL. No apparent added risk in using this technique. The stone clearance and complication rates are within the accepted values compared to previous studies. 
MP05-18 Correlation between peripheral blood inflammatory indexes and Systemic Inflammatory Response Syndrome in patients received Percutaneous Nephrolithotomy

\section{An, Q Xu, X Huang}

Peking University Applied Lithotripsy Institute/Peking University People's Hospital Urology and Lithotripsy Center

Introduction \& Objective: To investigate the correlation between peripheral blood inflammatory indexes and Systemic Inflammatory Response Syndrome (SIRS) in patients who received Percutaneous Nephrolithotomy (PCNL).

Methods: We retrospectively analyzed 175 patients who received PCNL in our department from January 2015 to June 2016. Of these 175 patients, 52 patients who suffered SIRS after surgery were classified as group SIRS-. And the other 123 patients without occurrence of SIRS were classified as group SIRS-. We analyzed correlation between preoperative and immediately postoperative peripheral blood inflammatory indexes and occurrence of SIRS after PCNL by Mann-Whitney U test, chisquare test and logistic regression. Peripheral blood inflammatory indexes included neutrophil-lymphocyte ratio (NLR), neutrophil-monocyte ratio (NMR), immediately postoperative NLR/preoperative NLR (postNLR/preNLR) and postNMR/preNMR. Other common risk factors associated with SIRS such as gender, age, ASA class, history of ipsilateral kidney surgery, preoperative placement of stent or percutaneous nephrostomy, preoperative serum creatinine, preoperative urine culture, operation time, number of access, simultaneous ureteroscopy, intraoperative tachycardia, and blood transfusion were also investigated in this study.

Results: Increase of immediately postoperative peripheral blood inflammatory indexes, female, positive preoperative urine culture, longer operation time, multiple accesses, and blood transfusion were correlated with occurrence of SIRS after PCNL. Multivariate logistic regression showed postNMR/preNMR was the only independent risk factor for the occurrence of SIRS after PCNL. PostNMR/preNMR was divided into four groups by quartile, risk for occurrence of SIRS after PCNL of group

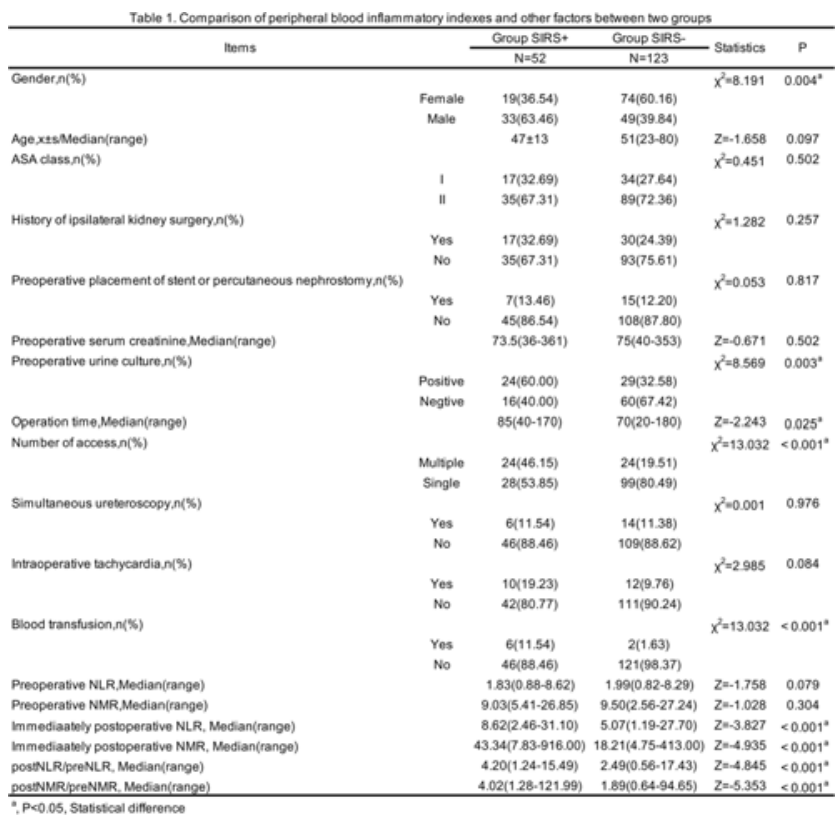

\begin{tabular}{|c|c|c|c|c|c|c|c|}
\hline \multirow{2}{*}{ nems } & \multirow{2}{*}{ B } & \multirow{2}{*}{ S.E. } & \multirow{2}{*}{ Wals } & \multirow{2}{*}{ Sig. } & \multirow{2}{*}{$\operatorname{Exp}(B)$} & \multicolumn{2}{|c|}{$\operatorname{EXP(B)} 95 \%$} \\
\hline & & & & & & Lower & Uper \\
\hline Gender & 0.964 & 0.342 & 7.958 & $.005^{2}$ & 2.623 & 1.342 & 5.126 \\
\hline Age & -0.023 & 0.013 & 3.022 & 0.082 & 0.977 & 0.952 & 1.003 \\
\hline ASA class & .0 .24 & 0.358 & 0.45 & 0.502 & 0.787 & 0.39 & 1.586 \\
\hline History of ipsilateral kidney surgery & 0.409 & 0.363 & 1.274 & 0.259 & 1.506 & 0.74 & 3.065 \\
\hline Preoperabve placement of stent or percutaneous nephrostomy & 0.113 & 0.491 & 0.053 & 0.817 & 1.12 & 0.428 & 2.932 \\
\hline Preoperative serum creatinine & .0 .002 & 0.004 & 0.379 & 0.538 & 0.998 & 0.99 & 1.005 \\
\hline Preoperatve unine culture & 1.133 & 0.394 & 8.258 & $.004^{\circ}$ & 3.103 & 1.433 & 6.719 \\
\hline Operation time.Median & 0.013 & 0.005 & 5.821 & $.016^{\circ}$ & 1.013 & 1.002 & 1.023 \\
\hline Number of access & 1.263 & 0.359 & 12.35 & $.000^{\circ}$ & 3.536 & 1.748 & 7.151 \\
\hline Simultaneous ureteroscopy & 0.015 & 0.519 & 0.001 & 0.976 & 1.016 & 0.367 & 2.807 \\
\hline Intraoperative tachycardia & 0.79 & 0.465 & 2.884 & 0.089 & 2.202 & 0.885 & 5.478 \\
\hline Blood transfusion & 2.066 & 0.835 & 6.125 & $.013 a$ & 7.891 & 1.537 & 40.52 \\
\hline Preoperative NLR & .0211 & 0.173 & 1.493 & 0.222 & 0.809 & 0.577 & 1.136 \\
\hline Preoperative NMR & .0 .033 & 0.044 & 0.559 & 0.455 & 0.968 & 0.888 & 1.055 \\
\hline Immediaately postoperasve NLR & 0.126 & 0.032 & 15.34 & $.000^{\circ}$ & 1.135 & 1.065 & 1.209 \\
\hline Immediaately postoperative NMR & 0.009 & 0.003 & 11.67 & $.001^{\circ}$ & 1.009 & 1.004 & 1.014 \\
\hline postNLR/preNLR & 0.252 & 0.062 & 16.74 & $.000^{\circ}$ & 1.287 & 1.14 & 1.452 \\
\hline postNMR/preNMR & 0.047 & 0.017 & 7.536 & $.006^{*}$ & 1.048 & 1.013 & 1.084 \\
\hline
\end{tabular}

\begin{tabular}{|c|c|c|c|c|c|c|c|c|}
\hline \multirow{2}{*}{ Items } & \multirow{2}{*}{ B } & \multirow{2}{*}{ S.E, } & \multirow{2}{*}{ Wals } & \multirow{2}{*}{ df } & \multirow{2}{*}{ Sig. } & \multirow{2}{*}{$\operatorname{Exp}(B)-$} & \multicolumn{2}{|c|}{ EXP(B) 95\% C.I. } \\
\hline & & & & & & & Lower & Upper \\
\hline postNLR/preNLR sQ25 & & & 4.399 & 3 & 0.221 & & & \\
\hline postNLR/preNLR Q25 Q50 & 1.664 & 0.916 & 3.295 & 1 & 0.069 & 5.278 & 0.876 & 31.809 \\
\hline postNMR/preNMR Q50 Q75 & 1.877 & 0.942 & 3.968 & 1 & $.046^{a}$ & 6.534 & 1.031 & 41.429 \\
\hline postNMR/preNMR > Q75 & 2.151 & 1.051 & 4.187 & 1 & $.041^{2}$ & 8.591 & 1.095 & 67.422 \\
\hline
\end{tabular}

Q50 75 and group Q $>75$ were 6.5 and 8.5 times beyond group $\mathrm{Q} \leq 25 \quad(\mathrm{OR}=6.534, \quad 95 \% \mathrm{CI}: \quad 1.031 \sim 41.429, \quad \mathrm{P}=0.046)$ $(\mathrm{OR}=8.591,95 \% \mathrm{CI}: 1.095 \sim 67.422, \mathrm{P}=0.041)$.

Conclusions: Immediately postoperative peripheral blood inflammatory indexes, gender, preoperative urine culture, operation time, number of access, and blood transfusion were correlated with occurrence of SIRS after PCNL. PostNMR/preNMR was independent risk factor for the occurrence of SIRS after PCNL.

\section{MP05-19 Risk factors for resistance to preservation treatment of calculus pyelonephritis}

N Hodotsuka, T Hayashi, Y Endo, T Ueda, J Akatsuka, H Takeda, S Osawa, G Kimura, Y Kondo

Nippon Medical School Hospital, Department of Urology

Introduction \& Objective: Urinary drainage for obstructive upper urinary tract infections is an effective treatment. However, there is no clear drainage criteria for calculus pyelonephritis. In this study, we examined the risk factors of patients who needed drainage in stone nephritis treated at our hospital.

Methods: We retrospectively examined 90 patients with calculus pyelonephritis treated in our hospital from April 2015 to December 2018. We defined hydronephrosis in abdominal CT with grade 0-4 from the degree of dilation of the renal pelvis and calices. Cases requiring immediate drainage using ureteral stent showed shock vital and hydronephrosis grade 4 , these cases were performed urinary drainage within 24 hours after admission. The preservation treatment cases had improved inflammatory response within 24 hours to 3 days by antibiotic administration. The drainage group using ureteral stent was defined as antibiotic administration without improvement of the inflammatory response, sustained fever and renal dysfunction. Standard antibiotics were not prescribed. We defined stone surface area as stone major diameter $\mathrm{x}$ minor diameter $\mathrm{x}$ TT $\mathrm{x} 1 / 4$. We used the following factors for analysis parameters, age, sex, Body mass index (BMI), Performance Status (PS), grade of hydronephrosis, DM, SSA, CT value, Stone skin distance (SSD), WBC, creactive protein, creatinine, potassium, albumin, platelets, and 
total bilirubin. Risk factors for resistance to conservative treatment were investigated using chi-square test and multivariate logistic analysis.

Results: The median age for all 90 cases was 64 and the male/ female ratio was 0.41 . Sixty-three patients improved with conservative treatment, while 20 required drainage treatment. After drainage, all cases improved promptly. The grade of hydronephrosis, serum albumin, c-reactive protein, creatinine, SSA, CT value, and SOFA score were significantly considered risk factors for drainage using chi-square test. Multivariate logistic analysis on these factors was significant in hydronephrosis grade and SOFA score.

Conclusions: $70 \%$ of calculous pyelonephritis improved with conservative treatment. Hydronephrosis grade and SOFA score were shown to be risk factors for resisting conservative treatment. These results demonstrate that high hydronephrosis grade and SOFA score were considered to be eligible for drainage.

\section{MP05-20 The impact of phone counseling on urinary stone prevention}

A Nevo, A Navaratnam, MT Keddis, MR Humphreys

Mayo Clinic

Introduction \& Objective: Urinary stones disease is characterized by recurrences and relapses. Medical treatment and dietary changes play an important role in preventing recurrent episodes. Because of the tertiary nature of our center, established patients are sometimes consulted with phone conversation and a letter entailing our recommendations. The aim of this study was to compare the impact of the standard multidisciplinary stone clinic consult and a non-physical consult on 24 hours urinary parameters.

Methods: A retrospective study of consecutive patient who completed a 24-hour collection from February 2019 to April 2019. Patient's previous consults and 24 urine collections were reviewed. The type of consult (clinic or phone) and the recommendations were compared to the results of the subsequent urine collection. The study endpoint was normalization of dietary urine parameters, categorized as "worsened", "improved", or "normalized.

Results: Eighty-four consults from 26 patients were included in the study. There were $63(75 \%)$ office consults and $21(25 \%)$ phone consults. There were no differences between the groups with respect to patient's age, sex, distance from hospital, stone composition, or prior stone episodes. Dietary parameters nor-

\begin{tabular}{|c|c|c|c|c|}
\hline & & Stone clinic & Phone consult & p \\
\hline \multicolumn{2}{|c|}{ Median Age $(q 1, q 3)$} & $72(63,74)$ & $73(59,75)$ & 0.85 \\
\hline \multicolumn{2}{|c|}{ Sex (\% male) } & $47(74.6)$ & $18(85.7)$ & 0.38 \\
\hline \multicolumn{2}{|c|}{ Mean distance from hospital (SD) } & $568(770)$ & $793(808)$ & 0.26 \\
\hline \multicolumn{4}{|c|}{ Number of past stone episodes/interventions } & 0.22 \\
\hline \multicolumn{2}{|c|}{0} & 4 & 0 & \\
\hline \multicolumn{2}{|l|}{1} & 7 & 0 & \\
\hline \multicolumn{2}{|c|}{$2-5$} & 27 & 10 & \\
\hline \multicolumn{2}{|c|}{ More than 5} & 25 & 11 & \\
\hline \multicolumn{4}{|c|}{ Main stone composition } & 0.09 \\
\hline \multicolumn{2}{|c|}{ Calcium oxalate monohydrate } & 37 & 11 & \\
\hline \multicolumn{2}{|c|}{ Calcium oxalate dehydrate } & 1 & 0 & \\
\hline \multicolumn{2}{|c|}{ Calcium Phosphate } & 0 & 2 & \\
\hline \multicolumn{2}{|c|}{ Uric acid } & 13 & 6 & \\
\hline \multicolumn{5}{|c|}{ Dietary primary endpoint } \\
\hline \multirow[t]{3}{*}{ Urine volume } & Normalized & 24 & 7 & 0.87 \\
\hline & Improved & 7 & 2 & \\
\hline & Worsened & 15 & 6 & \\
\hline \multirow[t]{3}{*}{ Sodium } & Normalized & 6 & 3 & 0.61 \\
\hline & Improved & 11 & 2 & \\
\hline & Worsened & 21 & 7 & \\
\hline \multirow[t]{3}{*}{ Oxalate } & Normalized & 7 & 1 & 0.47 \\
\hline & Improved & 3 & 0 & \\
\hline & Worsened & 11 & 4 & \\
\hline
\end{tabular}

malized, improved, and worsened in, $28.6 \%, 41.3 \%$, and $30.2 \%$ In the clinic consult group, and $28.6 \%, 28.6 \%$, and $42.9 \%$ in the phone consult group $(\mathrm{p}=0.49)$. Medical recommendations resulted in normalization, improvement, and worsening results in $40 \%, 18.2 \%$, and $41.8 \%$ in the clinic consult group, and $52.6 \%$, $26.3 \%$, and $21.1 \%$, in the phone consult group $(\mathrm{p}=0.266)$.

Conclusions: Established stone clinic patients who receive phone consultations have similar outcomes as patients who receive standard clinic consultation.

MP05-21 Value of urinary $\mathrm{pH}$ in assessment of highrisk stone patients: Outcomes from a dedicated metabolic stone clinic

\author{
M Ramachandra, M Saraogi, A Pietropaolo, BK Somani, \\ P Cook
}

University Hospital Southampton

Introduction \& Objective: The prevalence of kidney stone disease is progressively increasing. This has been associated with obesity, hypertension and smoking. The relationship between urinary $\mathrm{pH}$ and the type of nephrolithiasis has been widely studied, with the hypothesis that lower urinary $\mathrm{pH}$ may be related to uric acid nephrolithiasis.

Methods: Dataset was collected from a dedicated metabolic stone clinic. A retrospective analysis was performed on the demographics, medical co-morbidities, urinary $\mathrm{pH}$ levels and the type of kidney stones. The eligible patients were divided into two groups depending on the urinary $\mathrm{pH}$ levels (group 1 - urinary $\mathrm{pH}$ $<5.5$ and group $2-$ urinary $\mathrm{pH} \geq 6.5$ ).

Results: A total of 567 high-risk patients with renal stones were identified with a predominance of males $(67 \%)$. These patients were divided into the pre-designated $\mathrm{pH}$ groups. In group 1, there were 114 eligible patients with an average age of 54 years (range: 17-82 years) and in group 2 there were 145 patients with an average age of 45 years (range: $17-83$ years). The male to female ratio was $2: 1$ across both the groups. In group $1,70 \%$ of the patients had diabetes mellitus compared to $38 \%$ in group 2 . Over $50 \%$ of the patients were smokers and clinically obese across both the groups. Sub-analysis of the stones' composition confirmed Calcium oxalate in $28.9 \%$ and $29.6 \%$ across both groups. There was a pre-dominance of uric acid nephrolithiasis in Group $1(25.4 \%)$ compared to Group $2(1.3 \%)(\mathrm{P}<0.001)$.

Conclusions: Conclusion Acidic urine $\mathrm{pH}$ is an important precursor for uric acid stones and is associated with diabetes mellitus. It is an important biochemical marker for patients with kidney stones and its measurement should be mandatory in all patients.

\section{MP05-22 How much energy is needed to ablate one cubic millimeter of stone volume during flexible ur- eteroscopy using a low-power laser device? Results from a proof-of-principle clinical study}

\author{
E Ventimiglia, S Doizi, F Panthier, O Traxer \\ Division of Experimental Oncology/Unit of Urology; URI; \\ IRCCS Ospedale San Raffaele
}

Introduction \& Objective: We aimed to estimate the total energy needed to ablate $1 \mathrm{~mm}^{3}$ of stone volume during flexible ureteroscopy lithotripsy using a low-power (35W) Ho:YAG laser device, in order to estimate laser efficacy and to lay the basis to develop a pre-operative planning tool. 
Methods: We collected data regarding 25 patients submitted to flexible ureteroscopy for renal stones whose volume was bigger than $500 \mathrm{~mm}^{3}$. We excluded patients with solitary kidney, anatomical abnormalities, and positive urine culture. A $35 \mathrm{~W}$ Ho:YAG laser (Dornier Medilas H Solvo 35, Germany) was used for every procedure with a $272 \mu \mathrm{m}$ laser fiber. For each patient, we recorded laser parameters, the total energy delivered by the laser fiber, the time from the first laser pulse until the last one, and the active laser time as provided by the machine. Long pulse was set for all the procedures. In case of multiple procedures, the aforementioned data were additively cumulated. We then estimated values and predictors for the total amount of energy needed to ablate $1 \mathrm{~mm}^{3}$ of stone volume.

Results: Median (interquartile range, IQR) age and BMI were respectively 49 (36-62) years and $24(22-27) \mathrm{kg} / \mathrm{m}^{2}$. Median (IQR) stone volume and stone density were respectively 1410 (557-3023) $\mathrm{mm}^{3}$ and 1200 (793-1300) Hounsfield units (HU). In terms of laser parameters, median (IQR) energy and frequency were $0.6(0.4-0.8)$ $\mathrm{J}$ and 15 (15-18) Hz. Median (IQR) delivered energy per $\mathrm{mm}^{3}$ of stone volume was 19 (15-23) J. Median (IQR) total lithotripsy time was 68 (36-88) minutes. Laser was active during 84\% (70-95\%) of the total lithotripsy time. At multivariable linear regression analysis, higher stone density in HU (beta: $0.02, \mathrm{p}=0.03$ ) and larger stone volume (beta: $0.1, p=0.04$ ) emerged as independent predictors of the energy value needed for ablating $1 \mathrm{~mm}^{3}$ of stone volume.

Conclusions: It is possible to perform laser lithotripsy using a low-power laser device with a virtually continuous laser activity. The estimation of the pre-operative parameters as well as the total energy needed to ablate $1 \mathrm{~mm}^{3}$ of stone volume are fundamental for a proper pre-operatory planning.

\section{MP06: EDUCATION, SIMULATION \& VIRTUAL REALITY}

\section{MP06-01 Construction and Assessment of an inno- vative indigenous TURP Simulator}

A Rawandale-Patil, LG Patni, PA Rawandale, A Hindustanwala, A Gade, S Kaushik

Tejnaksh Hospital's Institute of Urology

Introduction \& Objective: Transurethral resection of the prostate (TURP) has a significant learning curve. Improper approach and techniques may have unwanted postoperative complications. We describe and validated our own, portable, sausage based TURP simulator.

Methods: A short anatomical study of urethra, sphincter and prostate was conducted using CT sections and 10 endoscopic videos of TURPs. The simulator frame was designed, patented and constructed using silicon. The frame is designed to accept and allow resection of sausages or meat roles using any technique. Visual assessment by the mentor facilitates training. Evaluation done using a three-step test, GRS score and trainee feedback was analyzed using SPSS.

Results: 16 urology trainees, 3 experts participated in this single center study. Face and content validity evaluated by experts demonstrated satisfactory replication of the prostate and bladder
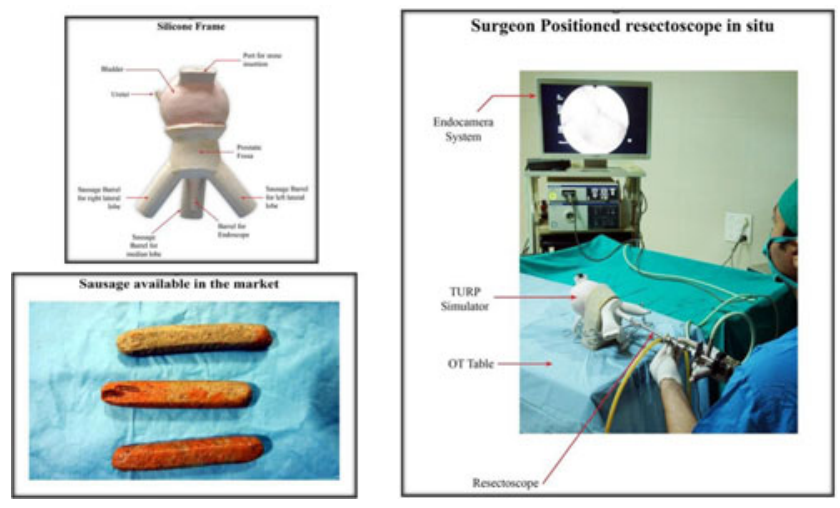

anatomy. The simulator could differentiate novices from the experts. All subjects demonstrated statistically significant betterment in their GRS scores and total resection time. Parameters of trainees showed a shift, towards the control demonstrating the training capabilities. Subjective simulator assessment indicated high degree of satisfaction on effectiveness.

Conclusions: Our portable TURP simulator is the first of its kind. It uses sausages as a substrate for resection using any resection technique. It allows evaluation and supervised, repetitive tailored learning in a controlled, low stress environment. It needs no preparation, has a low initial and no maintenance cost. Further studies would be aimed at further assessment of training and proficiency abilities.

\section{MP06-02 Effective Utilization of Legos to Create a Ureteroscopic Trainer for Urology Residents}

SL Best, KA Richards, BJ Lewis, DR Gralnek, CJ Kozler, TG Leffler, BL Miller, DH Williams, SY Nakada, BV Le

Department of Urology, University of Wisconsin School of Medicine \& Public Health

Introduction \& Objective: Concerns about urology residents practicing skills on patients are driving an interest in surgical simulation worldwide. Models for flexible ureteroscopy (fURS) range from low cost box trainers to high-cost virtual reality systems. It appears that low-cost, low-fidelity trainers made of readily available materials can be effective in teaching junior residents fURS, which also supports a solution which is more internationally feasible. At our institution, first year residents learn fURS in a surgical skills bootcamp setting, but rarely can practice fURS. Our goal was to create a low-cost, portable trainer to practice fURS any time.

Methods: Using interlocking Legos ${ }^{\mathrm{TM}}$, we built chambers of different colors which the trainee passed the fURS into and through. We cut a hole $(43 \times 36 \mathrm{~mm})$ for insertion of the fURS in the plastic storage bin housing the trainer. We purchased an indefinitely-reusable fURS (Neoflex ${ }^{\text {TM }}$, Neoscope2020 LLC, San Jose, CA) with a USB connector that can be connected to any 


Table 1: Time trials and \% reduction in time
\begin{tabular}{|c|c|c|c|c|}
\hline Resident & $\begin{array}{c}\text { Time 1 } \\
\text { (seconds) }\end{array}$ & $\begin{array}{c}\text { Time 2 } \\
\text { (seconds) }\end{array}$ & $\begin{array}{c}\text { Time } 3 \\
\text { (seconds) }\end{array}$ & $\begin{array}{c}\% \text { reduction in time } \\
\text { with practice }\end{array}$ \\
\hline A & 231 & 160 & 93 & $60 \%$ \\
\hline B & 131 & 127 & NA & $3 \%$ \\
\hline C & 143 & 108 & NA & $24 \%$ \\
\hline D & 152 & 121 & 112 & $26 \%$ \\
\hline E & 87 & 50 & 39 & $55 \%$ \\
\hline F & 217 & 130 & 102 & $53 \%$ \\
\hline G & 126 & 82 & 124 & $2 \%$ \\
\hline
\end{tabular}

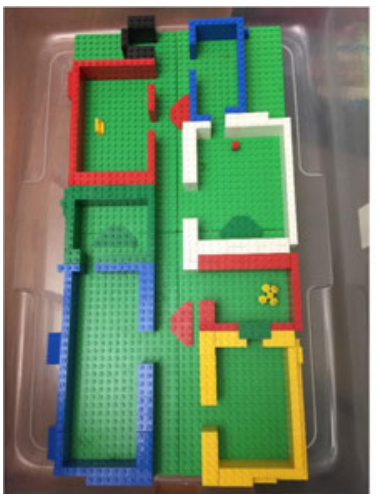

Sample configuration of box trainer. Further details can be found at https://urology.wisc.edu/education-and. training/urology-simulation-education-program/ureteroscopy-box-trainer/

computer for viewing. To purchase all supplies new, the cost would be $\sim \$ 60$ USD. The fURS with USB connector cost $\sim \$ 1500$ USD. We trialed the box trainer with 7 residents who were instructed to maneuver the scope to a series of targets in a specific order. They were timed for 2 or 3 attempts.

Results: With practice, the residents demonstrated an average of $32 \%$ reduction in time to complete the trainer tasks. The trainer can be reconstructed into different patterns to address weaknesses. Once a resident is proficient with a configuration, the arrangement can be easily changed. The time trials indicate that residents' overall time decreased with subsequent attempts indicating improvement with practice. (Table 1).

Conclusions: This apparatus met our criteria for the model, which are reproducible results, low cost and portability. While this model has low realism, training can then advance to more complex and realistic models, both inanimate and animate.

\section{MP06-03 Immersive Virtual Reality for Percutaneous Nephrolithotomy Training}

E Parkhomenko, S Resad, D Wang, S Wason

Boston Medical Center

Introduction \& Objective: Stone clearance for percutaneous nephrolithotomy (PCNL) is dictated by percutaneous access into the desired renal calyx, however establishing renal access is difficult and current training methods outside of the operating room are limited. Immersive virtual reality (iVR) technology with haptic feedback may provide a training platform to augment surgical skill acquisition. We sought to evaluate the efficacy of a novel iVR simulator for PCNL tract access.

Methods: Urology and interventional radiology (IR) residents and attendings evaluated an iVR simulator for percutaneous tract access (Table 1). Using a head mounted display (Oculus Rift) and a robotic arm (Marion Surgical), participants were instructed to gain access into the lower pole calyx in a prone patient in a VR operating room (Figure 1). A Likert type scale $(1=$ not at all, $5=$ extremely, and $1=$ poor, $5=$ excellent) was used to record the
Table 1: Demographics, Virtual reality experience, image control, and economy of motion of an immersive virtual reality simulator for percutaneous nephrostomy tract access

\begin{tabular}{|c|c|c|c|c|}
\hline \multicolumn{5}{|c|}{ Demographics $(\mathrm{n}=\mathbf{2 0})$} \\
\hline Age (year) & \multicolumn{4}{|c|}{$35(26-66)$} \\
\hline Gender (male) & \multicolumn{4}{|l|}{$65 \%$} \\
\hline Participants & \\
\hline Attendings & \multicolumn{4}{|c|}{$6(\mathbb{I R}=2$, Urology $=4)$} \\
\hline Residents & 14 & & & \\
\hline \multicolumn{5}{|c|}{ Virtual Reality Experience ( $1=$ not at all, 5 = extremely) } \\
\hline & Total $(\mathrm{n}=20)$ & Novice $(\mathrm{n}=17)$ & Expert $(\mathrm{n}=3)$ & p \\
\hline Realistic (yes) & $95 \%$ & $94.1 \%$ & $100 \%$ & \\
\hline $\begin{array}{l}\text { Realism of instrument } \\
\text { handling vs live } \\
\text { surgery }\end{array}$ & 2.7 & 2.6 & 3.3 & 0.57 \\
\hline $\begin{array}{l}\text { Realism of targets (i.e. } \\
\text { calyx, collecting } \\
\text { system, etc.) }\end{array}$ & 3.4 & 3.2 & 4.3 & 0.24 \\
\hline $\begin{array}{l}\text { Realism of haptic } \\
\text { feedback }\end{array}$ & 2.4 & 2.2 & 3.3 & 0.20 \\
\hline Overall realism & 3.25 & 3.3 & 3.0 & 0.77 \\
\hline $\begin{array}{l}\text { Usefulness for hand- } \\
\text { eye coordination }\end{array}$ & 3.8 & 3.7 & 4.3 & 0.49 \\
\hline Useful rehearsal tool & 3.8 & 3.6 & 4.3 & 0.44 \\
\hline $\begin{array}{l}\text { Likelihood to } \\
\text { recommend }\end{array}$ & 3.6 & 3.5 & 4.3 & 0.40 \\
\hline \multicolumn{5}{|c|}{ Image Control ( 1 = poor, 5 = excellent $)$} \\
\hline & Total $(\mathrm{n}=20)$ & Novice $(\mathrm{n}=17)$ & Expert $(n=3)$ & $\mathrm{p}$ \\
\hline Quality & 4.1 & 4.1 & 4.3 & 0.51 \\
\hline Brightness & 4.3 & 4.3 & 4.3 & 0.94 \\
\hline Resolution & 3.95 & 3.9 & 4.3 & 0.46 \\
\hline $\begin{array}{l}\text { Delay in image display } \\
(5=\text { no delay) }\end{array}$ & 4.05 & 4.1 & 4.0 & 0.92 \\
\hline Overall comfort & 3.9 & 3.9 & 4.0 & 0.87 \\
\hline Overall performance & 3.95 & 3.9 & 4.3 & 0.46 \\
\hline \multicolumn{5}{|c|}{ Economy of Motion } \\
\hline & Total $(\mathrm{n}=20)$ & Novice $(\mathrm{n}=17)$ & Expert $(n=3)$ & p \\
\hline Cumulative time (s) & 180 & 174 & 214 & 0.55 \\
\hline Path length $(\mathrm{cm})$ & 273 & 275 & 261 & 0.87 \\
\hline Average velocity $(\mathrm{cm} / \mathrm{s})$ & .0019 & .020 & 0.015 & 0.36 \\
\hline
\end{tabular}

participant's VR experience. Additionally, the robotic arm recorded kinematics parameters.

Results: Majority of the participants (95\%) rated the VR simulation as a realistic experience. The instrument handling $(2.7 / 5)$, targeting (3.4/5), haptic feedback (2.4/5) and overall realism $(3.25 / 5)$ were rated as slightly to somewhat realistic (Table 1). The image quality (4.1/5), brightness (4.3/5), resolution (3.95/5), delay (4.05/5), overall comfort (3.9/5) and overall performance $(3.95 / 5)$ were rated as very good. The usefulness of the simulator for hand-eye coordination (3.8/5), as a rehearsal tool (3.8/5), and likelihood to recommend it to colleagues for training $(3.6 / 5)$ were rated as very good. There were no significant differences between the novices and experts for their VR experience nor economy of motion.

Conclusions: The iVR experience for PCNL tract access was rated favorably by participants, however it was unable to differentiate novices from experts. Future software upgrades may improve the participant's experience, and it is likely that future trainees will benefit from iVR training.

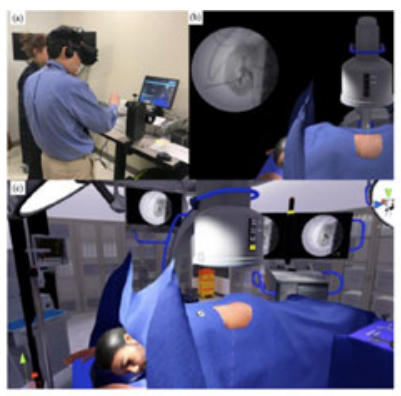

Figure 1: Virtual Surgery Simulator (a) VR simulator set up and use (b) fluoroscopic image with target access (c) virtual operating room seen by participant. 
MP06-04 A vascularized peritumor area model based on quantitative three-dimensional reconstruction facilitates precise partial nephrectomy: FACS scoring criteria and four defining steps for surgery planning

W Xiong, H Liu, Y Du, J Yang, P Guo, J Chen, J Lyu, X Huang, K Dou, Q Ran

Introduction \& Objective: The numeric nephrometries had been developed from 2-dimensional cross-sectional imaging to assist the semi-quantitative description of the reno-tumor anatomy and clinical outcomes comparison. Herein, we describe a new model and scoring criteria to quantify the renal vasculature and contact area based on quantitative three-dimensional reconstruction of reno-tumor imaging for designing the precise renal surgery.

Methods: The vascularized peritumor area (VPA) model was summarized based on 71 cases three-dimensional reconstruction guided surgery for renal cell carcinoma and the scoring criteria was designed based on standard 2D computerized tomography and three-dimensional reconstruction data set between November 2017 and April 2019. The feeder artery of the tumor, angle of maximum cross section, clamping segments number and surface of contact area were evaluated and integrated into a scoring system which was developed upon the anatomic characterization of renovascular-tumor 3D reconstruction data and literature review.

Results: The 71 cases were performed evenly, in which 67 cases experienced the precise partial nephrectomy, 60 robotic, 5 laparoscopic, 2 open, 1 auto-transplant and 3 radical nephrectomies. The renal arterial hierarchy is used to rank the weightiness of the feeder artery of the renal tumor and assigned the score. The feeder artery of the tumor was defined and confirmed by 2D CT imaging 3D reconstruction, and the planned clamping or ischemic segments was defined during the renal segmentation and virtual resection of renal tumor on the affected kidney on 3D reconstruction. The scoring criteria was developed in the new scoring system, nominated as FACS system, in which the feeder artery of the tumor $(\mathrm{F})$, angle of maximum cross section(A) clamping segment number $(\mathrm{C})$ and surface of tumor-parenchyma contact area(S). The FACS scores were calculated equates to the vascularized peritumor area that is the unique feature of the renovascular-tumor anatomy. As the FACS score increases, the tumor peripheric area becomes more proximal to the renal sinus with bigger tumor diameter, larger feeder artery and larger surface of contact area. Multivariate regression analysis revealed an association of the FACS score with warm ischemia time $(\mathrm{p}<0.05)$ which is a surrogate for technical demanding.

Conclusions: The FACS score system provides a clinically useful characterization of the renal tumor and cognitive assistance for precise nephron sparing surgery, which may allow improved surgeon's intuition and risk assessment of partial nephrectomy planning. VPA model also is a reproducible tool for designing the precise robotic renal surgery in clinical scenario.

\section{MP06-05 Machine Learning using a Multi-task Con- volutional Neural Networks Can Accurately Provide Robotic Skills Assessment}

\author{
JC Gahan, RL Steinberg, A Garbens, X Qu, BA Johnson, \\ E Larson
}

University of Texas Southwestern

Introduction \& Objective: Surgical skill evaluation relies on either direct observation or video review. Both are time consuming and do not scale well. We aim to train and evaluate multi-

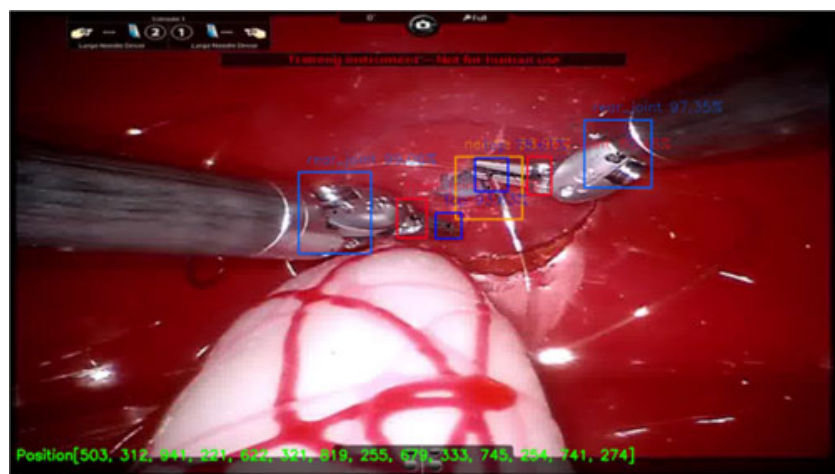

task convolutional neural network to predict surgical proficiency score using a validated robotic assisted suturing model.

Methods: Twenty-three videos of surgeons with varying robotic skill levels completing a validated urethrovesical anastomosis model were utilized. Global evaluation assessment of robotic skills (GEARS) scoring was assigned to each video by expert reviewers and used as training data for the machine learning algorithm. The front wrist joint, rear wrist joint, and needle driver tip of each instrument, and needle, were manually labelled in 300 frames to train a YOLO object detector (Figure 1). Each video was randomly broken down into 6 second clips, providing multiple representative clips for each video. A heatmap representing object movement based on coordinate differences over time was generated (Figure 2). Using a multi-task convolutional neural network, each clip was assigned a GEARS score. A composite GEARS score for each video was then calculated using a novel video pooling method. Model training was performed using leave-one-out cross validation. The output was the predicted score for each GEARS domain. Success was defined when the model accurately predicted the domain score within the range assigned by human experts.

Results: Algorithm training for 23 videos of 1-2 minute duration took 4 hours using a supercomputer. The multi-task model successfully predicted 6 of 6 domains in $65 \%$ of videos, 5 of 6 domains in $74 \%$ of videos and 4 of 6 domains in $74 \%$ of videos. Conclusions: With limited training data, a novel multi-task convolutional neural network can accurately predict surgical proficiency as assessed by GEARS scoring in a urethrovesical model. Further testing using actual operative videos is warranted.

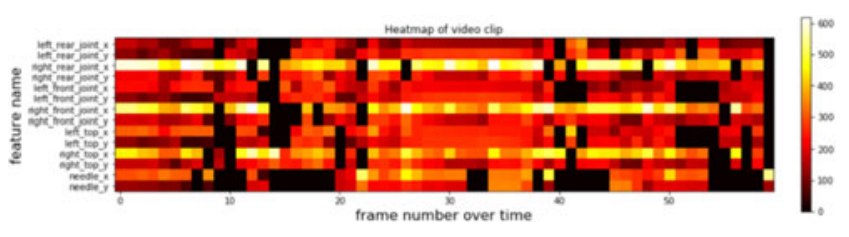

MP06-06 Training in urolithiasis procedures using simulation-based models: A comparative evaluation

U Baig, A Aydin, A Al-Jabir, N Raison, T Abe, J Ramón Cansino Alcaide, Y Farahat, K Sarica,

P Dasgupta, K Ahmed

MRC Centre for Transplantation, King's College London, Guy's Campus, King's Health Partners, London, United Kingdom

Introduction \& Objective: With urolithiasis conditions being one of the most common presentations in urology, it is becoming 
increasingly important to provide a structured simulation-based training regime using validated training models. The aim of this study is to evaluate the existing simulation models and identify their strengths and drawbacks via expert and user feedback.

Methods: This prospective, international, multi-center, comparative observational study recruited participants of varying experience $(n=134)$ ranging from newly-appointed residents to consultant level. Each participant used the simulator for suitable ureteroscopy training and completed a survey feedback scoring various parameters for ureteroscopy, on Likert scale. The results for each model were collected and a mean for each parameter was calculated and tabulated using a traffic light system for effective comparison.

Results: The Scope Trainer (Mediskills) and Boston Scientific models both received $100 \%$ of mean feedback scores between 4-5 in all measured parameters (mean: 4.25 vs 4.44 , respectively). The Advanced Scope Trainer (Mediskills) was amongst the least favored models (mean: 3.68). As the exclusive virtual reality model, The Uro-Mentor had integrated fluoroscopy features and presented with strengths in simulating flexible ureterenoscopy (fURS) and stone extraction (mean: 4.08 vs 4.02 , respectively). Cadaveric simulation incorporated real time fluoroscopy and excelled principally in C-arm control and instrument handling (mean: $4.72 \mathrm{vs}$ 4.61, respectively). Other models had their scores evaluated and recommendations were given based on their areas of proficiency. Conclusions: The Scope Trainer (Mediskills) was amongst the most favored models for trainees and consultants receiving 100\% of mean feedback scores between 4-5 in all measured parameters, making it the best model to simulate complete procedures. Further investigation into the transferability of skills is required, with long-term follow up studies to establish whether there is genuine skill development and retention using simulation models for surgical training, and whether this helps to reduce surgical complications in the operating room.

\section{MP06-07 Can video-based learning support the im- provement of surgery safety by reducing surgeon er- ror? An experimental study}

S Anacleto, V Fernandes, P Mota, N Carvalho, M Costa, J Torres, N Morais, P Passos, R Rodrigues, E Dias, E Lima, J Correia-Pinto

Introduction \& Objective: Laparoscopy revolutionized surgical practice and the Halstedian apprenticeship model became outdated and insufficient. As video-based surgical learning and simulation-based training are emerging as valuable strategies to overcome this necessity, the aim of this study was to develop two video-mentored tutorials (VMT) concerning the Peg-Transfer (PT) and Needle Guidance (NG) exercises, of the European Basic Laparoscopic Urologic Skills examination (E-BLUS) and evaluate the impact on participants performance.

Methods: An experimental, unblinded, parallel, 2-intervention, 2-period, with an allocation ratio of 1:1, randomized clinical trial was conducted. A total of 42 medical students were enrolled on a voluntary basis and randomized into two groups (group $1=21$; group $2=21$ ). Both groups performed two trials for each exercise: group 1 watched the VMT in both trials and group 2 watched firstly the original E-BLUS examination video and then the VMT. Outcome measures were task time and total number of errors. A post-experiment inquiry of preference was delivered. Results: In the first period, participants who visualized the PT and NG VMT had a decreased total number of errors than the participants who visualized the E-BLUS video $(p=0.001$ and $\mathrm{p}=0.014$, respectively). In the second period, after watching the
Figure 1
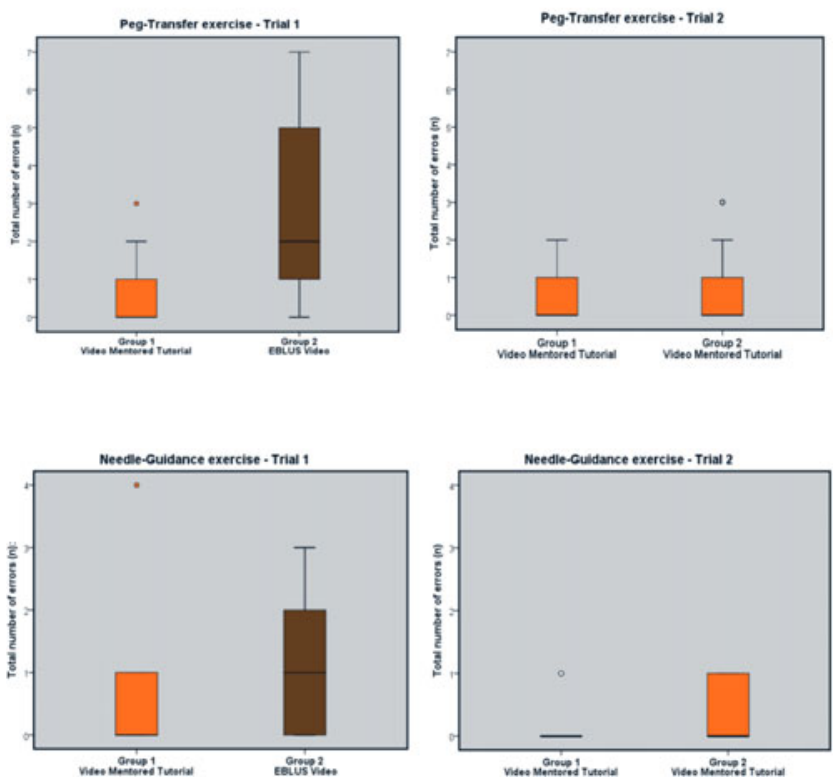

VMT a decrease in the total number of errors in PT and NG exercises was observed in the participants who previously watched the E-BLUS video $(p=0.001$ and $p=0.002$, respectively) (figure 1). In the second period, a decrease in median task time was observed for group 1 and 2 after watching the PT VMT $(\mathrm{p}=<$ 0.001 and $p=0.003$, respectively) and NG VMT $(p=0.005$ and $\mathrm{p}=0.01$, respectively). Most valued video feature was the presence of "tips and tricks".

Conclusions: These findings suggest that the use of VMT can lead to a smaller number of errors and, if allied to deliberate practice, could lead to a shorter task time in exercise performance among participants with no previous laparoscopic experience. Ultimately, these skills could be transferable to the surgical environment in the operating room, raising surgeons' performances and patients' outcomes.

\section{MP06-08 Development and Validation of a Novel Skills Training Model for PCNL}

G Bozzini, U Besana, G Pini, E Liatsikos, AS Gozen, C Buizza, M Sighinolfi, B Rocco

\section{ASST Valle Olona - Busto Arsizio VA}

Introduction \& Objective: The aim of this study is to validate a totally non biologic training model that combines the use of ultrasound and X ray to train Urologists and Residents in Urology in PerCutaneous NephroLithotripsy (PCNL).

Methods: The training pathway was divided into three modules: Module 1, related to the acquisition of basic UltraSound (US) skill on the kidney; Module 2, consisting of correct Nephrostomy placement; and Module 3, in which a complete PCNL was performed on the model. Trainees practiced on the model first on Module 1, then in 2 and in 3. The pathway was repeated at least three times. Afterward, they rated the performance of the model and the improvement gained using a global rating score questionnaire.

Results: A total of 150 Urologists took part in this study. Questionnaire outcomes on this training model showed a mean 4.21 (range 1-5) of positive outcome overall. Individual 
constructive validity showed statistical significance between the first and the last time that trainees practiced on the PCNL model among the three different modules. Statistical significance was also found between residents, fellows and experts scores. Trainees increased their skills during the training modules.

Conclusions: This PCNL training model allows for the acquisition of technical knowledge and skills as US basic skill, Nephrostomy placement and entire PCNL procedure. Its structured use could allow a better and safer training pathway to increase the skill in performing a PCNL.

\section{MP06-09 Utility and feasibility of video-based as- sessment of residents performing ureteroscopy and laser lithotripsy}

Y Wang, K Dore, D Russell, J Hoogenes, B Shayegan, NC Wong, ED Matsumoto

\section{McMaster University}

Introduction \& Objective: Assessment plays a central role in competency-based medical education. The operating room (OR) is a demanding environment with many competing priorities placed on the primary surgeon, one being the assessment of resident performance. Previous studies show that assessment reliability decreases with increased task complexity. The objective was to explore the utility of video-based assessment of resident performance for ureteroscopy and laser lithotripsy.

Methods: Over a 6-month period, 15 ureteroscopy and laser lithotripsy cases performed by urology residents under staff surgeon guidance were captured on video using a 3-camera setup capturing the surgeon's view, the endoscopic view, and a 360degree view of the OR. Following each case, the staff surgeon assessed resident performance using the 11-item entrustmentbased Ottawa Surgical Competency Operating Room Score Evaluation (O-SCORE) and evaluated his or her own cognitive load during the case using the Surgical Task Load Index (SURGTLX). Two independent staff urologists reviewed each video using the same assessment tools. Raters' results were compared those of the case surgeons.

Results: Over 15 cases, 630 minutes of video was collected of 7 residents and 5 staff surgeons. Six cases were completed by junior residents and 9 by senior residents. The interrater reliability between video assessors was highly correlated $(\mathrm{k}=0.82)$. Comparisons between intraoperative assessment, video-based assessment, and mean O-SCOREs were also highly correlated $(\mathrm{r}=0.7)$. Evaluation of O-SCORE domains for intraoperative performance showed a slight decrease in correlation between the intraoperative assessment and video-based assessment $(\mathrm{r}=0.68)$. All assessors (video and staff surgeons) expressed low cognitive load, with mean SURG-TLX scores of 5 (out of a possible 100) ( $\mathrm{SD} \pm 1.8)$. Video-based assessors frequently rewound the video during critical steps of each case to evaluate resident performance.

Conclusions: We showed that video-based assessment of resident performance during ureteroscopy and laser lithotripsy using the O-SCORE is useful and feasible, with high interrater reliability among the case surgeon and independent video reviewers. Use of the SURG-TLX provided new insight into the cognitive load of both case surgeons and video assessors. Additional research with other types of surgical cases is required to further explore the use of video-based assessment in the OR.

\section{MP06-10 Early Exposure to Robotic Surgery is Im- proving Urology Resident Robotic Skills}

T Rogers, S Bhat, M Covas Moschovas, F Onol, T Larson, V Patel, D Thiel

Global Robotic Institute

Introduction \& Objective: To evaluate whether urology resident trainee robotic skills are improving due to earlier exposure during training and widespread acceptance of robotic technology into urologic practice.

Methods: Urologic resident trainees from across the southeast attended a 2-day robotic training course. While in attendance the trainees filled out questionnaires which obtained information about their individual access to robotic simulation, general robot experience, and time spent on the console. Each trainee completed a set of specific tasks on the Mimic Robotic Simulator (Mimic Technologies, Inc., Seattle, WA). Baseline scores were obtained on 4 specific exercises and these scores were compared across four different groups. These groups included all resident course participants in 2012, 2015, and 2019, as well as, a fourth group which consisted of practicing surgeons new to robotics who had their skills evaluated on day 1 of a 5-day training course. These surgeons participated in the same tasks on the Mimic Simulator as the residents. A total of 83 resident trainees from across the Southeast Section of the American Urological Association, participated in a 2-day robotic training course in Celebration, Fl. There were also 28 novice international robotic surgeons attending a separate 5-day robotic training course.

Results: The metric values were normalized by looking at the maximum and minimum metric values for all exercise attempted. These were analyzed and mapped to a $0-1$ scale. Mean and median values were determined for each group and decreasing raw metric values signified improved performance (less time, less motion of instruments, fewer errors, etc.). The 2019 resident trainees outperformed the 2012 and 2015 course participants on every evaluated task. The 2019 residents had an average score of 0.110 , while 2012 and 2015 had scores of 0.143 and 0.119 . The improvement for 2019 participants was seen in earlier PGY groups and without a reported increase in simulator access or actual robotic console time. The 2019 residents also outperformed the international surgeons with scores of 0.110 and 0.126 , respectively. Conclusions: Resident trainees are achieving better scores on basic simulator exercises in 2019 when compared to two previous groups of residents, 2012 and 2015. Current Urology residents are also outperforming practicing international surgeons new to robotics. This linear progression is displayed by residents that are in an earlier stage of their urological training and is not dependent on simulator access or console time.

\section{MP06-11 Evaluation of the Non-Technical Skills for Urological Surgeons (NTS) Curriculum and Assess- ment Scale}

CM Griffin, A Aydin, O Brunckhorst, C McIlhenny, J Brewin, N Raison, A Al-Jabir, T Brouwers, J Palou Redorta, M Khan, P Dasgupta, K Ahmed

King's College London

Introduction \& Objective: In the last decade non-technical skills (NTS) have emerged as a vital area for improvement within surgery: they are a major cause for error and have often been overlooked in training. Although training for NTS is now 
receiving greater attention, there is still a lack of standardized, validated training programs and high-quality studies to evaluate them. This study aimed to develop and evaluate a Nontechnical Skills for Urological Surgeons (NoTSUS) training curriculum and assessment scale.

Methods: Delphi methodology was conducted with experts in urolithiasis and NTS training to refine the curriculum content and NoTSUS rating scale. Four independent one-day training sessions were delivered as a HoT course as part of an international longitudinal observational study. In groups of four, 62 participants underwent four independent scenarios within the previously validated Full Immersion / Distributed Simulation 'igloo' environment. Each candidate was assessed using both the (Non-technical Skills for Surgeons) NoTSS and NoTSUS rating scales by NTStrained and non-trained experts in urolithiasis. After the session, candidates were asked to fill out an evaluation questionnaire.

Results: 43 trainee surgeons and 19 specialists from the United Kingdom, North America and Europe underwent the NoTSUS course. The NoTSUS curriculum received positive feedback from participants and demonstrated educational value, feasibility and acceptability, however did not demonstrate construct validity. The NoTSUS scale was significantly correlated to the NoTSS scale $(\mathrm{p}<0.0001)$, and there was significant correlation between the NoTSUS scores of two expert examiners $(\mathrm{p}=0.012)$.

Conclusions: The NoTSUS curriculum is a useful component of NTS training for urological surgeons, with all participants stating they benefitted from the course. Correlation of the NoTSUS scale with the extensively validated NoTSS scale proves its suitability for evaluating NTS in future training. Demonstration of interrater reliability indicates that the scale is reliable for use in assessment. Furthermore, qualitative feedback from participants suggests the skills learnt over the course will be transferred to practice to continue to improve patient outcomes.

MP06-12 Construction And Assessment Of An Innovative Indigenous All-Endo-Uro-Sim - A One Stop Solution For Endourology Training

A Rawandale-Patil, LG Patni, A Gade, S Kaushik, PA Rawandale, A Hindustanwala

Tejnaksh Hospital's Institute of Urology

Introduction \& Objective: Endourology is evolving at fast pace. Simulation based effective endourology training of the trainee at a low cost is the mandate of the day. To provide a one stop solution for endourology training.

Material and Methods: The All-Endo-Uro-sim was designed and manufactured after a brief anatomical study of the human genitourinary system. It consists of a silicone model representing the penis, scrotum, bladder, ureters and pelvicalyceal system. This model was used for endourological procedures such as Cystolithotripsy, ureterolithotrity and flexible retrograde intrarenal surgery using routine endoscopes and accessories. Evaluation using a 3-step test, GRS score and trainee feedback was analyzed using Spearman rank order correlations and paired to test.

Results: There was overall improvement in the pre and post test GRS scores of all the trainees. The procedure time, instrument handling and injuries to the organs showed a statistically significant betterment (Spearman rank order correlations) $\mathrm{p}<0.001$. Face and content validity as evaluated by the experts was satisfactory. Subjective simulator assessment of the trainees indicated a high degree of satisfaction on effectiveness of the simulator.
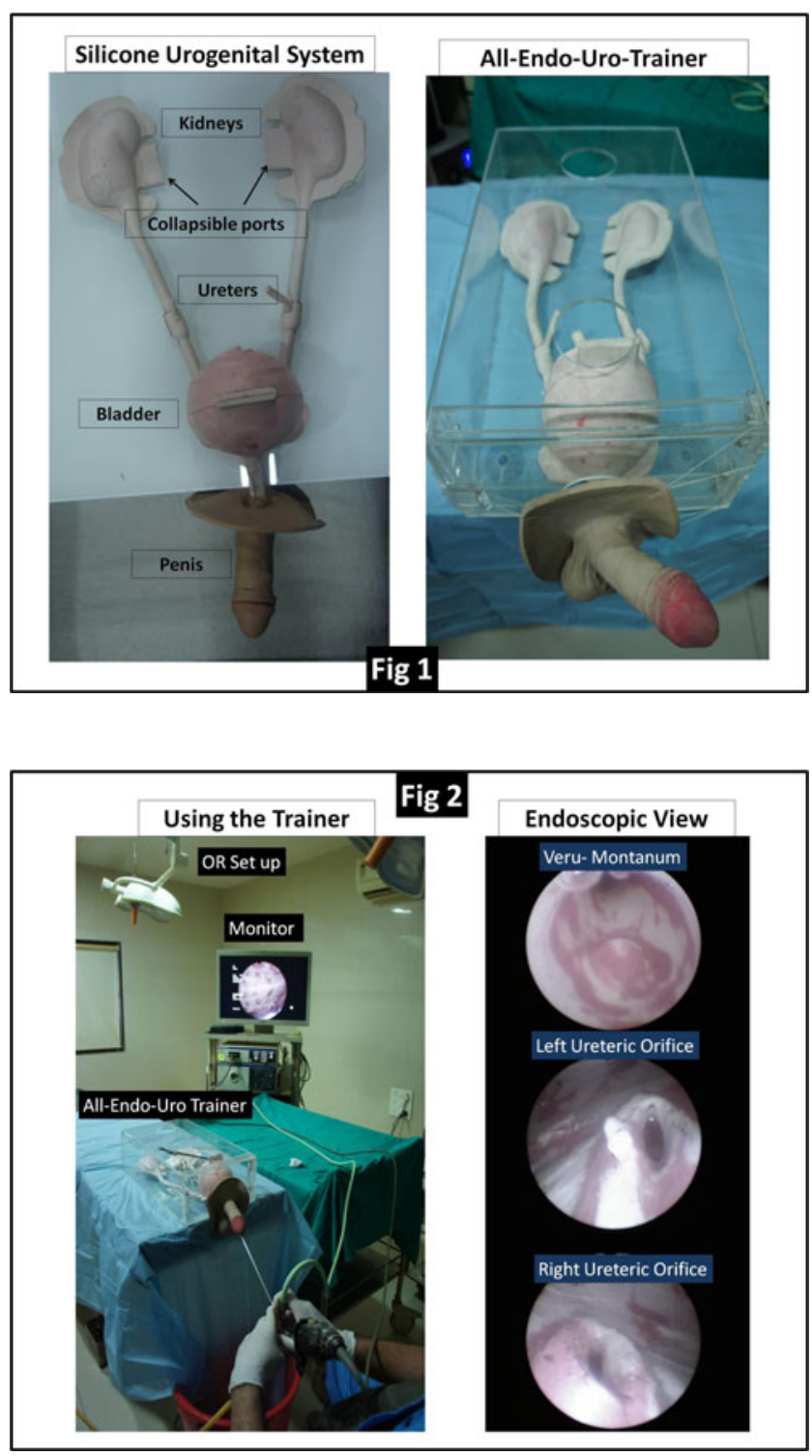

Conclusions: Our portable All-Endo-Uro-sim is a cost-effective training model for endourologic training. It uses routine endoscopic instruments and accessories. It is portable and washable. It allows evaluation and supervised repetitive tailored learning in a controlled, low stress environment. The simulator has the potential to be used as a percutaneous trainer which would make it one of its kind; a complete solution for most endourological procedures.

MP06-13 Evaluation of The Learning Curve of the European Training In Basic Laparoscopic Skills (EBLUS) Validated Training Model: A Prospective Cohort Study

MS Amin, N Abbud, A Aydin, B Van Cleynenbreugel, D Venenziano, BK Somani, AS Gozen, J Palou Redorta, M Khan, P Dasgupta, J Makanjuola, K Ahmed

MRC Centre for Transplantation, King's College London, London, UK 
Introduction \& Objective: Despite the advances of robotic surgery, it remains necessary to training surgeons in laparoscopy. Training curriculums such as the European Training in Basic Laparoscopic Skills (E-BLUS) which aim to achieve this by improving performance and time efficiency of training. This study aims to map the learning curve of the individual tasks of the E-BLUS curriculum to identify plateaus and limiting factors in training.

Methods: Novices were recruited randomized into the cohort. Participants completed the 4 E-BLUS tasks (peg transfer, circle cutting, needle guidance, suturing) ten times. The learning curve was mapped by using a performance improvement score (Piscore) as a measure of skill.

Results: 20 novices were recruited of which 6 participants completed tasks all ten times. Initial data suggests it took an

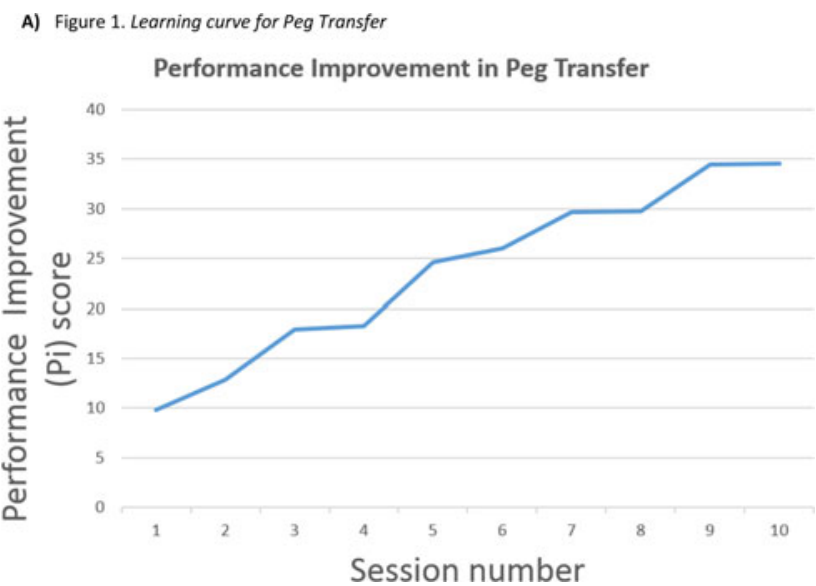

B) Figure 2. Learning curve for Circle Cutting

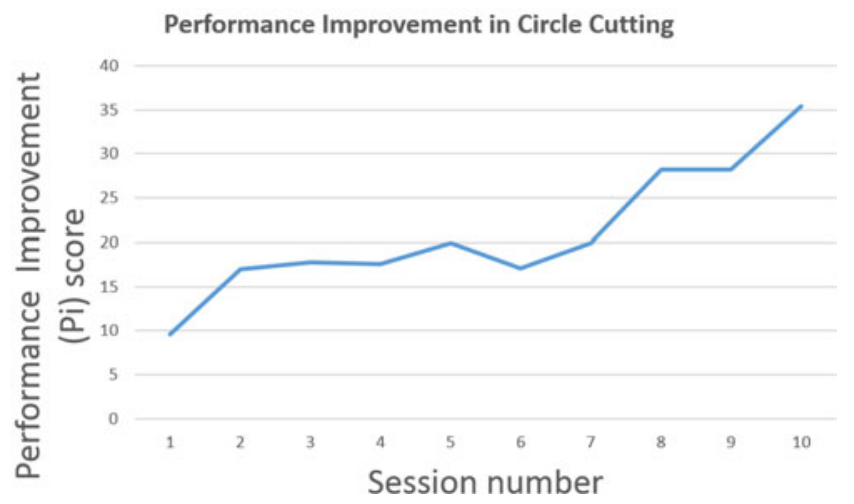

C) Figure 3. Learning curve for Needle Guidance

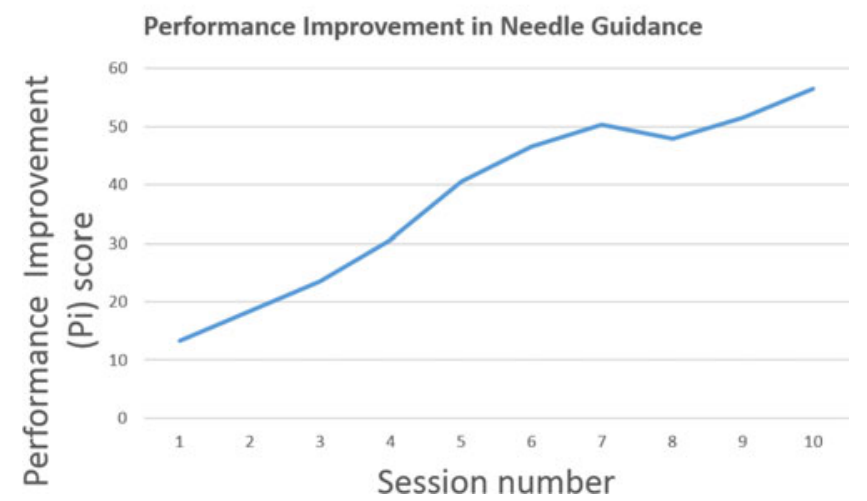

D) Figure 4. Learning curve for Peg Laparoscopic Suturing

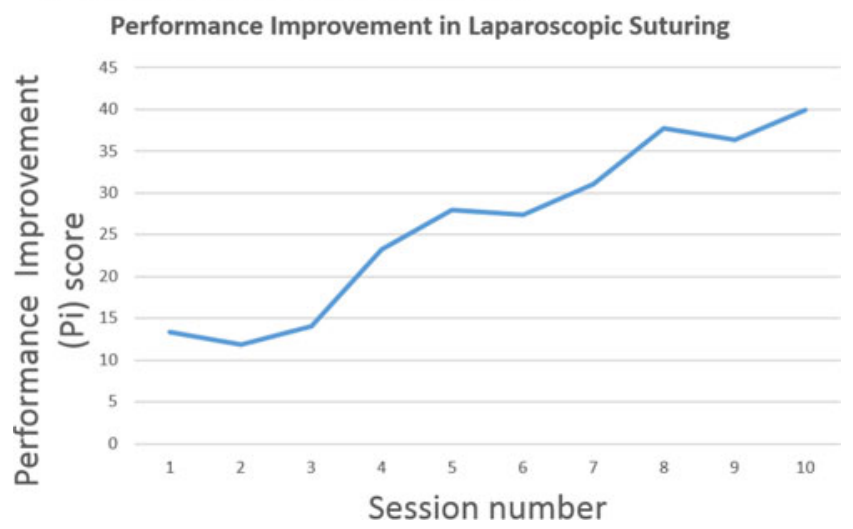

average of 5.8 sessions pass the peg transfer task and 8.5 sessions to successfully complete the circle cutting task. The needle guidance task was passed in an average of 4.6 sessions and an average of 6.5 sessions to pass laparoscopic suturing.

Conclusions: The circle cutting trial limited the time at which EBLUS competency was achieved to 8.5 sessions. This stems from an early plateau in skill identifying the need to improve guidance in the initial stages of the task. After reaching competency in all tasks novices continued to improve, thus terminal plateaus in skill was not reached. This can be identified in future trials by increasing the number of skills sessions received. Further data is required to increase accuracy of findings.

\section{MP06-14 Assessment of Construct validity of virtual reality TURP simulator}

E Lim, A Sim, H Ho, w Lau, K Chen

Singapore General Hospital

Introduction \& Objective: To assess construct validity and potential training benefits of a virtual reality TURP simulator.

Methods: There were a total of 40 participants recruited to complete a virtual simulation median lobe prostate resection task twice on the TURP simulator (VirtaMed UroS tm). Participants were distributed according to their level of experience as follows: 12 experts (> 300 TURPs performed), 11 Residents (Year 1 to Year 6) and 17 novices (no prior experience). Performance

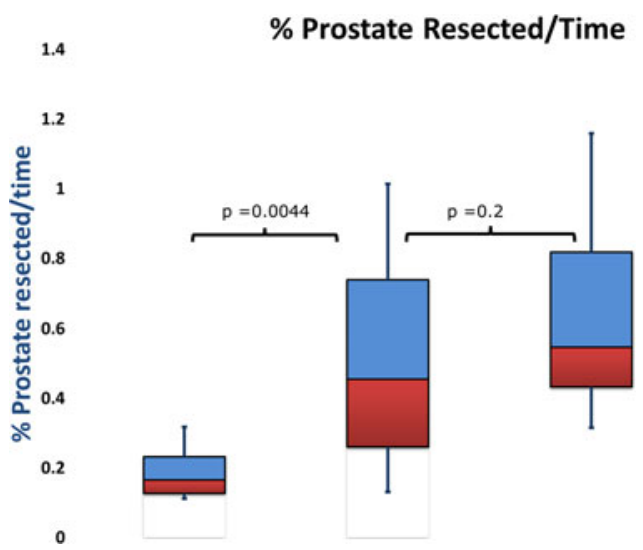



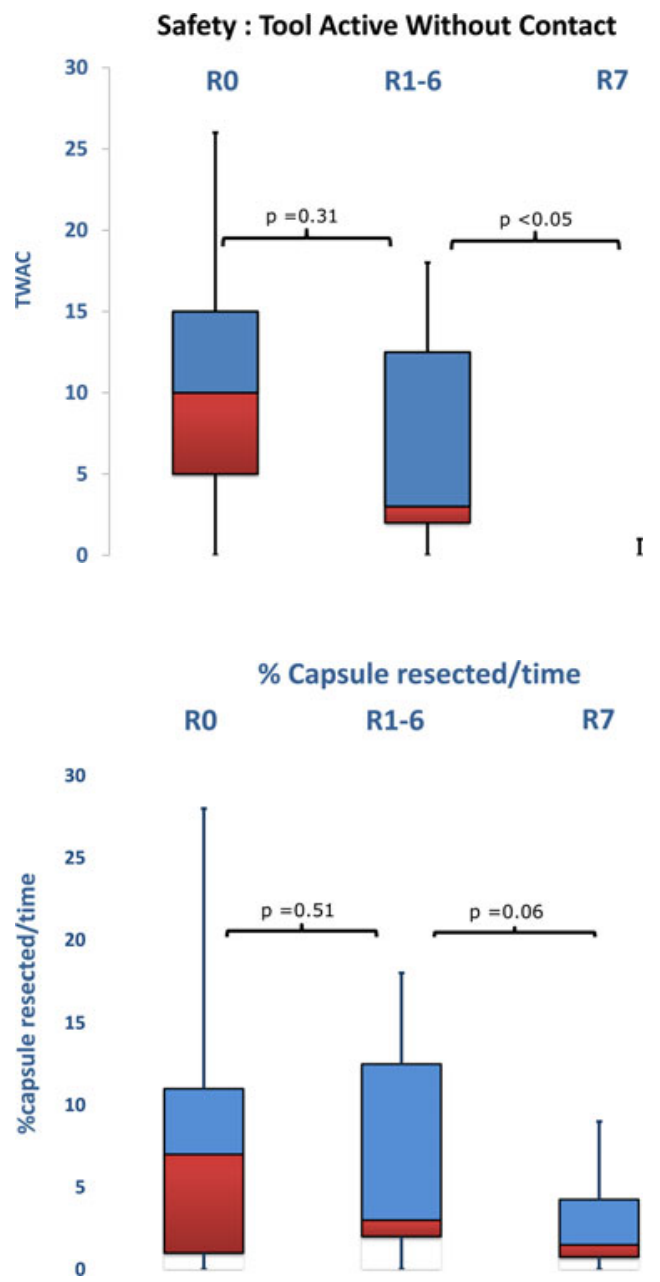

Performance : \% Prostate Resected/Time - 1st vs 2nd Attempt

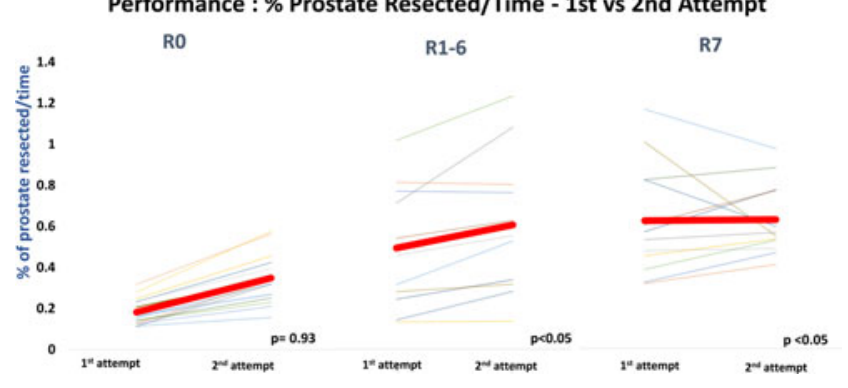

indicators such as percentage of prostate resected, percentage of capsule resected, time diathermy loop active without tissue contact (TAWC) were recorded via TURPsim and compared across all levels of experience (novice to experts) to assess construct validity. Repeated attempts of the task by the novices and residents were analyzed to assess the training benefits of the TURPsim.

Results: Experts resected a significantly greater percentage of prostate per second $(\mathrm{p}<0.001)$ (Fig1) and had significantly less active diathermy time without tissue contact (TWAC) than novices and trainees (Fig2). Percentage of capsule resected was also significantly greater in the novice and resident group compared to the expert group (Fig3). Following a repeated attempt, all groups had improvement in all performance indicators, with

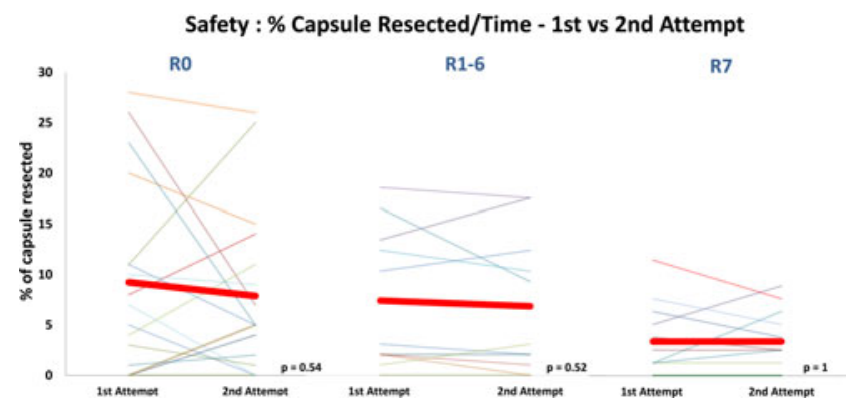

an expected greater increase in the novice and resident group compared to the expert group (Fig 4). There was also a decrease in \% capsule resected/time noted in the novice group and junior residents (R0 to R3) as opposed to the senior residents (R4-R6) and expert group. (Fig 5).

Conclusions: This study has demonstrated the construct validity for the virtual reality TURP simulator. It has also shown potential benefit in utilizing this simulator in training, particularly in the novice and junior resident population.

\section{MP06-15 Objective Mental Workload Evaluation Using Functional Brain Network Metrics and Artifi- cial Neural Network}

S Besharat Shafiei, AA Hussein, NA Aldhaam, KA Guru

Roswell Park Comprehensive Cancer Center

Introduction \& Objective: We sought to find the relationship between visual metrics and mental health measurements of pain, hope, anxiety, and mental well-being.

Methods: Sixteen patients, in the recovery phase after major oncologic surgeries, wear Tobii eyeglasses and were exposed to three categories of art paintings (abstract, figurative, and landscape; totally 18 artworks) at the Roswell Park Art Gallery, developed in collaboration with the Albright Knox Art Gallery, for 15 minutes. Patients completed a survey assessing baseline art knowledge, and 4 post-study questionnaires assessing pain (Pain Rating Scale), hope (Herth Hope Index), anxiety (State-Trait Anxiety Inventory for Adults), and mental wellbeing (WarwickEdinburgh Mental Wellbeing Scale). Extracted eye gaze metrics. Trajectory volume: Volume encompassed by the trajectory of pupil movement, Fixation: A period in which patient's eyes are locked toward a specific area of the art piece, Saccade: The eye gaze points between fixations are known as saccades.

Results: Eye fixation has been suggested as a measure of cognitive engagement. The negative association between pain severity level and fixation rate may suggest that tolerating pain decreases cognitive engagement to the task. While the increase of $\mathrm{HHI}$ is associated with a higher fixation rate, indicating higher engagement to task in patients with a higher level of hope. Saccade feature has been proposed to measure the subject's attention shift with different events and awareness. The negative correlation between saccade and STAI shows the loss of awareness by anxiety increase. While cognitive wellbeing increases awareness. Pupil diameter has been frequently proposed as an indicator of cognitive mental workload, as the pupil dilates under the mental workload. Our results also showed that higher wellbeing and HHI is associated with a lower pupil diameter of both left and right eyes, showing lower mental resources are 
Table 1: Correlation (and p-value) between mental health status metrics and visual metrics.

\begin{tabular}{|c|c|c|c|c|}
\hline Eye Gaze Features & Current pain & Hope & Anxiety & Mental Wellbeing \\
\hline Trajectory volume, left pupil & $-0.05(0.35)$ & $-0.50\left(1 \times 10^{-24}\right)$ & $0.25\left(1 \times 10^{-5}\right)$ & $-0.30\left(7 \times 10^{-5}\right)$ \\
\hline Trajectory volume, right pupil & $0.16\left(2 \times 10^{-3}\right)$ & $-0.56\left(2 \times 10^{-32}\right)$ & $0.26\left(5 \times 10^{-7}\right)$ & $-0.39\left(6 \times 10^{-15}\right)$ \\
\hline $\begin{array}{l}\text { Average change of left pupil } \\
\text { diameter }\end{array}$ & $0.01(0.87)$ & $-0.01(0.90)$ & $0.01(0.9)$ & $0.01(0.78)$ \\
\hline $\begin{array}{l}\text { Average change of right pupil } \\
\text { diameter }\end{array}$ & $-0.01(0.90)$ & $-0.01(0.91)$ & $0.02(0.63)$ & $-0.01(0.85)$ \\
\hline Average left pupil diameter & $-0.16\left(2 \times 10^{-3}\right)$ & $-0.52\left(2 \times 10^{-27}\right)$ & $0.31\left(2 \times 10^{-9}\right)$ & $-0.36\left(8 \times 10^{-13}\right)$ \\
\hline Average right pupil diameter & $0.05(0.34)$ & $-0.58\left(1 \times 10^{-35}\right)$ & $0.29\left(1 \times 10^{-8}\right)$ & $-0.45\left(3 \times 10^{20}\right)$ \\
\hline Fixation & $-0.25\left(8 \times 10^{-7}\right)$ & $0.25\left(7 \times 10^{-7}\right)$ & $0.04(0.49)$ & $-0.05(0.30)$ \\
\hline Saccade & $0.03(0.61)$ & $0.18\left(5 \times 10^{-4}\right)$ & $-0.28\left(1 \times 10^{-8}\right)$ & $0.36\left(98 \times 10^{-13}\right)$ \\
\hline
\end{tabular}

loaded (mental workload) to process the same task in patients with higher wellbeing and hope level. However, anxiety level (STAI) increases mental workload needed to process task. Conclusions: Mental health status may be evaluated by visual metrics.

\section{MP06-16 Trial of A Near-Miss Resident Conference}

J Ahn, B Dalkin, H Wessells, M Sorensen

University of California San Francisco

Introduction \& Objective: Present day morbidity and mortality conferences have several limitations. They are typically conducted in a large public forum amongst peers and superiors, they can be an intimidating environment for the clinician responsible, extensive presenter preparation is frequently required, and only complications meeting a certain severity (death, readmission, etc.) are discussed. Safety experts in other high-risk professions seriously value learning from near-miss and unsafe events which arguably happen exponentially more frequently than serious complications (Figure 1). We introduced a new conference format separate from our department's morbidity and mortality conference to address these limitations.

Methods: We implemented a new quality improvement conference where near-miss clinical events and personal medical errors could be disclosed in a safe environment. This 1-hour conference, held quarterly, permits only residents to attend with the exception of one trusted faculty member to serve as a moderator. Residents were asked to each present one case in a 2minute synopsis or less (no PowerPoint slides required) with an additional 3 minutes for group discussion per case. Presenters would disclose what happened, what he or she learned, and what could have been done differently. Group discussion served to support the reporter, analyze the case, and propose solutions for prevention. Residents were administered surveys after the first session.

Results: The conference was very well received with 11 of 11 $(100 \%)$ resident respondents highly recommending that the conference continue and recur at bimonthly intervals. Cases presented ranged in severity from near-inadvertent ureteral stent placement on the wrong side to forgetting about patient follow up. None of these cases would have been discussed at our morbidity and mortality conference based on their criteria. Residents felt that the conference was more educational than expected, a safer environment for disclosing personal error, and sparked numerous quality improvement project ideas.

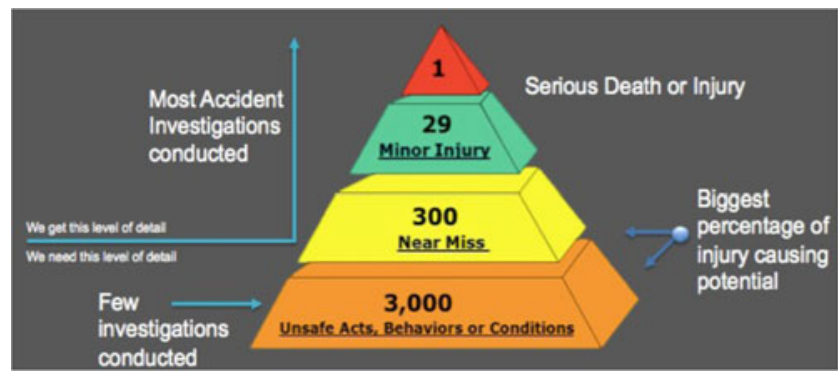

Conclusions: Disclosing personal medical errors can be difficult and near miss clinical complications in our field are likely under reported and analyzed. We propose a new, more resident centered quality improvement conference to better prevent recurrent clinical error.

\section{MP06-17 Evaluation of the Microsoft HoloLens as a Training Tool in Urological Laparoscopic Procedures - a Randomized Control Trial}

N Abbud, A Aydin, MS Amin, C Scott, D Venenziano, B Van Cleynenbreugel, BK Somani, AS Gozen,

J Palou Redorta, M Khan, P Dasgupta, K Ahmed

MRC Centre for Transplantation, King's College London, London, UK

Introduction \& Objective: As the popularity of minimally invasive surgery grows, it is no surprise that there is much interest surrounding training in laparoscopy. The Microsoft HoloLens is a novel mixed reality head-mounted display that, to date, has not been extensively researched. This study aims to evaluate the use of the HoloLens as a training tool for basic laparoscopic skills and assess its impact on the learning curve of participants.

Methods: 40 novices were randomly assigned to either conventional training methods or use of the HoloLens. Each participant, after basic training, was required to complete ten sessions, in which they performed four tasks taken from the validated E-BLUS curriculum. The control group had access to

a) Suturing - Performance Improvement

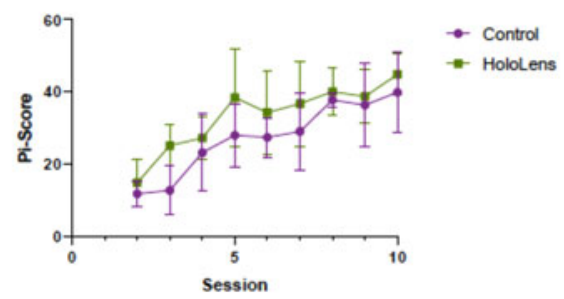

b) Suturing - Sessions To Reach Target

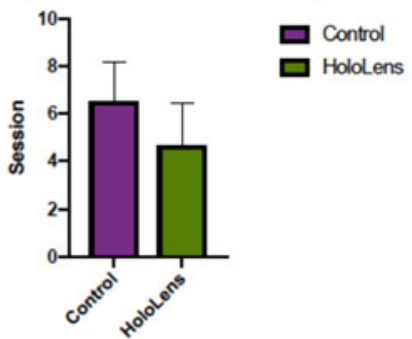

Figure 1 - (a) Performance improvement scores for suturing task. (b) Number of sessions required to reach proficiency for the suturing task. 
the conventional monitor and stack system. The interventional group used the HoloLens to replace the monitor; they could adjust screen size, positioning and closeness, as well as personalize their surroundings to view videos and instructions of the task in real time in the form of 3D holograms. A timer could be viewed, and the footage could also be recorded. A performance improvement score (Pi-Score) was recorded and learning curves for the four tasks were mapped. A comprehensive evaluation survey concerning the HoloLens was distributed and completed for analysis.

Results: No statistical significance was demonstrated in the number of sessions needed to reach proficiency for the peg transfer $(p=0.12)$, circle cutting $(p=0.38)$ and needle guidance tasks $(p=0.23)$. There was however a steeper learning curve for the suturing task. Users reached their target performance in significantly fewer sessions when using the HoloLens compared to conventional methods $(4.6 \pm 1.6$ vs $6.5 \pm 1.75, p=0.045)$. More than $95 \%$ of participants agreed or strongly agreed that the HoloLens has a potential role within surgical education.

Conclusions: The HoloLens has demonstrated faster skill acquisition in knot tying, and its portable design provides a fantastic alternative to the often cumbersome set up of conventional training monitors. The HoloLens provides ergonomic benefits through realignment of the visual-motor axis and allows for a personalized learning environment to be created. Logistical factors, including comfort and battery life, need to be addressed for this device to reach its full potential.

\section{MP06-18 Development and testing of an online motor imagery training tool}

M Goble, N Raison, J Chong, M Khan, P Dasgupta, K Ahmed

King's College London Medical School

Introduction \& Objective: Motor imagery (MI), also known as mental training or mental imagery, is the act of imagining oneself performing a task without physically moving. Current literature indicates it has the potential to help surgeons develop their technical abilities, but no standardized training tool using this concept exists. This research aims to design and test a flexible, accessible training tool using motor imagery.

Methods: A literature review of existing MI protocols and frameworks was undertaken to develop a MI training tool. A pilot randomized controlled trial (RCT) to determine feasibility of testing the tool and evaluate its preliminary efficacy and acceptability was conducted. Medical students were taught suturing skills and randomly assigned to an intervention group with access to the tool or a control group, the primary outcome measures were improvement in MI ability using the Mental Imagery Questionnaire Revised 2 (MIQ-RS) and improvement in suturing skills using a validated 9-point checklist.

Results: The Motor Imagery Training for Surgical Skills (MITSS) tool was developed as an online resource. Median number of log-in times to use the tool was 3 (range 2-10), the median time spent online in one session was 10 minutes (range 267). $3 / 5$ participants rated the platform as easy to use, $4 / 5$ felt there was a benefit to using the tool versus only completing practical training and were likely or very likely to use the tool for other skills in the future. 13 medical students completed the MIQ-RS preintervention, mid-intervention and post-intervention (mean score was respectively $87.0(\mathrm{SD}=14.7)$ in control group, $\mathrm{n}=7$, vs 79.2 $(\mathrm{SD}=16.1)$ in intervention group, $\mathrm{n}=6 ; 87.5(\mathrm{SD}=18.0)$ vs 84.2 $(\mathrm{SD}=17.6) ; 87.5(\mathrm{SD}=19.3)$ vs $85.2(\mathrm{SD}=15.1))$. There was no statistically significant effect of group allocation on changes in
MIQ-RS scores over time $(\mathrm{F}(2,18)=1.845, \mathrm{p}=0.187$. $)$ The mean suturing ability score pre-intervention was $-68.0(\mathrm{SD}=51.7)$ in the control group and $-97.4(\mathrm{SD}=64.2)$ in the intervention group; and $-37.0(\mathrm{SD}=29.0)$ vs $-13.0(\mathrm{SD}=41.76)$ post-intervention. There was no significant effect of the intervention on score over time $\mathrm{F}$ $(1,9)=2.405, p=0.155$, the relative increase in score was $86.6 \%$ in the intervention group and $45.6 \%$ in the control group. Sample size required for a fully powered RCT based on these results is 52 participants.

Conclusions: The MITSS tool is adaptable, accessible, costeffective and user-friendly. A fully powered randomized control trial evaluating its effectiveness is feasible, preliminary results indicate that use of the tool may improve MI ability and technical skill.

\section{MP06-19 Intrinsic Streaming Platform for Remote Live Surgical Mentoring and Review - Advanced Mentoring in a High Volume Robotic Urological Training Centre}

\author{
J MacKinlay, E Latif, MM Quraishi, B Osman, M Thomas, \\ EH Streeter, BA Eddy, SS Kommu
}

Department of Urology. Kent \& Canterbury Hospital. East Kent Hospitals University NHS Foundation Trust

Introduction \& Objective: With the near exponential uptake of Robotic Surgical Procedures and evolving trends in the need for optimal skills acquisition and delivery, there remains an ever expanding need to develop advanced tools to meet the demands. Our aim was to develop and assess the feasibility of an Intrinsic Streaming Platform for Remote Live Surgical Mentoring and Review. The platform was developed to serve a pentad of roles (1) To enable the Senior Surgeon to Mentor in a real-time two way feedback system to facilitate optimal surgical outcomes for the patient (2) To enable the Junior Surgeon or Robotic Fellow to dial in and liaise with the Senior Surgeon at the time of surgery for guidance in case of difficulty (3) To enable Remote Teleconsultation for patient after care and follow-up (4) To enable video conference capabilities to facilitate Local, Regional and International dialogue during cases with ability to log into multiple sites and (5) To enable visitors, fellows and global faculty to take part in dialogue in the Urology Media Room while live surgery in being performed. All the factors are either directly or indirectly related to potentially providing optimal patient outcomes in patients undergoing Robotic Urological Surgical Procedures in a High Volume Robotic Fellowship Training Centre. Herein, we present our experience.

Methods: The platform was set up with the aid of the NHS Information Technology and Unified Communications Team. The schematic consisted of Large Screen Monitors coupled with both web based and hard wired platforms for direct visualization of the Robotic Surgeon's Console View on a real-time basis. The entire platform was achieved by connections using the daVinci Slave Screen, Robot Console, patient cart, Coax P2P, with a LAN directed connection and communications interface coupled with an Antrika Encoder [Fig 1].

Results: Thirty live cases were successfully tested with image transfer and two-way communications between console surgeon and senior mentor with the full written consent of the patients. Furthermore, all five facets of the pentad of aims were successfully carried out with excellent feedback.

Conclusions: The Intrinsic Streaming Platform for Remote Live Surgical Mentoring and Review proved to be a workable and welcome tool for engagement of surgeon-mentor, surgeon- 


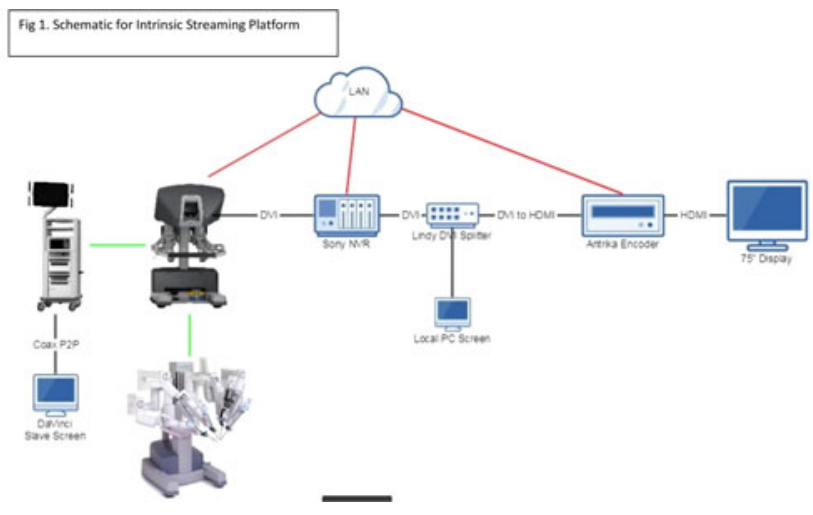

fellow, surgeon-patient and interhospital interface and for optimal interaction in a high volume robotic fellowship training center. Similar platforms could be set up in other robotic training centers.

\section{MP06-20 Evaluation of effective urological ward rounds}

\section{S Murali-Krishnan, J Krishnan, N Osman, R Inman \\ Sheffield Teaching Hospitals}

Introduction \& Objective: Urological ward rounds are covering increasingly large numbers, with more complex medical needs in a limited time. This issue, coupled with trainee's desire to rush to other clinical commitments and an ever changing team due to shift patterns, can lead to an unsatisfactory ward round experience with patient care being affected. Our aim was to investigate if a simple World Health Organization (WHO) style checklist based off the Royal College of Surgeons Edinburgh Shine report in 2014 before and after the ward round, could improve staff satisfaction and improve efficiency.

Methods: Our initial audit cycle used a questionnaire to highlight staff members' impression of how the ward round was conducted, including organization, clarity of plans, staffing, if their issues were addressed and overall satisfaction. The questionnaire used a 5point scale ranging from 1 equating to "strongly disagree" and 5 being "strongly agree". We also recorded the total time of the ward round, number of patients on the ward round, number of interruptions and number of times team members were not actively engaging in the ward round. This was performed over a week and the audit was repeated for a further week after the implementation of the ward round checklist before and after the ward round. During the second cycle we also measured our documentation compliance against General Medical Council standards.

Results: A Wilcoxon Signed-Rank test was used to investigate if a there was a statically significant difference in the overall satisfaction of ward round between the first cycle and the second. The data demonstrated a statistically significant increase of satisfaction of 1.38 points $(\mathrm{p}<0.05)$ of the two groups. Interruptions of the ward round had also reduced from between 3 and 5 per day to almost none with the exception of one cardiac arrest on the ward. Conclusions: Our results show that with a simple framework and cultural change implemented in a firm, team satisfaction and efficiency improved. This study could not account for the complexity of each patient however the overall ward round time was also reduced despite an increased patient load. Interruptions were minimized by addressing issues of split duties of registrars whom had to run the ward round with the on call bleep as well and the nursing staff were better incorporated in to the ward round. Despite the profound impact on the firm that these changes made, they were very easy to implement and due to this we would hope that it is applicable to other units where they face similar issues with ward rounds.

\section{MP06-21 Withdrawn}

\section{MP06-22 Residency Training for Robotic Partial Nephrectomy with a Porcine Model}

K Du, N Badhiwala, S Monda, A Sivaraman, E Kim, R Venkatesh, J Vetter, A DeClue, R Figenshau

Washington University in St. Louis

Introduction \& Objective: Residency robotics training increasingly relies on simulations. We present our experience with a porcine model for robotic assisted partial nephrectomy (RAPN) training.

Methods: A quarter-size circular area was marked on porcine kidneys as the area of the "tumor", and RAPN was performed by residents in a standardized manner using Intuitive Surgical Da Vinci Si robot at a surgical education center. Each resident partook in 4 training sessions in pairs of a senior (PGY 4-5) with a junior (PGY 2-3) evenly spread across the 2017-2018 academic year. Each session was video recorded and scored using the global evaluative assessment of robotic skills (GEARS), a validated standardized assessment tool for robotic surgical skills, by a robotics faculty member. A survey of the residents was conducted at the beginning and end of the program.

Results: Twelve residents, ranging from PGY 2 to 5, completed the program. Mean excision, renorrhaphy, and total times were 8.2, 13.9, and 22.1 minutes, respectively, and mean GEARS score was 16.7. Pearson correlation coefficients between GEARS score and excision, renorrhaphy, and total times were $-0.682,-0.504$, and -0.702 , respectively (all $\mathrm{p}<0.001$ ). On average, residents reduced their excision, renorrhaphy, and total times by 1.6, 2.0, and 3.6 minutes (all $\mathrm{p}<0.001$ ), respectively, while improving their GEARS score by $1.4(\mathrm{p}=0.008)$ with each subsequent session. Comparing the $4^{\text {th }}$ and $1^{\text {st }}$ sessions, PGY 4 residents improved their GEARS score significantly more than any other class, while no significant difference was found between other classes or in mean change in total time (Table 1). Residents' self-reported confidence level at performing RAPN and robotic surgery increased over the course of the program $(\mathrm{p}=0.012$ and $\mathrm{p}<0.001$, respectively). There was no difference in change in confidence level between junior and senior residents $(p=0.213$ for RAPN; $\mathrm{p}=0.140$ for robotic surgery). Overall, residents rated that this program has greatly contributed to their skill (4 out of 5) and confidence (4.1 out of 5 ) in performing robotic surgery.

Conclusions: Porcine kidney model is an effective training and evaluation instrument for resident education on RAPN and robotic surgery. Adoption of this simulation in a urology residency curriculum may improve residents' skill and confidence in robotics.

Table 1
\begin{tabular}{|l|r|r|}
\hline PGY Year & $\begin{array}{l}\text { Mean Change in GEARS score } \\
\text { Session 1 to Session 4 }\end{array}$ & $\begin{array}{l}\text { Mean Change in Total Time in } \\
\text { Minutes Session 1 to Session 4 }\end{array}$ \\
\hline PGY 2 & 3.0 & -12.6 \\
\hline PGY 3 & 1.7 & -8.0 \\
\hline PGY 4 & 9.0 & -12.9 \\
\hline PGY 5 & 2.0 & -7.5 \\
\hline
\end{tabular}




\section{MODERATED POSTER SESSION 07: CLINICAL STONES: PCNL (I)}

\section{MP07-01 Withdrawn}

MP07-02 Mini versus standard percutaneous nephrolithotomy for treatment of pediatric renal stones: Is smaller enough?

Sn Mahmood, BO Mohammed, HM Toffeq, SS Fakhraldin

Univesrsity of Sulaimania, College of Medicine, Department of Surgery

\section{Introduction \& Objective:}

Introduction: In an effort to lower perioperative morbidity while maintaining high stone-free rates, miniaturized percutaneous nephrolithotomy (PCNL) has gained increasing popularity in recent years. However, its potential advantages in terms of safety and efficacy when compared to conventional PCNL is still under debate.

Objective: We retrospectively assessed the safety and efficacy of pediatric renal stones treated by mini-percutaneous nephrolithotomy (MPCNL) versus standard percutaneous nephrolithotomy (SPCNL).

Methods: We performed a retrospective data analysis of 134 consecutive patients under the age of 17 years who underwent PCNL between January 2014 and July 2018. The patients were categorized into two treatment groups depending on the size of the tract and the instruments used. Seventy-five patients were treated with SPCNL using adult instruments via a 22-26 Fr tract, and 59 patients were treated with MPCNL using pediatric instruments via a 16-20 Fr tract. In both groups, the patients were operated on under general anesthesia in the prone position by a single experienced endourologist, and the stones were fragmented by pneumatic lithotripsy. Critical aspects were analyzed in both groups, including stone clearance, perioperative complications, operative time, and duration of hospitalization.

Table 1. patients' preoperative characteristic

\begin{tabular}{|l|c|c|c|}
\hline Variable & Mini-PCNL & Standard PCNL & p-value \\
\hline Number of patients & 59 & 75 & \\
\hline Age (y) & $6.91 \pm 4.982$ & $6.20 \pm 4.138$ & 0.376 \\
\hline Gender (M/F) n, (\%) & $31(52.5) / 28($ & $51(68) / 24(32)$ & 0.16 \\
\hline Previous stone related surgery & $47.5)$ & & \\
\hline Stone size ,cm , mean (SD) & $8(13.8 \%)$ & $11(14.7)$ & 0.886 \\
\hline Stone location & $1.9 \pm 1.162$ & $2.2 \pm 1.424$ & 0.188 \\
pelvis & & & \\
middle calyx & $21,(36.2 \%)$ & $34,(45.3)$ & \\
lower calyx & $5,(8.6)$ & $3,(4.0)$ & \\
pelvis +lower calyx & $6,(10.3 \%)$ & $5,(6.7)$ & \\
upper +lower calyx & $14,(24.1)$ & $23,(30.7)$ & 0.285 \\
pelvistmiddle calyx & $1,(1.7 \%)$ & $1,(1.3)$ & \\
pelvis+upper calyx+middle calyx+ lower calyx & $2,(3.4)$ & $2,(2.7)$ & \\
pelvis +upper calyx +lower calyx & $4,(6.9)$ & $4,(5.3)$ & \\
\hline Stone complexity & $5,(8.6)$ & $3,(4.0)$ & \\
Single stone & & & \\
Multiple stone & $22(37.3)$ & $30(40.1)$ & \\
Partial staghorn & $26(44.1)$ & $31(41.3)$ & \multirow{2}{*}{0.492} \\
Complete staghorn & $6(10.2)$ & $6(8)$ & \\
& $5(8.5)$ & $8(10.7)$ & \\
& & & \\
\hline GSS ,n,\% & & & \\
1 & & & \\
2 & $20(34.5)$ & $30(40)$ & \\
3 & $27(46.6)$ & $31(41.3)$ & 0.439 \\
4 & $8(13.8)$ & $6(8)$ & \\
\hline Preoperative creatinine & $3(5.2)$ & $8(10.7)$ & \\
\hline & $0.056 \pm 0.206$ & $0.05 \pm 0.275$ & 0.878 \\
\hline
\end{tabular}

Table 2. Operative and postoperative characteristics

\begin{tabular}{|c|c|c|c|}
\hline Variables & MPCNL & SPCNL & p-value \\
\hline Operative duration, $\min$, mean (SD) & $41.53 \pm 10.597$ & $37.52 \pm 6.747$ & 0.073 \\
\hline Hospital stay, days, mean (SD) & $1.91, \pm 1.154$ & $2.41, \pm 1.14$ & 0.01440 \\
\hline $\begin{array}{l}\text { Punctures, } \boldsymbol{n}(\%) \\
\text { Single puncture } \\
\text { Multiple punctures }\end{array}$ & $\begin{array}{l}52,(88.1) \\
7,(11.9)\end{array}$ & $\begin{array}{l}74,(98.7) \\
1,(1.3)\end{array}$ & 0.001 \\
\hline $\begin{array}{l}\text { Operative and postoperative complication,, } \boldsymbol{n}(\%) \\
\text { Blood transfusion, } \boldsymbol{n}(\%) \\
\text { Postoperative fever, } \boldsymbol{n}(\%) \\
\text { Post-operative transient hematuria , } \boldsymbol{n}(\%) \\
\text { Sepsis } \\
\text { Plural injury } \\
\text { Colon injury } \\
\text { Mortality } \\
\text { Urinoma } \\
\text { Prolonged urine leakage ( } 224 \text { hour) }\end{array}$ & $\begin{array}{l}11(18) \\
1(1.69) \\
9(15.5) \\
1(1.69) \\
0 \\
0 \\
0 \\
0 \\
0 \\
0\end{array}$ & $\begin{array}{l}22(29) \\
4(5.3) \\
14(18.9) \\
4(5.3) \\
0 \\
0 \\
0 \\
0 \\
0 \\
0\end{array}$ & $\begin{array}{l}0.389 \\
0.609 \\
0.257\end{array}$ \\
\hline Mean postoperative $\mathrm{Hg}$ drop $(\mathrm{g} / \mathrm{S} \mathrm{SD})$ & $0.354 \pm 0.299$ & $0.568 \pm 0.332$ & 0.001 \\
\hline Mean Post-operative creatinine $(\mathrm{mg} / \mathrm{dl} \pm \mathrm{SD})$ & $0.8210 \pm 0.1733$ & $0.8120 \pm 0.1700$ & 0.763 \\
\hline $\begin{array}{l}\text { Stone clearance (PCNL-single session),, } \boldsymbol{n}(\%) \\
\text { Complete clearance } \\
\text { Stone residual, } \\
\text { CIRF }(\leq 4 \mathrm{~mm})\end{array}$ & $\begin{array}{l}51(89.5) \\
5(8.8) \\
1(1.8)\end{array}$ & $\begin{array}{l}71(94.7) \\
3(4.0) \\
1(1.3)\end{array}$ & 0.513 \\
\hline
\end{tabular}

Results: A total of 134 children, including 75 in the SPCNL group and 59 in the MPCNL group, underwent PCNL and were evaluated. Patient demographics and stone characteristics were comparable between the two groups. The mean stone size ranged from $1.9 \pm 1.162 \mathrm{~mm}$ in the MPCNL group to $2.2 \pm 1.424 \mathrm{~mm}$ in the SPCNL group, and the overall stone-free rate was $89.5 \%$ in the MPCNL group and $94.7 \%$ in the SPCNL group. While the stone-free rate, the incidence of perioperative complications, and the rate of bleeding requiring blood transfusions were higher in the SPCNL group, the operative time was shorter. However, the differences were not statistically significant. Conversely, the mean postoperative hemoglobin decrease was statistically significantly lower in the MPCNL group compared with the SPCNL group, at $0.354 \pm 0.299 \mathrm{~g}$ versus $0.568 \pm 0.332 \mathrm{~g}$, respectively $(\mathrm{p}=0.001)$. In addition, the mean duration of hospitalization was statistically significantly lower in the MPCNL group compared

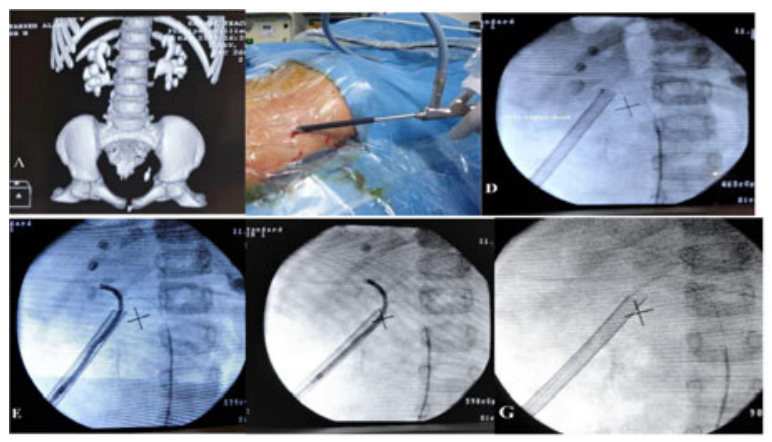

Figure 1

A: preoperative native $\mathrm{CT}$ showing bilateral complete staghorn stone in 6 years old boy.

B\&C: MPCNL of the left staghorn calculi through $20 \mathrm{Fr}$ access, single lower pole access clear majority of the stone.

D\&E: to avoid $2^{\text {nd }}$ and $3^{\text {rd }}$ tract flexible URS with laser lithotripsy used to cleared middle and upper calyx stones.

F: complete stone clearance using single MPCNL $20 \mathrm{Fr}$ access. 
with the SPCNL group, at $1.91 \pm 1.154$ days compared to $2.41 \pm 1.14$ days, respectively $(\mathrm{p}=0.014)$.

Conclusions: MPCNL represents a valuable way of treating simple and complex renal stones in children, with a comparable operative time, stone-free rate, and overall complication rate to SPCNL. MPCNL resulted in shorter hospitalization and fewer hemoglobin drops.

MP07-03 Percutaneous Nephrolithotomy with intraoperative CT-guided evaluation of stone free rate a feasibility study

\section{Zhu, A Kusters, H Langenhuijsen, F d'Ancona}

Radboudumc

Introduction \& Objective: Percutaneous nephrolithotomy (PCNL) has been accepted as a highly effective treatment for large renal calculi. The stone free rate is $87-95 \%$, depending on the definition. Residual fragments are important as they pose a risk for stone-related events. Currently, fluoroscopic imaging is used during PCNL which in turn leads to overestimation of stone free rate. The aim of this prospective study is to demonstrate the feasibility of intra-operative CT-guided evaluation to ensure an actual stone free situation and explore its advantages.

Methods: Adult patients with kidney stones were included, in whom PCNL is indicated according to the Dutch and EAU guideline. Patients are positioned in prone position on a full carbon-operating table. Puncture was performed with ultrasound and fluoroscopy guidance. The Zeego CT scan, positioned in the operating room, is able to make an intraoperative rotation around the patient. The PCNL procedure is performed as usual, directly followed by an intra-operative CT scan. When observed, residual fragments visible on CT scan were removed if possible. Primary outcome was stone free rate. Complications need for reintervention were secondary outcomes. Follow-up occurred 6 weeks after discharge.

Results: Between June 2017 and May 201915 patients were included in the study ( 9 men and 6 women). The mean age was 59.7 years. The mean surgery time was 139 minutes. The stone size was between $9-43 \mathrm{~mm}$. In 1 patient the puncture was unsuccessful. In the remaining 14 patients, we performed miniPCNL in 10 and PCNL in 4. The intra-operative CT scan showed residual fragments (stone size 3, 8,10,10,12 and $16 \mathrm{~mm}$ ) in 6 of the 14 patients. In 1 (with a fragment of $8 \mathrm{~mm}$ ) the PCNL pro-

\begin{tabular}{|c|c|c|c|c|c|c|c|c|}
\hline $\begin{array}{l}\text { Parti- } \\
\text { cipant }\end{array}$ & $\begin{array}{l}\text { STONE } \\
\text { score }\end{array}$ & $\begin{array}{l}\text { Number } \\
\text { of } \\
\text { stones }\end{array}$ & $\begin{array}{l}\text { Stone } \\
\text { size } \\
(\mathrm{mm})\end{array}$ & Approach & $\begin{array}{l}\text { Residual stone } \\
\text { and size on CT }\end{array}$ & $\begin{array}{l}\text { Stone } \\
\text { free }\end{array}$ & Tube & Re-intervention \\
\hline 1 & $7 / 8$ & 4 & 43 & Mini-PCNL & Yes, $16 \mathrm{~mm}$ & No & $\begin{array}{l}\text { Nephro- } \\
\text { stomy }\end{array}$ & No \\
\hline 2 & $7 / 8$ & 1 & 14 & Mini-PCNL & Yes, $8 \mathrm{~mm}$ & Yes & $\begin{array}{l}\text { Nephro- } \\
\text { stomy }\end{array}$ & No \\
\hline 3 & $5 / 6$ & 1 & 19 & Mini-PCNL & No & Yes & $\begin{array}{l}\text { Nephro- } \\
\text { stomy }\end{array}$ & No \\
\hline 4 & $7 / 8$ & 1 & 25 & Mini-PCNL & No & Yes & $\begin{array}{l}\text { Nephro- } \\
\text { stomy }\end{array}$ & No \\
\hline 5 & $7 / 8$ & 4 & 30 & Mini-PCNL & No & Yes & $\begin{array}{l}\text { Nephro- } \\
\text { stomy }\end{array}$ & No \\
\hline 6 & $11 / 12 / 13$ & 1 & 24 & Mini-PCNL & No & Yes & Tubeless & No \\
\hline 7 & $7 / 8$ & 1 & 21 & Mini-PCNL & Yes, $10 \mathrm{~mm}$ & No & $\begin{array}{l}\text { Nephro- } \\
\text { stomy }\end{array}$ & No \\
\hline 8 & $9 / 10$ & 1 & 30 & PCNL & Yes, $10 \mathrm{~mm}$ & No & $\begin{array}{l}\text { Nephro- } \\
\text { stomy }\end{array}$ & No \\
\hline 9 & $9 / 10$ & 1 & 46 & PCNL & Yes, $12 \mathrm{~mm}$ & No & $\begin{array}{l}\text { Nephro- } \\
\text { stomy }\end{array}$ & Yes, URS \\
\hline 10 & $5 / 6$ & 1 & 13 & $\begin{array}{l}\text { Puncture } \\
\text { failed }\end{array}$ & - & - & $\begin{array}{l}\text { Nephro- } \\
\text { stomy }\end{array}$ & No \\
\hline 11 & $5 / 6$ & 1 & 14 & Mini-PCNL & No & Yes & $\begin{array}{l}\text { Nephro- } \\
\text { stomy }\end{array}$ & No \\
\hline 12 & $5 / 6$ & 1 & 11 & Mini.PCNL & No & Yes & $\begin{array}{l}\text { Nephro- } \\
\text { stomy }\end{array}$ & No \\
\hline 13 & $7 / 8$ & 1 & 14 & PCNL & No & Yes & $\begin{array}{l}\text { Nephro- } \\
\text { stomy }\end{array}$ & No \\
\hline 14 & $5 / 6$ & 1 & 9 & Mini-PCNL & No & Yes & $\begin{array}{l}\text { Nephro- } \\
\text { stomy }\end{array}$ & No \\
\hline 15 & $7 / 8$ & 2 & 36 & PCNL & Yes, $3 \mathrm{~mm}$ & No & $\begin{array}{l}\text { Nephro- } \\
\text { stomy }\end{array}$ & No \\
\hline
\end{tabular}

cedure was extended with removal of the fragments. In the other 5 patients the existing access route was insufficient to reach the stones with the nephroscope/cystoscope and a secondary puncture was not carried out due to prolonged surgery time. One (with residual fragment of $12 \mathrm{~mm}$ ) was scheduled for an URS and in others (stone size 3,10,10 and $16 \mathrm{~mm}$ ) conservative approach was applied. Two patients had complications: 1 post-operative pulmonary embolism and 1 clot retention.

Conclusions: Intra-operative CT during PCNL is feasible and provides more certainty regarding stone free rate, which appeared to be lower when compared to literature. In case of residual stones, intra-operative CT provides a window of opportunity to extract them during the same surgery but often they are inaccessible via the same tract. Nevertheless, a plan regarding follow-up, additional imaging or auxiliary procedure can immediately be made.

\section{MP07-04 Supine Percutaneous Nephrolithotomy in Horseshoe Kidneys? Results of a Multicentric Study}

FC Vicentini, E Mazzucchi, M Gokce, M Sofer, Y Tanidir, T Sener, P Melo, B Eisner, T Batter, T Chi, M Armas-Phan, C Scoffone, C Cracco, B Manzo, O Angerri Feu, E Emiliani, O Maugeri, K Stern, C Batagello, M Monga

\section{Brigadeiro Hospital}

Introduction \& Objective: To report on the outcomes of Percutaneous Nephrolithotomy (PCNL) in Horseshoe Kidneys (HSK) in 12 different institutions worldwide and evaluate the impact of positioning during surgery.

\begin{tabular}{|c|c|c|c|c|}
\hline & TOTAL & PRONE & SUPINE & $\mathbf{p}$ \\
\hline $\mathbf{N}$ & 106 & 67 & 39 & \\
\hline AGE (mean - SD) & $50.5(13.3)$ & $51(14.4)$ & 49.5 (11.2) & 0.580 \\
\hline Gender \% Male (n) & $80.2 \%(85)$ & $76.1 \%(51)$ & $87.2 \%(34)$ & 0.211 \\
\hline ASA Score (median - range) & $2(1-3)$ & $2(1-3)$ & $2(1-3)$ & 0.093 \\
\hline Right Side \% & $51.9 \%$ & $52.2 \%$ & $51.3 \%$ & 0.99 \\
\hline BMI (mean - SD) & $29.2(5)$ & $30.1(5,5)$ & $27.7(3.5)$ & 0.024 \\
\hline Previous ESWL Rate \% & $36.8 \%$ & $41.8 \%$ & $28.2 \%$ & 0.085 \\
\hline Previous PCNL Rate\% & $32.4 \%$ & $33.3 \%$ & $30.8 \%$ & 0.99 \\
\hline Preoperative CT Rate \% & $91.8 \%$ & $100 \%$ & $74.1 \%$ & 0.229 \\
\hline Stone Size (mm) (median - range) & $25(19-35)$ & $23(18-35)$ & $27(21-35)$ & 0.367 \\
\hline Stone Density (HU) (median - range) & $1100(836-1321)$ & 1075 (710-1334) & $1200(1000-1300)$ & 0.21 \\
\hline Guy's Stone Score (median - range) & $3(2-4)$ & $3(2-4)$ & $3(2-4)$ & 0.062 \\
\hline Surgical Time (min) (median - range) & $120(80-167)$ & $126.5(85-178)$ & $100(75-139)$ & 0.040 \\
\hline Fluoro time (min) (median - range) & $3(1.2-6)$ & $4(3-6)$ & $1.9(1-10.2)$ & 0.120 \\
\hline Number of Punctures (median - range) & $1(1-2)$ & $1(1-2)$ & $1(1-2)$ & 0.141 \\
\hline \multicolumn{4}{|l|}{ Calyx of Puncture } & $<0.001$ \\
\hline \% Upper Calyx & $66.7 \%$ & $80.3 \%$ & $43.6 \%$ & \\
\hline \% Middle Calyx & $28.6 \%$ & $18.2 \%$ & $46.2 \%$ & \\
\hline \% Lower Calyx & $4.8 \%$ & $1.5 \%$ & $10.3 \%$ & \\
\hline \multicolumn{4}{|l|}{ Dilation Technique } & 0.002 \\
\hline \% Serial & $52.8 \%$ & $40.3 \%$ & $74.4 \%$ & \\
\hline \% Balloon & $47.2 \%$ & $59.7 \%$ & $25.6 \%$ & \\
\hline Nephrostomy rate - \% Yes & $59.8 \%$ & $62.1 \%$ & $55.6 \%$ & 0.664 \\
\hline Double J Rate - \% Yes & $65 \%$ & $60.3 \%$ & $73 \%$ & 0.756 \\
\hline \multicolumn{4}{|l|}{ Lithotriptor } & 0.001 \\
\hline \% Ultrasonic & $71.8 \%$ & $84.4 \%$ & $51.3 \%$ & \\
\hline \% Ballistic/Laser & $28.2 \%$ & $15.6 \%$ & $48.7 \%$ & \\
\hline Delta $\mathrm{Hb}$ (mean - SD) & $1.5(0.9)$ & $1.5(1)$ & $1.6(0.8)$ & 0.575 \\
\hline Transfusion rate $\%$ & $3.8 \%$ & $4.5 \%$ & $2.6 \%$ & 0.999 \\
\hline Complication rate \% & $17.5 \%$ & $16.9 \%$ & $18.4 \%$ & 0.999 \\
\hline Immediate Success $(<=4 \mathrm{~mm}) \%$ & $59.2 \%$ & $52.5 \%$ & $69.2 \%$ & 0.148 \\
\hline Auxiliary procedures \% & $24.5 \%$ & $26.6 \%$ & $21.1 \%$ & 0.637 \\
\hline Final Success rate $(<=4 \mathrm{~mm}) \%$ & $72.4 \%$ & $66.1 \%$ & $82.1 \%$ & 0.086 \\
\hline
\end{tabular}


Methods: We performed a retrospective analysis of prospective databases, between 2008 and 2018, that included all PCNL in HSK. Pre, peri and postoperative data were collected and a subgroup analysis according the patient position was performed. Success rate was defined as absence of $>4 \mathrm{~mm}$ fragments on the control study. A p value $<0.05$ was considered statistically significant.

Results: A total of 106 surgeries were analyzed. Demographic data, stone characteristics and operative parameters are reported in Table 1. The transfusion, complication and immediate success rates were $3.8,17.5$ and $54.7 \%$, respectively. Final success rate increased to $72.4 \%$ after a mean of 0.24 secondary procedures. Multivariate analysis showed that higher BMI and stone size were significantly associated with residual stones $>=4 \mathrm{~mm}$. When analyzing surgeries according to the patient position, 67 patients $(63.2 \%)$ were operated in prone and $39(36.8 \%)$ in supine. Prone patients had a significantly higher BMI than supine group ( 30.1 vs $27.7, p=0.024)$. The transfusion, complication and immediate success rates between prone and supine groups were $4.5 \%$ vs $2.6 \%(p=0.99), 16.9$ vs $18.4 \%(p=0.99)$ and 52.5 vs $69.2 \%(p=0.151)$, respectively. Surgical time was significantly higher for Prone group (126.5 vs $100 \mathrm{~min}, \mathrm{p}=0.04$ ). Upper pole was the preferred access in $80.3 \%$ of Prone group and in $43.6 \%$ in Supine group $(\mathrm{p}<0.001)$. Prone group had significantly more Clavien 2 complications than supine one $(p=0.013)$. Final success rate for prone and supine groups increased to 66.1 and $82.1 \%$ after 0.26 and 0.21 secondary procedures respectively. There were no higher than Clavien 3 complications in this series. Conclusions: Our paper shows that PCNL in HSK is a relatively low frequency procedure. However, it is a safe and effective treatment with a low rate of complications. Higher BMI and stone size impacted negatively outcomes and Supine positioning was associated to a lower operative time. Moreover, it breaks the paradigm that PCNL in HSK should only be done in prone positioning through the upper pole, since supine and prone groups had similar outcomes. Patient positioning during PCNL in HSK could be chosen according surgeon preference.

\section{MP07-05 Practice of Percutaneous Nephrolithotripsy and Outcomes at the Philippine General Hospital}

\section{J Flor, A Cabungcal, M Lapitan \\ University of the Philippines- Philippine General Hospital}

Introduction \& Objective: There have been refinements on the technique of PCNL over the years, but the Philippine General Hospital has just recently acquired the equipment and materials for this complex procedure. The outcomes and practice of PCNL at a tertiary hospital was analyzed.

Methods: Medical records of 57 patients who were treated with PCNL from July to December 2018 were retrospectively scrutinized.

Results: Fifty-seven patients underwent percutaneous nephrolithotripsy in the Philippine General Hospital, with a mean age of 48 years (range 21-78 years). Stone burden significantly predicts length of operative time (mean 103 minutes), lithotripsy time (mean 638 seconds), nephroscopy time (mean 43 minutes), fluoroscopy time (mean 10 minutes) and major complications (sepsis and bleeding requiring blood transfusion) but no association was found with the amount of irrigation fluid utilized which ranged from $200 \mathrm{ml}$ to 50 liters (mean of 6.61 liters). Complete stone clearance was achieved in 44/57 (77 \%) patients. Three patients $(5.26 \%)$ succumb to urosepsis and one $(1.75 \%)$ had delayed hematuria. The average length of hospital stay and postoperative length of stay was 8.6 and 5.2 days, respectively.
The morbidity rate was $5.3 \%$, mortality rate was $0 \%$, reoperation rate was $3.5 \%$, and readmission rate was $8.8 \%$.

Conclusions: As a tertiary hospital that caters to about 120 patients for PCNL per year, PGH can be a considered a highvolume center for this procedure. Stone clearance rate is acceptable which can be improved with time as residents achieve maximum learning curves with the techniques of the operation. Practice and outcomes need to be monitored to deliver the best quality of care tailored to the patient characteristics and stone demographics. The figures presented in this study may be quoted in counseling patients who will undergo PCNL.

MP07-06 Partial tubeless with single step dilatation percutaneous nephrolithotomy with a novel technique of zero degree $c$ arm angel puncture without a single blood transfusion: 1000 cases from single center in rural INDIA

\section{H Tanwar, Y Kaje, A Pardeshi}

Introduction: Practicing endourology with keeping pace in recent advances is a challenging job for a practicing urologist in rural India. With economical constraints of patient even having at least adequate imaging modalities like CT IVP in patients with renal stone disease is at times is difficult. Operating 1000 cases with partial tubeless and single step dilatation without a single patient needing blood transfusion in such scenario was a challenge for us.

Objectives: To access the safety and efficacy of tubeless with single step dilatation percutaneous nephrolithotomy (PCNL) in patients of rural India where due to economical constraints patients can't afford preoperative modern imaging facilities like CT scan.

Methods: Since January 2016, 1000 consecutive Partial tubeless with single step dilatation PCNLs irrespective of guy stone score performed at our hospital were enrolled into this prospective. The study followed the tenets of the Declaration of Helsinki, with an informed consent signed by all the patients who were included. PCNL included a large stone burden, multiple stones, calyceal stones, urinary tract anatomic anomalies, and extracorporeal shock wave lithotripsy or ureteroscopy failure for patients with upper urinary tract stones. All of the PCNLs were performed by the same experienced urology team. All the punctures of PCNL were taken by A NOVEL TECHNIQUE OF ZERO DEGREE C ARM ANGEL. After puncture the renal dilatation was done over a glidewire and alkens guiderod with single step amplatz dilators ranging from $22 \mathrm{fr}$ to $28 \mathrm{fr}$.

Results: The average age of the patients (640 males and 360 females) was $54.4 \pm 12.6$ years, and 80 patients were 70 years old or older. The stone characteristics were 590 non-complete staghorn kidney stones, 218 ureteral stones, 119 kidney + ureteral stones and 70 complete staghorn stones. The mean stone size was $3.0 \pm 1.5 \mathrm{~cm}$, and 90 patients had stone size of more than $5.0 \mathrm{~cm}$. The average operative time was $75.0 \pm 20.4$ minutes. The stone free rates were $88.8 \%, 100 \%, 84.9 \%$ and $74.0 \%$ for kidney, ureter, kidney + ureter and complete staghorn stones, respectively, with an overall stone free rate of $82.3 \%$. The highlight of this study was even with partial tubeless with single step dilatation pcnl none of the patient required blood transfusion.

Conclusions: The novel technique of zero degree $\mathrm{c}$ arm puncture, partial tubeless with single step dilatation PCNL is safe, decreases operative time as well as radiation exposure and can be performed in patients without preoperative CT imaging. 


\section{MP07-07 Withdrawn}

MP07-08 Infra-costal Puncture for Upper Pole Calyceal Access for Percutaneous Stone Extraction in Both Prone and Supine Positions

H Burns, N Smyth, S Nalagatla

University Hospital Monklands

Introduction \& Objective: An upper pole puncture is the best access for upper pole stones and some staghorn stones during percutaneous nephrolithotomy (PCNL). Supra-costal and infracostal approaches are the two described techniques with a higher risk pneumothorax, hydrothorax and bleeding for supra-costal punctures. This study discusses the safety and efficacy of treating upper pole renal stones using an infra-costal puncture in both prone and supine positions by displacing the kidney inferiorly.

Methods: Data was collected retrospectively for all infra-costal punctures to the upper pole of the kidney to assess stone clearance, complications and length of stay for all patients over a 6-year period at a single institution. The kidney was displaced caudally to allow infra-costal puncture either by causing prolonged deep inspiration or mild traction on a ureteric balloon catheter. Alternatively, a flexible ureteroscopic guided puncture was used. The standard procedure involved either prone or supine position, a 30 French (Fr) access sheath with a 27Fr nephroscope and insertion of a 4.8Fr stent. Stone clearance was assessed with a plain Xray KUB. Results: 15 patients underwent infra-costal puncture for the percutaneous treatment of upper pole stones. 7 patients underwent a prone procedure and 6 had a supine PCNL. 14 patients had a postoperative stent and one required a nephrostomy due to residual stone. The overall stone free rate was $93.3 \%$ (all but one patient). $100 \%$ of the prone group were stone free following the procedure. $87.5 \%$ of the supine group had a complete clearance. The mean stone size was $24.4 \mathrm{~mm}(19-41 \mathrm{~mm})$ with a mean density of 937.1 $\mathrm{HU}(517 \mathrm{HU}-1390 \mathrm{HU})$. Average length of stay was 3-4 days. Of the 15 patients, 3 developed post-operative pyrexia, 1 developed sepsis and 3 patients required a blood transfusion. There were no other complications identified, including thoracic injury. There was no difference in complication rate or length of stay in patients with an upper pole or non-upper pole puncture.

Conclusions: Upper pole calyceal stones can be safely and effectively treated percutaneously using direct upper pole puncture via an infra-costal approach in both prone and supine positions with renal displacement with no increase in complication rate and no thoracic injury. A larger patient cohort will further validate these results.

\section{MP07-09 Trends of PCNL in Saudi Arabia}

\section{WK Kamal, A Alhazmy, M Alharthi, A Al-Solumany}

Consultant Urology and Endourology King Fahd Hospital Jeddah

Introduction \& Objective: To present the current practice patterns on percutaneous nephrolithotomy (PCNL) in a Saudi Arabia.

Methods: A survey consisting of 28 questions was sent to urologists working in Saudi Arabia using a Google Forms questionnaire. The first four questions were related to demographic data while the 24 remaining were directed to urologists who performed PCNL. Questions summarized in table 1.

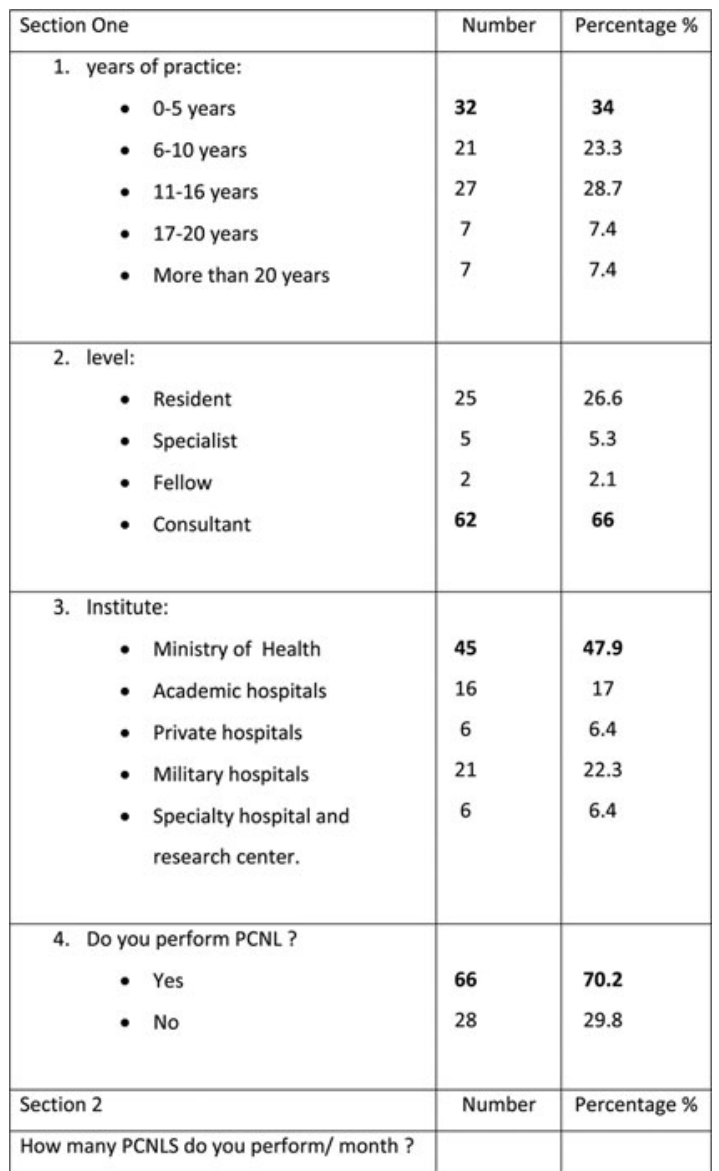

\begin{tabular}{|c|c|c|}
\hline - $0-5$ & 62 & 93.9 \\
\hline - 6-11 & 3 & 4.5 \\
\hline - $12-17$ & 0 & 0 \\
\hline - $18-23$ & 0 & 0 \\
\hline - more than 23 & 1 & 1.5 \\
\hline \multicolumn{3}{|l|}{ To what percentage does the cases of the } \\
\hline PCNL contributes to your general practice? & & \\
\hline - Less than $10 \%$ & 22 & 33.3 \\
\hline - $10-20 \%$ & 25 & 37.9 \\
\hline - $20-30 \%$ & 7 & 10.6 \\
\hline - $30-40 \%$ & 6 & 9.1 \\
\hline - $40-50 \%$ & 3 & 4.5 \\
\hline - $50 \%$ & 3 & 4.5 \\
\hline \multicolumn{3}{|l|}{ How did you learn to perform PCNL ? } \\
\hline - During residency & 39 & 59.1 \\
\hline - Fellowship in Endourology & 13 & 19.7 \\
\hline - From a colleague & 9 & 13.6 \\
\hline - From workshops & 5 & 7.6 \\
\hline \multicolumn{3}{|l|}{ Who performs the puncture of the PCNL? } \\
\hline - Yourself & 53 & 80.3 \\
\hline - Interventional Radiology & 13 & 19.7 \\
\hline \multicolumn{3}{|l|}{$\begin{array}{l}\text { What imaging modality do you obtain prior } \\
\text { to PCNL? }\end{array}$} \\
\hline - X-Ray Kidny Ureter Bladder (KUB) & 11 & 16.7 \\
\hline
\end{tabular}




\begin{tabular}{|c|c|c|}
\hline $\begin{array}{l}\text { - C.T without contrast } \\
\text { - Ultrasound } \\
\text { - Intravenous Pyelogram }\end{array}$ & $\begin{array}{l}60 \\
4 \\
0\end{array}$ & $\begin{array}{c}90.9 \\
6.1 \\
0\end{array}$ \\
\hline $\begin{array}{l}\text { Do you perform Renogram before PCNL? } \\
\text { In all cases } \\
\text { - Only if radiological investigations } \\
\text { suggests decreased renal function } \\
\text { - In patients with Staghorn calculus } \\
\text { - Inever perform Renogram prior to } \\
\text { PCNL } \\
\text { - Answer B and C } \\
\text { - In all cases }\end{array}$ & $\begin{array}{c}2 \\
6 \\
18 \\
6\end{array}$ & $\begin{array}{c}54.5 \\
3 \\
9.1 \\
\\
27.3 \\
9.1\end{array}$ \\
\hline $\begin{array}{l}\text { The regiment for Prophylactic antibiotics is } \\
\text { No prophylaxis } \\
\text { - One shot at induction } \\
\text { - Less than } 24 \text { hours pre operative } \\
\text { - } 24 \text { hours pre operative } \\
\text { - } 1-3 \text { days pre operative } \\
\text { - More than } 3 \text { days }\end{array}$ & $\begin{array}{c}39 \\
10 \\
12 \\
2 \\
2\end{array}$ & $\begin{array}{c}59.1 \\
15.2 \\
18.2 \\
3 \\
3\end{array}$ \\
\hline $\begin{array}{l}\text { You perform the puncture under } \\
\text { - Fluoroscopy guidance } \\
\text { - Ultrasound guidance } \\
\text { - Combined fluoroscopic and } \\
\text { ultrasound } \\
\text { - C.T guidance }\end{array}$ & $\begin{array}{l}45 \\
3 \\
6\end{array}$ & $\begin{array}{r}68.2 \\
4.5 \\
9.1\end{array}$ \\
\hline
\end{tabular}

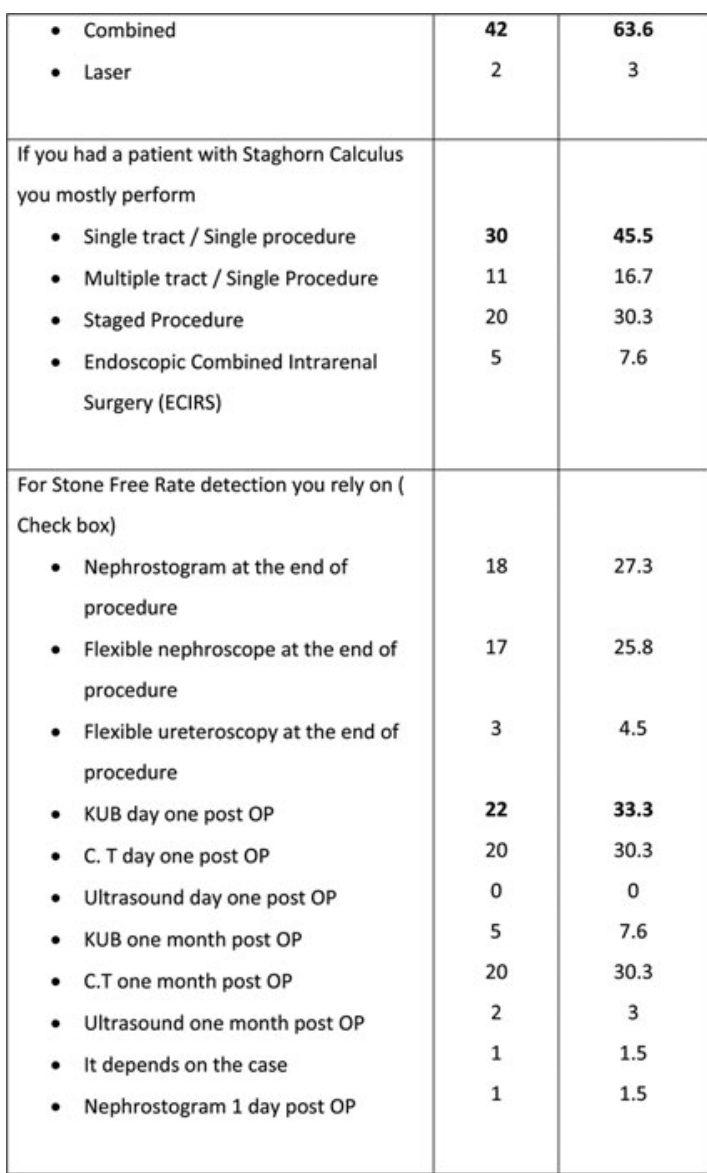

\begin{tabular}{|c|c|c|}
\hline $\begin{array}{l}\text { - Retrograde Puncture } \\
\text { - The intervention radiologist does the } \\
\text { puncture }\end{array}$ & $\begin{array}{c}2 \\
10\end{array}$ & $\begin{array}{c}3 \\
15.2\end{array}$ \\
\hline $\begin{array}{l}\text { In which position do you perform PCNL } \\
\text { - Prone position only } \\
\text { - Supine position only } \\
\text { - More than } 50 \% \text { Prone position } \\
\text { - More than } 50 \% \text { Supine } \\
\text { - } 50-50 \% \text { Prone and Supine }\end{array}$ & $\begin{array}{l}53 \\
3 \\
4 \\
5 \\
1\end{array}$ & $\begin{array}{c}80.3 \\
4.5 \\
6.1 \\
7.6 \\
1.5\end{array}$ \\
\hline $\begin{array}{l}\text { Method of Dilators } \\
\text { - Amplatz dilators } \\
\text { - Balloon Dilators } \\
\text { - Alken Dilators } \\
\text { - Others }\end{array}$ & $\begin{array}{l}11 \\
45 \\
8 \\
1\end{array}$ & $\begin{array}{c}16.7 \\
69.7 \\
12.1 \\
1.5\end{array}$ \\
\hline $\begin{array}{l}\text { To which size do you dilate } \\
\text { - } \mathrm{XL}(25 \mathrm{Fr} \text { and more }) \\
\text { - } \mathrm{L}(20-24 \mathrm{Fr}) \\
\text { - } \mathrm{M}(15-19 \mathrm{Fr}) \\
\text { - } \mathrm{S}(10-14 \mathrm{Fr}) \\
\text { - } \mathrm{XS}(5-9 \mathrm{Fr}) \\
\text { - } \mathrm{XXS}(<5 \mathrm{Fr})\end{array}$ & $\begin{array}{c}48 \\
14 \\
3 \\
0 \\
1 \\
0\end{array}$ & $\begin{array}{c}72.7 \\
21.2 \\
4.5 \\
0 \\
1.5 \\
0\end{array}$ \\
\hline $\begin{array}{l}\text { Energy Sources most frequently used for } \\
\text { Stone fragmentation } \\
\text { - Pneumatic } \\
\text { - Ultrasonic }\end{array}$ & $\begin{array}{c}15 \\
7\end{array}$ & $\begin{array}{l}22.7 \\
10.6\end{array}$ \\
\hline
\end{tabular}

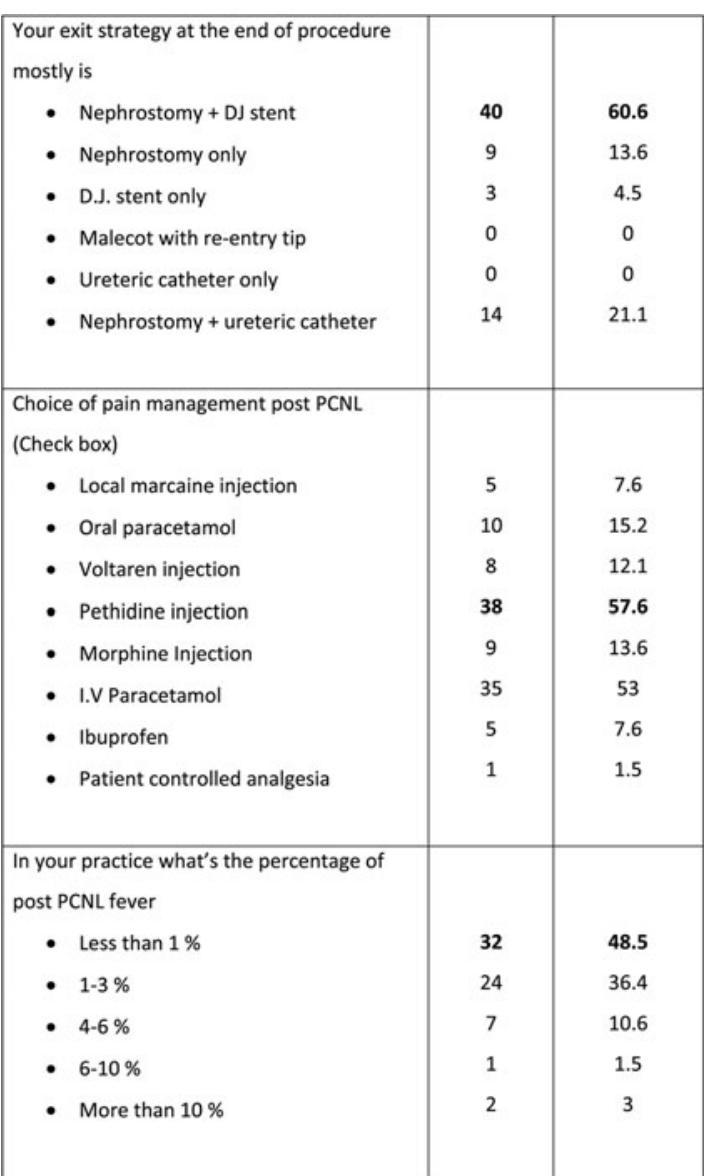




\begin{tabular}{|l|c|c|}
\hline In your practice what's the percentage of & & \\
hydropneomthorax & 60 & 90.9 \\
- Less than $1 \%$ & 5 & 7.6 \\
- $1-3 \%$ & 0 & 0 \\
- $4-6 \%$ & 1 & 1.5 \\
- $6-10 \%$ & 0 & 0 \\
- More than $10 \%$ & & \\
\hline & & \\
\hline In your practice what's the percentage for & & \\
bleeding requiring transfusion post PCNL? & 42 & 63.3 \\
- Less than $1 \%$ & 16 & 24.2 \\
- $1-3 \%$ & 6 & 9.1 \\
- $4-6 \%$ & $2-10 \%$ & 3 \\
- More than $10 \%$ & 0 & 0 \\
& & \\
\hline In your practice what's the percentage of A- & & \\
V fistula post PCNL requiring & & \\
angioimbolization & & \\
- Less than $1 \%$ & 60 & 90.9 \\
- $1-3 \%$ & 6 & 9.1 \\
- $4-6 \%$ & 0 & 0 \\
- $6-10 \%$ & 0 & 0 \\
- More than $10 \%$ & 0 & 0 \\
\hline & & \\
\hline In your practice what's the percentage of & & \\
febrile UTI ? & & \\
\hline & & \\
\hline & & \\
\hline
\end{tabular}

\begin{tabular}{|c|c|c|}
\hline - $1-3 \%$ & 22 & 33.1 \\
\hline - $4.6 \%$ & 4 & 6.1 \\
\hline - $6-10 \%$ & 2 & 3 \\
\hline More than $10 \%$ & 1 & 1.5 \\
\hline \multicolumn{3}{|l|}{ In your practice what's the percentage of } \\
\hline \multicolumn{3}{|l|}{ neighboring organs injury } \\
\hline - Less than $1 \%$ & 63 & 95.5 \\
\hline - $1-3 \%$ & 3 & 4.5 \\
\hline - $4.6 \%$ & 0 & 0 \\
\hline - $6-10 \%$ & 0 & 0 \\
\hline - More than $10 \%$ & 0 & 0 \\
\hline \multicolumn{3}{|l|}{ How much did the introduction of Flexible } \\
\hline \multicolumn{3}{|l|}{ Ureteroscopy decreased your rate of doing } \\
\hline \multicolumn{3}{|l|}{ PCNL } \\
\hline - Did not affect it at all & 10 & 15.2 \\
\hline - Decreased it by $5 \%$ & 11 & 16.7 \\
\hline - Decreased by $10 \%$ & 15 & 22.7 \\
\hline $\begin{array}{l}\text { - More than } 20 \% \text { decrease in the rate } \\
\text { of PCNL }\end{array}$ & 30 & 45.5 \\
\hline \multicolumn{3}{|l|}{ Do you perform $P C N L$ in pediatrics? } \\
\hline - Yes & 14 & 21.2 \\
\hline - No & 52 & 78.8 \\
\hline
\end{tabular}

Results: 94 replied to the survey. $70.2 \%$ performed PCNL, $59.1 \%$ of them learned PCNL during residency. The access was obtained by the surgeon in $80.3 \%, 68.2 \%$ uses fluoroscopy. The majority $(80.3 \%)$ performs PCNL in the prone position. $69.7 \%$ uses the balloon dilators and $16.7 \%$ uses the amplatz dilators. For kidney drainage, $60.6 \%$ place a nephrostomy tube and a Double J stent together and $4.5 \%$ uses DJ stent only. $45.5 \%$ stated that the introduction of Flexible Ureteroscopy decreased the rate of doing PCNL for more than $20 \%$. Results shown in table 1 .

Conclusions: From a selected group of urologists, we observe that urologists practicing in Saudi Arabia usually learn PCNL during residency. The majority gain their own access for PCNL guided by fluoroscopy. They predominantly prefer the prone position, use balloon dilators to mostly dilate to $\geq 25 \mathrm{Fr}$. The majority uses combined ultrasonic/pneumatic lithotripters. Most of the respondents insert nephrostomy tube with DJ stent as the exit strategy. The complications rate encountered among the participants is consistent with the international figures.45.5\% stated that the introduction of Flexible Ureteroscopy decreased the rate of doing PCNL for more than $20 \%$. Performing PCNL in pediatrics is rare among the participants.

\section{MP07-10 Stone Related Preoperative factors affect- ing postoperative PCNL Stone Free Rate (SFR)}

G Bozzini, G Pini, U Besana, S Proietti, G Giusti, C Buizza, M Sighinolfi, B Rocco

\section{ASST Valle Olona - Busto Arsizio VA}

Introduction \& Objective: The aim of this study was to evaluate the impact of stone related preoperative factors in the PCNL treatment of patients who received this indication according to EAU Guidelines.

Methods: We evaluated 192 consecutive patients who underwent PCNL due to multiple, partial staghorn or staghorn stones between June 2013 and June 2018 in our department. Patients' preoperative factors recorded were stones number, measured larger diameter, position of the stone and Hunsfield number based on contrast enhanced CT. Demographic variables, CROES scores and routine laboratory tests, as well as peri-and post-operative findings, as fluoroscopy and operative times, hemoglobin level drop, stone free rates (SFR) and complication rates according Calvien-Dindo classification were recorded. PCNL was performed in prone position and access was obtained using combined intraoperative US and fluoroscopy guidance by the operating Urologist. Fragmentation and stone removal were accomplished in all patients using pneumatic and ultrasound energy and retrieval graspers through a rigid 24Fr nephroscope. Stone clearance were assessed with low impact CT postoperative third day. All stones were evaluated as residual fragments.

Results: Total SFR was $68.75 \%$ (132 pts). SFR obtained was much higher in patients with a singular stone (117 pts, SFR $76.07 \%$ ) instead of patients with multiple stone (SFR $57.33 \%$ ) $(p<0.05)$. A measured larger diameter do not directly affected SFR ( $p>0.05)$ while the position of the stone played a role with an higher SFR for pelvic stones (77 pts, SFR $88.31 \%$ vs 115 pts, SFR $55.65 \%)(p<0.05)$, Hunsfield number is not directly related with a worst SFR $(\mathrm{p}>0.05)$ while played an important role on duration of the procedure $(\mathrm{p}<0.05)$. Complications observed were mainly of grade I and II. There were 12 Grade IIIa pts and 2 pts with Grade IIIb that required embolization. In all this Grade III pts SFR was not reached.

Conclusions: Our study demonstrated that SFR is compromised by the presence of multiple and caliceal stones. Larger Stone and Harder stone do not directly affect SFR. Patients with major complication do not reach the Stone free status. 
MP07-11 The Outcome of Ultrasound-Guided Percutaneous Nephrolithotomy in the Treatment of Horseshoe Kidney with Calculi: A Decade's Experience

Y Hong, Q Xu, X Huang, L Xiong, F Zhang, L An, K Ma

Peking University People's Hospital

Introduction \& Objective: To evaluate the safety and efficacy of percutaneous nephrolithotomy (PCNL) in the treatment of horseshoe kidney with calculi.

Methods: Clinical data of 43 patients (50 kidney units) with horseshoe kidney stones performed PCNL from January 2007 to January 2017 were retrospectively analyzed. There were 33 males and 10 females, the mean age was 41.1 years (range, 21-72 years), 20 cases on the left side, 16 cases on the right side, 7 cases on both sides. The group included single stone in 4 kidney units, multiple stones in 39 kidney units, and staghorn stones in 7 kidney units. The mean maximum stone size was $32.8 \mathrm{~mm}$ (Range, $10-70 \mathrm{~mm}$ ). Fifteen patients received extracorporeal shock wave lithotripsy (ESWL) before surgery. The procedure of puncture and dilation were guided by ultrasound solely.

Results: The mean operative time was 80.7 min (range, 35$210 \mathrm{~min}$ ). The mean nephrotomy tube removed was 3.7 days. The postoperative hospital stay was 7.2 days. The postoperative hemoglobin concentration decreased in 34 patients, and the mean hemoglobin decrease was $12.0 \mathrm{~g} / \mathrm{L}$ (Range, 0.7-26.8 g/L). The stone-free rate at hospital discharge is $80 \%$ (40/50). Postoperative complications occurred in 3 patients including fever $\left(>38.5^{\circ} \mathrm{C}\right)$ in $3(7.0 \%)$ cases. There were no patients who received blood transfusion in our study. No sepsis, kidney loss, and adjacent organ injury were observed.

Conclusions: Despite the abnormal anatomy of the kidney, PCNL also has an acceptable stone-free rate and complications in the treatment of horseshoe kidney stones.

MP07-12 Outcome of Ultrasound-Guided Percutaneous Nephrolithotomy in the Treatment of Medullary Sponge Kidney with Symptomatic Stones: ten years' experience

Y Hong, Q Xu, X Huang, L Xiong, L An, H Ye

Peking University People's Hospital

Introduction \& Objective: To assess the safety and efficacy of percutaneous the treatment of Medullary Sponge Kidney with symptomatic stones.

Methods: From March 2006 to February 2016, the clinical data of 77 patients of Medullary Sponge Kidney with symptomatic stones who underwent percutaneous nephrolithotomy in our institute were retrospectively reviewed. The group included 33 men and 44 women with mean age of $42.1 \pm 13.2$ years. And the type of stone included multiple stone in 74 cases and staghorn stone in 3 cases. The mean stone burden was $14.80 \pm 21.18 \mathrm{~cm} 3$ (range, 2.16-126.75 cm3) which was measured by 3 dimensions reconstructed computerized tomography. Preoperative urinary tract infectious was recorded in 15 patients.

Results: 159 percutaneous renal tracts were established in the 91 kidney units of medullary sponge kidney patients included 42 tracts through upper pole, 71 tracts through middle pole and 46 tracts through lower pole. The mean operative time was $88.1 \pm 37.5 \mathrm{~min}$. The mean hemoglobin drop was $15.2 \pm 12.5 \mathrm{~g} / \mathrm{L}$. The mean postoperative hospital stay was $9.5 \pm 6.1 \mathrm{~d}$. The hospital discharge stone-free rate was $67.0 \%$ and the final stone-free rate was $78.0 \%$ at 3 months after procedure. The complications of Clavien grade I and II occurred in $22(28.6 \%)$ cases included fever in 16 cases and blood transfusion in 8 cases. No sepsis, kidney loss, and adjacent organ injury were observed.

Conclusions: Percutaneous nephrolithotomy is an effective procedure for medullary sponge kidney with symptomatic stones with a lower incidence of high-grade complications.

\section{MP07-13 Percutaneous Nephrolithotomy: Is Mono- planar better than Biplanar Access?}

D Irani, M Hosseini, A Eslahi, A Haghpanah

Shiraz Nephrology Urology Research Center

Introduction \& Objective: The aim of this study was to compare the clinical outcomes and complications of monoplanar and biplanar access techniques for percutaneous nephrolithotomy (PCNL)

Methods: In a prospective study, between May and November 2018, the data from patients who underwent monoplanar or biplanar fluoroscopy-guided access for percutaneous were compared. In monoplanar technique, a C-arm fluoroscope was brought into vertical position, the collecting system was visualized with a contrast agent, and the most appropriate calix was selected for access. In biplanar technique, puncture is adjusted based on different fluoroscopic projections, including vertical and 30 degree positions.

Results: The monoplanar technique was performed for renal access in 101 patients (group 1), and the biplanar technique was used for renal access in 102 patients (group 2). There were no statistically significant differences between the two groups for demographic data, mean operative times, hospital stay, nephrostomy removal time and degree of hydronephrosis $(\mathrm{P}>0.05)$. While, the mean puncture time and fluoroscopy screening time were significantly lower in monoplanar group when compared with that of biplanar group $(\mathrm{P}=0.000)$. The monoplanar and biplanar groups had similar success rates of $81 \%$ and $84 \%$, respectively $(\mathrm{P}>0.05)$, and the rates of complications also were similar for both groups. Conclusions: There is similar success rates for monoplanar and biplanar access techniques, while monoplanar access technique is a safe technique with decreased puncture time and minimized surgical team and the patient's radiation exposure time.

MP07-14 Which one is the easiest and quickest scoring system to predict percutaneous nephrolithotomy outcomes by Junior surgeons?

N Iqbal, T Bhatty, S Akhter, J Rahim

Pakistan Kidney and Liver Institute and Research Center

Introduction \& Objective: To compare the time taken by young urologists for application and the capacity of the nomograms to predict the success of S.T.O.N.E. Nephrolithometry (STONE), Guy's Stone Score (GSS) and Clinical Research Office of the Endourological Society nephrolithometric nomogram (CROES) of percutaneous nephrolithotomy (PCNL).

Methods: We studied 50 patients who underwent PCNL by 2 senior Surgeons (Experience of 10 years in endourology) between 2017 and 2018. A radiologist calculated STONE and CROES based on preoperative non-contrast computed tomography (CT) images and clinical data. Then 10 junior surgeons were given task to review all images and assigned scores. We compared the application time of each nomogram. We used annova t test to compare the time taken for scoring the by different methods describe an analysis of variance ford above. 
Results: The stone free rate was $82.7 \%$ and complications occurred in $8.7 \%$ of cases (mostly clavien grades $1-2$ ). The average operative time was 109 minutes. Mean application time was significantly lower for the GSS (34.5 seconds) when compared to 236.2 seconds for STONE and 245.4 seconds for CROES $(\mathrm{p}<0.001)$. There was no significant difference among the GSS $(\mathrm{AUC}=0.653)$, STONE $(\mathrm{AUC}=0.563)$ and $\mathrm{CROES}(\mathrm{AUC}=$ 0.641 ) in the ability to predict immediate success of PCNL.

Conclusions: All three nomograms had shown similar ability to predict success of PCNL, however the GSS was the quickest to be applied, that is an important aspect for routine clinical use when counseling patients who are candidates to PCNL.

\section{MP07-15 Supine mini-percutaneous nephrolithotomy using a novel access sheath with negative pressure aspiration system: A prospective evaluation from a Swedish tertiary center}

M Popiolek, D Al-Rammahi, P Georgouleas, M Wagenius, S Andersson, O Andrén

Department of Urology, Univeristy Hospital Örebro

Introduction \& Objective: Evacuation of disintegrated stone material during mini-percutaneous nephrolithotomy (mPCNL) usually takes place through a so-called "vacuum cleaner effect" which is both time-consuming and imposes a risk for dislocating the stone fragments. A new working sheath with active extraction assisted by vacuum (ClearPetra ${ }^{\circledR}$, Well Lead Medical Co.) has recently been introduced for $\mathrm{mPCNL}$ the aim was to present our initial experience and evaluate safety and efficacy of mPCNL with ClearPetra ${ }^{\circledR}$.

Methods: All consecutive patients operated with supine mPCNL utilizing ClearPetra ${ }^{\circledR}$ between March 2018 and April 2019 were included in the analysis. Patient data and variables related to the surgery were obtained from a local, prospective register. Stone complexity grade was assessed according to Guy's stone score (GSS). Results: In 52 patients (25 women, 27 men) ClearPetra ${ }^{\circledR}$ (size: $16 \mathrm{Ch}$ ) access sheath was utilized for mPCNL in flank-free modified supine position. The median age was $62 \pm 15.9$ years (16-86) and mean Body Mass Index was 27.5 \pm 4.7 (14.5-40). Median stone size and density were $22 \pm 4.8 \mathrm{~mm}(13-42)$ and $1126 \pm 286$ HU (440-1516), respectively. In 17 cases (34\%) GSS was 3 or higher. Percutaneous access was created with combined ultrasound and fluoroscopy technique (11 upper calyx, 13 middle calyx, 32 lower calyx). Multiple accesses were used in 6 cases $(11 \%)$. ClearPetra ${ }^{\circledR}$ sheath along with a one-step dilator was introduced over a stiff wire without any predilation. HolmiumYAG laser with $550 \mu$ laser fiber was used for stone disintegration. The median operating time was $75 \pm 23$ minutes (38-171). In total, 48 (94\%) received only JJ stent (tubeless) as an exit strategy, which was removed during the first postoperative day in 29 cases $(57 \%)$. Of all, $41(80 \%)$ were discharged within 24 hours. Three patients $(6 \%)$ developed postoperative fever (Clavien II) whereas one needed nephrostomy insertion (Clavien IIIa). Median hemoglobin (Hb) drop was $9 \pm 8,3 \mathrm{~g} / \mathrm{L}$ (0-29). No blood transfusion was required in any of the cases. The surgery was successful (no residuals $>4 \mathrm{~mm}$ ) in 48 cases $(92 \%$ ) and complete stone clearance (no residuals) was achieved in 40 cases (77\%) according to a computed tomography scan performed on the first poroperative day in all patients.

Conclusions: Our study shows high efficacy and safety of mPCNL with ClearPetra ${ }^{\circledR}$ system even in complex, large stone burden. It appears to be a promising adjunct to mPCNL, which might have the potential to additionally improve the outcomes, but further evaluation in larger cohorts is required.

\section{MP07-16 Ideal Candidates for the Tract Dilation Monitored by Ultrasound in Percutaneous Ne- phrolithotomy}

S Wang, X Zhang, Y Tang, B Xiao, J Li

University of Maryland School of Medicine

Introduction \& Objective: Ultrasound guided PCNL is gaining its reputation because of its convenience and being free of X-ray exposure. One issue that would keep urologists away from this technique is the lack of monitoring during the tract dilation since most of the previous ultrasound-guided PCNL studies have focused on the puncture, with the dilation completed either under fluoroscopy or blindly. If the tract dilation could also be monitored under ultrasound, these two vital steps, puncture and dilation, can be perfectly combined. In our study, we aimed at investigating the feasibility of ultrasound guided balloon dilation technique in PCNL, and risk factors for its failure.

Methods: A retrospective study was conducted on patients underwent PCNL with only one access using balloon dilation monitored by ultrasound from December 2014 to May 2018 in Beijing Tsinghua Changgung Hospital. Patients' demographic information, intra and postoperative findings were collected. Risk factors for the failure of this technique were investigated by regression analysis.

Results: 207 patients (115 males and 92 females) with a mean age of $51 \pm 10$ years. Mean BMI was $25.2 \pm 3.3 \mathrm{~kg} / \mathrm{m}^{2}$. Mean size of stone was $3.6 \pm 1.2 \mathrm{~cm}, 47.3 \%$ of which were staghorn stones. $78.3 \%$ of patients manifested hydronephrosis of the targeted calyx. Tract dilation failed in $24(11.6 \%)$ cases at first attempt. Postoperative outcomes were comparable between successful and failed groups. Multivariate analysis revealed that the presence of staghorn stones $(\mathrm{p}=0.032)$, history of ipsilateral open nephrolithotomy lower pole access $(\mathrm{p}=0.026)$, and lower pole access $(p=0.039)$ were risk factors for the failure of access establishment, while the presence of hydronephrosis of target calyx $(p=0.001)$ would significantly increase the success rate.

Conclusions: Tract dilation using the balloon catheter can be safely monitored by ultrasound. Patients with staghorn stones, history of open nephrolithotomy, and lower pole access had a lower success rate while hydronephrotic target calyx will increase the success rate.

\section{MP07-17 Withdrawn}

MP07-18 Mini-Percutaneous Nephrolithotomy versus Retrograde Intrarenal Surgery for the treatment of 10-20mm lower pole renal stones. A Systematic Review and Meta-Analysis

JD Cabrera, B0 Manzo, JE Torres, FC Vicentini, H Sánchez, E Rojas, E Lozada

Hospital Regional de Alta Especialidad del Bajío

Introduction \& Objective: To compare the efficacy and safety of the Mini-Percutaneous nephrolithotomy (Mini-PCNL) and retrograde intrarenal surgery (RIRS) for the treatment of 10$20 \mathrm{~mm}$ lower pole renal stones.

Methods: A comprehensive literature search of PubMed, Scopus, Cochrane, and Web of Science was conducted to identify all 


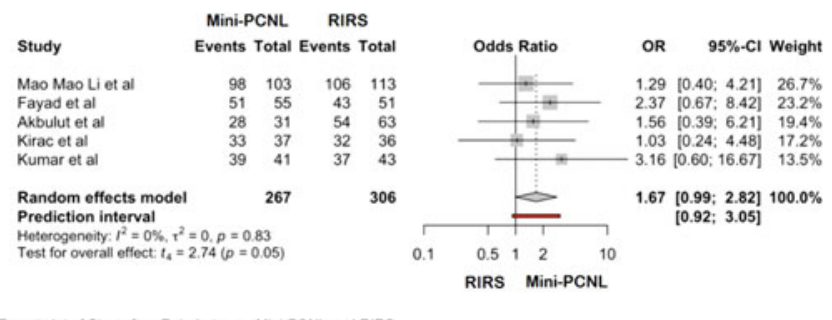

Forest plot of Stone-tree Rate Denween Mini.PCNL and RIRS

studies comparing Mini-PCNL and RIRS for 10-20 mm lower pole renal stones before March 2019. Articles selection proceeded according to the search strategy based on Preferred Reporting Items for Systematic Reviews and Meta-analysis criteria. The meta-analysis was done with the R program version 3.5.1. Results: A total of 5 studies were included, two randomized controlled trials and three retrospective case-controlled studies, with an overall 587 patients included for the analysis. The stonefree rate (SFR) was significantly higher in the Mini-PCNL Group (OR: $1.67 ; 95 \% \mathrm{CI} ; \mathrm{p}=0.05$ ). Operative and fluoroscopy time were similar for both groups (MD: $2.45 ; 95 \% \mathrm{CI} ; \mathrm{p}=0.87$ ) and (MD: $2.11 ; 95 \% \mathrm{CI} ; \mathrm{p}=0.09$ ) respectively. The hospital stay was similar in both groups (MD: 41.94; 95\% CI; $\mathrm{p}=0.18$ ). Concerning the overall complications rate, both procedures showed no difference (OR: $1.76 ; 95 \% \mathrm{CI} ; \mathrm{p}=0.11$ ).

Conclusions: Our analysis showed that both procedures are safe for the treatment of $10-20 \mathrm{~mm}$ lower pole renal stones with similar complications rate, operative time, fluoroscopy time and hospital stay, but Mini-PCNL was significantly superior in effectiveness with a higher SFR. Also, our systematic review showed that randomized controlled trials are necessary to give strength to our conclusion.

\section{MP07-19 Preoperative factors associated with perio- perative complications in Percutaneous Ne- phrolithotomy}

V Grajales, D Miller, A Mohapatra, A Delmonte, J Bigley, T Averch, M Semins

\section{UPMC}

Introduction \& Objective: Percutaneous nephrolithotomy (PCNL) is a widely used procedure for large stone burden and in patients were retrograde approach is compromised. Our study aimed to analyze complication rates using the clavien-dindo modified system in regard to preoperative factors including frailty, renal function, hematocrit, stone burden and BMI.

Methods: We conducted a retrospective study of 149 out of 165 patients who underwent percutaneous nephrolithotomy between January 2016 and January 2018 and met our inclusion criteria at our institution. In total there were 171 PCNL procedures done within our study population since 2010 . Chi square and t tests were used to compare demographics and surgery characteristics for the two groups.

Results: Overall the complication rate in our study was $30.4 \%$, of that $32.6 \%$ were UTIs with a $14 \%$ readmission rate. There were no significant differences in mean age, gender, BMI, preoperative Hct or frailty score index and complication rate. Most of the complications seen were low grade and associated with longer mean operative time (138 vs $117 \mathrm{~min}, \mathrm{p} \mathrm{0.005),} \mathrm{hospital}$ length of stay (2.8 vs 1.4 days, $\mathrm{p} 0.01$ ) and stone burden (4 vs $3 \mathrm{~cm}, \mathrm{p}$ 0.005). Preoperative CKD (11.5 vs 4.2\%, p 0.0000001)

\begin{tabular}{|c|c|c|c|c|c|}
\hline \multirow[t]{3}{*}{ Demographics } & \multirow{2}{*}{\multicolumn{2}{|c|}{ Patients with complications }} & \multirow{2}{*}{\multicolumn{2}{|c|}{$\begin{array}{l}\text { Patients without } \\
\text { complications }\end{array}$}} & \multirow{3}{*}{$P$ value } \\
\hline & & & & & \\
\hline & $N$ & $\%$ & $N$ & $\%$ & \\
\hline Mean Age (years) & 55 & & 56.4 & & 0.6 \\
\hline \multicolumn{6}{|l|}{ Gender } \\
\hline Male & 21 & $24.70 \%$ & 64 & $75.30 \%$ & \multirow{2}{*}{0.11} \\
\hline female & 31 & $36 \%$ & 55 & $64 \%$ & \\
\hline \multicolumn{6}{|l|}{ Laterality } \\
\hline Right & 29 & $55.80 \%$ & 53 & $44.50 \%$ & \multirow{2}{*}{0.001} \\
\hline Left & 22 & $42.30 \%$ & 66 & $55.50 \%$ & \\
\hline Mean BMI & 28.5 & & 29.4 & & 0.7 \\
\hline $\mathrm{Cr}>2$ & 6 & $11.50 \%$ & 5 & $4.20 \%$ & 0.0000001 \\
\hline Het $<35$ & 14 & $26.90 \%$ & 32 & $26.90 \%$ & 0.63 \\
\hline Stone burden (cm) & 4 & & 3 & & 0.005 \\
\hline Operative time (min) & 138 & & 117 & & 0.005 \\
\hline LOS (days) & 2.8 & & 1.4 & & 0.01 \\
\hline Second procedure & 27 & $51.90 \%$ & 33 & $27.70 \%$ & 0.023 \\
\hline \multicolumn{6}{|l|}{ RAI } \\
\hline $0-20$ & 28 & $36.80 \%$ & 48 & $63.20 \%$ & \multirow{2}{*}{0.3} \\
\hline$\geq 21$ & 9 & $26.50 \%$ & 25 & $73.50 \%$ & \\
\hline \multicolumn{6}{|c|}{ Modified Clavien-Dindo } \\
\hline None & 119 & $69.60 \%$ & & & \\
\hline Grade 1 & 15 & $28.80 \%$ & & & \\
\hline Grade 2 & 18 & $34.60 \%$ & & & \\
\hline Grade 3 & 13 & $25.00 \%$ & & & \\
\hline Grade 4 & 6 & $11.50 \%$ & & & \\
\hline Grade 5 & $\mathbf{0}$ & $0 \%$ & & & \\
\hline
\end{tabular}

and secondary procedures (51.9 vs $27.7 \%$, p 0.023 ) were also associated with greater complication rates.

Conclusions: Overall, PCNL is a safe procedure with mostly lower grade clavien complications. Prolonged surgery time must be balanced with secondary procedures to render a patient stone free as both factors were associated with perioperative complications. Preoperative chronic kidney disease and a larger stone burden also carried a higher risk.

MP07-20 Serum Cystatin C, 24 Urine Creatinine Clearance, and Renal scan (DTPA) as a markers of renal function, pre and postoperative percutaneous nephrolithotomy (PCNL)

C Martinez-Arroyo, G Veliz-Cabrera,

P Cortes-Raygoza, M Cantellano-Orozco,

G Fernandez-Noyola, G Morales-Montor,

C Pacheco-Gahbler

Hospital General Dr. Manuel Gea Gonzalez

Introduction \& Objective: In PCNL, there has been an interest in kidney damage caused by the performance of one or multiple tracts and overdistention of the renal pelvis. There are multiple serum, urinary and imaging studies that help identify and compare kidney function. Among them we find the urine creatinine clearance in 24 hours ( $\mathrm{ClCr}$ urine 24), serum Cystatin $\mathrm{C}$ and the renal scan with diethylene triamine pentaacetic acid marker (DTPA). Describe the pre and postoperative evaluation of renal function with different renal markers and images in the PCNL. Methods: Observational, descriptive and transversal study. 127 patients undergoing PCNL. At the evaluation of renal function pre and postoperative exist diferent primary variables, $\mathrm{ClCr}$ urine 24 , serum cystatin C and renal scan DTPA, the secondary variables related with different comorbidities to the disease, unique renal unit, number of tracts, lithiasic volume and number of procedures. 

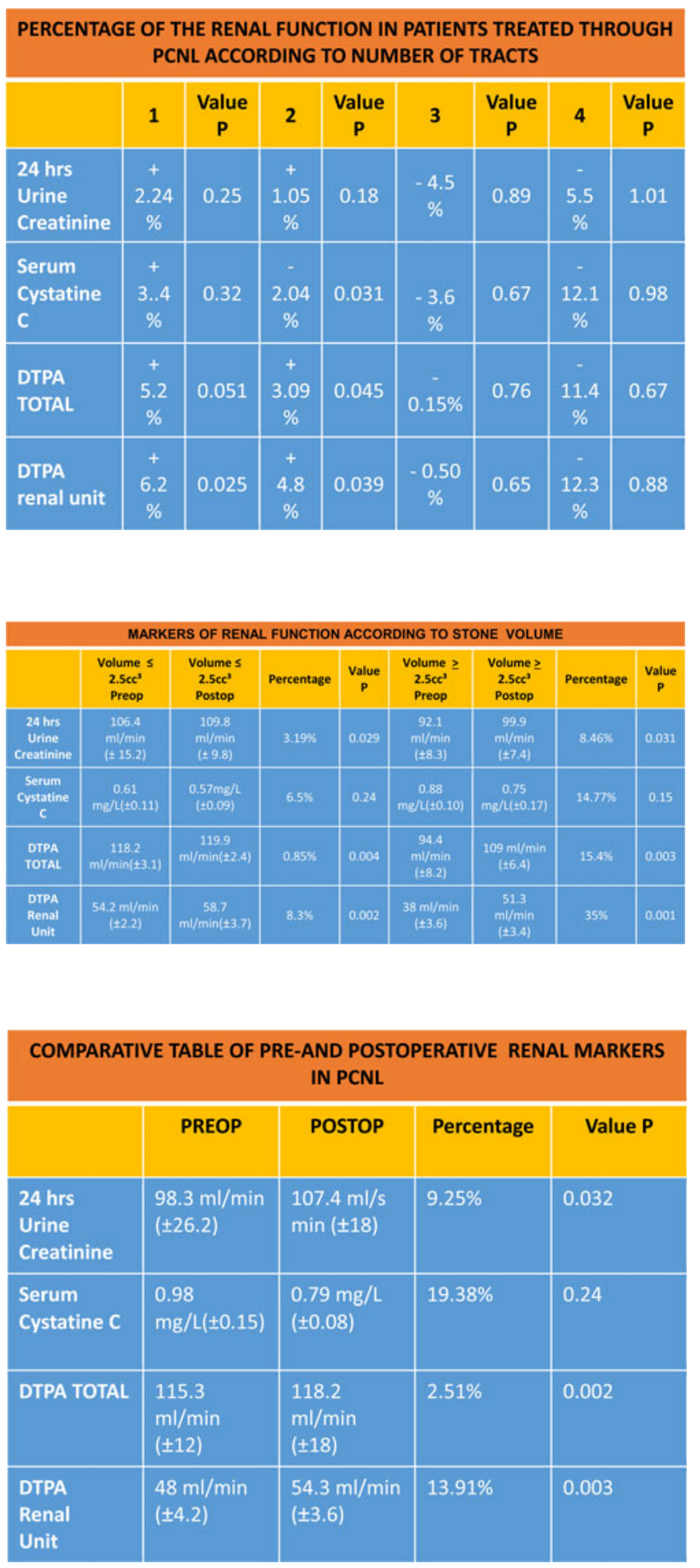

Results: 63 patients were evaluated with pre- and postoperative markers. Of which the $\mathrm{ClCr}$ urine 24 pre and pos was $94 \mathrm{ml} / \mathrm{min}$ \pm 11 and $98 \mathrm{ml} / \mathrm{min} \pm 8$, serum cystatin C pre and pos was $0.9 \mathrm{mg}$ / $1 \pm 0.3$ and $1.1 \mathrm{mg} / 1 \pm 0.4$, pre-global renal DTPA and 3 months pos was $100 \mathrm{ml} / \mathrm{min} \pm 7$ and $111 \mathrm{ml} / \mathrm{min} \pm 8$. On the treated kidney, the results before and after were $42 \mathrm{ml} / \mathrm{min} \pm 4$ and $51 \mathrm{ml} / \mathrm{min}$ \pm 6 . The most common comorbidity was diabetes (38\%). There is no decrease in renal function with: number of tracts, lithiasic volume or number of procedures.

Conclusions: PCNL is a safe procedure that isn't modified the kidney function

\section{MP07-21 Outcomes of Percutaneous Nephrolitho- tomy with or without Nephrostomy Tube: A Com- parative Study}

\author{
M Ali, P Saha, S Chowdhury, M Rahman, N Kamal \\ Dhaka Medical College Hospital
}

Introduction \& Objective: To compare the outcomes of PCNL with or without a nephrostomy tube.

Methods: This prospective comparative study intended to compare the outcomes between PCNL without nephrostomy tube and PCNL with nephrostomy tube. As per statistical sample size calculation, a total of 50 cases of renal stone disease planned for PCNL in Dhaka Medical College Hospital from July 2015 to June 2017, included in this study according to the inclusion and exclusion criteria. Cases were randomly allocated to group A (PCNL without nephrostomy tube) and group B (PCNL with nephrostomy tube). Each group consisted of 25 patients. The outcome variables were postoperative pain, the requirement of analgesic, Leakage of urine and post-operative hospital stay. Data were analyzed and compared by statistical tests.

Results: The mean age of group A was $44.04( \pm 10.97)$ years and that of group B was $44.24( \pm 12.75)$ years. The age differences between the two groups were not statistically significant. $(\mathrm{p}=0.95)$. The sex distribution between the two groups was not statistically significant $(\mathrm{p}=0.55)$. The mean operation time of group A (79.24 \pm 21.50$)$ and group B $(84.64 \pm 20.09)$ was not statistically significant $(\mathrm{p}=0.36)$. The mean pain intensity of group A was $20.00 \pm 20.95 \mathrm{~mm}$ in and that of group B was $41.88 \pm 21.82 \mathrm{~mm}$ in visual analog scale $(\mathrm{p}<0.0007)$. The mean dose of pethidine that was required in group A and group B was $66 \pm 14.21 \mathrm{mg}$ and $120 \pm 27.95 \mathrm{mg}$ respectively. The analgesic requirement of PCNL without nephrostomy tube group was significantly lower than the PCNL with nephrostomy tube group $(\mathrm{p}<0.0001)$. The mean urinary leakage through the puncture site in group A cases was $7.68 \pm 4.88$ hours and in group B cases was $16.16 \pm 6.05$ hours $(\mathrm{p}<0.0001)$. The postoperative hospital stay in group A was $1.92 \pm 0.49$ days and in group B was $2.84 \pm 0.68$ days $(\mathrm{p}=<0.0001)$.

Conclusions: Percutaneous nephrolithotomy without nephrostomy tube is better than percutaneous nephrolithotomy with nephrostomy tube in selective cases. It significantly reduces postoperative pain, analgesic requirement $\&$ postoperative hospital stay. So percutaneous nephrolithotomy without nephrostomy tube is safe and effective.

\section{MP07-22 Percutaneous Nephrolithotomy for Renal Stones in Chronic Kidney Disease and Poorly Func- tioning Kidney: A Prospective Study}

M Abdulrahman, M Gadelmoula, EO ElGanainy, YM Abdelsalam

\section{Assiut University}

Introduction \& Objective: Removal of stone in patients with concurrent renal insufficiency improves the function of the kidney in most of the cases and stabilizes the others. Percutaneous nephrolithotomy (PCNL) provides the most suitable option in these patients with minimum morbidity and mortality. Herein we prospectively evaluate the short-term outcome and safety of PCNL on renal function in chronic kidney disease (CKD) patients and patients with unilateral poorly functioning kidney (PFK). 
Methods: From March 2015 to March 2018, 42 patients underwent standard PNL were prospectively evaluated. We divided them into two groups, $21 \mathrm{CKD}$ and 21 unilateral PFK patients. Renal function estimation for all patients was done preoperatively, before discharge and three months postoperatively. Renal function estimation for CKD patients was measured by estimated glomerular filtration rate (eGFR), while for unilateral PFK patients by DMSA and DTPA renal scan.

Results: For all patients, complete clearance and complications rates (according to the modified Clavien-Dindo classification) were 26 patients $(61.9 \%)$ and 17 patients $(40.5 \%)$, respectively. For CKD patients, the mean eGFR preoperatively and three months postoperatively was $43.23( \pm 11.86)$ and $45.32( \pm 13.92)$ $\mathrm{ml} / \mathrm{min} / 1.73 \mathrm{~m}^{2}$, respectively $(\mathrm{P}$-value $=0.249)$. For unilateral PFK patients the median GFR by renal scan was 21 (10-30) and
$17.6(10.8 \rightarrow 34) \mathrm{ml} / \mathrm{min} / 1.73 \mathrm{~m}^{2}$ respectively $(\mathrm{P}$-value $=0.217)$. For CKD patients, 16 patients $(76.2 \%)$ were improved or stable and five patients $(23.8 \%)$ deteriorated. For PFK patients 15 patients $(71.4 \%)$ improved or stable and six patients $(28.6 \%)$ deteriorated. The predictor(s) of renal function deterioration in $\mathrm{CKD}$ patients was post-operative urine leakage $(\mathrm{P}$-value $=$ 0.048), while in PFK patients, were operative time ( $\mathrm{P}$ value $=0.019)$, multiple renal punctures $(\mathrm{P}$-value $=0.038)$, intra and postoperative complications $(\mathrm{P}$-value $=0.012)$ and blood transfusion $(\mathrm{P}$-value $=0.002)$.

Conclusions: PCNL has a safe impact and accepted outcome in relation to renal function and stone clearance in CKD patients and patients with unilateral PFK. However, a safe profile regarding other complications remains questionable for further research to make clear.

\section{MODERATED POSTER SESSION 08: BPH/LUTS: ELECTROSURGERY, LASERS \& OTHER TECHNOLOGY (II)}

\begin{abstract}
MP08-01 Could RezumTM water vapor ablation therapy for benign prostate enlargement be an option for patients with urinary retention? The first UK center experience
\end{abstract}

S Sarkar, M Johnston, T Farmer, T Gehring, G Rajkumar, T Nedas, A Emara, R Hindley

Introduction \& Objective: $\operatorname{Rezum}^{\mathrm{TM}}$ is a welcome addition to the portfolio of minimally invasive treatment options for symptomatic benign prostatic enlargement (BPE). The initial experience of this technology has however not included men in urinary retention. We assessed the outcomes of this group of men from our prospective database having now performed over 300 cases. This study investigated the possible extended role for this quick to perform day-case procedure in this patient group.

Methods: Since introducing Rezum ${ }^{\mathrm{TM}}$ to the UK in March 2017, over 300 men had treatment in our center. We report early outcomes on $25(10.4 \%)$ patients who were catheter dependent prior to undergoing Rezum ${ }^{\mathrm{TM}}$ treatment (using either intermittent selfcatheterization or with a permanent catheter). All men had their procedure as a day case and were discharged with an indwelling catheter for a minimum of 14 days after which they were invited for a trial without catheter (TWOC).

Results: The average age was 67 years with a mean gland volume of $72 \mathrm{cc}$ (range $22-100$ ). Minimum post treatment initial catheterization time was 14 days up to 21 days. 14/25 (56\%) passed the initial TWOC. A further 5 men (20\%) passed a subsequent TWOC. 19 patients $(76 \%)$ were catheter free within 3 months post treatment. The remaining 6 patients are all currently performing clean intermittent self-catheterization (CISC). The maximum flow rate (Qmax) and residual volume (PVR) were measured at 4-6 weeks postoperatively (for the 15 men with spontaneous voiding) with Qmax of $16.4 \mathrm{ml} / \mathrm{sec}$ and PVR of $94.4 \mathrm{ml}$ respectively. Average IPSS was 9 and QoL secondary to urinary symptoms of 2 for the catheter free cohort at 6 weeks. At 3 months their mean IPSS had improved to 5.6 and their QoL of 1.4. 1 patient was readmitted with hematuria after TWOC and was managed conservatively and another was treated with antibiotics for a urinary tract infection post TWOC.
Conclusions: Rezum ${ }^{\mathrm{TM}}$ water vapour therapy can be used successfully for the treatment of men in urinary retention. Rezum may be a good option or alternative to medication for men who fail an initial TWOC having presented in acute retention. We are hoping soon to participate in a UK multi-center study which aims to further establish whether the benefits of this minimally therapy can be extended to include this population of patients.

MP08-02 A Novel Technique of Morcellation Using a Pneumovesicum After Holmium Laser Enucleation of the Prostate in Complicated Situations: Our Initial Experience and Tips

H Choi

Department of Urology, Korea University Ansan Hospital, Korea University College of Medicine

Introduction \& Objective: To describe our initial experience with a novel method of adenoma retrieval using a pneumovesicum (PNV) after Holmium laser enucleation of the prostate (HoLEP).

Methods: From January 2016 to April 2018, a total of 93 consecutive patients treated with HoLEP were enrolled. For tissue morcellation, we used the PNV morcellation technique for an initial series of 21 patients and the conventional technique ( $\mathrm{Lu}$ menis VersaCut) for a consecutive series of 72 patients. We compared efficiency and safety between the novel technique and the traditional technique. From January 2016 to April 2018, a total of 93 consecutive patients treated with HoLEP were enrolled in this study. For tissue morcellation, we used the PNV technique for an initial series of 21 patients and the conventional technique for a consecutive series of 72 patients.

Results: The mean morcellation efficiency was higher $(8.50 \pm 1.94$ minutes vs. $1.76 \pm 0.45$ minutes, $\mathrm{P}<0.05)$ and the time of morcellation $(7.81 \pm 1.25$ minutes vs. $34.04 \pm 11.14$ minutes, $\mathrm{P}<0.05$ ) was shorter in the PNV group. Moreover, there were no significant differences between groups in hospitalization 
period ( $2.62 \pm 1.10$ days vs. $2.90 \pm 1.26$ days, $\mathrm{P}=0.852)$ and any other postoperative events, including recatheterization, reoperation, clot retention, and urethral stricture (P-value range, 0.194-0.447). In the PNV group, there were some cases of procedure-related complications, including postoperative extravesical leakage (5th case), clot retention (8th case), and recatheterization (9th case). There were significant differences in mean age and prostate volume between the 2 groups. However, there were no significant differences in the baseline characteristics and preoperative parameters in the subgroup analysis of large prostates $(>70 \mathrm{~mL})$. The mean morcellation efficiency was higher $(8.50 \pm 1.94$ minutes vs. $1.76 \pm 0.45$ minutes, $\mathrm{P}<0.05)$ and the time of morcellation $(7.81 \pm 1.25$ minutes vs. $34.04 \pm 11.14$ minutes, $\mathrm{P}<0.05$ ) was shorter in the PNV group. Moreover, there were no significant differences between groups in hospitalization period ( $2.62 \pm 1.10$ days vs. $2.90 \pm 1.26$ days, $\mathrm{P}=0.852)$ and any other postoperative events, including recatheterization, reoperation, clot retention, and urethral stricture (P-value range, 0.194- 0.447). In the PNV group, there were some cases of procedure-related complications, including postoperative extravesical leakage, clot retention, and recatheterization.

Conclusions: This method has a higher tissue retrieval efficacy, with the advantage of excellent visibility compared to conventional morcellation. The current method can be applied when a transurethral morcellator is out of order or cannot be used.

MP08-03 A matched comparison between $120 \mathrm{~W}$ Moses and $100 \mathrm{~W}$ standard holmium laser enucleation of the prostate in treating symptomatic benign prostate hyperplasia

\section{Meskawi, J Alamiri, ME Rivera}

Introduction \& Objective: Holmium laser enucleation of the prostate (HoLEP) is the most efficient and durable minimally invasive technique in treating men with lower urinary tract symptoms secondary to benign prostate hyperplasia (BPH) independent of prostate size. The Lumenis PulseTM 120 Holmium laser system, using Moses technology, was developed to optimize energy delivery through water to the target tissue. Herein, we performed a matched analysis of outcomes between $120 \mathrm{~W}$ Moses system and $100 \mathrm{~W}$ standard HoLEP in treating men with symptomatic BPH.

Methods: Between October 2017 and January 2019, 162 patients underwent either Moses or standard HoLEP with a pre-operative intention for an outpatient procedure. All patients had a preoperative prostate volume $<150$ gram. $2: 1$ propensity score matched analyses were performed. Primary endpoints included safety and efficacy outcomes at 3 months (AUASS, QoL, uroflowmetry parameters and PSA). Our secondary endpoint tested the hemostatic effect of Moses HoLEP compared to standard HoLEP. Thus, a multivariable logistic regression analyses were fitted after adjustment for patients' age, ASA score and prostate volume.

Results: After propensity score matching, 96 (67\%) standard and $48(33 \%)$ Moses HoLEP patients remained. Operative characteristics and postoperative complications recorded at 3 months after surgery were comparable between the two groups. Conversely, Length of stay was statistically significant shorter for Moses HoLEP, Specifically, $96 \%$ of patients treated with Moses laser had an outpatient procedure compared to $67 \%$ for the standard laser group $(\mathrm{p}<0.001)$. At 3 months follow-up, both groups had statistically significant improvement in their AUASS, QoL, Qmax and PVR (all p < 0.001) with no differences between the two groups. In multivariable logistic regression analyses, patients treated with Moses laser had statistically significant
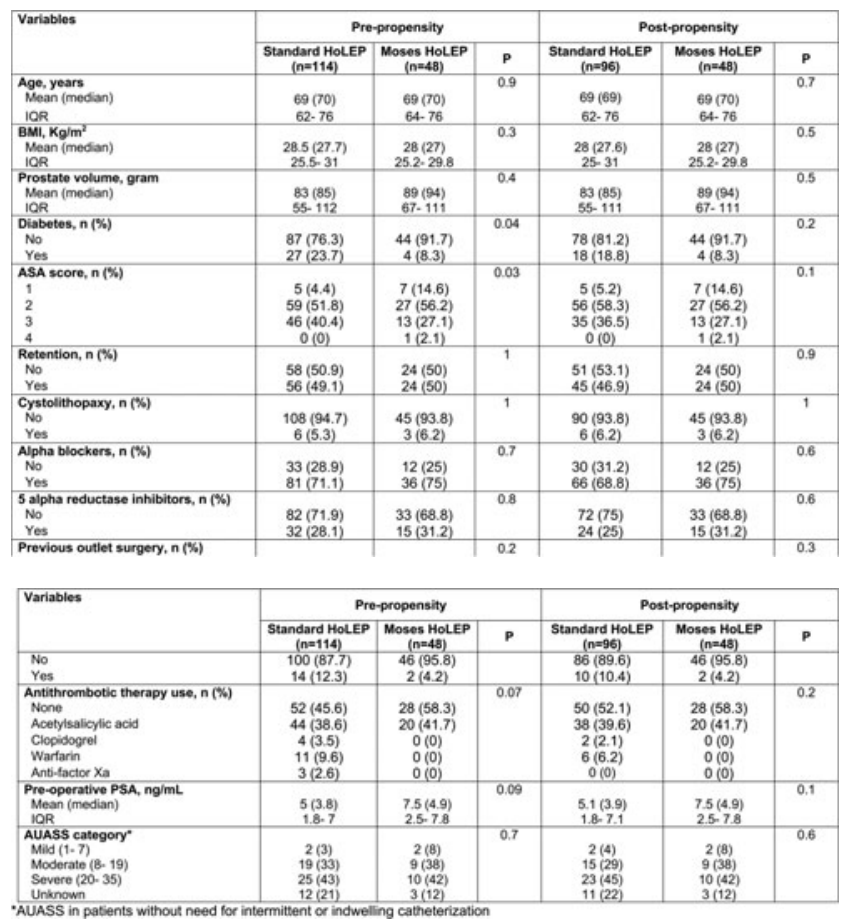

Table 3. Post-propensity postoperative adverse events

\begin{tabular}{|c|c|c|c|}
\hline Variables & $\begin{array}{c}\text { Standard HoLEP } \\
(n=96)\end{array}$ & $\begin{array}{c}\text { Moses HoLEP } \\
(n=48)\end{array}$ & $\mathbf{P}$ \\
\hline $\begin{array}{l}\text { Hospital stay (days) } \\
\text { Mean (median) } \\
\text { IQR } \\
\text { Range (Min, Max) }\end{array}$ & $\begin{array}{c}0.4(0) \\
0-1 \\
0,3 \\
\end{array}$ & $\begin{array}{l}0(0) \\
0-0 \\
0,1\end{array}$ & $<0.001$ \\
\hline $\begin{array}{l}\text { Hospital stay } \\
\text { Same day surgery } \\
\text { Overnight stay } \\
\geq 2 \text { days }\end{array}$ & $\begin{array}{c}64(66.7) \\
29(30.2) \\
3(3.1)\end{array}$ & $\begin{array}{c}46(96) \\
2(4) \\
0(0)\end{array}$ & $<0.001$ \\
\hline Transfusion rate, $\mathbf{n}(\%)$ & $2(2.1)$ & $0(0)$ & 0.8 \\
\hline Overall 90-day complications & $15(15.6)$ & $3(6.2)$ & 0.2 \\
\hline $\begin{array}{l}\text { Clavien II, n (\%) } \\
\text { Overall } \\
\text { Hematuria } \\
\text { UTI } \\
\text { Retention } \\
\text { Others } \\
\end{array}$ & $\begin{array}{l}9(9.4) \\
4(4.2) \\
3(3.1) \\
1(1) \\
1(1)\end{array}$ & $\begin{array}{l}2(4.2) \\
1(2.1) \\
0(0) \\
1(2.1) \\
0(0)\end{array}$ & $\begin{array}{c}0.4 \\
0.9 \\
0.5 \\
1 \\
1\end{array}$ \\
\hline $\begin{array}{l}\text { Clavien IIla, n (\%) } \\
\text { Overall } \\
\text { Retention } \\
\text { Stricture }\end{array}$ & $\begin{array}{c}2(2.1) \\
1(1) \\
1(1)\end{array}$ & $\begin{array}{l}0(0) \\
0(0) \\
0(0)\end{array}$ & $\begin{array}{c}0.8 \\
1 \\
1\end{array}$ \\
\hline $\begin{array}{l}\text { Clavien IIIb, n (\%) } \\
\text { Overall } \\
\text { Stricture } \\
\text { Bladder neck contracture }\end{array}$ & $\begin{array}{c}4(4.2) \\
0(0) \\
4(4.2)\end{array}$ & $\begin{array}{c}1(2.1) \\
1(2.1) \\
0(0)\end{array}$ & $\begin{array}{l}0.9 \\
0.7 \\
0.4\end{array}$ \\
\hline 30-day re-admission , $\mathrm{n}(\%)$ & $9(9.4)$ & $3(6.2)$ & 0.7 \\
\hline
\end{tabular}

\begin{tabular}{|c|c|c|c|c|}
\hline \multirow{2}{*}{ Variables } & \multicolumn{2}{|c|}{ Univariable analyses } & \multicolumn{2}{|c|}{ Multivariable analyses } \\
\hline & $\mathrm{OR}(95 \% \mathrm{Cl})$ & P-value & $\mathrm{OR}(95 \% \mathrm{Cl})$ & $P$-value \\
\hline Age & $1.06(1.01-1.11)$ & 0.03 & $1.04(0.98-1.11)$ & 0.2 \\
\hline Prostate volume & $1.04(1.02-1.06)$ & $<0.001$ & $1.05(1.03-1.07)$ & $<0.001$ \\
\hline $\begin{array}{l}\text { ASA score } \\
1 \\
2 \\
3 \\
4\end{array}$ & $\begin{array}{c}\text { Ref. } \\
3.5(0.42-28.7) \\
5.5(0.65-46.4) \\
-\end{array}$ & $\begin{array}{l}\text { Ref. } \\
0.2 \\
0.1 \\
-\end{array}$ & $\begin{array}{c}\text { Ref. } \\
0.8(0.76-7.95) \\
0.89(0.79-9.86)\end{array}$ & $\begin{array}{l}\text { Ref. } \\
0.8 \\
0.9 \\
--\end{array}$ \\
\hline $\begin{array}{l}\text { Laser type } \\
\text { Standard } \\
\text { Moses }\end{array}$ & $\begin{array}{c}\text { Ref. } \\
0.12(0.35-0.42)\end{array}$ & $\begin{aligned} & \text { Ref. } \\
< & 0.001\end{aligned}$ & $\begin{array}{c}\text { Ref. } \\
0.08(0.02-0.3)\end{array}$ & $\begin{array}{c}\text { Ref. } \\
<0.001\end{array}$ \\
\hline
\end{tabular}

better hemostasis compared to patient treated with the standard holmium laser (OR: 0.08; $\mathrm{p}<0.001$ ).

Conclusions: Moses HoLEP is safe and effective in treating patients with symptomatic BPH. Additionally, Moses HoLEP showed improved hemostatic potential compared to standard HoLEP. Thus, it is safe when performed as an outpatient procedure up to 150 gram prostate size. 
MP08-04 Prostatic Urethral Lift Study: Experiences in Northern Ireland (PULSE NI)

J Morrow, D Curry, F Farag, R Fiala, A Pahuja

NHS

Introduction \& Objective: The UroLift device is a novel technology in the surgical management of male lower urinary tract symptoms, with many reported benefits. The main advantages over traditional treatment modalities include; day case procedures under local anesthetic, minimal recovery time, no requirement for catheter post-operatively and preservation of sexual and ejaculatory function. However, there remains little published data on this new procedure to date. This study reviews the early experiences of UroLift in Northern Ireland.

Methods: A multicenter retrospective cohort study was performed with evaluation of all patients undergoing UroLift procedure in Northern Ireland to date. Data was collected from patient notes and the Northern Ireland Electronic Care Summary. Outcome measures include; improvement in International Prostate Symptom Score (IPSS), Quality of Life (QoL) score, maximal urinary flow rate (Qmax), post void residual (PVR) volumes, patient reported symptomatic improvement, procedural safety and complications.

Results: 60 patients underwent UroLift under 4 consultant Urologists across 3 Health Trusts, between March 2016 and May 2019. 12 procedures were performed under local anesthetic $+/$ - sedation, with an average of 3.6 implants per patient (range 2-6). Mean follow up was 6 months (range 1-22 months). There were statistically significant improvements in mean IPSS, QoL scores, flow rates and PVR volumes (see Table 1). Means were compared using paired t-test. $80 \%$ of patients reported a subjective improvement in symptoms. No patients reported deterioration in symptoms, or new sexual or ejaculatory dysfunction. 2 patients subsequently underwent TURP. Complications encountered included dysuria (2\%), pelvic pain $(2 \%)$, hematuria $(12 \%)$, urinary infection $(2 \%)$, acute renal impairment (2\%), acute urinary retention $13 \%$.

Conclusions: There were significant improvements in objective measurements of lower urinary tract symptoms and patient reported satisfaction following UroLift. There were no cases of de novo sexual or ejaculatory dysfunction. Adverse events were largely in keeping with the British Association of Urological Surgeons (BAUS) quoted complication rates for this procedure, however there were higher than expected rates of urinary retention. Results from this study are in line with the published evidence. Long term follow-up data is required to establish the longevity of the treatment.

\begin{tabular}{|c|c|c|c|c|c|c|c|c|c|}
\hline \multirow{2}{*}{$\begin{array}{l}\text { Outcome } \\
\text { Measure }\end{array}$} & \multicolumn{4}{|c|}{ Pre-Operative } & \multicolumn{5}{|c|}{ Post-Operative } \\
\hline & Mean & SD & Range & $n$ & Mean & SD & Range & $n$ & $p$ value \\
\hline IPSS & 20 & 5.46 & $7-29$ & 35 & 12 & 6.38 & $1-31$ & 37 & $p<0.0001$ \\
\hline QOL & 4 & 1.02 & $2-5$ & 35 & 2 & 1.23 & $0-4$ & 37 & $p<0.0001$ \\
\hline $\operatorname{Qmax}(\mathrm{m} / \mathrm{s})$ & 10.1 & 3.5 & 3-18 & 42 & 15.1 & 5.7 & $3-30$ & 33 & $p<0.0001$ \\
\hline PVR (ml) & 168 & 183.7 & $0-1200$ & 54 & 80 & 81.3 & $0-332$ & 46 & $p=0.0029$ \\
\hline
\end{tabular}

MP08-05 Postoperative Storage Symptoms of Holmium Laser Enucleation versus GreenLight Vaporization of the Prostate for Symptomatic Benign Prostatic Hyperplasia

A Ibrahim, M Elkoushy, M Aube, S Carrier

McGill University
Introduction \& Objective: To compare Greenlight laser plasmavaporisation of the prostate (PVP) versus Holmium laser enucleation of the prostate (HoLEP) in improving postoperative storage lower urinary tract symptoms (LUTS) in a large series of patients.

Methods: A retrospective review for patients undergoing HoLEP or PVP for symptomatic BPH between March 2002 and July 2016 was performed. Patients with urethral stricture, previous prostate surgery, prostate cancer or known history of neurogenic voiding dysfunction were excluded from the study. Patients were followed-up at 1, 3, 6, and 12-months postoperatively including prostate volume, international prostate symptoms score (IPSS), Quality of Life (QoL), peak flow rate (Qmax), residual urine (PVR) and prostatic specific antigen (PSA). The subtotal storage symptom score of the International Prostate Symptom Score (IPSS), was used to compare within and between groups, including the frequency, urgency, and nocturia scores. Moderate or severe storage symptoms were defined as IPSS storage score $\geq 9$.

Results: Out of 1755 HoLEP and PVP procedures, 1100 were included in the final analysis including 809 HoLEP vs. $291 \mathrm{PVP}$ procedures. HoLEP group had significantly larger prostates, higher total IPSS and longer operative time. Postoperatively, HoLEP patients had significantly lower IPSS than PVP group up to 12 -months $(7.7 \pm 5.9$ vs. $9.7 \pm 6.1, p=0.02)$ Storage score was significantly higher after PVP and did not improve until 3 months postoperatively $(2.8 \pm 2.8$ vs. $8.7 \pm 24.6, p=0.004)$. After then, the storage symptoms improved and became comparable with that of the HoLEP group ( $3.1 \pm 2.1$ vs. $3.6 \pm 2.6, p=0.67)$. In contrast, the subtotal storage symptom score was significantly decreased at one month after HoLEP compared to the baseline values $(5.4 \pm 3.2$ vs. $8.2 \pm 3.8, p=0.001)$. The number of patients with IPSS-storage score $\geq 9$ were significantly higher in the PVP group at 1 and 3 months of follow-up ([37.3\% vs. $15.1 \%$, $\mathrm{p}<0.001]$ and $[26.6 \%$ vs. $17.5 \%, \mathrm{p}=0.004]$, respectively).

Conclusions: Storage LUTS significantly improved immediately after HoLEP when compared with PVP. Patients undergoing PVP may have to wait at least for 3 months postoperatively to have their storage symptoms relieved. This should be considered when choosing the appropriate option for management of patients presenting with severe storage LUTS, and should be divulged to the patient before surgery.

\section{MP08-06 Utility of Post-Operative Day (POD) Zero Labs in Bipolar Trans-urethral Resection of Prostate (TURP)}

\section{B Patel, S Ekbal}

Introduction \& Objective: Obtaining immediate post-operative labs is part of routine post-TURP management, even in the era of bipolar resection. While checking lab values for post-TUR syndrome is relevant in the monopolar setting, performing these tests in the bipolar setting may have a significantly lesser role.

Methods: We retrospectively reviewed TURPs performed at a single academic center by two surgeons over 18 months. Pre- and post-operative electrolyte values, case duration, weight of resected tissue and information on medical co-morbidities were collected. Data was organized according to case duration and weight of resected tissue and analyzed for incidence of electrolyte abnormalities.

Results: 60 patients underwent TURP, with mean age 69.5 years. 26 patients had hypertension, 6 patients had diabetes mellitus, and 5 patients had chronic kidney disease. Mean case duration was $74.5 \pm 30$ minutes ( $\mathrm{min}$ ), and mean weight of resected tissue 
was $18.9 \pm 12$ grams $(\mathrm{g})$. No instances of sodium or potassium abnormality was observed. 23 instances of hyperchloremia (max $113 \mathrm{mEq} / \mathrm{L}$ ) were observed, of which 16 normalized on POD1, 3 worsened $(\max 112 \mathrm{mEq} / \mathrm{L})$, and 4 remained the same. No significant difference in frequency of electrolyte abnormality was seen when cases under 74 min were compared to cases over this time $(\mathrm{p}=0.83, \mathrm{RR}=1.07,95 \%$ CI $0.56-2.01)$, or when cases with resection weight over $18 \mathrm{~g}$ were compared to those under $18 \mathrm{~g}(\mathrm{p}=1.0, \mathrm{RR}=1.0,95 \% \mathrm{CI} 0.53-1.87)$. No patients reported neurologic complaints or had abnormal neurologic exam findings.

Conclusions: Checking laboratory values in POD\#0 bipolar TURP may ultimately not alter post-operative management due to low reported incidence of post-TUR syndrome. Our preliminary analysis of 18 out of 60 months of TURPs substantiates this and further specifies that case duration and weight of resected tissue are not associated with development of electrolyte abnormalities. Thus, it may be safe to defer checking electrolytes on POD\#0 after TURP.

\section{MP08-07 Evaluation of the New Holmium Laser Xpeeda $^{\mathrm{TM}}$ Side-firing Fiber versus GreenLight XPS 180W Vapoenucleation of the Prostate for Benign Prostatic Obstruction: An Early Clinical Experience}

A Ibrahim, M Aube, H Elmansy, S Carrier

\section{McGill University}

Introduction \& Objective: Recently, Holmium Lumenis Pulse $^{\mathrm{TM}} 120 \mathrm{~W}$ was introduced and it stands apart from other available technologies as a combination of power and efficiency, which minimizes vaporization time. It delivers $120 \mathrm{~W}$, which represent the highest holmium power on the market with better energy utilization through the new Xpeeda ${ }^{\mathrm{TM}}$ side-firing fiber. This technology seems to revolutionize utilization of the Holmium power and delivering more energy directly to the tissue, due to its capability of being in contact with the tissue. On the other hand, introduction of the Xcelerated Performance System (XPS) $180 \mathrm{~W}$ in 2010 with the Moxy fibers represents the highestpowered system currently in use for this type of laser. It encourages the adoption of the enucleation principle, making it a real contender to HoLEP in treating large adenomas. Herein, we report our early clinical experience comparing both procedures in improving bothersome LUTS secondary to BPH.

Methods: A prospective study comparing Holmium laser Xpeeda $^{\mathrm{TM}}$ side-firing fiber of the Lumenis Pulse $120 \mathrm{~W}$ to photoselective vapo-resection of the prostate-XPS $180 \mathrm{~W}$ was conducted. I-PSS, flow rate, residual urine, prostate specific antigen and prostate volume changes as well as perioperative complications were collected and compared.

Results: Overall 26 and 30 patients were included in the holmium laser ExpeedaTM side-firing (group A) and photoselective vaporesection group B, respectively. There were no significant difference in terms of operative time, hospital stay and time to catheter removal between both groups; $\mathrm{P}>0.05$. There was a significant comparable improvement in I-PSS and post-void residual urine volume at 1 , and 4 months. Re-intervention was needed in 1 and 2 cases in group $\mathrm{A}$ and group $\mathrm{B}$, respectively $(\mathrm{P}=0.1)$. There were no significant difference between both groups in terms of interoperative complications; ( 1 vs. $2, \mathrm{P}>0.05$ ).

Conclusions: Our early clinical experience suggests that the new Holmium laser Xpeeda ${ }^{\mathrm{TM}}$ side-firing fiber is safe, noninferior and effective in treatment of benign prostatic hyperplasia when com- pared with GreenLight XPS. Long-term follow-up and randomized clinical trials are definitely warranted to assess whether this new technology is more effective and more cost effective.

\section{MP08-08 A good bye to High Power Settings! A single center experience on Ultra Low Power HoLEP}

S Prabhakar, Y KK

Introduction \& Objective: Holmium Laser Enucleation of Prostate (HoLEP) is traditionally done at energy settings ranging from 80-120 Watt (W). The feasibility of HoLEP at 50W has been reported by some researchers. The cost of procuring high power laser equipment and recurring expenses are the major obstacles in wider acceptance of this procedure. This aim of this study is to assess feasibility of ultra Low Power Holmium Laser Enucleation of Prostate (uLP-HoLEP) at 30W.

Methods: The study included 453 consecutive patients from June 2015 to January 2019. The procedure was done using Lumenis Laser system at an energy setting of $30 \mathrm{~W}(2 \mathrm{~J} \times 15 \mathrm{~F})$. All procedures were done by three lobe technique, by a surgeon experienced in standard HoLEP. Age, indication, Pre-operative data [prostate volume, maximum flow (Qmax), residual volume (RV), International Prostate Symptom Score (IPSS) and Quality of Life (QoL) score], Peri-operative data [enucleation, morcellation, total procedure time and weight of enucleated tissue] and Post-operative data [hospital stay, catheterization time, complications] were recorded. Patients were followed up at 12 weeks for Qmax, RV, IPSS and QoL scores. The number of procedures per laser fiber were recorded. All variables were statistically analyzed by SPSS v.20 software. Paired t-test was used to compare difference of means between pre-operative and post operative parameters.

Results: The mean age of cohort was $65.6 \pm 8.4$ years. 243 patients were operated for urinary retention and the rest had bothersome symptoms. Mean size of prostate was $67.7 \pm 21.2 \mathrm{~g}$. Mean enucleation, morcellation and total procedure times were $50.0 \pm 12.3,9.5 \pm 3.5$ and $64 \pm 18.6 \mathrm{~min}$ respectively. Mean weight of enucleated tissue was $41.0 \pm 14.8$. Mean catheterization time and hospital stay was $2.1 \pm 0.7$ and $3.1 \pm 0.6$ days respectively. The mean pre and post-operative Qmax noted were $5.0 \pm 1.2$ and $19.2 \pm 2.8 \mathrm{ml} / \mathrm{s}$ respectively and the improvement was statistically significant ( $\mathrm{P}$ value $<0.001$ ). There was significant reduction in IPSS and QoL scores post-operatively with a mean reduction of 12.8 and 2.8 respectively. The complications noted were urinary tract Infection in 10 , failure to void in 4 , bleeding requiring cystoscopy in 5 , temporary incontinence in 4 and orchitis in 3 patients. The laser fiber utilization rate was 0.011 fibre per procedure.

Conclusions: The results of uLP - HoLEP are equivalent to standard HoLEP but it has the advantage of low cost device procurement, better fibre life and low incontinence rates.

\section{MP08-09 Thuvep of large prostates: a prospective single-center study}

F Germinale, L Tosco, A Giacobbe, D Collura, M Kurti, G Muto, E Berdondini

Department of Urology, Humanitas Gradenigo, Turin, Italy

Introduction \& Objective: We described our experience with THUVEP for large prostates, analyzing immediate outcomes and early and late complications. 
Methods: From 2011 we prospectively included in this study 252 patients undergoing Thuvep with prostate volume $>80 \mathrm{~mL}$ We evaluated intraoperative parameters, early and late complications and post-operative outcomes.

Results: Median prostate size was 92 (80-255 mL). Median operative time was 60.7 (30-160) minutes with a median enucleation time of $25(12$ - 65) minutes and median morcellation time of 8.2 (4 - 80) minutes. Median tissue weight was 65 (35 230) g. Median catheterization time was $55(24-216)$ hours and median length of hospital stay was 3.6 (2-14) days. Median maximum urinary flow rate ( $8 \mathrm{vs} 20 \mathrm{ml} / \mathrm{s}$ ), postpaid residual urine volume $(128-10 \mathrm{ml})$ changed significantly. The serum concentration of hemoglobin was 14.5 and 13.1 before and after the procedure. The rate of early complications was $12.6 \%$ (acute urinary retention $3.9 \%$, incontinence $3.5 \%$, clot retention $2.3 \%$, UTI $1.9 \%$, blood transfusion $0.7 \%$ ). Only 3 patients $(1.1 \%)$ reported late complications such as urinary incontinence. The functional outcomes in terms of IPSS score showed a significant improvement ( 20.1 vs $5.8 \mathrm{p}<0.05)$.

Conclusions: THUVEP is an effective treatment for BPH of high volume. We observed low peri- and post-operative complications and an improvement of the functional outcomes.

\section{MP08-10 Withdrawn}

MP08-11 WATER vs WATER II: Aquablation Therapy for Benign Prostatic Hyperplasia

A Kasraeian, N Bhojani, D Nguyen

Kasraeian Urology

Introduction \& Objective: Surgical options are limited when treating large $(>80 \mathrm{cc})$ prostates for lower urinary tract symptoms (LUTS) due to benign prostatic hyperplasia (BPH); there is a need for novel surgical approaches with shorter learning curves and effective treatment. Aquablation (AquaBeam System, PROCEPT BioRobotics, Inc., USA), an ultrasound-guided, robotically executed waterjet ablative procedure, could be this novel tool. This analysis compares the outcomes of Aquablation in $30 \mathrm{cc}$ to $80 \mathrm{cc}$ prostates with the outcomes in $80 \mathrm{cc}$ to $150 \mathrm{cc}$ prostates.

Methods: WATER (NCT02505919) is a prospective, doubleblind, multicenter, international clinical trial comparing the safety and efficacy of Aquablation and TURP in the treatment of LUTS/BPH in men 45 to 80 years old with a prostate between $30 \mathrm{cc}$ and 80cc. WATER II (NCT03123250) is a prospective, multicenter, single-arm international clinical trial of Aquablation in men with a prostate between $80 \mathrm{cc}$ and $150 \mathrm{cc}$. We herein report baseline parameters and 6-month outcomes in 116 WATER (W-I) and 101 WATER II (W-II) study subjects undergoing Aquablation. Students' t-test or Wilcoxon tests were used for continuous variables and Fisher's test for binary variables.

Results: Mean operative time was $33 \pm 17$ minutes in W-I and $37 \pm 13$ minutes in W-II. The average length of stay postprocedure was $1.4 \pm 0.7$ days (W-I) vs. $1.6 \pm 1.1$ days (W-II). Mean changes in IPSS and IPSS quality of life were substantial, occurring soon after treatment and averaging (at 6 months) 16.9 and 3.5 points, respectively, in $\mathrm{W}-\mathrm{I}$ and 17.4 and 3.2 points in $\mathrm{W}$ II ( $\mathrm{p}=.6046$ and .2607 respectively). By 3 months, ClavienDindo grade 2 or higher events occurred in $19.8 \%$ of W-I subjects and $34.7 \%$ of W-II subjects $(\mathrm{p}=.4680)$. One W-I subject $(0.9 \%)$
Table 1. Characteristics and Outcomes of each cohort

\begin{tabular}{|l|c|c|c|}
\cline { 2 - 4 } \multicolumn{1}{l|}{} & $\begin{array}{c}\text { WATER } \\
(\boldsymbol{n}=116)\end{array}$ & $\begin{array}{c}\text { WATER II } \\
(\boldsymbol{n}=101)\end{array}$ & P-Value \\
\hline Age, years; mean (SD) & $65.9(7.3)$ & $67.5(6.6)$ & .0854 \\
\hline Prostate specific antigen, g/dL; mean (SD) & $3.7(3.0)$ & $7.1(5.9)$ & $<.0001$ \\
\hline Prostate size (TRUS), cc; mean (SD) & $54.1(16.2)$ & $107.4(22.1)$ & $<.0001$ \\
\hline Operative time, min; mean (SD) & $33(17)$ & $37(37)$ & .0275 \\
\hline Resection time, min; mean (SD) & $3.9(1.4)$ & $8(3)$ & $<.0001$ \\
\hline Length of stay, days; mean (SD) & $1.4(0.7)$ & $1.6(1.1)$ & .4630 \\
\hline $\begin{array}{l}\text { CD Grade 2+ Subjects by 3 months; } \% \\
\text { (number) }\end{array}$ & $19.8(23)$ & $34.7(35)$ & .0206 \\
\hline $\begin{array}{l}\text { Maintenance of antegrade ejaculation; } \% \\
\text { (number) }\end{array}$ & $93.1(108)$ & $81(82)$ & .0057 \\
\hline
\end{tabular}

-Two-sided

and $6 \mathrm{~W}$-II subjects $(5.9 \%)$ required postoperative blood transfusion $(\mathrm{p}=.0517)$.

Conclusions: Aquablation clinically normalizes outcomes between patients with a $30 \mathrm{cc}$ to $80 \mathrm{cc}$ prostate and patients with an $80 \mathrm{cc}$ to $150 \mathrm{cc}$ prostate treated for LUTS/BPH with an expected increase in the risk of complication. It is effective in patients with large prostate glands $(>80 \mathrm{cc})$ with acceptable complications.

\section{MP08-12 A Comparison of Outcomes After Holmium Laser Enucleation of the Prostate in Patients with Chronic Retention, a History of Acute Retention, and No Prior Retention}

A Martin, BB Whiles, A Brevik, J Thompson, KL Thurmon

University of Kansas Medical Center

Introduction \& Objective: The purpose of this study is to compare outcomes of holmium laser enucleation of the prostate (HoLEP) in patients with and without urinary retention, specifically those with a remote history of acute urinary retention vs. those with chronic urinary retention.

Methods: In this retrospective review, patients who underwent HoLEP between 06/2016 - 02/2018 at an academic medical center were identified. Preoperative voiding method including any history of acute or chronic urinary retention was determined via chart review. Preoperative, intraoperative, and postoperative variables were compared for all three groups.

Results: A total of 199 patients met inclusion criteria. This included 66 patients $(33 \%)$ with chronic urinary retention, 42 patients $(21 \%)$ with a history of acute urinary retention, and 91 patients $(46 \%)$ without urinary retention. Patients with no history of retention had the smallest prostates by volume compared to patients with acute urinary retention and chronic urinary retention ( $85 \mathrm{~g}$ vs $115 \mathrm{~g}$ vs $111 \mathrm{~g}$; $\mathrm{p}<0.01$ ). Preoperative International Prostate Symptom Score (IPSS) and bother scores were similar in all three groups $(\mathrm{p}>0.05)$. Total hospital length of stay was also similar for all three groups. All patients had six-week follow up data, and 134(68\%) had three-month follow up data. Postoperatively, there was no difference in rates of complications, including postoperative urinary retention, incidences of reoperation, or formation of urethral strictures $(\mathrm{p}>0.05)$. While all groups showed improvements in IPSS and bother scores (all $\mathrm{p}<0.05)$ patients with current urinary retention showed significantly greater improvements in IPSS and bother scores compared to patients with a history of acute retention $(-11.6$ vs $-6.8,-4.4$ vs -0.4 , respectively; $\mathrm{p}=0.03$ ). 
Conclusions: To our knowledge, this study represents the first comparison of HoLEP in patients with and without urinary retention. Interestingly, though it is often taught "size doesn't matter in BPH" those patients with larger glands were significantly more likely to have urinary retention than those with smaller glands. Surgical outcomes were similar in all three groups. Subjective symptoms improved for all patients, with the biggest improvement in those with chronic urinary retention. This study does not support the common misconception that patients with urinary retention will have a suboptimal outcome after outlet surgery. Those patients with retention are actually the most benefited from HoLEP from a quality of life standpoint.

MP08-13 Is RezumTM Water Vapour ablation therapy a suitable option for men with larger prostate glands?

S Sarkar, M Johnston, T Farmer, T Gehring, G Rajkumar, T Nedas, A Emara, R Hindley

Introduction \& Objective: Rezum ${ }^{\mathrm{TM}}$ water vapour ablation therapy is a minimally invasive treatment for symptomatic benign prostatic enlargement (BPE). The surgical treatment options for men with larger prostate glands are rather limited to long term pharmacological treatment or surgery and invariably involve a significant in-patient stay.

Methods: This study involves a prospective analysis of data for men undergoing Rezum comparing pre-treatment assessments with 3 months follow-up data. Gland volumes in the cohort ranged from 20 to $120 \mathrm{ml}$. We compared men with smaller glands $(<80 \mathrm{mls})$ and larger glands $(>80 \mathrm{mls})$. Pre and post procedure assessments included validated questionnaires (IPSS with QoL, IIEF-5), urinary flow rate (Qmax), prostate volume and length of stay. 236 patients were included of whom 41 had gland volumes $>80 \mathrm{mls}(17.3 \%)$

Results: Mean volume for glands $<80 \mathrm{mls}$ was $49.5 \mathrm{mls}$ and larger glands was $93 \mathrm{mls}$ (range $80-127) .68 .2 \%(n=28)$ of men with larger glands passed their initial trial without catheter (TWOC). $82 \%(n=128)$ of men with smaller glands passed this TWOC. The mean IPSS before and 3 months after treatment for the whole cohort was 20.8 and 4.3 respectively ( 21 for small glands and 20.0 for large glands pre, $\mathrm{p}=0.44$ and 5.7 vs. 5.8 post, $\mathrm{p}=0.89$ ). The QoL scores in small and large glands were 4.4 and $4.3, \mathrm{p}=0.83$ pre-treatment and 1.4 vs. 1.5 post, $\mathrm{p}=0.44$. There were no significant differences in flow rate or length of stay. A single patient in each group required bladder washout in theatre due to secondary hemorrhage. 6 patients in the small gland group were treated for UTI compared to 1 in the large gland group.

Conclusions: In this series there was no significant overall difference in early post treatment outcomes when comparing both groups rendering it a suitable minimally invasive technique for BPH management and as an alternative to medication. Further studies are necessary to determine a sensible 'upper limit' in gland size for those wishing to consider this new therapy.

\section{MP08-14 Aquablation for Benign Prostatic Hyper- plasia: Early Outcomes from a Tertiary Care Center in the Middle East}

N Abdallah, A Mailhac, M Bulbul, H Tamim, R Jaafar, A Hajj

American University of Beirut Medical Center
Introduction \& Objective: Aquablation is a new, minimally invasive technique in the treatment of benign prostatic hyperplasia. It relies on a robotic platform that delivers a high-pressure waterjet for tissue ablation guided by a transrectal ultrasound probe. Our aim was to evaluate the 3 months postoperative outcomes.

Methods: We included patients who underwent aquablation at our center between March 2018 and June 2019. Preoperative, intraoperative and postoperative data were recorded prospectively (table 1). Variables were compared, and a p-value $<0.05$ was considered significant.

Results: During the aforementioned period, 51 patients underwent aquablation at our center, mean age was $68.8 \pm 7.5$. Mean prostate size was $69 \pm 30.1$ and IPSS was $20 \pm 7.7$. 14 patients were in retention. Hemostasis was secured by cautery in 25 patients and a novel double balloon catheter in the last 10. Four patients had postoperative urinary retention, and 2 had capsular perforation, all resolved after catheterization. One patient had recurrent hematuria and clotting which resulted in prolonged hospital stay. Hemoglobin level was significantly decreased at discharge $-1.7 \mathrm{~g} / \mathrm{dl}(\mathrm{p}<0.0001)$, but none of the patients required transfusion. At 3 months after the surgery, one patient required transurethral resection for residual symptoms. Overall, the procedure resulted in a PSA reduction 4.4 to $1.77 \mathrm{ng} / \mathrm{mL}(\mathrm{p}=0.001)$, Postvoid Residual Volume reduction 270.9 to $120.7 \mathrm{~mL}$ $(\mathrm{p}=0.033)$, prostate volume reduction of $21.43 \mathrm{~g}(\mathrm{p}<0.0001)$, IPSS reduction from 20 to $8(\mathrm{p}=0.003)$, and increase in Qmax from 12.5 to $23.47 \mathrm{~mL} / \mathrm{sec}(\mathrm{p}=0.028)$. (Figure 1) Changes in

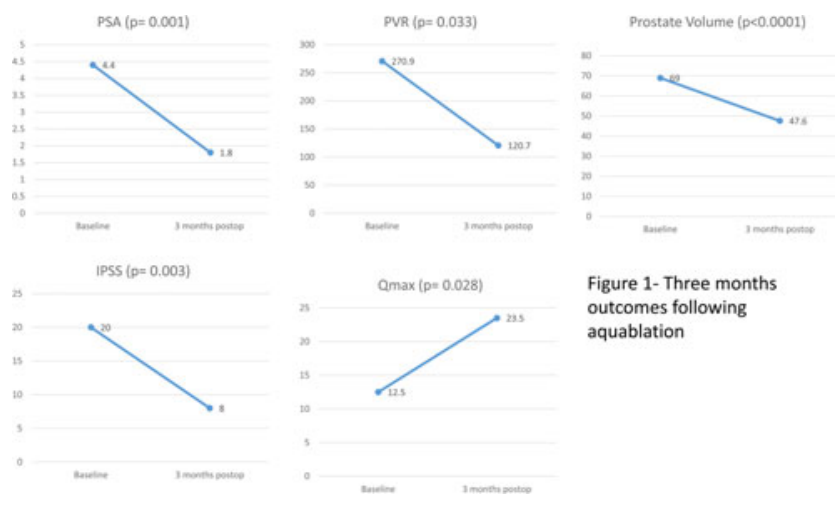

Table 1-Baseline characteristics and procedure details.

\begin{tabular}{|l|l|}
\hline N=51 & Mean \pm Standard Deviation \\
\hline Pre-operative data & \\
\hline Age (years) & $68.8 \pm 7.5$ \\
\hline Prostate-Specific Antigen (PSA) (ng/ml) & $4.4 \pm 4$ \\
\hline Hemoglobin (Hb) (g/dL) & $14.4 \pm 1.5$ \\
\hline Peak flow (Qmax) (mL/sec) & $12.5 \pm 8.4$ \\
\hline Postvoid Residual Volume (PVR) (mL) & $270.9 \pm 204.9$ \\
\hline Prostate Volume (cc) & $69 \pm 30.1 ;$ (Range 30-148) \\
\hline International Prostate Symptom Score (IPSS) & $20 \pm 7.7$ \\
\hline International Index of Erectile Function (IIEF-15): & \\
\hline \multicolumn{1}{|c|}{-Erectile function } & $15.4 \pm 10.5$ \\
\hline \multicolumn{1}{|c|}{-Orgasmic function } & $5 \pm 3.7$ \\
\hline \multicolumn{1}{|c|}{-Intercourse satisfaction } & $6.4 \pm 2.4$ \\
\hline \multicolumn{1}{|c|}{ Overall satisfaction } & $6.4 \pm 5$ \\
\hline $\begin{array}{l}\text { Male Sexual Health Questionnaire-Ejaculatory } \\
\text { Dysfunction (MSHQ-EjD) }\end{array}$ & $6.7 \pm 1.8$ \\
\hline Intra-operative data & $6.3 \pm 5.1$ \\
\hline $\begin{array}{l}\text { Duration of the procedure (minutes) } \\
\text { (from induction to Foley insertion) }\end{array}$ & \\
\hline Post-operative data & $79 \pm 29.4$ \\
\hline Time with Foley in (hours) & \\
\hline Discharge (Postoperative day) & $41.5 \pm 16$ \\
\hline & $2.1 \pm 0.8$ \\
\hline
\end{tabular}


International Index of Erectile Function and Male Sexual Health Questionnaire scores were not statistically significant.

Conclusions: The aquablation procedure is safe and reproducible with good functional outcomes. Longer follow up is needed to monitor the long-term treatment effect in our center.

\section{MP08-15 Ablation Efficiency Utilizing a Modulate Pulse Platform for Holmium Laser Ablation of the Prostate}

BB Whiles, A Martin, A Brevik, KL Thurmon

University of Kansas Medical Center

Introduction \& Objective: Holmium laser ablation of the prostate (HoLAP) is one tool in the urologist's armamentarium for the treatment of benign prostatic hyperplasia. Multiple fiber and holmium laser energy delivery options exist. Based on our experiential evidence from using the modulated pulse platform of the Moses laser for prostate ablation, we hypothesized that this technology would allow for faster tissue ablation.

Methods: In this retrospective cohort study, we identified patients who underwent HoLAP between $01 / 2018$ - 10/2018 by a single urologic surgeon at an academic medical center. Preoperative prostate size was determined by transrectal ultrasound or other prior cross-sectional imaging. Ablation parameters, as well as energy usage, were determined for patients who underwent HoLAP with a $550 \mu$ m laser fiber via either the Pulse $120 \mathrm{H}$ vs the Moses Pulse $120 \mathrm{H}$ laser system and fiber with modulated pulse settings. Since prostate ablation is typically carried down to the prostatic capsule, intraoperative ablation rate was estimated by pre-procedure prostate size and the amount of time required for ablation. Data analysis was completed in SAS.

Results: HoLAP was completed in 26 patients. Of these, 11 procedures were completed with the Moses platform. There was no difference in prostate size or ablation time $(\mathrm{p}=0.56$ and 0.39 , respectively). Ablation rate was similar between these two groups with 1.06 grams/min with Moses vs 1.04 grams $/ \mathrm{min}$ without Moses $(\mathrm{p}=0.93)$. Although laser energy usage was higher in the Moses group (260.6KJ vs $168.8 \mathrm{KJ}$; $\mathrm{p}=0.75$ ), there was no difference in energy usage rate $(\mathrm{KJ} / \mathrm{min})$ or energy efficiency $($ grams $/ \mathrm{KJ})(\mathrm{p}=0.75$ and $\mathrm{p}=0.88$, respectively).

Conclusions: No differences were seen in intraoperative ablation parameters with the use of the Moses laser and its fiber. This is discordant to our experiential evidence via subjective surgeon assessment. Additional studies are needed to further define the utility of using this technology in tissue ablation.

\section{MP08-16 HoLEP as a day case procedure}

N Raison, H Godbole, N Kale, MK Bhattacharyya

North Middlesex University Hospital

Introduction \& Objective: Holmium laser enucleation of the prostate (HoLEP) is rapidly gaining recognition as the gold standard for managing bladder outflow obstruction secondary to $\mathrm{BPH}$. A retrospective cohort study was performed to identify the relevant clinical and patient factors for day case HoLEP.

Methods: All patients were identified who underwent a HoLEP procedure by a single surgeon in a District General Hospital. Case notes and electronic health records were reviewed. A variety of potential factors to support or hinder day case surgery including pre-surgical haemoglobin (as a marker of fitness for surgery), gland size on best available imaging (Ultrasound or MRI), operation time, past medical history and age at time of surgery. Day case procedures were classified as those in patients who discharged on the day of surgery. Non-parametric data analysis was performed used SPSS statistics version 25 .

Results: 68 HoLEP procedures was performed between 2009 2019. 17 were day cases. Preoperative haemoglobin was higher in day case procedure but this did not reach significance $(135 \mathrm{~g} / \mathrm{dL}$ vs $127 \mathrm{~g} / \mathrm{dL}, \mathrm{p}=0.38) .70 \%(\mathrm{n}=12)$ of day case patients had a normal Haemoglobin prior to procedure. Gland size ranged from $18-116 \mathrm{ml}$ with day case patients have a significant smaller gland size than inpatients, $\mathrm{p}=0.024$. Operation time was significantly lower in day case patients, $36 \mathrm{~min}$ vs $54 \mathrm{~min} \mathrm{p}=0.02$. Most of the cohort had a number of co-morbidities which did not exclude them from being operated on. The cohort age range was between 54-86 with no significant difference in ages between patients managed as days case and inpatients (69 years vs 73 $\mathrm{p}=0.18$ ). Of those that were not discharged home on the same day often it was haematuria and the need for continuous bladder Irrigation post op which prevented them from leaving hospital sooner. Conclusions: This study demonstrates that HoLEP can be performed safely as a day case in a DGH. The main barrier to day case surgery is prostate gland size and consequently operation time. Notably age, preoperative haemoglobin and patients' comorbidities per se are not causes for exclusion from day case surgery. These results support the expansion in day case HoLEP surgery and may be used to counsel more patients to undergo this novel care pathway.

MP08-17 Outcomes of transurethral resection and holmium laser enucleation in more than $60 \mathrm{~g}$ of prostate: A prospective randomized study

\author{
RJ Sinha, V Singh, S Mehrotra, S Mehdi, S Pandey, R Sinha
}

Department of Urology, King Georges Medical University, Lucknow, India

Introduction \& Objective: The management of BPH has witnessed a dramatic change in recent years and the modality of management has shifted from open to minimally invasive procedures. Lasers are now being used more liberally, particularly holmium laser as the skills of surgeons and technology have advanced over years. Although transurethral resection of prostate (TURP) is continues to be the gold standard surgical procedure holmium laser enucleation of prostate (HoLEP) is seen as close rival of TURP. The study aims to study the long- and short-term outcomes of transurethral resection and holmium laser enucleation in the prostate of more than $60 \mathrm{~g}$.

Methods: A total of 164 patients were included in this prospective randomized study. Inclusion criteria were age $<75$ years after failed or poor response to medical therapy, prostatic size $>60 \mathrm{~g}$, gross hematuria secondary to $\mathrm{BPH}$, recurrent urinary tract infection, acute urinary retention, postvoid residual $>150 \mathrm{ml}$, and Schafer Grade II or more. BPH associated with neurogenic bladder, stricture urethra, and carcinoma prostate were excluded from the study. Group 1 comprises patients who underwent TURP and Group 2 comprises who underwent HoLEP. Follow-up was done at 1, 3, 6, 12, and 24 months after the surgery.

Results: Data of 144 patients were analyzed. The mean age of patients in TURP and HoLEP group was $66.78 \pm 7.81$ and $67.70 \pm 7.44$ years, respectively $(P=0.47)$, mean prostatic volume was $74.5 \pm 12.56$ and $75.6 \pm 12.84 \mathrm{~g}$, respectively $(P=$ 0.60 ), operative time was $73.10 \pm 10.49$ and $89.56 \pm 13.81 \mathrm{~min}$, 
respectively $(P=0.0001)$. Mean resected tissue was $44.80 \pm 9.87$ and $48.49 \pm 10.87$, respectively $(P=0.03)$. The sexual function did not change significantly in postoperative follow-up.

Conclusions: Although HoLEP is associated with longer operative time and postoperative dysuria, it offers the advantages of less blood loss, lower transfusion rates, and a shorter hospital stay.

\section{MP08-18 Telementored bipolar enucleation of the prostate: A magic way to teach surgery}

M Amato, R Milandri, S Ciarlariello, G Guarino, M Sighinolfi, A Meneghini, B Rocco, S Micali

Department of Surgical, Medical and Dental Morphological Sciences related to Transplant, Oncology and Regenerative Medicine - Section of Urology, University of Modena and Reggio Emilia, Modena, Italy

Introduction \& Objective: Telemedicine is the use of medical information exchanged from one site to another via telecommunication to improve a patient's clinical health status. The evolution of Telemedicine includes a growing variety of applications and services using two-way video, smart phones, wireless tools and other forms of technology. Surgical telementoring is a concept within telemedicine consisting of an expert surgeon guiding a less experienced surgeon through advanced or novel cases from a remote location, it is an evolving technology which has potential to become an integral part of surgical practice. The aim of this study is to evaluate the feasibility of telementoring for endoscopic enucleation of the prostate.

Methods: In our preliminary study we recruited 5 patients who were candidates for endoscopic enucleation of the prostate. We developed a software that allowed us to communicate live audio video between the surgeon in the operating room (Modena) and the mentor from his workstation (Adria Hospital). Through telestration the mentor can give allows visual advice on an additional monitor in the operating room. Through a questionnaire submitted to the mentor and to the surgeon we evaluated the feasibility of remote teaching and the training impact. As secondary outcomes we evaluated the surgical outcome: length of hospital stay, blood loss, catheter duration, uroflowmetry at three months.

Results: Out of 5 operations started in Telementoring, all cases were successfully performed, in 2 procedures a signal delay of about 1 second was observed which did not compromised the procedure. Operating time was slidely longer related with learning curve. The post-operative course was regular. No complication was observed, and patients were discharged within 4 days. In the evaluation form both the mentor and the surgeon described this experience as positive and promising.

Conclusions: Telementored Endoscopic Enucleation of the prostate appears safe and feasible. Some limitations due to current technologies must be overcome as delay connection in order to enter into clinical practice. This teaching tool has the potential to ensure availability of surgical expertise in remote locations for difficult or rare operations, and to improve endoscopic training worldwide in a low-cost way.

\section{MP08-19 Antegrade Ejaculation Preserving Techni- que with Green Light XPS}

PN Contreras, N Bonanno, L Blas, N villasante, H Ríos Pita, C Ameri

Hospital Alemán
Introduction \& Objective: Retrograde ejaculation is one of the most common complication in benign prostatic hyperplasia (BPH) surgery and has been reported in up to $70 \%$ of cases. Ejaculatory sparing technique (EST) has been described using Transurethral Resection and Holmium Laser. Our objective was to analyze the results of EST with Green Light XPS.

Methods: Between March 1st, 2016 and May 30 ${ }^{\text {th }}, 2018,117$ patients underwent photovaporization with Green Light (GL) XPS. Procedures were performed by a single surgeon with 9 years of experience with GL. Anatomical vaporization, with 3 lobe technique was used. A " $U$ " shape incision $1 \mathrm{~cm}$. proximal to the veru montanum was made at the beginning of the procedure. Incision were made using $120 \mathrm{Watts}$, blunt dissection was used and vaporization of dissected prostate tissue was made with 180 Watts. Bladder neck was widely opened in all the patients. The four items Male Sexual Health Questionnaire (MSHQ-EjD Short Form) for assessing ejaculatory dysfunction (EjD). was sent to the patients. We received 43 questionnaires completed.

Results: Median age was 67 years (48-85). Median prostate size was $57 \mathrm{~g}$. (19 - $177 \mathrm{gr})$. Antegrade ejaculation (AE) was found in $37(86 \%)$. Of them $29(78.4 \%)$ had antegrade eyaculation all the time or most of the time and $8(21.6 \%)$ had AE about half of the time or less than a half of time. Sixt patients (14\%) had none AE. About strength, $22(59.4 \%)$ had as strong as it always was or a little less, $11(29.7 \%)$ had somewhat less strong or much less strong and 4 (10.8\%) had none AE. About volume 18 (48.6\%) had as much as it always was or a little less, $15(40.5 \%)$ had somewhat less that it used to be or much less than it used to be and $4(10.8 \%)$ had none AE. About bother or satisfaction, for those patients with $\mathrm{AE}$ the average rate was 1.08. and for those without $\mathrm{AE}$ the average rate was 3.2 (0 means No problem with ejaculation and 5 extremely bothered).

Conclusions: Using a preserving antegrade ejaculation technique with Green Light XPS, antegrade ejaculation was found in most of treated patients which had a strong impact in quality of life.

MP08-20 Clinical Comparison of Holmium Laser Enucleation of the Prostate and Bipolar Transurethral Enucleation of the Prostate: A short term followup

A Tuncel, C Aykanat, E Gazel, S Akdemir, T Oksay, Y Aslan, M Balci, O Guzel, M Arslan, L Tunc

University of Health Sciences, Ankara Numune Research and Training Hospital

Introduction \& Objective: To assess the efficacy and morbidity of holmium laser enucleation of the prostate (HOLEP) and bipolar transurethral enucleation of the prostate (B-TUEP) in patients with benign prostatic hyperplasia (BPH).

Methods: Between August 2017 to December 2019, 60 (55\%) and $49(45 \%)$ patients who underwent HOLEP (Lumenis ${ }^{\mathrm{TM}}$ $100 \mathrm{~W}$, Israel, Jena Surgical ${ }^{\mathrm{TM}} 140 \mathrm{~W}$, Germany) and B-TUEP (Olympus ${ }^{\mathrm{TM}}$ ESG-400, Japan) in four different clinics. All cases were evaluated preoperatively and at $3^{\text {th }}$ months after surgery. Clinical parameters were compared among the groups.

Results: The mean age of the study cohort $65.3 \pm 8.3$ (46-80) years. Table 1 details the pre, and intraoperative characteristics of both groups. Groups did not alter in terms of baseline serum prostate specific antigen (PSA) level, international prostate symptom score (IPSS) and maximum urine flow rate $\left(\mathrm{Q}_{\max }\right)$. However, baseline prostate volume and postvoid residual urine 
Table 1: Baseline and perioperative data.

Data presented as mean \pm standard deviation with minimum and maximum value

\begin{tabular}{|l|c|c|c|}
\hline \multicolumn{1}{|c|}{ PARAMETERS } & HOLEP(n=60) & B-TUEP (n=49) & p \\
\hline Age (yr) & $64.4(46-80)$ & $66.4(47-80)$ & 0.209 \\
\hline PSA (ng/mL) & $3.9 \pm 2.9(0.96-20.3)$ & $5.3 \pm 5.3(0.9-21.2)$ & 0.094 \\
\hline IPSS & $25.4 \pm 4.4(14-33)$ & $20.1 \pm 5(11-30)$ & 0.076 \\
\hline Qmax (mL/s) & $9.3 \pm 3.5(2-18)$ & $8.1 \pm 2.5(4-13)$ & 0.180 \\
\hline PVR (mL) & $168 \pm 85.2(25-450)$ & $84.7 \pm 69.5(0-280)$ & $<0.001^{*}$ \\
\hline Prostate volume (mL) & $75.3 \pm 17.5(41.6-130)$ & $68.1 \pm 25.3(30-110)$ & $0.021^{*}$ \\
\hline Operation time (min) & $67.2 \pm 20.6(32-120)$ & $77.2 \pm 29.8(23-170)$ & 0.130 \\
\hline Catheterization time (hr) & $40.4(17-72)$ & $42.5(16-120)$ & 0.614 \\
\hline Haemoglobin drop (g/dL) & $1.01 \pm 0.86$ & $1.06 \pm 0.95$ & 0.232 \\
\hline Hospital stay (d) & $1.3(1-2)$ & $2.1(1-5)$ & $<0.001^{*}$ \\
\hline $\begin{array}{l}\text { Enucleated prostate weight } \\
\text { (gr) }\end{array}$ & $66.4 \pm 20.1(30-108)$ & $39.5 \pm 15.2(18-90)$ & $<0.001^{*}$ \\
\hline * Significant & & & \\
\hline
\end{tabular}

Table 2. Postoperative data.

Data presented as mean \pm standard deviation with minimum and maximum value

\begin{tabular}{|l|c|c|c|}
\hline \multicolumn{1}{|c|}{ PARAMETERS } & HOLEP $(\mathbf{n = 6 0 )}$ & B-TUEP $(\mathbf{n = 4 9 )}$ & p \\
\hline PSA (ng/mL) & $1.25 \pm 1.01(0.1-4.1)$ & $1.34 \pm 1.07(0.4-3.9)$ & 0.667 \\
\hline IPSS changes & $-19.5 \pm 4.8(3-29)$ & $-14.9 \pm 6(2-26)$ & $0.001^{*}$ \\
\hline Qmax $(\mathbf{m L} / \mathbf{s})$ & $33.2 \pm 9.3(11-51)$ & $19.8 \pm 7(6-45)$ & $0<001^{*}$ \\
\hline Qmax changes (mL/s) & $+24.4 \pm 10(2-47)$ & $+11.4 \pm 7.3(2-35)$ & $0<001^{*}$ \\
\hline PVR $(\mathbf{m L})$ & $25.5 \pm 27.7(0-150)$ & $32 \pm 18.8(0-135)$ & 0.738 \\
\hline Prostate volume (mL) & $17.23 \pm 4.6(9-30)$ & $20.49 \pm 7.7(10-42)$ & $0.008^{*}$ \\
\hline $\begin{array}{l}\text { Decrease in PSA ratio } \\
\text { (\%) }\end{array}$ & $64.7 \pm 20.1$ & $67.1 \pm 21.6$ & 0.594 \\
\hline
\end{tabular}

* Significant

Table 3. Complications.

\begin{tabular}{|l|c|c|c|}
\hline \multicolumn{1}{|c|}{ PARAMETERS } & HOLEP (n=60) & B-TUEP (n=49) & p \\
\hline Blood transfusion, n (\%) & $0(0)$ & $3(6.1)$ & 0.088 \\
\hline Stress urinary incontinence, n (\%) & $0(0)$ & $2(4.1)$ & 0.200 \\
\hline Capsule perforation, n (\%) & $2(3.3)$ & $5(10.2)$ & 0.146 \\
\hline Re-operation, n (\%) & $1(1.7)$ & $2(4.1)$ & 0.587 \\
\hline Urethral stricture, n (\%) & $1(1.7)$ & $3(6.1)$ & 0.324 \\
\hline Complication, n (\%) & $3(5)$ & $7(14)$ & 0.095 \\
\hline
\end{tabular}

volume (PVR) significantly higher in HOLEP group. Except for hospital stay and enucleated prostate weight, other intraoperative parameters were comparable between the groups. Postoperative parameters significantly improved in all patients. In HOLEP group, IPSS and $\mathrm{Q}_{\max }$ changes better than B-TUEP group (Table 2). Complication rates were comparable between the groups (Table 3 ). There were no conversions to open surgery.

Conclusions: Our short-term results showed that both HOLEP and B-TUEP are safe and effective treatment options for men with BPH. When compared to B-TUEP, HOLEP provided shorter hospital stay, more prostatic tissue enucleation, and better improvements in IPSS and $\mathrm{Q}_{\max }$
MP08-21 Preliminary data on prospectic randomized trial on the comparison of holmium laser enucleation of the prostate (HOLEP) vs. prostate enucleation with Moses technology (MOLEP)

\author{
Y Hussein M.I., F Petrelli, S Corti, R Milesi, F Ceresoli, \\ I Vavassori
}

ASST Bergamo Ovest

Introduction \& Objective: BPH is a common condition in older men. Several techniques use laser energy to resect, enucleate or ablate hyperplastic prostate tissue. Holmium laser is used for $\operatorname{HoLEP}(\mathrm{H})$. MoLEP $(\mathrm{M})$ is a usage of HoLEP technique that endorsea modified laser pulse with Moses technology (Lumenis ${ }^{\circledR}$ MOSES Pulse $\left.{ }^{\mathrm{TM}} 120 \mathrm{H}\right)$. We are conducing this phase $3 \mathrm{~b}$ study of HoLEP vs. MoLEP to expand the post-marketing data on safety and efficacy of MoLEP enucleation in BPH treatment.

Methods: We are conducting an ongoing single center phase $3 \mathrm{~b}$ study in men with moderate to severe BPH-associated symptoms refractory or intolerant to medical therapy or with acute or chronic urinary retention and prostate volume of $>80 \mathrm{ml}$. Patients were excluded if they received previous surgery, there is a suspect of prostate cancer, there is an untreated urinary infection, there are cardiovascular comorbidities or patients suffered of neurological bladder. 55 patients $(28 / 27 \mathrm{H} / \mathrm{M})$ were randomized preoperatively in a 1:1 fashion (Setting $2 \mathrm{~J} ; 50 \mathrm{~Hz}$ ) to H-Group or M-Group. All the procedures were performed by a single experienced operator using the traditional 3 lobes technique. The primary endpoint is the evaluation of the difference in average treatment duration between the two procedures. Comparisons of means in the paired sample was performed with a two-tail T-test ( $\alpha$ power of 0.05 to observe a $10 \%$ difference in time of enucleation).

Results: Mean age was 70 in both groups. Average baseline volume of prostates were 92 and $95 \mathrm{ml}$ in group $\mathrm{H}$ and $\mathrm{M}$ respectively. Comparison of the 2 modalities lead to a reduced time of enucleation for $\mathrm{M}(\mathrm{H} / \mathrm{M}: 30.5 / 23$ minutes; $\mathrm{P}=0.03)$, time of treatment $(\mathrm{H} / \mathrm{M}: 52 / 46 ; \mathrm{P}=0.7)$ and time to hemostasis $(\mathrm{H} / \mathrm{M}: 5.5 /$ 4.6 minutes; $\mathrm{P}=0.28$ ). Total energy for Hemostasis or enucleation was similar in both arms ( $\mathrm{P}=0.85$ and 0.33 for the 2 comparisons $)$. No bleeding was observed in both groups $(\mathrm{P}=0.34)$. Decrease in $\mathrm{Hb}$ levels postoperatively was 0.99 and 1.08 in $\mathrm{H}$ and $\mathrm{M}$ $\operatorname{arms}(\mathrm{P}=0.69)$. Fiber consumption length was $2.97 \mathrm{~mm}$ and $1.87 \mathrm{~mm}$ in groups $\mathrm{H}$ and $\mathrm{M}(\mathrm{P}=0.05)$. Weight/energy ratio was similar in 2 arms $(\mathrm{P}=0.78)$. Interestingly, weight/time ratio (grams of morcellated prostatic tissue/minute of enucleation) was favor in M group: 2.14 vs.2.82, $\mathrm{P}=0.32$.

Conclusions: This ongoing phase $3 \mathrm{~b}$ study of HOLEP vs MOLEP is showing significant reduction in enucleation time, however demonstrating similar short-term outcomes in terms of total timing of treatment and hemostasis, energy used, bleeding (hematuria and postoperative $\mathrm{Hb}$ levels). There is, however, lower fiber consumption with MOLEP and a more efficient weight of enucleated tissue/time unit in the MOLEP group.

\section{MP08-22 Single Surgeon Experience with Procept Aquabeam Aquablation of Prostate: First 40 Cases}

\section{A Kasraeian}

Kasraeian Urology

Introduction \& Objective: Procept Aquabeam Aquablation is a novel, minimally invasive technology for the management of $\mathrm{BPH}$, regardless of prostate size or shape. The Aquabeam system 
is an innovative combination of robotic technology, multidimensional real-time imaging, and a heat-free waterjet to precisely and accurately remove obstructive prostate tissue in men with $\mathrm{BPH}$. We report our initial experience with our first 40 cases.

Methods: Between July 2018 and May 2019, data was prospectively collected on 40 men who underwent Aquablation of the prostate. Evaluation of LUTS included cystoscopy, urodynamics (UDS) studies, and transrectal ultrasound (TRUS) to measure of prostate volume. Pre-operative, intra-operative and post-operative outcomes were prospectively collected and reported.

Results: Pre-operative demographics include a mean age of 67 ( 50 to 84 ) and a mean prostate volume of $92 \mathrm{cc}$ (27 to 201). Of our 40 patients, 29 had prostates larger than $80 \mathrm{cc}, 15$ greater than $100 \mathrm{cc}$ and three greater than $150 \mathrm{cc}$. An obstructing median lobe was noted in $33(83 \%)$ men in our study. Pre-operative UDS demonstrated severe bladder outlet obstruction (BOO) in 37 of the 40 men $(93 \%)$. Detrusor instability was noted in 23 men $(58 \%)$, and 19 of the $40(48 \%)$ suffered from urinary retention with $13(33 \%)$ requiring catheterization prior to their procedure. Mean operative time was 57 minutes (25-108). Twenty-five of the 40 men were discharged on post-operative day (POD) 1 , with 11 discharged on POD 2 and three on the third post-operative day. No patients received transfusions. Mean pre-operative hemoglobin ( $\mathrm{Hgb}$ ) was 14.3 (8.6 to 16.7) and mean immediate postoperative and day of discharge Hgb levels were 13.2 (10 to 16) and 11.8 (7.2 to 15), respectively. Of note, 33 of the 40 men reported erectile dysfunction at baseline. Post-operatively, a 16 point (5-27) mean decrease in AUA symptom score (AUASS) was noted from 22 (10 to 35), pre-operatively, to 6.6 (1-15), post-operatively. Post-operative uroflow studies after 4-6 weeks demonstrated a mean maximum flow rate (Qmax) of $21 \mathrm{ml} / \mathrm{s}(4.3$ to 44 ), an increase of 13 points from $7.6 \mathrm{ml} / \mathrm{s}$ ( 2.1 to 15$)$, preoperatively. Erectile function was maintained post-operatively with a mean SHIM score of 12.2 (range 1 to 25) versus 11.7 (1 to 25), pre-operatively. All patient's completed successful voiding trials post-operatively, and of the patients with baseline urinary retention, none currently require any form of catheterization.

Conclusions: The PROCEPT AQUABEAM Aquablation is an innovative technology that offers predictable and reproducible outcomes, independent of prostate size. Aquablation has a short learning curve ad is easily reproduced regardless of prostate size or shape.

\section{MODERATED POSTER SESSION 09: BPH/LUTS: OUTCOMES \& COMPLICATIONS}

\author{
MP09-01 A novel one-stop LUTS clinic model from a \\ tertiary referral university hospital in the United \\ Kingdom \\ U Walters, T Latimer, S Dean, M Morgan, V Jeram, \\ T El-Husseiny \\ Charing Cross Hospital, Imperial College Healthcare NHS Tust
}

Introduction \& Objective: Lower urinary tract symptoms (LUTS) are a substantial reason for presentation to Urology clinics in the UK. This places a significant pressure on the overstretched available resources. In the current pathway at our university hospital, patients wait for 20 weeks for new appointments and 55 weeks for follow up appointments, when definitive decisions are made regarding management. Enabling definitive plans on the day of the first visit can reduce this 35-week gap. This quality improvement project evaluated a new one-stop clinic pathway compared to our current LUTS clinic pathway.

Methods: A retrospective audit was carried out, comparing the current LUTS pathway (March-May 2018) with those seen in a newly designed one-stop LUTS clinic pathway (OctoberNovember 2018). The one-stop clinic comprised of consultations before and after any required diagnostic tests (flow rate, post-voiding bladder scan, flexible cystoscopies, urodynamic studies and trans-rectal ultrasound scans) on the same day. This resulted in definitive management decisions being made on the same day.

Results: There were 298 patients analyzed in the current clinic pathway and 109 patients in the new one-stop clinics. In the current clinic pathway, only 55\% of patients had LUTS, with high variation amongst the clinics. The one stop model reduced follow up appointments from $60 \%$ (current) to 5\% (one-stop).
There was an increase in patients being offered surgical management (from $10 \%$ to 57\%) and an increase in clinical discharge (from $25 \%$ to $32 \%$ ). Vetting and calling patients beforehand resulted in reducing the Did Not Attend (DNA) rate from 19\% to $2 \%$ and also increasing the number of LUTS patients (55\% to $96 \%$ ). There was an increase in the use of diagnostics (urodynamic studies from $15 \%$ to $22 \%$ and flexible cystoscopies from $22 \%$ to $39 \%$ ), with review suggesting appropriateness of ordering these tests. The feedback from patients was positive with $98 \%$ preferring the one-stop clinic model and $100 \%$ reporting feeling satisfied or extremely satisfied.

Conclusions: Employing a one-stop clinic model for LUTS patients can reduce the current pathway waiting times by 35 weeks, while providing more consistent, higher quality care. Vetting and calling patients beforehand can significantly reduce the DNA rate, optimizing clinic appointment utilization. Definitive plans regarding surgical management or discharge are made at the first visit, due to the availability of all investigations. Both patients and staff preferred the one-stop clinic model.

\section{MP09-02 Analysis of the Factors Influencing Hol- mium Laser Enucleation of the Prostate Training}

BA Penev, A Henderson, E Peneva, M Cynk

Maidstone and Tunbridge Wells NHS Trust

Introduction \& Objective: Attempt to quantify cases volume load needed to reach satisfactory experience plateau, as well as other factors that influence the effectiveness. Enucleation and morcellation efficiency can be used as an index of expertise. 
Methods: Prospectively collected data of 100 consecutive HoLEP cases was retrospectively analyzed. A $100 \mathrm{~W}$ Holmium Laser at settings 2 Joules and $50 \mathrm{~Hz}$ with Mocellator Lumenis VersaCut were used. Data is presented in groups of 25 consecutive cases, in order to compare the differences in the effectiveness between each group. Data acquisition: Electronic theatre records of operative time; Pathology specimen weight. Efficacy is defined as enucleated weight over total surgical time.

Results: Results are presented in graphs. During the first two periods of total of 50 cases there was an improvement in the efficiency coefficient from average of $0.14 \mathrm{~g} / \mathrm{min}$ to the double this of $0.29 \mathrm{~g} / \mathrm{min}$. Over the next two periods of the study the efficiency rose more slower from $0.3 \mathrm{~g} / \mathrm{min}$ in the first subperiod to $0.48 \mathrm{~g} / \mathrm{min}$ at end of the last period. This represents statistically slower rate of improvement $(\mathrm{p}=0.045)$ in the second period. Therefore, it can be said that the plateau was reached at the end of the first subgroup of 50 cases. In the last 5 cases of the study it was applied two lobe enucleation technique, that reduced the operative time. The prostates with size over 80 grams were more efficiently operated than those with smaller size, $0.45 \mathrm{~g} / \mathrm{min}$ versus $0.21 \mathrm{~g} / \mathrm{min}$, respectively.

Conclusions: It appears that approximately 50 consecutive cases were needed to reach a plateau of efficiency. Two lobes Enucleation technique improved the efficacy further and the larger

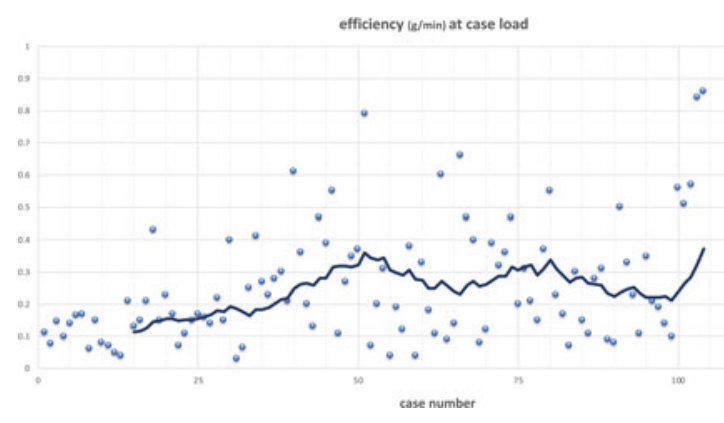

Efficiency in relation to prostate volume

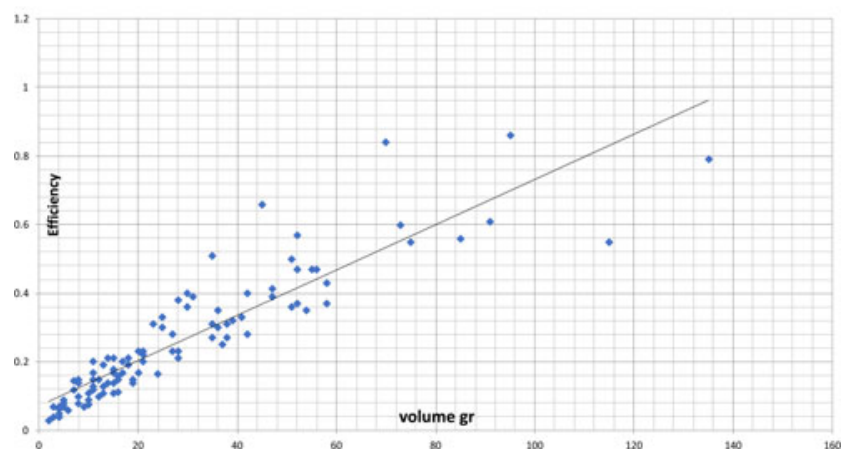

Relation of removed volume and operative time

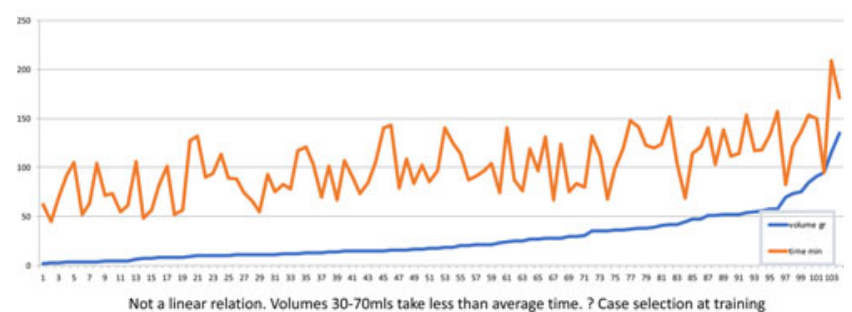

the glands, the higher the efficacy was demonstrated. Drawbacks of the study are that the morcellation time was not recorded separately and some cases have additional pathology like bladder stones, adding to the overall surgical time.

\section{MP09-03 The incidence of Urethral complications with using 26-French versus 28-French resectoscope sheaths in Holmium Laser Enucleation of the Prostate (HoLEP)}

\author{
KH Thai, JC Smith, J Stutz, J Sung, M El Tayeb
}

\section{Baylor Scott \& White}

Introduction \& Objective: HoLEP is gaining popularity for management BPH. Long-term complications of HoLEP include urethral strictures (US) and bladder neck contracture (BNC). Studies report rates of $2.8-4.4 \%$ and $3.6-5.4 \%$ for US and BNC, respectively. To date, there are no studies that have shown the difference between resectoscope sheath size and urethral complications. Our objective is to determine the rate of US and BNC between patients who undergo HoLEP surgery with $26 \mathrm{Fr}$ vs. 28Fr resectoscope sheaths (RS).

Methods: After IRB approval, a retrospective chart review of patients who had HoLEP surgery by a single surgeon (MET) between August 2015 to June 2018. Patients with prior history of US or BNC were excluded. The operative set-up for a HoLEP includes Ho:YAG laser, urethral dilation to 28 or $30 \mathrm{Fr}$, use of a $26 \mathrm{Fr}$ or $28 \mathrm{Fr}$ continuous flow RS, and use of a tissue morcellator. Standard post-op care included overnight observation on continuous bladder irrigation and voiding trial on the post-op day (POD) 1 if minimal hematuria. Primary endpoints were the presence of postoperative urethral stricture or bladder neck contracture. Secondary endpoints include post-operative catheterization time, the success of voiding trial and stress urinary incontinence (SUI). Statistical analysis was performed using appropriate methods.

Results: Out of 502 HoLEP patients, 163 consecutive patients had surgery with a 26Fr RS (group A), and 339 consecutive patients had surgery with 28Fr RS (group B). Three patients (A) and 12 patients $(B)$ had post-op urethral strictures $(\mathrm{p}=0.41)$. Zero (A) and eight (B) patients had post-op bladder neck contracture $(\mathrm{p}=0.0585)$. Mean catheterization time was 1.66 days $(\mathrm{A})$ and 1.84 days $(\mathrm{B})(\mathrm{p}=0.97)$. 14(A) vs 33(B) patients failed their POD1 voiding trial requiring re-catheterization $(p=0.68)$. SUI at 6 weeks, 3-6 months, and 1 year, was present in 54(A) vs $118(\mathrm{~B})$ $(\mathrm{p}=0.71), 9(\mathrm{~A})$ vs 10(B) $(\mathrm{p}=0.157)$, and $1(\mathrm{~A})$ vs 22(B) $(\mathrm{p}=0.003)$, respectively.

Conclusions: Resectoscope sheath size resulted in no statistical significance in terms of urethral strictures, bladder neck contracture and short-term urinary incontinence. Use of 28Fr resectoscope sheath was associated with long-term SUI.

\begin{tabular}{|c|c|c|c|}
\hline & $\mathbf{2 6} \mathrm{Fr}(\mathbf{A})$ & $\mathbf{2 8} \mathrm{Fr}(\mathbf{B})$ & p-value \\
\hline N & 163 & 339 & \\
\hline Age (mean) & 71.1 & 71.9 & 0.19 \\
\hline Post op urethral stricture (n, \%) & $3(1.8 \%)$ & $12(3.5 \%)$ & 0.405 \\
\hline Bladder neck contracture (n, \%) & 0 & $8(2.4 \%)$ & 0.059 \\
\hline Mean Catheterization Time (days) & 1.66 & 1.84 & 0.97 \\
\hline POD 1 Failed voiding trial (n, \%) & $14(8.6 \%)$ & $33(9.7 \%)$ & 0.68 \\
\hline 6 weeks incontinence (n, \%) & $54(33.1 \%)$ & $118(34.8 \%)$ & 0.71 \\
\hline 3-6-month incontinence (n, \%) & $9(5.5 \%)$ & $10(2.9 \%)$ & 0.157 \\
\hline 1-year incontinence (n, \%) & $1(0.6 \%)$ & $22(6.5 \%)$ & $0.003 * * *$ \\
\hline
\end{tabular}


MP09-04 Prostatic amyloidosis- What is the clinical significance?

A Nevo, ML Stanton, A Navaratnam, MR Humphreys

Mayo Clinic

Introduction \& Objective: Amyloidosis is characterized by extracellular deposition of an abnormal proteinaceous material in various organs. Involvement of the urogenital system is rare, and most frequently reported in the bladder or seminal vesicles. Prostatic involvement is even less common, reported in only several case reports. Amyloidosis can be localized or systemic, and because of its rarity, whether other organs should be investigated if the prostate is involved is unknown. We present the largest series to date of prostatic amyloidosis in order to determine the clinical implication of this finding.

Methods: A retrospective chart review of all patients who were diagnosed with prostatic amyloidosis on prostate biopsy or minimally invasive surgery, at Mayo Clinic in Arizona between 2000 and 2019. Positive specimens were formally reviewed by a uropathologist. Clinical information including age, presenting symptoms, PSA, pathology results, and the diagnosis of systemic amyloidosis before or after the biopsy was collected.

Results: We identified 12 patients with prostatic amyloidosis, of which 2 were diagnosed with systemic amyloidosis prior to the biopsy. Median age was 76 years. Nine patients had undergone prostate biopsy, 2 patients had undergone robotic assisted radical prostatectomy. Holmium laser prostatectomy and transurethral prostatectomy were performed in one patient each. Prostate cancer was diagnosed in 5 patients, and HGPIN and ASAP were diagnosed in 1 patient each. Follow up data was available for 10 patients. Over a median follow up of 4 years, 4 patients were diagnosed with cardiac amyloidosis, and one patient underwent bone marrow transplantation.

Conclusions: Amyloidosis is a rare finding on a prostate biopsy and is associated with systemic amyloidosis in $50 \%$ of the cases. In one third of the patients, systemic disease was diagnosed after the biopsy. We recommend further investigation in patients with incidental amyloid on prostate biopsy.

\section{MP09-05 Urethra and ejaculation preserving robot- assisted simple prostatectomy: near infrared fluores- cence imaging-guided Madigan technique}

G Simone, L Misuraca, U Anceschi, F Minisola, M Ferriero, S Guaglianone, G Tuderti, M Gallucci, R Mastroianni

Introduction \& Objective: With the increasing adoption of novel technologies and anatomical techniques, the surgical management of benign prostatic hyperplasia (BPH) provides significant benefits in terms of obstruction relief, early urethral catheter removal and faster return to daily activities. However, the main pitfall of BPH surgery in sexually active men remains ejaculatory dysfunction (ED) which permanently affects quality of life (QoL). Our objective is to detail a novel technique for marking intraprostatic urethra through a retrograde injection of indocyanine green (ICG) to enhance a selective dissection of prostatic lobes during urethra-sparing robot-assisted simple prostatectomy (US-RASP) with the use of Near Infrared Fluorescence Imaging (NIFI).

Methods: Between January-September 2017, 12 consecutive male patients with BPH, sexually active and motivated to preserve ejaculatory function, underwent US-RASP with NIFI to enhance identification and preservation of prostatic urethra.
Clinical data were prospectively collected into our institutional RASP dataset. Perioperative and functional outcomes of USRASP were both graded and assessed according to Clavien Grading System and validated questionnaires postoperatively (IPSS; MSHQ-EjD Short Form) at 3,12 months.

Results: Median preoperative prostate size was 102 cc (IQR 88115). Median operative time was 150 minutes (IQR 145-170). Median estimated blood loss was 250 (IQR 200-350). Continuous bladder irrigation was avoided in $83.4 \%$ of patients. Median time to catheter removal was 7 days (IQR 7-7) with a median hospital stay of 3 days (IQR 2-3). At 1-yr follow-up median IPSS score, IIEF score and MSHQ-EjD Short Form were 5 (IQR 4-8), 27 (IQR 26-28) and 12 (IQR 3-14), respectively. A satisfactory anterograde ejaculation was reported in 8 patients (66\%).

Conclusions: We described a novel NIFI-guided technique to perform US-RASP. This technique showed promising early functional results suggesting a significant role of intraprostatic urethral integrity for the preservation of ejaculatory function.

\section{MP09-06 Risk factors for febrile complications after} holmium laser enucleation of the prostate

Y Kyono, F Endo, M Sakurai, M Sano, K Komatsu, K Narimoto, M Shimbo, K Ohwaki, K Hattori

Introduction \& Objective: Although febrile complications (FCs) after HoLEP occasionally develop even when prophylactic antibiotics are prescribed, the prevalence thereof is not accurately known because of a lack of data. Here we report the incidence of FC and the associated pre- and peri-operative risk factors.

Methods: Of 1,237 patients with benign prostate hyperplasia who underwent HoLEP in our tertiary hospital from June 2006 to December 2018, 863 were enrolled in the present study. FC (body temperature $\geqq 38.0^{\circ} \mathrm{C}$ within 30 postoperative days) was the outcome of interest. We explored possible explanatory variables including patient age and body mass index, preoperative urine culture and laboratory data, operative parameters (operation time, resected specimen weight, irrigation fluid volume, and type of anesthesia), and immediate postoperative vital signs (body temperature, heart rate, respiratory rate, and blood pressure).

Results: Prophylactic antibiotics were administered for 857 cases $(99.3 \%)$. We encountered 90 cases $(10.4 \%)$ of FCs, 56 cases $(6.48 \%)$ of urinary tract infections (including bacteremia), but no case of fatal complications. On multivariate regression analysis, a positive urine culture (odds ratio [OR] 1.893: 95\% confidence interval [CI] 1.170-3.063), the preoperative serum albumin level (OR 0.402: 95\% CI 0.192-0.843), the operation time (OR 1.006: 95\% CI 1.002-1.011), and the immediately postoperative body temperature (OR 2.534: 95\% CI 1.568-4.095) were significant risk factors for FCs.

Conclusions: We found that a preoperative positive urine culture and a low serum albumin level were significant risk factors for FCs. Surgeons should evaluate these parameters prior to HoLEP, to prevent FCs.

MP09-07 Outcome of Unilateral bladder neck incision in comparison with Bilateral incision incase of Primary bladder neck obstruction

M Alam

Bangabandhu Sheikh Mujib Medical University (BSMMU) 
Introduction \& Objective: Primary bladder neck obstruction is not uncommon in middle aged men. Patients with primary bladder neck obstruction usually present with voiding and storage symptoms. They are evaluated by history, physical examination, investigations, and confirmed by urodynamic study. There are different modalities of treatment. If medical

\section{RESULTS}

This Quasi experimental study was carried out in the Department of Urology, Dhaka Medical College Hospital, Dhaka from the period of July 2013 to June 2015. In this study 60 patients with lower urinary tract symptoms (LUTS) due to primary bladder neck obstruction with clear urodynamic evidence were enrolled as sample taken from Department of Urology, Dhaka Medical College and Hospital, Dhaka. The patients were divided into two groups: 30 patients underwent U-BNI (Group I) and the other 30 underwent B-BNI (Group II). According to analysis of age distribution, there was no significant difference among both groups.

Table I: Distribution of patients according to age

\begin{tabular}{|c|c|c|c|}
\hline \multirow[b]{2}{*}{ Age } & \multicolumn{2}{|c|}{ Group } & \multirow[b]{2}{*}{$p$ value } \\
\hline & $\begin{array}{c}\text { Group I } \\
\text { (UBNI) } \\
\text { n (\%) }\end{array}$ & $\begin{array}{c}\text { Group II } \\
\text { (BBNI) } \\
\text { n (\%) }\end{array}$ & \\
\hline $20-25$ & $10(33.3)$ & $11(36.7)$ & \\
\hline $26-30$ & $11(36.7)$ & $10(33.3)$ & \\
\hline $31-35$ & $6(20.0)$ & $5(16.7)$ & \\
\hline $36-40$ & $3(10.0)$ & $4(13.3)$ & \\
\hline Total & $30(100.0)$ & $30(100.0)$ & 0.954 \\
\hline Mean \pm SD & $28.33 \pm 5.33$ & $28.20 \pm 5.49$ & 0.824 \\
\hline
\end{tabular}

$\mathrm{t}$ test was done to measure the level of significance.

Table I shows distribution of patients by age in two groups. In group I, maximum $11(36.7 \%)$ patients were in age group 26-30 years followed by $10(33.3 \%), 6(20.0 \%)$ and $3(10.0 \%)$ were in age groups $20-25$ years, $31-35$ years and $36-40$ years respectively.

Table II: Distribution of patients according to duration of operation and hospital stay

\begin{tabular}{lccc}
\hline \multirow{2}{*}{ Variables } & \multicolumn{2}{c}{ Group } & \\
\cline { 2 - 3 } & $\begin{array}{l}\text { Group I } \\
\text { (UBNI) } \\
\text { Mean } \pm \text { SD }\end{array}$ & $\begin{array}{c}\text { Group II } \\
\text { (BBNI) } \\
\text { Mean } \pm \text { SD }\end{array}$ & p value \\
\hline Duration of operation (Minutes) & $19.9 \pm 3.26$ & $27.16 \pm 2.65$ & $<0.001$ \\
Hospital stay (days) & $2.10 \pm 0.30$ & $2.40 \pm 0.56$ & 0.013 \\
\hline
\end{tabular}

$\mathrm{t}$ test was done to measure the level of significance.

Table II shows per and post operative findings. Mean (SD) duration of operation was 19.9 (3.26) minutes and 27.16 (2.65) minutes in group I and group II respectively. The difference between these two groups was statistically significant $(\mathrm{p}<0.05)$. Mean (SD) hospital stay was $2.10(0.30)$ days and $2.40(0.56)$ days in group I and group II respectively. The difference between these two groups was statistically significant $(\mathrm{p}<0.05)$.

Table III: Distribution of patients according to voiding time

\begin{tabular}{lccc}
\hline & \multicolumn{2}{c}{ Group } & p value \\
\cline { 2 - 3 } Voiding time (Second) & $\begin{array}{c}\text { Group I } \\
\text { (UBNI) } \\
\text { Mean } \pm \text { SD }\end{array}$ & $\begin{array}{c}\text { Group II } \\
\text { (BBNI) } \\
\text { Mean } \pm \text { SD }\end{array}$ & (between groups) \\
\hline Pre operative & $51.63 \pm 10.71$ & $53.83 \pm 4.37$ & $0.302^{*}$ \\
$1^{\text {st follow up }}$ & $35.33 \pm 6.83$ & $29.86 \pm 4.40$ & $0.001^{*}$ \\
$2^{\text {nd }}$ follow up & $32.33 \pm 6.83$ & $27.93 \pm 4.43$ & $0.004^{*}$ \\
$3^{\text {rd } \text { follow up }}$ & $25.33 \pm 6.83$ & $24.90 \pm 4.41$ & $0.772^{*}$ \\
$\begin{array}{l}\text { p value (with in groups between } \\
\text { pre operative and } 3^{\text {rd }} \text { follow up) }\end{array}$ & $<0.001^{*}$ & $<0.001^{*}$ & \\
\hline
\end{tabular}

*Independent $t$ test was done to measure the level of significance.

"Paired $t$ test was done to measure the level of significance.

Table III shows voiding time at different follow ups in groups. Pre operative mean (SD) voiding time was $51.63 \pm 10.71 \mathrm{sec}$ and $53.83 \pm 4.37 \mathrm{sec}$ in group I and group II respectively. The time was $51.63 \pm 10.71 \mathrm{sec}$ and $53.83 \pm 4.37 \mathrm{sec}$ in group 1 and group 11
difference between these two groups was not statistically significant $(\mathrm{p}>0.05)$. treatment failed, they are treated with unilateral or bilateral bladder neck incisions. Among them, per and postoperative outcome like operation time, hospital stay, voiding time, PVR, Qmax and retrograde ejaculation were assessed in both the group.

Objectives: The purpose of the study was to compare of unilateral and bilateral incision in patients with primary bladder neck obstruction and to see the operation time, postoperative hospital stay, PVR, Qmax, voiding time and retrograde ejaculation between two groups.

Table IV: Distribution of patients according to post void residual (PVR)

\begin{tabular}{lccc}
\hline & \multicolumn{2}{c}{ Group } & \\
\cline { 2 - 3 } Post void residual(ml) & $\begin{array}{c}\text { Group I } \\
\text { (UBNI) } \\
\text { Mean } \pm \text { SD }\end{array}$ & $\begin{array}{c}\text { Group II } \\
\text { (BBNI) } \\
\text { Mean } \pm \text { SD }\end{array}$ & $\begin{array}{c}\text { p value } \\
\text { (between groups) }\end{array}$ \\
\hline Pre operative & $101.10 \pm 10.82$ & $105.73 \pm 11.50$ & $0.114^{*}$ \\
$1^{\text {st }}$ follow up & $26.16 \pm 5.96$ & $24.16 \pm 5.66$ & $0.188^{*}$ \\
$2^{\text {nd }}$ follow up & $22.30 \pm 3.44$ & $21.00 \pm 3.36$ & $0.145^{*}$ \\
$3^{\text {rd }}$ follow up & $18.03 \pm 3.31$ & $17.03 \pm 3.31$ & $0.248^{*}$ \\
$\begin{array}{l}\text { p value (with in groups between } \\
\text { pre operative and 30 }\end{array}$ & $<0.001^{*}$ & $<0.001^{*}$ & \\
\hline
\end{tabular}

*Independent $t$ test was done to measure the level of significance.

"Paired $t$ test was done to measure the level of significance.

Table IV shows post void residual (PVR) at different follow ups in groups. Pre operative mean (SD) PVR was $101.10 \pm 10.82 \mathrm{ml}$ and $105.73 \pm 11.50 \mathrm{ml}$ in group I and group II respectively.

Table V: Distribution of patients according to maximum urinary flow rate (Qmax)

\begin{tabular}{|c|c|c|c|}
\hline \multirow[b]{2}{*}{ Qmax (ml/sec) } & \multicolumn{2}{|c|}{ Group } & \multirow[b]{2}{*}{$\begin{array}{c}\text { p value } \\
\text { (between groups) }\end{array}$} \\
\hline & $\begin{array}{c}\text { Group I } \\
\text { (UBNI) } \\
\text { Mean } \pm \text { SD } \\
\end{array}$ & $\begin{array}{c}\text { Group II } \\
\text { (BBNI) } \\
\text { Mean } \pm \text { SD } \\
\end{array}$ & \\
\hline Pre operative & $7.96 \pm 0.99$ & $8.43 \pm 1.57$ & $0.172^{*}$ \\
\hline $1^{\text {st }}$ follow up & $20.38 \pm 5.25$ & $22.26 \pm 3.95$ & $0.123^{*}$ \\
\hline $2^{\text {nd }}$ follow up & $22.51 \pm 5.10$ & $23.85 \pm 4.06$ & $0.266^{*}$ \\
\hline $3^{\text {rd }}$ follow up & $24.37 \pm 4.79$ & $26.16 \pm 3.69$ & $0.111^{*}$ \\
\hline $\begin{array}{l}\text { p value (with in groups between } \\
\text { pre operative and } 3^{\text {rd }} \text { follow up) }\end{array}$ & $<0.001^{\prime \prime}$ & $<0.001^{\prime \prime}$ & \\
\hline
\end{tabular}

*Independent $\mathrm{t}$ test was done to measure the level of significance.

"Paired $t$ test was done to measure the level of significance.

Table V shows Qmax at different follow ups in groups. Pre operative mean (SD) Qmax was 7.96 $\pm 0.99 \mathrm{ml} / \mathrm{sec}$ and $8.43 \pm 1.57 \mathrm{ml} / \mathrm{sec}$ in group I and group II respectively. The difference between these two groups was not statistically significant $(p>0.05)$.

Table VI: Distribution of patients according to AUA symptom score

\begin{tabular}{lccc}
\hline \multirow{2}{*}{ AUA symptom score } & \multicolumn{2}{c}{ Group } & p value \\
\cline { 2 - 3 } & $\begin{array}{l}\text { Group I } \\
\text { (UBNI) } \\
\text { Mean } \pm \text { SD }\end{array}$ & $\begin{array}{c}\text { Group II } \\
\text { (BBNI) } \\
\text { Mean } \pm \text { SD }\end{array}$ & (between groups) \\
\hline Pre operation & $17.06 \pm 3.86$ & $16.70 \pm 3.95$ & $0.718^{*}$ \\
$1^{\text {st }}$ follow up & $2.23 \pm 1.73$ & $2.13 \pm 1.83$ & $0.821^{*}$ \\
$2^{\text {nd }}$ follow up & $2.10 \pm 1.58$ & $1.96 \pm 1.62$ & $0.749^{*}$ \\
$3^{\text {rd }}$ follow up & $2.10 \pm 1.58$ & $1.86 \pm 1.56$ & $0.569^{*}$ \\
$\begin{array}{l}\text { p value (with in groups between } \\
\text { pre operative and } 3^{\text {rd }} \text { follow up) }\end{array}$ & $<0.001^{*}$ & $<0.001^{*}$ & \\
\hline
\end{tabular}

*Independent $t$ test was done to measure the level of significance.

"Paired $t$ test was done to measure the level of significance.

Table VI shows pre operative and post operative AUA symptoms score in groups. Pre operative mean AUA (ml/sec) was $17.06 \pm 3.86$ and $16.70 \pm 3.95$ in group I and group II respectively. The difference between these two groups was not statistically significant $(\mathrm{p}>0.05)$. 
Table VII: Distribution of patients according to retrograde ejaculation

\begin{tabular}{lccc}
\hline & \multicolumn{2}{c}{ Group } & p value \\
\cline { 2 - 3 } Retrograde ejaculation & $\begin{array}{c}\text { Group I } \\
\text { (UBNI) } \\
\text { Mean } \pm \text { SD }\end{array}$ & $\begin{array}{c}\text { Group II } \\
\text { (BBNI) } \\
\text { Mean } \pm \text { SD }\end{array}$ & \\
\hline $1^{\text {st }}$ follow up & $2(6.7)$ & $8(26.7)$ & 0.038 \\
$2^{\text {sd }}$ follow up & $2(6.7)$ & $8(26.7)$ & 0.038 \\
$3^{\text {rd }}$ follow up & $2(6.7)$ & $8(26.7)$ & 0.038 \\
\hline
\end{tabular}

Chi-square test was done to measure the level of significance.

Table VII shows retrograde ejaculation at different follow ups in groups. Retrograde ejaculation was observed among $2(6.7 \%)$ patients and $8(26.7 \%)$ patients in group I and group II respectively at the time of $1^{\text {st }}$ follow up. There was statistical significant difference between these two groups $(\mathrm{p}<0.05)$. No change was observed at the time of $2^{\text {nd }}$ and $3^{\text {td }}$ follow up among the patients regarding retrograde ejaculation.

Methods: This quasi experimental study was carried out in the department of urology, Dhaka Medical College, Dhaka, from July 2013 to June 2015. Male patients with lower urinary tract symptoms due to primary bladder neck obstruction were the study population. Sixty patients were selected from urology out patients as sample according to inclusion and exclusion criteria. Then, they were divided into two groups purposely, designated as group I, and group II for unilateral and bilateral bladder neck incision respectively to see the per and postoperative outcome like operating time, hospital stay, voiding time, PVR, Qmax and retrograde ejaculation.

Results: Total 60 patients were recruited in this study, of which 30 patients were in group I and the rest of 30 patients were in group II. The mean age with a SD was $28.33 \pm 5.33$ years and $28.20 \pm 5.49$ years in group I and group II respectively $(\mathrm{p}>0.05)$. In group I, the mean \pm SD of operative time was $19.9 \pm 3.26 \mathrm{~min}$ within a range $15-25 \mathrm{~min}$. In group II, the mean \pm SD of operation time was $27.16 \pm 2.65 \mathrm{~min}$ within a range $20-30 \mathrm{~min}(\mathrm{p}<0.05)$. In group I mean (SD) hospital stay was $2.10 \pm 0.30$ day, in group II it was $2.40 \pm 0.56$ day $(\mathrm{p}<0.05)$. In this study $2(6.7 \%)$ patients and $8(26.7 \%)$ patients developed retrograde ejaculation in group I and group II respectively $(\mathrm{p}<0.05)$. In group I, mean $\pm \mathrm{SD}$ of voiding time was $25.33 \pm 6.83 \mathrm{sec}$. In group II, mean $\pm \mathrm{SD}$ of voiding time was $24.90 \pm 4.41 \mathrm{sec}$. $(\mathrm{p}>0.05)$. In group I, the mean $\pm(\mathrm{SD})$ Qmax was $24.37 \pm 4.79 \mathrm{ml} / \mathrm{sec}$ where in group II, it was $26.16 \pm 3.69 \mathrm{ml} / \mathrm{sec},(\mathrm{p}>0.05)$. Mean (SD) PVR in group I was $18.03 \pm 3.31 \mathrm{ml}$, in group II, it was $17.03 \pm 3.31 \mathrm{ml}(\mathrm{p}>0.05)$. Conclusion: Unilateral bladder neck incision is better than conventional bilateral bladder neck incision in primary bladder neck obstruction of young aged men.

\section{MP09-08 Is TURP safe even in super-aged patients over 85 years of age?}

J Kang, S Shin, J Cho, K Moon, D Cho, J Choi, m Kim, J Park, T Yoo

Eulji Medical center

Introduction \& Objective: The world is aging at a rapid rate, and also South Korea already became aged society in 2018 where people 65 years or older account for more than $14 \%$ of the population and expected to become a super-aged society in 2026 when this ratio reaches $20.8 \%$. Therefore, a countermeasure for these super-aged population may be necessary. The purpose of
Table 1. Comparison of parameters before and after TURP

\begin{tabular}{|c|c|c|c|}
\hline & Pre-operative & Post-operative & Pvalue $^{\circ}$ \\
\hline Max. urine flow $(\mathrm{m} / \mathrm{s})^{n}$ & $4.8 \pm 2.8$ & $10.0 \pm 5.4$ & $<0.01$ \\
\hline Residual urine volume $(\mathrm{ml})^{*}$ & $135.9 \pm 189.5$ & $26.8 \pm 24.7$ & 0.03 \\
\hline IPSS total score ${ }^{b}$ & $21.1 \pm 9.5$ & $10.3 \pm 9.8$ & 0.03 \\
\hline QOL score & $4.1 \pm 2.1$ & $2.4 \pm 1.4$ & 0.08 \\
\hline
\end{tabular}

this study is to analyze the results of transurethral resection of prostate gland (TURP) in super-aged patients over 85 years of age.

Methods: From January 2004 to December 2017, 92 patients aged 85 years or older who underwent TURP at five hospitals in northeastern areas of Seoul were included. The age of the patients, co-morbidities, and the reason for the operation were retrospectively investigated, and the preoperative and postoperative clinical parameters were compared. We also assessed complications and alpha-blockers medication after TURP.

Results: The mean age of the patients on the operation was $87.2 \pm 2.3$ years. Hypertension was present in 51 patients $(58.6 \%)$, diabetes mellitus in 19 patients $(21.8 \%)$ and cerebrovascular disease in 14 patients $(16.3 \%)$. Forty-five patients $(52.3 \%)$ underwent TURP owing to recurrent acute urinary retentions. The average preoperative prostate volume was $64.0 \pm 33.1 \mathrm{cc}$, the mean transitional volume was $34.6 \pm 25.1 \mathrm{cc}$, the mean operation time was $75.6 \pm 43.1$ minutes, and the resected prostate volume was $23.6 \pm 13.6 \mathrm{~g}$. Significant improvements were observed after TURP in maximal flow rate, residual urine volumes and IPSS scores (Table). Two patients $(2.2 \%) \mathrm{did}$ not void post-operatively and had an indwelling catheter and 12 patients $(13.0 \%)$ continued to take alpha-blockers for facilitating voiding. A blood transfusion was needed in 2 patients $(2.2 \%)$ due to post-operative hemorrhage and 4 patients $(3.3 \%)$ developed infectious complications but soon improved. Postoperative delirium occurred in 7 patients (7.6\%) without any complication. No mortality or serious complications including TURP syndrome occurred in any patient.

Conclusions: In our study, over 50\% of super-aged patients underwent TURP due to recurrent acute urinary retentions. TURP have shown effective in improving the lower urinary tract symptoms in super-aged patients without any serious complications. Deliriums may occur in these age groups; therefore, appropriate measures should be taken during the admission periods.

MP09-09 A randomized controlled study to compare the efficacy of thulium laser $200 \mathrm{~W}$ enucleation versus Greenlight laser $180 \mathrm{~W}$ vaporesection of the prostate for the treatment of benign prostatic obstruction: Midterm report

F Hsu, C Chou, H Chang

Taipei City Hospital

Introduction \& Objective: Transurethral laser treatment is considered to be an alternative treatment to transurethral resection of the prostate for patients with benign prostate obstruction (BPO). For further investigation of the efficacy of laser in treating $\mathrm{BPH}$, we organized a prospective randomized control study. We enrolled patients with BPO, treated with either 


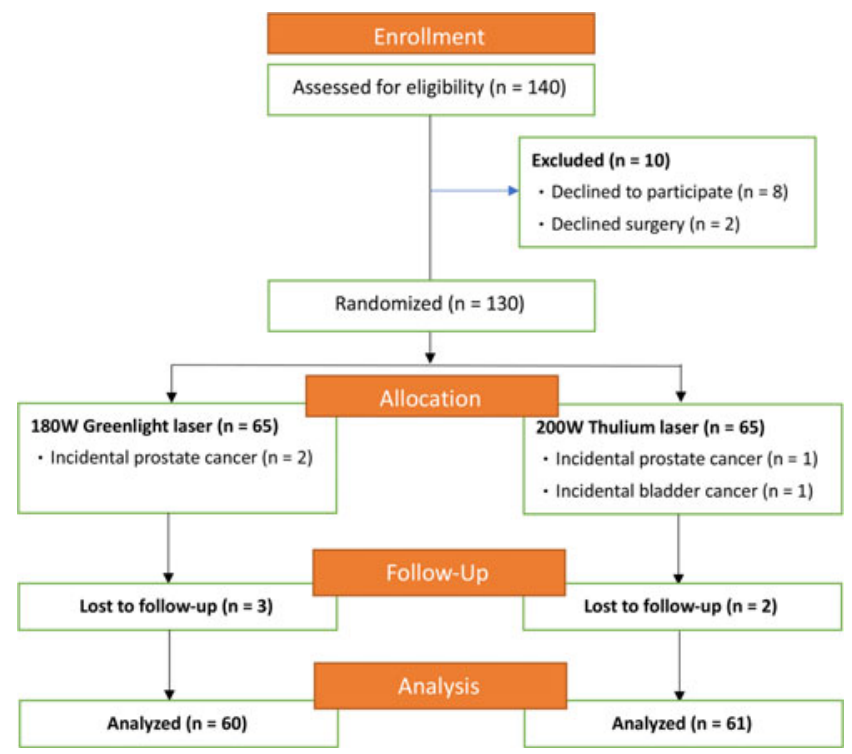

GreenLight XPS laser vaporesection of the prostate (GLVP) or thulium laser vapoenucleation (ThuVEP), and compare their safety and efficacy.

Methods: This study is a prospective, randomized, singleblinded, clinical trials (RCT) to compare outcomes of GLVP versus ThuVEP. Between October 2017 and October 2018, a total of 130 patients with BPO were assigned under randomization to treat with the GLVP (65 cases) or the ThuVEP (65 cases). Only the surgeons knew the operation procedures. Inclusion criteria are patient age greater than 50 years, maximum flow rate $(\mathrm{Qmax})<15 \mathrm{ml} / \mathrm{second}$ and an International Prostate Symptom Score (IPSS) $\geq 10$. Exclusion criteria are known prostate cancer, bladder cancer, previous transurethral surgery,

Table 1. Preoperative patients' demographic characteristics. GLVP = GreenLight XPS laser $180 \mathrm{~W}$ vaporesection of the prostate, ThUVEP $=$ thulium laser $200 \mathrm{~W}$ vapoenucleation, IPSS = international prostate symptom score, $\mathrm{Qmax}=$ maximum flow rate, $\mathrm{QoL}=$ the quality-of-life scores, PVR = postvoid residual

\begin{tabular}{lccc} 
& GLVP $(\boldsymbol{n}=63)$ & ThuVEP $(\boldsymbol{n}=63)$ & $p$ value \\
Age & $66.2 \pm 7.9$ & $67.9 \pm 8.5$ & 0.239 \\
Prostate volume (mL) & $59.5 \pm 18.9$ & $54.7 \pm 17.1$ & 0.132 \\
IPSS & $21.9 \pm 5.5$ & $21.5 \pm 6.1$ & 0.634 \\
\hline Qmax (mL/sec) & $8.5 \pm 4.4$ & $9.7 \pm 5.3$ & 0.185 \\
\hline QoL & $5.1 \pm 0.7$ & $5.0 \pm 0.7$ & 0.382 \\
\hline PVR volume (mL) & $88.3 \pm 120.2$ & $57.9 \pm 60.4$ & 0.076 \\
\hline PSA (ng/mL) & $4.5 \pm 5.1$ & $4.3 \pm 3.6$ & 0.793 \\
\hline Indwelling catheter & $9(14.2 \%)$ & $5(7.9 \%)$ & 0.198 \\
\hline Anticoagulants use & $16(25.4 \%)$ & $20(31.7 \%)$ & 0.277 \\
\hline Diabetes & $21(33.3 \%)$ & $28(44.4 \%)$ & 0.136 \\
\hline Charlson comorbidity index & $0.94 \pm 0.82$ & $1.16 \pm 1.24$ & 0.240
\end{tabular}

Continuous variables are shown as the mean \pm standard deviation.

Categorical variables are shown as the number (percent).
Table 2. Perioperative parameters (GLVP vs. ThuVEP).

\begin{tabular}{|c|c|c|c|}
\hline & $\operatorname{GLVP}(n=63)$ & ThuVEP $(n=63)$ & $p$ value \\
\hline Operative time ( $\mathrm{min}$ ) & $27.9 \pm 9.8$ & $26.8 \pm 10.1$ & 0.515 \\
\hline Catheterization time (hr) & $21.4 \pm 3.8$ & $20.4 \pm 5.0$ & 0.221 \\
\hline Hospital stay (day) & $2.4 \pm 0.6$ & $2.5 \pm 0.9$ & 0.255 \\
\hline Resected adenoma weight* & $9.83 \pm 6.1$ & $11.3 \pm 6.7$ & 0.210 \\
\hline Hemoglobin drop (gm/dl) & $0.38 \pm 0.57$ & $0.46 \pm 0.71$ & 0.507 \\
\hline Laser energy (joule) ${ }^{*}$ & $64,730 \pm 44,886$ & $29,793 \pm 13,369$ & $<0.001$ \\
\hline
\end{tabular}

- Statistically significant

interstitial cystitis and neurogenic bladder. The local institutional review board committee has approved the study (106002-F, New Taipei City Hospital). We registered this RCT on clinicaltrials.gov (NCT03318991). The primary outcome measurement is International Prostate Symptom Score (IPSS) at 1 year. The secondary outcome measurements were Qmax and QoL. The sample size was determined based on an expected mean IPSS of $5.8 \pm 2.6$ at 1-year follow up. The investigators considered a change of deference of IPSS $\leq 2$ as an accepted equivalence between the two groups. The sample size was calculated to be at least 21 patients in each group with $\alpha=0.05, \beta=0.80$ and a desired statistical power level of $80 \%$. Considering the possibility of patients drop out or lost to follow up, 40 patients or more will be enrolled in each arm.

Results: Preoperative data, including age, prostate volume, IPSS, catheterization time, Charlson comorbidity index were similar in two groups. Peri-operative parameters, including operative time, the duration of catheterization, and length of hospital stay were comparable. The laser energy used, were significantly lower in the ThuVEP group. The voiding parameters improved significantly in both groups postoperatively. There were significantly better outcomes in QoL and IPSS at 6-month in the GLVP group. There were no significant differences in improvement of Qmax, residual prostate volume and PSA at the 6-months.

Table 3. Improvement of functional outcomes at 6 months after surgery (GLVP vs. ThuVEP).

\begin{tabular}{|c|c|c|c|c|c|}
\hline & $\begin{array}{c}\text { GLVP } \\
\text { preoperative }\end{array}$ & $\begin{array}{c}\text { GLVP } \\
6 \text { months }\end{array}$ & $\begin{array}{c}\text { ThuVEP } \\
\text { preoperative }\end{array}$ & $\begin{array}{l}\text { ThuVEP } \\
6 \text { months }\end{array}$ & $p$ value \\
\hline & & 60) & $(n=$ & & \\
\hline IPSS & $21.9 \pm 5.5$ & $4.7 \pm 2.2^{*}$ & $21.5 \pm 6.1$ & $5.7 \pm 3.5^{*}$ & $0.044^{* *}$ \\
\hline QoL & $5.1 \pm 0.7$ & $0.7 \pm 0.8^{*}$ & $5.0 \pm 0.7$ & $1.1 \pm 0.9^{*}$ & $0.008^{* *}$ \\
\hline $\mathrm{Qmax}(\mathrm{mL} / \mathrm{sec})$ & $8.5 \pm 4.4$ & $20.9 \pm 6.0^{*}$ & $9.7 \pm 5.3$ & $19.9 \pm 7.7^{*}$ & 0.405 \\
\hline PVR (mL) & $88.3 \pm 120.2$ & $10.4 \pm 10.4^{*}$ & $57.9 \pm 60.4$ & $13.7 \pm 12.5^{*}$ & 0.118 \\
\hline $\begin{array}{l}\text { Prostate volume } \\
(\mathrm{mL})\end{array}$ & $59.5 \pm 18.9$ & $24.2 \pm 8.8^{*}$ & $54.7 \pm 17.1$ & $24.8 \pm 8.7^{*}$ & 0.722 \\
\hline PSA (ng/mL) & $4.5 \pm 5.1$ & $2.0 \pm 1.8^{*}$ & $4.3 \pm 3.6$ & $2.4 \pm 1.6^{*}$ & 0.302 \\
\hline
\end{tabular}

${ }^{*} p<0.001 ; p$-value of parameters at 6 months compared to preoperative data.

** statistically significant 
Conclusions: Both treatments led to comparable symptomatic improvements. GLVP demonstrates better outcome at 6-month follow-up. However, the final result should be shown at 1-year.

\section{MP09-10 Correlation of Frailty Scores and Stress Incontinence post Holmium laser enucleation of the prostate for Benign Prostatic Hyperplasia}

\section{J Smith, J Stutz, J Sung, H Hashim, M El Tayeb}

Baylor Scott \& White

Introduction \& Objective: Elderly patients tend to be at increased risk for postoperative complications. As the population continues to age, older patients present for more surgical evaluations and frailty indices were created as a method to assess the physiologic reserves and vulnerability in older surgical patients. our institution implemented a Modified Fried Frailty Index which was obtained on patients prior to Holmium laser enucleation of the prostate (HoLEP). Our goal is to evaluate any correlation between frailty indices and rates of incontinence, specifically stress urinary incontinence (SUI).

Methods: A retrospective chart review was performed for a single surgeon at our facility for all HoLEPs from October 2015 to June 2108. Preoperative, perioperative, postoperative and demographic data was analyzed, including follow-up at 6 weeks, 3-6 months, and 1-1.5 years. All patients with a frailty score were included but excluded from final analysis if no postoperative incontinence information documented.

Results: A total of 610 patients were in the database and 403 had a frailty score. Patients stratified into frailty 0-1 (low risk) and 25 (moderate/high risk). Most patients were low risk $(n=379)$, but there were 24 with frailty scores 2-5 (19 with frailty 2 and 5 with frailty 3). As with previous studies, there is a transient increase in stress incontinence at 6 weeks that decreases over time. There was no statistical significance in the overall incontinence rate, specifically SUI, over any follow-up between the 2

\begin{tabular}{|c|c|c|c|}
\hline & \multirow{2}{*}{\multicolumn{2}{|c|}{ Frailty Score }} & \multirow{3}{*}{ p-Value } \\
\hline & & & \\
\hline & $0-1$ & $2-5$ & \\
\hline Number & 379 & 24 & \\
\hline Age & 71 & 76.25 & 0.0044 \\
\hline BMI & 28.2 & 27.4 & 0.0817 \\
\hline Grip Strength Average (in pounds) & 38 & 26 & $<0.0001$ \\
\hline Race & & & 0.9999 \\
\hline White & $318(88.58 \%)$ & $21(91.3 \%)$ & \\
\hline Black / African America & $33(9.19 \%)$ & $2(8.7 \%)$ & \\
\hline Asian & $5(1.39 \%)$ & 0 & \\
\hline ASA Scores & & & $<0.0001$ \\
\hline 1 & $90(24.19 \%)$ & 0 & \\
\hline 2 & $186(50 \%)$ & $7(30.43 \%)$ & \\
\hline 3 & $88(23.66 \%)$ & $11(47.83 \%)$ & \\
\hline 4 & $8(2.15 \%)$ & $5(21.74 \%)$ & \\
\hline Prostate Weight & $45 \mathrm{~g}$ & $44 \mathrm{~g}$ & 0.6317 \\
\hline 6 weeks Follow Up & & & \\
\hline All Type Incontinence at 6 weeks & $139(36.7 \%)$ & $8(33 \%)$ & 0.9797 \\
\hline Stress Incontinence at 6 weeks & $58(15.3 \%)$ & $1(4.2 \%)$ & 0.2212 \\
\hline Pads per day at 6 weeks & 2 & 1.5 & \\
\hline 3-6 Month Follow Up & & & \\
\hline All Type Incontinence at 3-6 months & $43(11.3 \%)$ & $3(12.5 \%)$ & \\
\hline Stress Incontinence at 3-6 months & $21(5.5 \%)$ & 0 & \\
\hline Pads per day at 3-6 months & 1 & 1 & \\
\hline 1 Year Follow Up & & & \\
\hline All Type Incontinence at 1 year & $15(3.96 \%)$ & $2(8.3 \%)$ & \\
\hline Stress Incontinence at 1 year & $10(2.6 \%)$ & 0 & \\
\hline
\end{tabular}

stratified frailty groups (Table 1). SUI rates were $15.3 \%$ for frailty $0-1$ and $4.2 \%$ for $2-5$ at 6 weeks $(p=0.22)$. At $3-6$ months, SUI was $5.5 \%$ for frailty $0-1$ and $0 \%$ for $2-5(\mathrm{p}=0.63)$. At $1-1.5$ year follow-up, rate of SUI was $2.6 \%$ in frailty $0-1$ and $0 \%$ in $2-5(\mathrm{p}=1.0)$. As expected, there is a statistically significant difference between the age of the cohorts, grip strength, and ASA scores.

Conclusions: The data shows no significant difference between incontinence rates, specifically SUI rates, for those with increasing frailty scores. This suggests the more frail population does not appear to have increased risk for SUI as compared to the general population. Due to small sample size of higher risk patients, more studies need to be conducted to further elucidate this outcome.

MP09-11 Robot-assisted Total Prostatectomy: A New Technique for the Management of Benign Prostatic Hyperplasia

\section{R Pathak, A Hemal}

\section{Wake Forest University}

Introduction \& Objective: Simple prostatectomy, by definition, involves removal of the obstructive adenoma component by finger via the open method or use of laparoscopic or robotic instruments. Removal of just the obstructive adenoma leaves behind a significant portion of prostate tissue, up to 22 to $32 \%$ of the pre-operative TRUS volume. Sole removal of adenomatous tissue fails to account for the peripheral zone explaining the low detection of cancer in some series (4\%). Therefore, we introduce a novel technique (Robotic Total Prostatectomy, RTP) which involves removal of the totality of prostate tissue including the transition, central and peripheral zones while sparing the pseudocapsule, seminal vesicles and vas deferens. Our main objective is to validate RTP as a safe, efficacious and durable treatment for $\mathrm{BPH}$ and compare it to an institutional series of robotic simple prostatectomy (RSP).

Methods: After obtaining IRB approval (IRB 00049371) a total of 65 patients with a prior negative work-up for elevated PSA underwent robotic prostatectomy for the management of advanced BPH. Of these 65 patients, 21 underwent RTP. Indications for surgery included the typical indications for simple prostatectomy, namely obstructive LUTS and elevated prostate gland volume. Primary endpoints of resection volume, postoperative PSA and incidental cancer detection rate was examined. Resection efficiency score (RES) was calculated by measuring resected weight/pre-operative TRUS/MRI volume. Patients were followed for at least three months post-operatively and preoperative, perioperative and postoperative outcomes were studied. Results: A total of 21 and 44 patients underwent RTP and RSP, respectively. Patients were comparable to several preoperative variables including BMI, ASA, IPSS score, SHIM score, postvoid residual (PVR), TRUS-volume, and PSA. RTP patients were significantly older than RSP (74.0 vs. $69.4, p=0.0019)$. OR time, LOS and EBL were similar. RES for RSP and RTP was $67 \%$ and $92 \%$, respectively. Presence of aberrant pathology was statistically significant in favor of RTP at $p=.05$. Post-operative variables such as SHIM, IPSS, Qmax, and PVR were comparable. Postoperative PSA was significantly lower in the RTP cohort (0.09 vs. 0.65)

Conclusions: By resecting the totality of prostate tissue, the incidental cancer detection rate may increase. Moreover, the 3month PSA difference among both cohorts (.09 vs .65) further validates our notion of the removal of the totality of prostate 
tissue. Lastly, our RTP RES further validates our ability for maximal resection of prostate tissue without compromise to functional outcomes.

\section{MP09-12 Ejaculation Sparing Thulium Laser En- ucleation of the Prostate (ES-ThuLEP): Outcomes on a large cohort}

G Bozzini, U Besana, A Calori, A Govorov, G Pini, C Buizza, M Sighinolfi, B Rocco

ASST Valle Olona - Busto Arsizio VA

Introduction \& Objective: To assess the effect of ES-ThuLEP on sexual function in a group of patients with LUTS secondary to BPH. To evaluate how surgical technique in ES-ThuLEP can lead to ejaculatory function sparing.

Methods: Prospective study that analyzes changes in sexual function and urinary symptoms in a group of 283 patients that underwent ES-ThuLEP. To assess the changes on erection and ejaculation, urinary symptoms and their interference on quality of life, two validated questionnaires were used: ICIQMLUTSsex and MSHQ-EjD. Patients were evaluated before surgery and 3 and 6 months after ES-ThuLEP. Patients with previous prostatic surgery and evidence of ED were excluded. Statistical analysis was performed by the Student $t$ and chisquare Test and logistic regression analysis. For all statistical comparisons significance was considered at $\mathrm{p}<0.05$.

Results: Mean age was 64.21 years. Erectile function was conserved in $95.05 \%$ and $100 \%$ of the pts at three and six months respectively. Ejaculatory function was spared in 203 pts $(71.73 \%)$ at three months and in 219 pts $(77.38 \%)$ at six. No significant differences were observed about erectile function before and after surgery. The ES-THULEP surgical technique do not lead to any obstructive LUTS in the follow up period.

Conclusions: ES-ThuLEP can spare ejaculatory function in a selected cohort of patients with no effects on disobstruction. Although endoscopic management of BPH (TURP and new technologies) causes retrograde ejaculation, ES-ThuLEP can offer a postoperative conserved ejaculation in the majority of cases.

\section{MP09-13 Initial experience with intraoperative fluid warming during Holmium Laser Enucleation of the Prostate (HoLEP)}

T Large, CU Nottingham, A Krambeck

Introduction \& Objective: Core body temperature is a tightly controlled process. Maintaining normothermia throughout surgical procedures and during the perioperative period is critical, as prior research has linked perioperative hypothermia to significant complications including, cardiovascular events (bradycardia, myocardial ischemia), protein catabolism and infection related complications. The FluidSmartTM System by Thermedx (Solon, OH) is a fluid management system that allows for warmed, pressurized (40-250 $\mathrm{mm} \mathrm{Hg}$ ) saline to circulate through the urinary system during endourological procedures. We sought to determine if the FluidSmart system resulted in higher core body temperatures compared to standard room temperature saline after HoLEP.

Methods: This was a prospective randomized trial comparing outcomes including intra and postoperative body temperature,

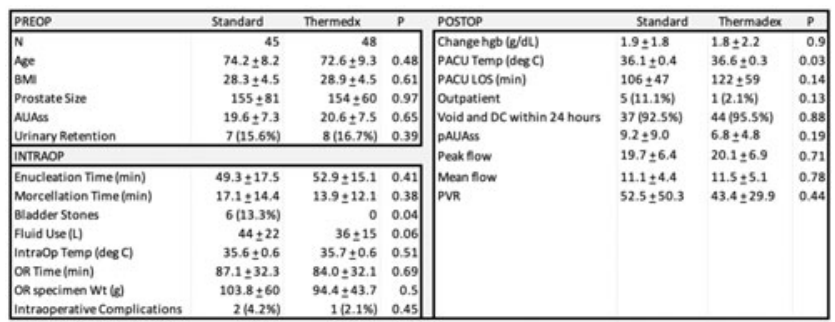

hospital stay and complications after HoLEP using standard fluid irrigation and the FluidSmartTM system. Men with LUTS/BPH and $100 \mathrm{gm}$ prostate or larger based on TRUS, CT, or MRI scan were enrolled in the study. The room was kept at 69 degrees $F$ for all cases and an upper body Bair hugger was utilized. Single tail t-tests were used to compare outcomes.

Results: 93 patients, 45 standard and 48 FluidSmartTM underwent HoLEP (table 1). Core body temperature was higher after surgery in FluidSmartTM patients (36.6 vs $36.1 \mathrm{p}=0.03$ ). The post anesthesia care unit length of stay times were similar between groups. Complications were all clavian II or less $(3.2 \%)$ with 1 patient in the FluidSmartTM identified as having a bladder perforation from over pressurizing the bladder. Additionally, 1 patient in each group had clot urinary retention that required readmission and foley catheter irrigation.

Conclusions: The FluidSmart system does provide continuous warmed pressurized fluid safely during HoLEP. Patients who underwent a FluidSmartTM HoLEP had higher core body temperatures than standard HoLEP; however, this finding has limited clinical significance emphasized by the similar PACU stay time.

\section{MP09-14 Safety of Holmium Laser Enucleation of the Prostate in the Outpatient Setting}

D Agarwal, K Hebert, ME Rivera

Indiana University

Introduction \& Objective: Limited data is available assessing holmium laser enucleation of prostate (HoLEP) performed in the outpatient setting. We performed a retrospective review of our outpatient HoLEP experience to assess feasibility of the approach. We retrospectively reviewed single surgeon data of men undergoing HoLEP in the outpatient setting between July 2017 and March 2018 at Mayo Clinic (Rochester, MN). Detailed preoperative, perioperative, and postoperative information was obtained. Statistical analysis was performed to evaluate for significant associations in men discharged the same day versus men admitted for overnight observation.

Methods: We retrospectively reviewed single surgeon data of men undergoing HoLEP in the outpatient setting between July 2017 and March 2018 at Mayo Clinic (Rochester, MN). Detailed preoperative, perioperative, and postoperative information was obtained. Statistical analysis was performed to evaluate for significant associations in men discharged the same day versus men admitted for overnight observation.

Results: A total of 52 men (median age 69.1 years) underwent HoLEP with plan for same-day dismissal. Median prostate size was $58.5 \mathrm{~cm}^{3}$ (IQR 41-82.3), morcellated tissue volume $36.4 \mathrm{~cm}^{3}$, time on CBI 212 minutes (IQR 173-353), and total postoperative time prior to discharge 316 minutes (IQR 243-414). At 3-month follow-up, average improvement in flow rate was $12.4 \mathrm{ml} / \mathrm{s}$ and reduction in AUA symptom score was 13 points. No 
complications were noted however, four patients experienced postoperative retention requiring catheterization between 2-11 days. Only $5(9.6 \%)$ patients required overnight admission for prolonged CBI. On univariate analysis, no statistically significant associations were noted between men discharged same day versus men admitted for overnight observation. There was a nonstatistically significant association between CBI time compared to prostate size $(\mathrm{p}=0.067)$.

Conclusions: Appropriately selected patients can undergo HoLEP in the outpatient setting. Further data is needed to assess maximum size that can be safely performed as an outpatient.

\section{MP09-15 Withdrawn}

\section{MP09-16 Convective water vapor thermal prostate ablation: experience at a tertiary center}

G Greear, T DiPina, K Kader, T Hsieh, J Buckley, J Woo, $\mathrm{S}$ Bechis

UC San Diego

Introduction \& Objective: Convective water vapor energy ablation, i.e. Rezūm, is a new minimally invasive treatment for benign prostatic hyperplasia (BPH). We present initial results from a tertiary academic center.

Methods: A retrospective review of 83 consecutive patients treated with Rezūm by 5 multi-specialty urologists from August 2017 to July 2018 was conducted. Patient demographics, prior medical treatment, international prostate symptoms scores (IPSS), maximum flow rates (Qmax), post-void residual (PVR) and adverse events were evaluated. Data were analyzed at baseline, 0-1, 1-3, 3-6 and 6-12 months after treatment. All procedures were performed under anesthesia as outpatient surgery. Descriptive and comparative statistics were performed.

Results: Patient characteristics are presented in Table 1. The mean number of treatments delivered was 4.2. A median lobe was noted in 50 men $(60 \%)$ and was treated in 37 men $(74 \%)$. Mean post-operative catheter duration was 4.3 days. Mean follow-up was 4 months. Maximal benefit was noted at the 3-6 months interval with IPSS improved from baseline 20.5 to 10.3 and QoL from 3.9 to 2.1 (Table 2). Median (mean) Qmax increased by $2.5(2.8) \mathrm{cc} / \mathrm{s}(\mathrm{p}=0.07)$ and PVR decreased by $0(42)$ $\mathrm{cc}(\mathrm{p}=0.23)$ at a median interval of 2 months post-op. Two $(2 / 5)$ patients in retention pre-operatively were able to resume spontaneous voiding. Rates of adverse events included: UTI 6 (7\%),

\begin{tabular}{|l|l|l|}
\hline $\begin{array}{l}\text { Table 1. Baseline } \\
\text { characteristics, } n=83\end{array}$ & $\begin{array}{l}\text { Mean ( } \pm \text { Std } \\
\text { Dev) } \\
\text { Median (range) }\end{array}$ & $\mathbf{n}(\%)$ \\
\hline Age, years & $\begin{array}{l}67.3(9.1) \\
67.0(48-94)\end{array}$ & $83(100)$ \\
\hline ASA status & 1 & \\
& 2 & $5(6)$ \\
& 3 & $43(52)$ \\
& 4 & $34(41)$ \\
\hline Prostate cancer & & $1(1)$ \\
\hline Pre-op alpha blocker & & $8(10)$ \\
\hline Pre-op 5-AR inhibitor & & $62(75)$ \\
\hline Prior BPH procedure & & $26(31)$ \\
\hline $\begin{array}{l}\text { Catheter dependent } \\
\text { Preop }\end{array}$ & & $7(8)$ \\
\hline PSA, ng/dL & $2.89(2.83)$ & $62(75)$ \\
\hline Prostate volume (cc) & $1.93(0.01-10.6)$ & $63(21)$ \\
\hline & $50(14-115)$ & $60(72)$ \\
\hline
\end{tabular}

\begin{tabular}{|c|c|c|c|c|c|c|}
\hline $\begin{array}{l}\text { Time after } \\
\text { surgery }\end{array}$ & $\begin{array}{l}\text { Baseline } \\
\text { (preop) }\end{array}$ & $0-1$ month & $\begin{array}{l}1-3 \\
\text { months }\end{array}$ & $\begin{array}{l}3-6 \\
\text { months }\end{array}$ & $\begin{array}{l}\text { 6-12 } \\
\text { months }\end{array}$ & $\begin{array}{l}\text { Last } \\
\text { recorded }\end{array}$ \\
\hline \multicolumn{7}{|l|}{ IPSS } \\
\hline $\begin{array}{l}\mathrm{n} \text { (paired } \\
\text { subjects) }\end{array}$ & 66 & 15 & 35 & 23 & 7 & 49 \\
\hline Baseline ( $\pm S D)$ & $20.1(7.0)$ & $20.7(6.3)$ & $20.1(6.6)$ & $20.5(7.0)$ & $19.4(7.8)$ & $19.9(7.3)$ \\
\hline $\begin{array}{r}\text { Mean change } \\
( \pm \mathrm{SD})\end{array}$ & & $-2.4(6.5)$ & $-9.3(7.3)$ & $\begin{array}{l}-10.2 \\
(9.2)\end{array}$ & $-7.1(12.1)$ & $-9.4(9.1)$ \\
\hline$\%$ change & & $-11.6 \%$ & $-46.2 \%$ & $-49.6 \%$ & $-36.4 \%$ & $-88.8 \%$ \\
\hline $\begin{array}{r}p \text { value (paired } \\
\text { t-test) }\end{array}$ & & 0.18 & $<0.01$ & $<0.01$ & 0.17 & $<0.01$ \\
\hline \multicolumn{7}{|l|}{ QoL } \\
\hline $\begin{array}{l}\mathrm{n} \text { (paired } \\
\text { subjects) }\end{array}$ & 63 & 15 & 33 & 22 & 7 & 46 \\
\hline Baseline $( \pm S D)$ & $3.8(1.2)$ & $3.7(1.0)$ & $3.9(1.2)$ & $3.9(1.2)$ & $4.0(1.3)$ & $3.8(1.2)$ \\
\hline $\begin{array}{r}\text { Mean change } \\
( \pm \mathrm{SD})\end{array}$ & & $-0.7(1.1)$ & $-1.9(1.4)$ & $-1.8(1.5)$ & $-1.8(2.5)$ & $-1.7(1.7)$ \\
\hline$\%$ change & & $-17.9 \%$ & $-48.4 \%$ & $-45.3 \%$ & $-44.6 \%$ & $-83.3 \%$ \\
\hline $\begin{array}{r}p \text { value (paired } \\
\text { t-test) }\end{array}$ & & 0.03 & $<0.01$ & $<0.01$ & 0.11 & $<0.01$ \\
\hline
\end{tabular}

urinary retention requiring catheter reinsertion $12(15 \%)$, and persistent dysuria requiring adjunct therapy $>30$ days 9 (11\%). The number of ER visits within 30 days was 16 (19\%) and was due to urinary retention in the majority of cases. Post-operative urinary retention after initial void trial was associated with a higher baseline PSA (5.85 vs $2.39 \mathrm{ng} / \mathrm{dL}, \mathrm{p}=0.03$ ) but did not correlate with prostate size $(50.7$ vs $61.1 \mathrm{cc}, \mathrm{p}=0.14)$. No covariates were associated with these adverse events on multivariate analysis.

Conclusions: Rezūm improves lower urinary tract symptoms and yields satisfactory short-term outcomes in the initial experience of a tertiary academic center.

\section{MP09-17 Perioperative Outcomes of Bladder Neck Sparing Robot-assisted Simple Prostatectomy}

M Shahait, K Patel, J Kim, DI Lee

University of Pennsylvania

Introduction \& Objective: To present perioperative and functional outcomes of Bladder Neck Sparing Robot-assisted Simple Prostatectomy.

Methods: Between March 2015 and December 2018, a total of 30 consecutive non-randomized patients with BPH underwent bladder neck sparring RASP. This technique includes the following: preserving the pubo-prostatic ligament as well as DVC complex, bladder neck sparing, and circumferential vesicourethral anastomosis.

Results: The median age was 66.5 (59.3-72.3), and median body mass index was $27.6(24.5-72.3) \mathrm{kg} / \mathrm{m} 2$. The median preoperative IPSS was 23 (17.5-27), and median prostate size was 97 (74-148.75) ml; The mean (SD) operative time was 107.5 (22.2) minutes, and the mean (SD) estimated blood loss was 132.4(35.4) $\mathrm{ml}$. All cases were completed robotically, no intraoperative complications were encountered, continuous bladder irrigation was not needed in any patient; all the patients were discharged with 24 hours of the procedure. All the patients were continent ( 0 pad), and able to void after catheter removal except one patient with underlying neurogenic bladder who resumed clean intermittent catheterization. Of the 19 patients who had antegrade ejaculation before surgery, 8 patients (42\%) reported of enjoying antegrade ejaculation after surgery. No patient required additional surgical nor medical treatment during the follow-up, and no bladder neck contracture or urethral stricture were developed in these patients. The median follow-up of the cohort was 16.5 (9.5-24.8) months. 
Conclusions: We demonstrate a simplified approach to bladder neck sparring RASP that is reproducible with a short learning curve, favorable perioperative and intermediate functional outcomes. This technique obviates the need for continuous bladder irrigation as well as intraperitoneal drain.

MP09-18 Randomized Comparison of Moses Enucleation of the Prostate to Standard Holmium Enucleation of the Prostate (HoLEP)

T Large, CU Nottingham, J Williams, A Krambeck

Introduction \& Objective: We compared outcomes for patients undergoing Holmium Laser Enucleation of the Prostate (HoLEP) for lower urinary tract symptoms secondary to benign prostate hyperplasia (LUTS/BPH) using 3 different laser fibers and two different laser energy modes.

Methods: This is a retrospective review of a clinic registry of men with LUTS/BPH who underwent HoLEP between August 2018 and January 2019. Patients were assigned to group 1 (50 patients), group 2 (50 patients) and group 3 (52 patients) based on their HoLEP being completed with a Slimline $550 \mu \mathrm{m}$, Slimline $1000 \mu \mathrm{m}$, or MOSES ${ }^{\circledR} 550 \mu \mathrm{m}$ laser, respectively. The groups were compared using SSPS for ANOVA comparison of means and multivariate logistic regression.

Results: There were no differences between the groups in age, comorbidities, American Society of Anesthesia (ASA) classification, BPH medication or antiplatelet/anticoagulation (AP/AT) status between the groups. There were 10 patients who underwent concomitant stone surgery (2 PCNL, 8 URS, 3 bilateral cases) and 11 patients had bladder stones removed; ancillary procedures did not significantly differ between groups (group 1 $5(10 \%)$, group $2-5(10 \%)$, group $3-1(1.9 \%), p=0.2)$. Prostate enucleation times differed significantly $(22.5+7.3,16.4+6.9$, $18.1+8.6 \min \mathrm{p}=<0.001$ ) between the groups; however, statistical significance was lost once enucleation time was indexed against enucleated tissue weight. Time to achieve hemostasis (min) was statistically different between the different groups $(10.6+6.1,7.7+5.2,6.3+4.8 \mathrm{p}<0.001)$. This difference in hemostasis time was maintained on multi-logistic regression which was independent of patient age, presence of foley catheter and AP/AT status. Time to achieve homstasis was less affected by increasing gland size (fig 1) and was associated with a 3.9-minute decrease for Moses compared to the Slimline 550 HoLEP $(\mathrm{p}<0.001)$.

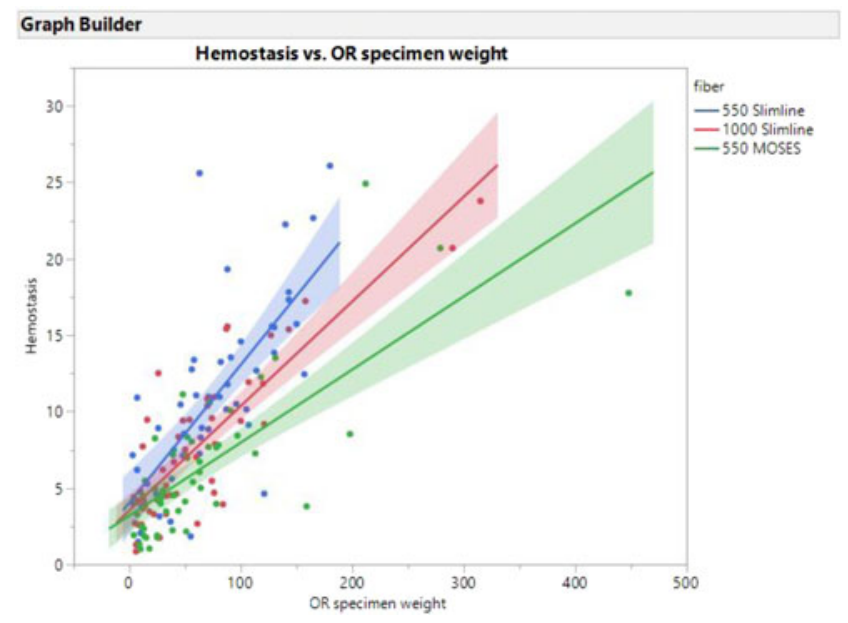

\begin{tabular}{|c|c|c|c|c|}
\hline Table 1 & Slimline 550 & Slimline 1000 & MOSES 550 & $\mathbf{P}$ \\
\hline & 50 & 50 & 52 & 0.6 \\
\hline Age & $71 \pm 8.2$ & $70.3 \pm 8.8$ & $72 \pm 9.0$ & 0.9 \\
\hline BMI & $27.7 \pm 5.3$ & $27.9 \pm 4.3$ & $28 \pm 5.9$ & 0.4 \\
\hline Prostate Size & $110.5 \pm 85.5$ & $118.3 \pm 92.4$ & $155.6 \pm 5.3$ & 0.5 \\
\hline Urinary Retention & $22(44 \%)$ & $19(38 \%)$ & $16(31 \%)$ & 0.4 \\
\hline Hx or Urinary Tract Infections & $5(10 \%)$ & $4(8 \%)$ & $4(8 \%)$ & 0.9 \\
\hline Diabetes Mellitus & $7(14 \%)$ & $4(8 \%)$ & $9(17 \%)$ & 0.4 \\
\hline ASA Score & 0 & $1(2 \%)$ & 0 & \\
\hline " & $17(34 \%)$ & $8(16 \%)$ & $9(17 \%)$ & \\
\hline III & $33(66 \%)$ & $39(78 \%)$ & $41(79 \%)$ & \\
\hline IV & 0 & $2(4 \%)$ & $2(4 \%)$ & 0.2 \\
\hline Antiplatelets/Anticoagulants & $15(30 \%)$ & $12(24 \%)$ & $15(29 \%)$ & 0.8 \\
\hline Discontinued/Bridged/Continued & $9 / 4 / 2$ & $10 / 1 / 1$ & $9 / 5 / 2$ & \\
\hline Concomitant Stone Surgery & $4(8 \%)$ & $5(10 \%)$ & $1(1.9 \%)$ & 0.4 \\
\hline Concomitant Cystolitholopaxy & $5(10 \%)$ & $5(10 \%)$ & $1(1.9 \%)$ & 0.2 \\
\hline Enucleation Time (min) & $47.1 \pm 17.9$ & $41.5 \pm 14.6$ & $39.9 \pm 15.1$ & 0.01 \\
\hline Laser Cutting Time (min) & $22.5 \pm 7.3$ & $16.4 \pm 6.9$ & $18.1 \pm 8.6$ & $<0.001$ \\
\hline Hemostasis Time (min) & $10.6 \pm 6.1$ & $7.7 \pm 5.2$ & $6.3 \pm 4.8$ & $<0.001$ \\
\hline Morcellation Time (min) & $11.6 \pm 10.9$ & $10.2 \pm 13.5$ & $10.3 \pm 13.4$ & 0.7 \\
\hline Ave [range] OR specimen Wt (g) & $72.5[3-265]$ & $65.9[4-315]$ & $76.7[4-448]$ & 0.7 \\
\hline Change in $\mathrm{Hgb}(\mathrm{mg} / \mathrm{dL})$ & $1.5+1.2$ & $1.5+1.6$ & $1.0+1.1$ & 0.02 \\
\hline Same day discharge & 0 & 4 & 3 & 0.02 \\
\hline Void and Discharge within 24 hours & $46(92 \%)$ & $41(89 \%)$ & $45(92 \%)$ & 0.7 \\
\hline
\end{tabular}

Conclusions: Our findings suggest that modulated pulsed laser energy, such as MOSES, can improve hemostasis during the enucleation phase of a HoLEP resulting in shorter procedure times.

\section{MP09-19 Long-Term Functional Outcomes of Robot- Assisted Simple Prostatectomy in Patients with $>3$ Year Follow-Up}

\section{Z Lee, A Keehn, AM Asghar, D Strauss, M Lee, DD Eun}

Temple University

Introduction \& Objective: Although open simple prostatectomy is the gold standard surgical treatment for men with lower urinary tract symptoms secondary to a large prostate, the robotic approach has been increasingly utilized. Compared to the open approach, robot-assisted simple prostatectomy (RASP) has been associated with reduced blood loss, shorter length of stay, and similar functional outcomes. Despite this, reported functional outcomes associated with RASP have been limited by short-term follow-up. We report our long-term functional outcomes of RASP in patients with at least 3-year follow-up.

Methods: We retrospectively reviewed our institutional RASP database to identify all patients who underwent the procedure between May 2013 and May 2016 with at least 3-year follow-up. The indication for RASP was bothersome lower urinary tract symptoms refractory to medical management and a prostate volume ${ }^{3} 80$ milliliters. Our technique involved a transvesical enucleation of the prostate adenoma followed by a 360-degree circumferential urethrovesical anastomosis. All Foley catheters were removed within 10 days postoperatively. The severity of lower urinary tract symptoms was assessed in the pre- and postoperative setting using the American Urological Association Symptom Score (AUASS) score. Mann-Whitney U test was used to compare pre- and post-operative AUASS scores.

Results: There were 34 patients included in our analysis and the median preoperative estimated prostate size was 133 milliliters (IQR 92-151). Preoperatively, the median AUASS was 18 (IQR 15-24). The median operative time was 165 minutes (IQR 117188), estimated blood loss was 213 milliliters (IQR 112-438), and there were no intraoperative complications. The median length of stay was 1 day (IQR 1-1). There were 2/34 (5.9\%) major postoperative complications (Clavien $>2$ ). Two-weeks postoperatively, the median AUASS was $6(\mathrm{IQR} 4-7)(\mathrm{p}<0.001)$ and none of the patients developed urinary incontinence. 
Although 26/34 (76.5\%) patients were Foley dependent preoperatively, all patients were catheter-free 2 weeks after RASP. At a median follow-up of 48 (IQR 39-53) months, the median AUASS was $2($ IQR 2-5) $(\mathrm{p}<0.001)$ and none of the patients had urinary incontinence.

Conclusions: RASP is an effective and durable treatment option for men with bothersome lower urinary tract symptoms and a large prostate. Men can expect their improvement in urinary function after RASP to remain stable for $>3$ years postoperatively.

MP09-20 Predicting outcomes of robotic assisted simple prostatectomy in patients with large prostate volumes

RL Steinberg, H Trivedi, BA Johnson, A Garbens, C Roehrborn, JC Gahan

Introduction \& Objective: Robotic assisted simple prostatectomy (RASP) has shown to be effective and safe in treating large prostate glands. Though, little data exists regarding the predictive nature of pre-operative patient or disease-related characteristics on the improvement in patient symptoms. Our objective was to assess which pre-operative variables can predict improvement in IPSS after RASP.

Methods: A retrospective, single institution review was performed of 231 RASP procedures performed by 2 surgeons from December 2014 to October 2018. Only patients with prostate glands measured to be greater than 80 grams and IPSS scores both pre- and post-surgery were included for analysis. Baseline patient demographics, pre-operative (pre-op) functional data (maximum flow rate (Qmax), post void residual (PVR), IPSS) and post-operative data were collected. Linear regression analysis was performed using SPSS (IBM Corp, Armonk, NY).

Results: After exclusions, 145 men (mean age 69.2 \pm 7.3 years) with mean prostate volume of $130 \pm 42 \mathrm{~g}$ and mean PSA $6.7 \pm 4.7 \mathrm{ng} / \mathrm{dL}$ were included in the analysis. Simple regression identified older age $(\mathrm{p}=0.014)$, higher ASA score (score 3 vs. 2 , $\mathrm{p}=0.01)$, and greater pre-op $\mathrm{Qmax}(\mathrm{p}=0.008)$ as predictive of worse IPSS improvement (Table 1). Specifically, a 2 point increase in pre-op Qmax predicted a 1 point decrease in IPSS improvement. Conclusions: In men with $>80$ gram prostates undergoing RASP, older age, ASA status of 3 and a higher pre-op Qmax predicted worse IPSS improvement after surgery. This may be useful in counselling patients prior to RASP.

Table 1. Simple linear regression of functional predictors for change in IPSS after RASP

\begin{tabular}{lcccc}
\hline & \multicolumn{2}{c}{ Simple } & \multicolumn{2}{c}{$95 \% \mathrm{Cl}$} \\
Predictive Factors & B & p-value & Lower & Upper \\
\hline Age & 0.287 & 0.014 & 0.058 & 0.517 \\
BMI & -0.018 & 0.927 & -0.409 & 0.373 \\
ASA & 4.923 & 0.010 & 1.192 & 8.655 \\
Baseline PSA & -0.194 & 0.318 & -0.577 & 0.189 \\
Prostate Volume & 0.015 & 0.471 & -0.026 & 0.056 \\
Pre-op Qmax & 0.527 & 0.008 & 0.139 & 0.916 \\
Pre-op PVR & 0.000 & 0.966 & -0.009 & 0.009 \\
\hline
\end{tabular}

MP09-21 HoLEP learning curve - is there a plateau after 50 cases?

A Coscione, S Kampantais, D Cohen, K Gordon, P Acher

Southend University Hospital NHS Trust

Introduction \& Objective: The initial learning curve for HoLEP is reported at an average of 50 cases. However, there is a paucity of data on learning beyond this phase. We report the effect of operator experience beyond 50 cases.

Methods: A single center UK surgeon series of consecutive HoLEPs from May-2012 to August-2017 was analyzed. Patients were excluded if they had previous TURP or limited resection. Primary outcomes were Enucleation-Efficiency (EE) (tissue weight/enucleation time) and Laser to Prostate Ratio (L/PR) (laser energy/enucleation weight). Secondary outcomes were PSA analysis and length of hospital stay.

Results: 323 cases were identified. The first 50 were excluded to account for the initial learning curve and a further 7 for meeting exclusion criteria leaving 276 cases. Cases were divided into 5 cohorts (Group A: 1-50, Group B:51-100, Group C: 101-150, Group D:151-200 and Group E 201-276). Mean patient age was $70.41( \pm 8.43)$. As the series progressed there was a statistically significant improvement in both median EE: $0.95 \mathrm{~g} / \mathrm{min}$ Group A - $1.45 \mathrm{~g} / \mathrm{min}$ Group E $(\mathrm{p}=0.001)$, and median L/PR: $3.79 \mathrm{~kJ} / \mathrm{g}$ Group A - 1.87kJ/g Group E $(\mathrm{p}=0.016)$. There was improvement in Efficiency of $0.125 \mathrm{~g} / \mathrm{min}$ per 50 cases and L/P-ratio of $0.48 \mathrm{~kJ} / \mathrm{g}$ per 50 cases. There was no plateauing in either EE or $\mathrm{L} / \mathrm{P}$ ratio in the series. No statistical difference was found in secondary outcomes.

Conclusions: Our study suggests that performance continues to improve beyond 50 cases and did not plateau. The reported learning curve may be an underestimate which may have implications for performance, mentoring programs and theatre utilization.

MP09-22 Comparison of Different Types of Catheters for Intermittent Self-Catheterization: A Systematic Review and Network Meta-analysis

D Ye, Y Chen, Z Jian, H Li, K Wang

West China Hospital

Introduction \& Objective: Intermittent catheterization (IC) is considered the standard treatment for neuro-urological patients who cannot empty their bladders. Several catheter designs and techniques are evolved to decrease the risk of complications, but which type of catheter is best is still under controversial. We aim to conduct a systematic evaluation and network meta-analysis of different types of catheter for IC.

Methods: We searched MEDLINE, Cochrane Central Register of Controlled Trials, and Web of Science databases to identify relevant studies published up to September 2018. Search terms used were intermittent catheterization, neurogenic, urinary catheters, and urethral catheterization in several logical combinations. Randomized clinical trials or randomized cross-over trials comparing at least 2 of the following types of catheters were selected: sterile non-coated, gel-lubricated sterile noncoated, hydrophilic-coated, pre-activated hydrophilic-coated and clean non-coated catheter. Outcomes were the incidence of asymptomatic bacteriuria, symptomatic UTI and hematuria. A Bayesian network meta-analysis was performed.

Results: A total of 13 RCTs and 6 randomized crossover trials with 851 participants were included in this meta-analysis. Gellubricated sterile non-coated catheter had the highest SUCRA on asymptomatic bacteriuria (84.2\%) and symptomatic UTI (51.4\%). For hematuria, there was a $73.2 \%$ chance that hydrophilic-coated catheter was the preferred option. The pooled odds ratio for the incidence of symptomatic UTI of two ready-to-use design catheters (gel-lubricated and pre-activated hydrophilic-coated catheter) compared to sterile non-coated catheter was 0.36 (95\% CI $0.13-$ 
$0.94)$ and 0.40 (95\% CI 0.19-0.85), respectively. It was not able to demonstrate any statistically significant difference between sterile and clean non-coated catheters.

Conclusions: Gel-lubricated non-coated catheter is likely to be the preferred option to decrease the risk of UTI for IC. The readyto-use technique shows advantages compared to techniques that allow patients to lubricate catheter or activate hydrophilic coating by themselves. Considering both safety and economic factors, there is still no convincing conclusion as to whether the sterile or clean catheter is better. More well-designed trials with strict definition of outcomes are needed, preferably involving cost-effectiveness analysis.

\section{MODERATED POSTER SESSION 10: NEW TECHNOLOGY: STONES}

\author{
MP10-01 Cascade of Allium ureteral stent for ureteral \\ stenosis after radiotherapy for gynecologic cancer \\ W Qi \\ Peking university People's Hospital
}

Introduction \& Objective: Ureteral stricture is a wellrecognized adverse side effect after surgery or radiation therapy for gynecologic tumors. Endoscopically the ureters are often not just intrinsically narrow but also have completely lost their motility and thus propulsive function resulting in obstructive hydronephrosis. We evaluate the feasibility and effectiveness of a self-expanding, large caliber ureteral stent (URS) (Allium, ${ }^{\circledR}$ Allium LTD, Caesarea, Israel), in patients with ureteral stenosis after radiotherapy for gynecologic tumors.

Methods: Between August 2018 and April 2019, we placed Allium stents in 10 patients for ureteral stricture with history of radiotherapy for gynecologic cancer. The ureteral stenosis of 5 patients were bilateral and the others were unilateral. The basic demographic, clinical, laboratory, and radiologic data were reviewed. The Allium URS in our center comes in calibers $(30 \mathrm{~F})$ and in lengths $(12 \mathrm{~cm})$. The ureters were contrasted antegradely via the nephrostomy tube or retrogradely by a ureteral catheter with iodine injected under fluoroscopic control showing the site of narrowing. If the length of stricture was longer than the stent $(>12 \mathrm{~cm})$, another Allium stent was inserted overlapping with the first one in the way of cascade. We used fluoroscopy to show the vanish of obstruction or not. We also used the sonography, serum creatinine, and radionuclide renal dynamic imaging to look for changes in renal function. Primary patency was defined as a complete resolution or downgrading of hydronephrosis shown by imaging studies or success in the removal of a preexisting nephrostomy tube, otherwise the procedure was considered a primary failure.

Results: The length of stenosis in 11 ureters with 7 patients was found longer than $12 \mathrm{~cm}$ which need to be inserted 2 Allium stents in cascade. The other 4 ureters were inserted one Allium stent. All stents were inserted successfully and positioned correctly. The radiography in the surgery showed the patency of the ureters. During a mean follow-up time of 94 days (range 30-200 days), 4 patients with 7 ureteres were failed according to the definition, and they were all inserted the third Allium URS again. For the last follow up, all the patients including the ones undergoing the second surgery had patency ureteral stents. No stent migration or occlusion occurred. The renal function was improved in all patients.

Conclusions: Cascade of Allium ureteral stent was an effective way to treat the ureteral stenosis after radiotherapy for gynecologic cancer.

\section{MP10-02 The First UK experience of Super-Mini Percutaneous Nephrolithotomy}

H Abboudi, G Celentano, S Bilal, G Kazantis, F Schoofs, V Arumuham, S Choong

University College London Hospital

Introduction \& Objective: We report the first UK experience of the novel 14Fr super-mini PCNL (SMP) access sheath for the treatment of renal stones. The novelty relates to a combination of a small sheath and the ability to remove fragments using a combination of active (intermittent high pressure) and passive (continuous low pressure) suction.

Methods: Patients with renal stones not suitable for ESWL or retrograde intrarenal surgery (RIRS) and those with stones requiring a PCNL underwent SMP and data was collected retrospectively. Only patients who had completed a minimum of 3 months follow up were included. Ultrasound or CT-scan was performed 6-8 weeks post-operatively to determine stone-free status defined as no residual fragments of any size.

Results: Forty-six patients underwent SMP between January 2016 and December 2018. 76\% of patients (35/46) were totally tubeless. $24 \%$ of patients $(11 / 46)$ had tubes sited (six - nephrostomy only, two stents only, two nephrostomy and stent, one a ureteric catheter). Tubes were left in situ because of bleeding (6/ 11), anatomical variations such as PUJO, duplex systems and transplant kidneys (4/11) or risk of residual fragments (1/11). $61 \%$ of patients $(28 / 46)$ had $<24$ hours admission and the mean hospital stay was 2.5 days (median 1 day). The mean $\mathrm{Hb}$ drop was 14g/L

\begin{tabular}{|l|l|}
\hline Mean age & 51 years \\
\hline Mean BMI, kg/ m² & 28.1 \\
\hline Mean stone size & $\begin{array}{l}13.1 \mathrm{~mm} \text { (range } \\
6 \mathrm{~mm}-21 \mathrm{~mm})\end{array}$ \\
\hline $\begin{array}{l}\text { Mean stone } \\
\text { Hounsfield units }\end{array}$ & 1017 \\
\hline $\begin{array}{l}\text { Median operative } \\
\text { time, mins }\end{array}$ & $\begin{array}{l}46 \text { mins (range } \\
32 \text { mins-120 } \\
\text { mins) }\end{array}$ \\
\hline $\begin{array}{l}\text { Mean length of } \\
\text { hospital stay }\end{array}$ & 2.5 days \\
\hline Median length of stay & 1 day \\
\hline
\end{tabular}


and no patients required a blood transfusion. One patient $(2.2 \%)$ required an embolization procedure for an arteriovenous fistula. Stone free rate at three months was $82.6 \%(38 / 46)$.

Conclusions: Our early experience of SMP demonstrates that this technique is safe and effective in the treatment of stones. It is associated with a low level of blood loss, short operative times, minimal need for drainage tubes and short length of stay.

\section{MP10-03 Use of analgesia used for shockwave litho- tripsy (SWL): Twitter survey from EULIS/ESUT}

A Pietropaolo, K Sarica, A Skolarikos, E Liatsikos, BK Somani

Introduction \& Objective: Shock wave lithotripsy (SWL) is a recommended treatment option for ureteric and renal stones. However, there is no consensus on the type of analgesia used for this procedure. We wanted to do a twitter-based survey to find out the common analgesia used by urologists worldwide.

Methods: A twitter survey was set up with 4 commonly used treatment options and a free text box for other treatments offered by centers. Data was collated for analgesia used, total twitter visualizations, interactions and responses received.

Results: A total of 136 responses were received over a period of 6 days. However, the number of visualizations and interactions were 2676 and 167 respectively. A fifth of all responders did not use any analgesia with two thirds using either oral or rectal nonsteroidal medications ( $40 \%$ oral non-steroidal anti-inflammatory drugs and 24\% rectal Diclofenac). Opiates were used in 15\% of all responders. Oral or intravenous paracetamol was used in $6 \%$ patients either on its own or in combination with other analgesics.

Conclusions: There seems to be a wide variation in the use of analgesia offered for lithotripsy and no current protocol exists for this. It is debatable on whether this might have an effect on patient comfort, quality of life and treatment outcomes. There is a need for this issue to be addressed in the urolithiasis guidelines for a protocol based standardized analgesia for SWL.

\section{MP10-04 Initial Experience with Swiss Lithoclast Trilogy Demonstrates Reliable and Rapid Lithotripsy during Percutaneous Nephrolithotomy}

CU Nottingham, T Large, K Cobb, RL Sur, NE Canvasser, C Stoughton, A Krambeck

Indiana University School of Medicine

Introduction \& Objective: Several currently-available lithotrites have stone clearance rates of $24-32 \mathrm{~mm}^{2} / \mathrm{min}$. The objective of this study was to critically evaluate the initial experience with the Swiss Lithoclast Trilogy lithotrite during percutaneous nephrolithotomy (PCNL).

Methods: We prospectively enrolled patients at three locations (Indiana University, University of California Davis, and University of California San Diego) scheduled to undergo PCNL for nephrolithiasis. Patients had a minimum of $15 \mathrm{~mm}$ of stone in axial diameter. We assessed objective measures of stone clearance time, stone clearance rate, device malfunction, stone-free rate, and complications. Each surgeon also evaluated subjective parameters from each case related to use of Trilogy on a $1-10$ scale $(10=$ Extremely Effective) and compared it to their usual lithotrite on a $1-5$ scale $(5=$ much better $)$.

\begin{tabular}{|c|c|}
\hline Mean (SD) Age in Years on Date of Surgery & $59(18)$ \\
\hline Mean (SD) BMI & $32.3(10.8)$ \\
\hline Mean (SD) ASA Score & $2.8(0.8)$ \\
\hline Female Sex, n (\%) & $22(51.2)$ \\
\hline Left Sided Only, n (\%) & $25(58.1)$ \\
\hline Bilateral PNL, n (\%) & $7(16.3)$ \\
\hline Mean (SD) Maximal Stone Diameter in Millimeters & $22(12)$ \\
\hline Mean (SD) Stone Surface Area in Millimeters ${ }^{2}$ & $345(387)$ \\
\hline Mean (SD) Stone Hounsfield Units & $780(362)$ \\
\hline Mean (SD) Stone Clearance Time in Minutes & $9.3(10.1)$ \\
\hline Mean (SD) Stone Clearance Rate in Millimiters ${ }^{2} /$ Minute & $68.9(108.4)$ \\
\hline Mean (SD) Highest Ultrasound Setting & $92(15)$ \\
\hline Mean (SD) Highest Ballistic Setting & $6(3)$ \\
\hline Mean (SD) Highest Suction Setting & $55(11)$ \\
\hline $\begin{array}{l}\text { Mean (SD) Ergonomics Impression Score }(1-10,10=\text { Extremely } \\
\text { Comfortable) }\end{array}$ & $6.7(1.4)$ \\
\hline $\begin{array}{l}\text { Mean (SD) Impression of Ultrasound Effectiveness (1-10, } 10= \\
\text { Extremely Effective) }\end{array}$ & $7.3(1.7)$ \\
\hline $\begin{array}{l}\text { Mean (SD) Impression of Ballistic Effectiveness (1-10, } 10= \\
\text { Extremely Effective) }\end{array}$ & $8.1(1.4)$ \\
\hline $\begin{array}{l}\text { Mean (SD) Impression of Combination Effectiveness (1-10, } 10= \\
\text { Extremely Effective) }\end{array}$ & $8.7(1.2)$ \\
\hline $\begin{array}{l}\text { Mean (SD) Impression of Suction Effectiveness (1-10, } 10= \\
\text { Extremely Effective) }\end{array}$ & $8.4(1.0)$ \\
\hline $\begin{array}{l}\text { Mean (SD) Overall Impression of Trilogy Relative to Usual } \\
\text { Lithotriptor ( } 1-5,1=\text { much worse, } 2=\text { slightly worse, } 3=\text { the same, } \\
4=\text { slightly better, } 5=\text { much better) }\end{array}$ & $3.6(1.0)$ \\
\hline \multicolumn{2}{|l|}{ Device Malfunctions, $\mathrm{n}$ (\% of patients) } \\
\hline Hand Piece & 0 \\
\hline Probe & $1(2.3)$ \\
\hline Suction & $1(2.3)$ \\
\hline Other & 0 \\
\hline Need to Use Another Lithotrite, $n$ & 0 \\
\hline $\begin{array}{l}\text { Mean (SD) Impression of Ease of Set Up before Procedure (1-10, } \\
10=\text { Extremely Easy) }\end{array}$ & $8.3(1.4)$ \\
\hline $\begin{array}{l}\text { Mean (SD) Impression of Ease of Troubleshooting (1-10, } 10= \\
\text { Extremely Easy) }\end{array}$ & $8.6(1.1)$ \\
\hline $\begin{array}{l}\text { Mean (SD) Impression of Ease of Managing Settings (1-10, } 10= \\
\text { Extremely Easy) }\end{array}$ & $9.2(0.7)$ \\
\hline Patients with No Stones Present on POD1 Imaging, n (\%) & $24 / 29(82.8)$ \\
\hline Complications, n (\%) & \\
\hline Renal Pelvis Perforation & $1(2.3)$ \\
\hline Blood Transfusion & $1(2.3)$ \\
\hline Pneumothorax Requiring Chest Tube Placement & $1(2.3)$ \\
\hline Renal Artery Pseudoaneurysm & $1(2.3)$ \\
\hline
\end{tabular}

Results: All data is detailed in Table 1 . We included 43 patients and had 7 bilateral $(16.3 \%)$ cases, for a total of 50 renal units. One case was a mini-PCNL. Two cases experienced device malfunctions through which the team was able to troubleshoot, and no case required transition to another lithotrite. The mean stone clearance rate was $68.9 \mathrm{~mm}^{2} /$ minute. The stone-free rate on post-operative day 1 imaging was $82.3 \%$ ( 24 of 29 patients with available imaging). The lowest subjective rating was the ergonomics score of 6.7, and the highest subjective rating was the ease of managing settings score of 9.2. Importantly, the surgeon impressions of ultrasound (7.3), ballistic (8.1), combination of ultrasound and ballistic (8.7), and suction (8.4) were high. One patient experienced an intraoperative renal pelvis perforation, one patient required a blood transfusion, one patient had a pneumothorax requiring chest tube placement, and one patient had a renal artery pseudoaneurysm requiring endovascular embolization.

Conclusions: Trilogy offers a reliable and efficient lithotrite, with stone clearance rates over twice as fast as other commerciallyavailable devices.

\section{MP10-05 Comparison of Small Core Diameter Op- tical Laser Fibers Used for Holmium:YAG and Super Pulse Thulium Laser Lithotripsy}

\author{
BE Knudsen, WR Molina, BH Chew
}

The Ohio State University

Introduction \& Objective: Small core $(240$ - $300 \mu \mathrm{m})$ sized optical laser fibers are typically used for Holmium:YAG 
(Ho:YAG) laser lithotripsy. The introduction of the Super Pulse Thulium Fiber (SPTF) laser permits the use of smaller $150 \mu \mathrm{m}$ core sized fibers while still permitting high power output. This study evaluates the performance characteristics of a $150 \mu \mathrm{m}$ core sized fiber used with SPTF laser in comparison to a $242 \mu \mathrm{m}$ core sized fiber used for Ho:YAG laser lithotripsy.

Methods: Fiber flexibility, mechanical breakdown, and thermal breakdown testing were performed using a Boston Scientific Accumax 200 Ho:YAG fiber (Core diameter of $242 \mu \mathrm{m}$ ) and an Olympus 150 SPTF laser fiber (Core diameter $150 \mu \mathrm{m}$ ). For fiber flexibility, a "3-point bend test" was performed for each fiber size five times and the results recorded in N/mm. A "2 plate break test" was performed to measure the tightest diameter the fiber could be bent to before mechanical failure $(\mathrm{N}=5)$. For thermal breakdown testing, the fibers were bent $180^{\circ} \mathrm{C}$ at a diameter of $3 \mathrm{~cm}$ and the lasers activated at pulse energies of 0.2 , $0.5,1$, and $2 \mathrm{~J}$, all at $10 \mathrm{~Hz}$, in successive order for 15 seconds or until fiber failure. If the fiber did not fail, the bend diameter was reduced, and the testing repeated until failure or $0.2 \mathrm{~cm}$ reached. Bend diameters of $3,2,1,0.5,0.4,0.3$, and $0.2 \mathrm{~cm}$ were sequentially tested. Five trials for each fiber were performed.

Results: The Olympus 150 was less stiff than the Accumax 200 (Mean 0.055 vs $0.0139 \mathrm{~N} / \mathrm{mm}$ ). (Figure 1) The mean bend diameter before mechanical failure was $0.099 \mathrm{~mm}$ for the Olympus $150 \mu \mathrm{m}$ fiber and $1.74 \mathrm{~mm}$ for the Accumax 200. (Figure 2) For thermal breakdown testing, the Olympus $150 \mu \mathrm{m}$ was successfully bent to a diameter of $0.3 \mathrm{~cm}$ prior to failing in $3 / 5$ trials, with a bend diameter of $0.2 \mathrm{~cm}$ reached in $2 / 5$, at pulse energy of $0.2 \mathrm{~J}$. The Accumax 200 failed at bend diameters ranging from $0.4-$ $1.0 \mathrm{~cm}$ and pulse energies of $0.2-1.0 \mathrm{~J}$ (Table 1 ).

Conclusions: The Olympus 150 SPTF fiber has greater flexibility and is able to bend to a tighter diameter before thermal breakdown compared to the Accumax 200. This may provide clinical benefits during laser lithotripsy procedures, especially in the difficult to reach lower pole.
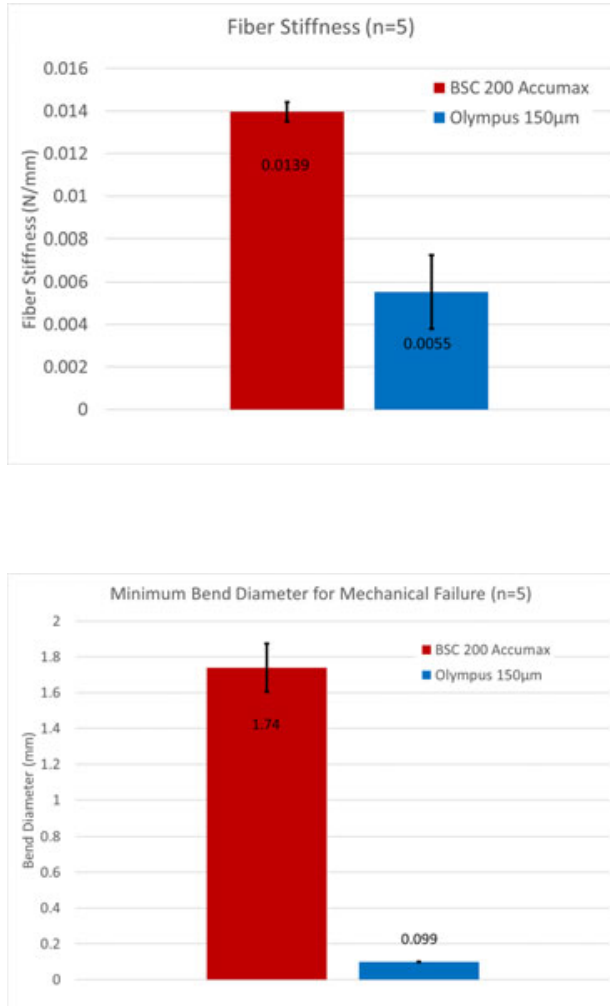

\begin{tabular}{|c|c|c|c|}
\hline Fiber Manufacturer & Trial $t$ & Pulse Energy Needed to Break Fiber ( $\mathrm{mJ}$ ) & Bend Diameter $(\mathrm{cm})$ \\
\hline BSC 200 Accumax & 1 & 200 & 0.4 \\
\hline BSC 200 Accumax & 2 & 1000 & 0.4 \\
\hline BSC 200 Accumax & 3 & 200 & 0.4 \\
\hline BSC 200 Accumax & 4 & 2000 & 1.0 \\
\hline BSC 200 Accumax & 5 & 200 & 0.5 \\
\hline 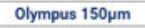 & 1 & 200 & 0.2 \\
\hline Olympus $150 \mu \mathrm{m}$ & 2 & 200 & 0.3 \\
\hline Olympus $150 \mu \mathrm{m}$ & 3 & 200 & 0.3 \\
\hline Olympus $150 \mu \mathrm{m}$ & 4 & 200 & 0.3 \\
\hline Olympus $150 \mu \mathrm{m}$ & 5 & 200 & 0.2 \\
\hline
\end{tabular}

MP10-06 Comparison of Stent-Related Symptoms Between Conventional Metal Stent and Allium Ureteral Stent

W Qi

Peking University People Hospital

Introduction \& Objective: Metal stents revolutionized the management of ureteral strictures but have significant morbidity. We compare stent-related symptoms and quality of life between conventional metal stent and Allium ureteral stent in patients with ureteral strictures.

Methods: Eighty patients with a conventional metal stent or an Allium stent for ureteral strictures were mailed a validated ureteral stent symptom questionnaire. We evaluated scores on the visual analog scale pain, the SF-36 (36-Item Short-Form Health Survey), the I-PSS (International Prostate Symptom Score) and the OABSS (Overactive Bladder Symptom Score) on postoperative days 3 and 14. Statistical analysis compared the differences in these parameters between the two groups.

Results: Overall the records of 80 patients were analyzed. Pain scores were significantly lower in the Allium stent placement group vs the conventional metal stent placement group (postoperative days 3 and $14,3.81$ vs $9.62, \mathrm{p}<0.05$, and 1.05 vs 8.20 , $\mathrm{p}<0.05$, respectively). The total I-PSS score (postoperative days 3 and $14,5.01$ vs $13.85, \mathrm{p}<0.05$, and 4.67 vs $15.10, \mathrm{p}<0.05$, respectively) and total analgesic use was significantly lower in the Allium group. The quality of life index and OABSS scores were significantly better in the Allium stent placement group than the metal stent placement group on postoperative days 3 and 14 (all $\mathrm{p}<0.05)$. There was no difference in the complication rate between the 2 groups.

Conclusions: Allium stent placement may be associated with less ureteral stent related discomfort than conventional stent placement.

MP10-07 Evaluation of a single use flexible cystoscope: a multi-institutional international study

K Scotland, ER Tawfiek, A Chiura, BH Chew, D Bagley

University of British Columbia

Introduction \& Objective: The introduction and widespread use of flexible cystoscopy in the developing world has been challenging. Acquisition costs and prohibitively expensive technical support for endoscope maintenance, repair and reprocessing have limited its use in developing nations. An additional challenge in areas with limited resources is a lack of appropriate space or funding for monitors and towers. This study evaluated the performance of a novel single use digital cystoscope which directly connects to a laptop or computer monitor with free uploaded software for visualization. 
Methods: The performance characteristics of the Neoscope flexible single use cystoscope was prospectively evaluated versus a reusable flexible cystoscope (Olympus CYF-VH) in three clinical cases and two independent benchtop testing episodes in Canada. Cystoscope maneuverability, imaging, deflection and ease of use of instrumentation were investigated. Additional investigations were performed during clinical use in Zimbabwe (7 patients), Egypt (10 patients) and Dominica (5 patients).

Results: Bench testing revealed average smaller tip diameter and shorter single use cystoscopes $(4.05 \mathrm{~mm}$ and $35.35 \mathrm{~cm})$ versus reusable cystoscopes $(6.09 \mathrm{~mm}$ and $38 \mathrm{~cm})$. Deflection of the single use scope was superior with an empty working channel (230 up/220 down) versus the reusable (195 up/95down) but was inferior on placement of instruments including a 365 um laser fibre ( 85 up/ 85 down versus 175 up/85 down). Clinical use revealed excellent maneuverability and ease of use of both instruments. Satisfactory deflection, visualization and image color fidelity were noted for single use scopes and light distribution was deemed good in 22/25 clinical cases with dark edges noted in $3 / 25$ cases.

Conclusions: Clinical and benchtop testing performance of the single use digital flexible cystoscopes were inferior compared to reusable digital cystoscopes. However, these single use endoscopes offer adequate illumination, imaging and maneuverability in patient procedures. Direct connection to any computer monitor allowed truly portable use, allowing for treatment of patients in a variety of clinical settings without the need for sophisticated equipment.

MP10-08 Initial human experience with a novel stone aspiration device used during ureteroscopic lithotripsy for renal stones

S Agrawal, A Singh, RL Sur, B Eisner, G Haleblian, G Preminger, A Ganpule, R Sabnis, M DESAI

Muljibhai Patel Urological Hospital, Nadiad Gujarat, India

Introduction \& Objective: Recent evidence suggests that basket extraction of stone fragments after ureteroscopic lithotripsy is time-consuming and may result in poorer-thanexpected stone free rates. Here in we report the first-in-human experience using a novel stone aspiration device designed to more efficiently and effectively remove stone fragments after ureteroscopy.

Methods: A randomized, controlled trial was performed comparing two techniques during ureteroscopy with laser lithotripsy group 1 underwent standard fragment extraction via basket retrieval and group 2 underwent stone fragment aspiration only with a novel device (K-VACÔ; Kalera Medical, San Diego, CA). Inclusion criteria was renal stones $5-15 \mathrm{~mm}$. Patients with multiple stones were included as long as the largest stone was $<15 \mathrm{~mm}$ in diameter. For group 1, stones were laser fragmented and then basket extraction was performed until surgeons felt the subject was stone free. For group 2, stones were laser fragmented and the ureteroscope replaced by K-VAC. K-VAC was passed systematically through all calyces of the kidney using fluoroscopic guidance, and stone fragments were aspirated for approximately 2 minutes in each area where stones were located. All procedures utilized a 12/14 Fr ureteral access sheath and also a ureteral stent at the end of the procedure. Non-contrast CT scan was performed post-operative day 1 and 30 . Stone free was defined as no stone fragments.
Results: Eight (8) subjects were randomized to the basket arm of the study and nine (9) subjects were randomized to the K-VAC arm. There were no differences in demographics or pre-operative stone volume (mean basket group $=435 \mathrm{~mm}^{3}, \mathrm{~K}-\mathrm{VAC}$ group $=$ $550 \mathrm{~mm}^{3}, \mathrm{p}=0.6$ ). Mean \% stone volume removed during the procedure was significantly higher in the K-VAC group $(88.7 \%$ versus $58.8 \%, \mathrm{p}=0.03)$. There was no difference in the mean total fluoroscopy (318 seconds versus 295 seconds, $\mathrm{p}=0.8$ ). At 1 month follow up CT scan, $100 \%$ of subjects in the K-VAC group were stone free compared to $75 \%$ of subjects in the basket group.

Conclusions: Use of $\mathrm{K}-\mathrm{VAC}$, a novel stone aspiration device, demonstrated superior stone free rates compared to conventional basketing during ureteroscopy with lithotripsy. Larger clinical trials are planned to confirm the efficacy of this innovative device.

MP10-09 Comparing dusting and fragmenting efficiency using the new Super Pulse Thulium Fiber Laser vs a 120W Holmium:YAG laser

\section{BH Chew, BE Knudsen, WR Molina}

University of British Columbia

Introduction \& Objective: Holmium:YAG laser is the current lithotrite of choice. Although improvements such as higher pulse frequency, longer pulse duration, and multi-pulse technology have advanced the platform, inherent limitations remain include high amperage power requirements, upper limits of pulse frequency, and limitations regarding fiber size. A new technology utilizing Thulium Fiber, which is completely different from Thulium:YAG, offers low pulse energy settings and pulse frequencies over $600 \mathrm{~Hz}$. We compared the novel Super Pulse Thulium Fiber laser (SPTF) to a commercially available $120 \mathrm{~W}$ Ho:YAG laser.

Methods: Standard, homogeneous $125 \mathrm{~mm}^{3}$ Begostones were used for all testing $(n=10)$. To test ablation, stones were reduced using a commercially available $120 \mathrm{~W}$ laser vs the SPTF laser until remaining particles were $<1 \mathrm{~mm}$. To test fragmentation and dusting, resulting particle sizes were measured after delivering a total of $0.5 \mathrm{~kJ}$ and $2 \mathrm{~kJ}$, respectively, at optimal laser settings for each laser for fragmenting or dusting.

Results: Ablation to particles $<1 \mathrm{~mm}$ was significantly faster using the SPTF laser $2.23 \pm 0.22 \mathrm{mg} / \mathrm{s}(0.6 \mathrm{~J} 30 \mathrm{~Hz} \mathrm{SP})$ compared to the $120 \mathrm{~W}$ laser $1.78 \pm 0.44 \mathrm{mg} / \mathrm{s}(0.8 \mathrm{~J} 10 \mathrm{~Hz} \mathrm{SP}), \mathrm{p}=0.01$

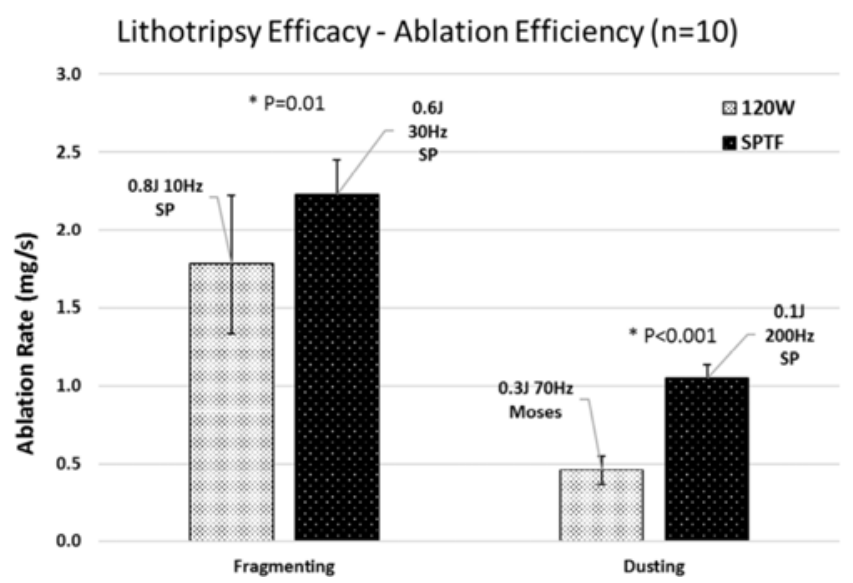




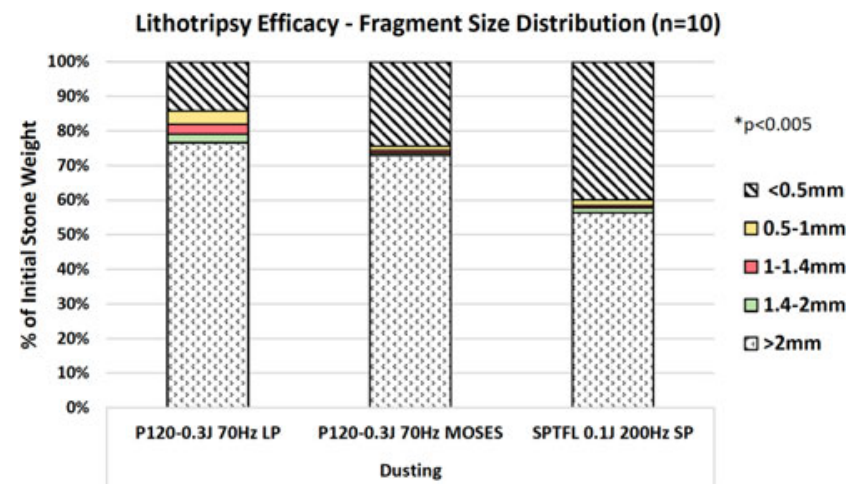

(Fig 1). After delivering $0.5 \mathrm{~kJ}$, fewer particles $>2 \mathrm{~mm}$ remained for SPTF than for the $120 \mathrm{~W}$ laser (2.1 vs 7.2 fragments). Clinically, this would equate to fewer basketing passes for SPTF. After delivering $2 \mathrm{~kJ}$, the dusting rate $(1.05 \pm 0.08 \mathrm{mg} / \mathrm{s})$ was significantly faster using SPTF $(0.1 \mathrm{~J} 200 \mathrm{~Hz}$ SP) compared to $120 \mathrm{~W} 0.46 \pm 0.09 \mathrm{mg} / \mathrm{s}(0.3 \mathrm{~J} 70 \mathrm{~Hz}$ Moses $), \mathrm{p}<0.001$ (Fig 1). The SPTFL $(0.1 \mathrm{~J} 200 \mathrm{~Hz})$ produced $40 \%$ dust $<0.5 \mathrm{~mm}$ compared to $24 \%$ produced by the $120 \mathrm{~W}$ laser at $0.3 \mathrm{~J} 70 \mathrm{~Hz}$ Moses and $14 \%$ at $0.3 \mathrm{~J} 70 \mathrm{~Hz} \mathrm{LP}, \mathrm{p}<0.005$ (Fig 2). The settings evaluated in this study are considered the optimal settings for each laser. Tests at identical dusting settings on both platforms still showed an increase in ablation rate for the SPTF technology. The setting tested was a default preset for stone dusting on the 120W laser.

Conclusions: The new Super Pulse Thulium Fiber laser is more efficacious in bench testing than a commercially available $120 \mathrm{~W}$ laser in fragmenting and dusting stones. In tests of particle sizes, it produced smaller particles of dust. Fragmentation produced fewer fragments using SPTF (and more dust), thus making basketing more efficient.

MP10-10 To investigate the clinical efficacy of transurethral ureteroscopic laser lithotripsy in the treatment of ureteral calculi under ultrasound guided paravertebral nerve block anesthesia

Y Wu, Y Lu, H Hu, S Wang, Q Wang

Tongji Hospital

Introduction \& Objective: To explore the clinical efficacy and safety of ultrasound-guided multilevel paravertebral nerve block anesthesia in transurethral ureteroscopic laser lithotripsy. Methods: We retrospectively analyzed 170 patients who underwent transurethral ureteroscopic laser lithotripsy at our institution from July 2017 to July 2018. According to the different anesthesia methods, 69 cases were in the study group and 101 cases were in the control group, the study group underwent ultrasound-guided multilevel paravertebral block anesthesia, and the control group received general anesthesia. The intraoperative hemodynamic parameters, anesthesia induction time, operation time, intraoperative and postoperative complications, stone clearance rate, hospital stay, and lower limb muscle strength were compared.

Results: The anesthesia induction time, postoperative complications, and hospitalization time of the study group were significantly lower than those of the control group $(\mathrm{P}<0.05)$. The hemodynamic parameters of the study group were stable compared with the control group and the lower limb muscle strength of the study group was significantly higher than that of the control group $(\mathrm{P}<0.05)$. The operation time of the study group was shorter than that of the control group, but there was no statistical difference $(P>0.05)$. There was no significant difference in anesthetic effect and stone clearance rate between the two groups.

Conclusions: In transurethral ureteroscopic laser lithotripsy, ultrasound-guided multilevel paravertebral nerve block anesthesia and general anesthesia can achieve good anesthesia, however, intraoperative hemodynamics are more stable in patients with paravertebral nerve block anesthesia. Reducing the use of general anesthetics and perioperative pulmonary complications and postoperative muscle strength recovery is faster, shorter hospital stay and less medical expenses.

\section{MP10-11 Integrated, Real-Time Digital Stone Mea- surement in Ureteroscopic Stone Procedures: A Workflow Feasibility Study}

\author{
K Koo, T Aro, S Lim, D Petrisor, D Stoianovici, B Matlaga \\ Johns Hopkins University School of Medicine
}

Introduction \& Objective: Accurately estimating fragment size is an important task during ureteroscopic stone management, as it can provide determination of the adequacy of dusting or the ability to retrieve fragments through a ureteral access sheath. We previously reported an ex vivo validation of a novel software that can accurately measure small stone fragments. This study assesses the feasibility of integrating the software into operating room workflow.

Methods: Mock stone fragments were selected to mimic a variety of non-ideal fragmentation conditions. Using a commercial model of the kidney and ureter designed for ureteroscopy training, we performed two versions of a timed basketing task to simulate real-time software measurement. In the conventional version, the ureteroscope was navigated from the UPJ to the stone in a superior pole calyx, then the stone was retrieved with the basket back to the UPJ. In the measurement version, the ureteroscope was again navigated from the UPJ to the stone, then real-time measurement of the stone was performed using the software, followed by stone retrieval to the UPJ. Differences in task completion time and fragment characteristics were compared.

Results: A total of 20 stone fragments were selected with different combinations of internal characteristics: size (mean $7.1 \mathrm{~mm}$, range $3.2-10.3 \mathrm{~mm}$ ), 3-dimensional shape (50\% pyramidal, $30 \%$ ovoid, $20 \%$ cuboid), surface contour (50\% smooth, $50 \%$ rough), and planar symmetry (60\% asymmetric, $40 \%$ symmetric). In the conventional version of the task, mean completion time was $16.5 \mathrm{sec}$ (range 10.1-33.6 sec), compared with a mean completion time of $38.8 \mathrm{sec}$ (range 27.2-60.0 sec) in the measurement version. The additional procedure time required to perform real-time measurement averaged $22.2 \mathrm{sec}$ (range 8.8$42.6 \mathrm{sec}$ ). Variation in these times for certain stones was typically due to more challenging planar alignment of the ureteroscope and basket tip, which is required for accurate calculation using the software.

Conclusions: Integrating a novel software for real-time stone measurement during ureteroscopy is feasible and has a modest effect on the time required for stone manipulation. These findings support in vivo trials of the software to enhance clinical decisionmaking in ureteroscopic stone management. 
MP10-12 Comparison of Mini PCNL v/s Retrograde intrarenal surgery (RIRS) v/s Extracorporeal shockwave lithotripsy (ESWL) for the treatment of renal stones up to 2.5 centimeters

P. Pattnaik

Bombay Hospital PG Institute

Introduction \& Objective: To evaluate the safety and efficacy of Mini PCNL v/s Retrograde intrarenal surgery (RIRS) v/s Extracorporeal shockwave lithotripsy (ESWL) for the treatment of renal stones up to 2.5 centimeters.

Methods: This prospective randomized study was conducted between March 2016 to February 2018. After taking written informed consent, 195 patients with renal calculi up to 2.5 centimeters were included in the study. Patients were divided into three groups by computer generated random numbers. Patients in Group A $(n=68)$ underwent Mini PCNL, Group B $(n=61)$ underwent RIRS, Group $C(n=66)$ underwent ESWL. A ureteric stent was placed in all patients undergoing RIRS and ESWL. All patients were operated under regional/intravenous anesthesia. STORZ 12.5 Fr Mini Nephroscope with $15 \mathrm{Fr}$ sheath was used for mini PCNL. WOLF (COBRA) Flexible uretero-renoscope was used for RIRS. Siemens Modularis Variostar Lithotripter was used for ESWL. Mini PCNL and ESWL were totally Ultrasound guided procedures.

Results: There were 103 Males and 92 Females. Patients were in the age group of 17 to 68 years. 72 patients had calculi in renal pelvis, 36 in upper calyx, 22 in middle calyx and 65 patients had stones in lower calyx. The mean duration of procedure was $35 \pm 10.14 \mathrm{~min}$ in the Mini PCNL group, $80.55 \pm 15.27 \mathrm{~min}$ in the RIRS group and $45 \pm 10.15$ min in ESWL group. The hospital stay was shortest in the ESWL group (Daycare) followed by RIRS ( $45 \pm 2.5$ hours) and ( $35 \pm 1.5$ hours) for Mini PCNL. Stone-free rates after one session were $99.2 \%$ for Mini PCNL, $87.5 \%$ for RIRS and $82.4 \%$ for ESWL Group. The stone-free rate of the RIRS group improved to $97.7 \%$ after the second session. No patients developed severe complications.

Conclusions: This study revealed that Mini PCNL had the highest stone clearance rates in a single session. However, RIRS and ESWL had comparable stone free rates.

MP10-13 Rapid, Efficient Crowdsourcing Using Social Media for the Surgical Management of Nephrolithiasis

K Koo, T Aro, B Matlaga

Johns Hopkins University School of Medicine

Introduction \& Objective: The surgical management of urinary stone disease can be challenging due to complexity and multiple potential approaches. One advantage of urologists' increasing social media use is the opportunity for crowdsourcing these challenges. This study assesses urologists' use of Twitter to crowdsource dilemmas in surgical stone disease.

Methods: Twitter was queried with 22 stone-related keywords during a 60-day study period. All public tweets were reviewed to identify original posts and associated reply posts by urologists seeking clinical advice or feedback about surgical stone management. Crowdsourcing posts were reviewed to assess the nature and content of the requests. Content analysis was performed by 2 reviewers; differences were resolved by consensus.

Results: Urologists contributed a total of 399 posts to 29 crowdsourcing requests during the study period. On average each crowdsourcing request generated 12.8 replies from 9.6 unique users.
Crowdsourcing requests were answered rapidly; the mean elapsed time from index post to final reply was 2.8 days. In 18 (62\%) of the crowdsourcing requests, the index author responded directly to replying users or posted a follow-up question, indicating a bidirectional exchange of ideas. The majority of crowdsourcing threads engaged a multinational audience; index posts originated from 27 urologists in 12 countries, and all but one of the 29 requests $(97 \%)$ had replies from users outside the origin country. Among crowdsourcing requests that received $\geq 1$ reply, the mean number of Twitter followers of the index author was 795, but users with as few as 59 followers who initiated crowdsourcing requests also received numerous replies, suggesting that novice Twitter users may nonetheless be able to benefit. Of the 29 index requests, the most common reasons for crowdsourcing were to define an optimal surgical approach to the stone (206 posts, 52\%) and request colleagues' experiences with a device, technique, or approach (86 posts, $22 \%$ ). A novel use of crowdsourcing emerged from 2 quality improvement requests; the authors described their surgical complications and sought feedback about managing the problem and avoiding it in the future. Finally, protection of patient privacy may be a concern; among the 17 (59\%) crowdsourcing requests that were about specific patients or cases, all included radiographic images or videos, but none stated whether the patient's permission had been obtained. Conclusions: Twitter can facilitate crowdsourcing a range of general, patient-specific, and quality improvement challenges in surgical stone management. Urologists should consider privacy concerns when sharing imaging or patient data.

MP10-14 Operator-assisted vs self-achieved basketing during ureteroscopy: results from an in-vitro preference study

E Ventimiglia, S Doizi, T Besombes, O Traxer

Division of Experimental Oncology/Unit of Urology; URI; IRCCS Ospedale San Raffaele

Introduction \& Objective: Basketing is one of the fundamental yet most challenging step during flexible ureteroscopy. A recently introduced device (LithoVue Empower ${ }^{\mathrm{TM}}$ or LE, Boston Scientifics, USA) allows the surgeon to directly control the basket without the need of an assistant. We aimed to evaluate the impact of this device on stone retrieval time and surgeon's mental/physical demand.

Methods: We used a portable bench-training model for flexible ureteroscopy, the Key-box (K-Box ${ }^{\circledR}$, Porge's-Coloplast, France), in order to compare the LE configured with a 1.9F stoneretrieval tipless basket (ZeroTip TM, Boston Scientific, USA) and a traditional assistant-maneuvered $1.9 \mathrm{~F}$ stone-retrieval tipless basket (ZeroTip TM, Boston Scientific, USA). Six experienced endourologists ( $>100$ ureteroscopies each) and 7 residents in training in urology retrieved a fake stone $(5 \times 5 \times 5 \mathrm{~mm})$ from 3 different cavities of the K-Box simulating three renal cavities with increasing access complexity $(\mathrm{C} 1$ the simplest, $\mathrm{C} 2$ the intermediate, and $\mathrm{C} 3$ the hardest) first with the traditional basket (Task 1) and then with the LE device (Task 2). A single use ureteroscope (LithoVue ${ }^{\mathrm{TM}}$, Boston Scientific, USA) was used in all cases. None of the subjects had ever used before the LE device. We recorded time from ureteroscope insertion until stone retrieval and defined a failure in case time exceeded 300 seconds. All the operators filled in the NASA Task Load Index (TLI) for self-evaluation of their performance. We then compared results from Task 1 and Task 2 in terms retrieval time, failure rates, and NASA TLI scores using non-parametric descriptive statistics. 
Results: Overall, stone retrieval times and failure rates were comparable between Task 1 and Task 2 (all p >0.05) for all analyzed cavities. NASA TLI scores revealed lower effort $(\mathrm{p}=0.001)$ and frustration $(\mathrm{p}=0.04)$ for the Task 2 vs Task 1 . When stratifying the analyses according to surgical experience, fully trained urologists performed faster stone retrieval than residents in training for the $\mathrm{C} 1$ and $\mathrm{C} 2$ cavities (both $\mathrm{p}<0.01$ ) overall, but no difference was found according to the retrieval device used. Residents showed lower efforts $(p=0.005)$ and frustration $(p=0.04)$ scores at NASA TLI when performing Task 2, whereas this pattern was not observed in experienced urologists.

Conclusions: The individually controlled retrieval system is an effective device assisting stone retrieval and does not seem to necessitate specific training even among unexperienced endourologists, who might instead benefit from it in terms of procedure-related anxiety and exertion.

\section{MP10-15 Two-step precise method with transurethral flexible ureteroscopy for treatment of parapelvic cyst combined with renal calculi}

G Jingyang, Y Wenzeng, Z Yanqiao, S Xiaoqiang, A Feng, $G$ Deqiang

Affiliated Hospital of Hebei University

Introduction \& Objective: To observe Two-step precise method with transurethral flexible ureteroscopy for treatment of parapelvic cyst combined with renal calculi.

Methods: 23 patients in our hospital who underwent a Two-step precise method with transurethral flexible ureteroscopy for treatment of parapelvic cyst combined with renal calculi, analyzed the clinical data of patients, observation of therapeutic effect.

Results: All patients underwent successful surgery I, 4 patients puncture the level of urine confirmed calyx diverticulum, ureteral diverticula mouth soft lenses expand the drainage, the remaining 19 cases were confirmed as parapelvic cysts, cut inside wall line the success of open internal drainage, decreased hemoglobin before and after surgery showed no significant change. Without blood transfusions, mean postoperative hospital stay two days, the first day after the review KUB observed DJ tube position, after 1-month review ultrasound or CT, cyst volume were significantly reduced after followed up for 6 months to 1 year, all patients had no long-term complications.

Conclusions: transurethral flexible ureteroscopy for Two-step precise method treatment of parapelvic cyst and renal pelvis lamp beside diverticulum, a clear direction for the next operation, simple operation, no significant complications, quick recovery, treatment parapelvic cysts ideal treatment.

MP10-16 Super Pulse Thulium Fiber Laser Compared to 120W Holmium:YAG Laser: Impact on Retropulsion and Laser Fiber Burn Back

BE Knudsen, BH Chew, WR Molina

The Ohio State University

Introduction \& Objective: Thulium fiber laser technology is an emerging field for laser lithotripsy that addresses inherent limitations of the Holmium:YAG laser including high current re- quirements, limited pulse frequency, and poor coupling with sub$200 \mu \mathrm{m}$ core sized fibers. The Super Pulse Thulium fiber (SPTF) laser system was compared to a $120 \mathrm{~W}$ Holmium:YAG system with respect to retropulsion and laser fiber burn back.

Methods: A force sensor was used to measure retropulsion when laser energy was applied to a Begostone phantom. Both fragmentation and dusting settings were measured $(n=15)$. The retropulsion force was recorded both per pulse and per second at each setting. Laser fiber burn back was measured with the Begostone phantom in contact with the laser fiber tip. Two hundred micron fibers were utilized for the testing.

Results: The SPTF laser produced significantly less retropulsion compared to long pulse dusting settings of the 120W Ho:YAG
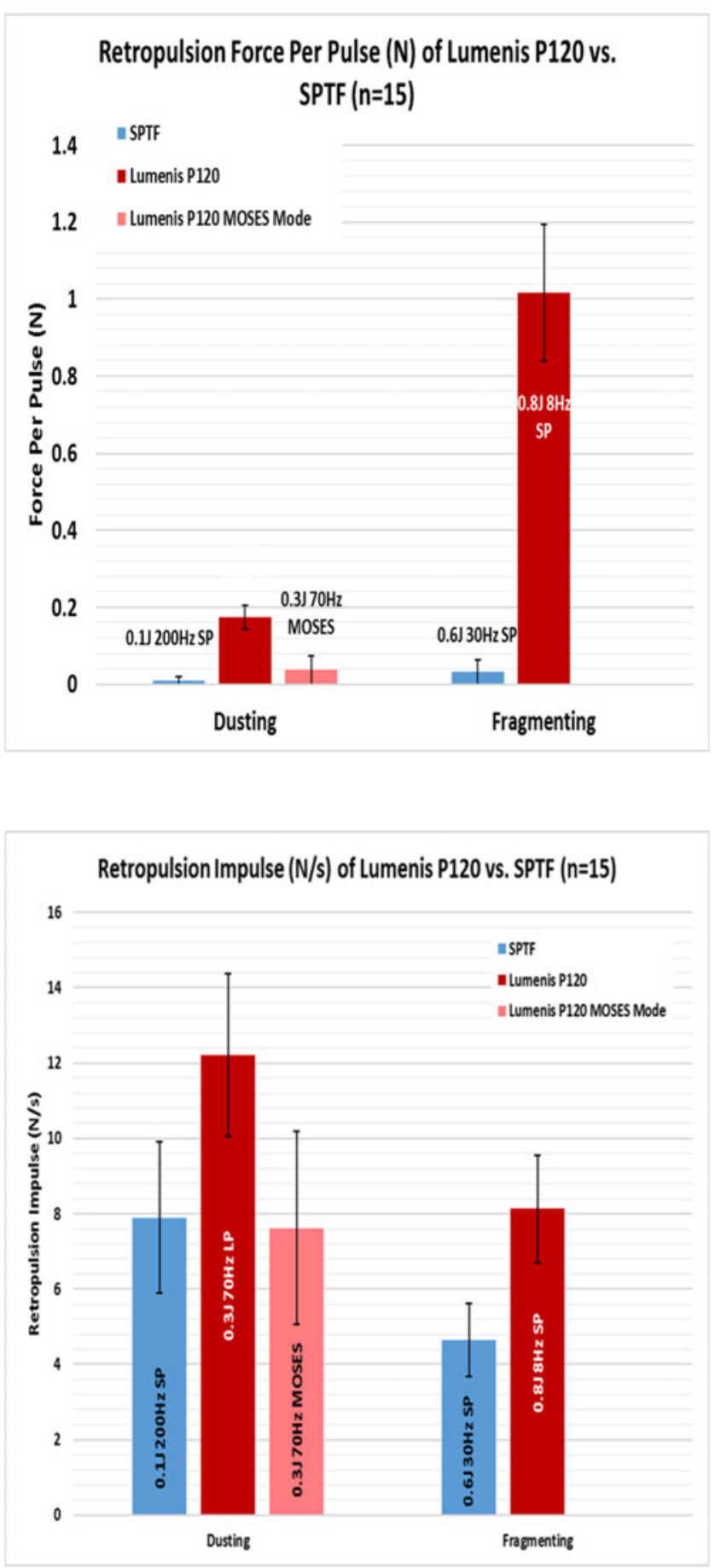


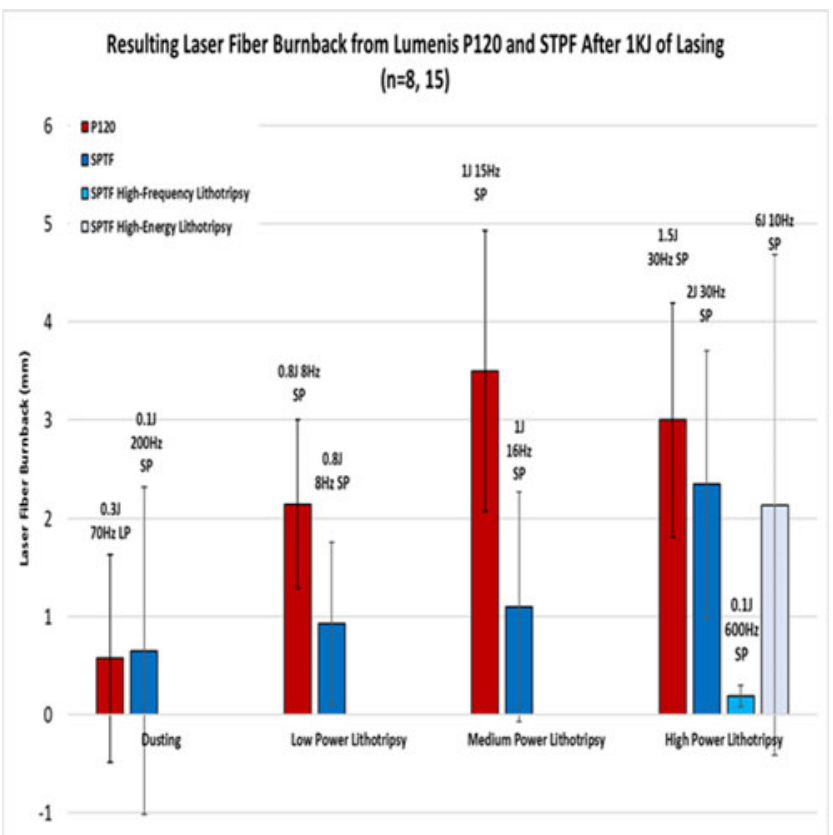

$\cdot 2$

laser both in terms of retropulsion force per pulse $(\mathrm{N})$ and retropulsion impulse. $(\mathrm{N} / \mathrm{s})(\mathrm{p}<0.001)$ The SPTF laser produced significantly less retropulsion compared to short pulse fragmentation settings of the 120W Ho:YAG laser $(\mathrm{p}<0.001)$ both in terms of retropulsion force per pulse $(\mathrm{N})$ and retropulsion impulse. $(\mathrm{N} / \mathrm{s})(\mathrm{p}<0.001)$ Retropulsion of the SPTF laser was significantly less than the Ho:YAG laser in Moses mode in terms of retropulsion for per pulse $(\mathrm{N})$ but similar for retropulsion impulse (N/s). (Figs 1 \& 2) Laser fiber burn back was significantly less for the SPTF laser as compared to the Ho:YAG laser at all fragmentation settings tested. Burn back was similar at dusting settings. $(\mathrm{p}<0.001$, Fig 3$)$.

Conclusions: The SPTF laser created less retropulsion in comparison to a $120 \mathrm{~W}$ P120 Holmium:YAG laser at all tested settings with the exception of retropulsion impulse testing in Moses mode, which was equivalent. The SPTF system had diminished fiber burn back in comparison to the 120w Holmium:YAG system.

MP10-17 RIRS for lower pole small renal stones: Novel SuperPulse Thulium (Tm) fiber laser lithotripsy. First clinical results

D Korolev, R Klimov, D Enikeev, D Tsarichenko, M Enikeev, A Dymov, S Ali, G Akopyan, V Lekarev, L Rapoport

Institute for Urology and Reproductive Health, Sechenov University

Introduction \& Objective: The SuperPulse Thulium (Tm) fiber laser (IRE Polus, Russia) (wavelength of $1.94 \mu \mathrm{m}$ ) is a next generation laser technology that has a significant potential for urinary stones treatment. But still a small number of clinical studies does not allow to fully reveal the capabilities of this laser in the management of renal stones. To evaluate the effectiveness of the SuperPulse Tm fiber laser in the management of lower pole small calyceal stones during flexible ureteroscopy (F-URS).

Methods: The SuperPulse Tm fiber laser device has been cleared for clinical use by the Ministry of Health of Russian Federation and the protocol has been approved by the institutional Ethical Review committee. Between January 2018 and February 2019, 130 patients with kidney stones have undergone Thulium fiber laser lithotripsy during F-URS. We retrospectively analyzed 15 of these patients with a single radiopaque lower pole calculus which were included in the present study. Stone size, its density, lithotripsy time (from the first to last pedal press) and "lasering", (laser emission) time were measured. The SuperPulse Tm fiber laser was used for stone disintegration with different settings in dusting and fragmentation modes $(0.1-4 \mathrm{~J}, 7-300 \mathrm{~Hz}, 6-40 \mathrm{~W})$ via a fiber with a $200-\mu \mathrm{m}$ core diameter. Low dose CT scanning was performed on POD 90 to assess SFR.

Results: Stone size ranged from 4 to $17 \mathrm{~mm}$ and stone density varied from 350 to $1459 \mathrm{HU}$. The average lithotripsy time was $12 \mathrm{~min}$ (3-30 $\mathrm{min})$. The average "lasering" time was $1.3 \mathrm{~min}$ (0.4-2.5 $\mathrm{min})$ and the mean hospital stay was $1.1 \pm 0.3$ days. In all cases we reached the lower pole stone containing calyx with a laser fiber. The complication rates were evaluated by using the Clavien-Dindo grading system and did not exceed GII. The overall postoperative complication rate was $26.6 \%$ (GI 20\%; GII $6.6 \%$ ). SFR on POD 90 was achieved in $86.6 \%$ of cases.

Conclusions: F-URS with SuperPulse Tm fiber laser is safe and effective option in the management of lower pole small calyceal stones. The possibility of using small laser fibers gives better instrument deflection which make possible to reach lower pole calyces stones even with acute lower pole infundibulopelvic angle (IPA).

\section{MP10-18 Ultrasound guided paravertebral nerve block anesthesia and SVOF accurate puncture for percutaneous nephrolithotomy — an innovative am- bulatory surgery mode}

Y Wu, Y Lu, J Zhang, S Wang, Q Wang

Tongji Hospital

Introduction \& Objective: To explore the feasibility and safety of SVOF precise puncture PCNL as an innovative ambulatory surgery mode for patients with renal calculi under ultrasound guided paravertebral nerve block (PVB) anesthesia.

Methods: From March 2017 to July 2018, we performed SVOF precision puncture PCNL in 31 patients with kidney stones under paravertebral block anesthesia. All patients were located the vertebral segments by the "waist 1 disappearance method" in the lateral position. Real-time ultrasound guided T9-L1 five-point paravertebral nerve block and detected the anesthesia effect after 20 minutes. After successful anesthesia, we tried to choose the middle or upper part of the kidney and ultrasound-guided renal peri-kidney two-step method for precise puncture of target renal pelvis according to the SVOF principle, last ureteroscopic laser lithotripsy. No nephrostomy tube and catheter were placed after operation, we then assessed the patient's condition before leaving the hospital. Finally, we analyzed the perioperative and postoperative follow-up data.

Results: The average stone size was $2.1 \pm 0.6 \mathrm{~cm}$. All patients could achieve at least T9-L1 pain block by paravertebral block anesthesia, all patients had a smooth operation without blood transfusion and the average operation time was $32 \pm 9.1 \mathrm{~min}$. All patients had no pain complaints during surgery. Compared with preoperative, there were no significant differences in hemodynamic 
parameters during the operation $(\mathrm{P}>0.05)$. There were two cases of lower limb muscle strength II, 24 cases of grade III, five cases of grade IV, and grade V on the contralateral side. All patients could get out of bed about one hour after surgery, seven patients had mild pain after surgery but no analgesic drugs were used. All patients were discharged within one day. All patients did not show significant bleeding and fever after follow-up, only three patients have reddish urine for a longer duration, two patients presented with discomfort due to lumbar discomfort, after checking the plain film we did not see the double $\mathrm{J}$ tube position abnormal, all patients had symptom relief after rest. The stone clearance rate was $93.2 \%$.

Conclusions: SVOF accurate puncture of PCNL under paravertebral nerve block anesthesia is safe and feasible as a ambulatory operation. This new ambulatory surgery mode reduces postoperative pain in patients and the amount of opioids used during surgery. Hemodynamics parameters are more stable during surgery, postoperative muscle strength recovery is faster, shorter hospital stay and less medical expenses. It is ideal for large hospitals with mature PCNL technology.

MP10-19 Evaluation of safety and efficacy of combined Extracorporeal shockwave lithotripsy and retrograde intrarenal surgery for the treatment of large lower calyceal calculi

PK Pattnaik, SK Pattnaik, MP Pattnaik

S S Urological \& Research Insitute, Bombay Hospital PG Institute

Introduction \& Objective: We present our experience of combined Extracorporeal shockwave lithotripsy and retrograde intrarenal surgery for the treatment of impacted, large lower calyceal calculi and evaluate the safety and efficacy of this treatment modality.

Methods: Between January 2014 and December 2018, 196 patients with lower calyceal calculi more than 2 centimeters underwent combined Extracorporeal shockwave lithotripsy and retrograde intrarenal surgery. Calculus size, density (Hounsfield units), preoperative urinary culture, blood biochemistry and Pelvicalyceal anatomy (by pre-operative CT scan) were documented. Our protocol was to administer 2 sessions of ESWL at an interval of 48 hours and subsequently plan RIRS. All patients were stented prior to ESWL. Ureteral access sheath was used in $160(81.63 \%)$ cases. The final stone-free rates (SFRs) were assessed 3 weeks after the surgery by computed tomography (CT). Preoperative, operative and postoperative parameters were analyzed. Serum creatinine and glomerular filtration rate (GFR) were measured preoperatively, one month postoperatively.

Results: The mean stone burden was $25 \pm 1.2 \mathrm{~mm}^{2}$ (range 22$28 \mathrm{~mm}^{2}$ ). The mean operative time was $74.5 \pm 10.3 \mathrm{~min}$ (range 5590). 14 patients (7\%) required second-stage RIRS for residual stones. The SFRs after the procedures was $96.8 \%$. Minor complications (hematuria, post-operative dysuria, fever with chills) occurred in 15 patients $(7.65 \%)$. Two patients developed perinephric collection and were treated with percutaneous drainage.

Conclusions: Combined Extracorporeal shockwave lithotripsy and retrograde intrarenal surgery is a safe and effective procedure for the treatment of impacted, large lower calyceal calculi with various advantages such as faster clearance of stone fragments, least damage to the flexible endoscope, easier relocation of fragments created by previous ESWL, no damage to the infundibulum, lesser chances of sepsis and shorter operative time. Hence, we recommend this novel technique for management of large lower calyceal calculi.
MP10-20 Ureteroscopic Doppler Ultrasonography: Where is the Least Vascular Renal Access Site for Percutaneous Nephrolithotomy?

RM Patel, V Cooper, L Limfueco, S Tapiero, M O’Leary, D Regan, J Landman, RV Clayman

University of California, Irvine

Introduction \& Objective: Detailed knowledge of forniceal, papillary, and infundibular blood flow would allow placement of a nephrostomy track in the least vascular site during percutaneous stone removal. Herein, we provide the first report regarding in vivo porcine renal forniceal, papillary, and infundibular blood flow at the level of the urothelium.

Methods: A Doppler transducer (Vascular Technology Inc.) with a $1 \mathrm{~cm}$ depth of penetration was mounted to a $3 \mathrm{~F}, 120 \mathrm{~cm}$ catheter; the catheter was passed through the working channel of a dual lumen flexible ureteroscope. Forniceal and papillary blood flow was mapped in seven juvenile female Yorkshire pigs and infundibular blood flow was mapped in four adolescent female Yorkshire pigs. Blood flow measurements were categorized from 0 (no flow) to 3 (highest flow) based on auditory intensity.

Results: The infundibular blood flow was more often noted to be high (i.e. $40-55 \%$ of the readings) than that of the calyceal fornices $(11.9-15.8 \%$ of the readings $)(\mathrm{p}<0.001)$ (Table 1$)$. Distribution of blood flow did not differ significantly between anterior and posterior calyces nor along the length of the kidney. The 6 o'clock forniceal position had significantly more flow than the other forniceal locations $(\mathrm{p}<0.001)$. The center of each papilla consistently had significantly less blood flow $(p<0.001)$ than the forniceal locations. The data were collated and a 3D reconstruction was created to summarize the findings (Figure 1).

Table 1: Comparison of Blood Flow by Infundibular and Caliceal Location.

\begin{tabular}{|c|c|c|c|c|c|}
\hline Regions & Anatomy & $\begin{array}{l}\text { High Blood } \\
\text { Flow }\end{array}$ & $\begin{array}{l}\text { Low Blood } \\
\text { Flow }\end{array}$ & $P$ value & $\begin{array}{c}\text { Pairwise } \\
\text { Comparison* }\end{array}$ \\
\hline \multirow{2}{*}{ Anterior } & $\begin{array}{l}\text { Infundibulum (AI) } \\
(n=240)\end{array}$ & $\begin{array}{c}108 \\
(45.0 \%)\end{array}$ & $\begin{array}{c}132 \\
(55.0 \%)\end{array}$ & \multirow{4}{*}{$<0.001$} & \multirow{4}{*}{$\begin{array}{l}\text { Al>AC }(p<0.001) \\
\text { Al }>P C \quad(p<0.001) \\
P I>A C \quad(p<0.001) \\
P I>P C \quad(p<0.001)\end{array}$} \\
\hline & $\begin{array}{l}\text { Calyx (AC) } \\
(n=544)\end{array}$ & $\begin{array}{c}65 \\
(11.9 \%)\end{array}$ & $\begin{array}{c}479 \\
(88.1 \%)\end{array}$ & & \\
\hline \multirow{2}{*}{ Posterior } & $\begin{array}{l}\text { Infundibulum (PI) } \\
(n=240)\end{array}$ & $\begin{array}{c}116 \\
(48.3 \%)\end{array}$ & $\begin{array}{c}124 \\
(61.7 \%)\end{array}$ & & \\
\hline & $\begin{array}{l}\text { Calyx (PC) } \\
(n=512)\end{array}$ & $\begin{array}{c}81 \\
(15.8 \%)\end{array}$ & $\begin{array}{c}431 \\
(84.2 \%)\end{array}$ & & \\
\hline \multirow{2}{*}{ Upper Pole } & $\begin{array}{l}\text { Infundibulum (UPI) } \\
(n=160)\end{array}$ & $\begin{array}{c}88 \\
(55.0 \%)\end{array}$ & $\begin{array}{c}72 \\
(45.0 \%)\end{array}$ & \multirow{6}{*}{$<0.001$} & \multirow{6}{*}{ 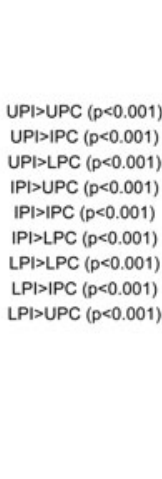 } \\
\hline & $\begin{array}{l}\text { Calyx (UPC) } \\
(n=368)\end{array}$ & $\begin{array}{c}49 \\
(13.3 \%)\end{array}$ & $\begin{array}{c}319 \\
(86.7 \%)\end{array}$ & & \\
\hline \multirow{2}{*}{ Interpolar } & $\begin{array}{l}\text { Infundibulum (IPI) } \\
(n=160)\end{array}$ & $\begin{array}{c}64 \\
(40.0 \%)\end{array}$ & $\begin{array}{c}96 \\
(60.0 \%)\end{array}$ & & \\
\hline & $\begin{array}{l}\text { Calyx (IPC) } \\
(n=352)\end{array}$ & $\begin{array}{c}51 \\
(14.5 \%)\end{array}$ & $\begin{array}{c}301 \\
(85.5 \%)\end{array}$ & & \\
\hline \multirow{2}{*}{ Lower pole } & $\begin{array}{l}\text { Infundibulum (LPI) } \\
(\mathrm{n}=160)\end{array}$ & $\begin{array}{c}72 \\
(45.0 \%)\end{array}$ & $\begin{array}{c}88 \\
(55.0 \%)\end{array}$ & & \\
\hline & $\begin{array}{l}\text { Calyx (LPC) } \\
(\mathrm{n}=336)\end{array}$ & $\begin{array}{c}46 \\
(13.7 \%)\end{array}$ & $\begin{array}{c}290 \\
(86.3 \%)\end{array}$ & & \\
\hline
\end{tabular}

" $\alpha$ adjusted using Bonferroni Correction to correct for multiple comparison. -Anterior vs Posterior total 4 comparisons, $\alpha<0.0125$ considered significant -Upper pole vs Interpolar vs Lower pole total 6 comparisons, $\alpha<0.00833$ considered significant 


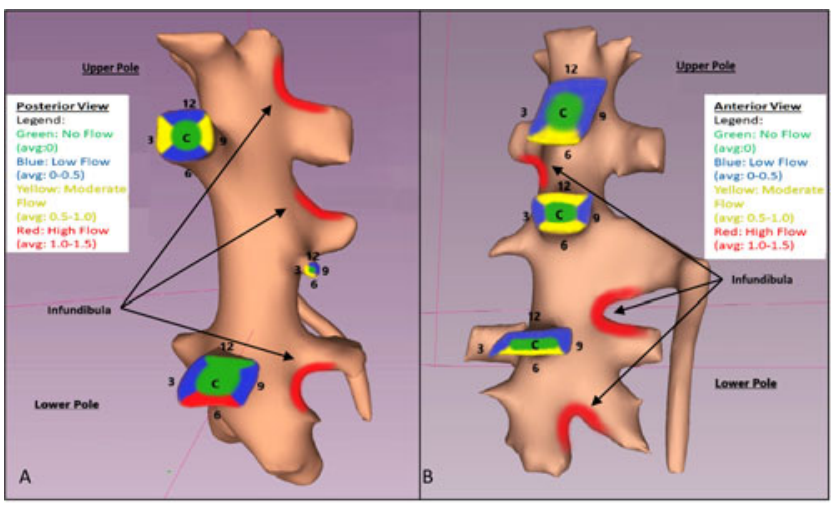

Figure 1: A) 3D reconstruction of the posterior calyces with blood flow outlined by color. B) 3D reconstruction of the anterior calyces with blood flow outlined by color.

Conclusions: Using a unique ureteroscopic Doppler transducer, areas of low and high blood flow within the porcine kidney were mapped. The center of the calcyeal papilla had the least vascular flow followed by the fornices. The infundibulae had the highest percentage of high blood flow.

MP10-21 Selective Utilization of the LithoVue SingleUse Digital Flexible Ureteroscope for the Management of Upper Urinary Tract Stones Results in Shorter Operative Times and Cost Savings

R Munver, J Tsui, B Desroches, M Degen, M Ahmed, G Lovallo, MD Stifelman

\section{Hackensack University Medical Center}

Introduction \& Objective: The judicious use of the LithoVue single-use digital flexible ureteroscope (LithoVue ${ }^{\mathrm{TM}}$, Boston Scientific Corporation, MA), can potentially reduce operative times for complex ureteroscopic (URS) procedures. With the average cost of operating room time ranging from $\$ 21$ to \$133 per minute (median approximately $\$ 79 /$ minute), this can result in significant savings for hospitals or ambulatory surgical centers. Methods: An IRB approved retrospective review was performed of all URS procedures at a single institution since the transition to digital flexible ureteroscopes in January 2016. The HackensackUMC Algorithm for using LithoVue was established in 2016 and instituted in January 2017, with indications: (1) large stone burden $(\geq 1.5 \mathrm{~cm})$, (2) multiple lower pole stones $(\geq 3),(3)$ extensive renal/ureteral tumor or laser use ( $\geq 1$ hour), (4) bilateral ureteroscopy, or (5) stones within complex/tortuous renal anatomy. Perioperative data for patients specifically with upper urinary tract stones were analyzed, including procedure indications and operative time. A control group was matched by age, sex, BMI, ASA score, stone size, location, complex anatomy, dusting versus fragmentation technique, and use of a ureteral access sheath. Prematurely completed procedures and intraoperative complications were excluded from the study.

Results: The total number of flexible URS procedures per year was 272 (2016), 318 (2017), and 308 (2018). In 2017, 63 singleuse URS procedures were performed for the indications: (1) 35 , (2) 9 , (3) 14 , (4) 5, (5) 6 . Of these, 14 procedures involved 2 $(\mathrm{n}=12)$ or $3(\mathrm{n}=1)$ indications. In 2018,91 single-use URS procedures were performed for the indications: (1) 62 , (2) 24, , (3) 5 , (4) 6 , (5) 6 . Of these, 20 procedures involved $2(n=16)$ or 3 $(n=4)$ indications. A total of 58 patients treated with upper urinary tract stones met inclusion criteria. The median age was 47, BMI 26, and ASA score 2. A ureteral access sheath was used in $84 \%$ of procedures. The mean operative time was 67 minutes (LithoVue group) and 84 minutes (control group) [ $p=0.007]$ for patients with large stones, multiple lower pole stones, or stones within complex/tortuous renal anatomy.

Conclusions: The implementation of an algorithm for LithoVue utilization resulted in an average $17 \mathrm{~min}$ operative time reduction and significant cost savings as compared to a matched pair cohort. This observation may be a result of improved scope maneuverability, use of a secondary monitor for URS, fewer equipment malfunctions, and allowance of more aggressive laser use. Select use of a single-use ureteroscope can reduce operative times with resultant cost savings at high volume institutions.

\section{MODERATED POSTER SESSION 11: NEW TECHNOLOGY: LAPAROSCOPY \& ROBOTICS/ MISCELLANEOUS}

\begin{abstract}
MP11-01 Implementation of a Single-Use Digital Flexible Ureteroscope Results in a Decrease in Reusable Digital Ureteroscope Repair Costs, and Increase in Reusable Digital Ureteroscope Life, and Prevention of Operative Case Cancellation
\end{abstract}

R Munver, J Tsui, B Desroches, M Degen, G Lovallo, M Ahmed, MD Stifelman

Hackensack University Medical Center

Introduction \& Objective: The transition from fiberoptic to digital flexible ureteroscopes has occurred at many institutions. Digital ureteroscopes offer enhanced optics, however they are associated with increased individual and total repair costs. Furthermore, the lack of an available flexible ureteroscope due to repairs or sterile processing can result in costly operative case cancellations. The availability of a single-use digital flexible ureteroscope for complex cases may potentially preserve the life of reusable digital ureteroscopes, and thus prevent case cancellation when a reusable scope is not available. We present our experience with selective implementation of the LithoVue $^{\mathrm{TM}}$ single-use digital flexible ureteroscope (Boston Scientific Corporation, Marlborough, MA) and evaluated the impact on reusable ureteroscope use and operative case cancellation prevention.

Methods: An IRB approved retrospective review was performed of all ureteroscopy procedures at our institution since January 1, 2016. The algorithm for using a single-use digital flexible ureteroscope specifically for complex cases was introduced in 2017. Perioperative data were analyzed, including number of procedures, repairs, and costs. 
Results: The total number of ureteroscopy procedures was 418 (272 flexible, 146 semirigid) in 2016, 398 (318 flexible, 80 semirigid) in 2017, and 403 (308 flexible, 95 semirigid) in 2018. The total number of ureteroscope repairs was 47 (2016), 35 (2017), and 22 (2018). The cost savings from 2016 to 2017 was $21 \%$ and from 2017 to 2018 was $34 \%$, for an overall cost savings of $48 \%$. The number of single-use ureteroscopy procedures performed for the algorithmic indications included 63 (2017) and 81 (2018). The average number of uses prior to requiring repair for the reusable digital flexible ureteroscope was 5.7 (2016), 6.6 (2017), 9.9 (2018), and >10 (2019 YTD). The single-use ureteroscope was used in 26 additional procedures in 2017 and in 10 additional procedures in 2018 when a reusable digital ureteroscope was not available, to prevent operative case cancellation, and which translated to a net profit for these cases. In 2019 YTD, no additional single-use ureteroscopes have been required to prevent case cancellation.

Conclusions: Implementation of the LithoVue ${ }^{\mathrm{TM}}$ Single-use Digital Flexible Ureteroscope resulted in a decrease in reusable digital ureteroscope repair costs and increase in uses of the reusable digital flexible ureteroscope, and prevention of operative case cancellations. A single-use ureteroscope may be a reasonable alternative to subjecting the more expensive reusable ureteroscope to extensive stress and damage, while resulting in cost savings.

MP11-02 Tissue incision, excision and ablation using Super Pulse Thulium Fiber (SPFL) LASER: Comparison with High Power 120W Holmium-YAG LASER (Ho:YAG)

BH Chew, BE Knudsen, WR Molina

University of British Columbia

Introduction \& Objective: Holmium -YAG (Ho:YAG) Laser system is an stablished energy technology for incision of tissue in urological surgery. The Super Pulse Fiber Laser (SPFL) is a new technology for use in stones and tissue. We aimed to compare the effects on tissue using SPFL compared to a commercially available 120W Ho:YAG laser.

Methods: Freshly harvested porcine kidneys were placed in a saline bath heated to $37^{\circ} \mathrm{C}$.Eachlaser was applied at a constant speed and distance from tissue using 2 different fiber sizes (SPFL - $150 \& 940 \mu \mathrm{m}, \mathrm{Ho}: \mathrm{YAG}-230 \& 955 \mu \mathrm{m}$ ) using default settings for incision, excision, or ablation $(n=3)$. For the larger fibers, enucleation and vaporization settings were also utilized. Tissue samples were analyzed for histopathology using $\mathrm{H} \& \mathrm{E}$ staining to determine the maximum depth of tissue effect by each laser. Measurements included the depth incised into tissue as

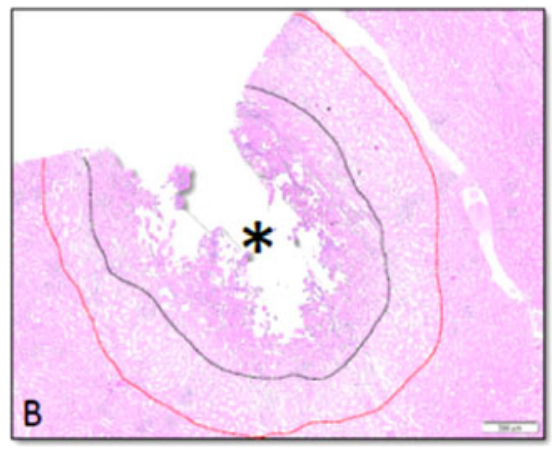

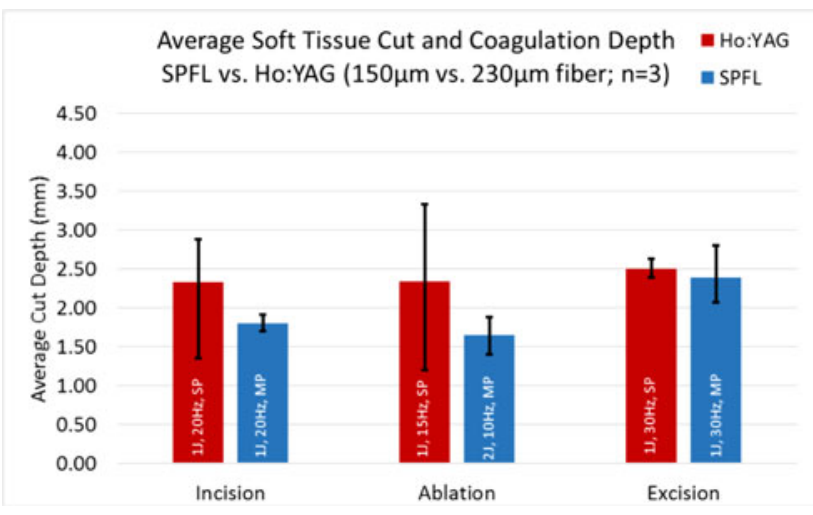

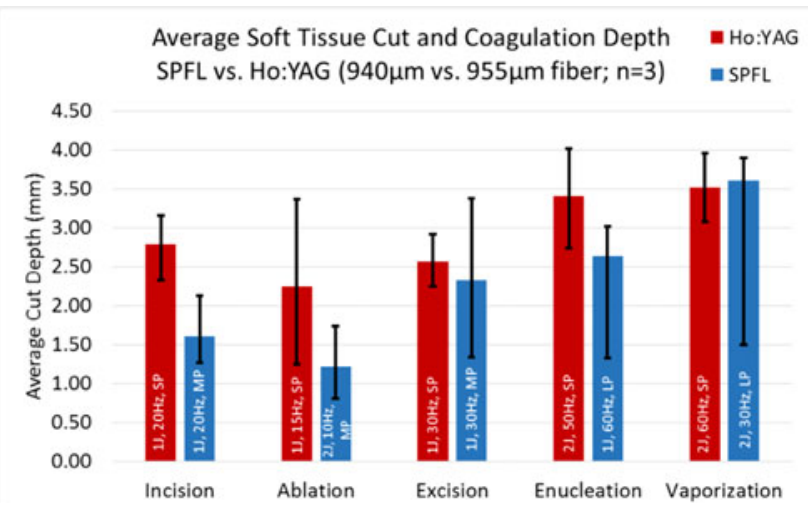

well as the region of coagulated tissue extending below the cut. The red line in the histopathology image below outlines the coagulated region of the tissue and was considered the depth of effect. (Fig 1).

Results: Tissue cutting, and coagulation depth were similar between both SPFL and 120W laser at all settings using both small and large fibers (Figs 2 and 3); there was a trend towards a narrower depth of cutting and coagulation using the SPFL, although it was not statistically significant $(\mathrm{p}=\mathrm{n}$.s.).

Conclusions: Initial results show SPFL in tissue has similar effects to a 120W Ho:YAG laser in cutting tissue at various settings in an ex vivo porcine kidney model.

\section{MP11-03 Withdrawn}

\section{MP11-04 Holmium laser versus thulium laser versus Thulium Fiber Laser enucleation of the prostate: a single center study}

PK Pattnaik, SK Pattnaik, DP Pattnaik

S S Urological \& Research Institute, Bombay Hospital PG Institute

Introduction \& Objective: The aim of our study was to compare the outcomes of three different newer generation lasers for prostatic enucleation performed at a single center. $60 \mathrm{~W}$ holmium laser (newer Generation) enucleation of the prostate (HoLEP), $200 \mathrm{~W}$ thulium laser (Dual mode) enucleation of the prostate (ThuLEP) and Super pulse Thulium Fiber Laser. 
Methods: This is a Prospective Randomized study performed on three homogeneous groups of 94 patients that underwent prostate laser enucleation by $60 \mathrm{~W}$ holmium laser (newer Generation) Group A ( $\mathrm{n}=31$ ), ThuLEP (Group B, $\mathrm{n}=38$ ) and TM Fiber Laser enucleation (Group C, $\mathrm{n}=25$ ) at a single center between January 2019 and May 2019, following the classical 'three lobes' enucleation technique. The IPSS and prostate volume were the main parameters considered for matching the patients between the three groups.

Results: Average enucleation and morcellation time was $65.5+12.5 \mathrm{~min}$, respectively, in the HoLEP group (Group A) versus $73+14$ min, respectively, in the ThuLEP group (Group B) and $69+12 \mathrm{~min}$ in TM Fiber (Group C). Enucleated adenoma weight was comparable ( $45.5 \mathrm{~g}$ v/s $44.6 \mathrm{~g}$ v/s $46.5 \mathrm{~g}$ ). Catheterization time was comparable (2.5 days in all 3 Groups). The International Prostate Symptom Score (IPSS) and Quality of Life (QOL) score significantly decreased, as well as maximal urinary flow rate (Qmax) was excellent and Blood loss negligible in Thulep (Group B), medium in TM Fiber Laser (Group C) and Average in HoLEP (Group A). Irritative lower urinary tract symptoms were maximum in Group A.

Conclusions: We recommend that Thulium or TM Fiber Laser are better options for Prostatic Enucleation than Holmium Laser enucleation.

\section{MP11-05 Digital application developed for evaluation of functional results following assisted robotic radical prostatectomy}

L Faria, S Reis, K Leite, J da Cruz, R Maia, R Pimenta, MM Xavier, D Ilias, G Xavier, F Fakhouri, M Srougi, C Passerotti, G Magalhaes, M Belkovsky

Hospital Alemão Oswaldo Cruz

Introduction \& Objective: The $21^{\text {st }}$ century brought new paradigms in information technology, with repercussions in the most diverse aspects of daily life. Mobile applications, websites, software developed for smartphones and tablets are increasingly used in healthcare, allowing remote patient support or selfpromotion of health care. The prostate cancer (PC) screening allowed PC diagnosis in early stages resulting in more curative procedures as radical prostatectomy. Among the main complications following surgery are urinary incontinence and erectile dysfunction. However, the exact numbers related to these morbidities are often missing due to the brief interview during consultation in the medical office. Therefore, the objective of the study was to build an app to collect data of patients regarding recovery after treatment in an informal and more comfortable environment.

Methods: The app was built using the IONIC Framework system and applied to patients through a prospective randomized study. We included 100 patients divided into two groups 1. Patients who used the app $(n=50) 2$. Patients who responded via validated printed questionnaires (control group) $(n=50)$. All patients received discharge counseling to respond to questionnaires $1,3,6$ and 12 months after the procedure. The app group after verbal guidance on how the monitoring would occur received an SMS with a user name and password allowing access to the system and sent alerts to users to respond the questionnaires.

Results: The app developed is called UroHealth and is available for download in the Apple store or at www.urohealth.com.br. The mean age of patients was 64.45 years-old $( \pm 8.33)$. Age, PSA, prostate volume, ISUP grade and pathological staging were
Table 1. Response rates for patients who used the app and for patients who responded via validated printed questionnaires

\begin{tabular}{|c|c|c|c|}
\hline \multicolumn{4}{|c|}{ Preoperative (\%) } \\
\hline & No & Yes & $\mathbf{p}$ \\
\hline App group & 56.3 & 43.8 & \multirow{2}{*}{0.003} \\
\hline Questionnaires group & 83.7 & 16.3 & \\
\hline \multicolumn{4}{|c|}{1 month (\%) } \\
\hline & No & Yes & $\mathbf{p}$ \\
\hline App group & 50.0 & 50.0 & \multirow{2}{*}{0.763} \\
\hline Questionnaires group & 46.9 & 53.1 & \\
\hline \multicolumn{4}{|c|}{3 months (\%) } \\
\hline & No & Yes & $\mathbf{p}$ \\
\hline App group & 57.8 & 42.2 & \multirow{2}{*}{0.419} \\
\hline Questionnaires group & 66.0 & 34.0 & \\
\hline \multicolumn{4}{|c|}{6 months (\%) } \\
\hline & No & Yes & $\mathbf{p}$ \\
\hline App group & 64.5 & 35.5 & \multirow{2}{*}{0.732} \\
\hline Questionnaires group & 68.4 & 31.6 & \\
\hline \multicolumn{4}{|c|}{12 months (\%) } \\
\hline & No & Yes & $\mathbf{p}$ \\
\hline App group & 71.4 & 28.6 & \multirow{2}{*}{0.390} \\
\hline Questionnaires group & 86.7 & 13.3 & \\
\hline
\end{tabular}

similar between groups. When we evaluated response rates, we found that $43.8 \%$ of the patients answered the preoperative questionnaire in the app group while $16.3 \%$ responded in the control group $(\mathrm{p}=0.003)$. The follow up in one, three, six and 12 months maintained higher response rates in the app group, but there was no statistical significance for any of them $(p>0.05)$ (table).

Conclusions: The app development, such as UroHealth may be of great value in monitoring patients after surgical procedures, allowing information of good quality regarding recovery and morbidity related to treatment. Our initial results indicate that it may become a useful tool in obtaining more frequent and realistic answers, helping the improvement of surgical techniques.

MP11-06 Hemostatic properties of Super Pulse Thulium Fiber (SPTF) LASER: Comparison with High Power 120W Holmium-YAG LASER (Ho:YAG) in an ex-vivo Porcine Kidney Model

\section{WR Molina, BH Chew, BE Knudsen}

University of Kansas Medical Center

Introduction \& Objective: The Holmium-YAG (Ho:YAG) Laser system is a well-established energy technology for tissue ablation and coagulation. The Super Pulse Thulium Fiber (SPTF) Laser has emerged as an attractive alternative. We aim to compare hemostatic properties of both systems in an ex-vivo reperfused porcine kidney model.

Methods: Porcine kidneys were re-perfused with blood using a closed-loop pressurized system. A $2 \mathrm{~cm}$ long incision on the kidney parenchyma was performed with a scalpel. Gauze was weighed before and after placement for 30 seconds on the incision. Hemostasis of the incision at $2 \mathrm{~mm} / \mathrm{s}$ was conducted using both SPTF and Ho:YAG lasers at default presets of $1 \mathrm{~J}, 20 \mathrm{~Hz}$, Long Pulse. 3 different fiber sizes were used for each platform (SPTF - 150, 365 and $940 \mathrm{~mm}$, Ho:YAG - 230, 365 and $955 \mathrm{~mm}$ ). Another pre-weighed gauze was placed for another $30 \mathrm{sec}$ at the incision site after the laser firing. Both gauzes were weighed, and the weight differences were calculated to determine the reduction in bleeding rate.

Results: Across all fiber sizes, SPTF achieved an average bleeding rate reduction of $73.4 \%$ vs $12.5 \%$ with the Ho:YAG 
Figure 1 - Overall Bleeding Reduction

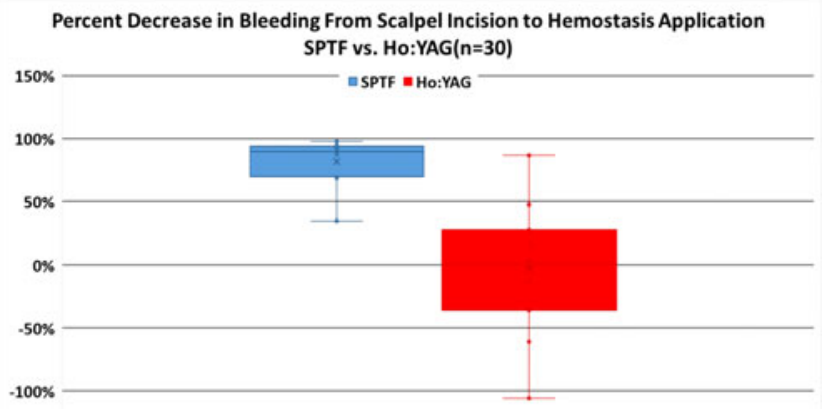

$-150 \%$

Figure 2 - Average Bleeding Reduction by Fiber Sizes

Percent Decrease in From Scalpel Incision to Hemostasis Application Ordered by Fiber Size

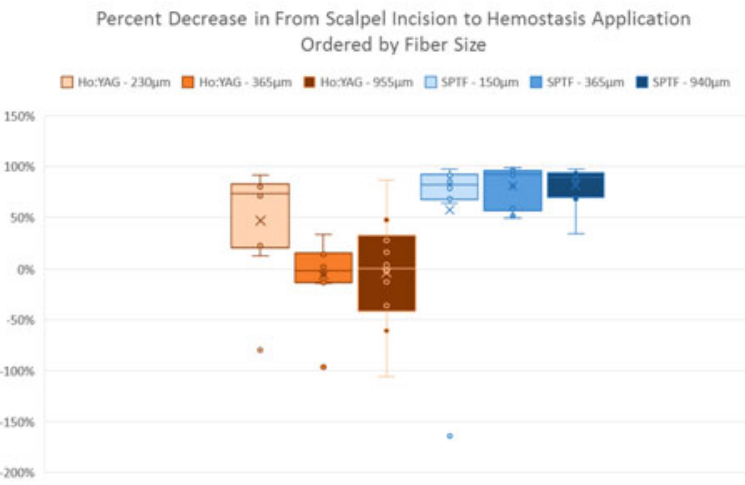

laser (Fig 1). For each fiber size, SPTF performs equivalently or better compared to Ho:YAG (Fig 2).

Conclusions: SPTF performs tissue hemostasis equal to or better than the Ho:YAG regardless of fiber sizes. The Ho:YAG laser had its best hemostasis performance when used with $230 \mathrm{~mm}$ fiber, but was still equivalent to SPTF.

\section{MP11-07 Initial Evaluation Of A Novel Modulated Radiofrequency-Based Bladder Denervation Device}

Z Okhunov, FA Jefferson, R Mao, RM Patel, S Tapiero, T Lee, G Ghoniem, G Li, J Landman

University of California, Irvine

Introduction \& Objective: We developed a novel ablation device (Denerblate) incorporating an intravesical balloon with selectively mounted electrodes, thermal and impedance sensors, a novel energy algorithm and insulation material for highly targeted and modulated radiofrequency ablation (RFA). We hypothesized that the application of carefully modulated RFA on the urinary bladder will reduce bladder nerve density which could result in a novel strategy in the management of overactive bladder (OAB).

Methods: We developed a prototype device with the RF antenna fabricated on an ultra-thin Teflon film ( $<20 \mathrm{um}$ in thickness) and mounted on the balloon (figure 1). A total of 15 domestic pigs were divided into four groups. Groups consisted of control $(n=3), 1$-week $(n=4), 4$-week $(n=4)$ and 12-week $(n=4)$ survival times. Denerblate was deployed on the trigone area of the bladder. Once the balloon was positioned, three 240-second cycles of modulated RFA were applied with 30 -seconds between cycles. All animals were euthanized at the end of their survival term, and the urinary bladders were harvested for histopathological evaluation. Transverse sections were made at $2 \mathrm{~mm}$ intervals from the distal urethra to the dome of the bladder. The nerve tissue of the ablated area was immunohistochemically identified using the neuronal marker, S100 protein. Nerve count and density were manually calculated, recorded and compared between the groups.

Results: All procedures were successfully completed, and all animals survived to the desired time points. The mean nerve density was highest in the control and 1-week survival group compared to the 4-week and 12-week survival groups, which demonstrated significant diminishment. The nerve density (nerves/mm2) in the bladder neck at control, 1 week, 4 weeks and 12 weeks were $1.8,1.35,0.87$ and 0.12 , respectively $(\mathrm{p}<0.001)$. Nerve density in bladder trigone area at control, 1 week, 4 weeks and 12 weeks were $1.5,0.98,0.65$ and 0.112 , respectively $(\mathrm{p}<0.001)$. Epithelial heat injury was observed in $14.3 \%$ at 1

Figure 1: The prototype device with the RF antenna fabricated on an ultra-thin Teflon film ( $<20$ um in thickness) and mounted on the balloon.

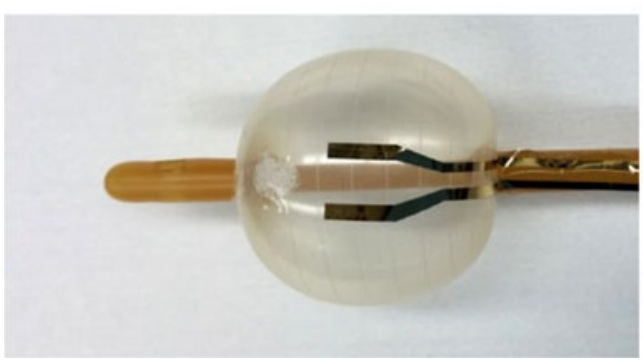

Table 1: Subjective histological assessment of epithelial injury at 1 week, 4 weeks and 12 weeks.

\begin{tabular}{|c|c|c|c|}
\hline Epithelial injury & $\cdot 1$ Week $(n-40)$ & 4 weeks $(n-40)$ & 12 weeks $(n-40)$ \\
\hline & $\%$ & $\%$ & $\%$ \\
\hline None & 19.0 & 66.7 & 77.7 \\
\hline Mild & 19.0 & 27.3 & 22.3 \\
\hline Moderate & 47.6 & 3.6 & 0.0 \\
\hline Severe & 14.3 & 2.4 & 0.0 \\
\hline Total & 100 & 100 & 100 \\
\hline \multirow[t]{2}{*}{$\begin{array}{c}\text { Epithelial } \\
\text { hemorrhage }\end{array}$} & 1 Week & 4 weeks & 12 weeks \\
\hline & $\%$ & $\%$ & $\%$ \\
\hline Absent & 66.7 & 85.7 & 100.0 \\
\hline Present & 33.3 & 14.3 & 0.0 \\
\hline Total & 100 & 100 & 100 \\
\hline \multirow[t]{2}{*}{$\begin{array}{c}\text { Nerve density } \\
\text { change }\end{array}$} & 1 Week & 4 weeks & 12 weeks \\
\hline & $\%$ & $\%$ & $\%$ \\
\hline No & 69.5 & 0.0 & 0.0 \\
\hline Yes & 30.5 & 100.0 & 100.0 \\
\hline Total & 100 & 100 & 100 \\
\hline \multirow[t]{2}{*}{$\begin{array}{l}\text { Nerve distribution } \\
\text { change }\end{array}$} & 1 Week & 4 weeks & 12 weeks \\
\hline & $\%$ & $\%$ & $\%$ \\
\hline Normal & 9.5 & 0.0 & 0.0 \\
\hline Mild loss & 71.4 & 100.0 & 100.0 \\
\hline Severe loss & 19.0 & 0.0 & 0.0 \\
\hline Total & 100 & 100 & 100 \\
\hline
\end{tabular}

* animals in each group; 10 histological slides per animal 
week, $10.7 \%$ at 4 weeks and completely resolved at 12 -week survival groups.

Conclusions: We successfully constructed and deployed a transurethral device and an associated energy algorithm for highly targeted delivery of modulated RFA to the bladder. Denerblate reduced nerve density in the bladder neck and trigone by $88.6 \%$ and $88.9 \%$ at 12 -week with initial moderate epithelial injury, which completely resolved in all animals at 12-week of follow-up.

MP11-08 Immersive Virtual Reality (iVR) Renal Models as an Educational and Preoperative Planning Tool for Laparoscopic Donor Nephrectomy: Initial Experience

FA Jefferson, E Parkhomenko, M O’Leary, J Sung, L Limfueco, S Tapiero, RM Patel, H Ichii, D Dafoe, J Landman

University of California, Irvine

Introduction \& Objective: It is challenging for surgeons to assimilate the more than 2,500 two-dimensional images provided by computed tomography (CT) into a 3D understanding of the renal anatomy optimal for laparoscopic donor nephrectomy (LDN). Immersive virtual reality (iVR) may enhance preoperative planning by allowing for an interactive, 3D immersive experience with the renal anatomy prior to LDN.

Methods: Fifteen patients scheduled for LDN were recruited. Using 3D Slicer, CT scans were translated into 3D models, which were viewed with a head-mounted display (Figure 1). Two surgeons assessed their preoperative understanding of the anatomy using CT alone vs. CT with iVR and performed a postoperative assessment rating the correlation of CT vs. iVR with the anatomy $(0=$ poor to $10=$ excellent $)$. Patients used a Likert-type scale to assess their comprehension and anxiety about their surgery before and after viewing the iVR. Patient outcomes were assessed by retrospectively matching the iVR cohort with a non-iVR cohort of LDN patients. Matching was based on age, BMI, ASA score, operating surgeon, and laparoscopic (LAP) vs. handassisted laparoscopic (HA) techniques.

Results: Preoperatively, iVR models enhanced surgeons' understanding of a patient's arterial $(9.9$ vs. $7.4, p<0.001)$ and venous $(9.9$ vs. $7.1, \mathrm{p}<0.001)$ anatomy and improved their overall confidence regarding the operation $(10$ vs. $7.6, p<0.001)$ vs. CT alone. Preoperative viewing of iVR altered the surgical approach in $14(93 \%)$ cases. Patients agreed that iVR decreased

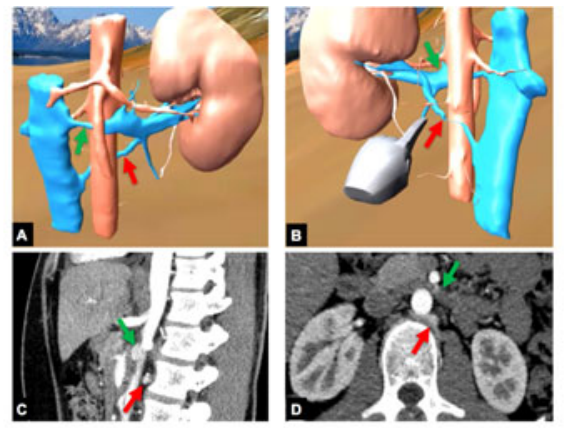

Figure 1. (A-B): iVR model of laparoscopic donor nephrectomy patient. (C-D): CT images of the same patient. A) Anterior coronal view of the anatomy demonstrating a circumaortic branch of the left renal vein as it passes anterior (green arrow) and posterior (red arrow) to the aorta. B) Posterior coronal view of anatomy with virtual hand pointing to retroaortic component of renal vein. C) Sagittal view showing branching of circumaortic left renal vein. D) Axial view.
.
Table 1. Patient characteristics and surgical outcomes by surgical approach.

\begin{tabular}{|l|c|c|c|c|c|c|}
\hline & \multicolumn{3}{|c|}{ Laparoscopic } & \multicolumn{3}{c|}{ Hand-Assisted Laparoscopic } \\
\hline & $\begin{array}{c}\text { Control } \\
n=14\end{array}$ & $\begin{array}{c}\text { iVR } \\
n=9\end{array}$ & P & $\begin{array}{c}\text { Control } \\
n=30\end{array}$ & $\begin{array}{c}\text { iVR } \\
n=6\end{array}$ & p \\
\hline Age & $38.8( \pm 11.7)$ & $46.0( \pm 10.0)$ & 0.14 & $40.2( \pm 12.4)$ & $45.3( \pm 18.3)$ & 0.40 \\
\hline Male/Female & $6 / 8$ & $3 / 6$ & 1.0 & $9 / 21$ & $5 / 1$ & 0.02 \\
\hline BMI & $24.9( \pm 3.3)$ & $26.6( \pm 2.1)$ & 0.13 & $25.6( \pm 3.3)$ & $25.0( \pm 2.5)$ & 0.63 \\
\hline ASA score & $1.4( \pm 0.5)$ & $1.6( \pm 0.5)$ & 0.37 & $1.4( \pm 0.6)$ & $1.7(0.5)$ & 0.32 \\
\hline Laterality (L/R) & $13 / 1$ & $8 / 1$ & 1.0 & $30 / 0$ & $5 / 1$ & 0.17 \\
\hline $\begin{array}{l}\text { Operative time } \\
\text { (min) }\end{array}$ & $192( \pm 45)$ & $161( \pm 22)$ & 0.04 & $284( \pm 53)$ & $225( \pm 21)$ & $<0.001$ \\
\hline EBL (ec) & $36.1( \pm 24.3)$ & $24.3( \pm 12.7)$ & 0.16 & $60.7( \pm 54.3)$ & $46.7( \pm 28.8)$ & 0.38 \\
\hline $\begin{array}{l}\text { Intraoperative } \\
\text { complications }\end{array}$ & 0 & 0 & 1.0 & $1(3 \%)^{\mathrm{t}}$ & 0 & 1.0 \\
\hline $\begin{array}{l}\text { 30-day } \\
\text { postoperative } \\
\text { complications }\end{array}$ & $2(14.3 \%)^{\mathrm{b}}$ & 0 & 0.50 & $8(27 \%)^{6}$ & $3(50 \%)^{\mathrm{d}}$ & 0.34 \\
\hline
\end{tabular}

Data expressed as mean $( \pm \mathrm{SD})$

Adrenal vein clip malfunction leading to increased bleeding

'Clavien grade 1

Six Clavien grade 1; one Clavien grade 2; one Clavien grade 3a

Two Clavien grade 1; one Clavien grade 2

R = immersive virtual reality; BMI = body mass index; ASA - American Society of Anesthesiologists; EBL estimated blood loss

their apprehension regarding their surgery (4.4/5). Compared to the matched cohort $(n=44)$, the iVR group had a $16 \%(p=0.04)$ reduction in operative time for LAP and a $21 \%(\mathrm{p}<0.001)$ reduction in operative time for HA approaches (Table 1). There were no significant differences in estimated blood loss nor operative or postoperative complications.

Conclusions: Initial experience with iVR prior to LDN improved the surgeon's understanding of the renal anatomy and was associated with a reduction in operative time. Furthermore, iVR improved patients' understanding of the procedure and reduced their anxiety.

MP11-09 Comparing the utilization trends and 90day outcomes for open, laparoscopic, and robotassisted radical nephrectomy cases in New York State between 2009-2014

KH Bilal, M Finkelstein, E Kaplan-Marans, M Palese

Mount Sinai Medical System

Introduction \& Objective: The aim of the study was to compare the utilization trends and 90-day post-operative outcomes of open (ORN), laparoscopic (LRN), and robot-assisted radical nephrectomy (RRN) in New York State between 2009-2014.

Methods: The Statewide Planning and Research Cooperative System database was used to conduct a retrospective cohort study of 16,533 patients undergoing radical nephrectomy (ICD-9 55.5) for treatment of for renal cell carcinoma (189.0) between 2009-2014. Patients with a minimally invasive modifier (54.21, $54.51)$ without the robot-assisted modifier (17.4X) were categorized in the laparoscopic cohort. Logistic regression was performed to assess 90-day outcomes by procedure type using ORN as control.

Results: Of the 16,533 patients, $64.6 \%(10,686 / 16,533)$ underwent ORN, $24.1 \%(3,968 / 16,533) \mathrm{LRN}$, and $11.3 \%(1,879 /$ 16,533) RRN. From 2009 to 2012, the proportion of patients treated with RRN increased significantly from $8 \%$ in 2009 to $23 \%$ in 2014. In comparison, ORN cases decreased from $70.9 \%$ in 2009 to $57.3 \%$ in 2014. Proportion of patients treated with 
LRN remained relatively unchanged during the observation period. When comparing 90-day outcomes, in comparison to ORN patients, patients undergoing RRN and LRN procedures were significantly less likely to experience a prolonged length of stay (OR 0.437, p $<0.0005$ and OR 0.491, p <0.0005), had a lower risk of requiring blood transfusion (OR $0.713, \mathrm{p}<0.0005$ and $\mathrm{OR}$ $0.587 \mathrm{p}<0.0005)$, and a lower risk sepsis in 90-day post-op pe$\operatorname{riod}(\mathrm{OR} 0.641, \mathrm{p}<0.0005$ and $0.692, \mathrm{p}<0.0005)$. Patients who underwent RRN also had a lower risk of inpatient readmission in the 90-day post-op period (OR 0.701, p<0.0005). RRN cases were associated with higher average costs when compared to ORN, however but there was no significant difference in cost between ORN and LRN cases.

Conclusions: Our results show that over the observation period, robot assisted procedures have increased in prevalence while during the same period, open radical nephrectomies have decreased in prevalence. The proportion of radical nephrectomies performed laparoscopically has remained relatively unchanged. When comparing outcomes in the 90-day post-op period, minimally invasive robotic and laparoscopic procedures confer lower risk to patients regarding prolonged length of stay, need for blood transfusion, and diagnosis of sepsis. Patients who underwent robot assisted radical nephrectomy were also at lower risk of requiring an inpatient admission within 90-days following the initial procedure.

MP11-10 Concomitant treatment of ureteropelvic junction obstruction and complicated renal calculi with laparoscopic pyeloplasy and $19.5 \mathrm{~F}$ rigid nephroscopy

\section{An, L Xiong, X Huang}

Peking University Applied Lithotripsy Institute/Peking University People's Hospital Urology and Lithotripsy Center

Introduction \& Objective: Concomitant treatment of ureteropelvic junction obstruction (UPJO) and complicated renal calculi with laparoscopic pyeloplasty and pyelolithotomy via flexible cystoscope or $9.8 \mathrm{~F}$ rigid ureteroscope have been reported. While we found that $19.5 \mathrm{~F}$ rigid nephroscope was more efficient. The aim of this study was to introduce our experience of laparoscopic pyeloplasy and $19.5 \mathrm{~F}$ rigid nephroscopy.

Methods: 10 patients underwent laparoscopic pyeloplasy and 19.5F rigid nephroscopy between April 2016 and September 2018. The pivotal procedures were described below: Dissociated dilated pelvis and performed a small pyelotomy, $19.5 \mathrm{~F}$ rigid nephroscope passed through the assistant port and the previously created pyelotomy to get into pelvis, pyelolithotomy or litho-

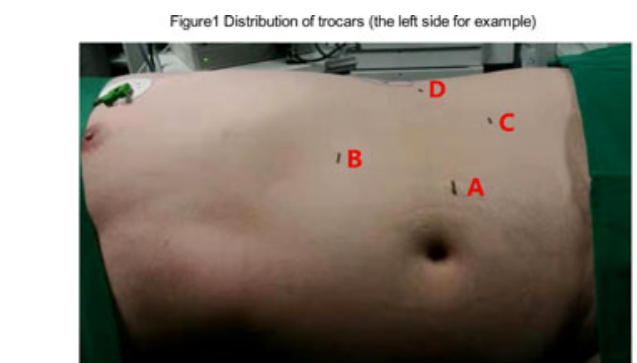

A : $3 \mathrm{~cm}$ lateral to the umbilicus at the lower margin of the umbilicus $(\varphi=10 \mathrm{~mm})$ B: $20 \mathrm{~mm}$ inferior to the costal margin at the lateral border of the left rectus muscle $(\phi=12 \mathrm{~mm}) \mathrm{C}: 6-8 \mathrm{~cm}$ lateral and superior to trocar $A(\varphi=12 \mathrm{~mm}) D$ : lateral to the umbilicus of the left collarbone midine.
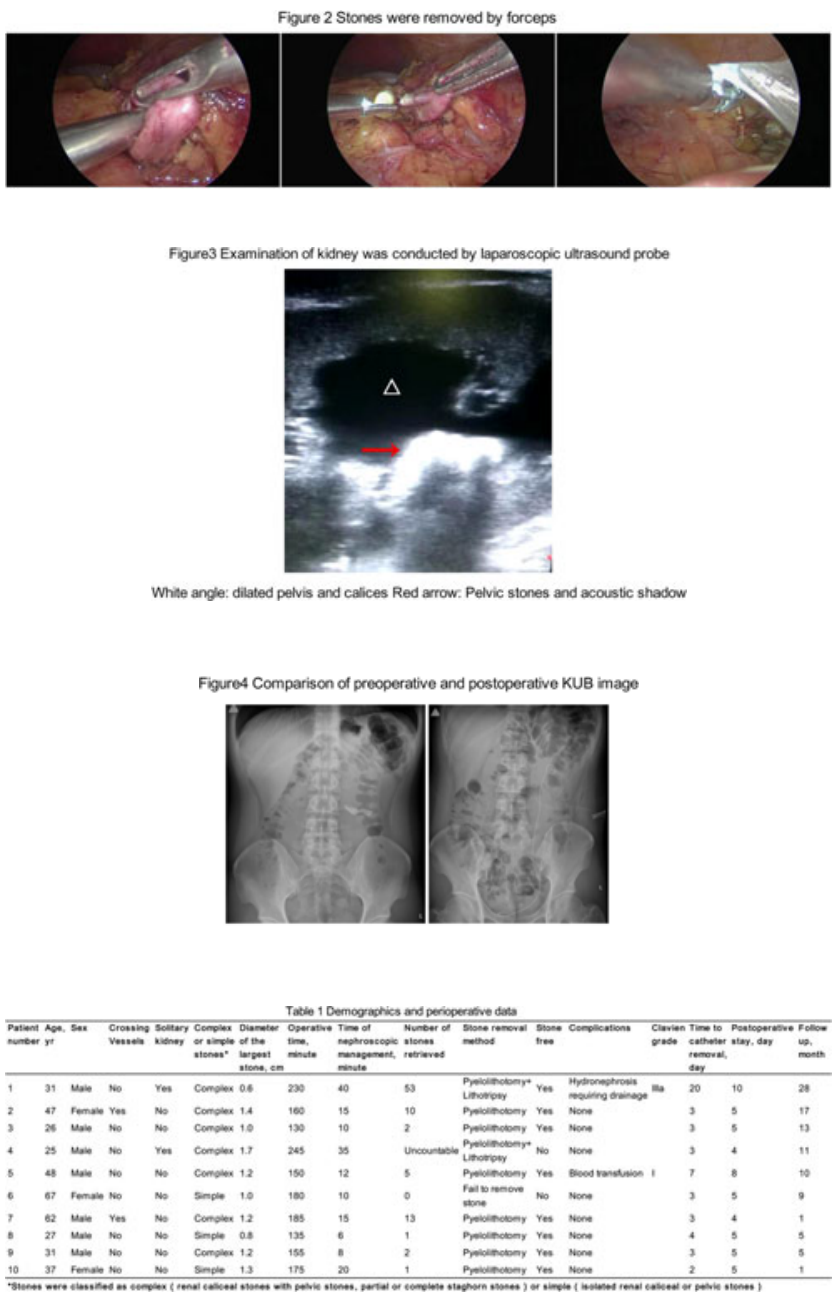

tripsy via nephroscopy were conducted. Data of the patients were analyzed.

Results: Of 10 patients, 7 patients suffered complex stones, 2 patients had anatomical solitary kidney. The mean diameter of the largest stone was $1.1 \pm 0.31 \mathrm{~cm}$. The mean operative time was $174.5 \pm 37.9 \mathrm{~min}$, the mean time of nephroscopic management was $17.1 \pm 11.5 \mathrm{~min}, 7$ patients received pyelolithotomy by rigid grasper and 2 patients received ultrasonic lithotripsy, the mean number of stones retrieved was $9.7 \pm 16.9$. Overall stone-free rate was $80 \%$. The mean time to catheter removal was $5.1 \pm 5.4 \mathrm{~d}$, and the mean postoperative stay was $5.6 \pm 1.9 \mathrm{~d}$. Complications included blood transfusion in one case and hydronephrosis requiring drainage in another case. At the mean follow-up of 10.0 \pm 8.1 months, no patients had obstruction or recurrent stones.

Conclusions: Laparoscopic pyeloplasty and 19.5F rigid nephroscopy is a safe and feasible option for the concomitant treatment of UPJO complicated with renal calculi.

MP11-11 Microwave assisted, off-clamp no renorrhaphy laparoscopic partial nephrectomy in a porcine model - A feasibility study

AK Chow, A Sivaraman, M Talcottt, S Greco, S Arafenian, $\mathrm{R}$ Venkatesh

Washington University School of Medicine, St. Louis, USA 
Introduction \& Objective: With continued rise in the number of robotic partial nephrectomies for small renal mass, an effective technique that prevents global renal ischemia without parenchymal renorrhaphy is lacking for laparoscopic or robotic use. We performed an off clamp, no renorrhaphy partial nephrectomy using a needle microwave technology and compared to standard on-clamp sliding-clip renorrhaphy partial nephrectomy.

Methods: In this acute non-survival study, we performed a microwave assisted, off-clamp laparoscopic lower pole partial nephrectomy (MAPN) in 6 renal units in 3 domestic adult pigs (approx. 25-30 kg.) and standard on-clamp sutured, laparoscopic partial nephrectomy (ONPN) in 2 renal units/1 pig. Renal resection was performed by laparoscopic shears after coagulating the line of resection ("cook \& cut") with a $17 \mathrm{G}, 22 \mathrm{~cm}$ percutaneous microwave probe (NEUWAVETM, Madison WI) at a setting of $90 \mathrm{~W}$ for $30 \mathrm{sec}$ or $45 \mathrm{sec}$ pulses in 3 renal units each respectively. Intra-operative laparoscopic ultrasound (IOUS) was used to map intra-renal vasculature and selective ablation of segmental vessels. Finally, bilateral nephrectomies were performed and integrity of the collecting systems (PCS) was verified using Encore ${ }^{\mathrm{TM}} 26$ pressure gauge by cannulating the ureter. Specimens were sent for histopathology evaluation for collateral tissue damage.

Results: All 6 MAPN without clamping was completed successfully with good hemostasis. In the ONPN group, mean warm ischemia time was $25 \mathrm{~min}$ while mean MAPN resection time was 12min. Mean EBL in MAPN and ONPN was 171(30 - 400) cc vs. $50(20-80)$ cc respectively. IOUS was used to identify cortical $0.5 \mathrm{~mm}$ intra-renal arteries and coagulate $0.8-1 \mathrm{~mm}$ diameter deeper vessels, including open arterial bleeders. Post resection PCS integrity showed MAPN leaks at $<0.5$ atm. while ONPN leaked at $1 \mathrm{~atm}$. Microscopically, the resection margin in MAPN kidneys demonstrated marked coagulative necrosis and degeneration of tubules, glomeruli and adjacent vessel up to a mean distance of $1.4 \mathrm{~cm}(0.6-1.8)$, while such effects were minimal in ONPN kidneys with $0.15 \mathrm{~cm}$ tissue damage.

Conclusions: Microwave assisted, off-clamp partial nephrectomy appears a feasible simple technique, \& intra-operative doppler ultrasound can assist in intra-renal vasculature mapping for precise coagulation and better hemostasis. Compared to on-clamp sutured partial renal resections, MAPN resection times were shorter albeit with higher collateral tissue damage. Variation in microwave energy settings with and probe modification can minimize collateral tissue damage for clinical application.

\section{MP11-12 Initial experience with Robotized Laparo- scopy at single center}

K Ramaswami, P Bhirud, R Raveendran, H Pothiyedath

Metromed Institute of Advanced Urology and Renal Translplant

Introduction \& Objective: Minimally invasive surgical approaches have proven to reduce abdominal incisions, bringing less patient discomfort and aesthetical benefits, shorter time of surgery and decreased recovery time. Robotic surgery gave the maximum advancement and comfort to the operating surgeon with good result and its main drawback is the high cost of investment and maintenance. Robotized laparoscopy with the $3 \mathrm{D}$ vision is a new alternative which is providing the benefits of robotic surgery at a lower cost and good result. We share the techniques, outcomes, feasibility and experience of 121 cases done with robotized laparoscopic instruments.
Methods: From March 2017 to April 2019, patients were admitted for Robotized laparoscopic surgery in our institute. All of them were explained regarding robotized laparoscopic surgery. This is a retrospective study based on medical records. All patients were operated in proper surgical room with 3D laparoscopic system. Time required for complete surgery and other parameters related to respected procedures such as ischemia time for partial nephrectomy or anastomosis time for radical prostatectomy and pyeloplasty were recorded. Perioperative details were recorded.

Results: This is a retrospective analysis of 121 cases of various urology procedures using robotized laparoscopy operated during March 2017 to April 2019 and follow up period from 1-24 months. Cases included Radical prostatectomy (15), Simple prostatectomy (2), Partial nephrectomy (12), ureteric reimplant (4), ureteroureterostomy (8), ureterolithotomy (16), Pyelolithotomy (10), Nephropexy (2), Pyeloplasty (22), Anatropic

\begin{tabular}{|l|l|l|l|l|l|}
\hline $\begin{array}{l}\text { Sr } \\
\text { No }\end{array}$ & Surgery & No of cases & Males & Females & $\begin{array}{l}\text { Mean Age } \\
\text { (Years) }\end{array}$ \\
\hline 1 & Radical Prostatectomy & 15 & 15 & 0 & $66.3+/-5.3$ \\
\hline 2 & Simple prostatectomy & 2 & 2 & 0 & 65.5 \\
\hline 3 & Partial Nephrectomy & 12 & 8 & 4 & $52.2+/-4.2$ \\
\hline 4 & Ureteric reimplantation & 4 & 2 & 2 & 32.4 \\
\hline 5 & Uretero-ureterostomy & 8 & 4 & 4 & $36.7+/-5.3$ \\
\hline 6 & Ureterolthotomy & 16 & 11 & 5 & $39.4+/-5.1$ \\
\hline 7 & Pyelolithotomy & 10 & 7 & 3 & $42.5+/-6.1$ \\
\hline 8 & Nephropexy & 2 & 0 & 2 & 43 \\
\hline 9 & Pyeloplasty & 22 & 8 & 14 & $38.5+/-5.3$ \\
\hline 10 & Anatrophic nephrolthotomy & 3 & 1 & 2 & 42.3 \\
\hline 11 & Davis intubatedureterostomy & 2 & 1 & 1 & 41.2 \\
\hline 12 & RadicalCystectomy & 2 & 2 & 0 & 62.1 \\
\hline 13 & PartialCystectomy & 1 & 1 & 0 & 54 \\
\hline 14 & Sacrocolpopery & 3 & 0 & 3 & $53.2+/-4.1$ \\
\hline 15 & Testicular vein ligation & 19 & 19 & 0 & $32.5+/-5.2$ \\
\hline
\end{tabular}

\begin{tabular}{|l|l|}
\hline PARTIAL NEPHRECTOMY & \\
\hline Total no of cases & 12 \\
\hline Males: Females & $8: 4$ \\
\hline Right : Left & $5: 7$ \\
\hline Mean Age (Years) & $52.2+/-4.2$ \\
\hline Mean Ischemic time (Min) & $23.4+/-3.6$ \\
\hline Mean operative duration (Min) & $80.6+/-12.3$ \\
\hline Mean Hospital Stay (Days) & $5.4+/-2.1$ \\
\hline Major complications & Pseudoaneyrysm - (Angioembolization) \\
\hline
\end{tabular}

\begin{tabular}{|l|l|}
\hline Radical Prostatectomy & 15 \\
\hline Total number of cases & $66.3+/-5.3$ \\
\hline Mean Age (Years) & $175.5+/-21.2$ \\
\hline Mean Operative Duration (Min) & $25.2+/-5.3$ \\
\hline Mean Anastomosis Duration (Min) & 14 \\
\hline Foley Catheter Duration (Days) & $5.8+/-2.4$ \\
\hline Mean Hospital Stay Duration (Days) & Intravenous Paracetamol for 48 to 72 \\
\hline Post operative Anagesia & hours \\
\hline Major Complications & Nil \\
\hline Continence rate at 1 month & $100 \%$ \\
\hline Transient Incontinence (Immediate) & $55 \%$ \\
\hline Post operative PSA at 1 month & $<0.1$ \\
\hline
\end{tabular}




\begin{tabular}{|l|l|}
\hline Pyeloplasty & 22 \\
\hline Total no of cases & $8: 14$ \\
\hline Males: Females & $38.5+/-5.3$ \\
\hline Mean Age (Years) & $6: 16$ \\
\hline Right: Left & 18 \\
\hline $\begin{array}{l}\text { TYPES } \\
\text { Anderson-Hynes } \\
\text { Y-V }\end{array}$ & 4 \\
\hline Mean anastomosis duration (Min) & $13.4+/-3.5$ \\
\hline Mean operative duration (Min) & $65.3+/-6.4$ \\
\hline DJ Stent & All cases \\
\hline Mean hospital stay (Days) & $4.2+/-2.1$ \\
\hline Post operative DTPA scan & No obstruction in all cases. \\
\hline Need for RevisionSurgery & Nil \\
\hline
\end{tabular}

nephrolithotomy (3), davis intubated ureterostomy (2), Radical cystectomy (2), Partial cystectomy (1), sacrocolpopexy (3), Testicular vein ligation (19). 3 cases were children below 10 yrs. The operating time and blood loss, hospital stay was similar to standard laparoscopic procedures but with more comfort for the surgeon of our own series. No major complication, conversion to open, blood transfusion, instrument malfunction was not observed in procedures done using robotized laparoscopic surgery. Ergonomically robotized laparoscopy instruments are far superior providing higher level of comfort to the operating surgeon, especially during the advanced tasks like scissor dissection and cutting in difficult angles, suturing and knotting which are involved in reconstructive procedures.

Conclusions: Robotic assistance is an adjunct to threedimension laparoscopy, being far superior ergonomically.

MP11-13 Non-Ischemic or Ischemic Laparoscopic Partial Nephrectomy Using a Newly Developed Hybrid Pencil Type Energy Device in a Porcine Model

K Nakagawa, S Irie, H Nishimatsu, J Inokuchi, M Eto, M Tanaka

Tokyo Dental College Ichikawa General Hospital

Introduction \& Objective: To compare the efficacy of a newly developed hybrid pencil type energy device (HD; Olympus Corp., Tokyo, Japan) generating simultaneously monopolar high-frequency electric energy and ultrasonic energy, with that of conventional device (CD) during laparoscopic partial nephrectomy (LPN).

Methods: A total of 16 female pigs were divided into four equal groups: non-ischemic and ischemic HD-LPN groups and nonischemic and ischemic CD-LPN groups. We performed bilateral LPN for each pig. HD alone was used in the HD-LPN group, while commercially available monopolar scissors and a soft coagulation system were used in the CD-LPN group. After observing the postoperative course for 14 days, we euthanized the animals and harvested the kidneys for histopathological observations.

Results: We completed an LPN on a total of 32 kidneys. There were no conversion cases to open surgery, nor were there any deaths or complications requiring treatment. For non-ischemic LPN, LPN time was significantly shorter in the HD-LPN group than in the CD-LPN group $(\mathrm{P}<0.05)$. Decrease in postoperative hemoglobin was equally low level in both groups. However, TachoSil $^{\circledR}$ was used significantly more often in the CD-LPN group $(\mathrm{P}<0.01)$. For ischemic $\mathrm{LPN}$, we found no significant differences in parameters such as LPN time between the 2 groups. The depth of thermal injury was most superficial in the non-ischemic HD-LPN group in comparison with other 3 groups. Conclusions: The use of a newly developed HD allowed nonischemic LPN to be performed safely in a short time with minimal blood loss in an animal model. It is expected that the HD can provide the superior non-ischemic LPN in actual clinical settings.

\section{MP11-14 In Vivo Porcine Evaluation of a Novel Self- Contained Bladder Irrigation System (MultiphzeTM)}

P Jiang, RM Patel, S Tapiero, J Landman, RV Clayman

UC Irvine Urology

Introduction \& Objective: Current standard bladder irrigation (SBI) methods are tedious and pose a biohazard risk due to spillage of blood and urine on the patient, environment, and provider. The Multiphze ${ }^{\mathrm{TM}}$ enclosed irrigation system (MIS) (Multiphze LLC), a newly developed self-contained system (Figure 1), was designed to eliminate spillage while improving the efficiency of clot evacuation.

Methods: While under inhalation anesthesia, two female, juvenile pigs were each injected with $100 \mathrm{mg}$ IV fluorescein. Via cardiac puncture, $60 \mathrm{cc}$ of blood was withdrawn and then instilled into the pig's bladder via a 24Fr 3-way catheter. After 10 minutes, 3 urologists (i.e. chief resident, endourology fellow, and junior faculty), with experience using the standard Piston Irrigation Tray (Bard Medical) each performed bladder irrigation with $4 \mathrm{~L}$ of sterile water; each operator performed a trial with the standard technique as well as with the Multiphze ${ }^{\mathrm{TM}}$ system. Time of each irrigation cycle and clarity of drainage fluid using a spectrophotometer were recorded after each liter of irrigation. Total areas of spillage on the procedural field and on the operator were identified with a Wood's lamp and then quantified.

Results: The mean irrigation time for the 4 liters of water with MIS and SBI were 17.9 and 23.1 minutes $(p=0.035)$, respectively (Table 1 ). The mean clarity measurements at the end of each trial were similar for both MIS and SBI: $82.7 \%$ and $82.9 \%$, respectively. Wood's lamp illumination revealed $98 \%$ reduction of spillage with MIS compared to SBI: mean spillage area of $19.8 \mathrm{~cm}^{2}$ and $1001.3 \mathrm{~cm}^{2}$, respectively $(p=0.034)$. Despite users having no experience with the novel system, the MIS was intuitive to use and all operators had a reduction in irrigation time (range 2 to 9 minutes).

Figure 1. Multiphze ${ }^{\mathrm{TM}}$ system diagram

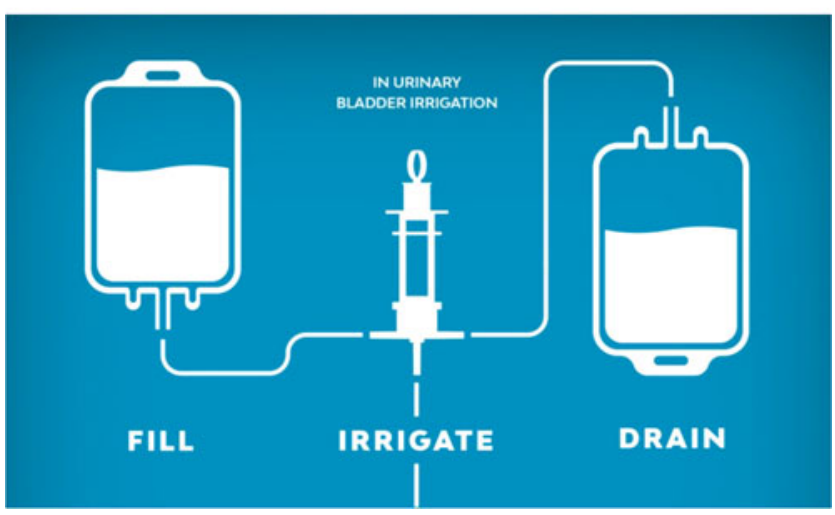


Table 1. Mean irrigation times among all operators

\begin{tabular}{|c|c|c|c|}
\hline & $\begin{array}{c}\text { Standard } \\
\text { (SBI) }\end{array}$ & $\begin{array}{c}\text { Multiphze } \\
\text { (MIS) }\end{array}$ & \\
\hline $\begin{array}{c}\text { Time to } \\
\text { irrigate 1 L } \\
\text { (min) }\end{array}$ & 5.8 & 4.5 & \\
\hline $\begin{array}{c}\text { Time to } \\
\text { irrigate 4 L } \\
\text { (min) }\end{array}$ & $23.1-7.4]$ & {$[3.7-5.5]$} & $\mathrm{p}<0.001$ \\
\hline
\end{tabular}

Conclusions: The newly developed Multiphze ${ }^{\mathrm{TM}}$ irrigation system reduced the time to successfully clear a clot filled bladder by $22 \%$ in a porcine model. Furthermore, the enclosed system demonstrated a $98 \%$ reduction in spillage of contaminated irrigant.

\section{MP11-15 A pilot study of the diagnostic accuracy using fluorescence confocal microscopy for urothelial carci- noma of the bladder during transurethral resection}

B Becker, E von Rohden, A Gross, B Feyerabend, D Scholz, C Netsch

Departement of Urology, Asklepios Hospital Barmbek, Hamburg, Germany

Introduction \& Objective: Real-time histology plays an increasingly important role in modern medicine. Flourescence Confocal Microscopy (FCM) is already well-established in differential diagnosis of skin lesions. However, FCM has yet not been tested during transurethral resection of bladder tumors (TURBT) for the detection of urothelial carcinoma. FCM is a novel technology that allows the surgeon to obtain instant digital images of freshly resected tissue without losing the specimens' architecture. The images are digitally developed mimicking Hematoxyclin \& Eosin staining. This study aimed to determine the diagnostic accuracy of FCM on the detection of urothelial carcinoma of the bladder during TURBT.

Methods: We prospectively collected specimens of 50 patients during TURBT with suspected urothelial cancer or during second-look procedure. For FCM, all samples were analyzed with the Mavig VivaScope ${ }^{\circledR}$ directly after surgery. The time from preparing the tissue sample to the storage of the picture is approximately three minutes. All images were digitalized and examined by our pathologist and urologist in a blinded manner. The diagnosis after FCM was compared to the final histopathology. Results: In 46 of the patients, one specimen was obtained for FCM and in 6 patients two specimens were used for diagnosed with FCM. Figure 1 shows an example of FCM with detection of urothelial carcinoma (marked with an arrow) next to physiological bladder mucosa. Of the 50 specimens included in analysis, urothelial carcinoma was present in 24 cases. In this study, FCM showed high rates of sensitivity and specificity in accordance to final histopathology. Furthermore, we have noticed an excellent intra- and interobserver reliability of the correct diagnosis using FCM.

Conclusions: Our data suggest that FCM leads to a high diagnostic accuracy for immediate diagnosis during TURBT. This might help to improve the procedures quality and might also lead

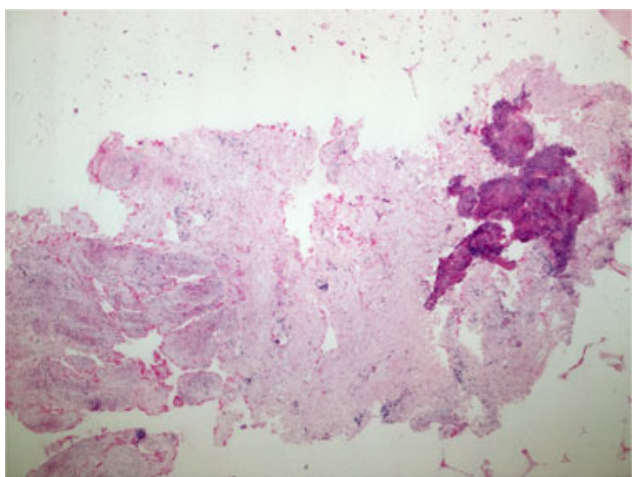

to less need of second-look TURBT due to guaranteed R0resection. However, further studies, especially for evaluation of the tumor grading are necessary to confirm the usefulness of FCM to detect bladder tumors.

\section{MP11-16 Evaluation of The Robotic Camera Holder on The Learning Curve for Basic Laparoscopic Skill: A Randomised Control Trial}

MS Amin, N Abbud, A Aydin, B Van Cleynenbreugel, D Venenziano, BK Somani, AS Gözen, JP Redorta, M Khan, P Dasgupta, J Makanjuola, K Ahmed

MRC Centre for Transplantation, King's College London, London, UK

Introduction \& Objective: Use of technological adjuncts forms the basis of modern surgical development yet barriers such as cost, and training are still common. The use of technology enhanced devices such as automated camera holders may improve ergonomic and performance benefits. Furthermore, the advantage given by making the camera assistant redundant is useful for surgical trainees as they can now actively assist in procedures. The objective of this study was to evaluate the use of the FreeHand ${ }^{\circledR}$ (Freehand 2010 Limited, Guildford, United Kingdom) camera holder on basic laparoscopic skill (BLS) compared to the conventional practice of using a human camera holder $(\mathrm{HCH})$.

Methods: Novices naive in laparoscopic skills were recruited and block randomized into 2 groups. The control group used the conventional practice of a $\mathrm{HCH}$ and the experimental group used the FreeHand ${ }^{\circledR}$ robotic camera holder $(\mathrm{RCH})$. BLS was determined by performance in laparoscopic suturing, a task from the European training in basic laparoscopic skill (E-BLUS) curriculum. Performance was measured using a performance improvement score (Pi-score) in comparison to E-BLUS competency criteria. Pi-scores were recorded from ten training sessions and used to map a learning curve to identify when competency was first reached.

Results: Novices $(n=40)$ were randomized into two groups $(n=20)$ with an allocation ratio of 1:1 with intention-to-treat analysis. 2 participants completed the ten sessions from the $\mathrm{RCH}$ group and 6 completed the sessions from the $\mathrm{HCH}$ group. The $\mathrm{RCH}$ group took an average of 2.6 sessions to reach competency compared to the 6.5 sessions needed for the $\mathrm{HCH}$ group which was significantly faster $(\mathrm{P}=0.0066)$. The learning curve showed both groups continued to increase in skill as time elapsed, but the $\mathrm{RCH}$ group's rate of increase and overall skill achieved was much greater. 


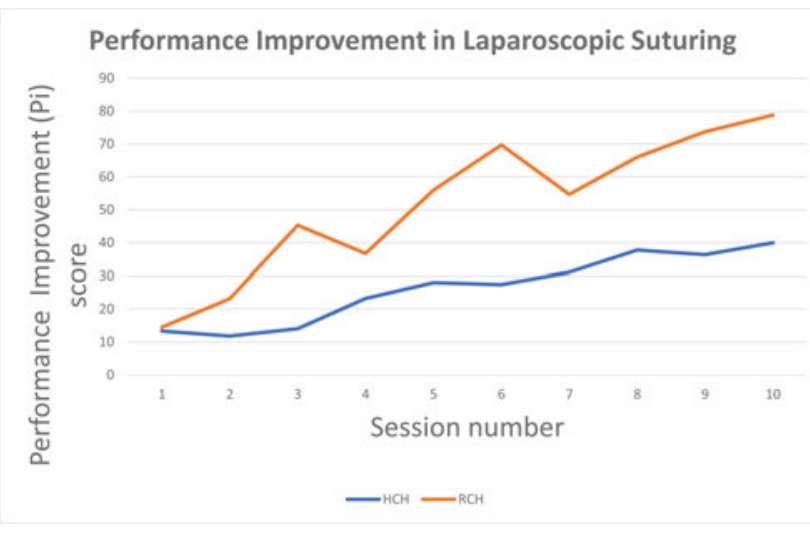

Conclusions: The group using the $\mathrm{RCH}$ performed significantly faster in the suturing task indicating potential benefit to operative outcomes e.g. duration and surgeon comfort in complex operations. These findings warrant future studies to evaluate intraoperative benefits of RCHs during complex tasks including suturing. More data is required to increase accuracy of results.

\section{MP11-17 Thulium-Holmium:YAG laser in conserva- tive treatment of upper tract urothelial cancer: 13 years-experience from a tertiary national referral center}

L Defidio, M Antonucci, M De Dominicis, N Russo, G Fuchs, A Patel

Introduction \& Objective: The study aimed to evaluate the safety and efficacy of Thulium-Holmium:YAG Duo laser as an ablative tool in retrograde intra-renal upper tract urothelial carcinoma (UTUC) conservative treatment. Use of Thulium laser was demonstrated might improve endo-visibility through photothermal coagulation, better fiber tip stability and less penetrative energy capability.

Methods: A retrospective study was performed on 178 consecutive patients who underwent uretero-renoscopy and renal conserving RIRS UTUC-eLA in a single national tertiary referral center (Dept. of Urology, "Cristo Re" Hospital, Rome, Italy), between January 2005 and December 2018. Using a standardized study proforma, key data including patients' demographic data were recorded.

Results: At first planned endo-surveillance follow up (136 pts), 51 patients $(37.5 \%)$ were recurrence-free, 50 patients $(36.8 \%)$ had recurrences, and 35 patients $(25.7 \%)$ underwent RNU and dropped out. At last follow-up (mean 28.7 mo.) a total 70 patients $(51.5 \%)$ were recurrence-free, 22 patients $(16 \%)$ had multiple recurrences treated conservatively, and a further 9 making a total of 44 patients $(32.5 \%)$ had undergone RNU. After first follow up, 101 patients were candidates for continued UTUC conservative management, $69 \%$ were recurrence free at last follow up, $22 \%$ still had recurrences and of this cohort, only $9 \%$ underwent deferred RNU during follow up. The global kidney preserving rate (KPR) was $67.5 \%$ for the starting population of 178 patients but after excluding those lost to follow-up immediately after diagnosis (42), it was $91 \%$ for patients in whom conservative endoscopic management was confirmed and continued after first follow up; in imperative indications patients with solitary kidney or bilateral tumors, KPR was $87.2 \%$. No major complication was registered intra- or post-operatively, and $90.4 \%$ were discharged
Fig. 1 shows the Consort diagram of the entire patient cohort
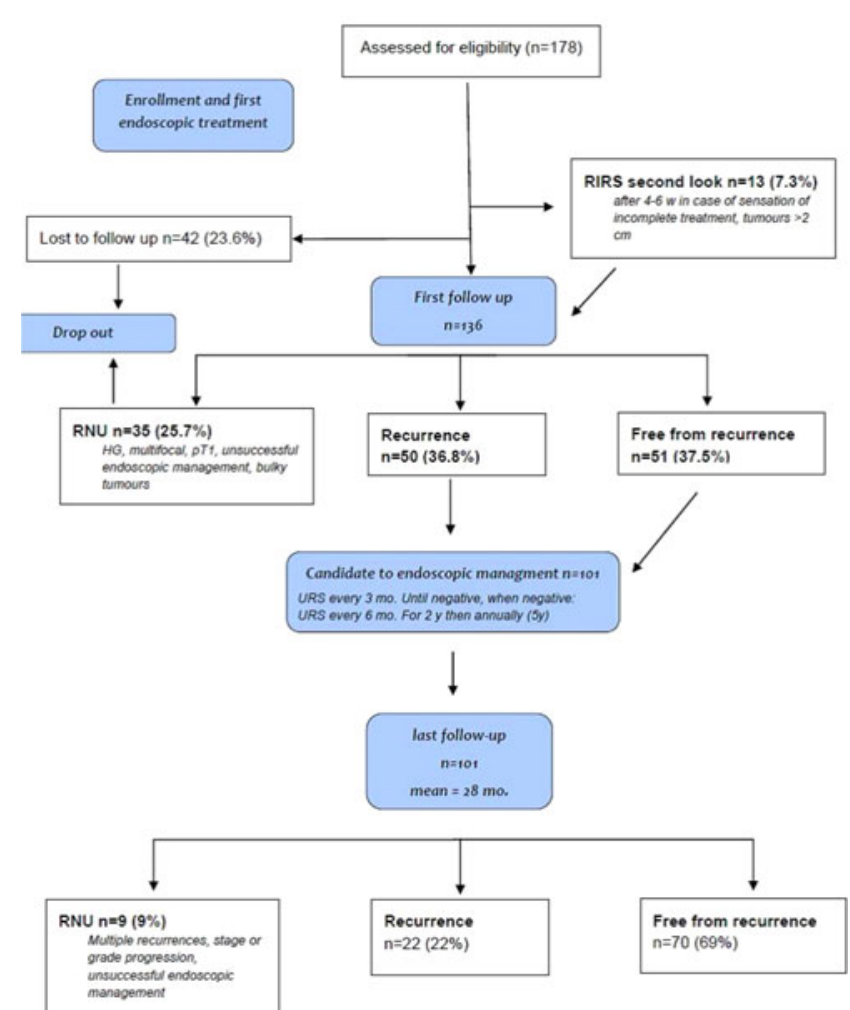

on the $1^{\text {st }}$ post-op day. Only Clavien Dindo grade I complications were reported in $10 \%$ of patients (self-limiting hematuria).

Conclusions: The conservative RiRS treatment of UTUC with Thulium-Holmium:YAG Duo laser was safe and oncologically effective over a thirteen year period and seemingly non-inferior to alternative combination laser energy technologies used for this indication.

MP11-18 Matched Comparison of Laparoscopic Vs Robotic Assisted Nephro-Ureterectomy and Bladder Cuff Excision

S Nalagatla, I Ibrahim, NO Umez-Eronini, OM Aboumarzouk

Queen Elizabeth University Hospital

Introduction \& Objective: Laparoscopic Nephro-ureterectomy (LNU) has become the 'gold standard' over open surgery for localized TCC of the upper urinary tract. LNU remains a challenging procedure, however with the recent introduction of Robot Assisted Nephro-ureterectomy (RANU), these challenges associated with laparoscopic surgery are believed to be minimized. We aimed to compare the intra-operative, post-operative and oncological outcomes between LNU and RANU in our unit.

Methods: A prospective evaluation comparing all patients undergoing LNU to RANU from October 2017 to December 2018 was conducted. Comparative measures were patient and operative demographics as well as oncological outcomes.

Results: Twenty-two patients underwent LNU and 25 patients had a RANU. Average age was 70 years with a total of 25 women and 23 men. No difference between the two groups regarding 
patient demographics was found. There was no difference with regards to operative time, blood loss, transfusion rate, conversion rate, or hospital stay $(\mathrm{p}>0.05)$. No major complications occurred. Oncological outcomes have shown that there was a higher proportion of bladder recurrences in the LNU group $(\mathrm{p}=0.02)$, however no difference regarding distant metastases. No peri-operative deaths occurred.

Conclusions: Efficacy of LNU and RANU appear to be equivocal in their role in the management of upper tract urothelial malignancy from an operative point of view. However, more bladder recurrences are seen in the LNU group as opposed to the RANU group. Further research comparing these procedures is needed to redefine the current roles for these techniques.

MP11-19 Multi-Porous Open-Ended Catheter Technique for Intraureteral Indocyanine Green (ICG) Injection During Surgery

E Kaplan-Marans, J Fulla, M Palese

Cleveland Clinic

Introduction \& Objective: ICG is a fluorescent dye that has traditionally been used intravenously to identify vascular perfusion and differentiate tissue density. ICG has recently been used intraureterally to visualize the urinary tract for ureteral surgery and assess viability of ureteral tissue. We prepared a multi-porous open-ended catheter technique for intraureteral ICG injection that can be used to easily identify the urinary tract during surgery and may also help prevent surgical complications. Methods: A 20-gauge needle is used to make small perforations every $2 \mathrm{~cm}$ along an open-ended catheter. Cystoscopy is performed and the open-ended catheter is inserted into the ureteral orifice and attached to a Foley catheter distally. A $25 \mathrm{mg}$ solution of ICG is prepared in $50 \mathrm{~mL}$ of distilled water. A $10 \mathrm{~mL}$ volume is injected through the open-ended catheter during the surgery. Near-infrared fluorescence, which is integrated into a number of surgical camera systems, is used to detect the ICG in real-time and can therefore be used in any robotic, laparoscopic, or open procedure that requires ureteral identification.

Results: The mulit-porous open-ended catheter technique provides simultaneous distribution of ICG throughout the entire ureter, allowing easy and quick ureteral identification. An ICG concentration of $25 \mathrm{mg}$ in $50 \mathrm{~mL}$ of distilled water, which is more dilute than that reported in other studies, minimizes venous back flow and spillage out of the ureter into the surrounding soft tissue. Conclusions: A multi-porous open-ended catheter technique for intraureteral ICG injection can aid minimally invasive ureteral surgery by identifying the urinary tract and may also help prevent surgical complications.
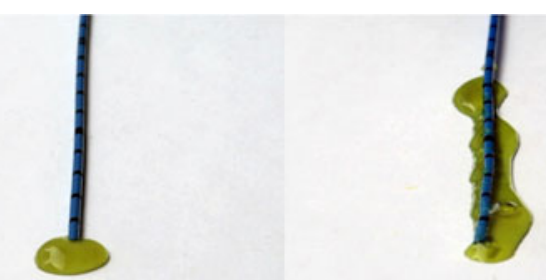

MP11-20 On-clamp versus purely off-clamp robotic partial nephrectomy in solitary kidneys: comparison of chronic kidney disease progression at two highvolume centers

U Anceschi, G Tuderti, A Brassetti, M Ferriero, S Guaglianone, R Mastroianni, R Flammia, M Gallucci, R Bertolo, F Minisola, M Costantini, J koauk, G Simone

"Sapienza" University

Introduction \& Objective: Solitary kidney is the optimal clinical model to assess the real impact of surgical technique on renal functional outcomes. The risk of developing chronic kidney disease (CKD) after robotic partial nephrectomy (RPN) in patients with solitary kidney has been poorly addressed. In this study, we aimed at assessing the impact of ischemia time on the risk of developing stage $3 \mathrm{a}$ (CKD3a) and stage $3 \mathrm{~b}$ (CKD3b) after RPN in solitary kidneys.

Methods: From December 2013 to October 2018, we analyzed 54 consecutive patients who underwent RPN for solitary kidneys with different surgical approaches (purely off-clamp vs conventional on-clamp). Kaplan-Meier method estimated probability of developing new-onset CKD3a and CKD3b after off-clamp vs on clamp RPN was compared. Univariable and multivariable Cox regression analyses were performed to identify predictive factors for the development of new onset CKD3a and CKD3b among the surgical variables. For all statistical analyses, a twosided $\mathrm{p}<0.05$ was considered significant.

Results: At a median follow-up of 13 months (IQR 6.3 - 34), newonset CKD-3a and CKD-3b stages were observed in $33.6 \%$ and $19.6 \%$ of patients, respectively. At Kaplan-Meier analysis, onclamp approach was associated with a significantly higher risk of developing both CKD-3a and CKD-3b stages (Figure 1A,1B; $\mathrm{p}=0.047,0.034$, respectively). At multivariable Cox regression analysis, eGFR at discharge (HR $0.94=95 \%$ CI $0.91-0.98$; $\mathrm{p}=0.005$ ) and continuously coded warm ischemia time (HR 1.0595\% CI 1.01-1.09; $\mathrm{p}=0.008)$ were independent predictors of developing both CKD-3a and CKD-3b (HR 0.89-95\% CI 0.82-0.96; $\mathrm{p}=0.004$ and HR 1.09-95\% CI 1.02-1.17; $\mathrm{p}=0.009$, respectively). Conclusions: Ischemia time and eGFR at discharge are independent predictors of renal functional outcomes after RPN in solitary kidney. With the limitation of the small sample size, ischemia time remains the only surgical modifiable variable significantly associated with renal function deterioration during follow-up.

\section{MP11-21 Robot assisted Supine Extraperitoneal Ap- proach for midline retroperitoneal masses}

GK Pooleri, A LAddha

Amrita Institute of Medical Sciences

Introduction \& Objective: Robot-Assisted Supine extraperitoneal (RASE) technique is a novel approach for midline retroperitoneal tumors. The supine extraperitoneal approach is described with laparoscopy. We adopted this technique and modified it for robotic surgery. The approach is very useful for RPLND and removal of retroperitoneal midline masses.

Methods: From January 2017 to March 2019, eight patients were attempted for RASE approach including five post chemotherapy retroperitoneal lymph nodes masses of testicular tumor, one post liver transplantation retroperitoneal mass. The patient was positioned in the flat supine position with a small pillow behind the 
iliac crest. The initial access to retroperitoneum was done with the open method through a $1.5 \mathrm{~cm}$ incision, two inches above iliac crest. The working ports were placed under finger guidance and the space was created under direct vision. All the procedures were done using da Vinci Xi system.

Results: Out of eight patients, six patients could be successfully completed through this approach. Two patients who underwent RPLND required conversion, (One in left-sided approach and one in right-sided approach) one to open surgery due to tumor-related issues and second to transperitoneal robotic approach due to loss of pneumoperitoneum. The procedure was very useful in patients with history of previous abdominal surgery where the transperitoneal approach is technically very challenging One of our patients had a history of previous liver transplantation. This approach is useful in In patients with a high risk of resuscitation and open conversion. One of our patients with post-chemotherapy testicular residual masses had IVC thrombus and severe bleomycin toxicity. The anesthetist was more comfortable for supine flat approach rather than a steep Trendelenburg position for this patient. In cases were open conversion is planned, there was no need for any change in position. Average blood loss was $150 \mathrm{ml}(100-250 \mathrm{ml})$ and average hospital stay was 4 days (2-10 days), One RPLND patient developed chylothorax which required drainage.

Conclusions: Robot-assisted supine extraperitoneal (RASE) approach is a new technique for accessing midline retroperitoneal masses. The possible advantages of this procedure are 1) Bowel related complications and postoperative ileus is minimized 2) Resuscitation and open conversion is easier and safer since the patient is positioned supine without any tilt. ${ }^{3}$ ) Useful in previous surgery case presenting with midline masses.

MP11-22 Decreased Risk of Upper Extremity Neuropraxia During Robotic Assisted Laparoscopic Prostatectomy: A Novel Technique

M Watson, B Koch, R Xu, G Heath, A Singh

Introduction \& Objective: Intraoperative neuropathic injury due to positioning in minimally invasive surgeries is established as a leading complication. Continuous upper extremity (UE) intraoperative neuromonitoring using somatosensory evoked potentials (SSEP) is a proven method to detect and prevent brachial plexus injury during neurosurgical procedures. Robotic-assisted laparoscopic prostatectomy (RALP) requires steep Trendelenberg which predisposes patients to the risk of UE neuropraxias. Our study is the first to describe the use of SSEP to decrease risk of UE neuropraxias due to positioning of patients undergoing RALP.

Methods: SSEP data was collected using a Cadwell Pro. Bilateral ulnar nerves were stimulated with wrist electrodes. Stimulation occurred at a $1.41 \mathrm{~Hz}$ rate, 200 usec pulse width and 10-30 mA current throughout the case. Latency and amplitude were prospectively recorded. Established SSEP criteria for intervention (50\% 10ss in amplitude, $10 \%$ increase in latency) was used to determine the need for intraoperative repositioning in order to decrease risk of neuropathic damage. Data between April 2016 and July 2018 was retrospectively reviewed to identify patients who satisfied intraoperative intervention criteria and analyzed using SPSS statistical software.

Results: 143 patients received RALP with nerve monitoring. Mean age was 65.2 years (SD 6.7), average weight was 205.6 pounds (SD 33.7) and mean BMI was 30.0 (SD 4.3). Mean operative time was 96.3 minutes (SD 30.2). 14 patients (8.9\%) met intervention criteria requiring intraoperative repositioning. Average duration of signal loss meeting intervention criteria was 22 minutes (SD 5.0). Following repositioning, baseline amplitudes were recovered in $92.9 \%$ of patients intraoperatively. One diabetic patient who did not fully recover baseline signals (BMI 42.3, 54 minutes amplitude loss) experienced transient postoperative UE weakness.

Conclusions: This study is the first to describe the use of SSEP to reduce the risk of UE neuropraxias due to positioning during RALP. Risk of UE nerve injury was identified intraoperatively and prevented in $92.9 \%$ of those patients meeting intervention criteria. High risk patients may benefit from pre-surgical selection for nerve monitoring and further analysis to preoperatively stratify patients for SSEP during RALP is currently ongoing. This study also provides proof of concept for the application of nerve monitoring during robotic surgery, which may translate to other urological robotic procedures to prevent UE neuropraxias.

\section{MODERATED POSTER SESSION 12: LAPAROSCOPIC/ROBOTIC: PROSTATE (I)}

\begin{abstract}
MP12-01 Effect of Perioperative Continuation of Antithrombotic Therapy on Outcome of RobotAssisted Radical Prostatectomy : A Prospective Clinical Trial at Single-Institution
\end{abstract}

M Kubota, T Matsuoka, S Murata, I Suzuki, N Makita, Y Tohi, Y Sugino, K Inoue, M Kawakita

Kobe City Medical Center General Hospital

Introduction \& Objective: To assess the impact on bleeding and complications due to the perioperative continuation of antithrombotic agents during undergoing robot-assisted radical prostatectomy (RARP), we prospectively investigated surgical outcomes of patients whose are all continuing antithrombotic therapies.
Methods: From January 2014 to September 2018, a total of 445 patients who underwent RARP with prostate cancer were included in this prospective clinical trial. All patients continued taking antithrombotic agents in overall perioperative phase. We compared outcomes including intraoperative blood loss, transfusion rate, and bleeding complications in patients with continuing administration of antithrombotic agents (AA group: $\mathrm{n}=65$ ) with those with patients without history of taking antithrombotic agents (NA group: $\mathrm{n}=380$ ). Multivariable analysis was used to identify independent risk factors for increasing bleeding in all patients.

Results: Among patients in AA group, 53 (82\%) patients used antiplatelet (aspirin: 45, clopidogrel: 6, others: 4, combination: 2), 16 patients (24\%) used anticoagulant (warfarin: 10, dabigatran etexilate: 2, others: 4), and 4 patients used both of them 
(aspirin + warfarin). As comparison of both group patients (AA vs NA group; median (min-max)), patients in AA group were older (72 (51-79) vs $68(45-82)$ years, $\mathrm{p}<0.0001)$ and had more severe comorbidity (charlson-comorbidity index; 2 (0-8) vs $2(0-5), \mathrm{p}<0.0001)$ than NA group. Nevertheless, there was no significant difference between both groups in intraoperative blood loss $(200(0-1338)$ vs $189(0-2055) \mathrm{ml}, \mathrm{p}=0.63)$, hemoglobin deficit $(2.3(0.6-4,7)$ vs $2.2(0.2-6.4) \mathrm{mg} / \mathrm{dl}, \mathrm{p}=0.61)$, rate of intraoperative transfusion ( $0 \mathrm{vs} 0.3 \%, \mathrm{p}=0.85)$, and rate of any high-grade complications (Clavien-Dindo Grade $\geqq \mathrm{III} ; 4.6$ vs $1.8 \%, \mathrm{p}=0.17)$. There was no patient who needed secondary procedure for postoperative hemorrhage in both groups. In multivatiate logistic regression analysis, predictors of intraoperative bleeding $>400 \mathrm{ml}$ (upper $25 \%$ tile of overall patients) $\mathrm{BMI} \geqq 25$ (OR $=2.55$, CI95\%: $1.43-4.50, \mathrm{p}<0.001)$, not expert surgeon $(\mathrm{OR}=2.21$, CI95\%: $1.19-4.15, \mathrm{p}=0.013)$, nerve sparing $(\mathrm{OR}=3.21$, CI95\%: $1.85-5.55, \mathrm{p}<0.001)$, and low trendelenburg position $\left(15^{\circ}\right)$ $(\mathrm{OR}=2.35, \mathrm{C} 195 \%: 1.40-3.95, \mathrm{p}=0.0013)$. The history of continuing antithrombotic therapies $(\mathrm{OR}=0.9$, IC95\%: $0.5-2.0$, $\mathrm{p}=0.89$ ) was not a significant risk factor of high volume bleeding. Conclusions: These results indicate that perioperative continuing use of antithrombotic agents is not a factor of increasing bleeding, and considered as safe in RARP.

\section{MP12-02 Institutional Learning Curves for Robotic Radical Prostatectomy using CUSUM Analysis}

F Endo, M Shimbo, K Ohwaki, K Hattori

St. Luke's International Hospital

Introduction \& Objective: CUSUM (Cumulative Sum) is an analysis method which allows instinctive comprehension of temporal changes in early stages of studies and therefore has begun to be used to analyze surgical learning curves (LC).

Methods: 595 cases of clinical stage $\mathrm{T} 1$ or $\mathrm{T} 2$ prostate cancer who underwent robotic radical prostatectomy (RARP) since the DaVinci was introduced in September 2011 through December 2017 at our tertiary hospital were enrolled in this study. We investigated the initial LC as a facility by using CUSUM analysis regarding RM1or positive surgical margins (PSM) which were below 20\%. We also analyzed surgeons up until the fourth generation who had become independent.

Results: The average age and BMI of the patients were 66.5 years and $23.7 \pm 2.8 \mathrm{~kg} / \mathrm{m}^{2}$, respectively. There were 3 cases of Clinical stage $\mathrm{T} 1 \mathrm{a} / \mathrm{b}, 140$ cases of T1c and 452 cases of T2. They consisted of 91 low risk cases, 286 intermediate risk cases and 218 high risk cases according to the D'Amico risk classification. There was a total of 101 cases $(16.7 \%)$ of RM1 and when grouped by generations of surgeons, First generation: 45/293 cases (15.4\%), Second generation: $19 / 121$ cases $(15.8 \%)$, Third generation 10/67 cases (15.1\%), Fourth generation 20/88 (23.0\%). The CUSUM for all cases shows that the first 54 cases were in the negative direction but after the 55th case, there was a change in trend and keeps increasing. The first 50 cases were mostly conducted by the first generation and could be approximated to the institutional LC. The surgeons after the second generation conducted procedures under the guidance of the first generation then an increase in CUSUM score was seen in all generations from the beginning. However, a change was seen in the fourth generation in the 46th case where the trend became negative and continued afterwards.

Conclusions: The LC regarding PSM for our institution after introducing RARP was 55 cases and the LC for the first generation was 44 cases. Thereafter, if the succeeding generations operated under the guidance of an adept surgeon, the LC was

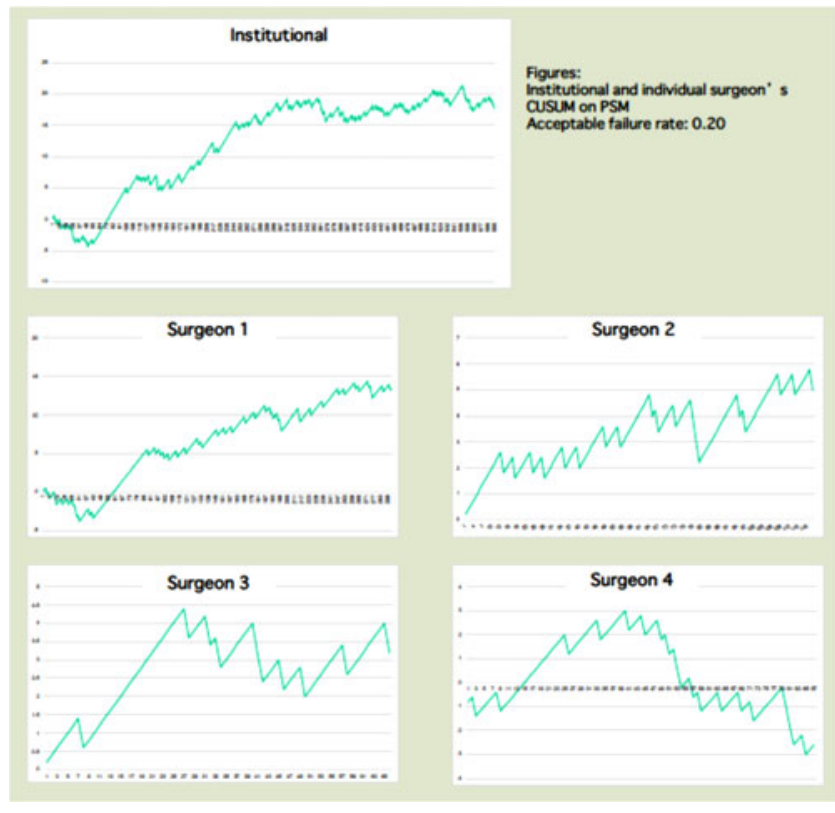

hardly seen, and therefore we have proven that the quality of the procedure as a facility can be assured. Also, by considering and monitoring the PSM of a surgeon who has become independent through CUSUM analysis, an attending surgeon can decide when to step in, if necessary, at an early stage and can be considered as an index for long-term quality control.

\section{MP12-03 Predictors of Length of Hospital Stay in Robotic Assisted Radical Prostatectomy: a Retro- spective Cohort Study}

MS Labban, J Zarka, NF Abou Heidar, A Mailhac, H Tamim, A Hajj

American University of Beirut Medical Center

Introduction \& Objective: Length of hospital stay (LOS) has become a measuring tool for medical resource consumption and an indicator of quality of health care delivered. Prolonged hospital stay has been associated with elevated economic burden and worse surgical outcomes. The ability to identify the preoperative predictors of extended LOS would help surgeons predict the postoperative course of the patient and perform the appropriate counseling. Through this paper, we aim to determine the preoperative predictors of composite morbidity and prolonged hospital stay.

Methods: We used the American College of Surgeons National Surgical Quality Improvement Program (ACS NSQIP) database. We selected patients who underwent robotic assisted radical prostatectomy (RARP) between 2008-2016. We collected anonymous data including demographics, co-morbidities, and laboratory values as potential predictive factors for length of hospital stay. Using multivariate logistic regression, we evaluated the predictors of composite morbidity (total in-hospital postoperative complications). Then, we assessed the predictors of LOS excluding composite morbidity as the latter proved to be on the causal pathway of extended stay.

Results: We obtained data for 31, 253 patients of which $66.5 \%$ stayed $\leq 1$ day, $22.4 \%$ stayed 2 days, and $11.2 \%$ stayed $>2$ days. Hypertension and diabetes were independent predictors of composite morbidity (odds ratio [OR] 1.42, 95\% CI 1.18-1.71) and $(1.42,1.15-1.77)$, respectively. Hematocrit (Hct) $<40 \%$ 
(2.05, 1.18-3.56) and Creatinine $(\mathrm{Cr})>1.2(1.34,1.07-1.68)$ were predictors of postoperative complications while leukocytosis $>11,000$ was not. Demographic variables including Body Mass Index (BMI) <25 (2.82, 1.65-4.82), smoking (1.21, 1.08$1.35)$, dialysis dependent $(2.64,1.39-5.00)$, and dependent functional status $(3.12,1.63-5.95)$ were predictors of extended length of stay ( $>2$ days). Unlike the predictors of composite morbidity, the most prominent comorbidities extending hospital stay were Chronic Obstructive Pulmonary Disease (1.78, 1.412.26), bleeding disorders (2.01, 1.46-2.74), and Congestive Heart Failure (4.64, 2.00-10.78). Abnormal preoperative laboratory values including $\mathrm{Cr}>1.2(1.28,1.14-1.43)$, leukocytosis $>11,000(1.32,1.07-1.62)$, and Hct $<40 \%(1.39,1.25-1.54)$ were also independent predictors of hospital stay of $>2$ days.

Conclusions: Preoperative patient characteristics and comorbidities can predict postoperative morbidity and prolonged length of hospital stay. Thus, these findings can be used for risk assessment and patient counseling prior to RARP.

\section{MP12-04 The Value of Nerve Sparing during RARP in Men with Low SHIM score}

M Shahait, J Kim, A Malhotra, RW Dobbs, DI Lee

University of Pennsylvania

Introduction \& Objective: The value of the neurovascular bundles (NVBs) preservation during RARP on erectile function is well documented, its influence on continence remains controversial. Actually, it is still contentious if NVBs contribute to the neural supply of the urethral sphincter. Intuitively, the benefit of sparing NVBs in patients with low SHIM score might not be well perceived. The aim of this study is to explore the value of nerve sparing during RARP on functional outcomes.

Methods: A total of 127 patients with SHIM score $<11$ who underwent RARP by a single high-volume surgeon (DIL) were analyzed. Functional data were assessed using self-administrated questionnaires. Data were collected prospectively into a database approved by the institutional review board. Patient were categorized into two groups based on the status if NVBs sparing into $\mathrm{NS}$, and non NS. Continence was defined as the use of zero or one safety pad per day.

Results: In unadjusted analysis, urinary continence rates at 3 months and 12 months after surgery were $68 \%$ and $88 \%$, respectively, in the NS group; $50 \%$ and $67 \%$, respectively, in the Non nerve sparing group. There was no significant difference in the mean EPIC-CP score between the two groups at 3 months (15 vs. $17, \mathrm{p}=0.24$ ). However, at 12 months NS group had lower EPIC-CP score compared to non-NS (11.6 vs. 18.3, $\mathrm{p}=0.0118$ ). The main effect of NS on urine incontinence domain recovery was significant such that NS group had shorter time to recover compared to non NS group $(\mathrm{F}=4.6, \mathrm{p}=0.038)$.

Conclusions: Our results indicate that NS in patients with low SHIM score might hasten urine continence recovery and improve long term quality of life outcomes.

MP12-05 The effect of USPSTF recommendation on post-operative outcomes in Robot-assisted laparoscopic prostatectomy (RALP) based on analysis of a single surgeon series

S Bhat, O Fikret, M Moschovas, T Rogers, C Jenson, B Rocco, V Patel

Global Robotics Institute, Celebration, FL

\begin{tabular}{|c|c|c|c|c|c|c|}
\hline \multirow[t]{2}{*}{ Parameters } & \multicolumn{2}{|c|}{ Till Dec $2012(n=3817)$} & \multicolumn{2}{|c|}{ From lanuary $2013(n=3451)$} & \multicolumn{2}{|c|}{ P value } \\
\hline & PNS & FNS & PNS & FNS & PNS & FNS \\
\hline PSM & $265(16)$ & $237(13.5)$ & $442(21.5)$ & $190(13.5)$ & $<.001$ & 0.147 \\
\hline Potency & $963(58.8)$ & $1587(79.7)$ & $834(40.7)$ & $1031(73.7)$ & $<.001$ & $<.001$ \\
\hline Continence & $1541(94)$ & $1908(95.8)$ & $1754(85.6)$ & $1315(94)$ & $<.001$ & 0.054 \\
\hline BCR & $240(7.7)$ & $154(7.7)$ & $216(10.5)$ & $56(4)$ & \multirow[t]{2}{*}{$<.001$} & \multirow[t]{2}{*}{$<.001$} \\
\hline PSA persistence & $87(5.3)$ & $20(1)$ & $135(6.6)$ & $13(1)$ & & \\
\hline Triffecta & $766(46.8)$ & $1405(70.6)$ & $676(33)$ & $947(67.7)$ & $<.001$ & 0.074 \\
\hline Pentafecta & $656(40)$ & $1231(61.8)$ & $538(26.2)$ & $805(57.5)$ & $<.001$ & 0.012 \\
\hline Total & 1638 & 1991 & 2950 & 1399 & & \\
\hline
\end{tabular}

Introduction \& Objective: US Preventive Services Task Force (USPSTF)'s recommendation against PSA-based screening has resulted in a change in practice pattern of patients receiving robot assisted radical prostatectomy (RALP). We evaluated the possible influence of these changes on surgical technique, trifecta and pentafecta outcomes following RALP.

Methods: We retrospectively analyzed all men that underwent RALP between 2002 to 2018 with minimum 6 months follow-up from a prospectively collected IRB approved database. Patients were sub grouped based on degree of nerve sparing. Subgroups were analyzed for differences in clinical parameters, trifecta and pentafecta rates before and after USPSTF's recommendation by using Chi square test.

Results: 7268 patients were available for analysis. Mean preoperative PSA increased from 6.0 to $7.41 \mathrm{ng} / \mathrm{ml}$ between the two subgroups. After the USPSTF recommendation, $\geq \mathrm{pT} 3$ disease undergoing Partial Nerve Sparing (PNS) and full nerve sparing (FNS) increased by $16 \%$ and $6 \%$. respectively. In patients with FNS, potency rate decreased from $79.7 \%$ to $73.7 \%$ without significant change in trifecta rates before and after 2012 (70.6\% vs. $67.7 \%, \mathrm{p}=0.074$, Table 1). There was a small but statistically significant decrease in pentafecta rates from $61.8 \%$ to $57.5 \%$ $(\mathrm{p}=0.012$, Table 1). In patients with PNS, PSM, potency and continence rates decreased significantly after USPSFT's recommendation (Table 1). This resulted in a significant decrease in trifecta rate from $46.8 \%$ to $33 \%$, and in pentafecta rate from $40 \%$ to $26.2 \%$.

Conclusions: After USPSTF recommendation, our practice has seen a trend towards operating more aggressive disease. In patients undergoing FNS, this only marginally affected the surgical outcomes. However, in patient with PNS this was associated with a statistically significant decrease in our trifecta and pentafecta rates despite increasing surgical experience.

MP12-06 Evaluation of lymphoceles after pelvic lymph node dissection in robot-assisted radical prostatectomy

Y Muro, H Iijima, C Nakamura, T Fujimoto, R Yabusaki, Y Shiraishi, M Imamura, K Yoshimura

Department of Urology, Shizuoka General Hospital

Introduction \& Objective: The most frequent complication of pelvic lymph node dissection (PLND) is lymphocele formation. The purpose of this study is to evaluate the incidence and characteristics of lymphoceles in patients undergoing PLND in robotassisted radical prostatectomy (RARP).

Methods: 63 patients who underwent PLND during RARP for high-risk prostate cancer from January 2016 to April 2018 were 
included in the study. RARP was performed using transperitoneal approach. All patients received pelvic computed tomography (CT) 3 and 12 months after the surgery and the presence of lymphoceles were evaluated. Lymphoceles were defined as cystic lesions over $10 \mathrm{~mm}$ in diameter.

Results: The mean (range) patients age was 67.7 (52-78) years. At 3 months post-surgery, lymphoceles were identified in $14 \%$ ( $9 / 63$ patients). All lymphoceles were unilateral. The mean (range) size of lymphocele in diameter was $35 \mathrm{~mm}(22-42 \mathrm{~mm})$. All lymphoceles were asymptomatic, and none required intervention. However, one patient who received CT for fever evaluation was discovered with an infected lymphocele at 1-month post-surgery but was successfully treated with antibiotics and free from lymphoceles at 3 months. Of 9 patients with lymphoceles at 3 months post-surgery, lymphoceles resolved spontaneously in 67\% (6/9 patients) at 12 months. Pathological Gleason score, resected prostate volume, number of lymph nodes dissected, pathological $\mathrm{T}$ stage were not associated with the formation of lymphoceles. There was also no correlation between duration of drain tube placement, drain output at the day of the removal, total drain output, and the formation of lymphoceles.

Conclusions: The incidence of lymphoceles was relatively low in our study, compared to other reported outcomes. Furthermore, duration of drain tube placement and output from drain tube was not related to the formation of lymphoceles. Although the formation of lymphoceles is the most frequent complication of PLND, it is mostly asymptomatic and resolve spontaneously within a year.

\section{MP12-07 Impact of Median Lobes on Urinary Func- tion after Robotic Radical Prostatectomy}

OE Martinez, R Abaza

OhioHealth Dublin Methodist Hospital

Introduction \& Objective: The presence of an enlarged median lobe (ML) presents a technical challenge and may affect the outcomes of robotic prostatectomy (RP). If known, the impact of a potentially larger bladder neck on continence and preoperative chronic obstruction on postoperative urinary symptoms might aid in patient counseling. We assessed the impact of intraoperativelyidentified median lobes (ML) on urinary function.

Methods: We reviewed our prospective RP database between 2013-18. AUA symptoms scores (AUA-SS) were assessed preoperatively and at one, three, and six months. We compared patients with and without ML (No-ML). Bladder-neck sparing was routinely performed to avoid reconstruction.

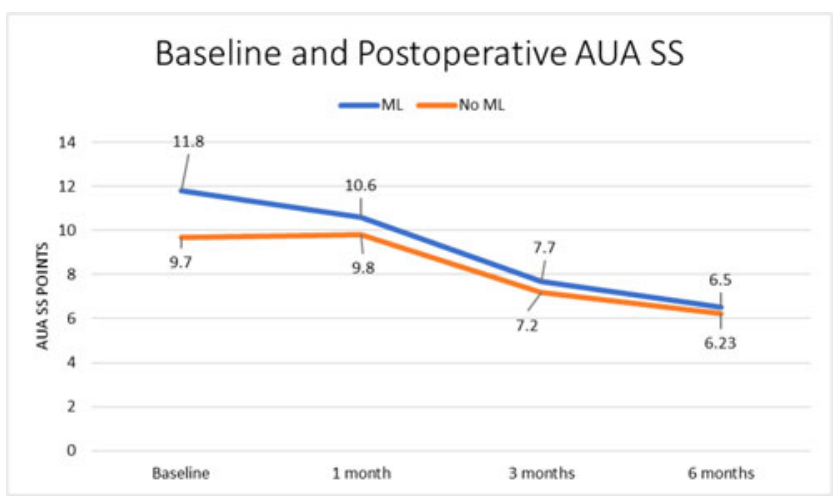

Results: Of 562 patients with complete data at all time points, 163 (29\%) had ML. There were no statistically-significant differences in demographics, PSA, or clinical stage. Mean operative time was $153 \mathrm{~min}$ with and $148 \mathrm{~min}$ without ML, respectively $(\mathrm{p}<0.05)$. Only two in each group required bladder-neck reconstruction $(1.2 \%$ versus $0.5 \%)$. There was no immediate or long-term difference in continence rates between groups. Baseline mean AUASS was higher in ML patients and showed more improvement postoperatively $(-5.3$ versus $-3.47,<0.05)$ with greatest improvement in ML patients with severe preoperative symptoms (-15.1) and no difference in AUA-SS between groups by 6 months. Conclusions: Patients with enlarged ML do not have an increased risk of incontinence after RP but appear to benefit more from RP in terms of postoperative urinary function

\section{MP12-08 Long-term Oncologic Outcomes After Limited Pelvic Lymph Node Dissection for Inter- mediate and High Risk Prostate Cancer}

AS Elsayed, NA Aldhaam, AA Hussein, G James, O Abdel-Razzak, I Saad, M Abdelhakim, KA Guru

\section{Roswell Park Comprehensive Cancer Center}

Introduction \& Objective: The role of pelvic lymph node dissection (PLND) during robot-assisted radical prostatectomy (RARP) for pathological staging and prognosis is clear but its extent and oncologic benefits remain uncertain. We investigated the long-term oncologic outcomes of patients who underwent a limited PLND at RARP for intermediate (IR) or high (HR) risk disease based on the NCCN guidelines classification.

Methods: A retrospective review of a prospectively populated and updated database of patients who underwent RARP and limited PLND at Roswell Park Comprehensive Cancer Center between 2005 and 2014. Pearson Chi-Square and Wilcoxon Rank Sum tests were utilized, and the Kaplan-Meier method was used to define disease-specific survival (DSS), and biochemical recurrence-free survival (BFS).

Results: Three hundred forty-five patients were identified, with a mean age of $60 \pm 7$, and a median (IQR) follow up of $60(30,90)$ months. 196 were (57\%) IR and 149 patients (43\%) were HR. Three percent received neoadjuvant androgen deprivation therapy. 142 (41\%) patients were pT2 and $203(59 \%)$ were $\geq$ pT3. The median (IQR) lymph node yield was $7(4,10)$, positive lymph disease was $5 \%$, and surgical soft tissue margins were $31 \%$ (Table 1). DSS and BFS were $98 \%$ and $87 \%$ at 5 years respectively (Figure 1).
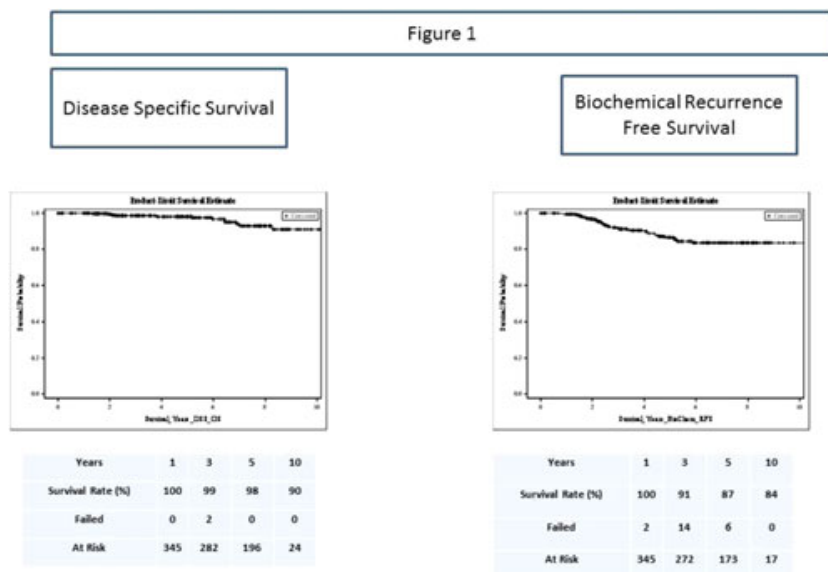
Table 1: Baseline characteristics

\begin{tabular}{|c|c|}
\hline Number of patients & $345(100 \%)$ \\
\hline Mean Age ( \pm SD) & $60( \pm 7)$ \\
\hline$<60$ & $192(56 \%)$ \\
\hline 260 & $153(44 \%)$ \\
\hline \multicolumn{2}{|l|}{ Race: } \\
\hline Caucasian American & $298(86 \%)$ \\
\hline African American & $38(11 \%)$ \\
\hline Other & $8(2 \%)$ \\
\hline Mean BMI ( $(\mathrm{SD}), \mathrm{kg} / \mathrm{m}^{2}$ & $30( \pm 5)$ \\
\hline Median Charlson Comorbidity Index (IQR) & $2(1,3)$ \\
\hline Pre-operative PSA (ng/ml), mean(SD) & $10( \pm 11)$ \\
\hline \multicolumn{2}{|l|}{ PSA } \\
\hline$<10 \mathrm{ng} / \mathrm{ml}$ & $239(69 \%)$ \\
\hline $10-20 \mathrm{ng} / \mathrm{ml}$ & $67(19 \%)$ \\
\hline$>20 \mathrm{ng} / \mathrm{ml}$ & $38(11 \%)$ \\
\hline \multicolumn{2}{|l|}{ Clinical T Stage,n(\%) } \\
\hline $\mathrm{T} 1$ & $182(53 \%)$ \\
\hline $\mathrm{T} 2$ & $140(41 \%)$ \\
\hline T3 & $23(7 \%)$ \\
\hline \multicolumn{2}{|l|}{ Clinical Gleason Score,n(\%) } \\
\hline 6 & $19(6 \%)$ \\
\hline 7 & $217(63 \%)$ \\
\hline $8-10$ & $109(32 \%)$ \\
\hline \multicolumn{2}{|l|}{ NCCN Risk Category,n(\%) } \\
\hline Intermediate & $196(57 \%)$ \\
\hline High & $149(43 \%)$ \\
\hline Neoadjuvant ADT, $n(\%)$ & $9(3 \%)$ \\
\hline \multicolumn{2}{|l|}{ Pathological T Stage, $n(\%)$} \\
\hline pT2 & $141(41 \%)$ \\
\hline $2 \mathrm{pT3}$ & $203(59 \%)$ \\
\hline
\end{tabular}

\begin{tabular}{|c|l|}
\hline \multicolumn{1}{|c|}{ 66 } & $12(3 \%)$ \\
\hline 7 & $260(75 \%)$ \\
\hline $8-10$ & $72(21 \%)$ \\
\hline Median Lymph Node Yield (IQR) & $7(4,10)$ \\
\hline Pathological Nodal Stage, $\mathrm{n}(\%)$ & \\
\hline $\mathrm{pN} 0$ & $323(94 \%)$ \\
\hline $\mathrm{pN} 1$ & $17(5 \%)$ \\
\hline $\mathrm{pNx}$ & $5(1 \%)$ \\
\hline Bladder Neck Invasion, $\mathrm{n}$ (\%) & $59(17 \%)$ \\
\hline Positive Surgical Margin Status, $\mathrm{n}(\%)$ & $107(31 \%)$ \\
\hline Adjuvant therapy (XRT, ADT, or both), $\mathrm{n}(\%)$ & $127(36 \%)$ \\
\hline Median Follow Up (IQR) (months) & $60(30,90)$ \\
\hline
\end{tabular}

Conclusions: Limited PLND in NCCN IR or HR prostate cancer patients shows comparable oncologic outcomes to reported outcomes of extended Lymph node dissection. Limited PLND in NCCN IR or HR prostate cancer patients shows comparable oncologic outcomes to reported outcomes of extended Lymph node dissection.

MP12-09 Impact of membranous urethral length on the urinary continence outcomes of patients after robotic-assisted radical prostatectomy

Y Murakami, K Tabata, H Tsumura, T Hirayama, $\mathrm{K}$ Matsumoto, M Iwamura

Introduction \& Objective: We investigated the association between membranous urethral length (MUL) using magnetic resonance imaging (MRI) and urinary continence after roboticassisted radical prostatectomy (RALP).
Methods: Clinical records were prospectively collected in 159 patients with RALP underwent preoperative and postoperative MRI between 2014 and 2018. Urinary continence was evaluated at 1, 3, 6, and 12 months after RALP. Of 159 patients, 80 patients were evaluated the quality of life (QOL) score regarding urinary continence pre and postoperatively using UCLA-PCI questionnaire. Urinary continence was defined as the use of one pad or less (safety pad). Logistic regression analysis was used to identify clinical factors associated with urinary incontinence including preoperative MUL, postoperative MUL, percent change of MUL.

Results: When identifying a cutoff point of MUL to be $16 \mathrm{~mm}$, MUL $16 \mathrm{~mm} \geqq$ patients showed significantly higher rate of continence patients than those of MUL $<16 \mathrm{~mm}$. In addition, MUL $16 \mathrm{~mm} \geqq$ patients scores significantly better at 1,3 months than those of MUL $<16 \mathrm{~mm}$. In multivariate analysis, postoperative MUL was significantly associated with urinary incontinence along with specimen weight at 3 months. However, only specimen weight was an independent predictor associated with urinary incontinence at 6 months, and BMI was the only factor associated with urinary incontinence at 12 months. Percent change of MUL was associated with urinary incontinence at 3 months in univariate analysis, but, association did not remain significant in multivariate analysis.

Conclusions: Postoperative MUL would be a predictive factor for early recovery of urinary continence at 3 months after RALP. These results suggest preservation of MUL should be considered during surgery for early urinary continence outcomes.

\section{MP12-10 Nerves at the posterior of the prostate base contribute to erectile function: An intraoperative electrical stimulation assessment}

Y Kaiho, J Ito, Y Kohada, J Mikami, G Anan, T Kudo, M Sato

Department of Urology, Tohoku Medical and

Pharmaceutical University

Introduction \& Objective: Nerve-sparing techniques in radical prostatectomy have evolved to improve postoperative erectile function. The cavernous nerve fibers have been revealed to create a periprostatic network distributing primarily in the posterolateral region of the prostate. Excision lines on the ventral side of the prostate in nerve-sparing techniques have been well established, including the veil technique, in which the prostate fascia is incised anteriorly. However, the excision line of nerve-sparing techniques at the posterior area of the prostate base has yet to be explored. To confirm the distribution of functional nerves involved in erectile function at the posterior of the prostate base, intraoperative nerve stimulation was performed during robotassisted radical prostatectomy (RARP).

Methods: Several points at the posterior of the prostate and the posterolateral typical neurovascular bundle (NVB) were electrically stimulated at the level of the prostate base during RARP in patients with clinically localized prostate cancer. The prostate pedicle (PP), medial side of the PP (MPP), Denonvilliers' fascia (DF) and typical NVB were stimulated using bipolar electrodes. The changes in pressure at the middle of the urethra were measured using an inserted balloon-catheter to detect the increase in cavernosal pressure.

Results: Although the study included only 11 patients, every stimulation at the PP, MPP and NVB evoked urethral pressure responses in all patients. The median amplitude of the pressure 


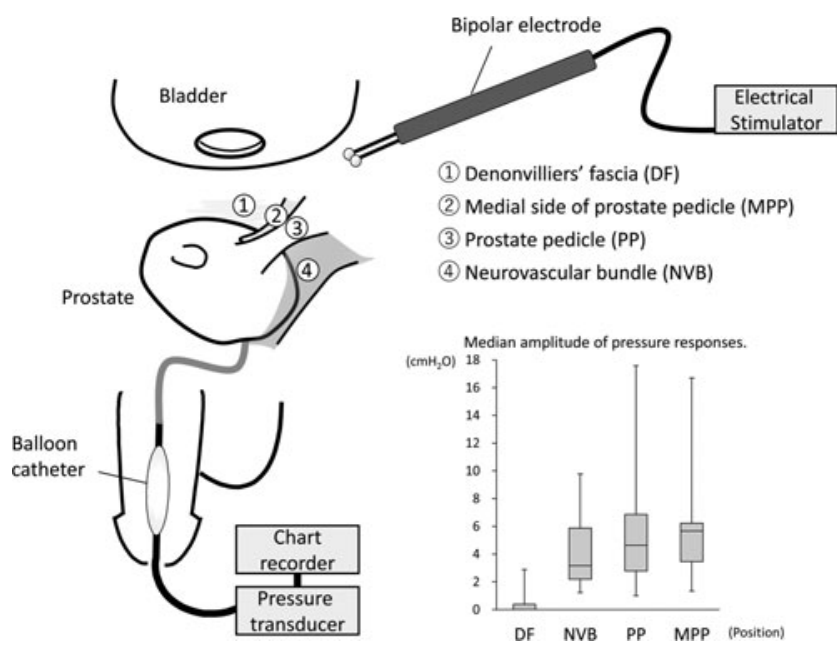

responses was 4.64 (IQR 2.78-6.86), 5.67 (IQR 3.46-6.22), and 3.17 (IQR 2.19-5.88) $\mathrm{cmH}_{2} \mathrm{O}$ at the PP, MPP, and NVB, respectively. There was not significant difference among the amplitude of responses at the PP, MPP and NVB. Stimulations at the DF induced unstable weak urethral response alone or no response in all patients.

Conclusions: We demonstrated that electro-stimulation at the PP and MPP increase cavernosal pressure similar to the typical NVB stimulation. These findings indicate that that maximal preservation of the tissues at the posterior area of the prostate base can contribute to optimal recovery of postoperative erectile function after nerve-sparing RARP.

\section{MP12-11 Use Of Transversus Abdominis Plane Block To Decrease Pain Scores And Narcotic Use Following Robot Assisted Laparoscopic Prostatectomy}

T Rogers, F Onol, S Bhat, M Covas Moschovas, C Jenson, S Roof, N Gallo, B Gallo, M Sandri, V Patel

Global Robotic Institute

Introduction \& Objective: To assess whether transversus abdominis plane (TAP) blocks can be utilized to decrease patient pain scores and narcotic use during the first 24 hours following robot assisted laparoscopic prostatectomy (RALP).

Methods: 100 patients received a TAP block with a mixture of $1.3 \%$ liposomal bupivacaine, $0.5 \%$ Marcaine and $0.9 \% \mathrm{NaCl}$ prior to RALP. This was in addition to an already established pain management regime, which included preoperative PO acetaminophen (650mg), celecoxib (200mg), and tolterodine ER (4mg). These patients were prospectively followed and then retrospectively compared to a match group of 100 patients that did not receive a TAP but did receive the preoperative PO medications. Pain scores were assessed on a scale from 1-10 in the PACU; as well as; the surgical floor at 8,16 , and 24 hours post-surgery. Intra/ post-operative narcotic use and time to ambulation following arrival to the surgical floor were also analyzed.

Results: Patient receiving TAP blocks had immediate post op pain scores of 2.23 vs. 4.26 for those not receiving TAP blocks $(p=0.000)$. The pain scores at 8,16 , and 24 hours for TAP patients were $2.68,2.62$, and 2.62 ; as compared to $2.89,2.87$, and 3.36 for non-TAP patients. The difference was statistically significant for immediate and 24-hour pain scores $(\mathrm{p}=0.000$,
0.001 respectively). On average TAP block patients ambulated faster than non-TAP patients, 2.68 vs. 4.91 hours $(p=0.000)$. Intra-operative narcotic use was decreased in the TAP group for each of the opioids that were used: Fentanyl 177.5 vs. $205 \mathrm{mcg}$ $(p=0.001)$, Morphine 5.5 vs. $10 \mathrm{mg}(p=0.000)$, and Hydromorphone 0.75 vs. $1.75 \mathrm{mg}(p=0.001)$. Narcotic usage in the PACU was limited to hydromorphone and TAP patients used $0.7 \mathrm{mg}$ compared to $1.36 \mathrm{mg}$ ( $p=0.003$ ) for non-TAP patients. Oral oxycodone/acetaminophen $(5 \mathrm{mg} / 325 \mathrm{mg})$ was used for pain control on the surgical floor and on average TAP patients received less, 2.4 vs. 5 tabs $(p=0.000)$. Average time to perform the TAP block was 3.5 minutes and total OR time for TAP vs. non-TAP patients was 107.41 vs. 106.58 minutes $(p=0.386)$.

Conclusions: TAP blocks as part of a perioperative pain management protocol can be utilized during RALPs to decrease patient pain scores at two different time intervals, immediately post-operative and 24 hours after surgery. Patients also ambulate sooner following surgery and require a decreased amount of narcotics during the intra-operative and post-operative periods. TAP blocks are quick, effective, and do not add a significant amount of OR time to RALPs.

\section{MP12-12 Transvesical Robot Assisted Radical Pros- tatectomy}

G Wang, X Zhou, B Fu, C Zhang

The First Affiliated Hospital of Nanchang University

Introduction \& Objective: To introduce the technique and report our initial experience of transvesical robot assisted radical prostatectomy (TvRARP).

Methods: 10 TvRARP for prostate cancer performed by a single surgical team between 2018 April and 2018 October were retrospectively reviewed. Preoperative data of patients [patient age $(58.6 \pm 9.4)$ years, BMI $(26.5 \pm 3.1) \mathrm{kg} / \mathrm{m}^{2}$, tPSA $(19.5 \pm 4.1)$ $\mathrm{ng} / \mathrm{ml}$, biopsy Gleason score $6(6 \sim 7)$, prostate volume $(33.4 \pm 15.8) \mathrm{ml}$ and IIEF-5 score $12(10 \sim 16)]$ were collected. Preoperative study revealed 8 cases of $\mathrm{cT}_{1 \mathrm{c}}, 1$ case of $\mathrm{cT}_{2 \mathrm{a}}$ and 1 case of $\mathrm{cT}_{2 \mathrm{~b}}$. All patients were continent preoperatively. Surgical techniques the surgery was performed with da Vinci Si system via 4 robotic ports and 2 assistant ports as the following: 1) cystotomy, further expended by bilateral suspension stitches.

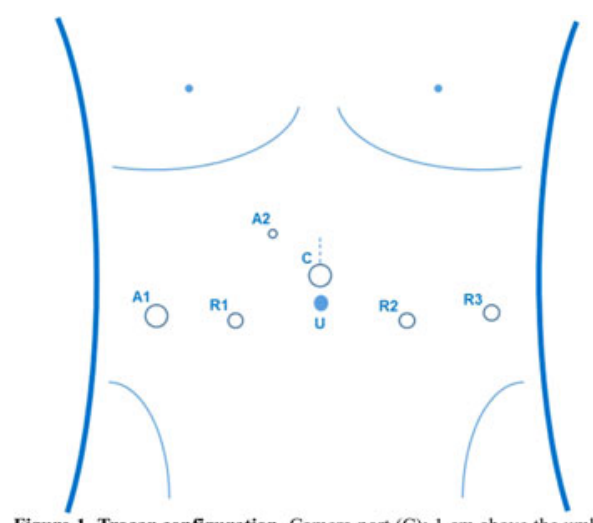

Figure 1. Trocar configuration. Camera port (C): $1 \mathrm{~cm}$ above the umbilical (U); $2^{\text {nd }}$ arm trocar (R1) and $3^{\text {rd }}$ arm trocar (R2): lateral margin of rectus abdominis, $8 \mathrm{~cm}$ from camera port; $4^{\text {th }}$ arm trocar (R3): lateral to and $8 \mathrm{~cm}$ from the $3^{\mathrm{rd}}$ arm trocar; $12-\mathrm{mm}$ assistant port (A1): lateral to and $8 \mathrm{~cm}$ from the $2^{\text {nd }}$ arm trocar; 5-mm assistant port (A2): between $2^{\text {nd }}$ arm trocar and camera port. The dash line marks the incision for specimen extraction. 

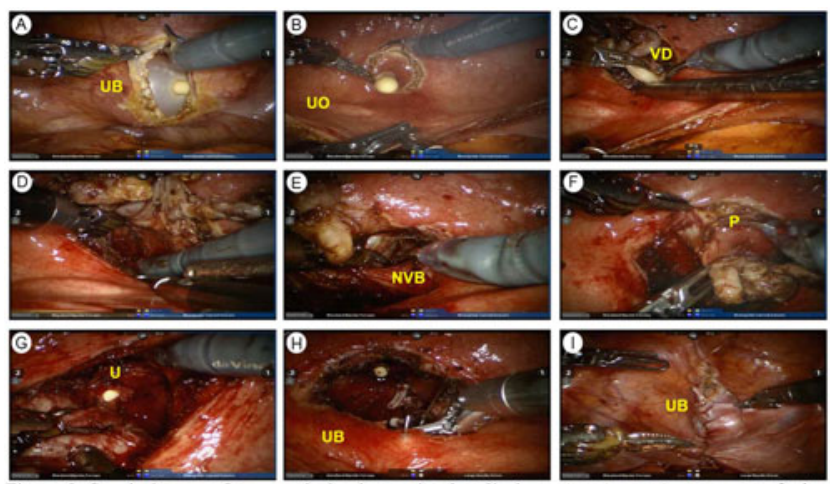

igure 2. Surgical steps of transvesical robot assisted radical prostatectomy. A: cystotomy, further expended by bilateral suspension stitches. B: a circumferential incision is made around the internal urethral orifice through the mucosa and muscular layer. $\mathrm{C}$ : initial dissection of the vas deferens and semi vesicles is carried out through the lower half of the circumferential incision around the internal urethral orifice. D: posterior dissection towards the apex of the prostate. E: dissection of bilateral NVB. F: anterior dissection towards the apex of the prostate. G: dissection and transection of the urethra. H: urethrovesical anastomosis. I: 2-layer closure of bladder. Abbreviations: UB (bladder), UO (ureteral orifice), VD (vas deferens), NVB (neurovascular bundle), P (prostate), U (urethra).

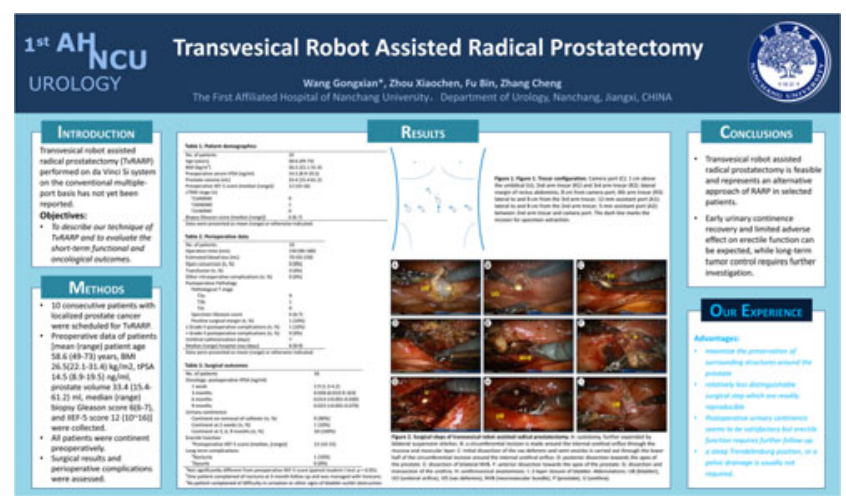

2) A circumferential incision is made around the internal urethral orifice through the mucosa and muscular layer. 3) Initial dissection of the vas deferens and semi vesicles is carried out through the lower half of the circumferential incision around the internal urethral orifice. 4) Posterior dissection towards the apex of the prostate. 5) Dissection of bilateral NVB. 6) Anterior dissection towards the apex of the prostate. 7) Dissection and transection of the urethra. 8) Urethrovesical anastomosis. 9) 2layer closure of bladder.

Results: All 10 cases were successfully performed robotically without conversion, transfusion or other major intraoperative and postoperative complications. Postoperative pathology confirmed 6 pT 2 a cases, 3 pT 2 b cases and 1 pT $2 \mathrm{c}$ case [Gleason score $6(6 \sim 7)] .1$ case reported positive surgical margin $(10 \%)$. Operative time was $(140.5 \pm 35.5) \mathrm{min}$. Estimated blood loss was $(65.5 \pm 35.5) \mathrm{ml}$. Urethral catheter was removed at postoperative 7-day. 9 patients achieved urinary continence ( 0 pads) immediately after the removal of urinary catheter, while 1 patient returned to full continence in 2 weeks postoperatively. During a mean follow up of 3 months ( $2 \sim 4$ months), no biochemical recurrence was noted (tPSA $<0.2 \mathrm{ng} / \mathrm{ml}$ ). At 2 -month postoperatively, IIEF-5 score was $11(8 \sim 13)$ and not statistically different from preoperative status $(\mathrm{p}>0.05)$.

Conclusions: Transvesical approach is a valid alternative of performing RARP in patients bearing localized low-risk prostate cancer. Tumor control and preservation of erectile function remains to be determined in long term follow up.
MP12-13 Can tumor volume percentage in radical prostatectomy predict risk of biochemical recurrence in prostate cancer? Determining a cut-off point and composite risk factors approach

\section{A Alenezi, C Eden, M Ismail}

Mubarak Hospital and Sabah Al Ahmad Urology Centre

Introduction \& Objective: Estimation of risks is paramount for specific management and investigating new trials. Incidence of biochemical recurrence (BCR) is relatively high and survival can be poor. Debate exists whether tumor volume is predictor of $\mathrm{BCR}$ and when treatments should be started after BCR. Our aims are to assess the impact of tumor volume percentage(TVP) as a predictor for BCR, determine TVP cut-off point for BCR and the relationship of TVP as a single and composite risk of BCR.

Methods: From March 2000-December 2013,1777 patients had laparoscopic radical prostatectomy for localized prostate cancer. None received neoadjuvant or adjuvant therapy. 146 had BCR (3 months-10 years). Using D'amico classification and age, matching control of 146 patients without BCR was created for comparison. Liu cut-point analysis was used to identify TVP value with optimal sensitivity and specificity. Single and composite BCR risk predictors were analyzed using Cox hazards regression.

Results: Mean TVP was $18.2 \%$ (1-90\%). TVP $\geq 8 \%$ (optimal cutpoint) was an independent predictor of BCR with $\mathrm{HR}$

\begin{tabular}{|c|c|c|}
\hline Parameter & Non recurrence & Recurrence \\
\hline Number & 146 & 146 \\
\hline Age & $61.5(41-75)$ & $63(46-76)$ \\
\hline \multicolumn{3}{|l|}{ Clinical stage } \\
\hline Tic & 66 & 32 \\
\hline T2a & 28 & 40 \\
\hline $\mathrm{T} 2 \mathrm{~b}$ & 6 & 20 \\
\hline $\mathrm{T} 2 \mathrm{c}$ & 36 & 28 \\
\hline T3a & 7 & 16 \\
\hline $\mathrm{T} 3 \mathrm{~b}$ & 3 & 8 \\
\hline Missing & 1 & 3 \\
\hline \multicolumn{3}{|l|}{ Pathological stage } \\
\hline T1c & 1 & 0 \\
\hline T2a & 16 & 10 \\
\hline $\mathrm{T} 2 \mathrm{~b}$ & 13 & 17 \\
\hline $\mathrm{T} 2 \mathrm{c}$ & 73 & 43 \\
\hline T3a & 24 & 28 \\
\hline T3b & 14 & 44 \\
\hline Missing & 6 & 4 \\
\hline Preoperative PSA & $9(1-31)$ & $14.2(1.253)$ \\
\hline Gleason Grade & & \\
\hline $\begin{array}{l}5 \\
6\end{array}$ & 1 & 0 \\
\hline \multirow{2}{*}{$\begin{array}{l}6 \\
7\end{array}$} & 57 & 25 \\
\hline & 78 & 86 \\
\hline $\begin{array}{l}7 \\
8\end{array}$ & 4 & 13 \\
\hline 9 & 3 & 17 \\
\hline 10 & 1 & 0 \\
\hline Missing & 3 & 6 \\
\hline D'Amico & & \\
\hline Low & 23 & 23 \\
\hline Intermediate & 55 & 55 \\
\hline \multirow{2}{*}{$\begin{array}{l}\text { High } \\
\text { Missing }\end{array}$} & 68 & 68 \\
\hline & 1 & 1 \\
\hline $\begin{array}{l}\text { Mean follow up } \\
\text { (months) }\end{array}$ & $108(63-194)$ & $103(30-194)$ \\
\hline Prostate weight (g) & $62(23-450)$ & $57(20-153)$ \\
\hline $\begin{array}{l}\text { Mean tumour volume } \\
\% \text { (range) }\end{array}$ & $12.8(1-90)$ & $23.6(0-80)$ \\
\hline Surgical Margin & 29 & 64 \\
\hline & 117 & 82 \\
\hline Missing & 1 & 1 \\
\hline & \\
\hline $\begin{array}{l}\text { Lymph Node Dissection } \\
\text { Performed: }\end{array}$ & 48 & 69 \\
\hline $\begin{array}{l}\text { - Invaded with } \\
\text { cancer }\end{array}$ & & \\
\hline $\begin{array}{l}\text { - Not invaded } \\
\text { with cancer }\end{array}$ & 43 & 36 \\
\hline $\begin{array}{l}\text { Lymph Node Dissection } \\
\text { Not Performed }\end{array}$ & 98 & 77 \\
\hline
\end{tabular}




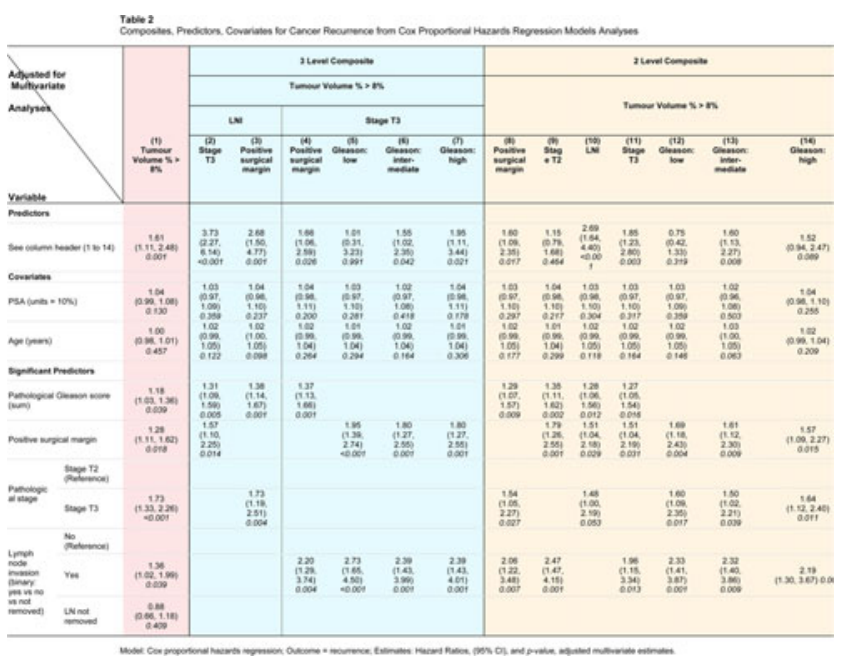

$1.6(\mathrm{p}=0.001,95 \% \mathrm{CI}=1.11-2.48) . \mathrm{TVP}$ of $8 \%$ was associated with the highest accuracy: sensitivity $74 \%$ and specificity $53 \%$ ( ROC curve $=0.7)$. At TVP $\geq 8 \%$, pathological stage T3 was associated with1.7 risk of BCR relative to T2. Lymph node invasion was associated with1.4 risk of BCR relative to no invasion. Combining TVP $\geq 8 \%$, pathological stage T3 and LNI were predictors of BCR with HR 3.73( $\mathrm{p}<0.001,95 \% \mathrm{CI}=2.27-6.14)$ and TVP $\geq 8 \%$, positive surgical margin and LNI HR $2.68(\mathrm{p}=001,95 \% \mathrm{CI}=1.50-4.77)$. Most of $\mathrm{BCR}$ peaked around 3 years follow up.

Conclusions: TVP can act as an independent predictor of BCR. TVP cut-point of $\geq 8 \%$ is an absolute value with the best discrimination. TVP should be considered in combination with other prognostic factors to aid the prediction of long-term oncological outcomes and to be used in risk stratification groups.

\section{MP12-14 Acute kidney injury and its impact on renal prognosis after robot-assisted laparoscopic radical prostatectomy}

S Kanda, S Narita, H Sato, T Inoue, T Nara, K Numakura, M Saito, T Habuchi

Akita University Graduate School of Medicine Department of Urology

Introduction \& Objective: The incidence of AKI and renal prognosis after AKI in robot-assisted laparoscopic radical prostatectomy (RALP) are largely unknown. The objective of this study is to assess the incidence and impact of AKI on renal prognosis after AKI in patients with prostate cancer who underwent RALP.

Methods: The medical records of 303 patients treated with RALP at Akita University Hospital between December 2012 and December 2017 were retrospectively reviewed. AKI was defined in accordance with the Kidney Disease Improving Global Outcomes criteria, and the patients with postoperative AKI were dichotomized into two groups including early AKI (immediately after surgery) and late AKI ( 1 to 7 days after surgery). The impact of clinical factors on two AKIs and the relationship between the presence of AKIs and clinical outcomes after surgery were statistically assessed.

Results: The mean age was 66 years. The mean level of serum creatinine (sCre) immediately after surgery was significantly higher than that at baseline ( $1.16 \mathrm{vs} 0.82 \mathrm{mg} / \mathrm{dl}, \mathrm{p}<0.01)$. The mean level of sCre on postoperative day 1 to 7 was not statistically different from those at 1 month, 3 months, 6 months, and 1 year after RALP. In total, the early and late AKIs were observed in 55.8\% (169/303) and 5.3\% (16/303) patients, respectively. In multivariate analysis, hypertension and console time were independent risk factors for early AKI, whereas diabetes mellitus was an independent risk factor of late AKI. The eGFR decline at 12 months after surgery in patients with early AKI tended to be lower than that in patients without early AKI $(\mathrm{p}=0.090)$. Among 172 patients with preoperative eGFR of $\geq 60 \mathrm{ml} / \mathrm{min}, 22$ patients $(12.8 \%$ ) developed eGFR of $<60 \mathrm{ml} / \mathrm{min}$ until 12 months after RALP. In patients with preoperative eGFR of $\geq 60 \mathrm{ml} / \mathrm{min}$, the eGFR decline at 12 months after surgery in patients with early AKI was significantly lower than that in patients without early AKI (6.1 vs. $3.1 \mathrm{ml} / \mathrm{min}, \mathrm{p}=0.048)$. On the other hand, the presence of AKIs were not associated with biochemical recurrence after surgery.

Conclusions: More than half of the patients who underwent RALP experienced AKI after surgery if renal function is measured immediately after surgery. Hypertension, diabetes mellitus and console time were associated with postoperative AKIs. The patients with AKI slightly reduced a renal function at 12 months after surgery. Therefore, long term renal outcomes after AKI in patients with prostate cancer treated with RALP need to be addressed in future studies.

\section{MP12-15 Comparison of clinical outcomes of lapa- roscopic and robotic radical prostatectomy}

H Nakanishi, K Ohba, S Gohno, K Kawada, T Matsuda, K Mitsunari, Y Shida, T Matsuo, T Hakariya, Y Miyata, H Sakai

Introduction \& Objective: Radical prostatectomy (RP) is a standard treatment for localized prostate cancer. Laparoscopic RP (LRP) was first performed in 1991 but was not accepted by urologists because the procedure was difficult and complex. The use of robot-assisted LRP (RALP) rapidly increased thereafter, and most RP procedures were performed by RALP. However, RALP is not adopted in many regions of the world because of its high cost. The most important points of RP are its perioperative, functional, and oncological outcomes. Therefore, this study was performed to compare the clinical outcomes between LRP and RALP.

Methods: This study included 163 patients who underwent LRP and 137 patients who underwent RALP from 2011 to 2017. LRP was performed using an extraperitoneal approach, and RALP was performed using a transperitoneal approach. Extended lymphadenectomy was performed for patients who underwent RALP with high risk according to the D'Amico classification. Only local lymphadenectomy was performed for all patients who underwent LRP.

Results: Although a history of open surgery was significantly higher in the LRP group than RALP group (18 vs. 5 patients, respectively; $p=0.046$ ), the patients' age, preoperative prostatespecific antigen level, and prostate volume were not different between the two groups. Perioperative blood loss was significantly lower in the LRP group than RALP group (615.6 vs. $193.9 \mathrm{ml}$, respectively; $\mathrm{p}<0.001)$. However, the operative time and number of nerve-sparing procedures were not different between the two groups. The oncological outcomes, including the pathologic tumor stage and positive surgical margin, were not significantly different between the two groups; however, more 
patients in the LRP than RALP group had a Gleason score of $<8$. The continence rate was not significantly different between the two groups. The frequency of late complications was not different between the two groups except for the appearance of inguinal hernia (LRP, 26 patients vs. RALP, 5 patients; $\mathrm{p}=0.002$ ).

Conclusions: In this study, the perioperative outcome, oncologic outcome, complications, and continence rate were comparable in both groups, although intraoperative blood loss and the appearance of inguinal hernia were worse in the LRP than RALP group.

MP12-16 Preservation of umbilical ligaments and Retzius space reconstruction improves early urinary incontinence after robot-assisted laparoscopic radical prostatectomy

\author{
H Hospital, K Shiga, K Miyoshi, S Naito \\ Harasanshin Hospital
}

Introduction \& Objective: A urinary incontinence is a most important complication in robot-assisted laparoscopic radical prostatectomy (RALP). Retzius space sparing (RSS) methods has been offered as an approach that contributes to early recovery of full urinary continence due to bladder anatomy preservation. However, RSS was reported to have more positive surgical margin rate on anterior surface of the prostate and more complicated procedure. Therefore, we established new methods to improve urinary incontinence by preservation of umbilical ligaments and Retzius space reconstruction (RSR) during RALP.

Methods: A retrospective registry database of 76 cases (34 of non-RSR versus 42 of RSR) consecutive transperitoneal RALP cases performed by a single surgeon was analyzed. The RSR contains the preservation of bilateral umbilical ligament and partially preservation of median umbilical ligament at the top of the bladder. After urethrovesicular anastomosis, anterior reconstruction was commonly performed in both groups, but bladder suspension to abdominal wall was performed only in RSR group.

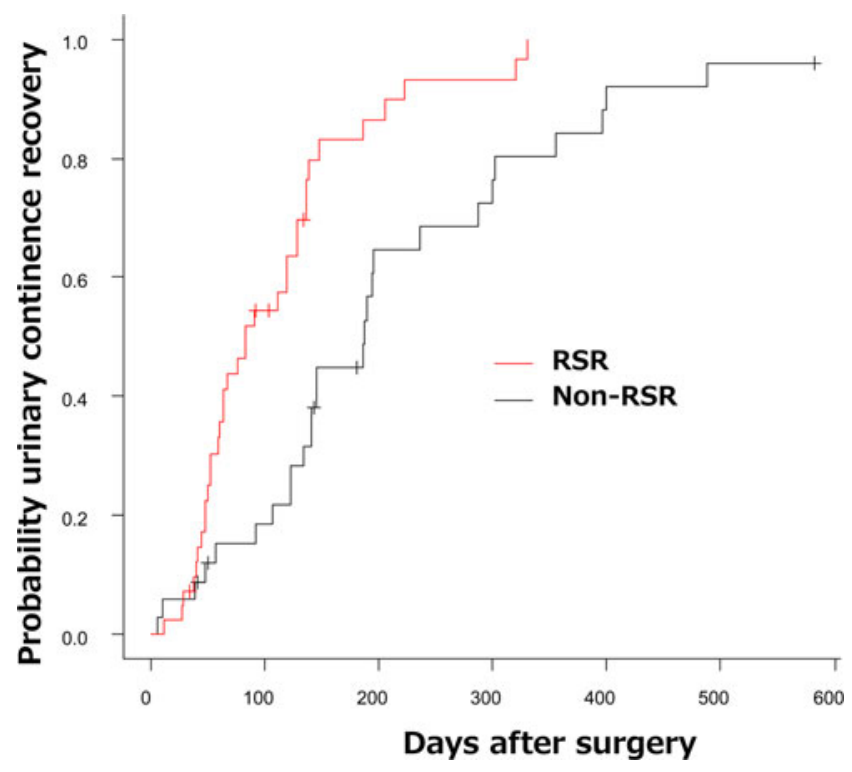

Results: Perioperative factors were analyzed and compared between the two groups. Demographic and perioperative data did not differ between the two groups. Kaplan-Meier analysis revealed that RSR group demonstrated significant faster urinary continence recovery ( 0 pads or 1 safety pad) (median days to recovery 83 days (95\% CI: 59-128 days) vs. 188 (95\% CI: 134 236 days), $p=0.0003)$. Furthermore, RSR was only a significant favorable factor that associated with postoperative continence (HR: $3.09,95 \%$ CI: $1.46-6.55, \mathrm{P}=0.0003$ ) in the Cox proportional hazard model analysis. The age, clinical stage by MRI, nerve sparing, pT and prostate volume did not significantly affect postoperative continence. There were no difference in operative and post-operative complication rate and grade between the RSR group and non-RSR group.

Conclusions: Preservation of umbilical ligaments and RSR in transperitoneal RALP is a simple and efficient methods that contribute to early recovery on urinary incontinence after surgery.

MP12-17 Predictive value of serum testosterone levels as early PSA recurrence after Robot-assisted laparoscopic radical prostatectomy in patients with higher Body Mass Index

\author{
S Koga, Y Horiguchi, A Ogata, N Sakamoto, F Tsukuda \\ Department of Urology, Edogawa Hospital
}

Introduction \& Objective: Current study is based on our previous consistent observation of paradoxical correlation of higher serum total testosterone levels (TT) among elder patients who undergone prostate biopsy. We hypothesized that higher preoperative TT would have a potential predictive value of aggressiveness of prostate cancer. We investigate the relationship between Body Mass Index (BMI), preoperative TT and postoperative PSA recurrence in patients undergoing robot-assisted laparoscopic radical prostatectomy (RALP).

Methods: We measured TT at 6 o'clock in the morning in all 719 patients who underwent transrectal/ transperineal prostate biopsy between July 2015 and March 2019 at our hospital. Of the 423 men with biopsy-proven prostate cancer, 150 underwent RALP. Results: The median age, serum PSA, BMI, and TT at diagnosis were 69 (41-80) years old, 8.8 (3.2-113.9) ng/mL, 24.5 (17.0 $36.6) \mathrm{kg} / \mathrm{m}^{2}$, and $4.16(0.73-9.86) \mathrm{ng} / \mathrm{mL}$, respectively. The median follow-up period was 20 (1-44) months. Twenty-two patients $(14.7 \%)$ showed early PSA recurrence postoperatively (median 4, range: 0-26 months). As previously observed, TT was positive correlation with age $(\mathrm{P}=0.006$, Linear regression analysis). While, TT was negatively correlated as BMI increases as generally observed in obesity populations $(\mathrm{P}<0.001)$. Among 40 patients in the higher BMI group stratified by BMI $>26$ (75 percentile), TT was significantly higher in 5 patients $(12.5 \%)$ with PSA recurrence (mean 3.28 vs $5.50, p=0.008$ ). On univariate analysis, pathological seminal vesicle invasion in the resected specimen as well as TT $>4.25$ (75 percentile of 40 higher BMI patients) were significant predictors of early PSA recurrence, whereas TT was the only significant predictor on multivariate analysis (Odds ratio 13.6, $\mathrm{p}=0.038$, Logistic regression analysis). Conclusions: Preoperative TT is a significant independent predictor of PSA recurrence after RALP in patients with a high BMI. The results suggest that preoperative TT may be a useful predictor of early PSA recurrence following RALP in patients with a high BMI. The limitation of our current study of short-range of follow-up period holds the current observation for future analysis of longer observation period which is currently on-going. 
MP12-18 Comparison of biochemical recurrence between open and robot-assisted radical prostatectomy in patients with positive surgical margins

T Ohashi, T Majima, R Ishida, Y Matsukawa, T Nishikimi, M Kato, T Tsuzuki, M Gotoh, H Kobayashi

Introduction \& Objective: Previous studies demonstrated that robot-assisted radical prostatectomy (RARP) was more associated with less blood loss and better functional outcomes, such as urinary continence and erectile function, compared with open radical prostatectomy (ORP). However, whether RARP leads to better oncological outcomes than ORP remains unclear. Therefore, we compared biochemical recurrence (BCR) between ORP and RARP in patients with positive surgical margins.

Methods: We retrospectively evaluated patients who underwent ORP or RARP at the Nagoya University Graduate School of Medicine and Japanese Red Cross Nagoya Daini Hospital between August 1996 and December 2016. Patients with patho-

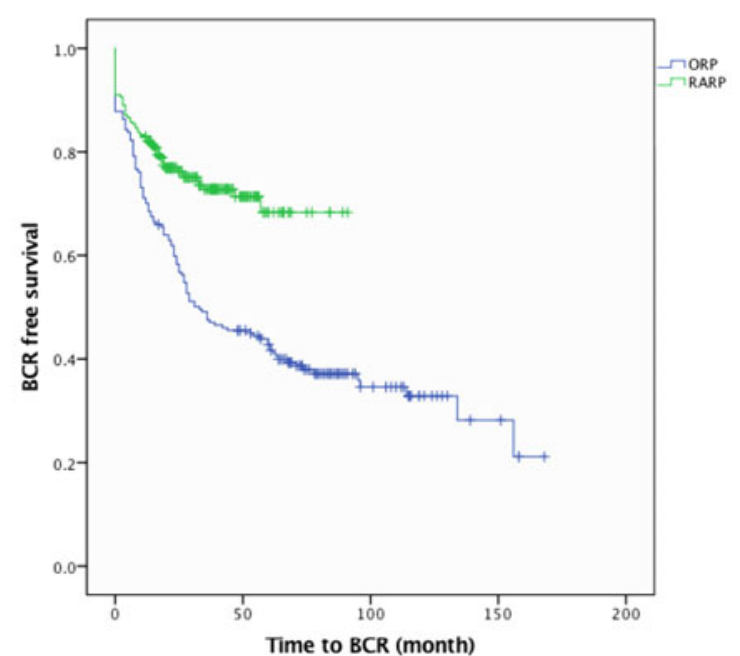

Fig. 1. Kaplan-Meier curve: BCR-free survival of patients who underwent RARP or ORP. BCR biochemical recurrence; RARP, robot-assisted radical prostatectomy; ORP, open radical prostatectomy.

\begin{tabular}{|c|c|c|}
\hline Variable & $\mathrm{HR}(95 \% \mathrm{Cl})$ & p Value \\
\hline \multicolumn{3}{|l|}{ Surgical approach } \\
\hline ORP & Reference & \\
\hline RARP & $0.412(0.300-0.565)$ & $<0.001$ \\
\hline \multicolumn{3}{|c|}{ Pathological T stage } \\
\hline pT2 & Reference & \\
\hline pT3a & $0.912(0.655-1.268)$ & 0.583 \\
\hline pT3b & $2.164(1.378-3.400)$ & 0.001 \\
\hline \multicolumn{3}{|l|}{ Gleason sum } \\
\hline$\leq 7$ & Reference & \\
\hline $8-10$ & $2.221(1.548-3.186)$ & $<0.001$ \\
\hline \multicolumn{3}{|l|}{ Pre-operative PSA } \\
\hline$<10$ & Reference & \\
\hline $10-20$ & $1.551(1.125-2.140)$ & 0.007 \\
\hline $20<$ & $1.857(1.139-3.028)$ & 0.013 \\
\hline
\end{tabular}

$\mathrm{HR}$, hazard ratio; $\mathrm{Cl}$, confidence interval. logically positive surgical margins were included. All prostatectomy specimens were reevaluated by a single central pathologist. Patients with pathological N1, metastatic disease, neoadjuvant hormonal therapy, adjuvant therapy, and follow-up of $<12$ months were excluded. The Kaplan-Meier method and multivariate Cox regression analysis were performed to determine the relationship between BCR and surgical approach, pathological T-stage (pT-stage), Gleason sum, preoperative PSA value, and length of surgical margins.

Results: In this study, 443 patients (197 ORP and 246 RARP) were included. The Kaplan-Meier analysis revealed BCR-free survival rates of $25.6 \%$ and $64 \%$ for the ORP and RARP patients, respectively $(p<0.001)$ (Figure 1$)$. In the multivariate Cox regression analysis, the predictive factors significantly associated with BCR were RARP (hazard ratio [HR], 0.412; $\mathrm{p}<0.001$ ) compared with ORP, pT3b (HR, 2.164; $p=0.001)$, Gleason sum of $\geq 8$ (HR, 2.221; $<<0.001)$, and preoperative PSA of $10-20$ $(\mathrm{HR}, 1.551 ; \mathrm{p}=0.07)$, PSA of $>20(\mathrm{HR}, 1.857 ; \mathrm{p}=0.013)$ (Table 1).

Conclusions: Our study demonstrated that RARP was significantly associated with BCR-free survival in comparison with ORP in patients with positive surgical margins. RARP possibly provides better oncological outcomes than ORP.

MP12-19 Comparison of outcomes in salvage roboticassisted laparoscopic prostatectomy for post-primary radiation vs. ablation therapies

O Fikret, S Bhat, T Rogers, M Covas Moschovas, H Ganapathi, S Roof, V Patel

Global Robotic Institute

Introduction \& Objective: We compared clinical and oncological outcomes of salvage robotic-assisted laparoscopic prostatectomy (sRALP) in post-primary radiation (RAD) vs. ablation (ABL) therapies.

Methods: Between 2008 and 2018, 126 patients underwent sRALP by a single surgeon. Of these, $94(74.6 \%)$ and $32(25.4 \%)$ patients had underwent RAD and $\mathrm{ABL}$, respectively; including external beam radiation (EBRT, $\mathrm{n}=39$ ), intensity modulated radiation $(n=15)$, proton beam $(n=3)$, brachytherapy $(n=23)$, combined EBRT and brachytherapy $(n=14)$, HIFU $(n=9)$, cryoablation $(n=20)$, and other (electroporation/microwave, $n=4)$ therapies. We analyzed the differences in clinical, oncological and functional outcomes between RAD and ABL groups. Results: Preoperative characteristics were similar except for potency rates $(46.9 \%$ vs. $22.6 \%$ in $\mathrm{ABL}$ and $\mathrm{RAD}$ group, respectively, $\mathrm{p}=0.013$ ). Operative times, complication rates, and hospital stay were not significantly different between the 2 groups. Postoperative catheter duration was shorter in the $\mathrm{ABL}$ group (mean 10 vs. 16 days, $\mathrm{p}=0.018$ ). ABL group had higher $\geq \mathrm{pT} 3$ disease $(71 \%$ vs. $50 \%, \mathrm{p}=0.042)$ and positive surgical margin rates (PSM $43.8 \%$ vs. $17 \%, \mathrm{p}=0.004$ ) compared to RAD group. However, estimated 5-year biochemical recurrence (BCR)-free survival rates were similar (59\% vs. $56 \%, \mathrm{p}=0.761)$. In multivariate analysis, only Gleason score $\geq 8$ at pathology was predictive for BCR (OR: 4.013, CI: 1.549-10.395). Postoperative continence recovery was significantly higher in the ABL group (Figure 1a). Full (no pads/day) and social (0-1 pad/day) continence rates in ABL and RAD groups were $77.3 \%$ vs. $39.2 \%$, and $87.5 \%$ vs. $51.3 \%$, respectively $(\mathrm{p}=0.002)$. Multivariate analyses showed ABL as primary treatment (OR: 2.644, CI: 1.066-6.559) and nerve sparing (OR: 2.415, CI: 0.99-5.891) as predictors of 


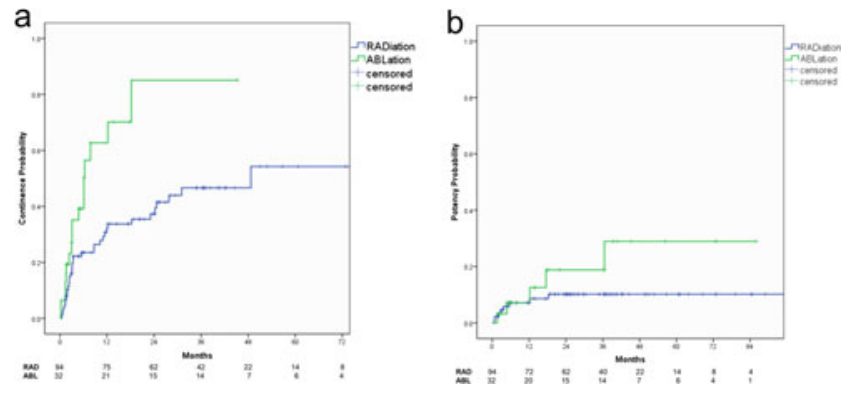

postoperative continence. There was a trend towards higher potency probability in the ABL group (Figure 1b), but the difference did not reach statistical significance $(p=0.179)$. Postoperative potency rate was $9.9 \%$ and $26.3 \%$ in the RAD and ABL group, respectively $(p=0.12)$. No independent predictors were identified for postoperative potency.

Conclusions: Patients who had ABL therapies as primary treatment have better erectile function at baseline. Radiation is associated with inferior functional outcomes after sRALP. Ablation therapies are associated with higher non-organ confined disease and PSM rates without a significant difference in shortterm BCR-free survival.

MP12-20 The Therapeutic Outcomes of Robotassisted Radical Prostatectomy (RARP) Following Neoadjuvant Chemohormonal Therapy for High-risk Prostate Cancer

T Yoneyama, H Yamamoto, A Imai, S Hatakeyama,

Y Hashimoto, C Ohyama

Hirosaki University Graduate School of Medicine

Introduction \& Objective: Robot-assisted Radical Prostatectomy (RARP) was introduced in Hirosaki University Hospital in July of 2011 and has been performed in 620 patients to date. Since January 2012, RARP has also been actively performed in patients with high-risk prostate cancer. In this study, We retrospectively examined efficacy and safety of neoadjuvant chemohormonal therapy with LHRH analogue + low-dose estramustine (LHRH+EMP) followed by RARP.

Methods: Subjects were 335 high-risk prostate cancer patients who underwent RARP at our hospital up to June 2018 and who were available for assessment. Mean age of 67.4 (50-77) years and a mean initial PSA level of $13.9(1.9-99.5) \mathrm{ng} / \mathrm{mL}$. A total of 86 patients were cT1c, 83 were cT2, 137 were cT3, and 9 were cT4. The mean Gleason score upon biopsy was 8.2 (6-10), and the mean follow-up period was 41.9 (4-81) months. The therapeutic method involves 6 months of neoadjuvant chemohormonal therapy using LHRH analogue and low-dose estramustine (EMP), followed by RARP.

Results: The mean surgical duration was 163.0 (95-291) and the mean console time was 110.0 (53-221) minutes, respectively. The mean blood loss was $25.0(1-1250) \mathrm{mL}$, respectively. Pathological evaluation revealed that 40 patients $(12.7 \%)$ were pT0. One patient was found to have pT2-positive surgical margin. The overall survival rate was $99.7 \%$ for 5 years, and PSA free survival was $98.7 \%$ for 1 year, $88.3 \%$ for 3 years, and $87.6 \%$ for 5 years. There was one case of prostate cancer death. Postoperative complications were observed in 11 cases with wound infection $(3.5 \%), 4$ cases with ileus $(1.2 \%)$.

\section{Overall survival}

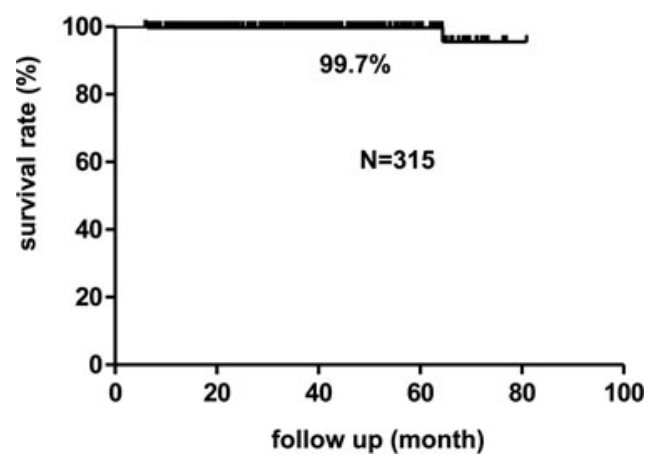

\section{PSA-free survival}

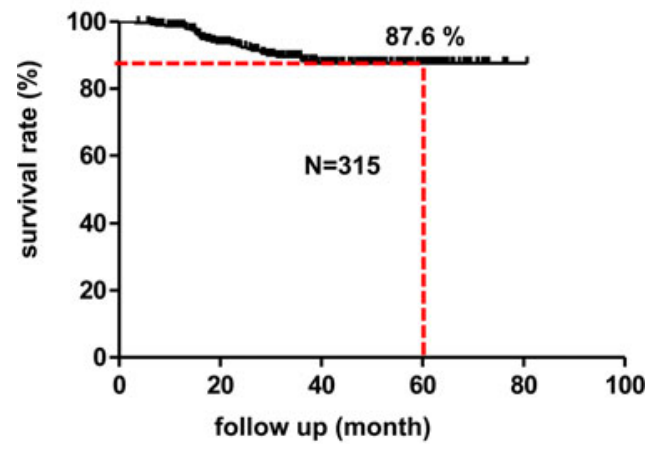

Conclusions: It was possible to safely perform RARP following neoadjuvant chemohormonal therapy for high-risk prostate cancer. However, a longer follow-up period than that of the present study is needed to establish cancer control.

MP12-21 Effect of Puboprostatic Ligaments Preservation during Robotic-Assisted Laparoscopic Radical Prostatectomy on Early Continence: Randomized Controlled Trial

W Ratanapornsompong, P Sungkum, S Pacharatakul, C Leenanupan, W Kongcharoensombat

Ramathibodi Hospital

Introduction \& Objective: Prostate cancer is the eighth most common cancer in Thailand. There are treatment options for localized disease, but robotic-assisted laparoscopic radical prostatectomy (RARP) is the most popular method at our institute. Post-radical prostatectomy incontinence is common. Continence is an important factor in improving patient quality of life. Most patients will regain continence in the first year after surgery but enhancing continence recovery is still attractive due to better quality of life. The aim of our study is to prove the effectiveness of puboprostatic ligament-preserving RARP on enhancing early continence compared with the standard technique RARP. Moreover, the margin status is also compared between the two groups.

Methods: Ninety-two patients with localized adenocarcinoma of the prostate scheduled for RARP from August 2017 to January 2019 were prospectively single-blinded and randomized into two 
groups: group A included patients for whom a standard RARP was performed $(n=46)$, and group B included patients for whom a puboprostatic ligament-sparing RARP was performed $(n=46)$. The primary outcome was continent status at Foley catheter removal day and 3 months after surgery using the score from the International Consultation on Incontinence QuestionnaireUrinary Incontinence Short Form (ICIQ-UI SF) and pad usage. The secondary outcomes were the pathological margin status, blood loss, operative time, and complications.

Results: Ninety-six patients were enrolled in this trial, with a mean age of $67.3 \pm 6$ years and median Prostate-Specific Antigen (PSA) of $9.77 \mathrm{ng} / \mathrm{ml}$ (range: 0.92-64). There were no significant differences in baseline characteristics between two groups including age, preoperative PSA, biopsy grade group and clinical risk. Continence status at 3 months postoperative was evaluated by the ICIQ-UI SF. The mean score was $8.74 \pm 4.28$ for group A and $6.93 \pm 3.96$ for group B. The score was better for group B $(p=0.038)$. There was one case in group A of complete or severe incontinence (more than 5 pads/day) at 3 months after surgery. Groups A and B did not exhibit significant differences regarding their margin status $(\mathrm{p}=0.828)$. Positive margins were detected in $34.78 \%$ of patients in group A and $36.96 \%$ in group B. There was also no difference in perioperative parameters: blood loss, operative time, and perioperative complications.

Conclusions: We propose the use of puboprostatic ligamentsparing RARP as a method to accelerate early continence without affecting the final oncological outcome.

\section{MP12-22 Salvage Pelvic Lymph Node Dissection (sPLND) for Nodal Recurrence of Prostate Cancer after Radical Prostatectomy}

V Berge, O Hopland, F Ottosson, S Fosså, E Hernes, K Hole, A Svindland, J Hoff, B Brennhovd, L Eri

Oslo University Hospital, Oslo University
Introduction \& Objective: There is no consensus about how to treat patients with biochemical recurrence (BCR) after radical prostatectomy, in whom metastases to lymph nodes are suspected by positron emission tomography/computerized tomography (PET/CT) or MRI (magnetic resonance imaging). The objective of this study was to study salvage sPLND on PSA kinetics and subsequent endocrine treatment (ET).

Methods: 80 consecutive sPLND patients, who had received no ET prior to sPLND from January 2013 to December 2018, are included. 35 patients ( $44 \%$ ) had undergone PLND during RALP and 39 patients ( $49 \%$ ) had received post-RALP radiation therapy. All patients had BCR and suspicious findings in pelvic lymphnodes on PET/CT and/or MRI. Biochemical response (BR) was defined as PSA $<0.2 \mathrm{ng} / \mathrm{ml} 6$ weeks $\left(1 \frac{1}{2}\right.$ month) after sPLND.

Results: Median age at RALP was 62 years (range $43-74$ years) and median pre-RALP PSA was $13 \mathrm{ng} / \mathrm{ml}$ (range $1.8-45.0 \mathrm{ng} / \mathrm{ml}$ ). 57 patients $(71.3 \%$ ) had PSA nadir $<0.2 \mathrm{ng} / \mathrm{ml}$ post RALP. Median age at SPLND was 67 years (range 47-79 years) and median PSA presPLND was $1.4 \mathrm{ng} / \mathrm{ml}$ (range $0.2-10.0 \mathrm{ng} / \mathrm{ml}$ ). Median time between RALP and sPLND was 47 months (range 4-205 months). There was agreement between MRI-PET/CT and malignant histology in 79\% of cases. Median lymph node yield in 38 patients with unilateral sPLND was 7.5 nodes (range 1-24 nodes), median metastatic node yield was 1 node (range $0-14$ nodes). Median lymph node yield in 42 patients with bilateral sPLND was 7.5 nodes (range 3-36 nodes), median metastatic node yield was 2 nodes (range 0-29 nodes).

Conclusions: The study suggests that sPLND can postpone initiation of endocrine treatment in some patients with BCR and positive lymph nodes on PET/CT and/or MRI after RALP. $19 \%$ of patients achieved a long-lasting BR

Table 1: Biochemical respons (BR) and endocrine treatment (ET) post-sPLND:
\begin{tabular}{|l|l|l|l|}
\hline & $\begin{array}{l}\text { FU months, median } \\
\text { (range) }\end{array}$ & N (\%) & $\begin{array}{l}\text { PSA, ng/ml } \\
\text { Median (range) }\end{array}$ \\
\hline BR & $1 \frac{1}{2}$ & $23(29)$ & $<0.2$ \\
\hline BR & $16(6-33)$ & $15(19)$ & $<0.2$ \\
\hline No ET & $14(4.0-51)$ & $41(51)$ & $0.5(0-7 \mathrm{ng} / \mathrm{ml})$ \\
\hline ET started & $9(1-45)$ & $39(49)$ & NA \\
\hline
\end{tabular}

\section{MODERATED POSTER SESSION 13: LAPAROSCOPIC/ROBOTIC: RENAL (I)}

\section{MP13-01 Robotic partial nephrectomy versus radical nephrectomy in elderly patients with large renal mass: a multi-institutional propensity score match paired analysis}

A Veccia, A Antonelli, A Mottrie, A Minervini, I Derweesh, C Lau, G Simone, J Kaouk, K Jacobsohn, DD Eun, $\mathrm{B}$ Challacombe, S Perdonà, J Porter, C Zang, U Capitanio, M Aron, C Simeone, L Hampton, F Porpiglia, R Autorino

Virginia Commonwealth University Health System

Introduction \& Objective: to perform a comparative study of the outcomes of patients older than 65 years undergoing robotic partial (RAPN) or radical nephrectomy (RARN).

Methods: retrospective analysis of a large multicenter, multinational dataset of patients with non-metastatic renal masses treated with robotic surgery. Patients' demographics, clinical staging features, perioperative variables, renal functional data, pathology, and oncological data were analyzed. A propensity score match analysis was performed adjusting for age, ASA $\geq 3$, R.E.N.A.L. Score and pre-operative eGFR. Univariable and multivariable logistic regression analyses assessed the association with the odds of overall complications and eGFR decrease $\geq 25 \%$ at discharge and at 6 months follow-up.

Results: a total of 445 elderly (over 65 yo) patients with large $(>4 \mathrm{~cm}$ ) renal mass were eligible for the analysis (after match pair analysis, RAPN $=184$ cases and RARN=79). In the RAPN group tumors were smaller $(6.55 \pm 2.14 \mathrm{cmvs} 8.21 \pm 1.68 \mathrm{~cm}$; $\mathrm{p}<0.001$ ), and with higher rate of low complexity R.E.N.A.L. score (32\% vs 5\%, p=< 0.001). Estimated blood loss was higher in the RAPN group $(301.31 \pm 427.23 \mathrm{~mL}$ vs $134.48 \pm$ 143.39; $\mathrm{p}<0.001)$. eGFR was higher in the RAPN group at both discharge $(62.03 \pm 22.01 \mathrm{~mL} / \mathrm{min}$ vs $47.52 \pm 15.24 \mathrm{~mL} / \mathrm{min}$; $\mathrm{p}<0.001)$ and 6 months follow-up $(54.54 \pm 17.72 \mathrm{~mL} / \mathrm{min}$ vs 

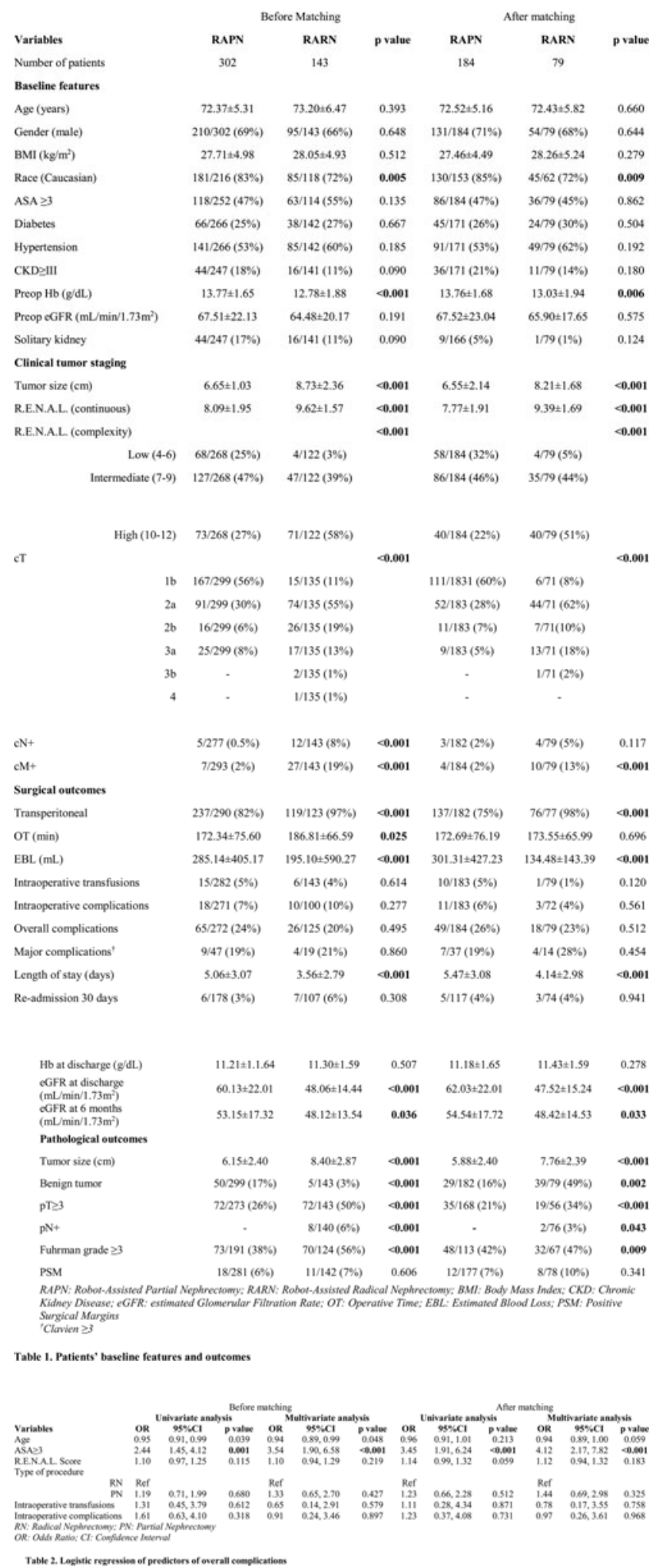

$48.42 \pm 14.53 \mathrm{~mL} / \mathrm{min}, \mathrm{p}=0.033)$. RARN group showed larger pathological tumor size $(7.76 \pm 2.39 \mathrm{~cm}$ vs $5.88 \pm 2.40 \mathrm{~cm}$; $\mathrm{p}<0.001$ ), worse pT (pT $\geq 3: 34 \%$ vs $21 \%$; $<<0.001$ ), and $\mathrm{pN}$ ( $\mathrm{pN}+: 3 \%$ vs $0 ; \mathrm{p}=0.043)$. At multivariate analysis, $\mathrm{ASA} \geq 3$ (OR: 4.12 ; 95\% CI: $2.17,7.82 ; \mathrm{p}<0.001)$ was the only independent predictor of overall complications.

Conclusions: RAPN represents a valid treatment option for elderly patients with large renal mass, as it provides better func- tional outcomes compared to RARN without increasing risk of complications. RARN can be reserved for more advanced disease.

\section{MP13-02 Retroperitoneoscopic partial nephrectomy - Trifecta outcome analysis in a single-institution series of 700 consecutive cases}

\author{
C Wülfing, D Marghawal, O Andura
}

Asklepios Klinik Altona

Introduction \& Objective: Minimally-invasive partial nephrectomy is increasingly offered to patients qualifying for nephron-sparing surgery for renal tumors. Reporting outcome in the literature is heterogenous but 3 key outcomes (1.) warmischemia time (WIT) below 20 minutes, 2.) negative surgical margins (SM) and 3.) no complications) are mostly mentioned. We report trifecta outcome in a single-surgeon series of retroperitoneoscopic partial nephrectomy (RPN).

Methods: 700 patients underwent RPN at our institution between April 2010 and April 2019 applying a standardized surgical procedure: After establishing the retroperitoneoscopic access, the renal hilum was dissected. Then, the tumor was dissected and - after placing a bulldog clamp on to the renal artery excised. The defect was sutured either using a double-layer VLoc running suture and additional Surgicel ${ }^{\circledR}$-bolster or applying the "sliding-clip technique" using hem-o-lok ${ }^{\circledR}$ clips. Perioperative parameters were collected, and Trifecta key outcome parameters were analyzed for the whole patient group.

Results: In all 700 patients, positive surgical margins occurred in 23 patients (3.2\%). WIT could be kept below 20 minutes in 525 cases $75 \%$ ). (Major) Complications happened in 33 patients $(4.7 \%)$. Trifecta of these three key parameters were achieved in 501 of 700 patients, accounting for a trifecta rate of $71.5 \%$ for the entire cohort. Technical modifications and evolution of the surgeon's learning curve lead to an improvement of Trifecta parameters to $87 \%$ for the most recent 200 cases of the cohort.

Conclusions: Retroperitoneoscopic Partial Nephrectomy using a standardized stepwise approach leads to favorable trifecta outcome. Trifecta should be a routine reporting system in future studies about minimally-invasive partial nephrectomy.

\section{MP13-03 Diagonal renorrhaphy technique in robot- assisted partial nephrectomy for endophytic mid-pole renal tumor of size $>\mathbf{4 m}$}

\section{G Wang, X Liu, X Zhou, B Fu, L Chen, Y Li}

The First Affiliated Hospital of Nanchang University; Jiangxi Institute of Urology

Introduction \& Objective: We developed a diagonal renorrhaphy technique for partial nephrectomy of endophytic midpole renal tumor of size $>4 \mathrm{~cm}$. The technique was tested in a porcine kidney model and compared with longitudinal suture and transverse suture technique. The safety and efficacy of diagonal renorrhaphy technique during robot-assisted partial nephrectomy (RAPN) for management of endophytic middle pole renal tumor of size $>4 \mathrm{~cm}$ was retrospectively evaluated.

Methods: 24 porcine kidneys were freshly harvested within 30 min of euthanasia from 12 healthy pigs. A standard renal 
defect was made in the mid-pole of porcine kidney opposite surface using a pair of custom-designed enucleation scissors. For renorrhaphy, a diagonal suture technique $(n=8)$, longitudinal suture $(n=8)$ and transverse suture $(n=8)$ were used. The decrease rate of the volume of intrarenal collecting system, the suture tension in perfusion states and the breakpoint pressure of renal artery when renorrhaphy ruptured were measured. Data of patients with endophytic mid-pole renal tumor of size $>4 \mathrm{~cm}$ who underwent RAPN from Jan 2016 to Jan 2019 were collected. All surgeries used diagonal renorrhaphy technique, following the same procedure: the renal artery was dissected and clamped, the tumor was enucleated from the kidney, defects of the vascular and collecting system were repaired, then the edge of the renal parenchyma was sutured diagonally along the border followed by unclamping. We retrospectively analyzed warm renal ischemic time, time required for renorrhaphy, blood loss.

Results: For animal study: The mean decrease rate of the volume of intrarenal collecting system was significantly less in the diagonal suture technique group than in the transverse suture technique $(30.5 \pm 18.3 \%$ vs $47.8 \pm 24.6 \%, \mathrm{P}<0.01)$. After simulated perfusion to $120 \mathrm{mmHg}$, the average suture tension to prevent observable leakage in diagonal suture technique group was lower than in the longitudinal suture technique $(3.5 \pm 1.3 \mathrm{~N}$ vs $6.8 \pm 2.6 \mathrm{~N}, \mathrm{P}<0.01)$. The mean breakpoint pressure of renal artery of renorrhaphy ruptures was significantly higher in the diagonal technique group than in the longitudinal suture technique $(476.5 \pm 104.3 \mathrm{mmHg}$ vs $276.8 \pm 95.6 \mathrm{mmHg}, \mathrm{P}<0.01)$. For clinical study: among the 21 patients, 15 were male (median age 62 years, range 39-73 years). Mean tumor diameter was $6.4 \pm 1.3 \mathrm{~cm}$. The mean warm ischemic time was $20.1 \pm 5.6 \mathrm{~min}$
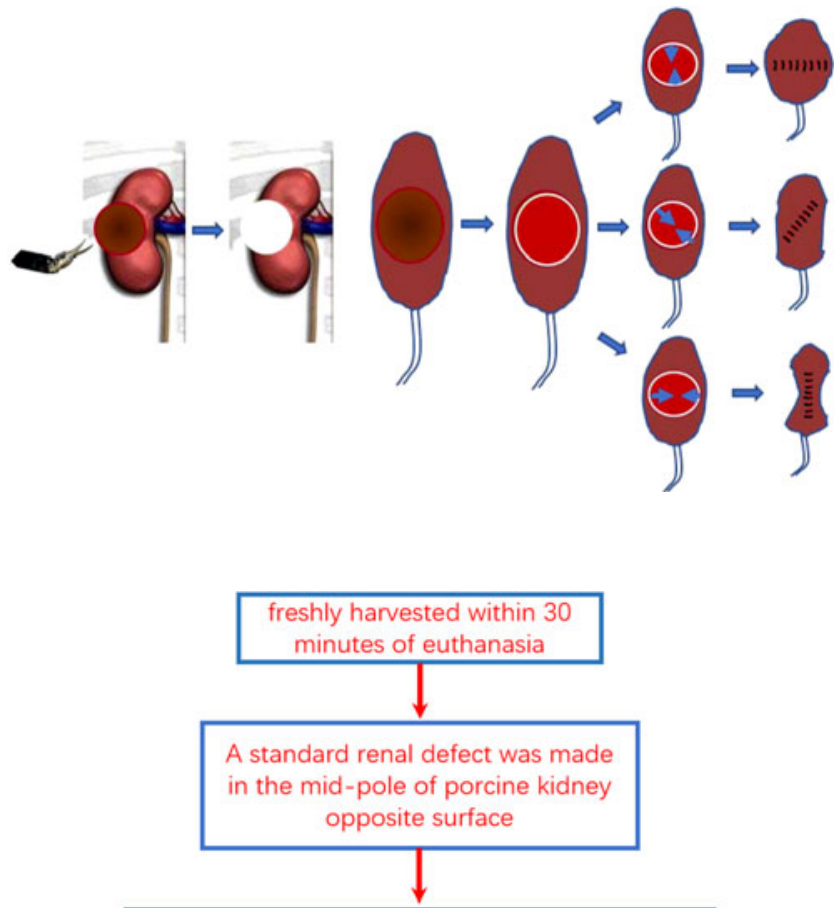

For renorrhaphy, a diagonal suture technique ( $\mathrm{n}$ $=8)$. longitudinal suture $(n=8)$ and transverse suture technique $(n=8)$ were used

The decrease rate of the volume of intrarenal collecting system, the suture tension in ischemic and perfusion states and the breakpoint and mean time required for renorrhaphy was $12.4 \pm 3.3 \mathrm{~min}$. Median blood loss during surgery was $130 \mathrm{ml}$ (range $50-360 \mathrm{ml}$ ). Conclusions: The diagonal renorrhaphy technique seems to be safe and effective for the management of endophytic mid-pole renal tumor of size $>4 \mathrm{~cm}$ during RAPN in selected patients.

\section{MP13-04 Is Robot-assisted Radical Nephrourete- rectomy with Bladder Cuff Excision Superior to Pure Laparoscopic Approach in The Treatment of Upper Urinary Tract Urothelial Carcinoma?}

Y Huang, E Huang, H Chung, M Tai, T Huang, T Wei, Y Fan, C Lin, T Lin, J Kuo, S Lu, Y Chang, A Lin, W Huang

Taipei Veterans General Hospital

Introduction \& Objective: Laparoscopic nephroureterectomy has been introduced to the treatment of upper urinary tract urothelial carcinoma for a long time, which could provide better cosmesis and comparable oncological outcomes to its open counterpart. Robotic approach was applied to such clinical scenario recently. However, there are few reports comparing laparoscopic and robotic approaches. We presented our experiences of robot-assisted (RANU) versus pure laparoscopic (LNU) radical nephroureterectomy with bladder cuff excision.

Methods: A retrospective review was performed for patients who underwent LNU or RANU from March 2012 to October 2017. Patients were matched for age ( \pm 3 years) and gender between the two-treatment arm. The perioperative and oncological outcomes were compared.

Results: A matched cohort of 174 patients who underwent RANU $(n=87)$ or LNU $(n=87)$ were compared. Each group consisted of 47 men and 40 women. There were no significant differences between the 2 groups with respect to BMI $(\mathrm{p}=0.63)$ and tumor location. The mean follow-up duration was 24.8 months in RANU group and 24.5 months in LNU group. No significant differences were noted regarding the total operative and nephroureterectomy time (335.8 vs 318.3 minutes, $\mathrm{p}=0.15$; 137.8 vs 147.4 minutes, $p=0.28)$, median post-operative hospital stay ( 8 vs 9 days, $\mathrm{p}=0.50$ ), median catheter time ( 5 vs 6 days, $\mathrm{p}=0.13)$, positive surgical margin rate $(4.6 \%$ vs $8 \%, \mathrm{p}=0.35)$ and peri-operative complications. However, the median estimated blood loss (EBL) was higher in the LNU cohort (150 vs $30 \mathrm{~mL}, \mathrm{p}<0.001)$. There were no significant differences with

\begin{tabular}{lccc}
\hline & RANU & LNU & $\mathrm{p}$ \\
\hline $\begin{array}{l}\text { Total operative } \\
\text { time (min) }\end{array}$ & $335.8(170-655)$ & $318.3(170-590)$ & 0.145 \\
$\begin{array}{l}\text { Nephroureterectomy } \\
\text { time (min) }\end{array}$ & $137.8(45-325)$ & $147.4(70-300)$ & 0.28 \\
$\begin{array}{l}\text { Median EBL (ml) } \\
\text { Median post-operative } \\
\quad \text { hospital stay (days) }\end{array}$ & $30(2-2500)$ & $150(30-1880)$ & $<0.001$ \\
$\begin{array}{l}\text { Median catheter time } \\
\quad \text { (days) }\end{array}$ & $5(3-16)$ & 9 & 0.496 \\
$\begin{array}{l}\text { Badder cuff excision } \\
\text { approach (\%) }\end{array}$ & 0 & $64(2-18)$ & 0.133 \\
$\quad \begin{array}{l}\text { Gibson Midline } \\
\text { Pure laparoscopic }\end{array}$ & $87(100 \%)$ & $11(12.6 \%)$ & \\
$\quad$ BCE & & & \\
$\begin{array}{l}\text { Intraoperative } \\
\quad \text { complication (\%) }\end{array}$ & $7(8)$ & $5(5.7)$ & \\
$\begin{array}{l}\text { Post-operative } \\
\text { complication (\%) }\end{array}$ & $11(12.6)$ & $9(10.3)$ & \\
$\begin{array}{l}\text { Clavien-Dindo score } \\
\quad \text { 2 3-5 }\end{array}$ & 1071 & 392 & 0.285 \\
\begin{tabular}{l} 
Median Clavien score \\
\hline
\end{tabular} & 1 & 2 & 0.305 \\
\hline
\end{tabular}



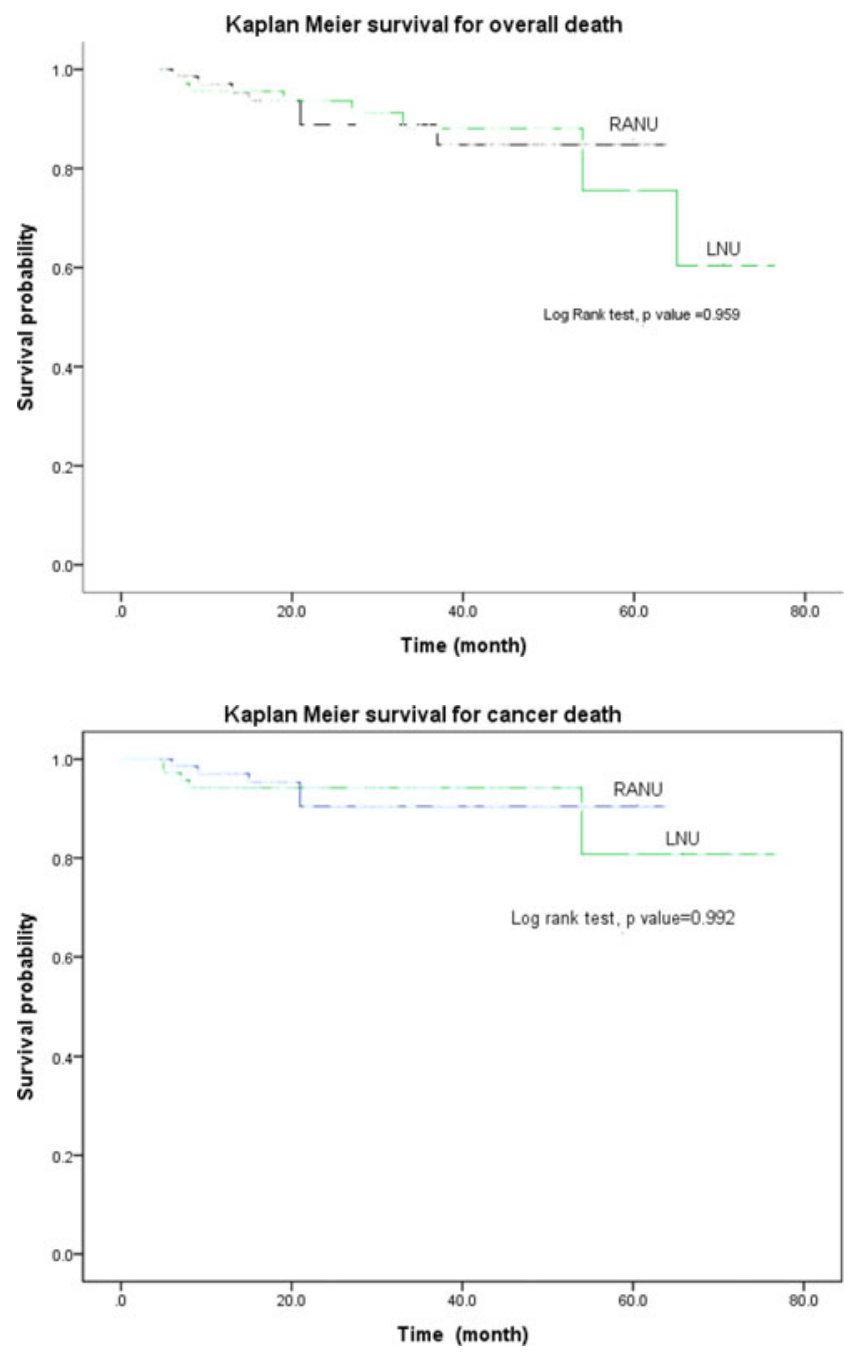

Perioperative results between RANU and LNU

\begin{tabular}{|l|c|c|c|}
\hline & RANU & LNU & $\mathrm{p}$ \\
\hline Total operative time (min) & $\begin{array}{c}335.8 \\
(170-655)\end{array}$ & $\begin{array}{c}318.3 \\
(170-590)\end{array}$ & 0.145 \\
\hline Nephroureterectomy time (min) & $\begin{array}{c}137.8 \\
(45-325)\end{array}$ & $\begin{array}{c}147.4 \\
(70-300)\end{array}$ & 0.28 \\
\hline Median EBL (ml) & $30(2-2500)$ & $150(30-1880)$ & $<0.001$ \\
\hline Median post-operative hospital stay (days) & 8 & 9 & 0.496 \\
\hline Median catheter time (days) & $5(3-16)$ & $6(2-18)$ & 0.133 \\
\hline Bladder cuff excision approach (\%) & & & \\
Gibson & & $64(73.6)$ & \\
Midline & & $12(13.8)$ & \\
Pure laparoscopic BCE & & $11(12.6)$ & \\
\hline Intraoperative complication (\%) & $7(8)$ & $5(5.7)$ & 0.55 \\
\hline Postperative complication (\%) & $11(12.6)$ & $9(10.3)$ & 0.635 \\
\hline Clavien-Dindo score & & & 0.285 \\
1 & 10 & 3 & \\
2 & 7 & 9 & \\
3-5 & 1 & 2 & \\
\hline Median Clavien score & 1 & 2 & 0.305 \\
\hline
\end{tabular}

respect to bladder recurrence, distant metastasis, cancer-specific death, and overall survival.

Conclusions: LNU and RANU had similar perioperative and oncological outcomes in our experiences. However, higher EBL was observed in the LNU cohort.
MP13-05 The Incidence and Pattern of Renal Cell Carcinoma Recurrence after Robotic Partial Nephrectomy

MW Salkini, A Lamoushi

West Virginia University

Introduction \& Objective: Robotic Partial Nephrectomy (RPN) is a rapidly growing treatment for small renal mass (SRM). In fact, RPN has shown good functional and oncologic outcome. In this manuscript, we are reporting on the incidence and pattern of recurrence of renal cell carcinoma (RCC) treated with RPN.
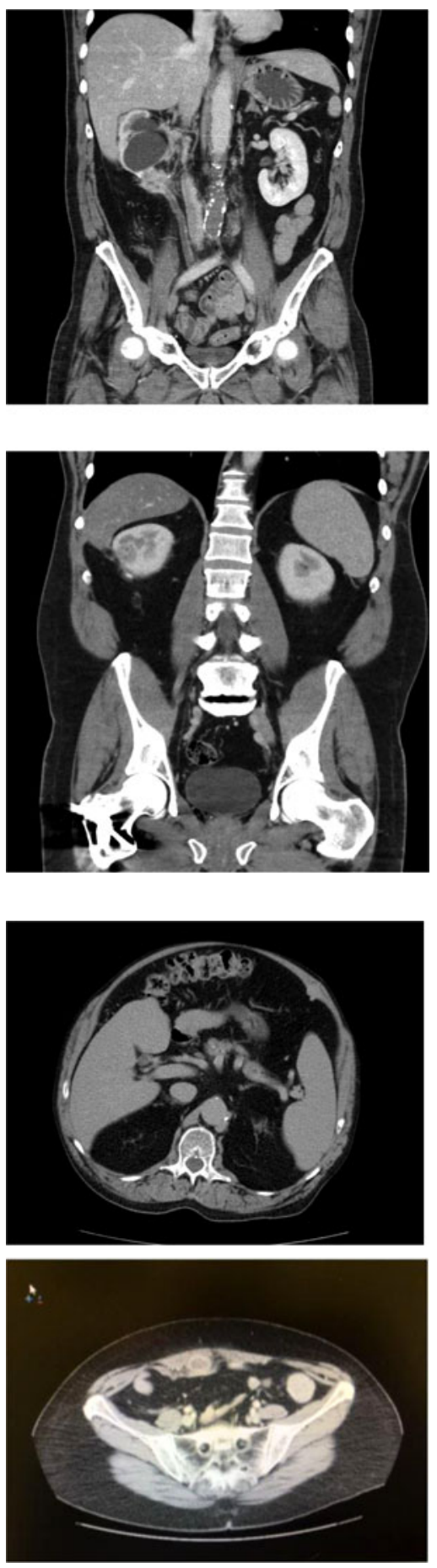


\begin{tabular}{|c|c|c|c|}
\hline Point given & 1 & 2 & 3 \\
\hline Radius of the Tumor $(\mathrm{cm})$ & $\leq 4$ & $>4$ but $<7$ & $\geq 7$ \\
\hline Exophytic/ Endophytic & $\geq 50$ & $\leq 50$ & $\begin{array}{l}\text { Completely } \\
\text { Endophytic }\end{array}$ \\
\hline $\begin{array}{l}\text { Nearness to the collecting } \\
\text { system }\end{array}$ & & & \\
\hline Anterior/Posterior & \multicolumn{3}{|c|}{ No points given, a for anterior and $\mathrm{p}$ for posterior tumor } \\
\hline $\begin{array}{l}\text { Location in relation to polar } \\
\text { lines }\end{array}$ & $\begin{array}{l}\text { Entirely above or below the } \\
\text { polar line }\end{array}$ & Crosses & $\begin{array}{c}\text { Entirely between } \\
\text { the polar lines }\end{array}$ \\
\hline
\end{tabular}

Table 2 Patient Demographics
\begin{tabular}{|l|l|}
\hline RPN & 335 patients. \\
\hline Studied RCC & $269(81 \%)$ \\
\hline Age & $67(28-81)$ Years \\
\hline Female/Male & $86 / 183(47 \%)$ \\
\hline
\end{tabular}

\begin{tabular}{|c|c|}
\hline R.E.N.A.L nephrometry score & $6.4(4-10)$ \\
\hline Clear Cell RCC & $192(71 \%)$ patients \\
\hline papillary RCC & $50(19 \%)$ patients \\
\hline Chromophobe RCC & $16(6 \%)$ \\
\hline unclassified RCC & $11(4 \%)$ \\
\hline Furman Grade 1 & $45(17 \%)$ \\
\hline Furman Grade 2 & $187(70 \%)$ \\
\hline Furman Grade 3 & $37(14 \%)$ \\
\hline Furman Grade 4 & 0 \\
\hline $\mathrm{Tla} 42$. & $215(80 \%)$ \\
\hline $\mathrm{Tl}$ & $42(16 \%)$ \\
\hline $\mathrm{T} 3 \mathrm{a}$ & $12(4 \%)$ \\
\hline
\end{tabular}

\begin{tabular}{|c|c|c|c|c|c|c|c|c|}
\hline Patient & 1 & 2 & 3 & 4 & 5 & 6 & 7 & 8 \\
\hline Pathologic Type & Clear cell & Papillary & $\begin{array}{l}\text { Clear } \\
\text { Cell }\end{array}$ & Papillary & Papillary & Papillary & Clear cell & Clear \\
\hline Tumor Grade & G2 & G3 & G2 & G3 & G2 & G2 & G3 & G2 \\
\hline Tumor & Tla & $\mathrm{TIb}$ & $\mathrm{T} 3 \mathrm{a}$ & $\mathrm{Tla}$ & $\mathrm{TIb}$ & Tla & $\mathrm{T} 3 \mathrm{a}$ & $\mathrm{TIb}$ \\
\hline $\begin{array}{l}\text { Timing of the } \\
\text { recurrence } \\
\text { (months) }\end{array}$ & 18 & 20 & 20 & 24 & 30 & 36 & 36 & 72 \\
\hline $\begin{array}{l}\text { Location of the } \\
\text { recurrence }\end{array}$ & Trocar site & Regional & Tumor bed & Tumor bed & $\begin{array}{l}\text { Second } \\
\text { primary }\end{array}$ & $\begin{array}{l}\text { Second } \\
\text { Primary }\end{array}$ & Tumor bed & $\begin{array}{c}\text { Trocar } \\
\text { site }\end{array}$ \\
\hline Age & 80 & 56 & 64 & 72 & 65 & 75 & 69 & 68 \\
\hline Sex & male & male & female & male & female & male & female & female \\
\hline
\end{tabular}

Methods: We reviewed prospectively collected data of patients who underwent RPN between September 2009 and March 2018. We selected patients with final pathologic diagnosis of RCC after the resection of their SRM. We described the incidence and pattern of recurrence in the patients who had it.

Results: Total of 335 patients with SRM underwent RPN. We found 269 patients to have RCC on the final pathologic evaluation of the SRM. 8 cases of recurrence were found with recurrence rate of $2.9 \%$ after mean follow up period of 31 months (range 18-72). The pattern of recurrence presented as the following: 2 patients $(0.7 \%)$ had trocar site recurrence (TSR), one patient $(0.37 \%)$ had loco-regional recurrence. 3 patients $(1.1 \%)$ had recurrence of the disease at the resection bed. Two patients $(0.7 \%)$ developed second primary tumor in the other kidney. No cancer related mortality occurred during the follow up period.

Conclusions: TSR, locoregional recurrence, tumor bed recurrence, and contralateral tumor development are the observed pattern of RCC recurrence after RPN. Recurrence was seen in up to 72 months. RPN provide great cancer control and high cure rate when utilized to treat RCC presenting as SRM,

MP13-06 Laparoscopic transperitoneal living donor nephrectomy early experience at a single center in Saudi Arabia

H El-Tholoth, T Alzahrani, H Al Akrash, R Zahid, A Al Zahrani

Urology Department, Prince Sultan Military Medical City

Introduction \& Objective: Renal transplantation is an appropriate treatment for end-stage renal disease. Laparoscopic donor nephrectomy has shown accretion in popularity as an effective way of kidney donation. Herein, we aim to present our early experience in laparoscopic donor nephrectomies.

Methods: All laparoscopic donor nephrectomies data collected from our first case on 25-1-2016 till December 2018 were collected. Their demographic data, transfusion requirement, ischemia time intraoperative finding and post-operative complications were reviewed. the data were analyzed using SPSS statistical package were conducted.

Results: 238 laparoscopic donor nephrectomies were done in this period 183 male 55 female with age range between (18-54) and average body mass index $30.8,233$ left side only 5 right side nephrectomies done. seventy-eight hand-assisted laparoscopic nephrectomy and 160 laparoscopic nephrectomies with a mean warm ischemia time 3 min $12 \mathrm{sec}$ and mean blood loss is $75 \mathrm{ml}$. no patients needed blood transfusion the mean operative time 75 mints. Regarding Post-operative complication 7 patients have grade I and II surgical complications and managed conservatively. No statistically significant difference between the two laparoscopic techniques regarding the operative time and warm ischemia time. The mean hospital stays for 2.4 days. Conclusions: Laparoscopic donor nephrectomy has an excellent surgical outcome and no significant postoperative complications and short hospital stay.

\section{MP13-07 Treatment outcomes of large angiomyoli- poma according to treatment method}

T Kwon, S Park, S Park, K Moon, S Cheon

Ulsan University Hospital

Introduction \& Objective: Selective arterial embolization (SAE) and nephron-sparing surgery (NSS) are acceptable treatment options for angiomyolipoma (AML) in the elective setting. SAE is an appealing option for management due to its less invasive nature; however, a comparative study of SAE and NSS is lacking. NSS is more invasive than SAE, but it might provide more durable results. In particular, NSS using robots can expect less invasive and effective treatment results. Therefore, the present study was performed to evaluate the treatment outcomes of large AML according to treatment method.

Methods: Between January 2011 and June 2018, we retrospectively reviewed 25 patients underwent robot-assisted partial 
nephrectomy (RAPN) or SAE for large AML. Ten patients underwent RAPN and 15 received SAE. Patient demographics, AML characteristics, operative and postoperative clinical outcomes were recorded and analyzed. To treatment outcomes were compared between patients who underwent RAPN and patients who received SAE, changes of renal function and decrease in size were evaluated after treatment.

Results: The mean age of the patients was 52.9 years and 22 out of 25 patients were female. The mean maximum diameter of the mass on CT was $8.9 \mathrm{~cm}$ and 8 were multiple masses. Twentytwo of 25 patients had moderate to high RENAL complexity. Patients who underwent SAE had more symptoms $(\mathrm{P}=0.018)$ and had higher RENAL complexity $(\mathrm{P}=0.013)$. The decrease in size after treatment, RAPN patients showed a reduction of 99\% in size and 58\% in SAE patients $(\mathrm{P}=0.001)$. Although the pre-treatment eGFR was higher in RAPN patients (99.8 vs. $80.0 \mathrm{~mL} / \mathrm{min} / 1.73 \mathrm{~m}^{2}, \mathrm{P}=0.043$ ), both groups were maintained without significant changes in eGFR after treatment. Complications occurred in one patient in the RAPN patient and postoperative ileus was improved without procedure.

Conclusions: Both RAPN and SAE were effective and feasible treatment options for large AML. The characteristics of AML and the condition of the patient might be important in determining the treatment method.

MP13-08 Discharge protocol for asymptomatic patients post pyeloplasty with satisfactory MAG 3 scans at annual review

N Kale, S Sangaralingam, Y Wagh, S Huq, Z Billoo, H Godbole

Department of Urology, North Middlesex Hospital

Introduction \& Objective: Retroperitoneoscopic Pyeloplasty (RP) for pelviureteric junction obstruction (PUJO) is postoperatively monitored with MAG3 scans for objective assessment. A retrospective analysis of the follow up of patients who underwent RP from 2005 till date is presented.

Methods: Adult patients presenting with symptomatic PUJO were evaluated by CT urogram and MAG3 scan preoperatively. On confirmation of diagnosis RP was performed. MAG3 scan was performed post operatively post stent removal. Follow up parameters included symptom relief and MAG3 scan comparison.

Results: Forty-two RPs were performed till date. Dismembered pyeloplasty was performed in 31 patients. Non dismembered pyeloplasty was performed in 11 patients. Crossing vessel was identified in 9 patients requiring anterior transposition of the PUJ. Thirty-six patients showed improvement in drainage postoperatively. Six patients had an obstructed curve 6 months post stent removal of which four improved by one year. One of the two patients with an obstructed curve maintained their split function and was managed conservatively. The other patient had postoperative pyonephrosis requiring an open redo pyeloplasty. At mean follow up of 65 months [6- 144 months] improved drainage remained sustained. Over a three-year period Hsi et al identified that most patients underwent functional imaging within 6 months of pyeloplasty. However, after 1 year only half of patients undergo follow up imaging which was usually non functional. [1] Nirmal et al followed up 138 patients over 8 years with repeat renal scans at 3.5 and 5.5 years after surgery showing stable split renal function. Of 138 patients, five had a significant deterioration in Split Renal Function. They conclude most renal units remain stable, thus repeat renal scans at five to seven-year follow up may be unjustified.[2] We performed MAG3 scan 6 months and one-year post pyeloplasty, with improvement in drainage in $95.2 \%$ cases at 1 year. Four patients with an obstructive curve at 6 months had improved clearance on a repeat scan at one year. Most patients had yearly scans after surgery. Only one of forty patients [2.5\%] with a non obstructive curve at one year follow up worsened.

Conclusions: In asymptomatic post RP patients MAG3 scan may be deferred till after one year. Despite a post-operative obstructive curve on MAG3 scan symptom relief with preservation of renal unit function is seen. Asymptomatic patients with satisfactory MAG 3 scans at annual review may be considered for discharge.

MP13-09 Outcomes and predictors of benign histology in patients undergoing robotic partial nephrectomy for renal masses: a multicenter study

A Veccia, A Antonelli, I Derweesh, A Mottrie, A Minervini, M Aron, G Simone, U Capitanio, C Simeone, DD Eun, S Perdonà, J Porter, C Sundaram, C Zang, R Uzzo, B Challacombe, L Hampton, J Kaouk, F Porpiglia, R Autorino

Virginia Commonwealth University Health System, Richmond, VA

Introduction \& Objective: To evaluate the incidence, outcomes, and predictive factors of benign renal masses in those patients who underwent robotic partial nephrectomy.

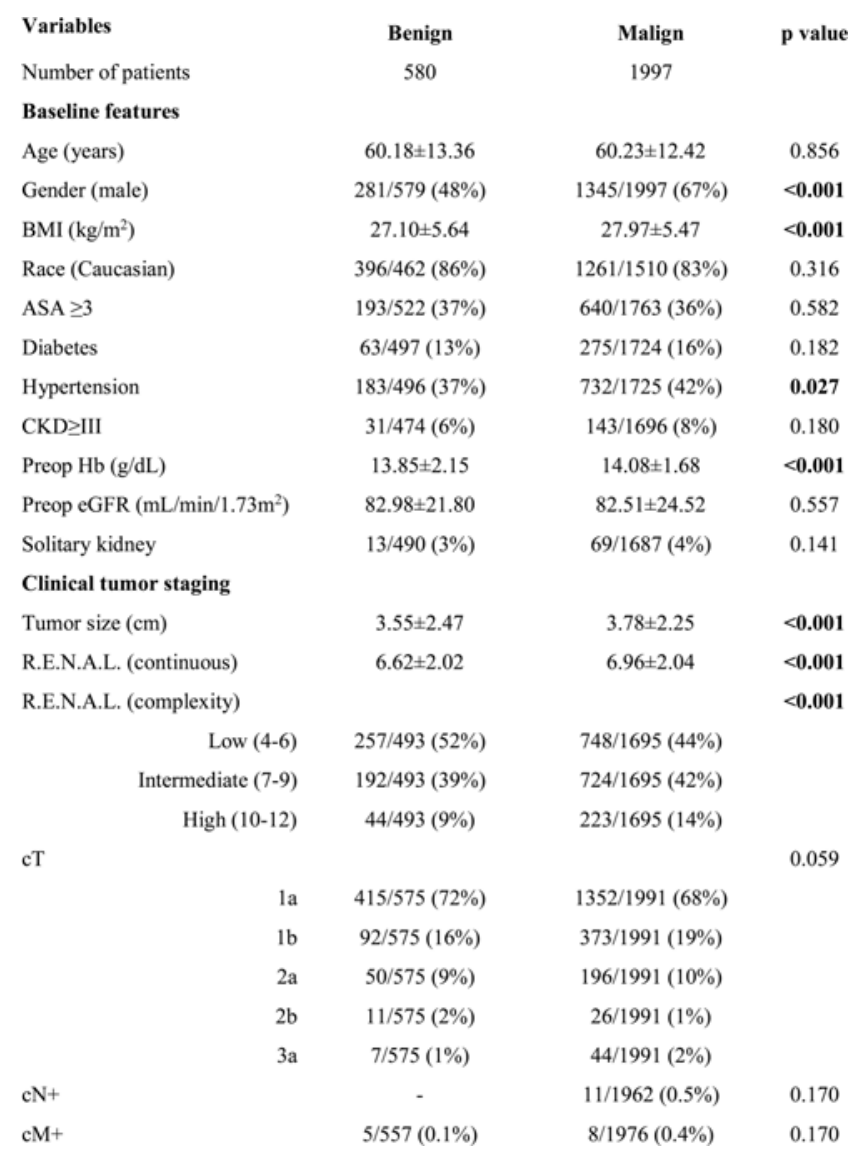




\begin{tabular}{|c|c|c|c|}
\hline \multicolumn{4}{|l|}{ Surgical outcomes } \\
\hline Retroperitoneal approach & $102 / 548(19 \%)$ & $311 / 1875(16 \%)$ & 0.267 \\
\hline OT (min) & $167.34 \pm 73.06$ & $175.92 \pm 74.83$ & 0.010 \\
\hline $\mathrm{EBL}(\mathrm{mL})$ & $152.32 \pm 209.37$ & $183.29 \pm 230.09$ & $<0.001$ \\
\hline Intraoperative transfusions & $8 / 542(1 \%)$ & $43 / 1868(2 \%)$ & 0.466 \\
\hline Intraoperative complications & $12 / 571(2 \%)$ & $54 / 1894(3 \%)$ & 0.331 \\
\hline Overall complications & $73 / 573(13 \%)$ & $293 / 1934(15 \%)$ & 0.151 \\
\hline Major complications ${ }^{\dagger}$ & $12 / 57(21 \%)$ & $34 / 227(15 \%)$ & 0.430 \\
\hline Length of stay (days) & $4.21 \pm 2.45$ & $4.42 \pm 2.56$ & 0.065 \\
\hline Re-admission 30 days & $6 / 352(2 \%)$ & $54 / 1194(4 \%)$ & 0.016 \\
\hline $\mathrm{Hb}$ at discharge $(\mathrm{g} / \mathrm{dL})$ & $11.77 \pm 1.55$ & $12.23 \pm 12.58$ & 0.207 \\
\hline $\begin{array}{l}\text { eGFR at discharge } \\
\left(\mathrm{mL} / \mathrm{min} / 1.73 \mathrm{~m}^{2}\right)\end{array}$ & $80.13 \pm 23.99$ & $74.90 \pm 26.44$ & $<0.001$ \\
\hline \multicolumn{4}{|l|}{ Pathological outcomes } \\
\hline Tumor size $(\mathrm{cm})$ & $3.57 \pm 2.67$ & $3.67 \pm 2.17$ & $<0.001$ \\
\hline $\mathrm{pT} \geq 3$ & $9 / 570(1.5 \%)$ & $171 / 1900(9 \%)$ & $<0.001$ \\
\hline $\mathrm{pN}+$ & - & $1 / 1784(0.05 \%)$ & 0.014 \\
\hline PSM & $23 / 517(4 \%)$ & $77 / 1952(4 \%)$ & 0.605 \\
\hline \multicolumn{4}{|c|}{$\begin{array}{l}\text { BMI: Body Mass Index; CKD: Chronic Kidney Disease; eGFR: estimated Glomerular } \\
\text { Filtration Rate; PN: Partial Nephrectomy; RN: Radical Nephrectomy; OT: Operative Time; } \\
\text { EBL: Estimated Blood Loss; PSM: Positive Surgical Margins } \\
\text { 'Clavien } \geq 3\end{array}$} \\
\hline
\end{tabular}

Table 1. Baseline features and outcomes

\begin{tabular}{|c|c|c|c|c|c|c|}
\hline \multirow[b]{2}{*}{ Variables } & \multicolumn{3}{|c|}{ Univariate analysis } & \multicolumn{3}{|c|}{ Multivariate analysis } \\
\hline & OR & $95 \% \mathrm{CI}$ & p value & OR & $95 \% \mathrm{CI}$ & p value \\
\hline Age & 0.99 & $0.99,1.00$ & 0.924 & 0.99 & $0.98,1.00$ & 0.609 \\
\hline Gender (male) & 0.45 & $0.37,0.55$ & $<0.001$ & 0.48 & $0.38,0.61$ & $<0.001$ \\
\hline BMI & 0.96 & $0.95,0.98$ & 0.002 & 0.96 & $0.94,0.98$ & 0.003 \\
\hline Pre-operative eGFR & 1.00 & $0.99,1.00$ & 0.701 & 0.99 & $0.99,1.00$ & 0.306 \\
\hline & \multicolumn{5}{|c|}{$\mathrm{cT}$} & 0.009 \\
\hline & Ref & & & Ref & & \\
\hline & 0.80 & $0.62,1.03$ & 0.091 & 0.81 & $0.59,1.13$ & 0.233 \\
\hline & 0.83 & $0.59,1.15$ & 0.271 & 0.81 & $0.51,1.24$ & 0.329 \\
\hline & 1.37 & $0.67,2.81$ & 0.378 & 1.07 & $0.41,2.77$ & 0.879 \\
\hline & 0.51 & $0.23,1.15$ & 0.110 & 0.56 & $0.23,1.38$ & 0.213 \\
\hline \multicolumn{7}{|c|}{$\begin{array}{l}\text { BMI: Body Mass Index; eGFR: estimated Glomerular Filtration Rate } \\
\text { OR: Odds Ratio; CI: Confidence Interval }\end{array}$} \\
\hline
\end{tabular}

Methods: Retrospective analysis of a large multicenter, multinational dataset of patients with non-metastatic cT2 masses treated with robotic surgery. Patients' demographics, clinical staging features, perioperative variables, renal functional data, pathology, and oncological data were analyzed. Univariable and multivariable logistic regression analyses assessed the association with the odds of benign or malignant tumor.

Results: A total of 2577 patients were included in the analysis (580 with benign tumor, and 1997 with malignant lesion). Patients with malignant tumor were mostly male (67\% vs $48 \%$, $\mathrm{p}<0.001)$, and with higher BMI $(27.97$ vs $27.10, \mathrm{p}<0.001)$ in the malignant group. The benign group showed lower rate of hypertension ( $37 \%$ vs $42 \%, \mathrm{p}=0.027)$. Benign group presented smaller renal masses $(3.55 \mathrm{~cm}$ vs $3.78 \mathrm{~cm}, \mathrm{p}<0.001)$ of lower complexity $(52 \%$ vs $44 \%, \mathrm{p}<0.001)$. The benign group demonstrated shorter operative time (167.34 minutes vs 175.92 minutes, $\mathrm{p}=0.010)$, lower estimated blood loss $(152.32 \mathrm{~mL}$ vs $183.29 \mathrm{~mL}, \mathrm{p}<0.001)$, and better eGFR at discharge $(80.13 \mathrm{~mL} / \mathrm{min}$ vs $74.90 \mathrm{~mL} / \mathrm{min}, \mathrm{p}<0.001)$. Malignant group showed worse pathological stage $(\mathrm{p}<0.001)$. At univariate and multivariate analysis none of the factors (age, male gender, BMI, preoperative eGFR, R.E.N.A.L. score and cT staging) was found to be predictor of benign histology.

Conclusions: in this study the rate of benign tumors was $29 \%$. Patients with benign tumors showed to present better surgical, functional, and oncological outcomes. None of the factors assessed showed to be independent predictor of benign tumor.

\section{MP13-10 Initial Experience with Robotic Assisted Left Renal Vein Extravascular Stent Placement in the Treatment of Nutcracker Phenomenon}

\section{RL Steinberg, A Garbens, BA Johnson, JA Cadeddu}

Introduction \& Objective: Nutcracker phenomenon of the left renal vein is a rare anatomic anomaly that can present with chronic flank/pelvic pain, pelvic congestion and hematuria. Treatment options include SMA transposition, endovascular stent placement, and auto-transplantation, all of which involve substantial risk or the need for chronic anti-coagulation. Extravascular left renal vein stent placement has also been reported. We now report our institution's experience with this procedure. Methods: A retrospective, single surgeon series from December 2016 to May 2019 was reviewed. After positioning and port placement ( 3 robotic ports, 1 assistant), the colon was reflected medially and Gerota's fascia was opened. The left renal vein was dissected free circumferentially and down to the inferior vena cava insertion. The distance between the renal vein ostium and adrenal vein was measured with a ruler. A $1 \mathrm{~cm}$ diameter ringed polytetrafluoroethylene vascular stent was cut to this length and bivalved. The stent was placed around the vein using traction sutures and the edges secured to itself with 3-0 polyglactin sutures. Demographics, surgical, and functional outcomes were collected. Descriptive statistics were calculated.

Results: Six patients with mean age of $45 \pm 6$ years and body mass index of $20.3 \pm 3.3$ grams underwent the procedure. Table 1 describes the baseline characteristics of the cohort, as well as surgical and functional outcomes. Mean operative time was $143 \pm 20$ minutes. Estimated blood loss was minimal. Mean graft length utilized was $2.25 \pm 0.3 \mathrm{~cm}$. Median day of discharge was 1.5 days (range 1-3). 2 patients sustained complications, both of which were Clavien Grade 1 (subcutaneous hematoma, chyle leak managed with dietary changes only). All patients received pain relief and $50 \%$ also saw other symptomatic improvements. Conclusions: Robotic assisted extravascular left renal vein stent placement appears safe and effective in a small cohort with short follow up. Further long-term follow-up for pain relief and graft related complications are needed.

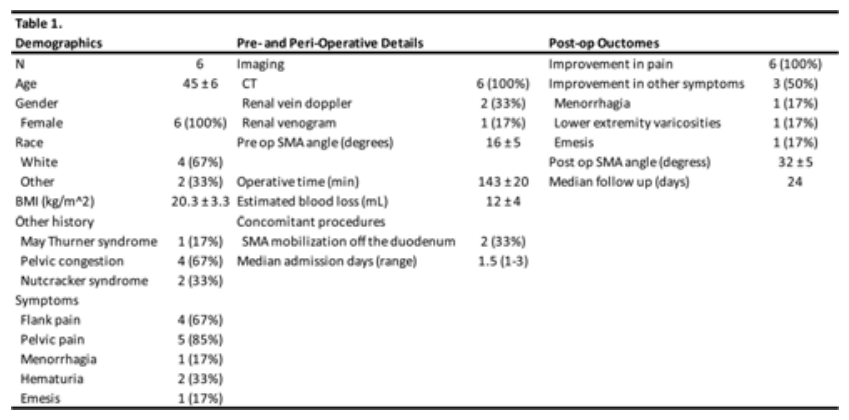

MP13-11 The role of intra-operative mitomycin $\mathrm{C}$ intravesical administration during robotic assisted nephroureterectomy

C Huang

Introduction \& Objective: The optimal timing of adjuvant mitomycin C bladder instillation is not well established. The aim of this study is to evaluate the safety and efficacy of intraoperative mitomycin $\mathrm{C}(\mathrm{MMC})$ intravesical administration during robotic nephroureterectomy (RALNUE). 
Methods: We retrospectively reviewed the patients who underwent RALNUE by single surgeon at tertiary referral center from November 2013 to February 2019. Intra-operative MMC administration was group A, and post-operative MMC administration was group B. Demographic information including age, gender, Charson cormobility index (CCI), ASA, and pathologic features were collected. Peri-operative outcome including operative time, blood loss (EBL), complication rate, and length of hospital stay and oncologic outcomes including bladder recurrence were evaluated by nonparametric statistics.

Results: There were 16 patients in group A while 28 patients in group B. Twenty-one patients were man. The mean (SD) age was 72.9(10.2), mean (SD) ASA was 2.6(0.49), mean (SD) CCI was 4.4(2.1). Among them $70.6 \%$ were stage I-III, and $29.4 \%$ were stage IV, and $95.2 \%$ were high grade urothelium carcinoma. There was no difference in demographic information and pathologic features between two groups. Regarding peri-operative outcomes, there were no difference in EBL (Group A: 50ml vs Group B: 100ml), operative time (Group A: 263 minutes, Group B:292 minutes), hospital stay (Group A: 7.6 days vs 7.6 days), and major complication rate (Group A: $6.3 \%$ vs Group B: $7.2 \%$ ). Though not significant, the bladder recurrence rate was lower than group $\mathrm{A}$ (6.3\% vs $21.4 \%, \mathrm{p}=0.2$ ).

Conclusions: Intraoperative MMC intravesical administration is a safe and feasible option for adjuvant treatment after RALNUE. Intraoperative MMC intravesical administration may reduce the rate of bladder recurrence. However further larger scale and longer followed up study was warranted.

MP13-12 Long-Term Stone Recurrence after ZeroFragment Laparoscopic Pyelolithotomy compared with Percutaneous Nephrolithotomy

H Pakmanesh, R Sharifian-Amiri, S Mohammad-Salehi

Kerman University of Medical Sciences

Introduction \& Objective: We compared percutaneous nephrolithotomy (PCNL) and laparoscopic pyelolithotomy (LP) in patients with large renal pelvis stone regarding success rate, complications as well as the recurrence free status.

Methods: From July 2015 to January 2019, 98 patients underwent PCNL or LP for single large renal pelvis stone $(\geq 2 \mathrm{~cm})$. Patient characteristics, preoperative and postoperative hemoglobin, creatinine as well as possible complications based on Clavien classification were recorded. Stone free status was evaluated using computed tomography scan one month after the surgery. Any particle bigger than $4 \mathrm{~mm}$ was considered as significant residual stone. During the next three years after the operation, renal ultrasonography was performed for all patients every six month to find any stone recurrence. Stone recurrence free status was compared using Kaplan-Meier estimate.

Results: Mean operation time was $72.35 \pm 24.89$ minutes and $136.12 \pm 31.41$ minutes in the PCNL group and LP group, respectively $(\mathrm{p}=0.001)$. Patients were hospitalized $2.06 \pm 1.31$

\begin{tabular}{|l|l|l|l|}
\hline Perioperative and Postoperative Data & LP $(\mathbf{n}=49)$ & PCNL (n=49) & P value \\
\hline $\begin{array}{l}\text { Perioperative finding } \\
\text { Mean operation time (min) }\end{array}$ & $136.12 \pm 31.41$ & $72.35 \pm 24.89$ & $0.00^{*}$ \\
$\begin{array}{l}\text { Mean hospital stay(day) } \\
\begin{array}{l}\text { Mean change in hemoglobin (g/dl) } \\
\text { Mean change in creatinine (mg/d) }\end{array}\end{array}$ & $3.33 \pm 1.13$ & $2.06 \pm 1.31$ & $0.00^{*}$ \\
\hline $\begin{array}{l}\text { Postoperative management } \\
\text { Stone free rate (1 month) }\end{array}$ & $1.60 \pm 1.05$ & $2.32 \pm 1.49$ & $0.002^{*}$ \\
\hline
\end{tabular}

days in the PCNL group and $3.33 \pm 1.13$ days in the LP group $(\mathrm{P}=001)$. Patients in the LP group showed less hemoglobin decrease $2.32 \pm 1.49$ vs $1.60 \pm 1.05(\mathrm{P}=0.002)$. Stone free rate was $79 \%$ in PCNL and $93.88 \%$ in LP group $(\mathrm{P}=0.03)$. Mean overall stone free status estimated to be 28.06 month in PCNL and 31.72 month in LP group $(\mathrm{P}=0.02)$.

Conclusions: PCNL has been the treatment of choice for large renal pelvis stones; however, in expert hands, LP is an appropriate substitute with superior stone free rate, less bleeding and remarkably less stone recurrence.

MP13-13 The " $N$ " component of R.E.N.A.L. nephrometry scores correlates with postoperative renal function after robotic-assisted partial nephrectomy

R Shimizu, E Moriyasu, N Sakamoto, T Hoshino, A Yamamoto, R Nishikawa, S Teraoka, Y Kimura, H Iwamoto, P Tsounapi, S Morizane, K Hikita, M Honda, A Takenaka

Department of Urology, Tottori University Faculty of Medicine, Yonago, Japan

Introduction \& Objective: R.E.N.A.L. nephrometry scores (RNS) has been used in robotic-assisted partial nephrectomy (RAPN) and is considered to be effective. The aim of this study was to evaluate the accuracy of RNS in evaluating perioperative outcomes of RAPN performed in our hospital.

Methods: RNS was evaluated in 95 patients who underwent RAPN in our hospital from August 2011 to January 2018. Of the five components, four (Radius, Exophytic/endophytic properties, Nearness of the tumor to the collecting system or sinus, Location relative to polar lines) were scored on 1, 2, or 3-point scale. Renal tumors were categorized by RNS complexity as low $(\mathrm{n}=39$; RNS sum, 4-6), moderate ( $\mathrm{n}=45$; sum, 7-9) and high ( $\mathrm{n}=11$; sum, 10 12). The relationships between the patients' scores and the outcomes of RAPN, warm ischemia time (WIT), tumor resection time, renorrhaphy time, postoperative complications, estimated blood loss (EBL), and percent change in estimated glomerular filtration rate (eGFR) were analyzed respectively. Trifecta was defined as achievement of negative surgical margin, no postoperative complications and WITof $\leq 25$ minutes.

Results: Significant differences were seen in RNS low, moderate and high in WIT (19.2 min vs. $21.1 \mathrm{~min}$ vs. $22.4 \mathrm{~min}: \mathrm{p}=0.026$ ), tumor resection time ( $7 \mathrm{~min}$ vs. $9 \mathrm{~min}$ vs. $11 \mathrm{~min}: \mathrm{p}=0.05$ ), renorrhaphy time (11 min vs. 14 min vs. $12 \mathrm{~min}$ : $\mathrm{p}=0.016$ ), but EBL had no statistically significant result. Significant declines in eGFR were observed 1-week post-surgery ( $3.5 \%$ vs. $14.0 \%$ vs. $15.7 \%$ : $\mathrm{p}<0.001)$ and 1-month post-surgery $(6.2 \%$ vs. $13.4 \%$ vs. $18.9 \%$ : $\mathrm{p}=0.001)$. The rates of achievement of Trifecta were $84.6 \%$ vs. $68.9 \%$ vs. $45.5 \%$ respectively $(\mathrm{p}=0.027)$. Examining the surgical outcomes of three components except the tumor size out of the four items of RNS, a significant difference was found among the 3 groups in the $\mathrm{N}$ component in the postoperative renal function (in 1 week; $\mathrm{p}<0.001$, in 1 month; $\mathrm{P}<0.001$, in 3 months; $\mathrm{p}=0.023$, in 1 year; $p=0.04$ ), but there were significant differences in the tumor size among the 3 groups. Between 1 point and 2 points groups of the $\mathrm{N}$ component with no significant difference in tumor size, there were significant differences in renal function between 1 week after surgery $(p=0.049)$ and 1 month after surgery $(p=0.024)$.

Conclusions: RNS proved to be useful for predicting the complexity of RAPN. Furthermore, it was associated not only with WIT, tumor resection time, renorrhaphy time and postoperative eGFR, but also with achievement of Trifecta. In addition, the $\mathrm{N}$ component of RNS can be a predictor of the early postoperative renal function. 
MP13-14 Comparative Analysis of Donor And Allograft Outcomes Based on Laterality of Kidney Donation: Proof of Equivalence of The Safety and Efficacy of Laparoscopic Left vs. Right Donor Nephrectomy

MA Dinno Francis, EL Gerial Jr., JA Abraham, DT Rubio

National Kidney and Transplant Institute

Introduction \& Objective: Laparoscopic right donor nephrectomy is avoided by most urologists because of the shorter vein and its proximity to the inferior vena cava. For them, dissection alongside the vena cava can be very treacherous. On the part of the implanting transplant surgeon, the shorter and thinner right renal vein is more challenging to anastomose than the lengthy thicker left. Further, these challenges with renal vein anastomosis may additionally impact on the renal arterial anastomosis which may result in kinking and subsequent thrombosis. We compared donor and recipient outcomes between laparoscopic left (LLDN) vs. right (LRDN) donor nephrectomy in a large volume transplant center in the Philippines.

Methods: A chart review was done on all laparoscopic donor nephrectomies performed from January 2011 to December 2015. The patients were grouped into LLDN (Group 1) and LRDN (Group 2). Donor demographics (age, gender, BMI) intraoperative [operative time (OT), length of hospital stay (LOS), estimated blood loss (EBL) and warm ischemia time (WIT)] and postoperative outcomes [mean creatinine rise, delayed graft function (DGF),] complication rate and one-year allograft survival were compared between the two groups.

Results: A total of 441 donors, 397(89\%) LLDN and 44(9\%) LRDN were performed during the study period. The donor characteristics were similar for both groups. There was no significant difference in OT in LLDN (178min[85-360]) vs. LRDN (176min[119-257]); LOS in LLDN (3days[2-8]) vs LRDN (3days[2-4]); EBL in LLDN (100mL[10-1600]) vs. LRDN (100mL[20-250]); WIT in LLDN (4min[1-32]) vs. LRDN (3min[1-12]) and DGF in LLDN 9/397(2.27\%) and LRDN 2/ $44(4.55 \%)$. There was no significant change in the mean donor and recipient creatinine up to one year. There was neither conversion to open nor mortality for both. The one-year allograft survival was also similar in both groups: LLDN 392/397(98.7\%) vs. LRDN 42/44(95.4\%).

Conclusions: Regardless of laterality, when performed by experienced surgeons, laparoscopic donor nephrectomy leads to equivalent outcomes for both donor and recipients in terms of safety profile and excellent allograft function.

MP13-15 Laparoscopic pyelolithotomy in a crossfused renal ectopia guided by 3 Dimensions imagine reconstruction

\section{Ye, M Hu, J Wu, Q Zu, Z Yang, J Wu}

Introduction \& Objective: Stone disease in cross-fused renal ectopia is rare, because of the abnormal anatomy, treatment options in such cases may be challenging. In this study, we present a case of right to left cross-fused renal ectopia with a $22 \mathrm{~mm}$ stone in the crossed kidney managed by transperitoneal Laparoscopic pyelolithotomy.

Methods: A 61-years-old woman presented with dull left lower abdomen pain and gross hematuria, CTU confirmed this was a right to left cross-fused renal ectopia with multiple stones, the largest one was $22 \mathrm{~mm}$ in the ectopic renal pelvis with suspicious

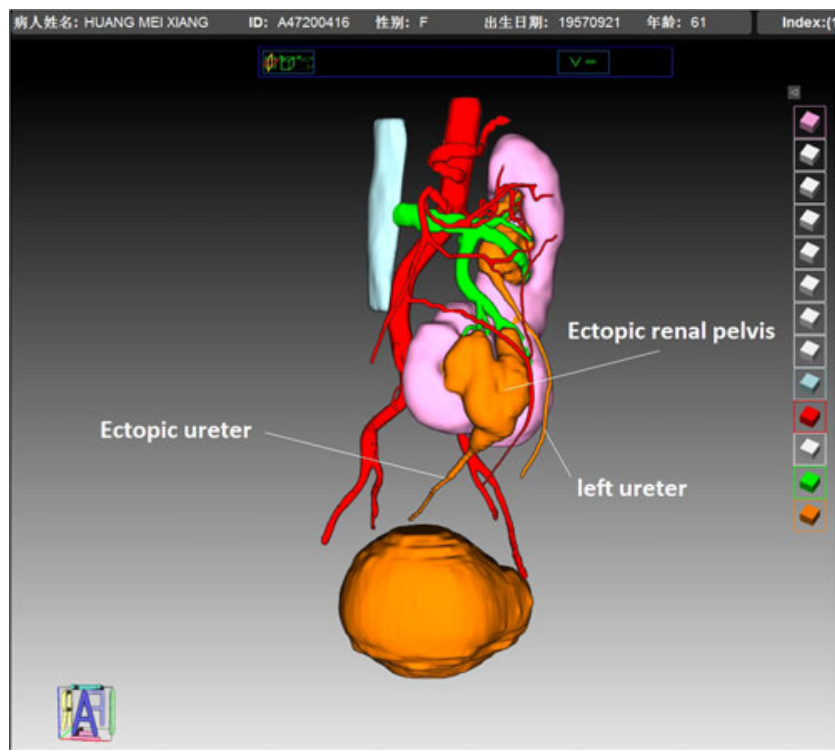

ureteropelvic junction obstruction. 3D imagine reconstruction was performed which provided anatomic details of crossed right kidney. Pros and cons of each treatment options including observation, ESWL, flexible ureteroscopic lithotripsy, PCNL, laparoscopic pyelolithotomy and open surgery were discussed, she was leaning towards a laparoscopic approach. Under general anesthesia, the patient was initially placed in a lithotomy position for bilateral ureteric catheters insertion, then she was replaced in a lateral position, transperitoneal and transmesocolic approach was used to give direct access to the renal pelvis, which was opened and calculi were removed from kidney, there was no obvious UPJ stricture, a double $\mathbf{J}$ stent was inserted antegrade and the pelvis was closed with continuous 4-0 absorbable sutures.

Results: The procedure was completed in 120 minutes without any intraoperative complications, estimated blood loss was less than $50 \mathrm{~mL}$. The patient had an uneventful postoperative recovery, she passed flatus and had diet on postoperative day (POD) 1, urethral catheter was removed on POD 4, drain tube was removed on POD5 and she was discharged on the sixth postoperative day. Postoperative KUB revealed no residual stone in the ectopic kidney and she was scheduled to remove the double J stent 6 weeks postoperatively. The patient would be followed up regularly for early detection of stone recurrence and surveillance of left kidney stones. Conclusions: Laparoscopic pyelolithotomy is a safe and feasible option in crossed fused renal ectopia that offers excellent outcome with low morbidity. Preoperative 3D imagine reconstruction is very helpful for surgical planning. Intraoperative ureteral cannulation on both sides should be considered to avoid iatrogenic injury to the contralateral ureter.

MP13-16 Laparoscopic Off-Clamp Partial Nephrectomy for Complex RENAL nephrometry score Tumors; its effects on peri-operative renal function

A Thwaini, M Evans, W Elbaroni, Z Aslam, K Randhawa

Craigavon Area Hospital

Introduction \& Objective: Off-clamp partial nephrectomy (OCPN) is advantageous for long term renal function but technically requires careful patient selection. We examine outcomes 
Figure 1: peri-operative outcome in the two groups:

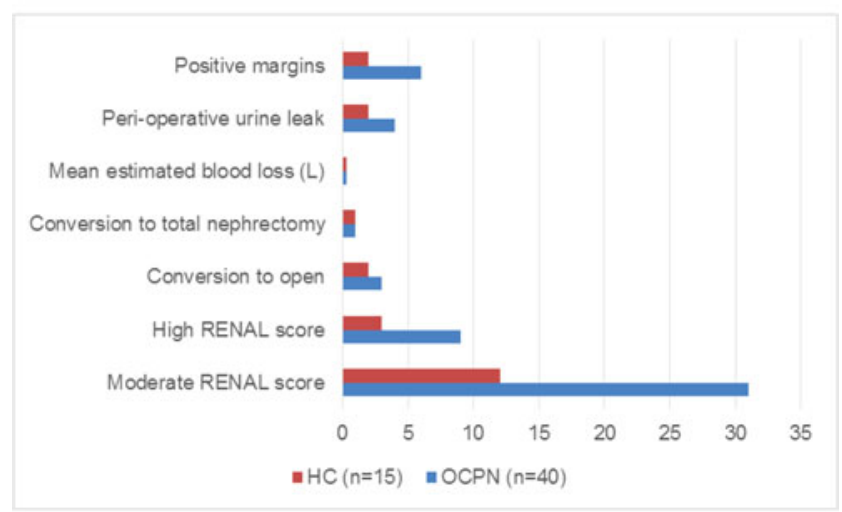

HC: Hilar clamping

OCPN: off-clamp partial nephrectomy

of OCPN in lesions with moderate to high RENAL nephrometry scores.

Methods: Retrospective review was performed of laparoscopic partial nephrectomy (LPN) procedures performed by a single surgeon between April 2014 and October 2016. Perioperative data and RENAL nephrometry score were collected. Cases with intermediate (score 7-9) and high-complexity (score 10-12) were compared against those of low nephrometry score and those with hilar clamping (HC). Renal function was assessed using the estimated glomerular filtration rate (eGFR). Postoperative oncological results and adverse events were analyzed. Data analysis was performed using t-test for. $p$ values of 0.05 or less were considered statistically significant.

Results: A total of 104 LPN procedures were performed. Low nephrometry score $(0-6)$ patients $(n=49)$ were excluded. HC $(n=$ 15) were used as control. Forty patients with moderate-to-high RENAL nephrometry scores underwent OCPN. The mean perioperative blood loss was lower in OCPN. The mean reduction in eGFR was less in OCPN group, however was not statistically significant. OCPN was also associated with a higher incidence of peri-operative urine leak. Positive margin rate was higher than that previously published, this maybe a reflection of the higher complexity of procedures being performed. On intermediate follow up, no patients developed any regional or distant recurrence.

Conclusions: Off-clamp partial nephrectomy can be safely achieved on renal tumors with intermediate and complex RENAL scores, with preservation of perioperative renal functional and satisfactory oncologic outcomes.

MP13-17 Necessity and feasibility of 4th arm for Robot-assisted partial nephrectomy by da Vinci Si

S Kira, T Mitsui, N Sawada, H Nakagomi, T Ihara, N Takahashi, M Takeda

Interdisciplinary Graduate School of Medicine and Engineering University of Yamanashi

Introduction \& Objective: It is unknown whether $4^{\text {th }}$ arm is feasible or necessary for Robot-assisted partial nephrectomy
(RAPN) by da Vinci Si. The aim of this study was to investigate the necessity and feasibility of $4^{\text {th }}$ arm for RAPN by da Vinci Si. Methods: This was a retrospective cohort study with 58 consecutive patients who underwent RAPN by da Vinci Si with same ports place between August 2016 and March 2019. After reviewing surgical videos and records, patients with usefulness of $4^{\text {th }}$ arm were categorized into group A and without it were into group B. Backgrounds of each patient (Height, BMI, and age), tumor characteristic (maximum diameter, R.E.N.A.L score, and tumor localization (upper, median, or lower pole/inner, median, or outer side on CT scan slice) in kidney), and operative outcomes (operative time, console time, warm ischemia time, estimated blood loss, and number of margin positive) were compared between two groups using Mann-Whitney's U test and chi-square test. Factors of patient and tumor characteristics for feasibility of $4^{\text {th }}$ arm was analyzed using multivariable logistic regression analysis.

Results: Group A and B consisted of 38 and 20 patients respectively. Group B had larger proportion of tumors located in the inner side of kidney than group A. (inner/median/outer; A 8/ $12 / 18$ vs B $11 / 6 / 3, p=0.015$ ) The console time was significantly longer in B group than in A group (185.5 vs $145 \mathrm{~min}, \mathrm{p}=0.036)$. There were no differences about other parameters including patient backgrounds, tumor characteristic, and operative outcomes between the two groups. Multivariable logistic regression analysis showed that tumor located in inner side of kidney was associated with useless of $4^{\text {th }}$ arm for RAPN by da Vinci Si (Odds ratio; 0.218, 95\% CI; 0.067-0.708, $\mathrm{p}=0.011$ ).

Conclusions: Our findings suggest that use of $4^{\text {th }}$ arm in RAPN by da Vinci Si should be considered for each tumor location.

\section{MP13-18 Three versus four arm robotic partial ne- phrectomy: Is it worth the hassle?}

J El-Asmar, R Sebaaly, A Mailhac, M Bulbul, R Khauli, H Tamim, A Hajj

\section{American University of Beirut Medical Center}

Introduction \& Objective: The addition of a $4^{\text {th }}$ arm during robotic partial nephrectomy (RPN) has been a debatable theme. Drawbacks in the 3-arm use implicate reliance on an experienced bedside assistant, a challenging hilar dissection and exposure varying according to surgeon's expertise. In contrast, a 4-arm use increases instrument clashing and patients' post-operative incisional pain. Despite the release of the Da Vinci Xi system which reduced instrument clashing, the Si model is still used in many centers worldwide. The outcomes of the two techniques are compared, and an innovative way to ease instrument collision with 4-arm use is described.

Methods: We report the results of a Middle Eastern tertiary referral center with RPNs from August 2013 to December 2017 performed by a single surgeon AEH. The data was retrospectively reviewed. A comparison study evaluated the differences between 3 versus 4 robotic arms in terms of surgical parameters, operative marks, and post-operative outcomes. Statistical analysis was performed with the Student's t-test and chi-squared test. To note, with 4-arm use longer bariatric ports were deployed to minimize arm collision and only one $12 \mathrm{~mm}$ assistant port was inserted.

Results: A total of 40 consecutive 3-arm RPNs and 40 consecutive 4-arm RPNs were retrospectively evaluated. Differences in tumor complexity (RENAL score) amongst the two groups were statistically insignificant ( $p$-value $>0.05$ ). Surgical 


\begin{tabular}{|c|c|c|c|c|}
\hline & & $\begin{array}{c}\begin{array}{c}\text { Number of } \\
\text { robotic arms }=3\end{array}\end{array}$ & $\begin{array}{c}\text { Number of } \\
\text { robotic arms }=4\end{array}$ & $\underset{\text { value }}{\mathrm{p}-}$ \\
\hline \multirow{3}{*}{ Renal score } & Low & $23(57.5)$ & $13(36.1)$ & \multirow{3}{*}{0.19} \\
\hline & Medium & $15(37.5)$ & $21(58.3)$ & \\
\hline & High & $2(5.0)$ & $2(5.6)$ & \\
\hline Biggest diameter Size & Mean $( \pm$ SD) & $3.14 \pm 1.19$ & $3.44 \pm 1.60$ & 0.351 \\
\hline Total OR time, min & Mean $( \pm \mathrm{SD})$ & $349.45 \pm 56.14$ & $307.33 \pm 51.91$ & 0.01 \\
\hline Surgeon hands on time & Mean $( \pm \mathrm{SD})$ & $286.35 \pm 56.72$ & $242.32 \pm 47.86$ & 0.000 \\
\hline Ischemia time, min & Mean $( \pm$ SD) & $17.40 \pm 6.16$ & $17.15 \pm 5.35$ & 0.852 \\
\hline Bld loss, mL & Mean $( \pm \mathrm{SD})$ & $247.50 \pm 135.38$ & $244.36 \pm 239.02$ & 0.943 \\
\hline Length of hospital, day & Mean $( \pm \mathrm{SD})$ & $3.98 \pm 1.05$ & $4.25 \pm 1.79$ & 0.405 \\
\hline GFR pre & Mean $( \pm \mathrm{SD})$ & $82.85 \pm 22.17$ & $86.175 \pm 21.79$ & 0.501 \\
\hline GFR post & Mean $( \pm$ SD) & $72.60 \pm 21.34$ & $77.36 \pm 23.29$ & 0.346 \\
\hline GFR difference & Mean $( \pm \mathrm{SD})$ & $-10.25 \pm 10.09$ & $-8.41 \pm 9.22$ & 0.401 \\
\hline \multirow{4}{*}{$\begin{array}{l}\text { Complication by Clavien } \\
\text { Dindo classification }\end{array}$} & 0 & $38(95.0)$ & $30(75.0)$ & \multirow{4}{*}{0.041} \\
\hline & 1 & $0(0.0)$ & $3(7.5)$ & \\
\hline & 2 & $2(5.0)$ & $4(10.0)$ & \\
\hline & 3 & $0(0.0)$ & $3(7.5)$ & \\
\hline \multirow{2}{*}{ Margin } & Negative & $38(94.9)$ & $37(92.5)$ & \multirow{2}{*}{1.00} \\
\hline & Positive & $2(5.1)$ & $3(7.5)$ & \\
\hline \multirow{6}{*}{ Pathology } & ccRCC & $24(60.0)$ & $23(57.5)$ & \multirow{6}{*}{0.024} \\
\hline & Papillary RCC Type 1 & $7(17.5)$ & $2(5.0)$ & \\
\hline & Papillary RCC Type 2 & $1(2.5)$ & $2(5.0)$ & \\
\hline & Angiomyolipoma & $4(10.0)$ & $0(0.0)$ & \\
\hline & Oncocytoma & $3(7.5)$ & $7(17.5)$ & \\
\hline & $\begin{array}{l}\text { Chromophobe RCC } \\
\text { Other }\end{array}$ & $\begin{array}{l}0(0.0) \\
1(2.5)\end{array}$ & $\begin{array}{l}2(5.0) \\
4(10.0)\end{array}$ & \\
\hline
\end{tabular}

margin positivity mean ischemia time, estimated blood loss, length of hospital stay, and mean change in serum creatinine all demonstrated no statistically significant difference between the two groups ( $\mathrm{p}$-value $>0.05$ ). Mean operative time was shorter by 38 minutes in the 4 -arm group $(\mathrm{p}=0.01)$. Post-operative complication rates according to the Clavien-Dindo classification scale was higher in the 4-arm group $(\mathrm{p}=0.04)$.

Conclusions: The addition of a $4^{\text {th }}$ arm in RPN can be of immense benefit especially in centers with limited resources. Our novel deployment of bariatric ports decreases arm collision. Whilst a higher complication rate with 4-arm use, the difference was small and likely related to increased tumor complexity observed in the 4- arm group. The ease of hilar dissection, retraction, and surgeon independence instigated a statistically significant decrease in operative time with 4 -arm use.

\section{MP13-19 Withdrawn}

MP13-20 Effect of Perioperative Continuation of Antithrombotic Therapy on Outcome of RobotAssisted Partial Nephrectomy : A Prospective Clinical Trial at Single-Institution

M Kubota, T Matsuoka, Y Tohi, S Murata, N Makita, I Suzuki, Y Sugino, K Inoue, M Kawakita

Kobe City Medical Center General Hospital

Introduction \& Objective: To assess the impact of the perioperative continuation of antithrombotic agents on bleeding and complications during undergoing robot-assisted partial nephrectomy (RAPN), we prospectively investigated surgical outcomes of patients.

Methods: From February 2015 to September 2018, a total of 127 patients who underwent RAPN for renal tumor were included in this prospective clinical trial. Twenty-eight patients continued taking antithrombotic agents in overall perioperative phase. We compared outcomes including intraoperative blood loss, transfusion rate, and bleeding complications in patients with continuing administration of antithrombotic agents (AA group: $\mathrm{n}=28$ ) with those with patients without history of taking antithrombotic agents (NA group: $n=99$ ). Multivariable analysis was used to identify independent risk factors for increasing bleeding in all patients.

Results: Among patients in AA group, 23 (82\%) patients used antiplatelet (aspirin: 13, clopidogrel: 5, others: 5, combination: 1), and 5 patients (18\%) used anticoagulant (rivaroxaban: 3 , dabigatran etexilate: 1, edoxaban tosilate hydrate: 1). As comparison of both group patients (AA vs NA group; median (IQR)), patients in AA group were older $(75(68-78)$ vs $63(52-72)$ years, $\mathrm{p}<0.001)$ and had more severe comorbidity (charlson-comorbidity index $\geqq 5 ; 7(25 \%)$ vs $4(4 \%), \mathrm{p}<0.001)$ than NA group. Nevertheless, there was no significant difference between both groups in intraoperative blood loss $(0(0-50)$ vs $50(0-150) \mathrm{ml}, \mathrm{p}=0.15)$, rate of intraoperative transfusion ( 4 vs $0 \%, p=0.22$ ), and rate of any high-grade complications (Clavien-Dindo Grade $\geqq$ III; 7.1 vs $2.0 \%, \mathrm{p}=0.21$ ). One patient in AA group was detected to develop a pseudoaneurysm postoperatively and performed angiographic embolization. None of the other patients in both groups had pseudoaneurysm and any other bleeding complications. In multivatiate logistic regression analysis, predictors of intraoperative bleeding $>150 \mathrm{ml}$ (upper $25 \%$ tile of overall patients) were transperitoneal approach $(\mathrm{OR}=4.3$, IC95\%: $1.3-12.1, \mathrm{p}=0.0025)$ and nearness of the tumor to the sinus $\leqq 4 \mathrm{~mm}$ (OR $=10.0$, IC95\%: $2.5-40.9, \mathrm{p}<0.001)$. The history of continuing antithrombotic therapies $(\mathrm{OR}=0.7$, IC95\%: $0.2-2.5, \mathrm{p}=0.92)$ was not a significant risk factor of high-volume bleeding.

Conclusions: These results indicate that perioperative continuing use of antithrombotic agents is not a factor of increasing bleeding and considered as safe in RAPN. Therefore, patients who have a risk of fatal thromboembolism should continue administrating their antithrombotic therapies during perioperative phase in RAPN.

\section{MP13-21 Laparoscopic donor nephrectomy: Strate- gies to reduce vascular complications and improve outcome}

Y Saifee, S Bhatia, C Chamania, J Kriplani, P Salgia, A Sepaha

\section{Choithram Hospital \& Research Centre}

Introduction \& Objective: Laparoscopic donor nephrectomy (LDN) has become the standard of care and popular among most of the transplant centers across the globe. However, for novices, it remains a challenge because of safety concerns during learning curve and many small volume centers are still hesitant to start laparoscopic donor program. At our institution, we adopted an approach that allowed us implementation of laparoscopic live donor nephrectomy without increased donor morbidity or graft failure, even during the early portion of a learning curve.

Methods: From December 2016 to May 2019, 242 Laparoscopic donor nephrectomy were performed by transperitoneal approach. All donors and recipients underwent extensive preoperative evaluation by a multidisciplinary team before the decision was made to proceed with the donation. To reduce beginner's fear, we made some technical modification in surgical technique. One important modification was use of additional ports and involvement of 2 nd consultant or chief surgical resident as assistant in some cases, depending on internal anatomy and the patient's body habitus. Second important modification was in sequence of hilar dissection. In our approach we completed dissection on both poles of kidney first and did the difficult lumbar vein and hilar dissection in the last. 
Results: Mean operation time was 107.6 (72-156) minutes and Mean warm ischemia time 186 (164-553) seconds. 27 donors (11.1\%) suffered a total of 31 postoperative complications. All complications were Class I and Class II only. So our initial data of 242 patients seems much better. Operative \& Warm ischemia time was lower. No major (Grade III and Grade IV) perioperative or postoperative complications and only two case requiring transfusion and conversion. We attribute this to various reasons. First reason for our success is proper planning and team approach. Involvement of experienced assistant and use of additional ports played a vital role. This strategy, facilitated maximum exposure of the renal hilum by simultaneous retraction and suction by assistant, leading to fast dissection, smooth progression and operative time reduction. Second reason in our opinion for our success is the sequence of hilar dissection in the last, after dissection of both poles of kidney. It made intrahilar dissection easier and safer because of opportunity of dissection in a relatively stretched hilum with a possible vision from both poles.

Conclusions: We propose that, proper planning, team approach and few technical modifications are the key strategies to reduce vascular complications and improve outcome of laparoscopic live donor nephrectomy program.

MP13-22 Prospective development of a prediction model for postoperative renal function evaluation after unilateral nephrectomies using preoperative risk factors and measured glomerular filtration rate with 51Cr-EDTA

HS Andrade, V Srougi, MA Arap, AI Mitre, CP Camargo, H Zargar, J Kaouk, WC Nahas, M Srougi, RJ Duarte

Clinicas Hospital - University of Sao Paulo

Introduction \& Objective: To analyze the preoperative variables associated to the postoperative glomerular filtration rate (GFR) outcomes after nephrectomy for benign and malignant conditions, measured by the reference isotopic technique ${ }^{51} \mathrm{Cr}$-EDTA and to create a model to predict the short-term postoperative GFR. Secondary aim was to evaluate which of the common equations for GFR estimation (Cockcroft-Gault, MDRD or CKD-EPI) has the best correlation with the ${ }^{51} \mathrm{Cr}$-EDTA.

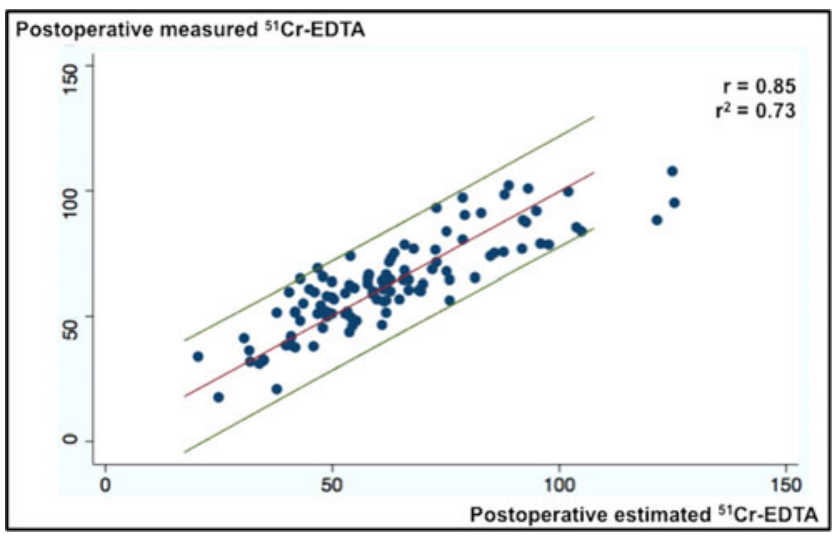

Methods: Patients undergoing unilateral nephrectomy from 2014 to 2018 were selected. Preoperative and postoperative variables were prospectively collected. Univariate and multivariate analyses were done to identify independent risk factors associated with renal function outcomes and to create a model to predict the postoperative GFR. Correlation analyses were performed to evaluate the performance of various serum creatininebased equation for GFR estimation compared to ${ }^{51} \mathrm{Cr}$-EDTA.

Results: In total, 107 patients were evaluated. After univariate and multivariate analyses, older age $(p=0,008)$, higher split function of the operated kidney on DMSA scintigraphy $(\mathrm{p}<0,001)$ and lower preoperative 51Cr-EDTA $(\mathrm{p}<0,001)$ were independent risk factors for higher GFR decline. Using these variables, a model was generated to estimate ${ }^{51} \mathrm{Cr}$-EDTA after unilateral nephrectomy: postoperative ${ }^{51} \mathrm{Cr}$-EDTA $(\mathrm{ml} / \mathrm{min} / 1.73 \mathrm{~m} 2)=$ $37.9-(0.29 *$ age $)-(0.42 *$ affected kidney DMSA $)+(0.67 *$ preoperative $51 \mathrm{Cr}$-EDTA). Significant correlations were observed between the calculated and observed ${ }^{51} \mathrm{Cr}$-EDTA six months after surgery $(r=0.85, \mathrm{p}<0.001$, Figure 1$)$. Correlation analyses showed that GFR estimated by CKD-EPI equation had the best concordance to GFR measured by $51 \mathrm{Cr}$-EDTA.

Conclusions: Based on our findings age, DMSA and lower preoperativen ${ }^{51} \mathrm{Cr}$-EDTA are predictors of postoperative renal function after unilateral nephrectomy. For the assessment of estimated GFR, CKD-EPI equation appears to have the best concordance with ${ }^{51} \mathrm{Cr}$-EDTA.

\section{MODERATED POSTER SESSION 14: IMAGING}

\author{
MP14-01 Can Preoperative Renal Mass Biopsy \\ Change Clinical Practice And Reduce Surgical Inter- \\ vention? \\ Z Okhunov, MA Gorin, FA Jefferson, M Allaf, P Pierorazio, \\ J Sung, RM Patel, LM Huynh, S Tapiero, LR Kavoussi, \\ RV Clayman, J Landman \\ University of California, Irvine
}

Introduction \& Objective: We performed a matched retrospective cohort analysis of patients with a cT1a or cT1b renal cortical neoplasm who underwent renal mass biopsy (RMB) before surgery versus patients who did not undergo pre-treatment biopsy.

Methods: We analyzed a multi-institutional, prospectively maintained database of patients who underwent an office-based, ultrasound-guided, RMB. A retrospectively matched cohort of patients who did not undergo a RMB served as a control. The groups were matched based on age, body mass index (BMI), Charlson Comorbidity Index (CCI), R.E.N.A.L Nephrometry score, and tumor size. Groups were analyzed for differences in treatment modality and surgical pathology results.

Results: A total of $91 \mathrm{RMB}$ and 107 control patients were analyzed. The groups were similar in regards to baseline characteristics. Overall RMB diagnostic rate was $80 \%$. Final surgical 
histopathology revealed that excision of benign tumors was significantly reduced in the RMB cohort compared to the control group ( $4 \%$ vs. $21 \%$; $<<0.0006)$. Additionally, the rate of active surveillance in the RMB cohort was $34.4 \%$ vs. $12.5 \%$ for the controls $(\mathrm{p}<0.001)$. Biopsy was concordant with surgical pathology in $94.7 \%$ of cases for primary histology, $97.1 \%$ for histologic subtype, and $78 \%$ for low (I or II) vs. high (III or IV) grade. On multivariate analysis patients who underwent surgical intervention without preoperative RMB were 14.5 times more likely to have benign histopathology compared to patients who underwent preoperative RMB (OR 14.5, 95\% CI=3.9 - 65.7). There were no intra-procedural or post-procedural complications.

Conclusions: For patients with cT1a or cT1b lesions, the implementation of routine office-based RMB led to a significant decrease in the rate of surgical intervention for benign tumors. This practice also resulted in a higher rate of active surveillance for the management of renal cortical neoplasms with benign histopathology compared to a control group.

\section{MP14-02 Prostate Artery Embolization for Lower Urinary Tract Symptoms - Prostatic Specific Antigen (PSA) Response Patterns}

I Ahmed, MM Quraishi, E Latif, B Osman, BA Eddy, EH Streeter, N Das, SS Kommu

Kent \& Canterbury Hospital. East Kent Hospitals NHS Foundation Trust

Introduction \& Objective: A quarter of men older than 70 years have moderate to severe Lower Urinary Tract Symptoms (LUTS) that impair their Quality of Life (QoL). Patients who cannot tolerate medical therapy or in whom medical therapy fails, may be considered for more invasive treatment options. Prostatic Artery Embolization (PAE) is now a recognized treatment modality. The Society of Interventional Radiology (SIR) stated that $\mathrm{PAE}$ is a safe and effective treatment for Benign Prostatic Hypertrophy $(\mathrm{BPH})$ and recommended further prospective comparisons with the available surgical options. Most of patients having PAE, are elderly patients with higher risk of developing prostatic Cancer. Herein, we aim to identify the impact of PAE on PSA patterns.

Methods: We retrospectively assessed cases referred from January 2017 till January 2019 for consideration of PAE. Patients were assessed for the impact of their LUTs using various tools (IPSS score, QoL score, Qmax, PVRs) and studied the impact on PSA before and after the procedure.

Results: Sixty patients were referred for PAE and the procedure was successfully performed in 14 patients while 32 patients were deemed unsuitable based on CT angiogram findings and a further 14 patients are still awaiting to have the procedure performed. Of patients who underwent PAE the mean age was 69.7yrs (range 63$77 \mathrm{yrs}$ ). The pre-procedural PSA level ranged from 0.4 to 9.1 (with average of 3.85), pre-procedural prostate volumes ranged from 55 grams to 135 grams (average 82.3 grams), post-procedure PSA was performed in 7 cases. Those who had successful bilateral PAE there was marked reduction in PSA. In our cohort, PSA had been assessed in 7 patients and they showed significant reduction in PSA (30\%) in 4 patients who had bilateral PAE. Whereas, patients with unilateral PAE $(n=3)$ showed less reduction of the PSA or no change compared to baseline PSA.

Conclusions: PAE when carried out bilaterally can predict PSA reduction by a factor of $30 \%$. This finding can aid in modification of protocols for age matched PSA guided biopsies. This is clearly a small cohort of patients and further large scale multi-centered studies are warranted as PAE uptake increases exponentially.

\section{MP14-03 Withdrawn}

\section{MP14-04 Pre-Biopsy Multiparametric MRI (mpMRI) In The Prediction of the Pathological Stage at Robotic Assisted Radical Prostatectomy - Results From a Series of 1,421 mpMRIs.}

MM Quraishi, M Stanowski, B Osman, N Lobo, I Morrison, E Latif, EH Streeter, SS Kommu, BA Eddy

Kent \& Canterbury Hospital. East Kent Hospitals University NHS Foundation Trust

Introduction \& Objective: The presence of Extra Capsular Extension (ECE) on final pathology confers a poorer prognosis than organ confined prostate cancer. It results in higher rates of progression, biochemical recurrence, prostate cancer specific mortality. Traditionally, risk of ECE is calculated by variables including PSA, Gleason grade, clinical stage, percentage of cores positive and volume of disease with the cores. Counselling patients appropriately over their individualized risk of ECE allows for better surgical planning for both achieving optimal and safe nerve sparing and oncological outcomes. It has been well established recently that pre-biopsy mpMRI both improves diagnostic accuracy of biopsy. mpMRI has also shown great promise in predicting the presence of ECE pre-operatively. We aim to retrospectively review our series of 1421 mpMRIs cross referenced to each histopathological specimen from the radical prostatectomy. We will assess our own positive and negative predictive values of mpMRI in predicting ECE in final histopathology. We will also assess if PSA Density (PSAD) can further improve the accuracy of the mpMRI assessment.

Methods: A retrospective review of 1,442 patients who were planned to undergo robotic assisted radical prostatectomy was undertaken from a regional high-volume tertiary referral center from 2011-2018. There were $1421 \mathrm{mpMRIs}$ with matching radical prostatectomy specimens 1.5 and 3T mpMRIs were utilized. PSA Density (PSAD) was calculated via presenting PSA value and TRUS volume at biopsy.

Results: Multiparametric MRI has a Positive Predictive Value of $74 \%$ and $65 \%$ and a Negative Predictive Value of $65 \%$ and $75 \%$ in diagnosing pT3 and pT2 respectively on final histology. Our results suggest that the higher the PSAD, the more likely the MRI may have under-staged locally advanced prostate cancer or suggest a higher likelihood of ECE on final histology. The difference in mean PSA between the various staging parameters was of statistical significance.

Conclusions: Multiparametric MRI in the hands of experienced readers is an important tool in both the diagnosis but in the surgical treatment of prostate cancer. mpMRI alone should not be blindly relied upon, although it has a reasonable PPV of $75 \%$ in diagnosing ECE. We have shown PSAD may be utilized in conjunction with mpMRI to create a model to enable more accurate prediction of ECE. We plan to assess other preoperative disease parameters to improve the accuracy of predicting ECE in order to improve our counselling of patients and pre-operative planning. Currently platforms are being developed to couple Artificial Intelligence with Machine Learning platforms to further aid evaluation of mpMRI Scans. 
MP14-05 Utility of prostate-specific antigen density in patients with inconclusive or negative multiparametric magnetic resonance imaging

L Rico, PN Contreras, L Blas, G Vitagliano, H Ríos Pita, C Ameri

Hospital Alemán

Introduction \& Objective: The Prostate-specific antigen density (PSAd) seeks to improve the sensitivity and specificity of PSA value and is obtained by dividing PSA and prostate volume. Although multiparametric prostate magnetic resonance imaging (MRI) is indicated, according to clinical practice guidelines, in patients with a previous negative biopsy, the rate of false negatives remains controversial ( 8 to $24 \%$ of clinically significant tumors). The aim of this work is to evaluate the sensitivity and specificity when adding the PSAd in patients with negative or inconclusive MRI.

Methods: A retrospective study was conducted, which included patients who underwent a transperineal prostate biopsy for suspected prostate cancer between October 2015 and June 2018. Variables analyzed were: age, PSA, PSAd, pre-procedure MRI and Pi-RADS (v2) score. Patients were divided into three groups: Pi-RADS score 1 or $2 ; 3$ and Pi-RADS score 4 or 5 . We analyzed the sensitivity and specificity. Subsequently, a PSAd value of 0.15 was plotted as a cut-off point, and two groups were divided $(\leq 0.15$ and $>0.15)$. We calculated the predictive values (negative and positive) this cut-off point and combining it with the result of the MRI.

Results: In all 281 patients were included, all of whom underwent a prostate biopsy for suspected prostate cancer. Of all of them, 105 patients presented a MRI before the procedure. The mean age was 64.7 years (SD \pm 7.6 ) and the mean PSA was $9.96 \mathrm{ng} / \mathrm{dL}(\mathrm{SD} \pm 7.18)$. The PSAd sensitivity and specificity for the diagnosis of clinically significant tumors, taking the cut-off point of 0.15 , were $89 \%$ and $55 \%$. Within the group of the score Pi-RADS 1 or 2, 10 patients were included, in the group of PiRADS score 3 there were 31 and 64 in the group of Pi-RADS score 4 or 5 . An $80 \%$ VPN was found for Pi-RADS 1 or 2 . When dividing according to PSAd value and comparing them with the result of the MRI, it was evidenced that the NPV (Pi-RADS 1-2 Pi-RADS 3), with a PSAd $\leq 0.15$, was $100 \%$ and a sensitivity of $100 \%$.

Conclusions: In our retrospective series, the PSAd $\leq 0.15$ increases the NPV of negative or indefinite MRI. In this way, unnecessary prostate biopsies can be avoided more accurately in patients with Pi-RADS score 1,2 or Pi-RADS 3.

\section{MP14-06 Effectiveness of Lead Aprons in Shielding the Surgeon during Percutaneous Nephrolithotomy}

J Smith, M Hajiha, A Amasyali, Z Campwala, H You, M Alsyouf, J Ewald, A Krause, D Baldwin, R Moghisi, D Baldwin

Introduction \& Objective: Although surgeons wear lead to protect themselves from scattered radiation, the effect of lead shielding upon scattered radiation has not been well characterized. In addition, conventional lead aprons still leave other body parts including the legs and head unprotected. The goal of this study is to determine the surgeon's radiation exposure at multiple sites with and without shielding during a simulated percutaneous nephrolithotomy (PCNL).

\begin{tabular}{|c|c|c|c|c|}
\hline & $\begin{array}{l}\text { Unshielded Radiation } \\
\text { Dose(mrem) }\end{array}$ & $\begin{array}{l}\text { Shielded Radiation } \\
\text { Dose(mrem) }\end{array}$ & $\begin{array}{l}\text { \% Reduction } \\
\text { In Radiation }\end{array}$ & P-value \\
\hline Head & 100.7 & 102.6 & $-2 \%$ & .938 \\
\hline Collar & 221.4 & 235.5 & $-6 \%$ & 0.754 \\
\hline Thyroid & 188.3 & 9 & $95 \%$ & $<0.001$ \\
\hline Sternum & 281.3 & 13.6 & $95 \%$ & .005 \\
\hline Lungs & 196.5 & 10.2 & $95 \%$ & .002 \\
\hline Male Gonads & 197.8 & 11 & $94 \%$ & $<0.001$ \\
\hline Kidney & 266 & 13 & $95 \%$ & .009 \\
\hline Sigmoid Colon & 430.6 & 19.6 & $95 \%$ & .003 \\
\hline Bladder & 380.1 & 15.6 & $96 \%$ & .001 \\
\hline Liver & 230.1 & 10.4 & $95 \%$ & .016 \\
\hline Tibia & 281.9 & 268.4 & $5 \%$ & .723 \\
\hline
\end{tabular}

Methods: A cadaver "patient" (BMI 24.6) was positioned supine and a cadaver "surgeon" (BMI 20.7) was placed upright 11 inches from the center of the radiation source to simulate the position of the surgeon during PCNL. Ten total trials of 20 minutes were completed, 5 trials with the surgeon wearing a $0.35 \mathrm{~mm}$ lead body apron and thyroid shield and 5 trials without lead. Dosimeters were placed in the "surgeon" over sensitive organs divided into three groups, upper body (thyroid, lung, sternum), lower body (liver, kidney, bladder, sigmoid and male gonads), and unshielded areas (Tibia, head). A T-test was used with statistical significance level set at $\mathrm{p}<0.05$.

Results: A 96\% reduction $(\mathrm{p}<0.001)$ in radiation was recorded between shielded (12.8 mrem) and unshielded (323.1 mrem) organs. For shielded trials, the organs with highest radiation exposure were the sigmoid colon (19.6 mrem), bladder (15.6 mrem), and sternum (13.6 mrem). Organs showing the lowest exposure were the thyroid ( 9 mrem), lungs $(10.2 \mathrm{mrem})$, and liver (10.4 mrem). In shielded groups, upper body organs had $22 \%$ lower radiation exposure than those in the lower body (10.9 mrem vs. $14.0, \mathrm{p}=0.02$ ). In the unshielded group, the upper body received $25 \%$ lower dose than the lower body (264.3 mrem vs. $356.3, \mathrm{p}=0.02$ ). When comparing shielded vs. unshielded groups, radiation exposure of the head $(102.6$ mrem vs. $100.4, p=.938)$ and tibia (268.4 mrem vs. 284.6 mrem, $\mathrm{p}=0.723$ ) was similar.

Conclusions: Although standard radiation protection measures dramatically reduce exposure, they may not be completely adequate in protecting certain covered radiation sensitive organs like the sigmoid and bladder. Furthermore, body parts like the tibia and head continue to receive large radiation exposure whether or not the surgeon wears conventional radiation protection. Therefore, in addition to wearing lead shielding, surgeons should take measures to reduce the fluoroscopy time and dose.

\section{MP14-07 Diagnostic Accuracy of Computed Tomo- graphy Urography (CTU) in Upper Tract Urothelial Transitional Cell Carcinoma (TCC)}

S Nalagatla, O Babawale, I Ibrahim, S Prattley, R Lockyer, NO Umez-Eronini, BK Somani, OM Aboumarzouk

Queen Elizabeth University Hospital

Introduction \& Objective: Transitional cell carcinoma (TCC) of the upper urinary tract is a rare disease accounting for only 5 to $6 \%$ of all urothelial tumors. The most commonly used imaging modality for diagnosis is a Computed Tomography Urography (CTU). A diagnostic ureteroscopy is performed to ratify the diagnosis as well as provide tissue diagnosis. However, in a selected number of patients, the tumor burden is such that proceeding to a Nephroureterectomy is required. We aimed to 


Table 1: Diagnostic Accuracy of CTU.
\begin{tabular}{|l|l|l|}
\hline & Disease Present & Disease Absent \\
\hline CTU Positive & 110 & 24 \\
\hline CTU Negative & 3 & 99 \\
\hline
\end{tabular}

study the diagnostic accuracy of CTUs in detecting UUT-TCC reported by an Uro-Radiologist.

Methods: Multicenter data was collected on all patients undergoing diagnostics for upper urothelial TCC over a 5-year period. Patient demographics, CTU report, ureteroscopy findings, biopsy and Nephroureterectomy pathology was recorded. Statistical analyses was performed using MedCalc for Windows, version 15.0 (MedCalc Software, Ostend, Belgium).

Results: A total of 236 patients were included in our study. The sensitivity and specificity of CTU was $97.3 \&(92.4 \%-99.5 \% \mathrm{CI})$ and $80.5 \%(72.4-87.1 \% \mathrm{CI})$ respectively. The positive likelihood ratio was 6.99 and negative likelihood ratio was 0.03 . The positive predictive value was $82.1 \%$ and negative predictive value was $97.06 \%$. The diagnostic accuracy of CTU in this study was found to be $88.6 \%(83.8 \%-92.3 \% \mathrm{CI})$.

Conclusions: The diagnostic accuracy of CTU in our study was higher in comparison to others reported. This is potentially due to all the reporting being performed by our Uro-Radiologists. Nonetheless, CTU detection of UUT-TCC was high. Potentially, CTU can thus be the definitive diagnostic test for large tumors that are not amenable to endoscopic ablation.

\section{MP14-08 Can Patients With Negative Multi- parametric MRI Avoid Prostate Biopsy? Results From 1000 Pre-Biopsy Multiparametric MRIs}

N Lobo, M Stanowski, MM Quraishi, E Latif, I Morrison, M Eraibey, SS Kommu, EH Streeter, BA Eddy

Kent \& Canterbury Hospital. East Kent Hospitals University NHS Foundation Trust

Introduction \& Objective: Pre-biopsy multiparametric MRI (mpMRI) is reported to have a negative predictive value of $89 \%$ in prostate cancer diagnosis. We determined the negative predictive value (NPV) of mpMRI alone and in combination with PSA density (PSAD) for significant prostate cancer (sPCa) in men undergoing prostate biopsy at our institution.

Methods: 1000 consecutive biopsy-naïve men undergoing 1.5T or 3T pre-biopsy from October 2015 to September 2018 were identified. All scans were reported by 2 dedicated uroradiologists using PIRADS version 2. Negative mPMRI was defined as PIRADS $<3$. Men with negative mpMRI underwent a minimum of 12-core systematic transectal ultrasound-guided biopsy. Significant prostate cancer was defined as Gleason $\geq 3+4$. The Mann-Whitney test, univariable and multivariable Cox regression models were performed for statistical analysis.

Results: sOf 1000 patients, 44\% $(\mathrm{n}=444)$ had negative mpMRI. Prostate cancer and $\mathrm{SPCa}$ was detected in $35 \%(\mathrm{n}=155)$ and $15 \%(n=66)$, corresponding to a NPV of $65 \%$ and $85 \%$ respectively. Using a PSAD cut-off of $0.15 \mathrm{ng} / \mathrm{ml} / \mathrm{ml}$ and $0.10 \mathrm{ng} /$ $\mathrm{ml} / \mathrm{ml}$ in combination with a negative mpMRI increased the NPV for sPCA to $89 \%$ and $91 \%$ respectively. Patients with sPCa had smaller prostate volumes $(\mathrm{p}<0.001)$ and higher PSAD $(\mathrm{p}<0.001)$ than non-sPCa patients. On univariate analysis, prostate volume $(\mathrm{p}<0.001)$ and increasing PSAD $(\mathrm{p}<0.001)$ were predictors for sPCa. On multivariable analyses, only PSA density was a predictor for SPCA.

Conclusions: At our institution, patients with a negative prebiopsy mpMRI and low PSA density can avoid a biopsy. However, a small number of significant cancers will be missed and patients should be given institution-specific negative predictive values when deciding whether or not to undergo biopsy.

\section{MP14-09 Factors influencing fluoroscopy use during Ureteroscopy at a residency training program}

SP Hager, S Desai, M Amster, N May

Main Line Health, Mercy Health Network

Introduction \& Objective: Ionizing radiation is used throughout urologic surgery and is known to cause a greater cancer risk with increasing exposure. The International Commission on Radiological Protection states that "it is the control of radiation dose that is important, no matter the source." There have been recent reports on the use of "fluoro-less" ureteral stent placement to decrease radiation exposure. However, there are few reports on the amount of radiation used by Urology residents during retrograde ureteroscopy (URS). We present the largest database evaluating fluoroscopy (fluoro) use during URS at a resident training program. Our objective is to assess the amount of fluoro use at varying levels of experience and to identify factors that may lead to increased fluoroscopy use during URS. Methods: Retrospective data from 242 URS performed at two community hospital training sites from 1/2015-5/2019 were collected. 105 surgeries were done by 2 attending physicians without and 137 with residents of varying training levels (Yr1Yr3). Patient demographics and intraoperative data were collected from the EMR. Statistical analyses were conducted using SPSS and include ANOVA, Spearman correlations, and multiple linear regression (MLR).

Results: Comparisons between $\mathrm{Yr} 1$ and $\mathrm{Yr} 2$ revealed significantly $(\mathrm{p}<0.05)$ decreased fluoro time $(16.4 \mathrm{~s})$ and operative time $(12.2 \mathrm{~m})$ for the $\mathrm{Yr} 2$ resident. Total operative time was significantly $(\mathrm{p}<0.05)$ decreased $(11.1 \mathrm{~m})$ for attending physicians operating on their own compared to a Yr1 resident, with no differences between $\mathrm{Yr} 2$ and $\mathrm{Yr} 3$ residents. For cases with resident involvement significant $(\mathrm{p}<0.05)$ correlations are shown in Table 1. MLR was performed on these variables; operative time, ureteral dilation and a pre- operative stent placement were significant predictors of fluoro time $(\mathrm{p}<0.05)$.

Conclusions: Fluoro time during retrograde URS was significantly reduced as residents gained more experience in the

Table 1: Significant Correlation with Fluoroscopy Time

\begin{tabular}{ll}
\hline Variable & Correlation Coefficient \\
\hline Urology Resident Month & -0.239 \\
Urology Resident Year & -0.225 \\
Preoperative stent placed & -0.180 \\
Access Sheath placement & 0.175 \\
Ureteral Dilation & 0.208 \\
Stone Size (mm) & 0.407 \\
Total Operative Time $(\mathrm{m})$ & 0.437
\end{tabular}


operating room from Yr1 to Yr2. An increase in fluoro time was also associated with the use of a ureteral dilator, the use of an access sheath, increasing stone size, and without pre- stenting. With knowledge of these factors, emphasis can be placed on using and teaching techniques that limit radiation exposure to both the surgeon and patient.

MP14-10 Do we protect ourselves? Review of radiological protection during endourological procedures with fluoroscopy

JM López, A Sierra, P Luque, A Alcaraz

Clínic Hospital Barcelona - University of Barcelona, Urology department Barcelona/Spain, Spain

Introduction \& Objective: The increasing use of endourology in the treatment of stone disease increases the use of fluoroscopy and places the urologist and the operating room staff at occupational risk of radiation exposure. This is due to the high levels of non-uniform radiation received mainly from the scattered radiation emitted by the patient. The main protection for the personnel is lead apron and lead screens and flaps wherever possible. It is also important to minimize the area of the fluoroscopy X-ray field by collimation of the X-ray beam, improving the quality of the image and reducing the scattered radiation by $25-50 \%$. The scatter radiation also varies with the direction of rotation of the arm, being greater when the primary beam is inclined at $30^{\circ} \mathrm{C}$. In 2014 there was a change in the European legislation on radioprotection (DIRECTIVE 2013/59/EURATOM OF THE COUNCIL). One of the most important changes was that the reduction of the limit dose in the crystalline; being of 20 $\mathrm{mSv}$ per year when previously it was $150 \mathrm{mSv}$ per year. The limit of the dose of radiation changes over time because we still do not know the injuries that can cause us, so the protection of the radio is mandatory. The main problem is that the knowledge of radioprotection is not standardized in urologic programs and, generally, we are overexposed unnecessarily. We review radioprotection during endourological procedures in different Hospitals, mainly from Europe, of videos published on the website of the European Association of Urology (EAU) and the Spanish Association of Urology (AEU).

Methods: Retrospective analysis of 95 videos of endourologic procedures with fluoroscopy published in the UROsource of the EAU website and the AEU website between 2011 and 2019. 14 countries were involved. The radioprotection measurements taken in each case were analyzed.: the use of radiation protection equipment (plumed aprons, thyroid protector, lead lens and

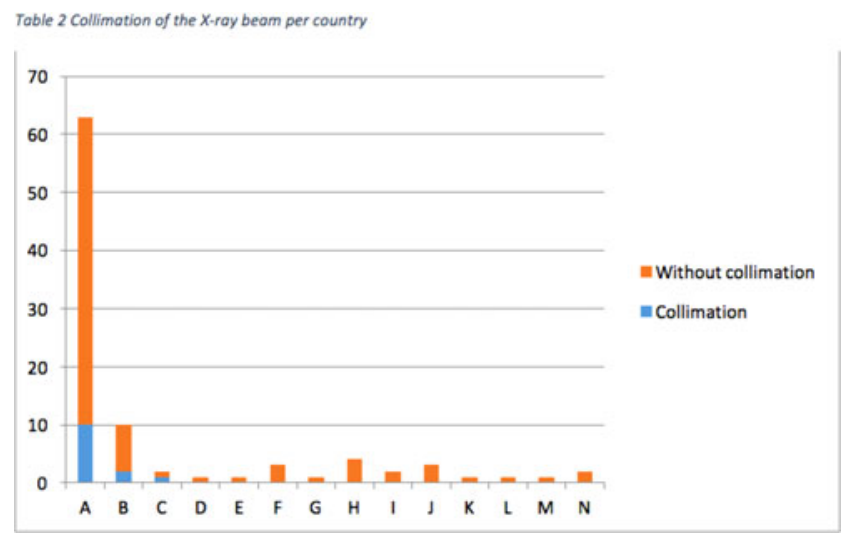

\begin{tabular}{|c|c|c|}
\hline \multicolumn{2}{|c|}{ RADIOPROTECTION MEASURES } & \multirow{2}{*}{$\begin{array}{c}\text { Correct radioprotection use } \\
48 / 51(94 \%)\end{array}$} \\
\hline \multirow{4}{*}{$\begin{array}{c}\text { Body } \\
\text { protection }\end{array}$} & $\begin{array}{l}\text { Rubber coated } \\
\text { lead apron }\end{array}$ & \\
\hline & Thyroid shields & $31 / 51(60.7 \%)$ \\
\hline & $\begin{array}{l}\text { Radio-protective } \\
\text { surgical gloves }\end{array}$ & $8 / 73(11 \%)$ \\
\hline & Leaded glasses & $13 / 52(25 \%)$ \\
\hline Technique & Collimation & $13 / 95(13,6 \%)$ \\
\hline \multicolumn{2}{|c|}{ Radiation exposure } & $0,28 \mathrm{~Sv}$ (range 0,$18 ; 0,3$ ) \\
\hline \multicolumn{2}{|c|}{ Exposure time } & $2,99 \min$ (range 1,$2 ; 10)$ \\
\hline
\end{tabular}

plumbed gloves), the placement of the X-ray tube, the collimation of X-ray beam and the exposure time.

Results: Table 1 and Table 2.

Conclusions: All occupational personnel should aim to "achieve a dose as low as reasonably possible" (ALARA) that complies with good radiation protection practices. Therefore, all personnel should routinely use safety gadgets such as lead equivalent thyroid shield and aprons, our results show that the use of radioprotective material and the knowledge is insufficient. We should be aware of the risks of maintained radiation without protection to solve these deficiencies in our centers. The follow of these rules should be considered when evaluating a video to be accepted in a scientific meeting

\section{MP14-11 Multiparametric Magnetic Resonance Imaging is a Cost Effective Method in the Evaluation of Renal Masses for Clear Cell Renal Cell Carcinoma}

RL Steinberg, BA Johnson, R Ghandour, R Rasmussen, A Garbens, Y Xi, T Yokoo, A Diaz de leon, I Pedrosa, JA Cadeddu

University of Texas Southwestern

Introduction \& Objective: Given its aggressive nature relative to other subtypes, identifying clear cell renal cell carcinoma (ccRCC) may lead to intervention over surveillance in some patients. Renal mass biopsy (RMB) has shown good diagnostic performance but suffers from a poor negative predictive value (NPV; 68\%). The clear cell likelihood score (ccLS) based on multiparametric magnetic resonance imaging (mpMRI) has also shown good diagnostic performance in identifying ccRCC. We now aim to compare the cost of RMB and mpMRI in identifying ccRCC in cT1a tumors.

Methods: All patients with cT1a tumors were identified in a retrospective database of mpMRIs with prospectively assigned ccLS for renal mass evaluation at two institutions between June 2016 and October 2018. Demographic, radiographic, and pathologic details were extracted. Confusion matrix analysis was performed with ccLS 4-5 considered positive, ccLS 1-2 negative and ccLS 3 indeterminant for ccRCC. Detailed cost information regarding professional, imaging, RMB-based procedure, and pathologic costs were obtained from our institution. Cost was defined as Medicare reimbursement for services.

Results: One hundred fourteen patients (mean age 57.5 \pm 13.0 years) with 115 renal masses were identified. Mean tumor size 


\begin{tabular}{lccc}
\multicolumn{4}{l}{ Table 1. Various Cost Associated with MRI and renal mass biopsy } \\
\hline $\begin{array}{l}\text { Costs } \\
\text { Radiology }\end{array}$ & mpMRI & US-guided RMB & CT-guided RMB \\
Professional & $\$ 112.95$ & $\$ 167.13$ & $\$ 192.66$ \\
Imaging & $\$ 456.37$ & - & \\
$\begin{array}{l}\text { Procedural } \\
\text { Professional }\end{array}$ & & $\$ 167.13$ & \\
Fixed Direct & - & $\$ 205.94$ & $\$ 192.66$ \\
Variable Direct & - & $\$ 255.72$ & $\$ 286.38$ \\
Indirect & & $\$ 157.72$ & $\$ 297.64$ \\
Pathology & & $\$ 208.84$ \\
Professional & - & $\$ 441.39$ & \\
Technical & - & $\$ 898.74$ & $\$ 441.39$ \\
\hline Total & $\$ 569.32$ & $\$ 2,126.64$ & $\$ 898.74$ \\
\hline
\end{tabular}

\begin{tabular}{|c|c|}
\hline Condition & Total Evaluation Cost (\$US Dollars) \\
\hline \multicolumn{2}{|l|}{ If all patients underwent... } \\
\hline CT guided biopsy & $\$ 267,449.75$ \\
\hline US guided biopsy & $\$ 244,563.60$ \\
\hline $50 \%$ CT / $50 \%$ US guided biopsy & $\$ 256,006.68$ \\
\hline mpMRI + CT guided biopsy for ccLS $3^{*}$ & $\$ 114,310.45$ \\
\hline mpMRI + US guided biopsy for ccLS $3^{*}$ & $\$ 110,131.24$ \\
\hline $\mathrm{mpMRI}+50 \% / 50 \% \mathrm{CT} / \mathrm{US}$ guided biopsy for $\mathrm{ccLS}^{*} *$ & $\$ 95,868.99$ \\
\hline
\end{tabular}

*21 patients had a ccLS 3 lesion in this cohort

was $2.8 \pm 0.8 \mathrm{~cm}$. ccLS diagnostic performance was $79 \%$ sensitivity, $77 \%$ specificity, $85 \%$ positive predictive value, and $96 \%$ NPV. Table 1 details the costs of each diagnostic modality. Assuming biopsy was reserved only for those with a ccLS 3 lesion, only $21 / 115(18 \%)$ would be candidates for a biopsy. Table 2 details the comparative costs of multiple scenarios in which mpMRI and RMB are used. Only a single patient with ccLS 1-2 was found to harbor ccRCC.

Conclusions: mpMRI confers similar diagnostic performance and significant cost savings over RMB in the identification of ccRCC; this technology may provide a way to more judiciously use RMB. Markov modeling including the cost of RMB complications (e.g. CT for pain, bleeding), active surveillance and missed malignancies is warranted.

MP14-12 Is characterization of renal stone composition by using fast kilovoltge switching dual-energy computed tomography can detect $100 \%$ of uric ucid stone?

U Romsaithong, K Jongjitaree, P Korpraphong,

V Woranisarakul, T Taweemonkongsap, C Nuanyong,

E Chotikawanich

Siriraj Hospital

Introduction \& Objective: To prospectively examine the diagnostic performance of fast kilovoltage switching dual-energy computed tomography (DECT) in characterization of in vivo renal stone composition compared with postoperative stone analysis.

Methods: Consecutive consenting patients scheduled for endoscopic kidney stone surgery in a tertiary referral hospital from June 2015 to January 2016, were enrolled. Patients were preoperatively scanned with single-source, fast kilovoltage switching DECT. Stone compositions were determined regarding to effective atomic number measurements. Results of the stone compositions from DECT were compared to postoperative infrared spectroscopy stone analysis as the standard reference.

Results: For the 39 patients enrolled in the study, DECT was able to detect uric acid stone with sensitivity of $88.9 \%$ and specificity of $100 \%$. There was $100 \%$ positive predictive value, $96.8 \%$ negative predictive value, and $97.4 \%$ accuracy. For non-contrast CT scan, sensitivity was $88.9 \%$, specificity was $96.7 \%$, positive predictive value was $88.9 \%$, negative predictive value was $96.7 \%$, and accuracy was $94.8 \%$. Of the 39 samples examined, 21 (54\%) were single composition whereas $18(46 \%)$ were combined. Single composition stones were correctly characterized by DECT in 100\% (8/8) for uric acid. Whereas, the result of uric acid stone containing stone discrimination in mixed composition was not so well with $\mathrm{Z}_{\text {eff }}$ alone, iodine imaging can compensate this fault.

Conclusions: DECT provides excellent accuracy in characterizing uric acid stone compositions. With the addition of iodine image, all of uric acid-containing stones can be determined by the DECT.

\section{MP14-13 First French experience of prostate targeted biopsies with URONAV system}

\section{BAILLY, P HENRY, C DARCQ, M HINTZY}

Centre d'Urologie, clinique Saint Vincent, Besançon FRANCE

Introduction \& Objective: The aim of this study is to report the first and only French experience to date of transrectal prostatetargeted biopsies with URONAV system, allowing elastic fusion of MRI and ultrasound images in real time.

Methods: This is a retrospective study conducted between January 1 and September 30,2018. The parameters studied are age, PSA level, history of biopsies and active surveillance of prostate cancer (PCa), prostatic volume, the number of MRI targets, the Pirads- 2 score, the location, the target volume, the Gleason score, the name of the radiologist interpreting prostate MRI. Targeted biopsies and systematic sextant cores were performed.

Results: 398 patients were included, mean age 67 years, mean PSA of 10 for a prostatic volume of $73 \mathrm{cc}$. 550 targets were biopsied. $7 \%$ of targeted areas were less than $5 \mathrm{~mm}$. $61 \%$ of the lesions were classified Pirads 4 or 5 and $39 \%$ Pirads $3.17 \%$ of the targets are Gleason $>6 \mathrm{PCa}$. One hundred and twenty-one patients $(22 \%)$ had systematic positive biopsies, of which eightyfive $(71 \%)$ were Gleason 6 . Thirty-three targets (14\%) Pirads 3 corresponded to an adenocarcinoma with a Gleason score $>6$. Biopsies of forty-four Pirads 5 targets (57\%) are negative. Only one Pirads 3 target located in transitional zone is associated with PCa. Twenty-four of the targets below $5 \mathrm{~mm}(63 \%)$ are a PCa. Thirty five of the 60 biopsied patients under active surveillance $(58 \%)$ had to leave the protocol and need to be treated because of a larger or more aggressive disease underdiagnosed with previous non-MRI targeted biopsies. PCa was diagnosed for sixtyseven patients (17\%) with previous negative cores. 
Conclusions: The transrectal freehand URONAV system is easy to use and very precise in its guidance for both small targets and those located in the anterior zone. The relevance of biopsy targeting leads in our study to a modification of initial management in more than one out of four cases. This tool reinforces the necessary collaboration between radiologists and urologists. This teamwork improves the quality of MRI interpretation and targeted cores. The short learning curve is enhanced with this expert team.

MP14-14 "The Dirty Prostate" - Background Peripheral Zone Apparent Diffusion Coefficient Does Not Appear to Differ Between False and True Negatives on Prostatic mpMRI

\author{
E Wajswol, Y Kuo, H Koster, A Bogomol, K Basralian, \\ MD Stifelman, M Naik \\ Hackensack Meridian Department of Urology
}

Introduction \& Objective: Multiparametric MRI (mpMRI) is an increasingly utilized imaging modality in urologic oncology that has allowed for more accurate diagnosis, staging, and management of prostate cancer. One of the greatest benefits of mpMRI is the ability to exclude clinically significant prostate cancer secondary to a high negative predictive value. It is believed that benign conditions may affect mpMRI signal on diffusion weighted imaging (DWI) and subsequently impact the negative predictive value. In particular, prostatitis, which has been described as causing a "dirty prostate" appearance, may result in restricted diffusion with lower or more heterogeneous apparent diffusion coefficient values throughout the peripheral zone. We examined if a significant difference exists in background ADC values between patients with false and true negative mpMRI findings.

Methods: Between May 2015 and January 2019, 72 men received an mpMRI read as negative (defined as a PI-RADSv2 score of 1 or 2) followed by a MR/US fusion biopsy (UroNav, Invivo, Gainesville, FL) alongside a systematic 12-core biopsy. The peripheral zone on prostatic mpMRI was retrospectively segmented using DynaCad software (Invivo, Gainesville, FL). The median, mean and standard deviation of ADC values were calculated for each segmented peripheral zone on DWI. ADC values between the false negative and true negative cohorts were compared with R statistical software (R Core Team, 2018) utilizing Student's t-test, F-test, and Wilcoxon Rank Sum Test.

Results: 72 men were included in the study, with 20 (28\%) being false negatives on mpMRI and $52(72 \%)$ being true negatives on mpMRI. Median age was 66 years (49 - 80) and median PSA was $6.2 \mathrm{ng} / \mathrm{mL}(1.04-20.3)$. Median ADC in the false negative cohort was 1252.4 and was 1274.6 in the true negative cohort

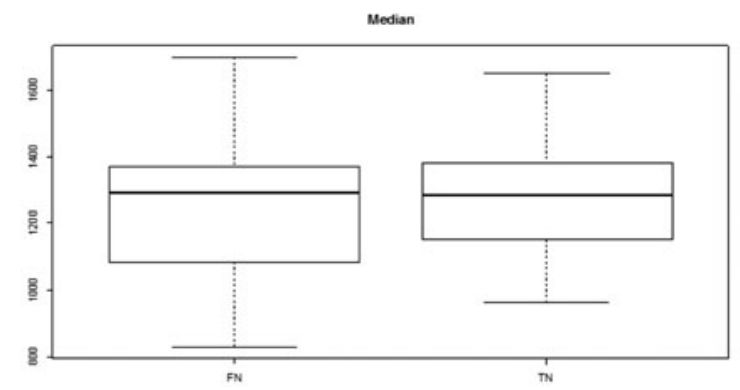

Figure 1: Box plot of median ADC for patients with false negative and true negative mpMRI results

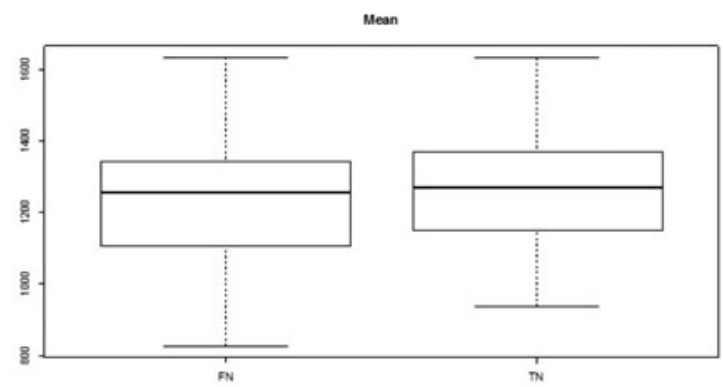

Figure 2: Box plot of mean ADC for patients with false negative and true negative mpMRI results

$(\mathrm{p}=0.64$, Figure 1). Mean ADC in the false negative cohort was 1235.6 and was 1260.0 in the true negative cohort $(\mathrm{p}=0.58$, Figure 2). Standard deviation of ADC in the false negative cohort was 216.0 and was 209.0 in the true negative cohort $(\mathrm{p}=0.60)$.

Conclusions: Background peripheral zone ADC values, including median, mean and standard deviation did not appear to significantly differ between the true and false negative cohorts on mpMRI. These findings suggest that pathologies that alter background peripheral zone signal on DWI may have a smaller impact on the negative predictive value of mpMRI than previously hypothesized.

MP14-15 Final pathology of radical prostatectomy specimens in patients with negative pre-biopsy multiparametric MRI

\author{
M Stanowski, N Lobo, EH Streeter, I Morrison, BA Eddy
}

Frimley Park Hospital

Introduction \& Objective: NICE guidelines now recommend pre-biopsy multiparametric MRI (mpMRI) as a first-line investigation for men with suspected prostate cancer. There are also departments who are beginning to use a normal mpMRI as a primary indication to avoid prostate biopsy. However, despite high sensitivity and excellent negative predictive values, some patients with negative mpMRI will harbor clinically significant disease and require radical treatment. We assess final histopathological specimens in men with negative mpMRI undergoing robot-assisted radical prostatectomy (RARP) at our institution.

Methods: A retrospective analysis of a single center institution of mpMRIs performed between 2015 and 2018 was completed. Routine investigation for suspicion of localized prostate cancer in this institution was mpMRI followed by TRUS Biopsy. We identified patients with a negative pre-biopsy mpMRI (defined as PIRADS <3) that underwent RARP between October 2015 and December 2018. RARP specimens were reviewed by a single genitourinary pathologist.

Results: 57 patients with negative pre-biopsy mpMRI underwent radical prostatectomy. Median age was 65 years (IQR: 55-66), median PSA was 6.1 (IQR 4.9-7.7) and median prostate volume was 46ml (IQR: 37-57). ISUP Grade Group (GG) 1 was detected in $1(2 \%)$ patient, GG 2 in $48(84 \%)$, GG 3 in $2(4 \%)$, GG 4 in 3 $(5 \%)$ and GG 5 in $3(5 \%)$. Pathological results of the prostatectomy specimens showed $7(12 \%)$ pT2a, $39(68 \%)$ pT2c, $11(19 \%)$ pT3a. 53\% of specimens had $</=5 \%$ of tumor occupying the prostate. $31(54 \%)$ patients had an index lesion that was $>10 \mathrm{~mm}$ 


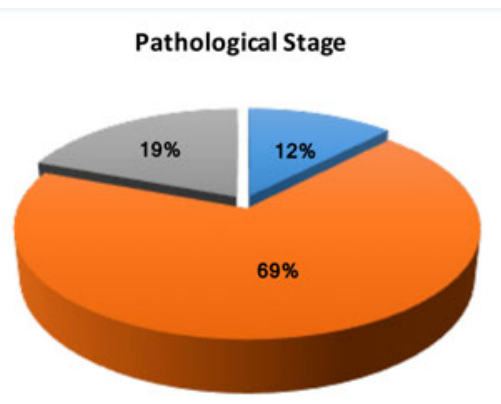

$\equiv \mathrm{pT2a}=\mathrm{pT} 2 \mathrm{c} \equiv \mathrm{pT3a}$

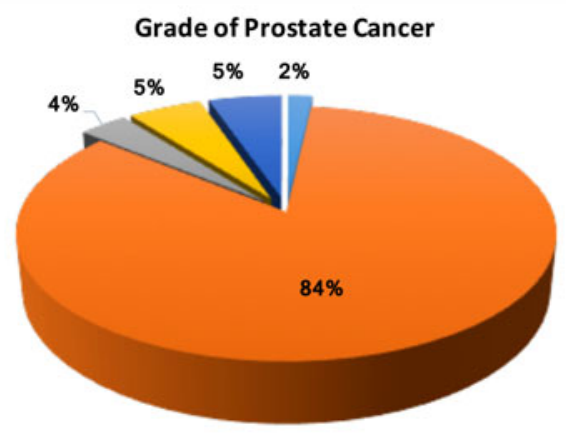

=ISUP $1=$ ISUP $2=$ ISUP $3=$ ISUP $4=$ ISUP 5

and $24(42 \%)$ patients had an index lesion $>15 \mathrm{~mm}$ in size. Extraprostatic extension was identified in $11(19 \%)$ patients and positive surgical margins were present in $12(21 \%)$.

Conclusions: Although mpMRI is highly reliable in excluding prostate cancer, a small proportion of significant tumors which require radical treatment will be missed. mpMRI should not be used in isolation as a decision-making tool. Other disease, diagnostic and patient parameters need to be considered and certainly patients should have their risks and benefits of biopsy following a negative mpMRI discussed with them so that they can make an informed decision.

\section{MP14-16 Phase II Multi-Center Trial: MR/US Fusion Guided Ultra-Focal Gold Nanoshell Directed Laser Ablation of Prostate Tumors: Reporting 3-Month Outcomes and Safety Data}

E Wajswol, AK George, S Diamond, M Davenport, V Tamisetti, JS Winoker, SK Doppalapudi, BA Taouli, SC Lewis, JA Schwartz, S Canfield, AR Rastinehad

Hackensack Meridian Department of Urology

Introduction \& Objective: Gold Silica Nanoshell (GSN) laser ablation is an investigational focal therapy (FT) modality for focal ablation of prostate tumors. GSN preferentially accumulate within tumors and are activated by near-infrared (NIR) light to selectively ablate areas with high GSN concentration, sparing normal surrounding tissue. Herein, we report the primary endpoint of a multi-institutional study of GSN-directed tumor ab-
Table 1

\begin{tabular}{|l|r|}
\hline Adverse Event (CTCAE Grade) & \# Events \\
\hline Hematuria (1) & 31 \\
\hline Failed TOV/Retention (2) & 10 \\
\hline Urinary Incontinence (2) & 2 \\
\hline Erectile Dysfunction (2) & 1 \\
\hline Catheter-OFD Melting (2) & 2 \\
\hline \begin{tabular}{l|} 
Transient Epigastric Pain - Self Resolving \\
(2)
\end{tabular} & 1 \\
\hline Abdominal Pain - Self Resolving (1) & 1 \\
\hline Blue Tongue - Self Resolving & 1 \\
\hline
\end{tabular}

lation using US and MRI/US fusion guidance (NCT02680535) via a transperineal approach.

Methods: All enrolled subjects had Gleason $\leq 7$ prostate cancer (PC) diagnosed by MR/US fusion-guided biopsy with disease limited to no more than two positive lesions. Participants received an intravenous infusion of GSN on day 1 . On day 2, laser trocars were placed into the prostate lesion transperineally using an electromagnetic-tracked MR/US fusion device (UroNav, Invivo Gainesville FL). Thereafter, the laser fiber was inserted into the trocars sequentially. Upon illumination at a sub-thermal energy level, the NIR laser activates the GSN, inducing thermal ablation in areas of high concentration. mpMRI was obtained 48 hours post-ablation and again at 3 months with MR/US fusion guided biopsy (primary endpoint). All patient demographics, PSA values, and biopsy details were recorded at baseline and 3 months.

Results: To date, 33 patients at 3 different sites have completed 3-month follow-up. All but one patient was treated for a single lesion. Median baseline PSA was $8.3 \mathrm{ng} / \mathrm{mL}$. Mean PSA at 3 months post-treatment was $4.0 \mathrm{ng} / \mathrm{mL}$ ( $51.8 \%$ reduction). 19 of 33 patients had a negative biopsy at 3 months, amounting to a $57.6 \%$ complete response rate following GSN FT. 24 of 33 patients $(72.7 \%)$ had a negative biopsy at 3 months according to clinical success criteria for focal therapy as defined by Delphi consensus. Following treatment, the most common adverse events experienced by patients were hematuria and transient urinary retention (Table 1); no patients experienced CTCAE grade 3 or higher events.

Conclusions: The short-term oncological outcomes of this multicenter trial are promising, suggesting that GSN-directed ablation in conjunction with MR/US fusion guidance is an effective, feasible, and safe FT modality that warrants further investigation.

MP14-17 Cancer detection rate at MPMRI-TRUS Fusion guided Prostate Biopsy: the PI-RADS version 2 and the role of the radiologist

G Bozzini, U Besana, A Calori, P Broggini, G Pini, C Buizza, M Sighinolfi, B Rocco

ASST Valle Olona - Busto Arsizio VA

Introduction \& Objective: Prostate fusion biopsy (PFB) improves cancer detection rate (CDR) in patients who performed Prostatic mpMRI. However, this aim is strictly connected with Radiologists' interpretation of Prostate Index - Reporting and Data System (PI-RADS) version 2 score. The aim of this study is to evaluate the PI-RADS 2 Radiologists' interpretation according to CDR of mpMRI-TRUS PFB. 
Methods: Two Independent Radiologists (Junior and Senior) for each involved center prospectively reviewed mpMRI studies of the prostate using the PI-RADS version 2 score, blinded to clinical and histological data. The Trinity Koelis ${ }^{\circledR}$ (Koelis, Grenoble, France) fusion system and software was used for mpMRI-TRUS PFB and biopsies were performed in a transrectal setting with 4 biopsies for each PI-RADS target. We evaluated the CDR according to PI-RADS scored by the two different Radiologist. Univariable (ULR) and multivariable logistic regression analyses (MLR) were performed to evaluate the predictors of CDR.

Results: 456 patients were enrolled. Median age was 68.7 years (R: 55-74), median total PSA was $9.3 \mathrm{ng} / \mathrm{ml}$ (R:4-51). The PIRADS scores were distributed as follow for the 2 Radiologists ( $\mathrm{J} \&$ S): 22 and 13 were PIRADS 2, 253 and 181 were PIRADS 3, 121 and 192 were PIRADS 4, 60 and 70 were PIRADS 5. Targeted biopsy CDR was $57.9 \%(n=264)$ and $34.4 \%(n=157)$ $(\mathrm{p}=0.03)$. There was a significative mismatch between the target indicated by the Senior Radiologist and the Junior one $(n=113$ targets in a different area). Cancer detection rate stratified according to PIRADS score was significantly higher in PIRADS 5 and 4 groups compared to PIRADS $2-3(\mathrm{p}<0.001)$. In a MLR model, accounting for total PSA, Senior Radiologist, PIRADS 4 and 5 were positive predictors of CDR.

Conclusions: Radiologists play a crucial role in reaching a high sensitivity and specificity results in CDR among their performances on PI-RADSv2 score. PI-RADSv2 and high-level Radiologists are positive predictors of CDR.

\section{MP14-18 Low dose CT KUB in urolithiasis}

\section{S EL RAI}

\section{Al Zahra hospital Sharjah}

Introduction \& Objective: Renal colic is a common condition, which is often investigated with a CT of the kidneys, ureters and bladder (CT KUB). Non-contrast (CT KUB) is the investigation of choice for renal colic; however, radiation exposure can be a concern for the patient as well as the patient incurred radiation dose particularly if we consider the amount of radiation needed in the management and the follow-up of the case. The objectives of our work is the following: review the current imaging guidelines in urolithiasis, to evaluate the value of CT KUB in renal stone disease, to provide an overview the technological advances in CT allowing low dose $\mathrm{CT}$ and to differentiate standard-dose CT KUB, LD CTKUB, ULD CTKUB.

Methods: Through our presentation, we will simplify the background and recent technological improvements, which have allowed a reduction in radiation dose with the emergence of lowdose CT KUB (LD-CT KUB) and ultra-low-dose CT KUB (ULD-CT KUB) techniques. We will review the literature regarding the diagnostic accuracy of LD-CT KUB and ULD CT KUB versus standard-dose in the diagnosis of clinically significant uroliths and will analyze their limits in identifying urinary tract stones and in detecting alternative diagnoses. Accordingly, will provide some take home messages regarding daily clinical practice.

Results: Unenhanced CT KUB is the recommended gold standard imaging investigation for patients who present with acute renal colic due to its high level of safety and sensitivity. We achieved in 2017 a significant decrease of radiation dose following the protocol of low dose CTKUB as shown in the table. ULD CT and LD CT are effective techniques and yield high

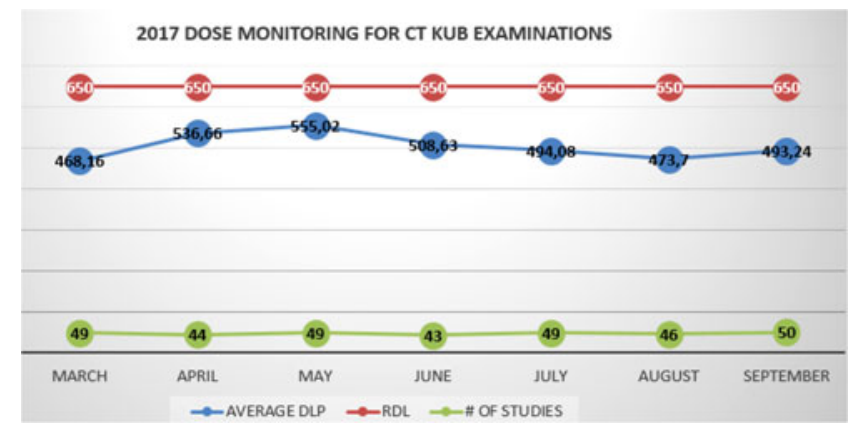

sensitivity and specificity. They may not be as effective in detecting stones $<3 \mathrm{~mm}$ in size or in patients with a body mass index of $>30 \mathrm{~kg} / \mathrm{m} 2$.

Conclusions: It is important to understand machine capabilities, include advanced technologies, to master the patient positioning, and develop good habits by automatic exposure control and applying new image reconstruction algorithms. The use of lowdose MDCT protocols and integration of newer iterative reconstruction algorithms into routine CT practice has resulted in a substantial decrease in ionizing radiation exposure. We recommend the use of LD-CT KUB when the pre-test probability of stone disease is significantly higher than the pre-test probability of alternative diagnoses, such as follow-up of known calculi. LD CT KUB should be the first-line investigation for the majority of renal colic patients in the modern era. Caution when the pre-test probability of alternative diagnoses is high.

MP14-19 Periprocedural and Diagnostic outcomes of Transrectal versus Transperineal US/MRI guided Fusion Prostate Biopsy: Multi-institutional Propensity Score Matched Pair Analysis

M Ferriero, M Oderda, V Forte, A Peltier, P Kumar, P Mozer, A Giacobbe, R Mastroianni, G Tuderti, U Anceschi, A Brassetti, J Roche, M Ciccariello, S Guaglianone, T Piechaud, M Rouprèt, P Gontero, G Muto, M Gallucci, G Simone

\section{"Regina Elena" National Cancer Institute}

Introduction \& Objective: Many software for fusion prostate biopsy (FPB) have been developed in the last years, most of them not providing the opportunity to combine transrectal (TR) and transperineal (TP) approach. Whereas one of these two approaches are beneficial in terms of complication rates or in terms of diagnostic accuracy is yet to be determined. The aim of this study was to compare periprocedural complication rates and diagnostic performance in propensity score matched pair cohorts to minimize the selection bias of non-random assignment of patients to TP as opposed to TR FPB.

Methods: Baseline, periprocedural and pathologic data of 1197 patients who received FPB were prospectively collected in 7 different centers. TR approach was performed in most patients $(1065,89 \%$, vs $132 \mathrm{TP})$. A 1:1 propensity score matched (PSM) analysis was employed to reduce covariate imbalances to $<10 \%$. The two selected PSM cohorts were compared for periprocedural and diagnostic performance. Chi-square or Fisher exact test and Student $\mathrm{t}$ test were used to compare categorical and continuous variables, respectively. Two-sided $\mathrm{p}$ values $<0.05$ were considered statistically significant. 

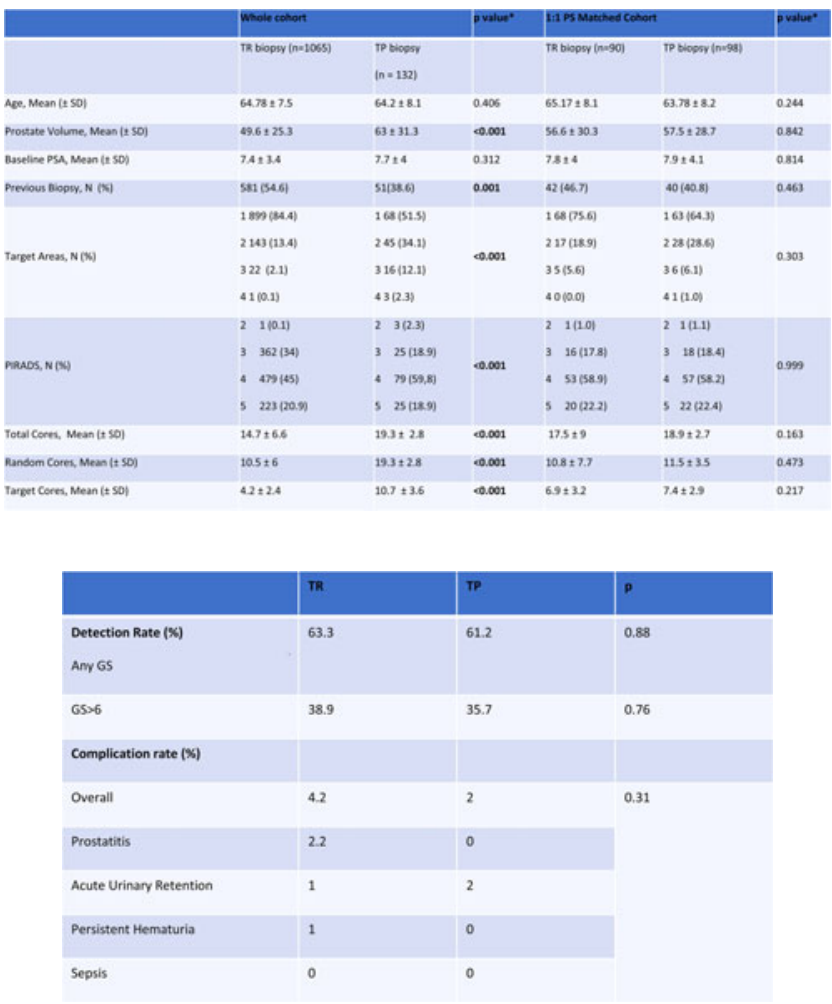

Results: In the overall cohort, patients treated with TR-FPB had significantly lower number of target areas, of target, random and total number of cores, lower prostate volume, higher incidence of PIRADS 3 lesions and of previous negative prostate biopsy (all $\mathrm{p}$ values $<0.001)$. After applying the PSM analysis, the two cohorts of 90 TR and 98 TP PFB did not differ for all covariates (Table 1; all $\mathrm{p}$ a $\% ¥ 0.16)$. TP vs TR PFB detection rates were comparable for any prostate cancer $(61.2 \%$ vs $63.3 \%$, respectively, $\mathrm{p}=0.88)$ as for clinically significant disease $(35.7 \%$ and $38.9 \%$, respectively $\mathrm{p}=0.76$ ). Complication rates were comparable between approaches ( $2 \%$ vs $4.4 \%$, respectively, $\mathrm{p}=0.31$ ).

Conclusions: Transrectal and transperineal Prostate Fusion Biopsy provide comparable diagnostic accuracy and periprocedural complication rates. Prospective randomized studies are needed to confirm these data.

MP14-20 Insertion of a Bioprotect balloon spacer between prostate and rectum prior to photon or proton beam radiotherapy in the treatment of carcinoma of prostate: early experience from a single UK center

JA Thomas, A Warlow, J Snell, J Clorely, R Davies, JF Lester

Rutherford Centre South Wales

Introduction and Objective: Approximately $38 \%$ of patients report RTOG grade 2 or worse bowel toxicity with hypofractionated prostate radiotherapy. We present a prospective review of the safety and efficacy of insertion of a biodegradable balloon spacer between rectum and prostate prior to proton (PBT) or photon therapy in a single UK center.

Materials and Methods: Between April 2018 and March 2019, 25 consecutive patients underwent insertion of a Bioprotect ${ }^{\mathrm{TM}}$ balloon prior to prostate radiotherapy. Mean age was 63 years (range 49 to 76). Median PSA was 7.6 (range 2.2 to 88 ). Twentytwo patients had localized disease (T1/2), 1 locally advanced (T3a) and 2 metastatic disease (T3b, M1). Twelve patients had hormone therapy prior to implantation. Prostate volumes ranged from 13.8 to $70 \mathrm{mls}$ (mean $35.5 \mathrm{mls}$ ). Anticoagulants (except aspirin) were stopped 7 days prior to implantation. Patients received antibiotics and an enema pre-procedure. A single surgeon implanted all balloons transperineally under general anesthetic in a dorsal lithotomy position using a bi-planar ultrasound probe and a brachytherapy stepper unit. Blunt dissection was employed to create a space for the balloon between the rectum and Denonvilliers' fascia. Post-implantation, 12 patients had hypofractionated radical PBT and 13 photon VMAT radiotherapy. Toxicity data were prospectively collected using RTOG scoring. Evaluation MRI imaging of the balloons was performed 24 hours post-implant.

Results: The first 10 patients were a 23-hour stay; the last 15 were day cases. All 25 patients had successful balloon implantation. Evaluation MRI showed a mean AP separation of rectum and prostate of $2.1 \mathrm{~cm}$ (range 1.7 to $2.5 \mathrm{~cm}$ ) and a mean balloon length of $4.5 \mathrm{~cm}$ (range $4.2-4.7 \mathrm{~cm}$ ). Post-operatively, one patient developed transient urinary retention that resolved within 48 hours. There were no wound or implant infections and no hematomas. Mild perineal discomfort occurred in $65 \%$ patients in the first 7 days. To date, there have been no reported grade 2 or worse acute rectal toxicities.

Conclusion: Initial results suggest insertion of a Bioprotect balloon between rectum and prostate is a safe and effective method of limiting acute radiation proctitis in men undergoing radiation therapy for prostate cancer.

MP14-21 Radiation dose and usage of CTKUB over a 5-year period (2014-2018): Observed trends in a University Hospital

A Pietropaolo, A Ho, T Bryant, I Wilson, F Wall, R Geraghty, BK Somani

Introduction \& Objective: CTKUB (CT scan of kidney, ureter and bladder) is the gold standard test for acute renal colic and diagnosis of kidney stone disease (KSD). We wanted to see the

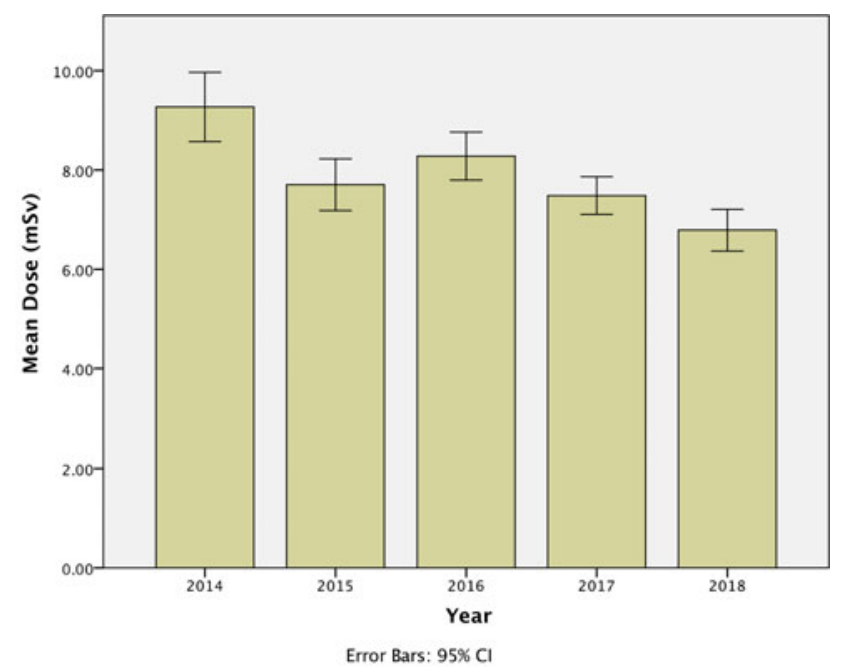

CT KUB radiation dose over the years 
TABLE 1 -trend rof radiation in CT KUB

\begin{tabular}{|l|l|l|l|l|l|}
\hline & 2014 & 2015 & 2016 & 2017 & 2018 \\
\hline $\begin{array}{l}\text { Number of } \\
\text { scans }\end{array}$ & 599 & 776 & 1040 & 1633 & 1078 \\
\hline $\begin{array}{l}\text { Radiation } \\
\text { dose (mSV) }\end{array}$ & 9.3 & 7.7 & 8.3 & 11.7 & 6.8 \\
\hline
\end{tabular}

trends of radiation dose and number of CT scans performed over a 5-year period.

Methods: Data for all CTKUB done from 2014-2018 was retrospectively recorded. We then analyzed the number of CT scans performed with the radiation dose over the study period. Data was collected and analyzed for trends to see if the radiation dose has changed with technological advances and the use of newer scanners. The original radiation dose was reported in DLP (dose length product) which was then converted to effective dose (mSV).

Results: A total of 5126 CTKUB was done over a 5-year period. The number of CT scans performed increased from 599 to 1078 in the study period. The radiation dose decreased from $9.3 \mathrm{mSV}$ in 2014 to $6.8 \mathrm{mSV}$ in 2018 . For each consecutive year the dose decreased achieving statistical significance $(p<0.001)$. The fluctuation of the CT scan dose was related to usage of new and old CT scanners in our hospital, with the later delivering a slightly higher dose.

Conclusions: The use of CTKUB has increased year on year over the last 5-years. Although more scans are being done, the radiation dose has steadily decreased over this period.

\section{MP14-22 Comparison of a Transperineal Versus Transrectal Targeted Biopsy with Magnetic-Resonance/ Ultrasound Fusion Guidance for Suspicious Lesions on Prostate Magnetic Resonance Imaging}

E Wajswol, JS Winoker, H Anastos, SK Doppalapudi, J Sfakianos, P Treacy, C Knauer, SC Lewis, BA Taouli, AR Rastinehad

Hackensack Meridian Department of Urology

Introduction \& Objective: The relative performance of the novel transperineal MR/US guided fusion biopsy approach in comparison to transrectal MR/US guided fusion biopsy is unknown. We evaluated and compared the accuracy of each of these targeted biopsy modalities to detect clinically significant prostate cancer (CS-PCa) in suspicious lesions identified on multiparametric MRI (mpMRI).
Table 1: Cancer detection rates (CDR) and clinically significant CDR (CS-CDR) for transperineal (TP) vs. transrectal (TR) biopsy approaches; overall and stratified by PI-RADS score.

\begin{tabular}{|cccc|ccc|}
\hline & TP: CDR & TR: CDR & p-value & TP:CS-CDR & TR:CS-CDR & p-value \\
\hline Overall & $135 / 176$ & $156 / 217$ & 0.279 & $103 / 176$ & $116 / 217$ & 0.315 \\
& $76.70 \%$ & $71.89 \%$ & & $58.52 \%$ & $53.46 \%$ & \\
PI-RADS 5 & $64 / 67$ & $52 / 58$ & 0.206 & $51 / 67$ & $44 / 58$ & 0.973 \\
& $95.52 \%$ & $89.65 \%$ & & $76.12 \%$ & $75.86 \%$ & \\
PI-RADS 4 & $58 / 76$ & $78 / 104$ & 0.839 & $44 / 76$ & $60 / 104$ & 0.978 \\
& $76.32 \%$ & $75 \%$ & & $57.89 \%$ & $57.69 \%$ & \\
PI-RADS 3 & $11 / 26$ & $23 / 48$ & 0.644 & $6 / 26$ & $10 / 48$ & 0.823 \\
& $42.31 \%$ & $47.92 \%$ & & $23.08 \%$ & $20.83 \%$ & \\
PI-RADS 2 & $2 / 7$ & $3 / 7$ & 1.000 & $2 / 7$ & $2 / 7$ & 1.000 \\
& $28.57 \%$ & $42.86 \%$ & & $28.57 \%$ & $28.57 \%$ & \\
& & & & & & \\
\hline
\end{tabular}

Methods: Between July 2015 and January 2019, 393 men enrolled in a phase III investigative device trial with suspicious lesions identified on mpMRI underwent concurrent targeted and systematic 12-core biopsy by either a transperineal $(\mathrm{tpBx})$ or transrectal (trBx) approach (UroNav, Invivo, Gainesville, FL). CS disease was defined as Gleason Grade Group (GGG) $\geq 2$. Cancer detection rates (CDR) and clinically significant CDR (CS-CDR) for each biopsy modality were stratified by PI-RADS v2 scores and compared with Chi-square, Fisher's exact, and Mann-Whitney U tests. Normality was tested with Shapiro-Wilk test.

Results: Median age was 66 years (37 - 89) and median PSA was $8.1 \mathrm{ng} / \mathrm{mL}(0.57-103.8)$. The groups were similar regarding digital rectal exam $(\mathrm{p}=0.303)$, family history of $\mathrm{PCa}(\mathrm{p}=0.99)$, previous negative biopsy $(\mathrm{p}=0.79)$, but differed regarding age $(\mathrm{p}=0.008)$. Of the 393 patients, $176(44.8 \%)$ and $217(55.2 \%)$ underwent tpBx and trBx, respectively. Overall, $135(76.7 \%)$ biopsies were positive for $\mathrm{PCa}$ in the tpBx group, of which 103 $(58.52 \%)$ were CS-PCa. By comparison, 156 (71.89\%) biopsies were positive for $\mathrm{PCa}$ in the $\operatorname{trBx}$ group with $116(53.46 \%)$ harboring CS disease. There was no significant difference between the two modalities with respect to overall CDR $(\mathrm{p}=0.279)$ or CS-CDR $(p=0.315)$. The same was true regardless of PIRADS suspicion score.

Conclusions: EM tracked MR/US fusion guided biopsies for transperineal and transrectal approaches did do not significantly differ in their ability to detect CS PCa while including the standard 12 core biopsy. Though additional research and larger patient cohorts are needed, these findings are encouraging and support the growing utilization of the novel transperineal fusion biopsy approach. In the absence of diagnostic superiority of one targeted biopsy modality over another, patient preference as well as procedural factors such as risk of infections may play a larger role in the choice of targeted biopsy modality. 


\section{MODERATED POSTER SESSION 15: LAPAROSCOPIC/ROBOTIC: ADRENAL, BLADDER \& LESS/NOTES}

\section{MP15-01 Withdrawn}

\author{
MP15-02 Is laparoscopic radical cystectomy really \\ suitable for the elderly?
}

G Kaneko, S Hattori, S Shirotake, K Kanao, S Hara, M Oyama

Saitama Medical University International Medical Center

Introduction \& Objective: Laparoscopic radical cystectomy (LRC) has been widespread due to less invasiveness and same oncological result compared with open radical cystectomy. Trendelenburg position is generally required for long time to perform LRC, therefore, whether LRC is really suitable for the elderly remains unclear. We compared surgical and oncological outcomes between younger and elderly patients to clarify it.

Methods: A total of 47 patients underwent laparoscopic radical cystectomy and extracorporeal urinary diversion at Kawasaki Municipal hospital and Saitama Medical University International Medical Center from December 2014 to May 2019. Patients were classified as elderly $(\geq 75$ years) or younger $(<75$ years). The clinical characteristics, operative outcomes, pathologic results, and survival were compared between two groups. Results: The outcomes of 33 younger and 14 elderly patients including 6 patients $\geq 80$ years old were assessed. No significant difference was found in sex, American Society of Anesthesiologists score, body mass index, with or without neoadjuvant chemotherapy, range of lymph node dissection between groups. The difference of operative time and pneumoperitoneal time was insignificant [508 minutes (younger) vs. 516 minutes (elderly), $P=0.824$ and 306 minutes (younger) vs. 320 minutes (elderly), $P=0.603$ ]. The estimated blood loss of the elderly group was larger than younger group, however, the difference was insignificant $[661 \mathrm{ml}$ (younger) vs. $821 \mathrm{ml}$ (elderly), $P=0.597]$. And the difference of time to discharge was insignificant [ 29 postoperative days (younger) vs. 29 postoperative days (elderly), $P=0.983$ ]. No difference was also found incidence of $\geq$ grade 3 complication by Clavien-Dindo classification between two groups. The proportion of $\geq$ pathological T2 stage and/or node positive in the elderly group was tend to higher than the younger group ( $50.0 \%$ (younger) vs. $78.6 \%$ (elderly), $P=0.070$ ), the difference of the proportion of adjuvant chemotherapy was insignificant $(20.0 \%$ (younger) vs. $23.1 \%$ (elderly), $P=0.558$ ). During follow up period of median 12.7 months, the difference of recurrence free survival and overall survival between groups was insignificant. Conclusions: The elderly does not appear to have any significant differences or compromises with regard to the perioperative and survival outcomes. Therefore, LRC can be offered as treatment option in select elderly patients.

MP15-03 Complete Hybrid Transvaginal Natural Orifice Transluminal Endoscopic Nephroureterectomy

Z Xiaofeng, Z Guoxi, X Tianpeng, Y Yuanhu,

$X$ Rihai, W Gengqing
Introduction \& Objective: To investigate the feasibility, safety and efficacy of complete hybrid transvaginal natural orifice transluminal endoscopic nephroureterectomy (TV-NOTES NU). Methods: We performed the first case of complete hybrid TVNOTES NU for a 40-year female patient with right renal pelvis carcinoma. Under general anesthesia, the patient was placed in improved side-seat riding position with the affected side elevated at $60^{\circ}$. A 5- $\mathrm{mm}$ and $10-\mathrm{mm}$ trocars were placed at the medial margins of umbilicus. A lengthened 5-mm trocar was placed through the posterior vaginal fornix into the pelvic cavity. The distal ureter was firstly dissected and blocked. Then the radical nephrectomy was performed. After that the ureter was isolated completely and bladder wall cuff resection was performed. All surgical procedures were laparoscopic performed. The specimen was placed inside a homemade bag and removed outside by extending the vaginal incision. Results: The first complete hybrid TV-NOTES NU was successfully performed. No other trocar was added during the operation and the operative time was $175 \mathrm{~min}$. The estimated blood loss was about $400 \mathrm{ml}$ without blood transfusion. There was no major intraoperative or postoperative complication occurred. At the end of the operation, gemcitabine was given intravesical instillation chemotherapy. The patient was discharged on the 8th day after operation. The umbilical scar was basically invisible 3 months after the operation, and the vaginal incision healed well. No recurrence or metastasis was observed after the review.

Conclusions: complete hybrid TV-NOTES NU was feasible, safe and effective with less injury, fast recovery and good cosmetic outcomes in properly selected patients. It is worth applying in urological clinical practice.

MP15-04 Long-term outcome of laparoscopic vesicouterine fistula repair: Experience from a tertiary referral centre

S Mehrotra, S Mehdi, V Singh, S Mehrotra, S Mehdi, S Pandey, R Sinha, V Singh

Department of Urology, King Georges Medical university, Lucknow, India

Introduction \& Objective: Vesicouterine fistulas (VUF) represent an uncommon type of female genito-urinary fistulas with lower segment uterine cesarean section (LSCS) being the underlying etiology in most of the cases. While open surgical repair constitutes the traditional treatment, a few case reports have been published describing laparoscopic repair of VUF as a minimally invasive technique along with their short term follow up. We are presenting the long- term outcome of laparoscopic repair of VUF in the present study.

Methods: The study involved retrospective analysis of 8 patients with VUF from 2010 to 2015 after obtaining approval from the Institutional Review Committee. All patients had history of LSCS of whom 3 had history of prolonged obstructed labor. Radiological imaging included ultrasound of kidney, ureter and bladder for all patients and hysterosalphingography in 4 patients and contrast enhanced computed tomography scan in 4 patients. 
Results: Median age of the patient was 25.5 years (range, 2232 ), and median follow up was 2.3 years (range, $1-4$ ). Cyclical menstrual bleeding through urine (menouria) was the most common presentation seen in all, associated amenorrhea was present in 6 patients while vaginal leakage of urine was seen in 2 cases. All patients underwent laparoscopic repair with successful outcomes. The mean operating time was $155 \pm 14.5 \mathrm{~min}$ (range, 135-186 min) with a median blood loss of $100 \mathrm{~mL}$ (range, 50 $210 \mathrm{~mL}$ ). Successful pregnancy was completed in 2 patients and other patients were taking contraceptives.

Conclusions: Laparoscopic repair of VUF represents a safe and effective minimally invasive technique with successful pregnancy in long- term follow up.

\section{MP15-05 Laparoscopic partial cystectomy for differ- ent benign bladder pathologies}

\section{AA ALSUNBUL, H El-Tholoth, T Alzahrani, A Al Zahrani}

Urology Department, Prince Sultan Military Medical City

Introduction \& Objective: Partial cystectomy may be indicated for localized bladder cancer or benign lesions such as diverticulum. The objective of this study is to on our initial experience with laparoscopic partial cystectomy (LPC) in four cases with bladder pathology.

Methods: We reviewed all cases of laparoscopic partial cystectomy performed in Prince Sultan Medical Military City (PSMMC) from May 2016 to September 2018. Pre-operative CT with contrast and cystoscopy were done for all patients to establish the diagnosis. Patients' demographic data, clinical presentation, preparative investigations, indications for the operation, intraoperative time, blood loss, perioperative complications, and hospital stay were reported. In addition, histopathology of the lesions removed and duration and methods of follow up were stated. Analysis of the data was done.

Results: The study included three females and one male with a mean age of 39.7 (range 28-67). Laparoscopic partial cystectomy was performed using the transperitoneal approach in all cases Laparoscopic partial cystectomy was completed in the four cases without open conversion. Clinical presentations were in the form of suprapubic pain, hematuria, and malignant hypertension. Cystoscopy-guided laparoscopic resection was done in paraganglioma case. The mean operative time in the four cases was 95 minutes (range 80-120), estimated blood loss was $80 \mathrm{ml}(50-100 \mathrm{ml})$, hospital stay was 4 days (2-6 day), and the Foley catheter was kept for a mean of 7.5 days (6-10). No major peri-operative complications were noted. Histopathology report revealed evidence of leiomyoma in two cases, paraganglioma in one and urachal cyst in the fourth. At last, follow up (range 9-21 months) no complications were reported. Conclusions: When partial cystectomy is indicated for bladder pathology, the laparoscopic approach is feasible and safe in carefully selected patients.

MP15-06 Robotic Cystectomy in the Over Eighties Study (RCOES) - A United Kingdom Multicenter Study

E Latif, J Noel, V Kusuma, D Moschonas, S Woodhams, M Swinn, P Pavlakis, H Mostafid, B Ayers, R Nair, M Perry, EH Streeter, HR Patel, R Issa, R Thurairaja, N Vasdev, K Patil, PD Rimington, S Khan, SS Kommu

Kent \& Canterbury Hospital. East Kent Hospitals NHS Foundation Trust
Introduction \& Objective: The treatment of Muscle Invasive Bladder Cancer (MIBC) in the aging population continues to present challenges both from an individualized approach and from a health burden point of view. Recent advances in minimally invasive approaches challenged previous age-related bias in deciphering optimal treatment strategies in this population. However, the risk to benefit ratio of surgical treatment of MIBC in patients aged 80 and over has not been objectively and comprehensively quantified. Herein, we aim to compare the outcomes of radical cystectomy between patients under and $\geq 80$ years.

Methods: Data from five United Kingdom tertiary referral urooncology centers was tallied and interrogated for demographic and outcome parameters. Between September 2010 and April 2019 , a total of 103 patients $\geq 80$ years underwent RC and ileal conduit formation. These patients were compared with 103 patients in the under $80 \mathrm{yrs}$ age group in the same time frame. Outcome parameters included total operative time, length of total hospital stay, impact of lymph node dissection, resection margins, in patient complications and 30-day complications.

Results: The median overall age for all patients was $82 \mathrm{yrs}$ (range 80-88) [69yrs (42-79) for under 80s \& 82yrs (80-88) for $\geq 80]$. There were a total of 103 patients [82 (79.6\%) Male \& 21 (20.4\%) Female]. The majority of patients had ASA grade of 2 or 3 . In the over 80 s group there were a total of 46 complications $(45 \%)$ within 30 days of which 17 were Clavien Dindo 3 or higher $(16.5 \%)$. There were no significant differences in rates of in-patient complications $(\mathrm{p}>0.46)$ and 30-day complications ( $p>0.20)$ between those were under 80 and $\geq 80$. There was no difference in survival at early and medium-term follow-up.

Conclusions: In patients 80 and over with MIBC, favorable outcomes can be achieved in dedicated tertiary referral pelvic uro-oncology centers. Robotic assisted radical cystectomy in over 80-year-old patients is safe and feasible in the carefully selected patient. Therefore, the risk to benefit ratio should be weighed carefully and age should not be considered a barrier to intervention. Further large-scale multicenter studies to compare the long-term outcome and overall survival are currently in progress to evaluate the overall impact of advanced age on outcomes of radical cystectomy.

\section{MP15-07 Comparison of synchronous bilateral transperitoneal and posterior retroperitoneal laparo- scopic adrenalectomy in high volume adrenal centers}

A Tuncel, J Langenhuijsen, A Erkan, T Mikhaylikov, M Arslan, Y Aslan, D Berker, Y Ozgok, E Gallyamov, AS Gozen

University of Health Sciences, Ankara Numune Research and Training Hospital

Introduction \& Objective: To report our multicenter results of the lateral transperitoneal and posterior retroperitoneal synchronous bilateral laparoscopic adrenalectomy experience in patients who had different bilateral adrenal pathologies and to compare the outcomes of these two different operative procedures.

Methods: Between 2012 and 2018, a total of 52 patients with a mean age of 43.5 years underwent simultaneous bilateral laparoscopic adrenalectomy at 6 different centers. Twenty-seven and 25 patients underwent bilateral lateral transperitoneal and posterior retroperitoneal laparoscopic adrenalectomy, respectively. Patients' age, gender, body max index, operative indications, mass size, operation time, blood loss, length of hospitalization, 
intraoperative and postoperative complications and pathology reports were analyzed.

Results: Transperitoneal group was younger than retroperitoneal group (37 yr vs. 50.4 yr.) (p:0.001). BRLA group had significantly decreased operating time and less blood loss than BTLA group. No significant difference was found with regard to postoperative hospital stay, perioperative and postoperative complications between two groups. Majority of the histopathological results were adrenal hyperplasia associated with Cushing's disease $(61.5 \%)$. Less frequent pathological results were adrenal adenoma and pheochromocytoma $(15.4 \%$ and $13.5 \%$, respectively). During the follow-up period, no recurrence or disease related mortality was observed in the patients.

Conclusions: Our results show that shorter operative time and less bleeding can be achieved with posterior retroperitoneal approach in synchronous bilateral LA. In our series, intraoperative and postoperative complication rates were similar between both surgical approaches.

\section{MP15-08 Suprapubic-assisted laparoendoscopic single- site surgery (SA-LESS) for secondary ureteral stricture}

Z Guoxi, Z Xiaofeng, Y Yuanhu, X Rihai, W Gengqing, W Xiaoning

Introduction \& Objective: To introduce the experience of Suprapubic-assisted laparoendoscopic Single-site Surgery (SALESS) in the treatment of secondary ureteral stricture.

Methods: 27 patients involved in this group (15 males and 12 females). The mean age was 37.8 (range: 21-67). The patients were all unilateral, 17 cases on the left and 10 cases on the right. Among them, there were 20 cases with the history of ureteroscopic holmium laser lithotripsy, 5 cases with the history of retroperitoneoscopic ureterotomy, and 2 cases with the history of extracorporeal shock wave lithotripsy. The stenosis was located in the upper ureter in 7 cases, the middle ureter in 9 cases, and the lower ureter in 11 cases. All patients had moderate or serious hydronephrosis in the kidney. All relevant radiographic examinations were performed before surgery to identify the ureteral stricture. The patient, under general endotracheal anesthesia, was secured on the operating table in lateral decubitus with the affected side elevated by $70^{\circ}$ or supine in the Trendelenburg position. A $5 \mathrm{~mm}$ and $10 \mathrm{~mm}$ trocars were inserted at the medial margin of the umbilicus, and a $10 \mathrm{~mm}$ trocar was inserted below the pubic hairline. A $5 \mathrm{~mm}$ flexible-tip $0^{\circ}$ laparoscope was used. Patients with stenosis in the middle and upper ureter underwent stenosis resection and anastomosis. We found ureteral strictures based on imaging data during surgery, if necessary, we cut longitudinally near the ureteral stricture to avoid unnecessary waste by cutting the ureter transversely. Patients with stenosis in the lower ureter underwent a ureteral bladder replantation. The operation time, intraoperative blood loss, postoperative hospital stay, intraoperative and postoperative complications, and follow-up were recorded.

Results: Twenty-six cases were successfully completed without ancillary trocars or additional instruments. One patient was converted to open surgery due to excessive tension during ureteral anastomosis. The median operation time was 170 (130-230) min, and the median blood loss was 50 (20-90) ml. No serious complications occurred during or after surgery. The drainage tube was removed 2 to 3 days after surgery. The drainage tube was removed on postoperative day 7 , and the double $\mathrm{J}$ tube was removed 1 to 2 months postoperatively. After follow-up of 3 to 6 months, the umbilical and pubic scars were not obvious. The results of intravenous urography showed that the ureter is unobstructed or the hydronephrosis was significantly reduced than before.

Conclusions: The suprapubic-assisted laparoendoscopic singlesite surgery is safe, effective. It also has a good cosmetic effect for the treatment of secondary ureteral stricture.

\section{MP15-09 Impact of a Complex Pelvic Operative Field on Robot-Assisted Radical Cystectomy Morbidity}

AS Elsayed, NA Aldhaam, B Hull, A Nagra, A Siam, M Durrani, Z Jing, AA Hussein, KA Guru

Roswell Park Comprehensive Cancer Center

Introduction \& Objective: The stigma of a previous surgery is hindering many patients to benefit from the advancement of technology associated with robot-assisted surgery. We hereby report the perioperative and oncologic outcomes of previous pelvic interventions in patients undergoing robot-assisted radical cystectomy (RARC).

Methods: A retrospective review of our prospectively maintained departmental database between 2005 and 2019. 586 RARC patients were identified. Patients were divided into three groups, having no previous abdominal surgery and/or upper abdominal surgery (320), having one pelvic surgery or radiation (186), and having 2 or more pelvic surgery and/or radiation (80). All groups were compared in terms of perioperative characteristics, pathologic and oncologic outcomes. A multivariate linear regression model was used to depict the predictors of blood loss and operative time. Multivariate logistic regression was used to depict the predictors of high-grade complications and readmissions. The Kaplan-Meier method was used to depict recurrence-free (RFS), disease-specific (DSS) and overall survival (OS).

Results: Mean age was $69 \pm 11$ years. Group 3 were more likely to have an $\mathrm{ASA} \geq 3$ (73\% vs $59 \%$ vs $49 \%, \mathrm{p}<0.01)$, receive an intracorporeal urinary diversion ( $83 \%$ vs $73 \%$ vs $70 \%, p=0.1)$, however they were less likely to receive a neobladder (3\% vs $7 \%$, vs $13 \%, \mathrm{p}<0.01)$ compared to groups 2 and 1 respectively. There

Table 1. Demographics, clinical characteristics, perioperative and pathological outcomes of patients.

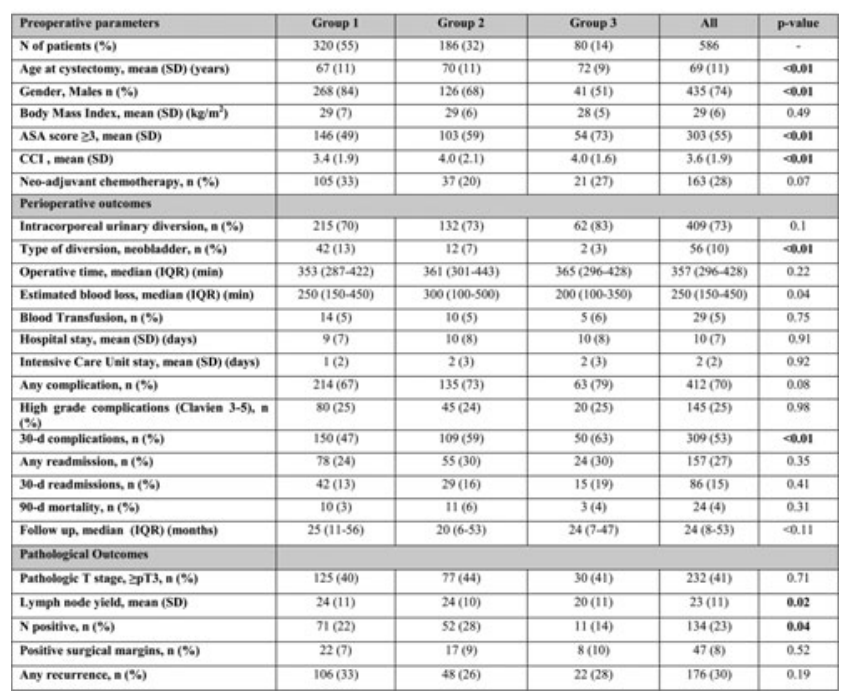


was no statistical significant difference between the groups in terms of operative time $(p=0.22)$, transfusion rates $(p=0.75)$, length of ICU stay $(p=0.93)$, hospital stay $(p=0.91)$, complications $(0.08)$, readmissions $(\mathrm{p}=0.41)$ and perioperative mortality $(p=0.31)$. Statistically significant difference was found in mean estimated blood loss $(\mathrm{p}=0.04)$, early complications $(\mathrm{p}<0.01)$, and lymph node yield $(\mathrm{p}=0.02)$ (Table 1$)$. On multivariate linear and logistic regression models previous pelvic surgery and/or radiation were not predictors for estimated blood loss, operative time, highgrade complications and any readmissions. The three groups showed a similar RFS (log-rank, $\mathrm{p}=0.33$ ), DSS (log-rank, $\mathrm{p}=0.96$ ), and OS (log-rank, $\mathrm{p}=0.57$ ).

Conclusions: Robot-assisted radical cystectomy should be provided for all patients who are candidates for cystectomy despite their previous surgical history. RARC seems to have a comparable safety profile compared to open radical cystectomy.

\section{MP15-10 The mid-long-term follow up after trans- vaginal natural orifice transluminal endoscopic sur- gery in urology} Z Xiaofeng, Z Guoxi, Y Yuanhu, X Rihai, W Gengqing,
W Xiaoning

Introduction \& Objective: To investigate the complication, sexual function and fertility after transvaginal natural orifice transluminal endoscopic surgery (NOTES) and analysis the longterm outcome of this procedure.

Methods: From May 2010 to May 2015, patients who underwent transvaginal NOTES were selected. Patients were informed to return to the hospital regularly by the means of telephone, message and wechat filled the follow-up questionnaire, female sexual function index (FSFI), and fertility function questionnaire. And the complication, sexual function and fertility of all subjects were analyzed.

Results: A total of 162 patients received complete clinical data and were included in the study, and 25 patients were lost to follow-up, 137 cases accepted the invitation, including 126 hybrid TV-NOTES procedures and 11 pure TV-NOTES procedures were performed. The various transvaginal hybrid NOTES procedures included nephrectomy, partial nephrectomy, adrenalectomy, heminephroureterctomy for duplex kidney (3 cases) and hybrid endoscopy for nephroureterectomy (6 cases). And the pure transvaginal NOTES procedures included nephreetomy, 23cases of intraoperative and postoperative complications were occurred, the mean age were 49.25 years. The mean body mass index was $21.90 \mathrm{~kg} / \mathrm{m}^{2}$. The follow-up time was 12 to 86 months. 137 patients completed the follow-up. Among them, 28 patients did not have sex, 34 invalid questionnaire, and the final 75 patients met the inclusion criteria and received a valid female sexual function score questionnaire. The mean FSFI of 75 cases preoperatively and $3 \sim 12$ months and 1 year postoperatively were $27.61 \pm 4.68,26.59 \pm 4.99$ and $28.04 \pm 4.41$ respectively. There was no statistically significant difference among them. Among the transvaginal hybrid NOTES and pure NOTES, there were no significant differences in the FSFI scores and scores of each dimension between the two groups ( $<48$ years old and $\geqq 48$ years old). 137 patients were married, and 136 cases have been educated, 133 had birth control. 3 cases of childbirth wish after surgery, 4 postoperative reproductive childbirth again, one case of unintended pregnancy, all of them are pregnant naturally and did not take drugs and artificial fertility After the start of sex life after pregnancy was 1 to 14 months, an average of 5.5 months; the average pregnancy was 280 days. Three patients had spon- taneous vaginal delivery, one patient was cesarean because of fetal umbilical cord around the neck, Four patients produced 2 boys and 3 girls after surgery.

Conclusions: transvaginal NOTES surgery does not have a negative impact on mid-to-long term sexual function.

\section{MP15-11 Withdrawn}

\section{MP15-12 Single-Port Robotic Surgery Allows Same- Day Discharge in Majority of Cases}

R Abaza, OE Martinez

OhioHealth Dublin Methodist Hospital

Introduction \& Objective: Further evolution of robotic surgery to a single point of access may extend the benefits of minimallyinvasive surgery even beyond the traditional robotic approach. We hypothesized that single-port (SP) robotic surgery may reduce pain and thereby allow earlier discharge than our typical overnight stay after robotic prostate and kidney surgery. We report our initial experience using the da Vinci $\mathrm{Sp}^{\circledR}$ robotic surgical platform for three major urologic surgeries and its influence on length of stay (LOS).

Methods: We evaluated our single surgeon (RA) prospective data database for the initial $25 \mathrm{SP}$ procedures performed between January and May 2019. Patient LOS was compared between SP and multiport procedures after September 2016 since we began offering same day discharge (SDD) to all patients undergoing robotic prostatectomy and kidney surgery.

Results: Among 25 SP robotic surgeries performed were 13 prostatectomies, 5 partial nephrectomies and 7 pyeloplasties. Overall mean age was 52.7 years (19-77 years), mean BMI was $26.7 \mathrm{~kg} / \mathrm{m}^{2}\left(19-38 \mathrm{~kg} / \mathrm{m}^{2}\right)$, mean estimated blood loss was $46.4 \mathrm{cc}$ (10-100cc) and mean operative time was $147 \mathrm{~min}$ (75-216min). Overall, 22 of 25 SP patients were discharged the same day as surgery $(88 \%)$. The rate of same day discharge (SDD) in all three types of SP procedures was higher compared to our historical SDD for multiport robotic surgeries. Among prostatectomies, 77\% of SP patients were discharged the same day versus $49 \%(\mathrm{p}=0.05)$. Among partial nephrectomies, $100 \%$ of SP patients went home the same day versus $12 \%(\mathrm{p}<0.001)$ as well as $100 \%$ of pyeloplasty patients versus $54 \%(\mathrm{p}=0.031)$. There were no postoperative complications or readmissions among any SP patients.

Conclusions: Although the purpose-built single-port robotic surgery platform is still very new, our initial experience suggests that earlier discharge is possible with the large majority of patients able to go home the same day as surgery so far. Further experience will be necessary to confirm this as well as larger number of patients to allow analysis of pain scores and analgesic usage as potential factors.

MP15-13 Extraperitoneal Single Port Robotic Prostatectomy: Initial Experience with Same Day Discharge

RJ Yau, GV Sawczyn, RJ Valero Carrion, M Eltemamy, J Kaouk

Cleveland Clinic Foundation

Introduction \& Objective: To present our experience of single port robotic assisted laparoscopic prostatectomy for prostate 
cancer in the outpatient setting with the new da Vinci SP ${ }^{\circledR}$ robotic platform.

Methods: We performed extraperitoneal single port robotic assisted laparoscopic radical prostatectomy (ESRRP) on 33 consecutive patients using the da Vinci $\mathrm{SP}^{\circledR}$ robot which is a purpose-built platform for single port robotic surgery. This offers advantages compared to traditional multi-arm robotic surgery which include avoiding bowel injury, flat supine patient position, no surgical drains, and a single incision with no accessory laparoscopic ports. We also practice an enhanced recovery protocol when combined with the advantages of single port robotic surgery, allow patients to go home the same day of surgery. Data was collected in a prospectively maintained institutional review board approved database.

Table1 - Preoperative Data

\begin{tabular}{|c|c|c|}
\hline \multicolumn{2}{|c|}{ Outpatient SP ${ }^{\mathscr{E}}$ Prostatectomy } & $n=29$ \\
\hline \multicolumn{2}{|c|}{ Age (median) } & 61.5 \\
\hline \multicolumn{2}{|c|}{ BMI (median) } & 25.9 \\
\hline \multirow{2}{*}{ ASA Classification } & 2 & $12(41 \%)$ \\
\hline & 3 & $17(59 \%)$ \\
\hline \multicolumn{2}{|c|}{ Previous Abdominal Surgery } & $7(26 \%)$ \\
\hline \multirow{2}{*}{$\begin{array}{l}\text { Charlson } \\
\text { Comorbidity Index }\end{array}$} & $\leq 4$ & $18(62 \%)$ \\
\hline & $\geq 5$ & $11(38 \%)$ \\
\hline \multicolumn{2}{|c|}{ PSA (Median) } & 5.72 \\
\hline \multirow{2}{*}{$\begin{array}{c}\text { NCCN } \\
\text { Risk Group }\end{array}$} & $\leq$ Intermediate & $23(79 \%)$ \\
\hline & $\geq$ High risk & $6(21 \%)$ \\
\hline
\end{tabular}

Table 2 - Operative Data

\begin{tabular}{|c|c|c|}
\hline \multicolumn{2}{|c|}{ Outpatient SP Prostatectomy } & $\mathrm{n}=\mathbf{2 9}$ \\
\hline \multicolumn{2}{|c|}{ Operative time (mean) } & $207 \pm 34 \mathrm{~min}$ \\
\hline \multirow{2}{*}{ Estimated blood loss (mean) } & $188 \pm 114 \mathrm{ml}$ \\
\hline \multirow{3}{*}{ Nerve Sparing } & Bilateral & $27(94 \%)$ \\
\cline { 2 - 3 } & Unilateral & $1(3 \%)$ \\
\cline { 2 - 3 } & Non-sparing & $1(3 \%)$ \\
\hline
\end{tabular}

Table 3 - Pathology Data

\begin{tabular}{|c|c|c|}
\hline \multicolumn{2}{|c|}{ Outpatient SP Prostatectomy } & $n=29$ \\
\hline \multicolumn{2}{|c|}{ Specimen Weight (mean) } & $55 \pm 27 g$ \\
\hline \multirow{2}{*}{ Surgical Pathology Gleason } & $\leq 7$ & $24(83 \%)$ \\
\hline & $\geq 8$ & $5(17 \%)$ \\
\hline \multicolumn{2}{|c|}{$\begin{array}{l}\text { Extraprostatic } \\
\text { Extension }\end{array}$} & $13(45 \%)$ \\
\hline \multicolumn{2}{|c|}{ Positive Margins } & $6(20 \%)$ \\
\hline \multirow{2}{*}{ pT } & $\leq T 2$ & $17(59 \%)$ \\
\hline & $\geq \mathrm{T} 3 \mathrm{a}$ & $12(41 \%)$ \\
\hline \multicolumn{2}{|l|}{ pN1 } & $1(3.5 \%)$ \\
\hline \multicolumn{2}{|c|}{$\begin{array}{l}\text { Number of regional lymph nodes examined } \\
\text { (median) }\end{array}$} & 5 \\
\hline
\end{tabular}

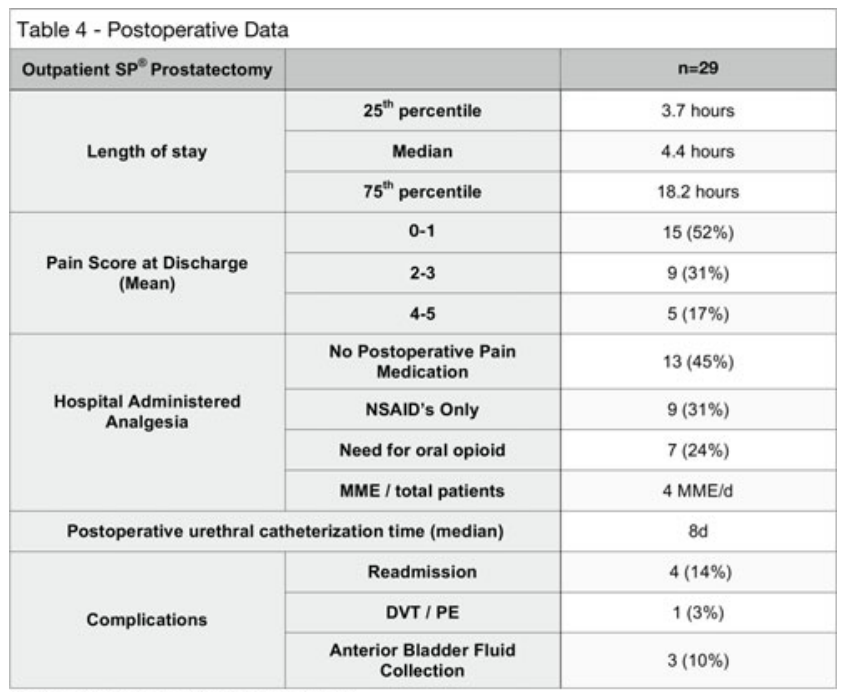

NSAID - Non-Steroidal Anti-Inflammatory Drugs

\begin{tabular}{|c|c|c|}
\hline Table 5 - 30 day Follow-up & \multicolumn{2}{|c|}{} \\
\hline Outpatient SP Prostatectomy & & $n=14$ \\
\hline \multirow{2}{*}{ First PSA follow-up } & Undetectable & $10(72 \%)$ \\
\cline { 2 - 3 } & Detectable & $4(28 \%)$ \\
\hline \multirow{3}{*}{ Continence Status } & 0-1 Pads/d & $5(36 \%)$ \\
\cline { 2 - 3 } & 2-3 Pads/d & 0 \\
\cline { 2 - 3 } & 4-5 Pads/d & $3(22 \%)$ \\
\hline \multirow{3}{*}{ Erectile Function Status } & $>5$ Pads/d & $6(42 \%)$ \\
\cline { 2 - 3 } & SHIM Score 5 & $7(50 \%)$ \\
\cline { 2 - 3 } & SHIM Score 6-10 & $4(28 \%)$ \\
\hline & SHIM Score >11 & $3(22 \%)$ \\
\hline
\end{tabular}

Results: Since February 5, 2019 we performed ESRRP on 33 consecutive patients. 29 patients were discharged before 48 hours. 20 patients were discharged the same day of surgery. Median length of stay was 4.4 hours. Mean patient reported pain scale was 1 out of 10 . Opioid use was only $24 \%$. There were no intraoperative complications or blood transfusions. Postoperative complications included three anterior bladder fluid collections, and one DVT/PE. Positive surgical margins were found in 6 patients (20\%). Our maximum follow-up is 90 days. Of those patients $60 \%$ are completely continent and $40 \%$ are having satisfactory erections.

Conclusions: With our extraperitoneal approach, the da Vinci $\mathrm{SP}^{\circledR}$ robotic platform, and enhanced recovery anesthesia protocol, we have been able to perform a virtually painless 
prostatectomy where the majority of patients can go home within 5 hours of the procedure without opioid use.

MP15-14 RARC and U-configuration ileal neobladder with totally intracorporeal procedure after neoadjuvant chemotherapy in patients with muscleinvasive bladder cancer

C Ohyama, Y Hashimoto, T Yoneyama, S Hatakeyama, A Imai

Hirosaki University Graduate School of Medicine

Introduction \& Objective: Radical cystectomy (RC) is the gold standard of treatment for patients with muscle-invasive bladder cancer (MIBC) and robot-assisted RC (RARC) have demonstrated acceptable surgical and perioperative outcomes. However, frequency of use of neoadjuvant chemotherapy and intracorporeal neobladder reconstruction remains low. We previously reported our original U-configuration ileal neobladder reconstruction using $40 \mathrm{~cm}$ of a cross-folded ileal loop. (Int J Urol 2006, Urol 2010) We applied this simple procedure for intracorporeal neobladder reconstruction after RARC with neoadjuvant chemotherapy. Here, we report our initial experiences.

Methods: We retrospectively reviewed the charts of 37 consecutive patients who underwent RARC and bilateral pelvic node dissection between May 2012 and May 2019. This study included patients with MIBC who had histologically confirmed stage T2-T4a urothelial carcinoma of the bladder without lymph node or distant metastases and received neoadjuvant chemotherapy (NAC) prior to RARC. We compared surgical outcomes of intracorporeal U-ileal neobladder to those of extracorporeal U-ileal neobladder.

Results: The mean surgical duration for RARC with extracorporeal and RARC with intracorporeal neobladder was $365 \mathrm{~min}$ (standard deviation $78 \mathrm{~min}$ ) and $498 \mathrm{~min}$ (standard deviation 58 min), respectively. The mean estimated blood loss for RARC with extracorporeal and RARC with intracorporeal neobladder was $439 \mathrm{ml}$ (standard deviation $254 \mathrm{ml}$ ) and $390 \mathrm{ml}$ (standard deviation $381 \mathrm{ml}$ ), respectively. No open conversions nor $90-\mathrm{d}$ mortality were experienced for both procedures. There were no complications greater than grade 2 Clavien-Dindo classification for both procedures. Two patients $(40 \%)$ experienced renal dysfunction and one (20\%) experienced ileus after extracorporeal neobladder. While, there were no such complications after intracorporeal neobladder.

Conclusions: Our initial experience with the totally intracorporeal U-ileal neobladder after NAC appears to have favorable clinical outcomes. Totally intracorporeal procedure may be associated with less severe complications compared to extracorporeal neobladder procedure.

MP15-15 Global assessment of outcomes of Robotassisted radical cystectomy with orthotopic neobladder: Trifecta vs Pentafecta

A Brassetti, U Anceschi, G Tuderti, S Guaglianone, M Ferriero, F Minisola, R Mastroianni, R Flammia, M Gallucci, G Simone

\section{"Regina Elena” National Cancer Institute}

Introduction \& Objective: Robot-assisted radical cystectomy (RARC) with orthotopic neobladder $(\mathrm{ON})$ is associated with heterogeneous surgical, oncologic and functional outcomes. Two dif- ferent pentafecta have been already proposed to optimize outcomes reporting after cystectomy but their external validation is still missing, and both did not include functional outcomes. In this study, we developed a new Trifecta in patients who underwent RARC with intracorporeal ON and we validated the available Pentafecta. Methods: Our prospectively maintained bladder cancer (BC) database was queried for "RARC" and "intracorporeal ON". Only patients with a minimum 1-yr follow-up were included. The following data were collected and analyzed: baseline demographic, clinical, perioperative and pathologic data, functional and oncologic outcomes. The "trifecta" was defined as the combination of: daily urinary continence, recurrence-free status and Clavien-Dindo $\geq 3$-free status at 12 months. The Pentafecta designed by the PROMETRIC group (PentaPRO) 1 and the other proposed by the University of Southern California (PentaUSC) 2 were also calculated. A receiver operating characteristic (ROC) analysis was performed to assess the predictive accuracy of any model on 2-yr overall survival (OS) probability.

Results: Overall, 137 patients were included in the analysis (Table 1). The trifecta was achieved by $72(53 \%)$ of them, while $64(47 \%)$ and $99(72 \%)$ obtained the PentaPRO and the PentaUSC, respectively (Figure 1). On ROC analysis, trifecta was the only significant predictor of 2-yr OS $(\mathrm{AUC}=0.685$, 95\%CI:0.55-0.82; $\mathrm{p}=0.018)$, while both PentaPRO (AUC $=$ 0.599; 95\%CI: 0.453-0.764; $\mathrm{p}=0.205$ ) and PentaUSC models $(\mathrm{AUC}=0.645 ; 95 \% \mathrm{CI}: 0.492-0.798, \mathrm{p}=0.064)$ were not significantly associated with 2-yr OS probability (Figure 2).

Conclusions: Our trifecta is the first standardized and reproducible system specifically designed to provide a comprehensive outcome assessment after RARC with ON. Although external validation studies are required, this novel tool may optimize outcomes reporting and, at 1-yr follow-up evaluation, can be used to design a patient tailored follow-up.

MP15-16 Perioperative efficacy and long-term survival benefit of Robotic-assisted radical cystectomy in elderly patients: a nationwide multi-institutional study in Japan

H Iwamoto, S Morizane, T Koie, R Shiroki, M Kawakita, T Gondo, K Matsumoto, T Habuchi, A Takenaka, H Kanayama

Tottori University Hospital

Introduction \& Objective: To determine the perioperative safety and oncological value of robotic-assisted radical cystectomy (RARC) for older and younger patients in an initial Japanese RARC series.

Methods: We retrospectively analyzed the demographics, complications, perioperative and oncologic outcomes of 253 consecutive patients with bladder cancer who underwent RARC at 34 institutions in Japan between April 2009 and March 2017. The patients were assigned to groups according to ages at surgery of $<70$ (younger; $n=125$ ) and $\geq 70$ (older; $n=128$ ) years.

Results: Mean Charlson Comorbidity scores $(p=0.045)$ and the incidences of a history of previous abdominal surgery $(p=0.002)$ were significantly higher, whereas a history of neoadjuvant chemotherapy $(\mathrm{p}=0.028)$ and neobladder $(\mathrm{p}<0.001)$ were significantly lower in the older group. Mean total operative time was significantly shorter $(\mathrm{p}=0.019)$ and mean estimated blood loss $(\mathrm{p}=0.013)$ was significantly lower in the older group. Postoperative Grade $\geq$ II complications were comparable at 0 - 30, 3190 and 91 days after surgery despite urinary tract associations between the two groups. Rates of positive surgical margins and 

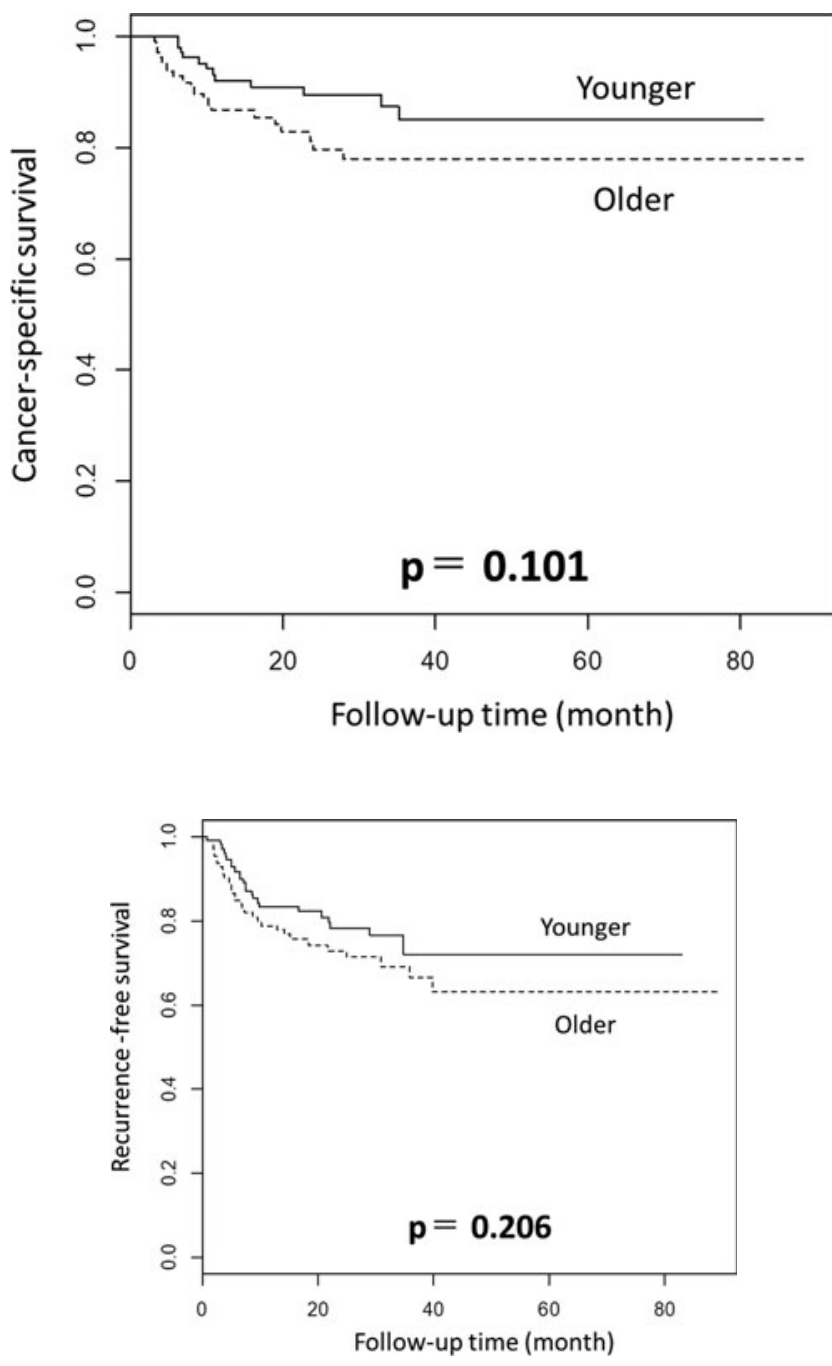

mean numbers of lymph nodes removed were comparable between the two groups. Although 5-year overall survival rates were significantly lower $(\mathrm{p}=0.03)$ for older patients, 5-year cancerspecific $(p=0.10)$ and recurrence-free survival rates were comparable $(\mathrm{p}=0.20)$ between the groups.

Conclusions: Using RARC potentially allows less-invasive procedures and cancer control for septuagenarian patients that is equivalent to that of younger patients.

\section{MP15-17 Laparoscopic urethrectomy following lapa- roscopic radical cystectomy}

T Yoshida

Fukuoka Wajiro Hospirtal, JAPAN

Introduction \& Objective: Urethrectomy is performed in radical cystectomy for invasive bladder cancer. As the operative field of the perineum in open urethrectomy is deep and narrow, it often increases blood loss to penetrate the urogenital diaphragm blindly using forceps for dissection of the urethra. In spite of the laparoscopic technique in radical cystectomy, intraperitoneal pressure decrease, and hemorrhage increase from urogenital diaphragm or stump of DVC (dorsal vein complex) in open urethrectomy. It

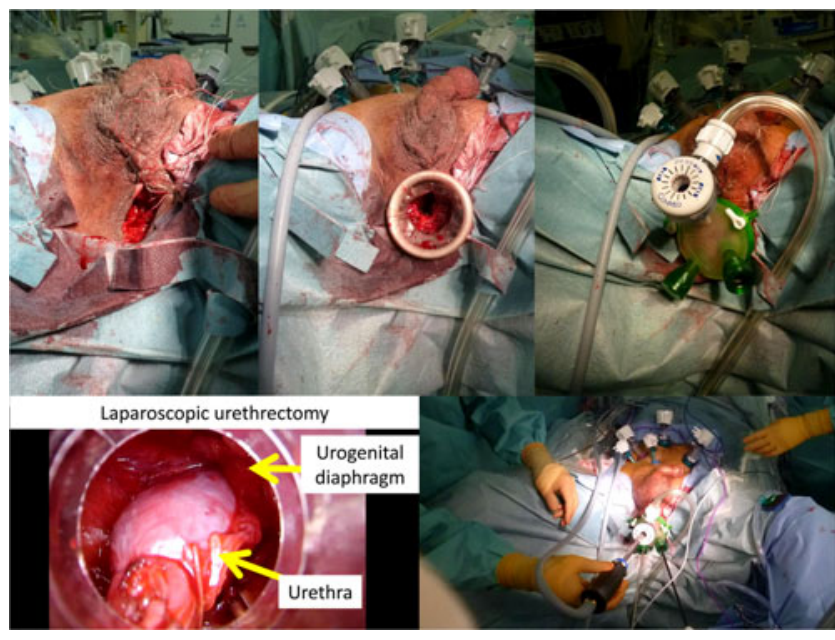

sometimes damages and rupture the wall of the urethra and may disseminate cancer cells. In this report, we introduce the procedure of laparoscopic urethrectomy following laparoscopic radical cystectomy. This is inspired by the technique of transanal total mesorectal excision (TaTME) for very low rectal cancer.

Methods: A male patient with muscle-invasive, non-metastatic and multiple bladder tumors (T2N0M0) received LRC (laparoscopic radical cystectomy). Around the end of cystectomy, an assistant started urethrectomy. After dissection of pendulum urethra to bulbar urethra by conventional open technique, we attached a trocar (GelPOINT ${ }^{\circledR}$ Mini, instruments for single port surgery) to the perineal surgical site and performed laparoscopic urethrectomy. The free urethra was pushed into the pelvic cavity. Gel POINT was removed, and the surgical site was closed with the conventional procedure. The devices we used are below; Laparoscopes: ENDOEYE ${ }^{\mathrm{TM}}$ HD II $10 \mathrm{~mm}$ (Rigid type) (OLYMPUS, Japan) Insufflation device: AirSeal $^{\text {TM }}$ (CONMED, US) Electrosurgical unit:VIO ${ }^{\mathrm{TM}}$ 200D (Erbe, Germany) Vessel sealing device: LigaSure ${ }^{\mathrm{TM}}$ (Medtronic, Ireland) Laparoscopic trocar: GelPOINT ${ }^{\mathrm{TM}} \mathrm{Mini}$ and $\mathrm{Kii}^{\mathrm{TM}}$ access system (Applied Medical, US) Laparoscopic forceps and scissors: CLICK LINE $^{\text {TM }}$ (KARL STORZ, Germany).

Results: Sure hemostasis was performed using the vessel sealing device in laparoscopic view under insufflation pressure. The insufflation time was about 20 minutes with a very small volume of blood loss. No damage to urethra occurred. Laparoscopic urethrectomy reduces the possibility of dissemination of cancer cells. This procedure is simple and required no experience of single port surgery.

Conclusions: Laparoscopic urethrectomy is a safe and easy technique and reduces blood loss, operative time and the possibility of dissemination of cancer cells.

MP15-18 Sex sparing vs standard Robot Assisted Radical Cystectomy with Intracorporeal Padua Ileal Neobladder in female: perioperative, oncologic and functional outcomes

G Tuderti, R Flammia, R Mastroianni, S Guaglianone, F Minisola, U Anceschi, L Misuraca, A Brassetti, M Ferriero, M Gallucci, G Simone

"Sapienza" University

Introduction \& Objective: Despite the widespread use of Robot assisted radical cystectomy (RARC), there is paucity of data 
concerning outcomes of Sex-sparing RARC with intracorporeal ileal neobladder (iN) performed in female patients. The aim of this study was to compare perioperative, oncologic and functional outcomes of two cohorts of female patients who underwent either Sex-sparing or Standard RARC-iN.

Results: Out of 47 patients included, 11 underwent sex-sparing RARC $(23.4 \%)$. The two cohorts were homogeneous for all baseline, clinical and pathologic features (all $\mathrm{p} \geq 0.14$ ), except for age, being sex-sparing patients significantly younger (47.1 vs $61.7 \mathrm{yrs}, \mathrm{p}<0.001)$. Perioperative complications and hospital stay were comparable between groups $(p=0.25$ and $p=0.67$, respectively). With regard to functional outcomes, no significant differences were observed for last estimated glomerular filtration rate $(p=0.64)$, neobladder stone formation rate $(p=0.93)$ and 1 -yr incidence of ureteroileal strictures $(p=0.56)$. Day-time continence recovery probability was significantly higher in Sex-sparing cohort (1-yr rate $90.9 \%$ vs $74 \%$, log-rank $\mathrm{p}=0.02$ ). A trend towards a significantly higher adoption of intermittent self-catheterization was observed in Sex-sparing cohort $(\mathrm{p}=0.07)$. At a median follow-up of 19.9 months, no patients of Sex-sparing cohort developed recurrences.

Conclusions: In select cohort of patients, Sex-sparing RARC-iN seems an oncologically sound procedure, associated with favorable perioperative and functional outcomes. Due to small sample size and intrinsic selection biases, oncologic and functional outcomes of sex-sparing RARC-iN should be assessed in larger cohorts, while outcomes comparison with conventional RARC-iN technique requires prospective randomized trials.

\section{MP15-19 Innovation and development of laparoendo-} scopic nephroureterectomy: one-center experience

\author{
Z Xiaofeng, Z Guoxi, Y Yuanhu, X Rihai, \\ W Gengqing, W Xiaoning
}

Introduction \& Objective: To describe our experience with six kinds of innovational laparoendoscopic nephroureterectomy, and to evaluate the clinical value.

Methods: Between March 2005 and April 2018, a total of 127 patients were underwent five kinds of laparoscopic nephroureterectomy in our center. Patients consisted of 87 males and 40 females, including 75 cases of renal pelvis tumors, 32 cases of ureteral cancer, 6 cases of multiple cancers, 13 cases of renal tuberculosis, and a case of pyonephrosis and non-functional kidney. All the lesions occurred unilaterally, $\underline{59}$ cases got the left side, 68 cases were on the right side. Cystoscopic electrocision assisted retroperitoneal laparoscopy nephroureterectomy was completed in 26 patients. Pneumovesicum Cystoscopic electrocision assisted laparoscopy nephroureterectomy was completed in 18 patients. 61 patients underwent suprapubic-assisted laparoendoscopic singlesite surgery (SA-LESS) nephroureterectomy. 10 patients underwent transvaginal natural orifice transluminal endoscopic surgery (NOTES) hybrid endoscope nephroureterectomy. 11 patients underwent suprapubic-assisted laparoendoscopic single-site surgery (SA-LESS) nephroureterectomy with transvaginal natural orifice specimen extraction (NOSE) (SA-LESS +TV-NOSE NU. A patient underwent transvaginal hybrid NOTES nephroureterectomy. Results: All the 127 cases procedures were successfully performed. The median operative time was 180 (range 95 to 265) minutes, and the median estimated blood loss was 210 (range 50 to 375) $\mathrm{ml}$. No patients required a blood transfusion. There was no major perioperative complication occurred. Each patient underwent irrigation of bladder with Pirarubicin after performed suprapubic-assisted laparoendoscopic single-site surgery. All the patients resumed ambulation on postoperative day 1. Pelvic drainage tube was removed on postoperative day 2-5. Each patient underwent irrigation of bladder with Pirarubicin and the urethral catheter was removed on postoperative day 7. The patients were discharged on postoperative day 7-9. Pathology indicated that 105 cases were upper tract urothelial carcinoma, 8 cases were squamous cell carcinoma, 13 cases were renal tuberculosis, and a case was pyonephrosis and non-functional kidney. During the follow up $3 \sim 13$ years, six reported case had shown distant metastasis on postoperative month 6 .

Conclusions: Innovative laparoscopic is safe and feasible for upper urinary tract resection with faster postoperative recovery, less pain, shorter hospitalization time, better cosmetic results, which is worthy of clinical application.

\section{MP15-20 Robotic-Assisted Radical Cystectomy: a multi-centric Brazilian experience and learning curve}

M Covas Moschovas, D Chade, MA Arap, Á Sarkis, W Nahas, L Tanure, G Ebaid, A Fazoli, G Guglielmetti, C Moreira,

M Cordeiro, P Carvalho, R Coelho

Global Robotic Institute

Introduction \& Objective: Radical cystectomy $(\mathrm{RC})$ is the gold standard treatment for muscular invasive and recurrent high-grade non-muscle invasive bladder cancer. The robot benefit on that surgery such as less bleeding and shorter hospital length is described in the literature. Our objective is to report a multicentric Brasilian experience and outcomes since the first case performing a robotic-assisted radical cystectomy (RARC) on bladder cancer. Methods: We performed a retrospective database review of 37 patients who underwent RARC for bladder cancer from 2013 to 2019 in four different centers in São Paulo, Brazil. The surgeons already had expertise in robotic prostatectomies before started the first RARC case. We analyzed the patient's gender, age, bladder biopsy, pathological tumor analysis, as well as peri- and postoperative outcomes. The statistical analyses were performed with the IBM SPSS version 25 software.

Results: Overall, $86 \%$ were male patients, and the mean age was 67 years old (range, $45-86$ years). Six patients (16\%) underwent BCG treatment, and 11 (29\%) underwent chemotherapy before the surgery. The time between the bladder biopsy and cystectomy was 118 days (range, 6-682 days). Thirty-five patients (95\%) were operated with the Si console while the other $5 \%$ had the procedure done by Xi console. The mean operative time was 432 minutes (range, 210-690) and the console time was 294 minutes (range,135-490 minutes). The mean blood loss was $379 \mathrm{ml}$ (range, 50-1300ml). Four patients $(10 \%)$ underwent an intraoperative blood transfusion. In $60 \%$ of the cases, we performed Bricker urinary diversion. We established the complications according to the Clavien-Dindo scale. Six patients (16\%) had Clavien 1 or 2 , three patients $(8 \%)$ had Clavien 3 , one $(2,5 \%)$ Clavien 4 and two (5\%) Clavien 5.The final pathology report described $95 \%$ of free surgical margin, the mean number of nodes resected was 27 (range 0-62), eight patients (21\%) was diagnosed with compromised nodes with metastasis, and seven (19\%) had concomitant prostate adenocarcinoma. Three patients (8\%) underwent reoperation while nine $(24 \%)$ underwent ICU admission. The mean hospital length of stay was 10,5 days (range, 2-59 days) with 15 readmissions (38\%) in less than 90 days. The survival in 30 and 90 days was $98 \%$ and $95 \%$ respectively.

Conclusions: Although RARC is challenging and the complications are common, the procedure is safe and feasible when performed by experienced robotic surgeons. We described our 
results since the first case in Brazil and conclude that, during the learning curve, it is possible to reproduce the technique and outcomes of referral centres with similar peri- and post-operative results.

\section{MP15-21 Robotic Revision of Uretero-Iliac Anasto- motic Stricture: Technique and Outcome}

R Madi

Augusta University Health at the Medical College of Georgia

Introduction \& Objective: Stricture at the uretero-iliac anastomosis is a well-known complication of radical cystectomy. We report our experience with robotic revision of uretero-iliac stricture in 5 patients who developed an anastomotic stricture that failed endoscopic intervention.

Methods: From January 2012 to May 20195 patients underwent robotic revision of uretero-iliac anastomotic stricture. All those patients had previous radical cystectomy for bladder cancer and subsequently developed stricture that failed endoscopic intervention. Four patients had ileal conduit and one patient had neobladder. All anastomosis was Bricker type except of one Wallace. Results: All five patients had successful repair of the stricture. The diseased ureteral segment was excised, and a fresh uretero-iliac anastomosis was made. There were no intra-or peri-operative complications in all 5 patients. Median console time was 130 mins and median length of stay was one day. One patient had recurrence of the stricture which was managed by endoscopic dilatation.

Conclusions: Robotic revision of uretero-iliac anastomotic stricture is safe, feasible, and should be considered as a permanent solution for strictures that fail endoscopic intervention.

\section{MP15-22 Laparoscopic adrenalectomy for pheochro- mocytoma in the hemodialysis patient.}

S Tatarano, H Hayami, M Yonemori, H Enokida, M Nakagawa, H Yoshino

Department of Urology, Graduate School of Medical and Dental Sciences, Kagoshima University

Introduction \& Objective: Pheochromocytoma in the hemodialysis patient is considered rare. In general, patients on hemodialysis are controlled to keep dry weight avoiding extra fluid on their body. Therefore, it is crucial to make perioperative hemodynamic management safer. We herein report the outcome of laparoscopic adrenalectomy for pheochromocytoma in the hemodialysis patient. Methods: Six cases on hemodialysis underwent laparoscopic adrenalectomy for pheochromocytoma at Kagoshima University from 2007 to 2018. After the patient pretreated with an alpha adrenergic receptor blocker and volume expansion, the patient underwent surgery. All patients' dry weight increased slowly from base weight to plus $0.5-1.0 \mathrm{~kg}$ for a week increasing the intravascular volume. We compared the clinical parameter and outcome between the hemodialysis group $(n=6)$ and non-hemodialysis group $(n=37)$.

Results: All 6 cases of laparoscopic adrenalectomy were performed safely and without intraoperative complications and needed neither blood transfusion nor conversion to open surgery. In the hemodialysis group, the median age was 60 years (44-67). The patient had been receiving hemodialysis for 19 years (5-30). BMI was $21.3 \mathrm{~kg} / \mathrm{m}^{2}(19.3-28.2)$ and tumor diameter was $3.5 \mathrm{~cm}$ (2.2-6.0). Pneumoperitoneum time was 217 minutes (163-321) and blood loss was $40 \mathrm{ml}(20-320)$. Intraoperative hypertension (systolic blood pressure $>180 \mathrm{mmHg}$ ) occurred in 50\% (3/6) of cases. The median length of hospital stay was post-operatively 8 days. The clinical parameter and outcome demonstrated that there was no significant difference between the hemodialysis group and the non-hemodialysis group.

Conclusions: Laparoscopic adrenalectomy for pheochromocytoma in the hemodialysis patient was feasible as well as the nonhemodialysis patient. The increasing intravascular volume before pheochromocytoma surgery in a patient on hemodialysis might make the perioperative management safer, although further study is required to determine the adequate level of increment in the preoperative dry weight.

\section{MP15-23 Pathological Staging of Bladder Cancer, What Lies Beneath? Results from the International Robotic Cystectomy Consortium}

NA Aldhaam, AS Elsayed, C Wijburg, L Richstone, A Wagner, J Palou, M Stoeckle, M Menon, F Gaboardi, T Maatman, A Hemal, B Yuh, Z Jing, M Roupret, P May, J Kaouk, P Wiklund, J Peabody, KA Guru

\section{Roswell Park Comprehensive Cancer Center}

Introduction \& Objective: Pathological reclassification is not uncommon after robot-assisted radical cystectomy (RARC). We sought to correlate the final pathology with preoperative diagnostic pathology with a subset analysis of non-muscle invasive bladder cancer (NMIBC).
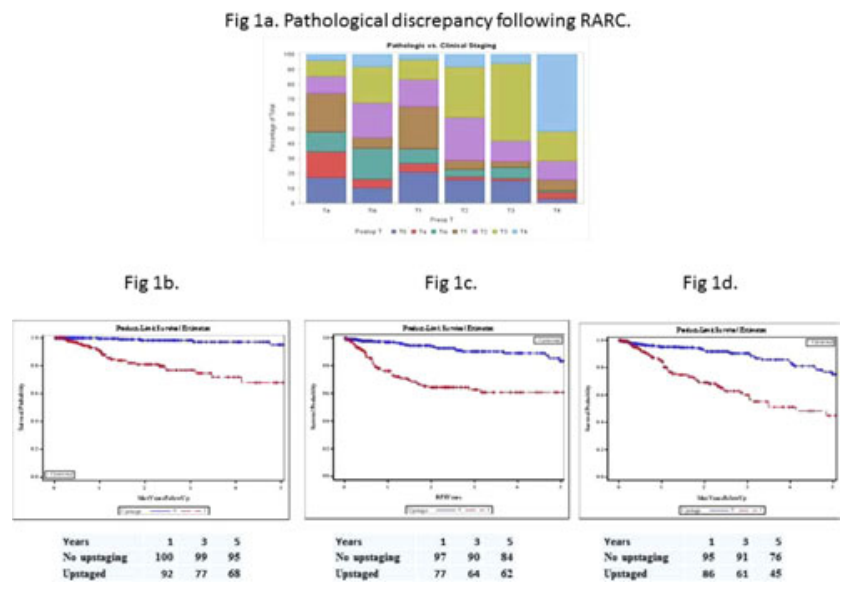

Table 1. Clinical data of patients with NMIBC.
\begin{tabular}{|l|l|l|l|r|}
\hline Variable & Not Upstaged & Upstaged & \multicolumn{1}{|l|}{ All } & P Value \\
\hline Number of Patients, n (\%) & $395(59)$ & $275(41)$ & 670 & $<0.01$ \\
\hline Age, mean (SD) & $67 \pm 10$ & $69 \pm 11$ & $68 \pm 10$ & 0.03 \\
\hline Body mass index, mean (SD) & $28 \pm 5$ & $27 \pm 5$ & $28 \pm 5$ & $<0.01$ \\
\hline $\begin{array}{l}\text { Preoperative T stage, } n(\%): \\
\text { Ta } \\
\text { Tis }\end{array}$ & $101(26)$ & $36(13)$ & $137(20)$ & $<0.01$ \\
T1 & $91(23)$ & $123(45)$ & $214(32)$ & \\
\hline Intrcorporeal urinary diversion, n (\%) & $163(51)$ & $116(42)$ & $319(48)$ & \\
\hline Neobladder diversion, n (\%) & $106(27)$ & $146(55)$ & $312(49)$ & 0.01 \\
\hline $\begin{array}{l}\text { Charlson comorbidity Index, mean } \\
\text { (SD) }\end{array}$ & $2.5 \pm(1)$ & $59(22)$ & $165(25)$ & 0.11 \\
\hline Adjuvant treatment, n (\%) & $12(4)$ & $45(21)$ & $57(11)$ & $<0.01$ \\
\hline Hospital stay, mean (SD) & $11 \pm(10)$ & $13 \pm(10)$ & $12 \pm(10)$ & $<0.0001$ \\
\hline $\begin{array}{l}\text { Pathological lymph node positive, n } \\
\text { (\%) }\end{array}$ & $4(1)$ & $10(4)$ & $14(2)$ & 0.02 \\
\hline Pathological T3 or T4, mean (\%) & $0(0)$ & $146(53)$ & $146(22)$ & $<0.01$ \\
\hline Positive surgical margin & $11(3)$ & $19(7)$ & $30(4)$ & 0.01 \\
\hline
\end{tabular}


Methods: We reviewed our prospectively maintained database from the International Robotic Cystectomy Consortium between 2006 and 2017. Upstaging was defined as the identification $\geq p$ T2 at final pathology from initial pathology $<$ pT2. Multivariate stepwise regression models were fitted to elicit predictors of upstaging. Kaplan Meier (KM) curves were used to depict disease-specific survival (DSS), recurrence-free survival (RFS), and overall survival (OS). Multivariate Cox Regression Models were used to identify predictors.

Results: 1830 patients who underwent RARC were identified. Initial pathology was compared to the final pathology (Fig1a). Out of 1830 patients, 670 had NMIBC and underwent RARC, upstaging occurred in 41\%. Patients who upstaged were older (69 vs 67 years), had longer hospital stay (13 vs 11 days), were more likely to be readmitted $(\mathrm{p}<0.03)$, and receive adjuvant treatment after surgery $(\mathrm{p}<0.01)$. Additionally, post-operative pathology for pa- tients who upstaged showed statistically significant positive lymph nodes $(\mathrm{p}=0.035)$ and higher soft tissue margins $(\mathrm{p}=0.01)$. Predictors of upstaging were BMI $(\mathrm{p}<0.01)$, preoperative Tis $(\mathrm{p}<0.01)$, and $\mathrm{T} 1(\mathrm{p}<0.01)$. KM curves showed a statistically worse DSS (68\% vs 95\%) (Fig 1b), RFS (62\% vs 84\%), (Fig 1c), and OS (45\% vs 76\%) (Fig 1d), for patients who upstaged at 5 years. Multivariate cox regression model proved that upstaging was an independent risk factor for RFS ( $p=0.01)$, but not DSS and OS. Predictors of DSS were $\mathrm{pT} \geq 3(\mathrm{p}<0.01)$, lymph node positive disease $(p=0.02)$, and soft tissue margins $(p=0.01)$. Predictors of RFS were $\mathrm{pT} \geq 3(\mathrm{p}<0.01)$, and soft tissue margins $(\mathrm{p}=0.02)$. $\mathrm{pT}$ $\geq 3(\mathrm{p}<0.01)$, was the only predictor for OS.

Conclusions: The pathological discrepancy is common after robot-assisted radical cystectomy. Patients with NMIBC need to be carefully counseled regarding their disease aggressiveness and future treatment options.

\section{MODERATED POSTER SESSION 16: ENDOSCOPIC}

\author{
MP16-01 Super-Mini Laser Cystolitholapaxy: A use- \\ ful approach in those with challenging retrograde \\ access
}

H Abboudi, G Celentano, K Adasonla, F Schoofs, S Choong

University College London Hospitals

Introduction \& Objective: To describe our technique using a Super-Mini PCNL (SMP) sheath to access the bladder through existing drainage tracts in patients with impossible or difficult urethral access.

Methods: The SMP access sheath traditionally used for percutaneous nephrolithotomy procedures was used to clear bladder stone fragments. The SMP 14Fr or 18Fr access sheaths were passed over an Amplatz stiff guidewire through existing access into the bladder - either via a suprapubic catheter or into a mitrofanoff channel. In the case of mitrofanoff access, semirigid ureteroscope $(6 / 7.5 \mathrm{Fr})$ and guidewire placement was used to determine entry into bladder. A semi rigid ureteroscope and 550-micron Holmium laser fibre was used to facilitate fragmentation of the bladder calculi in all cases. Fragments were then removed using a combination of active and passive suction.

Results: Between November 2018 to March 2019 four patients underwent super-mini laser cystolitholapaxy: three via existing suprapubic catheter tracts, 1 via a mitrofannof channel. Each patient had multiple bladder stones with combined stone burdens ranging from $22 \mathrm{~mm}-51 \mathrm{~mm}$. There were no reported complications, with all patients visually stone free at the end of the procedure. The Mitrofanoff patient had one small residual fragment at US follow up four weeks post operatively. Utilizing existing access obviated the need to re-puncture the bladder or create any new access tracts thereby minimizing morbidity.

Conclusions: Cystolitholapaxy using a supermini sheath for bladder access, whether suprapubically or through a mitrofanoff channel, is a safe and effective option for managing bladder stones in those with challenging urethral access.
MP16-02 Laparoscopic nephroureterectomy is safe and established procedure, but it needs to be paid attention to intravesical recurrence.

S Shimura, K Matsumoto, M Ikeda, D Ishii, K Tabata, Y Taoka, T Utsunomiya, D Matsuda, N Okuno, A Irie, M Iwamura

Kitasato University

Introduction \& Objective: Localized upper urinary tract cancer is treated with surgical intervention, including laparoscopic and open procedures. Recently, laparoscopic radical nephroureterectomy has been used as a minimally invasive modality instead of the open procedure. Recent multi-institutional studies and a meta-analysis have shown that perioperative and oncological outcomes are comparable between open and laparoscopic surgery in patient with upper urinary tract cancer. The aim of this study was to evaluate the differences of clinicopathological findings between two procedures in patients with upper urinary tract cancer.

Methods: From 1990 to 2015, 441 non-metastatic upper urinary tract cancer patients underwent nephroureterectomy at 6 Kitasato university-affiliated hospitals. Two hundred forty-seven patients were performed open procedure (Open) and 194 patients were treated with laparoscopic procedure (Lap). Laparoscopic nephroureterectomy was performed via a retroperitoneal approach following an open partial cystectomy. This study was approved by the institutional review board at each institution (B15-25). Median follow-up period was 35.7 months.

Results: Lap group showed that elderly patients, prolonged operative time, less blood loss and less lymphovascular invasion (LVI) compared to Open group. Irrespective of patients with organ-confined disease $(<\mathrm{pT} 3)$ or with extra-organ disease, there were no significant differences between the groups in terms of progression-free, cancer-specific and overall survival. In multivariate analyses, pT3, positive LVI, positive surgical margin and high-grade lesion were independently predictive factors for cancerspecific survival, but surgical procedure was not. Intravesical 
recurrences were observed in $96(39 \%)$ and $90(46 \%)$ patients in Open and Lap groups with median recurrent time of 9.7 and 7.4 months, respectively. Patients with non-muscle invasive cancer $(<\mathrm{pT} 2)$ in Lap group were higher intravesical recurrence than those in Open group $(\mathrm{p}=0.02)$. However, there was no significant difference in patients with muscle invasive cancer $(>\mathrm{pT} 1)$ between the groups.

Conclusions: Nephroureterectomy can be safely performed and procedure was not independent prognostic factor. However, we need to pay attention to intravesical recurrences within 1 year postoperatively in patients with non-muscle invasive cancer who underwent laparoscopic procedure.

\section{MP16-03 The Endockscope: A Disruptive Endoscopic Technology}

S Lu, R Yoon, C Cottone, FA Jefferson, J Sung, Z Okhunov, S Tapiero, RM Patel, J Landman, RV Clayman

University of California, Irvine

Introduction \& Objective: The Endockscope (ES) combines a smartphone, lens system, and a rechargeable LED light source to provide a low-cost alternative $(\$ 45)$ to the standard camera and high-powered light source $(\$ 45,000)$ used in endoscopic procedures. Originally designed for flexible fiberoptic endoscopy, we evaluated the ES performance compared to the standard highpowered light source and camera viewing system using a broad range of rigid/semi-rigid endoscopes in fresh-tissue cadaver models.

Methods: Videos of rigid nephroscopy, semi-rigid ureteroscopy, rigid cystoscopy, and laparoscopy in two adult male cadavers were recorded using the ES combined with either the Apple iPhone X (iPX) or Samsung Galaxy S9+ (GS9+), the top performers from our earlier favorable fiberoptic endoscopic study, and then with the standard high-definition camera system (Karl Storz) and high-powered light source. A total of 16 urologists

\begin{tabular}{|c|c|c|c|c|c|c|}
\hline \multicolumn{7}{|c|}{ Rigid Cystoscope (n=15) } \\
\hline & Resolution & Brightness & Color & Sharpness & $\begin{array}{l}\text { Overall } \\
\text { Image } \\
\text { Quality }\end{array}$ & $\begin{array}{c}\text { Acceptability } \\
\text { for Diagnosis } \\
\text { (\%) }\end{array}$ \\
\hline iPhone X & $\begin{array}{c}3.13 \pm 0.64 \\
(p>0.05)\end{array}$ & $\begin{array}{c}3.47 \pm 0.92 \\
(p>0.05)\end{array}$ & $\begin{array}{c}3.47 \pm 0.92 \\
(p>0.05)\end{array}$ & $\begin{array}{c}3.00 \pm 0.76 \\
(p>0.05)\end{array}$ & $\begin{array}{c}3.27 \pm 0.88 \\
(p>0.05)\end{array}$ & 80 \\
\hline $\begin{array}{c}\text { Galaxy } \\
\text { S9+ }\end{array}$ & $\begin{array}{l}3.60 \pm 0.83 \\
(p>0.05)\end{array}$ & $\begin{array}{l}3.53 \pm 0.74 \\
(p>0.05)\end{array}$ & $\begin{array}{l}3.40 \pm 0.83 \\
(p>0.05)\end{array}$ & $\begin{array}{c}3.33 \pm 0.82 \\
(p>0.05)\end{array}$ & $\begin{array}{l}3.40 \pm 0.83 \\
(p>0.05)\end{array}$ & 66.7 \\
\hline Standard & $3.27 \pm 1.03$ & $3.73 \pm 0.70$ & $3.29 \pm 0.91$ & $3.33 \pm 0.90$ & $3.33 \pm 0.72$ & 80 \\
\hline \multicolumn{7}{|c|}{ Rigid Nephroscope (n=13) } \\
\hline & Resolution & Brightness & Color & Sharpness & $\begin{array}{l}\text { Overall } \\
\text { Image } \\
\text { Quality }\end{array}$ & $\begin{array}{c}\text { Acceptability } \\
\text { for Diagnosis } \\
\text { (\%) }\end{array}$ \\
\hline iPhone X & $\begin{array}{c}3.85 \pm 0.69 \\
(p>0.05)\end{array}$ & $\begin{array}{c}3.85 \pm 0.90 \\
(\mathrm{p}>0.05)\end{array}$ & $\begin{array}{c}3.62 \pm 0.65 \\
(p>0.05)\end{array}$ & $\begin{array}{c}3.46 \pm 0.78 \\
(p>0.05)\end{array}$ & $\begin{array}{l}3.50 \pm 0.65 \\
(p<0.03)\end{array}$ & 92.3 \\
\hline $\begin{array}{c}\text { Galaxy } \\
\text { S9+ }\end{array}$ & $\begin{array}{c}3.92 \pm 0.64 \\
(p>0.05)\end{array}$ & $\begin{array}{c}3.69 \pm 0.75 \\
(p>0.05)\end{array}$ & $\begin{array}{c}3.62 \pm 0.77 \\
(p>0.05)\end{array}$ & $\begin{array}{c}3.85 \pm 0.69 \\
(p>0.05)\end{array}$ & $\begin{array}{c}3.90 \pm 0.64 \\
(p>0.05)\end{array}$ & 84.6 \\
\hline Standard & $4.00 \pm 0.58$ & $4.08 \pm 0.64$ & $4.08 \pm 0.76$ & $3.85 \pm 0.69$ & $3.98 \pm 0.58$ & 100 \\
\hline \multicolumn{7}{|c|}{ Semi-Rigid Ureteroscope $(n=13)$} \\
\hline & Resolution & Brightness & Color & Sharpness & $\begin{array}{l}\text { Overall } \\
\text { Image } \\
\text { Quality }\end{array}$ & $\begin{array}{c}\text { Acceptability } \\
\text { for Diagnosis } \\
\text { (\%) }\end{array}$ \\
\hline iPhone X & $\begin{array}{c}4.15 \pm 0.69 \\
(p>0.05)\end{array}$ & $\begin{array}{c}4.00 \pm 0.91 \\
(p>0.05)\end{array}$ & $\begin{array}{c}4.23 \pm 0.83 \\
(p>0.05)\end{array}$ & $\begin{array}{c}4.31 \pm 0.75 \\
(p>0.05)\end{array}$ & $\begin{array}{c}4.10 \pm 0.76 \\
(p>0.05)\end{array}$ & 84.6 \\
\hline $\begin{array}{c}\text { Galaxy } \\
\text { S9+ }\end{array}$ & $\begin{array}{l}4.77 \pm 0.44 \\
(\mathbf{p}<0.02)\end{array}$ & $\begin{array}{c}4.23 \pm 0.73 \\
(p>0.05)\end{array}$ & $\begin{array}{c}4.69 \pm 0.63 \\
(p>0.05)\end{array}$ & $\begin{array}{l}4.69 \pm 0.63 \\
(\mathbf{p}<0.01)\end{array}$ & $\begin{array}{c}4.52 \pm 0.77 \\
(p>0.05)\end{array}$ & 100 \\
\hline Standard & $3.96 \pm 1.09$ & $4.23 \pm 0.83$ & $4.19 \pm 1.03$ & $3.77 \pm 1.01$ & $4.06 \pm 1.04$ & 84.6 \\
\hline \multicolumn{7}{|c|}{$30^{\circ}$ Laparoscope $(n=13)$} \\
\hline & Resolution & Brightness & Color & Sharpness & $\begin{array}{l}\text { Overall } \\
\text { Image } \\
\text { Quality }\end{array}$ & $\begin{array}{c}\text { Acceptability } \\
\text { for Diagnosis } \\
\text { (\%) }\end{array}$ \\
\hline iPhone X & $\begin{array}{l}2.46 \pm 1.05 \\
(\mathrm{p}<0.01)\end{array}$ & $\begin{array}{l}2.83 \pm 0.72 \\
(\mathrm{p}<0.01)\end{array}$ & $\begin{array}{l}2.77 \pm 0.83 \\
(\mathbf{p}<0.01)\end{array}$ & $\begin{array}{l}2.17 \pm 0.58 \\
(\mathrm{p}<0.01)\end{array}$ & $\begin{array}{l}2.46 \pm 0.88 \\
(p<0.01)\end{array}$ & 23.1 \\
\hline $\begin{array}{c}\text { Galaxy } \\
\text { S9+ }\end{array}$ & $\begin{array}{l}3.92 \pm 0.90 \\
(p>0.05)\end{array}$ & $\begin{array}{l}3.83 \pm 0.83 \\
(p<0.05)\end{array}$ & $\begin{array}{l}3.85 \pm 0.90 \\
(p>0.05)\end{array}$ & $\begin{array}{l}4.00 \pm 1.00 \\
(p>0.05)\end{array}$ & $\begin{array}{l}3.85 \pm 0.90 \\
(\mathbf{p}<0.05)\end{array}$ & 92.3 \\
\hline Standard & $4.46 \pm 0.78$ & $4.38 \pm 0.65$ & $4.15 \pm 0.99$ & $4.42 \pm 0.67$ & $4.38 \pm 0.65$ & 100 \\
\hline
\end{tabular}

(12 attendings and 4 residents) blinded to the recording specifics, assessed the image resolution, brightness, color, sharpness, and overall image quality on a $1-5$ Likert scale $(1=$ poor, $5=$ excellent); they also judged whether each video was acceptable for diagnostic purposes (yes or no).

Results: Image parameters: For rigid cystoscopy, all image parameters with the ES plus the iPX or the GS9+ were equivalent to the standard system $(n=15)$. For rigid nephroscopy, the ES plus the GS9+ performed similarly to the standard system, with similar results for the ES plus the iPX except in overall quality $(n=13)$. The ES plus the GS9+ performed superiorly in image resolution and sharpness in semi-rigid ureteroscopy, while the ES plus iPX performed equally $(n=13)$. For laparoscopy, the ES plus the GS9+ performed nearly as well as the standard system in all areas except brightness and overall quality $(\mathrm{p}<0.05)(\mathrm{n}=13)$. In contrast, the ES plus iPX for laparoscopy was uniformly inferior for all image parameters compared to the standard system. Diagnostic assessment: For diagnostic purposes, the ES plus GS9+ was equally acceptable to the standard system for all four endoscopes; the ES plus iPX fell short for laparoscopic diagnostic assessment (Table 1).

Conclusions: The Endockscope system plus the Samsung Galaxy S9+ provides similarly sufficient imaging for diagnostic assessment to the standard system for rigid endoscopy of the kidney, ureter, bladder and abdomen.

\section{MP16-04 Impact of Photodynamic Diagnosis and Narrow Band Imaging on Detection Rate of Urothelial carcinoma in Transurethral Resection of Non-Muscle Invasive Bladder Cancer}

N Makita, S Murata, I Suzuki, M Kubota, Y Tohi, Y Sugino, $\mathrm{K}$ Inoue, M Kawakita

Kobe City Medical Center General Hospital

Introduction \& Objective: The detection rate of urothelial cancer at transurethral resection of non-muscle invasive bladder tumor (TURBT) can be increased by combining photodynamic diagnosis (PDD) with 5-Aminolevulinic acid or narrow band imaging (NBI) with white light (WL), but it is not known which of these two modalities is better. The aim of this study is to compare the detection rates of urothelial carcinoma among WL, PDD and NBI at TURBT.

Methods: This study prospectively enrolled 32 patients who underwent TURBT for bladder cancer with at least one or more papillary lesions from June 2018 to March 2019. Patients with history of instillation of BCG into bladder within 3 months or patients who received second TURBT were excluded. At TURBT, all the patients were inspected under WL, PDD, and $\mathrm{NBI}$, then all lesions were recorded. TUR or cold biopsy of all the lesions was carried out. We compared the detection rate, the sensitivity and the specificity of urothelial carcinoma by each modality, referring to the pathological diagnosis.

Results: From 32 patients with bladder cancer, 111 lesions were resected at TURBT. The median age was 72 years, the ratio of male was $84 \%$, and the ratio of patients with newly diagnosed bladder cancer was $62 \%$. For urine cytology, $19 \%$ was positive, $28 \%$ was suspect of positive, $53 \%$ was negative. Of all 111 lesions, the sensitivity and specificity of WL/PDD/NBI/ WL+PDD/WL+NBI were 81.6/81.8/80.5/97.4/89.6\%, and 58.8/ $50.0 / 44.1 / 32.4 / 29.4 \%$ respectively. Of 46 flat lesions, the sensitivity and specificity of WL/PDD/NBI/WL+PDD/WL+NBI was $50.0 / 73.1 / 61.5 / 88.5 / 69.2 \%$, and $85.0 / 50.0 / 55.0 / 40.0 / 40.0 \%$ 
respectively. The PDD and NBI group were comparable for sensitivity of urothelial carcinoma in all lesions $(\mathrm{p}=0.968)$, or flat lesions $(\mathrm{p}=0.375)$. And the $\mathrm{WL}+\mathrm{PDD}$ and $\mathrm{WL}+\mathrm{NBI}$ group were comparable for sensitivity of urothelial carcinoma in all lesions $(p=0.102)$, or flat lesions $(p=0.174)$. On the other hand, sensitivity of urothelial carcinoma in WL+PDD group was superior to WL group in all lesions $(\mathrm{p}=0.003)$, or flat lesions $(\mathrm{p}=0.006)$. In addition, the sensitivity of pathologically diagnosed carcinoma in situ(CIS) in WL+PDD group was $100 \%$, and superior to WL group $(\mathrm{p}=0.024)$.

Conclusions: There is no statistical difference between sensitivities of urothelial carcinoma in PDD and NBI group. With combination of PDD and WL at TURBT, the sensitivity of urothelial cancer could be significantly increased. In addition, WL+PDD can detect more CIS lesions which are often overlooked only with WL.

MP16-05 Narrow Band Imaging reduces persistence of cancer in patients with pT1 high grade bladder cancer

R Giulianelli, B Gentile, G Mirabile, L Albanesi, L Mavilla, G Rizzo, P Tariciotti, A Lopes Mendes, P Aloisi, M Vermiglio, R Lombardo

Introduction \& Objective: To evaluate persistence rate on repeated transurethral resection of the bladder (re-TURB) 6 weeks after the first TURB in patients with pT1HG disease undergoing resection of the margins and bed on Narrow Band Imaging.

Methods: A consecutive series of patients undergoing TURB and a diagnosis of pT1 high grade disease were prospectively enrolled. On initial TURB patients underwent classic white light resection of the tumor followed by narrow band image (NBI) resection of margins and bed. After 6 weeks from the initial TURB, patients underwent a re-TURB under white light. Persistence rates on re-TURB were recorded.

Results: Overall 797 patients underwent TURB, out of them 126 patients with pT1 high grade disease were included in the study. The total number of lesions was 226 meaning 1.79 lesions per patient. On re-TURB 24/126 (19\%) of the patients presented residual disease with a total of $28 / 226(12 \%)$ lesions identified. All these patients presented a pTa residual disease. Out of them $8 / 21(38 \%)$ presented bladder cancer on the resection bed and 13/ $21(62 \%)$ presented bladder cancer on margins.

Conclusions: Narrow Band Imaging trans-urethral resection of the bladder is an oncological effective procedure in the treatment of pT1HG disease. The procedure has a $19 \%$ of persistence rate which is inferior when compared to the available evidence on white light TURB. Further multicenter studies are needed in order to validate our results.

MP16-06 Role of endoscopic management in synthetic sling/mesh erosion following previous incontinence surgery: A systematic review

S Sulaiman, BK Somani, A Pietropaolo, T Hughes

University Hospital Southampton

Introduction \& Objective: Over the last two decades the treatment of stress urinary incontinence (SUI) has shifted to a mid-urethral sling (MUS) or a mesh-based bladder neck procedure. Mesh erosion is now recognized as a major long-term complication of MUS surgery. Although the true extent of mesh erosion seems to be under-reported, due to an alarming rise of reported cases in 2018, there was a temporary ban on all MUS procedures in UK. The aim of our systematic review was to ascertain the outcomes of endoscopic management in synthetic sling/mesh erosion following previous incontinence surgery.

Methods: A systematic literature search was conducted according to the Cochrane review and Preferred Reporting Items for Systematic Reviews and Meta-Analyses (PRISMA) protocol. The population examined was adults who underwent synthetic sling procedures for incontinence. There was no comparative group, and the outcomes were to ascertain the role of endoscopic management following sling erosion.

Results: Our search resulted in 931 articles and 20 articles (198 patients) met our inclusion criteria and were included in our review. The mean age across studies varied from 48-68 years. 149 (75\%) had tension-free vaginal tapes (TVT) or tension-free obturator tapes (TOT) as their initial procedure. The endoscopic procedures patients received included laser incision, TUR with electrode loop or laparoscopic scissors/forceps. $108(55 \%)$ patients were treated exclusively with laser incision. The initial and final success rate with this technique was $67 \%$ (72 patients) and $92 \%$ (99 patients) respectively. Post-operative complications were seen in 26 (24\%) patients. These included SUI in 23 $(21 \%)$, urinary tract infection in $2(1.9 \%)$ and voiding difficulty in 1 patient. $90(45 \%)$ patients were managed using TUR with electrode loop or laparoscopic scissors/forceps. The initial and final success rate with this technique was $80 \%$ ( 72 patients) and $98 \%$ (88 patients) respectively. Post-operative complications were seen in 25 (28\%) patients. These included SUI in 19 $(21 \%)$, vesicovaginal fistula in $3(3 \%)$, intra-peritoneal bladder perforation needing further procedure in $2(2 \%)$ and retroperitoneal bladder perforation in 1 patient.

Conclusions: Endoscopic management of foreign body erosion is an effective minimally invasive technique with good outcomes and minimal morbidity. Management with the use of holmium laser is gaining momentum and should be attempted before open surgical removal. A close cooperation between endourologists and urogynecologists with appropriate patient counselling is necessary as some patients may need repeat procedures to achieve a successful outcome.

MP16-07 Factors impact on intravesical recurrence of endoscopic management for high-grade upper urinary tract urothelial cell carcinoma followed by radical nephroureterectomy with bladder cuff excision.

\author{
A Sawangchareon, E Chotikawanich \\ Division of Urology, Department of Surgery, Faculty \\ of Medicine Siriraj Hospital, Mahidol University
}

Introduction: Upper urinary tract urothelial carcinoma (UTUC) is an infrequent disease, radical nephrourerectomy with bladder cuff excision (RNU+BCE) is a standard of care. Endoscopic management has been established to have definite diagnosis in conjunction with an imaging as well as to have minimally invasive surgery for some circumstances. Intravesical recurrence has been an issue while using this procedure before radical surgery especially for high-grade UTUC, therefore, we study the factors impact on bladder recurrence after endoscopic management of high-grade UTUC followed by radical surgery.

Objective: To study the factors impact on intravesical recurrence after endoscopic management for high-grade UTUC initially and then followed by radical nephroureterectomy with bladder cuff excision. 
Methods: A retrospective reviewed of patients diagnosed of UTUC at Siriraj Hospital from 2002 to 2019. Inclusion criteria was localized high-grade UTUC. The study included patients underwent ureteroscopy (URS) with tumor biopsy or laser ablation to know tumor grade, high-grade tumor was considered RNU+BCE subsequently. Parameters such as tumor location (intrarenal, upper ureter, lower ureter and multiple locations tumors), tumor size $(<1 \mathrm{~cm}, 1-2 \mathrm{~cm}$ and $>2 \mathrm{~cm})$, pathological staging (pT1 or lower, pT2, pT3 or higher), type of endoscopic management (URS with biopsy, URS with laser ablation or URS with concurrent RNU+BCE) and time interval between URS and RNU+BCE ( $<2$ weeks, $2-4$ weeks and $>4$ weeks) were evaluated to have factors impact on bladder recurrence.

Results: There were 42 patients met criteria of high-grade UTUC underwent URS and then RNU+BCE. Intravesical recurrence was found $36.8 \%$ for intrarenal tumor, $25.0 \%$ for proximal ureteral tumor, $53.8 \%$ for distal ureteral tumor, and $100 \%$ for multiple locations tumors. Multiplicity of tumor location was a significant factor for intravesical recurrence $(p=0.017)$. URS with biopsy had higher rate of intravesical recurrence than URS with laser ablation significantly $(66.7 \%$ vs $20.0 \%, p=0.028)$. Longer time interval between URS and $\mathrm{RNU}+\mathrm{BCE}$ tend to have higher incidence of intravesical recurrence but not statistically significant. Incidence of intravesical recurrence was not different among various tumor size or staging. This study had no distant metastasis developed during the follow up period, and all bladder recurrence was treated by TURBT successfully.

Conclusions: Multiple tumor locations and URS with biopsy beforehand were found to be the factors impact to intravesical recurrence after RNU+BCE for high-grade UTUC.

\section{MP16-08 Endoscopic Bladder Suture after ureteral and bladder cuff excision during laparoscopic Ne- phroureterectomy}

CA Oliveira, AA Barros, R Reis, J Correia-Pinto, E Dias, E Lima

Life and Health Sciences Research Institute, School of Medicine, University of Minho, Braga, Portugal. ICVS/3B's, PT Government Associate Laboratory, Braga / Guimarães, Portugal. Department of Urology, Hospital de Braga, Braga, Portugal.

Introduction \& Objective: Upper urinary tract urothelial carcinomas are usually managed by radical nephroureterectomy (RNU), often followed by intravesical chemotherapy to minimize recurrence. Open surgery is the gold standard procedure for RNU, but it associates with high morbidity, and it has been increasingly replaced by minimally invasive strategies, such as laparoscopy and endoscopy. Although effective, endoscopic ureteral excision leaves the bladder unsutured, precluding the immediate administration of intravesical chemotherapy. The objective of this work is to describe a new method to close the bladder wall after ureteral excision, using barbed sutures via the endoscopic access in patients being submitted to Radical Nephroureterectomy.

Methods: Seven patients with upper urinary tract urothelial carcinoma were submitted to endoscopic bladder wall closure in addition to standard laparoscopic nephroureterectomy. Ureteral disinsertion was performed using a bipolar resectoscope. The bladder wall defect was closed endoscopically using a 2/0 barbed suture. (figures 1 e 2) The patients underwent standard laparo-

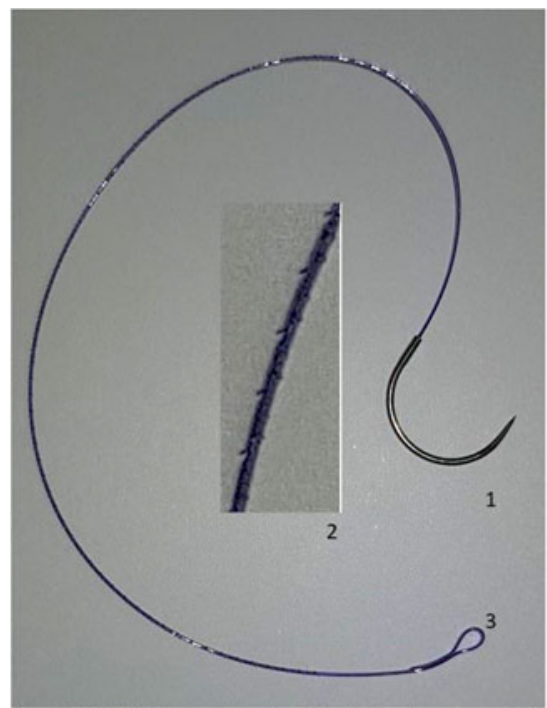

Figure 1-V-locTM90 suture: 1-needle, 2-Ampliation to show the anchoring projections on the surface of the suture, 3. blind loop at the distal part of the suture.
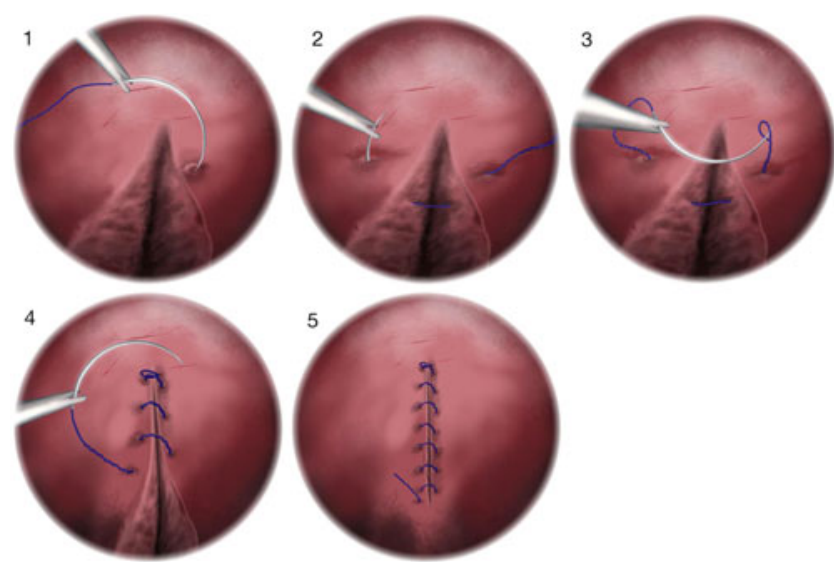

Figure 2 - Illustration of the steps (1-5) to close bladder incision using barbed sutures

scopic nephroureterectomy and were submitted to intravesical instillation of $40 \mathrm{mg}$ mitomycin $\mathrm{C}$ in the $12 \mathrm{~h}$ pos-surgery.

Results: The procedure was performed in a simple fast and fluid manner. The total time of the endoscopic procedure was under 15 minutes. The bladder defect was completely closed. There were no complications to register. Patients underwent mitomycin bladder instillation without any adverse events to register.

Conclusions: We describe a simple, fast and safe technique of endoscopic bladder wall closure. With this method it is possible to maintain a minimal invasive approach and simultaneously administer early intravesical chemotherapy

MP16-09 Short term outcome of mini-percutaneous Holmium laser endopyelotomy for treating secondary ureteropelvic junction obstruction

O Elgebaly, H Rashad, A Balah, A Zahran

Urology Department, Alexandria University

Introduction \& Objective: To evaluate the efficacy and safety of mini-Percutaneous laser endopyelotomy in secondary ureteropelvic junction obstructions (UPJO). 
Methods: Twenty-five cases diagnosed with secondary UPJO were included, 12 cases following laparoscopic pyeloplasty and 8 cases following pyelolithotomy. Antegrade mini-Percutaneous laser endopyelotomy was performed using $14 \mathrm{~F}$ nephroscope and holmium laser. Operation related parameters were studied with follow up after 6 months was done using symptomatic assessment and renal ultrasound.

Results: Average procedure duration was $70 \mathrm{~min}$. Mean discharge was 1.4 days. There was no notable complication except one case $(4 \%)$ of intraoperative bleeding managed by catheter balloon nephrostomy for 2 days. The success rate was $80 \%$ (20 cases), with 5 patients needed redo pyeloplasty. Failure cases were found to have stricture segments more than $1 \mathrm{~cm}$ in the retrograde contrast study, preoperative renal split function less than $25 \%$, and advanced hydronephrosis.

Conclusions: Mini percutaneous laser endopyelotomy is considered a safe and effective option for secondary UPJO in cases with short stricture segment, renal function more than $25 \%$ and absence of advanced hydronephrosis.

\section{MP16-10 Is single-use flexible endoscope Uscope Pu- sen performant enough for Endoscopic Stones Recognition?}

V Estrade, I Jour, P Meria, J Ferriere, H Bensadoun, J Bernhard, G Capon, F Bladou, G Robert, O Traxer

Hospital Pellegrin

Introduction \& Objective: Endoscopic recognition of kidney stones by the urologist constitutes a new diagnostic modality that could replace conventional microscopic and spectrophotometric recognition in most cases. The flexible video ureteroscope URFV by Olympus, with a simultaneous multicolor CCD chip, delivers a high definition view of the morphology of stone surface and a cross section allowing very high concordance with microscopic and spectrophotometric recognition. Since 2015, there is a surge of a single-use digital flexible ureteroscope (Pusen Uscope) that possess CMOS imager. The objective of this paper is to validate a list of kidney stones recognised using the Uscope Pusen.

Methods: Between June 2015 and June 2018, we systematically compared the endoscopic recognition provided by the Olympus URF-V or by the Pusen Uscope with the microscopic recognition and spectrophotometric analysis of stone fragments. All procedures were performed by a single surgeon with expertise in endourology and stone recognition. This clinical study was approved by our local ethic committee.

Results: Endoscopic recognition was performed on 512 kidney stones using the Olympus URF-V(HD) and on 29 using the Pusen Uscope.

When compared with microscopic and spectrophotometric recognition the endoscopic recognition provided by the Olympus URF-V (HD) adequately identified 167 stones (32;6\%) from group I (whewellite), 192 (37,5\%) from group II (weddellite), 81 $(15,8 \%)$ from group III (Uric acid), $62(12,1 \%)$ from group IV (carbapatite and struvite), 5 (1\%) from group V (cystine), and $5(1 \%)$ in group VI (other stones). The Pusen Uscope adequately identified pure stones Ia 3(10;3\%), IIb 3(10;3\%), IIIb 2(6;9\%) and mixed IIb+Ia $2(6,9 \%)$ were recognized (fig 1-4). It was impossible to recognize stone morphology in 19 cases $(65,5 \%)$ with Uscope Pusen.

Conclusions: High definition digital imaging with CCD chip allows an endoscopic recognition of all the different types of pure and mixed kidney stones. The endoscope Uscope Pusen with CMOS imager provides digital images allowing the recognition of certain more typical stones and visualizing the morphology of some mixed stones. The quality of the images provided by the latter needs to be improved in order to allow for better endoscopic recognition of all kidney stones prior to their destruction.

\section{MP16-11 En-bloc resection of bladder tumors (ERBT) for pathological staging: the value of lateral margins analysis}

R Milandri, S Ciarlariello, C Del Prete, L Sarchi, A Eissa, S Puliatti, M Rizzo, M Sighinolfi, G Bianchi, B Rocco, S Micali

Department of Surgical, Medical and Dental Morphological Sciences related to Transplant, Oncology and Regenerative Medicine - Section of Urology, University of Modena and Reggio Emilia, Modena, Italy

Introduction \& Objective: The gold standard treatment of nonmuscle invasive bladder cancer (NMIBC) is the conventional transurethral resection of the tumor (C-TURBT). This technique is associated with several limitations that might affect the final pathological interpretation of the specimens. Thus, en-bloc resection of bladder tumor (ERBT) was introduced to overcome the limitations of C-TURBT. The aims of the study are to show the pathological advantages obtained from ERBT and to assess the value of the peritumoral margins in ERBT on tumor recurrence.

Methods: We retrospectively collected the data of 50 patients diagnosed as NMIBC, treated with ERBT in the period between January and December 2017. The inclusion criteria are: patients with bladder tumor size $\leq 20 \mathrm{~mm}$ with clinical stage $\leq \mathrm{cT} 1$ and no a previous history of bladder cancer.

Results: The mean operative time and the median hospitalization period were $29.08 \pm 10.11$ minutes and 2 (IQR 1), respectively. Two patients developed extra peritoneal bladder perforation and one patient developed acute urinary retention. Specimens with an Absent detrusor muscle were 5/50(10\%). Tumor staging of the resected specimens showed that $\mathrm{pTa} / \mathrm{LG}$ was detected in $11(22 \%)$ patients, $\mathrm{pTa} / \mathrm{HG}$ was detected in $3(6 \%)$ patients, $\mathrm{pT} 1 /$ LG was detected in $30(60 \%)$ patients, and pT1/HG detected in $6(12 \%)$ patients. The lateral margins were evaluated in 43 patients (86\%) and these margins showed dysplasia in 16 patients and malignancy in 3 patients. Local recurrence occurred in $14(28 \%)$ patients. Early recurrence $(<12$ months) occurred in $8(16 \%)$ patients, whereas, the late recurrence ( $>12$ months) was observed in $4(8 \%)$ patients. It was noted that $57 \%$ of patients with recurrence showed some degree of dysplasia or malignancy in the lateral margin; however, on multivariate logistic regression lateral margins lesions were not significantly associated with recurrence (OR 3.13, 95\% CI 0.704-13.913, $\mathrm{p}=0.13$ ). Moreover, $5 / 14(35.7 \%)$ of the recurrences occur in the same location of the primary tumor with only $3(6 \%)$ patients showing tumor stage upgrading.

Conclusions: ERBT is a feasible and reproducible technique for endoscopic resection of bladder tumors, it allows to obtain a correct orientation of the pathological specimens. This is useful to improve the tumor staging and reducing the possibility to obtain a pathological specimen without muscular layer. There was a trend toward increased rate of recurrence in patients with dysplasia or malignancy in their lateral margins; but it isn't statistically significant. 
MP16-12 Ureteral stent failures and complications in patients with malignant ureteral obstruction

NA Pickersgill, K Du, R Figenshau, A Desai, J Vetter, R Venkatesh

Barnes Jewish Hospital/Washington University School of Medicine

Introduction \& Objective: Management of malignant ureteral obstruction (MUO) with ureteral stents remains a clinical challenge, often involving frequent stent exchanges due to stent failure or other urological complications. We report our institutional experience with ureteral stents for management of MUO including analysis of clinical factors associated with stent-related issues.

Methods: We performed a retrospective review of patients treated with indwelling ureteral stents for MUO in non-urothelial malignancies at our tertiary-care institution between 2008 and 2019 (Table 1). Multivariate logistic regression was performed to identify clinical variables predictive of stent failure or complications. Stent failure was defined as need for unplanned stent exchange, placement of percutaneous nephrostomy (PCN), or tandem stents.

Results: In our cohort of 78 patients, the median (range) number of stent exchanges was $2(0-17)$ during a total stent dwell time of 4.3 (0.1-40.3) months. Thirty-four patients (43.6\%) developed a culture-proven urinary tract infection (UTI) during stent dwell time. Thirty-five patients $(44.8 \%)$ had stent failure. Twenty-two patients $(28.2 \%)$ underwent unplanned stent exchanges, 23 (29.5\%) required PCN following initial stent placement, and 6 $(7.7 \%)$ required tandem stents. Ten $(28.6 \%)$ patients with stent failure were treated with upsized stents, which led to resolution in 7 patients. Stent failure occurred with 20/44 (45.4\%) Percuflex $^{\mathrm{TM}}, 15 / 27(55.6 \%)$ polyurethane, and $2 / 3(66.7 \%)$ metal

Table 1. Demographics of patients with MUO.

\begin{tabular}{|c|c|c|}
\hline \multicolumn{3}{|c|}{ Patient Characteristics } \\
\hline Mean Age (years) & & \\
\hline Sex & $45 \mathrm{fer}$ & \\
\hline Mean BMI & & \\
\hline Race & $\begin{array}{l}\text { Caucasian } \\
\text { African American } \\
\text { Other }\end{array}$ & $\begin{array}{l}75.6 \%(59) \\
23.1 \%(18) \\
1.3 \%(1)\end{array}$ \\
\hline Primary Cancer Site & $\begin{array}{l}\text { Colorectal } \\
\text { Prostate } \\
\text { Uterine } \\
\text { Ovarian } \\
\text { Cervical } \\
\text { Breast } \\
\text { Appendiceal } \\
\text { Testicular } \\
\text { Gallbladder } \\
\text { Pancreatic } \\
\text { Gastric } \\
\text { Leiomyosarcoma } \\
\text { Leukemia } \\
\text { Lung } \\
\text { Unspecified squan }\end{array}$ & $\begin{array}{l}25.6 \%(20) \\
19.2 \%(15) \\
14.1 \%(11) \\
10.3 \%(8) \\
6.4 \%(5) \\
5.1 \%(4) \\
3.8 \%(3) \\
3.8 \%(3) \\
2.6 \%(2) \\
2.6 \%(2) \\
1.3 \%(1) \\
1.3 \%(1) \\
1.3 \%(1) \\
1.3 \%(1) \\
1.3 \%(1)\end{array}$ \\
\hline History of Radiation & & \\
\hline Chronic Kidney Disease & & \\
\hline Bilateral Stents & & \\
\hline $\begin{array}{l}\text { Stent Size (French) at Initial } \\
\text { Stent Placement }\end{array}$ & $\begin{array}{l}\text { Less than } 6 \\
\text { Six } \\
\text { Seven } \\
\text { Greater than } 7\end{array}$ & $\begin{array}{l}3.8 \%(3) \\
47.4 \%(37) \\
37.2 \%(29) \\
11.6 \%(9)\end{array}$ \\
\hline $\begin{array}{c}\text { Reason for } \\
\text { Unplanned Stent Exchange }\end{array}$ & $\begin{array}{l}\text { Hydronephrosis } \\
\text { Urosepsis } \\
\text { Ureteral injury } \\
\text { Stent migration } \\
\text { Ureteroiliac fistula }\end{array}$ & $\begin{array}{l}72.7 \%(16) \\
13.6 \%(3) \\
4.5 \%(1) \\
4.5 \%(1) \\
4.5 \%(1)\end{array}$ \\
\hline
\end{tabular}

Table 2. Multivariate logistic regression analysis for clinical predictors of stent failure and UT

\begin{tabular}{|c|c|c|}
\hline \multicolumn{3}{|c|}{ Stent Failure } \\
\hline Variable & Hazards Ratio & p-value \\
\hline Age (continuous) & 1.00 & 0.91 \\
\hline Male vs. Female Sex & 1.24 & 0.54 \\
\hline African-American vs. Caucasian Race & 1.25 & 0.57 \\
\hline BMI (continuous) & 0.99 & 0.68 \\
\hline History of Radiation & 1.30 & 0.49 \\
\hline Chronic Kidney Disease & 1.65 & 0.23 \\
\hline Bilateral vs. Unilateral Stent & 1.23 & 0.54 \\
\hline Stent French: $<6$ vs. 6 & 1.22 & 0.79 \\
\hline Stent French: 7 vs. 6 & 0.73 & 0.41 \\
\hline Stent French: $>7$ vs. 6 & 0.84 & 0.75 \\
\hline \multicolumn{3}{|c|}{ UTI } \\
\hline Variable & Hazards Ratio & p-value \\
\hline Age (continuous) & 1.01 & 0.60 \\
\hline Male vs. Female Sex & 1.14 & 0.71 \\
\hline African-American vs. Caucasian Race & 0.98 & 0.96 \\
\hline BMI (continuous) & 0.99 & 0.73 \\
\hline History of Radiation & 2.58 & 0.019 \\
\hline Chronic Kidney Disease & 1.32 & 0.47 \\
\hline Bilateral vs. Unilateral Stent & 2.26 & 0.032 \\
\hline Stent French: $<6$ vs. 6 & 0.34 & 0.31 \\
\hline Stent French: 7 vs. 6 & 0.73 & 0.44 \\
\hline Stent French: $>7$ vs. 6 & 1.33 & 0.58 \\
\hline
\end{tabular}

stents. In patients with $\geq 2$ exchanges $(\mathrm{N}=45)$, mean time between exchanges was $4.3 \pm 2.0$ months. Bilateral stenting and history of radiation predicted UTI development (Table 2). Median overall patient survival after initial stent placement was 19.9 months (95\% CI 16.5-37.9 months).

Conclusions: Ureteral stent failure poses a significant medical and financial burden to patients with MUO. Better methods to minimize stent-related issues and improve patient quality of life are needed.

MP16-13 A Systematic Review of Transurethral Resection of Ejaculatory Ducts for the Management of Obstructive Azoospermia: Outcomes and Predictors of Success

A Mekhaimar, M Goble, O Brunckhorst, HM Alnajjar, D Ralph, A Muneer, K Ahmed

King's College London

Introduction \& Objective: Ejaculatory duct obstruction (EDO) is an uncommon cause of male factor infertility but can potentially be treated endoscopically by transurethral resection of the ejaculatory ducts (TURED). At present, there is limited data relating to the patient population who can benefit from TURED and fertility outcomes. A systematic review of the literature was conducted to assess the success of TURED in treating infertility due to EDO and identify patient subgroups who benefit from the procedure.

Methods: This review was conducted following PRISMA guidelines and prospectively registered on PROSPERO (CRD42019123024). A database search of PubMed, Embase, and Scopus (up to January 2019) was performed to identify all 
primary studies assessing infertile males with EDO undergoing TURED. Primary outcome measures extracted were semen parameters and post-operative natural pregnancies. Secondary outcomes included complications, symptomatic improvement and a change from in-vitro fertilization to intrauterine insemination.

Results: Out of 3277 articles screened, 29 studies, including 6 prospective studies, (27 single cohort and 2 comparative studies) with a total of 634 patients were included. While outcomes varied considerably between the studies, there was a general increase in all semen parameters post-operatively. Semen volume was reported in 23 studies with a median of $0.93 \mathrm{ml}$ (IQR 0.37 ) pre-operatively, and $2.90 \mathrm{ml}$ (IQR 1.09 ) post-operatively and a median of $83.0 \%$ (IQR 37.5) patients showed improvement. Sperm motility $(\mathrm{n}=10$ studies) increased from a median of $10.0 \%$ (IQR 9.2) to $34.8 \%$ (IQR 9.9) and an improvement was seen in a median of $63.0 \%$ (IQR 15.0 ) patients. Sperm concentration $(\mathrm{n}=21)$ increased from $3.2 \times 10^{6} / \mathrm{ml}(\mathrm{IQR} 12)$ to $17.0 \times 10^{6} \%$ $\mathrm{ml}$ (IQR 19.6) and 62.5\% (IQR 16.5) patients showed improvement. Natural pregnancy rates were low, with a median of 25\% (IQR 15.7). Improvement in semen parameters and pregnancy rates were found to be greater in those with congenital aetiologies and only partial EDO whereby patients showed features of obstruction with variable degrees of oligozoospermia. Differences in surgical technique did not appear to impact outcomes. Symptomatic improvements were seen in 50-100\% of patients with a median complication rate of $10 \%$ overall.

Conclusions: TURED is associated with improvements in both semen parameters and also symptoms across the studies. However, translation into natural pregnancies is not well demonstrated. While results are promising, the current evidence remains limited due to predominantly retrospective studies with small sample sizes, meaning further investigation is required.

\section{MP16-14 Evaluation of a novel 3-D printed portable cystoscopy device: The "GoScope"}

M Landin, A Aydin, B Smith, F Kum, M Khan, P Dasgupta, K Ahmed

King's College London

Introduction \& Objective: There is a demand for portable cystoscopy devices utilizing widely available technology that allow for prompt investigation in resource limited settings. The aim of this study was to ergonomically assess a new 3D printed smart phone-mounted cystoscopy device called the "GoScope". Methods: This prospective randomized controlled trial compared ergonomics of the GoScope with traditional rigid cystoscopy equipment in a simulated cystoscopy task. Novice medical students with no prior experience of cystoscopy were recruited and included. Participants $(n=28)$ were evaluated using a modified Surgery Task Load Index (SURG-TLX) questionnaire and through continuous electromyography (EMG) recording of the trapezius muscle. The primary outcome measures were the subjective ergonomic perspective from each group and the objective electromyography (EMG) readings. Consultant and trainee urologists were approached for their opinion on the device and were given the opportunity to perform the task.

Results: EMG recording showed a statistically significant difference in trapezius muscle activity between the control and the experimental arm $(\mathrm{P}=0.0439)$. However, analysis of participants' subjective view of the strain experienced revealed there was no statistically significant difference between the two groups

\begin{tabular}{|l|l|l|}
\hline & Control & GoScope \\
\hline Mean EMG range & 846.5 & 991.1 \\
\hline P Value & & $\mathbf{0 . 0 4 3 9}$ \\
\hline
\end{tabular}

Table 1. Mean EMG amplitude in arbitrary units ranges for control and experimental (GoScope) arm.

\begin{tabular}{|c|c|c|c|c|}
\hline & \multirow[t]{2}{*}{ Question } & \multicolumn{2}{|l|}{ Mean } & \multirow[t]{2}{*}{ P-value } \\
\hline & & Control & GoScope & \\
\hline 1 & $\begin{array}{l}\text { The device felt } \\
\text { comfortable to } \\
\text { hold and use }\end{array}$ & 3.429 & 3.071 & 0.3379 \\
\hline 2 & $\begin{array}{l}\text { The device } \\
\text { required } \\
\text { significant time } \\
\text { to get used to }\end{array}$ & 3.000 & 2.286 & 0.1342 \\
\hline 3 & $\begin{array}{l}\text { The device got } \\
\text { in the way while } \\
\text { performing the } \\
\text { task }\end{array}$ & 2.357 & 2.571 & 0.6274 \\
\hline
\end{tabular}

in the following parameters: ease of use, pain/discomfort, mental demand, physical demand, task complexity, degree of difficulty and distractions. EMG data collected from urology consultants and trainees showed no statistically significant difference in muscle activity exhibited by the experts and students of the experimental arm. Questionnaire responses reached statistical significance in the ease of use and degree of difficulty sections. Overall, experts felt that the device got in the way of performing the task to a greater degree than the students $(\mathrm{P}=0.0217)$. The students reported a greater degree of difficulty compared to the experts $(\mathrm{P}=0.0045)$.

Conclusions: Although there is a clear demand for portable cystoscopy devices, no studies to date have evaluated the use of a 3D-printed smart-phone adapter device from an ergonomic standpoint. Subjective evaluation shows no difference in ergonomics between the GoScope and rigid cystoscopy, however, objectively, the GoScope appears to incur more muscle activity. Future research is warranted in evaluating flexible cystoscopy with more accurate objective measures.

\section{MP16-15 Withdrawn}

\section{MP16-16 Evaluation Of Single-Use Flexible Uretero- scopes Available In The Saudi Market: In Vitro Comparison Between Four Disposable Ureteroscope Clinically Oriented Approach.}

WK Kamal, M Abuzenada, A Alhazmy, A Al-Solumany, M Alharthi, Y Alobairi

Consultant Urology and Endourology King Fahd Hospital Jeddah

Introduction \& Objective: In vitro comparison between four single-use flexible ureteroscopes available at the Saudi market. Methods: Four single-use flexible ureteroscopes; Wiscope ${ }^{\circledR}$ (OTU Medical), Pusen (1rs. generation; Zhuhai Pusen Medical Technology Company Limited, China), LithoVue (Boston Scientific) and EU-Scope (Innovex Medical) were tested and compared in an vitro setting mimicking clinical based scenarios. Parameters which could potentially affect surgical technique were compared between the four scopes. 
Results: Wiscope was the lightest ureteroscope (185g) while the Lithovue was the heaviest $(260 \mathrm{~g})$. Wiscope and EU-Scope had the highest deflection with an empty channel reaching up $285^{\circ}$, followed by Lithovue at $273^{\circ}$, then Pusen with $270^{\circ}$. Wiscope and EUscope had the lowest deflection loss with the $200 \mu \mathrm{m}$ laser, 2.2 Fr basket and $365 \mu \mathrm{m}$ laser. EU-Scope had the longest working length $(790 \mathrm{~mm})$ and Pusen had the shortest working length $(630 \mathrm{~mm})$. Even though the working channel diameter is the same for all scopes (3.6 Fr), with an empty channel; Pusen, Wiscope and EU-Scope had the highest irrigation rate at $50 \mathrm{ml} / \mathrm{minute}$ while Lithovue had an irrigation rate of $42 \mathrm{ml} /$ minute. Nonetheless, Wiscope was the least scope to be affected in terms of irrigation with the introduction of $200 \mu \mathrm{m}$ laser fiber $(25.5 \mathrm{ml} / \mathrm{min})$ and $2.2 \mathrm{Fr}$ basket $(10 \mathrm{ml} / \mathrm{min})$, while the

\begin{tabular}{|c|c|c|c|c|}
\hline & Lithovue & Pusen & WiScope & EU-Scope \\
\hline Weight & $260 \mathrm{~g}$ & $220 \mathrm{~g}$ & $185 \mathrm{~g}$ & $240 \mathrm{~g}$ \\
\hline $\begin{array}{l}\text { Monitor } \\
\text { Weight }\end{array}$ & $6.85 \mathrm{~kg}$ & $4.35 \mathrm{~g}$ & Not applicable & Not applicable \\
\hline $\begin{array}{l}\text { Processor } \\
\text { weight }\end{array}$ & Not applicable & Not applicable & $2.7 \mathrm{~kg}$ & $2.2 \mathrm{~kg}$ \\
\hline $\begin{array}{l}\text { Processor } \\
\text { Dimension }\end{array}$ & Not applicable & Not applicable & $\begin{array}{l}32.5 \\
\mathrm{~cm} \times 6.5 \times 30.5\end{array}$ & $\begin{array}{l}160 \times 109 \times 33 \mathrm{~m} \\
\mathrm{~m}\end{array}$ \\
\hline Tip Diameter & $7.7 \mathrm{Fr}$ & $9.0 \mathrm{Fr}$ & $\begin{array}{l}\text { 7.4 Fr } \\
\text { Deflective part } \\
9.5 \mathrm{Fr}\end{array}$ & $9.3 \mathrm{Fr}$ \\
\hline $\begin{array}{l}\text { Shaft } \\
\text { Diameter }\end{array}$ & $9.5 \mathrm{Fr}$ & $9.0 \mathrm{Fr}$ & $8.6 \mathrm{Fr}$ & $8.7 \mathrm{Fr}$ \\
\hline $\begin{array}{l}\text { Working } \\
\text { channel } \\
\text { diameter }\end{array}$ & $3.6 \mathrm{Fr}$ & $3.6 \mathrm{Fr}$ & $3.6 \mathrm{Fr}$ & $3.6 \mathrm{Fr}$ \\
\hline $\begin{array}{l}\text { Working } \\
\text { channel } \\
\text { position }\end{array}$ & 4 o'clock & 4 o'clock & 9 o'clock & 9 o'clock \\
\hline $\begin{array}{l}\text { Deflection } \\
\text { angle EMPTY }\end{array}$ & 272 & 270 & 285 & 285 \\
\hline $\begin{array}{l}\text { Deflection } \\
\text { angle } \\
\text { Laser } 200 \\
\text { micron }\end{array}$ & 270 & 268 & 283 & 283 \\
\hline $\begin{array}{l}\text { Deflection } \\
\text { angle } \\
\text { Laser } 365 \\
\text { micron }\end{array}$ & 230 & 235 & 250 & 250 \\
\hline $\begin{array}{l}\text { Working } \\
\text { channel } \\
\text { length }\end{array}$ & $680 \mathrm{~mm}$ & $630 \mathrm{~mm}$ & $670 \mathrm{~mm}$ & $790 \mathrm{~mm}$ \\
\hline $\begin{array}{l}\text { Irrigation } \\
\text { EMPTY }\end{array}$ & $42 \mathrm{ml} / \mathrm{min}$ & $50 \mathrm{ml} / \mathrm{min}$ & $50 \mathrm{ml} / \mathrm{min}$ & $50 \mathrm{ml} / \mathrm{min}$ \\
\hline $\begin{array}{l}\text { Irrigation } \\
\text { LASER } \\
200 \mu\end{array}$ & $18.6 \mathrm{ml} / \mathrm{min}$ & $18.6 \mathrm{ml} / \mathrm{min}$ & $25.5 \mathrm{ml} / \mathrm{min}$ & $22 \mathrm{ml} / \mathrm{min}$ \\
\hline $\begin{array}{l}\text { Irrigation } \\
\text { BASKET } \\
2.2 \mathrm{Fr}\end{array}$ & $6 \mathrm{ml} / \mathrm{min}$ & $7 \mathrm{ml} / \mathrm{min}$ & $10 \mathrm{ml} / \mathrm{min}$ & $9 \mathrm{ml} / \mathrm{min}$ \\
\hline $\begin{array}{l}\text { Optical } \\
\text { System }\end{array}$ & $\begin{array}{l}\text { Digital } \\
\text { CMOS }\end{array}$ & $\begin{array}{l}\text { Digital } \\
\text { CMOS }\end{array}$ & $\begin{array}{l}\text { Digital } \\
\text { CMOS }\end{array}$ & $\begin{array}{l}\text { Digital } \\
\text { CMOS }\end{array}$ \\
\hline
\end{tabular}

\begin{tabular}{|l|l|l|l|l|}
\hline $\begin{array}{l}\text { Direction of } \\
\text { View }\end{array}$ & 0 & 0 & 0 & 0 \\
\hline $\begin{array}{l}\text { Ball tip } \\
\text { passing test } \\
\text { with 365 }\end{array}$ & no & no & no & yes \\
\hline Field of view & $87^{\circ}$ & $75^{\circ}$ & $100^{\circ}$ & $110^{\circ}$ \\
\hline $\begin{array}{l}\text { deflective } \\
\text { part length }\end{array}$ & $6 \mathrm{~cm}$ & $7.5 \mathrm{~cm}$ & $5.7 \mathrm{~cm}$ & $7.1 \mathrm{~cm}$ \\
\hline $\begin{array}{l}\text { Loop } \\
\text { diameter of } \\
\text { the } \\
\text { deflective } \\
\text { part }\end{array}$ & $220 \mathrm{~mm}$ & $300 \mathrm{~mm}$ & $200 \mathrm{~mm}$ & $270 \mathrm{~mm}$ \\
\hline
\end{tabular}

The summary of the technical features of the comparison between the four single use flexible ureteroscopes
Pusen scope showed the greatest loss in the irrigation rate between the scopes $(18.6 \mathrm{ml} / \mathrm{min})$ with the $200 \mu \mathrm{m}$ and $(7 \mathrm{ml} / \mathrm{min})$ with the 2.2 basket. EU-Scope had the highest field of view, while the pusen had the lowest. The Lithovue and Pusen requires their specific monitor, while EU-Scope and Wiscope are connected to a processor with outlets that could be connected to any monitor. Both Wiscope and Pusen had an autolock mechanism with deflection.

Conclusions: Wiscope was the lightest scope, had the lowest shaft and tip diameter, the shortest loop diameter of the deflective part and showed the least irrigation loss with instruments in the working channel. Wiscope and EU-scope had the highest deflection angle. EU-scope had the longest working length.

\section{MP16-17 Impact of refractive errors on Da Vinci SI robotic system}

MB Tuna, AE Kılavuzoğlu, TS Doğanca, Ö Argun, İ Tufek, o ozisik, C Obek, A Kural

\section{Acibadem Maslak Hospital}

Introduction \& Objective: The aim of this study is to investigate the impact of refractive errors (myopia, hyperopia, astigmatism, and presbyopia) on binocular visual acuity while using Da Vinci SI robotic system console.

Methods: Eighty volunteer people with refractive errors were included in this study. Ophtalmologic examination of the volunteers were performed by the same ophthalmologist. Refractive errors, anisometropia status (the condition in which the two eyes have unequal refractive power) and Titmus fly test (a test that measures the ability to perceive depth) scores were recorded. Prescriptions for glasses or contact lenses were provided for all volunteers. After this stage; all 80 volunteers were examined on Da Vinci SI robotic system console by using a near vision chart; which is placed $35 \mathrm{~cm}$ distance from the 0 -degree robotic camera. Binocular uncorrected and best corrected visual acuity values of the volunteers were recorded. Logarithm of the minimal angle of resolution (LogMAR) method was used for scaling visual acuity. Mann-Whitney U test was used to determine the impact of the refractive error correction (with proper glasses or contact lenses) on binocular visual acuity.

Results: Eighty volunteer people (53 female, 27 male) with a mean age of $32.25 \pm 10.4$ years were included in this study. Myopia was recorded in 59 people $(73.75 \%)$ with a mean of $-1.44 \pm 1.41$ diopters. Hyperopia was recorded in 13 people (16.25\%) with a mean of $+0.22 \pm 0.62$ diopters. On the other hand; astigmatism was recorded in 68 people $(85 \%)$ with a mean of $0.57 \pm 0.93$ diopters. Anisometropia and presbyopia (mean $+0.36 \pm 0.74$ diopters) were recorded in $13(16.2 \%)$ and $17(21.2 \%)$ people, respectively. Mean Titmus Fly test score was $54.65 \pm 26.1$ seconds of arc. In anisometropia; correction of refractive errors with proper glasses or contact lenses enhanced the visual acuity with a mean of $0.34 \pm$ $0.09 \log M A R$ value $(\mathrm{p}<0.05)$. In addition; correction of presbiyopia also enhanced the visual acuity with a mean of $0.25 \pm 0.17$ LogMAR value $(\mathrm{p}<0.05)$. For myopia, hyperopia, and astigmatism $-2.375,+1.0$, and 2.375 diopters were determined as cut-off values respectively $(\mathrm{p}<0.05)$. Any deterioration from this cut-off values resulted in poorer binocular visual acuity while using Da Vinci SI robotic system console.

Conclusions: For better view on the console deemed, robotic surgeons who have anisometropia, presbyopia, myopia $\geq-2.375$ diopters, hyperopia $\geq 1.0$ diopters and astigmatism $\geq 2.375$ diopters should better use proper glasses or contact lenses at the console during surgery. 
MP16-18 Comparison of Multi-tract minimally invasive percutaneous nephrolithotomy and Endoscopic Combined Intrarenal Surgery for Staghorn Renal Calculi: a single institution experience

$\mathrm{K} \mathrm{Xu}, \mathrm{Z} \mathrm{Li}$

Introduction \& Objective: To compare the outcomes of multitract minimally invasive percutaneous nephrolithotomy (MPCNL) and simultaneous combined MPCNL and flexible ureteroscopic lithotripsy (Endoscopic Combined Intrarenal Surgery, ECIRS) as treatment for staghorn stones.

Methods: We retrospectively analyzed data from 135 patients with staghorn stones who underwent MPCNL using multiple

\begin{tabular}{|c|c|c|c|}
\hline Variable & $\begin{array}{l}\text { Multi-tract PCNL } \\
(n=74)\end{array}$ & $\begin{array}{l}\text { ECIRS } \\
(n=61)\end{array}$ & $p$ \\
\hline \multicolumn{4}{|l|}{ Gender, $\mathrm{n}(\%)$} \\
\hline Female & $41(55.40)$ & $29(47.54)$ & 0.363 \\
\hline Male & $33(44.60)$ & $32(52.46)$ & \\
\hline Age (years), mean \pm SD & $52.2 \pm 11.8$ & $47.4 \pm 10.9$ & 0.053 \\
\hline BMI $\left(\mathrm{kg} / \mathrm{m}^{2}\right)$, mean \pm SD & $22.6 \pm 3.7$ & $23.6 \pm 3.7$ & 0.21 \\
\hline \multicolumn{4}{|l|}{ Stone laterality, n (\%) } \\
\hline Rt & $43(58.11)$ & $28(45.90)$ & 0.157 \\
\hline $\mathrm{Lt}$ & $31(41.89)$ & $33(54.10)$ & \\
\hline \multicolumn{4}{|l|}{ Stones classification, n (\%) } \\
\hline Partial staghorn & $23(31.08)$ & $24(39.34)$ & 0.316 \\
\hline Complete staghorn & $51(68.92)$ & $37(60.66)$ & \\
\hline \multicolumn{4}{|l|}{ Comorbidities, n (\%) } \\
\hline Hypertension, n (\%) & $12(16.22)$ & $8(13.11)$ & 0.614 \\
\hline Diabetes mellitus, $\mathrm{n}(\%)$ & $7(9.46)$ & $6(9.84)$ & 0.941 \\
\hline Surgery history, $\mathrm{n}(\%)$ & $18(24.32)$ & $20(32.79)$ & 0.277 \\
\hline \multicolumn{4}{|l|}{ Hydronephrosis, n (\%) } \\
\hline Nil or mild & $40(54.05)$ & $35(57.38)$ & 0.699 \\
\hline Moderate or severe & $34(45.95)$ & $26(42.62)$ & \\
\hline $\begin{array}{l}\text { Stone diameter }(\mathrm{mm}) \\
\text { mean } \pm S D\end{array}$ & $58.5 \pm 11.4$ & $57.7 \pm 11.6$ & 0.658 \\
\hline
\end{tabular}

Table 2. Intraoperative outcomes

\begin{tabular}{|c|c|c|c|}
\hline Parameters & Multi-tract PCNL & ECIRS & $\mathrm{p}$ \\
\hline Number of tracts, $n(\%)$ & & & - \\
\hline 1 & $0(0)$ & $61(100)$ & \\
\hline 2 & $56(75.68)$ & $0(0)$ & \\
\hline 3 & $18(24.32)$ & $0(0)$ & \\
\hline \multicolumn{4}{|l|}{ 1st calyx of puncture, $n(\%)$} \\
\hline Upper pole & $6(8.11)$ & $3(4.92)$ & $<0.001$ \\
\hline Middle pole & $38(51.35)$ & $6(9.84)$ & \\
\hline Lower pole & $30(40.54)$ & $52(85.24)$ & \\
\hline \multicolumn{4}{|l|}{2 nd calyx of puncture, $n(\%)$} \\
\hline Upper pole & $30(40.54)$ & - & \\
\hline Middle pole & 27 (36.49) & & \\
\hline Lower pole & $17(22.97)$ & & \\
\hline \multicolumn{4}{|l|}{ 3rd calyx of puncture, $n(\%)$} \\
\hline Upper pole & $10(55.55)$ & - & \\
\hline Middle pole & $5(27.78)$ & & \\
\hline Lower pole & $3(16.67)$ & & \\
\hline \multicolumn{4}{|l|}{ 1st tract size, n (\%) } \\
\hline $18 \mathrm{~F}$ & $34(45.95)$ & $35(57.38)$ & 0.186 \\
\hline $22 \mathrm{~F}$ & $40(54.05)$ & $26(42.62)$ & \\
\hline \multicolumn{4}{|l|}{ 2nd tract size, n (\%) } \\
\hline $16 \mathrm{~F}$ & $4(5.40)$ & - & \\
\hline $18 \mathrm{~F}$ & $50(67.57)$ & & \\
\hline $22 \mathrm{~F}$ & $20(27.03)$ & & \\
\hline \multicolumn{4}{|l|}{ 3rd tract size, $\mathrm{n}(\%)$} \\
\hline $16 \mathrm{~F}$ & $1(5.55)$ & - & \\
\hline $18 \mathrm{~F}$ & $14(77.78)$ & & \\
\hline $22 \mathrm{~F}$ & $3(16.67)$ & & \\
\hline Operative time ( $\mathrm{min})$, mean \pm SD & $144.0 \pm 57.8$ & $118.9 \pm 44.7$ & 0.006 \\
\hline Blood transfusion, n (\%) & $4(5.41)$ & $0(0)$ & 0.447 \\
\hline $\begin{array}{l}\text { Estimated blood loss }(\mathrm{ml}) \text {, } \\
\text { mean } \pm \text { SD }\end{array}$ & $175.0 \pm 186.3$ & $78.4 \pm 74.7$ & $<0.001$ \\
\hline
\end{tabular}

Table 3. Postoperative outcomes

\begin{tabular}{|c|c|c|c|}
\hline Parameters & Multi-tract PCNL & ECIRS & $p$ \\
\hline $\mathrm{Hb}$ drop $(\mathrm{g} / \mathrm{L})$, mean $\pm \mathrm{SD}$ & $23.2 \pm 14.5$ & $12.5 \pm 9.9$ & $<0.001$ \\
\hline $\begin{array}{l}\text { Serum creatinine increase } \\
(\mu \mathrm{mol} / \mathrm{L}) \text {, mean } \pm S D\end{array}$ & $12.6 \pm 26.3$ & $3.3 \pm 18.1$ & 0.075 \\
\hline $\begin{array}{l}\text { Nephrostomy tube extraction } \\
\text { time (days), mean } \pm \text { SD }\end{array}$ & $4.9 \pm 2.0$ & $3.3 \pm 2.0$ & $<0.001$ \\
\hline $\begin{array}{l}\text { Postoperative hospital stay } \\
\text { (days), mean } \pm \text { SD }\end{array}$ & $8.8 \pm 3.3$ & $6.7 \pm 3.1$ & 0.003 \\
\hline \multicolumn{4}{|l|}{ Stone-free rate, $n(\%)$} \\
\hline One-stage SFR & $60(81.08)$ & $49(80.33)$ & 0.912 \\
\hline Final SFR & $68(91.89)$ & $55(90.16)$ & 0.726 \\
\hline Auxiliary procedures, $n(\%)$ & $13(17.57)$ & $10(16.39)$ & 0.857 \\
\hline PCNL & $5(6.76)$ & $4(6.55)$ & 1.0 \\
\hline SWL & $8(10.81)$ & $6(9.84)$ & 0.863 \\
\hline \multicolumn{4}{|l|}{ Complication, $\mathrm{n}(\%)$} \\
\hline Fever $\left(>38^{\circ} \mathrm{C}\right)$ & $13(17.6)$ & $7(11.5)$ & 0.321 \\
\hline Postoperative pain & $2(2.70)$ & $1(1.64)$ & 1.0 \\
\hline Bleeding & $9(12.16)$ & $1(1.64)$ & 0.046 \\
\hline Blood transfusion & $9(12.16)$ & $0(0)$ & 0.013 \\
\hline Embolization & $2(2,70)$ & $1(1.64)$ & 1.0 \\
\hline Hydrothorax & $1(1.35)$ & $0(0)$ & 1.0 \\
\hline Urinary tract infection & $3(4.05)$ & $3(4.92)$ & 1.0 \\
\hline Urosepsis & $2(2.70)$ & $0(0)$ & 0.501 \\
\hline \multicolumn{4}{|l|}{ Clavien-Dindo Grade, n (\%) } \\
\hline I & $15(20.27)$ & $8(13.11)$ & 0.271 \\
\hline II & $14(18.92)$ & $3(4.92)$ & 0.015 \\
\hline IIIla & $3(4.05)$ & $1(1.64)$ & 0.754 \\
\hline
\end{tabular}

(two or more) access tracts or single tract ECIRS. A total of 74 patients were managed by multi-tract PCNL; while 61 patients were managed by ECIRS. Data on demographics, clinical characteristics, perioperative complications and stone free rate (SFR) were recorded and statistically analyzed.

Results: Neither the one-stage SFR $(81.1 \%$ vs $80.3 \%, p=0.912)$ nor the final SFR after ancillary procedures $(91.9 \%$ vs $90.2 \%$, $\mathrm{p}=0.726$ ) were significantly different between the groups. The mean operating time was significantly higher $(144.0 \pm 57.8 \mathrm{~min})$ in the multi-tract MPCNL group compared with the ECIRS group (118.9 $\pm 44.7 \mathrm{~min}, \mathrm{p}=0.006)$. Overall, complications were significantly higher in the multi-tract MPCNL group than the ECIRS group ( $43.2 \%$ vs $19.7 \%, \mathrm{p}=0.004)$. The number of blood transfusions was $9(12.2 \%)$ in the multi-tract MPCNL group and $0(0.0 \%)$ in the ECIRS group $(\mathrm{p}=0.013)$. Patients undergoing ECIRS required shorter postoperative hospitalization time than those undergoing multi-tract MPCNL ( $8.8 \pm 3.3$ days vs $6.7 \pm 3.1$ days, $\mathrm{p}=0.003$ ).

Conclusions: Both the procedures were effective and resulted in comparable SFR. However, ECIRS had better post-operative outcomes including lower rate of complications and shorter hospitalization time compared with multi-tract MPCNL.

MP16-19 Sequential Layered Muscle Inclusive Relook TURBT (SeL-MIRT) In High Grade Bladder Cancer - Results from a High Volume United Kingdom Centre

MM Quraishi, B Osman, A Simoes, I Ahmed, E Latif, M Jones, J Hale, OA Ramadan, A Rajagopalan, B Varga, N Shrotri, M Thomas, P Keirandish, BA Eddy, R Krishnan, EH Streeter, SS Kommu, G Papadopoulos

Department of Urology. Kent \& Canterbury Hospital. East Kent Hospitals University NHS Foundation Trust

Introduction \& Objective: Bladder cancer carries with it a considerable morbidity and mortality. Non-muscle invasive 
bladder cancers (NMIBC: pTa, pT1) are characterized by a high risk of recurrence and/or progression. Optimal resection of the primary tumor and early re-resection in high grade disease is often mandated. However, the technique of early re-resection varies considerably. One of the issues is lack of adequate muscle sampling in either the primary tumor or early re-resection. Herein, we describe the technique of Sequential Layered Muscle Inclusive Relook TURBT (SeL-MIRT) in high grade bladder cancer in a high-volume center.

Methods: This was a prospective study of bladder tumor resections over 6 consecutive months in 2018 with a minimum follow-up of 12 months. The technique of re-resection involved a systematic and deep resection in a sequential layered format starting with superficial elements being resected first then being extracted endoscopically. This was then followed by intermediate level resection of estimated tumor(s) base. This was again followed by a deep resection to include muscle in the resection specimen as opposed to random tumor base biopsies.

Results: A total of 145 patients were identified to have undergone an initial bladder tumor resection with histologically proven malignancy. Mean age of patient at resection was 72 years (Range 18-94). Male to Female prevalence was seen at $82 \%$ versus $18 \%$. High grade transitional cell (TCC) non muscle invasive cancer (HG-NMIBC) and muscle invasive bladder cancer constituted $57(39 \%)$ and $20(14 \%)$ of the cohort, respectively. Non transitional cell carcinoma bladder cancer was seen in $5 \%$ $(n=7)$ of patients. In patients with HG-NMIBC histological presence of muscle was confirmed and complete resection achieved in the primary resection in $68 \%(n=39)$ and $88 \%(n=50)$ of patients respectively. Early relook was performed in $60 \%(n=34)$ of HG NMIBC patients, of whom benign histology was found in $56 \%$ on resection. Patients with CIS did not undergo routine re-resection. Recurrences for HG-NMIBC at 12 months were identified in 10 patients, of whom 8 had undergone an early re-resection.

Conclusions: Sequential Layered Muscle Inclusive Relook TURBT (SeL-MIRT) Technique is achievable and safe and offers good results. We are currently setting up large scale multi-center studies to further ratify this. We also aim to further evaluate outcomes with improved skill acquisition with larger case load. Trainee development in this particular technique will facilitate its dissemination under the care of a high-volume surgeons.

\section{MP16-20 Correspondence between contrast enhanced computed tomography and ureteroscopy in the man- agement of upper tract urothelial carcinoma}

A Gallioli, A Territo, F Sanguedolce, F Regis, R Boissier, J Gaya, M Martinez, P Gavrilov, D Vanacore, J Palou, A Breda

Department of Urology. Fundació Puigvert. Autonomous University of Barcelona. Barcelona. Spain.

Introduction \& Objective: Contrast enhanced computed tomography (CT) is the gold standard imaging to detect upper tract urothelial carcinoma (UTUC). Recently, a higher rate of bladder recurrence in patients submitted to nephroureterectomy after diagnostic ureteroscopy (URS) for UTUC has been described. The aim of the study is to report on the correspondence between CT and URS findings and how it influences the therapeutic indication.

Methods: From 01/2015 to 11/2018, we prospectively collected 268 consecutive patients submitted to URS for suspicion of UTUC with available CT. All CTs were performed within 60 days before surgery at 1.5 -slice thickness. CT result was categorized as positive, suspicious, unlikely and negative. Correspondence between imaging, URS and histology was analyzed. Number, diameter and site of lesions were reported. Therapeutic indication after negative or positive CT, stratified for low and high risk UTUC according to EAU Guidelines, was recorded. Chi-square test was used for categorical variables. Statistical significance was set at 0.05 . This study adhered to the Declaration of Helsinki, all patients signed an informed consent for research purposes at recovery.

Results: One-hundred and fifty (56\%) patients had a UTUC on histology. CT sensitivity and specificity were $90 \%$ and $42 \%$, respectively. A filling defect corresponded to UTUC in 104/151 $(69 \%)$ cases, while urinary thickness in 16/42 (38\%) and stenosis plus thickness in $6 / 11(55 \%)$ patients. Patients with positive/suspicious/unlikely/negative CT scan had an UTUC in 79\%/ $37 \% / 25 \% / 37 \%$ cases, respectively. Out of the positive CTs, URS detected 49/103 (48\%) UTUCs with different characteristics compared to CT imaging (21 bigger/smaller, 7 multifocal and 20 in other sites). So, the sensitivity of CT to detect and characterize UTUC lesions dropped to $47 \%$. In case of positive or negative CTs $(n=163)$, therapeutic indication was changed after URS in 48/163 (29\%) patients. According to CT findings, 76/163 (47\%) high risk cases should have been treated surgically (nephroureterectomy/ureterectomy). Out of these, surgical treatment was not indicated in 28/76 (37\%) cases. On the other hand, 20/87 (23\%) patients with no CT indication for surgery were managed with radical intervention $(x 2=26.9 ; \mathrm{p}<0.001)$.

Conclusions: CT accuracy to characterize UTUC lesions is low. URS should be considered a crucial step in the diagnostic pathway of UTUC even in case of CT-documented high risk tumors, since it may significantly change the treatment indication.

\section{MP16-21 Withdrawn}

\section{MP16-22 Treatment of iatrogenic ureteric lesions af- ter abdomino-pelvic surgery with metallic stents}

S Martín Martín, J Torrecilla García-Ripoll, F Lara Pérez, j Diaz Romero, M Bedate Núñez, J Soto Rodríguez,

A Ruano Mayo, A Zamora Horcajada, F Natal Alvarez, J Cortiñas González

\section{HOSPITAL CLINICO UNIVERSITARIO DE VALLADOLID}

Introduction \& Objective: There is few information available on literature about minimally invasive treatment of iatrogenic ureteric injuries, where only isolated cases are described. Our objective is to present our experience using metallic stents to treat these rare lesions.

Methods: We performed a retrospective review of all patients with iatrogenic ureteric injuries treated with metallic stents from 2006 to 2018.

Results: Thirteen stents $\left(9\right.$ Viabahn ${ }^{\circledR}, 3$ Uventa $^{\circledR}, 1$ Memokath $\left.{ }^{\circledR}\right)$ were placed in 12 patients ( 2 male and 10 female), with a mean age of 59.86years (Range from 33 to 86yo.). In 50\% of the patients the injury was secondary to a hysterectomy; the other half to colorectal surgery. The most frequent localization was at the sacral ureter $(84.62 \%)$. Different length of stents were used, being $10 \mathrm{~cm}$ the most frequent $(30.7 \%)$. 11 patients had previously been treated with temporal nephrostomies for urinary 
diversion, and 6 with double-pig tail catheters. Stents were retired after a mean time of 4.8 months. Mean follow-up time was 26.3 months (range from 9 to $144 \mathrm{mo}$ ). Complications observed, were: migration of the stent $(23.1 \%)$, persistent urinary infection $(7.7 \%)$ and mucosal hypertrophy $(15.4 \%) .6$ patients $(50 \%)$ are currently cured (ureter patent without needing stenting), 3 patients persist stented with periodic changes ( 2 of them died be- cause of tumoral progression), 2 patients (15.4\%) still need Double-pig tail catheters and 1 needed ureteral reimplantation as a definitive maneuver after stent migration and presence of vaginal-ureteric fistulae.

Conclusions: Ureteric metallic stents are a safe, minimally invasive and effective option to treat iatrogenic injuries of the ureters, especially in non-oncological patients.

\section{MODERATED POSTER SESSION 17: LAPAROSCOPIC/ROBOTIC: PROSTATE (II)}

\section{MP17-01 Impact of Modified Bladder Neck Suspen- sion on Early Recovery of Continence after Robot Assisted Radical Prostatectomy}

H Moon, J Yang, Y Park, W Bae, H Cho, U Ha, S Hong, S Kim, J Lee

Seoul St. Mary's Hospital

Introduction \& Objective: The incontinence after RARP significantly decrease the quality of life in prostate cancer patients. A number of techniques have been introduced for the recovery of continence after RARP. Although, the mechanism of the continence recovery is still unclear. We aimed to evaluate the early recovery of continence after RARP by inducing early anterior adhesion and reducing the hypermobility of the urethra through the modified bladder neck suspension (BNS) procedure.

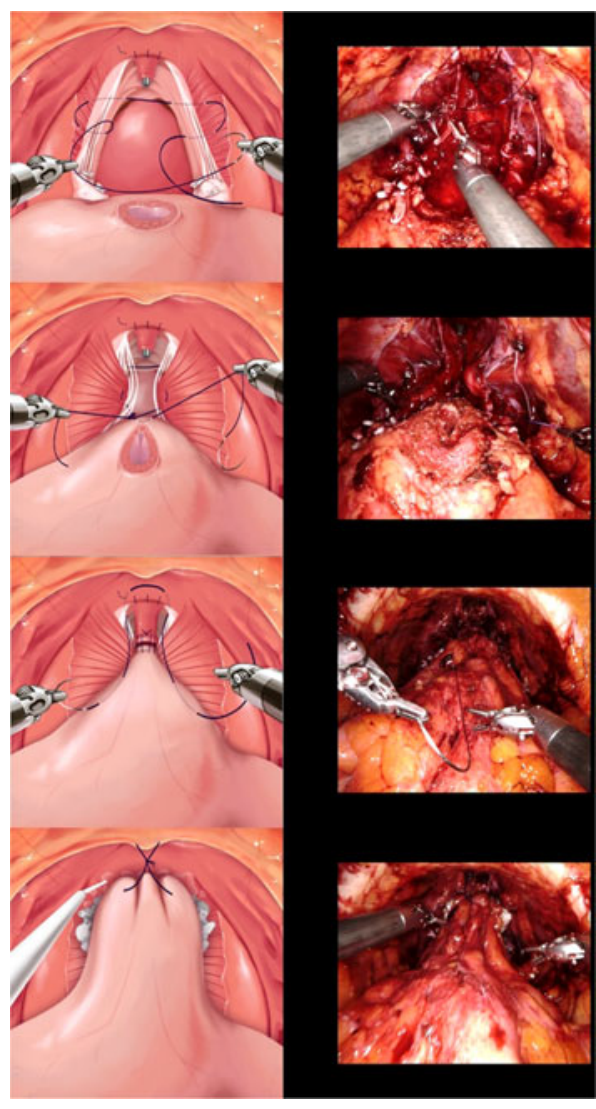

Methods: From March 2017 to June 2018, a total of 186 consecutive patients who underwent RARP (by single surgeon) were included. Patients were divided into two groups based on operation procedure (Standard procedure vs BNS procedure). Demographics, perioperative variables and pathologic outcome were analyzed. We assessed recovery of continence at 1, 3, 6, 9 and 12 months after surgery. Postoperative recovery of continence defined as the use of no pad during 24hr. Multivariable logistic regression analyses were performed to evaluate independent predictors of the early recovery of continence at 1 month.

Results: We performed RARP with standard procedure $(n=95)$ or BNS procedure $(n=91)$. There were no statistically difference in perioperative variables between the two groups except anastomosis time $(22.1 \pm 13.4$ vs $17.0 \pm 7.3, \mathrm{p}=0.002)$. The pad free continence rate was $90.5 \%$ (standard group) and $94.5 \%$ (BNS group) at 12 month after RARP ( $>>0.05$ ). However, early continence rate $(1 \mathrm{mo})$ were significantly higher in the BNS group $(12.8 \%$ vs $26.7 \%, \mathrm{p}=0.026)$. On multivariate logistic analyses, BNS procedure (odds ratio [OR]:2.77, 95\% confidence interval [CI]: 1.06-7.24, p=0.0376), age (OR:0.92, CI: 0.86-0.98, $\mathrm{p}=0.0157$ ) were independent factor for early recovery of continence after RARP.

Conclusions: The modified bladder neck suspension procedure showed significantly better outcomes than the standard procedure in terms of the early recovery of urinary continence.

Table 3. Univariate and multivariate logistic regression of early continence recovery after RARP.

\begin{tabular}{l|c|c|c|c|c|c}
\hline \multicolumn{1}{c|}{ Stone Free } & \multicolumn{3}{c|}{ Univariate analysis } & \multicolumn{3}{c}{ Multivariate analysis } \\
\hline & OR & $\mathbf{9 5 \%} \mathbf{C I}$ & $\boldsymbol{p}$ & $\mathbf{O R}$ & $\mathbf{9 5 \%} \mathbf{C I}$ & $\boldsymbol{p}$ \\
\hline Modified bladder neck suspension & 2.58 & $1.18-5.67$ & 0.0180 & 2.77 & $1.06-7.24$ & 0.0376 \\
\hline Age & 0.93 & $0.88-0.98$ & 0.0115 & 0.92 & $0.86-0.98$ & 0.0157 \\
\hline Nerve sparing & 1.48 & $1.00-2.21$ & 0.0514 & & & \\
\hline Preoperative IPSS & 1.03 & $0.97-1.08$ & 0.3230 & 1.04 & $0.99-1.10$ & 0.1399 \\
\hline BMI & 0.87 & $0.77-0.99$ & 0.0286 & & & \\
\hline
\end{tabular}

MP17-02 The Impact of Body Mass Index (BMI) on Pelvic Lymph Node Yield in High Risk Prostate Cancer

K Parikh, R Bateh, R Pak

Mayo Clinic Florida

Introduction \& Objective: The number of lymph nodes removed during extended pelvic lymphadenectomy (ePLND) for high risk prostate cancer (PCa) has been documented as an important diagnostic and prognostic outcomes predictor. From our 
Figure 1. Total Number of Nodes According to Body Mass Index Group

The median number of nodes was 14 for those with a normal BMI, 15 for overweight, and 18 for obese. The horizontal lines of the box plots represent the median, $25^{\text {th }}$ percentile, and $75^{\text {th }}$ percentile.

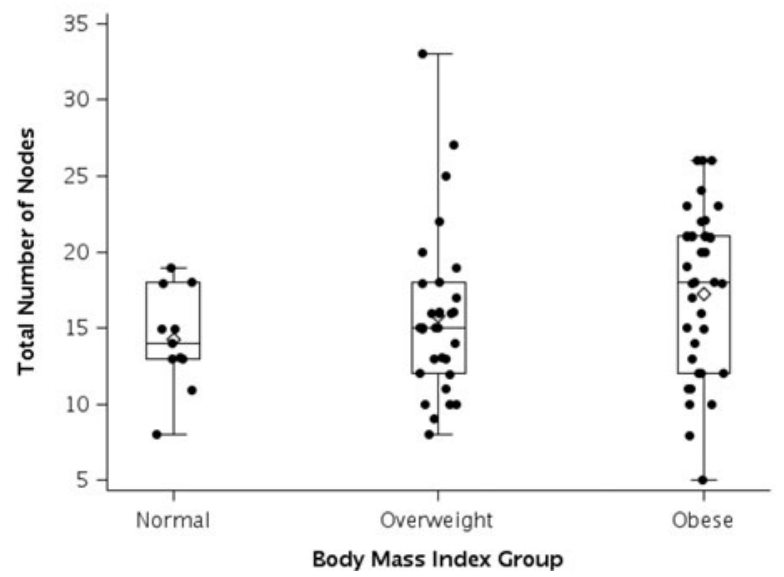

personal experience, ePLND can be time consuming and technically challenging, especially in overweight patients. The goal of our study was to evaluate the impact of BMI on lymph node yields during robotic prostatectomy with ePLND.

Methods: A retrospective review was performed of patients who underwent bilateral ePLND at the time of robotic prostatectomy for high risk PCa between 2017 and 2019. This was a single surgeon series with a standardized template. High risk PCa was defined following NCCN criteria (PSA >20 and/or Gleason Score $>7$ and/or T3a). Bilateral pelvic lymph node samples were collected with a single laparoscopic retrieval bag and extracted at the end of the case. BMI was classified as normal $(18.5-24.9 \mathrm{~kg} /$ $\mathrm{m}^{2}$ ), overweight $\left(25.0-29.9 \mathrm{~kg} / \mathrm{m}^{2}\right)$, or obese $\left(30 \mathrm{~kg} / \mathrm{m}^{2}\right.$ or higher). Single variable Poisson regression model was used for analysis.

Results: A total of 76 males were included in with a median BMI of $29 \mathrm{~kg} / \mathrm{m}^{2}$ (range, 22 to $41 \mathrm{~kg} / \mathrm{m}^{2}$ ); $11(14 \%$ ) were classified as normal BMI, $31(41 \%)$ were classified as overweight, and 34 $(45 \%)$ were classified as obese. The median number of lymph nodes removed during surgery was 16 (range, 5 to 33). Figure 1 shows a boxplot with individual observations of lymph nodes removed according to their BMI. The difference in the expected number of lymph nodes removed per $5 \mathrm{~kg} / \mathrm{m}^{2}$ increase in BMI was 1.07 (95\% CI, 1.00-1.14; $\mathrm{P}=.047)$. The difference in the expected number of lymph nodes removed between those who were overweight and those who had a normal BMI was 1.11 (95\% CI, 0.92-1.32). The difference in the expected number of lymph nodes removed between those who were obese and those who had a normal BMI was 1.21 (95\% CI, 1.02-1.44).

Conclusions: As the BMI increased, the lymph node yield increased for patients with high risk PCa. The observed number of lymph nodes removed for normal weight patients had less variation when compared to overweight and obese patients.

\section{MP17-03 Combined Anterior and Posterior Re- construction during Robotic Assisted Radical Prosta- tectomy - Efficacy and Early Continence Rates}

M Stanowski, N Lobo, N Petrides, MM Quraishi, B Osman, E Latif, SS Kommu, EH Streeter, BA Eddy

Department of Urology. Kent \& Canterbury Hospital. East Kent Hospitals University NHS Foundation Trust
Introduction \& Objective: Early urinary continence is a key goal in patients undergoing Robot Assisted Radical Prostatectomy (RARP). Herein, we assess the impact on early continence of combined anterior and posterior reconstruction.

Methods: Data was collected prospectively for an experienced single surgeon (>1000 RARPs) series for 53 cases with the use of a single anterior suspension stitch combined with posterior rocco reconstruction $(\mathrm{CR})$ compared to the immediately previous 53 cases where only posterior reconstruction (PR) was performed in each of the cases. Continence was assessed pre and post operatively with ICIQ-UI Short Form as well as patient reported postoperative pad usage data. Patients with a pre-op ICIQ of $>0$ and previous TURP were excluded. If nerve sparing was performed, it was recorded for each patient. Data has been collected at 6 weeks and 3 months post operation.

Results: The mean age of patients was 64 (PR) and 63 (CR) years. Non nerve sparing was performed in 21(PR) and 24(CR) cases. Bilateral nerve spare was performed in 24 (PR) and 23 (CR) cases. The mean pad number at 6 weeks and 3 months was 1.06 and 0.43 for $C R$ versus 1.55 and 0.75 for PR only. The percentage of patients pad free at 6 weeks and 3 months was $51 \%$ and $66 \%(\mathrm{CR})$ versus $42 \%$ and $56 \%(\mathrm{PR})$. Mean ICIQ at 6 weeks and 3 months was 8.15 and 5.83 (CR) vs 9.43 and 7.61 (PR).

Conclusions: In this series, combined anterior and posterior reconstruction improved the early return of continence in patients with a pre-op ICIQ of 0 . We are assessing the progress of these patients at 6,9 and 12 months. We will continue to perform combined anterior and posterior reconstruction in our patients. Further large scale multi-centered studies could add further information on the potential benefits of the combined approach.

\section{MP17-04 Impact of prior Transurethral Resection Of The Prostate on the outcomes of Robotic-Assisted Laparoscopic Prostatectomy: A Systematic Review}

M Belkovsky, G Magalhaes, J da Cruz, D Ilias, MM Xavier, AL Sousa, CM Silva, F Fakhouri, R Maia, M Srougi, C Passerotti

Faculdade de Medicina da Universidade de São Paulo

Introduction \& Objective: Transurethral resection of the prostate is the gold standard for the treatment of benign prostatic hyperplasia. Some of these patients may develop prostate cancer and underwent robotic-assisted laparoscopic prostatectomy (RALP). This systematic review aims to verify the association of prior TURP and surgical, oncological and clinical outcomes in patients that underwent RALP for prostate cancer.

Methods: From February 2014 to December 2018, 623 patients from Oswaldo Cruz German Hospital, Brazil, were submitted to RALP of which 15 had prior TURP. Anatomopathological, surgical, and clinical data were collected. Following guidelines of Preferred Reporting Items for Systematic Reviews and MetaAnalyses, a systematic literature search in Web of Science, PubMed, Cochrane Library, EMBase and Scopus was carried out to identify relevant studies published up to May 2019. Articles published in the English language including patients that underwent RALP with and without prior TURP were selected, and the results compared with our study sample.

Results: The patients were divided into 2 groups: one with history of prior TURP and the other without. The groups were analyzed regarding to age $(72.23$ vs $65.88 \mathrm{p}=0.003), \mathrm{BMI}(\mathrm{p}=$ $0.552)$, preoperative PSA $(\mathrm{p}=0.230)$, prostate size $(\mathrm{p}=0.076)$, seminal vesicle invasion $(\mathrm{p}=0.893)$, extracapsular invasion 
$(p=0.741)$, potency $6(p=0.671)$ and $12(p=0.802)$ months after the surgery, continence $6(p=0.533)$ and $12(p=0.671)$ months after the surgery, operative time $(\mathrm{p}=0.187)$ and aspirated blood loss $(p=0.333)$. Our literature search generated 9 studies that compare patients submitted to RALP with and without prior TURP. One study with 158 patients, 26 with prior TURP, associated TURP with greater bleeding $(494 \mathrm{~mL}$ vs $324 \mathrm{~mL}$, $\mathrm{p}=0.03$ ). One study with 380 patients, 20 with prior TURP, used matched pair analysis to select patients with the same clinical characteristics to compare both groups. It showed an increase in console ( $195 \mathrm{~min}$ vs $160 \mathrm{~min}, \mathrm{p}=0.016)$ and operative time ( $238 \mathrm{~min}$ vs $203 \mathrm{~min}, \mathrm{p}=0.028$ ) as well as increased bleeding $(187 \mathrm{~mL}$ vs $116 \mathrm{~mL}, \mathrm{p}=0.001)$ and biochemical recurrence $(12 \%$ vs $11 \%, \mathrm{p}=0.04)$. One study found a significant increase in positive margins, one with 2041 patients, 51 with prior TURP (35.3\% vs $17 \%, \mathrm{p}=0.015)$. One study, with 200 patients, 16 with prior TURP, found less nerve sparing in patients with prior TURP $(33.3 \%$ vs. $92.0 \%, \mathrm{p}=0.001)$.

Conclusions: The technical difficulty of performing RALP in a patient with previous TURP is reflected by worse intraoperative and postoperative parameters. Therefore, such cases should be handled by experienced surgeons with adequate expertise.

MP17-05 Does prostate size influence the outcomes of Robotic-Assisted Laparoscopic Radical Prostatectomy (RALP)?

MM Xavier, G Magalhaes, M Belkovsky, CM Silva, AL Sousa, J da Cruz, R Maia, S Reis, C Passerotti

Hospital Alemão Oswaldo Cruz

Introduction and Objective: Considering that smaller prostates may show a less clear anatomical delimitation and larger prostates lead to reduced mobility and worse visualization of the operative field, poorer results are to be expected in these groups after Radical Prostatectomy. The use of robot-assisted technique, however, could overcome such difficulties as it provides a stereoscopic and magnified vision, also a greater precision of surgical movements. The purpose of this study is to evaluate perioperative, oncological and functional differences between three groups of different prostate sizes in patients submitted to RALP. Methods: Retrospective data was collected from 437 patients submitted to RALP between 2014 and 2018, then divided into groups according to the pathological specimen: weight $\leq 35 \mathrm{~g}$ $(\mathrm{n}=131)$, weight between 35 and $70 \mathrm{~g}(\mathrm{n}=270)$ and weight $\geq 70 \mathrm{~g}$ $(\mathrm{n}=36)$. The analysis compared Age, Body Mass Index, Initial PSA, Biopsy Gleason, Operative Time, Bleeding, Surgical Specimen Gleason, Tumoral Volume, Proximal, Distal and Circumferential Margins, Extra-Prostatic Extension, Lymph nodes and also PSA, Incontinence and Potency at 3, 6 and 12 months after surgery.

Results: Results: Groups with larger prostates required longer operative time $(128 \times 144 \times 168 \mathrm{~min}, \mathrm{p}=0.006)$ and were associated with higher blood loss $(180 \times 215 \times 310 \mathrm{ml}, \mathrm{p}<0.001)$. Larger glands showed greater absolute tumor volume $(5,8 \times 8,6 \times 12,7 \mathrm{~g}$, $\mathrm{p}<0.001$ ), although the proportion of tumoral involvement was smaller $(20 \times 15 \times 10 \%, \mathrm{p}=0.01)$, as well as positive distal margins $(10.7 \times 6.0 \times 0 \%, p=0.047)$, respectively. There was no significant difference between the groups regarding the postoperative PSA levels, Incontinence and Potency at 3, 6 or 12 months.

Conclusions: Men with smaller glands tend to lose less blood and have faster procedures; however, despite less absolute tumor volume, smaller prostate shows higher proportion of disease relatively to weight, which may justify higher rates of positive distal margins. Such findings may be due to a lower clarity of anatomical limits when dealing with small prostates. Regardless of the size of the operated gland, RALP provided the same functional results and pos-operative PSA values.

\section{MP17-06 Outcomes of Salvage Robotic Pelvic Lym- phadenectomy for Recurrent Prostate Cancer De- tected on Fluciclovine Positron Emission Tomography (PET) Scan}

\author{
K Parikh, A Shumate, R Pak
}

Introduction \& Objective: With the emergence of new generation PET scans to detect recurrent prostate cancer, there is a rising interest in metastatic directed therapy options compared to systemic therapy. Our goal is to share our initial experience with robotic salvage pelvic lymphadenectomy for recurrent prostate cancer detected on imaging.

Methods: We retrospectively reviewed salvage robotic pelvic lymphadenectomy cases for recurrent prostate cancer when fluciclovine PET scan suggested recurrence as evidenced by metabolically active pelvic lymph nodes. All salvage lymphadenectomy cases utilized an extended pelvic lymph node dissection template. We excluded patients with a suspicion for recurrence outside of the pelvis or bone metastasis. We reviewed the indications for surgery, PET images, surgical parameters, and postoperative outcomes.

Results: A total of 3 patients underwent salvage robotic pelvic lymphadenectomy for recurrent prostate cancer detected on PET imaging. All patients underwent a prior initial robotic radical prostatectomy. Two of these patients had pelvic lymphadenectomy at the time of initial prostatectomy. Of these 2, 1 had salvage radiation for detectable PSA post-operatively. The patient without pelvic lymphadenectomy at time of prostatectomy also had salvage radiation for detectable post-operative PSA. All salvage radiation was prior to salvage lymphadenectomy. The average age at salvage lymphadenectomy was 63. PSA prior to salvage lymphadenectomy ranged from 0.16 to $7 \mathrm{ng} / \mathrm{mL}$. Average lymph node yield was 16 nodes (range 12-21). There was a median of 4 nodes positive for metastatic prostate cancer. Two of 3 patients had more nodes positive on final pathology compared to metabolically active nodes on PET scan. There were no complications. Average blood loss was $42 \mathrm{cc}$. Average length of hospital stay was 0.67 days. Two of 3 patients had delayed cancer progression and delayed initiation of systemic therapy. One patient required post-operative androgen deprivation. Average length of follow up was 19 weeks.

Conclusions: As emerging imaging modalities provide better methods of localizing recurrent prostate cancer, salvage surgical options need to be further evaluated. In our study, fluciclovine PET scan underestimates the number of metastatic nodes compared to final pathology. Salvage robotic pelvic lymphadenectomy is a safe surgical option and provides oncologic benefit when measuring success based on PSA progression and a delay to additional systemic treatments.

\section{MP17-07 Lessons Learnt From 1010 Consecutive Robotic Assisted Radical Prostatectomies (RARP)}

MM Quraishi, B Osman, N Petrides, N Lobo, m stanowsk, SS Kommu, B Eddy

Kent Sussex and Surrey 
Introduction \& Objective: RARP is considered a gold standard treatment of Prostate Cancer by many. Lack of prospective studies on long term Oncological and Functional outcomes. To our Knowledge - it's one of the Largest prospective study with almost 8 years follow up.

Methods: Prospective Bespoke Purpose Built Database created on FileMakerPro ${ }^{\mathrm{TM}}$. Data collected Real-Time at point of care between May 2011 - April $2019(\mathrm{~N}=1010)$ RARP patients. Data - Patient demographics, preoperative diagnostics, operative and oncological outcomes.

Results: Mean Age 63.78 yrs (42-76), Mean PSA 8.67 ug/l (0.855), BMI 27.6 (19-40) TURP 6.8\% (69 out of 1010 patients) Surveillance patients were 84 out of 713 recorded $8.5 \%$ Console Time 119 mins (43-240 mins) Mean Total Op time 151 mins (64300 mins) Training cases (39\%) 319/814 recorded Mean blood loss of $233 \mathrm{mls} 0 \%$ intraoperative transfusion with no conversion. Mean length of stay LOS 1.13 days with $94 \%$ home day one. $3.2 \%$ hospital readmission with $1.7 \%$ retention. $1 \%$ Calvin Dindo complications grade 3-5 with no death in 30 days. $3.4 \%$ strictures $72 \%$ of cases had no lymphadenectomy. $68.7 \%$ ISUP group 2 with $75.9 \%$ Gleason 7,3.2\% Gleason 6 and $20.9 \%$ Gleason 8-10. final pathology shows $44.9 \% \mathrm{~T} 3,54.5 \% \mathrm{~T} 2$ and $0.4 \% \mathrm{~T} 4.22 \%$ had positive surgical margins with $75 \% \mathrm{~T} 4,33 \%$ $\mathrm{T} 3$ and $11 \% \mathrm{~T} 2.8 \%$ cases are non-training. functional outcome $44 \%$ pads free by 6 weeks and $92 \%$ pad free by 24 months. $26 \%$ cases had pre op ICIQ $>0.4 .5 \%$ patients referred to tertiary center for incontinence management. 95\% data collection for preoperative sexual function with $54 \%$ had erectile dysfunction with SHIM of 20 or less $.33 \%$ had SHIM of 15 or less.

Conclusions: 1) Largest prospective RARP series of a single surgeon with maintained low complication rates and good functional outcome. 2) Positive Margins rate are stable and difficult to improve. 3) All patient must have preoperative \& postoperative validated measurement tools of continence and erectile function. 4) Importance of Surgical planning of nerve sparing surgery with validated measurement tools. 5) Training cases closely monitored to ensure no detrimental outcome. 6) Operative times take around 700 cases to plateau.

\section{MP17-08 10-Year Oncological Outcomes of Prostate Cancer after Robotic-assisted Laparoscopic Radical Prostatectomy : Single Center Experience}

N Yodkhunnatham, V Woranisarakul, T Taweemonkongsap, T Hansomwong, S Leewansangtong, S Srinualnad

Introduction \& Objective: There is no comparative study about impact of neurovascular bundle (NVB) sparing on oncological outcomes after robotic-assisted laparoscopic radical prostatectomy (RALRP) in Thailand. The purpose of this study was to evaluate oncological outcomes after RALRP with or without NVB sparing and to study associating factors that affect biochemical recurrence and survival outcome.

Methods: All charts of prostate cancer patients who underwent RALRP between 2007 and 2017 were reviewed. Data consisted of demographic information, initial PSA, operative technique, pathological report, peri-operative data, complication, biochemical recurrence (BCR) rate, time to recurrence, and overall survival rate. Comparative analysis was used to analyzed.

Results: 1,048 patients were included in this study. Pathological staging was $2.3 \%$ of pT1, $57.1 \%$ of pT2, and $40.6 \%$ of pT3. Median follow up time was 60 months. BCR rates were $17.3 \%$, $20.0 \%$, and $21.4 \%$ in non-NVB sparing, unilateral NVB sparing, and bilateral NVB sparing group respectively $(p=0.339)$. Uni- lateral or bilateral NVB sparing had no effect on mean time to BCR (8.83 vs 8.89 years; $p=0.544$ ). There was no significant different in term of 10-year overall survival between three groups (98.6\%, 98.7\%, 99.4\%, respectively; $p=0.562)$. Multivariate analysis confirmed that NVB sparing did not affect biochemical recurrence rate (Hazard ratio 1.108, 95\%CI 0.827-1.942; $\mathrm{p}=0.549$ )

Figure 1. 10-year overall survival $(\mathrm{p}=0.562)$

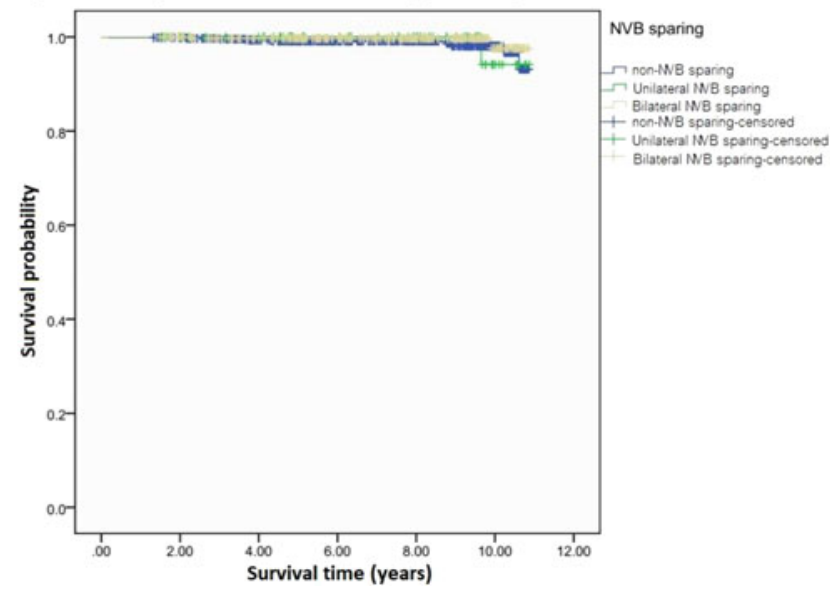

Figure 2. $B C R$ free survival $(p=0.339)$

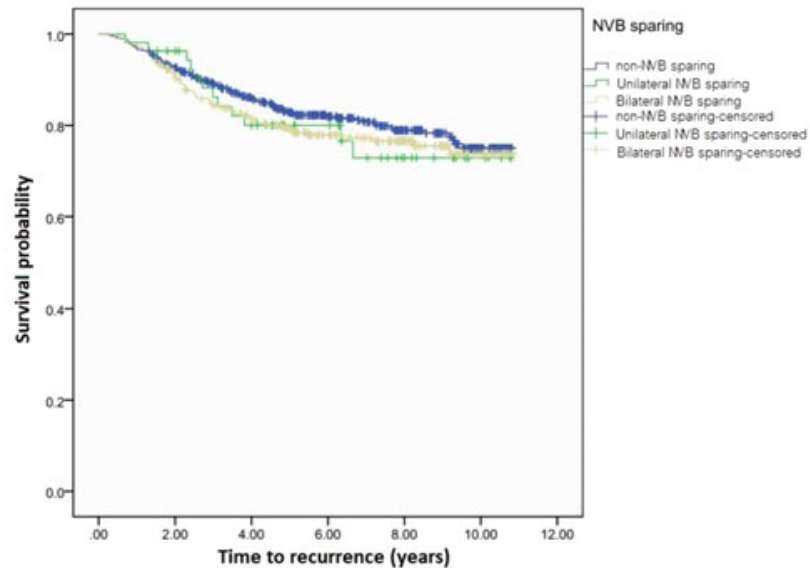

Figure 3. BCR free survival of pT2 stage $(\mathrm{p}=0.130)$

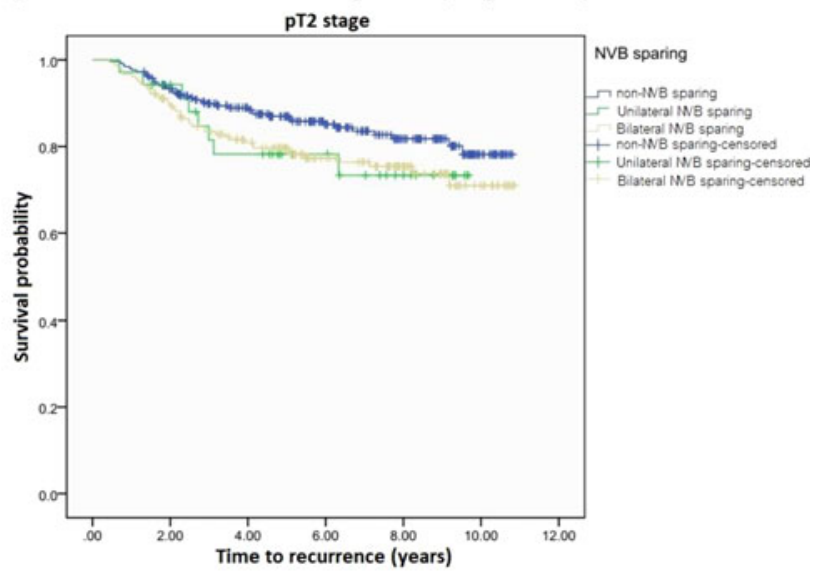


Figure 4. BCR free survival of $\mathrm{pT} 3$ stage $(\mathrm{p}=0.825)$

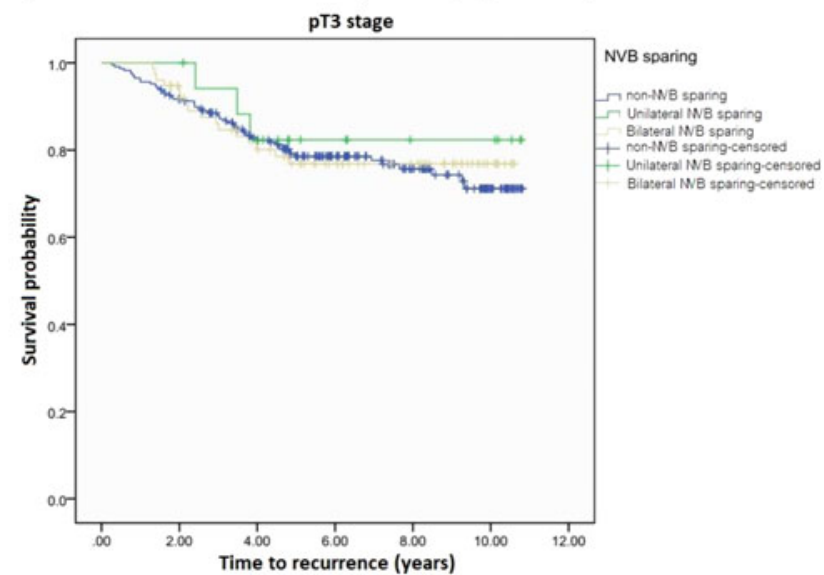

\begin{tabular}{|c|c|c|c|c|}
\hline Characteristics & $\begin{array}{l}\text { Non-NVB sparing } \\
(\mathrm{n}=636)\end{array}$ & $\begin{array}{l}\text { Unilateral NVB sparing } \\
(\mathrm{n}=75)\end{array}$ & $\begin{array}{l}\text { Bilateral NVB sparing } \\
(\mathrm{n}=337)\end{array}$ & $p$ value \\
\hline Age (years) & & & & $<0.001$ \\
\hline Mean (SD) & $68.53(7.33)$ & $64.84(7.25)$ & $63.64(6.91)$ & \\
\hline $95 \% \mathrm{Cl}$ & $67.95-69.1$ & $63.17-66.5$ & $62.90-64.38$ & \\
\hline ASA & & & & 0.164 \\
\hline $1(\mathrm{n},(\%))$ & $68(10.7)$ & $13(17.3)$ & $49(14.5)$ & \\
\hline $2(\mathrm{n},(\%))$ & $471(74.1)$ & $55(73.3)$ & $245(72.7)$ & \\
\hline $3(\mathrm{n},(\%))$ & $97(15.3)$ & $7(9.3)$ & $43(12.8)$ & \\
\hline BMI & & & & 0.553 \\
\hline Mean & 24.52 & 24.31 & 24.70 & \\
\hline SD & 3.23 & 2.98 & 3.04 & \\
\hline $95 \% \mathrm{Cl}$ & $24.27-24.78$ & $23.62-25.00$ & $24.37-24.76$ & \\
\hline iPSA & & & & $<0.001$ \\
\hline$<10(\%)$ & $256(41.9)$ & $35(51.5)$ & $214(66.5)$ & \\
\hline $10-20(\%)$ & $179(29.3)$ & $29(42.6)$ & $88(27.3)$ & \\
\hline$>20(\%)$ & $176(28.8)$ & $4(5.9)$ & $20(6.2)$ & \\
\hline $\begin{array}{l}\text { Gleason Grade } \\
\text { group }\end{array}$ & & & & 0.001 \\
\hline $1(\%)$ & $94(16)$ & $9(13.2)$ & $78(24.6)$ & \\
\hline $2(\%)$ & $232(39.5)$ & $35(51.5)$ & $143(45.1)$ & \\
\hline $3(\%)$ & $104(17.7)$ & $8(11.8)$ & 44 (13.9) & \\
\hline $4(\%)$ & $74(12.6)$ & $9(13.2)$ & $27(8.5)$ & \\
\hline $5(\%)$ & $84(14.3)$ & $7(10.3)$ & $25(7.9)$ & \\
\hline Pathologic stage & & & & $<0.001$ \\
\hline pTl(\%) & $13(2.1)$ & $1(1.3)$ & $10(3)$ & \\
\hline $\mathrm{pT} 2(\%)$ & $318(50.2)$ & $44(58.7)$ & $235(69.7)$ & \\
\hline pT3(\%) & $302(47.7)$ & $30(40)$ & $92(27.3)$ & \\
\hline
\end{tabular}

\begin{tabular}{|c|c|c|c|c|}
\hline Outcome & $\begin{array}{l}\text { Non-NVB sparing } \\
(\mathrm{n}=561)\end{array}$ & $\begin{array}{l}\text { Unilateral NVB sparing } \\
(\mathrm{n}=60)\end{array}$ & $\begin{array}{l}\text { Bilateral NVB sparing } \\
(\mathrm{n}=280)\end{array}$ & $p$ value \\
\hline \multicolumn{5}{|l|}{ BCR rate $(\mathrm{n},(\%))$} \\
\hline $\begin{array}{l}\text { Overall } \\
\text { pT2 }\end{array}$ & $\begin{array}{l}97(17.3) \\
40(14.5)\end{array}$ & $12(20.0)$ & $\begin{array}{l}60(21.4) \\
43(22,9)\end{array}$ & $\begin{array}{l}0.339 \\
0.061\end{array}$ \\
\hline $\begin{array}{l}\mathrm{pT2} \\
\mathrm{pT} 3\end{array}$ & $\begin{array}{l}40(14.5) \\
55(20.2)\end{array}$ & $\begin{array}{l}8(22.2) \\
3(13.0)\end{array}$ & $\begin{array}{l}43(2.29) \\
17(20.2)\end{array}$ & 0.704 \\
\hline $\begin{array}{l}\text { Detectable PSA } \\
(\mathrm{n},(\%))\end{array}$ & $61(10.9)$ & $6(9.8)$ & $16(5.7)$ & 0.064 \\
\hline PSM rate (n, (\%)) & $294(45.8)$ & $28(37.3)$ & $149(44.2)$ & 0.264 \\
\hline $\begin{array}{l}\text { Mean time to BCR } \\
\text { (years) } \\
\text { SD }\end{array}$ & $\begin{array}{l}9.16 \\
0.15\end{array}$ & $\begin{array}{l}8.83 \\
0.49\end{array}$ & $\begin{array}{l}8.89 \\
0.22\end{array}$ & 0.544 \\
\hline $95 \% \mathrm{Cl}$ & $8.86-9.46$ & 7.87 & $8.45-9.34$ & \\
\hline \multicolumn{5}{|l|}{ Mean survival time } \\
\hline (years) & 9.75 & 9.8 & 9.81 & 0.562 \\
\hline SD & 0.03 & 0.07 & 0.03 & \\
\hline $95 \% \mathrm{Cl}$ & $9.68-9.82$ & $9.66-9.93$ & $9.73-9.83$ & \\
\hline 10-year survival & 98.6 & 98.7 & 99.4 & 0.522 \\
\hline
\end{tabular}

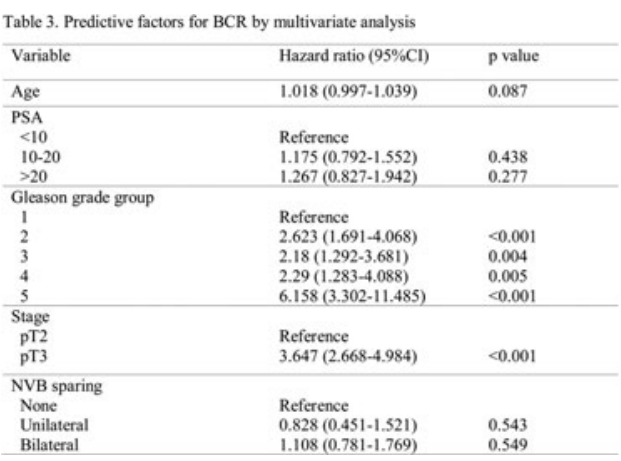

\begin{tabular}{|c|c|c|c|c|}
\hline Dutcome & Non-NVB sparing & Unilateral NVB sparing & Bilateral NVB sparing & p value \\
\hline \multicolumn{5}{|l|}{ perative time (min) } \\
\hline Mean & 199.72 & 196.23 & 185.26 & 0.064 \\
\hline SD & 71.71 & 50.05 & 49.42 & \\
\hline \multicolumn{5}{|l|}{ stimated blood loss (ml) } \\
\hline Median $(\min , \max )$ & $300(20,2810)$ & $300(30,2500)$ & $300(50,2000)$ & 0.635 \\
\hline erioperative complication & & & & \\
\hline lavien-Dindo score $\geq 3$ & $12(2.2)$ & $1(1.8)$ & $3(1.2)$ & 0.601 \\
\hline Delay complication (n, (\%)) & $92(17.2)$ & $12(20.7)$ & $34(13.3)$ & 0.243 \\
\hline
\end{tabular}

Conclusions: This study demonstrated that NVB sparing in RALRP had no significant difference in term of biochemical recurrence and survival outcome compare to non NVB sparing group.

\section{MP17-09 Comparison of Retzius sparing vs standard robot-assisted radical prostatectomy for prostate cancer}

A Tahra, U Sen, A Inkaya, E Kucuk, U Boylu

University of Health Sciences, Umraniye Teaching

Hospital, Urology

Introduction \& Objective: The aim of this study is to compare the oncological and functional outcomes of Retzius-sparing robot-assisted radical prostatectomy (Rs-RARP) vs standard RARP (RARP).

Methods: After Ethical committee approval, we prospectively collected the data from 92 patients who underwent robotic radical prostatectomy between April 2017 - April 2018 in our clinic by a single surgeon. We compared oncological and functional outcomes of 46 patients who underwent Rs-RARP with 46 patients who underwent RARP who had at least a one-year followup. Potency was assessed preoperatively and postoperatively using the Sexual Health Inventory for Men (SHIM) score. The potency was considered to achieve the preoperative level of SHIM score. Patients were assessed with PSA, pathology and for continence (no pad vs $\geq 1$ pad usage) after the first month of the

\begin{tabular}{l} 
Table 1. Patients' demographics. \\
\begin{tabular}{|l|l|l|l|}
\hline & Rs-RARP & RARP & p-value \\
\hline Number of patients & 46 & 46 & - \\
\hline Mean age & 61.2 & 60.8 &, 769 \\
\hline Mean BMI $\left(\mathrm{kg} / \mathrm{m}^{2}\right)$ & 26,8 & 27,0 &, 749 \\
\hline $\begin{array}{l}\text { Preop mean PSA } \\
\text { levels(ng/ml) }\end{array}$ & 8.28 & 12.34 & 0,113 \\
\hline Preop SHIM & 16.3 & 15.6 & 0,531 \\
\hline $\begin{array}{l}\text { Preop ISUP Gleason grade } \\
\text { group }\end{array}$ & & & 0,043 \\
1 & 26 & 19 & \\
2 & 19 & 16 & \\
3 & 1 & 7 & \\
4 & 0 & 2 & \\
5 & 0 & 2 & \\
\hline $\begin{array}{l}\text { EAU risk (Classification) } \\
\text { Low }\end{array}$ & 24 & 19 & \\
Intermediate & 21 & 17 & \\
High & 1 & 10 & \\
\hline
\end{tabular} \\
\hline
\end{tabular}


Table 2. Perioperative and postoperative comparison of the patients

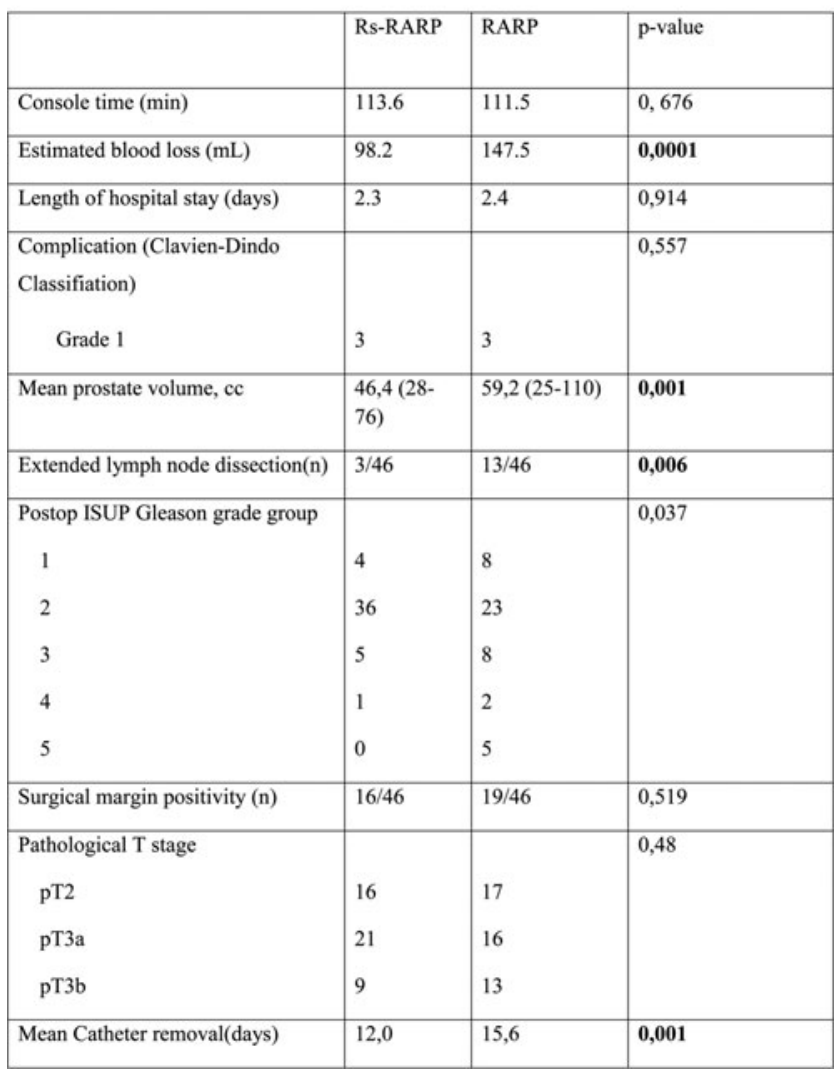

surgery. Patients were also assessed bi-monthly with PSA, continence and SHIM score.

Results: Patients' demographics are seen in table 1. There were no significant differences in the mean age, mean BMI, preop PSA, preop shim score, preop ISUP grade group and EAU prostate cancer risk classification when comparing the Rs-RARP group with s-RARP groups. Mean prostate volume and the mean estimated blood loss is lower, catheter removal time are significantly shorter and in Rs-RARP group. One month after surgery, 41 patients (\%89) were continent (use no pads) in Rs-RARP group while 26 patients (\%56) were continent in RARP group and it's statistically highly significant $(\mathrm{p}=0.001)$ (Table 3$)$.

After a one-year follow-up, there were no differences in the continence rates and potency between two groups. 43 patients (\%93) were continent (use no pads) in Rs-RARP group, 41 patients $(\% 89)$ were continent in the s-RARP group. potency rates were $26 \%$ in Rs-RARP vs $23 \%$ in RARP respectively. No biochemical recurrence was observed in two groups after one-year follow-up.

Table 3. Continence and potency rates of surgical procedures

\begin{tabular}{|c|l|l|l|}
\hline & Rs-RARP & RARP & p-value \\
\hline $\begin{array}{c}\text { 1. month Continence } \\
\text { no pads } \\
\geq 1 \text { pad used }\end{array}$ & 41 & 26 & $\mathbf{0 , 0 0 1}$ \\
\hline $\begin{array}{l}\text { 1. year Continence } \\
\text { no pads } \\
\geq 1 \text { pad used }\end{array}$ & 53 & 20 & \\
\hline $\begin{array}{l}\text { Potency (Achieve Preop } \\
\text { SHIM score) (n) }\end{array}$ & 3 & 41 & 0,459 \\
\hline
\end{tabular}

Conclusions: We demonstrated that Rs-RARP is a feasible surgical technique as much as standard RARP. There is no question about Rs-RARP is better early continence rates while no significant difference was observed after the first year follow up.

\section{MP17-10 Robot-Assisted Simple Prostatectomy: Does Gland Size Affect Outcomes?}

AM Asghar, Z Lee, D Strauss, A Keehn, DD Eun

Temple University

Introduction \& Objective: Robot-assisted simple prostatectomy (RASP) is a surgical option for patients with symptomatic benign prostatic hyperplasia (BPH) for prostate size $\geq 80$ grams. Although RASP has been increasingly utilized, it is unclear whether prostate size affects perioperative or functional outcomes. Herein, we compared perioperative and functional outcomes in patients with prostate size $<150 \mathrm{~g}$ versus those with prostate size $\geq 150 \mathrm{~g}$.

Methods: A retrospective review of our robotic surgery database (5/2013-12/2018) identified patients who underwent RASP. All patients underwent Retzius-sparing approach with posterior cystotomy, excision of adenoma, and complete urethrovesical anastomosis. Prostate volume was measured using US, CT, or MRI. Patients were stratified by prostate size $(<150 \mathrm{~g}$ and $\geq 150 \mathrm{~g}$ ). For functional outcomes, we used the total IPSS and Quality of Life (QOL) scores. Mann-Whitney U and ChiSquared tests were utilized to compare variables between the cohorts.

Results: Of the 148 patients who underwent RASP, 146 had imaging studies available for analysis. We found $89(60.9 \%)$ patients with glands $<150 \mathrm{~g}$ and $57(39.1 \%)$ with glands $\geq 150 \mathrm{~g}$. At the time of analysis, our average length of follow-up was $18(\mathrm{SD}=19.5)$ months. We found no significant difference in console time, Length of Stay (LOS), 30-day post-operative complications (>Clavien Grade II) between the two cohorts. Estimated Blood Loss (EBL) was significantly higher in glands $\geq 150$ g (200cc vs $275 \mathrm{cc}, \mathrm{p}=0.008$ ); however, no patient required a blood transfusion in the larger gland cohort (Table 1). The median pre-operative and post-operative IPSS/QOL were significantly improved for both cohorts: $18 / 5$ to $4 / 1(\mathrm{p}<0.00001)$

\begin{tabular}{|c|c|c|c|c|}
\hline \multirow[t]{2}{*}{ Variable } & \multicolumn{4}{|c|}{$\begin{array}{c}\mathrm{N}(\mathrm{\%}) \\
\text { Median (IQR) }\end{array}$} \\
\hline & All Patients & $\begin{array}{l}\text { Glands < 1508 } \\
\mathrm{N}=89(60.9 \%)\end{array}$ & $\begin{array}{l}\text { Glands } \geq 1508 \\
N=57 \text { (39.1\%) }\end{array}$ & \\
\hline Age & $71(65-75)$ & $70(65-75)$ & $71(65-74)$ & - \\
\hline BMI & $28(26-31)$ & $28(26-31)$ & $28(26-31)$ & - \\
\hline Pre- Operative Gland Size (g) & $129(98-168)$ & $102(89-119)$ & $181(161-228)$ & - \\
\hline Console Time (min) & $165(123-203)$ & $157(120-198)$ & $168(139-212)$ & $p=0.168$ \\
\hline EBL (cc) & $213(150-350)$ & $200(100-300)$ & $275(200-463)$ & $p=0.008$ \\
\hline Blood Transfusion & $4(2.7 \%)$ & $4(4.5 \%)$ & $0(0 \%)$ & $p=0.156$ \\
\hline 30-days Complication (> Clavien Grade 2) & $4(2.7 \%)$ & $3(2.0 \%)$ & $1(0.7 \%)$ & $p=0.341$ \\
\hline LOS (Days) & $1(1-1)$ & $1(1-1)$ & $1(1-1)$ & $p=0.575$ \\
\hline Post-Operative Catheter Duration (Days) & $6(6-8)$ & $6(6-7)$ & $6(6-8)$ & - \\
\hline Pre-Operative IPSS & $18(12-23)$ & $18(13-24)$ & $18(12-23)$ & - \\
\hline Pre-Operative QOL & $5(3-6)$ & $5(3.5)$ & $5(3-6)$ & - \\
\hline Post-Operative IPSS & $2(0-4)$ & $4(2-6)$ & $3(2-5)$ & $p=0.407$ \\
\hline Post-Operative QOL & $0(0-1)$ & $1(0-1)$ & $0(0-1)$ & $p=0.177$ \\
\hline
\end{tabular}


and $18 / 5$ to $3 / 0(p<0.00001)$, respectively. Comparison of postoperative IPSS/QOL between the 2 cohorts found no statistically significant difference: $4 / 1$ vs. $3 / 0(p=0.40 / p=0.23)$, respectively. Importantly, no patient reported new onset stress urinary incontinence (SUI) or pad use for SUI.

Conclusions: RASP is an effective surgical option for men with symptomatic BPH and large glands. Using the Retzius-sparing approach and complete urethrovesical anastomoses, we find that patients have similar peri-operative outcomes with significant reduction in lower urinary tract symptoms and no reported SUI regardless of prostate size at 18-month follow-up.

MP17-11 Robotic Salvage Radical Prostatectomy. An efficient yet underutilized treatment for prostate cancer recurrence

R Madi

Augusta University Health at the Medical College of Georgia

Introduction \& Objective: Salvage radical prostatectomy is an efficient method to treat prostate cancer recurrences following primary radiation therapy failure. Historically, it has been perceived as a difficult procedure that is associated with significant morbidity. Recent literature however suggested that robotic approach is less morbid and offers superior functional outcome than open approach. We evaluated a single center single surgeon experience with robotic salvage radical prostatectomy.

Methods: We performed a retrospective analysis of a prospectively collected prostatectomy database. We identified 15 robotic salvage radical prostatectomies that were performed from January 2012 until May 2019 by a single surgeon RM. All patients had a biopsy proven recurrence and metastatic work-up that was negative for metastasis. Pre-operative, intra-operative and postoperative characteristics were analyzed. Urinary continence is defined as using no pads or one precautionary pad per day.

Results: All 15 procedures were successfully completed. There were no intra or peri-operative complications reported. Patients were discharged home on POD 1, and none required transfusion or re-admission. Out of the 15 patients, 10 had undergone primary X-RT, 3 had brachytherapy, 1 patient has cryotherapy and one patient had cyberknife treatment. Median operative time was 175 minutes (IQR :167- 232). Median estimated blood loss (EBL) was $100 \mathrm{ml}$ (IQR: 50-200). All patients underwent bilateral pelvic lymph nodes dissection (PLND), and 4 patients underwent a Retzius-sparing approach. Final pathology showed residual prostate cancer G.S 7 in 57\%, G.S 8 in 14\%, and G.S 9 in $29 \% .18 \%$ of the patients had positive surgical margins, and $71 \%$ of patients had an undetectable PSA upon 3 months postoperative follow-up. $34 \%$ and $67 \%$ of patients were continent at 3 , and 6 months post-op follow up.

Conclusions: Robotic salvage radical prostatectomy offers the best curative option for recurrences following primary radiation therapy. Contrary to open approach, robotic approach appears to be safer, and offers reasonable continence outcome.

MP17-12 Modified simple prostatectomy: an approach to address large volume BPH and associated prostate cancers.

M Covas Moschovas, S Bhat, O Fikret, T Rogers, V Patel

Global Robotic Institute
Introduction \& Objective: Patients with grossly enlarged prostates over $100 \mathrm{cc}$ may not be optimal candidates for endoscopic techniques or may have failed such techniques previously. Simple prostatectomy has traditionally been the option for management of these patients. Some of these patients with BPH are also known to harbor prostate cancer. This issue can be a challenge because, in the traditional simple prostatectomy, the posterior lateral zone of the prostate is often left in place, and that is usually the area of the prostate cancers. Our objective is to analyze the outcomes of modified simple prostatectomy (MSP) by the elimination of the entire prostate tissue.

Methods: Thirty-four patients underwent Robotic MSP from September 2010 to April 2019. We evaluated the pre- and postoperative clinical characteristics as well as pathological data.MSP was performed using the DaVinci robot; the entire prostate was removed with full nerve sparing bilaterally, minimal apical dissection, seminal vesicles sparing, and full vesicourethral anastomosis. The pre- and postoperative AUA scores were compared with a paired T-test.

Results: The average follow up was 29 months, mean patient age was 71 years (range 57 to 89 years), preoperative AUA Symptom score was 21 (range 6 to 35). The mean operative time and blood loss were 126 minutes and $160.5 \mathrm{ml}$, respectively. None of the patients had complications. The pathology report described 17 $(50 \%)$ with prostate adenocarcinoma. Thirteen patients $(38.2 \%)$ had Gleason 6 and four patients $(11.7 \%)$ Gleason 7, being two patients $4+3$. Pathologically, $5(14 \%)$ had pT2a, $3(8 \%)$ pT2b and $9(24 \%)$ had pT2c. None of them presented with extraprostatic extension or lymphovascular invasion. The mean prostate size reported was 145.6 grams (range 100-264 grams). Twenty-nine patients from thirty that followed in our clinic (96\%) had an AUA score improvement with symptom relief. The mean improvement in the AUA score after surgery was 13 points. Eighty percent of the patients who followed in our clinic (24) presented full continence after surgery. The mean days to continence were 68.5 days (range 4-224 days). Regarding the patients with a prostate cancer diagnosis, $100 \%$ who follow up in our clinic presented PSA lower than 0.01 in at least six months follow-up.

Conclusions: Modified simple prostatectomy is an acceptable treatment option for men with BPH, large prostates over 100cc, and significant obstructive symptoms. The procedure allows for significant symptom relief and removal of chronic catheters. Our modified approach was able to eliminate the entire prostate, which was significant because $50 \%$ of these patients had prostate cancer on the final pathology.

MP17-13 Comparison between robot-assisted radical prostatectomy and minimum incision endoscopic radical prostatectomy for high-risk prostate cancer following neoadjuvant therapy

A Imai, H Yamamoto, S Hatakeyama, T Yoneyama, Y Hashimoto, C Ohyama

Hirosaki University Graduate School of Medicine

Introduction \& Objective: The treatment outcomes of robotassisted radical prostatectomy (RARP) and minimum incision endoscopic radical prostatectomy (MIE-RP) for high-risk prostate cancer following preoperative endocrine chemotherapy were studied retrospectively.

Methods: In our department, RARP and MIE-RP were performed on 600 and 800 cases, respectively between 2005 and 2018. Of the total 1400 subjects, 556 (304 RARP; 252 MIE-RP) high risk 


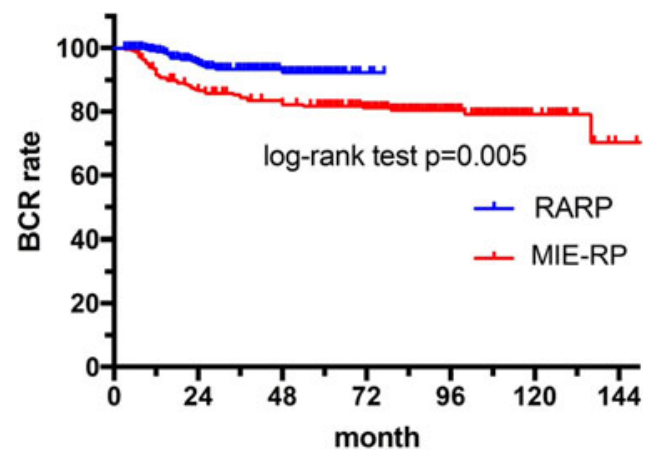

prostate cancer patients underwent surgery after neoadjuvant therapy (ADT+low dose estramustine). Operation time, blood loss volume, Gleason score (GS), resection margin (RM), whether there was leakage from the anastomotic site, whether or not complications occurred, and recurrence based on prostate specific antigen (PSA) levels were compared between the two groups. Neoadjuvant therapy was administered for at least for 6 months before surgery. Results: In the RARP and MIE-RP groups, the mean age of the patients was $67.1(50-77)$ years and $68.3(47-78)$ years $(\mathrm{p}=0.637)$. The mean PSA at the initial visit was $13.7 \mathrm{ng} / \mathrm{mL}$ $(6.0-16.3)$ and $18.1 \mathrm{ng} / \mathrm{mL}(7.1-24.5)(\mathrm{p}=0.001)$. The mean operation times were 167 minutes (142-181) and 121 minutes (99-183), mean blood loss volumes $54 \mathrm{~mL}(10-102)$ and $962 \mathrm{~mL}$ (550-1228), respectively, showing significant differences between the two groups $(\mathrm{p}<0.001)$. Both the RM-positive rate (RARP: $7.1 \%$ and MIE-RP: $10.2 \%$ ) and the PSA recurrence rate (RARP: $3.3 \%$ and MIE-RP: $14.2 \%$ ) were significantly lower in the RARP group $(\mathrm{p}<0.001)$.

Conclusions: As compared to MIE-RP, RARP for high-risk prostate cancer following preoperative endocrine chemotherapy was associated with longer surgical times, while the blood loss volume was significantly lower. Leakage from the anastomotic site and whether or not complications occurred did not differ between the two groups. Therefore, performing RARP with neoadjuvant therapy was considered to be safe. Furthermore, the RM-positive rate and the PSA recurrence rate were significantly lower, showing RARP to potentially play an important role as a highly radical surgery.

MP17-14 Early comparison of the Single Port robotic-assisted radical prostatectomy vs its multiport predecessor

S Crivellaro, WR Halgrimson, YJ Lee, RW Dobbs

University of Illinois at Chicago

Introduction \& Objective: The da Vinci Single Port ${ }^{\mathrm{TM}}$ platform (Intuitive Surgical, Sunnyvale, CA) offers the potential to minimize patient morbidity by conducting a flexible camera and three flexible instruments through a single $3 \mathrm{~cm}$ incision. Given the prevalent use of the Multi-Port (MP) platform in radical prostatectomy (RP), we sought to compare our initial operative and patient experiences between the Single Port (SP) and legacy MP platforms.

Methods: A retrospective review was performed of the initial 22 consecutive patients undergoing SP-RP at our institution. Similar data were obtained from a comparison cohort of the 22 consecutive MP-RP cases immediately preceding introduction of the SP platform. All cases were performed by a single experienced robotic surgeon. Continuous variables were studied using T-Test with $\mathrm{p}<0.05$ indicating significance.

\begin{tabular}{|c|c|c|c|c|c|}
\hline & \multicolumn{2}{|l|}{ Single Port } & \multicolumn{2}{|l|}{ Multi-Port } & \multirow[b]{2}{*}{ Pvalue } \\
\hline & $\underline{N}$ & $\underline{\not}$ & $\underline{N}$ & ж & \\
\hline Number of cases & 22 & & 22 & & \\
\hline Nerve sparing & 12 & $55 \%$ & 5 & $23 \%$ & \\
\hline \multirow[t]{2}{*}{ Lymph node dissection } & 10 & $45 \%$ & 11 & $50 \%$ & \\
\hline & Mean (Ranqe) & $\underline{S D}$ & Mean (Ranqe) & $\underline{S D}$ & \\
\hline Age (Years) & $64(52-77)$ & 6.9 & $61(46-75)$ & 6.6 & 0.19 \\
\hline Body Mass Index $\left(\mathrm{kg} / \mathrm{m}^{2}\right)$ & $30.0(23.3-42.7)$ & 4.4 & $29.2(21.9-41.8)$ & 5.2 & 0.60 \\
\hline Ethnicity & $\underline{N}$ & $\underline{\alpha}$ & $\underline{N}$ & $\underline{\mathscr{\alpha}}$ & \\
\hline Black/African American & 11 & $50 \%$ & 13 & $59 \%$ & \\
\hline Caucasian & 2 & $9 \%$ & 3 & $14 \%$ & \\
\hline Hispanic & 4 & $18 \%$ & 0 & & \\
\hline Native American / Other & 4 & $18 \%$ & 6 & $27 \%$ & \\
\hline
\end{tabular}

\begin{tabular}{|c|c|c|c|c|c|}
\hline & \multicolumn{2}{|c|}{ Single Port } & \multicolumn{2}{|c|}{ Multi-Port } & \multirow[b]{2}{*}{$P$ value } \\
\hline & Mean & $\underline{S D}$ & Mean & $\underline{S D}$ & \\
\hline \multicolumn{6}{|l|}{ Peri-Operative Outcomes } \\
\hline Estimated total blood loss (mL) & $78^{\circ}$ & 64 & 143 & 91 & 0.01 \\
\hline Total operative time (Min) & $245^{\circ}$ & 37 & 251 & 52 & 0.67 \\
\hline \multicolumn{6}{|l|}{ Post-Operative Outcomes } \\
\hline Length of stay (days) & $2^{\circ}$ & 2 & 2 & 2 & 0.40 \\
\hline Total number of days with drain & 3 & 5 & 4 & 9 & 0.72 \\
\hline Total number of days with catheter & 11 & 6 & 13 & 8 & 0.49 \\
\hline \multicolumn{6}{|l|}{ Opiate use - cumulative (TME mg) } \\
\hline PODO 0 & 9.4 & 8.1 & 10.3 & 7.1 & 0.71 \\
\hline POD 1 & 22.2 & 17.6 & 22.0 & 16.2 & 0.95 \\
\hline Full admission period & $32.0^{\circ}$ & 29.5 & 28.8 & 23.6 & 0.70 \\
\hline Clavien-Dindo Complications & $\underline{N \text { (events) }}$ & $N$ (patients) & $N$ (events) & $\underline{N \text { (patients) }}$ & \\
\hline Grade I or II & 17 & 13 & 24 & 16 & \\
\hline Grade III or higher & 8 & 5 & 4 & 2 & \\
\hline \multicolumn{6}{|c|}{$\begin{array}{l}\text {-Excludes one SP patient who underwent combined prostatectomy and partial nephrectomy } \\
\text { "Excludes one SP patient with exceptional length of stay due to social placement issues }\end{array}$} \\
\hline POD - Post-operative Day; RP - Radic & Prostatector & y; TME - Tota & & nts (mg) & \\
\hline
\end{tabular}

Results: 22 SP-RP cases were performed between December 2018 through April 2019, and 22 MP-RP cases were performed between April and November 2018 (Table 1). Estimated total blood loss (EBL) was the only perioperative parameter that varied significantly between SP and MP cases (78 vs $143 \mathrm{cc}$; $\mathrm{p}=0.01$ ). Operative time, drain duration and catheter duration favored SP but the differences were not statistically significant. One SP patient experienced an intraoperative complication with serosal injury that was repaired immediately with no further untoward effect. No patients died. Mean follow-up was 94 (Range 27 - 160) and 219 (Range 2 - 349) days among the SP and MP patients. One patient was lost to follow-up after discharge following his MP-RP.

Conclusions: The SP platform demonstrated equivalent outcomes in several peri and post-operative domains, with potentially lower EBL and equivalent total opiates use for pain control. Limitations include a small sample size, short term follow-up and our early learning curve on the SP. Further research is needed to address these limitations, but the SP platform appears comparably safe and effective to MP in our early experience.

\section{MP17-15 Retzius-sparing Robotic-assisted Radical Prostatectomy versus Open Retropubic Radical Prostatectomy: A Prospective Comparative Study with 19-month Follow-up}

A Abdel Raheem, M Alenzi, K Chang, I Alowidah, A El-Bahnasy, Y Choi, K Rha

Urology Department, King Saud Medical City 
Introduction \& Objective: To compare the surgical outcomes of retzius-sparing robot-assisted radical prostatectomy (RSRARP) and open radical prostatectomy (ORP). So Far, this is the first study that compares both surgical techniques.

Methods: A prospective comparative study for patients with clinically localized prostate cancer (PCa) who underwent RSRARP or ORP from June 2013 to October 2016 was carried out. Our endpoints were to compare the perioperative, oncological, and continence results. Continence function was assessed using the validated International Consultation on Incontinence Questionnaire-Urinary Incontinence Short Form at 1, 3, 6, and 12-month. Urinary continence was defined as $\leq 1$ pad usage. Biochemical recurrence (BCR) was defined as two consecutive rise in serum prostate specific antigen $>0.2 \mathrm{ng} / \mathrm{ml}$.

Results: One-hundred twenty-five and 59 patients met our inclusion criterion in RS-RARP and ORP groups, respectively. Baseline patient's clinical and pathological features didn't differ between both groups $(\mathrm{p}>0.05)$. The median follow-up period was 19 months. The median blood loss $(1000 \mathrm{ml}$ versus $225 \mathrm{ml}$, $\mathrm{p}<0.001)$ and transfusion rate $(10.2 \%$ versus $2.4 \%, \mathrm{p}=0.029)$ were higher in ORP compared to RS-RARP, respectively. Postoperative visual analogue pain score was higher $(\mathrm{p}<0.05)$ with subsequent more analgesic consumption $(\mathrm{p}<0.05)$ and longer hospital stay $(\mathrm{p}=0.003)$ in the ORP group. Major complications ( $\geq 3$ b Clavien-Dindo grade) didn't differ between both groups $(\mathrm{p}=0.121)$. Overall positive surgical margin (PSM) rate was $28.8 \%$ in ORP and $24.8 \%$ in RS-RARP ( $p=0.494)$. At last

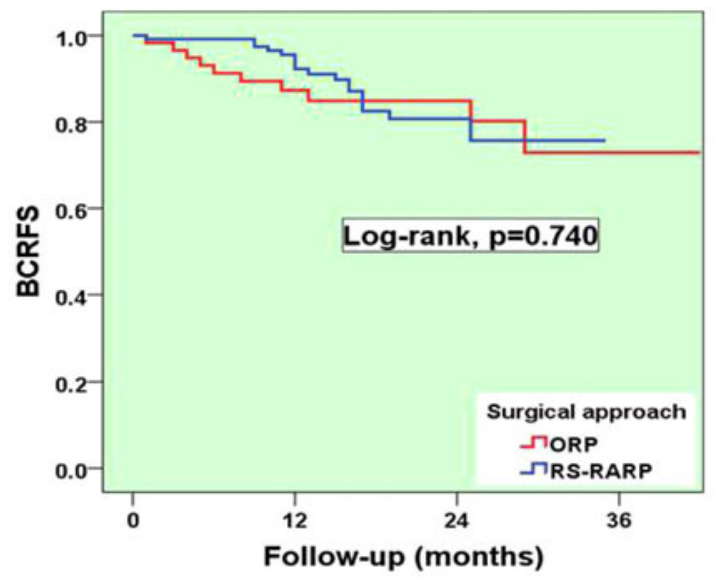

Figure 1a- BCRFS probability stratified by the surgical approach

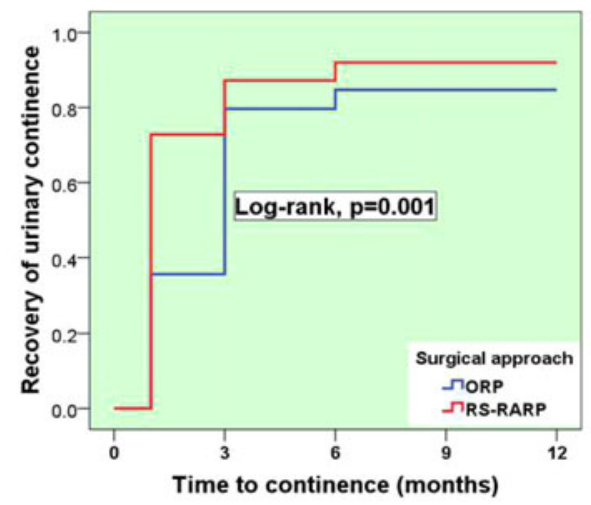

Figure 1b-Recovery of continence function stratified by the surgical approach follow-up, BCR rate was comparable (16.9\% versus $13.6 \%$, $\mathrm{p}=0.656)$ in ORP and RS-RARP groups, respectively. The actuarial BCR free survival rate at 1 -year was $(87.3 \%$ and $92.3 \%)$, and at 3 -year was $(72.9 \%$ and $75.7 \%)$ in ORP and RS-RARP groups, respectively (Log-rank, $\mathrm{p}=0.740$ ) (Fig.1a). Urinary continence rate at 1-month, 3-month, 6-month, and 12-month in ORP compared to RS-RARP was (42.4 versus $72.8 \%, \mathrm{p}<0.001)$, (62.7 versus $82.4 \% \mathrm{p}=0.003),(79.7 \%$ versus $90.4 \%, \mathrm{p}=0.044)$, and $(84.7 \%$ versus $92 \%, \mathrm{p}=0.131)$, respectively. Patients in RSRARP group achieved faster recovery of urinary continence than those in ORP group (Log-rank, p=0.001) (Fig.1b).

Conclusions: RS-RARP has fewer transfusions, less pain, and shorter hospital stay compared to ORP. Moreover, RS-RARP has better recovery of urinary continence in the first 6 months after surgery. Short-term oncological outcomes were comparable between both surgical approaches.

\section{MP17-16 The Impact of Low Free Testosterone on Prostate Cancer: High Risk Disease, Biochemical Recurrence, and Testosterone Replacement After Radical Prostatectomy}

LM Huynh, M Towe, KJ See, J Tran, F el Khatib, H Su, F Yafi, TE Ahlering

\section{UC Irvine Health}

Introduction \& Objective: Historically, high serum testosterone was feared to exacerbate prostate cancer (PC); however, recent studies now link low testosterone to significant metabolic complication. The present study seeks to evaluate the impact of low free testosterone (FT) on PC risk and recurrence following radical prostatectomy (RP).

Methods: 830 patients underwent RP, with prospectively-drawn total testosterone (TT), sex hormone binding globulin (SHBG) and calculated FT. Logistic regression was used to assess impact of FT on Gleason Grade Group (GGG), stage, and biochemical recurrence (BCR). A subset of 152 hypogonadal men with lowrisk PC were placed on testosterone replacement therapy (TRT) and were proportionately matched to 419 controls. Impact of TRT on BCR was assessed with stepwise multivariable analysis.

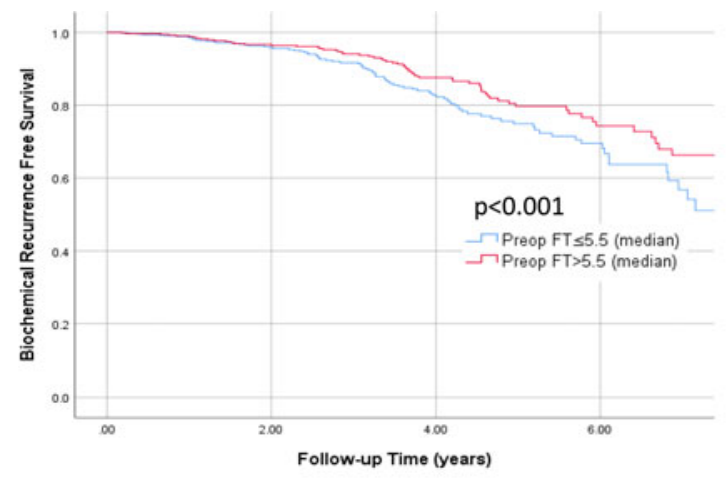

\begin{tabular}{|l|l|l|l|l|l|l|l|}
\hline & B & S.E. & \multirow{2}{*}{ Wald } & \multirow{2}{*}{ Sig. } & \multirow{2}{*}{ OR } & \multicolumn{2}{|l|}{ 95\% C.I. } \\
\cline { 7 - 10 } & & & & & Low & High \\
\hline Age, cont. & 0.02 & 0.016 & 1.534 & 0.216 & 1.02 & 0.989 & 1.052 \\
\hline Preoperative PSA, cont. & 0.101 & 0.018 & 31.526 & $<0.001$ & $\mathbf{1 . 1 0 6}$ & 1.068 & 1.146 \\
\hline GGG [<4+5 (ref) vs. 9-10] & 1.734 & 0.244 & 50.583 & $<0.001$ & $\mathbf{5 . 6 6 1}$ & 3.511 & 9.128 \\
\hline p-stage [pT2 (ref) vs. pT3/T4] & 1.531 & 0.237 & 41.655 & $<0.001$ & $\mathbf{4 . 6 2 5}$ & 2.905 & 7.364 \\
\hline Preoperative FT, cont. & -0.449 & 0.235 & 3.639 & $\mathbf{0 . 0 4 6}$ & $\mathbf{0 . 6 3 8}$ & 0.402 & 0.999 \\
\hline
\end{tabular}


Figure 2: Cox Regression of BCR-free survival, stratified by testosterone replacement therapy ( $N=152$ TRT patients matched by $G G G$ and $p$-stage to $N=472$ control patients)

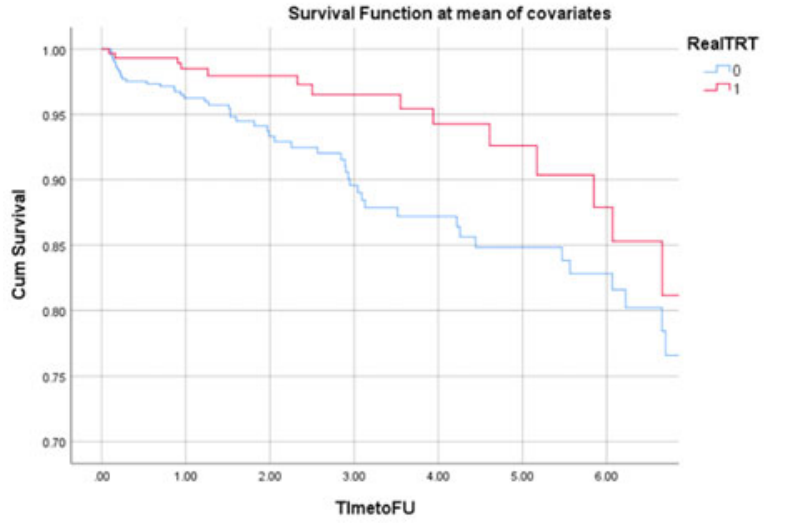

\begin{tabular}{|l|l|l|l|l|l|l|l|}
\hline & \multirow{2}{*}{ B } & \multirow{2}{*}{ S.E. } & \multirow{2}{*}{ Wald } & \multirow{2}{*}{ Sig. } & \multirow{2}{*}{ OR } & \multicolumn{2}{|l|}{$\mathbf{9 5 \%}$ C.I. } \\
\cline { 7 - 9 } & & & & & & Low & High \\
\hline Preoperative PSA, cont. & 0.058 & 0.012 & 23.651 & $<0.001$ & 1.06 & 1.035 & 1.085 \\
\hline GGG [<4+5 (ref) vs. 9-10] & 1.664 & 0.311 & 28.673 & $<0.001$ & 5.28 & 2.872 & 9.708 \\
\hline p-stage [PT2 (ref) vs. pT3/T4] & 1.407 & 0.268 & 27.638 & $<0.001$ & 4.084 & 2.417 & 6.901 \\
\hline Preoperative FT, cont. & -0.14 & 0.063 & 4.911 & $\mathbf{0 . 0 2 7}$ & 0.869 & 0.768 & 0.984 \\
\hline TRT [TRT (ref) vs. control] & -0.616 & 0.313 & 3.88 & $\mathbf{0 . 0 4 9}$ & 0.54 & 0.292 & 0.997 \\
\hline
\end{tabular}

Results: After adjusting for preoperative PSA, BMI, and age, low FT was significantly associated with increased likelihood of GGG 9-10 ( $\mathrm{p}=0.036)$, stage pT3/T4 ( $\mathrm{p}=0.047)$, and BCR within 3 -years post-RP $(\mathrm{p}<0.0001)$ [Figure 1]. Further, hypogonadal patients on TRT were 53\% less likely to experience a BCR, after accounting for GGG, stage, preoperative TT, cFT, and PSA. In patients destined to recur, TRT increased time to BCR by an average of 1.5 years $(\mathrm{p}<0.0001)$ [Figure 2].

Conclusions: Low FT contributes to high-risk PC via increased GGG, stage, and likelihood of recurrence. Men with biochemically low FT benefit oncologically with normalization via TRT both via a $53 \%$ reduction in rate of BCR and a 1.5-year delay in time to BCR. These results argue against previous notions that high testosterone furthers PC progression and suggests the need for prospective studies assessing benefit of TRT in PC patients.

\section{MP17-17 Clinical study on Extraperitoneal approach in Robot-Assisted Radical Prostatectomy - Compared to Transperitoneal approach}

S Murata, R Yamaguchi, H Kokubun, N Makita, I Suzuki, R Suzuki, A Fukunaga, Y Abe, M Kubota, Y Tohi, N Tsutsumi, K Inoue, M Kawakita

\section{Kobe City Medical Center General Hospital}

Introduction \& Objective: To compare extraperitoneal (Ep) to transperitoneal (Tp) approach on the perioperative outcomes in robot-assisted radical prostatectomy (RARP) for localized prostate cancer.

Methods: From January 2014 to September 2018, a total of 460 patients underwent RARP with prostate cancer. Among them, 204 patients in Tp who underwent extended lymph-node dissection (ELND) and 224 patients in Ep who did not underwent ELND were chosen. Tp without ELND and Ep with ELND were excluded. RARP in Tp was underwent in $30^{\circ}$ Trendelenburg position, and in Ep was underwent in $15^{\circ}$ Trendelenburg position. ELND was underwent for patients whose risk of lymph node metastases was $>5 \sim 10 \%$ according to the preoperative Briganti nomogram. We evaluated perioperative outcomes including operative time, intraoperative blood loss, and complication rates. Results: There were no significant differences in preoperative median age (68.7 vs 68.3 years, $\mathrm{p}=0.52)$, BMI (23.6 vs $23.9 \mathrm{~kg} / \mathrm{m}^{2}, \mathrm{p}=0.31$ ), Charlson Comorbidity Index (2 vs 2 , $\mathrm{p}=0.02$ ) between Ep and Tp patients. Owing to preoperative selecting criteria, iPSA (8.97 vs 19.5$) \mathrm{ng} / \mathrm{ml}, \mathrm{p}=0.0001$ ), rate of high Gleason Score ( $\geqq 8 ; 9.38 \%$ vs $66.7 \%, \mathrm{p}<0.0001)$, rate of high cT stage ( $\geqq$ cT3; $3.57 \%$ vs $28.4 \%, \mathrm{p}<0.0001)$ were significantly lower in Ep than in Tp patients. In addition, the rate of nerve sparing $(32.6 \%$ vs $9.8 \%, \mathrm{p}<0.0001)$ was significantly greater in Ep approach than in Tp. Overall operative time, including ELND time in Tp patients, is significantly shorter (266 vs $361 \mathrm{~min}, \mathrm{p}<0.0001$ ), but console time without ELND is significantly longer (194 vs $173 \mathrm{~min}, \mathrm{p}=0.00005$ ). Significantly more intraoperative blood loss (354.8 vs $191.7 \mathrm{ml}, \mathrm{p}<0.0001)$ was seen in Ep patients. The rate of any high-grade complications (Clavien-Dindo Grade $\geqq$ III) was significantly greater in $\mathrm{Tp}$ (3.3\% vs $0 \%, \mathrm{p}=0.006)$, including $1(0.5 \%)$ rectal injury, 1 $(0.5 \%)$ ileal injury, $3(1.4 \%)$ abdominal wall scar hernia, 2 $(1.0 \%)$ port site hernia, and $1(0.5 \%)$ lymphorrhea.

Conclusions: These results indicate that RARP in Ep approach was safe and feasible, although it had 20 min longer operative time and $160 \mathrm{ml}$ more blood loss for prostatectomy than $\mathrm{Tp}$ approach.

\section{MP17-18 Management of urethro-vesical anastomotic disruption: Strategies for managing delayed healing of urethro-vesical anastomosis post robotic radical prostatectomy}

\section{LM Alzweri, EJ Shaw, R Thomas}

Tulane University School of Medicine

Introduction \& Objective: Radical prostatectomy remains a well-established treatment option for patients with localized prostate adenocarcinoma. Nevertheless, there is no consensus in the recent published literature on the management of UrethroVesical Anastomotic Leak (UVAL) following radical prostatectomy. Multiple factors have been associated with increased risk of this seemingly under reported complication, including and not limited to pelvic anatomy variations, developing pelvic hematoma, obesity, surgeons level of surgical skills and experience, the size of the prostate gland, TNM stage of the prostate cancer and potentially the surgical approach ; open, laparoscopic or robotic, in addition to the surgical technique and suture material used to reconstruct the Urethro-Vesical anastomosis (UVA).

Methods: We performed retrospective analysis of our Department's experience performing minimally invasive radical prostatectomy since 2002 and how me managed the rare cases of UVAL. We highlighted two recent cases of UVAL and their surgical management strategies, while reviewing the related recent published literature.

Results: It seems that the under reported UVAL incidence in the literature varies significantly across different studies (4.5\%-7.5\%) and $(25.7 \%)$ post salvage radical prostatectomy procedures, it used to be $(0.3 \%-15.4 \%)$ for the period from $1985-2011$.

We recommend following our suggested flow chart with risk stratification of cases into low and high risk, early recognition of UVAL in the post-operative course and prompt utilization of surgical options to minimize risk of long-term complication and improve outcomes. 


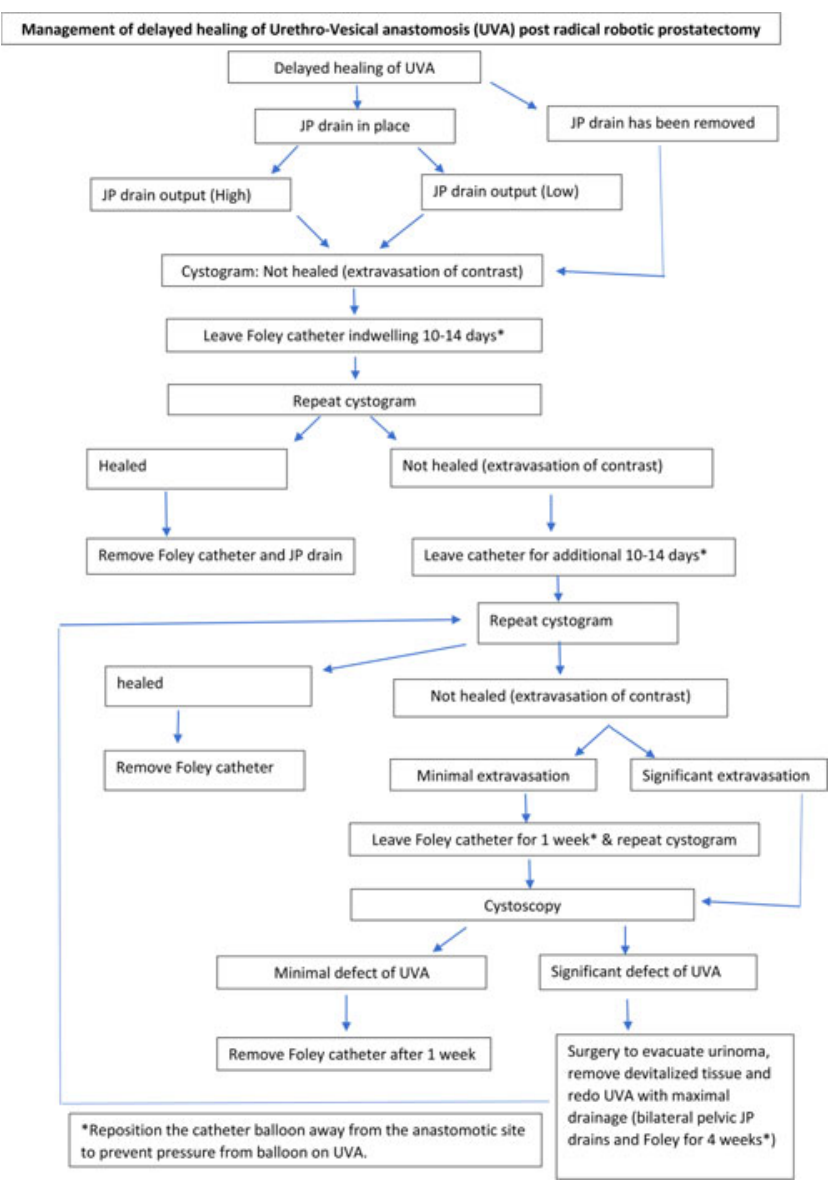

Conclusions: UVAL could be under reported due to lack of consensus on definitions and subsequent management. Partial disruption of the UVA is monumentally different from complete disruption. Any adverse outcome of the UVA poses a significant long-term consequence for the patient. Minimal leaks can be managed with maximum drainage and prolonged catheterization, while significant leak and disruption would require surgical intervention to fully drain the urinoma, removed the devitalized tissue affected by the urinoma and redo the anastomosis.

\section{MP17-19 Single Port radical prostatectomy versus Xi Multi-Port radical prostatectomy: a human factor analysis follow up study}

SM Talamini, WR Halgrimson, RW Dobbs, S Crivellaro

University of Illinois at Chicago

Introduction \& Objective: The process of learning a given task can be understood through the context of cognitive load theory. Cognitive load measures the number of informational elements that need to be processed to complete a task. It has been shown that an increase in overall cognitive load can impede the process of learning, resulting in decreased efficiency and suboptimal surgeon performance. The da Vinci Single Port (SP) robotic platform (Intuitive Surgical, Sunnyvale, CA) is a novel technology which offers new capabilities to the urologic surgeon, but its impact on surgeon cognitive load and performance is unknown. To better understand the impact of this new technology,

\begin{tabular}{lcccccccc} 
Operative Times for SinglePort and Multi-Port Radical Prostatectomy \\
\hline \multicolumn{7}{c}{ SinglePort } & \multicolumn{7}{c}{ Multi-Port } \\
\hline & $N$ & Mean & SD & $N$ & Mean & SD & P value \\
Operative Times (min) & 20 & 225 & 18.0 & 20 & 247 & 29.0 & 0.008 \\
Total Operative Time & 20 & 191 & 29.0 & 20 & 220 & 23.0 & 0.001 \\
Total Robotic Console Time & 20 & 176 & 29.0 & 20 & 205 & 22.0 & 0.001 \\
Total "Active" Console Time & 20 & 16 & 4.0 & 20 & 16 & 4.0 & 0.816 \\
Total Work Disruption Time & 20 & 34 & 23.0 & 20 & 26 & 18.0 & 0.264 \\
Setup, Docking and Closure Time & & & & & & & \\
& & 7 & & & & &
\end{tabular}

we have compared the SP and Multi-Port (MP) platforms through objective measurement of cognitive load during the crucial steps of a robotic-assisted laparoscopic prostatectomy (RALP).

Methods: We prospectively compared the first 20 SP and MP RALPs performed at our academic institution, all performed by a single experienced robotic surgeon over the same period. Comparisons were made with respect to mental workload (NASA Task Load Index), surgical workload (Surgery Task Load Index), team work (Observational Teamwork Assessment for Surgery) and workflow disruptions. Statistical analysis was performed using SPSS ${ }^{\circledR} 24$ (IBM, Armonk, NY) with $\mathrm{p}<0.05$ considered statistically significant. The mean is used to report performance metrics.

Results: All 20 SP and MP RALP procedures were performed between December 2018 and June 2019 by the same surgeon. No statistically significant difference was found between the MP and SP platforms in the cognitive workload experienced by the surgeon during the performance of a RALP for the NASA Task Load Index, Surgery Task Load Index or Observational Teamwork Assessment analyses. Workflow disruptions were similar between the two cohorts. The total robotic console time on the SP was notably less than the MP.

Conclusions: There was no significant difference with respect to cognitive load, human factor or workflow disruption measures between SP and MP platforms. Furthermore, operative time was decreased on the SP, suggesting that in the hands of an experienced robotic surgeon the adaptation of this technology may improve surgical efficiency.

MP17-20 Diagnostic and staging performance of mpMRI-US fusion prostate biopsy: prospective analysis on consecutive radical prostatectomy specimens from a multicentre series

M Ferriero, R Flammia, G Tuderti, R Mastroianni, U Anceschi, A Brassetti, M Oderda, P Gontero, A Giacobbe, G Muto, G Malossini, M Puglisi, A Peltier, K Pradeep, T Piechaud, J Descotes, R Papalia, S Guaglianone, M Gallucci, G Simone

"Regina Elena" National Cancer Institute

Introduction \& Objective: Despite the high accuracy of MRI in detecting prostate cancer, some clinically significant lesions remained still missed. In this study we assessed the diagnostic and staging performance of mpMR comparing Fusion (US/MRI guided) biopsy findings with radical prostatectomy specimens in a multicentric series.

Methods: Data of 399 consecutive patients with prostate cancer diagnosis at mp-MRI/US guided fusion biopsy who underwent minimally invasive radical prostatectomy were prospectively collected in a multicentre data set. All biopsies were performed 


\begin{tabular}{|c|c|}
\hline Table 1 & Median (IQR) or N (\%) \\
\hline Age & $66(60.75-70)$ \\
\hline Baseline PSA & $7.7(5-11)$ \\
\hline Volume & $40(32-56)$ \\
\hline Number of MRI suspicious foci & $1(1-2)$ \\
\hline Number of cores taken & $16(14-19)$ \\
\hline Number of target cores & $4(2-6)$ \\
\hline PIRADS score & $4(4-5)$ \\
\hline Pirads $<3$ & $9(2.3)$ \\
\hline Pirads 3 & $62(15.5)$ \\
\hline Pirads 4 & 207 (51.9) \\
\hline Pirads 5 & $121(30.3)$ \\
\hline \multicolumn{2}{|l|}{ Gleason score } \\
\hline 6 & $155(38.8)$ \\
\hline 7 & $187(46.9)$ \\
\hline 8 & $43(10.8)$ \\
\hline 9 & $14(3.6)$ \\
\hline \multicolumn{2}{|l|}{ Pathologic data } \\
\hline \multicolumn{2}{|l|}{ Gleason score } \\
\hline 6 & $120(32.8)$ \\
\hline 7 & $233(58.4)$ \\
\hline 8 & $27(6.8)$ \\
\hline 9 & $19(4.8)$ \\
\hline \multicolumn{2}{|l|}{ pT stage } \\
\hline pTza & $32(8)$ \\
\hline pIzb & $17(4.3)$ \\
\hline przc & $244(61.2)$ \\
\hline prзa & $83(20.8)$ \\
\hline prзb & $22(5.5)$ \\
\hline pT4 & $1(0.3)$ \\
\hline \multicolumn{2}{|l|}{$\mathrm{pN}$} \\
\hline pNx & $262(65.7)$ \\
\hline pNO & $89(24.6)$ \\
\hline $\mathrm{nN}+$ & $48(13.9)$ \\
\hline
\end{tabular}

using the UroStation (Koelis, France). MRI and Fusion Biopsy findings were compared with final pathologic report. Diagnostic performance of MRI-US fusion biopsy was evaluated in terms clinically significant PCa diagnosis, Gleason score upgrading at final pathology, presence of extraprostatic extension and of nodal involvement. In a subgroup of 60 patients with whole mount assessment of pathologic specimens a per core biopsy analysis was performed to assess exact correspondence between core biopsies and final pathologic findings and proper identification of the index lesion.

Results: Clinic and pathologic data of the whole cohort are reported into Table 1. Overall, MRI-US fusion biopsy missed 35 $(8.7 \%)$ clinically significant PCa. Biopsy Gleason score was confirmed on final pathology in 257 (64.4\%), upgraded in 87 (21.8\%) and downgrade in $55(13.8 \%)$ cases. The staging accuracy of MRI in predicting tumor side, extraprostatic extension and nodal involvement was $77.1 \%, 76.9 \%$ and $86.2 \%$, respectively. In the subgroup analysis of patients with whole mount pathologic assessment, at per core evaluation, 202/1504 (13.4\%) cores were positive for GS $>6$ out of the suspicious ROI at MRI. The mean ratio of suspicious ROI/ pathologic tumor foci was 0.63 (SD 0.30). The pathologic index lesion was missed by mpMRI-US fusion biopsy in 25\% (15/60) of the patients.

Conclusions: mpMRI and Fusion US/MRI guided prostate biopsy provided a reliable diagnostic and staging performance in detecting prostate cancer in patients receiving a surgical treat- ment. Despite significant improvements in diagnosis and staging, in $25 \%$ of cases the index lesion can be missed by mpMRI and Fusion US/MRI guided prostate biopsy

\section{MP17-21 Urinary and sexual function outcomes at 12 months post-radical prostatectomy: robotic-assisted versus laparoscopic approach}

\author{
C Moore, J Hoogenes, J Saini, A Molawi, B Shayegan, \\ ED Matsumoto
}

\section{McMaster University}

Introduction \& Objective: Radical prostatectomy (RP) is performed using open, robotic-assisted (RARP), and laparoscopic (LRP) approaches. The objectives of this study were to examine urinary and sexual function in prostate cancer (PC) patients at the 12-month mark post-RARP or LRP to identify any significant differences between groups. All cases were conducted by a single surgeon who has expertise with both approaches (>1000 LRPs and $>500$ RARPs).

Methods: Eligible patients underwent RARP or LRP and were followed regularly in clinic for at least 12 months. Patients must have completed the 20-item UCLA PC Index and the 5-item International Index of Erectile Function (IIEF-5) at the 12-month mark. These validated tools assess sexual, urinary, and bowel function using 5-point anchored Likert scales. Scores for each tool (UCLA Index out of 100 and IIEF-5 out of 25; higher scores indicate more favorable outcomes) were evaluated between groups, with additional focus on 3 domains: sexual bother, urinary bother, and daily pad usage. Along with descriptives, independent $t$-tests compared clinical characteristics and scores between groups.

Results: 90 patients were eligible (RARP $n=45$; LRP $n=45)$. Mean (SD) age was $62.2( \pm 6.7)$, baseline PSA was $7.7( \pm 5.5)$, 12 -month UCLA PC Index score was 58.5 ( \pm 10.2$)$. An equal percentage $(64.4 \%)$ of patients in both groups received erectile dysfunction (ED) treatment post-operatively. No significant differences were found between groups for the above variables. Bilateral nerve sparing (NS) was performed in $55.6 \%$ of RARP vs. $40.0 \%$ of LRP cases. Unilateral NS was used in RARP (28.9\%) vs. LRP (46.7\%). 12-month IIEF-5 scores were significantly different between groups $(\mathrm{RARP}=5.9 \quad( \pm 1.9)$; $\mathrm{LRP}=7.0( \pm 4.8) ; \mathrm{p}<0.05)$. RARP patients used fewer pads than LRP $(\mathrm{p}<0.05)$, and LRP patients had higher levels of sexual bother $(\mathrm{p}<0.05)$. Bilateral NS patients in both groups had significantly higher scores on the IIEF-5 $(\mathrm{p}=0.042)$. There were no differences between groups for the urinary bother domain.

Conclusions: RARP patients had significantly lower IIEF-5 scores, lower levels of sexual bother, used fewer pads, and had a higher rate of bilateral NS than LRP patients. The high rate of erectile dysfunction treatment and bilateral nerve sparing in all patients may relate to higher IIEF-5 scores, despite surgical approach. Continued follow-up past the 12-month post-operative mark will allow for a greater understanding of urinary and sexual issues in these patients. 


\section{MODERATED POSTER SESSION 18: LAPAROSCOPIC/ROBOTIC: RENAL (II)}

\section{MP18-01 Laparoscopic Donor Nephrectomy Without Drainage Does Not Increase Postoperative Morbidity}

\author{
D An, D You, J Han, K Kim, J Aum, Y Kim
}

Introduction \& Objective: Some authors addressed whether or not routine drainage is always necessary after partial nephrectomy or nephroureterectomy. However, the benefit of drainage versus their potential harm after laparoscopic donor nephrectomy has been questioned. Therefore, we conducted a comparative study to assess the need for routine drainage after laparoscopic donor nephrectomy. Methods: A prospectively maintained database of 136 donor nephrectomies consecutively performed by a single surgeon (DY) from July 14, 2014 to November 24, 2017, was reviewed. Since October 5, 2016, we decided to omit routine drainage after surgery. The former 80 donor nephrectomies drained routinely (Drainage group) and the latter 56 intended not to drain (Non-drainage group). Laparoscopic donor nephrectomy was performed as previously described, with minor modifications, using the umbilical incision as the kidney extraction site. Operative and convalescence parameters, and intraoperative and postoperative complications were compared between the groups. Intraoperative and postoperative complications within 90 days of surgery were graded using the Satava and modified Clavien classifications, respectively.

Results: Baseline characteristics were similar in the two groups, except for American Society of Anesthesiologists score II

\begin{tabular}{|c|c|c|c|}
\hline Characteristics & Drainage & Nondrainage & p Value \\
\hline Mean age $\pm \mathrm{SD}$, years & $44.2 \pm 11.3$ & $47.5 \pm 12.7$ & 0.122 \\
\hline Sex, male/female & $33 / 47$ & $24 / 32$ & 0.852 \\
\hline Relationship to recipient (\%) & & & 0.498 \\
\hline Related & $44(55.0)$ & $31(55.4)$ & \\
\hline Spouse/partner & $26(32.5)$ & $22(39.3)$ & \\
\hline Distantly related & $5(6.3)$ & $1(1.8)$ & \\
\hline Unrelated & $5(6.3)$ & $2(3.6)$ & \\
\hline Mean body mass index $\pm \mathrm{SD}, \mathrm{kg} / \mathrm{m}^{2}$ & $24.2 \pm 3.2$ & $24.3 \pm 3.3$ & 0.870 \\
\hline Diabetes mellitus (\%) & $0(0)$ & $3(5.4)$ & 0.068 \\
\hline Hypertension (\%) & $3(3.8)$ & $7(12.5)$ & 0.091 \\
\hline History of abdominal surgery (\%) & $18(22.5)$ & $16(28.6)$ & 0.421 \\
\hline Concomitant surgery $(\%)$ & $1(3.3)$ & $1(3.3)$ & 1.000 \\
\hline ASA score (\%) & & & $<0.001$ \\
\hline 1 & $68(85.0)$ & $29(51.8)$ & \\
\hline II & $12(15.0)$ & $27(48.2)$ & \\
\hline Laterality, right/left & $34 / 46$ & $26 / 30$ & 0.650 \\
\hline \multicolumn{3}{|l|}{ No. artery $(\%)$} & 0.581 \\
\hline 1 & $64(80.0)$ & $41(73.2)$ & \\
\hline 2 & $13(16.3)$ & $14(25.0)$ & \\
\hline 3 & $3(3.8)$ & $1(1.8)$ & \\
\hline \multicolumn{3}{|l|}{ No. vein (\%) } & \multirow{5}{*}{0.551} \\
\hline 1 & $65(81.3)$ & $45(80.4)$ & \\
\hline 2 & $11(13.8)$ & $11(19.6)$ & \\
\hline 3 & $3(3.8)$ & $0(0)$ & \\
\hline 4 & $1(1.3)$ & $0(0)$ & \\
\hline Mean preoperative hemoglobin $\pm \mathrm{SD}, \mathrm{g} / \mathrm{dL}$ & $13.8 \pm 1.6$ & $13.6 \pm 1.6$ & 0.455 \\
\hline Mean preoperative GFR $\pm \mathrm{SD}, \mathrm{mg} / \mathrm{dL}$ & $106.6 \pm 12.8$ & $103.0 \pm 15.0$ & 0.127 \\
\hline
\end{tabular}

(Drainage vs Non-drainage group $=15.0 \%$ vs $48.2 \% ; \mathrm{P}<0.001$ ). One patient in the Non-drainage group suffered from massive bleeding due to endoscopic stapler malfunction and drained unintentionally. There were no significant between-group differences in total operation time, warm ischemia time, visual analogue scale pain scores on postoperative day 1 and at discharge, and interval to return to a regular diet. The Drainage group showed less estimated blood loss $(477.8 \mathrm{~mL}$ vs $614.3 \mathrm{~mL}$; $\mathrm{P}=0.007)$, whereas the Non-drainage group showed shorter interval to removal of the drain $(3.4$ days vs 0.1 day; $\mathrm{P}<0.001)$ and overall hospital stay (5.4 days vs 4.9 days; $\mathrm{P}=0.026$ ). The overall intraoperative complication rate was similar in the two groups (22.5\% vs $30.4 \%$; $\mathrm{P}=0.302)$. The overall postoperative complication rate was similar in the two groups $(57.5 \%$ vs. $51.8 \%$; $\mathrm{P}=0.510$ ).

Conclusions: Laparoscopic donor nephrectomy without routine drainage is feasible and safe. Donors not to drain might benefit from a shorter hospital stay without an increase in postoperative morbidity.
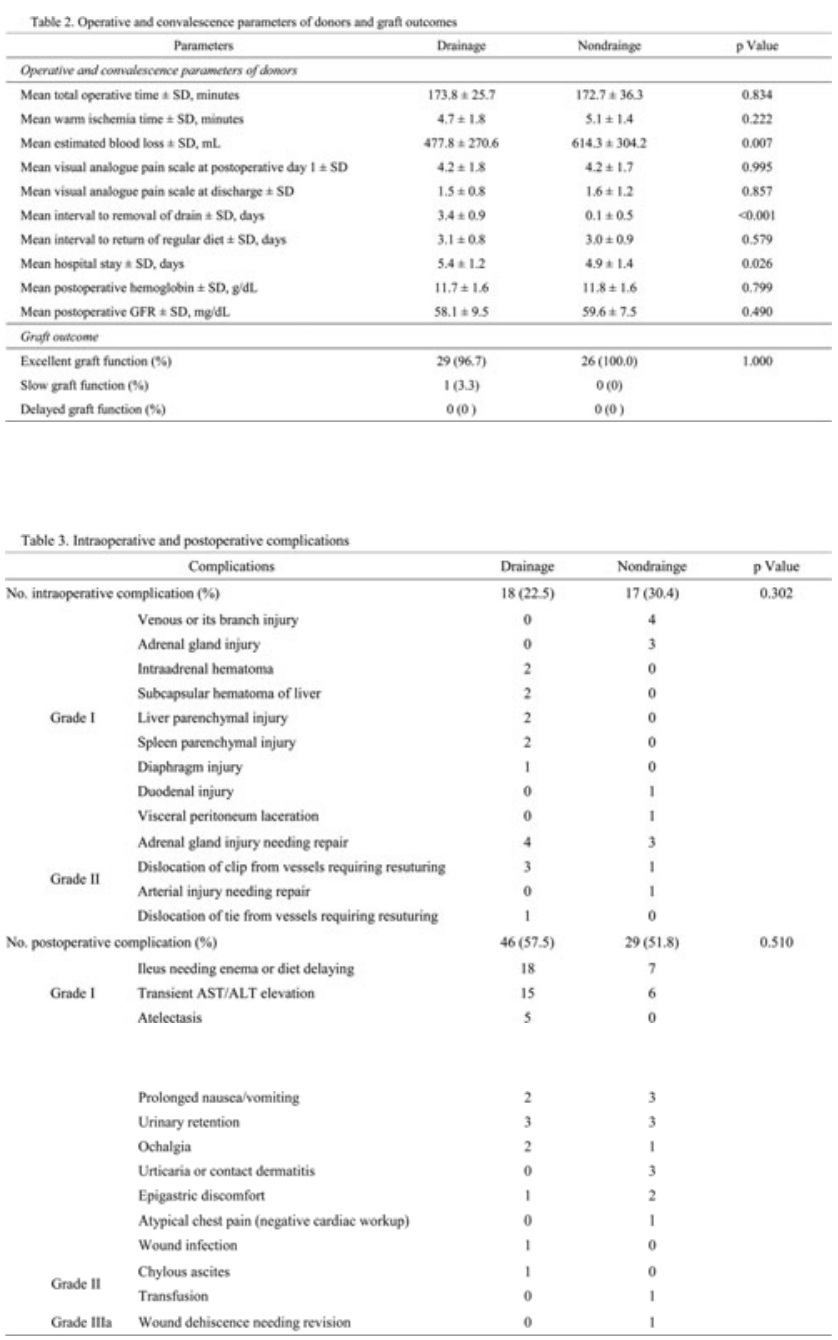
MP18-02 Local Regional Recurrence of Renal Cell Carcinoma after Robotic Partial Nephrectomy

PM Youssef, JC Deolankar, PD Kothari, SS Komaravolu, D Kim, N Diab, H Koster, M Ahmed, R Munver, G Lovallo, MD Stifelman

Introduction \& Objective: Robotic partial nephrectomy (RPN) has been established as an important minimally invasive nephron-sparing technique that is implemented for patients with renal cell carcinoma (RCC). The objective of this study is to determine the incidence of local regional recurrence of disease in patients who have undergone an RPN for the treatment of RCC. Methods: Using our IRB-approved prospective and retrospective databases, we analyzed all patients who underwent an RPN for the treatment of RCC between the years of 2011 and 2019. We excluded all patients who were found to have benign pa-

\begin{tabular}{|c|c|c|}
\hline \multicolumn{2}{|l|}{ Variable } & Value \\
\hline \multirow{2}{*}{\multicolumn{2}{|c|}{$\begin{array}{l}\text { Mean age, year (SD) } \\
\text { Mean bodv mass index, kg/m2 (SD) }\end{array}$}} & $63.3(12.1)$ \\
\hline & & $29.5(5.9)$ \\
\hline \multicolumn{2}{|l|}{$\begin{array}{l}\text { Mean body mass index, kg/m2 (SD) } \\
\text { Pre-op Creatinine (SD) }\end{array}$} & $0.96(0.27)$ \\
\hline \multirow[t]{2}{*}{ Gender, $\mathbf{n}(\%)$} & Male & $125(71.0)$ \\
\hline & Female & $51(29.0)$ \\
\hline \multirow[t]{5}{*}{ Race, n (\%) } & White & $136(77.3)$ \\
\hline & Black or African American & $13(7.4)$ \\
\hline & Asian & $6(3.4)$ \\
\hline & Other & $7(4.0)$ \\
\hline & Unknown / Not Reported & $14(8.0)$ \\
\hline \multirow[t]{3}{*}{ Ethnicity, n (\%) } & Hispanic or Latino & $32(18.2)$ \\
\hline & Not Hispanic or Latino & $143(81.3)$ \\
\hline & Unknown & $1(0.6)$ \\
\hline \multirow[t]{4}{*}{ Smoking status, n (\%) } & Active & $16(9.1)$ \\
\hline & Former & $58(33.0)$ \\
\hline & Never & $101(57.4)$ \\
\hline & Unknown / Not Reported & $1(0.6)$ \\
\hline \multirow{9}{*}{ Nephrometry Score, n (\%) } & 4.0 & $9(5.1)$ \\
\hline & 5.0 & $13(7.4)$ \\
\hline & 6.0 & $9(5.1)$ \\
\hline & 7.0 & $11(6.3)$ \\
\hline & 8.0 & $21(11.9)$ \\
\hline & 9.0 & $21(11.9)$ \\
\hline & 10.0 & $10(5.7)$ \\
\hline & 11.0 & $3(1.7)$ \\
\hline & Unknown / Not Reported & $79(44.9)$ \\
\hline \multirow{3}{*}{$\begin{array}{l}\text { Robotic/Laparoscopic Approach, n } \\
\text { (\%) }\end{array}$} & Transperitoneal & $150(85.2)$ \\
\hline & Retroperitoneal & 21 (11.9) \\
\hline & Unknown / Not Reported & $5(2.8)$ \\
\hline \multirow[t]{2}{*}{ Laterality, n (\%) } & Right & $89(50.6)$ \\
\hline & Left & $87(49.4)$ \\
\hline \multirow[t]{5}{*}{ Fuhrman Grade, n (\%) } & 1 & $1(0.6)$ \\
\hline & 2 & $106(60.2)$ \\
\hline & 3 & $39(22.2)$ \\
\hline & 4 & $5(2.8)$ \\
\hline & Unknown / Not Reported & $25(14.2)$ \\
\hline \multirow[t]{4}{*}{ T Stage, n (\%) } & Tla & $135(76.7)$ \\
\hline & $\mathrm{Tlb}$ & $31(17.6)$ \\
\hline & $\mathrm{T} 2 \mathrm{a}$ & $2(1.1)$ \\
\hline & T3a & $8(4.5)$ \\
\hline \multirow[t]{3}{*}{ N Stage, n (\%) } & NX & $167(94.9)$ \\
\hline & No & $9(5.1)$ \\
\hline & N1 & $0(0)$ \\
\hline \multirow[t]{2}{*}{ M Stage, n (\%) } & M0 & $176(100)$ \\
\hline & MI & $0(0)$ \\
\hline \multirow[t]{2}{*}{ Margin Status, n (\%) } & Negative & $166(94.3)$ \\
\hline & Focally Positive & $10(5.7)$ \\
\hline
\end{tabular}

Table 1. Demographic and tumor characteristics for patients who underwent RPN for solitary RCC.

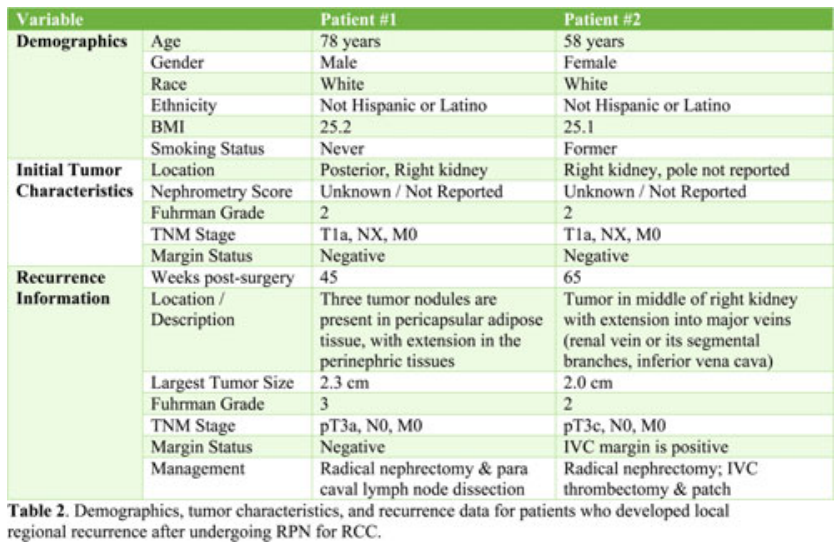

thology. Local regional recurrence was defined as recurrent disease in the partial nephrectomy bed or the surrounding operative field. We report patient demographics and tumor characteristics along with data on recurrence in Tables $1 \& 2$.

Results: A total of 176 patients were extracted from the database with a mean follow-up period of $74.4+/-51.7$ weeks. Table 1 reveals a mean age of 63.3 years, $25 \%$ rate of grade 3 tumors or higher, and $94.3 \%$ rate of stage T1a-b tumors. Only 2 patients developed local regional recurrence, yielding an incidence of $1.1 \%$. Table 2 reveals that both patients initially had grade 2, T1a tumors and developed recurrence at 45 and 65 weeks of follow-up that were both managed with radical nephrectomies.

Conclusions: The rate of local regional recurrence is quite low $(1.1 \%)$ with utilization of RPN for the treatment of RCC. Studies in which the rate of stage T1a-b tumors were above $90 \%$, similar to our study, have reported local recurrence rates between $0-$ $1.2 \%$ after laparoscopic techniques for the treatment of RCC, compared to $1.9 \%$ with the open technique (Hafez et al., 1997 \& Permpongkosol et al., 2006). Therefore, our study shows that $\mathrm{RPN}$ is at least comparable to the open technique with respect to local regional recurrence rates. Interestingly, both patients who developed recurrence originally had low grade T1a tumors, which highlights the importance of close follow-up for all patients regardless of initial tumor features.

\section{MP18-03 The Impact of Postoperative AKI after Robotic PN on Patient Outcomes and Predictors to Ascertain Risk}

\author{
O Omidele, M Finkelstein, M Palese
}

Icahn School of Medicine

Introduction \& Objective: Post-operative acute kidney injury (AKI) is a common complication of renal surgery. One of the goals of robotic PN is to maintain kidney function in both the short and long term. Irrespective of cause, AKI has been associated with progression to CKD and irreversible renal injury. Thus, it is important to identify predictors and patient demographics associated with new-onset AKI after robotic PN.

Methods: We retrospectively reviewed all robotic (ICD-9 17.4) partial nephrectomies (PN; ICD-9 55.4) reported in the Statewide Planning and Research Cooperative Systems (SPARCS) database of New York State (NYS) from 2008 to 2015. Patients were divided into two groups based on the presence or absence of a postoperative AKI. Patient characteristics included patient age, race, ethnicity, Charlson Comorbidity Index (CCI), and source of payment. Outcome measures included length of stay, 30-day readmission rate, and mortality at 1 year.

Results: There were 124 (3\%) patients who suffered a postoperative AKI following robotic PN and 4287 (97\%) patients without AKI. The average age of a patient with a postoperative AKI was 65.56 compared to $59.11(\mathrm{p}<.001)$. Of the patients who had an AKI, $72 \%$ were male compared to just $62 \%$ in the nonAKI group $(\mathrm{p}<.001)$. There were a higher percentage of Medicaid patients in the AKI group compared to the non-AKI group $(\mathrm{p}<.001)$. Patients with AKI had longer lengths of stay, higher proportion of 30-day readmissions, and increased mortality at 1 year $(\mathrm{p}<.001)$. Positive predictors of postoperative AKI following robotic PN include elevated CCI, increased age between 64-79, and procedure conducted by a physician who graduated more than 30 years ago $(\mathrm{p}<.001)$. The only negative predictor of postoperative AKI was medium volume hospital status $(\mathrm{p}=0.026)$. 


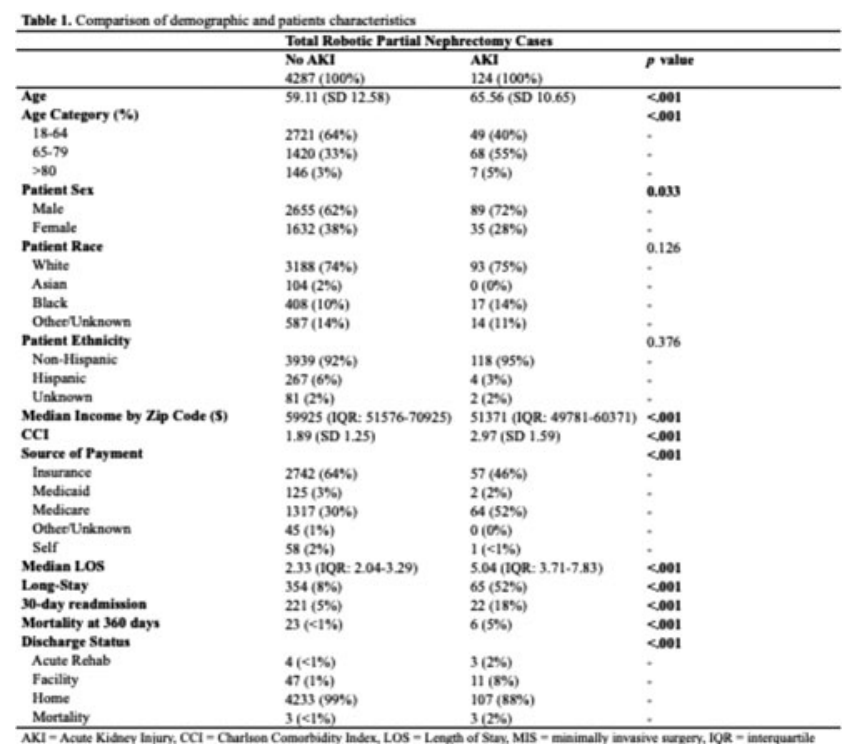

\begin{tabular}{|c|c|c|c|}
\hline \multirow[b]{2}{*}{ Variable } & \multicolumn{3}{|c|}{ AKI } \\
\hline & $\mathrm{OR}$ & $\mathrm{Cl}(2.5 \%-97.5 \%)$ & $p$ value \\
\hline CCI & 1.63 & $1.46-1.83$ & $<.001$ \\
\hline \multicolumn{4}{|l|}{ Patient Race } \\
\hline White & Reference & & \\
\hline OtheoUnknown & 0.95 & $0.51-1.64$ & 0.851 \\
\hline Black & 1.71 & $0.96-2.92$ & 0.054 \\
\hline \multicolumn{4}{|l|}{ Source of Payment } \\
\hline Insurance & Reference & & \\
\hline Medicaid & 0.68 & $0.11-2.29$ & 0.603 \\
\hline OtheoUnknown & 1.10 & $0.67-1.80$ & 0.713 \\
\hline \multicolumn{4}{|c|}{ Physician Volume Level } \\
\hline Low & Reference & & \\
\hline Medium & 0.69 & $0.43-1.11$ & 0.130 \\
\hline High & 0.87 & $0.46-1.66$ & 0.668 \\
\hline \multicolumn{4}{|c|}{ Hospital Volume Level } \\
\hline Low & Reference & & \\
\hline Medium & 0.57 & $0.34-0.93$ & 0.026 \\
\hline High & 0.80 & $0.42-1.49$ & 0.493 \\
\hline \multicolumn{4}{|c|}{ Patient Agge Category } \\
\hline $18-64$ & Reference & & \\
\hline $64-79$ & 2.24 & $1.37-3.68$ & $<001$ \\
\hline$>80$ & 2.24 & $0.84-5.27$ & 0.081 \\
\hline \multicolumn{4}{|c|}{ Physician Time From Graduation } \\
\hline $0-15$ & Reference & & \\
\hline $16-30$ & 1.36 & $0.89-2.11$ & 0.161 \\
\hline $31-100$ & 2.83 & $1.51-5.13$ & $<001$ \\
\hline
\end{tabular}

Conclusions: The study suggests that postoperative AKI following robotic PN can have a significant impact on patient outcomes such as length of stay, readmission, and mortality. Additionally, patients at risk of postoperative AKI include patients with higher comorbidity index and elevated age; whereas, factors protective of a postoperative AKI include medium hospital volume.

MP18-04 Retroperitoneoscopic or laparoscopic? which is the best for posterior tumors partial nephrectomy?

J Correia, C Tavares, P Principe, R Versos, R Borges, M Silva-Ramos

Department of Urology, Centro Hospitalar Universitário do Porto; Hospital Lusíadas Porto
Introduction \& Objective: In the past years several publications have focused on the role of retroperitoneoscopic kidney surgery. The retroperitoneal route provides a more direct and rapid access to the kidney and the renal hilum and avoids intra-peritoneal organs manipulation, nevertheless it is seldomly used in partial nephrectomies. Posterior tumors seem to be the more suitable to this approach. In this study we investigated the peri-operatory and oncological outcomes of both laparoscopic and retroperitoneoscopic partial nephrectomy for posterior tumors.

Methods: We reviewed the medical charts of the 112 minimallyinvasive partial nephrectomies performed by the same group of surgeons in two institutions between April 2011 and April 2019. Baseline demographic characteristics, perioperative and oncological outcomes were compared between patients in the laparoscopic group (LG) and those in the retroperitoneoscopic group (RG). The RENAL nephrometry score was used to grade surgical complexity and register tumor location.

Results: Forty-three patients (38\%) had posterior tumors, 31 were operated using a laparoscopic approach and 12 using a retro-

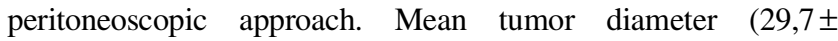
$10,7 \mathrm{~mm}$ in $\mathrm{LG}$ and $26,7 \pm 10,1 \mathrm{~mm}$ in RG) mean RENAL score $(6,9 \pm 1,8$ in $\mathrm{LG}$ and $6,3 \pm 1,3$ in RG) were similar in both groups. Mean warm ischemia time (18,7 $\pm 5,2$ in $L G$ and 20,2 $\pm 6,6$ in RG); mean operative time $(145,2 \pm 39,4$ in $\mathrm{LG}$ and $135,9 \pm 43,6$ minutes in $\mathrm{RG})$ and mean hospitalization time $(4,71 \pm 2,51$ in $\mathrm{LG}$ and $4,45 \pm 5,9$ days in $R G$ ) were also similar. Blood loss was significantly less in the RG, $69,0 \pm 34,8 \mathrm{ml}$ versus $160,0 \pm 150 \mathrm{ml}$ in LG. Tumor histology, marginal status and Clavien class ${ }^{3}$ III were similar in both groups.

Conclusions: The retroperitoneal approach showed lesser blood loss and generally equivalent perioperative results to the laparoscopic approach. Retroperitoneoscopic partial nephrectomy may be a safer option for the treatment of posterior renal masses.

\section{MP18-05 The Effect of Adhesive Perirenal Fat on Operative Outcomes}

KE Okhawere, AA Elbakry, BD Dayal, A Beksac, K Meilika, J Daza, J Sfakianos, DD Eun, A Hemal, R Abaza, A Bhandari, R Mehrazin, J Porter, KK Badani

Icahn School of Medicine at Mount Sinai

Introduction \& Objective: Partial nephrectomy for management of renal tumors can be challenging for reasons not directly related to the tumor itself or the degree of its complexity. The aim of our study is to understand the effect of the adhesive perirenal fat on the outcome of robotic partial nephrectomy.

Methods: We reviewed our prospectively maintained multicenter database to identify patients with available data about reported adhesive perirenal fat during robotic partial nephrectomy procedures. Of the 326 patients, baseline data, perioperative and post-operative data were compared among 36 (11.04\%) patients with reported presence of adhesive perirenal fat (based on intra-operative finding) vs 290 (88.96\%) without. The two groups were analyzed using Mann-Whitney U test, Chi Square test, and Fisher's exact. Unadjusted and adjusted multivariable linear regression models were used to assess significant perioperative outcomes which were log transformed.

Results: Patient with adhesive perirenal fat were more likely to be males ( 80.6 vs $19.4 \%$; $=0.012$ ), have a higher body mass index (BMI) (31.0 vs $\left.27.7 \mathrm{~kg} / \mathrm{m}^{2} ; \mathrm{p}=0.007\right)$, higher Charlson comorbidity index (CCI) (4 vs 3; p=0.004), higher ASA score (ASA 2, 30.0 vs $57.6 \%$; ASA 3, 63.3 vs $40.6 \%$; ASA 46.7 vs 
Table 1: Baseline demographic and tumor characteristics by adhesive perirenal fat

\begin{tabular}{|c|c|c|c|}
\hline & \multicolumn{2}{|c|}{ Perirenal fat } & \multirow[b]{2}{*}{ P-value } \\
\hline & No adhesive & Adhesive & \\
\hline & $290(88.96)$ & $36(11.04)$ & \\
\hline Age (years) & $64(54,70)$ & $65(60,74)$ & 0.163 \\
\hline \multicolumn{4}{|l|}{ Sex } \\
\hline Male & $169(58.9)$ & $29(80.6)$ & 0.012 \\
\hline Female & $118(41.1)$ & $7(19.4)$ & \\
\hline $\mathrm{BMI}\left(\mathrm{kg} / \mathrm{m}^{2}\right)$ & $27.7(22.1,32.2)$ & $31.0(27.3,37.7)$ & 0.007 \\
\hline CCI & $3(2,4)$ & $4(3,6)$ & 0.004 \\
\hline \multicolumn{4}{|l|}{ ASA Score } \\
\hline 1 & $2(0.9)$ & $0(0.00)$ & 0.005 \\
\hline 2 & $133(57.6)$ & $9(30.0)$ & \\
\hline 3 & $94(40.6)$ & $19(63.3)$ & \\
\hline 4 & $2(0.9)$ & $2(6.7)$ & \\
\hline Tumor size $(\mathrm{cm})$ & $3.0(2.1,4.4)$ & $3.3(2.0,4.8)$ & 0.547 \\
\hline Renal score & $7(5,8)$ & $7(6,8)$ & 0.799 \\
\hline Baseline eGFR ( $\mathrm{ml} / \mathrm{min}$ ) & $76.7(66.2,91.1)$ & $68.1(59.3,79.1)$ & 0.016 \\
\hline
\end{tabular}

\begin{tabular}{|c|c|c|c|}
\hline & \multicolumn{2}{|c|}{ Perirenal fat } & \multirow[b]{2}{*}{ P-value } \\
\hline & No adhesive & Adhesive & \\
\hline Warm ischemia time (min) & $12(10,15)$ & $15(14,18)$ & $<0.001$ \\
\hline Operative time (min) & $145(126,171)$ & $169(157,199)$ & 0.001 \\
\hline Estimated blood loss (ml) & $50(50,100)$ & $50(50,100)$ & 0.481 \\
\hline \multicolumn{4}{|l|}{ Blood Transfusion } \\
\hline No & $181(98.4)$ & $12(100.0)$ & 1.000 \\
\hline Yes & $3(1.6)$ & $0(0.0)$ & \\
\hline \multicolumn{4}{|l|}{ Intra operative complication } \\
\hline No & $288(99.3)$ & $34(94.44)$ & 0.062 \\
\hline Yes & $2(0.69)$ & $2(5.56)$ & \\
\hline \multicolumn{4}{|l|}{ Conversion to open } \\
\hline No & $285(98.6)$ & $36(100.0)$ & 1.000 \\
\hline Yes & $4(1.4)$ & $9(0.0)$ & \\
\hline Conversion to radical & & & 0.604 \\
\hline No & $281(96.9)$ & $36(100.0)$ & \\
\hline Yes & $9(3.1)$ & $0(0.0)$ & \\
\hline \multicolumn{4}{|l|}{ Major complication } \\
\hline No (Clavien <3) & $142(95.9)$ & $25(96.2)$ & 1.000 \\
\hline Yes (Clavien $\geq 3$ ) & $6(4.0)$ & $1(3.8)$ & \\
\hline Length of stay (days) & $1(1,2)$ & $1(1,1)$ & 0.270 \\
\hline
\end{tabular}

$0.9 \% ; \mathrm{p}=0.005)$, lower baseline eGFR (68.1 vs $76.7 \mathrm{ml} / \mathrm{min}$; $\mathrm{p}=0.016)$, longer warm ischemia time (WIT) $(15 ; \mathrm{IQR}-14,18 \mathrm{vs}$ 12; IQR 10, $15 \mathrm{~min} ; \mathrm{p}=<0.001)$ and prolonged operative time (169 vs $145 \mathrm{~min} ; \mathrm{p}=0.001)$. Estimated blood loss $(\mathrm{EBL})(\mathrm{p}=0.481)$, blood transfusion $(\mathrm{p}=1.000)$, intraoperative complication $(\mathrm{p}=0.062)$, conversion to open $(\mathrm{p}=1.000)$, conversion to radical $(\mathrm{p}=0.604)$, major complication $(\mathrm{p}=1.000)$ and length of hospital stay (LOS) $(\mathrm{p}=0.270)$ were not significantly associated with the presence of adhesive perirenal fat. Controlling for age, sex, BMI, CCI, tumor size, surgeon, total R.E.N.A.L score, baseline eGFR, and ASA score in the multivariate analysis of the different perioperative outcomes, adhesive perirenal fat was significantly associated with longer WIT $(=17.4 \%, 95 \% \mathrm{CI}-4.1 \%, 36.6 \%)$ and longer operative time $(=15.0 \%, 95 \% \mathrm{CI}-(5.1 \%, 27.1 \%)$.

Conclusions: We found that adhesive perirenal fat was associated with lower baseline GFR and was more prevalent among male patients who had more comorbidities and a higher BMI. Length of hospital stay, complication rates and estimated blood loss were similar. Therefore, tools for pre-op characterization of adhesive fat are needed.

MP18-06 The effect of retroperitoneal approach at robotic partial nephrectomy for post-operative VAS pain score: Match-Paired Case-Control Study

B Kim, T Shin, W Jung, J Ha, c Park, C Kim

Department of Urology, Keimyung University School of Medicine, Dongsan Hospital

Introduction \& Objective: We compared the perioperative and functional outcomes between trans-peritoneal robotic partial nephrectomy (TRPN) and retro-peritoneal robotic partial nephrectomy (RRPN).

Methods: We retrospectively reviewed the data of 64 patients who underwent robotic partial nephrectomy (RPN) between July 2013 and July 2018. TRPN was conducted 50 patients and RRPN was conducted 14 patients. Patient pairs were matched based on age at operation, RENAL nephrometry score and pre-operative GFR. Patient demographics, operative and post-operative outcomes were compared.

Results: From 65 patients with RPN, 14 pairs were matched. There were no significant differences in mean age (58.64 vs 60.29yr), gender (M:F; 8:6 vs 8:6), body mass index $\left(24.56 \pm 2.82\right.$ vs $\left.24.93 \pm 2.74 \mathrm{~kg} / \mathrm{m}^{2}\right)$, tumor size $(29.57 \pm 13.92$ vs $28.50 \pm 12.65 \mathrm{~mm})$, RENAL nephrometry score $(6.29 \pm 1.68 \mathrm{vs}$ $6.29 \pm 1.68)$, and pre-operative GFR $(92.96 \pm 24.74$ vs $86.23 \pm$ $23.36 \mathrm{~mL} / \mathrm{min} / 1.73 \mathrm{~m}^{2}$ ). For perioperative outcomes, the operative time (174.79 \pm 48.57 vs $158.57 \pm 49.05 \mathrm{~min})$, Warm ischemic time (15.86 \pm 4.62 vs $15.99 \pm 6.44 \mathrm{~min})$ and estimated blood loss $(234.29 \pm 234.71$ vs $157.86 \pm 156.40 \mathrm{ml})$ were not different between both groups. The rate of eGFR $<90 \%$ that compared to preoperation (28.6\% vs $28.6 \%$ ) also no significant different. But, at RRPN group showed significantly low post-operative maximal VAS pain score $(6.43 \pm 1.09$ vs $4.79 \pm 2.08, \mathrm{p}=0.015)$.

Conclusions: There were no differences for perioperative and functional outcomes between TRPN and RRPN. But RRPN group has low post-operative VAS pain score and has been felt more comfortable.

\section{MP18-07 Surgical treatment of local retroperitoneal recurrence after retroperitoneal laparoscopic radical nephrectomy for renal cell carcinoma}

H Kim, H Choe, D Lee, S Lee

\section{St. Vincent's Hospital}

Introduction \& Objective: Local retroperitoneal recurrence of renal cell carcinoma after radical nephrectomy is known to have a poor prognosis. Aggressive surgical excision offers the highest survival rate but not without high morbidity, especially bowelrelated complications. Retroperitoneal approach for the initial radical nephrectomy may have advantage in this aspect, as bowel adhesion may be less substantial when subsequent surgery of retroperitoneal recurrence is undertaken. We describe our experience of laparoscopic treatment of local retroperitoneal recurrence after retroperitoneal laparoscopic radical nephrectomy (LRN) for renal cell carcinoma.

Methods: Retrospective chart review of all patients who underwent laparoscopic excision of local retroperitoneal recurrence after retroperitoneal LRN for renal cell carcinoma between

Table 1. Baseline information of the initial retroperitoneal laparoscopic radical nephrectomy

\begin{tabular}{|l|l|l|l|l|l|}
\hline Patient & $a$ & $b$ & $c$ & $d$ & $e$ \\
\hline Age & 42 & 49 & 53 & 74 & 81 \\
\hline Sex & Male & Male & Male & Female & Male \\
\hline BMI $\left(\mathrm{kg} / \mathrm{m}^{2}\right)$ & 26.6 & 20.4 & 29.9 & 25.6 & 24.1 \\
\hline ASA class & 1 & 2 & 2 & 2 & 2 \\
\hline Tumor laterality & Right & Right & Left & Right & Left \\
\hline Tumor stage & & & & & \\
T & $3 \mathrm{a}$ & $3 \mathrm{a}$ & $2 \mathrm{a}$ & $3 \mathrm{a}$ & $1 \mathrm{~b}$ \\
N & 0 & 0 & 0 & 0 & 0 \\
M & 0 & 0 & 0 & 0 & 0 \\
\hline Tumor size & 7.5 & 3.7 & 5.5 & 9.4 & 6.5 \\
\hline Tumor pathology & Clear cell & Clear cell & Clear cell & Clear cell & Clear cell \\
Cell type & 2 & 4 & 3 & 2 \\
Fuhrman grade & 2 & 2 & & \\
\hline
\end{tabular}


Table 2. Details of the retroperitoneal recurrence

\begin{tabular}{|l|l|l|l|l|l|}
\hline Patient & $a$ & $b$ & $c$ & $d$ & \\
\hline $\begin{array}{l}\text { Tumor size } \\
\text { (cm) }\end{array}$ & 1.0 & 8.8 & 3.4 & 1.2 & 1.8 \\
\hline Multiplicity & Single & Single & Multiple & Multiple & Single \\
\hline Location & Pararenal fascia & Psoas muscle & $\begin{array}{l}\text { Psoas muscle } \\
\text { Renal fossa } \\
\text { Pararenal fascia }\end{array}$ & $\begin{array}{l}\text { Psoas muscle } \\
\text { Abdominal wall }\end{array}$ & Psoas muscle \\
\hline $\begin{array}{l}\text { Interval* } \\
\text { (months) }\end{array}$ & 19 & 11 & 7 & 3 & 20 \\
\hline $\begin{array}{l}\text { Operation } \\
\text { modality }\end{array}$ & Robotic & Robotic & Robotic & Laparoscopic & Laparoscopic \\
\hline $\begin{array}{l}\text { Operation time } \\
\text { (minutes) }\end{array}$ & 155 & 395 & 450 & $\begin{array}{l}205 \\
\text { (co-op with TS) }\end{array}$ & 125 \\
\hline $\begin{array}{l}\text { Lengh of stay } \\
\text { (days) }\end{array}$ & 6 & 8 & 5 & 6 & 7 \\
\hline Complication & None & $\begin{array}{l}\text { Clavien-Dindo } \\
\text { II (transfusion) }\end{array}$ & None & None & None \\
\hline Pathology & Granuloma & Cancer & Cancer & Cancer & Cancer \\
\hline $\begin{array}{l}\text { Follow up after } \\
\text { recurrence op }\end{array}$ & 50 & 5 & 51 & 33 & 3 \\
\hline $\begin{array}{l}\text { Metastatic } \\
\text { progression }\end{array}$ & None & $\begin{array}{l}\text { Liver } \\
\text { Lung } \\
\text { Peritoneum }\end{array}$ & None & Lung & Peritoneum \\
\hline $\begin{array}{l}\text { Adjuvant } \\
\text { therapy }\end{array}$ & None & $\begin{array}{l}\text { Sunitinib } \\
\text { Afinitor }\end{array}$ & None & Pazopanib & Pazopanib \\
\hline CSS (months) & 70 & 28 (expired) & 58 & 37 & 24 \\
\hline interval between the initial nephrectomy and retroperitoneal recurrence excision & \\
\hline
\end{tabular}

TS = thoracic surgery
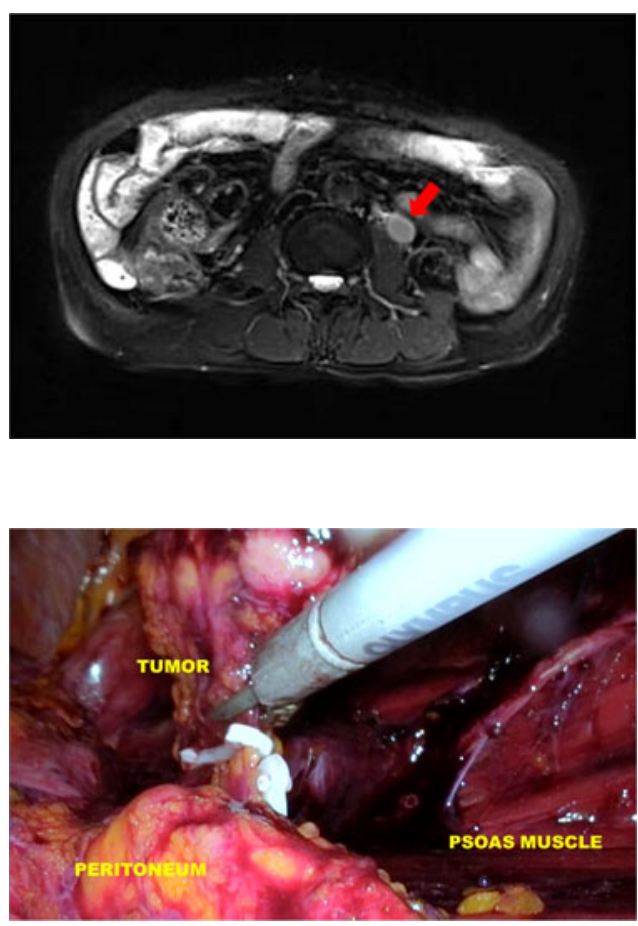

November 2009 and February 2019 was done. Baseline patient characteristics and information of the initial LRN was recorded. Details of the subsequent surgery for local retroperitoneal recurrence were also recorded, including tumor size, multiplicity, location, operation time, complications, pathology, metastatic progression, etc. The surgeries for retroperitoneal recurrence were all done laparoscopically through a transperitoneal approach.

Results: Of the 183 retroperitoneal radical nephrectomies (165 laparoscopic, 18 robotic) performed during the period, there were 5 cases with suspected local retroperitoneal recurrences who subsequently underwent surgical excision. None of them had lymph node or distant metastasis at the time of the initial LRN. The size of the recurrent tumors ranged between 1.0 and $8.8 \mathrm{~cm}$ and most of them were found abutting the psoas muscle. Three patients underwent the surgery robotically and two laparoscopically. Operation time ranged between 125 and 450 minutes. There was one Clavien-Dindo II complication (transfusion) in one patient. No bowel-related complications occurred.
Three patient showed metastatic progression after the surgery and subsequently underwent target therapy.

Conclusions: When the initial LRN is done through a retroperitoneal approach, transperitoneal laparoscopic excision of local retroperitoneal recurrent tumor can be done with little bowelrelated complications.

\section{MP18-08 Learning Curve In Robotic Assisted Kidney Transplantation: Results From The ERUS RAKT Working Group}

A Territo, A Gallioli, R Boissier, R Campi, D Vanacore, A Alcaraz, M Musquera, K Decaestecker, V Tugcu, S Serni, A Breda

Fundació Puigvert. Autonomous University of Barcelona. Barcelona. Spain

Introduction \& Objective: Robotic assisted kidney transplantation (RAKT) has been recently described, providing similar functional outcomes to Open KT with advantages of minimal invasive surgery. RAKT requires a learning curve and timing of surgery is crucial considering the difficulty to keep the graft cold in the abdomen until unclamping. In this study, we analyze the learning curve and its reproducibility in RAKT, evaluating surgical/functional results and complications.

Methods: Data from five centers of ERUS RAKT working group accounting for $>20$ RAKTs each were selected. RAKTs were performed by one single surgeon each center, expert in both KT and robotic surgery ( $>100$ procedures). Shewart Control Charts analyzed the learning curve in vascular anastomosis, ureterocystoneostomy and rewarming time; values between target and standard deviation (SD), +2SD, +3SD defined "mastery", "proficiency, "competence" of the surgery, respectively. Intraoperative and 30-day post- operative complications were reported. To evaluate them, RAKTs were divided in three groups: group 1 (first 10 surgeries), group 2 (11-20), group 3 (> 20). Cadaver RAKTs were excluded by functional result analysis.

Results: On arterial and venous anastomosis competence was achieved after 9 surgeries, proficiency after 12 . The competence in rewarming time was attained after 25 procedures; 16 out of the first 25 surgeries were above the alarm level $(+3 \mathrm{SD})$. On ureterocystoneostomy the surgeons were proficient after 18 RAKTs. In all technical variables, "mastery" could not be obtained. On linear regression, all the anastomoses time curves were comparable between the surgeons; rewarming time showed a slightly difference $\left(r^{2}=0.1 ; p=0.004\right)$. Intraoperative complications occurred in $3 / 50(6 \%)$ patients of group 1 , and in $1 / 50$ $(2 \%)$ of group 2 and $3 / 82(3.7 \%)$ of group 3 . In group 1, postoperative Clavien-Dindo 3-4 were reported in 7/50 (14\%) cases, while in group 2 and 3 were 1/50 (2\%) and 3/82 (3.7\%) in cases. Three graft loss occurred in group 1, none in the other groups. Mean eGFR on postoperative day 3,7,30 was comparable between groups. Delayed graft function was observed in $4.4 \%$ (2/ $45), 2.4 \%(1 / 42)$ and $1.3 \%(1 / 80)$ of cases respectively in group 1,2 and 3.

Conclusions: The learning curve of RAKT needs 25 cases to achieve positive technical results in terms of rewarming time. To do so, a synergy between the surgeon and the assistant must be created to reduce time between vascular anastomoses. Technical learning curves on anastomoses are reproducible on linear regression. All graft loss occurred in the first 10 RAKTs. Functional results of successful RAKT are not affected by learning curve. 


\section{MP18-09 Robot Assisted Renal Allograft Ne- phrectomy}

H Abdul-Muhsin, S McAdams, A Syal, R Nunez-Nateras, A Navaratnam, KM Rose, K Farraj, E Castle

Mayo Clinic, Arizona

Introduction \& Objective: To evaluate the outcomes and perioperative complication rates following robot assisted transplant nephrectomy (RATN).

Methods: All patients who underwent RATN at our institution were included. No exclusion criteria were applied. Clinical records were retrospectively reviewed and reported. This included preoperative, intraoperative and postoperative outcomes. Complications were reported utilizing the Clavien-Dindo classification system. Descriptive statistics were reported using frequencies and percentages for categorical variables, means and standard deviation for continuous variables.

Results: Between July 2014 and April 2018, fifteen patients underwent RATN. Most patients had the transplant in the right iliac fossa (13/15). Ten patients underwent a concomitant procedure. The total operative time for the entire cohort was 336 $( \pm 102)$ minutes (including concomitant procedures) and 259 $( \pm 46 \mathrm{~min})$ when concomitant procedures were excluded. Mean estimated blood loss was $383( \pm 444) \mathrm{ml}$. Postoperatively, three
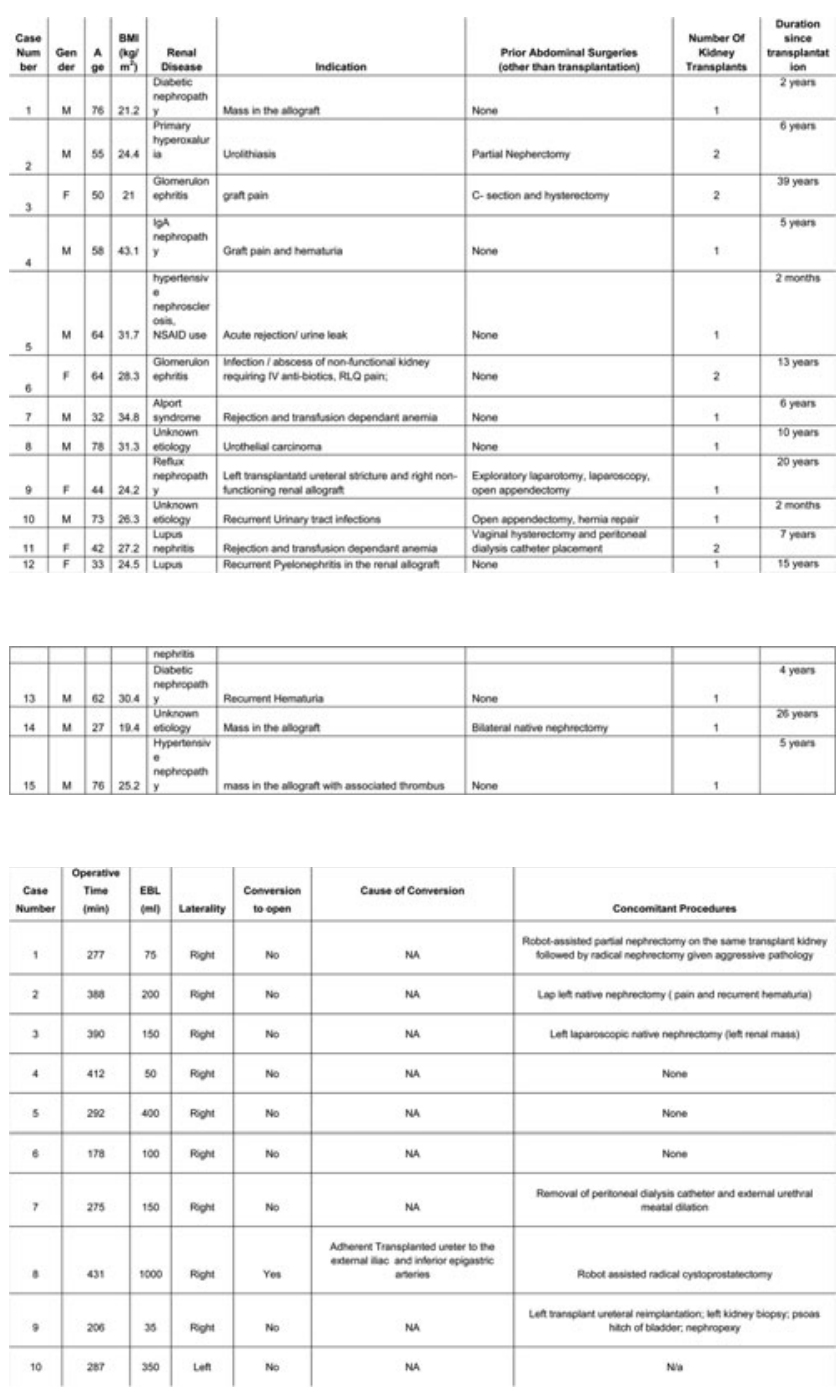

\begin{tabular}{|c|c|c|c|c|c|c|}
\hline$"$ & sss & 1500 & Len & rem & 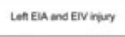 & 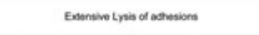 \\
\hline 12 & 204 & $2 \infty$ & Rot & to & in & ne \\
\hline , & 200 & $\infty \infty$ & Rot & No & $\mathrm{m}$ & Feneron onterosery \\
\hline n & 2n & $2 \infty$ & Rot & No & in & 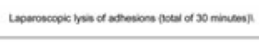 \\
\hline 15 & ass & 1000 & Rote & "o & n & 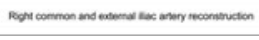 \\
\hline
\end{tabular}

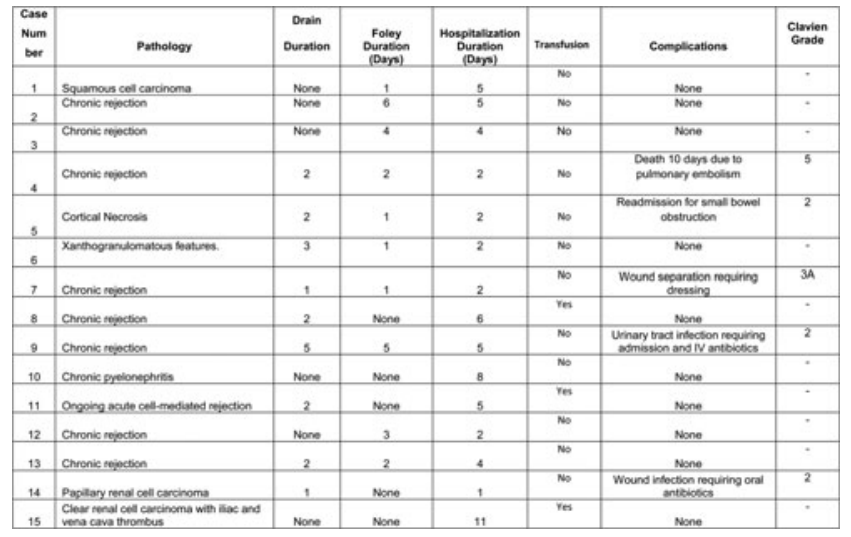

patients required blood transfusion. Average hospital stay was 4 $( \pm 2.7)$ days. Most patients had finding consistent with graft rejection on final pathology. There were five complications; three of which were minor (grade $2=2$ and grade $3=1$ ); one patient had a wound infection requiring dressing (3A) and one patient died due to pulmonary embolism following discharge.

Conclusions: This case series demonstrate that RATN is technically feasible. With continued experience and larger case series, the robotic approach may provide a minimally invasive alternative to open allograft nephrectomy.

\section{MP18-10 Outcomes of Laparoscopic Donor Ne- phrectomy in presence of double renal arteries}

R Mohsin, B Ali, A Shahzad, M Hussain

Sindh Institute of Urology and Transplantation

Introduction \& Objective: Laparoscopic donor nephrectomy has gained widespread acceptance, since it's description in 1995. Difficult vascular anatomy, such as two renal arteries, can pose a challenge to the surgeon. The multiplicity of donor artery may have an adverse effect, not only on safety of donor but also on graft outcomes.

Methods: Data was retrieved retrospectively. Patients with single and double renal artery who underwent laparoscopic donor nephrectomy were evaluated for laterality of side, operation time, ischemia time and hospitals stay.

Results: A total of 110 donors were included in the study, 55 with single and 55 with double renal arteries. The male to female ratio was 3:1. Majority of kidneys were left sided. Maximum warm ischemia time recorded was $6 \mathrm{~min} 25 \mathrm{sec}$ in double artery group. There was no conversion to open surgery in double renal artery donors. There was no primary graft failure. Mean warm ischemia time was higher in double artery group, however serum creatinine of recipients at 6 months was comparable to single renal artery grafts.

Conclusions: Outcomes of laparoscopic donor nephrectomy with double renal artery is comparable to laparoscopic donor nephrectomy with single renal artery in experienced surgical hands. 
MP18-11 Parenchymal Volume Loss Affects Postoperative Contralateral Kidney Volume Following Partial Nephrectomy

A Beksac, KE Okhawere, AA Elbakry, BD Dayal, J Daza, K Meilika, KK Badani

Icahn School of Medicine at Mount Sinai

Introduction \& Objective: Ipsilateral parenchymal volume loss (PVL) and contralateral kidney hypertrophy are two factors that affect kidney function post partial nephrectomy (PN). We sought to analyze the effect of PVL on contralateral hypertrophy following PN.

Methods: From a single surgeon series, we have analyzed the data of 119 robotic PN patients between 2014 and 2017. Patients with end stage renal disease, bilateral tumors, and solitary kidney were excluded from analysis. A semi-automatic tissue segmentation tool was used to calculate kidney and tumor volume before and after PN. Axial venous phase images of cross-sectional imaging were used for analysis. Simple linear regression models were used to determine the possible predictors of contralateral hypertrophy; and a multivariable linear regression model was used to evaluate the association between PVL and contralateral hypertrophy, controlling for confounders.

Results: Patient characteristics are presented in Table 1. The median follow-up was 11.40 months. The mean PVL was $16.99 \pm 13.49 \%$ and the mean change in contralateral kidney volume was $-1.12 \pm 11.9 \%$. In the simple linear regression model, contralateral kidney volume was associated with age $(\beta=-0.28$, $\mathrm{p}=0.03$ ), Charlson comorbidity index (CCI) (OR:-21.55, $\mathrm{p}=0.001)$ and PVL $(\beta=-0.23, \mathrm{p}=0.015)$. In the multivariable linear model, contralateral kidney volume was associated with $\operatorname{PVL}(\beta=-0.32, \mathrm{p}<0.001), \mathrm{CCI}(\mathrm{OR}:-10.63, \mathrm{p}=0.049)$, and high tumor complexity (OR: 9.89, $\mathrm{p}=0.001)$. (Table 2 ).

\begin{tabular}{|c|c|c|c|}
\hline \multirow[b]{2}{*}{ Variables } & \multirow[b]{2}{*}{$\mathrm{N}(\%)$} & \multicolumn{2}{|c|}{$\%$ volume loss mean $\pm S D$} \\
\hline & & $P$ value & rho \\
\hline Age, years mean $\pm S D^{a}$ & $60 \pm 12$ & 0.402 & 0.078 \\
\hline Gender ${ }^{b}$ & & 0.243 & \\
\hline Female & $60(50.42 \%)$ & & \\
\hline Male & $59(49.58 \%)$ & & \\
\hline Charlson Comorbidity index ${ }^{a}$ & & 0.606 & 0.048 \\
\hline 0 & $6(5.04 \%)$ & & \\
\hline 1 & $10(8.40 \%)$ & & \\
\hline$\geq 2$ & $103(86.55 \%)$ & & \\
\hline Body Mass Index, $\mathrm{kg} / \mathrm{m}^{2}$ mean $\pm S D^{a}$ & $28.59 \pm 5.60$ & 0.663 & -0.05 \\
\hline Hypertension & & 0.813 & \\
\hline No & $64(53.78 \%)$ & & \\
\hline Yes & $55(46.22 \%)$ & & \\
\hline Diabetes $^{b}$ & & 0.228 & \\
\hline No & $91(76.47 \%)$ & & \\
\hline Yes & $28(23.53 \%)$ & & \\
\hline Baseline eGFR, $\mathrm{ml} / \mathrm{min}$ mean $\pm S D^{a}$ & $78.25 \pm 18.90$ & 0.308 & -0.09 \\
\hline Chronic kidney disease ${ }^{b}$ & & 0.533 & \\
\hline No & $100(84.03 \%)$ & & \\
\hline Yes & $19(15.97 \%)$ & & \\
\hline Tumor size, $\mathrm{cm}$ & $2.5(2.0,3.6)$ & $<0.001$ & 0.378 \\
\hline $\begin{array}{lll}\text { R.E.N.A.L. } & \text { Nephrometry } & \text { Score } \\
\text { median(IQR) } & \end{array}$ & $6(5,9)$ & $<0.001$ & 0.423 \\
\hline Tumor complexity*a & & $<0.001$ & 0.376 \\
\hline Simple & $55(50.93 \%)$ & & \\
\hline Intermediate & $43(39.81 \%)$ & & \\
\hline High & $10(9.26 \%)$ & & \\
\hline Warm Ischemia Time, mins mean $\pm S D^{a}$ & $12.09 \pm 4.40$ & $<0.001$ & 0.439 \\
\hline Last follow-up eGFR, $m l / \min$ mean $\pm S D^{a}$ & $75.86 \pm 22.54$ & 0.013 & -0.249 \\
\hline$\%$ Change in eGFR,ml/min mean $\pm S D^{a}$ & $-2.59 \pm 20.08$ & 0.006 & -0.273 \\
\hline$\%$ volume loss mean \pm SD & $16.99 \pm 13.49$ & - & - \\
\hline Median follow-up, months median(IQR) & $11.40(6.24,19.97)$ & - & - \\
\hline $\begin{array}{l}\text { SD - Standard deviation; \% volume loss } \\
\text { centimeter; eGFR - Estimated glomerula } \\
\text { "missing data } \\
\text { a Spearmann correlation was used to com } \\
\text { b Student } t \text { test was used to compare cova } \\
\text { bStudent } t \text { test } \\
\text { Significance was determined at } \mathrm{p}<0.05\end{array}$ & $\begin{array}{l}\text { percentage volume } \\
\text { filtration rate; mins - } \\
\text { are covariates with } \% \\
\text { ates with } \% \text { volume ch }\end{array}$ & $\begin{array}{l}\text { ss in the or } \\
\text { inutes } \\
\text { olume cha } \\
\text { inge }\end{array}$ & kidney; $\mathrm{cm}$ \\
\hline
\end{tabular}

Table 2: Linear regression models predicting the percentage contralateral hypertrophy among patients who underwent RPN

\begin{tabular}{|c|c|c|c|c|}
\hline & \multicolumn{4}{|c|}{ Contralateral hypertrophy } \\
\hline & Unadjusted & & Adjusted & \\
\hline & $\beta(95 \% \mathrm{Cl})$ & Pvalue & $\beta(95 \% \mathrm{Cl})$ & $P$ value \\
\hline Age, years & $-0.28(-0.46,-0.10)$ & 0.030 & $-0.02(-0.16,0.19)$ & 0.844 \\
\hline \multicolumn{5}{|l|}{ Gender } \\
\hline Female & Ref. & & & \\
\hline Male & $1.82(-2.51,6.15)$ & 0.406 & & \\
\hline \multicolumn{5}{|c|}{ Charlson } \\
\hline \multicolumn{5}{|c|}{ Comorbidity index } \\
\hline 0 & Ref. & & Ref. & \\
\hline 1 & $-19.97(-31.24,-8.70)$ & 0.001 & $-11.53(-22.25,-0.81)$ & 0.039 \\
\hline$\geq 2$ & $-21.55(-30.72,-12.39)$ & $<0.001$ & $-10.63(-21.23,-0.03)$ & 0.049 \\
\hline & $-0.07(-0.61,0.48)$ & 0.809 & & \\
\hline \multicolumn{5}{|l|}{$\begin{array}{l}\text { Index }\left(\mathrm{kg} / \mathrm{m}^{2}\right) \\
\text { Hypertension }\end{array}$} \\
\hline No & Ref. & & & \\
\hline Yes & $-1.61(-5.95,2.73)$ & 0.464 & & \\
\hline \multicolumn{5}{|l|}{ Diabetes } \\
\hline No & Ref. & & & \\
\hline Yes & $-2.57(-7.66,2.53)$ & 0.321 & & \\
\hline \multicolumn{5}{|l|}{$\begin{array}{l}\text { Chronic kidney } \\
\text { disease (CKD) }\end{array}$} \\
\hline No & Ref & & & \\
\hline Yes & $-3.84(-9.73,2.04)$ & 0.198 & & \\
\hline$\%$ volume loss & $-0.23(-0.39,-0.08)$ & 0.015 & $-0.32(-0.45,-0.20)$ & $<0.001$ \\
\hline \multicolumn{5}{|c|}{ Tumor complexity } \\
\hline Simple & Ref. & & Ref. & \\
\hline Intermediate & $0.40(-3.16,3.77)$ & 0.824 & $2.65(-0.67,5.97)$ & 0.117 \\
\hline High & $5.01(-1.01,11.03)$ & 0.102 & $9.89(4.07,15.71)$ & 0.001 \\
\hline
\end{tabular}

Conclusions: PVL affects contralateral kidney volume change negatively. Therefore, efforts must be made to minimize the ipsilateral kidney volume loss during PN. CCI and tumor complexity are additional factors that factor in contralateral hypertrophy.

\section{MP18-12 Enhanced Recovery After Surgery (ERAS) Programme for Robotic Assisted Partial Nephrectomy (RAPN) - Outcomes in a High Volume Robotic Urol- ogy Centre.}

MM Quraishi, B Osman, E Latif, N Lobo, M Jones, SS Kommu, BA Eddy, M Thomas, S Matthew

Kent \& Canterbury Hospital. East Kent Hospitals University NHS Foundation Trust

Introduction \& Objective: Enhanced Recovery After Surgery (ERAS) programs have been shown to improve patient recovery, length of stay and minimize complications. There is a paucity of information on the implementation and success of ERAS in RAPN patients. A variety of tools and healthcare professionals input coupled with optimal commucation aids in ensuring optimal outcomes in an ERAS pathway. We aimed to study the outcomes of our ERAS pathway for RAPN patients from a single surgeon in a high volume robotic urological center.

Methods: Retrospective review of medical records were conducted on all patients undergoing RAPN at our specialist center from January 2017 to December 2018, in order to obtain a minimum of 6 months follow up data. Patient demographics, operative complications, length of stay, tumor histology were amongst the features recorded.

Results: Thirty-nine patients were identified to have been listed for a RAPN by a single surgeon during the study period. Mean age of patients was 61 years (Range 40-76) at time of operation. Conversion to radical nephrectomy was seen in $4(10 \%)$ patients. None of the patients required conversion to open surgery. The median length of stay was 1 day, with a maximum length of stay of 4 days. On $62 \%$ of cases, patients were discharged on day 1 post op. The mean size of tumor was identified as $29 \mathrm{~mm}$ (range $12-$ $60 \mathrm{~mm}$ ). Four patients were readmitted within 10 days, with only a single Clavien Dindo 2 or above complication identified. Positive 
margin was only identified in a single case. The median Leibovich score when applicable was found to be 1 . Histological subtypes included angiolmyolipoma $(n=3)$, papillary $(n=7)$, oncocytoma $(n=6)$, Clear Cell $(n=19)$, chromophobe $(n=3)$, Bosniak 3 Enhancing cyst $(n=1)$.

Conclusions: We report our findings of a successful ERAS program for RAPN. The median discharge date is on the first postoperative day, with minimal operative complications. The successful outcomes can be attributed to a combination of features including early mobilization, catheter removal, drain removal, prophylaxis analgesia, consistent team approach with an aim to discharge patient early and safely. We are currently looking at novel platforms for patient engagement with dedicated mobile phone and tablet-based apps to further aid patient recovery and monitoring once discharged from hospital.

\section{MP18-13 Operative and functional outcomes for se- lective clamping in robotic partial nephrectomy for patients with a solitary kidney}

KK Badani, PD Kothari, JC Deolankar, PM Youssef, D Kim, SS Komaravolu, M Billah, KE Okhawere, H Koster, R Munver, M Ahmed, G Lovallo, MD Stifelman

\section{Hackensack University Medical Center}

Introduction \& Objective: We aim to evaluate the role of selective clamping on kidney function during robotic partial nephrectomy in a solitary kidney model by assessing intraoperative factors, complications, and postoperative kidney function.

Methods: Data from IRB approved retrospective and prospective databases from 2006 to 2019 at multiple institutions was evaluated. Patients with a solitary kidney were identified and stratified based on whether selective or full renal artery clamping was performed. Both groups were analyzed for demographics, risk factors, intraoperative complications, and postoperative outcomes using Chi-square test, Fisher's exact test, t-test, and Mann Whitney U's test.

Table 1: Demographic data of patients with solitary kidney undergoing robotic partial nephrectomy

\begin{tabular}{|c|c|c|c|c|}
\hline & & Selective cl & ping & \\
\hline & & No & Yes & $P$ value \\
\hline & & $51(70.83)$ & 21 (29.17) & \\
\hline Age years ${ }^{8}$ & $62 \pm 12$ & $62 \pm 13$ & $63 \pm 9$ & 0.882 \\
\hline Gender $^{b}$ & & & & 0.630 \\
\hline Female & $20(27.78)$ & $15(29.41)$ & $5(23.81)$ & \\
\hline Male & $52(72.22)$ & $36(70.84)$ & $16(76.19)$ & \\
\hline BMI $\mathrm{kg} / \mathrm{m}^{2}$ & $\begin{array}{l}30.72(26.28, \\
35.90)\end{array}$ & $\begin{array}{l}30.64(26.28 \\
34.60)\end{array}$ & $\begin{array}{l}31.00(27.30, \\
36.86)\end{array}$ & 0.949 \\
\hline ASA & $3(3,3)$ & $3(3,3)$ & $3(3,3)$ & 0.692 \\
\hline Hypertension ${ }^{b}$ & & & & 0.965 \\
\hline No & $34(47.22)$ & $24(47.06)$ & $10(47.62)$ & \\
\hline Yes & $38(52.78)$ & $27(52.94)$ & $11(52.38)$ & \\
\hline Diabetes $^{b}$ & & & & 0.528 \\
\hline No & 57 (79.17) & $39(76.47)$ & $18(85.71)$ & \\
\hline Yes & $15(20.83)$ & $12(23.53)$ & $3(14.29)$ & \\
\hline Tumor size $\mathrm{cm}$ & $3.0(2.1,3.8)$ & $2.9(2.0,3.8)$ & $3.2(2.1,3.6)$ & 0.477 \\
\hline $\begin{array}{l}\text { Nephrometry } \\
\text { Score }\end{array}$ & $7.0(5.0,9.0)$ & $6.0(5.0,8.5)$ & $7.0(5.0,9.0)$ & 0.081 \\
\hline Baseline eGFR & $\begin{array}{l}58.70(50.28, \\
70.84)\end{array}$ & $\begin{array}{l}60.61(49.34, \\
71.92)\end{array}$ & $\begin{array}{l}56.35(52.92, \\
63.17)\end{array}$ & 0.639 \\
\hline WIT mins & $13(10,20)$ & $13(9,20)$ & $14(11,21)$ & 0.424 \\
\hline
\end{tabular}

BMI - Body Mass Index; ASA - American Society of Anesthesiologists; CCI - Charlson comorbidity index; WIT - Warm Ischemia time

${ }^{b}$ Categorical variables presented as frequency and percentage and analyzed using Chisquare and Fisher's exact

s Continuous variable presented as mean \pm Standard deviation and analyzed using $t$ test Other variables are presented as median and interquartile range and analyzed using Mann Whitney U's test
Table 2: Perioperative and functional outcomes of patients with solitary kidney who had selective clamping during robotic partial nephrectomy

\begin{tabular}{|c|c|c|c|}
\hline & \multicolumn{2}{|c|}{ Selective clamping } & \multirow[b]{2}{*}{$\begin{array}{l}P \\
\text { value }\end{array}$} \\
\hline & No & Yes & \\
\hline EBL $m L$ & $100(75,200)$ & $100(75,300)$ & 0.985 \\
\hline Operative time mins & $160(133,220)$ & $162(116,218)$ & 0.593 \\
\hline \multicolumn{4}{|c|}{ Intraoperative complication, $\mathrm{n}(\%)$} \\
\hline No & $51(100.00)$ & $20(95.24)$ & 0.292 \\
\hline Yes & $0(0.00)$ & $1(4.76)$ & \\
\hline \multicolumn{4}{|l|}{ Minor complication, n(\%) } \\
\hline No & $42(82.35)$ & $19(90.48)$ & 0.491 \\
\hline Yes & $9(17.65)$ & $2(9.52)$ & \\
\hline \multicolumn{4}{|c|}{$\begin{array}{l}\text { Major complication (Clavien } \geq 3 \text { ), } \\
\text { n(\%) }\end{array}$} \\
\hline No & $48(94.12)$ & $21(100)$ & 0.551 \\
\hline Yes & $3(5.88)$ & $0(0.00)$ & \\
\hline First 30days after surgery & & & 0.528 \\
\hline Patients, n & 48 & 19 & \\
\hline$\%$ change eGFR & $\begin{array}{l}-18.25(-26.58,- \\
4.87)\end{array}$ & $\begin{array}{l}-17.54(-39.24,- \\
2.66)\end{array}$ & 0.959 \\
\hline $\mathrm{AKI}, \mathrm{n}(\%)$ & $15(29.41)$ & $8(38.10)$ & 0.476 \\
\hline \multicolumn{4}{|l|}{ 3-12 months after surgery } \\
\hline Patients, $\mathrm{n}$ & 27 & 10 & \\
\hline Follow-up, months & $6(4,9)$ & $7(3,9)$ & 1.000 \\
\hline$\%$ change eGFR & $-3.38(-19.38,8.60)$ & $-22.54(-26.51,5.86)$ & 0.281 \\
\hline De novo CKD, n(\%) & $3(10.71)$ & $2(22.22)$ & 0.577 \\
\hline \multicolumn{4}{|c|}{$\begin{array}{l}\text { AKI- acute kidney injury; EBL -estimated blood loss; eGFR - estimated glomerular filtration rate; } \\
\text { CKD - chronic kidney disease, defined at eGFR <60mL/min; } \mathrm{n} \text { - frequency } \\
\text { Categorical variables presented as frequency and percentage and analyzed using Chi-square } \\
\text { and Fisher's exact } \\
\text { Other variables are presented as median and interquartile range and analyzed using Mann } \\
\text { Whitney U's test }\end{array}$} \\
\hline
\end{tabular}

Results: A total of 72 patients had undergone partial nephrectomy on a solitary kidney, 51 with full clamping and 21 with selective. Both groups had no significant difference in demographics, tumor size, baseline eGFR, or warm ischemia time (WIT) (Table 1). Of note, median WIT was less than 15 minutes in both groups. Intraoperative outcomes, including estimated blood loss (EBL, ) operative time, and intraoperative complications were similar. Short and long term postoperative percentage change in eGFR, frequency of acute kidney injury (AKI), and frequency of de novo chronic kidney disease (CKD) were also not significantly different (Table 2).

Conclusions: We have evaluated a large cohort of solitary kidney patients and found that selective clamping results in similar intraoperative and postoperative outcomes compared to full clamping and confers no additional risk of harm. Selective clamping does not appear to provide any functional advantage since there was no difference observed in the frequency of AKI, CKD or change in eGFR. Short WIT in both groups may have prevented identification of benefits in selective clamping; a similar study analyzing cases with longer WIT may possibly elucidate any beneficial effects of selective clamping.

\section{MP18-14 Oncological Outcomes of distal ureter man- agement followed by Laparoscopic/Robotic nephrou- reterectomy for Upper Tract Urothelial Carcinoma}

\section{W Kuo, J Lin, C Yu \\ Kaohsiung Veteran General Hospital}

Introduction \& Objective: To evaluate the impact of distal ureter management on oncological results after Laparoscopic/Robotic nephroureterectomy comparing intra-corporeal versus open extra-vesical bladder cuff excision.

Methods: We retrospectively collected data from 250 patients who underwent Laparoscopic/Robotic nephroureterectomy for upper urinary tract urothelial carcinoma from 2003 to 2013. Patients were divided into two subgroups according to the type of operation performed. Group A comprised 130 patients who had 


\begin{tabular}{|c|c|c|c|}
\hline Significant factors & $\begin{array}{c}\text { Group A } \\
\text { Open BCE } \\
\mathrm{N}=130\end{array}$ & $\begin{array}{c}\text { Group B. } \\
\text { Intra-corporeal BCE } \\
\mathrm{N}=120\end{array}$ & P value \\
\hline Operating time & $181\left(122^{\sim} \sim 248\right)$ & $165\left(118^{\sim} 257\right)$ & 0.01 \\
\hline Length of hospital stay & $4.6\left(3^{\sim} 7\right)$ & $3.8\left(3^{\sim} 6\right)$ & 0.25 \\
\hline Blood loss & $143\left(58^{\sim} 366\right)$ & $86\left(35^{\sim} 264\right)$ & 0.01 \\
\hline Bladder recurrence & 13 & 10 & 0.51 \\
\hline Local recurrence & 6 & 4. & 0.5 \\
\hline
\end{tabular}
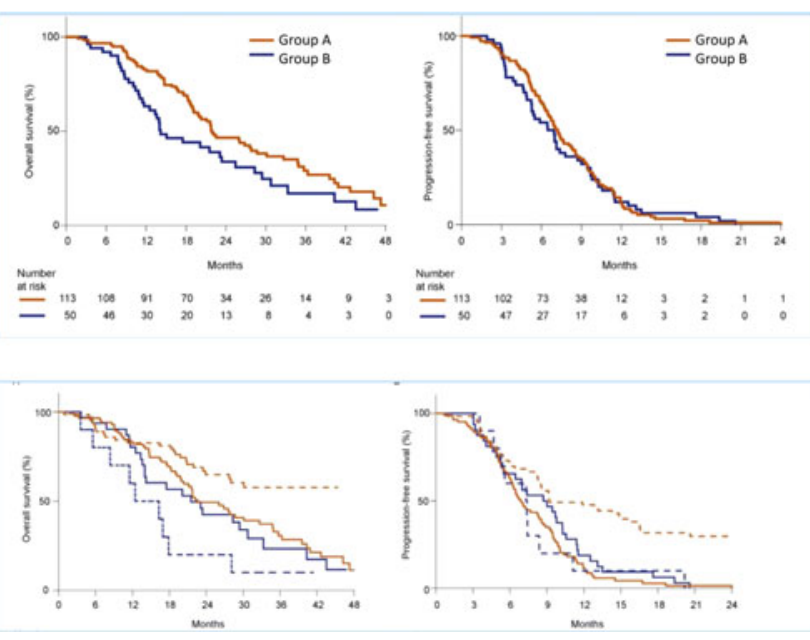

laparoscopic nephroureterectomy with open resection of the bladder cuff. Group B comprised 120 patients in whom received intra-corporeal bladder cuff excision. The mean operative time, hospital stay, duration of catheterization, bladder recurrence rates, and cancer-specific survival (CSS) were assessed.

Results: The total operative time was statistically significantly less in the Group B (intra-corporeal BCE). There was no statistical difference between Groups $\mathrm{A}$ and $\mathrm{B}$ for the bladder recurrence rate (Group A $10 \%$ vs $8 \%$ in Group B, $P=0.51$ ). There was no difference in CSS at the 5-year follow-up.

Conclusions: Laparoscopic/Robotic nephroureterectomy with intra-corporeal bladder cuff excision is an oncologically safe and technically feasible operation.

MP18-15 Zero Ischemia Robot-Assisted Partial Nephrectomy: Trifecta Outcomes

MB Tuna, TS Doğanca, İ Tufek, Ö Argun, C Obek, A Kural

Acibadem Maslak Hospital

Introduction \& Objective: "Trifecta" in partial nephrectomy consists of negative surgical margins, minimal decrease of renal function and absence of complications. Zero ischemia nephron sparing surgery is increasingly preferred when technically feasible and better "Trifecta" outcomes are projected.

Methods: This is a retrospective analysis of "Trifecta" outcomes of robotic zero ischemia partial nephrectomy patients in our single center series operated between April 2008 and May 2019. Postoperative renal function was evaluated with estimated glomerular filtration rate (eGFR) at postoperative 1st month and compared with preoperative eGFR. Complications were defined based on Clavien-Dindo grading system. Patients with absence of grade $>2$ Clavien-Dindo complications, $<15 \%$ postoperative estimated glomerular filtration rate (eGFR) decrease and nega- tive surgical margins were reported to achieve Trifecta outcomes.

Results: A total of 248 patients underwent robotic partial nephrectomy (RPN). Zero ischemia robotic partial nephrectomy was performed in 49 patients. Mean age and BMI of the patients was $57.4 \pm 13.2$ years and $27.1 \pm 4.3 \mathrm{~kg} / \mathrm{m}^{2}$, respectively. Mean tumor size in surgical specimen was $29.91 \pm 13.07 \mathrm{~mm}$. Mean operative time was $82.28 \pm 39.34$ minutes. Mean estimated blood loss was $279.59 \pm 278.7 \mathrm{ml}$. Mean preoperative and postoperative eGFR values were $85 \pm 21.7$ and $84.5 \pm 21.6 \mathrm{ml} / \mathrm{min}$. per $1.73 \mathrm{~m}^{2}$, respectively. Two patients had solitary kidney. Four patients had $>15 \%$ postoperative eGFR decrease. One patient had focal positive surgical margin. One patient had both Clavien IIIB complication and $>15 \%$ postoperative eGFR decrease. In the series, 43 of $49(88 \%)$ zero ischemia patients achieved Trifecta outcomes.

Conclusions: Zero ischemia partial nephrectomy can achieve Trifecta outcomes in technically feasible cases.

\section{MP18-16 Long-term oncologic outcomes after robotic assisted partial nephrectomy for renal cell carcinoma}

K Du, J Vetter, A Paradis, R Venkatesh, R Figenshau

Washington University in St. Louis

Introduction \& Objective: We assessed the long-term oncologic outcomes in patients who have undergone robot-assisted partial nephrectomy (RAPN) for renal cell carcinoma.

Methods: We retrospectively reviewed a prospectively maintained database of patients who underwent RAPN at our institution from 2007 to 2012. We summarized baseline characteristics, operative data, and oncologic outcomes and assessed for potential predictors of overall survival (OS) and disease-free survival (DFS). We also evaluated positive surgical margin (PSM) and its impact on oncologic outcomes.

Results: Overall, 408 patients underwent RAPN and had renal cell carcinoma on pathology. There were 50 deaths $(12.3 \%)$ and 10 recurrences $(2.4 \%)$ at a mean follow up of 73 months. There was 15 PSM (3.7\%), which was associated with longer operative time and estimated blood loss (Table 1). Worse DFS was associated with PSM and high-grade tumors but not BMI, gender, tumor histology, or tumors $>4 \mathrm{~cm}$ (Figure 1). Worse OS was

\begin{tabular}{|c|c|c|c|c|}
\hline Variable & Total cohort (408) & No PSM (n=393) & $\operatorname{PSM}(n=15)$ & p-value \\
\hline Age in years, mean (SD) & $57.6(12.5)$ & $57.4(12.5)$ & $64.1(11.0)$ & 0.055 \\
\hline BMI, mean (SD) & $31.2(7.3)$ & $31.1(7.2)$ & $32.7(9.1)$ & 0.645 \\
\hline Operative time in minutes, mean (SD) & $161.8(52.2)$ & $160.9(52.3)$ & $186.3(45.8)$ & 0.032 \\
\hline Estimated blood loss in mL, mean (SD) & $221(280)$ & $211.9(263.0)$ & $456.7(536.1)$ & 0.001 \\
\hline Nephrometry score, mean (SD)* & $7.5(1.8)$ & $7.5(1.8)$ & $7.7(1.6)$ & 0.751 \\
\hline Clinical tumor size in cm, mean (SD) & $3.1(1.5)$ & $3.1(1.5)$ & $3.2(1.3)$ & 0.461 \\
\hline Charlson comorbidity index, mean (SD) & $1.1(1.4)$ & $1.1(1.5)$ & $0.9(0.7)$ & 0.879 \\
\hline Gender, $\%$ & & & & 0.689 \\
\hline Female & 41.7 & 41.5 & 46.7 & \\
\hline Male & 58.3 & 58.5 & 53.3 & \\
\hline Off-Clamp, \% & & & & 0.207 \\
\hline No & 77.2 & 77.0 & 93.3 & \\
\hline Yes & 22.3 & 23.0 & 6.7 & \\
\hline Pelvicaliceal Repair, \% & & & & 0.559 \\
\hline No & 44.6 & 45.7 & 53.3 & \\
\hline Yes & 52.4 & 54.3 & 46.7 & \\
\hline Laterality, \% & & & & 0.441 \\
\hline Right & 49.8 & 50.1 & 40.0 & \\
\hline Left & 50.2 & 49.9 & 60.0 & \\
\hline TStage, \% & & & & 0.221 \\
\hline 1a & 83.8 & 84.1 & 73.3 & \\
\hline $1 \mathrm{~b}$ & 13.2 & 13.1 & 20.0 & \\
\hline $2 a, 2 b, 3 a$ & 2.9 & 2.8 & 6.7 & \\
\hline Fuhrman Grade, \% & & & & 0.555 \\
\hline 1 or 2 & 76.0 & 76.2 & 66.7 & \\
\hline 3 or 4 & 24.0 & 23.8 & 33.3 & \\
\hline High Volume Surgeons (>50 cases/year), \% & & & & 0.614 \\
\hline No & 7.1 & 7.4 & 0.0 & \\
\hline Yes & 92.9 & 92.6 & 100.0 & \\
\hline
\end{tabular}


Figure 1. Disease free survival Kaplan-Meier curves
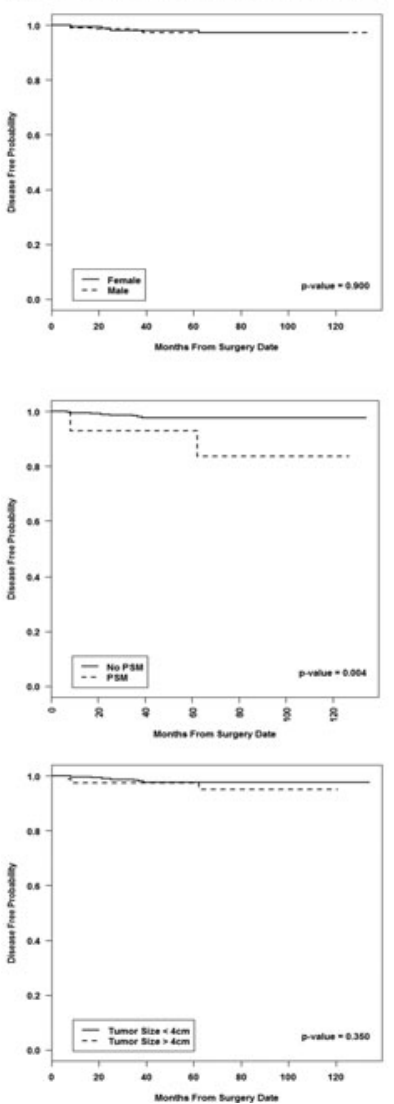

Figure 2. Overall survival Kaplan-Meier curves
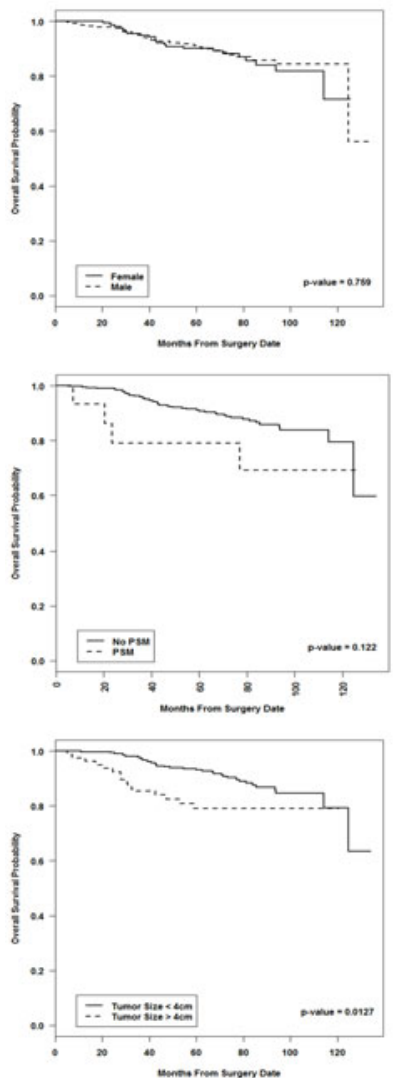
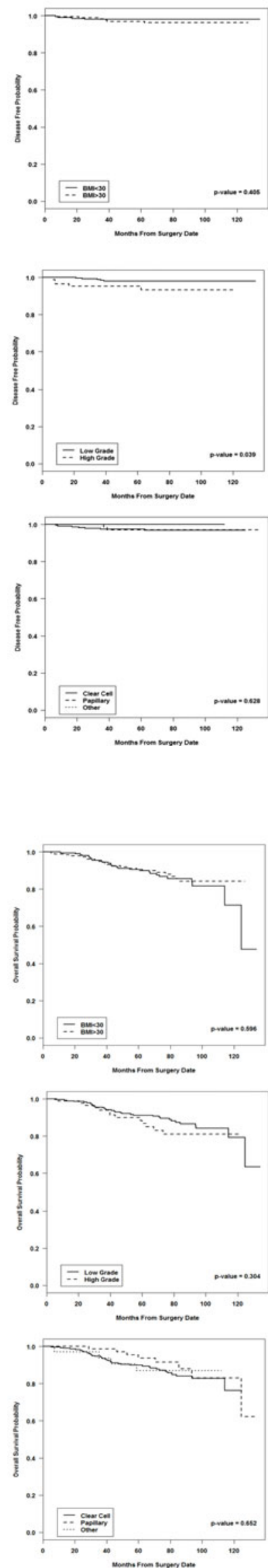

associated with tumors $>4 \mathrm{~cm}$ but not BMI, gender, tumor histology, high grade, or PSM (Figure 2).

Conclusions: Robotic assisted partial nephrectomy offers high OS and DFS for treatment of localized renal cell carcinoma. However, larger tumor size, high Fuhrman grade, and PSM may reduce patient survival in this long-term report.

\section{MP18-17 Robotic Partial Nephrectomy Outcomes in Highly Complex Kidney Cancers: Are We Pushing the Boundaries Too Far?}

D Kim, NH Patel, PM Youssef, JC Deolankar, PD Kothari, SS Komaravolu, M Billah, H Koster, Y Kuo, M Ahmed, G Lovallo, R Munver, MD Stifelman

Hackensack Meridian Department of Urology

Introduction \& Objective: We evaluated the distribution of nephrometry score for patients undergoing surgery for a known renal mass over the last 3 years. We then evaluated the incidence of robotic partial vs radical nephrectomy for each tumor complexity. Finally, we compared peri-operative outcomes of robotic partial nephrectomy (PN) based on tumor complexity.

Methods: Data from our IRB retrospective and prospective database from January 2016 to October 2018 was evaluated. Nephrometry scores were broken into 3 categories: simple (4-6), moderate (7-9), and highly complex tumors (10-12). We evaluated the incidence of PN based on nephrometry score and compared their outcomes. Krushal-Wallis one-way ANOVA by rank analysis and Fischer exact test were then performed to analyze significance of outcomes among tumor complexities.

Results: 299 procedures from 294 patients were evaluated with the percentage of highly complex nephrometry scored tumors increasing each year $(18 \%, 30 \%, 38 \%, \mathrm{p}=0.031)$. Over this 3-year period, the use of PN remains high for simple and moderately complex tumors $(89.4$ and $85.3 \%)$ and selectively for highly complex tumors $(33.7 \% ; \mathrm{p}<0.0001)$. For patients with highly

\begin{tabular}{|c|c|c|c|c|}
\hline & \multicolumn{3}{|c|}{ Level of Nephrometry Score } & \multirow[b]{2}{*}{ P-value } \\
\hline & 1:4-6 & 2: 7.9 & 3:10-12 & \\
\hline & $\mathrm{n}=85(28.4 \%)$ & $n=122(40.8 \%)$ & $\mathrm{n}=92(30.7 \%)$ & \\
\hline \multicolumn{5}{|l|}{ Patient Information } \\
\hline Age (years) & 62.3 (SD 11.9) & 62.6 (SD 11.7) & 61.3 (SD 14.7) & 0.755 \\
\hline Female & $32(32 \%)$ & $41(41 \%)$ & $27(27 \%)$ & 0.221 \\
\hline Male & $52(27 \%)$ & $78(40 \%)$ & $64(33 \%)$ & 0.073 \\
\hline BMI & 29.5 (SD 5.9) & 29.4 (SD 6.5) & 28.4 (SD 5.6) & 0.341 \\
\hline Greatest Tumor size $(\mathrm{cm})$ & 2.5 (SD 1.4) & 3.6 (SD 1.9) & 7.2 (SD 3.7) & $<0.0001$ \\
\hline Nephrometry Score & $5.0(S D 0.8)$ & 8.2 (SD 0.7) & 10.8 (SD 0.8) & $<0.0001$ \\
\hline $\begin{array}{l}\text { Incidence of Partial } \\
\text { Nephrectomy }\end{array}$ & $n=76(89.4 \%)$ & $\mathrm{n}=104(85.3 \%)$ & $n=31(33.7 \%)$ & \\
\hline Operating Time (mins) & 117.7 (SD 54.5) & 126.2 (SD 76.7) & 129.5 (SD 48.1) & 0.165 \\
\hline $\begin{array}{l}\text { Warm ischemia time } \\
\text { (mins) }\end{array}$ & 15.5 (SD 5.4) & 17.5 (SD 5.7) & 20.2 (SD 8.9) & 0.002 \\
\hline Estimated blood loss (cc) & 126.8 (SD 104.2) & 132.5 (SD 126.3) & 154.4 (SD 178.5) & 0.624 \\
\hline Post Op LOS & $1.5(\mathrm{SD} 0.7)$ & 1.9 (SD 1.5) & 2.0 (SD 1.2) & 0.055 \\
\hline $\begin{array}{l}\text { Positive Surgical } \\
\text { Margin* }\end{array}$ & $1(1.3 \%)$ & $2(1.9 \%)$ & $0(0 \%)$ & 1.000 \\
\hline $\begin{array}{l}\text { Intra-0perative } \\
\text { Complications }\end{array}$ & $0(0 \%)$ & $0(0 \%)$ & $3(9.7 \%)$ & 0.003 \\
\hline $\begin{array}{l}\text { Complications with } \\
\text { Clavien Score } 23\end{array}$ & $1(1.3 \%)$ & $3(2.9 \%)$ & $0(0 \%)$ & 0.810 \\
\hline Type of Tumor: RCC & $56(74 \%)$ & $94(90 \%)$ & $28(94 \%)$ & 0.006 \\
\hline
\end{tabular}


complex tumors undergoing PN, outcomes were similar except for warm ischemia time (WIT), intraoperative complication rate, and incidence of malignancy compared with moderate and simple complexity tumors. (Table 1 ) WIT was $<20$ minutes for all groups, and there was no statistical difference in positive margins.

Conclusions: In a high-volume center with experienced surgeons, the use of PN for both simple and moderate complex tumors is over $85 \%$. With appropriate selection, PN can be performed in highly complex tumors with a slight increase in WIT and intra-operative complications. Our data suggests that there has not been an over utilization of PN in surgeons with significant experience.

\section{MP18-18 Are There Any Functional Benefits From Ischemia Sparing Techniques among Patients with Chronic Kidney Disease?}

A Beksac, KE Okhawere, D Rosen, D Paulucci, AA Elbakry, BD Dayal, J Sfakianos, R Abaza, PD Kothari, DD Eun, A Bhandari, A Hemal, J Porter, MD Stifelman, KK Badani

Icahn School of Medicine at Mount Sinai

Introduction \& Objective: We sought to compare the renal functional benefit and perioperative outcomes between main arterial clamping (MAC), selective arterial clamping (SAC) and off-clamping (OC) techniques in stage III-V CKD patients who underwent robotic partial nephrectomy (RPN).

Methods: Data was obtained from our multi-institutional database and included patients with available data on clamping technique, baseline eGFR and follow-up between 3-24 months. Median follow-up was 12.1 months (IQR 6.9-17.2; Range 3.124.4 Months). The bivariate relationship between clamping technique, baseline characteristics and perioperative outcomes was assessed using Chi-square, Fisher's exact and Kruskal Wallis tests. A linear mixed effect model was used to evaluate

\begin{tabular}{|c|c|c|c|c|}
\hline & $\begin{array}{l}\text { Main Arterial } \\
\text { Clamping RPN }\end{array}$ & $\begin{array}{l}\text { Selective Arterial } \\
\text { Clamping RPN }\end{array}$ & Off-Clamp RPN & $\mathbf{P}$ \\
\hline Ischemia Time & $17(13,22)$ & $15(12,21)$ & $0.0(0.0-0.0)$ & $<0.001$ \\
\hline $\begin{array}{l}\text { Estimated Blood } \\
\text { Loss }\end{array}$ & $100(50,150)$ & $100(75,250)$ & $100(50,150)$ & 0.114 \\
\hline Operative Time & $171(132,217)$ & $162(130,205)$ & $174(132,233)$ & 0.478 \\
\hline $\begin{array}{l}\text { Post-Op } \\
\text { Complications }\end{array}$ & $70(18.87 \%)$ & $6(12.50 \%)$ & $6(15.79 \%)$ & 0.522 \\
\hline $\begin{array}{l}\text { Positive Surgical } \\
\text { Margin }\end{array}$ & $23(6.89 \%)$ & $3(6.67 \%)$ & $0(0.0 \%)$ & 0.550 \\
\hline Length of Stay & $2(1,3)$ & $2(1,2)$ & $2(1,2)$ & 0.120 \\
\hline
\end{tabular}

\begin{tabular}{|c|c|c|}
\hline Variable & $\beta(95 \% \mathrm{Cl})$ & P-value \\
\hline Intercept ${ }^{3}$ & $42.69(33.49,51.9)$ & $<0.001$ \\
\hline Time (Months) ${ }^{b}$ & $-0.17(-0.45,0.10)$ & 0.219 \\
\hline SAC & $-1.32(-5.61,2.98)$ & 0.473 \\
\hline$O C^{c}$ & $-1.34(-5.01,2.32)$ & 0.547 \\
\hline SAC*Time ${ }^{d}$ & $-0.34(-0.85,0.18)$ & 0.201 \\
\hline OC*Time ${ }^{d}$ & $0.22(-015,0.58)$ & 0.251 \\
\hline \multicolumn{3}{|c|}{$\begin{array}{l}\text { SAC -Selective arterial clamping; OC- Off clamping } \\
\text { a. Intercept=Preoperative eGFR for the SAC and OC groups combined } \\
\text { b. Time=Average increase in eGFR per month for the SAC and OC groups combined } \\
\text { c. SAC and OC =Difference in preoperative eGFR for the SAC and OC vs. MAC patients. } \\
\text { d. SAC and OC * Time Postoperative=Difference in the average decline in eGFR per } \\
\text { month for the SAC and OC groups vs. MAC group } \\
\text { " Model adjusted for body mass index, hypertension, and presence of solitary kidney, } \\
\text { tumor size, R.E.N.A.L. Nephrometry score, endophytic, hilar, and tumor laterality. }\end{array}$} \\
\hline
\end{tabular}

change in eGFR over time in patients with baseline CKD 3-5 who underwent RPN with main arterial clamping (MAC) $(\mathrm{n}=375$, $81.2 \%)$, SAC $(n=48,10.4 \%)$ or OC $(n=39,8.4 \%)$.

Results: In bivariate analysis, clamp type was associated with ischemia time $(\mathrm{p}<0.001)$ but was not associated with estimated blood loss $(E B L)(p=0.114)$, operative time $(p=0.478)$, postoperative complication $(\mathrm{p}=0.522)$ and length of hospital stay $(\mathrm{p}=0.120)$. In the multivariable linear mixed effects model adjusting for body mass index, hypertension, and presence of solitary kidney, tumor size, R.E.N.A.L. nephrometry score, endophytic, hilar, and tumor laterality, the change in eGFR over time was not significantly different between SAC and MAC RPN $(\mathrm{b}=-0.34 ; 95 \% \mathrm{CI}=-0.85,0.18 ; \mathrm{p}=.201)$ and $\mathrm{OC}$ and MAC RPN ( $b=0.22 ; 95 \% \mathrm{CI}=-0.15,0.58 ; \mathrm{p}=.251)$.

Conclusions: Selective clamping and off-clamp techniques during RPN were not associated with benefit in preservation of eGFR in patients with baseline CKD.

MP18-19 Robot assisted radical nephrectomy and inferior vena cava thrombectomy: surgical technique, perioperative and mid-term oncologic outcomes

U Anceschi, G Tuderti, A Brassetti, M Ferriero, F Minisola, M Costantini, S Guaglianone, R Flammia, R Mastroianni, M Gallucci, G Simone

"Regina Elena” National Cancer Institute

Introduction \& Objective: Radical nephrectomy with Inferior vena cava (IVC) thrombectomy for renal cancer represents one of the most challenging urologic surgical procedures. The aims of this series are to detail the surgical technique and to report perioperative and oncologic outcomes of 30 consecutive cases of completely intracorporeal robot-assisted radical nephrectomy with IVC level I (20\%) II (30\%) III (46.7\%) and IV (3.3\%) tumor thrombectomy.

Methods: Thirty consecutive patients with renal tumor and IVC thrombus were treated between July 2011 and October 2018. Baseline, perioperative and follow-up data were collected into a prospectively maintained IRB approved databases. Surgical technique has been previously described. We report perioperative and oncologic outcomes of 30 consecutive patients treated in a tertiary referral center.

Results: All procedures were successfully completed. No conversion to open or laparoscopic surgery occurred. Median operative time was 3600 minutes. Eleven patients (47.8\%) did not experience any complication. Eleven patients $(43,4 \%)$ required blood transfusion (Clavien grade 2); one patient $(3,3 \%)$ had a Clavien grade $3 \mathrm{a}$ complication (gastroscopy); two patients $(3,3 \%)$ had Clavien grade $3 b$ complication (reintervention due to bleeding from adrenal gland; cardiac cardioversion); Two patients $(6,6 \%)$ required ICU admission (Clavien 4a), for PRESS syndrome and atrial fibrillation, respectively. Out of 12 patients who underwent cytoreductive nephrectomy and IVC thrombectomy, at a median follow-up of 20 months (IQR 12-35), 2-yr cancer specific and overall survival rates were $50 \%$. Eighteen patients received surgery with curative intent and 8 of these experienced disease recurrence. three patients died of disease progression; 2-yr disease-free and cancer specific survival rates were $55.6 \%$ and $60 \%$, respectively.

Conclusions: Robotic IVC tumor thrombectomy has demonstrated to be a feasible and safe surgical procedure in tertiary referral centers. Favorable perioperative outcomes represent a 
rational base to expand indications also in the cytoreductive setting.

\section{MP18-20 Evaluation of postoperative parenchymal reduction with or without renorrhaphy using com- puted tomography volumetry in robotic-assisted par- tial nephrectomy}

R Sagehashi, T Inoue, S Nakajima, S Kanda, T Nara, K Numakura, M Saito, S Narita, S Satoh, T Habuchi

Akita University School of Medicine

Introduction \& Objective: The addition of suturing renorrhaphy to partial nephrectomy may damage intraparenchymal vessels and compress the parenchyma, which may cause an increase in damaged parenchymal volume. Pre- and post-operative parenchymal volumes were measured using computed tomography $(\mathrm{CT})$ volumetry and compared between the renorrhaphy and the non-renorrhaphy groups to evaluate if renorrhaphy increases parenchymal reduction volume in robot-assisted partial nephrectomy (RAPN).

Methods: A total of 69 patients with a small renal mass $<4 \mathrm{~cm}$ in diameter underwent RAPN between November 2013 and November 2018 at Akita University Hospital. The data of 26 patients who underwent suturing renorrhaphy (renorrhaphy group) and that of 43 patients who underwent medullary hemostatic suturing alone (non-renorrhaphy group) were retrospectively compared. The tumor volume and pre- and post-operative parenchymal volumes were measured with contrast-enhanced CT before and 6 months after the surgery using Synapse Vincent ver.4 software (Fujifilm, Tokyo, Japan). The parenchymal reduction volume was calculated as pre- operative minus postoperative parenchymal volume.

Results: The median pre-operative parenchymal volume and tumor volume were not significantly different between the renorrhaphy and non-renorrhaphy groups. The median parenchymal reduction volume was not significantly different between the renorrhaphy and non-renorrhaphy groups $(13.8$ vs. $15.2 \mathrm{~mL}$, $p=0.642)$. The median reduction rate of the estimated glomerular filtration rate (eGFR) was not significantly different between the renorrhaphy and non-renorrhaphy groups (7.5 vs. $8.2 \%$, $\mathrm{p}=0.602$ ). Postoperative complications of Clavien-Dindo grade $\geq 3$ were observed in $3.8 \%$ (1/26) of patient in the renorrhaphy group and in $11.6 \%(5 / 43)$ of patients in the non-renorrhaphy group.

Conclusions: The addition of suturing renorrhaphy to RAPN neither significantly increased the parenchymal reduction volume nor significantly decreased kidney function when compared with the findings of the non-renorrhaphy technique.

Table: Comparison of numerical values measured by CT volumetry between the renorrhaphy and non-renorrhaphy groups in robot-assisted partial nephrectomy.

\begin{tabular}{|c|c|c|c|}
\hline & renorthaphy group ( $n=26$ ) & non-renorthaphy group $(n=43)$ & D \\
\hline Preoperative parenchymal volume (mL) & 166.3 & 161.1 & 0.732 \\
\hline Tumor dameter $(\mathrm{cm})$ & 24.3 & 25.0 & 0.746 \\
\hline Tumor volume (mL) & 9.6 & 10.7 & 0.887 \\
\hline Postoperative parenchymal volume $(\mathrm{mL})$ & 150.6 & 140.2 & 0.887 \\
\hline Parenchymal reduction volume $(\mathrm{mL})$ & 138 & 15.2 & 0.642 \\
\hline Decreasing rate of eGFR (s) & 7.5 & 8.2 & 0.602 \\
\hline Complications with Clavien-Dindo grade 23 & $1 / 26(3.85)$ & $5 / 43(11.6 \mathrm{~V})$ & 0.266 \\
\hline
\end{tabular}

MP18-21 Head to head impact of MIC vs a novel TRIFECTA score on oncologic and functional outcomes after robotic assisted partial nephrectomy: results of a multicentric series

U Anceschi, R Bertolo, A Brassetti, G Tuderti, U Capitanio, M Ferriero, A Larcher, A Veccia, A Mottrie, A Antonelli, A Minervini, P Dell'Oglio, I Derweesh, F Porpiglia, F Montorsi, J koauk, R Autorino, M Gallucci, G Simone, R Mastroianni

Introduction \& Objective: The optimal achievement of perioperative outcomes for robot-assisted partial nephrectomy (RAPN) is usually assessed through standard trifecta or MIC. However, there is paucity of data describing the ability of these tools in predicting oncologic and functional outcomes of RAPN. In this study we performed a head-to-head comparison of our novel trifecta definition with the standard MIC (margin, ischemia, complications) for predicting oncologic and functional outcomes on a large multicenter series of RAPN.

Methods: A multicentric database was queried for patients with non-metastatic renal masses who underwent RAPN at 8 participating institutions between September 2006 and September 2017. Baseline demographic, clinical, pathologic and perioperative data were collected. MIC achievement was defined as the fulfillment of the following criteria: negative margins; ischemia time $<20$ minutes; absence of major complications (Clavien-Dindo $\geq 3$ ). Our novel trifecta proposal was defined as negative margins, no severe complications (Clavien Dindo $\geq 3$ ) and estimated glomerular filtration rate (eGFR) reduction at discharge $\leq 30 \%$. Kaplan-Meier method was used to assess the predictive role of MIC and TRIFECTA on newly onset chronic kidney disease stages 3a (CKD-3a), 3b (CKD-3b), 4-5, (CKD4,5 ), on recurrence-free survival (RFS) and on overall survival (OS) probabilities. The same analyses were subsequently performed in a subgroup cohort of patients receiving off-clamp (OC) RAPN. For all statistical analyses, a two-sided $\mathrm{p}<0.05$ was considered significant.

Results: Mean follow-up was 26.7 months. (IQR 10.3-47). Overall, 1807 patients were included in the analysis. 1285 patients achieved MIC (71.1\%) while $1492(82.6 \%)$ achieved trifecta. On Kaplan-Meier analysis patients achieving MIC displayed significantly higher OS $(\mathrm{p}=0.004)$, and lower newly onset CKD 3a, 3b, 4-5 (all $\mathrm{p}<0.001$ ) probabilities; achieving MIC did not predict RFS probability $(\mathrm{p}=0.355)($ Fig.1,2). Patients achieving trifecta had higher OS $(\mathrm{p}=0.014)$, RFS $(\mathrm{p}=0.009)$ and lower newly onset CKD 3a, 3b, 4-5 (all p <0.001) probabilities. In a subgroup analysis on patients receiving OCRAPN, MIC score did not predict newly onset of CDK-3a, 3b, and 4,5 stages, while Trifecta was a significant predictor of all newly onset of CKDstages (all p values $\leq 0.002$ ). (Fig.2).

Conclusions: Compared to MIC, the newly defined trifecta was a significant predictor of all major oncologic and functional outcomes, including recurrence free survival probabilities. Moreover, the newly defined trifecta is easily and widely applicable in all patients after RAPN, regardless clamping technique.

MP18-22 Fate of Residual Ureteral Stump in Patients Undergoing Incomplete Robot-assisted Radical Nephroureterectomy for High-risk Upper Tract Urothelial Carcinoma

R Pathak, A Hemal

Wake Forest University 
Introduction \& Objective: Management of the distal ureter in radical nephroureterectomy and bladder cuff excision (RNUBCE) is paramount, directly influencing oncologic outcomes, particularly local recurrence. Herein, we analyze the natural history of patients who have undergone radical nephroureterectomy and retained ureteral stump and compare this cohort with patients undergoing RNUBCE for high-risk upper tract urothelial carcinoma (UTUC).

Methods: From January 2006 to October 2018, all patients who underwent robotic RNUBCE for high-risk UTUC were reviewed. For patients who did not undergo formal bladder cuff excision, preoperative, perioperative, and postoperative variables were investigated. Overall-survival and cancer-specific survival were calculated using Kaplan-Meier curves. Follow-up consisted of abdominopelvic/chest imaging and cystoscopy at regular intervals consistent with the NCCN.

Results: A total of 105 patients underwent robotic RNU during the above time period. Of patients with documented 6-month follow-up, approximately $6.6 \%$ of patients had retained ureteral stump. Median follow-up for the entire cohort was 31.5 months with a range of 6 to 114.2 months. Factors that precluded formal BCE were densely, fibrotic reaction near the ureterovesical junction due to prior vascular or pelvic surgery in 5 patients, severe pyonephrosis and continued anesthetic risk in one patient, and surgeon choice (patient co-morbidities) in another patient. Three patients died with metastatic disease and one patient succumbed to cardiovascular compromise. 2 additional patients developed local recurrence only at the level of the ureteral stump, with one patient undergoing eventual distal ureterectomy, contralateral RNUBCE and radical cystectomy with urinary diversion.

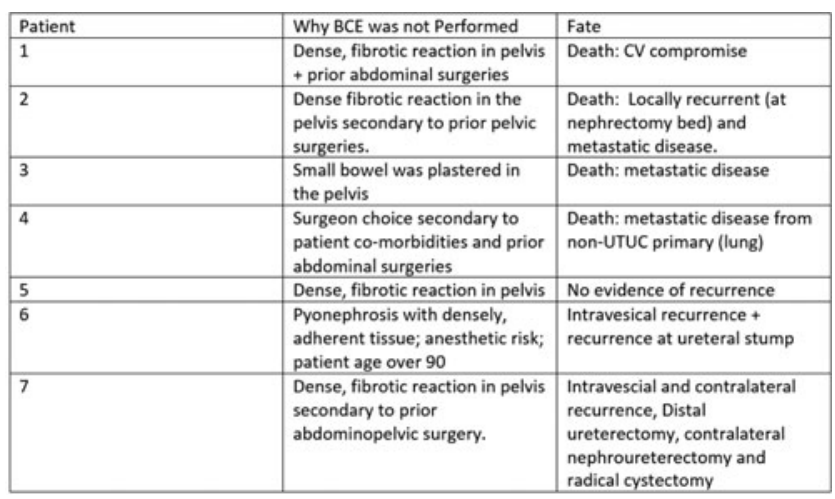

\begin{tabular}{|l|l|l|}
\hline \multicolumn{3}{|c|}{ Table 2: Comparison of Patients Undergoing RNU and Formal BCE with those with Retained Ureteral } \\
\multicolumn{1}{|c|}{ Stump } \\
\hline & p-value & HR and $95 \% \mathrm{Cl}$ \\
\hline Overall Survival & 0.0009 & $0.19(0.07,0.51)$ \\
\hline Cancer-specific Survival & 0.0001 & $0.09(0.02,0.30)$ \\
\hline Local Recurrence-free Survival & 0.0033 & $0.16(0.05,0.54)$ \\
\hline Distant Recurrence-free Survival & 0.0004 & $0.17(0.06,0.45)$ \\
\hline Bladder Recurrence-free Survival & 0.14 & $0.31(0.07,1.46)$ \\
\hline
\end{tabular}

Conclusions: In these cases, responsibilities assumed by the surgeon demand the utmost in judgement and skill, however, at times circumstances prevail such as patient factors and nature/biology of the disease. These factors may prevent adequate excision of bladder cuff at the time of RNU. In this robotic cohort of patients undergoing RNUBCE for UTUC, not excising the bladder cuff directly translates to inferior oncologic outcomes. BCE should be performed where possible as this is an integral part of the surgery. Also, if feasible, adjunctive treatments should be considered.

\section{MODERATED POSTER SESSION 19: LAPAROSCOPIC/ROBOTIC: OTHER}

\begin{abstract}
MP19-01 Evaluation of a routine clinical prostate multiparametric MRI and Decipher Score for prediction of Biochemical Recurrence in patients who underwent robotic-assisted laparoscopic prostatectomy
\end{abstract}

P Ratnani, U Falagario, I Jambor, A Martini, S Sobotka, A Beksac, P Wiklund, S Nair, A Tewari, T Bashorun, D Phillip, R Weil

Icahn School of Medicine

Introduction \& Objective: Biochemical recurrence (BCR) affects a large proportion of patients who undergo robotic-assisted laparoscopic prostatectomy (RALP). The study aims to evaluate the performance of a routine clinical prostrate multiparametric magnetic resonance imaging (mpMRI) and Decipher score for prediction of biochemical recurrence in patients who underwent RALP.

Methods: This is a retrospective single institutional cohort study. The subjects included nightly-one patients who underwent RALP with available mpMRI and Decipher post-operative. All patients had PSA follow-up of at least 3 years or biochemical recurrence (BCR) within 3 years, defined as PSA $>0.2 \mathrm{mg} / \mathrm{ml}$ in two consecutive. The mpMRI was performed at 27 different institutions and included T2w, DWI, DCE-MRI. All mpMRI studies were reported by one reader using PI-RADsv2.1 without knowledge of other findings. Univariate and multivariate analysis using greedy feature selection and tournament leave-pairout cross-validation (TLPOCV) were used to evaluate the performance of different combination of imaging, biopsy, and Genomic variables.

Results: Forty-eight 48 (53\%) patients developed BCR. The best performing individual features with AUC of 0.73 (IQR: $0.62-$ $0.82)$ were RALP GGG, mpMRI-vol followed by biopsy GGG $(0.72,0.62-0.82)$, and Decipher score $(0.71,0.60-0.82)$. The best combination of MRI and surgical variables performed the same (TLPOCV AUC of 0.82) as the best combination of Decipher and surgical variables.

Conclusions: Relative lesion volume measured on a routine clinical mpMRI performed similarly to Decipher score in BCR prediction and their combination did not lead to further improvements. 
Table 1 Patients' demographics of 91 included patients

\begin{tabular}{|c|c|c|c|c|}
\hline & All patients & $\begin{array}{c}\text { Patients } \\
\text { without } \mathrm{BCR}\end{array}$ & $\begin{array}{l}\text { Patients with } \\
\text { BCR }\end{array}$ & P-value \\
\hline Sample size & 91 & 43 & 48 & \\
\hline Age, years; median (IQR) & $64(58-68)$ & $64.4(57.0,67.8)$ & $64.1(59.7,68.3)$ & 0.5 \\
\hline PSA, ug/L; median (IQR) & $6.9(5.2-10.0)$ & $6.5(5.3,9.0)$ & $7.2(5.2,11.6)$ & 0.3 \\
\hline dPSA\%; median (IQR) & $14.6(9.8-21.3)$ & $16.7(9.8-22.4)$ & $13(9.5-18.2)$ & \\
\hline DRE positive; n (\%) & $46(51)$ & $15(34.9 \%)$ & $31(64.6 \%)$ & 0.005 \\
\hline \multicolumn{5}{|l|}{$\begin{array}{c}\text { Biopsy Gleason Grade } \\
\text { Group }(\%)\end{array}$} \\
\hline 1 & 11 & $8(18.6 \%)$ & $3(6.2 \%)$ & 0.006 \\
\hline 2 & 26 & $17(39.5 \%)$ & $9(18.8 \%)$ & \\
\hline 3 & 26 & $12(27.9 \%)$ & $14(29.2 \%)$ & \\
\hline 4 & 14 & $4(9.3 \%)$ & $10(20.8 \%)$ & \\
\hline 5 & 14 & $2(4.7 \%)$ & $12(25.0 \%)$ & \\
\hline \multicolumn{5}{|l|}{$\%$ of PCa cores } \\
\hline$<50 \%$ & 40 & 19 & 21 & \\
\hline$\Rightarrow>50 \%$ & 51 & 24 & 27 & \\
\hline \multicolumn{5}{|l|}{ eT } \\
\hline Tlc & 42 & $28(65.1 \%)$ & $17(35.4 \%)$ & 0.017 \\
\hline $\mathrm{T} 2$ & 39 & $13(30.2 \%)$ & $28(58.3 \%)$ & \\
\hline $\mathrm{T} 3$ & 4 & $2(4.7 \%)$ & $3(6.2 \%)$ & \\
\hline \multicolumn{5}{|l|}{$\begin{array}{l}\text { RALP Gleason Grade } \\
\text { Group }\end{array}$} \\
\hline 1 & 4 & $3(7.0 \%)$ & $1(2.1 \%)$ & 0.001 \\
\hline 2 & 43 & $28(65.1 \%)$ & $15(31.2 \%)$ & \\
\hline 3 & 28 & $11(25.6 \%)$ & $17(35.4 \%)$ & \\
\hline 4 & 1 & $0(0.0 \%)$ & $1(2.1 \%)$ & \\
\hline 5 & 15 & $1(2.3 \%)$ & $14(29.2 \%)$ & \\
\hline ECE positive; n (\%) & 39 & $15(34.9 \%)$ & $24(50.0 \%)$ & 0.15 \\
\hline SVI positive; n (\%) & 16 & 4 & 12 & \\
\hline $\begin{array}{c}\text { NeuroSAFE PSM } \\
\text { positive; n (\%) }\end{array}$ & 17 & 4 & 13 & \\
\hline PSM positive; n (\%) & 11 & $7(16.3 \%)$ & $4(8.3 \%)$ & 0.2 \\
\hline \multicolumn{5}{|l|}{ pT } \\
\hline $\begin{array}{l}1 \\
3 \\
\end{array}$ & $\begin{array}{c}4 \\
41 \\
\end{array}$ & $\begin{array}{c}3(7.0 \%) \\
23(53.5 \%)\end{array}$ & $\begin{array}{c}1(2.1 \%) \\
18(37.5 \%)\end{array}$ & 0.11 \\
\hline 4 & 28 & $13(30.2 \%)$ & $15(31.2 \%)$ & \\
\hline 5 & 16 & $4(9.3 \%)$ & $12(25.0 \%)$ & \\
\hline
\end{tabular}

Table 4 Area under the curve values ( $95 \%$ confidence interval) for all individual parameters

\begin{tabular}{|l|l|c|}
\hline Variable Group & Best Set of Variables & TLPOCV AUC \\
\hline Clinical & DRE, PSA, Age & 0.70 \\
\hline Biopsy & GGG-Bx & 0.72 \\
\hline Surgery & $\begin{array}{l}\text { GGG-RALP, PSM-neurosafe, } \\
\text { PSM-prostatectomy }\end{array}$ & 0.78 \\
\hline Genes & Decipher-test-score & 0.71 \\
\hline MRI & MRI-lesion-percentage & 0.72 \\
\hline Clinical and MRI & MRI-lesion-percentage, DRE, PSA & 0.75 \\
\hline Biopsy and MRI & MRI-lesion-percentage, GGG-Bx & 0.76 \\
\hline Surgery and MRI & $\begin{array}{l}\text { GGG-RALP, MRI-lesion-percentage, } \\
\text { PSM-neurosafe, PSM-prostatectomy }\end{array}$ & 0.81 \\
\hline Genes and MRI & MRI-lesion-percentage, Decipher-test-score & 0.75 \\
\hline Genes and Surgery & $\begin{array}{l}\text { GGG_RALP, PSM-neurosafe, } \\
\text { Decipher-test-score, PSM-prostatectomy }\end{array}$ & 0.82 \\
\hline Clinical, Biopsy and MRI & MRI-lesion-percentage, GGG-Bx & 0.76 \\
\hline $\begin{array}{l}\text { Clinical, Biopsy, Surgery, } \\
\text { Genes and MRI }\end{array}$ & $\begin{array}{l}\text { GGG-RALP, MRI-lesion-percentage, } \\
\text { PSM-neurosafe, PSM-prostatectomy }\end{array}$ & 0.79 \\
\hline
\end{tabular}

MP19-02 Outcomes of radical prostatectomy in transplanted- kidney patients who have localized prostate cancer

W Kongcharoensombat, W Kongchareonsombat

Mahidol University

Introduction \& Objective: To present the outcomes and method of radical prostatectomy in end stage renal disease patients who have transplanted kidney in iliac fossa.
Methods: We have collected the results of surgery in radical prostatectomy in localized prostate cancer in patients who have kidney transplantation. We choose three methods for surgery open ( 2 cases), laparoscopic ( 2 cases) and robotic (3cases) assisted radical prostatectomy The seven cases in our series and the preoperative and out comes are reported in estimated blood loss (cc), operative time (minutes), hospital stay (day), and blood for kidney function (serum creatinine level)

Results: The result are report in

\begin{tabular}{|c|c|c|c|c|c|c|c|}
\hline & ORP & ORP & LRP & LRP & RARP & RARP & RARP \\
\hline $\operatorname{EBL}(\mathrm{CC})$ & 800 & 1,000 & 300 & 300 & 350 & 200 & 250 \\
\hline $\begin{array}{l}\text { OP TIME } \\
\text { (MIN.) }\end{array}$ & 360 & 360 & 180 & 180 & 180 & 120 & 180 \\
\hline $\begin{array}{l}\text { Hospital } \\
\text { stay(day) }\end{array}$ & 14 & 15 & 7 & 7 & 5 & 5 & 5 \\
\hline $\begin{array}{l}\text { serum } \\
\quad \text { creatinine }\end{array}$ & 0.9 & 1.1 & 1.1 & 0.9 & 1.1 & 0,9 & 0.8 \\
\hline
\end{tabular}

Conclusions: All seven patents have operation in different options and outcomes are similar. So the radical prostatectomy is the good choice for localized prostate cancer even they have transplanted kidney that obstructed the operative field and no harm for the patients and transplanted kidneys.

\section{MP19-03 A Multicentre United Kingdom Study of Robot Assisted Ureteric Reimplantation}

E Latif, J Chong, J Pain, M Wright, EH Streeter, RA Persad, PD Rimington, SS Kommu, BA Eddy

Kent \& Canterbury Hospital. East Kent Hospitals NHS Foundation Trust

Introduction \& Objective: Ureteric reimplantation is a procedure indicated in a variety of situations, in both pediatric and adult populations, including benign and malignant disorders of the ureter, and transplant surgery. This has conventionally been performed using open operative techniques. In the past decade, published reports of ureteric reimplantation using the daVinci Surgical System have emerged. A number of case series have demonstrated successful outcomes in a variety of indications. Published comparisons of open vs. robotic procedures have demonstrated shorter hospital stay and reduced opiate requirement, though longer operative times. Further European and American series in transplantation have also been presented with good outcomes. Herein, we present a United Kingdom Multicenter experience of robot assisted ureteric reimplantation.

Methods: We retrospectively reviewed all patients who have undergone robotic ureteric reimplantation in several centers across the United Kingdom. Patient charts were reviewed for demographics, indication, operative time, and complications.

Results: A total of 70 patients underwent robotic assisted ureteric reimplantation across three centers. Indications included ureteric strictures secondary to pelvic endometriosis, stone disease, bladder diverticula and iatrogenic ureteric injury. The procedures included distal ureteric resections with reimplantation and psoas hitch, bladder diverticulectomy with reimplantation, and uretero-ureterostomy. The median operative time was 155 minutes, and the median hospital stay was 1 day. No intraoperative complications were observed, and no cases required surgical conversion. Post-operative complications include urinary tract infection in one patient at 4 weeks, and residual reflux 
pain on voiding in three patients. There was one readmission due to pain. The mean follow-up time was 4.7 months, with all patients having no evidence of urinary tract obstruction.

Conclusions: Our series demonstrates robotic-assisted ureteric reimplantation is safe, feasible and effective and offers a minimally invasive alternative to traditional open surgery. Outcomes have been favorable in short-term follow-up.

MP19-04 Decreased Short-Term Complications in Minimally-Invasive Approaches to Nephrectomy in the Elderly

PC Yong, A Kaldany, KK Badani

Icahn School of Medicine at Mount Sinai

Introduction \& Objective: Greater than $20 \%$ of new renal cell carcinoma (RCC) cases are diagnosed in elderly patients above the age of 75. Minimally-invasive approaches to partial and radical nephrectomy (MIPN and MIRN) have been shown to result in fewer complications compared to open approaches, but few studies specifically investigate outcomes in the elderly. We used the National Surgical Quality Improvement Program (NSQIP) dataset to compare 30-day post-operative outcomes based on surgical approach to nephrectomy in patients age 75 and older.

Methods: We identified patients age 75 and older who underwent PN and RN using NSQIP data from 2005 to 2014. Surgical complications were grouped according to the Clavien-Dindo classification as minor (Clavien I-II), major (Clavien III-IV), and death (Clavien V). Differences in post-operative complications based on surgical approach were evaluated using student's Ttests and Chi-Square tests as appropriate. Multivariable regression was used to adjust for comorbidities. All statistical analyses

Table 1. Comparison of 30-day complications of partial and radical nephrectomy by procedure approach.

\begin{tabular}{|c|c|c|c|c|c|c|}
\hline & $\begin{array}{l}\text { MIPN } \\
n(\%)\end{array}$ & $\begin{array}{r}\text { OPN } \\
n(\%)\end{array}$ & P-value & $\begin{array}{l}\text { MIRN } \\
n(\%)\end{array}$ & $\begin{array}{l}\text { ORN } \\
n(\%)\end{array}$ & P-value \\
\hline \multicolumn{7}{|l|}{ Minor } \\
\hline$\overline{\text { SSI }}$ & 0 & $4(1.0)$ & 0.03 & $17(1.2)$ & $18(2.4)$ & 0.05 \\
\hline PNA & $6(1.0)$ & $13(3.4)$ & 0.02 & $31(2.2)$ & $35(4.7)$ & 0.001 \\
\hline PE & $3(0.5)$ & $3(0.8)$ & 0.61 & $6(0.4)$ & $11(1.5)$ & 0.02 \\
\hline UTI & $7(1.2)$ & $11(2.9)$ & 0.09 & $45(3.1)$ & $27(3.6)$ & 0.54 \\
\hline Blood & $46(8.0)$ & $80(20.9)$ & $<0.0001$ & $153(10.6)$ & $249(33.2)$ & $<0.0001$ \\
\hline Transfusion & & & & & & \\
\hline DVT & $1(0.2)$ & $5(1.3)$ & 0.04 & $6(0.4)$ & $11(1.5)$ & 0.02 \\
\hline Overall & $60(10.4)$ & $104(27.2)$ & $<0.0001$ & $229(15.9)$ & $290(38.70)$ & $<0.0001$ \\
\hline \multicolumn{7}{|l|}{ Major } \\
\hline$\overline{\mathrm{DSI}}$ & 0 & $1(0.3)$ & 0.22 & $3(0.2)$ & $3(0.4)$ & 0.41 \\
\hline $\begin{array}{l}\text { Organ space } \\
\text { infection }\end{array}$ & $1(0.2)$ & $2(0.5)$ & 0.34 & $5(0.4)$ & $8(1.1)$ & 0.07 \\
\hline Dehiscence & $2(0.4)$ & $2(0.5)$ & 0.68 & $4(0.3)$ & $1(0.1)$ & 0.50 \\
\hline Reintubation & $6(1.0)$ & $14(3.7)$ & 0.009 & $21(1.5)$ & $26(3.5)$ & 0.003 \\
\hline $\begin{array}{l}\text { Ventilator } \\
>48 \mathrm{~h}\end{array}$ & $2(0.4)$ & $7(1.8)$ & 0.03 & $4(0.3)$ & $31(4.1)$ & $<0.0001$ \\
\hline $\begin{array}{l}\text { Acute renal } \\
\text { failure }\end{array}$ & $1(0.2)$ & $8(2.1)$ & 0.004 & $8(0.6)$ & $17(2.3)$ & 0.0009 \\
\hline CVA & 0 & $2(0.5)$ & 0.08 & $1(0.1)$ & $2(0.3)$ & 0.23 \\
\hline Cardiac arrest & $5(0.9)$ & $6(1.6)$ & 0.32 & $1(0.1)$ & $9(1.2)$ & 0.0004 \\
\hline MI & $3(0.5)$ & $10(2.6)$ & 0.009 & $8(0.6)$ & $10(1.3)$ & 0.08 \\
\hline Sepsis & 0 & $12(3.1)$ & $<0.0001$ & $16(1.1)$ & $18(2.4)$ & 0.03 \\
\hline Overall & $14(2.4)$ & $39(10.2)$ & $<0.0001$ & $55(3.8)$ & $80(10.7)$ & $<0.0001$ \\
\hline Death & $6(1.0)$ & $8(2.1)$ & 0.18 & $12(0.8)$ & $22(2.9)$ & 0.0003 \\
\hline $\begin{array}{l}\text { Prolonged } \\
\text { Length of Stay } \\
\text { (>5 days) }\end{array}$ & $61(10.6)$ & $147(28.4)$ & $<0.0001$ & $314(21.8)$ & $391(52.2)$ & $<0.0001$ \\
\hline $\begin{array}{l}\text { Return to OR } \\
\text { within } 30 \text { days }\end{array}$ & $6(1.0)$ & $18(4.7)$ & 0.0005 & $38(2.6)$ & $30(4.0)$ & 0.09 \\
\hline
\end{tabular}

$\mathrm{SSI}=$ superficial skin infection, $\mathrm{PNA}=$ pneumonia, $\mathrm{PE}=$ pulmonary embolism, $\mathrm{UTI}=$ urinary tract infection, DVT = deep vein thrombosis, DSI = deep skin infection, CVA = cerebrovascular accident, $\mathrm{MI}$ = myocardial infarction.

Minor $=$ Clavien I-II, Major $=$ Clavien III-IV, Death $=$ Clavien V
Table 2. Adjusted logistic regression comparing of 30-day complications of partial and radical nephreetomy by procedure approach.

\begin{tabular}{|c|c|c|}
\hline & $\begin{array}{c}\text { Partial Nephrectomy } \\
\text { Robotic vs. Open } \\
\text { OR }(95 \% \mathrm{Cl})\end{array}$ & $\begin{array}{c}\text { Radical Nephrectomy } \\
\text { Robotic vs. Open } \\
\text { OR }(95 \% \text { Cl })\end{array}$ \\
\hline $\begin{array}{l}\text { Minor } \\
\text { SSI } \\
\text { PNA } \\
\text { PE } \\
\text { UTI } \\
\text { Blood Transfusion } \\
\text { DVT } \\
\text { Overall }\end{array}$ & $\begin{array}{l}- \\
\mathbf{0 . 3 6}(\mathbf{0 . 1 3}, \mathbf{0 . 9 7}) \\
0.66(0.13,3.36) \\
0.45(0.17,1.20) \\
\mathbf{0 . 3 5}(0.23,0.52) \\
0.14(0.02,1.21) \\
\mathbf{0 . 3 3}(\mathbf{0 . 2 3}, \mathbf{0 . 4 8})\end{array}$ & $\begin{array}{c}0.49(0.25,0.96) \\
0.45(0.28,0.75) \\
0.26(0.10,0.71) \\
0.84(0.51,1.4) \\
0.23(0.18,0.29) \\
0.28(0.10,0.75) \\
0.29(0.23,0.35)\end{array}$ \\
\hline $\begin{array}{l}\text { Major } \\
\text { DSI } \\
\text { Organ space infection } \\
\text { Wound dehiscence } \\
\text { Reintubation } \\
\text { Ventilator }>48 \mathrm{~h} \\
\text { Acute renal failure } \\
\text { CVA } \\
\text { Cardiac arrest } \\
\text { MI } \\
\text { Sepsis } \\
\text { Overall }\end{array}$ & $\begin{array}{c}- \\
0.26(0.02,3.03) \\
0.46(0.04,5.02) \\
\mathbf{0 . 3 2}(\mathbf{0 . 1 2}, \mathbf{0 . 8 4}) \\
\mathbf{0 . 1 5}(\mathbf{0 . 0 3}, \mathbf{0 . 8 0}) \\
\mathbf{0 . 1 0}(\mathbf{0 . 0 1}, \mathbf{0 . 8 3}) \\
- \\
0.65(0.19,2.20) \\
\mathbf{0 . 2 3}(\mathbf{0 . 0 6}, \mathbf{0 . 8 5}) \\
- \\
\mathbf{0 . 2 6}(\mathbf{0 . 1 4}, \mathbf{0 . 4 9})\end{array}$ & $\begin{array}{l}0.53(0.10,2.67) \\
0.32(0.10,0.98) \\
2.2(0.24,20.35) \\
\mathbf{0 . 4 3}(0.24,0.78) \\
\mathbf{0 . 0 6}(0.02,0.18) \\
\mathbf{0 . 2 5}(0.11,0.61) \\
0.26(0.02,2.91) \\
\mathbf{0 . 0 6}(0.01,0.49) \\
0.43(0.17,1.10) \\
\mathbf{0 . 4 6}(0.23,0.90) \\
\mathbf{0 . 3 4}(0.23,0.48)\end{array}$ \\
\hline Death & $0.62(0.21,1.88)$ & $0.28(0.14,0.58)$ \\
\hline $\begin{array}{l}\text { Prolonged Length of } \\
\text { Stay }(>5 \text { days) }\end{array}$ & $0.21(0.15,0.29)$ & $0.25(0.20,0.30)$ \\
\hline $\begin{array}{l}\text { Return to } O R \text { within } \\
30 \text { days }\end{array}$ & $0.25(0.10,0.64)$ & $0.69(0.42,1.12)$ \\
\hline
\end{tabular}

were performed using SAS (version 9.4) with significance defined as $\mathrm{p}<0.05$.

Results: In total, we identified 961 elderly patients who underwent PN and 2193 elderly patients who underwent RN. Of the PN and RN performed, 60\% (578/961) and 66\% (1444/2193) were via the MI approach, respectively. After adjusting for comorbidities, elderly patients undergoing MI procedures had a significantly lower rate of both minor and major complications and prolonged length of stay compared to elderly patients undergoing open procedures (Tables 1,2). Patients undergoing MIPN had a lower 30-day return to OR rate compared to open $\mathrm{PN}$, and patients undergoing MIRN had lower 30-day mortality compared to open $\mathrm{RN}$.

Conclusions: $\mathrm{MI}$ approaches to $\mathrm{PN}$ and $\mathrm{RN}$ in the elderly are associated with a favorable short-term complication profile compared to open approaches. In the context of an aging population and increasing incidence of RCC in the elderly, these findings are important in determining the optimal treatment modality for this group.

\section{MP19-05 Open Retrieval Post Retroperitoneoscopic Radical Nephrectomy does not increase risk of port site recurrence.}

N Kale, Y Wagh, H Godbole, F Harris

Department of Urology, North Middlesex Hospital

Introduction \& Objective: Laparoscopic \& Robotic radical nephrectomy is an acceptable minimally invasive treatment with acceptable long-term oncological outcomes. We report the incidence of port site recurrence following open retrieval post retroperitoneoscopic radical nephrectomies.

Methods: Prospective data collection was performed for all patients who underwent a retroperitoneoscopic radical nephrectomy at our hospital from 2005. Information was accessed from a specially created excel sheet. Further information was obtained from PACS system. Patients were followed up as per local network guidelines. Data was manually compiled \& a retrospective 
analysis was performed. Recurrences were identified on surveillance scans during MDT discussions. With patients in a lateral position a retro peritoneal space was created using a disposable balloon port placement was done with open technique. Care was taken not to breach the peritoneum. After development of space the ureter was identified which was traced up to the hilum. Artery and vein dissection once completed. Artery and vein were clipped with hemolock clips. Kidney was dissected all around and ureter cut. There was no space to open a retrieval bag in the retro peritoneum. Hence the kidney was removed through an incision placed by joining the anterior port sites.

Results: 102 patients underwent a Retroperitoneoscopic Radical Nephrectomy. 3 patients had local recurrence, not a single patient had port site recurrence. The use of specimen retrieval bags has been described after laparoscopic radical nephrectomies. These were essentially performed inside the peritoneal cavity allowing a large space for movements of instruments. In comparison the retro peritoneum affords a limited space to open the retrieval bag and place a specimen within. Our results don't show any worsening of oncological efficacy after radical nephrectomies.

Conclusions: Open retrieval without use of a specimen bag does not lead to port site recurrence after retroperitoneoscopic radical nephrectomy.

\section{MP19-06 Withdrawn}

\section{MP19-07 The Role of the Prostatic Vasculature as a Landmark for Bladder Neck Sparing during Robot- assisted Radical Prostatectomy.}

M Shahait, A Sandberg, DI Lee

University of Pennsylvania

Introduction \& Objective: Bladder neck sparing during Robotassisted Radical Prostatectomy is a daunting task with a controversial effect on early continence and has been linked to an increase in the positive surgical margin rate. Prostate artery gives perforating branches to the prostatic gland, with apparent penetration made at the 2 and 10 o'clock positions for the anterolateral pedicles and at the 5 and 7 o'clock positions for the posterior-lateral pedicles. The antero-lateral pedicles runoff could provide macroscopic landmarks for bladder neck dissection during RARP.

Methods: We prospectively evaluated 53 consecutive patients who underwent RARP between March and May 2019 for the presence of this landmark artery on each side of the prostate gland during bladder neck dissection. A modified lateral approach for bladder neck sparing was performed on all patients. The length of the surgical margin at the bladder neck was assessed by a pathologist who was blinded to the landmark artery status. All bladder neck dissections were graded between 1 and 4; higher grades corresponded to an increasing degree of complete bladder neck preservation.

Results: We could identify a LA in $83.9 \%$ (89 of 106) of the operated sides. Bladder neck sparing (grade $\geq 3$ ) was achieved in $79.24 \%$ of the patients. Positive surgical margin was noted in $37.7 \%$ of the patients. Only 2 patients in the total cohort had PSM at the bladder neck, both of them had pT3 disease and their bladder neck spared. The linear length of both margins was $<3 \mathrm{~mm}$.
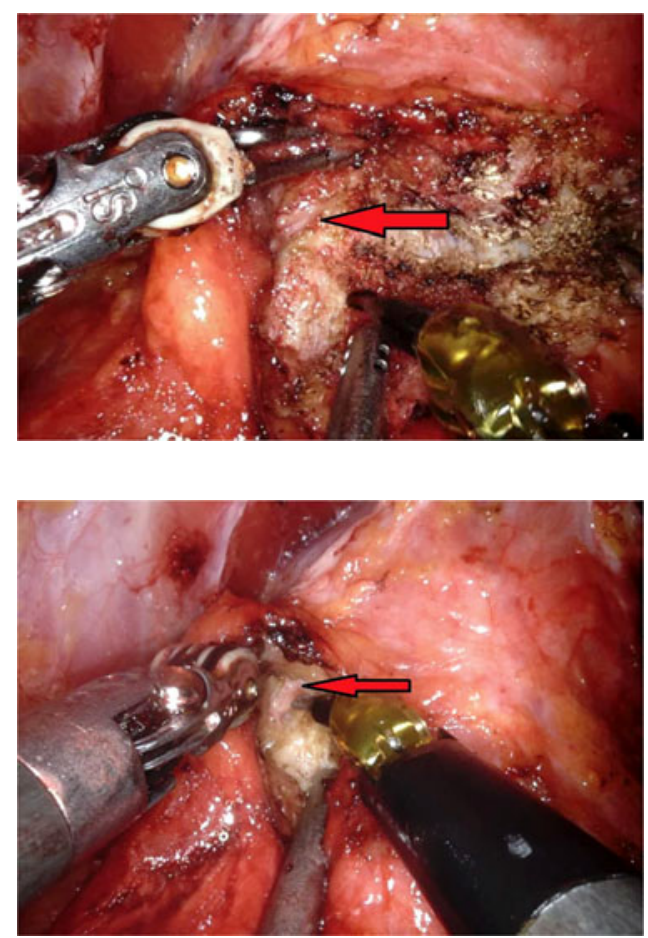

Conclusions: The Prostatic Vasculature provide an identifiable landmark during bladder neck dissection, which help to maximize bladder neck sparing without compromising oncological outcome.

MP19-08 Do Dialysis and Transplant Patients have Worse Surgical Outcomes with Laparoscopic Nephrectomy Compared to Normal Controls?

K Parikh, A Kahn, D Thiel

Mayo Clinic Florida

Introduction \& Objective: Among patients undergoing laparoscopic nephrectomy, there is an inherent concern of the negative effects of losing a portion of renal function. Nephrectomy patients with a history of end stage renal disease are a special patient cohort given that they already have suboptimal renal function prior to undergoing nephrectomy. Our goal was to compare our renal transplant and dialysis patient population to normal controls to see if they have comparable or worse surgical outcomes.

Methods: Data was collected from 412 patients who underwent laparoscopic nephrectomy at Mayo Clinic in Jacksonville, FL from July 2007 to October 2018. Among the 412 patients, 62 were on dialysis, 20 had a renal transplant in place, and 330 were considered normal controls. Surgical outcomes of dialysis and transplant patients were compared to controls using Wilcoxon rank sum test, Fisher exact sum test, and the Holm step down method for statistical analysis. Specific surgical outcomes studied included operative time, postoperative complications, length of hospital stay and 90-day readmission rate.

Results: Comparing dialysis patients to normal controls: There was evidence to suggest that dialysis patients compared to controls had a shorter total operative time (median: 133 vs. 149; $\mathrm{P}=0.022$ ). Dialysis patients compared to controls did not have 
any significant differences in postoperative complication rate $(8 \%$ vs $5 \% ; \mathrm{P}=0.34)$, greater than 3 -day hospital stay $(23 \%$ vs $16 \% ; \mathrm{P}=0.20)$, or 90 -day readmission rate ( $8 \%$ vs $6 \% ; \mathrm{P}=0.56)$. Comparing transplant patients to normal controls: Transplant patients compared to normal controls had a statistically significant higher rate of 90 -day readmission $(20 \%$ vs $6 \%$; $\mathrm{P}=0.034)$. Transplant patients compared to normal controls did not have any significant differences in median operative time (134 vs $149 ; \mathrm{P}=0.47)$, postoperative complication rate ( $15 \%$ vs $5 \%$; $\mathrm{P}=0.075)$, or greater than 3 -day hospital stay $(30 \%$ vs $16 \%$; $\mathrm{P}=0.12$ ).

Conclusions: When comparing dialysis patients to normal controls undergoing laparoscopic nephrectomy, dialysis patients did not have worse surgical outcomes. In fact, dialysis patients actually had a shorter operative time than their normal control counterparts. Transplant patients, however, did have a higher 90day readmission rate after laparoscopic nephrectomy when compared to normal controls.

MP19-09 The use of multiple video assessment methods to determine the influence of surgical skill on potency and continency in patients after robot-assisted radical prostatectomy

A Beulens, W Brinkman, R Meijer, E Koldewijn, J van Basten, $\mathrm{H}$ van der Poel, J van Merriënboer, C Bangma, C Wagner

Catharina Hospital Eindhoven

Introduction \& Objective: A combination of surgical skill and perioperative events have its effect on postoperative outcome. Different video assessment methods can be used to assess surgical skills alone. This group developed the PROTEST method in order to assess both surgical skill and perioperative events. In order to evaluate the effectiveness of the different assessment method our research question is: Which aspects of surgical skill and perioperative events can be related to postoperative outcome? Is it possible to measure surgical skill using the PROTEST method?

Methods: Patients who underwent a RARP in the Antoni van Leeuwenhoek Hospital were eligible for this study. Two cohorts containing two arms of patients were selected and matched. Surgical skills were measured using, the global evaluative assessment of robotic skill (GEARS), the Prostatectomy Assessment and Competence Evaluation (PACE). and the PROTEST method developed by this research group. As a fourth assessment method the videos were evaluated by two experts surgeon (the surgeon who performed the surgery and an independent expert in RARP, both with experience in >500 RARP). All assessors were blinded for the selected study arm and group of patients and were asked to evaluate all surgery on continence and erectile function.

Results: The GEARS and PACE methods showed no difference in either cohorts. The results of the PROTEST method showed a shorter duration of the nerve preservation phase of the surgery in the erectile dysfunction group versus the group with adequate erections. There were no differences in the other phases of the surgery in all groups of the study. One of the experts who evaluated the surgeries was able to determine continence in $90 \%$ of the patients and incontinence in $75 \%$ of the patients. The erectile function was accurately determined by this expert in $75 \%$ of the group with adequate erections and $83.3 \%$ in the group with erectile dysfunction. The assessments included the apical dissection of the prostate and the vesicle-urethral anastomosis phase of the surgery. The verdict was based on the length and thickness of the urethra and the thickness of the neuro-vascular bundles. Conclusions: The results of this study showed the assessment of continence and erectile function by an expert reviewer gives insight into perioperative factors which could influence postoperative results. These factors were not assessed in the different assessment methods used in this study. Further research into the specific influence of the length and thickness of the urethra and the thickness of the neuro-vascular bundles is needed to clarify the relation between these aspects of the surgery and continence and or erectile function.

\section{MP19-10 Face and Content Validity of a novel chicken model for Laparoscopic Neobladder Reconstruction.}

A Singh, Z Kareem, GR TAK, A Ganpule, R Sabnis, M DESAI

Muljibhai Patel Urological Hospital

Introduction \& Objective: Learning curve of complex urological procedures can be shortened by simulation-based training. We present a cadaveric chicken and bovine intestine model for training in laparoscopic neobladder reconstruction.

Methods: It is a prospective observational study. Twenty novice and twenty trained laparoscopic surgeons were included in the study. The relevant chicken anatomy and surgical steps were described to all the surgeons. The culled chicken was placed on its back; evisceration was done and proventriculus was used as urethra and $70 \mathrm{~cm}$ of bovine intestine was used to reconstruct the neobladder. The chicken esophagus and trachea were excised and attached to the chicken thigh and mimicked as right and left ureter. This assembly was placed in a self-designed endotrainer. The surgeons were asked to fill a nine-point questionnaire comprising of: bowel organization, ability to do urethro neovesical anastomosis, suturing time, suturing similarity, quality of suturing, tissue texture, integrity of anastomosis, realism and usefulness of model after finishing the procedure and score it on a scale of $1-5$.

Results: All the participants in the study gave a mean score of 3 or more to all the questions asked in the questionnaire. Both the groups rated the usefulness of the model highly with a mean score of 4.6 and 4.4 respectively. The mean score of the questionnaire was 35.9 and 36 respectively for both the groups.

Conclusions: The Chicken model for laparoscopic neobladder reconstruction is a useful and effective training tool. This model has Face and Content validity to be used as a teaching and training tool in laparoscopic urology.

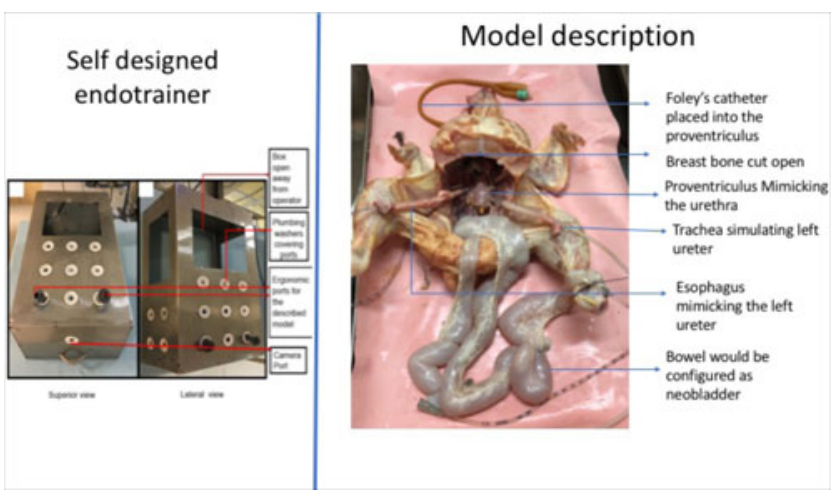


MP19-11 Laparoscopy has better conditional survival than open radical nephroureterectomy in patients with stage pT3 advanced UUTC: a retrospective multicenter conditional survival analysis

\section{Song, B Hong, S Kang, B Jeong, J Ku, H Seo, S KIM}

Introduction \& Objective: We aimed to propose a comparison of conditional cancer-specific survival (CSS) between open (ONU) and laparoscopic (LNU) radical nephroureterectomy (RNU) in Korean population with locally advanced upper urinary tract urothelial carcinoma (UTUC) with pT3-4N0M0 and TxN+M0.

Methods: We retrospectively reviewed 723 patients who underwent ONU $(n=388)$ or LNU $(n=260)$ from 5 tertiary institutions between 2000 and 2012. Cox proportional hazard regression model and the Kaplan-Meier estimator were used to identify the effect of surgical approach as a predictor of additional 5-year CSS suggested by the 1- to 5-year survivorship model, and to estimate the conditional survival using the multiplicative law of probability. Results: During the median follow-up duration of 49.00 months, 255 deaths were observed. Among the significant risk factors for CSS, including age, presence of concomitant bladder tumor, pathological TN stage, and presence of lymphovascular invasion in multivariate analysis, the following year of CSS showed that additional tumor nuclear grade and baseline significant factors were significant factors for 1 st-year CSS, carcinoma in situ presence and baseline tumor location significant for 2nd-year CSS, and adjuvant chemotherapy significant for 3rd-year CSS after RNU. The CSS curves according to tumor staging showed that CSS was significantly different between LNU and ONU from postoperative 4 years for T3, 3 years for T4, and 4 years for $\mathrm{pN}+$.

Conclusions: This study showed not only insignificantly better trends of survival rate of LNU over ONU in locally advanced UTUC but also significantly better survival rate of LNU, especially in patients with stage pT3 UTUC.

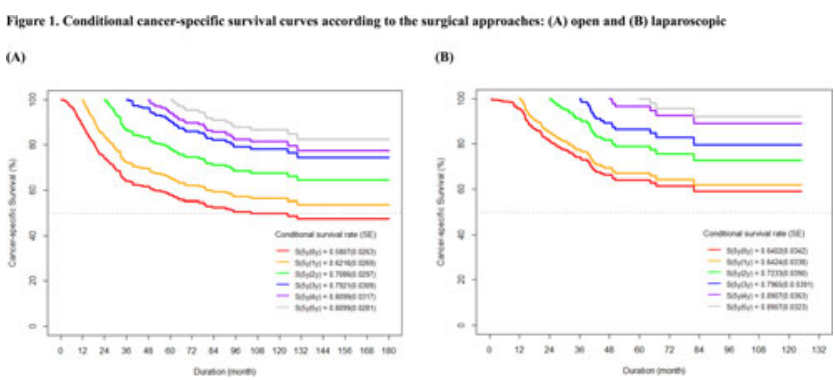

Figure 2. Coeditional probulality of cancet-specific survival in (A, D) stage pT3, (B, E) stage pT4, and (C, F) stage pN+ upper uriney trat urothelial carcinoma groups accoerding to surgical approach including previous history of bladder cancer (A, B, and C) or without hissory of bladder cancer (D, E, and F) .

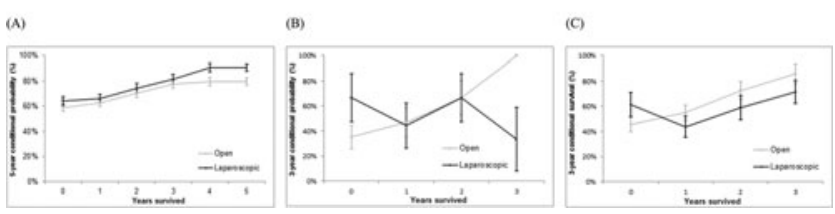

MP19-12 Indocyanine Green Angiography for Use in Robotic Spermatic Cord Denervations

NV Halstead, SP Catarinicchia, GL Lloyd

Introduction \& Objective: Once patients have failed more conservative therapies for orchialgia they may elect to undergo
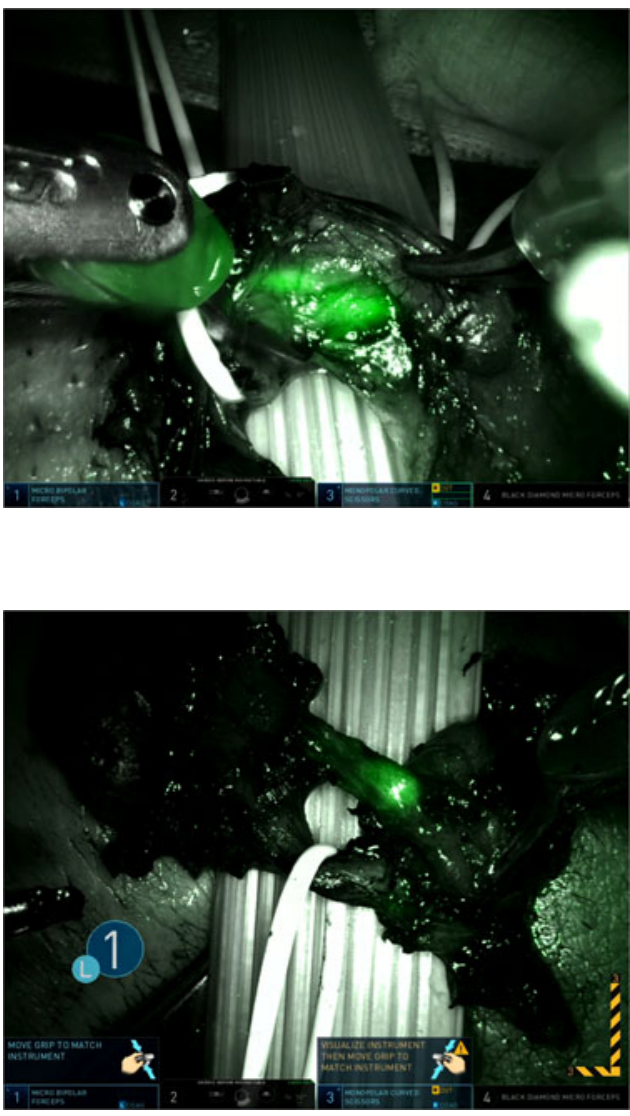

surgery in the form of a spermatic cord denervation. One challenge in doing so is ensuring the arterial blood supply is preserved throughout the case. Currently there is a paucity of data surrounding these surgical approaches. Our goal is to describe our experience using indocyanine green (ICG) for intra-operative angiography during robotic cord denervations as a means to help identify and preserve the testicular artery.

Methods: We describe our use of ICG for testicular artery identification during a robotic spermatic cord denervation in two patients with chronic orchialgia, performed by a single surgeon. Both had failed conservative management with medications and scrotal support. Other treatable causes of orchialgia were ruled out. They both underwent a spermatic cord block in clinic prior to surgery to test temporary effects prior to permanent irreversible surgical denervation. The cord was delivered in an open fashion and dissection was completed using the da Vinci Xi Robotic Surgical System. We then injected $0.5 \mathrm{cc}$ to $1 \mathrm{cc}$ of ICG intravenously to identify the testicular artery, which was then confirmed using a Doppler probe. This was repeated at the end of the case to confirm preservation of the artery.

Results: Each patient was administered ICG twice. The first patient received $1 \mathrm{cc}$ initially and then $0.5 \mathrm{cc}$ to confirm. The second patient receive $0.5 \mathrm{cc}$ twice. In all instances, the testicular artery illuminated green in Firefly mode within minutes of administration (Figures 1 and 2). This was then confirmed with use of a Doppler probe, which produced strong arterial signals. The remainder of the dissection was carried out with care to avoid the artery.

Conclusions: The use of ICG angiography intra-operatively for robotic spermatic cord denervations appears to be a reasonable approach that aids in the identification and preservation of the testicular artery. 
MP19-13 Factors associated with Increasing trend in benign pathology at partial nephrectomy for cT1 tumors in the era of advanced renal imaging

AK Chow, A Sivaraman, R Figenshau, J Ippolito, J Vetter, R Venkatesh

Washington University School of Medicine, St. Louis, USA

Introduction \& Objective: Factors associated with finding a being lesion at partial nephrectomy for renal mass is not clear and this continues to increase in spite of significant advances in the imaging for renal mass including appropriate phasing protocols and more recently functional scans. The aim of this study was to evaluate factors associated with finding a benign tumor on imaging among patients undergoing partial nephrectomy for a cT1 renal mass in a large tertiary center.

Methods: Between 2007 and 2017, 1068 partial nephrectomies were performed for suspected malignant renal mass in a large tertiary center. Patients with established familial renal cancer syndromes and imaging consistent with benign lesions were excluded. Pre-operative imaging (computer tomography [CT]

Table 1. Baseline demographics

\begin{tabular}{|c|c|c|c|}
\hline \multicolumn{4}{|c|}{ Cohort: All Partial Nephrectomies from 2007 - 2017} \\
\hline Variable & $\begin{array}{l}\text { Benign } \\
(\mathrm{N}=153)\end{array}$ & $\begin{array}{l}\text { Malignant } \\
\text { (N=915) }\end{array}$ & p-value \\
\hline Age & & & 0.008 \\
\hline Mean & 60.7 & 57.9 & \\
\hline St. Dev. & 11.2 & 12.3 & \\
\hline Clinical Tumor Size & & & $<0.001$ \\
\hline Mean & 2.7 & 3.2 & \\
\hline St. Dev. & 1.5 & 1.5 & \\
\hline Pathologic Tumor Size & & & 0.014 \\
\hline Mean & 2.7 & 3.0 & \\
\hline St. Dev. & 1.4 & 1.6 & \\
\hline $\mathrm{CCl}$ & & & 0.177 \\
\hline Mean & 1.0 & 1.2 & \\
\hline St. Dev. & 1.4 & 1.6 & \\
\hline BMI & & & 0.022 \\
\hline Mean & 29.7 & 31.2 & \\
\hline St. Dev. & 5.4 & 7.2 & \\
\hline Laterality & & & 0.361 \\
\hline Right (553) & $48.4 \%$ & $52.3 \%$ & \\
\hline Left (515) & $51.6 \%$ & $47.7 \%$ & \\
\hline Imaging & & & 0.203 \\
\hline СT (586) & $58.8 \%$ & $54.3 \%$ & \\
\hline MRI (301 & $27.5 \%$ & $28.3 \%$ & \\
\hline $\mathrm{U} / \mathrm{S}(61)$ & $7.2 \%$ & $5.5 \%$ & \\
\hline & Benign (153) & Malignant (915) & p-value \\
\hline Tumor Size Subsets & & & 0.001 \\
\hline $0-1.5 \mathrm{~cm}(112)$ & $25.9 \%$ & $74.1 \%$ & \\
\hline $1.5-2 \mathrm{~cm} \mathrm{(178)}$ & $17.4 \%$ & $82.6 \%$ & \\
\hline $2-3 \mathrm{~cm}(320)$ & $14.1 \%$ & $85.9 \%$ & \\
\hline $3.4 \mathrm{~cm}(239)$ & $11.3 \%$ & $88.7 \%$ & \\
\hline $4-7 \mathrm{~cm}(198)$ & $9.1 \%$ & $90.9 \%$ & \\
\hline
\end{tabular}

Figure 1: a) Trend of benign pathology at partial nephrectomy from 2007 - 2017; b) Percent of benign and malignant pathology noted at partial nephrectomy by year; c) Comparison of case volume and corresponding incidence of benign pathology at partial nephrectomy by year.
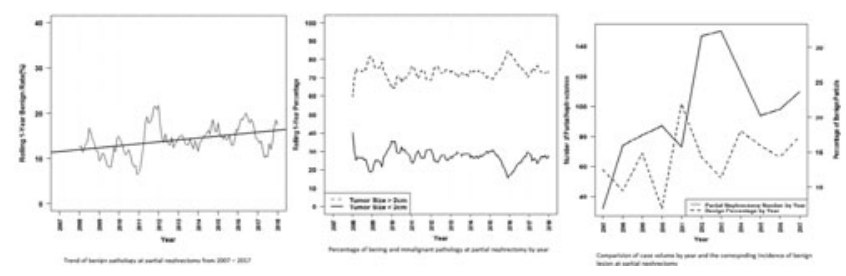

and magnetic resonance imaging [MRI]) and imaging protocols evolved per established practice over the study period. Patient population was divided into benign $(n=153)$ and malignant $(\mathrm{n}=915)$ based on final histopathology. Baseline demographics were compared between the two groups and the incidence of finding benign lesion across tumor size up to $7 \mathrm{~cm}$ and the trend over the 10-year period was evaluated.

Results: Respective mean age (years) in benign and malignant group was 60.7 vs $57.9(\mathrm{p}=0.008)$ and clinical tumor size $(\mathrm{cm})$ was 2.7 vs 3.2 ( $\mathrm{p}<0.001)$. Pre-operative imaging employed were CT in $54.9 \%$, MRI in $28.12 \%$ and US in $5.75 \%$. Incidence of benign lesion ranged from $25.9 \%$ in $<1.5 \mathrm{~cm}$ lesions to $9.1 \%$ in 4-7 cm renal lesions (Table 1). Over the 10-year period, incidence of benign lesions continues to increase from $12.5 \%$ in 2007 to $17.3 \%$ in 2017 while percent of small tumors $(<2 \mathrm{~cm})$ operated did not increase significantly. Volume of partial nephrectomies performed by year was not associated with the incidence of being lesions. (Figure 1) Multivariable logistic regression showed increasing age $(\mathrm{p}<0.001)$ and decreasing tumor size $(\mathrm{p}=0.001)$ were significantly associated with finding benign pathology.

Conclusions: Finding benign pathology at partial nephrectomy continues to rise in spite of advances in renal imaging. Case volume by year did not contribute to this increase and percent of small tumors $(<2 \mathrm{~cm})$ operated did not increase. Improving imaging methods and developing a robust nomogram potentially incorporating functional imaging and genetic information may reduce misclassified preoperative evaluation.

\section{MP19-14 The Efficiency and Safety of Different An- algesic Drugs for patients with Postoperative Pain in Department of Urology: A Randomized Controlled Trial}

D Ye, L Ma, L Liu, B Liao, H Li, K Wang

West China Hospital

Introduction \& Objective: The management of postoperative pain is an important part of ERAS (Enhanced recovery after surgery). The present study is to investigate the efficiency and safety of parecoxib or traditional on-demand opioids in various types of urology surgery, and to evaluate its application in ERAS. Methods: A total of 278 patients underwent urology surgery and were hospitalized in the Department of Urology, West China Hospital of Sichuan University from August 2016 to April 2018 were enrolled. The patients were randomly divided into the parecoxib sodium group and the conventional opioid analgesia group. According to the type of surgery, it is divided into four subgroups: open surgery, laparoscopic surgery, transperitoneal urethral stricture surgery, and transurethral prostate surgery. Postoperative pain scores, adverse effects, hospital stay, and postoperative recovery time were analyzed in the two groups.

Results: The pain scores $(1.67 \pm 1.84,1.47 \pm 1.77,0.6 \pm 0.83)$ at the $6 \mathrm{~h}, 24 \mathrm{~h}$, and $48 \mathrm{~h}$ after open surgery (36 patients) in the parecoxib group were significantly lower than those in the opioid group $(4.19 \pm 1.47,2.88 \pm 1.75,1.93 \pm 1.24, \mathrm{p}<0.001, \mathrm{p}=0.023$, $\mathrm{p}=0.001)$. For Laparoscopic surgery (173 cases), the postoperative pain scores $(2.23 \pm 1.55,1.58 \pm 1.279,0.85 \pm 1.10)$ in parecoxib group at $6 \mathrm{~h}, 24 \mathrm{~h}, 48 \mathrm{~h}$ were significantly lower than those in the opioid group $(3.14 \pm 2.14,2.29 \pm 1.70,1.28 \pm 1.52, p=0.002$, $\mathrm{p}=0.003, \mathrm{p}=0.041$ ), and the number of postoperative rescues $(p<0.001)$, the number of nighttime awakenings $(p=0.048)$, the postoperative exhaust time $(\mathrm{p}<0.001)$ and feeding time $(\mathrm{p}=0.013)$ in the parecoxib group were significantly superior to the control 
group. The pain scores $(2.29 \pm 2.39,1.74 \pm 2.07,1.24 \pm 1.44)$ at $6 \mathrm{~h}, 24 \mathrm{~h}$, and $48 \mathrm{~h}$ after transperitoneal urethral stricture surgery in the parecoxib group were significantly lower than those in the opioid group $(2.48 \pm 2.04) .2 .4 \pm 1.73,1.52 \pm 1.37, \mathrm{p}=0.011$, $\mathrm{p}=0.003, \mathrm{p}=0.006)$, the postoperative exhaust time $(\mathrm{p}=0.002)$ and feeding time $(\mathrm{p}<0.001)$ were significantly superior to the control group. For transurethral prostate surgery (23 cases), only the postoperative exhaust time in the parecoxib group was significantly superior than the control group $(p=0.031)$, but there was no significant difference in postoperative pain scores $(\mathrm{p}=0.119, \mathrm{p}=0.444, \mathrm{p}=0.546)$.

Conclusions: Parecoxib has a superior effect than opioid analgesia on the management of postoperative analgesia in patients with urinary surgery and has certain advantages in shortening the recovery time of postoperative intestinal function.

\section{MP19-15 Robot-Assisted Nephrouretercomy - A Case Series.}

S Nalagatla, I Ibrahim, J Hendry, OM Aboumarzouk

Queen Elizabeth University Hospital

Introduction \& Objective: Robotic surgery is becoming more frequently used within Urology and across many other surgical specialties. Robot-Assisted Nephro-ureterectomy (RANU) is becoming increasingly popular as an alternative to Laparoscopic Nephro-ureterectomy (LNU) for upper tract urothelial tumors. The aim of our study was to report our experience in RANU for upper tract urothelial carcinoma (UTUC).

Methods: All patients who underwent a RANU from October 2017 to December 2018 at our institute were identified retrospectively through a surgical database (Opera). Patient information including age, gender, operation time, console time, blood loss, transfusion requirements, hospital stay, complication rates, conversion rates, evidence of bladder recurrence, evidence of distant metastasis and any deaths were recorded.

Results: A total of 24 patients underwent RANU. The mean age was 70 years old. The average operative time and console time was 293 (range: 210-375) and 225 (range: 140-315) minutes respectively. RANU was performed without re-docking or the repositioning of the patient. The average blood loss was $198 \mathrm{mls}$ (range: 0-2000). No complications occurred. 2 patients were converted to open procedure due to more extensive disease found intra-operatively (data excluded from calculations). RANU was associated with one bladder recurrence and no distant metastases and no peri-operative deaths.

Conclusions: RANU is a safe and efficient option for upper tract urothelial tumors. The learning curve at the console can be relatively short however with successive procedures, the operative times and blood loss appear to improve, resulting in reduced hospital stays. Our operative data presented is also comparable with other studies.

\section{MP19-16 Drains Are No Longer Necessary in Ur- ologic Surgery}

R Abaza, OE Martinez

OhioHealth Dublin Methodist Hospital

Introduction \& Objective: Routine drain placement after surgery involving violation of the urinary tract is based on tradition rather than evidence. While most often drains are harmless, they may contribute to discomfort, infections, and closed suction drains may potentiate urine leakage or hemorrhage. Since the advent of robotic surgery, the use of drains has decreased possibly reflecting an increased confidence with obtaining water-tight reconstruction of the urinary tract. We have performed robotic urologic surgery with routine omission of drains even when the urinary tract was knowingly violated for over a decade. We reviewed our experience to determine if this is a safe practice.

Methods: We reviewed a single-surgeon (RA) prospective database of 4,835 robotic procedures, all performed from 2008-2019. Those involving violation of the urinary tract were included in the study, including prostatectomy, partial nephrectomy, pyeloplasty, nephroureterectomy, ureteral resections/reconstructions, and partial cystectomy or other surgery opening the bladder (e.g. simple prostatectomy). Complications including fluid collections and urine leaks requiring intervention as well as readmissions, emergency room visits, or unscheduled clinic visits were evaluated.

Results: Of 4,835 robotic procedures, 4,284 met the study criteria and involved urinary tract violation. Among these were 3,421 prostatectomies, 559 partial nephrectomies, 126 pyeloplasties, 86 nephroureterectomies, 81 robotic ureteral procedures, and 11 other bladder procedures. Mean patient age was 65 yrs (10-90), mean BMI was $30 \mathrm{~kg} / \mathrm{m}^{2}$ (15-63), mean blood loss was $111.1 \mathrm{~mL}$ (5-1500), and mean length of stay was 0.9 days $(0$ $15)$. No drain was placed in $4244 / 4284$ patients $(99 \%)$, including $99.6 \%$ of prostatectomies, $96.8 \%$ of partial nephrectomies, $96 \%$ of pyeloplasties, $98.8 \%$ of nephroureterectomies, $93.8 \%$ of ureteral procedures, and $100 \%$ of other bladder cases. All 43 patients who had drains placed during surgery had these removed prior to discharge, indicating they were only precautionary. Within 90 days, 7 patients without drains later required intervention for urine leak $(0.16 \%)$, all without infection, that included percutaneous drain placement in 2, ureteral stent placement in one, and Foley catheter alone in 4 patients. All-cause readmission rate was $2.4 \%(101 / 4,284)$.

Conclusions: Routine placement of drains can be safely avoided after the majority of urologic procedures despite urinary tract violation with a low rate of clinically significant urine leaks and a very low overall readmission rate.

\section{MP19-17 Withdrawn}

\section{MP19-18 Enigma of the crossing vessel in pelviure- teric junction obstruction.}

S Sangaralingam, H Godbole, C Carr, H SafarAly, N Kale

Department of Urology, North Middlesex Hospital

Introduction \& Objective: AIM: A retrospective analysis to confirm the accuracy of CT Urogram (CTU) in diagnosing a crossing vessel $(\mathrm{CV})$ in patients with pelviureteric obstruction (PUJO) was performed.

Methods: 42 patients had confirmed PUJO on pre-op investigations which included a CTU and MAG3 scan. All patients underwent laparoscopic retroperitoneal pyeloplasty. Dissection was performed to identify structures from the upper ureter leading on to the renal hilum including freeing up of the renal pelvis and delineation of the PUJ. A retrospective review of the preoperative CTU was performed by two Uro-Radiologists independently aiming to identify a CV. Intra operative findings from the operation notes were compared with these observations. 
Results: 15 patients were confirmed to have CV on CTU by one uro-radiologist while 7 patients were identified by the other uroradiologist. Of these 6 patients were common to both uroradiologists. 9 patients had confirmed CV intra-operatively. Only two out of 42 patients had confirmed $\mathrm{CV}$ both intra-operatively as well as in CTU.

Conclusions: CT angiography allows adequate identification of the presence and location of crossing vessels. The detection however is dependent on the arterial phase and a CTU is not protocoled as an arteriogram, therefore the disparity in identification. There is no correlation observed in this study between two expert opinions and with intraoperative findings. Despite thorough preoperative image analysis, a high index of suspicion and diligent intraoperative dissection is mandatory to identify $\mathrm{CV}$.

MP19-19 Aim: To assess the feasibility of Robotic assisted kidney transplantation in Graft with multiple vessels (GMV).

K Thekke Adiyat, V Raveendran

\section{ASTER MEDCITY}

Introduction \& Objective: Aim: To assess the feasibility of Robotic assisted kidney transplantation (RAKT) in Graft with multiple vessels (GMV).

Methods: We evaluated a total of 15 patients who underwent RAKT with GMV from living donor performed by a single surgeon in a single institution. The retrospective data obtained was compared to recipients who underwent RAKT with single vessel and open kidney transplant (OKT) with GMV.

Results: There were no significant differences in graft function outcome and perioperative parameters in all three groups. In comparison with OKT in GMV, we found that the RAKT with GMV had less pain score on post-operative $4^{\text {th }}$ day. There was also a significant statistical difference in mean analgesic requirement and incision length.

Conclusions: With increasing experience, grafts with challenging vascular anatomy can be taken up for RAKT and GMV should not be considered as a contraindication for RAKT.

MP19-20 Prospective comparative study on robotassisted vs open kidney transplantation: trend to less perioperative inflammatory response and similar functional results

A Territo, J Subiela, G Theil, L Gausa, F Regis, R Boissier, D Vanacore, M Nasreldin, P Fornara, A Gallioli,

L Guirado, A Breda

Fundació Puigvert. Autonomous University of Barcelona. Barcelona. Spain.

Introduction \& Objective: Open kidney transplantation (OKT) is the preferred approach in kidney transplantation (KT), but robot-assisted kidney transplantation (RAKT) has been recently standardized. The main aim of this study is to prospectively quantify the inflammatory response and the functional results of OKT vs RAKT.

Methods: We prospectively compared the inflammatory response between standard OKT and RAKT. 30 patients underwent pre-emptive KT between January and December 2017
(15 RAKT, 15 OKT). Blood levels of inflammatory markers (NGAL, CRP, IL-6) were measured at several time points: T0 (preoperative/baseline), T1(H1), T2(H6), T3(H12), T4(H24), T5(D2), T6(D3) and T7(D5) after KT. Serum creatinine and estimated glomerular filtration rate (eGFR) were evaluated at postoperative days 1, 3 and 7. A point-to-point analysis was performed, the differences in clinical variables between RAKT and OKT were evaluated using the unpaired t-test or non parametric Mann Whitney U test.

Results: IL-6 and CRP significantly increased in both groups after surgery compared with baseline $(\times 9$ and $\times 15$ respectively, all $\mathrm{p}<0.01)$. There was a significant difference in the mean level of IL-6 at T1 (H1) and T3(H12) in the advantage of RAKT $(\mathrm{p}<0.01)$. Significant differences in the mean level of CPR were found at T3(H12) and T5(D2) in the advantage of RAKT $(\mathrm{p}<0.01)$. Compared to baseline NGAL significantly decreased in both RAKT $(\mathrm{p}<0.01)$ and OKT $(\mathrm{p}<0.01)$. Significant differences in the mean level of NGAL were found at T3(H12), T4(H24) and T6(D3) in the advantage of RAKT. Serum creatinine and eGFR at postoperative days 1,3 and 7 were similar in RAKT and OKT (all p >0.05).

Conclusions: RAKT trended to induce a lower inflammatory response compared with OKT. Short term functional outcomes and complications rate were similar in RAKT vs OKT.

\section{MP19-21 Initial Outcomes of Minimally Invasive versus Open Inguinal Lymph Node Dissection}

SS Pandya, NJ Farber, R Patel, KD Radadia, J Sterling, A Tabakin, RE Weiss, IY Kim, TL Jang,

EA Singer, SE Elsamra

Introduction \& Objective: Squamous cell carcinoma of the penis (SCCP) is a rare genitourinary malignancy, which spreads in a predictable manner from superficial inguinal lymph nodes (LN) to deep inguinal $\mathrm{LN}$ and ultimately to pelvic $\mathrm{LN}$, with pelvic LN involvement portending limited survival. High grade and higher stage ( $\geq$ pT1b) SCCP have a greater likelihood of metastasizing to the inguinal LN including micro-metastases, which can be present in non-palpable/clinically negative inguinal LN. Thus, the current NCCN guidelines recommend inguinal lymph node dissection (ILND) including prophylactic ILND for these patients as it is an important predictor of survival. Historically, open ILND has been performed; however, it is associated with significant morbidity. Recent data has suggested that minimally-invasive ILND may offer the same oncologic benefits with the added benefit of reduced morbidity compared to an open approach. Therefore, we aimed to evaluate the initial outcomes of minimally-invasive ILND at our institution.

Methods: We retrospectively evaluated the outcomes of laparoscopic and robotic ILND at a single institution between 2015-2019. LN yield, estimated blood loss (EBL), hospital length of stay, and complications (using Clavien-Dindo system) were assessed. We compared outcomes between minimally invasive ILND and 11 historical open ILND patients at our institution.

Results: ILND was performed laparoscopically on 2 thighs and robotically on 22 thighs in 13 total patients. Mean EBL for minimally invasive ILND did not differ significantly from open ILND (56.3 cc vs. 78.6 cc respectively; $\mathrm{p}=0.223$ ). Mean LN yield for minimally invasive ILND trended toward an increase over open ILND (9.1 vs. 5.9 respectively; $p=0.055$ ). LN size ranged from 0.2 to $5 \mathrm{~cm}$. Majority of the patients were discharged home on a postoperative day 1 for both minimally-invasive 
ILND vs. open ILND with no significant differences in the length of hospital stay ( 1.3 vs. 1.2 respectively; $p=0.713$ ). Average drain removal time was 19.6 days for minimally invasive. $0 \%$ of minimally-invasive vs. $30 \%$ of open ILND patients experienced a Clavien grade III/IV complication $(\mathrm{p}<0.05)$.

\begin{tabular}{|c|c|c|c|}
\hline & $\begin{array}{l}\text { Minimally } \\
\text { Invasive }\end{array}$ & Open & p-values \\
\hline $\begin{array}{l}\text { Mean Estimated Blood } \\
\text { Loss (EBL) }\end{array}$ & $56.3 \mathrm{cc}$ & $78.6 \mathrm{cc}$ & $\mathrm{p}=0.223$ \\
\hline Hospital Length of Stay & 1.3 days & 1.2 days & $\mathrm{p}=0.713$ \\
\hline Mean LN Yield & $9.1 \mathrm{LN}$ & $5.9 \mathrm{LN}$ & $\mathrm{p}=0.055$ \\
\hline $\begin{array}{l}\text { Clavien Grade III/IV } \\
\text { Complications }\end{array}$ & $0 \%$ & $30 \%$ & $\mathrm{p}<0.05$ \\
\hline
\end{tabular}

Conclusions: Our initial series shows that minimally invasive ILND is comparable to open ILND in terms of hospital length of stay and EBL, while potentially superior with respect to morbidity and lymph node yield.

\section{MP19-22 Risk factor for erectile dysfunction after robot-assisted radical prostatectomy}

Y Nakano, J Furukawa, K Harada, K Shigemura, T Ishimura, N Hinata, M Fujisawa

Introduction \& Objective: Robot-assisted radical prostatectomy (RARP) is one of the most frequently selected therapeutic modalities for patients with localized prostate cancer; however,
RARP has been shown to accompany significant morbidities, including erectile dysfunction (ED) and urinary incontinence. In this study, therefore, we assessed the sexual function in patients treated for prostate cancer with RARP, using the erection hardness score (EHS).

Methods: This study included 144 patients with potency undergoing nerve-sparing RARP between January 2010 and December 2018. Of the 144 patients, $48(33 \%)$ had undergone a bilateral nerve-sparing (NS) procedure, $96(67 \%)$ had undergone a unilateral NS procedure. Sexual function was evaluated using International Index of Erectile Function (IIEF) and EHS before and 12 months after RARP. In this series, potency was defined as the ability to have an erection sufficient for intercourse, corresponding to EHS $\geq 3$, while patients with EHS $\geq 2$ were regarded as those with erectile function. Several factors associated with erectile dysfunction after RARP were identified by univariate analysis.

Results: After surgery, the rates of sufficient for intercourse in the bilateral NS group, and unilateral NS group were 71\% (34/ 48 ), and 63\% (60/96), respectively. and the difference of erectile function between two groups was no significant. In a multivariate analysis, risk factor affected postoperative ED was only age. Furthermore, examining sexual function after surgery in patients younger than 65 years, the recovery rates of potency in the bilateral NS group, and unilateral NS group were $76 \%$ (35/46), and $79 \%$ (28/33), respectively.

Conclusions: Younger men have better recovery of erectile function after nerve-sparing prostatectomy. This has led to the suggestion that, preservation of postoperative erectile function is highly expected even after radical prostatectomy for younger patients with good sexual function.

\section{MP20: EPIDEMIOLOGY, HEALTH POLICY, SOCIOECONOMICS \& OUTCOMES}

\author{
MP20-01 Trends of urolithiasis management in the \\ Chilean private health system from 2008 to 2018 \\ GM Astroza, VI Giraudo
}

Urology Department, Pontificia Universidad Catolica de Chile

Introduction \& Objective: During the last years have been described an increase in urolithiasis specially in women and children. Along this, endourologic techniques had become the cornerstone of its management. Before the 2000's, shock wave lithotripsy was the treatment of choice for over the $60 \%$ of kidney and ureteral stones in USA, but now the endoscopic interventions count for more than the $60 \%$ of stone management in developed countries. There's no information of this changes in south America other than Brazil. Our objective is to describe the evolution of treatment choices in Chile, a developing country. Methods: We analyzed the surgical records of all private insurance's companies in Chile for the last 10 years. The codes for percutaneous nephrolithotomy, anatrophic nephrolithotomy, endoscopic ureterolithotomy, endoscopic nephrolithotomy and shock wave lithotripsy were recorded. We analyzed the rate of each surgery and its progression over time. The total number of procedures and the relation between gender were also calculated.
Results: In the year 200811,013 procedures were performed. $66.9 \%$ in men and $33,1 \%$ in women, the mean age was 43.7 years. SWL counted for the $60.52 \%$, URS $32.26 \%$, PCNL $4.02 \%$ and open surgery 3.25 . In 201814,150 procedures were performed, with an increase of $28.48 \%$ during the 10 years period. In $201870.73 \%$ were men and $29.27 \%$ in women, the mean age was 47.34 years. URS counted for $62.43 \%$, SWL $31.87 \%$, PCNL $4.97 \%$ and open surgery $0.73 \%$. The gender distribution was

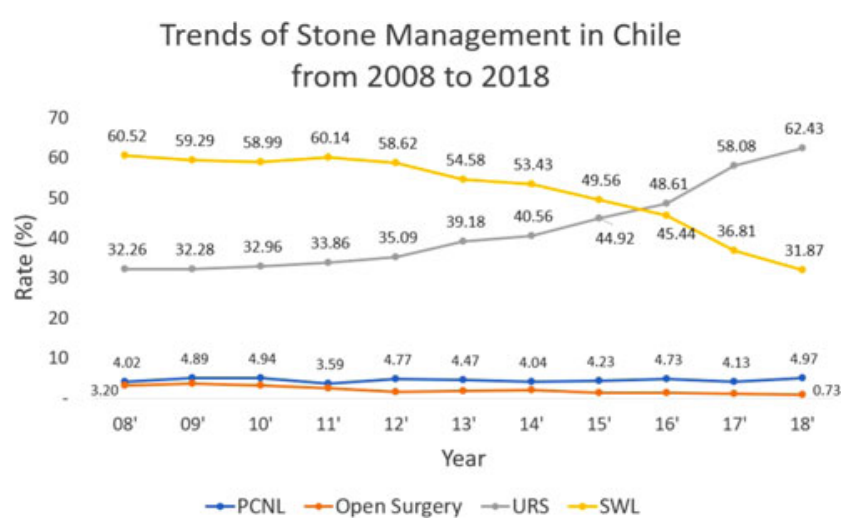


higher in men (3:1) similar to previous reports in the literature. An important increase of URS was observed since 2013, being the most common treatment modality over SWL since 2015. PCNL rate had no major variations, ranging from $4 \%$ to $5 \%$ of the procedures in the analyzed period. Open surgery initially accounted for $3.2 \%$ but after 2013 the rate went below $1 \%$, with a diminution of $77.18 \%$ from 2008 to 2018 .

Conclusions: In Chile as well as the rest of the developed countries, the rate of use of SWL and URS have inverted completely. This trend supports the hypothesis that the use of endourology procedures had taken over as the preferred stone management modality counting for more than the $60 \%$ of the interventions. PCNL had been stable over time, as it's used for precise indications of kidney stones that can't be removed with URS. Lastly, the open surgery had come to lost it's relevance over new techniques available

\section{MP20-02 Incorrect imaging for hematuria workup} across specialties is leading to poor value of healthcare

\author{
B Adamic, J Aizen, C Labbate, S Park
}

Introduction \& Objective: Value-based healthcare aims to decrease costs while increasing patients' health outcomes and satisfaction. Hematuria is a very common presenting complaint to providers across many specialties, but we suspect that patients are receiving very poor value in hematuria workups because incorrect diagnostic imaging tests are widely performed. We aimed to identify the patterns of imaging ordered for hematuria working and its disparity with published AUA guidelines.

Methods: We identified all Computerized Tomography Stone (CT-S), CT Urogram (CTU), CT Abdomen/Pelvis with contrast (CTAP w) and retroperitoneal ultrasound (RUS) scans performed for the diagnosis of hematuria at our large community health system during a 60-day period in 2018. Studies ordered for a concomitant diagnosis of renal colic were excluded. The ordering physician's specialty, patient age, sex, smoking status, renal function and need for additional imaging were recorded. Chi-squared tests were utilized for statistical analysis.

Results: In the two-month study period, non-urologists ordered $201(58 \%)$ of the 347 total radiographic studies for hematuria. Although $323(93 \%)$ patients had a GFR $>30$ or normal serum creatinine, only $191(59 \%)$ underwent CTU. Emergency physicians and nephrologists did not order a single CTU for the work up of hematuria, the former inappropriately ordering CT-S and the latter preferring RUS. Twenty-nine $(8.5 \%)$ patients underwent additional imaging for hematuria and 24 (82.8\%) appropriately underwent CTU. Interestingly, while their numbers were small, urogynecologists were most likely to correctly order CTU, followed by urologists. Physician specialty, younger age, higher GFR and gross vs. microscopic hematuria were associated with correct CTU imaging $(\mathrm{p}<0.05)$ but smoking status was not associated with undergoing CTU $(\mathrm{p}=0.361)$.

Conclusions: Among patients presenting with hematuria, over half undergo imaging tests ordered by non-urologists such as ER, internal medicine and family practitioners. Unfortunately, only $59 \%$ of them underwent CTU despite having normal GFR. Thus, $41 \%$ of the remaining who underwent insufficient imaging will be told they require another CT scan using contrast to appropriately complete their hematuria workup, leading to increased costs, more patient dissatisfaction, unnecessary re-exposure to ionizing radiation and therefore decrease healthcare value. We believe more education across specialties is required to reduce these avoidable imaging errors.
MP20-03 Post-operative narcotics prescribing patterns following endoscopic procedures: How much is too much?

\author{
C Tabib, K Cwach, P Mistry, M Schwartz
}

Arthur Smith Institute for Urology

Introduction \& Objective: Prescription narcotic abuse has become a national crisis with state and federal government officials devising laws to limit the number of unused narcotics from being obtained and used for non-medical reasons. While these restrictions will help slow down the opioid epidemic, they leave room for improvement in terms of understanding which patients benefit most from narcotics and the role providers play in ensuring pain is appropriately controlled. Following a study done at our institution showing minimal amounts of narcotics were being used following urologic ambulatory cases, we sought to identify and understand practice patterns of providers following ambulatory cases.

Methods: We retrospectively reviewed adult patients undergoing ambulatory transurethral resection of bladder tumor (TURBT) and ureteroscopy (URS). Patients who were admitted, had concurrent surgery, or had prior diagnosis of chronic pain were excluded. Narcotic prescriptions including type and number prescribed were quantified. Prescribers included both physician assistants (PA) and physicians (MD). Data was analyzed using chi-square, Fischer's exact, and Mann-Whitney U tests.

Results: From January to November 2017, 297 patient charts were reviewed. 177 and 120 patients underwent TURBT and URS, respectively. Of the URS group, 110 patients received a narcotics prescription post-operatively from either an MD or PA. The PA group tended to give more total pills compared to MD (25.5 vs 17.8 pills, $\mathrm{p}<0.001)$. For the patients undergoing TURBT, 80 of 177 received narcotics with increased tablets being given by PAs vs MDs (19.6 vs 14.7 pills, $\mathrm{p}=0.035$ ). None of the patients required refills within 30 days of their procedure. Conclusions: This study identifies the disparity in prescription patterns between providers within the same practice. Presumably those not involved directly with the procedure tended to be overly cautious regarding pain control leading to a larger number of pills being prescribed. Given none of these patients refilled their prescriptions it is safe to say that the lower number of pills should be the absolute maximum with a cap set below this number. However, further studies regarding the use and effectiveness of narcotics for these particular populations needs to be completed to determine which group, if either, is managing postoperative pain best.

\section{MP20-04 Clinical, fiscal and environmental benefits of a specialist led virtual ureteric colic clinic: a report of a prospective study}

MJ Connor, S Miah, M Edison, J Brittain, M Kondjin-Smith, C Gan, M Hanna, R Dasgupta, T El-Husseiny

Imperial College Healthcare NHS Trust, Charing Cross Hospital

Introduction \& Objective: Virtual clinic (VC) is a clinical consultation without a face-to-face (FTF) meeting. Incorporating innovative telehealth strategies such as a $\mathrm{VC}$ in the follow-up pathway for patients is one such method to clinically and fiscally accommodate the increasing service demands of uncomplicated 
ureteric colic. This study objective was to evaluate the clinical, fiscal and environmental impact of a specialist-led acute ureteric colic virtual clinic (VC) pathway.

Methods: All patients with uncomplicated acute ureteric colic referred to a single tertiary center were prospectively entered into the study over a four year period ( Jan 2015-Dec 2018). Inclusion criteria: low-dose non-contrast CT KUB, WBC $<16 \times 10^{9} / \mathrm{L}$, pain controlled, normal renal function, no clinical concern. Primary outcomes: time (days) from referral to virtual clinic outcome, virtual clinic outcome (Discharge, Further VC, Face-to-face (FTF) clinic, ESWL, URS, PCNL), adverse event (sepsis or obstruction). Secondary outcomes: patient and stone demographics, cost and environmental analysis. Minimum follow-up 3 months.

Results: 1,008 patients entered into the study, $91.5 \%$ (922) were of working age. Median time from presentation to virtual clinic outcome was 2 days (IQR 4). Virtual clinic outcomes were: $16.3 \%$ (164) discharged, $18.2 \%$ (183) discharged following further VC, $17.2 \%$ (173) intervention, 48.4\% (488) FTF clinic. Interventions: PCNL 0.5\% (5), ESWL 7.7\% (78), URS $8.9 \%$ (90). Stone demographics: 570 (56.5\%) lower-, 157 (15.6\%) upper-, $96(9.5 \%)$ mid-ureteric, $163(16.2 \%)$ renal calculi and 22 (2.2\%) recently passed. Mean stone size of $3.5 \mathrm{~mm}$ (SD 2.3). Two $(0.2 \%)$ adverse events reported. Introducing a VC saved $£ 145,152$ for clinical commissioning groups. The equivalent NHS tariff payment of performing 106 URS or 211 ureteric stent insertions. Overall, 9,374 patient journey miles were avoided, equal to $0.70-2.93$ metric tons of $\mathrm{CO} 2 \mathrm{e}$ production and the need to plant 14.7 trees to achieve carbon balance.

Conclusions: Specialist-led virtual acute ureteric colic clinic reduces time to treatment decision to a median of 2 days. This creates additional clinic capacity, reduces the fiscal burden of traditional clinics and their associated carbon footprint.

\section{MP20-05 High Fidelity Registry Demonstrates Dif- ferences in Stone Presentation Between China and the United States: Results from ReSKU}

J Ahn, T Chi, T Chi, J Li, W Hu, M Stoller, Y Tang

University of California San Francisco

Introduction \& Objective: Stone composition of nephrolithiasis patients can vary across regions and countries based on environmental and innate factors. The ReSKU database is a high fidelity data collecting system integrated into the electronic medical record for tracking nephrolithiasis patients. ReSKU has been implemented at 2 high volume endourologic medical centers in Beijing, China and San Francisco, United States. Our aim was to examine differences in patient demographics and stone analysis composition between multinational settings.

Methods: Demographic and stone analysis data were prospectively collected from consecutive patients into the ReSKU databases at the University of California, San Francisco Medical Center (UCSF) and Beijing Tsinghua Changgung Hospital $(\mathrm{BCH})$ in Beijing. Stones with heterogenous compositions were assigned type based on the majority composition. Differences in stone analysis and body mass index (BMI) were assessed using Fisher's exact test.

Results: At the time of analysis, the UCSF and $\mathrm{BCH}$ databases contained 1,198 and 1,525 nephrolithiasis patients, of which $53 \%$ and $62 \%$ which were male, respectively. The top 3 common ethnicities at UCSF were 66\% White 14\% Latino/Hispanic, and 7\% eastern Asian, while the $\mathrm{BCH}$ population was almost completely of Chinese ethnicity (99.8\%). Mean BMI at UCSF and BCH was 28 and 25 respectively $(\mathrm{p}<0.05)$. Educational data was collected in $717(47 \%)$ and $1047(89 \%)$ patients of the BCH and UCSF population. Differences were apparent in educational levels with the majority of the $\mathrm{BCH}$ population having the highest level of education as grade school (47\%), undergraduate (19\%), or technical school (15\%), compared to undergraduate $(30 \%)$, graduate $(23 \%)$, or technical school $(20 \%)$ in the UCSF group. Stone analyses were collected in 297 (25\%) and 1226 (80\%) of the UCSF and $\mathrm{BCH}$ population respectively. Primary stone composition for the UCSF group was $53 \%$ calcium oxalate monohydrate (COM), $14 \%$ calcium oxalate dihydrate (COD), 11\% Apatite, $8 \%$ Uric Acid (UA) and 3\% Struvite, while the $\mathrm{BCH}$ group was $57 \%, 2 \%$, $0.2 \% 11 \%$, and $13 \%$ respectively. The differences in rates of COD, Struvite, and Apatite stones was statistically significant.

Conclusions: Nephrolithiasis patients presenting to high volume stone centers in the United States and China demonstrate differences in gender, ethnicity, BMI, and level of education. COD, apatite, and struvite stone analysis rates differed between the 2 groups while COM and UA did not. These results demonstrate multiple areas of further study enabled by high quality prospective data collection using ReSKU as a common registry tool.

\section{MP20-06 Is the introduction of single use flexible ureteroscopes capable of preventing reusable scopes breakages? Results from a high-volume center}

\section{E Ventimiglia, S Doizi, L Villa, O Traxer}

Division of Experimental Oncology/Unit of Urology; URI; IRCCS Ospedale San Raffaele

Introduction \& Objective: Single-use flexible ureteroscopes (SUfURS) have clear advantages in terms of availability, sterility, and lack of maintenance costs compared to reusable flexible ureteroscopes (fURS). We aimed to assess whether the introduction of SUfURS at our high-volume center had an advantageous impact on the turn-over and breakage rates of reusable fURS.

Methods: We retrospectively analyzed data related to reusable fURS (URF-P5 and URF-V, Olympus, Japan) number of usages and breakages at our center between February 2015 and December 2018. We kept track of the number of usages for each analyzed scope between the first usage until a breakage requiring reconditioning was recorded. Usage count was restarted following reconditioning. Since SUfURS (Lithovue, Boston Scientific, USA), were introduced and routinely employed at our center in September 2016, we had the chance of comparing different reusable ureteroscope life cycles according to both SUfURS availability and usage intensity (i.e. number of SUfURS used during each reusable fURS life cycle). We then graphically explored the relationship between SUfURS usage intensity and reusable scopes survival (i.e. number of utilizations before any breakage requiring reconditioning) using locally weighted scatterplot smoothing (LOWESS) approach. Moreover, we used Poisson regression model in order to estimate relative risks (RR) for reusable scope survival according to single-use scope usage intensity.

Results: During the analyzed time frame, 5 different fURSs (4 URF-P5 and 1 URF-V) were employed at our center, for a total of 1820 usages and 40 breakages requiring reconditioning. Median (interquartile range, IQR) number of usages before breaking was 38 (25-51) overall. A total of 98 SUfURS were used during the study period. After SUfURS introduction, median (IQR) reusable fURS number of usages increased from 28 
(19-46) until 45 (34-59), (+62\%, p=0.01). The graphical relationship between SUfURS usage intensity and reusable scopes survival showed a linear survival increase after 10 or more SUfURS scopes were used per each life cycle. At Poisson regression analysis, the SUfURS usage intensity was positively associated to increased reusable fURS survival, RR (95\% CIs) 1.02 (1.01$1.03), \mathrm{p}<0.001$.

Conclusions: Life-time cycle of reusable fURS increased by $62 \%$ after the introduction of SUfURS. Ten or more used SUfURS per life cycle were associated to increased fURS survival.

\section{MP20-07 New Persistent Opioid Use after Outpatient Ureteroscopy for Upper Tract Stone Treatment}

C Tam, CA Dauw, K Ghani, V Gunaseelan, T Kim, D Leavitt, J Raisky, JM Hollingsworth

University of Michigan

Introduction \& Objective: Over 100 Americans die every day from opioid overdose. Recent studies suggest that many opioid addictions surface after surgery. In this context, we measured the incidence of persistent opioid use following ureteroscopy (URS). Methods: Using claims data, we identified adults who underwent outpatient URS for treatment of upper tract stones between January 2008 and December 2016 and filled an opioid prescription attributable to URS. We then measured the rate of new persistent opioid use-defined as continued use of opioids 91 to 180 days after URS among those who were previously opioidnaive. Finally, we fit multivariable models to assess whether new persistent opioid use was associated with the amount of opioid prescribed at the time of URS.

Results: In total, 47,247 patients underwent outpatient URS, $53.4 \%$ of whom were opioid-naïve. Nearly 1 in 15 (6.4\%) opioidnaïve patients developed new persistent opioid use after URS. Four months following surgery, beneficiaries with new persistent opioid use continued to fill prescriptions with daily doses of 3 oral morphine equivalents (Figure). Adjusting for measured sociodemographic and clinical differences, patients in the highest tercile of opioids prescribed at the time of URS had 79\% higher odds of new persistent opioid use compared to those in the lowest tercile (odds ratio, $1.79 ; 95 \%$ confidence interval, 1.56 to 2.04 ). Conclusions: Nearly 1 in 15 opioid-naive patients develop new persistent opioid use after URS. New persistent opioid use is associated with the amount of opioid prescribed at the time of

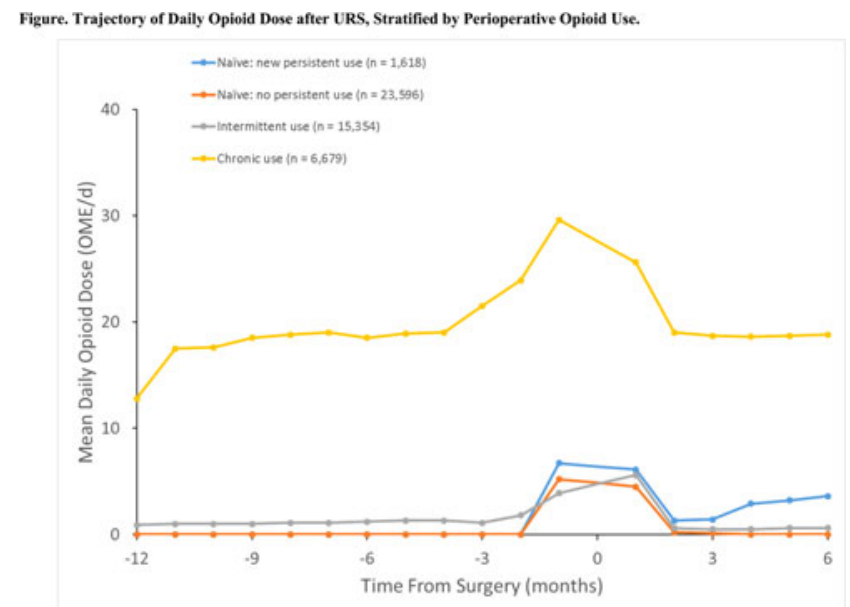

URS. Given these findings, urologists should re-evaluate their post-URS opioid prescribing patterns.

\section{MP20-08 Streamlining Costs of Robot-Assisted Ra- dical Prostatectomy: Can We Optimize the Financial Burden?}

AA Hussein, AM Toenniessen, NA Aldhaam, AS Elsayed, Z Lone, P May, S Tauber, K Sniadecki, R Grady, KA Guru

Roswell Park Comprehensive Cancer Center

Introduction \& Objective: The robot-assisted approach to radical prostatectomy (RARP) has gained worldwide acceptance and has become a continuously-emergent standard procedure. It is estimated $>85 \%$ of radical prostatectomies are performed with robotic assistance. However, the financial burden of robotic surgeries to health administrations and patients is known to be higher than the traditional approaches, such as Laparoscopic and Open procedures. The average cost of RARPs in the US is approximately $\$ 14,000$, compared to $\$ 8,600$ for open radical prostatectomy, according to one study comparing prices at hospitals across the US (Tomaszewski, 2012). We sought to identify drivers of cost at our institution and implement changes that could reduce the financial burden associated with RARPs.

Methods: A retrospective review of annual cost data of all RARPs performed by four experienced robotic surgeons during the last fiscal year was performed at our site (2017-2018). Significant drivers of cost were identified $(>10 \%$ variability among all surgeons). After educating the surgical team, a pilot process, instituting a series of changes, was evaluated with the most economical surgeon. Implemented changes included reducing 6 robotic instruments to 4 , switching from a V-Lock to a standard suture, and the incorporation of non-robotic reusable trocars (Table 1). Our team subsequently compared the impact these changes had on costs (two-sample t-test).

Results: 106 procedures were reviewed and compared. Significant variability in the cost associated with the procedure was observed. Supplies were identified as the chief driver of operative costs $(44 \%)$ followed by console time $(22 \%)$. Evaluation of the pilot process revealed 11 procedures before the changes were made compared to 9 procedures after the changes were implemented for this fiscal year (Table 2). A decrease in console time by 30 minutes was observed. There was also a statistically significant decrease in medical supply costs $(p=0.0016)$.

Conclusions: Open discussion of costs related to operating room expenses will initiate the process to reduce the financial burden associated with Robotic-Assisted Radical Prostatectomies. This process has the potential to be economically instrumental for

Table 1: Change in Instrument Usage
\begin{tabular}{|l|l|l|}
\hline Instrument & Before & After \\
\hline Needle Driver & 2 & 1 \\
\hline $\begin{array}{l}\text { Graspers (Maryland and/or } \\
\text { Fenestrated) }\end{array}$ & 1 & 1 \\
\hline Cobra Grasper & 1 & 1 \\
\hline Scissors & 1 & 1 \\
\hline Cautery Hook & 1 & 0 \\
\hline Suture & V-Lock & Standard \\
\hline Trocars (Non-Robotic) & Disposable & Reusable \\
\hline Total Instruments Used & 6 & 4 \\
\hline
\end{tabular}

Table 2: Changes in Costs

\begin{tabular}{|l|l|}
\hline Category & $\%$ Decrease \\
\hline Console Time Cost & $11 \%$ \\
\hline Supply Cost & $23 \%$ \\
\hline $\begin{array}{l}\text { Maintenance and } \\
\text { Depreciation Cost }\end{array}$ & $13 \%$ \\
\hline Total Cost & $18 \%$ \\
\hline
\end{tabular}


health care facilities across the US who perform RARP. Further analysis of this process will aid in applicability to other large health care facilities.

\section{MP20-09 Management of Rare PCNL Complications - is a Fellowship Required?}

D Mikhail, L Griffiths, Z Kozel, C Tabib, A Rai, D Hoenig, Z Okeke, AD Smith

Introduction \& Objective: Rates of PCNL have steadily risen internationally over the past couple of decades as indications expand and technological advancements make it more accessible. Percutaneous access is a complex skill set with 60 cases needed for competence. Although safe, PCNL still has a 10-15\% complication rate. Major complications such as visceral organ injury is rare but can be fatal. We investigated the international community's experience with rare complications and evaluate the effect of training and experience on management approaches. Methods: We created an anonymous 26-question survey that was distributed to the Endourological Society. The survey included questions on respondent demographics, training, experience, practice, surgical technique, rare complications experienced and their management. The complications of interest were splenic, liver and colon injury. Data was analyzed using SPSS (IBM, 2017). Fisher's exact, Chi-squared tests were used to assess colonic injury management. Goodman $\&$ Kruskal's $\lambda$ was used to evaluate diagnosis and management of splenic and liver complications. We predicted differences based on practice level, previous training, and annual PCNL volume.

Results: A total 323/2000 members (16\%) began the survey, with a completion rate of $89 \%(\mathrm{n}=288)$. Most reported a predominantly academic practice $(59 \%)$, were from North America $(36 \%)$ or Europe $(16 \%)$ and had a minimum of 10 years of independent practice $(63 \%)$. The largest group (40\%) performed 20-60 PCNLs per year, while $26 \%$ did less than 20 per year. In terms of training only $42 \%$ had done any fellowship training in PCNL, while almost half $(43 \%)$ of respondents reported no formal courses or training in PCNL. When asked to recall injuries at their institution over the past 15 years - respondents recalled a total of 36 splenic, 39 liver and 133 colonic injuries. Analysis of management options for each of these injuries showed that current practice level was predictive of splenic injury management and time of diagnosis $(\mathrm{p}<0.05)$, while number of PCNLs performed per year was predictive of diagnosis and management of all three injuries $(\mathrm{p}<0.05)$. Interestingly, the most common complication (colonic injury) was more likely to be appropriately managed by fellowship trained urologists $-27.3 \%$ vs. $13.9 \%(\mathrm{p}=0.006)$.

Conclusions: As PCNL becomes more commonly performedurologists need to be prepared to recognize and manage potentially fatal complications. Our results show that experience and formal training lead to improved recognition and appropriate management of visceral complications. As surgical volumes during training decrease - formal endourology training might be necessary for safe practice of PCNL.

\section{MP20-10 Over-investigation of Non-visible Hema- turia - Optimizing Current Platforms to Reduce Ex- cessive Investigations.}

MM Quraishi, A Simoes, B Osman, J Dorrell, G Papadopoulos, M Thomas, P Kheirandish, R Krishnan, BA Eddy, EH Streeter, SS Kommu, N Shrotri
Kent \& Canterbury Hospital. East Kent Hospitals University NHS Foundation Trust

Introduction \& Objective: Upper tract investigation of non visible hematuria $(\mathrm{NVH})$ via the urgent cancer pathways has been a controversial issue recently, with evidence of very low incidence of upper tract malignancies. In cash strapped healthcare systems or self-funded patients, an objective thorough assessment needs to be made if computed tomography (CT) is truly justified to irradiate patients or its financial ramifications. We assessed the impact of investigation of NVH in a teaching hospital.

Methods: Patients were identified from the Hematuria clinics across all the Hospital related satellite sites over a 10-week period.

Results: Of the 337 patients attending the rapid access clinics, 100 patients were found to have presented with NVH. Every patient underwent a flexible cystoscopy, with 3 patients having a further cystoscopy. Ultrasound scanning of the renal tract was performed in 63 patients, whilst CT scanning was performed in 78 patients. Only 7 patients were identified to have significant urological abnormalities on CT Urogram, that was missed on flexible cystoscopy and Ultrasound scanning (Stones $>5 \mathrm{~mm}$, Bosniak 2 cysts, possible bladder malignancy). Three patients were found to have significant non urological abnormalities solely identified on CT ( 2 patients with gynecological abnormalities, later proved to be malignancy, and 1 deep vein thrombosis). CT scans requested on ultrasound findings were only found to be justified in 3 cases (focal hydronephrosis, septated renal cyst, irregular kidney). Stratifying CT scans performed on smokers/ ex-smokers, did not identify any significant pathology. In addition to the flexible cystoscopies, 13 general anesthetic procedures were undertaken to reach a diagnosis (incl optical urethrotomy) and 6 procedure performed to treat conditions found (transurethral resection of prostate, flexible ureteroscopy). Of the $86 \mathrm{CT}$ scans performed, only 10 identified additional abnormalities. This reveals that $76 \mathrm{CT}$ scans did not yield any benefit, at the expense of $\$ 12,773$ to the hospital. Furthermore 37 patients did not have ultrasound but instead had CT scans directly. If only ultrasounds scans had been performed instead of CT scans, a cost saving of $\$ 4,365$ would have noted. Since the study period covered only 10 weeks, potential cost savings could be extrapolated to 5 times more per annum.

Conclusions: There is a paucity of updated guidelines on the management of NVH. A better triage system to avoid multiple investigative modalities may yield a larger cumulative cost saving. Our results depict the poor positive findings of upper tract imaging for $\mathrm{NVH}$ and call upon a discussion/emergence of guidelines to better direct financially feasible patient care.

\section{MP20-11 Time Trends in Metabolic Diagnoses of Recurrent Stone Formers}

S Dianatnejad, K Piranviseh, S Tapiero, P Jiang,

$\mathrm{R}$ Youssef Yaacoub

University of California, Irvine

Introduction \& Objective: Metabolic factors underlying the recent increase in stone prevalence over the past decades are not well understood. Herein, we evaluate chronological trends in metabolic risk factors in recurrent kidney stone formers.

Methods: We systemically reviewed literature in the last 4 decades comparing 10 old studies before year 2000 (1578 patients) 

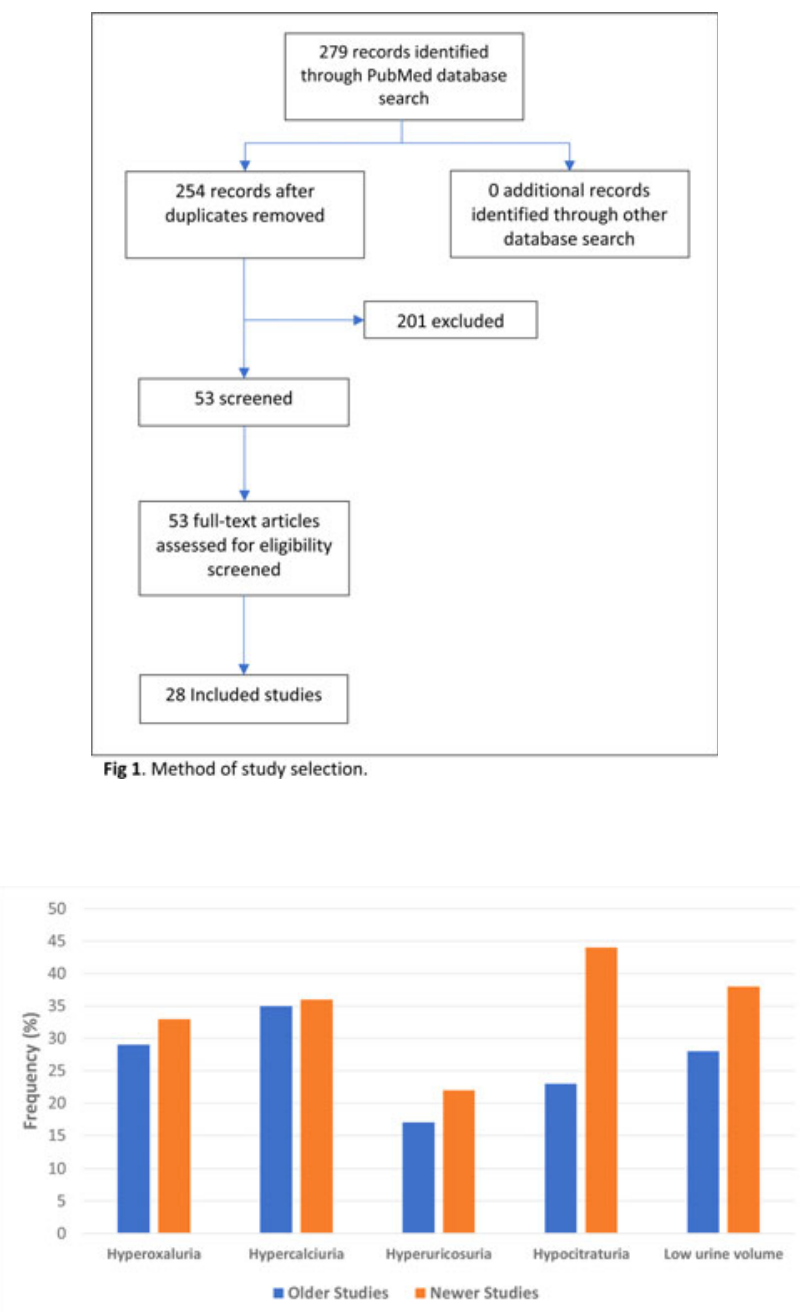

Fig. 2. Frequencies (in \%) of metabolic abnormalities in recurrent stone formers by older studies (1980-1999) vs. newer studies (2000-2018).

to 18 recent studies ( 8747 patients) after year 2000. Studies with inadequate 24-hour urine metabolic data or $<50$ patients; were excluded. Gender differences were compared only in recent studies due to lack of data in old studies.

Results: Comparing old to recent studies, the prevalence of hyperoxaluria, hypercalciuria, hyperuricosuria, low urine volume and hypocitraturia has increased from $29 \%$ to $33 \%, 35 \%$ to $36 \%, 17 \%$ to $22 \%, 28 \%$ to $38 \%$ ( $p>0.05$ for all) and $23 \%$ to $44 \%$ $(\mathrm{p}=0.032)$; respectively. Comparing non USA to USA studies, hyperoxaluria was significantly higher $(39 \%$ vs $19 \% ; \mathrm{p}=0.048)$ in old studies. However, with rising hyperoxaluria in USA, there was no significant difference in recent studies (33\% vs $32 \%$ ). Hyperuricosuria was significantly higher in USA (20\% vs $45 \%$; $\mathrm{p}=0.017)$ in recent studies. Hyperoxaluria and hyperuricosuria ( $35 \%$ vs $22 \%$; $\mathrm{p}=0.047 ; 22 \%$ vs $15 \%$; $\mathrm{p}=0.021$; respectively) were significantly higher in men.

Conclusions: The prevalence of metabolic risk factors including hyperoxaluria, hypercalciuria, hyperuricosuria, low urine volume has increased; with significant rise of hypocitraturia. Hyperoxaluria and hyperuricosuria had significantly increased in USA and has been significantly higher in males than females. Dietary modifications and innovative medical therapies are needed to decrease metabolic risk factors underlying nephrolithiasis.

\section{MP20-12 Withdrawn}

\section{MP20-13 Kidney stones in black women in the United States: Data from the Black Women's Health Study}

\author{
M D'Amico, S Wason, L Rosenberg, Y Cozier \\ Boston Medical Center Department of Urology
}

Introduction \& Objective: Nephrolithiasis is a common urologic condition and a significant source of patient morbidity. There has been an increase in the prevalence of kidney stones in the United States in recent years, especially among black and female patients. There are few epidemiologic studies of kidney stones focusing on black women. We present data on the prevalence and clinical characteristics of women with self-reported kidney stones among participants in the Black Women's Health Study (BWHS).

Methods: The BWHS, initiated in 1995, is a prospective, epidemiologic study of 59,000 US black women (age 21-69) followed via biennial postal and web questionnaires. The 2005 questionnaire asked whether participants had ever been diagnosed with kidney stones as well as data on patient characteristics (age, geographic region) and medical factors (body mass index, type-2 diabetes, hypertension, high cholesterol, gallstones). In 2017, a subset of BWHS participants $(n=2,570)$ completed a questionnaire focusing on urinary tract health questions regarding undergoing metabolic work-up and imaging related to the diagnosis of kidney stones. Chi-square tests were used to compare characteristics between participants with and without a history of nephrolithiasis.

Results: Among the 43,179 participants who completed the 2005 survey, 836 (2\%) reported ever being diagnosed with kidney stones. Women with and without a history of kidney stones were similar in terms of geographic location and health insurance coverage. Respondents with a history of kidney stones were more likely to be older $(\mathrm{P}<0.0001)$, to have smoked $(\mathrm{P}=0.04)$, to be obese $(\mathrm{P}=0.01)$, and to have a comorbid condition (type-2 diabetes $(\mathrm{P}<0.0001)$, hypertension $(\mathrm{P}=0.01)$, hyperlipidemia $(\mathrm{P}<0.0001)$, gallstones $(\mathrm{P}<0.0001)$. Of the 2,570 sub-study participants in $2017,8 \%$ reported a history of kidney stones of which $40 \%$ experienced $\geq 2$ stones in their lifetime, $32 \%$ completed a metabolic work up, $70 \%$ had undergone a CT scan, and $29 \%$ had undergone a surgical procedure.

Conclusions: BWHS participants who reported a history of kidney stones were more likely to have other medical comorbidities, including components of metabolic syndrome and gallstones. These data are consistent with hypotheses relating lifestyle-associated risk factors with nephrolithiasis. Further study is needed to establish the temporal sequence between nephrolithiasis and common comorbid conditions, as well as to identify the barriers and facilitators of diagnostic work up of kidney stones in black women.

\section{MP20-14 Utilization and 5-Year treatment free rates for Radical Nephrectomy and Partial Nephrectomy for the diagnosis of Renal Cell Carcinoma between 2000-2014 in New York State}

KH Bilal, M Finkelstein, M Palese

Mount Sinai Medical System

Introduction \& Objective: To observe the utilization trends of radical nephrectomy $(\mathrm{RN})$ and partial nephrectomy $(\mathrm{PN})$ for the 
treatment of renal cell carcinoma (RCC). Also, to compare the proportion of patients who require no further treatment in the 5year post-operative period after initial RN and PN.

Methods: Retrospective-cohort study of 34,287 records from Statewide Planning and Research Cooperative Systems database, of patients diagnosed with renal cell carcinoma (ICD-9:189.0) between 2000-2014. Patient records were stratified based on the treatment procedure, either RN (ICD-9:555.1) or PN (ICD-0:554.0). Results: The proportion of RCC cases treated with RN decreased significantly from $84.33 \%$ in 2000 to $49.29 \%$ in 2014 ( $p<0.005$ ). While, the proportion of RCC cases treated with PN increased significantly from $15.67 \%$ in 2000 to $50.71 \%$ in 2014 ( $\mathrm{p}<0.005)$. On average $97.46 \%$ of patients treated annually with RN required no further treatment for RCC for a period of 5 years. However, the 5 -year treatment free rate for PN significantly increased from $86.38 \%$ in 2000 to $96.44 \%$ in 2009 ( $\mathrm{p}<0.005)$.

Conclusions: A significant shift has occurred in the utilization trends of RN and PN for treatment of RCC. While RN was the procedure of choice for majority of cases of RCC in 2000, PN has significantly increased in popularity and now stands equal in proportion of cases of RCC. Furthermore, the 5-year treatment
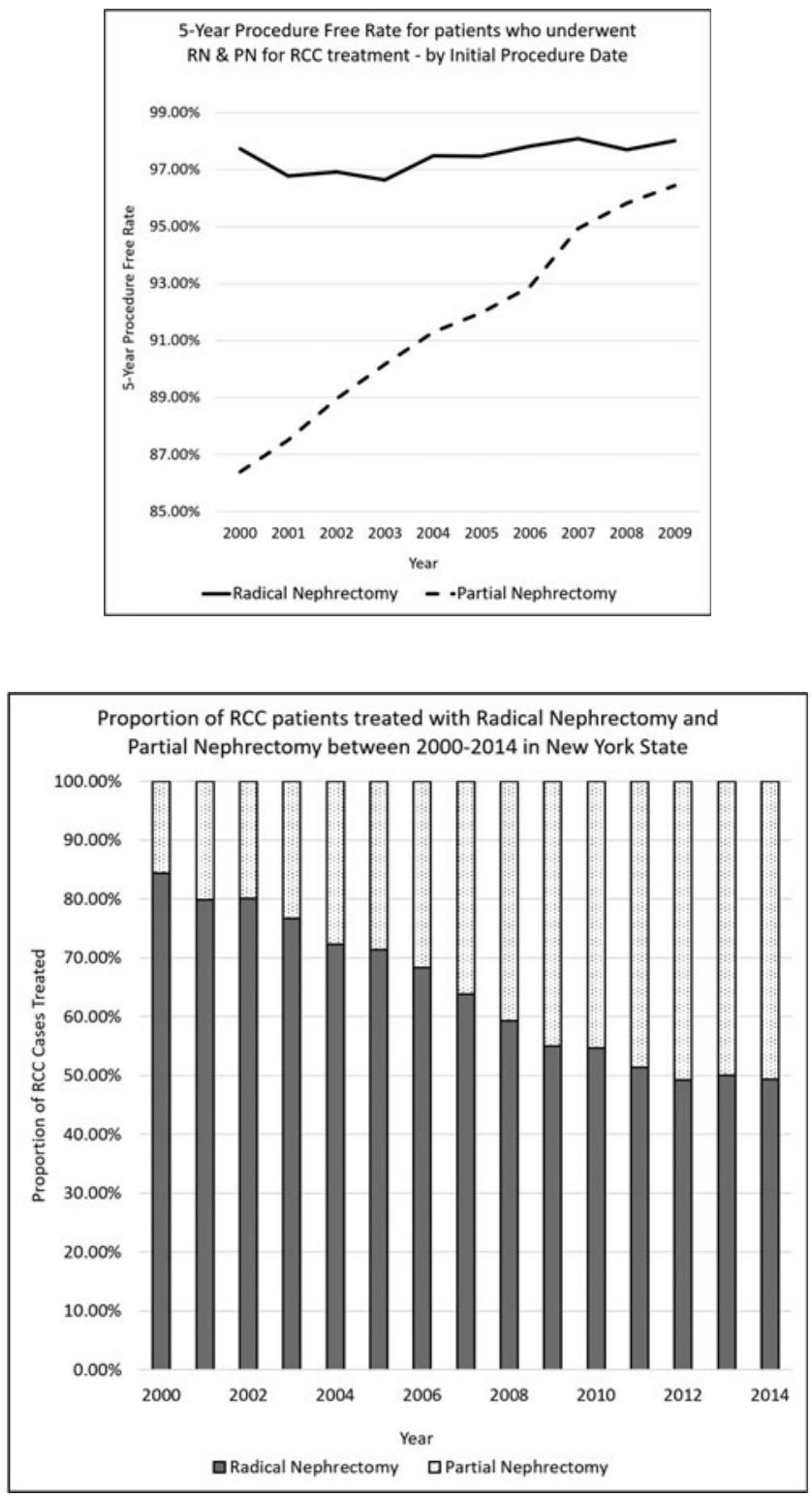

free rate of PN has increased significantly to be on par with the treatment free rate of $\mathrm{RN}$.

\section{MP20-15 The Impact of "Freeing Up" The On-Call Consultant From Elective Activity on Emergency Urological Admissions, Length of Hospital Stay and Re-Admission Rates.}

A Simoes, N Shrotri, P Keirandish, J Hale, D Bogard, G Papadopoulos, M Thomas, BA Eddy, EH Streeter, R Krishnan, SS Kommu

\section{Kent \& Canterbury Hospital}

Introduction \& Objective: The number of patients attending the hospital emergency units are exponentially on the rise and the reasons behind this upsurge are multifactorial. Hence, units are embarking on novel ways of working to address these challenges and one such approach is to provide consultant-led care. Traditionally, emergency unit attendees did not receive prompt consultant input in the management of their care, as the consultants were busy delivering elective services when oncall. "Freeing up" on-call consultants from elective activities allows early senior input to make important decisions regarding their management and the need for hospital admission.

Methods: This is a retrospective pre-intervention and postintervention observational study based at a university teaching hospital with an Emergency Care Centre (ECC) attending to all urological emergencies with a catchment population of 750,000. Data was extracted from the Hospital Information System on ECC attendances of patients with urological conditions for two 6months periods, April 2017 to September 2017 and April 2018 to September 2018. The patient outcome measures were analyzed to assess the effect of freeing up the consultant's elective commitments when on-call. Prior to the intervention, the on-call consultant conducted three elective sessions during the on-call week and these were reduced to two as a phased reduction on elective clinical activity. The patient outcome measures adopted to assess the effect of the intervention included admission rates, length of hospital stay and re-admission to the hospital within 30 days of discharge.

Results: Prior to the change in elective clinical activity, for a sixmonth period the total attendances to the ECC were 1389 of which 610 (44\%) admitted to hospital. Following the change, 1462 patients attended the ECC and $533(36 \%)$ were admitted. The average length of hospital stay before and after the change was implemented the in both groups was 4.9 days and on statistical analyses utilizing the two-sample t-test, the p-value of 1 indicated that there was no difference. Similarly, the readmission rates were 31 in the cohort before the change and 32 in the subsequent cohort.

Conclusions: The new on-call arrangement for the consultants was associated with a reduction of the number of hospital admissions, but it did not reduce the length of hospital stay or the 30-day re-admission rates. These findings could be used to further develop prediction tools for streamlining an endourological service.

MP20-16 A pattern of increased presentation of patients with kidney stones during Abu Dhabi's summer months.

Z Almallah, D Delaney, A Alaqqad, A Burham

Cleveland Abu Dhabi 
Introduction \& Objective: It has been noticed that there is an increase in the number of patients presented to the emergency department and to urology outpatient clinics with kidney stones, ureteric stones and renal colic during summer months. Furthermore, there is mounting evidence to suggest that the incidence of kidney stones is higher in areas with a hot climate. To study objective observational evidence of increased incidence of kidney stones presented to large tertiary institute in Abu Dhabi during summer months.

Methods: Initial review of Epic electronic patient record data for the diagnoses of kidney stones, ureteric stones and renal colic (ICD10 N20-N23) in the Emergency and Urology departments for summer months (Jul-Sep) of 2016 and 2017 (2018) and compare them with the preceding and following winter months in the same period.

Results: In the Emergency Department (ED), there was a trend beginning to emerge, year on year, where Q3 (Jul-Sep) displays a higher incidence rate (diagnoses per 1,000 ED visits) when compared with Q1 (Jan-Mar) in consecutive years from 2016 to Q1 2018. There was a suggestion that the null hypothesis, or no difference between the rates in these periods, can be rejected when comparing Q1'18 (6.4) vs Q3'17 (8.8) (prop.test: x2=4.4, $95 \% \mathrm{CI}=0.000-0.005, \mathrm{p}=0.035)$ however this level of significance was not repeated in every comparison. Reviewing coded data for the same period for patients attending Urology Clinics suggested a similar trend for the same period although with a potential outlier in Q3' 16 (71.5) where the rate was at its lowest for the period. Comparing Q3'17 (116) to Q1'17 (94.2) and Q1'18 (99), as with ED, displays differences in rates similar to ED respectively $([\mathrm{x} 2=6.795 \% \mathrm{CI}=-0.04--0.005 \mathrm{p}=0.009]$, $[\mathrm{x} 2=4.4,95 \% \mathrm{CI}=0.001-0.032 \mathrm{p}=0.036])$.

Conclusions: This initial observational study suggests a potential pattern of increased incidence of kidney stones presenting to ED at large tertiary institute in Abu Dhabi during the warmest months of the year (Jul-Sep) compared with winter months (Jan-Mar). Further collection of data over the coming months in the emergency department and urology clinics are needed and collaboration with other centers, to establish further evidence of a trend, if any before work on potentially implementing a prevention program is to be recommended.

\section{MP20-17 A Systematic Review of the Effects of In- traoperative Stress on Surgical Performance}

\author{
E Good, N Raison, M Khan, P Dasgupta, K Ahmed
}

King's College, London

Introduction \& Objective: Surgery requires a large amount of concentration, which can easily be disrupted by intraoperative stressors. Subsequent disruption of surgical flow may therefore impair surgical performance and patient outcome. This systematic review will identify key stressors and explore the effects they have on performance and patient outcome.

Methods: The systematic review search was conducted using the MEDLINE, EMBASE and PubMed databases. 4475 results were initially identified however, after removal of irrelevant articles and applying the inclusion and exclusion criteria, 23 papers were obtained for inclusion in the review. These papers were evaluated and the relevant data extracted from them.

Results: Seventeen types of stressors were identified in the articles such as; background noise, laparoscopic surgery, complex procedures and bleeding. Lack of experience was the most commonly reported stressors, showing a rise in intraoperative stress levels and worse performance with reduced surgical experience. Background noise was also often highlighted as a common stressor, which impaired performance. The methods of stress assessment were both subjective and objective. They varied from heart rate variability (HRV) and salivary cortisol levels, to questionnaires and State-Trait Anxiety Inventory (STAI) scores. STAI was used most frequently to assess stress however, this score is designed to measure changes in anxiety over a long period of time, not acutely. Conversely, heart rate, which was the second most commonly used measurement, correlated well with increased stress in all but one article where used. Furthermore, due to the wide variety of study designs, many performance assessment methods were used. Two of the most commonly reported measures were time taken to complete a task and the accuracy of the performance. These were found to increase and decrease respectively with raised task stress. Quality analysis was also performed via the Methodological Index for Non-Randomized Studies (MINORS) tool.

Conclusions: There is a wide variety of intraoperative stressors that may impair a surgeon's performance, many of which are shown to increase intraoperative stress, resulting in impaired performance. Lack of experience is particularly noted for increasing levels of intraoperative stress and reducing performance. However, more extensive research is required to investigate the effects that this intraoperative stress and impaired performance has on patient outcomes.

\section{MP20-18 The Cost of Gross Hematuria following Robotic-Assisted Laparoscopic Partial Nephrectomy}

O Kott, DW Sobel, B Golijanin, C Mueller-Leonhard, O ALALAO, C Tucci, G Pareek, D Golijanin

Brown University

Introduction \& Objective: Gross hematuria $(\mathrm{GH})$ following robotic-assisted laparoscopic partial nephrectomy (RaLPN) is often a serious surgical complication. GH in this setting can often indicate an arteriovenous malformation or pseudoaneurysm. GH is a major driver of readmission, and the overall healthcare burden associated have not been studied from a cost analysis perspective. We present a case series of patients readmitted for gross hematuria following RaLPN the associated costs as well as risk factors for development postoperatively.

Methods: A retrospective review was conducted of patients who underwent RaLPN from between January 1, 2012 and May 31, 2017 and had postoperative complications involving gross hematuria. We measured the costs associated with their postoperative hospital visits, admissions and procedures. Characteristics of patients readmitted with gross hematuria evaluated.

Results: A total of 223 patients who underwent RaLPN were reviewed and 21 patients were identified as having postoperative complications involving gross hematuria. 21/223 (8.5\%) presented with hematuria. 11/223 (4.9\%) underwent percutaneous angiography and embolization. For all readmitted patients the overall median cost of readmission was $\$ 62,392$. The highest cost driver was operating room time. Other notable costs associated with these cases were radiology services, room board, emergency room services and implants. Univariate analysis of patient characteristics identified off clamp technique $(\mathrm{OR} 0.13, \mathrm{p}=0.05)$ protective of gross hematuria, while postoperative Coumadin (OR 7.28, $\mathrm{p}=0.04$ ) was associated with gross hematuria and readmission. Conclusions: The overall incidence of gross hematuria following RaLPN is low, however, it carries significant healthcare 


\begin{tabular}{|c|c|c|c|c|c|}
\hline \multicolumn{2}{|c|}{$\begin{array}{l}\text { Patient } \\
\text { Charaeteristics }\end{array}$} & $\begin{array}{c}\text { Total } \\
(\mathrm{N}=223)\end{array}$ & $\begin{array}{c}\text { No Hematuria } \\
(\mathrm{N}=202)\end{array}$ & $\begin{array}{c}\text { Hematuria } \\
(\mathbb{N}=21)\end{array}$ & P-value \\
\hline \multicolumn{6}{|c|}{ Preoperative Features } \\
\hline Age, years & Median (IQR) & $61(53,69)$ & $61(53,69)$ & $60(55,68)$ & $0.95^{*}$ \\
\hline Sex, $N(\%)$ & Male & $118(53)$ & $106(52)$ & $12(57)$ & $0.86^{6}$ \\
\hline \multicolumn{6}{|c|}{ Body Mass Index, $\mathrm{kg} / \mathrm{m}^{2}$} \\
\hline & Median (IQR) & $29(26,32)$ & $29(26,32)$ & $29(26,31)$ & $0.75^{*}$ \\
\hline & $<18.5$ & $3(1)$ & $2(1)$ & $1(5)$ & \\
\hline & $18.5 \cdot 25$ & $33(15)$ & $29(14)$ & $4(19)$ & \\
\hline & $25 \cdot 30$ & $96(43)$ & $87(43)$ & $9(43)$ & \\
\hline & $>30$ & $91(41)$ & $84(42)$ & $7(33)$ & $0.35^{r}$ \\
\hline \multicolumn{6}{|c|}{ Smoker Classification at Surgery } \\
\hline & Current & $33(15)$ & $30(15)$ & $3(14)$ & $1.00^{r}$ \\
\hline & Ever & $72(36)$ & $63(35)$ & $9(50)$ & $0.21^{\prime}$ \\
\hline & Missing & 24 (11) & $21(10)$ & $3(14)$ & $0.48^{\circ}$ \\
\hline \multicolumn{6}{|c|}{ Renal Score, $\mathrm{N}(\%)$} \\
\hline & Median (IQR) & $7(5,8)$ & $6.5(5,8)$ & $8(6,9)$ & $0.18^{*}$ \\
\hline & $4-5$ & $68(30)$ & $64(32)$ & $4(19)$ & \\
\hline & $6-7$ & $51(23)$ & $47(23)$ & $4(19)$ & \\
\hline & $8-11$ & $77(35)$ & $68(34)$ & $9(43)$ & \\
\hline & Missing & $27(12)$ & $23(11)$ & $4(19)$ & $0.44^{r}$ \\
\hline \multicolumn{6}{|c|}{ Intraoperative Features } \\
\hline \multicolumn{6}{|c|}{ Estimated Bloed Loss, mL } \\
\hline & Median (IQR) & $100(50,150)$ & $100(50,150)$ & $100(70,200)$ & $0.37^{*}$ \\
\hline \multicolumn{2}{|c|}{ Clamping Technique, N (\%) } & & & & $0.11^{f}$ \\
\hline & Off Clamp & $53(24)$ & $52(26)$ & $1(5)$ & \\
\hline & Hilar Clamp & $135(61)$ & $118(58)$ & $17(81)$ & \\
\hline & $\begin{array}{l}\text { Super Selective } \\
\text { Clamp }\end{array}$ & $24(11)$ & $22(11)$ & $2(10)$ & \\
\hline \multicolumn{6}{|c|}{ Operation Time, minutes } \\
\hline & Median (IQR) & $220(189,263)$ & $220(189,260)$ & $251(194,271)$ & $0.31^{*}$ \\
\hline \multicolumn{2}{|c|}{ ASA Score, N (\%) } & & & & $0.47^{\mathrm{t}}$ \\
\hline & 1 & $4(2)$ & $3(1)$ & $1(5)$ & \\
\hline & 2 & $183(82)$ & $166(82)$ & $17(81)$ & \\
\hline & 3 & $36(16)$ & $33(16)$ & $3(14)$ & \\
\hline & 4.5 & $0(0)$ & $0(0)$ & $0(0)$ & \\
\hline \multicolumn{6}{|c|}{ Anticoagulant, $\mathrm{N}(\%)$} \\
\hline & None & $155(70)$ & $142(70)$ & $13(62)$ & \\
\hline & Any (ref. - None) & $60(27)$ & $52(26)$ & $8(38)$ & $0.40^{\circ}$ \\
\hline & $\begin{array}{l}\text { Coumadin Alone } \\
\text { (ref. = None) }\end{array}$ & $3(1)$ & $2(1)$ & $1(5)$ & $0.24^{t}$ \\
\hline & Missing & $8(4)$ & $8(4)$ & $\mathbf{0}$ & \\
\hline
\end{tabular}

Table 2: Univariable logistic regression analysis of patient characteristics and the development of any hematuria event after RALPNx surgical procedure betwee 2011 and 2017.

\begin{tabular}{|c|c|c|c|}
\hline \multirow{2}{*}{ Patient Characteristics } & \multicolumn{3}{|c|}{ Hematuria Event } \\
\hline & OR & $95 \% \mathrm{Cl}$ & P-value \\
\hline \multicolumn{4}{|l|}{ Preoperative Features } \\
\hline \begin{tabular}{|l|l|} 
Age (years) \\
\end{tabular} & 1.00 & $0.96,1.04$ & 0.98 \\
\hline Sex (ref = female) & 1.21 & $0.49,2.99$ & 0.68 \\
\hline BMI $\left(\mathrm{kg} / \mathrm{m}^{2}\right)$ & 1.00 & $0.92,1.08$ & 1.00 \\
\hline Current Smoker & 1.01 & $0.27,3.69$ & 0.99 \\
\hline Ever-Smoker & 1.87 & $0.71,4.96$ & 0.21 \\
\hline History of HTN & 0.94 & $0.37,2.37$ & 0.89 \\
\hline Cardiac Arrhythmia & 1.21 & $0.14,10.19$ & 0.86 \\
\hline Coronary Artery Disease & 0.79 & $0.10,6.41$ & 0.83 \\
\hline Diabetes & 1.39 & $0.48,4.04$ & 0.54 \\
\hline Antihypertensive Meds & 1.41 & $0.54,3.65$ & 0.48 \\
\hline \multicolumn{4}{|l|}{ Renal Score } \\
\hline \begin{tabular}{l|l} 
Continuous \\
\end{tabular} & 1.16 & $0.92,1.46$ & 0.21 \\
\hline $4-5$ & ref. & & \\
\hline $6-7$ & 1.36 & $0.32,5.73$ & 0.67 \\
\hline $8-11$ & 2.12 & $0.62,7.22$ & 0.23 \\
\hline Missing & 2.78 & $0.64,12.05$ & 0.17 \\
\hline \multicolumn{4}{|l|}{ Intraoperative Features } \\
\hline Estimated Blood Loss (per 200mL) & 1.11 & $0.60,2.05$ & 0.74 \\
\hline \multicolumn{4}{|l|}{ Clamping Technique } \\
\hline Hilar Clamp & ref. & & \\
\hline Off Clamp & 0.13 & $0.02,1.03$ & 0.05 \\
\hline Super Selective Clamp & 0.63 & $0.14,2.93$ & 0.56 \\
\hline Operation Time (hour) & 1.29 & $0.82,2.05$ & 0.28 \\
\hline \multicolumn{4}{|l|}{ ASA Score } \\
\hline 1 & ref. & & \\
\hline 2 & 0.31 & $0.03,3.12$ & 0.32 \\
\hline 3 & 0.27 & $0.02,3.50$ & 0.32 \\
\hline \multicolumn{4}{|l|}{ Anticoagulant } \\
\hline \begin{tabular}{l|l} 
& None
\end{tabular} & ref. & & \\
\hline Coumadin (alone or in combination) & 7.28 & $1.11,47.59$ & 0.04 \\
\hline Other (alone or in combination) & 1.34 & $0.48,3.71$ & 0.58 \\
\hline
\end{tabular}

costs. Off clamp renal hilar technique was protective of gross hematuria, while postoperative Coumadin was associated with increased risk of readmission. Further research is required to prevent this complication and prevent the associated costs.

\section{MP20-19 Fate of normal hematuria investigations patients in the immediate to long term - outcomes and clinical implications}

\author{
G KANDA SWAMY, R David, B Rai, B James, K Thomas, \\ P Bose
}

Swansea Bay University Health Board

Introduction \& Objective: Hematuria evaluation is one of the commonest referral to Urology most of which have normal investigation results. We studied the fate of these patients in the immediate to long term over the following years for development of urological issues, mortality if any and its causes.

Methods: Records of patients who had normal hematuria investigations in a single center between Jan 2012 - Dec 2013 were reviewed. Jan 31st, 2019 was taken as reference point ensuring at least 60 months of follow up each patient reviewed. Normal hematuria evaluation is defined as normal flexible cystoscopy \& Ultrasound KUB. Patient demographics, hematuria type, additional investigations, re-referral to urology, evaluation outcomes, mortality and causes were studied.

Results: The study identified 573 patients with male: female ratio of 1.3:1. Ratio of visible: non visible hematuria at presentation was 2.74:1. Additional IVU and CT was done in 59\% and 9\% respectively which picked up no urological malignancy. $3.5 \%$ of patients remained under Urology follow up for benign conditions and rest were discharged. As of reference point the median duration of follow up is 72 months. Re-referral rate was $18 \%$ during this period, out of which $53 \%$ was for hematuria. 4 Urological cancers were picked up in the re-referred cohort all of whom had Visible hematuria. As of reference point, $87.1 \%$ were still alive, while old age is the commonest cause among those died.

Conclusions: A high proportion of patients are likely to be rereferred to urology in the following 6 years after having normal hematuria investigation. However, mortality due to a urological cause is very low in the same period. Persistent non visible hematuria do not need any further evaluation but visible hematuria does. Until long term, prospective, multicenter, risk factor-based evidence is available this data could be used to counsel patients.

\section{MP20-20 The Impact of Video Visits on Follow-Up Care for Robot Assisted Radical Prostatectomy Pa- tients}

J Kim, c GU, E Kim, M Shahait, RW Dobbs, DI Lee

University of Pennsylvania

Introduction \& Objective: As the demand for healthcare resources continues to increase, healthcare systems have begun adapting various technological measures to maximize care efficiency and access to providers. One such advance has been the replacement of office visits with video visits. A recent clinical trial of post-robotic assisted radical prostatectomy (PRARP) patients, showed no difference in patient satisfaction and a significant decrease in cost and travel time for patients. We are 
recruiting randomized clinical trial to assess the impact of video visits in a denser urban setting on patient satisfaction, difference in waiting time, and cost per visit. Given shifting reimbursement landscape for telemedicine, we also sought to assess patient response to a self-pay model for telemedicine visits.

Methods: Post-RARP patients, who were due to follow-up visit as per guidelines, were block randomized to two groups: telemedicine visit group and standard office visit group. Patients were informed during the consent process that the video visit would be self-pay, which was initially set at $\$ 99$ but lowered to $\$ 49$ after the first month of recruitment. Patients in both groups were asked to complete questionnaires to assess travel time, costs related to the visit, time spent waiting to see a provider, and overall patient satisfaction.

Results: At this point, 24 patients have been accrued: 13 patients in the office group and 11 patients in the telemedicine group. Both groups had comparable age, education level, general health status, and comfort with technology. The recruitment rate during the $\$ 99$ self-pay period was $5.67 \%$ (6/106) vs. $8.95 \%$ (53/592) once lowered to $\$ 49$. The average waiting time for the video visit arm was shorter compared to the office visit (6.5 min vs. 29.23, $\mathrm{p}=.003$ ). The average cost for video visits was lower (\$9 vs. $\$ 24.2$, $p=0.001$ ), and the average travel time for office visits was longer compared to the video visits $(61.15 \mathrm{~min}$ vs. $0.5 \mathrm{~min}, \mathrm{p}<0.0001)$.

Conclusions: Early results from this RCT indicate that telemedicine improves the post-RARP patient experience by decreasing travel time and waiting time, as well as decreasing the associated costs of the visit. Though patients had significant savings in both costs and time, there appeared to be a marked increase in willingness to pay once the fee was lowered from $\$ 99$. As telemedicine becomes increasingly common, more research is needed to examine the factors that affect patients, providers, and social willingness to pay for this service.

MP20-21 Cystoscopic follow-up of low-risk bladder Transitional Cell Carcinoma-Are the National Institute of Health and Care Excellence guidelines from the United Kingdom appropriate in suggesting discharge after 12 months of follow up?

\section{Alsawi, H Burns, R Sohail Khan, J De Souza \\ Department of Urology -University Hospital Monklands}

Introduction \& Objective: Non-muscle invasive bladder tumors can be stratified into three risk groups: Low, intermediate and high risk. This classification is based on prognostic factors that include risk of recurrence and tumor progression. In intermediate and high-risk tumors recurrences after 10 years are not unusual, therefore the European Association of Urologists (EAU), American Urological Association (AUA) and National Institute of Health and Care Excellence (NICE) guidelines recommend lifelong cystoscopic follow up. However, with low-risk tumors the risk of recurrence after 5 years is low. The EAU and AUA guidelines recommend continuing cystoscopic surveillance of these tumors for up to 5 years. However, NICE guidelines recommend discharging these patients from follow up if their check cystoscopy at 1 year shows no recurrence. In our clinical practice we found that these tumors do recur beyond 1 year. Therefore, the aim of our study was to review if the NICE recommendations are safe and clinically appropriate.

Methods: We performed a retrospective analysis of patients who underwent a Transurethral Resection of Bladder Tumor (TURBT) over a 1-year period from May 2014 to May 2015. Patients with low risk bladder Transitional Cell Carcinoma (TCC) (Solitary G1pTa $<3 \mathrm{~cm}$, Solitary Low-Grade G2pTa $<3 \mathrm{~cm}$ ) were specifically reviewed to assess their recurrence status, timing of recurrence after initial TURBT, and their subsequent histology and its correlation to the patient's smoking status.

Results: 130 patients who underwent TURBT within a 1-year period were investigated. $82 / 130$ patients $(63 \%)$ were found to have low risk bladder TCC on initial TURBT. 33/82 patients $(40 \%)$ had a recurrence of their tumor, reassuringly none of these recurrences were upgraded to intermediate or high-risk groups. $22 / 33$ patients $(66 \%)$ had a recurrence within the first 12 months of the initial TURBT. However, $11 / 33$ patients $(33 \%)$ had a recurrence beyond 1 year (Range 14-52 months). Of those 11 patients $8(72 \%)$ were either current or Ex-Smokers.

Conclusions: In our study, $33 \%$ of all recurrences were beyond the 1-year period from initial TURBT. These recurrences would have been missed if these patients were discharged after 1 year as recommended by NICE. We therefore recommend a risk adjusted follow up especially in Ex or current smokers, with a cystoscopy at least 2 years and 5 years from the initial TURBT prior to considering discharge.

\section{MP20-22 Recommendations for Opioid Prescribing after Endourological Surgery: An Expert Panel Con- sensus}

K Koo, F Faisal, N Gupta, A Meyer, H Patel, P Pierorazio, B Matlaga

Johns Hopkins University School of Medicine

Introduction \& Objective: Opioids are frequently overprescribed after surgery. The 2018 American Urological Association position statement on opioid use suggests using the lowest dose and potency to achieve pain control, but a lack of procedure-specific prescribing guidelines contributes to wide variation in prescribing patterns. To address this gap, this study aims to develop opioid prescribing recommendations through an expert panel consensus.

Methods: A 15-member multidisciplinary expert panel included representatives from five stakeholder groups. A three-step modified Delphi method was used to develop recommendations for postsurgical opioid prescribing. Recommendations were made for opioid-naïve patients without chronic pain conditions. The panel used oxycodone $5 \mathrm{mg}$ equivalents to define the number of prescribed tablets.

Results: Procedure-specific recommendations were developed for 16 endourological and minimally-invasive urological procedures. The panel agreed that not all patients desire or require opioids; thus, the minimum recommended number of opioid tablets for all procedures was zero. Consensus ranges were identified to allow alignment of prescribed quantities with expected needs. The maximum recommended quantity varied by procedure, from zero tablets (three procedures) to 15 tablets (six procedures), with a median of 10 tablets. Attending urologists typically voted for higher opioid quantities than non-attending panel members. The panel identified eight overarching strategies for opioid stewardship, including contextualizing postsurgical pain management with patients' goals and preferences and maximizing non-opioid therapies.

Conclusions: Procedure-specific guidelines for postsurgical opioid prescribing may help to align individual urologists' prescribing habits with consensus recommendations. These guidelines can aid quality improvement efforts to reduce overprescribing in urology. 


\section{MODERATED POSTER SESSION 21: CLINICAL STONES: EQUIPMENT (I)}

\begin{abstract}
MP21-01 Introduction of a nurse led stent removal service using the single use ISIRIS in a tertiary referral stone unit and the effect on patient waiting times.
\end{abstract}

J Collie, S Shah, J Durrant, S Al-Hayek,

K Saeb-Parsy, O Wiseman

Cambridge University Hospitals NHS Trust

Introduction \& Objective: JJ stenting represents one of the most significant causes for patient discomfort and dissatisfaction. At a large tertiary referral center standard stent removal was undertaken with a flexible cystoscope (FC) in the Endoscopy Department by a doctor. The 'Isiris $\alpha$ ' (Coloplast) is a single use stent removal system consisting of a 'camera on chip' disposable FC with integrated grasper. We examine the impact on waiting times of a nurse led stent removal service using this system.

Methods: A specialist stone nurse undertook training in FC approved by The British Association of Urological Surgeons (BAUS). A nurse led service was offered to patients upon reaching competency for stent removal. Data between July and December 2016 showed 54 stent removals booked to a doctor service offered in the endoscopy department (Group A). In comparison a prospective database revealed that 94 stent removals were booked to a nurse led service (Group B). The delays in stent removal compared to the "ideal" stent removal date (plus or minus 3 days tolerance allowed) were compared between the two pathways.

Results: 94 stent removals were booked to Group B (Isiris), of which 87 were undertaken. In three of these the nurse was unable to remove the stent. In one case due to poor views in the bladder with debris, in one case because of encrustation, and in the remaining case due to inability to navigate through a tortuous prostatic urethra. Only 16 of 54 patients had their stents removed on time in Group A, whereas 74 of 87 patients in Group B (Isiris) had their stent removed on time ( $p<0.0001$, Fischer's exact test). In Group A 33 patients (61\%) experienced a delayed stent removal of more than 7 days. In Group B (Isiris) there were 5 patients $(6 \%)$.

Conclusions: This study has shown it is possible to introduce a nurse led stent removal service using the ISIRIS system which has led to a reduction in delays of stent removal. This will likely translate into significant quality of life improvements for patients. Further work is being undertaken to investigate emergency department attendance to identify if timely stent removals can reduce stent related hospital episodes.

\begin{tabular}{|l|rr|cc|}
\hline \multirow{2}{*}{ Number of delay days } & \multicolumn{2}{|c|}{ Pre Isiris Group A } & \multicolumn{2}{c|}{ Post Isiris Group B } \\
& Number & $\%$ & Number & $\%$ \\
\hline $0-3$ & 16 & 30 & 74 & 85 \\
\hline $4-7$ & 5 & 9 & 8 & 9 \\
\hline $8-21$ & 21 & 39 & 2 & 2 \\
\hline $22+$ & 12 & 22 & 3 & 4 \\
\hline Median delay days (above 3) & \multicolumn{2}{|c|}{13.5} & \multicolumn{2}{|c|}{7} \\
\hline
\end{tabular}

\section{MP21-02 The Importance of Stone Retrieval In The Modern Management Of Urolithiasis: The UroPro Stone catcher vs Traditional Sieving}

A Harbias, M Ravindraanandan, W Mahmalji

Hereford County Hospital

Introduction \& Objective: World urolithiasis is increasing with a 70\% increase in hospital admissions between 2000 and 2015. Stone analysis can allow for the identification of metabolic conditions and stone retrieval may decrease the need for followup imaging, reducing costs and patient radiation.

Methods: Between December 2018 and May 2019 patients receiving SWL or being conservatively managed for a ureteric stone were discharged with a Paramount Medical solutions UroPro Stone Catcher (Figure 1). Both groups of patients were asked to complete a patient survey regarding the product.

Results: 19 patients were recruited. 9 from SWL and 10 from acute admissions. 16 responses have been received: 8 each from each group. 3 patients were uncontactable. Chart 1 shows the outcome of the acute patients, of the 8 undergoing ESWL only 1 patient has caught fragments (Figure 2). Graph 1 outlines patients' responses to "how easy did you find the stone catcher to use" and Graph 2 highlights how only 4 of the 16 patients preferred using the UroPro Stone catcher over other methods. Common complaints about the stone catcher were that it was unhygienic due to the short handle and overflow of urine due to the mesh not being porous enough.
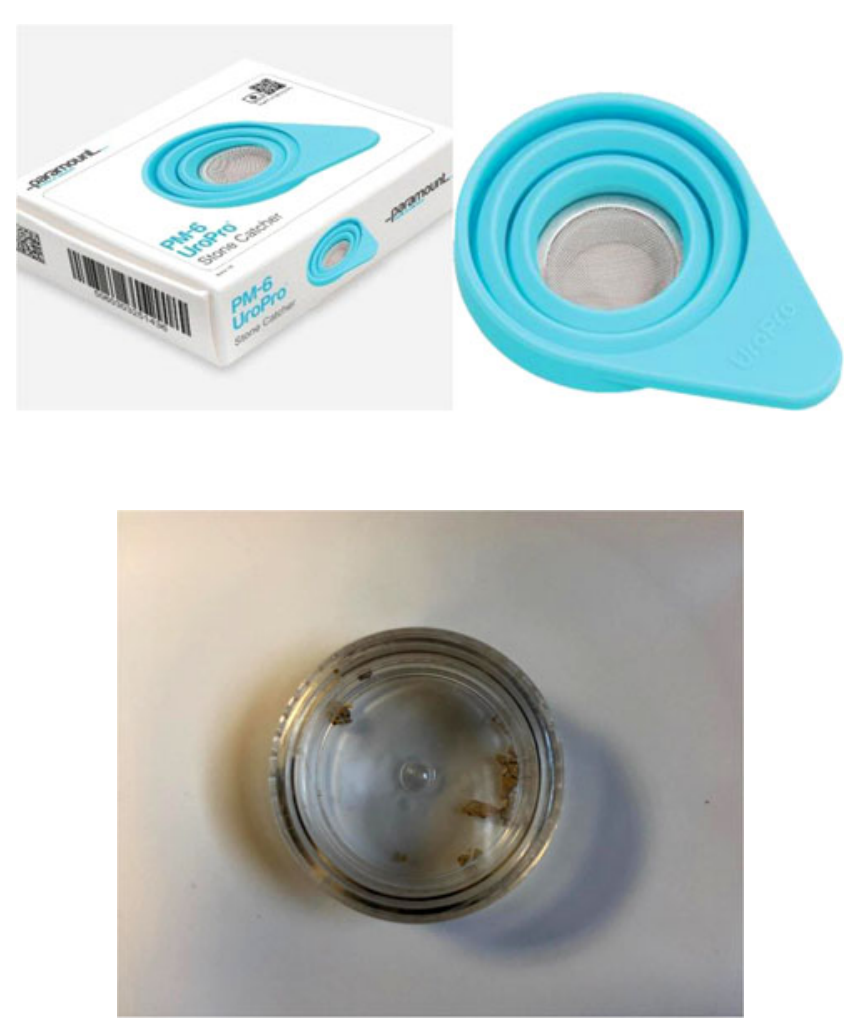

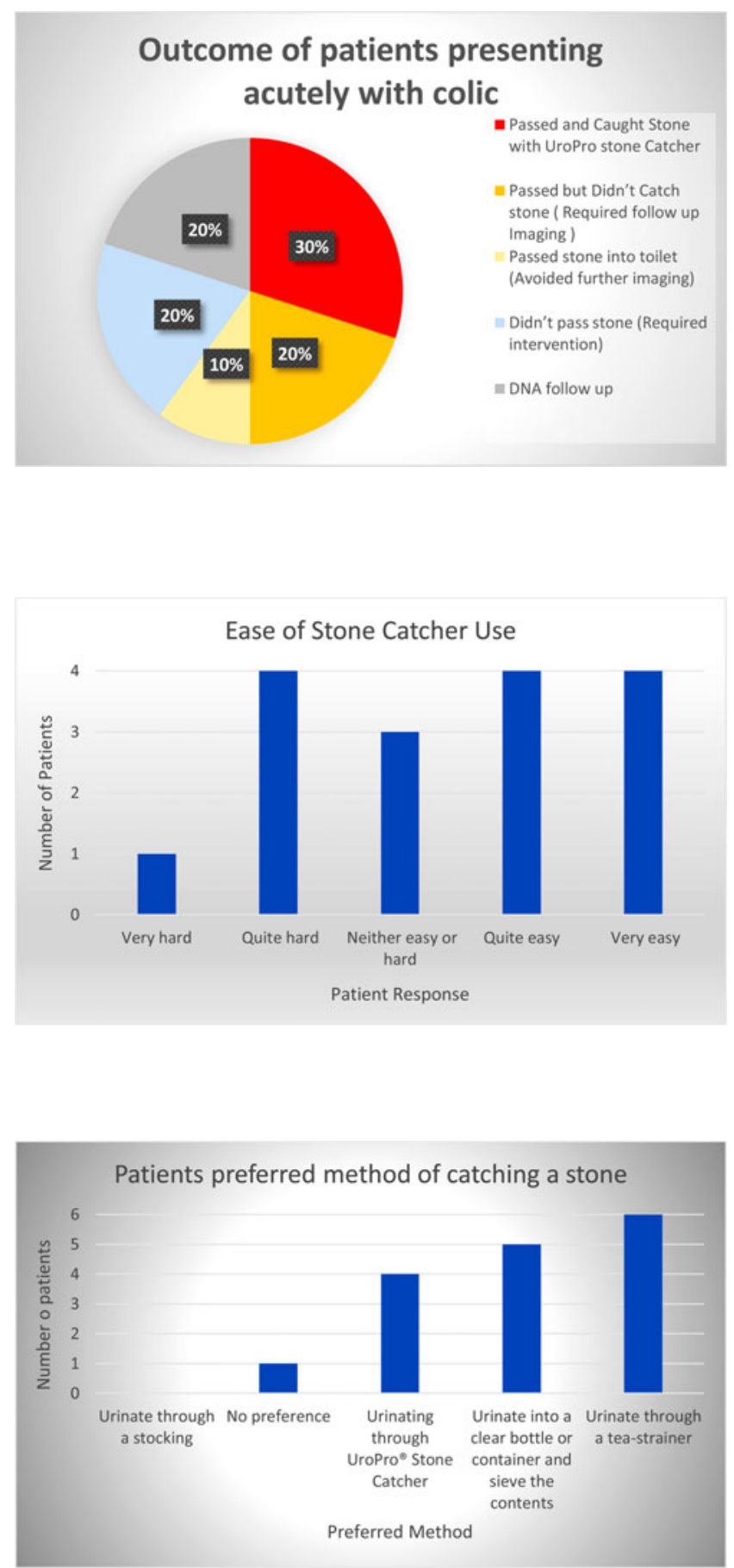

Conclusions: The UroPro stone catcher did increase the number of stones caught and sent for analysis. However, many patients preferred the older 'sieving' methods. Stone catchers retail at $£ 5.50$, therefore 'sieving' remains more cost effective. Stone retrieval in any form remains advantageous as confirming stone passage saves the patient 0.5 to $2.8 \mathrm{mSv}$ of radiation from follow-up imaging, the NHS circa $£ 100$ per unnecessary follow-up CT scan and aids metabolic analysis. Over time both factors may be substantial. Therefore, emphasizing the benefits and importance to patients to sieve their urine regardless of the techniques is paramount in the modern management of urolithiasis.
MP21-03 Ureteral Access Sheath-Related Injuries VS Post-Operative INFECTIVE conditions: is sheath insertion always needed?

G Bozzini, U Besana, A Calori, S PROIETTI, G Pini, C Buizza, M Sighinolfi, B Rocco

\section{ASST Valle Olona - Busto Arsizio VA}

Introduction \& Objective: To compare intraoperative ureteral injuries inflicted during retrograde intrarenal surgery (RIRS) with ureteral access sheath (UAS) insertion compared to the rate of postoperative infections after RIRS without UAS insertion, were evaluated.

Methods: Patients who received an indication for a RIRS were divided into two groups. Group A performed a RIRS without UAS insertion and Group B performed RIRS with UAS insertion. Post Ureteroscopic Lesion Scale (PULS) grading was performed after UAS or flexible ureteroscope removal. Ureteral lesions in distal, middle, proximal, and multiple locations were evaluated and compared according to the PULS scale. On the same hand patients were controlled postoperatively to assess any infective condition in both groups.

Results: The evaluation comprised 181 patients, 89 for Group A and 92 for Group B. In Group B a 9.5/11.5 French UAS device was used. The overall stone-free rate, clinical insignificant residual fragments, and final stone-free rate were $41.4 \%, 53.5 \%$, and $95 \%$, respectively. In $58.7 \%$ of the patients, no lesions were present according to PULS grading in both groups. No lesions of Grade 3 and above were found in; however, there were lesions of Grade 1 and 2 in $38.6 \%$ and $2.9 \%$ of the patients, respectively with no statistical difference between the two groups. On the other hand, the overall presence of postoperative infection rate was much higher for Group A $(37.1 \%$ vs $16.3 \% \mathrm{p}=0.03)$.

Conclusions: UAS insertion does not show a higher number of ureteral injuries. UAS insertion during RIRS allows a less rate of postoperative infections.

\section{MP21-04 Ureteral stenting after routine uretero- scopy: is earlier stent removal feasible?}

S Hill, A Boyko, S Merheb, M Biebel, M Katz, R Babayan, S Wason, D Wang

\section{Boston Medical Center Department of Urology}

Introduction \& Objective: Ureteroscopy is a standard treatment option for urinary tract calculi. Common protocol is to place an internal ureteral stent after ureteroscopy for up to one week. The minimal length of time required for stenting after ureteroscopy is not well defined. Ureteral stents are associated with significant morbidity, including pain and discomfort. The objective of this study was to determine if there were any difference in postoperative unplanned clinic or ER visits based on duration of stent placement. We sought to determine if earlier stent removal was feasible.

Methods: This is a single-institution IRB approved retrospective review of 231 ureteroscopy cases with laser lithotripsy or basket extraction for urinary tract calculi performed during 2018 by multiple surgeons. The main outcome measure was number of unplanned ED or outpatient visits within 30 days following surgery. Log rank test and Cox regression modeling (adjusted for sex, age, and presence of an impacted stone) were used to analyze if there were an association between unplanned visits and length of stent placement (number of days). The patients were separated 
into three groups based on stent duration: 1 (0-3 days), 2 (4-6 days), and 3 (7+ days).

Results: Of the 223 patients, there were 59 in group 1 (26.5\%), 80 in group $2(35.9 \%)$, and 84 in group $3(37.7 \%) .218(97.8 \%)$ were event free within the 30 -day post-operative period. $5(2.2 \%)$ patients had an unplanned visit after stent removal (2 in groups 1 and 2, 1 in group 3). There was no statistical difference between groups ( $\mathrm{p}$ value $=0.667$ ), and Cox regression showed no association between duration of stent placement and unplanned visits, when adjusted for sex, age, and the presence of impacted stones ( $\mathrm{p}$ value $=0.674) .18(8 \%)$ patients $(3(1.3 \%)$ in group $1,8(3.6 \%)$ in group 2, and $7(3.1 \%)$ in group 3 ) had an unplanned visit during the 30-day post-operative period, including pre- and poststent removal. Cox regression showed no association between duration of stent placement and unplanned visit, adjusted for sex, age, and presence of impacted stones $(\mathrm{p}$ value $=0.610)$.

Conclusions: We found no difference in unplanned clinic or ER visits in patients based on the duration of stent placement following routine ureteroscopy. Stent removal after 3 days appears to be sufficient to minimize morbidity and complications after uncomplicated ureteroscopy. Further prospective studies are required to further define optimal duration of stent placement.

\section{MP21-05 Benchtop Comparison of 13 Different Guidewires in 3 Different Impacted Stone Models}

A Amasyali, J Groegler, M Shah, M Hajiha, J Maldonado, M Alsyouf, P Stokes, D Baldwin

Loma Linda University Health

Introduction \& Objective: This study compares the ability of 13 different urologic guidewires to bypass a tightly impacted ureteral stone.

Methods: We tested 3 stone impaction models (resisting flow rate of water 300,362 , and $444 \mathrm{mmHg}$ ) using silicone ureters. In the first and second models, we recorded the maximum force required to bypass the stone. In the first model $(300 \mathrm{mmHg})$, ten different new 0.038 straight-tipped guidewires of 13 different types were advanced past a ureteral stone using a Series 5 digital force gauge. In the second model $(362 \mathrm{mmHg})$, the top 5 performing guidewires were similarly tested using ten trials for each wire type. In the third model ( $444 \mathrm{mmHg}), 5$ attending urologists and 5 urology residents performed three blinded trials with each of the 13 guidewire types, and rated their preferences using the Likert scale. Statistical analysis was performed with ANOVA, Chi-square, and the Mann-Whitney U test.

Table 1: Results of digital force gauge and surgeon's hands-on trial.

\begin{tabular}{|c|c|c|c|c|c|}
\hline & & $\begin{array}{l}\text { Force (pound) } \\
\text { (mean } \pm S D)\end{array}$ & $\begin{array}{l}\text { Success } \\
\text { rate" }(\%)\end{array}$ & $\begin{array}{l}\text { Time for wire } \\
\text { passage }(\mathrm{sec})^{*} \\
(\text { mean } \pm \text { SD) }\end{array}$ & $\begin{array}{l}\text { Likert } \\
\text { Scale* } \\
\text { (mean } \pm \text { SD) }\end{array}$ \\
\hline \multirow[t]{3}{*}{ Olympus } & PTFE & $0.395 \pm 0.10$ & 0 & NA & $1.0 \pm 0.0$ \\
\hline & Glidewire & $0.117 \pm 0.02$ & 100 & $5.18 \pm 5.34$ & $5.0 \pm 0.0$ \\
\hline & Ultratrack & $0.171 \pm 0.05$ & 33.3 & $94.09 \pm 42.8$ & $2.6 \pm 1.07$ \\
\hline \multirow[t]{2}{*}{$\begin{array}{l}\text { Boston } \\
\text { Scientific }\end{array}$} & Zipwire & $0.145 \pm 0.06$ & 76.7 & $42.19 \pm 45.6$ & $3.1 \pm 0.87$ \\
\hline & Sensor & $0.185 \pm 0.05$ & 16.7 & $102.24 \pm 40.6$ & $1.8 \pm 1.03$ \\
\hline \multirow[t]{4}{*}{ Cook } & Fixed core & $0.678 \pm 0.35$ & 0 & NA & $1.1 \pm 0.31$ \\
\hline & Hiwire & $0.130 \pm 0.01$ & 76.7 & $48.54 \pm 44.0$ & $3.0 \pm 0.94$ \\
\hline & Roadrunner & $0.256 \pm 0.08$ & 100 & $10.94 \pm 13.9$ & $3.9 \pm 0.31$ \\
\hline & Motion & $0.201 \pm 0.08$ & 43.3 & $72.57 \pm 55.7$ & $2.5 \pm 1.1$ \\
\hline \multirow[t]{4}{*}{ Bard } & Fixed core & $0.632 \pm 0.10$ & 0 & NA & $1.0 \pm 0.0$ \\
\hline & Hydroglide & $0.526 \pm 0.21$ & 0 & NA & $1.2 \pm 0.42$ \\
\hline & Solo Plus & $0.235 \pm 0.17$ & 40 & $78.38 \pm 52.5$ & $2.1 \pm 1.19$ \\
\hline & Solo Hydro & $0.149 \pm 0.06$ & 50 & $66.77 \pm 54.8$ & $2.9 \pm 1.28$ \\
\hline
\end{tabular}

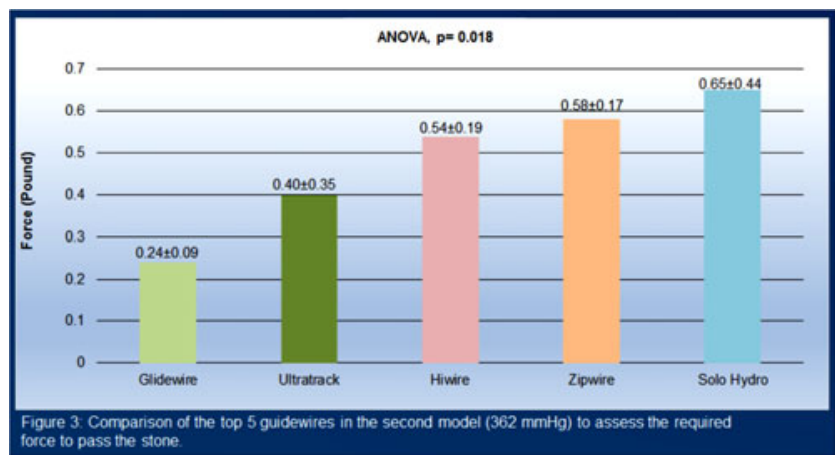

Results: In the first model, the Glidewire and HiWire passed the impacted stone with the lowest mean force $(\mathrm{p}<0.001)$. Of the 5 wires tested in the second model, the Glidewire required the least force to bypass the impacted stone $(\mathrm{p}=0.018)$ (Figure 1$)$. In the third model, only two wires (Glidewire and Roadrunner) bypassed the impacted stone in $100 \%$ of trials (Table 1). Hydrophilic wires had the highest success rate (standard:0\%, hybrid:36.67\%, and hydrophilic: $70.67 \%, \mathrm{p}=0.000$ ), Likert score (standard:1.03, hybrid:2.38, and hydrophilic:3.24, $\mathrm{p}=0.000$ ), and shortest required time to pass the stone (hybrid:82.81 seconds vs. hydrophilic:45.37 seconds, $\mathrm{p}=0.000$ ).

Conclusions: In this benchtop study, standard wires performed poorly and hybrid wires were not as effective as hydrophilic wires. The Glidewire required the least force, the shortest insertion time, and had the highest surgeon satisfaction rating.

\section{MP21-06 Retrospective study of the effect of preop- erative indwelling double $\mathbf{J}$ tube on ureteroscopic lithotripsy}

\section{Li, G Bao, C Li, Z Gao}

Introduction \& Objective: To investigate the effect of preoperative indwelling double $\mathbf{J}$ tube and non - indwelling double $\mathbf{J}$ tube on the efficacy of ureteral soft lithotripsy (FURL).

Methods: A retrospective study was performed on 67 patients who underwent FURL from January 2016 to June 2018 in our hospital. The patients who underwent double $\mathrm{J}$ tube before operation were included in the study group, 46 cases; the double $\mathrm{J}$ tube was not placed as a control group, 21 For example, the contrast between the two groups of patients with stone size, stone clearing rate, intraoperative soft tissue channel sheath success rate, lithotripsy time and postoperative complications (fever, pain, blood routine, etc.) were compared.

Results: There were 46 cases in the study group and 21 cases in the control group. There was no significant difference in the stone size between the two groups $(\mathrm{P}=0.83)$. There was no significant difference in the time between the test group and the control group $(\mathrm{P}=0.79)$; no double $\mathrm{J}$ tube was left. The success rate of patients with soft-optic channel sheath was lower than that of preoperative indwelling double-J tube $(\mathrm{P}<0.001)$. The postoperative complications of the experimental group were less than that of the control group, which was statistically significant $(\mathrm{P}=0.03)$. There were 46 cases in the study group and 21 cases in the control group. There was no significant difference in the stone size between the two groups $(P=0.83)$. There was no significant difference in the time between the test group and the control group $(\mathrm{P}=0.79)$; no double $\mathrm{J}$ tube was left. The success rate of patients with soft-optic channel sheath was lower than that of preoperative indwelling double-J tube $(\mathrm{P}<0.001)$. The 
postoperative complications of the experimental group were less than that of the control group, which was statistically significant $(\mathrm{P}=0.03)$.

Conclusions: Preoperative indwelling double $\mathrm{J}$ tube can improve the success rate of FURL. Double J tube may stimulate the diameter of ureter and increase the success rate of soft lens channel sheath, but there is no significant difference between the two groups in the effect of intraoperative lithotripsy.

MP21-07 Prospective Randomized Comparison of Standard Hand Pump Infuser Irrigation and an Automated Irrigation Pump for Operating Room Efficiency During Percutaneous Nephrolithotomy and Ureteroscopy

FA Jefferson, J Sung, L Limfueco, S Lu, C Cottone, S Tapiero, RM Patel, P Ghamarian, R Youssef Yaacoub, RV Clayman, J Landman

University of California, Irvine

Introduction \& Objective: The objective of this study was to determine if the use of an automated irrigation pump (AIP) during ureteroscopy (URS) and percutaneous nephrolithotomy (PCNL) improves procedural efficiency and alters surgeon and nurse satisfaction vs. a manual hand pump (HP) irrigation.

Methods: Eighty adult patients undergoing URS $(n=51)$ or PCNL $(n=29)$ for unilateral stone disease were randomized to HP or AIP irrigation. Preoperative pump setup time, intraoperative pump maintenance time, and the number of irrigation-related complaints verbalized by the surgeon intraoperatively were recorded by an independent observer; postoperatively, surgeons and nurses rated their satisfaction with the irrigation system ( $1=$ highly dissatisfied to $10=$ highly satisfied $)$. Results: For patients undergoing URS, body mass index (BMI) and the proportion of patients with $>1$ stone were higher in the HP group (both $\mathrm{p}=0.04$ ). On univariate analysis, AIP resulted in reduced pump setup and maintenance time for both URS and PCNL $(\mathrm{p}<0.001)$ (Table 1). Irrigation-related complaints were lower in the AIP group during URS (1.2 vs. 2.8, p<0.001) and trended to be lower during PCNL (1.9 vs. 4.0, $\mathrm{p}=0.07$ ). Surgeon satisfaction trended towards a significantly improved rating when AIP was used during URS (8.8 vs. $7.9, \mathrm{p}=0.07)$ and PCNL

\begin{tabular}{|c|c|c|c|c|c|c|}
\hline \multirow[b]{2}{*}{ Outcome } & \multicolumn{3}{|c|}{ Ureteroscopy } & \multicolumn{3}{|c|}{ Percutaneous Nephrolithotomy } \\
\hline & $\underset{(n=26)}{H P}$ & $\underset{(\mathrm{n}=25)}{\operatorname{AIP}}$ & $\mathbf{p}^{*}$ & $\underset{(n=15)}{H P}$ & $\underset{(\mathrm{n}=14)}{\operatorname{AIP}}$ & $\mathbf{p}^{*}$ \\
\hline $\begin{array}{l}\text { Pump setup time } \\
\text { (min) }\end{array}$ & $3.7 \pm 1.1$ & $2.3 \pm 1.2$ & $<0.001$ & $9.8 \pm 4.0$ & $2.2 \pm 1.0$ & $<0.001$ \\
\hline $\begin{array}{l}\text { Pump maintenance } \\
\text { time (min) }\end{array}$ & $2.3 \pm 2.3$ & $0.6 \pm 0.5$ & $<0.001$ & $24.0 \pm 29.9$ & $2.5 \pm 1.6$ & $<0.001$ \\
\hline $\begin{array}{l}\text { Intraoperative } \\
\text { irrigation-related } \\
\text { complaints }\end{array}$ & $2.8 \pm 2.1$ & $1.2 \pm 1.8$ & $<0.001$ & $4.0 \pm 3.3$ & $1.9 \pm 1.2$ & 0.07 \\
\hline $\begin{array}{l}\text { Surgeon } \\
\text { satisfaction" }\end{array}$ & $7.9 \pm 2.3$ & $8.8 \pm 2.0$ & 0.07 & $7.3 \pm 2.7$ & $8.9 \pm 2.4$ & 0.05 \\
\hline Nurse satisfaction" & $6.5 \pm 2.9$ & $9.2 \pm 1.3$ & $<0.001$ & $4.4 \pm 3.5$ & $9.4 \pm 1.0$ & 0.001 \\
\hline
\end{tabular}

Table 2. Primary outcomes for patients undergoing ureteroscopy; multivariate analysis

\begin{tabular}{|c|c|c|c|c|}
\hline & \multicolumn{2}{|c|}{ Ureteroscopy } & \multirow[b]{2}{*}{ Estimated difference (B) $(95 \%$ CI) } & \multirow[b]{2}{*}{$\mathrm{p}^{*}$} \\
\hline Outcome $e^{2}$ & $\underset{(\mathrm{n}=26)}{\operatorname{HP}}$ & $\underset{(\mathrm{n}=25)}{\mathrm{AIP}}$ & & \\
\hline \multirow[t]{2}{*}{ Pump setup time (min) } & $3.7 \pm 1.1$ & $2.3 \pm 1.2$ & $-1.67(-2.4--1.0)$ & $<0.001$ \\
\hline & \multicolumn{2}{|c|}{ Ureteroscopy } & & \\
\hline Outcome ${ }^{b}$ & $\underset{(\mathrm{n}=26)}{\mathrm{HP}}$ & $\underset{(\mathrm{n}=25)}{\text { AIP }}$ & Estimated OR $(95 \% \mathrm{CI})$ & $\mathrm{p}^{*}$ \\
\hline $\begin{array}{l}\text { Pump maintenance time } \\
<1 \mathrm{~min} \\
>1 \mathrm{~min}\end{array}$ & $\begin{array}{c}7(26.9 \%) \\
19(73.1 \%)\end{array}$ & $\begin{array}{c}20(80.0 \%) \\
5(20.0 \%)\end{array}$ & $15.8(1.2-4.3)$ & $<0.001$ \\
\hline $\begin{array}{l}\text { Intraoperative irrigation- } \\
\text { related complaints } \\
\text { None } \\
1 \text { or more }\end{array}$ & $\begin{array}{c}2(7.6 \%) \\
24(92.4 \%)\end{array}$ & $\begin{array}{l}11(44.0 \%) \\
14(56.0 \%)\end{array}$ & $6.9(0.2-3.7)$ & 0.03 \\
\hline $\begin{array}{l}\text { Surgeon satisfaction" } \\
\text { Favorable }(8-10) \\
\text { Unfavorable }(0-7)\end{array}$ & $\begin{array}{c}19(73.1 \%) \\
7(26.9 \%)\end{array}$ & $\begin{array}{c}20(80.0 \%) \\
5(20.0 \%)\end{array}$ & $1.6(-1.0-1.9)$ & 0.6 \\
\hline $\begin{array}{c}\text { Nurse satisfaction" } \\
\text { Favorable (8-10) } \\
\text { Unfavorable (0-7) }\end{array}$ & $\begin{array}{l}11(42.3 \%) \\
15(57.7 \%)\end{array}$ & $\begin{array}{c}23(92.0 \%) \\
2(8.0 \%)\end{array}$ & $25.4(1.4-5.1)$ & $<0.001$ \\
\hline $\begin{array}{l}\text { HP = hand pump irrigation; } \\
\text { "Linear regression used for at } \\
\text { "Logistic regression used for } \\
\text { "alpha }<0.01 \text { considered stat } \\
\text { of } 5 \text { outcome variables } \\
\text { "Irrigation satisfaction scorin }\end{array}$ & $\begin{array}{l}\text { automated } \\
\text { is (normally } \\
\text { ysis (data not } \\
\text { ally significa }\end{array}$ & $\begin{array}{l}\text { pp irrigation; } \\
\text { tributed data) } \\
\text { rmally distrib } \\
\text { ffter adjustme }\end{array}$ & $\begin{array}{l}\mathrm{R}=\text { odds ratio: } \mathrm{Cl} \text { = confidence interv } \\
\text { thed) } \\
\text { th with Bonferroni correction given com } \\
\text { ly satisfied }\end{array}$ & parison \\
\hline
\end{tabular}

( 8.9 vs. $7.3, p=0.05$ ), while AIP was associated with significantly higher nurse satisfaction during both URS (9.2 vs. 6.5) and PCNL (9.4 vs. 4.4) (both $\mathrm{p} \leq 0.001)$. On multivariate analysis of URS cases, controlling for BMI and number of stones, use of the AIP had decreased setup time $(B=-1.67,95 \%$ CI $-2.4-1.0$; $\mathrm{p}<0.001$ ), reduced maintenance time $<1$ minute (OR 15.8, 95\% CI $1.2-4.3 ; \mathrm{p}<0.001)$, and more favorable (8-10) nurse satisfaction rating (OR 25.4, 95\% CI 1.4-5.1; p<0.001) (Table 2).

Conclusions: During URS and PCNL, AIP was associated with reduced pump setup time, less nurse maintenance time and higher nurse satisfaction compared to standard HP irrigation.

\section{MP21-08 The Safety of Ureteral Stenting with The Use of Potassium Citrate for Management of Renal Uric Acid Stones}

M El Shazly, N Alenezi, F Zanaty, E El Sherif, A Badawy, M Elgharabawy

Introduction \& Objective: To evaluate the relative risks of ureteric stents application while managing Uric acid stones with potassium citrate in terms of stone encrustations and urinary tract infection (UTI).

Methods: We prospectively enrolled patients with renal uric acid stones who received potassium citrate from 2013-2018. Patients demographics were collected. All patients were evaluated using non-contrast CT scan to measure the stone size and density. JJ ureteric stent was inserted prior to the initiation of treatment. At follow up, all patients underwent urine analysis for $\mathrm{pH}$ and to detect UTI. CT was repeated at one month and those patients who showed incomplete stone resolution underwent another course of treatment for another month. CT was repeated prior to stent removal. The presence of encrustations was inspected and collected.

Results: We collected 59 patients with a median age of 36 years (18-73) and median stone burden of $26 \mathrm{~mm}^{3}$ (15-50). The median stone density was $310 \mathrm{HU}(175-498)$. Twenty-one patients 


\begin{tabular}{|l|l|c|}
\hline \multicolumn{2}{|c|}{ Parameter } & Findings \\
\hline \multicolumn{2}{|l|}{ Stone burden after treatment median (range) } & $6 \mathrm{~mm}^{3}(0-32)$ \\
\hline \multirow{2}{*}{ Duration of treatment } & One-month $n(\%)$ & 21 patients (35.6) \\
\cline { 2 - 3 } & Two months $n(\%)$ & 38 patients (64.4) \\
\hline \multirow{2}{*}{$\%$ decrease of stone burden median (range) } & $77.4 \%(25.6-100)$ \\
\hline \multirow{2}{*}{ Response to treatment } & $\mathbf{1 0 0 \% n ( \% )}$ & $16(27.1)$ \\
\cline { 2 - 3 } & $\mathbf{5 0 - 9 9 \% n ( \% )}$ & $41(69.5)$ \\
\cline { 2 - 3 } & $<\mathbf{5 0} \% n(\%)$ & $2(3.4)$ \\
\hline Incrustation $n(\%)$ & & $2(3.4)$ \\
\hline UTI $n(\%)$ & & $4(6.8)$ \\
\hline
\end{tabular}

Shows post-K citrate management outcomes.

$(35.6 \%)$ received $\mathrm{K}$ citrate treatment for one-month while the remaining patients had 2 months treatment. Sixteen patients $(27.1 \%)$ had a complete stone dissolution, 41 patients $(69.5 \%)$ had more than $50 \%$ decrease of stone burden while only 2 patients $(3.4 \%)$ had stones with poor dissolution. Four patients $(6.8 \%)$ experienced UTI while 2 patients $(3.4 \%)$ had visible JJ encrustations. Most of these complications occurred when the treatment was offered for a second month.

Conclusions: Short-term use of ureteral stents is safe during management of uric acid stones with potassium citrate

\section{MP21-09 The Japanese linguistic validation of the Ureteral Stent Symptoms Questionnaire (USSQ).}

J Matsuzaki, T Matsuda, A Yamaguchi, S Mugiya, K Wada, J Miyazaki, S Yamamoto

Department of Urology, Ohguchi Higashi General Hospital, The section meeting for ureteral stent in Japanese Society of Endourology

Introduction \& Objective: We validated the Japanese version of the Ureteral Stent Symptoms Questionnaire (USSQ) in patients with an indwelling ureteric stent.

Methods: The English version of the USSQ was translated into Japanese using a multi-step process by 3 urologists and 2 independent translators. A total of 70 patients with indwelling ureteral stents completed the Japanese USSQ as well as the validated questionnaires International Prostate Symptom Score (IPSS), Overactive Bladder Symptom Score (OABSS), and Euro-QOL score (EQ-5D) at 2weeks after stent insertion and 4 weeks after stent removal. In addition, 87 healthy subjects as a control group completed the same questionnaires. The psychometric properties of the questionnaires were compared and analyzed between the patients with stent inserted and those after stent removal, as well as between the patients and the control groups.

Results: Between the control and patient groups, IPSS, OABSS and USSQ domains for urinary symptoms showed significant differences, while domains for general health and work performance showed significant but moderate differences. In contrast, USSQ domains for body pain of USSQ did not significantly change between the two groups. Further, similar observations were obtained between the patients with stent inserted and after stent removal.

Conclusions: The Japanese version of USSQ proved to be a reliable and robust instrument for the evaluation of ureteral stentassociated morbidity for both male and female patients. It is expected to be a valid outcome measure in the future stent research.

\section{MP21-10 Ureteric stent with extraction string - strategies to reduce complications}

\author{
S Mukherjee, C Popanes, A Raza \\ Dartford and Gravesham NHS Trust
}

Introduction \& Objective: A ureteric stent with extraction string eliminates the need for cystoscopy assisted stent removal and reduces the duration of stent symptoms as stents can be removed sooner. We present suggestions from our experience which could potentially reduce morbidity associated with this stent with extraction string.

Methods: We prospectively collected data of patients who had ureteric stent with extraction string in our trust between April 2018 and March 2019. We audited our initial data after four months and compared this to our re audit data of new cases over the next 8 months. We also devised a protocol to identify appropriate cases for insertion of such stent, formulated a safe stent removal pathway, set up a nurse lead clinic for stent removal, increased awareness about this stent amongst the nurses and health care assistants, and finally created a patient information leaflet.

Results: In the first audit cycle over four months $(n=10)$ the morbidity rate was high $(50 \%)$ with accidental stent dislodgement in three cases, string retraction in one case and pulling out of the string by a recovery nurse in error in one case. In the re-audit over the next eight months $(n=16)$ the outcome was better with only one accidental stent dislodgement $(6 \%)$. Over the total 12 -month period 4 out of 26 patients (15\%) in total had stent dislodgement - three with thigh fixation and one with pubic fixation. Our complication rate was reduced by $44 \%$.

Conclusions: Careful case selection, proper string fixation with suprapubic fixation in female patients rather than thigh fixation, a defined stent removal pathway and increased awareness amongst the theatre and ward staff can considerably reduce the complications associated with stent with extraction string.

\section{MP21-11 The Utility of Instruments and Disposables during Endourologic Practice among Egyptian Urol- ogists}

M Omar, K Sayedahmed, YA Noureldin

Menoufia University

Introduction \& Objective: Our aim was to investigate the prevalence of utilization of different endourological instruments and disposables among Egyptian urologists and see how availability could affect the deviation from the universal standards that might result in reduced patient safety.

Methods: We surveyed members of the Egyptian Urological Association (EUA) by a questionnaire including different demographic and practice characteristics with concern regarding the importance of different instruments and disposables used during ureteroscopy (URS) and percutaneous nephrolithotomy (PCNL). Also, we surveyed them regarding the incidence of different endourological complications in their work environment. All responses were collected by a commercially available Internet-based survey host www.surveymonkey.com over a period of 8 weeks.

Results: One hundred and fifty-two responses were received. All respondents $(100 \%)$ perform ureteroscopy and $79 \%$ perform 
PCNL. For ureteroscopy, the most mandatory instruments and disposables included the C-arm device (83\%), dormia basket $(61 \%)$, ureteral stone forceps $(58 \%)$, pneumatic lithotripter $(50 \%)$ and regular PTFE guide wire (49\%). The instruments and disposables described as optional included 4.5 Fr ureteroscope (74\%), flexible ureteroscope (70\%) and hydrophilic guidewire $(67 \%)$. For PCNL, , the most mandatory instruments and disposables were pneumatic lithotripter (78\%), Alken metal dilator (75\%), Fascial dilator (45\%) and regular PTFE guidewire (42\%); while instruments and disposables described as optional included flexible cystoscope (70\%), hydrophilic guidewire (67\%), balloon dilator $(57 \%)$ and laser machine $(52 \%)$. An incidence of more than $3 \%$ complications rate were reported for ureteral perforation, post ureteroscopy and post PCNL sepsis by 34\%, 34\% and $27 \%$ of survey respondents respectively.

Conclusions: The prevalence of utilization of newly introduced instruments and disposables which are considered for the universal standard of care is low among Egyptian urologists. This might result in a deviation from the universal standards of care in endourologic interventions which might affect the patient safety in terms of decreasing the success rate or increasing the risk for complications.

MP21-12 Randomized clinical trial on urinary pH monitoring and nutraceutical intervention in the prevention of ureteral stent incrustation.

J Fernández-Concha Schwalb, C Torrecilla Ortiz,

J Ramón Cansino Alcaide, J Antonio Mainez,

J H. Amón Sesmero, S Costas Ochoa, O Angerri Feu,

E Emiliani, M Arrabal Martin, M A. Arrabal Polo,

A García Romero, M Carlos Reina Ruiz,

J Francisco Sánchez García, A Budía Alba, D Pérez-Fuentes,

F Grases Freixedas, A Costa-Bauzá, J Cuñé Castellana

La Fe University and Polytechnic Hospital

Introduction \& Objective: The use of double $\mathrm{J}$ ureteral stents is widely extended in the management of urolithiasis. A potentially severe complication associated with its use is incrustation, which is known to be related to urinary $\mathrm{pH}$. The main objective was to evaluate the efficacy of a medical device and a nutraceutical (NC) in the control of urinary $\mathrm{pH}$ and prevention of ureteral stent incrustation.

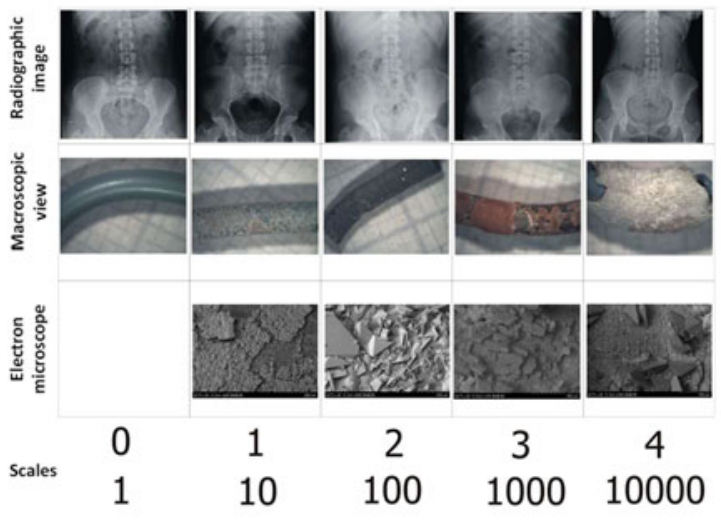

Methods: A multicenter, controlled, double blind and randomized clinical trial was conducted in 9 hospitals throughout Spain. Main inclusion criteria were: patients with a recent (less than a week) double $\mathrm{J}$ ureteral stent implantation with an expected indwelling catheter time of 3 to 8 weeks. Exclusion criteria were: patients with radiolucent stones or known acidic stone formers. Intervention arm consisted in tid oral administration of $\mathrm{NC}$ and control arm in oral administration of placebo tid and daily selfmonitoring of urinary $\mathrm{pH}$. The $\mathrm{NC}$ combines a urine acidifier (Lmethionine) and crystallization inhibitors. Renal and vesical ends of the stents were analyzed separately $(n=198)$. Ordinal (calcification degree 0-IV) and the equivalent exponential scales were employed due to the asymptotic behavior of the calcification process (analytically confirmed in terms of calcium concentration). The change in urinary $\mathrm{pH}$ was studied by comparing the mean value of the first 3 days with the $\mathrm{pH}$ at day 21 (minimum stent indwelling period for inclusion).

Results: No differences in type of stent and relative percentage of use among them were detected between groups. Three adverse reactions were described in each group, none of them severe or with a clear relation with the treatment received.

Conclusions: Urine acidification plus crystallization inhibitors in patients indwelling a double $\mathrm{J}$ ureteral stent significantly decreases incrustation rate (OR 8.8), reducing the need for additional surgical maneuvers as well as risk for the patient.

Table 1. Demographics and samples characteristics

\begin{tabular}{|c|c|c|c|}
\hline & Experimental & Control & \\
\hline & Mean (SD) & Mean (SD) & $\mathbf{p}$ \\
\hline $\mathbf{n}$ & 53 & 52 & $\mathrm{~ns}$ \\
\hline Age & $51.7(13.3)$ & $51.5(13.2)$ & $\mathrm{ns}$ \\
\hline BMI & $28.2(6.1)$ & $27.4(4.9)$ & $\mathrm{ns}$ \\
\hline Sex (male) & $\%$ & $\%$ & $\mathrm{~ns}$ \\
\hline $\begin{array}{c}\text { Previous obstructive } \\
\text { uropathy }\end{array}$ & 36.6 & 53.8 & $\mathrm{~ns}$ \\
\hline & 39.6 & 36.5 & \\
\hline Indwelling period & 35.42 & 39.65 & $\mathrm{~ns}$ \\
\hline
\end{tabular}

Table 2. Comparative analysis of the incrustation, $\mathrm{pH}$ reductions between groups.

\begin{tabular}{|c|c|c|c|c|}
\hline & Experimental & Control & \multicolumn{2}{|c|}{} \\
\hline & $\begin{array}{c}\text { Double J } \\
\text { Ends }\end{array}$ & Double J Ends & p & (OR) \\
\hline $\begin{array}{c}\text { Grade IV } \\
\text { calcifications }\end{array}$ & 1 & 8 & 0.018 & 8.8 \\
\hline $\begin{array}{c}\text { Degree of incrustation } \\
\text { (exponential) }\end{array}$ & $\begin{array}{c}187.57 \\
(1022.96)\end{array}$ & $847.92(2746.33)$ & 0.02 & $0.32(0.04-0.06)$ \\
\hline $\begin{array}{c}\text { pH reduction: } \\
\text { days 1-3 vs. mean pH }\end{array}$ & $0.48(0.44)$ & $0.2(0.32)$ & 0.002 & $0.85(0.39-1.31)$ \\
\hline $\begin{array}{c}\text { pH reduction: } \\
\text { days 1-3 vs. day 21 }\end{array}$ & $0.65(0.58)$ & $0.37(0.55)$ & 0.018 & $0.33(0.0-0.81)$ \\
\hline
\end{tabular}

MP21-13 Impact of Ureteral Access Sheath on Renal Stone Treatment: Prospective Comparative NonRandomized Outcomes over a 7-year Period

A Lima, T Reeves, R Geraghty, A Pietropaolo, L Whitehurst, BK Somani, T Hughes

University Hospital Southampton

Introduction \& Objective: The use of ureteric access sheaths (UAS) for treatment of renal stones has risen over the last decade. However, questions still remain regarding the safety and clinical 


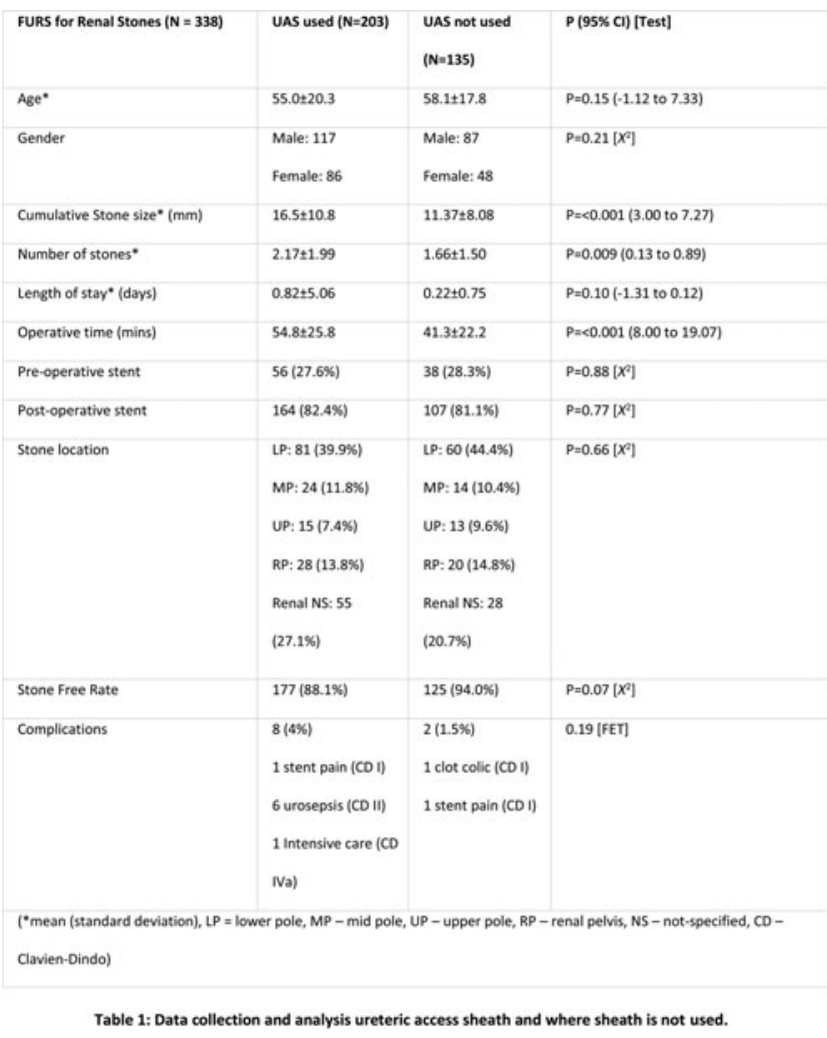

outcomes with use of access sheaths. This study looks at the role of UAS for ureteroscopic treatment of consecutive renal stones over a period of 7-years at a specialized center.

Methods: Outcomes of flexible ureteroscopy and stone treatment (FURS) for renal stones with and without the use of UAS was prospectively compared from March 2012 to July 2018. Patients were divided into two groups for stone treatment; group1 (UAS use) and group-2 (no UAS use. Data was collected prospectively on consecutive patients including demographics, stone size, location and number of stones, pre and post-operative stent usage, operative time duration, stone free rate (SFR), length of stay and complications.

Results: During the study period 338 patients underwent FURS for renal stones, of which a UAS was used for $203(60 \%)$ patients. The mean age of patients was 56 years (range $2-89$ years) with a male: female ratio of 204:134. The mean cumulative stone size and the mean number of stones were $16.5 \pm 10.8 \mathrm{~mm}$ and $11.37 \pm 8.08 \mathrm{~mm}(\mathrm{P}<0.001)$, and $2.17 \pm 1.99$ and $1.66 \pm 1.50$ $(\mathrm{P}=0.009)$ for groups 1 and 2 respectively. The pre and postoperative stent insertion rates were similar in both groups. Procedural time was longer in group-1 $(54.8 \pm 25.8$ minutes $)$ compared to group-2 $(41.3 \pm 22.2$ minutes $)(\mathrm{P}<0.001)$. The SFR for group-1 (88\%) was reduced in comparison to group-2 $(94 \%)$, but not statistically significant $(\mathrm{p}=0.07)$. There were no intra-operative complications in either group. Postoperative complications were seen in 8 patients in group-1 (7 Clavien I/II; stent pain/urinary tract infections and 1 Clavien IVa; urosepsis requiring intensive care management) and 2 patients in group-2 (Clavien I; clot retention, stent pain) $(\mathrm{P}=0.19)$.

Conclusions: The use of UAS for renal stones is safe with no intra-operative complications noted in our series. Good stone free rates were obtained for large and multiple renal stones with a small risk of minor complications post-operatively.

\section{MP21-14 PCNL Tract Dilation Efficiency - Balloon vs. Sequential Dilators?}

D Mikhail, V Vasudevan, A Rai, A Perez-Orozco, S Patwa, D Hoenig, AD Smith, Z Okeke

Introduction \& Objective: Percutaneous Nephrolithotomy (PCNL) continues to be the preferred treatment for large kidney stones - with mini-PCNL becoming more common in the endourology community. As the technology, indications \& number of PCNL procedures performed continue to increase - rising costs must be considered. The CROES study showed that North American urologists mostly used balloon dilators as opposed to sequential dilators used in the rest of the world. Balloon dilation is believed to be easier and faster, however $11 \%$ more in cost and only a single use vs. multi-use device. With ongoing controversy in the literature about the benefits of balloon dilation as compared to sequential dilation, we did a pilot study to assess whether the balloon dilators showed intra-operative benefit over sequential dilators.

Methods: We are a high-volume center with a large referral base \& complex patient population. We assessed 30 adult patients requiring PCNL. Patients with a nephrostomy tube were excluded. PCNL was performed by 2 fellowship trained endourologists using fluoroscopic access \& establishment of wire access prior dilation. One surgeon used a balloon dilator (XForce 30f Dilator, Bard), while the other chose sequential (Amplatz, Cook) or balloon dilator. We recorded dilation time from 1st instrument touching wire tip until last instrument removed from wire. Factors such as primary dilator \& technique variations were recorded. If multiple dilations or tracts were performed, only the first dilation was recorded. Statistics were performed using Excel 2013 (Microsoft).

Results: In our 30 patients - a total of 21 (15 balloon, 6 sequential) had full intra-operative data. Patients were mostly male $(67 \%)$ with a median age of $58( \pm 15)$ years \& average BMI of 33 $( \pm 8.5) \mathrm{kg} / \mathrm{m}^{2}$. Patients with sequential dilation had higher average BMI (35 vs. 32). The average time for balloon dilation was significantly lower than sequential dilation by about 90 seconds $(136 \pm 65$ vs. $226 \pm 68$ seconds, $p=0.02)$. Whether the primary dilator was a trainee $(n=13)$ or surgeon $(n=8)$ did not statistically effect dilation time $(\mathrm{p}=0.18)$, although surgeons averaged 51 seconds less. Each group had 4 patients that required multiple dilations or tracts - much easier to perform with sequential dilators. Conclusions: Our pilot cohort found a benefit of 90 seconds of balloon over sequential dilators - a benefit we predict would be less without trainees. However, single-use balloon dilators lack versatility: single size (30f) \& significant added cost, especially with multiple dilations or tracts. Given a minor time benefit \& increased costs with balloons - our data supports high volume centers with complex patients to use sequential dilators.

\section{MP21-15 Does ureteral balloon dilatation for ureteral access sheath (UAS) placement during retrograde in- trarenal surgery (RIRS) increase risk of de novo ure- teral strictures? : a retrospective comparative study}

J Choi, S Jeon, M Baek, H Jeon, H Sung, M Kang, D Han

Samsung Medical Center, Sungkyunkwan University School of Medicine, Seoul, Korea

Introduction \& Objective: The purpose of this study was to investigate the effect of ureteral balloon dilatation for facilitating ureteral access sheath (UAS) insertion during retrograde 


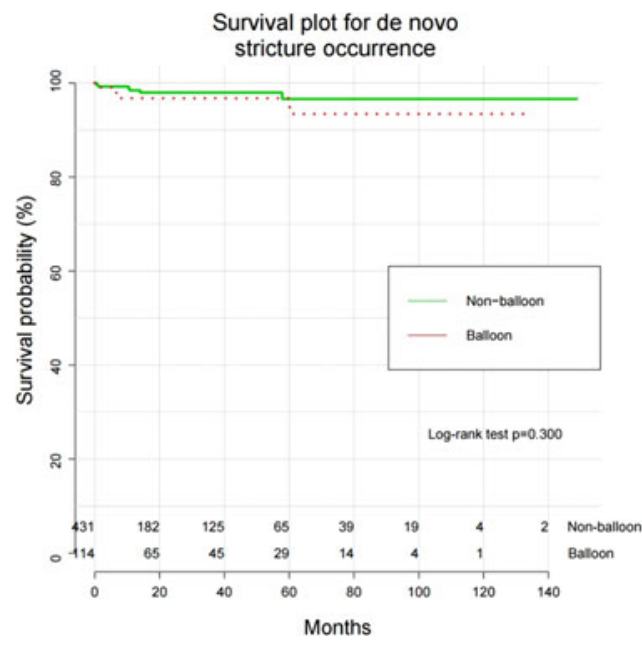

Table 1 Perioperative safety outcomes.

\begin{tabular}{lccc}
\hline Characteristics, n (\%) & $\begin{array}{c}\text { Non-balloon } \\
(\mathrm{UUs}=435)\end{array}$ & $\begin{array}{c}\text { Balloon } \\
(\mathrm{UUS}=114)\end{array}$ & $P$ value \\
\hline Follow up duration, months (mean $\pm \mathrm{SD})$ & $29.0 \pm 32.0$ & $37.4 \pm 32.6$ & 0.013 \\
Operation time, min (median, IQR) & $73.0(51.0-101.5)$ & $77.0(55.0-96.5)$ & 0.368 \\
Ureteral access sheath, n (\%) & $435(100.0)$ & $114(100.0)$ & 1.000 \\
Hospital stay, day (median, IQR) & $2.80(1.88-3.85)$ & $2.84(1.99-3.90)$ & 0.197 \\
D-J maintenance period, day (median, IQR) & $16.0(13.0-20.0)$ & $19.0(15.0-29.0)$ & 0.004 \\
Unexpected visit (ER or OPD) & $15(3.4)$ & $9(7.9)$ & 0.039 \\
Perioperative Complications & & & \\
\multicolumn{1}{c}{ Acute pyelonephritis ${ }^{\dagger}$} & $10(2.3)$ & $2(1.8)$ & 0.719 \\
$\quad$ Transfusion & $6(1.4)$ & $2(1.8)$ & 0.771 \\
$\quad$ Prolonged urine leakage & $0(0)$ & $0(0)$ & \\
De novo ureteral stricture & $7(1.6)$ & $4(3.5)$ & 0.198 \\
\hline
\end{tabular}

UUs, ureter units; PCN, percutaneous nephrostomy; D-J, double-J stent; ER, emergency room; OPD, outpatient clinic. "Within 1 weeks after surgery. ${ }^{\dagger}$ Over $38.0 \mathrm{C}$ with pyuria within 2 weeks after surgery.

intrarenal surgery (RIRS) on the occurrence of de novo ureteral stricture.

Methods: Patients who underwent RIRS using UAS for renal stones between June 2003 and August 2018 were included in this study. Patients were excluded if they had a history of ureteral stricture and/or upper urinary tract malignancy. Patients who had accompanying ureteral stone and/or ureteral stricture during RIRS were also excluded. The rates of de novo ureteral stricture were compared between patients who needed balloon dilatation during RIRS and those who did not. All patients were followed up by diagnostic imaging including CT and sonography. Suspected cases of post-operative ureteral stricture on imaging study were verified by diuretic renography or retrograde urography.

Results: A total of 549 ureter units (UUs) from 479 patients were included in this study, among which 114 (20.8\%) underwent balloon dilatation to facilitate UAS placement. With respect to basic clinical characteristics, there was no difference between the balloon dilatation and non-balloon dilatation groups except age. There was no difference in perioperative outcomes between the two groups except D-J stent maintenance period that was longer in the balloon group (16.0 and 19.0 days, $P<0.001)$. There was no statistically significant difference in the rate of de novo ureteral stricture $(1.5 \%$ and $3.4 \%$, $P=0.177)$. Other complications including acute pyelonephritis, prolonged urine leakage, and transfusion were not different between the two groups.

Conclusions: Balloon dilatation during RIRS to facilitate UAS placement did not adversely affect the incidence of de novo stricture. Balloon dilatation can be considered a safe and feasible option for RIRS.

\section{MP21-16 Holmium Laser versus thulium fiber laser for treatment of urinary calculus disease.}

PK Pattnaik, SK Pattnaik, MP Pattnaik

S S Urological \& Research Institute, Bombay Hospital PG Institute

Introduction \& Objective: Aim: To compare the fragmentation, dusting and retropulsion during laser lithotripsy using Holmium v/s Thulium fiber Laser system Introduction: Holmium:YAG laser operates at high pulse energy and low pulse rates during lithotripsy. On the other hand, the thulium fiber laser can effectively operate high pulse rate and low pulse energy. When used for lithotripsy of ureteric calculi, the retropulsion can be minimized at pulse rates less than $150 \mathrm{~Hz}$ but rapidly increased at higher pulse rate. Minimal retropulsion requires pulse energy less than $150 \mathrm{mj}$ at $8-10 \mathrm{~Hz}$. Thulium fiber laser operates at 5$35 \mathrm{mj}$ of pulse energy with a pulse duration of 300-500 micro seconds and pulse rate of $50-300 \mathrm{~Hz}$. Retropulsion with Holmium:YAG Laser linearly increases with pulse energy whereas with Thulium fiber Laser is minimal at pulse rate less than $100 \mathrm{~Hz}$ and rapidly increases with higher pulse rate. Dusting mode requires low pulse energy ( 0.2 to 0.5 Joules) and higher pulse rate (50-80Hz).

Methods: In this study we compared the fragmentation, dusting and retropulsion during laser lithotripsy using Holmium v/s Thulium fiber Laser system. We included 50 patents with ureteric and Renal calculi up to 2 centimeters and comparable stone density (1000 - 1400 Hounsfield Units) in this study after taking written, informed consent. The patients were divided into two Groups, Group A (Holmium Laser Lithotripsy) and Group B (Thulium fiber Laser Lithotripsy). Fragmentation settings in Group A (Holmium Group) was Pulse rate ranging from $10 \mathrm{~Hz}-$ $150 \mathrm{~Hz}$ and Pulse energy less than $150 \mathrm{~mJ}$. Fragmentation settings in Group B (Thulium Fiber Laser Group) was Pulse energy ranging from $5-35 \mathrm{~mJ}$, pulse rate $10-500 \mathrm{~Hz}$ and Pulse duration of 500 micro seconds.

Results: In Group A (Holmium Laser) the dusting and fragmentation rate was slower ( $15.5 \pm 5.5$ minutes $)$ and calculus fragment retropulsion was more. In Group B (TM Fiber Laser) the dusting and fragmentation rate was faster $(7.5 \pm 2.5$ minutes $)$, fragment retropulsion was negligible and dusting was better.

Conclusions: We conclude that TM Fiber Laser is a better modality for urinary calculus Lithotripsy since its capable of faster dusting and fragmentation, negligible fragment retropulsion.

MP21-17 JJ stent attached to a string - reasons for low uptake by urologists despite benefits to patients and improved utilization of hospital resources
S Mukherjee, C Popanes, A Raza
Dartford and Gravesham NHS Trust

Introduction \& Objective: A ureteric stent with extraction string obviates the need for cystoscopy assisted stent removal and thus avoids the potential complications of flexible 
cystoscopy, saves additional procedure costs and reduces the duration of stent symptoms as stents can be removed sooner than cystoscopic removal. However, uptake of this stent amongst urologists is low. We explored the concerns of the urologists regarding the use of a stent with extraction string.

Methods: All consultants and registrars in our department were requested to complete a questionnaire on stent with extraction string.

Results: 14 urologists completed the questionnaire, 8 (57\%) mentioned stent dislodgement as their main concern regarding the use of stent with extraction string. In male patients, $8(57 \%)$ urologists preferred penile fixation and $1(7 \%)$ preferred pubic fixation. However, in females the pubis was the preferred site of fixation (7 urologists, 50\%) followed by lateral aspect of thigh (3 urologists, 21\%). Regarding the likeliness of using this stent in appropriate cases (uncomplicated ureteroscopy where JJ stent can be removed within 7 days), only 4 (29\%) urologists scored $\geq 8$ in a scale of 10 ( 1 being very unlikely and 10 being very likely), however the rest $10(71 \%)$ urologists scored $\leq 6$ including $3(21 \%)$ urologists scoring $\leq 2.10(71 \%)$ urologists were prepared to change practice if convinced by further evidence scoring $\geq 7$ in a scale of 10 ( 1 being very unlikely and 10 being very likely).

Conclusions: The majority of urologists were concerned about stent dislodgement and therefore did not use a stent with extraction string despite the obvious benefits of decreased stent time, decreased potential risk to the patient through additional cystoscopy and decreased hospital costs. The variability of stent fixation technique with 3 urologists fixing to the thigh, with a higher risk of stent dislodgement, highlights the need for a formal fixation policy and standardization of technique. Increased education on the method of fixation and increased awareness of appropriate indications will lead to an increase in use in the future.

\section{MP21-18 Impact of the Ureteral Stent Diameter on Ureteral Stent-Related Symptoms.}

M Taguchi, K Yoshida, M Sugi, H Kinoshita, T Matsuda

Department of Urology, Osaka Saiseikai Izuo Hospital

Introduction \& Objective: To investigate the correlation between the ureteral stent diameter and stent-related symptoms.

Methods: We analyzed 71 patients who underwent ureteral stent placement before ureteroscopic lithotripsy (URSL) and in whom the ureteral stent tail was positioned inside the bladder without crossing the midline. The patients comprised 53 (74.6\%) men and $18(25.4 \%)$ women with a mean age of $59.3 \pm 14.2$ years. All stents were Inlay Optima stents, and the diameter $(6$ or $4.7 \mathrm{Fr})$ and length $(24$ or $26 \mathrm{~cm})$ were chosen at the surgeon's discretion. We classified the patients into two groups: Group 1=6-Fr stent and Group 2=4.7-Fr stent. We assessed urinary symptoms before insertion of the ureteral stents and the day before URSL using the International Prostate Symptom Score (IPSS) and the Overactive Bladder Symptom Score (OABSS). We also compared the patients' backgrounds and changes in the IPSS and OABSS. Furthermore, we investigated factors that affected ureteral stent-related symptoms using multivariate analysis.

Results: Compared with Group 2, Group 1 had a worse total IPSS $(p=0.02)$; IPSS subscores including the intermittency subscore $(p=0.009)$, urgency subscore $(p=0.008)$, voiding symptom subscore $(\mathrm{Q} 1+\mathrm{Q} 3+\mathrm{Q} 5+\mathrm{Q} 6, p=0.046)$, and storage symptom subscore (Q2+Q4+Q7, $p=0.017)$; total OABSS $(p=0.045)$; and OABSS urgency subscore $(p=0.002)$. In the multivariate analysis, the stent diameter was significantly associated with the total IPSS $(p=0.007)$ and OABSS $(p=0.036)$. Conclusions: This is the first study to show that ureteral stents with larger diameters induce significantly worse urinary symptoms. Ureteral stents with smaller diameters are recommended to improve ureteral stent-related symptoms.

\section{MP21-19 Temperature rise during Laser Lithotripsy: Comparison of Super Pulse Thulium Fiber LASER (SPTF) vs High Power 120W Holmium-YAG LASER (Ho:YAG)}

WR Molina, BH Chew, BE Knudsen

University of Kansas Medical Center

Introduction \& Objective: The holmium -YAG (Ho:YAG) Laser system is the current standard of care for laser lithotripsy (LL). Super Pulse Thulium Fiber Laser (SPTF) has emerged as an effective alternative. We compared the temperature profile of $120 \mathrm{~W}$ Ho:YAG laser system and a $60 \mathrm{~W}$ SPTF system during lithotripsy.

Methods: Anterograde semirigid ureteroscopy with LL was performed in ex-vivo porcine urinary tracts and with $3 \mathrm{~mm}$ Begostones. Room temperature (22C) saline was instilled using a hand pump for irrigation. Intraureteral temperature was measured using one probe proximal and one distal to the site of lithotripsy on the stone. LL was performed using a $200 \mathrm{~m}$ core fiber at dusting (SPTF- 0.1J, 200hz, SP; Ho:YAG- 0.3J, 80hz, LP) and fragmenting $(0.8 \mathrm{~J}, 8 \mathrm{~Hz}, \mathrm{SP}$ for both) settings for 5 secs. Fifteen repetitions were recorded for each laser at each setting. Tissue samples of the ureter for each setting and system at each site of lasering were collected for histological analysis.

Results: There was a temperature rise for both laser systems at both settings, but was greater at dusting settings; however, both systems remained below the threshold for cellular injury (42C). The temperatures were greater for the Ho:YAG on the dusting setting $(41.13 \mathrm{C}$ vs $40.46 \mathrm{C}, \mathrm{p}=0.011)$. On the fragmenting setting, the temperature was greater for the SPTF (31.87C vs $29.3 \mathrm{C}$, $\mathrm{p}=0.001$ ), Fig 1. Histological analysis did not show any signs of injury or necrosis in any of the tested settings.

Conclusions: Higher power settings used for dusting have a higher temperature rise in the ureter during lasering. Ureteral intra-luminal temperature rise during LL was more pronounced on the Ho:YAG during dusting and higher in the SPTF during fragmentation, but neither reached the threshold for thermal injury and are considered safe.

Average Maximum Temperature Measured by Thermocouples in Ex. Vivo Porcine Ureter

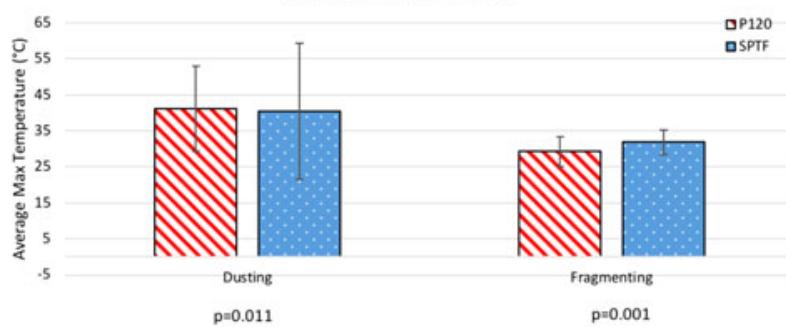


MP21-20 In Vitro Assessment of the Olympus URFP7 and V3 Flexible Ureteroscopes and Comparison to Prior Generation Scopes

RS Terry, B Winship, K Boydston, P Whelan, E Carlos, G Preminger, M Lipkin

Duke Urology

Introduction \& Objective: Olympus recently updated its portfolio of fiber-optic and digital flexible ureteroscopes with the addition of DuraBend ${ }^{\mathrm{TM}}$ technology to the distal scope shaft. DuraBend increases the length of the insertion-tube portion of the scope which is amenable to passive flexion and is designed to reduce mechanical stress and increase overall scope durability. We assessed the physical and optical characteristics of the new URF-P7 fiber-optic and URF-V3 digital flexible ureteroscopes relative to their prior generation counterparts, the P6 and V2.

Methods: The Olympus URF-P6, P7, V2, and V3 scopes were assessed in vitro for angle of maximum active tip deflection with an empty working channel, a $200 \mu \mathrm{m}$ laser fiber, a 1.9fr basket, and a $2.4 \mathrm{fr}$ basket. The diameter of the curl created during maximum deflection with an empty channel was also measured. Optical parameters including image resolution, distortion, depth of field, and color representation were additionally assessed. All tests were performed in duplicate. Outcomes between scopes (P6 vs P7 and V2 vs V3) were analyzed using student's $t$-test with statistical significance defined as $\mathrm{p}<0.05$.

Results: The P7 fiber-optic ureteroscope demonstrated significantly improved maximum deflection relative to the older P6 with an empty working channel $\left(281.2^{\circ}\right.$ vs $\left.260.7^{\circ}, \mathrm{p}=0.04\right)$, a $200 \mu \mathrm{m}$ fiber $\left(255.5^{\circ} \mathrm{vs} 236.3^{\circ}, \mathrm{p}=0.001\right)$, and a $2.4 \mathrm{fr}$ basket $\left(270.3^{\circ}\right.$ vs $\left.250.2^{\circ}, p=0.02\right)$. There was no difference between the scopes with a 1.9fr basket in the working channel. The P7 also demonstrated a significantly smaller curl with maximum deflection relative to the $\mathrm{P} 6$ ( 34.7 vs $37.9 \mathrm{~mm}, \mathrm{p}=0.03$ ). The V3 digital ureteroscope demonstrated significantly improved maximum deflection relative to the older V2 with a $1.9 \mathrm{fr}$ basket in the working channel $\left(259.3\right.$ vs $\left.256.9^{\circ} \mathrm{p}=0.01\right)$. The digital scopes demonstrated similar performance in all other physical measures. The V2 and V3 digital scopes demonstrated improved resolution, depth of field and color representation with reduced distortion relative to the P6 and P7 fiber-optic scopes. There was no significant difference in optical parameters between the older and newer generations of the same scope type.

Conclusions: Olympus recently introduced updates to both its fiber-optic and digital flexible ureteroscopes. The new URF-P7 and V3 scopes appear to offer some physical performance enhancements relative to the previous generation scopes without compromising optical performance.

MP21-21 Analysis of ureteral stents encrustation and biofilm formation after 3-week dwell time: Results of a Prospective Randomized Multicenter Clinical Comparative Study of Silicone Hydrocoated (Imajin ${ }^{\circledR}$ hydro) versus Percuflex ${ }^{\mathrm{TM}}$ Plus.

O Wiseman, J Letendre, J Cloutier, M Daudon, F Kleinklauss, S Doizi, O Traxer

Cambridge University Hospitals NHS Trust

Introduction \& Objective: Silicone was one of the first materials to be used for ureteral stents. Importantly, silicone has been
Table 1

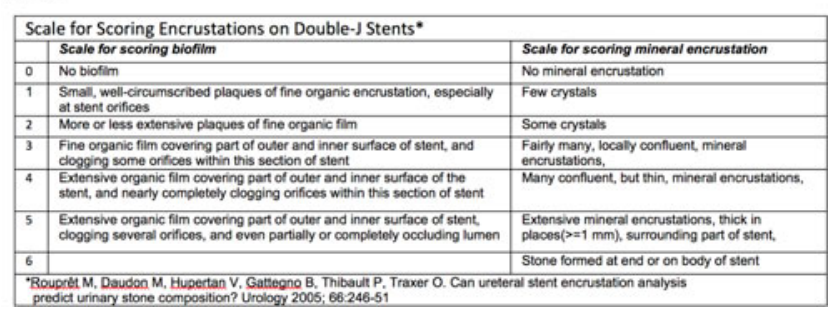

Table 2

\begin{tabular}{|c|c|c|c|c|c|}
\hline $\begin{array}{l}\text { Mineral } \\
\text { encrustation of } \\
\text { Double-J stents at } \\
\text { explantation }\end{array}$ & Ext renal tip & $\begin{array}{l}\text { Ext renal } \\
\text { angle }\end{array}$ & $\begin{array}{l}\text { Ext vesical } \\
\text { tip }\end{array}$ & $\begin{array}{l}\text { Ext vesical } \\
\text { angle }\end{array}$ & $\begin{array}{l}\text { Global Mineral } \\
\text { encrustation } \\
\text { (external \& interna } \\
\text { all sites) }\end{array}$ \\
\hline $\begin{array}{l}\text { Silicone Imajin } \\
\text { hydro } \\
\mathrm{N}=56\end{array}$ & $0.68 \pm 0.15$ & $0.87 \pm 0.13$ & $0.71 \pm 0.17$ & $1.09 \pm 0.17$ & $0.78 \pm 0.11$ \\
\hline $\begin{array}{l}\text { Percuflex plus } \\
N=63\end{array}$ & $1.19 \pm 0.14$ & $1.38 \pm 0.12$ & $1.68 \pm 0.16$ & $1.69 \pm 0.16$ & $1.22 \pm 0.10$ \\
\hline P & 0.0025 & 0.012 & 0.00006 & 0.0025 & 0.0048 \\
\hline $\begin{array}{l}\text { Biofilm level on } \\
\text { Double-J stents at } \\
\text { explantation }\end{array}$ & $\begin{array}{l}\text { Extern renal } \\
\text { tip }\end{array}$ & $\begin{array}{l}\text { Ext renal } \\
\text { angle }\end{array}$ & $\begin{array}{l}\text { Ext vesical } \\
\text { tip }\end{array}$ & $\begin{array}{l}\text { Ext vesical } \\
\text { angle }\end{array}$ & $\begin{array}{l}\text { Global Biofilm } \\
\text { (external } 8 \\
\text { internal all sites) }\end{array}$ \\
\hline $\begin{array}{l}\text { Silicone Imajin } \\
\text { hydro } \\
\mathrm{N}=56\end{array}$ & $0.91 \pm 0.12$ & $1.23 \pm 0.12$ & $0.66 \pm 0.12$ & $1.02 \pm 0.11$ & $0.93 \div 0.09$ \\
\hline $\begin{array}{l}\text { Percuflex plus } \\
\mathrm{N}=63\end{array}$ & $1.22 \pm 0.12$ & $1.49 \pm 0.11$ & $1.43 \pm 0.11$ & $1.71 \pm 0.11$ & $1.24 \pm 0.8$ \\
\hline P & 0.02 & 0.05 & 0.000001 & 0.00003 & 0.0021 \\
\hline
\end{tabular}

shown to be highly biocompatible and less prone to encrustation than polyurethane in a large series of removed ureteral stents. Since encrustation remains a significant concern when stents are implanted, we investigated these properties of silicone through a randomized multicenter study.

Methods: We performed a multicenter, prospective, randomized, single blind, comparative study of silicone hydrocoated ureteral stents versus PercuflexTM Plus, in patients treated by F-URS for a kidney stone. The studies had ethical committee approval in the respective hospitals. Primary endpoint was USSQ pain score after 3 -week dwell. Ureteral stent encrustation and biofilm formation at removal was evaluated through a scoring scale (Table 1). Comparison was performed using ANOVA.

Results: 119 stents were available after removal for analysis, 56 in the silicone and 63 in the Percuflex Plus group. Mean dwell duration was 21.8 days for silicone, 22.1 days for Percuflex Plus. Biofilm and mineral encrustations were evaluated through optical microscopy of inner and outer surfaces of tips, angles and along the stents body. There was significantly more mineral encrustation and biofilm (Table 2) on Percuflex Plus on both renal and vesical tips and angle surfaces compared to silicone. Mean global biofilm and mineral encrustation were also more significant on Percuflex Plus stents. Five silicone stents had no encrustation at all, compared to none stent for Percuflex Plus. Two silicone stents had neither biofilm nor encrustation (none for Percuflex Plus).

Conclusions: This multicenter randomized study evidences that silicone hydrocoated stents are less encrusted than PercuflexTM Plus after 3-week dwell time and confirms the low encrustation potential of silicone.

MP21-22 Comparison of Conventional 30, 120 Watt, and 120 Watt Moses Laser in a Benchtop Cystolitholapaxy Model

M Hajiha, A Amasyali, J Groegler, Z Campwala, J Smith, A Goyne, D Baldwin, R Moghisi, M Shah, M Alsyouf,

P Stokes, R Belay, D Baldwin 
Introduction \& Objective: Treating large bladder stones in a cost effective and minimally invasive manner can be challenging. A variety of laser machines and fibers are currently available to treat bladder stones. The aim of this study is to compare the performance and cost of a conventional $30 \mathrm{~W}$ and $120 \mathrm{~W}$ laser to a 120W Holmium laser with Moses technology in a benchtop cystolitholapaxy model.

Methods: In a benchtop cystolitholapaxy model, 15 identical $4 \mathrm{~cm}$ BegoStones were placed on a $5.3 \times 5.3 \mathrm{~mm}$ metal mesh in a 3D printed bladder model. Stones were fragmented using three different laser/fiber combinations: Lumenis Pulse $120 \mathrm{H}$ laser with $550 \mathrm{~mm}$ Moses D/F/L fiber at $4 \mathrm{~J}$ and $30 \mathrm{~Hz}$, Lumenis Pulse $120 \mathrm{H}$ laser with $550 \mathrm{~mm}$ SlimLine SIS laser fiber at $4 \mathrm{~J}$ and $30 \mathrm{~Hz}$, and a $30 \mathrm{~W}$ Dornier with $550 \mathrm{~mm}$ fiber at $2.5 \mathrm{~J}$ and $8 \mathrm{~Hz}$. Total costs were estimated by summing disposable fiber costs (Moses \$525/fiber, regular 120W \$425/fiber, and 30W \$330/ fiber) and operating room cost at $\$ 62 /$ minute. ANOVA was performed and subsequently, a Tukey's B post hoc was used to compare laser time, the time and number of laser strippings required, and initial and residual stone weights between groups.
Results: There was no difference in the initial stone weights between groups (mean $61.5 \mathrm{~g}, \mathrm{p}=0.867$ ). The Moses laser required $12.3 \%$ less fragmentation time compared to $120 \mathrm{~W}$ laser. The 30W laser required 3.6x longer fragmentation time compared to the Moses laser (Moses $=3651.80 \mathrm{sec}$ vs. $120 \mathrm{~W}=$ $4165.0 \mathrm{sec}$ and $30 \mathrm{~W}=16872.0 \mathrm{sec}, \mathrm{p}<0.001)$. The Moses group had less residual stone fragments $(10.76 \mathrm{~g})$ compared to the regular $120 \mathrm{~W}(19.10 \mathrm{~g}, \mathrm{p}<0.001)$ and the $30 \mathrm{~W}$ laser $(14.25 \mathrm{~g}$, $\mathrm{p}<0.001)$. The $120 \mathrm{~W}$ laser required more frequent stripping (mean 17.4) compared to the Moses $(6, \mathrm{p}<0.001)$ and $30 \mathrm{~W}$ laser $(7.8, \mathrm{p}<0.001)$. This resulted in longer surgical delays due to the additional stripping time $(120 \mathrm{~W}=1039.0 \mathrm{sec}$, Moses $=456.0$ and $30 \mathrm{~W}=690.0 \mathrm{sec}, \mathrm{p}<0.001)$. Using the $120 \mathrm{~W}$ Moses system resulted in a $\$ 430$ cost saving compared to regular $120 \mathrm{~W}$ and a $\$ 13466$ cost saving compared to the $30 \mathrm{~W}$ laser.

Conclusions: The Moses technology resulted in rapid and efficient bladder stone fragmentation and smaller fragments. The Moses system saved 8.5 minutes and $\$ 430$ /case compared to the regular 120W laser and 222 minutes and \$13469/case compared to the $30 \mathrm{~W}$ laser.

\section{MODERATED POSTER SESSION 22: CLINICAL STONES: MEDICAL MANAGEMENT}

\section{MP22-01 Urinary metabolic abnormalities in patients with pure vs. mixed uric acid stones}

RA Chandhoke, B Gallante, JN Bamberger, W Atallah, M Gupta, AM Zampini

\section{Mount Sinai}

Introduction \& Objective: Uric acid (UA) stones can occur in pure form or in combination with calcium oxalate $(\mathrm{CaOx})$. The association of $\mathrm{CaOx}$ with urinary metabolic abnormalities has been extensively investigated, however, little is known about the association between these metabolic disturbances and pure UA stones. We sought to better understand the differences between these patient populations by evaluating clinical and metabolic factors.

Methods: Data were prospectively obtained on patients with urinary tract calculi between 2012 and 2016. Patients were divided into two groups based on the degree of their UA stone composition: "pure UA stone formers" and "mixed UA stone formers." Variables compared between the two groups included the diagnosis of metabolic syndrome and 24-hour urine collection results.

Results: 92 patients with UA stones were identified. Pure UA stones were found in 26 patients and mixed UA stones in 66. Pure UA stone formers were clinically similar to those who formed mixed UA stones with no statistical difference in age, BMI, gender, total stone burden, stone location, and prior history of stones. Pure UA stone formers had a higher prevalence of metabolic syndrome (23.1\% versus $12.1 \%$ ) and diabetes $(42.3 \%$ versus $25.8 \%)$. When comparing metabolic profiles, the pure UA stone formers had significantly lower urine $\mathrm{pH}(5.43 \pm 0.39$ vs $5.72 \pm 0.58 ; \mathrm{p}=0.03)$,

\begin{tabular}{|c|c|c|c|c|}
\hline \multicolumn{2}{|c|}{ 24-hour Urine Results (Litholink) } & Pure UA Stones & Mixed UA Stones & \multirow[t]{2}{*}{ p-value } \\
\hline $\begin{array}{l}\text { Metabolic } \\
\text { Parameters }\end{array}$ & Normal Range & \multicolumn{2}{|c|}{ Mean ( \pm Std) } & \\
\hline Vol24 & & $1.98 \pm 0.68$ & $1.87 \pm 0.71$ & 0.52 \\
\hline SSCaOx & & $4.33 \pm 2.67$ & $5.40 \pm 3.19$ & 0.16 \\
\hline Ca24 & $\begin{array}{l}\text { Male }<250 \\
\text { Female }<200\end{array}$ & $122.98 \pm 77.95$ & $140.31 \pm 86.47$ & 0.41 \\
\hline $0 \times 24$ & $<40$ & $38.82 \pm 12.81$ & $41.14 \pm 13.45$ & 0.49 \\
\hline Cit24 & $\begin{array}{l}\text { Male }>450 \\
\text { Female }>550\end{array}$ & $500.43 \pm 308.09$ & $601.84 \pm 317.45$ & 0.19 \\
\hline SSCaP & & $0.29 \pm 0.49$ & $0.50 \pm 0.61$ & 0.15 \\
\hline pH & $5.8-6.2$ & $5.43 \pm 0.39$ & $5.72 \pm 0.58$ & $0.03^{*}$ \\
\hline SSUA & & $1.51 \pm 0.83$ & $1.38 \pm 0.93$ & 0.57 \\
\hline UA24 & $\begin{array}{l}\text { Male }<0.8 \\
\text { Female }<0.75\end{array}$ & $0.58 \pm 0.29$ & $0.65 \pm 0.29$ & 0.33 \\
\hline $\mathrm{Na24}$ & $<150$ & $175.17 \pm 79.57$ & $168.22 \pm 73.36$ & 0.71 \\
\hline K24 & $20-100$ & $74.82 \pm 36.84$ & $78.47 \pm 32.62$ & 0.67 \\
\hline Mg24 & $30-120$ & $85.56 \pm 33.88$ & $99.66 \pm 38.28$ & 0.13 \\
\hline P24 & $0.6-1.2$ & $1.03 \pm 0.48$ & $0.93 \pm 0.31$ & 0.29 \\
\hline $\mathrm{NH} 424$ & $15-60$ & $38.86 \pm 16.87$ & $29.71 \pm 14.02$ & $0.02^{*}$ \\
\hline $\mathrm{Cl} 24$ & $70-250$ & $187.68 \pm 82.34$ & $179.84 \pm 68.39$ & 0.67 \\
\hline Sul24 & $2-80$ & $53.23 \pm 21.75$ & $43.67 \pm 18.66$ & $0.05^{\circ}$ \\
\hline UUN24 & $6-14$ & $14.53 \pm 6.02$ & $12.25 \pm 4.71$ & 0.08 \\
\hline
\end{tabular}


higher urine ammonium levels (38.86 \pm 16.87 vs $29.71 \pm 14.02$; $\mathrm{p}=0.02)$, and higher urinary sulfate levels $(53.23 \pm 21.75$ vs $43.67 \pm 18.66 ; \mathrm{p}=0.05)$. Additionally, pure UA stone formers had lower urine uric acid levels $(0.58 \pm 0.29$ versus $0.65 \pm 0.29$; -0.33$)$ but this was not statistically significant.

Conclusions: Pure UA stone formers did not have any abnormality on metabolic workup that influenced stone prevention management. Treatment with potassium citrate and diet modification should address the underlying issues of their calculus growth kinetics (urine $\mathrm{pH}$ and hypocitraturia). Given this, metabolic evaluation in these patients is likely not necessary. Patients with mixed UA and $\mathrm{CaOx}$ stones have heterogeneous findings on metabolic evaluation and would benefit from 24-hour urine tests to identify exact risks for recurrence and inform treatment decisions.

\section{MP22-02 Buyer Beware: Evidence-Based Evaluation of Dietary Supplements for Nephrolithiasis}

K Koo, T Aro, B Matlaga

Johns Hopkins University School of Medicine

Introduction \& Objective: An increasing number of dietary supplements have recently been brought to market with the purported benefit of kidney stone risk reduction. These products may be purchased without a prescription and carry claims to reduce or even prevent kidney stone formation. However, the evidence for these claims is not clear. We performed a study to assess the scientific evidence supporting commercially available dietary supplements for patients with urinary stone disease.

Methods: The online marketplace Amazon.com was queried for dietary supplements to treat, alleviate, or prevent stone disease. A Google search was similarly performed to capture products not available on Amazon. Product labels were reviewed to compile specific stone-related claims and nonpharmacological active ingredients (ie, excluding vitamins and ion salts) in each supplement. The cost of a 30-day course of recommended treatment was assessed. We queried Google Scholar with all nonpharmacological active ingredients to assess the scientific evidence from published and lay sources. Non-English studies and conference proceedings were excluded. Two investigators independently performed the searches and analysis.

Results: Of 113 products reviewed in the searches, 27 dietary supplements containing 56 non-pharmacological active ingredients were analyzed. Products made a variety of claims: 12 (44\%) claimed to dissolve stones, 7 (26\%) claimed to prevent stone formation, $6(22 \%)$ claimed to reduce stone symptoms, and the majority $(19,70 \%)$ could be used to support kidney health. The mean 30-day cost was \$32 (range \$4-\$189), and 10 (37\%) products offered a money-back guarantee. Nearly all products were highly rated, with 21 products receiving $\geq 4$ stars on a 5-star scale (mean 4.2, range 3.4-5.0). Of the 56 non-pharmacological ingredients, $9(16 \%)$ had any published studies for use in stone disease, and $5(9 \%)$ had exclusively studies supporting their use. A total of 18 scientific publications about the ingredients were identified, of which 6 showed mixed or no benefit for stone disease. In the other 12 publications supporting use in stone formers, only 5 were human studies. Overall, among the 27 supplements, $18(67 \%)$ contained ingredients with conflicting, refuting, or absent evidence of benefit in stone disease.

Conclusions: In this analysis of commercially available dietary supplements claiming to treat or prevent kidney stones, twothirds contained ingredients with conflicting or no scientific ev- idence to support their claims. These findings may assist clinicians in counseling stone formers about the use of these unregulated products.

\section{MP22-03 Risk Factors for the Development of Multiple-Drug Resistant Bacteria in Patients With Urinary Stones}

\author{
S Wang, Y Zhang, X Zhang, Y Tang, B Xiao, J Li \\ University of Maryland School of Medicine
}

Introduction \& Objective: The empirical, prolonged, or incorrect usage of antibiotics contributes significantly to the emergence of bacteria resistant to multiple antimicrobial agents, which could lead to intractable infection and increase mortality rates, as well as medical costs. Moreover, MDR bacteria could result in an alarming public health issue that very few antimicrobial agents would be available for infections caused by such infection. In this study, we evaluated the prevalence of MDR bacteria in patients with urinary stone disease in a high-volume stone management center in Northern China and investigated the risk factors of its formation.

Methods: A retrospective study was performed among patients with urinary stones in Beijing Tsinghua Changgung Hospital from December 2014 to May 2018. Patients with positive urinary cultures and drug-sensitivity results were included. MDR were defined as any bacteria that have resistance to at least one agent in at least 3 classes of antibiotics. Clinical data of eligible patients were recorded, such as gender, age, diabetes mellitus, immunosuppressive status (renal transplantation, disorders treated with immunosuppressive medication), spinal abnormalities (scoliosis, paraplegia, etc.), renal abnormality (polycystic kidney, horseshoe kidney, ectopic kidney, etc.), indwelling catheters (ureteral stent or nephrostomy drainage $>3$ months), history of urinary tract diversion and surgery, antibiotics use of in the last 3 months and 4-12 months.

Results: 1655 patients with urinary stones were eligible for analysis, among which 367 patients had positive urinary culture, yielding 457 isolates of 45 species. $44.4 \%$ isolates were identified as MDR. The most 3 common Gram-negative bacteria were Escherichia coli, Proteus mirabilis, and Klebsiella pneumoniae, with a MDR rate of $84.33 \%, 62.5 \%$, and $48.39 \%$, respectively. In multivariate analysis, indwelling catheters (OR 3.1, 95\%CI 1.078.98) and antibiotics use in the last 3 months (OR 2.14, 95\% CI 1.04-4.38) were significantly associated with MDR formation.

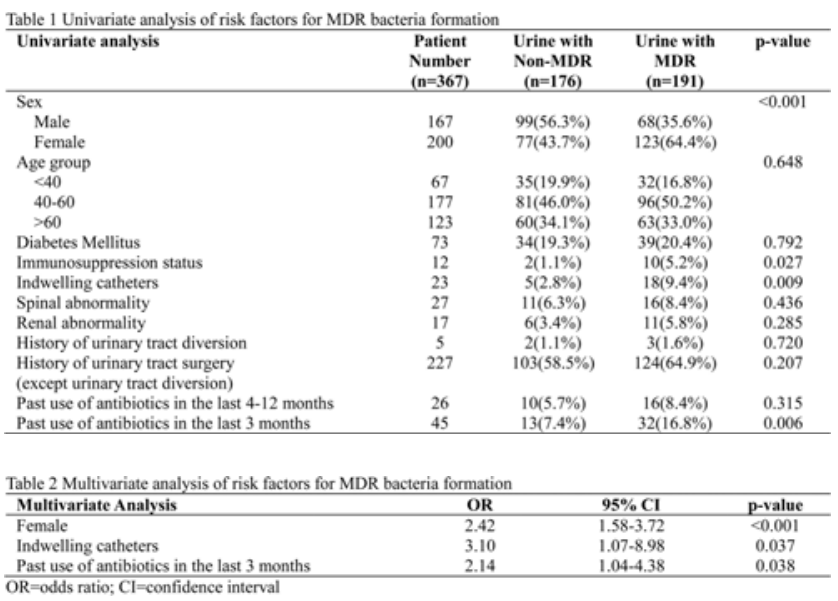


Conclusions: MDR-bacteria were common among patients with urinary stones in our center. Indwelling catheters and antibiotics use in the last 3 months were independent risk factors for MDR formation.

MP22-04 Impact of Demographic Factors and Systemic Disease on Urinary Stone Risk Parameters Amongst Stone Formers

K Wood, C Boyd, B Gower, W Poore, D Assimos

University of Alabama at Birmingham

Introduction \& Objective: Age, sex, and race are all known to influence kidney stone risk. Previous literature has demonstrated a clear link between kidney stone disease, obesity, and diabetes. Our objective was to examine in a multivariate analysis the associations between various demographic factors and systemic diseases on stone risk parameters in a stone forming population.

Methods: A retrospective chart review of adult kidney stone patients who completed 24-hour urine collections from April 2004 through August 2015 was performed. Demographic information was captured including age at collection, sex, race, and BMI. Chart review was performed to assess for a diagnosis of diabetes and hypertension. The results of CT Imaging, and renal/abdominal ultrasonography, performed with \pm 6 months were reviewed for a diagnosis of fatty liver disease. Statistical analysis included Pearson correlation analysis, Spearman correlation analysis, and linear and logistic regression analyses, both univariate and multivariate. Results: There were 589 patients included in the study. Numerous urinary parameters were significant in association with demographic factors or systemic diseases in a multivariate analysis. Older age was associated with decreased calcium $(\mathrm{Ca})$ excretion $(\mathrm{p}=0.0214)$, decreased supersaturation of calcium oxalate $(\mathrm{SSCaOx})(\mathrm{p}=0.0262)$, decreased supersaturation of calcium phosphate $(\mathrm{SSCaP})(\mathrm{p}<.0001)$, and decreased urinary $\mathrm{pH}$ $(\mathrm{p}=0.0201)$. Males excreted more $\mathrm{Ca}(\mathrm{p}=0.0015)$ and oxalate $(\mathrm{Ox})(\mathrm{p}=0.0010)$, had lower urine $\mathrm{pH}(\mathrm{p}=0.0269)$, and higher supersaturation of uric acid (SSUA) $(\mathrm{p}<.0001)$ than women. For race, African Americans had lower urine volume $(p=0.0023)$, less Ca excretion $(p=0.0142)$, less Ox excretion $(p=0.0074)$, and higher SSUA $(p=0.0049)$. Diabetes was associated with more Ox excretion $(\mathrm{p}<.0001)$, lower SSCaP $(\mathrm{p}=0.0068)$, and lower urinary $\mathrm{pH}(\mathrm{p}=0.0153)$. There were positive correlations between BMI and $\mathrm{Ca}$ excretion $(\mathrm{p}=0.0386), \mathrm{BMI}$ and $\mathrm{Ox}$ excretion $(\mathrm{p}=0.0177)$, and BMI and SSUA $(\mathrm{p}=0.0045)$.

Conclusions: These results demonstrate that both demographic factors and systemic disease are independently associated with numerous risk factors for kidney stones. These results highlight that there are differential risks for individuals to develop kidney stones based on these associations. The mechanisms responsible for these associations and disparities (racial differences) need to be further elucidated.

\section{MP22-05 Renal Stone Disease in Patients with Pri- mary Hyperparathyroidism - Findings in a Tertiary Referral Stone Centre}

MM Quraishi, T Olaomi, I Ahmed, SS Kommu, J Davis

Kent \& Canterbury Hospital. East Kent Hospitals University NHS Foundation Trust
Introduction \& Objective: Calcium based compounds form the commonest renal tract stones. Treatment of hyperparathyroidism (HPT) can reduce serum calcium levels, minimizing renal stone complications. Guidelines promote investigation of calcium levels in patients presenting with acute renal colic, as a screening tool to identify potential hyperparathyroidism. We aim to review the prevalence of renal tract stone disease in patients surgically treated for primary HPT, and their pathway to treatment.

Methods: A retrospective review of 134 patients who underwent a parathyroidectomy for primary HPT was performed. Computer and written based medical records were reviewed to identify relevant clinical features.

Results: Twenty-four primary hyperparathyroid patients were identified with confirmed renal tract stone disease, with adequate information available on twenty patients. Male to female predisposition for patients with stone disease was 1:3. Eleven patients presented with acute renal colic who were not yet diagnosed with hyperparathyroidism. The remaining 9 patients either were incidentally identified to have renal stones on imaging for alternative indications or in the work up for a raised calcium. Mean first calcium, phosphate and parathyroid hormone in patients presenting with renal colic was $2.83 \mathrm{mmol} / \mathrm{L}$, $0.76 \mathrm{mg} / \mathrm{dL}$ and $166 \mathrm{pg} / \mathrm{mL}$ respectively. Five patients underwent 2 or more urological interventions for stone disease prior to consideration of referral to an endocrine specialist. Calcium was recorded on first presentation in patients with undiagnosed HPT in $30 \%$ of patients. In renal tract stone patients' median number of days from diagnosis of primary HPT to ENT clinic review was 146(6-1454), whilst median number of days from ENT review to parathyroidectomy was 83(17-211). Mean calcium at postoperative week 4 was found to be $2.29 \mathrm{mmol} / \mathrm{L}$.

Conclusions: A proportion of patients with primary HPT may present acutely with renal colic, alluding to the underlying metabolic pathology. Caution must be exercised to ensure screening for primary HPT is performed to reduce stone burden/recurrences/complications for the patients. We highlight the variations in time to definitive surgery once diagnosed, which may have direct impact on patient care.

\section{MP22-06 Association between visceral adipose tissue loss post-bariatric surgery and 24-hour urinary profile changes}

M Uy, R DiLena, J Hoogenes, B Al-Harbi, A Woodward, S Gmora, B Shayegan, ED Matsumoto

\section{McMaster University}

Introduction \& Objective: Obesity is a known risk factor for abnormal urinary profiles and nephrolithiasis. Body mass index (BMI, $\mathrm{kg} / \mathrm{m}^{2}$ ) has been the traditional objective measure of obesity; however, visceral adipose tissue (VAT, $\mathrm{cm}^{2}$ ) has been shown to be more accurate. Post-operatively, bariatric patients often have a pronounced decrease in VAT, yet the association of VAT loss and changes to urinary profiles has not been wellstudied. We explored the relationship between the $\%$ of VAT loss post-bariatric surgery and metabolic urinary changes.

Methods: Records of patients with a history of nephrolithiasis who underwent bariatric surgery at our center were reviewed. Eligible patients had pre- and post-operative 24-hour urine (24HU) analysis and a CT KUB. A CT-based fat delineation program, AnalyzePro ${ }^{\circledR}$, was used to measure pre- and postoperative VAT at levels L4 and L5 (VAT1) (Figure 1), and L1L2 (VAT2). Univariate and multivariate analyses examined 


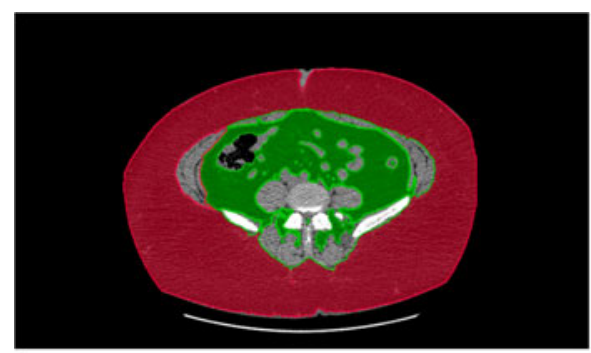

Figure 1. Axial computed tomography slice at the level of VAT 1 (L4 and LS). VAT is highlighted in green and subcutaneous adipose tissue is highlighted in red (extracted using AnalyzePro ${ }^{\circ}$ ).

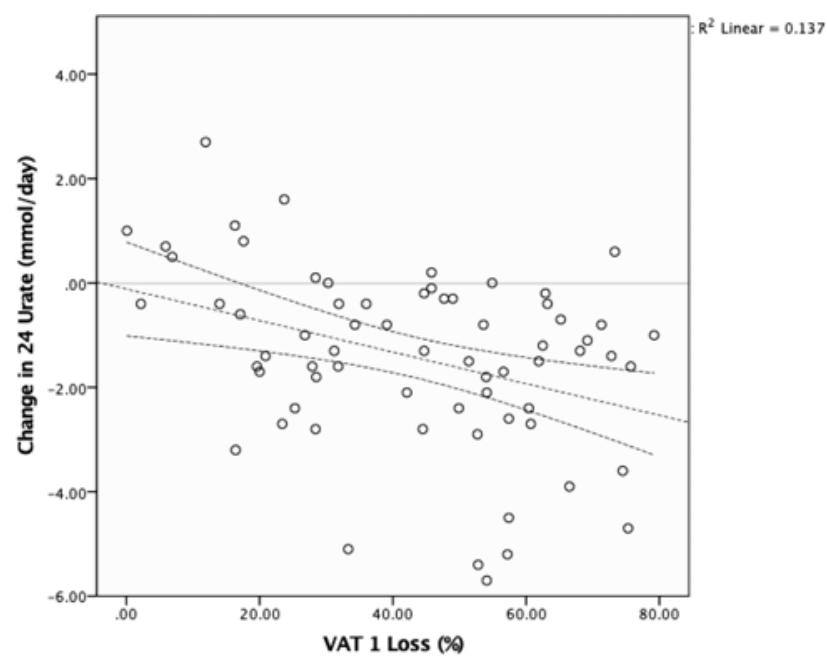

Figure 2. 24-hour urinary urate change by \% VAT 1 loss

associations between VAT and 24HU values, comorbidities, and urinary profiles.

Results: 88 patients met the inclusion criteria. The mean age was $51.9( \pm 9.2)$ and $68.2 \%$ were female. Pre-operatively, VAT1 was significantly correlated with hypertension, dyslipidemia, and metabolic syndrome $(\mathrm{p}=0.006 ; 0.015 ; 0.004$, respectively), and VAT2 was correlated with hypertension and metabolic syndrome $(\mathrm{p}=0.012$ and 0.014 , respectively). VAT2 was significantly associated with all $24 \mathrm{HU}$ values $(\mathrm{p}=<0.001-0.030)$, while VAT1 did not correlate with phosphate or calcium. The $\%$ of both VAT1 and 2 loss was a significant predictor for decrease in $24 \mathrm{HU}$ urate ( $\mathrm{p}=0.002$ and 0.003 , respectively) (Figure 2).

Conclusions: Within this patient cohort, VAT measurements were significantly correlated with pre-operative $24 \mathrm{HU}$ values and multiple comorbidities. The \% of VAT loss was able to predict post-operative $24 \mathrm{HU}$ urate changes. These findings indicate that VAT measurements can be used to inform the clinical management of bariatric patients with a history of nephrolithiasis.

MP22-07 Screening for Primary Hyperparathyroidism in a Tertiary Stone Clinic, a Useful Endeavor

K Wood, C Boyd, D Assimos

University of Alabama at Birmingham

Introduction \& Objective: Primary Hyperparathyroidism (1HPT) is associated with the risk of developing kidney stones. In addition, stone formers (SF) may have secondary hyperparathyroidism due to vitamin $\mathrm{D}$ deficiency or gastrointestinal malabsorption of calcium. Our objective was to determine the prevalence of 1HPT amongst SF evaluated at a tertiary stone clinic and determine if it is cost effective to screen for this condition.

Methods: We retrospectively reviewed 742 adult SF seen by a single urologic surgeon from 2012-2017 all of who were screened for 1HPT with an intact serum PTH (iPTH) and calcium. The diagnosis of 1HPT was based on the presence of hypercalcemia with an inappropriately elevated $\mathrm{PTTH}$ or a high normal serum calcium and an elevated iPTH. The diagnosis was confirmed by surgical neck exploration. Published cost data and stone recurrence rates were utilized to create a cost-effectiveness decision tree.

Results: Fifty-three (7.1\%) were diagnosed with 1HPT. Fifteen (28\%) had hypercalcemia and inappropriately elevated $\mathrm{PTTH}, 38$ (72\%) had high normal serum calcium levels and inappropriately elevated iPTH. The potential diagnosis was ignored/missed by primary care physicians in $9(17.0 \%)$ based on review of prior lab results. Cost modeling was undertaken for 5, 10, 15, and 20-year intervals after screening. Based on our prevalence data, historical risks for recurrence and published cost data for stone treatments, cost savings in screening are realized at 10 years.

Conclusions: These results support screening for primary hyperparathyroidism in patients evaluated in a tertiary referral setting.

\section{MP22-08 Withdrawn}

\section{MP22-09 Does Severity of Disease Impact Efficacy of Pharmacotherapy Versus Conservative Therapy in Reducing Stone Recurrence?}

BA Johnson, RL Steinberg, SL Best, SY Nakada, T Marien, C Tracy, N Miller, M Pearle, Y Lotan, JA Antonelli

University of Texas Southwestern

Introduction \& Objective: Despite proven effectiveness of drug therapy in preventing stone recurrence, compliance with pharmacotherapy is often poor. Using data from a multi-center collaboration to study medical management practices, we sought to compare the risk of stone recurrence between patients managed with conservative therapy versus pharmacotherapy controlling for aggressiveness of disease.

Methods: The Multi-center collaboration to Study Treatment Outcomes in Nephrolithiasis Evaluation (MSTONE) database contains demographics, management data, and outcomes for patients seen between July 2001 and April 2015 across four centers. The database was queried for patients managed with either conservative therapy (CT, fluid and dietary recommendations) or pharmacotherapy (PT). Stone events (new stone formation, stone growth, or an acute stone episode) were recorded. Events occurring within 3 months were censored. Patients were risk stratified according to number of previous stones spontaneously passed (low risk $=0$, moderate risk $\leq 10$, and high risk $>10$ ). Within each risk group, we compared those managed with CT vs PT with respect to rate of events and stone event-free survival (SEFS) via the Kaplan-Meier method. Student's t-test, Chi-square analysis, ANOVA, and Log-rank test were utilized.

Results: A total of 245 patients (median follow-up of 29 months, IQR 16 - 44) were identified (93 on CT, 152 on PT). Higher risk 


\begin{tabular}{|c|c|c|c|c|c|c|}
\hline & \multicolumn{3}{|c|}{ Risk Group } & \multirow[b]{2}{*}{$\begin{array}{l}\text { Entire } \\
\text { Cohort }\end{array}$} & \\
\hline & & $\begin{array}{l}\text { Low } \\
(n=62)\end{array}$ & $\begin{array}{c}\text { Intermediate } \\
(n=147)\end{array}$ & $\begin{array}{l}\text { High } \\
(n=36)\end{array}$ & & \\
\hline \multirow{3}{*}{ 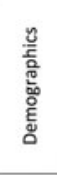 } & $\begin{array}{l}\text { Mean Age, years } \\
\pm \text { SD }\end{array}$ & $51 \pm 17$ & $53 \pm 14$ & $55 \pm 13$ & $53 \pm 15$ & $p=0.622$ \\
\hline & Gender & $29 \mathrm{M} / 33 \mathrm{~F}$ & $85 \mathrm{M} / 62 \mathrm{~F}$ & $22 \mathrm{M} / 14 \mathrm{~F}$ & $136 \mathrm{M} / 109 \mathrm{~F}$ & $p=0.260$ \\
\hline & $\begin{array}{l}\text { Mean Lifetime } \\
\text { Stone Surgeries } \pm \\
\text { SD }\end{array}$ & $1.7 \pm 2.3$ & $2.6 \pm 4.8$ & $5.4 \pm 4.7$ & $2.8 \pm 4.7$ & $p<0.001$ \\
\hline \multirow{3}{*}{ 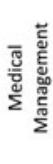 } & CT & $\begin{array}{l}32 / 62 \\
(52 \%)\end{array}$ & $53 / 147(36 \%)$ & $\begin{array}{l}8 / 36 \\
(22 \%)\end{array}$ & $\begin{array}{c}93 / 245 \\
(37 \%)\end{array}$ & \\
\hline & PT & $\begin{array}{l}30 / 62 \\
(48 \%)\end{array}$ & $94 / 147(64 \%)$ & $\begin{array}{l}28 / 36 \\
(78 \%)\end{array}$ & $\begin{array}{c}152 / 245 \\
(63 \%)\end{array}$ & \\
\hline & & & & & & $p=0.012$ \\
\hline \multirow{8}{*}{ 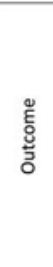 } & Stone Event Rate & & & & & \\
\hline & CT & $69 \%$ & $75 \%$ & $88 \%$ & $74 \%$ & \\
\hline & PT & $63 \%$ & $64 \%$ & $79 \%$ & $66 \%$ & \\
\hline & & $p=0.659$ & $p=0.148$ & $p=.556$ & $p=0.203$ & \\
\hline & $\begin{array}{l}\text { Event-free } \\
\text { Survival }\end{array}$ & & & & & \\
\hline & CT & $51 \%$ & $45 \%$ & $17 \%$ & $46 \%$ & \\
\hline & PT & $56 \%$ & $65 \%$ & $49 \%$ & $58 \%$ & \\
\hline & & $p=0.438$ & $p=0.005$ & $p=0.099$ & $p=0.006$ & \\
\hline
\end{tabular}

patients were more likely to receive PT (table). Patient risk correlated with frequency of previous stone surgeries. A stonerelated event occurred in $74 \%$ of patients in the CT group compared to $66 \%$ of patients in the PT group $(\mathrm{p}=0.203)$. Although the rate of events was lower with PT than CT in all risk groups, the differences were not significant. However, 30-month SEFS was significantly higher for PT (58\%) than CT (46\%) overall. When stratified by risk group, 30-month SEFS was statistically significantly higher for PT than CT in the intermediate risk group (65\% vs $45 \%$ for PT and CT, respectively) and trended higher in the high-risk group (table).

Conclusions: In a multicenter series, patients with aggressive stone disease were more often managed with PT. PT was more effective than CT in delaying stone-related events in patients with more aggressive stone disease, while CT appeared to be as effective as PT in lower risk patients. PT is best reserved for higher risk stone formers.

\section{MP22-10 Are we following NICE Guidelines for metabolic stone screening?}

M Ravindraanandan, P Jayawardena

Hereford County Hospital

Introduction \& Objective: Kidney stones are a common urological disease affecting approximately $13 \%$ of the global population. Identifying a preventable cause during follow-up for stone formation is a necessary factor in reducing recurrence. The majority of kidney stones are comprised of calcium, with oxalate and phosphate compounds accounting for approximately $80 \%$ of stone formers. Non-calcium forming stones such as uric acid account for $9 \%$ of presentations. Serum analysis can be used to identify the levels of calcium and uric acid in the blood, excluding metabolic causes for stones. Current NICE Guidelines recommend performing a metabolic screen prior to follow-up in clinic.

Methods: A retrospective audit was performed in a cohort of 124 patients from a single district general hospital between August 2017 to July 2018. Requests including serum calcium, phosphate and uric acid were analyzed in those who presented with renal colic. Data was captured using our online clinical portal system and patient notes, analyzing those who had presented to the Surgical Assessment Unit (SAU) with a clinical suspicion of kidney stones.

Results: From 124 patients $23.4 \%(n=29)$ had a complete serum analysis. 55.6\% $(n=69)$ had either bone profile (calcium and phosphate) or uric acid performed. $21.0 \%(n=26)$ had no serum analysis performed.

Conclusions: Only $23.4 \%$ met the standards set by NICE and over $50 \%$ had partially met national guidelines, whereas over a fifth of patients had no serum analysis at all. This demonstrates that the majority of clinicians are aware of adding a serum analysis to those presenting with renal colic, but not of what NICE recommends. Good education at induction to those working in Accident and Emergency and SAU departments may help improve this, as well as reminding urologists to check on post-take ward rounds. Grouping bloods together to create a "serum stone former" set may help improve results.

\section{MP22-11 24-hour Creatinine Excretion is a Poor In- dicator of Adequate 24-hour Urine Collection in Ob- ese Stone Formers}

BA Johnson, RL Steinberg, SL Best, SY Nakada, C Tracy, N Miller, T Marien, M Pearle, JA Antonelli, Y Lotan

University of Texas Southwestern

Introduction \& Objective: The diagnostic value of a 24-hour urine collection relies on proper collection technique, which is determined by comparing urinary creatinine (Ucr) to expected values based on body weight. However, clear standards for 24hour urinary creatinine in obese individuals have not been determined. We sought to analyze 24-hour urine collection data to evaluate the utility of Ucr in determining adequacy of a 24-hour urine collection.

Methods: The Multi-center collaboration to Study Treatment Outcomes in Nephrolithiasis Evaluation (MSTONE) database contains 24-hour urine samples collected from patients between July 2001 and April 2015 across four institutions. The traditional standard for 24-hour creatinine values was used to determine the adequacy of urine collections $(15-20 \mathrm{mg} / \mathrm{kg} / \mathrm{day}$ for females and $20-25 \mathrm{mg} / \mathrm{kg} /$ day for males) in patients with body mass index $(B M I) \geq 30.0 \mathrm{~kg} / \mathrm{m}^{2}$. For each patient actual body weight (ABW), ideal body weight (IBW), and adjusted body was (AjBW) were calculated. IBW was calculated utilizing the Broca Ideal Body Weight formula. AjBW $=\mathrm{IBW}+0.4(\mathrm{ABW}-\mathrm{IBW})$. Rates of adequate, under-collected, and over-collected urine samples were calculated.

Results: A total of 431 24-hour urine collections from 158 patients with $\mathrm{BMI} \geq 30.0 \mathrm{~kg} / \mathrm{m}^{2}$ were analyzed. The median age was 55 years (IQR 46-64). Mean height and BMI were 1.7 meters \pm 0.1 and $36.2 \mathrm{~kg} / \mathrm{m}^{2} \pm 6.1$, respectively. Using ABW to calculate expected urinary creatinine leads to the presumption of an "adequate" collection in $28.1 \%$, "undercollection" in $64.3 \%$ and "overcollection" in $7.7 \%$ of individuals. Using ideal body weight to calculate expected Ucr, "adequate" collection declines to $15.3 \%$, while "under-" and "overcollections" occur in 5.8\% and $78.9 \%$, respectively. Using AjBW yields more "adequate collections" than both ABW and IBW (40.6\% versus $28.1 \%$ and $15.3 \%$ ). Patients with $\mathrm{BMI} \geq 40.0 \mathrm{~kg} / \mathrm{m}^{2}$ demonstrate an even greater inconsistency with use of ABW and IBW. However, AjBW maintained a similar "adequate collection" rate $(41.6 \%)$. See table.

Conclusions: The determination of adequately collected 24-hour urine samples in obese stone formers is a significant challenge. 


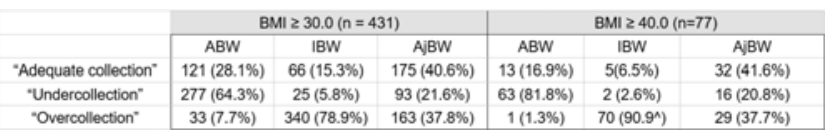

Using adjusted body weight yields the greatest number of "adequate collections for both for BMI $\geq 30.0 \mathrm{~kg} / \mathrm{m}^{2}$ and BMI $\geq 40$. Adjusted body weight should be considered when interpreting the adequacy of a 24-hour urine collection in an obese patient.

MP22-12 Healthcare utilization of alpha-blockers for medical expulsive therapy in patients with ureteral stones - a United States national claims database study

SP Argade, A Desai, J Vetter, M Keller, M Olsen, A Paradis, A Parker, R Venkatesh

Barnes Jewish Hospital/Washington University School of Medicine

Introduction \& Objective: Medical expulsive therapy (MET) has been widely utilized in the treatment of ureteral stones, however there is conflicting evidence as to its effectiveness. Many studies have evaluated the role of alpha-blocker therapy on stone passage rate, time to stone passage, and effects on pain, but few studies have elucidated its effects on healthcare utilization. This study aims to evaluate the use of MET in a large cohort of ureteral stone patients to determine its efficacy in reducing surgical intervention, imaging, adverse events, emergency room (ER) visits, and cost

Methods: Patients were selected from a United States national commercial claims database between 2011 and 2015 based on prescription of alpha-blocker therapy for ureteral stone diagnosis in an ER, urgent care, outpatient, or inpatient hospital setting. 58568 patients with and 78653 patients without prescription for alpha-blocker were identified. Patients were followed to 90 days after diagnosis to determine rates of surgical intervention, number of follow-up imaging studies, adverse events, ER visits, and 90-day total medical claims cost. Multivariate linear and logistic regression models were used to test associations between 90-day outcome variables and the use of MET.

Results: Alpha-blockers were prescribed to $43 \%$ of patients for a median length of 14 days. 24\% MET vs. 23\% non-MET $(\mathrm{p}<0.001, \mathrm{OR}=1.08)$ underwent a surgical procedure including stenting, ureteroscopy, extracorporeal shockwave lithotripsy, and percutaneous nephrolithotomy. 46\% MET vs. $38 \%$ nonMET $(\mathrm{p}<0.001, \mathrm{OR}=1.417)$ had a follow-up imaging study, most frequently $\mathrm{x}$-ray followed by computed tomography and ultrasound. 7.6\% MET vs. 9.2\% non-MET $(\mathrm{p}<0.001)$ had urinary tract infection (UTI) after ureteral stone diagnosis and $0.3 \%$ MET vs. $0.4 \%$ non-MET $(\mathrm{p}<0.001)$ had urosepsis. $24 \%$ on MET vs. $21 \%$ not on MET $(\mathrm{p}<0.001, \mathrm{OR}=1.261)$ presented to the ER within 90 days. Finally, 90-day total medical claims cost was $\$ 4597$ (IQR = \$2518-9519) for MET vs. \$4137 (IQR = \$18779112) for non-MET patients $(\mathrm{p}<0.001)$.

Conclusions: Patients prescribed MET for ureteral stone treatment had increased rates of surgical intervention, follow-up imaging studies, ER visits, and total cost, but had reduced rates of UTI and sepsis though these adverse events were infrequent. While recognizing the limitations of a medical claims database, these results should be considered along with the efficacy of MET in stone passage to determine the optimal utilization of resources in the already expensive treatment of stone disease.
MP22-13 Use of sipIT Intervention to Reduce Common Perceived Barriers to Increasing Fluid Intake Among Adult Patients with Kidney Stones

NM Streeper, D Brunke-Reese, E Thomaz, A Sanders, D Conroy

\section{Penn State Health}

Introduction \& Objective: Compliance with increasing fluid intake to produce at least $2.5 \mathrm{~L}$ of urine daily for stone prevention is commonly below $50 \%$. We have previously identified patient interest in the use of connected water bottles and demonstrated that wrist-worn sensors with accelerometers can detect drinking behavior and provide automated lapse detection in fluid intake in the lab setting. From these studies we developed the $\operatorname{sip}^{\mathrm{IT}}$ intervention which is a context-sensitive reminder system that incorporates a wrist-worn sensor (Fitbit Versa watch), connected water bottle (H20Pal) and self-monitoring through a smartphone application (app). The purpose of this study was to determine the feasibility and acceptability of $\operatorname{sip}^{\mathrm{IT}}$ intervention in the clinical setting. In addition, the changes in perceived barriers to increasing fluid intake were evaluated.

Methods: Patients with kidney stones were recruited from the community and a specialty kidney clinic to participate in a 1month feasibility trial. Patients were given a Fitbit Versa watch with the $\operatorname{sip}^{\mathrm{IT}}$ app installed and an H20Pal connected water bottle. They completed a questionnaire to determine perceived barriers to increasing fluid intake at baseline and at 1 month.

Results: 31 patients with a history of kidney stones were enrolled to participate ( $58 \%$ female, age $=40.0 \pm 14.3$ years). Findings are based on $n=23$ who have completed the 1 -month assessment ( $n=6$ are still being followed, $\mathrm{n}=2$ dropped out after enrollment). After the first month, patients reported that forgetting to drink and lack of thirst were less of a barrier to meeting fluid intake goals. Most participants perceived that the $\operatorname{sip}^{\mathrm{IT}}$ intervention helped them to achieve their fluid intake goals $(70 \%)$ and would recommend it to other patients with a history of kidney stones (70\%).

Conclusions: The $\operatorname{sip}^{\mathrm{IT}}$ intervention may be used to detect drinking behavior and provide automated lapse detection in fluid intake in the clinical setting. The system was acceptable to patients and there was reduction in common perceived barriers to fluid intake. Combining digital tools with behavioral science may help to improve adherence to fluid intake recommendations.

\section{MP22-14 Comparison of 24-hour Urinary Sodium and Potassium Excretion in U.S. Stone Formers vs. Non-Stone Formers from 2010-17}

KA Healy, O Shah, G Li, J Asplin

Columbia University Medical Center

Introduction \& Objective: Approximately $90 \%$ of sodium consumed is excreted in the urine. Based on NHANES 2013-14 data, the estimated mean sodium intake overall among U.S. adults was $3608 \mathrm{mg}$ per day. This study sought to compare 24 hour urinary sodium and potassium excretion between U.S. stone formers (SF) vs. non-SFs in the general adult population.

Methods: Retrospective review of adult SFs aged 20-69 years who submitted a baseline 24-hour Litholink urine collection from 2010-17. Patients with overt chronic kidney disease (serum creatinine $\geq 1.4 \mathrm{mg} /$ day men, $\geq 1.2 \mathrm{mg} /$ day women) or improperly collected specimens were excluded. Overall mean and median 


\begin{tabular}{|l|c|c|c|}
\hline & $\begin{array}{l}\text { Litholink } \\
\text { Stone Formers } \\
\text { Total N }=23,615\end{array}$ & $\begin{array}{l}\text { NHANES } \\
\text { Non-SFs } \\
\text { Total N =827 }\end{array}$ & P-value \\
\hline MEN (N) & 11,978 & 421 & \\
\hline N24 (mmol/day) & $197.0(80.02)$ & $182.8(111.96)$ & 0.010 \\
\hline K24 (mmol/day) & $64.9(24.46)$ & $61.5(39.19)$ & 0.078 \\
\hline Na/K Ratio & $3.29(1.46)$ & $3.32(3.66)$ & 0.867 \\
\hline WOMEN (N) & 11,637 & 406 & \\
\hline N24 (mmol/day) & $148.4(64.74)$ & $132.1(87.17)$ & $<0.001$ \\
\hline K24 (mmol/day) & $49.6(20.09)$ & $49.3(43.49)$ & 0.890 \\
\hline Na/K Ratio & $3.27(1.53)$ & $3.03(2.67)$ & 0.072 \\
\hline
\end{tabular}

24-hour urinary sodium and potassium excretion were calculated. SFs were then compared to sex and age-matched non-SFs using NHANES data $(\mathrm{n}=827)$. Among SFs, subgroup analysis was also done by sex and age (20-44 vs. 45-69). Statistical analysis was conducted using two-sample t-test.

Results: A total 23,615 SFs met study inclusion criteria $(50.7 \%$ men, $49.3 \%$ women). Mean 24-hour urinary sodium (197.0 vs. $148.4 \mathrm{mmol} /$ day) and potassium (64.9 vs. 49.6) excretion were both significantly higher in men vs. women SFs, respectively $(\mathrm{p}<0.001)$ aged 20-69; however, mean sodium-to-potassium molar ratios were equivalent ( 3.29 vs. $3.27, p=0.24)$. Compared to their non-SF counterparts, mean 24-hour urinary sodium excretion was significantly higher for both men and women SF (Table 1). No differences were found between SF vs. non-SF in mean 24-hour urinary potassium excretion or sodium-topotassium molar ratios; however, the latter trended toward significance for women $(\mathrm{p}=0.072)$.

Conclusions: Both SFs and non-SFs have high mean 24-hour urinary sodium, which reflects estimated intake above ADA daily recommendations. However, mean urinary sodium is markedly higher for SFs. Sex differences also exist across both groups with higher mean urinary sodium and potassium excretion in men. Future studies are needed to incorporate these measures into stone prediction tools.

\section{MP22-15 Withdrawn}

\section{MP22-16 Factors That Contribute to Inadequate 24- hour Urine Collections in Patients with Nephrolithiasis: a Multi-center Analysis}

BA Johnson, RL Steinberg, SL Best, SY Nakada, C Tracy, N Miller, T Marien, M Pearle, JA Antonelli, Y Lotan

University of Texas Southwestern

Introduction \& Objective: The 24-hour urine (24U) collection is a critical component of medical stone management. However, over- or undercollected urine might lead to inappropriate drug or dietary recommendations. Previous studies have indicated that 24-hour urine creatinine may not be an ideal measure of the adequacy of urine collection. Utilizing a multi-center collaborative study of medical management practices, $24 \mathrm{U}$ data was analyzed to determine factors that contribute to inadequate collections.

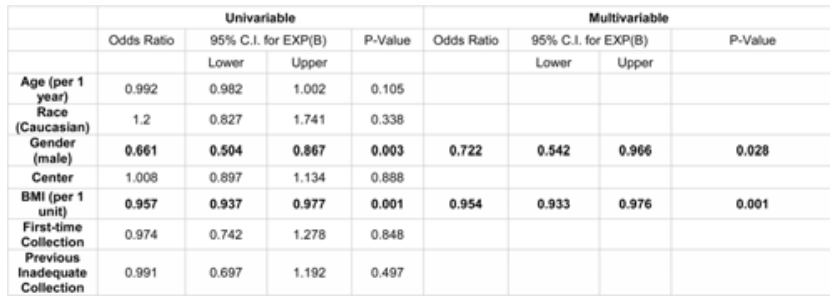

Methods: The Multi-center collaboration to Study Treatment Outcomes in Nephrolithiasis Evaluation (MSTONE) database contains the results 24U samples collected from patients between July 2001 and April 2015 across four institutions and analyzed for stone risk factors. The traditional standards for 24-hour creatinine values were used to determine the adequacy of urine collection $(15-20 \mathrm{mg} / \mathrm{kg} / \mathrm{day}$ for females and $20-25 \mathrm{mg} / \mathrm{kg} / \mathrm{day}$ for males). Univariable and multivariable binary regression was used for analysis.

Results: A total of 980 24U was collected from 379 patients ( $57 \%$ male). The median age of the cohort was 45 years (IQR 44 $-65)$ and mean BMI was $30.1 \mathrm{~kg} / \mathrm{m}^{2} \pm 7$. Among the $98024 \mathrm{Us}$, $33.7 \%$ of collections (330) were considered adequate. Of the 650 inadequate collections, $76.1 \%$ (495) were considered undercollected and 155 (23.9\%) were overcollected. On univariable analysis, neither age, race nor institution were predictive of inadequate collection. Patients were no more likely to have an inadequate collection if it was their first $24 \mathrm{U}$ or if they had previous inadequate collections. Male gender and higher BMI were predictive of inadequate collections on both univariable and multivariable analysis (Table 1). Patients over age 70 years had a $24.6 \%$ adequate collection rate, and of the poor collections, $95.7 \%$ were considered under-collections ( $72.1 \%$ of all collections).

Conclusions: While the $24 \mathrm{U}$ analysis supplies essential information to direct a stone prevention strategy, the determination of an adequate urine collection is problematic. Less than $34 \%$ of collections are considered adequate using standard accepted $24 \mathrm{H}$ creatinine ranges. Male gender, higher BMI, and older age increase the risk of "poor collection". Further research should attempt to clarify appropriate normal ranges, taking into account age and BMI, so as to provide more accurate indicators of poor patient compliance with urine collection.

MP22-17 Post-operative radiographic and 24-hour urine profile changes in bariatric patients with a history of nephrolithiasis: risk factors for recurrent stone formation associated with Roux-en $Y$ gastric bypass versus sleeve gastrectomy

M Uy, R DiLena, J Hoogenes, B Al-Harbi, S Gmora, B Shayegan, ED Matsumoto

\section{McMaster University}

Introduction \& Objective: Roux-en-Y gastric bypass (RYGB) and sleeve gastrectomy (SG) are the most common bariatric surgeries, yet it is not clear which is superior. ${ }^{1}$ Considering the propensity for the development of lithogenic urinary profiles and nephrolithiasis post-bariatric surgery is clinically important. ${ }^{2}$ To our knowledge, no studies have evaluated these changes in postbariatric surgery patients with a history of nephrolithiasis. We evaluated the differences in 24-hour urine (24HU) values and radiographic imaging post-RYGB and SG in patients with a history of nephrolithiasis. 


\begin{tabular}{|c|c|c|c|c|c|c|}
\hline \multirow[b]{2}{*}{$24 \mathrm{H}$ Urine Values } & \multicolumn{3}{|c|}{ RYGB } & \multicolumn{3}{|c|}{ os } \\
\hline & Pre & Post & p Value & Pre & Post & p Value \\
\hline Volume (mL) & 1808 & 1599 & 0.038 & 2001 & 1600 & 0.001 \\
\hline & 6.0 & 5.8 & 0.309 & 6.0 & 6.0 & 0.440 \\
\hline Urate (mmo/day) & 4.2 & 2.7 & $<0.001$ & 4.3 & 2.9 & $<0.001$ \\
\hline Sodium (mmol/day) & 185.7 & 140.3 & $\$ 0.001$ & 194.4 & 137.5 & 0.003 \\
\hline Phosphate (mmol/dary) & 31.5 & 23.2 & $<0.001$ & 30.0 & 22.1 & 0.001 \\
\hline Oxalate (umov/day) & 389.7 & 537.0 & 0.004 & 382.6 & 312.3 & 0.005 \\
\hline 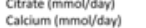 & $\begin{array}{l}4.4 \\
6.6\end{array}$ & $\begin{array}{l}3.2 \\
4.3\end{array}$ & $\begin{array}{l}0.001 \\
0.010\end{array}$ & $\begin{array}{l}3.92 \\
5.7\end{array}$ & $\begin{array}{l}4.0 \\
5.1\end{array}$ & $\begin{array}{l}0.959 \\
0.369\end{array}$ \\
\hline
\end{tabular}

Methods: We reviewed the records of 100 patients with a history of nephrolithiasis and underwent either RYGB or GS at our center. CT KUB imaging and $24 \mathrm{HU}$ profiles were performed preoperatively and at 1-year follow-up. The Wilcoxon rank sum test compared pre- and post-operative values, while multivariate regression analysis determined predictors of stones and hyperoxaluria.

Results: 60 patients underwent RYGB and 40 had SG. No baseline differences were found between groups. For $24 \mathrm{HU}$ profiles (Table 1), both groups had similar findings, although the RYGB group had a significant increase in oxalate and a decrease in citrate, while the SG group had a significant decrease in oxalate with citrate remaining stable. On multivariate analysis, RYGB (OR 6.4, 95\% CI 1.9-21.1, p=0.002) was the only significant predictor of post-operative hyperoxaluria. Radiographically, $26.7 \%$ of the RYGB group and $22.5 \%$ of the SG developed new stones. Post-operative stone procedure rate for each group was $10.0 \%$ and $7.5 \%$ respectively.

Conclusions: Patients with a history of nephrolithiasis who underwent RYGB had exacerbated lithogenic $24 \mathrm{HU}$ profiles, while those in SG patients improved. There were no significant differences in stone event rate, although this may be due to limited follow-up. The post-operative stone formation rate is higher than previously reported in similar studies. These findings support close urinary monitoring in patients with a history of nephrolithiasis who undergo RYGB. 1. Peterli R, Wölnerhanssen BK, Peters T, Vetter D, Kröll D, Borbély Y, et al. Effect of Laparoscopic Sleeve Gastrectomy vs Laparoscopic Roux-en-Y Gastric Bypass on Weight Loss in Patients With Morbid Obesity. JAMA [Internet] American Medical Association, 2018 [cited 26 Oct 2018];319(3):255. 2. Valezi AC, Fuganti PE, Junior JM, Delfino VD. Urinary evaluation after RYGBP: A lithogenic profile with early postoperative increase in the incidence of urolithiasis. Obes Surg 2013;

\section{MP22-18 Metabolic evaluation of adults with Ur- olithiasis: guidelines vs practice}

L Saunders, E Maher, F Hinds, N Thakare, SH Moochhala, R Kucheria, D Allen, L Ajayi, A Goyal

Royal Free Hospital

Introduction \& Objective: Patients with recurrent stone formation contribute to frequent hospital attendances and have higher risk of significant morbidity. Published guidelines from European Association of Urology define clear criteria and pathways for metabolic evaluation. We aim to assess our current practice with respect to metabolic workup of stone patients and its impact on stone recurrence.

Methods: All adult patients who attended stone clinic between August 2017 and November 2017 were retrospectively evaluated. High risk categories were identified as per EAU guidelines. Data was obtained to determine whether patients had basic evaluation and if extended evaluation were undertaken in high risk patients. Recurrent stone episodes including new stone formation were noted.

Results: A total of 399 patients were included; age range: 19 to 93, male $62 \%$ and female $38 \%$. Serum calcium, Urate and stone analysis were performed for 238 (60\%), 207 (52\%), and 105 (26\%) patients respectively. 4\% (10/238) had abnormal calcium, $17 \%(35 / 207)$ had abnormal Urate and $3(6 \%)$ had a further stone episode. 64/399 (16\%) were identified as high risk for stone formation. Only $35(55 \%)$ of high-risk patients had further metabolic assessment including 24-hour urine. Recurrent stone episodes occurred in 13 (20\%) of high-risk patients, 7 (54\%) of whom had an extended workup and $6(46 \%)$ of those were not fully investigated.

Conclusions: Compliance with metabolic evaluation can be improved. Recurrent stone episodes occurred in a small proportion with abnormal calcium and urate. Performing extended workup did not significantly impact stone recurrence in the short term. It will be interesting to observe the frequency of recurrent stones after a longer follow-up period and the impact of extended workup on stone recurrence in high risk patients in the long term.

\section{MP22-19 Magnesium Intake and the Risk of Incident Kidney Stone in Women Health Initiative (WHI)}

O Kott, C Chen, X Wen, B Nicholas, M Huang, J Li, J Wactawski-Wende, L Snetselaar, S Liu, J Tang

Nephrology, Alpert Warren School of Medicine, Brown University

Introduction \& Objective: Magnesium $(\mathrm{Mg})$ reduces intestinal oxalate and phosphorus absorption, which may reduce risk of calcium kidney stone formation. However, few prospective studies have directly examined the association of $\mathrm{Mg}$ intake with kidney stone risk in apparently healthy individuals particularly among women and ethnic minorities. Our objective was to examine the associations of total and dietary intakes of $\mathrm{Mg}$ on incident kidney stone formation in women.

Methods: Among 148,643 WHI Participants free of kidney stone history at baseline, we assessed their total daily $\mathrm{Mg}$ intake from diet using a semi-quantitative food frequency (FFQ) and supplement (via medication inventory). During the subsequent 11 years of follow up, incident kidney stone was self-reported on annual health surveys. Time dependent multivariable Cox proportional hazard model was used to estimate the effect of baseline and time-varying total $\mathrm{Mg}$ intake on the risk of kidney stone formation, adjusted for demographic, clinical, social, and dietary covariates.

Results: The mean age was 63 at baseline. Among them, $83 \%$ were Caucasian and $67 \%$ were above age 60 . A total of 5518 $(0.3 \%)$ women developed incident kidney stone during $1,601,750$ person-years of follow up. The mean total daily magnesium intake was $318 \mathrm{mg}$, with $26 \%$ in tertile 1 (less than $241 \mathrm{mg}$ per day), $41 \%$ in tertile 2 (241 mg-348 $\mathrm{mg}$ per day) and $33 \%$ in tertile 3 (above $348 \mathrm{mg}$ per day). Baseline characteristics associated with lower $\mathrm{Mg}$ intake included age younger than 60 (34\%, 32\% and $33 \%$ in tertiles 1,2 and 3 respectively), and African American race $(13 \%, 7 \%$ and $6 \%$ in tertiles 1, 2 and 3 respectively). The incidence of kidney stone report was 3.1, 3.3, and 3.9 per 1000 person-year in high $\mathrm{Mg}$, moderate $\mathrm{Mg}$ and low $\mathrm{Mg}$ groups, respectively. The corresponding multivariableadjusted hazard ratios were 0.82 (95\% CI: $0.76-0.88)$ for high vs 
low $\mathrm{Mg}$ intake, and 0.967 (95\% CI: 0.90-1.04) for high vs moderate $\mathrm{Mg}$ intake.

Conclusions: Higher intake of $\mathrm{Mg}$ was significantly associated with reduced risk of kidney stone formation among postmenopausal women from the Women's Health Initiative. The potential role for $\mathrm{Mg}$ in kidney stone prevention warrants further consideration and assessment.

\section{MP22-20 Radiological vs. Urological Determination of Ureteral Stone Density. Monocentric Retrospective Study}

JR Wießmeyer, J Ring, J Landmesser, T Ozimek, MW Kramer, AS Merseburger

Universitätsklinikum Schleswig-Holstein (Campus Lübeck)

Introduction \& Objective: Non-Contrast Computed tomography (NCCT) is recommended as a standard imaging for the work-up of ureteral stones. The stone density expressed as hounsfield units (HU) value is apart from stone size and location an important, however often neglected, factor for planning of individualized stone therapy, including chemolytholysis. The goal of our study was to investigate, if $\mathrm{HU}$ values can be adequately measured not only by radiologists but also by urologists on different training level, in order to simplify the stone therapy planning by the urologist, especially regarding indication to the chemolitholysis.

Methods: A monocentric retrospective analysis included 248 patients with singular ureteral stones treated from May 2014 to August 2016 in a tertiary urologic center. The mean HU values of the calculi of each patient were determined on the basis of NCCT by a consultant urologist, a senior (PGY5) and junior (PGY2) urology residents respectively. The inter-rater correlations between the values measured by urologists and the radiological findings as a reference were calculated using the intraclass correlation coefficient (ICC).

Results: The calculated inter-rater correlation between the the radiological $\mathrm{HU}$ values as reference and overall urological measurements was 0.739 (95\% CI 0.686-0.786, p <0,001). For the consultant urologist the ICC was 0.786 (95\% confidence interval $0.741-0.823, \mathrm{p}<0.001)$. For the final year resident (PGY5), the ICC was 0.695 (95\% CI $0.623-0.754, \mathrm{p}<0.001)$, for

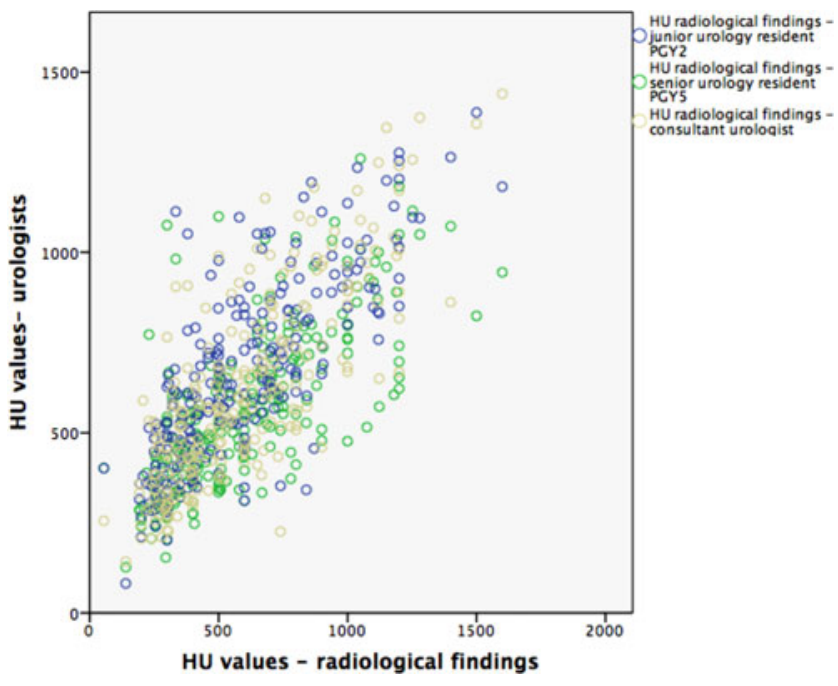

the second-year resident (PGY2), the ICC was 0,756 (95\% CI 0.667-0.818, $\mathrm{p}<0.001)$.

Conclusions: The stone density is an important radiological factor allowing to predict the success of oral chemolitholysis. The overall compliance of urological HU measurement with the radiological reference is high at every level of urological experience. The clinically relevant divergent measurements should be reviewed to find possible causes. In addition, it must be checked whether the values shown here can be confirmed by other raters. A prospective randomised validation of our results is warranted.

\section{MP22-21 Entering to 2.0 cystinuric management: medical digital solution to monitor and control uri- nary $\mathbf{p H}$}

O Angerri Feu, F Rousaud Baron, S Gracia Garcia, E Emiliani, A Kanashiro, F Sánchez-Martín, F Millán Rodriguez,

J Palou Redorta, J Cuñé Castellana

Fundacio Puigvert

Introduction \& Objective: Cystinuria, is a congenital orphan disease that causes an excess of cystine excretion in urine. Urinary alkalinization is a cornerstone of cystinuric patients to increase cystine solubility and making it difficult to crystallize. Hence the importance of urinary $\mathrm{pH}$ control to modulate the alkalizing treatments and maintain urine $\mathrm{pH}$ in alkaline range. Nowadays, urinary $\mathrm{pH}$ monitoring takes place with dipsticks, a subjective, non-standardized, semiquantitative colorimetric method (compare results with a scale). Some limitations in terms of diagnostic accuracy parameters were reported in them. The objective of the study is to know the acceptance of a new pHmeter, that has demonstrated superiority in terms of sensitivity, specificity, positive predictive value, negative predictive value and accuracy against dipsticks as a system to monitor the urinary $\mathrm{pH}$ by cystinuric patients.

Methods: A randomized, controlled, open trial in 28 cystinuric patients were performed in Puigvert Foundation after been approved by the ethical committee. Once the patient's eligibility is verified, those receiving alkalinizing therapy under routine clinical practice were randomized in 2 groups: those who measure the urinary $\mathrm{pH}$ twice a day with the medical device and those who do it with strips (standard method). The patients were randomized with a ratio $1: 1$ by blocks. Control visits were performed at baseline, 1, 3 and 6 months. Measures of urinary $\mathrm{pH}$, urine density, number of crystal and mean size were monitored. Questions about the facility of use and adherence to pharmacological treatment and nutritional recommendations were also studied.

Results: The 28 patients ( $32.1 \%$ women and $67.9 \%$ men) had an average age of $51.82 \pm 18.58$ (mean \pm standard deviation). Regarding the facility of use, the $\mathrm{pHmeter}$ was easier to learn how to manage $(\mathrm{p}=0.021)$, easier to prepare $(\mathrm{p}=0.034)$ and easier to use $(p=0.062)$ than strips. Regarding adherence to the pharmacological and dietary recommendations there were a tendency to be better in the pHmeter group. Regarding the acceptance, the pHmeter patients scored general satisfaction with a mean of $7.3 \pm 2.2$ (where 0 was the lowest score and 10 de highest). As expected, the measurement of urine $\mathrm{pH}$, its density, number of crystals and its mean size did not show statistically significant differences versus strips.

Conclusions: The pHmeter studied is an accepted medical device to monitor cystinuric patients easier to use than strips. This 2.0 solution opens a way to work on empowerment of patients. 
MP22-22 Adherence to pharmacologic treatment and its improvement in the twenty-four urine parameters in patients with kidney stones

B0 Manzo, E Emiliani, H Sánchez, JD Cabrera, JE Torres, E LOZADA

\section{Hospital Regional de Alta Especialidad del Bajío}

Introduction \& Objective: Compare the improvement in the twenty-four urine parameters in patients with adherence to pharmacologic treatment with those patients with no adherence. Methods: A retrospective observational study was conducted, and data from all patients with a diagnosis of urinary stones and at least two metabolic complete analysis from 2014-2018 were collected. Initial $24 \mathrm{~h}$-urine parameters were compared to a six months control. The adherence to pharmacologic treatment and the improvement in the urine parameters were evaluated. The Wilcoxon tests made this comparison. Finally, Delta differences were obtained with the initial and final parameters of each variable and the Mann-Whitney U test.

Results: A total of 90 patients met inclusion criteria of which 68 $(75.5 \%)$ showed adherence to pharmacologic treatment. The overall frequency of urine metabolic alterations was hypocitraturia $(41.26 \%)$, hyperoxaluria $(28.04 \%)$, hypercalciuria $(16.40 \%)$, and hyperuricosuria $(14.28 \%)$. Patients with adherence showed a significant increase of $235 \%$ in urine citrate levels in 24h-urine follow-up $(P=0.03)$ (Table 1). Urine uric acid and oxalate also decreased but with no statistical difference

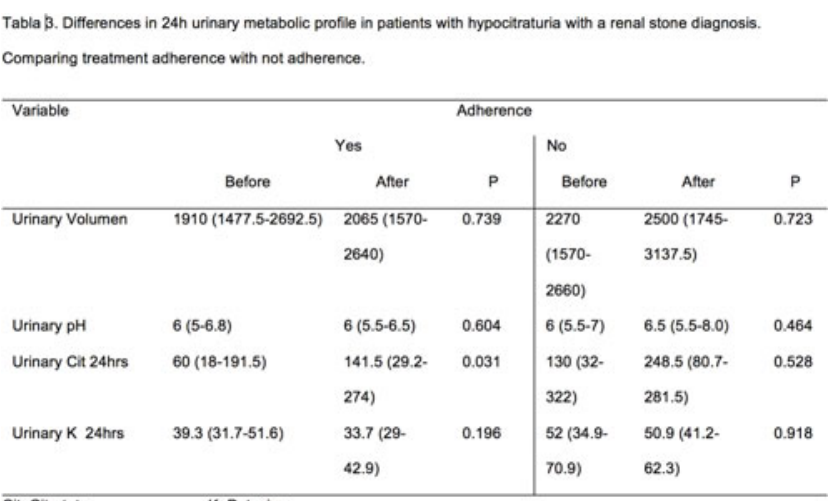

( $p=0.088$ vs. 0.327 and 0.286 vs. 0.374 respectively) in those patients with adherence. Urine calcium showed not changes ( $\mathrm{p}=0.889$ vs. 0.655$)$.

Conclusions: Hypocitraturia is the urine alteration that shows a better improvement after pharmacologic treatment in patients with renal stones and this improvement is most significant in patients with adherence to medical treatment. Long follow-up time is necessary to assess if these changes in urine parameters have a clinical impact on stone recurrence. Urologists must enforce adherence to pharmacological treatment and lifestyle modifications in patients with kidney stones.

\section{MODERATED POSTER SESSION 23: CLINICAL STONES: PCNL (II)}

\begin{abstract}
MP23-01 The use of immunization and probiotic therapy in the prevention of recurrent nephrolithiasis after puncture nephrolithotripsy
\end{abstract}

\section{A Pushkarev, V Pavlov \\ Bashkir State Medical University}

Introduction \& Objective: Treatment of recurrent infectious stones of the kidneys is quite a challenge. The formation of microbial biofilms with antibiotic-resistant microflora in the stone and on its surface, chronic pyelonephritis with lesions of the mucous membranes of the urinary tract and tubular kidney systems require an integrated approach. Prevention of intrahepatic hypertension during PCNL and, as a result, bacteremia, stone-free achievement, intensive antibacterial therapy to suppress microorganisms in biofilms. To increase the effectiveness of antibiotic therapy after PCNL in order to prevent recurrence of stone formation.

Methods: We have applied the Staphylo-Protein-Pseudomonas vaccine, which is a complex sorbed on aluminum hydroxide: the cytoplasmic antigen of Staphylococcus, staphylococcal anatoxin, proteic polyvalent chemical vaccine, and Pseudomonas toxoid. Immunization was carried out for 5-14 days prior to surgery by administering $0.5 \mathrm{ml}$ of the vaccine subcutaneously, re- vaccination was performed at a dose of $0.5 \mathrm{ml}$ not earlier than after 14 days. In some cases, the mucosal method of immunization was used. Immunization was performed by applying the vaccine directly to the mucous membranes of the renal pelvis system through nephrostomy drainage.

Results: The use of standard treatment regimens and the replacement of antibiotics did not give the expected effect against the background of high antibiotic resistance of the detected flora. The strains of P. aeruginosae, Proteus spp., Enterococcus spp., Klebsiella spp. Had the highest resistance to antibacterial drugs. At the same time, the resistance of these causative agents of infection extended even to reserve antibiotics and their combinations. In this situation, the use of an immunobiological scheme for the treatment of infection allowed in $72 \%$ of cases to achieve rehabilitation of the urinary tract. This indicator was 2 times higher than in the control group $(36.7 \%)$. At the same time, in the control group, as a result of treatment, a change of microbial agent prevailed (46.7\%), which did not always lead to the relief of the inflammatory process.

Conclusions: The combined immunobiological approach to the infection of the associated microbial biofilms has allowed to achieve good results in the eradication of antibiotic-resistant microbial pathogens, contributed to a significant reduction in the risk of the formation of infectious stones. 
MP23-02 The Comparison of Flexible Ureterorenoscopy and Percutaneous Nephrolithotomy in 20 $40 \mathrm{~mm}$ in Kidney Stones

A Albayrak, M Allahverdiyev, C Yesildal, A Yavuzsan, M Ilgi, S Kirecci

Introduction \& Objective: Retrograde Intrarenal Surgery and Percutaneous Nephrolithotomy are two important treatment modalities in $20-40 \mathrm{~mm}$ kidney stones. The aim of our study is to compare retrograde intrarenal surgery and percutaneous nephrolithotomy in $20-40 \mathrm{~mm}$ kidney stones.

Methods: A total of 800 patients, who applied to Urology Polyclinic of Etfal Training and Research Hospital of Şisli Hamidiye University of Health Sciences between May 2017 and June 2018 due to urinary system stone disease, were evaluated retrospectively. 282 patients, who have renal stones between $20 \mathrm{~mm}$ and $40 \mathrm{~mm}$ in size were included to the study. The demographic data of the patients are given in Table 1. Patients were randomized into two treatment groups: flexible ureterorenoscopy and percutaneous nephrolithotomy.

Results: The stone-free rate of the patients, who were applied one session of flexible ureterorenoscopy, was statistically significantly lower than the percutaneous nephrolithotomy group $(\mathrm{p}=0,005)($ Table2). There was a statistically significant difference in the complication rates of the groups $(p<0,001)$. The complication rate of the flexible ureterorenoscopy group was lower.

\begin{tabular}{lcccc}
\hline & & Flexible URS & PCNL & p \\
\hline Stone-free Rates n (\%) & No & $31(57,4)$ & $74(36,1)$ & 0,005 \\
& Yes & $23(42,6)$ & $131(63,9)$ & \\
Scopy Time (sn) Med. SD (Min-Max) & & $16,2 \pm 25,6(0-120)$ & $81,1 \pm 111,5(0-540)$ & 0,002 \\
Complication Rates n (\%) & 0 & $49(92,5)$ & $176(85,4)$ & 0,017 \\
& 1 & $0(0,0)$ & $7(3,4)$ & \\
& 2 & $1(1,9)$ & $20(9,7)$ & \\
& $3 \mathrm{a}$ & $3(5,7)$ & $1(0,5)$ & \\
& $3 \mathrm{~b}$ & $0(0,0)$ & $2(1,0)$ &
\end{tabular}

Operation Time (min) Med \pm SD (Min-Max) $85,5 \pm 24,7$ (30-150) $\quad 123,0 \pm 42,0(30-240)<0,001$ Hospitalization Time Med. \pm SD (Min-Max) $\quad 1,40 \pm 1,01(1-6) \quad 4,41 \pm 1,86(2-12) \quad<0,001$

\begin{tabular}{|c|c|c|c|c|}
\hline & & Flexible URS & PCNL & $\mathrm{p}$ \\
\hline \multicolumn{2}{|l|}{ Age Med \pm SD (Min-Max) } & $46,1 \pm 14,6(17-72)$ & $42,1 \pm 14,0(6-71)$ & 0,070 \\
\hline \multirow[t]{2}{*}{ Sex n $(\%)$} & Woman & $19(32,2)$ & $75(33,6)$ & \\
\hline & Man & $40(67,8)$ & $148(66,4)$ & \\
\hline \multicolumn{2}{|l|}{ Lenght Med \pm SD (Min-Max) } & $1,67 \pm 0,08(1,45-1,85)$ & $1,67 \pm 0,11(1,23-1,9)$ & 0,940 \\
\hline \multicolumn{2}{|l|}{ Weight Med \pm SD (Min-Max) } & $79,5 \pm 15,1(50-130)$ & $73,9 \pm 16,7(20-118)$ & 0,063 \\
\hline \multicolumn{2}{|c|}{ BMI (kg/m2) Med \pm SD (Min-Max) } & $28,4 \pm 5,2(18,7-43)$ & $26,2 \pm 5,2(8,3-45,8)$ & 0,012 \\
\hline \multicolumn{2}{|c|}{$\begin{array}{l}\text { Body surface area }(\mathbf{m} 2) \mathrm{Med} \pm \mathrm{SD} \\
\text { (Min-Max) }\end{array}$} & $1,88 \pm 0,18(1,50-2,41)$ & $1,45 \pm 0,33(0,99-1,82)$ & 0,012 \\
\hline \multicolumn{2}{|c|}{$\begin{array}{l}\text { Pre-op creatinine }(\mathrm{mg} / \mathrm{dl}) \mathrm{Med} \pm \mathrm{SD} \\
\text { (Min-Max) }\end{array}$} & $1,08 \pm 0,53(0,40-3,20)$ & $0,92 \pm 0,25(0,30-1,80)$ & 0,110 \\
\hline \multicolumn{2}{|l|}{ Hypertension n (\%) } & $19(32,2)$ & $48(21,7)$ & 0,094 \\
\hline \multicolumn{2}{|l|}{ Diabetes n (\%) } & $13(22,0)$ & $22(10,0)$ & 0,013 \\
\hline \multicolumn{2}{|l|}{ Hyperlipidemia n (\%) } & $10(16,9)$ & $12(5,4)$ & 0,011 \\
\hline \multirow[t]{2}{*}{ Side n (\%) } & Right & $23(39,7)$ & $50(41,7)$ & 0,798 \\
\hline & Left & $35(60,3)$ & $70(58,3)$ & \\
\hline \multicolumn{2}{|l|}{ Stone bilaterality n (\%) } & $18(36,0)$ & $23(20,9)$ & 0,043 \\
\hline $\begin{array}{l}\text { Stone Volume }(\mathrm{mm} 3) \\
\text { (Min-Max) }\end{array}$ & $\operatorname{Med} \pm$ SD & $\begin{array}{c}633,9 \pm 451,7 \\
(120,5-3254,4)\end{array}$ & $\begin{array}{l}2281,6 \pm 2078,4 \\
(295,8-14373,9)\end{array}$ & $<0,001$ \\
\hline \multicolumn{4}{|c|}{ Number of Stones Med \pm SD (Min- } & $<0,001$ \\
\hline
\end{tabular}

Conclusions: Although percutaneous nephrolithotomy is the gold standard in the treatment of renal stones larger than $20 \mathrm{~mm}$, it should be remembered that the similar success rate can be achieved with retrograde intrarenal surgical procedure with more than one session and greater than $20 \mathrm{~mm}$.

MP23-03 Management of Large Proximal Ureteral Calculi: A Comparative Study Between Simultaneous Supine Percutaneous Nephrolithotomy with Retrograde Ureterolithotripsy (ssPCNL) and Semi-rigid Ureteroscopic Lithotripsy

\section{Y Chen, H Chen, Y Juan, W Wu, C Li, T Huang}

Kaohsiung Medical University Hospital, Kaohsiung Medical University, Kaohsiung, Taiwan

Introduction \& Objective: The aim of the study was (1) to evaluate the treatment effectiveness of simultaneous supine percutaneous nephrolithotomy and retrograde ureterolithotripsy (ssPCNL) in the Galdakao-modified supine Valdivia position and (2) to compare the outcome of ssPCNL and semi-rigid (SRURS) ureteroscopic lithotripsy for treating large proximal ureteral calculus.

Methods: Between January 2015 and April 2019, all patients with large proximal ureteral stones $(>10 \mathrm{~mm})$ who underwent ssPCNL and SR-URS at three medical centers were retrospectively reported. The ssPCNL was performed by pushing back the ureter stone retrogradely with the ureteroscope and retrieving the stone using forceps with a nephroscope through an Amplatz sheath. The intraoperative and postoperative results, including operating time, hospital stay, the need of auxiliary procedure and complications, were compared between two groups. The stonefree clearance was defined as the absence of fragments or a single fragment of $\leq 4 \mathrm{~mm}$ on standard radiography.

Results: A total of 38 and 27 patients underwent ssPCNL and SR-URS. The mean stone size was $21 \mathrm{~mm}$ and $18.2 \mathrm{~mm}$ (Table 1); stone free rate was $97 \%$ and $33 \%$ in ssPCNL and SRURS group respectively. Regarding ssPCNL, there were 10 modified Clavien grade I and five grade II complications; no blood transfusions were necessary in this series. Compared with SR-URS, larger ureter stone $(\mathrm{P}<0.001)$, more operation time $(\mathrm{P}<0.001)$, prolonged hospital stay $(\mathrm{P}<0.001)$, higher stone free rate $(\mathrm{P}<0.001)$ and less patients requiring auxiliary procedure $(\mathrm{P}<0.001)$ were found in ssPCNL group (Table 2). No significant difference was observed regarding complications between two groups $(\mathrm{P}=0.1)$.

Conclusions: ssPCNL is a safe and effective treatment for large proximal ureteral stones. Even though larger stone size was found in ssPCNL group, ssPCNL represents significant higher stone free rate and less auxiliary procedure required, without

\begin{tabular}{|c|c|c|c|}
\hline Variables & $\begin{array}{l}\text { SsPCNL } \\
(n=38)\end{array}$ & $\begin{array}{l}\text { Semi-rigid Ureteroscopy } \\
\qquad(n=27)\end{array}$ & P.value \\
\hline Mean age, yr (range) & $55.9(32-74)$ & $60.3(33-86)$ & 0.2 \\
\hline Mean BMl, kg/m² (range) & $26.9(20.2-41.6)$ & $25.8(15.9-34.8)$ & 0.6 \\
\hline $\operatorname{Sex}, n(\%)$ & & & 0.3 \\
\hline Male & 29 (76) & 24 (88) & \\
\hline Stone side, $n(\%)$ & & & 1 \\
\hline Left & $18(47)$ & $12(45)$ & \\
\hline Right & $20(53)$ & $15(55)$ & \\
\hline Mean stone size, cm (range) & $2.1(1.2-3.7)$ & $1.82(1.0-4.7)$ & $<0.001$ \\
\hline Mean eGFR, mL/min/1.73m² (range) & $74(29-119)$ & $67(22-157)$ & 0.3 \\
\hline
\end{tabular}




\begin{tabular}{|c|c|c|c|}
\hline Variables & $\begin{array}{l}\text { ssPCNL } \\
(n=38)\end{array}$ & $\begin{array}{l}\text { Semi-rigid Ureteroscopy } \\
\qquad(n=27)\end{array}$ & P.value \\
\hline Mean operation time, min (range) & $82.1(30-150)$ & $57.1(30-105)$ & $<0.001$ \\
\hline Mean hospital stay, day (range) & $3.16(2-7)$ & $1.26(1-7)$ & $<0.001$ \\
\hline Postoperative stone-free status, no. (\%) & $30(97)$ & $9(33)$ & $<0.001$ \\
\hline Complications, n (\%) & $15(39)$ & $5(19)$ & 0.1 \\
\hline Modified Clavian classification grade 1, no. & 10 & 1 & 0.02 \\
\hline Modified Clavian classification grade 2, no. & 5 & 3 & 1 \\
\hline Modified Clavian classification grade 3, no. & 0 & 1 & 0.4 \\
\hline Patients needed auxillary procedure, $n$ (\%) & $1(4.3)$ & $12(44)$ & $<0.001$ \\
\hline Auxiliary ESWL & 1 & 8 & \\
\hline Auxiliary URSL & 0 & 4 & \\
\hline
\end{tabular}

major complications, compared to SR-URS group. More clinical studies are required to confirm the outcomes of the present study.

\section{MP23-04 The effectiveness and safety of four tract dilation methods of percutaneous nephrolithotomy: a meta-analysis}

Y Wu, Y Lu, Y Xun, S Wang, Q Wang

Tongji Hospital, Tongji Medical College, Huazhong University of Science and Technology

Introduction \& Objective: Percutaneous nephrolithotomy (PCNL) has become a routine surgical procedure for treating patients with large kidney stones; the fundamental step in this process is the creation of the nephrostomy tract. In the present study, a meta-analysis was performed to compare the effectiveness and safety of different tract dilation techniques for PCNL. Methods: We searched databases from database inception to 1 April 2019 to identify randomized controlled trials. We analyzed the X-ray exposure time, hemoglobin decrease, stone-free rate, transfusion rate, hospital stay, and the complication rate associated with the various techniques.

Results: A total of 11 studies with 1,415 cases were enrolled in the meta-analysis. Significant differences in X-ray exposure time [weighted mean difference (WMD), 30.67; 95\% confidence interval (CI), 20.08-41.26; $\mathrm{P}<0.001 ; \mathrm{I} 2=97 \%]$ and hemoglobin decrease (WMD, 0.19; 95\% CI, 0.15-0.23; $\mathrm{P}=0.65 ; \mathrm{I} 2=0 \%$ ) between metal telescopic dilation and one-shot dilation were reported. A significantly lower hemoglobin decrease was observed between balloon dilation (BD) and fascial Amplatz dilation (AD); the transfusion rate was similar between these techniques. Metal telescopic Alken type dilation had a notably higher successful dilation rate compared with one-shot dilation, but no significant differences in stone-free rate and transfusion rate were observed.

Conclusions: The present study determined that one-shot dilation was safer in almost every adult, including patients that had previously undergone renal surgery, compared with other methods. BD was also reported to be effective and safer in patients without a history of such surgery compared with other methods. The present study proposed AD and metal telescopic Alken type dilation as safer methods of dilation for patients who have undergone kidney surgery previously.

\section{MP23-05 Life threatening severe bleeding requiring angioembolization after percutaneous nephrolithotomy}

H Kim, S Lee, H Choe, K Lee, J Yoo, D Lee

St. Vincent's Hospital
Introduction \& Objective: To find out factors involved in severe bleeding requiring angioembolization after percutaneous nephrolithotomy (PCNL).

Methods: Retrospective analysis of patients who underwent angioembolization after PCNL in two teaching hospitals between December 2009 and May 2019 was done. Pre-operative patient and stone characteristics as well as procedural details of the PCNL were recorded. Pre-angiographic CT was reviewed to evaluate whether the puncture done during the PCNL procedure was appropriate and to calculate the degree of angulation in cases with UPJ involvement. Incorrect puncture was defined as any access pathway beyond Brodel's area (the area within $20^{\circ}$ posteriorly from the frontal plane of the kidney).

Results: Of the 1,554 PCNL cases performed during the period, $21(1.4 \%)$ patients underwent angioembolization. Mean BMI was 23.6 and any degree of hydronephrosis was present in $16(76.2 \%)$ cases. Mean operation time was 61 minutes, lower pole access was done in $17(81.0 \%)$ cases and single tract was used in $20(95.2 \%)$ cases. There were $14(66.7 \%)$ cases of incorrect puncture identified in the pre-angiographic CT. UPJ was involved in all the 7 cases with correct puncture. Lower pole puncture was made in all of the 7 cases with UPJ involvement and the mean degree of angulation was 64.4 degree.

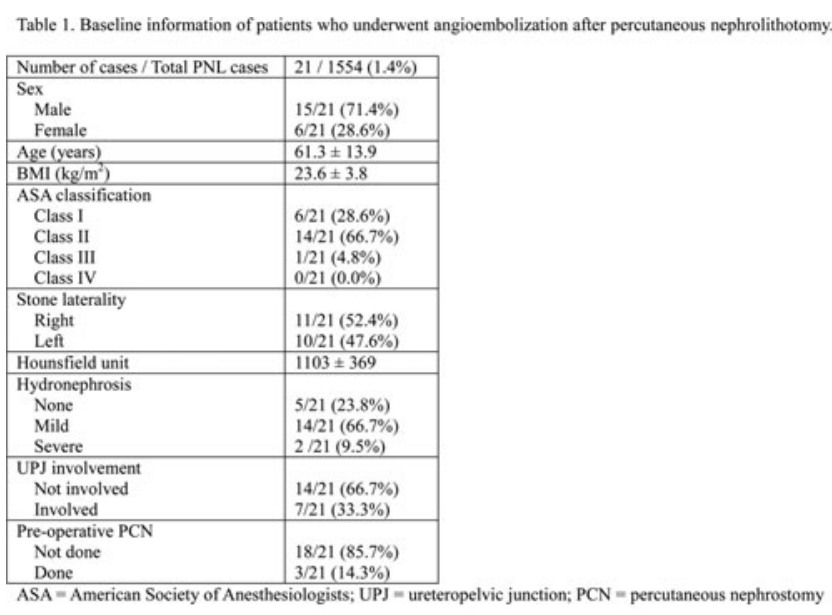

Results of continuous variables expressed as mean \pm standard deviation

Table 2. Procedural details of the percutaneous nephrolithotomy \& angioembolization

\begin{tabular}{|l|l|}
\hline Operation time (minutes) & $61.3 \pm 13.9$ \\
\hline $\begin{array}{l}\text { Location of access } \\
\text { Lower pole }\end{array}$ & $17 / 21(81.0 \%)$ \\
$\begin{array}{l}\text { Other (middle or upper) } \\
\text { Lower pole \& other }\end{array}$ & $3 / 21(14.3 \%)$ \\
\hline $\begin{array}{l}\text { Number of tracts } \\
1\end{array}$ & $20 / 21(4.8 \%)$ \\
2 & $1 / 21(4.8 \%)$ \\
\hline $\begin{array}{l}\text { Correctness of puncture } \\
\text { Correct puncture } \\
\text { Incorrect puncture }\end{array}$ & $7 / 21(33.3 \%)$ \\
\hline $\begin{array}{l}\text { Degree of angulation } \\
\text { (only cases with UPJ involvement) }\end{array}$ & $14 / 21(66.7 \%)$ \\
\hline $\begin{array}{l}\text { Interval between surgery and } \\
\text { angioembolization (days) }\end{array}$ & $7.4 \pm 6.4 \pm 12.9$ \\
\hline Angiographic findings & $15 / 21(71.4 \%)$ \\
Pseudoaneurysm & $5 / 21(23.8 \%)$ \\
AVF & $1 / 21(4.8 \%)$ \\
\hline Both & UPJ = ureteropelvic junction; AVF $=$ arteriovenous fistula \\
\hline
\end{tabular}

Results of continuous variables expressed as mean \pm standard deviation 
The mean interval between surgery and angioembolization was 7.4 days.

Conclusions: Incorrect renal puncture or excessive UPJ manipulation was involved in all cases of severe bleeding requiring angioembolization after PCNL. Extensive review of pre-operative $\mathrm{CT}$ scan is mandatory to appreciate the variations of calyceal anatomy before making a PCNL track. Additionally, a mid-polar puncture should be considered in cases of UPJ involvement.

\section{MP23-06 Endoscopic Combined IntraRenal Surger- $y(E C I R S)$ in treating complex high burden stone}

Y Tsao, T Huang

Kaohsiung Medical University Hospital

Introduction \& Objective: Although percutaneous nephrolithotomy (PCNL) is considered the standard procedure for the removal of large renal calculi, Endoscopic Combined IntraRenal Surgery (ECIRS), which is the combined use of flexible ureteroscopy (fURS), percutaneous nephrolithotomy (PCNL) usually under Galdakao-modified supine Valdivia (GMSV) position, has been proposed as a possible solution for the treatment of complex urinary tract calculi. In this study, we present our experiences with this surgical modality and to determine the efficacy and safety of this method to treat high burden urolithiasis.

Methods: From January 2018 to September 2018, 36 patients with complex high burden stone (Staghorn stone or multiple large calyceal stone) underwent preoperative non-contrast computed tomography (NCCT) examination and ECIRS surgery. The procedure has been standardized in our hospital after years of practice and was performed in Galdakao-modified supine Valdivia (GMSV) position with 20.8Fr mini-perc tract and $14 \mathrm{Fr}$ ureteral access sheath. Intraoperative double $\mathrm{J}$ stenting was performed in all patients. The status of stone free was determined within 3 months postoperatively using kidney-ureter-bladder (KUB) or NCCT. Average operation time, stone free rate, the need of further procedures, related complication or blood transfusion were documented.

Results: The mean operative time was $102.56 \pm 34 \mathrm{~min}$. The Stone free rate (SFR) after first operation was $68.3 \%$ and further increased up to $86.3 \%$ after further treatment including extracorporeal shock wave lithotripsy (ESWL), URSL, PCNL and RIRS. 1 out of 36 patients (3\%) has experienced major complication, which was urosepsis. The average hospitalization time after operation was 4.7 days.

Conclusions: Our study has showed that ECIRS is a safe and efficient procedure for complex urolithiasis under careful patient selection and preparation.

\section{MP23-07 Multi Institutional Experience with Single Access Percutaneous Nephrolithotomy for Staghorn Calculi}

T Large, C Forbes, K Scotland, D Agarwal, H Jarvis, R Yan, CU Nottingham, ME Rivera, BH Chew, A Krambeck

Introduction \& Objective: The treatment of complex stone disease has been revolutionized in the last 30 years-largely driven by advancing endoscopic technology. Despite published guidelines on the surgical management of kidney stones, a heterogeneous distribution of technology and physician training has inspired new interest in robotic assisted management of complex stones. Our objective is to demonstrate the efficacy

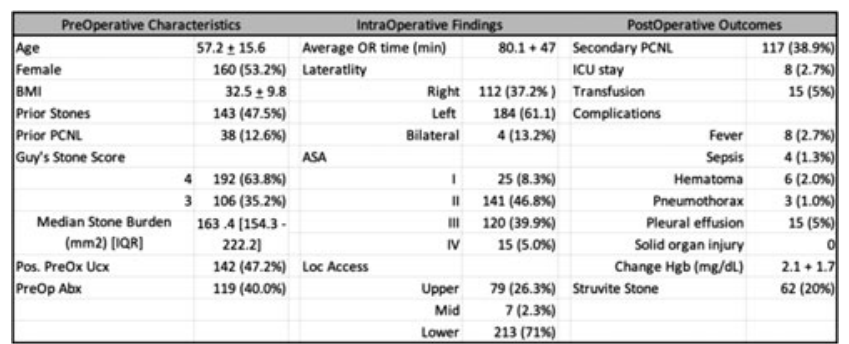

and overall outcomes of single access PCNL in patients with staghorn calculi.

Methods: This is an IRB approved multi-institutional retrospective review of patients with full or partial staghorn calculi treated with percutaneous nephrolithotomy (PCNL) between Jan 1, 2017 and 2019. Two tertiary and a quaternary referral center were included. We included only single access PCNL for final analysis. Stone free was defined as no calculi on postop CT scanning. Statistical analysis was performed utilizing SPSS statistical software to perform Fisher's exact and Wilcoxon Rank sum to identify predictive factors affecting stone free rates.

Results: A total of 340 staghorn patients were identified, of which $301(89 \%)$ patients were treated using a single access (table 1). Mean age was 57 (range 17-87). There were $160(53 \%)$ female patients and mean BMI of the entire cohort was 32.5 (range 9.5-75.9). All stones were either Guy's stone score 3 $(35.2 \%)$ or $4(63.8 \%)$ with a mean largest stone burden of $153 \pm 100 \mathrm{~mm}^{2}$. Mean OR time was $80 \pm 47$ minutes. Postoperative CT imaging was available in 297 patients with 118 (40\%) showing no residual stone. After secondary procedures for residual stones in 117 patients, the stone free rate (SFR) was $78 \%$ for staghorn stones treated through a single percutaneous access. Complications (table 1) were minimal. There were no preoperative factors that predicted SFR after primary PCNL.

Conclusions: High stone free rates with minimal morbidity is possible using a single access PCNL for staghorn calculi. This investigation reinforces the recommendation of PCNL as a first line therapy for staghorn calculi.

\section{MP23-08 Ultrasound-guided micro-percutaneous ne- phrolithotomy in the treatment of pediatric ne- phrolithiasis}

C Ning, W Wang, J Li

Beijing Friendship Hospital, Capital Medical University

Introduction \& Objective: The objective of this study was to demonstrate the efficacy and safety of ultrasound-guided micropercutaneous nephrolithotomy (micro-PNL) in the management of pediatric kidney stones.

Methods: We reviewed the records of pediatric patients with renal stones treated with micro-PNL technique in our center between October 2016 and April 2019. Micro-PNL treatments were performed under general anesthesia. Ureteral stent or catheter was indwelled in lithotomy position depended on the stone burden and CT value of the stone. Patients were then placed to a prone position, and visual puncture system (all-seeing needle) was used to puncture the target calyx under the guidance of ultrasound. The lithotripsy was performed with $4.8 \mathrm{~F}$ microsheath and $200-\mu \mathrm{m}$ holmium laser to disintegrate 1- to 2-mm 
fragments. Patients underwent abdominal radiography and ultrasound four weeks after operation. The double J-stent was removed under general anesthesia if retained intraoperatively. Perioperative and postoperative parameters along with operative data were collected.

Results: A total of 56 patients were included in the study. The group included 38 infants and 18 children; the patients' mean age was 4.1 years (range 1-8 years). Stone burden was $14 \pm 5 \mathrm{~mm}$ (range 5-25 mm), operative time was 30.0 $21.1 \mathrm{~min}$ (range 10$110 \mathrm{~min})$, and average dosage of flushing was $200 \pm 270 \mathrm{ml}(40$ $1500 \mathrm{ml})$. Complete stone-free status was achieved in $54(96.4 \%)$ patients. According to the modified Clavien classification, grade 1 and 2 postoperative complications occurred in three and three patients, respectively. The average hospital stay was 3 days (range 2-5 days). The number of anesthesia sessions was $1.4 \pm 0.5$.

Conclusions: Ultrasound-guided micro-perc was a safe and effective treatment option for pediatric kidney stones patients which has the advantage of preventing radiation hazard and reduce anesthesia times.

\section{MP23-09 Predisposing Factors Affecting Blood Loss During Percutaneous Nephrolithotomy (PCNL)}

JN Bamberger, B Gallante, RA Chandhoke, A Kapoor, H Kwun, W Atallah, M Gupta, AM Zampini

Mount Sinai

Introduction \& Objective: PCNL carries risks including increased chance of blood loss and infection. The objective of this study is to evaluate and compare clinical and surgical characteristics of patients who had significant blood loss during a PCNL vs. those who did not.

Methods: We queried our prospectively maintained database of 682 patients who underwent PCNL at our institution from 1/2014

\begin{tabular}{|c|c|c|c|}
\hline & $\begin{array}{l}\text { Significant Blood Loss } \\
(n=59)\end{array}$ & $\begin{array}{l}\text { No Significant Blood } \\
\text { Loss ( } n=176)\end{array}$ & $p$-value \\
\hline \multicolumn{4}{|c|}{ Clinical Characteristics and Demographics } \\
\hline Mean Age, years (SD) & $58.26(14)$ & $58.48(15)$ & 0.926 \\
\hline Gender, Male:Female & $28: 31$ & $103: 73$ & 0.269 \\
\hline Mean BMI, $\mathrm{kg} / \mathrm{m}^{2}$ & 27.87 & 28.74 & 0.464 \\
\hline Mean ASA & 2.15 & 2.12 & 0.736 \\
\hline Diabetes Mellitus & $9(15 \%)$ & $31(17 \%)$ & 0.521 \\
\hline Hypertension & $19(32 \%)$ & $60(34 \%)$ & 0.208 \\
\hline Hyperlipidemia & $16(27 \%)$ & $28(16 \%)$ & 0.013 \\
\hline Coronary Artery Disease & $7(12 \%)$ & $20(11 \%)$ & 0.525 \\
\hline Aspirin & $4(7 \%)$ & $15(9 \%)$ & 0.456 \\
\hline \multicolumn{4}{|l|}{ Labs } \\
\hline Mean Pre Hematocrit & 41.52 & 39.73 & \\
\hline Mean Post Hematocrit & 37.14 & 39.49 & \\
\hline Mean Pre Creatinine & 0.96 & 1.04 & 0.212 \\
\hline Mean Post Creatinine & 0.96 & 1.03 & 0.337 \\
\hline Mean $\mathrm{HgAlc}$ & 5.67 & 5.94 & 0.165 \\
\hline \multicolumn{4}{|c|}{ Intra-Operative Data and Stone Characteristics } \\
\hline Mean Number of Stones & 6.82 & 4.09 & 0.049 \\
\hline Mean Stone Burden (mm) & 44.07 & 37.65 & 0.108 \\
\hline Pre-Operative Hydronephrosis & $34(58 \%)$ & $99(56 \%)$ & 0.471 \\
\hline Staghorn & & & 0.000 \\
\hline Partial & $11(19 \%)$ & $51(29 \%)$ & \\
\hline Complete & $18(30 \%)$ & $14(8 \%)$ & \\
\hline \multicolumn{4}{|l|}{ Location } \\
\hline Upper & $27(46 \%)$ & $40(22 \%)$ & 0.001 \\
\hline Interpolar & $29(49 \%)$ & $44(25 \%)$ & 0.001 \\
\hline Lower & $42(71 \%)$ & $107(61 \%)$ & 0.100 \\
\hline Renal Pelvis & $32(54 \%)$ & $91(52 \%)$ & 0.427 \\
\hline UPJ & $13(22 \%)$ & $30(17 \%)$ & 0.250 \\
\hline Stent Placed & $31(52 \%)$ & $95(54 \%)$ & 0.881 \\
\hline Mean Number of Access Tracts & 1.51 & 1.08 & 0.000 \\
\hline Mean Surgery Time (min) & 116.73 & 98.43 & 0.057 \\
\hline
\end{tabular}

to $5 / 2019$. Significant blood loss was operationalized by a prevs. post-operative hematocrit (Hct) level decrease greater than $5 \%$. Data including demographics, relevant labs, number of punctures, stone location, and stone characteristics were collected and reviewed. ANOVA, chi square, and regression were then conducted to elucidate differences between the two patient groups.

Results: 235 patients had completed lab data and were included in our study. 59 of 235 (25\%) were classified as experiencing significant blood loss. Univariate analysis revealed a significant difference in proportion of patients with interpolar $(p=0.001)$ and upper pole stones $(p=0.001)$, mean number of stones $(p=0.049)$, presence of staghorn calculi $(\mathrm{p}=0.000)$, and mean number of access tracts $(\mathrm{p}=0.000)$ between the two blood loss groups. The only comorbidity found to be significantly associated with blood loss was hyperlipidemia (HLD) $(p=0.013)$. Within our regression model, the presence of interpolar calculi and HLD were found to independently predict blood loss $(\mathrm{p}=0.024)$ (Table 1).

Conclusions: During PCNL, the majority of access sites were in the upper and lower poles. Thus patients with interpolar calculi likely experienced significant blood loss likely due to torque to reach this isolated region of the collecting system. More liberal use of flexible nephroscopy and/or retrograde ureteroscopy during PCNL to access these stones may decrease the risk of blood loss. Additional studies analyzing how the anticoagulatory effects of statins may moderate the relationship between HLD and blood loss.

\section{MP23-10 The Experience of handling Channel Loss during the Procedure of Nephrostomy in Percuta- neous Nephrolithotripsy: Report of 17 Cases}

\section{Lin, L Guangyan, L Bingfeng}

Department of Urology Hong Kong University Shenzhen Hospital

Introduction \& Objective: percutaneous nephrolithotripsy is an important treatment for renal stones and impacted proximal ureteral stones. Establishing an effective and safe percutaneous access is the most important step in performing a successful and smooth percutaneous nephrolithotomy. We may lose channel during the Procedure of Nephrostomy. We share our experience of handling channel loss in percutaneous nephrolithotripsy.

Methods: A total of 656 patients that underwent percutaneous nephrolithotripsy from Mar 2013 to April 2019 in the hospital were retrospectively reviewed and analyzed. Channel loss happened in 17 cases during the procedure of nephrostomy. Methylene blue was injected into the ureteral catheter immediately and then the original channel was identified under the rigid ureteroscope. A Zebra or metal guidewire was put into the pelvicalceal system through the identified channel. Meanwhile, another metal guidewire was inserted parallel to the first guidewire and then a new channel could be established after dilation.

Results: One of the 17 cases was switched to open surgery and 16 cases were proceeded with percutaneous nephrolithotripsy successfully. There were no intraoperative or postoperative complications for all of the 17 cases.

Conclusions: Injection of methylene blue into the ureteral catheter is a feasible way to handle channel loss during the procedure of nephrostomy in percutaneous nephrolithotripsy. 


\section{MP23-11 Microperc-The Next Generation Of PCNL}

\section{A Soomro, M Murtaza}

Introduction \& Objective: To find out efficacy of microperc PCNL in selected pediatric stone disease patient.

Methods: Retrospective data for patient treated with microperc PCNL in one-year period were collected. Procedure was performed in prone position. Suitable calyx was punctured using 4.8 FR "all seeing needle "with ultrasound or fluoroscopic guidance. Stone fragmented with 200 micrometer holium laser fiber. Laser setting was 0.25 to 0.5 energy and frequency was 10 to 30 hertz. Stone fragments disintegrade up to 2 to $3 \mathrm{~mm}$.

Results: Mean age of studied children was $5.87+3.71$ and male to female ratio was $1.8: 1$. Hematuria $(34.8 \%)$ and UTI $(21.7 \%)$ were common presenting symptoms. $60.9 \%$ patients presented with stones in left kidney. Maximum stone diameter was

Table 1. Baseline Demographic and Stone Characteristics.

\begin{tabular}{|c|c|}
\hline Age & $5.87 \pm 3.71$ (range $1-12$ years) \\
\hline \multicolumn{2}{|l|}{ Gender } \\
\hline Male & $15(65.2)$ \\
\hline Female & $8(34.8)$ \\
\hline \multicolumn{2}{|l|}{ Presenting Complaint } \\
\hline Abdominal Pain & $3(13)$ \\
\hline Hematuria & $8(34.8)$ \\
\hline UTI & $5(21.7)$ \\
\hline Fever & $2(8.7)$ \\
\hline Combined & $5(21.7)$ \\
\hline \multicolumn{2}{|l|}{ Stone Laterality } \\
\hline Right & $9(39.1)$ \\
\hline Left & $14(60.9)$ \\
\hline \multicolumn{2}{|l|}{ Hydronephrosis (\%) } \\
\hline Yes & $13(56.5)$ \\
\hline No & $10(43.5)$ \\
\hline \multicolumn{2}{|l|}{ Urine Culture } \\
\hline Positive & $4(17.4)$ \\
\hline Negative & $17(73.9)$ \\
\hline Contaminated & $2(8.7)$ \\
\hline Maximum Stone Diameter & $1.28 \pm 0.15$ (range $1.0-1.5 \mathrm{~cm})$ \\
\hline Pre-op Hb & $12.77 \pm 1.07$ (range $11.1-14.5)$ \\
\hline
\end{tabular}

Table 2. Intra-operative study data and Stone Location.

\begin{tabular}{|l|l|}
\hline \multicolumn{2}{|l|}{} \\
\hline Stone Location & $4(17.4)$ \\
\hline Pelvis & $3(13.0)$ \\
\hline Lower Calyx & $3(13.0)$ \\
\hline Pelvis + Mid Calyx & $6(26.1)$ \\
\hline Pelvis + Lower Calyx & $3(13.0)$ \\
\hline Mid Calyx + Lower Calyx & $4(17.4)$ \\
\hline Upper Calyx + Lower Calyx & $41.4 \pm 5.39$ (range 32-50) \\
\hline Operating Time (mins) & $396.9 \pm 49.1$ (range 280-465) \\
\hline Irrigation Fluid (ml) &
\end{tabular}

Table 3. Post-operative Data.

\begin{tabular}{|c|c|}
\hline \multicolumn{2}{|l|}{ Double J Stent Placement (\%) } \\
\hline Yes & $9(39.1)$ \\
\hline No & $14(60.9)$ \\
\hline Post-op Hemoglobin (g/dL) & $11.29 \pm 1.0$ \\
\hline Hemoglobin Drop $(\mathrm{g} / \mathrm{dL})$ & $1.53 \pm 0.76$ (range $0.30-3.20$ ) \\
\hline \multicolumn{2}{|l|}{ Post-op Complications (\%) } \\
\hline Hematuria & $8(34.8)$ \\
\hline - Mild & $7(30.4)$ \\
\hline - Moderate & $1(4.3)$ \\
\hline Fever & $3(13.0)$ \\
\hline Blood Transfusion (\%) & $1(4.3)$ \\
\hline \multicolumn{2}{|l|}{ Stone Composition } \\
\hline Calcium Oxalate Monohydrate & $5(21.7)$ \\
\hline Calcium Oxalate Dihydrate & $10(43.5)$ \\
\hline $\begin{array}{l}\text { Mixed (Calcium Oxalate Monohydrate and } \\
\text { Dihydrate) }\end{array}$ & $7(30.4)$ \\
\hline Cystine & $1(4.3)$ \\
\hline Hospital Stay (Days) & $\begin{array}{l}3.13 \pm 0.69 \text { (Median } 3 \text { days) (range } \\
2-5 \text { days) }\end{array}$ \\
\hline Stone Free Rate at one Month (\%) & $18(78.3)$ \\
\hline - Residual Stone Size (cm) & $0.4 \pm 0.06$ (range $0.2-0.4$ ) \\
\hline Stone Free Rate at 3 Months (\%) & $22(95.7)$ \\
\hline
\end{tabular}

$1.28+0.15 \mathrm{~cm}$ (Table 1). Common stone location was Pelvis + Lower Calyx (26.1\%), Upper Calyx + Lower Calyx (17.4\%) and pelvis (17.4\%) (Table 2). Post-surgery mean $\mathrm{Hb}$ drop was $1.53+0.76 \mathrm{~g} / \mathrm{dL}$. Blood transfusion was required in only $4.3 \%$ patients. hematuria and fever were commonest post-op complications. Mean hospital stay was $3.13+0.69$ days. Double J stent was placed in $39.1 \%$ patient. Stone free rate after one month was $78.3 \%$ and stone free rate after 3 months was $95.7 \%$ (Table 3).

Conclusions: Microperc PCNL is a novel modification in endourology, which has high clearance rate. It's a safe, effective and new emerging weapon in stone war.

\section{MP23-12 Outcomes of non-lower pole puncture for supine PCNL: a multi-center study}

C Mejia Arbalaez, M Gokce, B Manzo, M Gonzalez, S Proietti, L Ajayi, J Torres Garcia, A Yaghoubian, G Giusti, P Daels, C Cristallo, G Saitta, T Birks, B Eisner

Massachusetts General Hospital

Introduction \& Objective: One of the criticisms of supine PCNL is that the surgeon is limited to lower pole access in the majority of procedures due to increased difficulty of supracostal access. We performed a multi-center retrospective review to evaluate the outcomes and complications of supine PCNL based on calyx of entry.

Methods: A multi-center, multi-national retrospective review was performed. Longitudinal medical records were reviewed. Centers were included from the following countries: Argentina, Italy, Mexico, United States, Turkey, United Kingdom.

Results: A total of 972 patients from 6 centers were included in the study. Puncture site was lower pole in 586 cases (60.2), middle calyx in 251 cases $(25.8 \%)$, and upper pole in 135 cases 
$(13.9 \%)$. Mean patient age and $\%$ female by location of puncture were as follows: Lower pole mean age $=50$ years, $41 \%$ female; middle calyx mean age 52 years, $37 \%$ female, upper pole mean age 53 years, $50 \%$ female. Mean stone size was similar between the groups (lower pole $2.9 \mathrm{~cm}$, middle calyx $2.8 \mathrm{~cm}$, upper pole $2.8 \mathrm{~cm}, \mathrm{p}=\mathrm{NS})$. Stone free rates were as follows: lower pole $62.9 \%$, middle calyx $80.1 \%$, upper pole $54.6 \%$ ( $p<0.001)$. Two patients in the study required chest tube placement for thoracic complications ( 1 in lower pole group, 1 in middle calyx group). Two patients in the study were admitted to the intensive care unit for septic physiology ( 1 in the lower pole group, 1 in the middle calyx group).

Conclusions: In this series middle calyx and upper pole puncture were not associated with a significant increase in complications or an unacceptable stone free rate. Surgeons considering supine PCNL should understand that middle and upper calyx puncture in the supine position is feasible, safe, and effective.

\section{MP23-13 Non-papillary mini-percutaneous nephroli- thotomy: Early experience}

P Kallidonis, A Vagionis, C Adamou, P Ntasiotis,
K Pagonis, E Liatsikos

University of Patras

Introduction \& Objective: This study aims the evaluation of the non-papillary puncture in mini-Percutaneous Nephrolithotomy (mini-PCNL) surgery in terms of safety and efficacy.

Methods: A total number of 32 patients was subjected to miniPCNL over a year. Non-papillary puncture was performed and followed by one- step track dilation up to 22Fr. A $18 \mathrm{Fr}$ nephroscope (Slender, Karl Storz GmbH, Germany) and an ultrasound lithotripter (Lithoclast Master, EMS S.A, Switzerland) with 9.9Fr probe was used. Demographics and perioperative data are prospectively collected in an institutional board approved database and the current data were retrospectively collected.

Results: The average cumulative stone size was $23.53 \pm 6.6 \mathrm{~mm}$. Mean operative time was $44.6 \pm 13.44$ minutes and primary stonefree rate after PCNL was $100 \%$ and $88.2 \%$ for single $(n=16)$ and multiple stones $(n=16)$, respectively. Second access was performed in 7 cases, all of which had multiple stones. Mean hemoglobin drop was $1.23 \pm 0.88 \mathrm{gr} / \mathrm{dL}$. The patients stayed $2.56 \pm 0.98$ days in the hospital. Overall complication rate was $9.37 \%$, without encountering any severe bleeding complication.

Conclusions: Using non-papillary puncture technique and an endoscope with a ultrasonic lithotripter allowed the removal of large stone burden with limited use of multiple accesses. The results regarding the stone free rate, operating time, hemoglobin loss and complications were similar to large scale studies which used papillary puncture.

\section{MP23-14 Factors Increasing the Likelihood of SIRS in} Patients Undergoing Percutaneous Nephrolithotomy

AR Omorogbe, JN Bamberger, E Rosenbluth, H Kwun, A Kapoor, M Gupta, AM Zampini

Mount Sinai

Introduction \& Objective: One of the most dreaded complications of PCNL is SIRS. Prior studies have shown that preexisting urinary tract infection and positive stone culture increase
Table 1:

Comparison of demographic, clinical, and operative parameters in patients who developed SIRS versus those who did not following percutaneous nephrolithotomy

\begin{tabular}{|c|c|c|c|}
\hline & $\begin{array}{l}\text { No SIRS } \\
N=256\end{array}$ & $\begin{array}{c}\text { SIRS } \\
\mathrm{N}=30\end{array}$ & $p$-value \\
\hline \multicolumn{4}{|l|}{ Demographics } \\
\hline Age, years & 57.40 & 55.80 & 0.558 \\
\hline Sex, Male:Female & $125: 131$ & $14: 16$ & 0.609 \\
\hline $\mathrm{BMI}, \mathrm{kg} / \mathrm{m}^{2}$ & 28.30 & 26.80 & 0.216 \\
\hline Mean ASA & 2.21 & 2.31 & 0.522 \\
\hline Diabetes Mellitus, \% & 21.09 & 25.93 & 0.561 \\
\hline Coronary Artery Disease, \% & 8.27 & 14.81 & 0.256 \\
\hline Hypertension, \% & 43.53 & 44.44 & 0.927 \\
\hline Hyperlipidemia, \% & 13.33 & 25.93 & 0.078 \\
\hline Hypercholesterolemia, \% & 24.80 & 23.10 & 0.842 \\
\hline \multicolumn{4}{|l|}{ Clinical and Operative Data } \\
\hline History of UTI within One year, \% & 28.90 & 40.00 & 0.249 \\
\hline Positive Preoperative Urine Culture, $\%$ & 26.30 & 34.60 & 0.365 \\
\hline Positive of Intraoperative Urine Culture, \% & 2.00 & 7.70 & 0.125 \\
\hline Positive Stone Culture, \% & 12.60 & 38.50 & 0.001 \\
\hline Immunocompromised, \% & 9.00 & 8.00 & 0.865 \\
\hline \multicolumn{4}{|l|}{$>60 \%$ of Stone Composition, $\%$} \\
\hline Calcium Oxalate & 51.00 & 38.50 & 0.224 \\
\hline Struvite & 3.20 & 7.70 & 0.246 \\
\hline Carbon Appetite & 14.70 & 30.80 & 0.035 \\
\hline Calcium Phosphate & 1.20 & 0.00 & 0.575 \\
\hline Uric Acid & 22.10 & 15.40 & 0.428 \\
\hline Cystine & 1.60 & 0.00 & 0.513 \\
\hline No Predominate Component & 5.60 & 7.70 & 0.660 \\
\hline \multicolumn{4}{|l|}{ Stone Location, \% } \\
\hline Upper Pole & 16.00 & 28.60 & 0.098 \\
\hline Interpolar & 11.40 & 25.00 & 0.042 \\
\hline Lower Pole & 62.00 & 64.30 & 0.815 \\
\hline Renal Pelvis & 34.20 & 35.70 & 0.871 \\
\hline \multirow[t]{4}{*}{ Number of Tracts, \% } & & & 0.000 \\
\hline & 51.00 & 56.00 & \\
\hline & 48.60 & 36.00 & \\
\hline & 0.40 & 14.29 & \\
\hline \multicolumn{4}{|l|}{ Outcomes } \\
\hline Mean Hospital Stay, days & 1.07 & 2.43 & 0.055 \\
\hline
\end{tabular}

risk of post-operative infectious complications. However, despite updated recommendations for preoperative cultures and antibiotic prophylaxis, reported cases of SIRS remains consistent across practices. We sought to elucidate other factors, infectious and non-infectious, that may increase the likelihood of SIRS following PCNL.

Methods: We queried our prospectively maintained database of 628 patients who underwent PCNL at a single comprehensive stone center from 2014 to 2018. Patients were included if their chart documented all SIRS criteria points including two or more of: temperature $>38 \mathrm{C}$ or $<36 \mathrm{C}$, pulse $>90 \mathrm{bpm}$, respiratory rate $>20$ breaths/min, and leukocyte count $>12,000$ or $<4,000$. X2 and independent samples t-test analyses were conducted to identify factors associated with infection between the two groups.

Results: Of the 628 patients, 286 were included in our analysis. Of this cohort, 30 (10.5\%) developed SIRS and $256(89.5 \%)$ did not. Significantly, higher rates of positive stone culture $(38.5 \% \mathrm{vs}$ $12.6 \%, p=0.001)$, presence of interpolar calculi $(25 \%$ vs $11 \%$, $p=0.042)$, predominate carbon apatite stone composition $(30.8 \%$ vs. $14.7 \%, p=0.035)$, and procedures requiring more than two tracts $(14.29 \%$ vs $0.4 \%, p=0.000)$ were associated with SIRS. No significant differences in comorbidities, history of UTI within one-year, pre-existing stent or nephrostomy tube, or change in hematocrit, hemoglobin, or creatinine were noted between the two groups.

Conclusions: Based on our analysis, both infectious factors positive stone culture - and non-infectious factors - interpolar stones, carbon apatite stone composition, and greater access tracts - were associated with an increased risk of post-operative SIRS. Pre-operative factors previously suggested to be predictive (positive urine culture or pre-existing stent/nephrostomy tube) were not significantly associated with SIRS in our study. 
MP23-15 The Effect of Continuing Aspirin on Postoperative Outcomes in Percutaneous Nephrolithotomy

E Rosenbluth, JN Bamberger, AR Omorogbe, B Gallante, RA Chandhoke, H Kwun, A Kapoor, M Gupta, AM Zampini

Mount Sinai

Introduction \& Objective: Percutaneous nephrolithotomy (PCNL) can be associated with significant blood loss and other complications. Special consideration must therefore be taken when performing PCNL in patients on chronic aspirin therapy. For these patients, it remains unclear whether aspirin should be discontinued prior to PCNL. This study attempts to determine whether a patient's aspirin status affects blood loss or other postoperative outcomes in PCNL.

Methods: We queried our prospectively maintained database of 682 patients who underwent PCNL at a tertiary stone center from January 2015 to May 2019. Patients whose aspirin status was included in the database were separated into three groups: No Aspirin (NA), Discontinued Aspirin (DA) 7 days before PCNL, and Continued Aspirin (CA). Demographic, operative, and outcome data were compared among the groups.

Results: From our cohort, 428 patients were included in the study. Of those, 334, 38, and 52 patients were included in the NA, DA, and CA groups, respectively. The CA group was older on average compared to DA and NA (66.4 years vs. 65.2 vs. 56.5 , $\mathrm{p}=0.001)$. The CA group also had significantly higher BMI, and higher rates of the documented comorbid medical conditions. There were no significant differences in post-op length of stay, operative time, serious complications (Clavien $\geq \mathrm{III}$ ), or change in hematocrit, hemoglobin, or creatinine from pre-op to PACU or post-op day 1 . The CA group had significantly higher transfusion rates compared to DA and NA $(7.9 \%$ vs. $0.0 \%$ vs. $0.4 \%$, $\mathrm{p}=0.001)$.

Conclusions: Continuing aspirin during PCNL was not associated with increased postoperative blood loss based on laboratory values alone. However, it was associated with an increased rate of blood transfusion postoperatively. There was no significant

Table 1.

Comparison of pre-operative factors and outcomes among patients who were never on aspirin. discontinued aspirin or continued aspirin prior to PCNL.

\begin{tabular}{|c|c|c|c|c|}
\hline & $\begin{array}{l}\text { Never } \\
\text { Aspirin } \\
\mathrm{N}=334\end{array}$ & $\begin{array}{c}\text { Discontinued } \\
\text { Aspirin } \\
\mathrm{N}=38\end{array}$ & $\begin{array}{c}\text { Continued } \\
\text { Aspirin } \\
\mathrm{N}=52\end{array}$ & $p$-value \\
\hline \multicolumn{5}{|l|}{ Demographics } \\
\hline Age, mean (years) & 56.5 & 65.18 & 66.44 & 0.001 \\
\hline Sex, Percent Male & 48.5 & 63.2 & 46.2 & 0.202 \\
\hline Mean BMI $\left(\mathrm{kg} / \mathrm{m}^{2}\right)$ & 27.96 & 29.74 & 29.92 & 0.049 \\
\hline Mean ASA Score & 2.15 & 2.5 & 2.5 & 0.000 \\
\hline Mean Pre-op Hematocrit & 40.281 & 40.208 & 39.083 & 0.355 \\
\hline Mean Total Stone Burden (mm) & 31.43 & 31.17 & 33.99 & 0.655 \\
\hline \multicolumn{5}{|l|}{ Comorbidities (\%) } \\
\hline Diabetes Mellitus & 18 & 34.3 & 49 & 0.001 \\
\hline Hyperlipidemia & 16.4 & 25.7 & 40.8 & 0.001 \\
\hline Coronary Artery Disease & 7 & 14.3 & 40 & 0.001 \\
\hline Hypertension & 36.8 & 54.3 & 74 & 0.001 \\
\hline Hypercholesterolemia & 13.2 & 28.9 & 38.5 & 0.001 \\
\hline \multicolumn{5}{|l|}{ Outcomes } \\
\hline \multicolumn{5}{|l|}{ Mean Change in Hematocrit } \\
\hline Pre-op to PACU & -1.61 & -1.48 & -1.62 & 0.988 \\
\hline Total (Pre-op to POD1) & -3.76 & -3.66 & -5.01 & 0.406 \\
\hline \multicolumn{5}{|l|}{ Mean Change in Hemoglobin } \\
\hline Pre-op to PACU & -0.55 & -0.3 & -0.44 & 0.683 \\
\hline Total (Pre-op to POD1) & -1.76 & -1.58 & -1.81 & 0.863 \\
\hline \multicolumn{5}{|l|}{ Mean Change in Creatinine } \\
\hline Pre-op to PACU & -0.04 & -0.02 & -0.02 & 0.897 \\
\hline Total (Pre-op to POD1) & -0.05 & -0.02 & -0.02 & 0.746 \\
\hline Mean Operative Time (min) & 97.02 & 89.27 & 99.06 & 0.774 \\
\hline Stent Placed (\%) & 48.8 & 39.5 & 42.3 & 0.520 \\
\hline Mean Length of Stay (days) & 1.27 & 1.04 & 1.3 & 0.686 \\
\hline Patients Transfused (\%) & 0.4 & 0 & 7.9 & 0.001 \\
\hline Patients with Clavien ₹Illa (\%) & 4.7 & 0 & 11.8 & 0.320 \\
\hline
\end{tabular}

difference in the rate of serious complications (Clavien $\geq$ IIIa) following PCNL among those that continued aspirin. While it may be safe to continue aspirin, surgeons should carefully weigh the risks and benefits of aspirin therapy in patients with cardiovascular disease undergoing PCNL.

MP23-16 Percutaneous nephrolithotomy in the transplant kidney - a prospective 20 year multicenter experience

T Birks, C Ferreiro, J Withington, M Moghul, C Torrecilla, F Vigués, O Angerri Feu, A Breda, L Ajayi, E Emiliani

Royal Free Hospital, London

Introduction \& Objective: Percutaneous nephrolithotomy (PCNL) in transplanted kidneys presents unique endourological challenges. By pooling the collective experience of three centers, each combining high volume transplant and high volume endourology practices, we aim to accurately and reliably demonstrate the safety and feasibility of this technique.

Methods: Prospective data from 3 high volume centers in 2 healthcare systems were combined for analysis, regarding patient, stone, technique and outcomes. Data were collected from 1998-2018; 17 transplant PCNLs were recorded.

Results: All patients were operated in a form of supine position (5 patients underwent surgery in a modified Valdivia position, 10 standard supine and 2 lithotomy). 7 procedures were performed on left iliac fossa (LIF) transplants, 10 in RIF transplants. 5 stones were located in the renal pelvis, 3 at the pelviureteric junction, 2 in the pelvis and proximal ureter, 3 in the ureter, 2 lower pole, 1 upper pole, 1 interpolar. Puncture was made with ultrasound alone in 5cases, 2 used fluoroscopy and in 10 a combination was used. 7 punctures were into lower pole, 3 upper pole, 3 interpolar, 1 inter- and upper pole and 1 not recorded. 6 cases used a combined approach with ureteroscopy. 3 were planned as staged procedures. Fragmentation was by Swiss lithoclast $^{\mathrm{TM}}$ in 7 cases and LASER in 5.5 were 'lift-out'. All patients had nephrostomies post-op; 3 had stents.

\begin{tabular}{lc}
\hline $\mathbf{N}=$ & $\mathbf{1 7}$ \\
\hline Male (\%) & $11(61)$ \\
Median age (range) & $51(36-74)$ \\
Median BMI (range) & $27.4(20.0-38.0)$ \\
Median CCI (range) & $4.5(2-7)$ \\
Median Stone Size,mm (range) & $16(7-60)$ \\
Median Procedure time (range) & $65(30-120)$ \\
Median Fluoroscopy time (range) & $84(3.1-372.0)$ \\
Stone clearance* (\%) & $14(82)$ \\
Complications (Clavien I-V) & 1 (Clavien IIIa) \\
Transfusion & \multicolumn{1}{c}{0} \\
\hline
\end{tabular}

Conclusions: In our collective experience, PCNL is a safe and effective technique in treating nephrolithiasis in transplant kidneys; stone clearance and complication rates compare favorably with contemporary 'native' renal PCNL data. Combination with endoscopic retrograde intrarenal surgery (ECIRS) is preferable to multiple punctures of the renal allograft. Supine PCNL both enables ease of access for ECIRS and the iliac fossa kidney. In our experience, patients benefit from surgical care within highvolume centers with dedicated transplant and endourological expertise. 


\section{MP23-17 Surgical outcomes of percutaneous ne- phrolithotomy in 3402 Patients and results of stone analysis in 1559 patients}

M Hussain, S Askari, A Hashmi, M Lal, M Jabeen, A Khalique

Sindh Institute of Urology and Transplantation

Introduction \& Objective: To report our experience of a series of percutaneous nephrolithotomy (PCNL) procedures in a single center over 18 years in terms of patient and stone characteristics, indications, stone clearance and complications, along with the results of chemical analysis of stones in a subgroup.

Methods: We retrospectively analyzed the outcomes of PCNL in 3402 patients, who underwent the procedure between 1997 and 2014, obtained from a prospectively maintained database. Data analysis included patients' age and sex, laboratory investigations, imaging, punctured calyx, duration of operation, volume of irrigation fluid, radiation exposure time, blood transfusion, complications and stone-free status at 1-month follow-up. For the present analysis, outcomes in relation to complications and success were divided in two eras, 1997-2005 and 2006-2014, to study the differences.

Results: Of the 3402 patients, 2501 (73.5\%) were male and 901 $(26.5 \%)$ were female, giving a male: female ratio of 2.8:1. Staghorn (partial or complete) calculi were found in $27.5 \%$ of patients, while $72.5 \%$ had non-staghorn calculi. Intracorporeal energy sources used for stone fragmentation included ultrasonography in 917 patients $(26.9 \%)$, pneumatic lithoclast in 1820 $(53.5 \%)$, holmium laser in $141(4.1 \%)$ and Lithoclast ${ }^{\circledR}$ master in $524(15.4 \%)$. In the majority of patients $(97.4 \%)$ a $18-22-\mathrm{F}$ nephrostomy tube was placed after the procedure, while 69 patients (2.03\%) underwent tubeless PCNL. The volume of the irrigation fluid used ranged from 7 to $37 \mathrm{~L}$, with a mean of $28.4 \mathrm{~L}$. The stone-free rate after PCNL in the first era studied was $78 \%$, vs $83.2 \%$ in the second era, as assessed by combination of ultrasonography and plain abdominal film of the kidney, ureter and bladder. The complication rate in the first era was $21.3 \%$ as compared with $10.3 \%$ in the second era, and this difference was statistically significant. Stone analysis showed pure stones in $41 \%$ and mixed stones in $58 \%$ of patients. The majority of stones consisted of calcium oxalate.

Conclusions: This is the largest series of PCNL reported from any single center in Pakistan, where there is a high prevalence of stone disease associated with infective and obstructive complications, including renal failure. PCNL as a treatment method offers an economic and effective option in the management of renal stone disease with acceptable stone clearance rates in a resource-constrained healthcare system.

MP23-18 PCNL: A safe alternative to guideline recommendations for stones less than $1 \mathrm{~cm}$

T Chi, M Armas-Phan, S Wiener, W Hu, B Xiao, B Su, M Stoller, T Chi, J Li

University of California, San Francisco

Introduction \& Objective: We hypothesize that percutaneous nephrolithotomy (PCNL) can be performed safely in patients with stones $<10 \mathrm{~mm}$ with minimal risk of complication and higher stone free rate compared to URS or SWL. Using an international stone registry, we investigated the stone free and complication rates after PCNL for stones $<10 \mathrm{~mm}$ relative to URS and/or SWL, and to determine international practice pattern differences in PCNL utilization.

\begin{tabular}{|l|c|c|}
\hline $\begin{array}{l}\text { Multivariate analysis of } \\
\text { characteristics predicting for } \\
\text { performance of PCNL }\end{array}$ & $\begin{array}{c}\text { Odds Ratio }(95 \% \text { Confidence } \\
\text { Interval) }\end{array}$ \\
\hline Age & $0.96(0.93-0.98)$ & 0.002 \\
\hline Female gender & $2.67(1.15-6.28$ & 0.022 \\
\hline BMI & $0.89(0.80-1.00)$ & 0.041 \\
\hline Lower pole stones & $1.51(0.60-4.00)$ & 0.377 \\
\hline Proximal ureteral stones & $1.23(0.27-5.58)$ & 0.786 \\
\hline Stone size & $0.90(0.76-1.06)$ & 0.214 \\
\hline Abnormal anatomy (Guy & $2.50(0.58-10.8)$ & 0.219 \\
\hline Stone Score) & $1.29(0.55-3.00)$ & 0.558 \\
\hline ASA score & $4.26(0.92-19.7)$ & 0.064 \\
\hline
\end{tabular}

Methods: Prospectively collected international data from the Registry for Stones of the Kidney and Ureter (ReSKU) composed of patients from the United States and China between September 2015 to July 2018 were investigated to evaluate characteristics of surgical patients treated for stones $<10 \mathrm{~mm}$. Data on patient age, gender, BMI, stone location, delay to surgery, complication and transfusion rates, and stone clearance efficiency were analyzed. Results: A total of 2329 surgical stone procedures were undertaken; 303 procedures for stones $<10 \mathrm{~mm}$ were identified. Multivariate analysis revealed that older age $(\mathrm{p}=0.002$, OR 0.96 per year) and higher BMI ( $p=0.041$, OR 0.89 per increase in BMI unit) was associated with lower likelihood of PCNL for stones $<10 \mathrm{~mm}$. Female gender was associated with higher rate of PCNL. There were no differences in complication or transfusion rates. Stone clearance was higher in patients undergoing PCNL (93\% vs $82 \% ; p=0.103$ ) but this was not statistically significant.

Conclusions: When controlling for other variables, PCNL for stones $<10 \mathrm{~mm}$ is more commonly performed in younger patients, men, and patients with a lower BMI. There is no difference in complications or transfusion rates when comparing patients undergoing PCNL versus URS or SWL. In centers with a high volume of PCNL procedures, using this approach for small stones can be performed safely with no difference in complication rates relative to other procedures.

\section{MP23-19 ULTRA-mini PERC under local anesthesia: a novel approach in select cases}

C Chalouhy, M Moukarzel, S Slaba, A Tabche, E Hanna, N Harake, T Smayra

Introduction \& Objective: Mini-Perc and super-mini Perc have successfully decreased the size of renal access catheters and sheaths to $14 \mathrm{fr}$ allowing the expansion of percutaneous nephrolithotomy to a special patient population i.e. pediatric etc. We have been evaluating the safety and efficacy of Ultra-mini Perc, a novel technique using antegrade flexible ureteroscopy through a $10 \mathrm{fr}$ renal access catheter under local anesthesia in high risk patients for general anesthesia and patients with complex lower tract anatomy.

Methods: Patients who were considered high risk for general anesthesia or spinal anesthesia underwent percutaneous antegrade flexible ureteroscopy as an alternative for RIRS or conventional PCNL/Mini Perc. A $10 \mathrm{fr}$ renal access catheter is placed under local anesthesia using fluoroscopic and ultrasound guidance. Lithovue - Boston Scientific flexible ureteroscopes with various deflections depending on the case were used for endoscopic laser fragmentation or dusting of the stones. Over distention of the renal pelvis by irrigation was avoided by continuous emptying through a side channel. Flexible ureteroscopy 


\begin{tabular}{|l|c|}
\hline Characteristics & \\
\hline Patient major comorbidities = avg & 2 \\
\hline Average stone size & $23 \mathrm{~mm}$ \\
\hline OR time & $210 \mathrm{~min}$ \\
\hline Stone free rate & $77 \%$ \\
\hline Estimated blood loss & $0 \mathrm{ml}$ \\
\hline Complex lower urinary tract anatomy & $67 \%$ \\
\hline
\end{tabular}

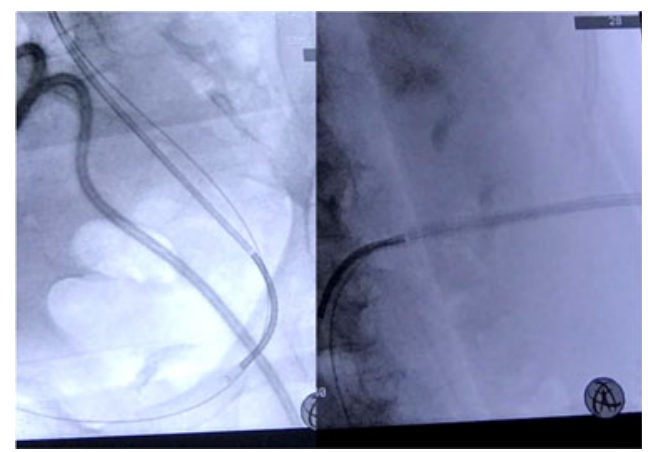

is carried out down the ureter to the uretero-vesical junction. A 6fr stent is placed at the end of the procedure.

Results: Our patient population $(n=3)$ average age was 52 and associated major comorbidities were 2 . They were considered high risk or very high risk to receive general anesthesia. The most determining risk factors for stratification were recent coronary stenting, congestive heart failure and severe COPD. The average stone size was $23 \mathrm{~mm}$. One of the patients had a solitary kidney and was started on hemodialysis before the procedure. The average procedure time was 210 minutes. a small dose of $1 \mathrm{mg}$ of morphine was given when allowed by the anesthesia team or if the patient complained of pain. The stone free rate approached $77 \%$. The JJ stent was placed in average for 6 weeks. The hospital stay was relatively prolonged up to 11 days irrelevant of the procedure. A nephrostomy was placed for 24 hours postoperatively in one patient who previously had it. No complications or transfusions were noted. Lithovue flexible ureteroscope with standard and reverse deflection were needed for efficient access to renal pelvis and calices in the antegrade fashion.

Conclusions: Ultra-mini percutaneous antegrade flexible ureteroscopy with laser fragmentation of kidney stones is a feasible, safe and a successful alternative for patients who are considered high risk for anesthesia and in need for treatment of obstructive kidney stones.

\section{MP23-20 Treatment of kidney stones by micro-NLPC in patients with urinary diversion.}

R Boissier, T Negre, F Michel, G Karsenty, E Lechevallier

Aix-Marseille University, Marseille, France; Department of Urology and Renal Transplantation, APHM, Conception University Hospital, Marseille, France

Introduction \& Objective: Flexible ureteroscopy is an effective and mildly morbid technique for the treatment of nephrolithiasis but in case of history of urinary diversion (bricker, Mitrofanoff) its effectiveness may be limited. Micro-NLPC is a minimally invasive percutaneous technique. The aim of this work was to evaluate the impact of a history of urinary diversion on the efficacy and feasibility of micro-NLPC for nephrolithiasis.
Methods: Retrospective bicentric study including 31 patients treated for nephrolithiasis by micro-NLPC. Group UD (5 patients) had urinary diversion (4 bricker, 1 enterocystoplasty with Mitrofanoff + Closure of the bladder neck) because of neurological diseases ( 2 multiple sclerosis, 3 spine trauma), were compared with patients with normal urinary anatomy (group N, 26 patients). All patients were operated in Valdivia position. Enlithotripsy was performed with a 200um laser fiber and a HolmiumYAG source in dusting mode.

Results: Clinical data for patients in groups $\mathrm{D}$ and $\mathrm{N}$ were : Mean age $45 \pm 12$ vs $53 \pm 18$ years $(\mathrm{p}=0.22)$, mean BMI $24 \pm 7$ vs $27 \pm 4(\mathrm{p}=0.38)$, Charlson median 2 [2-4] vs 0 [0-2] $(\mathrm{p}=0.02)$, mean GFR $135 \pm 45$ vs $98 \pm 28(\mathrm{p}=0.15)$, median number of stones 2 [1-5] vs1 [1-1] $(\mathrm{p}=0.04)$, mean density $800 \pm 258$ vs $1100 \pm 224(\mathrm{p}=0.06)$, mean size $(\mathrm{mm})$ calculation $13 \pm 4$ vs $14 \pm 6(\mathrm{p}=0.96)$. Preoperative urine culture was positive and preoperative antibiotic were significantly more common in group D $80 \%$ vs $4 \%(\mathrm{p}<0.01)$. Mean operating times $95 \pm 25$ vs $81 \pm 37 \mathrm{~min}(\mathrm{p}=0.29)$, hospitalization duration 3 [2-7] vs 1 [17], success rate (without fragment or fragments $<3 \mathrm{~mm}$ not requiring no e reprocessing) were $60 \%$ vs. $69 \%(\mathrm{p}>0.05)$. Infectious complication rates were similar $40 \%$ vs $27 \%(\mathrm{p}=0.30)$.

Conclusions: In this comparative study microNLPC for nephrolithiasis, a history of urinary diversion impacted nor the feasibility nor the efficacy of treatment (operative time, success rate and similar complications). The micro-NLPC is a minimally invasive alternative to ureteroscopy for the treatment of kidney stones with a history of urinary diversion.

\section{MP23-21 Withdrawn}

\section{MP23-22 It is Time For Supine PCNL Now: A Case Control Study}

PK Pandey, S Shukla

Hind Institute of Medical Sciences

Introduction and Objective: Conventionally, percutaneous nephrolithotomy (PCNL) is performed in prone position. However, lately increasing number of urologist are embarking on supine PCNL. This trend may be due to certain specific indications or in conjunction with patient safety and growing experience with this technique. The aim of the study is to evaluate feasibility and efficacy of supine PCNL compared to prone PCNL.

Methods: We conducted prospective case control study over a period of one year from Jan 2018 to $31^{\text {st }}$ Dec 2018. Patients with kidney stones admitted to Urology division of Surgery Department of tertiary care hospital were enrolled in the study. Patients were informed; consented and institutional ethical clearance was obtained. Twenty-five patients each underwent supine PCNL (cases or Group I) and prone PCNL (controls or Group II) during same period. Population in both the groups were matched in terms of stone location, stone burden, co-morbidities, body mass index, age groups and sex ratio. The variables noted and statistically analyzed included operative time, intra-operative and post-operative complications, stone clearance rates and duration of stay in the hospital. Results: The mean age of population of Group I was $26 \pm 17.4$ years and Group II was 29 \pm SD 15.2 years. Male to female ratio in Group I was $1.3: 1$ and 1.7:1 in Group II. The surgery in one patient of Group I was staged as pyonephrosis was encountered. One patient in Group II was shifted to Group I owing to anatomic 
abnormality leading to difficulty in positioning. The mean intraoperative time in cases was statistically lower when compared to controls $(p=0.01)$. None of the patients in group I and group II needed blood transfusions. The change in Haemoglobin levels in both the groups before and after the procedure was statistically insignificant $(p=0.10)$. Complete stone clearance was achieved in 24 patients in group I and 23 in group II. Subsequently, one patient in Group I and 2 patients in Group II needed extracorporeal shockwave lithotripsy at a later date.

Conclusions: Supine PCNL is equally effective and safe compared to prone PCNL, with added benefit of decreased intraoperative time.

\section{MODERATED POSTER SESSION 24: CLINICAL STONES: OUTCOMES (II)}

\begin{abstract}
MP24-01 Opiate Prescribing Patterns and Risk Factors for Prolonged Opiate Use in Patients with Acute Nephrolithiasis
\end{abstract}

B Cotta, S Bechis

UC San Diego

Introduction \& Objective: Over 2 million Americans abuse or are addicted to prescription opiates. In a urology practice, patients presenting with acute conditions such as renal stone colic may be at risk. We sought to analyze prescribing patterns at our institution and identify risk factors associated with continued use of opiates during episodes of acute nephrolithiasis.

Methods: We retrospectively reviewed patients presenting to our Comprehensive Kidney Stone Center with an acute stone episode. Data collected included patient demographics, size and location of stone, treatment plan, and time to stone passage or procedure. Opiate prescription data was obtained from the state of California's prescription drug monitoring program, CURES 2.0 (Controlled Substance Utilization Review and Evaluation System). Data collected included new and current opiate prescriptions as well as prescribers and quantity/strength of opiates. Results: 55 patients presented to clinic with both a stone confirmed on imaging and acute pain from 6/1/2017-1/31/2018. Median age was 53 years with 35 male patients. 53\% had history of nephrolithiasis. Median stone size was $6(2-28) \mathrm{mm}$ with $40 \%$ in the distal ureter. $44(80 \%)$ patients initially presented to the Emergency Department (ED) and $6(11 \%)$ went to their primary physician. Ultimately, $22(40 \%)$ patients passed their stones and $33(60 \%)$ underwent a procedure to clear their stone. $9(16.4 \%)$ patients had an existing opiate prescription at presentation, and $36(65.5 \%)$ filled an opiate prescription during their acute stone episode. 31 of $44 \mathrm{ED}$ patients $(70.5 \%)$ were given an opiate prescription with an average of 14 pills dispensed. 20 out of 55 patients refilled an opiate prescription prior to resolution of their stone episode; $6(30.0 \%)$ had an existing opiate prescription prior to the stone episode. $18(90.0 \%)$ ultimately underwent a procedure to clear their stone. Of the 35 patients who did not refill an opiate prescription prior to resolution, $3(9 \%)$ had an existing prescription, 17 (49\%) had a prior history of stones with median stone size $5.5 \mathrm{~mm}$, and 15 (43\%) ultimately underwent a procedure for the stone.

Conclusions: Despite the efficacy of non-opiate medications in treating acute renal colic, the majority of patients presenting to the ED are still prescribed opiates. Patients with a history of current opiate use, prior history of nephrolithiasis, and larger stones requiring interval stenting and definitive surgery are more likely to refill opiate prescriptions. These characteristics likely represent a group of patients who may be at risk for prolonged opiate use.
Further provider education and development of pain management algorithms for renal colic in the ED may be beneficial.

\section{MP24-02 Calcium Phosphate Stones Predominate Among Patients with Recurrent Nephrolithiasis Re- quiring Surgical Intervention}

\author{
A Arnouk, A Vig, MD White
}

Albany Medical College

Introduction \& Objective: Over the past 20 years, there has been a well-documented increase in the incidence and recurrence rate of patients with nephrolithiasis. Recurrence rates have reached approximately $50 \%$ at a 3 to 5 -year time interval. At our institution, a single surgeon has performed over 4,000 surgical procedures for stone disease over a 20 -year time period. During this time period, we have seen multiple patients requiring repeat non-staged surgical interventions and hypothesize that this cohort of patients have a different predominant stone composition than patients who have had a single stone procedure. We thus sought to evaluate the surgical recurrence rate and predominant stone composition of patients undergoing 3 or more non-staged surgical stone procedures over this time period.

Methods: We retrospectively evaluated patients who were surgically treated for stone disease from July 1998 to September 2017 by a single surgeon. Patients treated after 2007 were analyzed, given stone composition data were more readily available. Patients who underwent 3 or more non-staged procedures were included in our cohort. Data were collected including age, sex, procedure type, stone composition, time to recurrence, and other relevant factors. This cohort was compared with patients who underwent a single stone procedure during this time period, age and sex-matched. Surgical recurrence rate and most predominant component of stone compositions were analyzed.

Results: There were 44 patients who underwent 3 or more nonstaged stone procedures within the study time period. The mean rate of recurrence was 659 days ( 21 months). The predominant stone component in our 3 or more non-staged stone procedures group was calcium phosphate in $50 \%$, calcium oxalate monohydrate in $25 \%$, uric acid in $9 \%$, struvite (magnesium ammonium phosphate) in $6 \%$, calcium oxalate dihydrate in $5 \%$, and cysteine in $5 \%$. The predominant stone component in our control group was calcium oxalate monohydrate in $63 \%$, calcium oxalate dihydrate in $20 \%$, calcium phosphate in $11 \%$, uric acid in $5 \%$, and no patients with struvite or cysteine stones.

Conclusions: Patients within our cohort who underwent at least 3 non-staged stone procedures during study time period were 
noted to have a mean recurrence rate of 21 months. The most prevalent predominant stone component within this cohort was calcium phosphate, compared to calcium oxalate in our control group (Fisher's exact test $\mathrm{p}<0.001$ ). These data can then be used to guide surveillance management strategies and counsel patients at high risk for recurrent stone formation.

\section{MP24-03 Comparison of Mini PCNL v/s Retrograde intrarenal surgery (RIRS) v/s Extracorporeal shock- wave lithotripsy (ESWL) for the treatment of renal stones up to 2.5 centimeters}

\author{
PK Pattnaik, SK Pattnaik, MP Pattnaik \\ S S Urological \& Research Institute, Bombay Hospital \\ PG Institute
}

Introduction \& Objective: To evaluate the safety and efficacy of Mini PCNL v/s Retrograde intrarenal surgery (RIRS) v/s Extracorporeal shockwave lithotripsy (ESWL) for the treatment of renal stones up to 2.5 centimeters.

Methods: This prospective randomized study was conducted between March 2016 to February 2018. After taking written informed consent, 195 patients with renal calculi up to 2.5 centimeters were included in the study. Patients were divided into three groups by computer generated random numbers. Patients in Group A $(n=68)$ underwent Mini PCNL, Group B $(n=61)$ underwent RIRS, Group C $(n=66)$ underwent ESWL. A ureteric stent was placed in all patients undergoing RIRS and ESWL. All patients were operated under regional/intravenous anesthesia. STORZ 12.5 Fr Mini Nephroscope with 15 Fr sheath was used for mini PCNL. WOLF (COBRA) Flexible uretero-renoscope was used for RIRS. Siemens Modularis Variostar Lithotripter was used foe ESWL. Mini PCNL and ESWL were totally Ultrasound guided procedures.

Results: There were 103 Males and 92 Females. Patients were in the age group of 17 to 68 years. 72 patients had calculi in renal pelvis, 36 in upper calyx, 22 in middle calyx and 65 patients had stones in lower calyx. The mean duration of procedure was $35 \pm 10.14 \mathrm{~min}$ in the Mini PCNL group, $80.55 \pm 15.27 \mathrm{~min}$ in the RIRS group and $45 \pm 10.15$ min in ESWL group. The hospital stay was shortest in the ESWL group (Daycare) followed by RIRS ( $45 \pm 2.5$ hours) and ( $35 \pm 1.5$ hours) for Mini PCNL. Stone-free rates after one session were $99.2 \%$ for Mini PCNL, $87.5 \%$ for RIRS and $82.4 \%$ for ESWL Group. The stone-free rate of the RIRS group improved to $97.7 \%$ after the second session. No patients developed severe complications.

Conclusions: This study revealed that Mini PCNL had the highest stone clearance rates in a single session. However, RIRS and ESWL had comparable stone free rates.

MP24-04 The Risk of Post-Operative Sepsis following Ureteroscopy or Percutaneous Nephrolithotomy: An Analysis of NSQIP Data

\section{J Bjazevic, H Razvi}

Western University

Introduction \& Objective: Urinary stone disease is a highly prevalent condition worldwide with a rising incidence. While the mortality rate of urolithiasis is low, the development of sepsis following surgical intervention remains a leading cause of morbidity and mortality. However, risk factors for the develop- ment of sepsis and strategies to mitigate this risk remain poorly defined. We aimed to evaluate the rate of sepsis following both ureteroscopy (URS) and percutaneous nephrolithotomy (PCNL) over time and identify predictive patient and procedural factors associated with sepsis.

Methods: The American College of Surgeons National Surgical Quality Improvement Program (ACS NSQIP) is a multiinstitutional, validated, risk-adjusted program and was utilized to identify patients undergoing URS or PCNL from 2005 to 2017. Collected data included patient demographics, comorbidities, preoperative blood work, procedural and anesthetic characteristics, and the occurrence of post-operative sepsis defined as the presence of two or more systemic inflammatory response syndrome (SIRS) criteria. Chi squared test was used to evaluate changes in postoperative sepsis rates over time. A multivariate logistic regression was performed to identify factors associated with sepsis.

Results: 1517 patients were identified including 369 patients who underwent PCNL and 1148 patients who had URS. Patients were $53.1 \%$ male, had a mean age of $55 \pm 16$ years, and a mean BMI of $30.0 \pm 7.7$. The overall rate of post-operative sepsis in patients undergoing URS was $1.0 \%$, compared to $2.7 \%$ in patients undergoing PCNL. There was a trend towards an increased rate of sepsis over time in the URS cohort $(p=0.05)$. Factors associated with sepsis included higher American Society of Anesthesiologists (ASA) classification $(p=0.027)$, active smoking status $(p=0.008)$, and patients on dialysis $(p=0.000)$. A prior history of bleeding disorder $(\mathrm{p}=0.30)$, congestive heart failure $(p=0.019)$, and the number of days from hospital admission to operation $(\mathrm{p}=0.006)$ was associated with sepsis following URS but not PCNL.

Conclusions: The overall incidence of post-operative sepsis following URS or PCNL remains low; however, there is a trend towards increasing rates of sepsis in patients undergoing URS. This may be related to a rising prevalence of patient comorbidities and colonization with hospital acquired organisms. Several characteristics were associated with the development of sepsis following both URS and PCNL including ASA score, smoking, and renal failure requiring dialysis. Further investigation is required in order to better define factors predictive of postoperative sepsis, and to develop strategies to reduce this risk.

\section{MP24-05 Nephrolithiasis and Diabetes: Risk factors predicting stone disease}

V Talanki, K Ieong, A Zlatopolsky, S Eng, J Miller,

Y Sheynkin, D Schulsinger

Stony Brook University Hospital

Introduction \& Objective: Metabolic syndrome has been associated with an increased risk for nephrolithiasis. Obesity and Type 2 diabetes are well studied to be independent risk factors for stone events. These patients have higher urine oxalate and urine uric acid. We sought to better understand this association and identify modifiable risk factors in our patients with nephrolithiasis. We had particular interest in the role of Metformin, as it has been associated with an increase in stone events.

Methods: We performed an Institutional Review Board approved retrospective single-center review of all patients over a one-year period with a history of nephrolithiasis and underwent 24-hour urine testing. Univariate analysis and multivariate analysis were conducted in Statistical Analysis Software v9.4.

Results: On multivariate analysis among diabetics, Metformin users had 3.589 times higher odds of having a higher level of 
Urine oxalate when compared to those who do not use Metformin $(95 \% \mathrm{CI}=1.186-10.859, \mathrm{P}$-value $=0.0237)$. In our multivariate analysis we controlled for age, gender, body mass index, surgical history, plasma glucose, medication use, urine volume, urine $\mathrm{pH}$, urine uric acid. Diabetic patients were more likely to require percutaneous nephrolithotomy for stone management compared to nondiabetics ( $22.5 \%$ compared to $13.4 \%$, respectively). Diabetics were more likely to have uric acid stones $(17.2 \%$ compared to $9.0 \%$, respectively). Our data confirm previous studies that Type 2 diabetes and obesity were independently associated with higher urine uric acid and oxalate.

Conclusions: We identified that patients with Type 2 diabetics treated with Metformin have higher urine oxalate concentrations compared to diabetics managed with alternative antihyperglycemic medication. Our data suggest that diabetic stone formers with persistent hyperoxaluria should be managed with alternate medications. Prospective studies are needed to further characterize the role of Metformin in Type 2 diabetes and nephrolithiasis.

\section{MP24-06 How should the follow-up be done after stone surgery? CT scan vs Ultrasonography}

A Rivero, J Montero, J Adot, J Hernández, A Robles, E Gutierrez

Introduction: The target of the Stone surgery is to obtain a total calculi removal or finishing with a low rate of residual fragments. To define properly the concept of Stone free is very important to evaluate the accuracy of the different imaging techniques in the follow up of the surgery.

Objective: To evaluate the accuracy of ultrasonography (US) and non-contrast computed tomography (NCCT) after retrograde intrarenal surgery (RIRS).

Methods: A total of 110 patients who underwent RIRS were studied in the third month follow up after surgery. All of these patients were controlled with both, US and NCCT. The sensitivity, specificity, and stone size measured in US were validated by NCCT. Data of the stone size in US were classified into four groups (0-3.5, $3.6-5,5.1-10,>10 \mathrm{~mm})$ and then compared with NCCT data.

Results: In 110 patients, NCCT detected stones in 74 cases, while US could identify residual fragments in 66 . More differences were founded in the stone size, in this case, the US studies detected more or bigger fragments in 38 patients. About $46 \%$ concordance was obtained for the stone size measured by US and NCCT. The detection rate increased with the stone size, but sometimes the amounts of residual fragments were taken as a solitary Stone in the US. Factors such as hydronephrosis and urinoma were similar in the results of both techniques.

Conclusions: The accuracy of the US have a good rate of remaining stones detection but probably the TC could give us more information about the size and distribution of the residual fragments. Sometimes US could overestimate the Stone burden or confuse between the amount of dusted stones and real residual fragments. Despite the experience of the radiologist and the anatomy of the patient, there could be factors to choose the $\mathrm{CT}$ as a more sensitive study.

MP24-07 Can the qSOFA Score Predict the Need for ICU Admission and Outcomes in Patients with Obstructed Infected Ureteral Stones?

M Alsyouf, P Stokes, S Cho, Z Gilbert, A Amasyali, M Hajiha, M Shah, J Maldonado, J Groegler, D Baldwin

Loma Linda University Health
Introduction \& Objective: Infection associated with obstructing stones is a urologic emergency due to the risk of worsening sepsis and potential for mortality without prompt decompression. The Quick Sequential Organ Failure Assessment (qSOFA) score has been proposed as a predictor of severity of organ dysfunction in patients with sepsis but has not been validated in patients presenting with obstructive pyelonephritis requiring decompression. The purpose of this study is to evaluate peri-operative factors, including qSOFA scores, and their association with postoperative outcomes including the need for ICU admission. Methods: A retrospective review of 100 consecutive patients presenting to a single tertiary care academic medical center with obstructive pyelonephritis secondary to ureteral stones was performed. Obstructive pyelonephritis was defined as obstructing ureteral stone associated with urinary tract infection and/or signs of sepsis. All patients underwent emergent ureteral stent placement for decompression. ICU admission criteria included hypotension requiring vasopressor support and/or respiratory failure requiring mechanical ventilation. Univariate analysis and multivariate regression analysis were performed to identify factors associated with ICU admission, with $\mathrm{p}<0.05$ considered significant. Results: One hundred patients presented with obstructive pyelonephritis requiring emergent ureteral stent placement, with 15 patients requiring subsequent ICU admission. Patients requiring ICU admission were significantly older (average 63.4 vs. 49.1 years; $\mathrm{p}<0.01$ ), had higher pre-operative heart rate (average 136.0 vs. $102.6 ; \mathrm{p}<0.01$ ), higher initial WBC count (average 16.8 vs. $12.6 ; \mathrm{p}<0.01$ ), higher rate of pre-operative hypotension $(80.0 \%$ vs. $37.6 \% ; p<0.01)$, and qSOFA score $\geq 2(86.7 \%$ vs. $20.3 \% ; \mathrm{p}<0.01)$ compared to patients that did not require ICU admission postoperatively. Patients with pre-operative q-SOFA scores $\geq 2$ had significantly longer hospital stays (average 6.1 vs. 4.5 days; $\mathrm{p}=0.02$ ). On multivariate analysis, pre-operative $\mathrm{q}-$ SOFA score $\geq 2$ was the only significant predictor of ICU admission postoperatively (OR 11.8 [95\% CI 1.07-130.0]).

Conclusions: Higher qSOFA scores significantly predict the need for ICU admission in patients undergoing ureteral stent placement for obstructed infected stones. This study validates the qSOFA scoring system during the initial evaluation of patients presenting with obstructive pyelonephritis.

MP24-08 Utility of the POPVESL score for risk stratification of intrarenal vascular complications following percutaneous nephrolithotomy

P Shadpour, R Maghsoudi, N Yousefzadeh Kandevani, M Etemadian, N Abian

Hasheminejad Kidney Center (HKC), Iran University of Medical Sciences (IUMS), Tehran, Iran

Introduction \& Objective: Current practice guidelines favor percutaneous nephrolithotomy (PCNL) as the method of choice for larger renal stones, however, post-op hemorrhage remains an important complication of this procedure. Delayed bleeding heralds pseudoaneurysm (PA) or arteriovenous fistula (AVF) formation thus the costs and availability and timing of angioembolization become consequential. The aim of this study is to tap on our large volume single center experience, in pursuit of factors to predict the outcome of such bleeding.

Methods: We reviewed the records of all patients who were readmitted for gross hematuria after undergoing PCNL at our center between Dec 2011 and Jan 2016. All stable patients diagnosed with intra-renal vascular lesions underwent a period of 
conservative management. Those with a persistent drop in hemoglobin level and repeated transfusion were scheduled for elective angioembolization. Procedure-related, stone related and perioperative were analyzed to derive a risk score.

Results: From 4403 patients, 83 (1.9\%) were diagnosed to have intrarenal vascular lesions in the form of 54 arteriovenous fistulas (AVF 65\%) and 29 pseudoaneurysms (PA 35\%). Forty-nine patients responded to conservative treatment and 34 eventually required angioembolization. In multivariable analysis, the units of blood transfused after initial stabilization, PA, history of open renal surgery, the protracted interval from PCNL to the second admission, and size of the vascular lesion were ominous leading to our proposed POPVESL (short for Post $\boldsymbol{P N L} \boldsymbol{V}$ ascular $\boldsymbol{E m}$ bolization selection) score.

Conclusions: Based on our series the proposed POPVESL score have potential implications in updating future guidelines on the management of post-PCNL bleeding.

\section{MP24-09 Evaluation of $40 \mathrm{cmH} 20$ vs $100 \mathrm{~cm} \mathrm{H} 2 \mathrm{O}$ gravity irrigation system in fURS}

T Tefik, B Aydin, R Aydın, S Turan, F Ozcan, I Nane

Istanbul University, Istanbul Faculty of Medicine, Department of Urology

Introduction \& Objective: Introduction of high-power lasers in the endourological armamentarium has led to the increase in intrarenal temperatures and the need for higher intrarenal irrigant flow. In this preliminary study we present our data with high and low gravity irrigation and evaluate the postoperative outcomes. Methods: Between the dates January 2018 and May 2019 charts of patients undergoing fURS for kidney stone disease were retrospectively collected. Patients with recorded irrigation system height were evaluated for the study. Among them 9 patients were operated with $40 \mathrm{cmH} 2 \mathrm{O}$ (Group 1) and 17 patients with $100 \mathrm{cmH} 2 \mathrm{O}$ (Group 2) irrigation bag height. The height was defined as the distance in $\mathrm{cm}$ between the patient and the irrigation bag. All operations were performed by or under supervision of a single surgeon (TT). Postoperative values for sepsis and acute phase reactants were compared among groups.

Results: There was no statistical significance regarding age, sex, stone burden and operation time among groups. None of the patients experienced sepsis following the operation. Preoperative, postoperative values for CRP and CRP difference were $11.7 \pm 21.8$ vs $7.9 \pm 9.9,15.1 \pm 17.9$ vs $14.8 \pm 11.4$ and $3.3 \pm 8.1$ vs $6.8 \pm 10.5$ in Group 1 and 2, respectively, none reaching statistical significance. Similarly, preoperative, postoperative values for WBC and WBC difference were $8422 \pm 2201$ vs $8094 \pm 2000,8944 \pm 1416$ vs $9520 \pm 2809$ and $522 \pm 2414$ vs $1426 \pm 2795$ in Group 1 and 2, respectively, none reaching statistical significance. Visibility noted to be better by the surgeon in Group 2 without affecting the outcome.

Conclusions: Higher irrigation height of $100 \mathrm{~cm} \mathrm{H} 2 \mathrm{O}$ seems to be applied safely in fURS operations when treating kidney stone patients, without compromising the postoperative outcomes.

MP24-10 Bilateral Simultaneous Procedures for Bilateral Urolithiasis: The Future? Evidence from a Systematic Review

R Geraghty, A Pietropaolo, B Rai, P Jones, BK Somani
Introduction \& Objective: Bilateral urolithiasis is an uncommon presentation. Most operations carried out in this scenario are staged, however there is a growing body of evidence to suggest bilateral simultaneous (BS) procedures are safe. We performed a systematic review to investigate and evaluate the evidence.

Methods: Systematic review following Cochrane guidelines. Bias assessment performed using the GRADE assessment tool. Studies investigating BS procedures including PCNL, ureteroscopy (URS) and PCNL with contralateral URS (PCNL + URS) published after 2001 were included.

Results: In total 32 studies were identified for inclusion, 14 for BS-URS, 12 for BS-PCNL and 3 for PCNL + URS. 2. BS-PCNL: mean operative time was 157 minutes, mean SFR was $92 \%$, mean complication rates were $27 \%$ (Clavien I-II) and 6\% (Clavien $\left.{ }^{3} \mathrm{III}\right)$, and hospital stay was 4.9 days. PCNL+URS: mean operative time was 151 minutes, mean SFR was $76 \%$, mean complication rates were $6 \%$ (Clavien I-II) and $0 \%$ (Clavien ${ }^{3} \mathrm{III}$ ), and hospital stay was 6.1 days. Within the three groups there was one study comparing the outcomes above to staged procedures. BS-URS: all outcomes (apart from hospital stay, which was not measured) were non-significant $(p=0.12)$ in comparison to staged. BS-PCNL: SFR and complications were non-significant $(\mathrm{p}=0.91)$ and had significantly reduced hospital stay and operative time $(\mathrm{p}<0.001)$. PCNL+URS: SFR and complications were non-significant $(\mathrm{p}=0.72)$ and had significantly reduced hospital stay and operative time $(\mathrm{p}<0.001)$. There was a serious risk of bias amongst all studies examined.

Conclusions: There is limited evidence to suggest that bilateral simultaneous procedures are safe in the modern era. Further randomized trials are needed to clarify whether simultaneous or staged procedures should be utilized.

\section{MP24-11 Initial clinical experience of LithoVue ${ }^{\mathrm{TM}}$ in patients of Urolithiasis: An Indian perspective}

J Jagtap, L Sinha, S Veer, G Agrawal, P Rao, S Mistry

Gleneagles Global Hospitals, Mumbai

Introduction \& Objective: The use of LithoVue ${ }^{\mathrm{TM}}$ has rapidly gained traction owing to the high cost of procurement and maintenance of reusable flexible ureteroscopes. We present our prospective data on LithoVue ${ }^{\mathrm{TM}}$ (a single use digital flexible ureteroscope) in patients undergoing ureteroscopy for upper urinary tract lithiasis.

Methods: All patients of urolithiasis treated at a single tertiary referral hospital between August 2018 and June 2019 requiring the use of flexible ureteroscope were included in the study. Clinical parameters such as overall success rate, operative time, complication rates, hospital stay, etc. were analyzed. $1500 \$$ is the cost of each LithoVue ${ }^{\mathrm{TM}}$ in India.

Results: 33 consecutive cases utilizing LithoVue ${ }^{\mathrm{TM}}$ ureteroscope were included in this study. Mean patient age was 47.8 years and average stone size was $1.67 \mathrm{~cm}$. The average operation time was $22.66 \mathrm{~min}(3-70 \mathrm{~min})$ with average lasing time of $11.06 \mathrm{~min}$ (1$40 \mathrm{~min})$. No Scope failure occurred in any of the patients though in 2 of them reconnection of the ureteroscope to the processing module was necessitated due to onscreen artefacts. Balloon dilatation of a tight lower ureter was required in 3 cases $(9.1 \%)$. Also, three patients $(9.1 \%)$ had to be stented due to inability to pass the ureteroscope and the procedure was staged in them. Access sheath was utilized in $7 / 33$ cases $(21.2 \%)$ in view of multiple small stones. The rest $26(78.8 \%)$ were done sheathless. Overall stone free rate was $96.9 \%$ (32/33) and only 4/33 (12\%) 


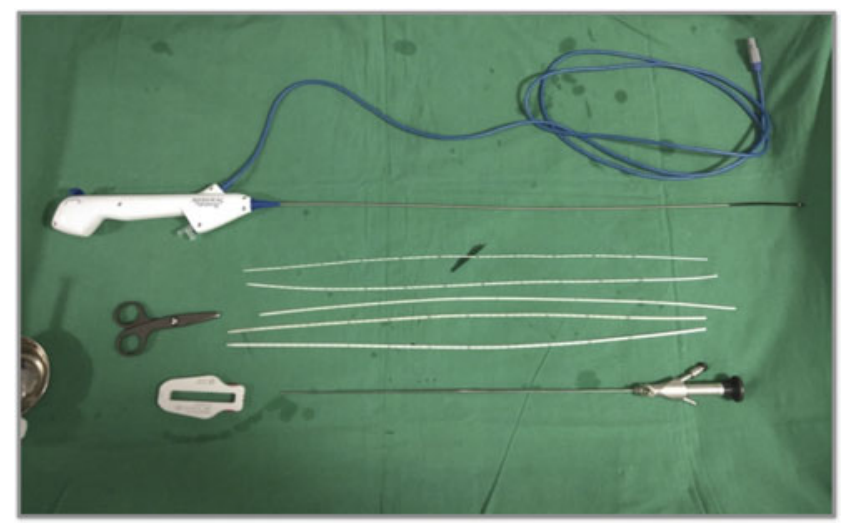

had Clavien Dindo grade I-II complications (3 had Grade I and 1 had Grade II). Average hospital stay was 1.24 days (1-3 days). Conclusions: LithoVue ${ }^{\mathrm{TM}}$ is a safe and viable alternative to reusable ureteroscopes with a high success rate. It has a negligible scope failure rate and advantages in terms of accessibility to new ureteroscopes with maximal deflection in each case.

\section{MP24-12 Body Mass Index impacts Percutaneous Nephrolithotomy and Retrograde Intra-renal Surgery results}

J Torres, P Pereira, N Morais, S Anacleto, R Rodrigues, P Passos, P Mota, R Leão, E Lima

Introduction \& Objective: Increasing prevalence of overweight and obese patients creates a unique challenge for choosing the ideal procedure for nephrolithiasis treatment. The aim of this study is to compare the outcomes and surgical complications of Percutaneous Nephrolithotomy (NLPC) and Flexible Ureterorenoscopy (RIRS) in patients with different Body Mass Index (BMI).

Methods: From January 2011 to May 2018, 331 patients $($ RIRS $=130 ;$ PCNL $=201)$ with nephrolithiasis were treated in a single center. Patients were divided in three groups according to BMI [obese $(\mathrm{BMI}>30)$; overweight $(30>\mathrm{BMI}>25)$ and normal weight $(\mathrm{BMI}<25)]$. Demographic characteristics, stone characteristics (size, number and location), surgical outcomes, stonefree rate and the intra and postoperative complications were evaluated and compared between groups.

Results: Sociodemographic variables were homogeneously distributed between both surgical groups. In general, when submitted to RIRS, overweight and obese patients displayed fewer postoperative complications when compared to normal weight patients( $p=0.046$ and $p=0.038$, respectively). In the PCNL group, obese patients suffered a lower hemoglobin drop $(\mathrm{Mdn}=1,40 \mathrm{~g} / \mathrm{dl})$ compared to normal $(\mathrm{Mdn}=2,00 \mathrm{~g} / \mathrm{dL})$ and overweight $(\mathrm{Mdn}=2,10 \mathrm{~g} / \mathrm{dL})$ patients $(\mathrm{p}=0,030)$. In this surgery, the overweight and obese patients had less risk of postoperative hematuria compared to normal weight patients $(\mathrm{p}=0,015$ and $p=0,026$, respectively). Stone-free rates were alike $(p=0.30)$ in both surgeries, with similar stone-free rates across the different BMI groups for each procedure. Additionally, in obese and overweight patients, PCNL procedure was associated with more frequent complications when compared to RIRS $(\mathrm{p}=0,004)$, namely hematuria $(\mathrm{p}=0,024)$ and hemoglobin drop $(\mathrm{p}=0,024)$ conditioning prolonged hospital stay $(\mathrm{p}<0,01)$ and operative time $(\mathrm{p}<0,01)$.
Conclusions: In this study, BMI variations do not impact the stone-free rates in patients submitted to RIRS and PCNL. Additionally, high BMI appears to have a protective effect in several postoperative outcomes, namely hemorrhagic complications in PCNL and overall complications in RIRS. Finally, these data show that RIRS is apparently safer than PCNL in selected patients with an elevated BMI. Further larger and prospective studies are needed to confirm these data.

\section{MP24-13 Emergent urologic consultation for renal colic-what factors lead to admission}

C Tabib, W Wu, P Samson, C Hartman

Arthur Smith Institute for Urology

Introduction \& Objective: Nephrolithiasis is a common condition affecting 1 in 10 patients with renal colic being a common presenting symptom. Poor pain control typically prompts the emergency department (ED) physicians to decide whether the patient warrants inpatient management. Excluding absolute indications for emergent decompression, the utility of an emergent urologic consultation is not well studied. We sought to examine factors associated with inpatient admission of patients with renal colic who had a urologic consultation in the ED at our institution. Methods: We retrospectively analyzed patients presenting to the ED with renal colic. All patients with confirmed diagnosis on computed tomography were evaluated by ED providers followed by a formal urologic consultation. Exclusion criteria included: prior ED visit within 30 days, planned admission by outpatient provider, and absolute indications for surgical management i.e. obstructive pyelonephritis, bilateral obstruction, or an obstructed solitary kidney. Patient demographics, laboratory data, imaging studies, medication requirements, ED course, and hospital course were analyzed. Univariate analysis was performed using $t$-test and chi-square test, and multivariate analysis was performed via logistic regression. One tailed alpha of 0.05 was considered significant.

Results: 172 patients between October 2014 and May 2015 had a formal urologic consultation with 87 patients meeting criteria for inclusion in this study. Of those, 55 were discharged from the ED while 32 patients were admitted. On univariate analysis, admitted patients were significantly older (57.4 vs 51.1 years, $\mathrm{p}=0.03)$, had worsened azotemia ( $1.57 \mathrm{vs} 1.21 \mathrm{mg} / \mathrm{dL}, \mathrm{p}=0.02)$, and had more proximal stones $(59.3 \%$ above midureter, $\mathrm{p}=0.032$ ). On multivariate analysis, only serum creatinine predicted admission (Table 1).

Conclusions: Due to increasing prevalence of stone disease combined with prolonged ED wait times, it is important to identify factors associated with admission for renal colic. Excluding situations of obstruction requiring emergent decompression, worsening azotemia is the only significant factor related to admission. This information can be used to help ED physicians with their decision making and could improve throughput and speed of likely admissions. This in turn would result in improved ED visit and appropriate disposition of patients.

\begin{tabular}{l|c|c|}
\hline Multivariate analysis & Odds ratio $(95 \% \mathrm{CI})$ & P-value \\
\hline Age & $1.02(0.981-1.07)$ & 0.286 \\
\hline White blood cell count & $0.965(0.821-1.13)$ & 0.664 \\
\hline Serum creatinine & $3.63(1.23-10.7)$ & 0.019 \\
\hline Urine white blood cell <25 per hpf & $5.34(0.232-122)$ & 0.294 \\
\hline Stone size & $1.08(0.914-1.27)$ & 0.368 \\
\hline Stone location (mid ureter and below) & $0.496(0.012-20.5)$ & 0.712 \\
\hline
\end{tabular}




\section{MP24-14 Identification of Stone Composition Based Upon Gross Appearance}

A Ng, V Vasudevan, C Hartman, D Hoenig, Z Okeke, AD Smith, C Tabib

Introduction \& Objective: Nephrolithiasis is a common and painful disorder, the lifetime risk estimated to be $10-15 \%$ in the United States. The prevalence of kidney stone disease increased from $3.8 \%$ in the 1970 's to $8.4 \%$ in 2010 . Accurate intraoperative identification of stone type provides the urologist valuable information, including deciding to dust or to leave fragments behind, and choosing the optimal energy settings for fragmentation. Additionally, identifying stones based on appearance lends to guidelines-based practice, specifically when rendering a patient stone free in the setting of suspected infectious residual fragments. As such, we examined the accuracy of surgeon identification of stone composition based on gross appearance.

Methods: This descriptive study was conducted on New York metropolitan area urology residents and attendings through convenience sampling. Data were collected by an online questionnaire consisting of two sections: questions that ask to identify the primary stone composition based on a series of pictures, and questions that ask the expected color of different stone subtypes. Results: 75 urologists answered the questionnaire, 22 residents, 29 attendings, 24 not listed. $40.5 \%$ correctly identified the uric acid stone, $33.3 \%$ correctly identified the primary mixed calcium phosphate stone, $44.6 \%$ correctly identified the calcium oxalate monohydrate stone, $23.0 \%$ correctly identified the calcium oxalate dihydrate stone, and $20.5 \%$ correctly identified the struvite stone. $57.5 \%$ correctly identified calcium oxalate stones as black or dark brown, $47.9 \%$ correctly identified calcium phosphate stones as off-white, $56.2 \%$ correctly identified uric acid stones as bright yellow or red, and $51.4 \%$ correctly identified struvite stones as off-white. On average, residents were better able to identify the composition of the stone compared to attendings $(34.5 \%$ vs $31.7 \%)$. Attendings were better able to predict the appearance of a stone based on stone subtype compared to residents $(62.1 \%$ vs $42.0 \%)$.

Conclusions: Urologists were best able to identify the calcium oxalate monohydrate stone $(44.6 \%)$ and were best at predicting the color of calcium oxalate stones, followed by uric acid, struvite, and finally calcium phosphate stones. Urologists are better at predicting the color of a stone type than identifying the stone composition based on appearance. Overall, this provides an area of opportunity for resident and faculty education.

MP24-15 Implementation of a Hospital-wide Protocol Reduces Time to Nephrostomy Tube and Length of Stay in Patients with Stone Related Obstructive Pyelonephritis.

\section{Haas, C Sayegh, J Pak, E Sebesta, E Hyams, O Shah}

Columbia University

Introduction \& Objective: Patients with obstructive pyelonephritis (OPN) require urgent decompression via retrograde ureteral stenting (RUS) or nephrostomy tube (NT). In 5/2016 the urology and IR departments at our institution enacted a protocol regarding the management of patients with OPN that enabled urologists to more swiftly engage IR for NT decompression should the urologist decide to pursue NT (Fig. 1). Our objective was to assess this protocol's impact on outcomes including time to NT and length of stay (LOS).

\begin{tabular}{|c|c|c|c|c|c|c|c|}
\hline & \multicolumn{3}{|c|}{ Pre-protocol } & \multirow[b]{2}{*}{$\begin{array}{l}\text { p-value NT } \\
\text { pre vs post }\end{array}$} & \multicolumn{3}{|c|}{ Post-Protocol } \\
\hline & $\begin{array}{l}\text { RUS } \\
n=44 \text { (71\%) }\end{array}$ & $\begin{array}{l}\text { NT } \\
n=18(29 \%)\end{array}$ & $\begin{array}{l}\text { P-value } \\
\text { RUS vs NT }\end{array}$ & & $\begin{array}{l}\text { RUS } \\
n=32 \text { (59\%) }\end{array}$ & $\begin{array}{l}\text { NT } \\
n=22(41 \%)\end{array}$ & $\begin{array}{l}\text { P-value } \\
\text { RUS vs NT }\end{array}$ \\
\hline \multicolumn{8}{|c|}{ Patient Characteristics } \\
\hline Age, mean \pm SD & $53 \pm 18$ & $66 \pm 15$ & $0.008^{*}$ & 0.32 & $50 \pm 16$ & $60.5 \pm 18$ & $0.031^{*}$ \\
\hline $\mathrm{CCl}$, mean $\pm \mathrm{SD}$ & $2.1 \pm 2.6$ & $4.8 \pm 2.6$ & $<0.001^{\circ}$ & 0.11 & $1.6 \pm 2.2$ & $3.3 \pm 3.1$ & $0.029^{*}$ \\
\hline Septic Shock & $9 \%$ & $44 \%$ & $0.002^{*}$ & $0.025^{\circ}$ & 3\% & $9 \%$ & 0.88 \\
\hline$>1 \mathrm{~cm}$ UPJ stone & $9 \%$ & $39 \%$ & $0.005^{\circ}$ & 0.22 & $32 \%$ & $19 \%$ & 0.27 \\
\hline \multicolumn{8}{|l|}{ Outcomes } \\
\hline $\begin{array}{l}\text { Decompression } \\
\text { time, median (IQR) }\end{array}$ & $\begin{array}{l}4.5 d \\
(3.2-7.6)\end{array}$ & $\begin{array}{l}7.2 \mathrm{hr} \\
(5.3 \cdot 14.1)\end{array}$ & $0.003^{*}$ & $0.002^{*}$ & $\begin{array}{l}5.4 \mathrm{hr} \\
(3.6-6.1)\end{array}$ & $\begin{array}{l}4.3 \mathrm{hr} \\
(3.7-6.6)\end{array}$ & 0.66 \\
\hline ICU admission & $9 \%$ & $67 \%$ & $<0.001^{*}$ & $0.0011^{*}$ & $13 \%$ & $14 \%$ & 0.90 \\
\hline $\begin{array}{l}\text { LOS s/p decompr. } \\
\text { Median (IQR) }\end{array}$ & $\begin{array}{l}2.7 d \\
(1.7-4.6)\end{array}$ & $\begin{array}{l}6.5 d \\
(4.2-13.8)\end{array}$ & $<0.001^{\circ}$ & $0.048^{*}$ & $\begin{array}{l}3.0 \mathrm{~d} \\
(1.8-4.0)\end{array}$ & $\begin{array}{l}3.9 \mathrm{~d} \\
(2.9-8.2)\end{array}$ & $0.027^{\circ}$ \\
\hline $\begin{array}{l}\text { ploS (>6d) s/p } \\
\text { decompression }\end{array}$ & $18 \%$ & $50 \%$ & $0.011^{\circ}$ & 0.24 & $16 \%$ & $32 \%$ & 0.16 \\
\hline Death & $1(5 \%)$ & 0 & 0.12 & 0.26 & $1(3 \times)$ & 0 & 0.40 \\
\hline
\end{tabular}

Methods: We retrospectively reviewed all patients at our institution who underwent NT from 2012 - 2017 or stent from 2014 2017 for stone related OPN meeting sepsis criteria. Univariate descriptive statistics (t-test or Mann-Whitney U test for continuous and chi-square for proportions) were used to compare patient characteristics and outcomes between RUS and NT and between NT pre- and post-protocol. Multivariable logistic regression assessed predictors of prolonged LOS ( $>6$ days).

Results: Utilization of NT increased after implementation of the protocol from $4.5 \mathrm{NT} / \mathrm{yr}$ to $18 \mathrm{NT} / \mathrm{yr}$ with a decrease in the median time from urologic consultation to NT from $7.2 \mathrm{hrs}$ to $4.3 \mathrm{hrs}(\mathrm{p}=0.002)$. Patients receiving NT were older and more comorbid than those receiving RUS (table 1). Median length of stay was shorter for those undergoing NT after the protocol compared to before the protocol ( $3.9 \mathrm{~d}$ vs $6.5 \mathrm{~d}, \mathrm{p}=0.05)$. On multivariable analysis controlling for sex, Charlson Comorbidity Score, and septic shock, hours to decompression increased odds of prolonged LOS (OR 1.09, $\mathrm{p}=0.007)$.

Conclusions: After implementing our OPN with sepsis protocol, NT utilization increased, time to decompression with NT decreased, and LOS decreased. Timely decompression reduced odds of prolonged hospital stay. A well-designed protocol engages both urology and IR in the management of these acutely ill patients expediting decompression and improving outcomes.
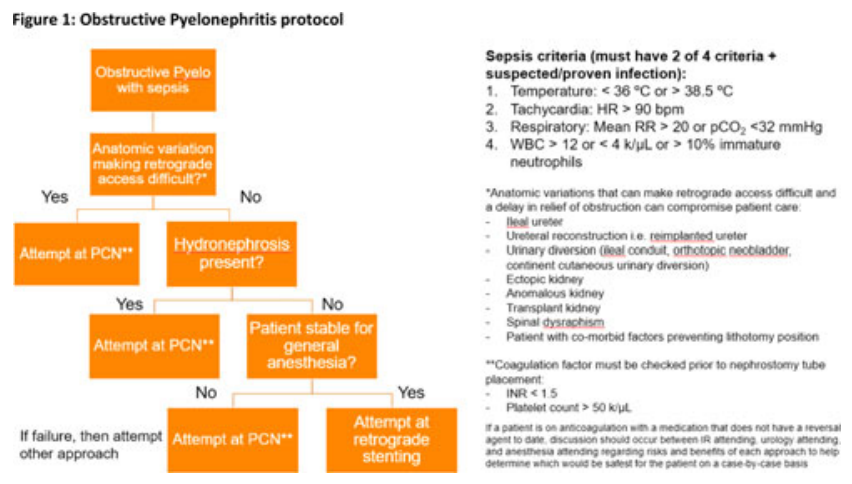

MP24-16 Prognostic performance of sepsis-3 and SIRS criteria for patients with urolithiasis-associated sepsis transferred to the ICU following surgical interventions: a single center study

\section{Y Huang, J Ding, B Shi}

Introduction \& Objective: The aim of the present study was to validate the prognostic effectiveness of Sepsis-3 criteria, including sequential organ failure assessment (SOFA) and quick SOFA (qSOFA), with systemic inflammatory response syndrome (SIRS) criteria among patients with urolithiasis- 

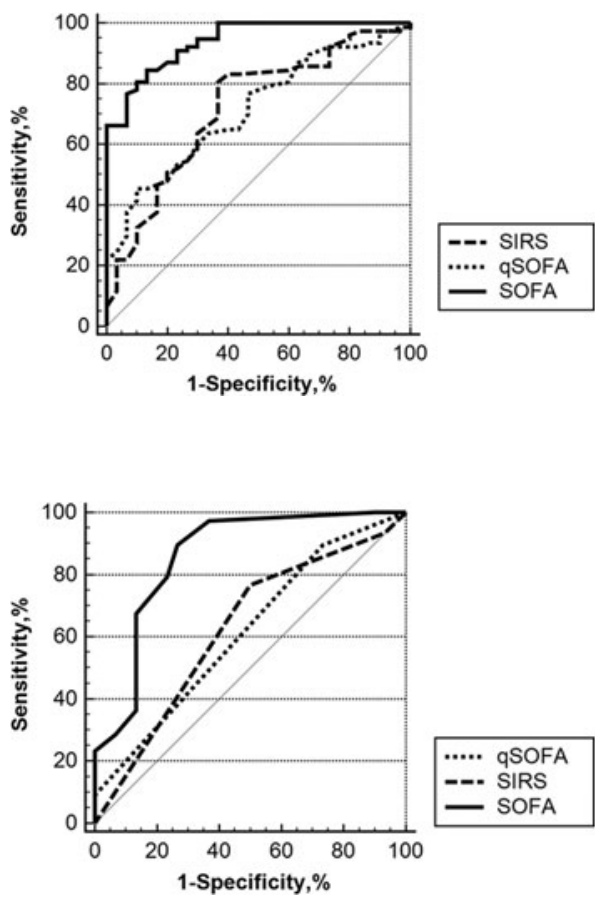

\begin{tabular}{|c|c|c|c|c|}
\hline & $\begin{array}{l}\text { ALL Patiens } \\
\text { ( } n=107)\end{array}$ & $\begin{array}{l}\text { Favorable } \\
\text { outcome }(n=30) \\
\text { SICU stay } \leqslant 3 \text { days }\end{array}$ & $\begin{array}{l}\text { Adverse } \\
\text { outcome( } n=77) \\
\text { In-hospital } \\
\text { mortality or SICU } \\
\text { stay > 3days }\end{array}$ & $P$ value \\
\hline $\begin{array}{l}\text { Age, median } \\
\text { (IQR),years }\end{array}$ & $66(54-77)$ & $64(54-73)$ & $67(54-80)$ & 0.19 \\
\hline Male, sex \% & $35(32.7)$ & $21(70.0)$ & $14(18.2)$ & $<0.001$ \\
\hline \multicolumn{5}{|l|}{$\begin{array}{l}\text { Surgical } \\
\text { procedures }(n, \%)\end{array}$} \\
\hline PCNL & $20(18.7)$ & $6(20.0)$ & $14(18.2)$ & 0.83 \\
\hline URL & $24(22.4)$ & $6(20.0)$ & $18(23.4)$ & 0.71 \\
\hline DJ stent & 63(58.9) & $18(60.0)$ & $45(58.4)$ & 0.88 \\
\hline \multicolumn{5}{|l|}{ Comorbidities $(n, \%)$} \\
\hline Hypertension & $50(46.7)$ & $12(40.0)$ & $38(49.4)$ & 0.384 \\
\hline $\begin{array}{l}\text { Cardiovascular } \\
\text { disease }\end{array}$ & $15(14.0)$ & $4(13.3)$ & $11(14.3)$ & 1.0 \\
\hline $\begin{array}{l}\text { Chronic obstructive } \\
\text { pulmonary disease }\end{array}$ & $14(13.1)$ & $4(13.3)$ & $10(13.0)$ & 1.0 \\
\hline Diabetes mellitus & $24(22.4)$ & $5(16.7)$ & $19(24.7)$ & 0.372 \\
\hline $\begin{array}{l}\text { Cerebrovascular } \\
\text { disease }\end{array}$ & $16(15.0)$ & $4(13.3)$ & $12(15.6)$ & 1.0 \\
\hline $\begin{array}{ll}\text { Chronic } & \text { kidney } \\
\text { disease } & \\
\end{array}$ & $12(11.2)$ & $5(16.7)$ & $7(9.1)$ & 0.265 \\
\hline Gout & $7(6.5)$ & $3(10.0)$ & $4(5.2)$ & 0.64 \\
\hline $\begin{array}{l}\text { History of } \\
\text { malignancy }\end{array}$ & $8(7.5)$ & $2(6.7)$ & $6(7.8)$ & 1.0 \\
\hline \multicolumn{5}{|l|}{$\begin{array}{l}\text { Laboratory results, } \\
\text { median (IQR) }\end{array}$} \\
\hline$W B C, \times 10^{9} / L$ & $16.21(11.30-23.54)$ & $13.95(7.07-15.51)$ & $\begin{array}{l}\text { 19.60(13.40-24.8 } \\
\text { 0) }\end{array}$ & $<0.001$ \\
\hline $\begin{array}{l}\text { C-reactive protein, } \\
\mathrm{mg} / \mathrm{L}\end{array}$ & $118(45-160)$ & $48(12-160)$ & $160(65-160)$ & 0.002 \\
\hline PCT, ng/mL & $17.91(3.43-96.23)$ & $2.18(0.23-7.77)$ & $\begin{array}{l}29.60(13.82-100 . \\
00)\end{array}$ & $<0.001$ \\
\hline $\begin{array}{l}\text { Vital signs, median } \\
\text { (IQR) }\end{array}$ & & & & \\
\hline
\end{tabular}

\begin{tabular}{|l|l|l|l|l|}
\hline Temperature, 'C & $38.5(38.0-38.9)$ & $38.65(38.0-39.0)$ & $38.5(38-38.9)$ & 0.32 \\
\hline $\begin{array}{l}\text { Systolic blood } \\
\text { pressure, mmHg }\end{array}$ & $78(72-83)$ & $84.5(79-90)$ & $76(71-81)$ & $<0.001$ \\
\hline $\begin{array}{l}\text { Mean arterial } \\
\text { pressure, mmHg }\end{array}$ & $59.3(56-63)$ & $63.3(60.0-66.3)$ & $58.3(55.7-60.3)$ & $<0.001$ \\
\hline $\begin{array}{l}\text { Respiratory rate, } \\
\text { /min }\end{array}$ & $27(23-32)$ & $26(22-32)$ & $27(23-31)$ & 0.89 \\
\hline Heart rate, /min & $114(103-125)$ & $116.5(105-127)$ & $113(103-125)$ & 0.55 \\
\hline $\begin{array}{l}\text { Altered } \\
\text { mentation(n, \%) }\end{array}$ & $11(10.3)$ & $0(0)$ & $11(14.3)$ & 0.032 \\
\hline $\begin{array}{l}\text { Evaluation criteria, } \\
\text { median (IQR) }\end{array}$ & $5(2-8)$ & $1(1-3)$ & $6(4-9)$ & $<0.001$ \\
\hline SOFA & $2(2-2)$ & $2(1-2)$ & $2(2-2)$ & 0.01 \\
\hline qSOFA & $4(3-4)$ & $3.5(3-4)$ & $4(4-4)$ & 0.04 \\
\hline SIRS & $86(80.4)$ & $11(36.7)$ & $75(97.4)$ & $<0.001$ \\
\hline SOFA $\geqslant 2(n, \%)$ & $90(84.1)$ & $21(70)$ & $69(89.6)$ & 0.034 \\
\hline qSOFA $\geqslant 2(n, \%)$ & $107(100)$ & $30(100)$ & $77(100)$ & NA \\
\hline SIRS $\geqslant 2(n, \%)$ & & & WBC & \\
\hline
\end{tabular}

PCNL percutaneous nephrolithotomy, URL ureteroscopic lithotripsy, WBC white blood count PCT procalcitonin, DJ double J, SOFA sequential (Sepsis-related) organ failure assessment, qSOFA quick sequential (Sepsis-related) organ failure assessment, SIRS systemic inflammatory response syndrome, IQR interquartile range, NA not available

associated sepsis that were transferred to the intensive care unit (ICU) following surgical interventions.

Methods: To achieve this, the records of all patients transferred to the ICU following surgical interventions and urolithiasisassociated sepsis between January 2010 and July 2017 at Xin Hua Hospital Affiliated to Shanghai Jiao Tong University were retrospectively reviewed. A total of 107 patients were enrolled. The prognostic performance of SOFA, qSOFA and SIRS for predicting in-hospital mortality (sepsis-related mortality during patients' hospitalizations) or prolonged length of ICU stay (>3 days) was compared using the area under the receiver operating characteristic curve (AUROC) and Z statistic.

Results: The results revealed that the overall in-hospital mortality rate was $8.4 \%$ and the percentage of in-hospital mortality or prolonged length of ICU stay ( $>3$ days) was $72.0 \%$ among the 107 patients. The favorable outcome group demonstrated significantly lower laboratory test results including white blood count, C-reactive protein, procalcitonin and higher systolic blood pressure and mean arterial pressure. The AUROC of qSOFA, SIRS and SOFA were $0.615,0.625$ and 0.860 , respectively. SOFA was significantly more effective at predicting adverse outcomes when compared with SIRS and qSOFA criteria. Following adjustments for patient age and comorbidities, the AUROC of qSOFA, SIRS and SOFA were $0.713,0.722$ and 0.940 , respectively.

Conclusions: In conclusion, the results of the present study indicate that the prognostic performance of SOFA for predicting in-hospital mortality or prolonged ICU stay among patients with urolithiasis-associated sepsis following surgical interventions, was significantly improved when compared with qSOFA or SIRS criteria. Based on these results it is recommended that urologists use the SOFA score for patients with urolithiasis-associated sepsis.

Table 2.Crude and adjusted AUROC
\begin{tabular}{|l|l|l|l|}
\hline & qSOFA & SOFA & SIRS \\
\hline Crude AUROC, $95 \% \mathrm{CI}$ & $0.615(, 0.516-0.707)$ & $0.860(0.780-0.920)$ & $0.625(0.526-0.716)$ \\
\hline Adjusted AUROC, $95 \% \mathrm{CI}$ & $0.713(0.618-0.797)$ & $0.940(0.877-0.977)$ & $0.722(0.627-0.804)$ \\
\hline
\end{tabular}
SOFA sequential (Sepsis-related) organ failure assessment, qSOFA quick sequential (Sepsis-related)
organ failure assessment, SIRS systemic inflammatory response syndrome


MP24-17 Creation and implementation of the DIRECT Pathway (Delayed Imaging to Reduce Excessive Computed Tomography) for the evaluation of patients with suspected renal colic

K Sternberg, DW Sobel, A Greenspun, N Moring, D Bak, R Bidad, P Weimersheimer

Brown University

Introduction \& Objective: Computed tomography (CT) remains the standard of care for the evaluation of acute renal colic in the United States despite concerns regarding radiation exposure and cost. Ultrasonography (US) has been proposed as an alternative initial approach. Considering the majority of ureteral stones ( $\sim 80 \%$ ) will pass without intervention, obtaining a CT on every patient with a suspected stone may not be necessary. We propose an algorithm using initial US for patients with high suspicion of uncomplicated ureterolithiasis that provides a mechanism for appropriate outpatient urologic follow-up with CT imaging if necessary.

Methods: An IRB approved prospective study and algorithm were developed defining patient eligibility, screening, diagnostic evaluation, discharge instructions, and follow-up. Patient eligibility is determined by the STONE score and clinical judgement of the likelihood of the presenting symptoms being secondary to a stone. For those with a high suspicion, an US is obtained. If symptoms are controlled with no change in suspected diagnosis, the patient is discharged home with a urinary strainer. Follow-up phone calls occur at day 1-2 and day 7-10 post discharge. If there is no definitive stone passage and/or persistent symptoms, an outpatient urology visit is initiated that includes a reduced dose non-contrast CT.

Results: 48 patients have been enrolled since January $2019.52 \%$ had a STONE score $<10($ mean $=9.6)$. Thus far, 9 patients $(18.8 \%)$ passed a stone after initial US and avoided CT. 16 patients had a CT after US, 3 while still in the ED. 12 of the CTs confirmed a ureteral calculus after ultrasound showed a stone or hydronephrosis, 2 had CT identifying a calculus after a normal initial US and one had a normal CT at follow up after US showed hydronephrosis. One patient is still awaiting follow up CT. 32 patients $(66.7 \%$ ) had US alone (9 passed a stone, 1 to OR from ED, 15 unable to be reached, 5 awaiting CT and urology follow up, and 2 patients refused CT and urology visit). Of the patients who could not be reached, review of the electronic health record did not reveal any ED return visits or CTs obtained.

Conclusions: A delayed CT imaging approach to suspected renal colic in the ED is feasible and initial results are promising regarding the reduction of upfront CT scans obtained. Over half of enrolled patients had a STONE score $<10$ indicating need for provider gestalt during evaluation. Future results are needed to evaluate the success of the pathway and determine whether this model can be expanded outside of our single center experience.

MP24-18 Bladder Lithiasis - Percutaneous Suprapubic or Transurethral Cystolithotomy: A Retrospective Study

S Anacleto, V Fernandes, J Torres, N Morais, P Passos, R Rodrigues, P Mota, E Dias, E Lima

Introduction \& Objective: Bladder stones account for 5\% of urinary calculi in the Western population. Despite the risk of urethral trauma is worrisome, evidence about the best treatment approach is scarce. Therefore, the aim of this study is to compare the safety and efficacy of transurethral cystolithotomy (TUC) and percutaneous suprapubic cystolithotomy (PSC) in adults' bladder lithiasis treatment.

Methods: We retrospectively evaluated120 patients (January 2012 to December 2017) who were treated surgically for bladder lithiasis with PSC $(n=20)$ and TUC $(n=100)$. Age, gender, calculi size, surgery duration, hospital stay, post-operative infections, hematuria, pain and urethral strictures were evaluated. Previous diagnosis of benign prostate hyperplasia, urethral strictures and neurogenic bladder were also considered for evaluation.

Results: Both groups were homogeneous according to the variables evaluated, including calculi dimensions and simultaneous diagnosis. Median follow-up time was 6 months. Median surgery time in PSC and TUC was 65 and 58 minutes, respectively $(\mathrm{p}=0.043)$. In the TUC, 3 patients $(3 \%)$ developed urethral stricture. None of the patients treated with PSC develop urethral strictures during the 6 months of follow up. Pain and hematuria were similar in both groups. Median hospital stay was 2,0 days in both groups. Bladder catheter was kept for a median time of 1,5 days in TUC and 1,8 days in the PSC group. Cystostomy catheter was not placed after PSC. Stone-free rates confirmed by cystoscopy at 3 to 6 months were similar in both groups.

Conclusions: PSC offers a significant advantage over TUC in terms of urethral trauma, and merits to be considered in our surgical armamentarium, especially in patients with known urethral strictures that do not allow transurethral access. However further prospective studies are needed to confirm our findings.

\section{MP24-19 Does Preoperative Turbid Urine Predict for Infectious Complication Following PCNL?}

D Mikhail, E Lynch, A Rai, S Mehta, A Greico, D Hoenig, Z Okeke, AD Smith

Introduction \& Objective: As complex stone incidence increases, rates of percutaneous nephrolithotomy (PCNL) for treatment continue to rise. Predicting and preventing common infectious complications from PCNL, including fevers and urosepsis, are a priority. Turbid or cloudy urine is often presumed by clinicians to be a sign of inflammation and UTI (urinary tract infection) - often affecting clinical management. We sought to establish whether preoperative turbid urine in stone patients carries intra- and postoperative implications.

Methods: We reviewed our prospectively collected PCNL database of patients undergoing PCNL between July 2015 and Dec. 2016. This database included pre- and intraoperative urine cultures, stone cultures and postoperative outcomes. We added preoperative urinalysis parameters for these same patients. We evaluated turbid urine preoperatively looking for causes as well as its predictive value in culture results and infectious outcomes. Univariate and multivariable statistical analysis was performed using Stata 15 (StataCorp, USA, 2017).

Results: A total of 150 patients were reviewed. Complete urinalysis data was found for 110 patients, 48 (44\%) of whom had turbid urine preoperatively. There were no significant differences in patient factors including age, sex, BMI or comorbidities between the two groups. Of patients with turbid urine, $40 \%$ had a positive preoperative urine culture and $44 \%$ experienced postoperative infectious complications - compared to $42 \%$ and $45 \%$ in those without turbid urine (not significant). Logistic regression analysis showed that turbid urine did not correlate with positive preoperative urine culture, intraoperative urine culture 
or infectious complications $(\mathrm{p}>0.05)$. Univariate and multivariable logistic regression found that turbid urine was associated with the presence of leukocyte esterase and nitrites on urinalysis, as well as indwelling stent $(\mathrm{p}<0.05)$. Interestingly, of 21 patients who developed sepsis and had turbid urine -11 (52\%) had 'contaminated' or negative urine cultures.

Conclusions: In our cohort of kidney stone patients awaiting PCNL, turbid urine alone on preoperative urinalysis did not predict having a positive pre- or intraoperative urine culture, stone culture, or postoperative infectious complications. Turbidity was associated with a patient having a stent preoperatively as well as nitrites and leukocyte esterase on urinalysis. Clinically, negative preoperative urine culture with the finding of turbid urine should be questioned as many of these patients developed infectious complications. Further prospective data is needed to clarify the clinical significance and prognosis of urine turbidity.

\section{MP24-20 Withdrawn}

\section{MP24-21 Evaluation of Bone Mineral Density in Kidney Stone Formers with Computed Tomography: A Single-Center Case-Control Study}

\author{
B Timuroglu, B Dolek, I Sari, N Gul, O Ozkan, \\ $\mathrm{T}$ Sener, Y Tanidir \\ Marmara University School of Medicine, Department \\ of Urology
}

Introduction \& Objective: The most common metabolic abnormality in patients with calcium-containing kidney stones is known to be hypercalciuria. Hypercalciuric patients exhibit decreased bone mineral density (BMD), however not much is known on the topic for the other stone types. The aim of this study is to determine the bone mineral density with a noncontrast computed tomography (NCCT) in patients with high stone kidney stone burden and to identify and compare the rate of osteoporosis in them.

Methods: The consecutive patients who underwent percutaneous nephrolithotomy (PCNL) between 2014 and June 2008 at the Department of Urology, Marmara University were identified. Through medical record, review details of procedures, outcomes and NCCT findings prior to the surgery were collected. NCCTs were evaluated with the 3D-Doctor software. Stone characteristics recorded and BMD of L1 vertebrae was evaluated according to Perry J. Pickhardt's method. Any patient with a BMD measurement under 160 Hounsfield Units (HU) was considered osteoporotic. Patients with a lesion of grade 2 or more according to Genant's semiquantitative grading scale for vertebral fractures and/or patients younger than 18 years of age were excluded.

Results: The data of 379 operations were reviewed and 248 patients $($ male/female rate $=1.6$ ) were identified as the study group. Mean age was $46.9 \pm 13.3$ years. The mean BMD value was found to be $184.3 \pm 46.8 \mathrm{HU}$. The rate of osteoporosis in males, females and in the whole study group was found to be $32.9 \%(\mathrm{n}=81), 33.3 \%(\mathrm{n}=49)$ and $32.7 \%(\mathrm{n}=32)$, respectively $(\mathrm{p}=0.511)$. The subanalysis of various age groups did not show any statistical difference in terms of BMD and osteoporosis rate. Calcium stone forming (CSF) patients found to have similar
BMD values with non-CSF patients. The mean BMD of patients above 50 years of age suggested osteoporosis for both CSF patients $(154.2 \pm 42.9 \mathrm{HU})$ and non-CSF patients $(127 \pm 38.0 \mathrm{HU})$. Interestingly, the subanalysis revealed CSF patients to have higher BMD values than non-CSF patients when they were older than 50 years $(p=0.049)$. No statistically significant difference was found between osteoporotic and non-osteoporotic patients in terms of number, volume, and density of stones. However, the stone surface area was found to be higher in patients with osteoporosis compared to non-osteoporotic ones $(p=0.045)$.

Conclusions: Our patient cohort was shown to have a 3-fold higher osteoporosis rate than the known. Although no significant difference in BMD was found between CSF and non-CSF forming groups, the non-CSF group seemed to have a tendency to low BMD. Thus, opportunistic scanning of BMD with NCCT in high kidney stone burden might help us to provide better health care.

\section{MP24-22 Do all patients with obstructing ureteric calculi and urinary nitrites need emergency decom- pression?}

T Marsden, A Pai, O Blach, A Alanbuki, A Symes

Brighton and Sussex University Hospitals

Introduction \& Objective: There is limited data available on the outcomes of patients with obstructing ureteric calculi with associated urinary nitrites. In the clinically septic patient, urgent decompression is mandatory. In patients who are clinically well, with nitrite positive urine, can conservative management be considered?

Methods: All cases of ureteric calculi referred to the Stone Clinic at our institution between January 2016 and May 2019 were retrospectively reviewed. All patients with a confirmed ureteric stone and nitrite positive urinalysis were included in the study. 57 patients met the inclusion criteria. The outcomes of these patients were compared with a control group of 412 patients with ureteric stones and urinalysis negative for nitrites who were referred to our stone clinic between January 2016 and December 2016.

Results: There was no significant difference in stone size characteristics between the two cohorts. Mean stone size was $4.2 \mathrm{~mm}$. $40 \%$ of stones were at the VUJ. $21.8 \%$ were distal-, $7.3 \%$ midand $31 \%$ proximal-ureteric. In the nitrite positive group, $14 \%$ of patients received empirical antibiotics at presentation. In the nitrite positive urine, $1.8 \%(n=1)$ required emergency decompression due to sepsis. $5.3 \%(n=3)$ were stented due to pain in the absence of sepsis. The overall emergency decompression rate in the nitrite positive group was $7.1 \%$ relative to $2.2 \%$ in the control group $(\mathrm{p}=0.076)$. In the nitrite positive group, $80.4 \%$ of stones passed spontaneously as opposed to $79.5 \%$ in the control group.

Conclusions: $1.8 \%(\mathrm{n}=1)$ of patients with confirmed ureteric calculi in the context of nitrite positive urinalysis required emergency decompression for sepsis. There was a high rate of spontaneous passage in the nitrite positive urine group, which was comparable to the control group. There was no significant difference in the rate of emergency decompression between the two groups. Prospective data is necessary to further guide the management of these patients. 


\section{MODERATED POSTER SESSION 25: CLINICAL STONES: URETEROSCOPY (I)}

\section{MP25-01 Can a disposable ureteroscope push up the 20-mm threshold? \\ R Takazawa, Y Waseda, S Yoshida, M Kobayashi, T Tsujii \\ Tokyo Metropolitan Ohtsuka Hospital}

Introduction \& Objective: Disposable flexible digital ureteroscopes (disposable-scopes) have become popular as an answer to the fragility problem of reusable flexible ureteroscopes (reusable-scopes). In our hospital, the LithoVue ${ }^{\mathrm{TM}}$ disposablescope has been used for scope-destroying cases i.e., large kidney stones ( $\geq 15 \mathrm{~mm}$ in diameter). There is currently no consensus on its cost-effectiveness. However, when used in practice, we have the impression that the treatment outcomes have improved in comparison to cases in which conventional reusable-scopes are used. Thus, we compared the outcomes of patients treated with disposable-scopes and those treated with reusable-scopes.

Methods: We analyzed the treatment outcomes of 35 cases (male, $\mathrm{n}=24$; female, $\mathrm{n}=11$ ) with kidney stones of $\geq 15 \mathrm{~mm}$ in diameter using a LithoVue ${ }^{\mathrm{TM}}$ scope during the period from April 2017 to March 2019. We also compared with the outcomes of 22 cases (matching stone conditions and male: female ratio) in which treatment was performed using a reusable-scope.

Table 1. Patient's characteristics and treatment outcomes.

\begin{tabular}{|c|c|c|c|}
\hline & Reusable $(\mathrm{n}=22)$ & Disposable $(\mathrm{n}=35)$ & Pvalue \\
\hline Male (n) & 16 & 24 & \multirow{2}{*}{0.74} \\
\hline Female (n) & 6 & 11 & \\
\hline Age (years) & $57.1 \pm 12.2$ & $56.7 \pm 12.0$ & 0.91 \\
\hline Height $(\mathrm{cm})$ & $163.2 \pm 7.7$ & $162.6 \pm 8.6$ & 0.80 \\
\hline Weight (kg) & $65.7 \pm 12.7$ & $65.8 \pm 13.5$ & 0.98 \\
\hline BMI $\left(\mathrm{kg} / \mathrm{m}^{*} \mathrm{~m}\right)$ & $24.6 \pm 4.0$ & $24.8 \pm 4.2$ & 0.89 \\
\hline Pre-stented (n) & 5 & 7 & 0.81 \\
\hline Obstructive pyelonephritis ( $\mathrm{n}$ ) & 2 & 4 & 0.82 \\
\hline Stone diameter $(\mathrm{mm})$ & $25.1 \pm 5.6$ & $26.0 \pm 9.4$ & 0.69 \\
\hline Stone volume by 3DCT $(\mathrm{cc})$ & $6.1 \pm 4.2$ & $6.6 \pm 4.3$ & 0.68 \\
\hline CT value $\max (\mathrm{HU})$ & $1386.5 \pm 204.5$ & $1334.5 \pm 281.8$ & 0.46 \\
\hline $\mathrm{CT}$ value mean $(\mathrm{HU})$ & $838.4 \pm 210.0$ & $843.8 \pm 192.2$ & 0.92 \\
\hline \multicolumn{4}{|l|}{ Stone composition } \\
\hline Calcium oxalate (n) & 21 & 28 & \multirow{5}{*}{0.47} \\
\hline Struvite (n) & 0 & 2 & \\
\hline Uric acid (n) & 1 & 2 & \\
\hline Calcium phosphate (n) & 0 & 1 & \\
\hline Cystine (n) & 0 & 2 & \\
\hline $1^{\text {st }}$ operation time $(\min )$ & $104.5 \pm 25.8$ & $108.2 \pm 27.1$ & 0.62 \\
\hline $\begin{array}{l}\text { Stone-free rate after } 1^{\text {st }} \text { session } \\
(<4 \mathrm{~mm})\end{array}$ & $50 \%(11 / 22)$ & $83 \%(29 / 35)$ & 0.01 \\
\hline Additional treatment rate & $32 \%(7 / 22)$ & $17 \%(6 / 35)$ & 0.20 \\
\hline $\begin{array}{l}\text { Total operation time, } 1^{\text {st }} \& 2^{\text {nf }} \\
\text { sessions (min) }\end{array}$ & $134.4 \pm 60.1$ & $127.4 \pm 60.1$ & 0.67 \\
\hline Complication rate & $14 \%(3 / 22)$ & $14 \%(5 / 35)$ & 0.95 \\
\hline Fever (n) & 3 & 3 & \\
\hline Stone street (n) & 0 & 1 & \\
\hline Ureteral damage G2 (n) & 0 & 1 & \\
\hline
\end{tabular}

Results: There were no significant differences in age or body size between the two groups (reusable group vs disposable group). The stone diameter $(25.1$ vs $26.0 \mathrm{~mm})$, stone volume calculated by a 3DCT analysis software program (6.1 vs $6.6 \mathrm{cc})$, CT values, operation time (104.5 vs $108.2 \mathrm{~min}$ ), and complication rate $(14 \%$ vs $14 \%)$ were statistically equal. However, the stone-free rate $(<4 \mathrm{~mm})$ after the 1 st session in the disposablescope group was superior to that in the reusable-scope group $(50 \%$ vs $83 \%, \mathrm{p}=0.01)$ (Table 1).

Conclusions: Disposable-scopes can improve the treatment outcomes of patients with large kidney stones. The disposablescope frees the surgeon from the limit of the breakability of the reusable scope, enabling more aggressive endoscopic operations and laser settings. Disposable-scopes have the potential to increase the $20-\mathrm{mm}$ stone diameter threshold for PCNL adaptation.

\section{MP25-02 Comparing Emergency and Elective Semi- Rigid Ureteroscopic Lithotripsy for Patients with Ureteric Calculi}

\section{SM Nazim, M Bangash, N Khan}

The Aga Khan University Hospital, Karachi Pakistan

Introduction \& Objective: Acute renal colic secondary to ureteric stones is a common presentation in urology practice. Failure of conservative management warrants stenting or nephrostomy tube placement. These measures provide prompt symptom relief and are followed by ureteroscopy (URS) or extracorporeal shockwave lithotripsy (ESWL). There is little available data regarding use of emergency ureteroscopy (EMURS). This study aims to compare safety and efficacy of the emergency versus elective ureteroscopic (ELURS) treatment of ureteric stones.

Methods: All adult patients with unilateral single radio-opaque ureteric stone who underwent semi-rigid URS from January 2007 to December 2017 were included. Patients with solitary kidney, Uro-sepsis, pregnancy or pre-operative drainage with nephrostomy or JJ stent were excluded. EMURS is defined as URS being performed within 24-48 hours of presentation, while ELURS is defined as URS performed after failed medical expulsive therapy. Patient, stone and outcome-related variables were compared in both groups. Stone free rate was defined as no evidence of stone on plain X-ray after 4 weeks.

Results: One hundred and twenty-five patients in the EMURS group versus 250 patients in ELURS group were compared. Age, sex and comorbidities and serum creatinine were comparable in both groups. Mean stone size was $6.54 \pm 3.282 \mathrm{~mm}$ in the EMURS group and $7.53 \pm 3.108 \mathrm{~mm}$ in the ELURS group $(p=0.0057)$. Majority of stones in EMURS group were located in distal ureter and uretero-vesical junction compared to proximal ureter in ELURS group $(\mathrm{p}<0.0001)$. EMURS had a comparable mean operative time versus ELURS (34.54 \pm 16.28 vs. $36.67 \pm 20.070$ minutes respectively). JJ stents were placed in $45.8 \%$ and $45.9 \%$ of EMURS and ELURS, respectively. Ancillary procedures (ESWL/Redo-URS) were performed in $17.92 \%$ $(n=28)$ of EMURS and in $18.69 \%(n=46)$ of ELURS 
$(p=0.8563)$. Overall complication rates were reported in $6.5 \%$ in EMURS and $14.5 \%$ in ELURS $(\mathrm{p}=0.0261)$ and they were mostly Clavein Grade 1. Stone free rate achieved was $75 \%$ in EMURS and $72.5 \%$ in ELURS, respectively.

Conclusions: Emergency URS for ureteric stones without other prior temporizing measures is a safe and effective one-stage definitive treatment option for patients with acute renal colic not responding to conservative management.

\section{MP25-03 Awake Flexible Ureteroscopy May Improve Patient-Urologist Communication and Stone Free Rate: A Single Surgeon Experience}

J Feghali

KMC Hospital, Department of Urology, American University of Beirut, Jounieh-Lebanon; SZUMC, Department of Urology, Lebanese University, North-Lebanon; NINI Hospital, Department of Urology, Beirut Arab University, North-Lebanon

Introduction \& Objective: During the last decade all efforts are made in order to have a simple and safe minimal invasive treatment for kidney stones and improve patient-urologist communication and stone free rate. Objective: Evaluate the Awake Flexible Ureteroscopy (AFURS) technique (Fig 1); defined as performing flexible ureteroscopy under spinal anesthesia (SA) while patient is watching live his procedure and communicating with his surgeon.

Methods: From February 2015 till May 2019, patients who accepted to be treated for AFURS (S/A with 25G or $27 \mathrm{G}$ spinal needle at L3-L4 interspace using heavy bupivacaine up to $15 \mathrm{mg}$ $+0.1 \mathrm{mg}$ morphine) were treated by single surgeon in three different centers. Patients characteristics; Patients impressions (5 hours after procedure) about AFURS; and Procedures characteristics were collected on prospective database.

Results: 122 patients ( 88 male and 34 female) performed the procedures with mean age 50.8 (24-89) years and mean stone size $2.44 \mathrm{~cm} ; 32.8 \%$ of patients had lower pole stone; $62.3 \%$ of patients had stent prior to surgery (Table1A). One patient felt anxious, painful and regret the procedure, so he was converted to General Anesthesia; 99.2\% of patients found AFURS improve patient-urologist communication and facilitate understanding of residual stone fragments and own kidney anatomy (Table1B). A total of 129 procedures were performed with mean number of procedures per case 1.06; mean operating room time $118 \mathrm{mns}$ (45-200); Stone Free Rate 95.9\% (fragment(s) $\leq 2 \mathrm{~mm}$ on standard radiograph and/or Uroscan) 1 month after treatment completion; UAS was used in $89.3 \%$ of cases $(40.2 \%(11 / 13))$. In $51.6 \%$ of cases a 6 Fr stent was used, and no stent used in one case. Complication rate was $3.1 \%$; one patient had pain, one had hematuria, one had mild ureteral perforation, and one had sepsis (Table1C).

Conclusions: AFURS is a safe and feasible technique for treating different sizes of kidney stones; it may improve patienturologist communication and stone free rate by avoiding patient's misinterpretation of residual stone fragments and in-

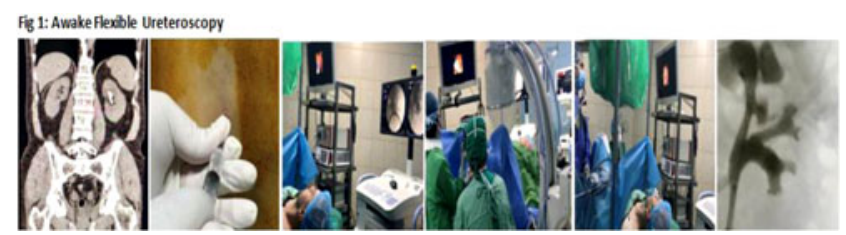

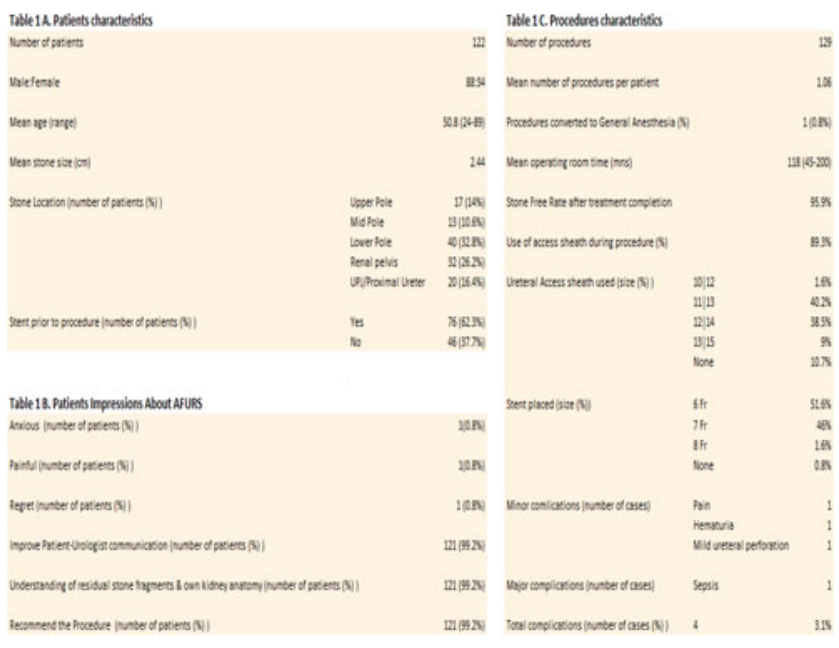

forming the patient about his own kidney anatomy, and best way to clear small residual stones.

MP25-04 Dedicated Day Case Ureteroscopy List: standardising the pathway and improving outcomes

\section{Bastianpilliai, S Mathew, A Golash, H Fernando}

University Hospitals North Midlands

Introduction \& Objective: Setting up a Dedicated Day Case Ureteroscopy (DDCU) List has inherent advantages in establishing a standard pathway for patients requiring this procedure. With finite resources and limited skilled staffing, dedicated DCU Lists increase efficiency, enhance training opportunities, and improve outcomes. We report the outcomes of DCU Lists in a University Hospital by a single surgeon who started this service. Methods: This is a retrospective study of all the ureteroscopies done in a dedicated DCU List, performed or supervised by a single consultant between May 2014 and March 2018, using electronic theatre and inpatient records. Data on patient demographics, procedure indication, intraoperative and postoperative outcomes and emergency re-attendances to hospital was recorded. The DDCU List is a fixed session once a week with a fixed urology theatre team and a radiographer. Patients are reviewed and discharged by the Day Case Staff / team if the discharge criteria are satisfied, with the necessary information booklet and follow-up plan.

Results: A total of 389 ureteroscopic procedures were performed over a 46-month period under a single surgeon, of which 347 were identified as elective planned day case procedures. Indications included the treatment of stones (84\%), evaluation and treatment of malignancy $(14 \%)$, and the treatment of strictures (2\%). $53 \%$ of the cases were coded for flexible ureterorenoscopy. Two-hundred and forty-six elective procedures $(71 \%)$ were discharged the same day while $21 \%$ were discharged within 24 hours from the day unit. The overall stone-free rate was $94 \%$. The re-attendance rate was $6.9 \%$ in the day case cohort with twothirds of cases due to stent symptoms.

Conclusions: The Dedicated DCU List is a model that is able to produce similar published outcomes with reduced morbidity and readmissions. We perceive this model to streamline the waiting lists in busy tertiary units, while also addressing training needs. The fixity of the lists ensures a reliably urologically trained theatre team and radiographer. 


\section{MP25-05 Defining thermally safe laser lithotripsy power and irrigation parameters: in vitro model}

A Aldoukhi, K Black, T Hall, K Ghani, AD Maxwell, B MacConaghy, WW Roberts

Department of Urology, Univeristy of Michigan

Introduction \& Objective: High-power laser settings are commonly employed for stone dusting techniques. Previous in vitro and in vivo studies have demonstrated that a toxic thermal dose can result from treatment within a renal calyx. Hence, both laser power and irrigation rate must be considered together to determine safe laser lithotripsy parameters. The objective of this in vitro study was to map the parameter safety boundaries and create guidelines for selection of safe laser lithotripsy settings and irrigation rates for simulated ureteral, calyceal, and pelvis model.

Methods: The experimental system consisted of in vitro models simulating ureter, renal calyx, and renal pelvis placed in a water bath maintained at $37^{\circ} \mathrm{C}$ (Figure 1A). A $242 \mu \mathrm{m}$ laser fiber (Flexiva, Boston Scientific) was inserted through a ureteroscope (Lithovue, Boston Scientific). Real time temperature was recorded by a wire thermocouple placed at the level of the ureteroscope with laser activation for 60 seconds. Trials were conducted at strategically selected power levels $(2.5-50 \mathrm{~W})$ and irrigation rates $(0-30 \mathrm{ml} / \mathrm{min})$; irrigation was provided with room temperature fluid. Thermal dose for each trial was calculated based on Dewey and Sapareto t43 methodology with

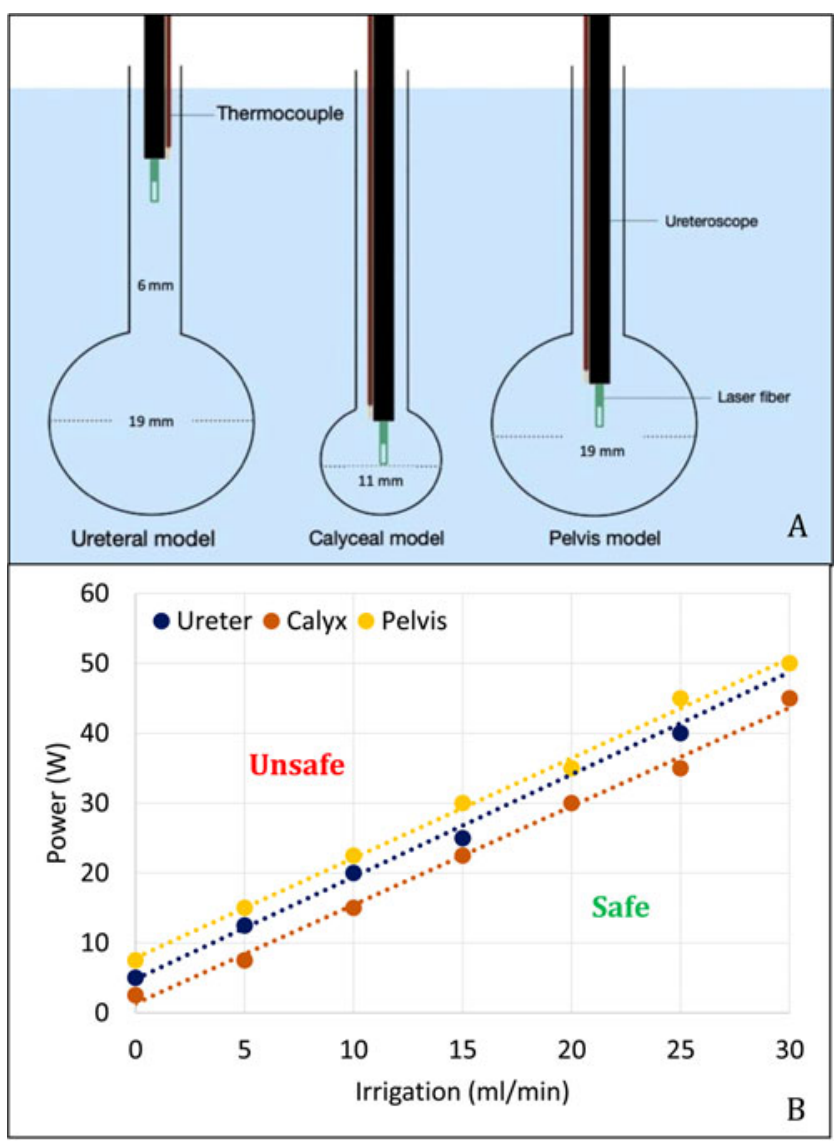

Figure 1: (A) Representation of the ureteral, renal calyx, and renal pelvis models and positioning of the ureteroscope, laser fiber, and thermocouple in each model. (B) Comparisons of the lower limit of the safety margins for the ureteral, calyceal, and pelvis model. thermal dose $>120$ equivalent minutes considered to result in thermal tissue injury. A parameter safety boundary was established by plotting the maximal safe power level for each irrigation rate.

Results: The parameter safety boundary was found to be linear for each scenario with the renal pelvis able to tolerate the highest laser power and the renal calyx the lowest power without injury. Figure $1 \mathrm{~B}$ presents the safety boundary for the simulated ureteral, calyceal, and pelvis model. For instance, when using a laser setting between $15-30 \mathrm{~W}$ an irrigation rate between 10 $20 \mathrm{ml} / \mathrm{min}$ is needed.

Conclusions: This study describes methodology to determine parameter safety boundaries that can be used to guide proper selection of thermally safe laser settings and irrigation rates during ureteroscopy with laser lithotripsy. This work provides a framework to assess the effectiveness of various strategies to control and mitigate thermal dose.

\section{MP25-06 Safety of Live Retrograde Intrarenal Sur- gery; Results from Society of Urological Surgery in Turkey-Stone Group (SUST-SG) Courses}

O Özman, S Çıtgez, C Basatac, H Akgül, C Yazici,

Y Tanidir, H Akpinar, B Onal

Gayrettepe Florence Nightingale Hospital

Introduction \& Objective: The aim of this study is to investigate the outcomes and complication rates of patients undergoing retrograde intrarenal surgery (RIRS) during the live surgery events (LSE) that were organized by Society of Urological Surgery in Turkey (SUST).

\begin{tabular}{|c|c|c|c|}
\hline Characteristic & LSE Group & Control Group & p value \\
\hline Number & 14 & 13 & \\
\hline Age (year, mean) & $45.9 \pm 17.9$ & $32.7 \pm 20.8$ & 0.09 \\
\hline (median, range) & $45(2-72)$ & $32(5-65)$ & \\
\hline \multicolumn{4}{|l|}{ Gender } \\
\hline Male & $5 / 14(36 \%)$ & $7 / 13(54 \%)$ & \multirow{2}{*}{0.34} \\
\hline Female & $9 / 14(64 \%)$ & $6 / 13(46 \%)$ & \\
\hline \multicolumn{4}{|l|}{ Side } \\
\hline Right & $10 / 14(71 \%)$ & $9 / 13(69 \%)$ & \multirow{2}{*}{1} \\
\hline Left & $4 / 14(29 \%)$ & $4 / 13(31 \%)$ & \\
\hline \multicolumn{4}{|l|}{ Stone Burden } \\
\hline Max. diameter (mm) & $11.6 \pm 2.17$ & $10.6 \pm 4.09$ & 0.52 \\
\hline Stone area $\left(\mathrm{cm}^{2}\right)$ & $1.23 \pm 0.36$ & $1.26 \pm 0.80$ & 0.88 \\
\hline Hunsfield Unit (mean) & $1069 \pm 308$ & $1090 \pm 193$ & 0.83 \\
\hline \multicolumn{4}{|l|}{ Localisation } \\
\hline Lower Calyx & $4 / 14$ & $3 / 13$ & \multirow{4}{*}{0.31} \\
\hline Other Calyces & $3 / 14$ & $5 / 13$ & \\
\hline Pelvis & $6 / 14$ & $2 / 13$ & \\
\hline Multiple Loc. & $1 / 14$ & $3 / 13$ & \\
\hline Hydronephrosis & $9 / 14(64 \%)$ & $5 / 13(38 \%)$ & 0.18 \\
\hline Preoperative Treatment for Same Stone & $8 / 14(57 \%)$ & $8 / 13(62 \%)$ & 0.82 \\
\hline Preoperative Double J Stent & $4 / 14(29 \%)$ & $4 / 13(31 \%)$ & 1 \\
\hline
\end{tabular}




\begin{tabular}{lccc}
\hline \multicolumn{3}{l}{ Table 2. Perioperative and Postoperative Outcomes of LSE Group According to Surgeons } \\
\hline Characteristic & Host surgeons & Guest surgeons & p value \\
\hline Number & 6 & 8 & \\
Operation Time (minute) & $63.5 \pm 28.1$ & $85.8 \pm 28.6$ & 0.17 \\
Floroscopy Time (second) & $11.3 \pm 3.8$ & $18.6 \pm 16.3$ & 0.31 \\
Length of Hospitality (day) & $1.33 \pm 0.82$ & $1.88 \pm 0.99$ & 0.30 \\
Stone Free & & & \\
Early & $83.3 \%(5 / 6)$ & $87.5 \%(7 / 8)$ & 1 \\
Late & $100 \%(6 / 6)$ & $87.5 \%(7 / 8)$ & N/A \\
Complication Rate & $0 \%(0 / 6)$ & $25 \%(2 / 8)$ & N/A \\
Need for Additional Procedure & $0 \%(0 / 6)$ & $\% 12.5(1 / 8)$ & N/A \\
\hline
\end{tabular}

\begin{tabular}{lccc}
\hline \multicolumn{3}{l}{ Table 2. Perioperative and Postoperative Outcomes } & \\
\hline Characteristic & LSE Group & Control Group & p value \\
\hline Operation Time (minute) & $76.2 \pm 29.6$ & $58.2 \pm 23.2$ & 0.09 \\
Floroscopy Time (second) & $15.5 \pm 12.8$ & $16.4 \pm 10.7$ & 0.84 \\
Length of Hospitality (day) & $1.64 \pm 0.92$ & $3.92 \pm 5.37$ & 0.13 \\
Perioperative Access Sheat & $14 / 14(100 \%)$ & $10 / 13(77 \%)$ & 0.09 \\
Stone Free & & & 1 \\
$\quad$ Early & $12 / 14(86 \%)$ & $12 / 13(92 \%)$ & 1 \\
$\quad$ Late & $13 / 14(93 \%)$ & $11 / 13(85 \%)$ & 0.59 \\
Complication Rate & $2 / 14(14 \%)$ & $2 / 13(15 \%)$ & 1 \\
Need for Additional Procedure & $1 / 14(7 \%)$ & $0 / 13(0 \%)$ & 1 \\
Postoperative Double J Stent & $13 / 14(93 \%)$ & $11 / 13(84 \%)$ & 0.59 \\
\hline
\end{tabular}

Methods: Five RIRS courses were organized by SUST in Turkey between November 2017 and May 2018. Data of 14 patients who underwent live RIRS (as LSE group) were compared with the data of 13 substitute patients (as control group) who underwent regular RIRS on the same day and at the same centers.

Results: Early and late stone free status of groups were similar (86-93\% in LSE and $92-85 \%$ in control group; $p=1.0$ and $\mathrm{p}=0.59$, respectively). There was no difference in complication rates between the two groups $(\mathrm{p}=1.0)$. The mean operation and fluoroscopy times were statistically similar for both groups $(\mathrm{p}=0.09, \mathrm{p}=0.84$ respectively).

Conclusions: RIRS, supported by new technologies, is implemented more frequently every day practice because of the increasing incidence of kidney stones and live surgical events on RIRS have become more popular. RIRS live surgery can be performed with low complication and high stone-free rates without jeopardizing patient safety.

\begin{tabular}{lcll}
\hline \multicolumn{3}{l}{ Table 3. Postoperative complication characteristics } \\
\hline Variable & Grade & LSE Group & Control Group \\
\hline Postoperative complications & 1 & $1(50 \%)$ & $1(50 \%)$ \\
according to the & 2 & $0(0 \%)$ & $1(50 \%)$ \\
Clavien Classification & 3 & $1(50 \%)$ & $0(0 \%)$ \\
$\begin{array}{l}\text { Postoperative } \\
\text { complications }\end{array}$ & 4 & $0(0 \%)$ & $0(0 \%)$ \\
\hline
\end{tabular}

MP25-07 Assessment of the clinical and economic relevance of the use of single-use digital flexible ureteroscopes: A systematic review

C McMartin, J Cloutier, S L'Espérance, R Drolet, A Nourissat, M Rhainds

CHU de Québec - Université Laval

Introduction \& Objective: Ureteroscopes (URS), especially digital flexible URS (dflURS), are very fragile and their use are associated with high rate of breakage due to transport, handling, reprocessing or use during a clinical situation with a high risk of breakage. Recently, single-use flexible digital URS (SUFDU) have been commercialized and could represent an interesting alternative to reusable dflURSs, particularly in terms of material resource management. We aimed to determine if the use of SUFDU may represent an effective device to implement in a high-volume hospital in order to reduce flURS breakage.

Methods: Systematic literature searche was conducted in several indexed databases and grey literature until Septembre 2018 in order to retrieve studies on clinical efficacy, safety and cost of SUFDU. Upon comparative analysis of efficacy of SUFDU, a cost minimization analysis was performed from our hospital annual ureteroscopy volume data. Budget impact related to SUFDU introduction was estimated according reduction of URS breakage and repair costs. Several scenarios comparing annual costs according to the proportion of single-use digital flexible SRUs used and the percentage of breakage avoided (25, 50, 75 and $100 \%$ ) was performed.

Results: Two non-randomized prospective studies aiming to assess SUFDU efficacy was included. Results suggested that efficacy of SUFDU for urinary lithiasis in adult population is similar to reusable fIURS. Results from 5 published economic studies suggested that global cost of SUFDU is higher than the cost of reusable dflURS. In 2017-2018, 328 flexible digital ureteroscopy have been performed in our hospital. Breakage rate of our dfIURS have been estimated to $6,4 \%$. Average cost per intervention of reusable dfIURS have been estimated at 353\$CAD while cost of SUFDU varied from $800 \$$ CAD to $1500 \$ C A D$ per unit depending of the brand of SUFDU used. Results from our cost analysis according breakage avoidance scenario suggested that less than $10 \%$ of our ureteroscopy should be performed with SUFDU in our hospital to stay cost effective.

Conclusions: Few evidence exist to support exclusive utilization of SUFDU in high volume tertiary hospital. Their equivalent efficacy with reusable dflURS and their higher cost should be considered into the decision processes relative to their introduction. Further studies should be made in order to assess their effectiveness and cost-effectiveness during clinical intervention with high risk of dfIURS breakage in high volume centers like ours.

MP25-08 The electron-microscopic characteristics of stones-new tool in the retrospective evaluation of laser settings applied in individualized lithiasis' treatment

BF Geavlete, G Balan, DA Georgescu, PA Geavlete

Saint John Emergency Clinical Hospital

Introduction \& Objective: The efficiency of holmium laser lithotripsy for urolithiasis depends upon several clinical factors, 
including stone size, composition, location and retropulsion. Different combinations of pulse energy, pulse frequency, and pulse width have specific effect on lithotripsy during ureteroscopy. The aim of our study was to evaluate how the stone composition influences the result of laser fragmentation.

Methods: We evaluated in vitro the effect of different Ho:YAG laser settings on different types of calculi. We used optical fiber of $200 \mu \mathrm{m}$. Different laser settings from 0.2 to $1.5 \mathrm{~J}$ and 10 to $30 \mathrm{~Hz}$ were used. Uric acid, calcium oxalate mono- and dehydrate and magnesium ammonium phosphate calculi were studied. The results were evaluated on electron microscopy by measuring the craters produced on the surface of stone.

Results: At same working parameters, but especially at low pulse energy of $0.2 \mathrm{~J}$, the volume of the crater for calcium oxalate monohydrate stones was significantly smaller than that obtained for uric acid and magnesium ammonium phosphate stones. With hard stones, higher energy is needed to obtain smaller fragments. Conclusions: By knowing stone composition we can adjust laser settings in order to obtain maximal fragmentation in shorter time.

\section{MP25-09 Compared endoscopic and microscopic rec- ognition of urinary stones: preliminary concordance study}

G Verzotti, M Daudon, P Meria, C Almeras, O Traxer, $\mathrm{V}$ Estrade

\section{Hospital Pellegrin}

Introduction \& Objective: The treatment of urinary stones is a matter of fragmentation or even dusting. The microscopic morpho-constitutional analysis (MCA) of a whole urinary stone is then no longer feasible. However, MCA has the best diagnostic concordance to the lithiasic mechanism that has produced the stone. We compared the endoscopic and microscopic stone recognition of urinary stones to validate the Endoscopic Stone Recognition (ESR).

Methods: Combined and independent urinary stone MCA was performed by two experts: a urologist and a biologist. The former examined the surface and cross-section of the stones with a ureteroscope before fragmentation. The ESR was performed according to the international MCA stones classification. Stone fragments were sent for MCA and infrared spectrometry. A comparative concordance study between the two approaches (endoscopic and microscopic) was carried out. $\mathrm{A} \mathrm{Chi}^{2}$ test was performed to assess this concordance.

Results: 268 files were examined. 7 files were excluded because images were not recognizable (fuzzy images). 7 more files were excluded because laboratory analysis could not be performed. In total, 254 files were included in the analysis. This comprised 34 types of stones, including 15 pure and 19 mixed. 15 stones had a specific endoscopic recognition that was not found on infrared examination. The $\mathrm{Chi}^{2}$ difference test found an excellent $(\mathrm{p}>\mathrm{or}=0.05)$ concordance for pure stones $(\mathrm{Ia}+\mathrm{Id})$ of whewellite $(85.7 \% \mathrm{n}=7)$, (IIb) weddellite $(88.2 \% \mathrm{n}=17)$, (IIIab) uric acid $(97,1 \% \mathrm{n}=34),(\mathrm{IVc})$ struvite $(100 \% \mathrm{n}=4)$ and mixed stones (Ia+IIb) whewellite/weddellite $(92.5 \% \mathrm{n}=40),(\mathrm{Ia}+\mathrm{III} \mathrm{Iab})$ whewellite/uric acid $(76.9 \% \mathrm{n}=13)$, (Ia+IIb+IVa), whewellite/weddellite/carbapatite $(87.5 \% \mathrm{n}=16)$. Other morphologies have an excellent concordance: Va cystine $(100 \% \mathrm{n}=1)$, VIa proteins $(100 \% \mathrm{n}=1), \mathrm{IIb}+\mathrm{IVd}$ weddellite/brushite $(100 \% \mathrm{n}=3)$ but too small a number to be considered representative.

Conclusions: This analysis confirms the reliability of ESR for the most frequent pure stones (Ia+Id) of whewellite, (IIb) wed-

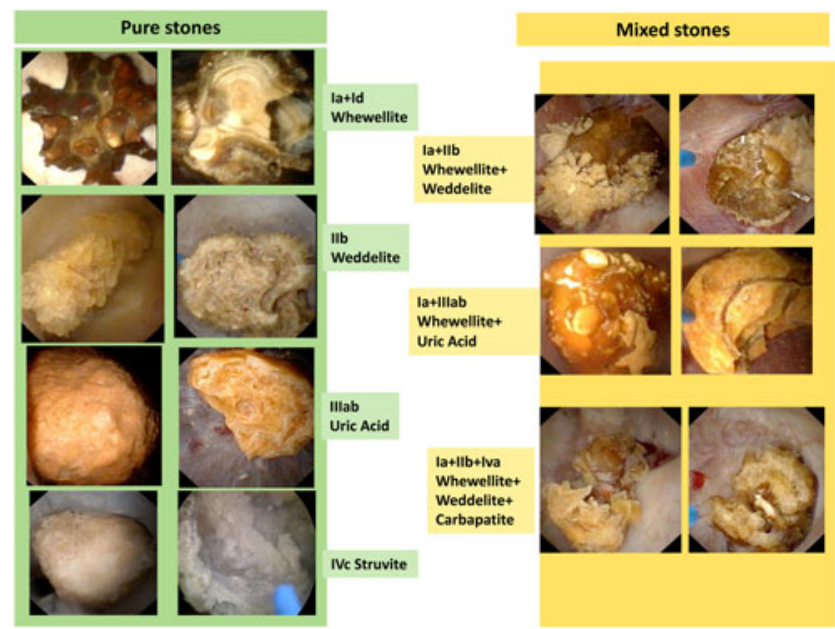

dellite, (IIIab) uric acid, (IVc) struvite and mixed (Ia+IIb) whewellite/weddellite, (Ia+IIIab) whewellite/uric acid, (Ia+IIb+IVa) whewellite/weddellite/carbapatite. ESR is a fundamental step in lithiasis. However, it must always be combined with infrared spectroscopy of the fragments.

\section{MP25-10 New proposed "NASA" score for mea-} surement of stone free rate in flexible ureteroscopy

P Cortes-Raygoza, C Martinez-Arroyo, M Ortega-Gonzalez, E Hernandez-Mendez, I Calvo-Vazquez, G Fernandez-Noyola, M Cantellano-Orozco, G Morales-Montor, C Pacheco-Gahbler

Hospital General Dr. Manuel Gea Gonzalez

Introduction \& Objective: Lithotripsy with flexible ureteroscopy has been considered the gold standard for the management of single renal stones and less than $2 \mathrm{~cm}$. There is no standardized practice for measuring Stone free rate. It is essential to perform a simple Score, easy to remember to provide a better expectation to

\begin{tabular}{|c|c|}
\hline $\begin{array}{c}\text { Score Predictor of } \\
\text { Stone Free Rate }\end{array}$ & $\begin{array}{c}\text { Stone Free Rate } \\
\text { (Percentage) }\end{array}$ \\
\hline Gea-NASA & $94.7 \%$ \\
\hline RUSS & $90.8 \%$ \\
\hline S-ReSC & $92.8 \%$ \\
\hline RIRS & $94.9 \%$ \\
\hline
\end{tabular}

\begin{tabular}{|c|c|c|}
\hline $\begin{array}{c}\text { Gea-NASA } \\
\text { Score }\end{array}$ & $\begin{array}{c}\text { Stone Free Rate } \\
\text { (surgeries/Stone free) }\end{array}$ & $\begin{array}{c}\text { Value } \\
\text { P }\end{array}$ \\
\hline 3 & $93.7 \%(30 / 32)$ & 0.005 \\
\hline 4 & $100 \%(42 / 42)$ & 0.001 \\
\hline 5 & $54.7 \%(39 / 45)$ & 0.029 \\
\hline 6 & $50.0 \%(3 / 6)$ & 0.635 \\
\hline 7 & $0 \%(0 / 1)$ & $<0.001$ \\
\hline
\end{tabular}




\begin{tabular}{|c|c|c|}
\hline Gea- NASA score & 1 & 2 \\
\hline (N) Number & 1 & $20+$ \\
\hline (A) Angle & $\begin{array}{c}>45 \text { or } \\
\text { outside of } \\
\text { inferior calix }\end{array}$ & $<45$ \\
\hline (S) Size & $<2 \mathrm{~cm}^{3}$ & $>2 \mathrm{~cm}^{3}$ \\
\hline (A) Anatomy & abnormal & $\mathrm{NA}$ \\
\hline
\end{tabular}

our patient. We provide a new option for the evaluation of stone free rate in flexible ureterenoscopy easy to remember and very accurate approach to stone free rate.

Methods: Retrospective, descriptive study of patients undergoing flexible ureteroscopy with laser lithotripsy in the period from January 2016 to May 2018 managed at the General Hospital Dr. Manuel Gea GonzÃ $\tilde{A}_{i}$ lez, we applied the RUSS (Resorlu-Unsal Stone Score). S-ReSc (Modified Seoul National University Renal Stone Complexity Score) and NASA (General Hospital Manuel Gea Gonz $\tilde{A}_{j}$ lez Renal Score of complexity in flexible ureteroscopy) we applied a multivariate analysis and performa detailed evaluation of the characteristics of the stones.

Results: 126 patients, average age $47( \pm 13.81)$, number of stones handled $1.64( \pm 3.41)$, morphometry $1.4( \pm 1.8 \mathrm{~cm} 3)$, location: Lower calyx $64(50.79 \%)$, of them an infundibulopelvic Angle less 45 degrees.

Conclusions: All proposed SCOREs are sufficient to perform an assessment that determines the stone free rate, an easy scale to apply for routine use in flexible ureteroscopy is necessary, with sufficient validity to predict the stone free rate.

\section{MP25-11 Large Ureteric Stones: No To Knife-The Laser Edge In Stone Age!}

SM Mahmud

Lifecare Hospital (Abu Dhabi Campus)

Introduction \& Objective: Urolithiasis is now one of the most common diseases ailing humanity with ever increasing worldwide incidence and prevalence. There are immense research and literature formulating guidelines in stone management. However, there is no standardization in treating large ureteric stones particularly more than $1.5 \mathrm{~cm}$ size. Large stones have conveniently been classified as Class I $(1.0-1.2 \mathrm{~cm})$, Class II (1.3$2.0 \mathrm{~cm})$ and Class III $(>2 \mathrm{~cm})$. The largest reported size is $11.5 \mathrm{~cm}$. Considering the high prevalence and recurrence in stone belt regions, it is essential to be as minimally invasive as possible. The technological advancement in terms of effective miniaturization of scopes and LASER technology, very large stones are now frequently fragmented, and the patient is set stone free in a single session without the use of a knife. We are hereby sharing our experience of managing Large Ureteric stones Ureteroscopy LASER Lithotripsy with an objective to emphasize on minimally invasive approach for even very Large Ureteric Methods: All Adult patients undergoing URS LASER Lithotripsy from October 2014 to April 30, 2019 at Lifecare Hospital Abu Dhabi, UAE. Prospectively maintained data for all patients undergoing Ureteroscopy LASER Lithotripsy were reviewed and patients with large Ureteric stones were selected for study assessing stone size, number of sessions of Ureteroscopy, stone freeing period, Stone free rate and Complications. The large stone is defined as size more than or equal to $1.3 \mathrm{~cm}$. Almost all patients underwent the procedure under Spinal Preoperatively Imaging included IVU or CT Abdomen/Pelvis unenhanced and first post of imaging as Xray KUB/ Ultrasound KUB performed in 1 week at first Follow up. All patients underwent LASER Lithotripsy with Ho:YAG LASER with defined Tissue Penetration $0.4 \mathrm{~mm}$. The procedure was performed with LASER Fiber $365 \mu \mathrm{m}$, Frequency $12-15 \mathrm{~Hz}$, Energy 1200-1500 mJ and Power 15 -25 Watts.

Results: In the study Period there were 465 patients who underwent Ureteroscopy and LASER Lithotripsy $(\mathrm{N}=465)$ and 35 were identified as having large ureteric stones $(n=35)$. Mean Stone Size was $1.8 \mathrm{~cm}(1.3-2.1)$. All patients had Double J Stenting. Twenty nine out of 35 were stone free in 1 month $(82.85 \%)$ and $31 / 35(88.57 \%)$ at two months. There were 4 patients who required retreatment. Complications rate was low with no ureteric perforation, sepsis or mortality.

Conclusions: URS LASER Lithotripsy can be the first line treatment for Large Impacted ureteric stones even up to $2 \mathrm{~cm}$ in size. There should always be an understanding of the limitations and readiness to review strategy

\section{MP25-12 Application of Soton ureteroscopy in the treatment of long Segment of Ureteral Stone Street}

W Xiaoning, Z Xiaofeng, Y Yuanhu, X Rihai, Z Guoxi, W Gengqing

Introduction \& Objective: To explore the clinical effect of Soton ureteroscopy on a long section of ureter stone street.

Methods: From March 2018 to June 2018, four patients with long ureteral stone street were treated by Soton ureteroscopy with holmium laser. There were three males and one female, aged 25 to 48 years, with an average age of 39 years. The length of the ureteral stone street was $5.2-10.8 \mathrm{~cm}$, with an average length of $7.4 \mathrm{~cm}$; maximum diameter of stone $0.4-4.0 \mathrm{~cm}$ (mean $1.1 \mathrm{~cm}$ ). Two cases developed a stone street after a previous flexible ureteroscopy, while two cases had no previous surgical treatment. The procedure was performed under general anesthesia and in the lithotomy position. A 12.5 French rigid ureteral passage sheath was inserted under zebra guide wire. A Soton ureteroscopy was inserted up the sheath until it reached the proximal stone. A negative pressure attractor was used to clear the stone followed by Holmium laser lithotripsy.

Results: The ureteral passage sheath was successfully implanted in all four cases and the lithotripsy was completed in one stage. The operative time ranged from $40-90$ minutes, with an average of 56 minutes. There were no complications such as ureteral perforation, mucosal avulsion, or massive bleeding found in any of the patients. After the operation, the ureter was patent and the removal rate of ureteral stone was 100 percent. There were no fevers or peri-renal hematomas formed in the postoperative hospitalization, occurring 1 - 3 days. One month after the operation, the ureteral stents were removed. Two months after the operation, B-ultrasound showed that hydronephrosis was decreased compared to preoperative imaging studies and there was no recurrence of stones.

Conclusions: Soton ureteroscopy should be considered as an option to treat a long segment stone street of the ureter in adults. The ureteral sheath combined with negative pressure suction can remove the stone simultaneously, improve the efficiency of the operation, and reduce the complications, such as ureteral injury and postoperative infection. These results suggest a safe and effective option worthy of clinical selection. 
MP25-13 “VaporTunnel” Ureteroscopic Holmium laser lithotripsy: intraoperative and early postoperative outcomes

G Bozzini, U Besana, A Calori, S Proietti, G Giusti, C Buizza, M Sighinolfi, B Rocco

ASST Valle Olona - Busto Arsizio VA

Introduction \& Objective: "VaporTunnel" technique is a result in pulse modulation during holmium laser lithotripsy in which the laser emits part of the energy to create an initial bubble, and the remaining energy is discharged once the bubble is formed so that it can pass through the already formed vapor channel. The aim of this study is to outline the outcomes of the Vapor Tunnel technology in ureteral stones.

Methods: A prospective trial was conducted for patients undergoing holmium laser lithotripsy for ureteral stones. Patients were randomly assigned to have holmium laser lithotripsy with or without Vapor Tunnel technology. All procedures were performed by four experienced urologists. Quanta Cyber Ho $100 \mathrm{~W}$ generator with $365 \mu$ was used for all cases. Demographic data, stone parameters, perioperative complications and success rates were compared. The degree of stone retropulsion was graded on a Likert scale from zero-no retropulsion to 3-maximum retropulsion.

Results: A total of 80 patients were included in the study ( 40 per each group). Both groups were comparable in terms of age, and preoperative stone size (1.2 vs. $1.1 \mathrm{~cm}, \mathrm{p}>0.05)$. When compared with the Regular mode, Vapor Tunnel Technology was associated with significantly lower fragmentation time (20.4 vs. $16.1 \mathrm{~min}, \mathrm{p}<0.05)$ and total procedural time (49 vs. $35.7 \mathrm{~min}, \mathrm{p}<0.05$ ). However, there were no significant differences in terms of total energy applied to the stones (9.9 vs. 10.7 KJ, p>0.05). Vapor Tunnel technology was associated with significantly less retropulsion. There was no significant difference between both modes in terms of intraoperative complications. The success rate at the end of 1 month was comparable between both groups ( 92.3 vs. $88.3 \%, \mathrm{p}>0.05)$.

Conclusions: Vapor Tunnel technology is associated with significantly lower fragmentation and procedural times. The reduced fragmentation time is a result of the significantly lower retropulsion of the stones during laser lithotripsy; thus, improving stone fragmentation efficiency.

\section{MP25-14 Comparison of early vs delayed uretero- scopy following obstructive pyelonephritis treated with urinary diversion}

S Lagacé, J Cloutier, I Fugaru, D Simonyan, N Laflamme

Université Laval

Introduction \& Objective: Urolithiasis is a common disease affecting approximately $8.8 \%$ of Americans. Obstructive urolithiasis and sepsis call for emergent decompression and antibiotic therapy. After treatment, definitive management can be achieved with ureteroscopy (URS) or percutaneous nephrolithotomy. There are no guidelines identifying the best moment to perform elective URS after urosepsis. Thus, our objective is to compare the outcomes, especially post-URS sepsis, of patients who underwent early vs delayed URS for obstructive pyelonephritis following decompression.

Methods: In this retrospective, noninferiority and single-center study, data was collected from patients who underwent elective ureteroscopy following decompression for obstructive pyelonephritis between years 2012 and 2017. Patients with the following criteria were excluded: obstruction unrelated to urolithiasis, other procedures performed during URS and unclear diagnosis. Early URS was defined as URS performed within 14 days after decompression while the patient was still on antibiotics.

Results: A total of 164 patients were included in the study. Of those patients, 61 of them had early URS, while 103 had delayed URS. There were a total of 10 post-URS sepsis including 1 in the early URS group and the other 9 in the delayed URS group. The adjusted odds ratio after multivariate analysis is $7.3(\mathrm{p}=0.0661)$ for post-URS sepsis when comparing delayed URS to early URS. The complication-free survival at 30 days is $98.4 \%$ for early URS and $91.3 \%$ for delayed URS $(\mathrm{p}=0.0665)$.

Conclusions: In conclusion, our study clearly shows the noninferiority of early URS for post-URS sepsis when compared to delayed URS. Furthermore, our data are showing a tendency that early URS might be better than delayed URS regarding post-URS sepsis although it is not statistically significant. Further studies will be needed to evaluate the superiority of early URS. However, our results showed that it is safe to perform early URS for treatment of urolithiasis following emergent decompression for obstructive pyelonephritis.

\section{MP25-15 Withdrawn}

MP25-16 Clinical effects of Poly- Flexible Ureteroscopy combined with ultrasound guided all seeing needle Microperc to treatment of Lower Pole Renal Calculi larger than $2 \mathrm{~cm}$ with infundibulopelvic angle less than 60 degrees

\author{
G Jingyang, Y Wenzeng, Z Yanqiao
}

Affiliated Hospital of Hebei University

Introduction \& Objective: To observation on Poly- Flexible Ureteroscopy (FURS) retrograde intrarenal surgery (RIRS) combined with ultrasound guided all seeing needle Microperc to treatment of Lower Pole Renal Calculi larger than $2 \mathrm{~cm}$ stone with infundibulopelvic angle (IPA) less than 60 degrees.

Methods: 63 patients with IPA angle less than 60 degrees in our hospital were treated with Poly-FURS retrograde intrarenal surgery combined with ultrasound guided all seeing needle Microperc, the patients stone sized more than $2 \mathrm{~mm}$ and located in the lower-pole calyx were retrospectively reviewed. Multiple stones were found in 37 cases and solitary stones in 26 . Male patients used $70 \mathrm{~cm}$ Poly-FURS, and the female $42 \mathrm{~cm}$. The clinical data including the operation time, the amount of bleeding, the stone free rate (SFR), the incidence of postoperative complications.

Results: The operation time was 30 60min, mean 45min, FURS lithotripsy time was $20 \sim 35 \mathrm{~min}$, ultrasound guided all seeing needle puncture time was $3 \sim 5 \mathrm{~min}$, and lithotripsy time was $8 \sim 12 \mathrm{~min}$. 48 cases mainly lithotripsy with FURS and 15 cases with microPercutaneous nephrolithotomy(micro-perc), 3 cases of fever as postoperative complications, 2 cases of pain; In non blood transfusion patients. The postoperative hospital stay was $2 \sim 3$ days, with an average of 2 days. Postoperative residual stones were observed in 3 cases (SFR 95.2\%) and treated with ESWL after 2 weeks. The stone clearance rate was $98.4 \%$ (62/63) after one month.

Conclusions: The results of our study demonstrated that PolyFURS retrograde intrarenal surgery combined with ultrasound guided all seeing needle Microperc had a significant effect on the success rate of lower Pole Renal Calculi larger than $2 \mathrm{~cm}$. 


\section{MP25-17 A single center prospective randomized controlled trial of two single use flexible ureteroscopes - LithoVue (Boston Scientific, USA) and Pusen PU3022a (Zhuhai Pusen, China)}

A Patil, S Agrawal, A Singh, A Ganpule, R Sabnis, M Desai

Muljibhai Patel Urological Hospital, Nadiad, Gujarat, India

Introduction \& Objective: The single use flexible ureteroscopes are now becoming equivalent to standard flexible ureteroscopes. The single use scopes have the benefit of decreasing infection transmission, avoiding the need for sterilization and no maintenance cost. The primary objective of this study was to compare the in vivo performance and surgical outcomes with these two single use ureteroscopes: LithoVue (Boston Scientific, USA) and Pusen PU3022a (Zhuhai Pusen, China) with the secondary objective being to compare their in vitro performance.

Methods: 50 patients having renal stones less than $2 \mathrm{~cm}$ and planned for RIRS were prospectively randomized to two groups: Group 1 had 25 patients in which LithoVue scope was used and group 2 had 25 patients in which Pusen scope was used. The various pre-, intra- and post-operative parameters were evaluated for both the scopes. The in vitro parameters included maximal tip deflection and irrigation flow rate with empty working channel, $200 \mu \mathrm{m}$ laser fiber and 1.9Fr basket. The vision of both the scopes without any accessory, with basket and with laser fiber was evaluated by an endourologist who was not part of surgical team and blinded to scope manufacturer.

Results: The preoperative parameters including demographic, stone size, stone location, stone HU, stone number, serum creatinine were comparable in both the groups. Intra-operative parameters including the need for ureteral dilatation $(\mathrm{p}=0.5)$, ureteral access sheath size $(p=0.38)$, accessibility $(p=0.66)$ and maneuverability $(p=0.66)$ were comparable in both the groups. The lower pole access was possible in all cases of Lithovue but was slightly difficult in 3 cases of Pusen (two with laser and one with basket), but statistically insignificant $(p=0.24)$. Intraoperative vision was hazy in 4 cases of Lithovue during lasing and in 3 cases of Pusen with accessory $(p=0.11)$ and eight cases during lasing $(p=0.3)$ but was not statistically significant. The blinded endourologist reported more cases with hazy vision in Pusen arm but was statistically insignificant. In vitro maximal tip deflection and irrigation flow rate with or without accessory were comparable in both the scopes. One case of device malfunction in Lithovue arm was noted. Complete stone clearance was achieved in all cases.

Conclusions: Both LithoVue and Pusen ureteroscopes have similar in vivo and in vitro performance. Larger clinical trials are needed to confirm the efficacy of these two single use scopes.

\section{MP25-18 Beware of Laser, Ureteral Stenosis after Ureteroscopy}

F Pauchard, N Calvo, D Gonzalez, C Ondarza, F Kramer, C Mülchi

Hospital Carlos Van Buren

Introduction \& Objective: The primary recommendation for management of ureteral stones is ureteroscopy. Ureteral stricture is believed to be a rare complication $(<1-4 \%)$. Risk factors are supposed to be perforation and stone impactation. Silent obstruction could end in renal failure. The objective of this study is to identify silent obstructions after ureteroscopy and possible predictors.

\begin{tabular}{|l|c|c|}
\hline $\mathrm{N}$ & No Stenosis & Stenosis \\
\hline Median age (IQR) & $83(95 \%)$ & $4(5 \%)$ \\
\hline Localization of the stone & $53(42,5-59,5)$ years & $56(41,75-60)$ years \\
\hline & $\begin{array}{c}\text { Distal } 60(72 \%) \\
\text { Mid } 14(17 \%) \\
\text { Proximal 7 }(8 \%) \\
\text { Combinations 7 (8\%) }\end{array}$ & $\begin{array}{c}\text { Mid 2 }(50 \%) \\
\text { Proximal 2 (50\%) }\end{array}$ \\
\hline Energy used & $\begin{array}{c}\text { Pneumatic 72 (86,7\%) } \\
\text { Laser 3 (4\%) } \\
\text { Other 8 (9\%) }\end{array}$ & Laser 4 (100\%) \\
\hline Mean stone size & $9,6 \mathrm{~mm}(\mathrm{SD} 4,22)$ & $11,75 \mathrm{~mm} \mathrm{(SD} \mathrm{4,19)}$ \\
\hline JJ postoperative & $55(63 \%)$ & $4(100 \%)$ \\
\hline Sessions & $1: 79(96 \%)$ & $1: 4(100 \%)$ \\
\hline Median follow up (IQR) & $26(14,5-40)$ months & $6(6-12)$ months \\
\hline
\end{tabular}

Methods: Patients who have been treated with semirigid ureteroscopy since 1/2014 until 4/2019 were searched. Clinical data were assessed and a renal ultrasonography (US) was made. If dilation was found, a contrast CT scan was performed. No further statistical analysis was made because of the small group with stenosis.

Results: 118 patients were included. 3 of them were dead and 28 wasn't possible to contact. Population of study was 82 patients. $52 \%$ female and $48 \%$ males. Age median was 53 years $(42,5-$ $59,5)$. Distal ureteral stones were found in $75 \%$ of patients. Stone free rate was $95 \%$. Median follow up was $24(12,5-36,75)$ months. 4 patients developed silent stenosis. None of the stenosis were in the distal ureter. Two of these patients had some degree of renal atrophy after diagnosis. The 4 patients had their stones fragmented with laser and 2 of them had an impacted stone.

Conclusions: The risk of stricture formation after ureteroscopy is well known, but not always well assessed. The costs associated with imaging post ureteroscopy are relatively low but considering the low frequency of obstruction there's not a clear consensus about follow up images. The concerns about radiation it's also taken into account. Predictors of stricture are not clear. Traxer's classification of ureteral damage did not correlate with clinical significant sequelae. Lesions produced with laser may behave different than blunt lesions to the ureter. Our results are similar to other reports. It's to be highlighted that all the strictures were produced after the use of laser and non with pneumatic energy, which is in line with a recent metaanalysis. We don't advocate to use more pneumatic energy, but to use laser cautiously and with a good control of the fiber in the ureter. Using low frequency could help to achieve better control of the laser shoots. We think that US as a follow up tool is available, safe and cost effective for all patients who had a ureteroscopy.

\section{MP25-19 Withdrawn}

MP25-20 Flexible ureteroscopy and holmium laser lithotripsy for ureteral stones in renal transplant recipients with ureteroureterostomy type anastomosis

\section{Ulker, O Celik, Y Ilbey}

Health Sciences University Tepecik Training and Research Hospital

Introduction \& Objective: Urinary stone formation after renal transplantation is very rare and the incidence has been reported between $0.17-1.8 \% .33 \%$ of the de novo urinary stones in renal allografts are seen in the ureter. However, ureteroscopic treatment of the ureteral stones in renal allografts could be challenging due to excessive elongation of the ureter, especially in ureteroureterostomy type anastomosis for which patient's native ureter is used. 
Here, we present the results of three cases of ureteral stone in renal transplant with ureteroureterostomy type anastomosis which treated by flexible ureteroscopy and holmium laser lithotripsy.

Methods: Between 2016-2018, three renal transplant patients with ureteroureterostomy type anastomosis who underwent flexible ureteroscopic laser lithotripsy with holmium:YAG laser for ureteral stone were evaluated retrospectively in terms of stone size, stone density, stone location, operation time, postoperative hospital stay, complications and stone free rate.

Results: The mean age of patients was 47.6 years and the mean time after renal transplantation was 5.2 years. The mean stone size and stone density was $8.8 \mathrm{~mm}$ and 700 Hounsfield units, respectively. The stone location was the middle ureter in two patients and the proximal ureter in one patient. One patient had percutaneous nephrostomy tube before the operation. In two patients we were able to reach the stone by retrograde access through a guide-wire and performed laser lithotripsy, while in the remaining patient laser lithotripsy was performed through antegrade way since the retrograde access was not successful. No complication was observed in any patient. The mean operation time was $66.6 \mathrm{~min}$ and the mean postoperative hospital stay was 1.6 days. Stone-free rate was revealed $100 \%$ at follow-up at 2 weeks.

Conclusions: Flexible ureteroscopy and holmium laser lithotripsy was an effective and feasible method in the treatment of ureteral stones in renal transplant patients with ureteroureterostomy type anastomosis. When the stone cannot be reached in retrograde way, percutaneous access and antegrade flexible ureteroscopy should be performed.

\section{MP25-21 Different morbidity using different flexible ureteroscopes - is it a reality?}

PA Geavlete, R Multescu, DA Georgescu, M Stan, C Bucur, BF Geavlete

Saint John Emergency Clinical Hospital

Introduction \& Objective: Nowadays, flexible ureteroscopy is associated with a reduced complications' rate, most of them being mild. The aim of this study is to evaluate the rate of complications stratified according to the model of the scope. Methods: We retrospectively analyzed the morbidity associated with the flexible ureteroscopy in 240 cases of pyelocaliceal lithiasis (May 2018-July 2018): Group I - 80 consecutive cases performed with Olympus URF-V (9.9F), Group II - 80 consecutive cases with Storz Flex-Xc (8.5F) and Group III - 80 consecutive cases with Olympus URF - V2 (8.5F).

Results: Complications' rate was $21.3 \%$ in Group I, $18.8 \%$ in Group II and $20 \%$ in Group III. Clavien I and II complications occurred in $15 \%, 15 \%$ and $16.3 \%$ respectively. Clavien III complications occurred in $6.3 \%, 3.8 \%$ and $3.8 \%$ respectively. No Clavien IV and V were registered.

Conclusions: The increased tip diameter may be associated with a similar overall complications rate, but with a higher severity.

\section{MP25-22 A Prospective Comparative Study Between Flexible and Semi-rigid Ureteroscopic Lithotripsy in the Management of Single Large Proximal Ureter Stone \\ H Chen, Y Chen, Y Juan, W Wu, C Li, T Huang \\ Kaohsiung Medical University Hospital, Kaohsiung Medical University, Kaohsiung, Taiwan}

Introduction \& Objective: The aim of the study was to analyze and compare the outcome and complications of flexible (F-URS) and semi-rigid (SR-URS) ureteroscopic lithotripsy in the management of surgically indicated single large ureter stone.

Methods: Patients with single large stone (large than $1 \mathrm{~cm}$ ) in the proximal ureter with slight to moderate hydronephrosis were prospectively selected and assigned into groups of SR-URS and F-URS on the basis of the surgeons' choice. Demographic data, operative duration, postoperative hospital stay, complication rate, and stone-free rate were recorded and compared. The primary outcome was stone-free clearance, which was defined as the absence of fragments or a single fragment of $\leq 4 \mathrm{~mm}$ on standard radiography at the follow-up examinations. Secondary outcomes included operating time, hospital stay, the need of auxiliary procedure and complications, which were graded according to the modified Clavien classification.

Results: From January 2018 to April 2019, 46 such patients (36 males and 10 females) were consecutively included into the study. No significant difference was found in preoperative demographics, including age, BMI, sex, stone size, stone side, preoperative renal function (Table 1). The mean stone size was $1.49 \mathrm{~cm}$ and $1.61 \mathrm{~cm}(\mathrm{P}=0.6)$, stone free rate $74 \%$ and $30 \%$ in F-URS and SR-URS group. Compared with SR-URS group, F-URS group has significantly higher stone free rate $(\mathrm{P}=0.007)$, less patients who required auxiliary procedure $(\mathrm{P}<0.001)$, but more operative time $(\mathrm{P}=0.001)$ and prolonged hospital stay $(\mathrm{P}<0.001)$ (Table 2$)$. There was no statistically significant difference between 2 groups in complication rate $(\mathrm{P}=1)$.

Conclusions: Regarding the treatment of single large proximal ureter stone, F-URS is associated with higher stone free rate, less patients who required auxiliary procedure, but more operative time and prolonged hospital stay than SR-URS. In addition, no severe complication was observed in F-URS. Further randomized control studies are required to confirm the outcomes of the present study.

\begin{tabular}{|c|c|c|c|}
\hline Variables & $\begin{array}{l}\text { F-URS } \\
(n=23)\end{array}$ & $\begin{array}{l}\text { SR-URS } \\
(n=23)\end{array}$ & P-value \\
\hline Mean age, yr (range) & $52.5(28-67)$ & $57.1(33-69)$ & 0.3 \\
\hline Mean BMI, kg/m² (range) & $26.2(21.1-33.9)$ & $26.7(18.9-34.8)$ & 0.4 \\
\hline $\operatorname{Sex}, n(\%)$ & & & 0.3 \\
\hline Male & $16(70)$ & $20(87)$ & \\
\hline Stone side, $n(\%)$ & & & 0.8 \\
\hline Left & $15(65)$ & $13(57)$ & \\
\hline Right & $8(35)$ & $10(43)$ & \\
\hline Mean stone size, cm (range) & $1.49(1.0-1.9)$ & $1.61(1.0-2.0)$ & 0.6 \\
\hline Mean eGFR, mU/min/1.73m² (range) & $68(28-109)$ & $68(27-157)$ & 0.9 \\
\hline
\end{tabular}

Table 2. Intraoperative parameters and postoperative outcomes

\begin{tabular}{|c|c|c|c|}
\hline Variables & $\begin{array}{l}\text { F-URS } \\
(n=23)\end{array}$ & $\begin{array}{l}\text { SR-URS } \\
(n=23)\end{array}$ & P-value \\
\hline Mean operation time, min (range) & $82.7(40-130)$ & $57.8(30-105)$ & 0.001 \\
\hline Mean hospital stay, day (range) & $1.91(1.4)$ & $1.04(1-2)$ & $<0.001$ \\
\hline Postoperative stone-free status, no. (\%) & $17(74)$ & $7(30)$ & 0.007 \\
\hline Complications, $n$ (\%) & $2(9)$ & $3(13)$ & 1 \\
\hline Modiffed Clavian classification grade 1, no. & 0 & $1(4)$ & 1 \\
\hline \multicolumn{4}{|l|}{ Modified Clavian classification grade 2, no. } \\
\hline Postoperative fever with IV form antebiotics & $2(9)$ & $1(4)$ & 1 \\
\hline Patients neoded auxiliary ESWL/URSL, n (\%) & $1(4.3)$ & $15(65)$ & $<0.001$ \\
\hline
\end{tabular}




\section{MODERATED POSTER SESSION 26: CLINICAL STONES: EQUIPMENT (II)}

MP26-01 Safety and Efficacy of Stone Dusting Utilizing a 30-Watt Laser system Versus a High Power (100/120W) System

O ALALAO, O Kott, DW Sobel, C Tucci, G Pareek

Brown University

Introduction \& Objective: There has been an increased utilization of ureteroscopy for treatment of kidney stone disease over the last two decades. Concurrently, lithotripsy utilizing various powers of the holmium:yttrium-aluminum-garnet (Ho:YAG) laser has popularized the dusting technique which reportedly leads to a reduction in operative time, ureteral access sheath (UAS) usage, and complications related to basketing stones. The aim of our study is to report the clinical outcome of our institution experience with a lower cost 30-Watt lasers compared to a costly high-power $100 \mathrm{~W}$ and $120 \mathrm{~W}$ Ho:YAG laser systems for URS lithotripsy.

Methods: We identified 246 adult patients who underwent URSL at our institution between March 1, 2017 to March 1, 2018. Flexible ureteroscopy was conducted using the Storz flex X2 with or without an access sheath $(9.5 \mathrm{~F} / 11.5 \mathrm{~F}$ or $12 \mathrm{~F} / 14 \mathrm{~F}$ Cook Flexor sheath). Cases were included if the pre-operative stone burden was measured using standard non-contrast CT imaging and SFR was defined as having complete absence of stones endoscopically or clinically insignificant fragments $\leq 2 \mathrm{~mm}$ on US or NCCT. All cases started with a similar stone fragmentation laser setting of $0.8 \mathrm{~J}$ and $8 \mathrm{~Hz}$. followed with a $0.4 \mathrm{~J}$ and $25 \mathrm{~Hz}$ dusting setting. We compared the operating time in our cohort to recently published data from two independent research groups that compared stone dusting using a high-energy holmium laser system (Tracey et al. J Endourol. 2018) and (Pietropaolo et al. Urolithiasis. 2018).

Results: 188 patients met the inclusion criteria (Table). The average stone size was $11.2 \mathrm{~mm}(1.2-37 \mathrm{~mm})$ the mean operative time was 33.2 minutes (5-155min) and similar to our comparison cohort (53 minutes (22-115). The mean operative time was 47.2 minutes (6-155) in our series compared to 51 minutes $(15-100)$ in the comparison cohorts. The $30 \mathrm{~W}$ laser system cost was $\$ 100,000$ less than if the high-power system utilized in the comparison groups. Conclusions: While dusting has been popularized with highpowered laser systems $(100 / 120 \mathrm{~W})$, utilizing a 30W laser maybe as effective. Considering the price difference between the high and low power lasers, we estimate the yearly costs savings in our institution to be around $\$ 100,000$. Operative time difference may be noticed in cases involving very large stone burden.

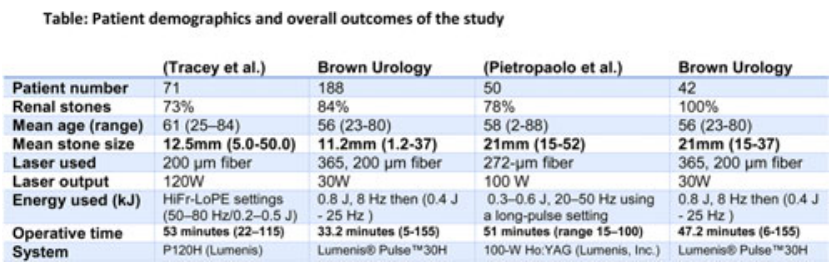

MP26-02 Analysis of ureteral stent's encrustations: comparison between silicone and Percuflex ${ }^{\mathrm{TM}}$

L Bevilacqua, M Amato, G Guarino, C Del Prete, R Milandri, M Sighinolfi, S Puliatti, B Rocco, S Micali
Department of Surgical, Medical and Dental Morphological Sciences related to Transplant, Oncology and Regenerative Medicine - Section of Urology, University of Modena and Reggio Emilia, Modena, Italy

Introduction \& Objective: Encrusted ureteral stent is a significant concern in endourology due to the possibility of serious injuries for removing them. Silicone has been shown to be highly biocompatible and less prone to encrustation. We retrospectively analyzed these properties of silicone compared to percuflex ${ }^{\mathrm{TM}}$ in a large series of implanted ureteral stents.

Methods: Since January 2017, a total of 382 ureteral stent have been inserted during endourological procedures for stones. Stent material was silicon-based in 148, percuflex in 234 cases. The primary endpoint of this retrospective study is to assess and compare the rate of encrustation of silicon based and percuflex ureteral stent; an analysis of factors associated with the need of an operative management has been provided as well and considered as the secondary endpoint. Data were analyzed with SPSS-24. Chi square test and non-parametric tests were used to compare the rate of calcification among different stents (silicon vs percuflex); a multivariate analysis was performed to assess the impact of each covariate (age, stone size, stone location, diameter and length of stent, stent material, indwelling time, presence of UTI) on the requirement of an operative approach for stent removal.

Results: Seventy-two $(18,8 \%)$ excretory units had stent encrustation at the time of stent removal. Among encrusted stent, 44 of them $(61 \%)$ required an operative approach to manage the case, that could be either a pure endourological (40) or a combined endourological and extracorporeal approach (4). The rate of encrustation of silicon-based ureteral stent was $8,7 \%(13 / 149)$; the rate of encrustation of Percuflex stent was 25,3\% (59/233) $(p=0.000)$. The rate of excretory units requiring an adjunctive operative procedure to manage the encrustation was $6 \%(9 / 149)$ for the silicon and 15\% (35/233) for the Percuflex stents $(p=0,005)$. The odd for developing encrustation is 2,43 times greater for Percuflex compared to silicon stent (95\% CI: 1,464,03 ); the odd for developing encrustation requiring an intervention is 2,02 for Percuflex compared to silicon stent (95\%CI: $1,15-3,67)$. As far as the secondary endpoint is concerned, none of the mentioned variables significantly relate to the need for an intervention in the subgroup of units with an encrusted ureteral stent. Conclusions: Our analysis shows that Silicone ureteral stents are safer and have a significant lower encrustation potential than Percuflex ${ }^{\mathrm{TM}}$. In a significant number of cases it was necessary a second procedure to remove the encrusted stent and treat the stone.

MP26-03 Is the irrigation method important for maintaining safe irrigation temperatures during flexible ureteroscopy when a high power Holmium:YAG laser is used? Evidence from an experimental study in porcine model

E Farsari, P Ntasiotis, C Adamou, A Vagionis, A Gerakaris, K Gkialas, GA Callas, E Liatsikos, P Kallidonis

University of Patras 


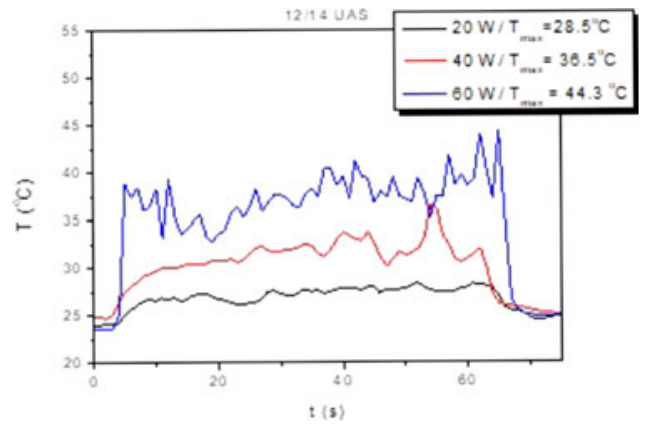

\begin{tabular}{cccc}
\multicolumn{4}{c}{ Forced irrigation } \\
& & $\mathrm{T}\left({ }^{\circ} \mathrm{C}\right)$ \\
& $20 \mathrm{~W}$ & $40 \mathrm{~W}$ & $60 \mathrm{~W}$ \\
no sheath & 35.5 & 41 & 42 \\
sheath 10-12 & 33.3 & 39.9 & 41.6 \\
sheath 12-14 & 28.4 & 36.7 & 44.3 \\
sheath 14-16 & 33.5 & 37.2 & 37
\end{tabular}

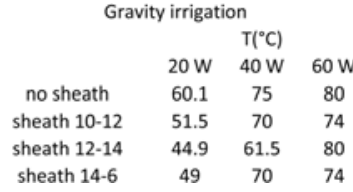

Introduction \& Objective: To evaluate the effect of high power Holmium:YAG laser in the temperature of the irrigation fluid under different irrigation methods during flexible ureteroscopy in a porcine model.

Methods: 22Fr percutaneous tracts were established in the upper calyces of the kidneys of a female pig. A custom-made balloon nephrostomy catheter containing a K-type thermocouple was inserted in the pelvicalyceal system. The thermocouple was connected to a data logger (TC08, Pico Technologies, UK) for real time temperature measurements. A Flex-X2 Ureteroscope (Karl Storz $\mathrm{GmbH}$, Germany) was used with gravity irrigation $\left(100 \mathrm{~cm} \mathrm{H}_{2} \mathrm{O}\right)$ or with forced irrigation with a manual pump (a pulse every $3 \mathrm{sec}$ ). Ureteroscopy was performed with a 12/ 14Fr UAS. A holmium:YAG laser (Moses Pulse 120H, Lumenis Ltd. Israel) was activated at powers of 20, 40 and $60 \mathrm{~W}$ for $60 \mathrm{sec}$. Temperature measurements were recorded.

Results: When gravity irrigation was used the respective highest temperatures developed in the irrigation fluid for 20,40 and $60 \mathrm{~W}$ were $45,61,5$ and $80^{\circ} \mathrm{C}$ respectively. The respective figures for the manual pump were $28.5,36.5$ and $44.3^{\circ} \mathrm{C}$ (Fig 1, Table 1).

Conclusions: The activation of the high-power laser with gravity irrigation at power $>40 \mathrm{~W}$ results in rapidly developing temperatures above the acceptable for safety limit despite the use of an UAS. When a manual pump and a 12/14Fr UAS are used, the temperatures were within safe limits.

MP26-04 Effectiveness of using thinner Double $\mathbf{J}$ stents following flexible ureterorenoscopy for stone fragment expulsion

E Fernandes, S Kanekar, H Maienkar

Vision Hospital, Healthway Hospital

Introduction \& Objective: Most Urologists prefer to deploy a Double J(DJ) stent following Flexible ureterorenoscopy (Furs) and laser lithotripsy (LL) to avoid untoward ureteric obstruction by calcular fragments. We present a comparative series of 15 patients(pts) each who were stented with standard (5French (Fr)/ 24-26) DJ stents and thinner (3Fr/24) DJ stents respectively to demonstrate the effectiveness of thinner DJ stents in stone fragment expulsion following Furs and LL.

Methods: Two groups with 15pts each in age group 20-50yrs without any co-morbidities. On preoperative Computed tomographic scan, pts either had a single pelvic or calyceal calculus

\begin{tabular}{|c|c|c|}
\hline & Standard (5/25-26) & Thinner(3/24-26) \\
\hline Ureteric colic & 0 & 0 \\
\hline Stent symptoms & 10 & 1 \\
\hline $\begin{array}{l}\text { Visible passage of } \\
\text { stone fragments }\end{array}$ & 0 & 8 \\
\hline $\begin{array}{l}\text { USG+Xray KUB } \\
\text { residual renal stones }\end{array}$ & 11 & 2 \\
\hline $\begin{array}{l}\text { USG }+ \text { Xray KUB residual } \\
\text { ureteric calculi }\end{array}$ & 4 & 0 \\
\hline $\begin{array}{l}\text { Relook furs at stent } \\
\text { removal }\end{array}$ & 5 & 0 \\
\hline
\end{tabular}

ranging from 11-13 millimeters $(\mathrm{mm})$ in size and 900-1500 Hounsfield unit (HU) in density. All pts were pre-stented with standard DJ stents. Flex-X2 Furs with 9.5Fr ureteric access sheath was used. All calculi were taken to the superior calyx and fragmented using holmium laser at 0.8 joules of energy and 1015 hertz frequency. All the procedures were completed within 60-80minuites. All pts was put on Tamsulosin $0.4 \mathrm{mgs}$ once at night for next 4 weeks. The Ultradound (usg) and xray KUB were done at 4 weeks postoperatively.

Results: None of the pts had ureteric colic postoperatively. In the standard DJ group, none of pts had visible passage of calculi. Post-operative usage and Xray showed residual Renal calculi in $11 \mathrm{pts}$ and also concurrent ureteric calculi in $4 \mathrm{pts} .5$ of the $15 \mathrm{pt}$ eventually required relook Furs at the time of DJ removal. In the thinner DJ group, 8pts visibly passed stone fragments. Only 2 pts had residual renal fragments $(<3 \mathrm{~mm})$ on post-operative usage and none of them needed relook Furs. The stent related symptoms were markedly less in thinner $\mathrm{Dj}$ group.

Conclusions: Stenting after Furs with LL is a good practice to avoid ureteric obstruction by stone fragments. It is worthwhile to deploy a thinner DJ stent since it allows the stone fragments to pass out by the side of stent, thus giving a good stone clearance and preventing the need for repeat procedures.

\section{MP26-05 Buried upper end of the double coil stent following perforation of the collecting system during percutaneous nephrolithotomy}

\section{F Hammad}

United Arab Emirates University and Tawam Hospital

Introduction \& Objective: Renal collecting system injury is a well-documented complication of percutaneous nephrolithotomy (PNL). Large disruption of the collecting system might require cessation of the procedure and adequate drainage using ureteral stent, nephrostomy tube or even percutaneous drain. Herein, we report on two cases in which the upper coil of the double coil (DC) stent got buried at the site of the collecting system perforation which occurred during PNL.

Methods: A 38-years-old male and 32-years-old female underwent left PNL for partial and total staghorn calculi, respectively. The collecting systems in both cases were dilated, however, the renal pelvis in the first case was relatively small (Figure-1). During the procedure in both cases, the renal pelvis was perforated by the tip of the nephroscope during lithotripsy which was performed using a combination of ultrasonic and pneumatic lithotripters. In both cases, the perforation occurred towards the end of the procedure and there was no significant bleeding, so the procedure was completed, and the stones were totally cleared. The collecting system was drained using a DC stent in both cases and the upper coils of the stents were placed in the renal pelvis. 


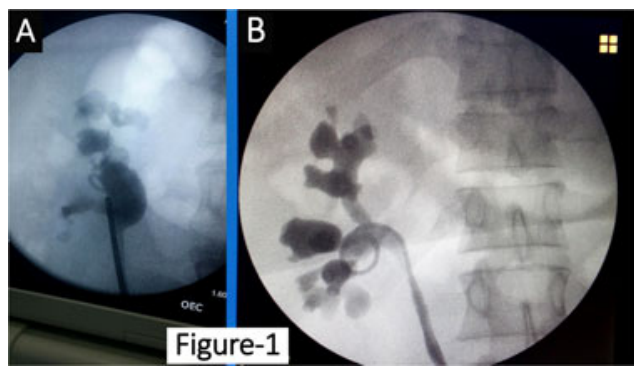

Results: Both patients did well in the immediate post-operative period. Trial to remove the DC stent failed six weeks following the procedure. Further imaging including retrograde pyelography (Figure-1) revealed that the upper ends of the DC stents were buried in the renal pelvis at the site of the perforation which occurred during the procedure. Using holmium laser endoscopically, the upper coils of the stents were released from the fibrous tissues which grew over them during the healing phase. The procedure was uneventful in both cases.

Conclusions: In this abstract, we reported for the first time on buried stents which occurred at the site of the renal collecting system perforation that occurred during PNL procedures. To prevent this complication in such cases, the authors suggest draining the collecting system using nephrostomy tube. Alternatively, every caution should be taken to place the upper coil of the DC stent away from the perforation site such as placing it in the upper calyx especially if the perforated renal pelvis is relatively small to accommodate the upper coil comfortably.

\section{MP26-06 Stent Syndrome. Does the diameter matter? Preliminary results of a prospective trial}

PN Contreras, N Bonanno, L Rico, M Ringa, F Frascheri, C Ameri

Hospital Alemán

Introduction \& Objective: We found some confusing data about catheter JJ sizes and Stent Syndrome scores. Our objective was to compare the stent syndrome scores with three different JJ sizes and evaluate their impact on the quality of life.

Methods: A prospective trial in patients that required a catheter JJ due to ureteral stones was started in June 2017 and it is still on going. Consecutive patients were allocated to 4,7; 6 and 7 French JJ catheter diameters using a randomization program. All procedures were done under general anesthesia by the same working team. Universa ${ }^{\circledR} 26 \mathrm{~cm} \mathrm{JJ}$ catheters were used in all patients. The ureteral stent symptoms questionnaire (USSQ) was completed between 7 and 14 days after de procedure. A minimum sample size of 180 patients was calculated to detect a difference of $15 \%$ between mean index scores for urinary symptoms, pain, and general health, respectively, with $80 \%$ power. Mean scores for USSQ domains scores were compared using one-way ANOVA. We present preliminary results.

Results: We randomized 123 patients. 14 were excluded (11 never delivered USSQ and 3 catheters where removed before 48 hours) leaving 109 for preliminary analysis. The average age was 47 years (SD 15.7) with a male/female ratio of 3.6/1. Most patients felt pain associated with catheter JJ, $75 \%$ of them stated that they would not be able to live with those symptoms and 50\% that it would be unpleasant to require a catheter JJ again. Better results of tolerance to $4,7 \mathrm{Fr}$ catheter were found, though no

\begin{tabular}{|c|c|c|c|c|}
\hline Total & $4.7 \mathrm{Fr}$ & $6 \mathrm{Fr}$ & $7 \mathrm{Fr}$ & $\mathrm{P}$ \\
\hline U & 12.4 & 16.8 & 16.5 & NS \\
\hline P & 11.6 & 15.2 & 17.8 & NS \\
\hline G & 6.1 & 10.3 & 6.7 & NS \\
\hline W & 7.8 & 8.6 & 9.4 & NS \\
\hline S & 0.29 & 0.41 & 0.57 & NS \\
\hline Otras & 4.7 & 5.45 & 5.21 & NS \\
\hline
\end{tabular}

significant intergroup difference were reached in the domain scores of urinary symptoms, pain, general health, work, sexual activity or additional problems. There was a tendency of less symptoms with thinner catheter diameter.

Conclusions: Ureteral stents were strongly associated with urinary symptoms that lowered life quality. In this preliminary report, no significant differences were found between different catheter JJ diameters.

MP26-07 FECal (forgotten, encrusted, calcified) grading system for forgotten ureteral stents. Is it a reliable system?

OE Fugita, J Amaro, F Marchant, N Bernardo, M Baptistussi, A Luna, M Gonzalez, J Campos, A Cardenaz-Ortiz

Faculdade De Medicina De Botucatu - UNESP

Introduction \& Objective: Ureteral stents (US) are indispensable tools in a wide range of elective and emergency urological scenarios. Most ureteral stents are placed for few weeks to few months, depending on the underlying cause, and they are associated with short and long-term complications. Despite meticulous vigilance, ureteral stents may be "forgotten" and tend to encrust, becoming difficult to remove, frequently entailing significant complications. Minimally invasive surgical management of overdue ureteral stent plays a key role in this scenario. In order to propose an algorithm in the management of the so called FECal (forgotten, encrusted, calcified) stent, Acosta-Miranda et al presented a grading system for these stents based on the stone size, location, and degree of stent encrustation. The FECal system grading was used to compare our choice of surgical approach and the one suggested by the FECal algorithm.

Methods: We retrospectively reviewed the charts of 5278 patients that had a ureteral stent managed between 2012 and 2016, at six different high-volume stone centers. All cases of forgotten ureteral stents (US) (defined as a catheter left in situ for more than 6 months after the insertion or that was already calcified even before that time) were identified. All patients had a KUB radiography and/or NCCT for determination of length and site of encrustation, and surgical planning.

Results: Forgotten US were identified in 81 patients of 5278 patients that had a ureteral stent managed between 2012 and 2016 at six different institutions. Some cases may have been lost as only those patients that returned to the departments were included. On the other hand, some of the patients had the US inserted somewhere else and were only referred to our institutions for complex stone management. Encrustation of DJ catheter at the KUB radiography or at NCCT scan was found in all cases. The mean extension of calcification was $5.35 \mathrm{~cm}$ (range $0,8-24$ ). According to the FECal system there were 22 grade I (27.1\%), 19 grade II (23.4\%), 11 grade III (13.5\%), 15 grade IV (18.5\%), and 14 grade $\mathrm{V}(17.2 \%)$. In 24 cases $(29.6 \%)$, the suggested surgical 
approach was not followed (19 PCNLs and 5 cystolitholapaxies). In all these 24 cases, flexible or semi-rigid ureteroscopies were performed instead. In all the other 57 cases $(70.3 \%)$, the surgical intervention was in accordance to the suggested by the FECal algorithm, including 3 cases $(3.7 \%)$ of nephrectomy.

Conclusions: The FECal stent grading system and management algorithm is a reliable tool to the surgical approach to this challenging clinical condition.

\section{MP26-08 A Novel Cost Effective Patient Engagement Based Educational Tool for Informed Stent Insertion Consent}

I Ahmed, OA Ramadan, B Osman, MM Quraishi, J Hale, P Keirandish, N Shrotri, R Krishnan, SS Kommu

Kent \& Canterbury Hospital. East Kent Hospitals NHS Foundation Trust

Introduction \& Objective: Ureteric stent insertion is a mainstay treatment for many emergency and elective urological conditions. Ureteric stents contribute a significant health burden and mandate optimal familiarity and education with all those involved especially patients. The urologist inserting the stent is responsible for informing the patient of the requirements, consequences, and complications associated with stent placement. Inappropriately consented patients lead to complaints, law suits, unnecessary visits to healthcare providers, poor quality of life and forgotten stents. Herein, we developed and conducted a preliminary test of a novel cost-effective patient engagement based educational tool for informed consent for stent insertion. Methods: A model was made utilizing readily available materials from the hardware store and supplemented with out of date stents and guide wires. A light weight board roughly with the dimensions of an adult human torso was implanted with plastic transparent tubing to replicate ureters in an ex vivo layout of anatomical expectation. Plastic bottles approximately the size of a human kidney and bladder were embedded to replicate kidneys, ureters and bladder. A small red triangular schematic was placed in the bladder to replicate a trigone. The model was further embedded with brief writings of a standardized consent material as published by the British Association of Urological Surgeons. At the time of consent for insertion of ureteric stent the patient is shown and taken through the steps of stent insertion with fit and well patients guided to insert the stent in the plastic ex vivo model. Once deployed, the surgeon goes through the necessary pros, relative complications and normal expectations of stent related symptoms.

Results: The model was developed at a cost of less than 20 US Dollars. Trial stents were deployed initially by 2 trainee urologists, 2 experienced consultant urologists, 2 laymen and 5 patients from a patient focus group. The stent deployment was noted to be informative and when assessed by the Single Ease Question (SEQ) Platform was noted to be of sufficient ease as to be utilized at all levels without issue. Questionnaire evaluation of the platform was conducted.

Conclusions: The novel, cost effective and replicable tool has been shown to be an effective alternative to the conventional paper consent and was noted to be highly informative as an educational patient engagement tool for optimal consent. This platform or similar tools could be used in urological centers to optimize consent prior to stent insertion. We are currently performing a large scale randomized trial of this novel patient engagement tool.

\section{MP26-09 How to place a loop type ureteral stent safely? From an early experience of a loop-type ureteral stent}

\section{H Byun, J Choi, D Han, H Ryoo, J Chung}

Introduction \& Objective: A ureteral stent can cause unpleasant side effects, such as urinary frequency, urgency, incontinence, hematuria, bladder pain and flank pain, which has a negative impact on a patient's quality of life. Recently, loop-type ureteral stents are used to minimize the amount of material in the bladder in order to decrease stent-related symptoms. However, there is little data on the functional and safety outcomes of looptype ureteral stent compared to the standard double pigtail ureteral stent. In this study, we report the initial experience of the loop-type stent.

Methods: This was a retrospective study conducted at a single center from April 2018 to April 2019. Patients who received unilateral ureteral stent placement after uncomplicated ureteroscopy for ureteral or renal stones were included. Conventional double pigtail stent and loop-type ureteral stent (Polaris loop ${ }^{\mathrm{TM}}$ ) were placed alternatively. Postoperative stent-related events and complications were assessed throughout the follow-up period after placement. The patient self-administered Ureteric Stent Symptoms Questionnaire (USSQ) was also used to assess stentrelated symptoms, that was completed by patients just prior to removal.

Results: A total of 77 patients were included: pigtail stent group $(n=37)$ and loop-type stent group $(n=40)$. The mean age of the patients was 57.5 years. Three patients with the loop-type ureteral stent needed stent exchange due to stent malfunction after initial placement. (blood clot in two patients, stone fragments in one patient). All these events occurred during initial 10 cases of loop group. In two cases with blood clot obstruction, clotinduced obstruction was observed in the loop segment of the stent (Fig 1.A). After these events, stent placement method was
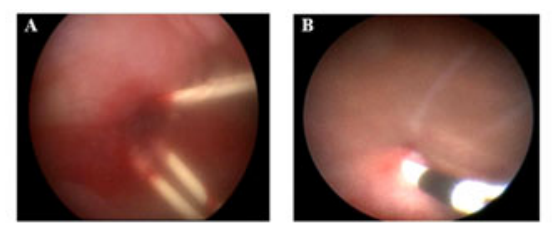

Fig. 1 Loop-type ureteral stent. A: Joint between shaft and loop segment is located in ureter. Ureter is obstructed by blood clot $\mathbf{B}$ : Joint between shaft and loop segment is located in bladder.

Table 1. Baseline characteristics of the patients

\begin{tabular}{|c|c|c|c|}
\hline & \multicolumn{2}{|c|}{ Number $(\%)$ or median } & \multirow{2}{*}{ p-valu } \\
\hline & $\begin{array}{c}\text { Double pigtail } \\
(\mathrm{N}=37)\end{array}$ & $\begin{array}{c}\text { Loop-type } \\
(\mathrm{N}=40)\end{array}$ & \\
\hline Mean age (years) & $58.2(59 \pm 9.0)$ & $56.4(57 \pm 10.0)$ & 0.401 \\
\hline \multicolumn{4}{|l|}{ Gender, $\mathrm{n}$} \\
\hline Male & $21(77.8 \%)$ & $29(72.5 \%)$ & \\
\hline Female & $16(22.2 \%)$ & $11(27.5 \%)$ & \\
\hline \multicolumn{4}{|l|}{ Side, $\mathrm{n}$} \\
\hline Right & $22(59.5 \%)$ & $18(45 \%)$ & \\
\hline Left & $15(40.5 \%)$ & $22(55 \%)$ & \\
\hline \multicolumn{4}{|l|}{ Ureteroscopy used, $\mathbf{n}$} \\
\hline Flexible & 23 & 25 & \\
\hline Rigid & 7 & 9 & \\
\hline Both Flexible and Rigid & 7 & 6 & \\
\hline Stent indwelling duration (days) & $15.1(15 \pm 4.3)$ & $13.2(12 \pm 4.1)$ & 0.053 \\
\hline \multicolumn{4}{|l|}{ Stent caliber, $\mathrm{n}$} \\
\hline $6 \mathrm{Fr}$. & 33 & 40 & \\
\hline $7 \mathrm{Fr}$. & 4 & 0 & \\
\hline Stent length $(\mathrm{cm})$ & 23.6 & 25.3 & $<0.001$ \\
\hline
\end{tabular}


changed so that the joint between the shaft and loop segment was located in the bladder (Fig 1.B). Stent malfunction did not occur after changing the stent placement method. There was one patient who experienced urinary tract infection in the double pigtail stent group. In both groups, USSQ scores showed no statistical difference.

Conclusions: From our initial experience with loop type ureteral stent, the risk of the malfunction was increased when the joint between the shaft and loop segment was located in the ureter. However, when the stent was inserted so that the joint of the stent was located in the bladder, the stent functioned safely. In order to safely place a loop-type stent and maximize the benefit of the stent, stent position should be considered. With regard to the optimal stent length of the loop-type stent, further study is needed.

MP26-10 Evaluation of the initial efficacy and safety in endoscopic combined intrarenal surgery (ECIRS)

Y Miyazawa, T Miyao, Y Fukuma, M Hatori, N Otake, T Sekihara, K Suzuki

Introduction \& Objective: To evaluate the efficacy and safety of endoscopic combined intrarenal surgery (ECIRS) using retrograde flexible ureteroscopy and percutaneous nephrolithotomy (PNL) for the treatment of patients with renal and ureteral calculi in our hospital at Initial introduction experience.

Methods: We retrospectively reviewed the records 42 cases of 30 patients with renal and ureteral calculi who underwent ECIRS in our hospital between April 2013 and April 2018. A flexible ureteroscope with a laser fiber was inserted through a ureteral access sheath, and lithoclast lithotripsy was performed through a mini-percutaneous tract. All procedures were conducted in modified Valdivia position, basically. Surgical parameters, including surgical time, stone-free (SF) rates, complication and decline of hemoglobin $(\mathrm{Hb})$ after surgery were analyzed. This study was approved by the institutional review board of Hidaka Hospital.

Results: The mean age of all patients was $63.5 \pm 10.6$ ( \pm S.D.) years. The mean BMI of all patients was $24.2 \pm 3.2$. Twenty-three patients were able to complete treatment with single operation. There were six patients requiring two operations to complete the treatment, and one patient requiring 7 times. The mean surgical time was $143.4 \pm 44.3$ minutes. The initial SF rate was $70.0 \%$, and the final SF rate was $93.3 \%$ after further treatment. The mean decline of $\mathrm{Hb}$ after surgery was $1.15 \pm 0.87$. No patient required a blood transfusion. We experienced one case of postoperative sepsis in all 42 cases.

Conclusions: We were able to introduce ECIRS safely in our hospital. From the experiences requiring a large number of surgeries in comparatively large stone cases, it seems necessary to identify cases in which multiple tracts should be considered.

\section{MP26-11 A Clinical Tracking Program for Ureteral Stents Integrated Into EMR}

F Ghali, D Papagiannopoulos, R Sur, S Bechis

UC San Diego

Introduction \& Objective: Retained ureteral stents are a common complication with an incidence near $10 \%$. Retained stents can be associated with significant complications and result in both cost and patient morbidity, and often necessitate more involved surgery to remove, largely due to encrustation. As many as $76 \%$ of stents are known to encrust after 12 weeks. Many approaches have been proposed to address this issue, including patient wristbands, phone apps, and external registries which have been met with varying limitations and success. These strategies involve increased documentation burden on the surgeon as well as separate electronic systems such as smartphone applications or registries, raising patient privacy concerns. We sought to design and implement an integrated stent tracking system in order to reduce the rate of retained ureteral stents while minimizing added work on the surgeon.

Methods: We designed and recently implemented a point-ofcare (POC), integrated, stent-tracking program within our hospital EMR (EPIC Hyperspace). At time of surgery, our system generates an alert within EPIC to input the 'expected explant date' of all ureteral stents (implants) placed during the surgery. This information is documented by the circulating nurse at the time that other implant information is entered. The expected explant date is determined by the surgeon and corresponds to the anticipated date of removal. The EPIC system then generates an alert when this expected date has passed, and messages can be sent to the appropriate clinical teams to contact the patient and schedule appropriate follow-up. We performed a retrospective review of the first 30 patients who underwent stent tracking.

Results: In a pilot study, 30 patients underwent stent placement using the new tracking system, and 26 have had stents successfully removed before the anticipated date of removal. Three patients had stents removed after the expected date, and 1 patient was flagged for missed stent removal and has been subsequently scheduled for a stent removal appointment. We are currently enrolling patients in a database to follow stent removal and assess the efficacy of our tracking system particularly with regard to timeliness of stent removal and incidence of retained or missed stents. Conclusions: Our integrated stent tracking system embedded within EPIC is a feasible mechanism for potentially reducing the incidence of retained stents while adding minimal administrative and financial burden on the surgeon and hospital. Current studies are ongoing to assess the efficacy of our system at preventing retained stents.

\section{MP26-12 Comparison of three different endoscopic treatment in the treatment of large bladder stone over $3 \mathrm{~cm}$}

J Choi, Y Kim, J Chang, Y Ko, P Song, K Moon, H Jung

Yeungnam University Medical Center

Introduction \& Objective: This study compared the following three endoscopic techniques used to treat large bladder stones sized over $3 \mathrm{~cm}$ : transurethral cystoscope used with a pneumatic lithoclast or cystoscope used with a holmium laser lithoclast and nephroscope used with an ultrasonic lithoclast.

Methods: A total 135 patients with bladder stones sized over $3 \mathrm{~cm}$ underwent endoscopic treatment. Patients were classified into 3 groups based on the endoscopic techniques and energy modalities used in each group as: group 1 (transurethral stone removal using a cystoscope with pneumatic lithoclast), group 2 (transurethral stone removal using a cystoscope with holmium laser lithoclast), and group 3 (transurethral stone removal using a nephroscope with ultrasonic lithoclast). Baseline and perioperative data were retrospectively compared between three groups.

Results: No statistically significant intergroup differences were observed in age, sex ratio, and stone size. A statistically significant intergroup difference was observed in the operation time- 
Table 1. Comparison of all the three groups for various variables

\begin{tabular}{lcccc}
\hline & Group 1 & Group 2 & Group 3 & P-value \\
\hline No of pationts & 63 & 38 & 34 & \\
Male : female & $55: 8$ & $33: 5$ & $30: 4$ & 0.526 \\
Age (years) & $66.7 \pm 13.5$ & $67.9 \pm 13.3$ & $63.5 \pm 13.7$ & 0.622 \\
Stone burden (cm) & $3.93 \pm 0.81$ & $4.11 \pm 1.08$ & $3.84 \pm 0.74$ & 0.513 \\
Operation time (mins) & $71.3 \pm 36.6$ & $38.0 \pm 13.7$ & $25.6 \pm 8.0$ & 0.017 \\
Mean urethral entries & $2.7 \pm 1.6$ & $1.13 \pm 0.12$ & $1.08 \pm 0.05$ & 0.081 \\
Hospital stay (days) & $2.2 \pm 0.9$ & $2.3 \pm 0.4$ & $2.1 \pm 0.3$ & 0.594 \\
\hline
\end{tabular}

group 1: $71.3 \pm 36.6 \mathrm{~min}$; group 2: $38.0 \pm 13.7 \mathrm{~min}$; and group 3: $25.6 \pm 8.0 \mathrm{~min}$. All patients showed complete stone clearance. The number of urethral entries was higher in group 1 than in the other groups. Significant complications did not occur in any patient.

Conclusions: Nephroscopy with ultrasonic lithoclast scores over cystoscopy for the removal of large bladder stones sized over $3 \mathrm{~cm}$ with respect to operation time. Ultrasonic lithoclast is a safe and efficacious modality that scores over a pneumatic lithoclast with respect to the operation time.

\section{MP26-13 Withdrawn}

\section{MP26-14 Analysis of Disposable Equipment Utiliza- tion on the Cost of Ureteroscopy: Impact of Technique and Training}

K Parikh, R Pak

Mayo Clinic Florida

Introduction \& Objective: Ureteroscopy (URS) for the management of kidney and ureteral stones utilizes a variety of disposables which can account for a significant portion of the cost of care. We sought to perform a utilization analysis to determine factors which impact the cost of performing URS.

Methods: In a single-institution, retrospective study, an analysis of disposable utilization was performed on all kidney and ureteral stone cases managed with URS and laser lithotripsy between July 1, 2016 and June 30, 2017. These cases were performed by six different surgeons, two of whom are fellowship trained in endourology. Disposable equipment was broken down into the following categories: baskets, ureteral dilators and bal-

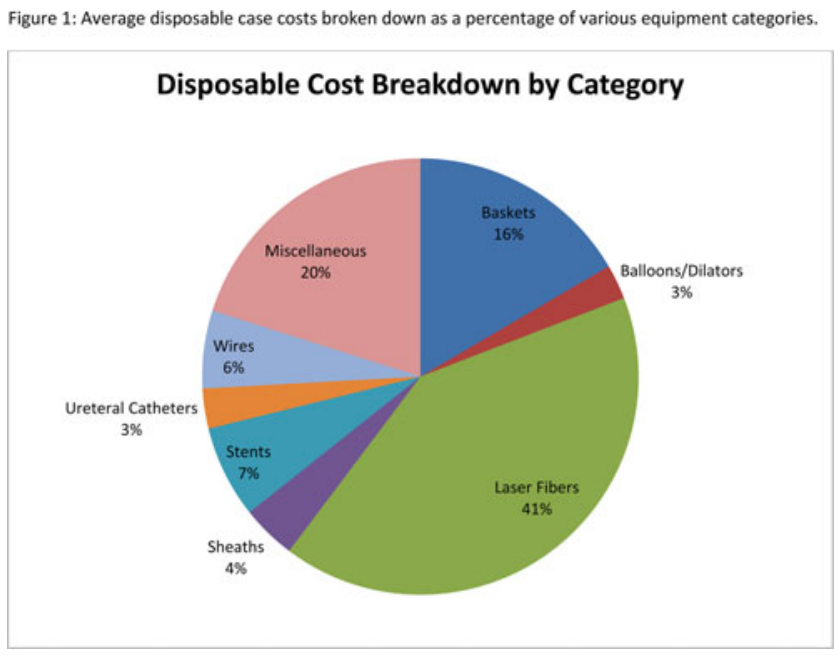

Figure 2: Average case costs, percentage of cases where a basket or access sheath is used, and selfidentified technique broken down per surgeon. The $(*)$ represents Society of Endourology trained urologist.

\begin{tabular}{|c|c|c|c|c|c|}
\hline Surgeon & \# of Cases & $\begin{array}{c}\text { Average Cost per } \\
\text { case }\end{array}$ & $\begin{array}{c}\text { Basket } \\
\text { Utilization }\end{array}$ & $\begin{array}{c}\text { Access Sheath } \\
\text { Utilization }\end{array}$ & Technique \\
\hline $\mathrm{A}^{*}$ & 27 & $\$ 871$ & $37 \%$ & $7 \%$ & Duster \\
\hline $\mathrm{B}^{*}$ & 100 & $\$ 1,084$ & $103 \%$ & $43 \%$ & Hybrid \\
\hline $\mathrm{C}$ & 20 & $\$ 1,163$ & $70 \%$ & $50 \%$ & Hybrid \\
\hline $\mathrm{D}$ & 62 & $\$ 1,055$ & $103 \%$ & $32 \%$ & Hybrid \\
\hline $\mathrm{E}$ & 28 & $\$ 1,029$ & $82 \%$ & $61 \%$ & Hybrid \\
\hline $\mathrm{F}$ & 32 & $\$ 1,340$ & $44 \%$ & $16 \%$ & Duster \\
\hline
\end{tabular}

loons, laser fibers, access sheaths, stents, ureteral catheters, wires, and miscellaneous.

Results: Among the 269 cases included in the analysis, the average cost of disposables used per case was $\$ 1,086.54$. Endourology Society fellowship trained physicians performed 127 $(47.2 \%)$ of these cases Average cost of disposables per case performed by a fellowship trained urologist was $\$ 1,038.72$ compared to $\$ 1,129.31$ for non-fellowship trained urologists. Figure 1 displays the breakdown of the disposables contributing to overall costs per case. Figure 2 displays the individual surgeon costs based on technique and training. Surgeon A, with fellowship training, and a preferred technique of dusting was the most cost effective overall with an average savings of $\$ 239.59$ per case. These savings were realized in the low utilization of baskets and sheaths. Although Surgeon F was similar to Surgeon A in technique, the costs were skewed by expensive laser fiber usage. Conclusions: Conclusion: Technique and training can impact utilization of disposables determining the costs of ureteroscopy.

\section{MP26-15 Comparison of Holmium Lithotripsy pulse duration on stone dusting}

W Kuo, C Yu, J Lin, J Tsai

\section{Kaohsiung Veteran General Hospital}

Introduction \& Objective: Holmium-YAG (Ho:YAG) laser lithotripsy is a multi-pulse treatment modality with certain effects on the fragmentation. Current Ho: YAG laser pulse duration are adjustable. In vitro investigations of Ho:YAG laser induced stone dusting and fragmentation were performed to identify potential impacts of different pulse durations on stone fragmentation characteristics.

Methods: A Ho:YAG laser system (RovoLix) with selectable long or short pulse mode was tested with regard to its fragmentation and dusting compatibility properties. Fragmentation tests on calcium oxalate stones $(\mathrm{N}=20)$ were performed under reproducible experimental conditions (fiber sizes: 365 ; laser settings: $10 \sim 18 \mathrm{~W}$ through combinations of $0.5,1,2 \mathrm{~J} /$ pulse and $20,10,5 \mathrm{~Hz}$, respectively).

Results: Differences in fragmentation and dusting rates between the different pulse duration regimes were measured. Using the long-pulse mode, the fiber burn back was trace, while in shortpulse mode, an increased burn back could be observed. Short pulse duration lithotripsy mode yield efficiency stone fragment, but stone fragments were larger then long pulse duration mode. Average stone dust rate was similar around $0.066 \pm 0.012 \mathrm{mg} / \mathrm{Joule}$ in $150 \mathrm{~S}$ mode vs $0.058 \pm 0.014 \mathrm{mg} / \mathrm{Joule}$ in $800 \mathrm{~S}$ mode. For similar stone burden, it takes longer stone treatment time and energy to complete fragment using longer pulse mode.

Conclusions: Depending on stone location, hardness and endoscopic type, we may adjust pulse duration and energy output in Ho:YAG laser lithotripsy base on our result for optimal clinic outcome. 
MP26-16 Impact on renal function and risk factors associated with the calcification of ureteral stents in a south hospital at México

KA Mendoza, A Ávila, E Cruz, MA Osorio, FE Navarrete, A Esqueda

Hospital Regional de Alta Especialidad de la Península de Yucatán

Introduction \& Objective: JJ stents used since 1967 with the purpose of establishing ureteral permeability, in more than $75 \%$ of patients with evidence of calcification after 3 months. With E. coli likely the main microorganism in the urine culture, $76 \%$ of the patients persist with positive results after removal. The FECal system associated grades IV and $\mathrm{V}$ with a retention time more than 2 years, in patients with advanced renal failure or solitary kidney require measures to correct complications that can be carried to renal function loss. Objective Determine the risk factors associated with JJ stent calcification, levels of $\mathrm{CrS}$ before and after the removal, identify changes in GFR in patients with CKD after removal and evaluate the staging after the surgical event.

Methods: Retrospective study of 44 patients with calcified JJ stents identified from a medical record of the service of our hospital (2016-2018). The generated list validated manually, and the data were collected and analyzed retrospectively.

Results: A total of 44 patients were included, with FECal II-V calcified stents, $70 \%$ were female and $29 \%$ male, mean age of 47 , $25 \%$ had a solitary kidney, $4.5 \%$ horseshoe kidney, the minimum time to carry the stent 3 months and maximum of 6 years. Among the associated comorbidities, $40 \%$ HBP, $20 \%$ DM2. 50\% of the patients with previous positive urine culture, $59 \%$ E. coli. With FECal II: $11 \%$, FECal III: $38 \%$, IV: $34 \%$ and V: 15 of the patients. All of them had previous history of lithiasis and JJ placement in $31 \%$, followed by previous renal surgeries with 3 surgeries in $22 \%$, with 2 in $13 \%$, 4 in $11 \%$, with more than 5 in $6.8 \%$. Within the estimation GFR and CKD-EPI in stage I:40\%, II: $15 \%$, IIIA and IIIB $6 \%$ respectively, IV: $18 \%$ and V: $11 \%, 5$ improved the GFR classified in a KDIGO of IV to IIIB, IIIA and B to II, from II to I in 2 patients, and 5 patients with worsening of the GFR reclassifying from stage IV to V, II to IIIA, I to II in 3 cases. A decrease in $\mathrm{CrS}$ values after surgery was observed in $43 \%$, a mean decrease of $0.8 \mathrm{ng} / \mathrm{dl}$, with an increase in $36 \%$ with a mean of 0.3 of the same rise. In patients with KDIGO IV CKD 6 with a mean $\mathrm{CrS}$ decrease of $0.6 \mathrm{ng} / \mathrm{dl}, 3$ with a mean increase of $0.3 \mathrm{ng} / \mathrm{dl}$, and 4 with no change, patients with KDIGO V with decrease in 3 patients with an average of $0.16 \mathrm{ng} / \mathrm{dl}$ and an increase in creatinine levels in 4 patients with a mean of 0.177 .

Conclusions: The factors that we found significative are: female sex, positive urine culture, previous history of lithiasis, HBP and DM2. In patients with ERC KDIGO IV CrS levels decrease after the procedure which translates into an improvement in the GFR, however in patients with CKD KDIGO V shows no benefit in improving GFR since CrS levels are maintained or increased.

MP26-17 Reusable Versus Single-use Cystoscopes for removal of DJ Stent: A Prospective Randomized Comparison and Cost Analysis

S Bin Hamri, MA Alkhamees, M Alzughabi, A Alothman, I Alshohayeb, A Almujallid, M Alsadhan, A Alasker, Y Ghazwani
Assistant Professor, Urology Department, College of Medicine, Majmaah University

Introduction \& Objective: Cystoscopy procedures require specific organization to perform them safely. With the technological development and medical equipment miniaturization, a new single-use cystoscopy designed to simplify the Double-J stents (DJ) removal is available. The objective of the present study is to compare reusable versus single-use cystoscopes for removal of DJ. Methods: This study is a prospective, randomized, controlled, single-center trial. We randomly assigned patients going for DJ removal from March 2018 until February 2019 into two groups, single-use Disposable cystoscope (DC) group (Coloplast Isiris Cystoscope) and Reusable cystoscope (RC) group (Olympus CYF-VH). A single RC was assigned for the study. All procedures were done by a senior urologist. Both procedures were done within the same settings (operating room and nursing staff). Surgeon, Nurse and patient satisfaction were measured postoperatively by a scale from 1 (Very easy/Extremely satisfied) to 5 (Very hard/Extremely unsatisfied). Other factors were also compared between the two modalities (operative time, cost, pain score, and complications).

Results: A total of 130 cases were performed (65 cases in each arm). The mean operative time per case for the DC and RC was 58 and 83 seconds. The surgeon was satisfied for $93 \%$ of removal acts with the $\mathrm{DC}$, and $79 \%$ with the $\mathrm{RC}(\mathrm{p}=0.06)$. There were two cases of Urinary Tract Infection after 7 days of the procedure in the $\mathrm{RC}$ group, and none in the DC group. Pain score was equivalent in both groups. No failure was reported for the RC. The total cost to perform 65 procedures in the endoscopy room was 34600 USD for the DC, while it was 29500 USD for RC group.

Conclusions: Both the DC and the RC provide good results in terms of surgeon's satisfaction, pain score, operative time and rate of UTIs. Interestingly, no cases of UTI was reported after removal by DC. The cost is subject to high variation for RC due to the frequency of use, type of procedure and repair cost related to device failures (did not occur within our study period). The incidence of several repairs may quickly reverse the trend in favor of DC. Moreover, the capacity to use DC in outpatient's area allows to free up expensive time slots of highly equipped rooms.

\section{MP26-18 Should the use of stent-on-strings become common practice during supine miniature percuta- neous renal stone extraction?}

H Burns, S Nalagatla

University Hospital Monklands

Introduction \& Objective: Nephrostomy insertion, ureteric catheters, antegrade or retrograde stenting and totally tubeless PCNLs have all been described as methods of managing the postoperative period following PCNL. A single center demonstrates the benefits of retrograde insertion of stent-on-strings following mini-PCNL which can safely be removed within 48 hours.

Methods: Data was collected retrospectively for all mini-PCNLs performed between July 2017 and August 2018. Age, co-morbidity, stone size, patient position, puncture site, length of admission, timing of stent removal and reason for delay were documented. The standardized procedure carried out in all patients included supine position, 17.5 Fr Karl Storz MIP-M sheath with retrograde stent insertion. The goal was to insert a tailed JJ stent at the end of the procedure to be removed prior to discharge within 48 hours. 
Results: 33 patients underwent mini-PCNL with an average admission of 2.9 days and $3 \%$ readmission rate. 30 patients had stent-on-strings inserted following the procedure. $53.3 \%$ had the stent removed within 2 days, $33.3 \%$ were removed between day $3-5$ and $13.3 \%$ were removed day $>5$. A delay of 3-5days was due to pyrexia or slow recovery secondary to poor mobility, pain or nausea. A delay of $>5$ days was due to frank hematuria or an infection requiring a course of IV antibiotics. Non-tailed stents were inserted in 3 patients due to residual stones requiring further intervention or IV antibiotics for post-operative pyrexia. These were removed between 1 - and 3-months post procedure. The recognized risk of dislodgement did not occur.

Conclusions: Stent-on-strings were removed after 48hours in $53.3 \%$ of cases limiting stent-related symptoms and duration of admission. Whereas removal of a stent without strings requires a further cystoscopy, or nephrostomy removal, which involves a nephrostogram, clamping of the tube prior to removal and wound closure. Therefore, this center demonstrates how early removal of stent-on-strings is a successful approach following mini-PCNL for renal calculi without an increased risk of complications or readmission rates and limiting troublesome side effects.

\section{MP26-19 The risk factors and complications of for- gotten double-J stents: a single center experience}

T Lin, W Lin, M Chen, S Jhang, T Yang, J Hsu

Mackay Memorial Hospital

Introduction \& Objective: Postoperative urology patients may require the insertion of a ureteral stent. However, the delayed removal or change of the ureteral stent may lead to serious consequences for some patients. This study primarily aimed to examine the risk factors and complications associated with forgotten double-J stents.

Methods: In this retrospective study, postoperative patients who underwent double-J stents insertion were recruited. Based on the brand of double-J stents, the maximal stent life was classified into 3-month, 6-month, and 12-month groups, and a forgotten double-J stent was defined as one that had yet to be removed 2 weeks past its maximal stent life. A total of 479 patients were analyzed. The reasons for the use of double-J stents use and the time and method of their insertion were recorded, and the risk factors and possible complications associated with forgotten double-J stents were analyzed.

Results: The primary reason for double-J stent insertion was urolithiasis $(69.7 \%)$, and insertions performed using ureterorenoscopy were the most common $(413 / 479,86.2 \%)$. Eighteen patients $(3.8 \%)$ had forgotten double-J stents, with an average overdue period of 63.17 days (18 189 days). Multivariate analysis revealed that patients older than 60 years $(\mathrm{OR}=3.432$, $95 \% \mathrm{CI}=1.049 \sim 11.236, \mathrm{p}=0.042)$, and double-J stents exchanged using fibrocystoscopy $(\mathrm{OR}=3.627,95 \% \mathrm{CI}=1.232 \sim$ $10.679, \mathrm{p}=0.019)$ were significantly associated with forgotten double-J stents. Three patients $(16.67 \%)$ experienced symptomatic complications, with one developing acute pyelonephritis, and the remaining two experiencing stone encrustation.

Conclusions: Patients older than 60 years were 3.4 times more likely to have forgotten double-J stents than patients aged 60 and below, and double-J stents exchanged using fibrocystoscopy were 3.6 times more likely to be forgotten than those inserted using ureterorenoscopy. Greater attention with regards to tracking and recalling double-J stents should be paid in high-risk patients to prevent forgotten double-J stents and associated complications.

MP26-20 Suctioning the dust out in mini PCNL, does it change the outcome?

\author{
A Singh, N REDDY, A Ganpule, R Sabnis, M DESAI
}

Muljibhai Patel Urological Hospital

Introduction \& Objective: Fragmentation of stone does not necessarily equate to stone clearance. Use of suction to remove stone dust as it is produced by laser would expedite the procedure. Aim of our study was to compare 'laser with suction' (LWS) and 'laser without laser' (LOS) in terms of safety, efficacy and feasibility in mini-PCNL.

Methods: Retrospective analysis of 200 patients (100 LWS vs 100 LOS). Mini-PCNL was done using energy source as either LWS or LOS. Results were tabulated and analyzed.

Results: Both the group were comparable in terms of demographic data. Mean stone size was $15.24 \pm 5.90 \mathrm{~mm}$ and $16.16 \pm 5.53 \mathrm{~mm}$ in LWS and LOS respectively. Mean HU of stone was 1285.64 and 1206.79 in LWS and LOS respectively. Operative time was less in LWS group $(56.89 \pm 19.65 \mathrm{~min})$ as compared to $\operatorname{LOS}(62.01 \pm 28.81 \mathrm{~min})$. Post-operative haemoglobin drop, hospital stay, and pain scores were comparable. LWS group had one patient with clavien - dindo grade III complication. At one-month follow-up radiological complete clearance was $96 \%$ in LWS and 92\% in LOS. One patient in LOS required repeat nephroscopy for residual calculi; other residual calculi were managed conservatively.

Conclusions: Laser is a powerful energy source for fragmentation of stone. Adding suction to laser should fasten the clearance of fragments thus decreasing the operative time, the same is seen in retrospective analysis. We propose a randomized control trial to prove the efficacy of this device.

\begin{tabular}{|l|l|l|}
\multicolumn{4}{c}{ ReSUltS } \\
\hline Parameter (meantSD) & LWS & LOS \\
\hline Age (years) & $41.15 \pm 18.52$ & $43.28 \pm 18.18$ \\
\hline Stone Size (mm) & $15.24 \pm 5.90$ & $16.16 \pm 5.53$ \\
\hline CT density (HU) & $1285.64 \pm 245.32$ & $1206.79 \pm 284.31$ \\
\hline Operative time (min) & $56.89 \pm 19.65$ & $62.01 \pm 28.81$ \\
\hline Tract size (Fr) & $16.87 \pm 3.78$ & $15.94 \pm 2.67$ \\
\hline VAS 6hrs & $5.02 \pm 2.13$ & $5.44 \pm 2.32$ \\
\hline VAS 12hrs & $3.14 \pm 1.42$ & $3.26 \pm 1.59$ \\
\hline VAS 24hs & $0.78 \pm 1.13$ & $0.81 \pm 1.06$ \\
\hline Stone Clearance rate (\%) & 96 & 92 \\
\hline Hb Drop (mg/dL) & $1.15 \pm 1.37$ & $1.16 \pm 0.95$ \\
\hline Ancillary procedure & 0 & 1 repeat nephroscopy \\
\hline Complication (clavien dindo & 1 & 2 \\
\hline grade III) & &
\end{tabular}

MP26-21 Thulium:YAG vs Holmium:YAG laser effect on soft tissue: evidence from an ex vivo experimental study

ME Rodriguez Socarras, S Proietti, R Luciano', F Scarfo', G Saitta, F Gaboardi, G Giusti

Instituto de Cirugía Urológica Avanzada (ICUA),

Madrid, Spain 
Introduction \& Objective: There are limited data regarding the effect of Thulium laser (Tm:YAG) and Holmium laser (Ho: YAG) on upper urinary tract (UUT). The aim of this study was to compare the effect in terms of incision depth and coagulation area of Tm:YAG and Ho: YAG laser on soft tissue at different laser settings.

Methods: An ex vivo experimental study was performed in a porcine model. Porcine kidneys were dissected in order to expose the UUT and the block samples containing urothelium and renal parenchyma were prepared. The $272 \mathrm{~mm}$ laser fiber, fixed on a robotic arm, perpendicular to the target tissue was used with both 100 W Ho:YAG and 200 W Tm:YAG (Quanta System, Italy). The incisions were made with the laser tip in contact with the urothelium and in continuous movement at a constant speed of $2 \mathrm{~mm} / \mathrm{s}$ for a length of $1.5 \mathrm{~cm}$, by using energy between $5-30 \mathrm{~W}$, in continuous/pulse mode with short/medium/long pulse. Then all porcine blocks were analyzed macroscopically and microscopically by two different pathologists. Incisions' shape was classified as follows: saccular, triangular, tubular and irregular. Incision Depth (ID), Vaporization Area (VA), Coagulation Area (CA) and Total Laser Area (TLA $=\mathrm{VA}+\mathrm{CA})$ of both lasers were evaluated (Figure 1). Experiments and measurements were repeated 3 times each. Statistical analysis was performed using the SPSS V23 package, $\mathrm{p}$ values $<0.05$ were considered statistically significant.

Results: A total of 132 experiments were performed. Incisions' shape was Saccular (46\%), Triangular (38\%) and Irregular (16\%) with the Ho:YAG; while it was mainly Tubular (89\%) with Tm:YAG $(p=0.001)$. ID was significantly deeper with Ho:YAG $(p=0.003)$, while CA and TA were wider with Tm:YAG

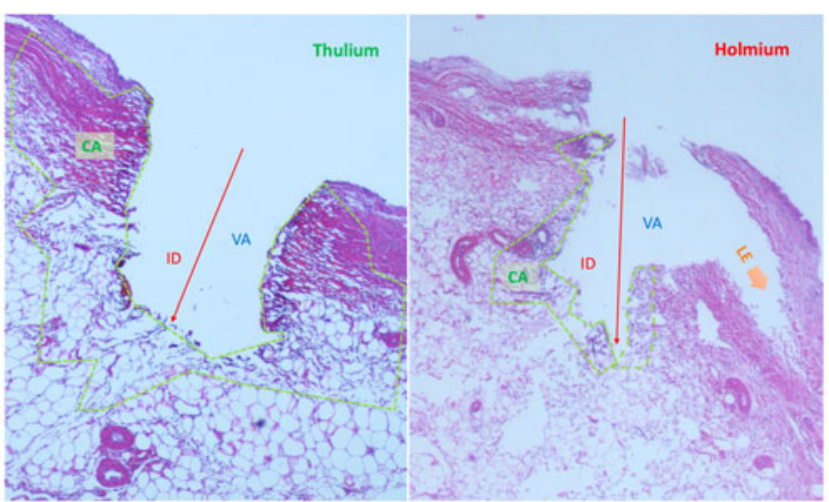

Fig 1: microscopic measurements Thulium and Holmium effect on soft tissue (swine kidney), showing: Incision Depth (ID), Vaporization Area (VA) and Coagulation Area (CA). Total Laser Area $(T L A)=I D+V A+C A$. Orange arrow show Lateral Energy (LE) dissection with Holmium laser.

\begin{tabular}{|c|c|c|c|}
\hline & Thulium & Holmium & $p$ value $e^{*}$ \\
\hline Watts, mean \pm SD & $15,17 \pm 7,85$ & $14,65 \pm 7,79$ & 0,939 \\
\hline $\mathrm{ID}(\mathrm{mm})$, mean $\pm \mathrm{SD}$ & $0,360 \pm 0,138$ & $0,432 \pm 0,211$ & $0,003^{*}$ \\
\hline $\mathrm{VA}\left(\mathrm{mm}^{2}\right)$, mean $\pm \mathrm{SD}$ & $0,070 \pm 0,45$ & $0,066 \pm 0,46$ & 0,572 \\
\hline $\mathrm{CA}\left(\mathrm{mm}^{2}\right)$, mean $\pm \mathrm{SD}$ & $0,183 \pm 0,103$ & $0,066 \pm 0,035$ & $0,001^{*}$ \\
\hline $\mathrm{TLA}\left(\mathrm{mm}^{2}\right)$, mean $\pm \mathrm{SD}$ & $0,264 \pm 0,146$ & $0,125 \pm 0,055$ & $0,005^{\star}$ \\
\hline
\end{tabular}

( $p=0.001$ and $p=0.005$ respectively). No statistical differences were found for VA $(\mathrm{p}>0.05)$ for both lasers in all settings tested (Table 1).

Conclusions: ID was deeper with Ho:YAG, while CA and TLA were wider with Tm:YAG. Tm:YAG laser seems to have better coagulation profile on upper urinary tract than Ho:YAG. Further studies are needed to evaluate which laser could better for ablation of soft tissue into UUT

\section{MP26-22 Initial In Vivo Experience with a Novel Single Probe Dual Energy Lithotriptor}

\section{Dunne, JE Abbott, JG Davalos}

University of Maryland Medical Center

Introduction \& Objective: Percutaneous Nephrolithotomy (PNL) surgery is a procedure that is traditionally used to treat large and complex stones. We are continually looking for ways to improve efficiency in treatment time. LithoClast Trilogy is a novel single probe dual energy device, which through ultrasonic vibration and electromagnetic impact forces, may offer a way to improve these parameters. PNL surgery is moving towards smaller tract surgery. One of the challenges that minipercutaneous nephrolithotomy (mPNL) presents is maintaining efficient treatment times using a smaller sheath and smaller scope. Up to this point, in our experience, we have not found an efficient lithotriptor for mPNL.

Methods: We present an eight-case initial experience with Trilogy. These cases were performed at one institution in November 2018 by one surgeon. Six cases were performed with standard tract (34 French) and two cases were performed with a mini-tract (17 French). A $3.9 \mathrm{~mm}$ probe was used during the standard tract cases and a $1.9 \mathrm{~mm}$ probe was used during the mPNL tract cases. All procedures were performed with the operative surgeon obtaining renal access and all procedures were performed tubeless (ureteral stent without a nephrostomy tube). All cases were reviewed, and demographic data and case details were analyzed. In particular, we focused on stone burden, stone density, skin to stone distance, treatment time and stone free rate. Results: Our 8 Trilogy cases were reviewed. Of the 6 standard PNL cases there was a mean stone burden of $42 \mathrm{~mm},(22-70)$, mean Houndsfield Units (HFU) of 975 (600-1400), mean skin to stone distance of $8.8 \mathrm{~cm}(7-12.7)$, and average treatment time of $21.8 \mathrm{~min}$ (15-34). Of the $2 \mathrm{mPNL}$ cases performed with Trilogy, there was a mean stone burden of $18.5 \mathrm{~mm}$, mean HFU of 825 , mean skin to stone distance of $16 \mathrm{~cm}$, and and average treatment time of $9.5 \mathrm{~min}$. Stone clearance rate was $2.1 \mathrm{~mm}^{3} / \mathrm{min}$ for both standard and mini PNL cases.

Conclusions: This experience of a small case series of 8 patients demonstrates that Trilogy proved to be an efficient lithotriptor. Stone treatment time and the rate of stone clearance exceeded expectations. Additionally, the efficiency demonstrated during the $\mathrm{mPNL}$ procedures was surprising. Interestingly the stone clearance rate was the same with both the $3.9 \mathrm{~mm}$ and $1.9 \mathrm{~mm}$ probes. In our experience, stone clearance rate with prior generation lithotriptors is about two-thirds of this rate. It appears that based off of our early experience that Trilogy may be a more efficient lithotriptor. Given these promising results of our initial experience, we plan to perform a much larger comparative series. 


\section{MODERATED POSTER SESSION 27: CLINICAL STONES: PEDIATRICS \& ESWL}

\author{
MP27-01 Stone composition in children: A single \\ center study in Pakistan.
}

N Iqbal, A Hasan, M Mughal, T Bhatty, S Akhter, J Rahim

Pakistan Kidney and Liver Institute and Research Center

Introduction \& Objective: The composition of pediatric kidney stones is said to be the same as that in adults with more than 75$80 \%$ being calcium oxalate $(\mathrm{CaOx})$ and the rest to include uric acid, cysteine and calcium phosphate stones. We wanted to assess the stone composition of children presenting to a biggest pediatric urology center in northern Pakistan.

Methods: A retrospective chart review of 288 patients was done, who presented at our pediatric stone clinic. Patient who had stone composition done and recorded in files were included. In case of more than one composition type, the first reported composition was included for the data analysis. Stones were defined as predominantly Calcium Oxalate, uric acid, cysteine and Calcium phosphate. Pearson Chi-Square test was used for comparing categorical variables. A p-value $<0.05$ was set as significant.

Results: One hundred and Fifty-two children were having stone analysis in charts records. There were 109 male, 43 female children Forty-three had a family history of stones. Mean age at initial clinic visit was $8.4 \pm 5.6$ years (range, $1.5-17.3$ years). Mean body mass index (BMI) was $18.6 \pm 7 \mathrm{~kg} / \mathrm{m}^{2}$. Out of these 152 children, $107(70.6 \%)$ were having predominantly Calcium Oxalate stones and $9(5.9 \%)$ were predominantly uric acid stones, $8(5.2 \%)$ were having cysteine stones. Twenty-eight (18.4\%) children Nine had mixed stone compositions (including calcium phosphate stones and others in mixed composition). Most of them had combination of Calcium Oxalate.

Conclusions: The majority of children at our center stone clinic had primarily calcium-based stones $(\mathrm{CaOx}$ along with calcium phosphate.

MP27-02 In vitro evaluation of a novel pediatric flexible cystoscope-A practical comparison of endoscopes for pediatric lower urinary tract evaluation

H Truong, J Rosado, J Hagerty, SG Hubosky, D Bagley

Introduction \& Objective: Adequate assessment of the lower urinary tract in patients' post-reconstructive surgeries, such as bladder neck reconstruction and continent catheterizable channels, presents a unique challenge for pediatric urologists. There is a need for a flexible cystoscope specifically designed for children. Herein, we compare in vitro performance of a novel single-use pediatric flexible cystoscope with commonly used endoscopes for pediatric cystoscopy.

Methods: We compared technical factors that affect surgical utilities including optics, deflection, and irrigation flow of a single-use Neoflex pediatric flexible cystoscope (Neoscope, USA), a Gyrus ACMI pediatric semirigid cystoscope (Olympus, USA), Flex-X flexible ureteroscope (Karl Storz, Germany), and an adult flexible cystoscope (Karl Storz, Germany).

Results: The technical characteristics are shown in Table 1. Neoflex has wider field of view $\left(85^{\circ}\right)$ than Storz cystoscope

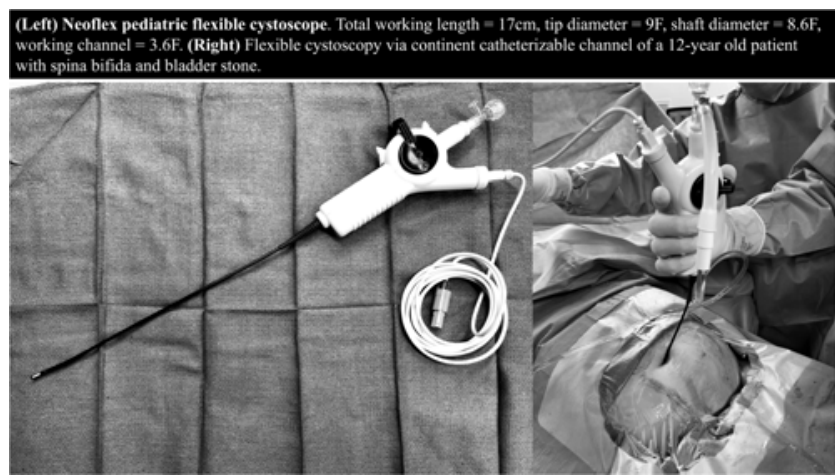

$\left(80^{\circ}\right)$, Flex-X $\left(75^{\circ}\right)$, and Gyrus ACMI $\left(40^{\circ}\right)$. Neoflex has the best angle and diameter of deflection with and without instruments in the working channel $(\mathrm{p}<0.03)$. Neoflex provides the highest image resolution compared to the other scopes at all distances tested $(p<0.0001)$. The color quality of each scope depends on the distance the scopes were positioned away from the target. Overall, Gyrus ACMI and Storz cystoscopes have highest color representation. Neoflex has the lowest color quality $(\mathrm{p}<0.0001)$. Gyrus ACMI has the highest irrigation flow with an empty working channel, followed by Storz cystoscope, Neoflex, and Flex-X at 286, 256, 65, and 42mL/min respectively. With the $1.9 \mathrm{~F}$ basket, the Storz cystoscope has more superior flow rate compared to Gyrus ACMI, Neoflex, and Flex-X.

Conclusions: The Neoflex single-use pediatric cystoscope is the only flexible cystoscope in the market designed for children. Neoflex outperforms available scopes used for pediatric cystoscopy in term of image resolution, field of view, and deflection capacity. The Gyrus ACMI pediatric semirigid cystoscope is superior in irrigation and color quality. A flexible cystoscope designed with pediatric specifications enhance lower urinary tract evaluation in children with bladder reconstruction and add to pediatric urologists' armamentarium.

MP27-03 Does Early Extracorporeal Shock Wave Lithotripsy (ESWL) Failure Predict Overall Treatment Failure-Results from A Tertiary Referral Stone Centre

E Latif, MM Quraishi, J Dorrell, J Hale, S Subramaniam, P Keirandish, OA Ramadan, B Osman, G Papadopoulos, I Ahmed, A Simoes, SS Kommu, R Krishnan, N Shrotri

Kent \& Canterbury Hospital. East Kent Hospitals NHS Foundation Trust

Introduction \& Objective: Extracorporeal Shock Wave Lithotripsy (ESWL) is a well-documented and widely utilized treatment for renal and ureteric calculi. Stone disease poses a significant burden on patients both physically and financially. Efficient and effective treatment is key to minimizing morbidity 
and returning patients to their regular activities. Our aim was to determine whether poor fragmentation of stones during initial ESWL treatment predicted failure with multiple sessions with a view to potentially implementing an early tailored treatment plan. The treatment plan being flexible uretero-renoscopic surgery (FURS) and laser fragmentation is indicated.

Methods: Fifty patients who underwent a total of 78 ESWL treatments were identified in a 6-month period. Patient records were consulted and additional data regarding previous and/or subsequent treatment(s) for the same stone, even if outside the study dates, was collected. One patient was excluded due to relocation to another country. Nine other episodes were identified of patients who attended for ESWL, but the stone could not be seen on pre-ESWL x-ray and/or could not be localized on the machine. These episodes have been excluded from the study as no treatment was given.

Results: Of the 50 patients, $14 \%(n=7)$ achieved stone-free status by use of ESWL alone, having undergone a total of 15 treatments. A further $38 \%(n=19)$ had their treatment plan changed to FURS with laser fragmentation of stone, 3 patients after only 1 session, 7 patients after 2 sessions and 9 patients after 3 sessions. A total of 44 treatments were performed on these 19 patients. Of these 26 patients treated until stone free 5 achieved this with 2 or less ESWL sessions. In 15 patients (30\%) a decision was made not to treat further (i.e. not to render radiographically stone free) after one treatment in 3 patients, 2 ESWL in 2 patients and $3 \mathrm{ESWL}$ in 10 patients. Of the total patients receiving 3 sessions or more of ESWL $(n=21)$ only 2 were rendered stone free by ESWL alone, a further 9 had definitive clearance by FURS and 10 received no further therapy. Upon completing this audit the patients lost to follow-up were seen in clinic and treated with a combination of ESWL or FURS.

Conclusions: Our study shows that early ESWL failure predicts likelihood of requirement of supplementary treatment. In those who did achieve stone clearance by ESWL alone, this was most likely to occur within 2 sessions. Likelihood of stone clearance rapidly decreased in patients requiring more than 2 treatments. This finding has led to a decision tree model for managing stones in our urology stone unit.

\section{MP27-04 Endoscopic hybridization in the treatment of complex upper urinary calculus in children}

L Zhong, J Sun

Shanghai Jiaotong University School of Medicine

Introduction \& Objective: Complicated upper urinary calculi in children, such as staghorn stones or renal calculi with congenital malformation, are difficult to treat. Laparoscopy, flexible ureteroscope and percutaneous nephroscope are not effective enough when they were performed alone. In this research we retrospectively review three kinds of cases which use two endoscopic in the operation procedure to study the feasibility and safety of endoscopic hybridization.

Methods: In the case of ureter distal stenosis complicated with renal calculi, the distal ureter was dissected under laparoscope then taken out from the laparoscope trocar. A 9.5/11.5F flexible ureteroscope sheath was inserted into uretral. Renal calculi was take out by flexible ureteroscope. In the case of staghorn stones complicated with hydronephrosis, the children was fixed on the oblique lithotomy position. The flexible ureteroscope and percutaneous nephroscope were perform at the same time to remove the calculi. In the case of duplicate kidney in which hydronephrosis of lower kidney with calculi, a nephropyeloplasty of lower kidney under laparoscope was performed firstly. And 12/ $14 \mathrm{~F}$ flexible ureteroscope sheath was inserted into collecting system of lower kidney through the trocar when the renal pelvis was open. Then the flexible ureteroscope was used to taken out the calculi.

Results: all cases were successfully operated without obvious stone residue and major complications.

Conclusions: Endoscopic hybridization can be applied safely in children.

MP27-05 The use of a virtual reality device (HypnoVR ${ }^{\circledR}$ ) during external shock wave lithotripsy for treatment of urinary stones: initial results from a feasibility study
E Ventimiglia, S Doizi, E Ayani, G Raynal, O Traxer
Division of Experimental Oncology/Unit of Urology; URI; IRCCS Ospedale San Raffaele

Introduction \& Objective: External shock wave lithotripsy (ESWL) is an outpatient procedure generally not requiring anesthesia or sedation. However, patients may experience pain during the procedure, as well as treatment-related anxiety. We aimed to test a virtual reality device (VRD, HypnoVR ${ }^{\circledR}$, Strasbourg, France) in order to assess its impact in terms of patientreported pain and anxiety.

Methods: We enrolled 15 patients submitted to ESWL for upper tract urinary stones. Patients with either epilepsy or migraine where excluded from the study. ESWL procedures were performed using the same lithotripter (Lithoskop; Siemens, AG Healthcare, Munich, Germany) at a frequency of $1 \mathrm{~Hz}$ delivering a total of 3000 shock waves per procedure. The VRD was installed and started 10 to 5 minutes before the procedure. Tolerability of pain and treatment-related anxiety represented the primary efficacy outcomes and were evaluated using a visual analogue scale (VAS), the short version of the McGill pain questionnaire (MPQ), and the short version of the Surgical Fear Questionnaire (SFQ). Secondary outcomes were represented by VRD ease of use (VAS) and patient satisfaction with the use of the device.

Results: Median (IQR) age and BMI were respectively 57 (51$60)$ years and $23(22-27) \mathrm{kg} / \mathrm{m}^{2}$. Stone location was renal in 10 (67\%) patients, and median stone size was 7 (6-12) $\mathrm{mm}$. Most of the patients were ESWL naïve $(10,67 \%)$ and were not prestented $(8,53 \%)$. Median extra time for the VDR installation was 6.5 (4-8) minutes. Median (IQR) pre-procedure pain (VAS) score and anxiety (VAS) scores were 1 (1-2) and 3 (2-4). Lower post-procedure anxiety levels were recorded, median (IQR) VAS $2(2-3)$, although not statistically significant, $p>0.05$. Procedure related median (IQR) pain was 3 (3-4). Similar results were reported for the MPQ and the SFQ. No procedure was stopped due to side effects from VR and one patient reported a negative experience due to an incorrect application of the device. Eight $(53 \%)$ patient reported that a greater VRD associated benefit in terms of pain tolerance rather than anxiety. All the patients who were not ESWL naïve reporter a better experience using the VRD compared to the recalled previous sessions. 14 out of the 15 (93\%) patients would use again the VRD if further ESWL session were needed in the future.

Conclusions: Our proof of principle study shows that VDR application during ESWL is safe and feasible. The initial report 
from patients are positive both in terms of pain and anxiety tolerance. Further comparative studies are needed.

\section{MP27-06 Withdrawn}

\section{MP27-07 Role of dusting and pop-dusting using high power $(100 \mathrm{w})$ laser in treatment of pediatric stones}

T Reeves, S Griffin, A Pietropaolo, S Chugh, BK Somani

Portsmouth Hospitals NHS Trust

Introduction \& Objective: The incidence of stone disease amongst pediatric population is increasing and stones are increasingly managed safely and effectively with minimally invasive endourological techniques. In the adult population a technique increasingly used is dusting and pop-dusting. The use of the holmium:yttrium-aluminum-garnet laser at high-power, high-frequency, long pulse allows for dust production that can evacuate spontaneously. The advantage of dusting appears to be a reduction in operative time, ureteral access sheath usage, fragment retrieval and complications relating to basketing them. Dusting and pop-dusting has been shown to be safe and effective in the adult population but there is a lack of evidence in the pediatric population. Here we present the results from a cohort of pediatric patients that underwent dusting and pop-dusting for stones using high powered laser.

Methods: Between February 2016 and July 2018, 12 patients were treated with dusting and pop-dusting at Southampton General Hospital. Outcomes were collected retrospectively, and complications graded using Clavien-Dindo criteria.

Results: Twelve patients underwent URS and stone dusting. The stone free rate (SFR) at time the of operation was $100 \%$. All patients were followed up with ultrasound (mean 3.5 months, range $2-5$ months) and stone free rate was $91.6 \%$ (11/12); this was due to failure to fully visualize lower pole calyx in one patient. There were no post-operative complications for any patient.

Conclusions: Advancements in endourological technique have made URS in the pediatric population safe and effective. Although our conclusions are based on small case series it shows dusting and pop-dusting can be performed with low morbidity and high SFR in the pediatric population.

MP27-08 Expulsive therapy using tamsulosin after shock wave lithotripsy of renal and ureteral stones: randomized prospective study

N Mouad

Clinique Achifaa Oujda

Introduction \& Objective: To evaluate if tamsulosin hydrochloride is effective as an adjunctive medical therapy to increase the effectiveness of extracorporeal shock-wave lithotripsy (ESWL) for treating ureteric and renal stones and minimizes the use of analgesic drugs after the procedure. To evaluate if tamsulosin hydrochloride is effective as an adjunctive medical therapy to increase the effectiveness of extracorporeal shock-wave lithotripsy (ESWL) for treating ureteric and renal stones and minimizes the use of analgesic drugs after the procedure. To evaluate if tamsu- losin hydrochloride is effective as an adjunctive medical therapy to increase the effectiveness of extracorporeal shock-wave lithotripsy (ESWL) for treating ureteric and renal stones and minimizes the use of analgesic drugs after the procedure. To evaluate if tamsulosin hydrochloride is effective as an adjunctive medical therapy to increase the effectiveness of extracorporeal shock-wave lithotripsy (ESWL) for treating ureteric and renal stones and minimizes the use of analgesic drugs after the procedure.

Methods: To treat ureteric and renal stones of $5-25 \mathrm{~mm}$ in diameter, 118 patients were treated with ESWL. After treatment, equal numbers of patients were randomly assigned to receive either the standard medical therapy alone (controls) or combined with $0.4 \mathrm{mg}$ tamsulosin daily for 12 weeks. All patients were followed up for 3 months or until an alternative treatment was offered.

Results: At 3 months the treatment was considered to be clinically successful in 50/60 (86.2\%) of those receiving tamsulosin and in $54 / 58(90 \%)$ of the controls $(\mathrm{P}=0.06)$. When patients were classified according to stone size the success rate was similar in both groups $(\mathrm{P}=0.21)$ for those with a stone of $>10 \mathrm{~mm}$. Ureteric colic was reported in $10 \%$ of patients treated with standard therapy and in $8 \%$ of those treated with tamsulosin $(\mathrm{P}=0.2)$. The mean cumulative diclofenac dose was $600 \mathrm{mg} /$ patient in the tamsulosin group and $650 \mathrm{mg} / \mathrm{patient}$ in the control group $(\mathrm{P}=0.06)$

Conclusions: This study showed the no effectiveness of tamsulosin as an adjunctive medical therapy after ESWL for ureteric and renal stones. it did not improve stone clearance and it did not decrease the use of analgesics.

MP27-09 Role of stone size, stone density, and skinto-stone distance by non- contrast computed tomography abdomen in predicting success of shock-wave lithotripsy for kidney stones.

\section{A Hamid, A Shah, N Choh}

\section{SKIMS, SRINAGAR, KASHMIR, INDIA}

Introduction \& Objective: Shock-wave lithotripsy (SWL) is a minimally invasive treatment procedure for kidney calculi, commonly employed as one of the first line therapy for properly selected renal and ureteric calculi. Multiple factors influence the outcome of shock-wave lithotripsy and assessment of certain CT characteristics of the these calculi prior to the procedure can help in predicting outcome of shock-wave lithotripsy. The aim of this study was to assess the influence of these factors in predicting the outcome of shock-wave lithotripsy for kidney calculi.

Methods: Between March 2016 and June 2018, 96 patients who underwent shock-wave lithotripsy for renal or proximal ureteric calculi at our center were included in the study. The CT characteristics of the calculi, shock-wave lithotripsy settings applied, and the final outcome observed were recorded in a proforma and subjected to statistical analysis.

Results: There were $58(60.42 \%)$ males and $38(39.58 \%)$ females in the study. The age of patients varied from 18 to 72 years with a mean of $36.4 \pm 13.8$ years. Majority of patients 69 (71.88\%) had renal (non-lower pole) calculi while $27(28.12 \%)$ had upper ureteric calculi. Stone size varied from $7.4 \mathrm{~mm}$ to $20.0 \mathrm{~mm}$ with a mean of $13.1 \mathrm{~mm} \pm 3.8 \mathrm{~mm}$. The stone density ranged from $536 \mathrm{HU}$ (Hounsfield unit) to $1232 \mathrm{HU}$ with a mean of $826.3 \pm 194.3 \mathrm{HU}$. The mean skin-to-stone distance (SSD) 
varied from $5.58-12.5 \mathrm{~cm}$ with a mean of $8.84 \pm 1.92 \mathrm{~cm}$. Complete stone fragmentation was observed in $65(67.70 \%)$ patients after first session; $16(16.67 \%)$ after second session and 7 $(7.30 \%)$ after third session. However, $8(8.33 \%)$ patients had failure of shock-wave lithotripsy and needed alternative treatment. Overall success rate of $91.67 \%$ (88/96) was observed. The mean number of shock-waves required to achieve complete stone fragmentation was observed to increase with the increased stone size and stone density, however, stone density had more significant influence than stone size and mean SSD.

Conclusions: From the study it was concluded that the use of NCCT-KUB for determining the stone size, stone density, and skin-to-stone distance of kidney calculi before shock-wave lithotripsy helps in predicting treatment outcome. The combination of these factors helps to identify ideal candidates for shock-wave lithotripsy. The increase in mean skin-to-stone distance is associated with an increase in the number of shockwave lithotripsy sessions required as well as an overall decreased success rate. However stone density appears to be more significant than stone size and skin-to-stone distance in predicting fragmentation of kidney calculi with shock-wave lithotripsy.

\section{MP27-10 Ultrasound Guided ESWL for VUJ Stones}

RS Bashetty, SB Alur, JB Alur, S Raju

\section{Matoshree Kidney Stone Center}

Introduction \& Objective: Vesicoureteric junction (VUJ) is the narrowest portion of the ureter. Majority of the stones gets stuck at the VUJ. Stones larger than $5 \mathrm{~mm}$ can cause significant symptoms and may not be expelled after MET and may require an intervention. Ureteroscopy (URS) is the most common procedure done for the treatment of lower ureteric stones. But it is invasive, requires an anesthesia $\&$ a second procedure is required if a stent is placed. ESWL is an alternative option to URS. We evaluated the efficacy of USG guided ESWL in the treatment of VUJ calculus.

Methods: Prospective study was conducted from Jan 2017 to Jan 2019. 91 patients with VUJ calculus after failed MET were included in the study. Informed consent was obtained. Inclusion criteria were calculus more than $5 \mathrm{~mm}$, failed MET, mild HUN. Exclusion criteria were calculus more than $15 \mathrm{~mm}$, moderate to severe HUN, untreated UTI, uncontrolled diabetes and hypertension, severe symptoms, pregnancy, bleeding diathesis \& BMI more than 30 . There were 71 males and 20 female patients within the age group of 22-56 years. The stones size ranged from $5 \mathrm{~mm}$ to $15 \mathrm{~mm}$. All the patents were treated with USG guided Siemens Modularis Variostar lithotripter. Treatment was carried out with the patient in semi lateral position with the therapy head in lateral position coupled to the Ipsilateral Iliac fossa with a 30-degree tilt. No anesthesia or analgesia was used. Patients received about 1200-1500 shocks at 60 shocks per minute $(1 \mathrm{~Hz})$ with Voltage Ramping. The Shock wave energy used was between 1.5 to 2.0 and a cumulative shock wave energy not exceeding $25 \mathrm{~J}$ per sitting. Post procedure all the patients were on prophylactic antibiotic, an alfa blocker, a diuretic and an analgesic if required. All the patients were followed up after a week with a USG scan and a second sitting is done if the calculus persisted.

Results: ESWL successfully cleared the VUJ stones in 85 of 91 patients. Stone was cleared in one sitting 55 patient, 29 patients required two sittings with a gap of 1 week and only one patient required 3 sittings. All these patients had mild symptoms post ESWL which responded well to oral medications. Stones didn't fragment in 3 patients after 3 sittings. These patients had an average stone size of $7.9 \mathrm{~mm}$ with a HU above 1200 . Subsequently these three patients were treated with Ureteroscopy. 2 patients underwent Ureteroscopy due to intractable colic not responding to conservative treatment within 3 days of ESWL.

Conclusions: USG guided ESWL is an excellent modality of treatment for VUJ stones less than $15 \mathrm{~mm}$ with a good success rates. ESWL should be offered as a treatment option to all the patents.

\section{MP27-11 Outcome of anterograde ureterography for ureteral stone detection in extracoporeal shock wave lithotripsy.}

J You, Y Shin, Y Kim

Department of Urology, Chonbuk National University Medical School and Research Institute of Clinical Medicine of Chonbuk National University-Biomedical Research Institute of Chonbuk National University Hospital, Jeonju, Korea.

Introduction \& Objective: When performing extracorporeal shock wave lithotripsy (ESWL), localization of the radiolucent ureteral stone is not easy. We report our experience that anterograde ureterography using a PCN for radiolucent ureteral stone localization in ESWL.

Methods: Between January 2012 and December 2018, a total of 129 patients underwent ESWL with anterograde ureterography for radiolucent ureteral stone management. A database was kept prospectively for all patients. Ultravist ${ }^{\circledR} 370 \mathrm{mgI} / \mathrm{mL}$ contrast medium was diluted $1: 1$ with saline. About 5 to $8 \mathrm{cc}$ of this solution was slowly injected through the PCN tract, paying attention to the patient's pain, contrast leakage, and contrast reflux. The filling defect area was aligned to the F2 zone and then lithotripsy was done in the same manner as a conventional ESWL. Three days to two weeks later, anterograde ureterography was performed through the PCN tract and additional ESWL was performed if a filling defect was present. Stone free was defined as the absence of filling defects on antegrade ureterography or absence of residual stones on CT scan.

Results: The mean age of the patients was $63.89 \pm 12.09$ years, 88 men and 41 women. Of the 129 patients, 80 underwent PCN for acute renal failure, 49 others had PCN for urinary tract infection. The mean stone size was $10.22 \pm 6.11 \mathrm{~mm}$. The locations of ureteral stones were 62,39 , and 28 in the upper, middle, and lower, respectively. The mean number of ESWL received until the stone was discharged was $1.13 \pm 0.40$ times. The success rate of ESWL was $97 \%$. There was no recurrence of urinary tract infection or acute renal failure.

Conclusions: When performing ESWL for radiolucent ureteral stone, anterograde ureterography using a PCN tract is useful and safe.

\section{MP27-12 Using Algorithm-Based Tools to Improve Shockwave Lithotripsy Outcomes}

BD Hamilton, R Seltzer, D Klett, SY Nakada, G Gerber

University of Utah 
Introduction \& Objective: Shockwave lithotripsy (SWL) treatment protocols vary widely based on a variety of factors. We incorporated algorithms that predict SWL outcomes based on pre- and intra-operative variables to drive real-time software applications, interactive reports, and work groups to guide urologists and technologists to make changes in patient selection and treatment delivery to improve SWL treatment outcomes. We tested a pre-post implementation model using these tools to standardize the treatment delivery evaluated the impact on treatment outcomes.

Methods: We analyzed 51,868 records from the International Stone Registry (ISR) from January 2015 to August 2018. Algorithm-based tools (ABT) were implemented in our system in June 2017. Treatments performed prior to the use of ABT comprised the pre-use group (Group $1, n=26,953$ ) and treatments performed after comprised the post-use group (Group 2, $\mathrm{n}=24,915)$. We used a Structural Equation Model to test the effect of ABT on 4 parameters (alignment to top performers, standardization of treatment delivery, accuracy of data entry, and adherence to maximum shock counts) and their effect on treatment success (stone free or fragments $\leq 4 \mathrm{~mm}$ ).

Results: Success rate (stone free $\& \leq 4 \mathrm{~mm}$ ) increased from $84.4 \%$ to $89.5 \%$ between pre and post use groups. There was a direct effect of the use of algorithm-based tools on all 4 parameters: alignment of treatment protocols to top performers (regression weight $b=.005, \mathrm{p} \leq .0001)$, standardization of treatment delivery $(b=-23.66, p<.0001)$, data input accuracy $(b=.19$, $\mathrm{p}<.0001)$, and adherence to maximum shock counts $(\mathrm{OR}=1.18$, $\mathrm{p}=.025)$. All of these represent improvement in these parameters. There was a direct effect of alignment of treatment protocols to top performers on treatment success $(\mathrm{OR}=1.17, \mathrm{p}=.005)$, indicating a $17 \%$ increase in the likelihood of treatment success. The model showed very good fit to the data $(\mathrm{X} 2=378.82$ (4), $\mathrm{p}<.0001 ; \mathrm{GFI}=.99 ; \mathrm{CFI}=.95 ; \mathrm{NFI}=.94 ; \mathrm{RMSEA}(90 \%$ $\mathrm{CI})=.03(.02, .04)$

Conclusions: Predictive analytics combined with interactive applications create Algorithm Based Tools that can help practitioners use SWL more effectively. The cumulative effect of using these tools over a national network of urologists resulted in greater treatment alignment with the top performers, decreased variation in treatment protocols, increased data input accuracy, and increased adherence to maximum shock counts. In turn, these effects led to a 17\% increased likelihood of SWL treatment success, and a real increase in patient stone-free rates.

\section{MP27-13 Analysis of infrared spectrum stone com- position of children in Gannan in Jiangxi Province: 52 cases report and clinical analysis}

X Ruiquan, Y Yuanhu, Z Xiaofeng, Z Guoxi, X Rihai, W Gengqing

Introduction \& Objective: To investigate the characteristics of pediatric calculus in Gannan in Jiangxi province, and explore the clinical characteristics of pediatric lithiasis.

Methods: A total of 52 pediatric urolithiasis specimens were collected by surgical removal, extracorporeal shock wave lithotripsy (ESWL) and drug treatment. The analysis of stone composition was performed by infrared spectroscopy. Collect clinical data of children, analyze the characteristics of stone composition in children of different ages, stones and history of disease, and explore the clinical characteristics of pediatric urinary calculi.
Results: This research included 52 children. There were 45 cases of upper urinary calculi and 7 cases of lower urinary calculi. The study included 52 children, 31 males and 21 females, with a male to female ratio of 1.48:1. There were 45 cases of upper urinary calculi and 7 cases of lower urinary calculi. 4 children were born to 1 years old. 5 children aged from 1 to 3 years old. 16 children aged from 3 to 6 years old; 27 children aged from 6 to 14 years old in school age; There was a significant difference in the sex ratio, composition of upper and lower urinary tract stones, proportion of upper and lower urinary tract stones between school age and preschool children $(\mathrm{P}<0.05)$. The results of infrared spectroscopic analysis showed that calcium oxalate stone and uric acid ammonium stone in school age and preschool group were statistically significant. $(\mathrm{P}<0.05)$.

Conclusions: Calcium oxalate and uric acid stones are the main components of children's stones. The incidence of ammonium urate stones was significantly higher than that in adults. Male children, school-age children and preschool children are children with high urinary tract calculi. Attention should be paid to prevention and treatment of urolithiasis in those children.

\section{MP27-14 Withdrawn}

MP27-15 Safety and efficacy of flexible ureteroscopy and laser stone fragmentation for pediatric renal stones: A systematic review of the literature

A Barr, S Chugh, A Pietropaolo, P Jones, OM Aboumarzouk, BK Somani

Portsmouth Hospitals NHS Trust

Introduction \& Objective: Flexible ureteroscopy (FURS) is becoming increasing popular for the treatment of pediatric renal stones. We conducted a systematic review to look at the outcomes of flexible ureteroscopy and laser fragmentation (FURSL) in the pediatric population.

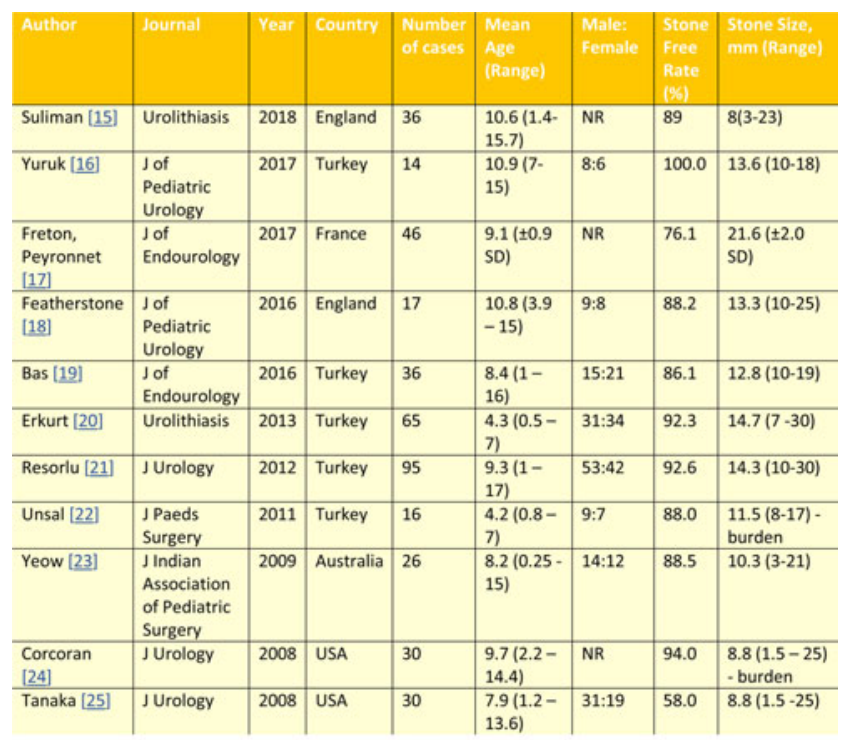

Table 1: Patient demographics of the included studies 


\begin{tabular}{|c|c|c|}
\hline Author & $\begin{array}{l}\text { Percentage complication } \\
\text { rates, } \%(n)\end{array}$ & Complication (n) \\
\hline Suliman [15] & $3.6(2)$ & $\begin{array}{l}\text { Post-operative UTI (1), Stent } \\
\text { symptoms (1) }\end{array}$ \\
\hline Yuruk [16] & $14.3(2)$ & $\begin{array}{l}\text { Post-operative fever (1), } \\
\text { Ureteral laceration (1) }\end{array}$ \\
\hline Freton, Peyronnet [17] & $21.7(10)$ & $\begin{array}{l}\text { Stent discomfort (3), } \\
\text { Haematuria (1), Post-operative } \\
\text { UTI (3), Urinoma (1), Urinary } \\
\text { retention secondary to stone } \\
\text { fragmentation (1), Urinoma (1) }\end{array}$ \\
\hline Featherstone [18] & $0(0)$ & \\
\hline Bas [19] & $16.7(6)$ & $\begin{array}{l}\text { Fever (2), Colic (1), Post- } \\
\text { operative UTI (2), Stent } \\
\text { insertion secondary to colic (1) }\end{array}$ \\
\hline Erkurt [20] & $27.7(18)$ & $\begin{array}{l}\text { Post-operative haematuria (6), } \\
\text { Post-operative UTI and fever } \\
\text { (10), Ureteral wall injury (2) }\end{array}$ \\
\hline Resorlu [21] & $8.4(8)$ & Unspecified (8) \\
\hline Unsal [22] & $31.3(5)$ & $\begin{array}{l}\text { Ureteral perforation (1), } \\
\text { Abdominal pain (2), Voiding } \\
\text { disturbances (2) }\end{array}$ \\
\hline Yeow [23] & $0(0)$ & \\
\hline Corcoran [24] & $0.3(3)$ & $\begin{array}{l}\text { Ureteral perforation (2), } \\
\text { Urinoma requiring } \\
\text { percutaneous drainage (1) }\end{array}$ \\
\hline Tanaka [25] & $2(1)$ & $\begin{array}{l}\text { Readmission due to nausea } \\
\text { and vomiting (1) }\end{array}$ \\
\hline
\end{tabular}

Methods: A systematic review carried out in a Cochrane style and in accordance PRISMA checklist using MEDLINE, Scopus, CINAHL, EMBASE and Cochrane library for all English language articles on FURSL in patients $\leq 18$ years from 19902018.

Results: A total of 11 studies were identified (431 patients) with a mean age of 8.5 years (range $0.25-17$ years). The mean stone size was $13 \mathrm{~mm}$ (range: $1.5 \mathrm{~mm}-30 \mathrm{~mm}$ ). The overall stone free rate (SFR) was $87 \%(58 \%-100 \%)$ with $76 \%$ needing a postoperative stent insertion, and a mean complication rate of $12.6 \%$ $(\mathrm{n}=55)$ (range: $0-31.3 \%)$. Of the complications, Clavien I/II included fever and UTI $(\mathrm{n}=19)$, stent discomfort/stent symptoms/post-operative pain $(n=8)$, hematuria $(n=7)$, voiding disturbance $(\mathrm{n}=2)$ and post-operative nausea and vomiting $(\mathrm{n}=1)$. Clavien III complications included urinoma $(n=1)$, ureteral injury which included perforation $(n=6)$ and acute urinary retention secondary to stone fragmentation $(n=1)$. Clavien IV complications were urinoma $(\mathrm{n}=2)$ and there were no Clavien $\mathrm{V}$ complications.

Conclusions: Our review suggests FURSL to be a safe and effective treatment with good SFR and a low risk of major complications.

\section{MP27-16 The prediction of treatment success and complications of shockwave lithotripsy using artificial intelligence}

R Seltzer, BD Hamilton, D Klett, Z Chen, SY Nakada, G Gerber

Introduction \& Objective: There continues to be a need for improvement in the delivery of shockwave lithotripsy (SWL) for treating urinary stones. Building on our previous model predicting outcomes for shockwave lithotripsy (SWL) from pre- and intra-operative characteristics, we have added a time series component for how SWL treatments are delivered. Furthermore, we have created this new model (Version 3.0) with a deeplearning time series analysis, which is a statistical way of accounting for multiple variables with different weights. This predictive algorithm may help urologists make personalized and evidence-based decisions on how effective SWL will be for a particular stone in a particular patient using a particular shock protocol.

Methods: An Artificial Intelligence platform (TensorFlow) with a Python deep learning library (Keras) was used to train and validate a time series neural network that predicted treatment success (stone free or $\leq 4 \mathrm{~mm}$ ) and complications based on patient and stone characteristics and SWL shock protocol, similar to our previous models. A 75/25 randomized split of 46,891 treatments sampled from the International Stone Registry (ISR) was used to create training and validation samples. The predictor variables in this model were stone size, location, and side; number of stones present; patient age, sex, preoperative blood pressure, anticoagulation; use of compression strapping; and sequence of power level, shock rate, and shock count delivered.

Results: The model predicting treatment success at each step during an SWL procedure yielded an accuracy of .88 and an Area Under the Curve (AUC) of .95 on the validation set. The model predicting complications yielded an accuracy of .77 and an AUC of .73 on the validation set.

Conclusions: This new version of predictive analysis for SWL treatment, incorporating patient and stone characteristics and now including the time sequence of shockwaves delivered using artificial intelligence results in an even more accurate predictor of treatment success and complications of SWL treatment. This algorithm can drive interactive software to help urologists make targeted decisions regarding the optimal treatment protocol for a particular patient and stone profile.

MP27-17 Pediatric urolithiasis: overview of treatment and outcomes from a single series of 50 patients

R Milandri, L Bevilacqua, C Di Pietro, P Ceccarelli, E Morini, A Elsherbiny, A Eissa, S Puliatti, M Sighinolfi, G Bianchi, S Micali, B Rocco

Department of Surgical, Medical and Dental Morphological Sciences related to Transplant, Oncology and Regenerative Medicine - Section of Urology, University of Modena and Reggio Emilia, Modena, Italy

Introduction \& Objective: Pediatric urolithiasis can cause significant morbidity and damage to the kidney, or even renal failure. Incidence is increasing both in western population and in developing countries as well. On contrary to adults, the symptomatology in children could be indefinite and vague. There is an abundance of literature on adult urolithiasis, but series from the pediatric population are still poor. We herein review our experience for the treatment of urolithiasis in pediatric patients.

Methods: We retrospectively collected the medical records of all children treated for urolithiasis at our center starting from January 2005 to June 2018. We reviewed and statistically analyzed demographic and preoperative patients' characteristics (i.e. sex, age, comorbidities, preoperative renal function), stone (stone size, location, and Hounsfield units), intraoperative (operative time, complications, and hemoglobin drop) and follow-up data (stone-free rate [SFR], defined as the absence of any stone fragment more than or equal to $2 \mathrm{~mm}$ ). Any patient 
with missing data was excluded from the analysis. The treatment modalities performed were ESWL (extracorporeal shock wave lithotripsy), ureteroscopy and percutaneous nephrolithotomy (PCNL).

Results: From January 2005 to June 2018 we treated 73 children. Age range was 1-17 (mean 10,2) and the male to female ratio was $3: 1$. Thirty-one $(42 \%)$ children were treated with PCNL, twenty-one (29\%) with ureteroscopy and 21 (29\%) underwent ESWL with DoLiS. Mean stone size treated with ureteroscopy was $11 \mathrm{~mm}(5-20 \mathrm{~mm}), 23 \mathrm{~mm}(15-35 \mathrm{~mm})$ with PCNL, and 9 (5-12) with ESWL. Mean operation time was 192 minutes for PCNL and 89.5 for ureteroscopy. Ureteroscopies were performed with Stortz XFlexC Ureteroscope (8 FR); the initial pediatric series of PCNL was carried out with $26 \mathrm{Fr}$ Nephroscope, and then changed to Minimally Invasive PCNL instruments (MIP) 9,5 and 15 Fr. Global stone free rate after the primary procedure is $88 \%$; the remaining cases underwent a second-look endourological approach. Clavien Dindo II complications (fever in all cases, responsive to antibiotic treatment) were observed in 10/73 patients.

Conclusions: Based on our experience in the treatment of pediatric urolithiasis, the introduction and availability of smaller instruments have led to safer use of percutaneous endoscopy and ureteroscopy in children, with results comparable to those in adults and an acceptable complication rate. The availability of an extracorporeal lithotripter may complete and improve the outcomes of pediatric stone treatment. As a consequence, the referral to a tertiary centre offering adequate instruments is of paramount importance to safely manage these delicate cases.

\section{MP27-18 Prospective analysis of the predictors of success after ESWL}

F Quadrini, A Boni, P Guiggi, M Del Zingaro, E Mearini

University of Perugia

Introduction \& Objective: Although the undoubted and remarkable development of endoscopic instruments and techniques, ESWL still has a role in the treatment of urolithiasis. The aim of this study is to identify predictors of success after ESWL procedure for ureteral and renal stones.

Methods: From January 2017 to June 2018 we prospectively enrolled 47 patients (pts) for a total of 51 calculi. In all cases stones were localized ultrasonographically and ESWL was performed with Piezolith $3000^{\circledR}$. We included either renal and ureteral stone diagnosticated by Non Contrast Computed Tomography (NCCT), we excluded pts with complicated urinary tract infection and pts with only ultrasound diagnosis. A maximum of 5000 shockwaves (mean $4000 \mathrm{SW}$ ) to a maximal power of $20 \mathrm{~J}$ and maximal frequency of $3 \mathrm{~Hz}$ were delivered. The limit of ESWL sessions was established to 3 and all treatments were performed or supervised by same urologist. In any case medical expulsive therapy was prescribed. We considered as NCCT factors predicting ESWL outcome: localization, maximum diameter in any dimension, volume, radiodensity (Hounsfield Unit, HU) and presence of ipsilateral hydronephrosis $\geq$ grade 2 $(H Y \geq 2)$. Success of the procedure, that is the stone-free status, was defined as no ultrasound evidence of residual fragment on the 2-month follow up. The Chi-squared test was used to compare categorical variables, the Mann-Whitney test was used for continuous ones. $\mathrm{p}<0.05$ was considered statistically significant. Data from the study population were divided into

\begin{tabular}{|c|c|c|c|c|}
\hline & SUCCESS GROUP & FAILURE GROUP & TOTAL & p value \\
\hline Cases, $n(\%)$ & $26(51 \%)$ & $25(49 \%)$ & 51 & \\
\hline Mean age $\pm S D(y r)$ & $54.61 \pm 13,36$ & $54 \pm 13.7$ & $54.31 \pm 13.4$ & 0.843 \\
\hline $\operatorname{sex} n(\%)$ & & & 47 & 0.541 \\
\hline M & $16(34.04)$ & $17(36.17)$ & $33(70.21)$ & \\
\hline F & $9(19.15)$ & $5(10.64)$ & $14(29.79)$ & \\
\hline Stone location, $n(\%)$ & & & 51 & 0.307 \\
\hline Upper calyx & 0 & $1(1.96)$ & $1(1.96)$ & \\
\hline Middle calyx & 0 & $3(5.88)$ & $3(5.88)$ & \\
\hline Lower calyx & $1(1.96)$ & $2(3.92)$ & $3(5.88)$ & \\
\hline Renal pelvis & $1(1.96)$ & $1(1.96)$ & $2(3.92)$ & \\
\hline Upper ureter & $7(13.72)$ & $7(13.72)$ & $14(27.45)$ & \\
\hline Middle ureter & $7(13.72)$ & $2(3.92)$ & $9(17.65)$ & \\
\hline Lower ureter & $10(19.61)$ & $9(17.65)$ & $19(37.25)$ & \\
\hline Diameter, mmะSD & $9.31 \pm 3.90$ & $9.72 \pm 3.93$ & $9.51 \pm 3.89$ & 0.622 \\
\hline Volume, $\mathrm{mm}^{2}+\mathrm{SD}$ & $364.03 \leftleftarrows 427.50$ & $393.76 \pm 446.19$ & $378.61 \pm 432.62$ & 0.932 \\
\hline Mean stone radiodensity, HU士SD & $1160.77 \pm 496.23$ & $1093.72 \pm 421.69$ & $1127.90 \pm 457.85$ & 0.510 \\
\hline Hydronephrosis (2 grade 2), n (\%) & & & 51 & $\underline{0.011}$ \\
\hline No & $1(1.96)$ & $8(15.69)$ & $9(17.65)$ & \\
\hline Yes & $25(49.02)$ & $17(33.33)$ & $42(82.35)$ & \\
\hline
\end{tabular}

two groups: treatment success (n 26, 51\%) and treatment failure (n25, 49\%).

Results: Table shows our results. $30(63.83 \%)$ of the 47 pts had a positive history of urolithiasis. Mean age was 54.31 yr. In 14 cases more than 1 treatment was performed and in 2 of these 3 sessions were necessary. Mean volume was $378.61 \mathrm{~mm}^{3}$ (12 mm3-2016 $\left.\mathrm{mm}^{3}\right)$. Mean HU was 1127.90 . In $42(82.35 \%)$ cases ultrasound detected $H Y \geq 2$. Expulsion of fragments was referred in 26 cases of 42 with associated $H Y \geq 2$. The presence of $\mathrm{HY} \geq 2$ was the only statistically significant factors that influenced the success of ESWL, being present in 25 of 26 stone-free (p 0.011). No statistically significant differences were found between two groups with respect to age, sex, localization, maximum diameter, volume and radiodensity.

Conclusions: The results of the present prospective study suggest that pretreatment $\mathrm{HY} \geq 2$ is associated with success of ESWL and significantly increases the stone free rate and reduced the time to stone clearance.

\section{MP27-19 Spectrum of pediatric urinary stone com- position in North-western India}

\section{R Saran, K Mirdha, S Saran}

Introduction \& Objective: Pediatric urolithiasis results in significant morbidity in later life. Incidence as well as site and chemical composition of calculi varies according to the changes in socio-economic conditions over time and the subsequent changes in dietary habits leading to a marked variation in the spectrum of urinary stone composition. This study evaluated the spectrum of urinary stone composition in pediatric population from North-western India.

Methods: A prospective observational study was conducted between October 2013 and February 2019 which included pediatric patients with urolithiasis. Demographic and epidemiological characteristics including age, sex, geography, religion, socio-economic status, dietary habits were recorded. The location and sizes of stones were documented with ultrasonography of kidney-ureter-bladder (USG-KUB) along with an X-ray KUB/ intravenous pyelogram (IVP). Fourier-transform infrared spectroscopy (FTIR) was performed using a Perkin-Elmer Fourier Transformer Infrared Spectrophotometer, with a wave number range from 7800 to $350 \mathrm{~cm}^{-1}$.

Results: A total of 163 patients with urolithiasis were enrolled, of which $86(53 \%)$ aged between 6 and 10 years, $49(30 \%)$ aged 
Table 1: Distribution of patients according to stone composition

\begin{tabular}{|l|c|c|}
\hline \multicolumn{1}{|c|}{ Stone composition } & Pure/ Mixed & N (\%) \\
\hline Calcium oxalate + calcium phosphate + uric acid & Mixed & $36(22)$ \\
\hline Calcium oxalate monohydrate + calcium oxalate dihydrate & Mixed & $29(18)$ \\
\hline Calcium oxalate + uric acid & Mixed & $25(15)$ \\
\hline Calcium oxalate + calcium phosphate & Mixed & $20(12)$ \\
\hline Calcium oxalate monohydrate & Pure & $16(10)$ \\
\hline Uric acid & Pure & $11(7)$ \\
\hline Struvite + calcium phosphate & Mixed & $10(6)$ \\
\hline Calcium oxalate dihydrate & Pure & $8(5)$ \\
\hline Cystine & Pure & $5(3)$ \\
\hline Struvite & Pure & $3(2)$ \\
\hline Total & & 163 \\
\hline
\end{tabular}

between 11 and 14 years and $28(17 \%)$ were aged between 0 and 5 years. The majority of patients were male $(n=134 ; 82 \%)$. The most common location of the stone was urinary bladder $(\mathrm{n}=106$; $65 \%)$ followed by kidney $(n=33 ; 20 \%)$, urethra $(n=16 ; 10 \%)$ and ureter $(n=8 ; 5 \%)$. The upper tract (kidney and ureter) to the lower tract (bladder and urethra) stone ratio was 1:4. Stones with mixed composition were more than pure stones $(73 \%$ versus 27\%). The most common composition was the mixed stone of calcium oxalate, calcium phosphate and uric acid $(n=36 ; 22 \%)$ followed by mixed stone of calcium oxalate monohydrate and dihydrate with uric acid $(n=29 ; 18 \%)$, calcium oxalate and uric acid $(\mathrm{n}=25,15 \%)$, calcium oxalate and calcium phosphate $(\mathrm{n}=20 ; 12 \%)$. Calcium oxalate was present in $80 \%$ of the stones, followed by uric acid in $7 \%$, struvite in $6 \%$, cystine in $3 \%$ and calcium phosphate in $2 \%$.

Conclusions: These observations indicate the prevalence of mixed stones with calcium oxalate as the predominant chemical component in the urinary stones of pediatric patients from Northwestern India.
MP27-20 Totally Ultrasound Guided ESWL Monotherapy for Impacted Uretero-Vesical junction (UVJ) calculi.

PK Pattnaik, SK Pattnaik, MP Pattnaik

S S Urological \& Research Institute, Bombay Hospital PG Institute

Introduction \& Objective: To evaluate the efficacy of Totally Ultrasound Guided ESWL Monotherapy for Impacted UreteroVesical junction (UVJ) calculi.

Methods: Between February 2016 and March 2018, 258 patients with impacted Uretero-Vesical junction (UVJ) calculi were treated by Ultrasound guided ESWL using Siemens Modularis Variostar (Germany). All patients with UVJ calculi were enrolled into the study after taking written, informed consent. There were 184 males and 74 females. The patients were in the age group of $15-48$ years. All patients were administered treatment under General anesthesia in supine position. Calculus localization was done by real time ultrasound with a rotatable therapy head. The outcome of treatment was evaluated after one week. 32\% (77 patients) required 2 sittings. The patients clinical and USG findings were reviewed and correlated with the stone-free rate (SFR). The patients with retained calculi after 2 sittings of ESWL were subjected to Ureteroscopy +/Laser fragmentation and retrieval of calculus fragments.

Results: After USG guided ESWL, 164 patients (68\%) were stonefree after one session and 77 patients $(32 \%)$ required two sittings. 16 patients $(6.2 \%)$ had retained fragments which required ureteroscopic retrieval. The stone free rate had no correlation with Body mass index (BMI), surface area of calculus. The only significant variable was calculus density (measured in Hounsfield units).

Conclusions: Totally Ultrasound Guided ESWL Monotherapy is a safe and effective form of treatment for impacted vesicoureteric junction calculi. The stone free rate is not influenced by BMI or calculus surface area. It has an added advantage of no ionizing radiation exposure to the patient (reproductive organs) and treating physicians.

\section{MODERATED POSTER SESSION 28: CLINICAL STONES: PCNL (III)}

\section{MP28-01 Comparison of S.T.O.N.E nephrolithometry and Guy's scoring systems for predicting stone clearance and complications after percutaneous ne- phrolithotmy}

SM Nazim, N Khan, M Farhan, B Salam, H Ather

The Aga Khan University Hospital, Karachi Pakistan

Introduction \& Objective: To validate and compare Guy's and S.T.O.N.E. scoring systems in predicting postoperative stone free status and complications following percutaneous nephrolithotomy (PCNL) for renal stones.

Methods: A total of 190 patients with renal stones who underwent unilateral single tract PCNL in prone position between November 2015 and June 2017 were included in the current prospective study. Guy's and S.T.O.N.E. nephrolithometry scores were calculated in each case and their association with stone-free status, operative time and length of hospital stay were evaluated. Postoperative complications were graded according to the modified Clavien classification, and the correlation of scoring systems with postoperative complications was also studied.

Results: Mean S.T.O.N.E. and Guy's stone scores were $8.76+2.29$ and $2.70+1.0$ respectively. When compared with patients with residual stones, stone free patients had significantly lower mean Guy's grade [ $2.58+1.01$ vs. $3.23+0.77(\mathrm{p}<0.001)]$ and S.T.O.N.E. scores $[8.44+2.24$ and $10.17+2.0(\mathrm{p}<0.001)]$ respectively. Both scoring systems were significantly correlated with stone-free status in univariate analysis; significant other factors included age $(\mathrm{p}=0.005)$ and stone size $(\mathrm{p}<0.001)$ and right side $(\mathrm{p}=0.04)$. On logistic regression analysis, both Guy's score $(\mathrm{OR}=0.48, \quad[95 \% \quad \mathrm{CI}=0.31-0.74] ; \mathrm{p}=0.001)$ and S.T.O.N.E score $(\mathrm{OR}=0.78,[95 \% \mathrm{CI}=0.59-0.84] ; \mathrm{p}=0.001)$ were found to be significantly associated with stone free status. 
No significant association was found between either S.T.O.N.E score $(p=0.22)$ and Guy's grade $(p=0.12)$ with respect to complications.

Conclusions: Both the scoring systems could effectively predict postoperative stone-free status for renal stones. However, they failed to predict complication rates.

MP28-02 Comparison of Quality of Life between standard tubeless PCNL and Mini Tubeless PCNL by using WISCONSIN Stone QOL Questionnaire.

\author{
N Iqbal, A Hasan, A Mughal, M Nazir, S Akhter, J Rahim \\ Pakistan Kidney and Liver Institute and Research Center
}

Introduction \& Objective: We wanted to compare the effect on quality of life of patients between standard size tubeless percutaneous nephrolithotomy (PCNL) and Mini tubeless PCNL at one week and 1 month after discharge using Wisconsin Stone Quality of Life questionnaire (WISQOL).

Methods: From April 2018 to August 2018, total of 80 patients were enrolled in this study having stone size between $15 \mathrm{~mm}$ to $30 \mathrm{~mm}$ and sterile urinary culture tests, having no cardio, pulmonary and coagulation abnormalities. They underwent PCNL and were randomized into 2 groups: 1 . Standard size tubeless PCNL group, $\mathrm{N}=40$ (tract size 24-26 Fr), 2. Tubeless Mini PCNL, $\mathrm{N}=40$ (tract size 16.5-17.5 Fr). We assessed for the quality of life differences between these groups' week before the procedure and at 1 week and 1month after surgery using WISQOL.

Results: No significant differences were present between the mean age (yrs.) $(38.35 \pm 11.57$ vs $39.14 \pm 12.62, \mathrm{P}=0.51)$, stone size $(\mathrm{mm})(26.50 \pm 9.28$ vs $24.36 \pm 8.45, \mathrm{P}=0.59)$, stone-free rate (37/40 vs $36 / 40, \mathrm{P}=0.61$ ), mean drop in hemoglobin $(\mathrm{g} / \mathrm{L})$ $(11.14 \pm 2.84$ vs $12.43 \pm 1.81, \mathrm{P}=0.67)$ and mean dosage of analgesics (opioids intravenous Nalbuphine) (3.6 $\pm 1.125 \mathrm{vs} 3.3 \pm$ $1.34, \mathrm{P}=0.19$ ). Operative time was longer in Mini PCNL group (115 min vs $133 \mathrm{~min} p=0.03)$. Assessment of changes in Quality of life using the WISQOL questionnaire showed significantly better result in tubeless mini PCNL at discharge group in energy, sleep, work and social, physical symptoms, and the general emotional well-being.

Conclusions: Tubeless Mini PCNL is comparable to standard size tubeless PCNL in terms of success rates and complications. Quality of life is better in Mini tubeless PCNL so it should be promoted in centers not doing it at moment with mentorship programs.

\section{MP28-03 Efficacy and safety of Ureteroscopic Lithot- omy and Percutaneous Nephrolithotomy for Upper Ureteral Calculi}

Q Xu, Y Hong, X Huang

Urology and Lithotripsy Center, Peking University People's Hospital

Introduction \& Objective: Both ureteroscopic lithotomy (URL) and percutaneous nephrolithotomy (PCNL) are the most commonly used minimal invasive procedure in treating upper ureteral calculi. Our objective is to investigate the efficacy and safety of URL and PCNL in the treatment of upper ureteral calculi.

Methods: Clinical data of 113 patients with unilateral upper ureteral calculus were analyzed retrospectively. PCNL was the preferential procedure for patients with distal ureteral stricture or circuity, urinary diversion, stone size $>1.5 \mathrm{~cm}$, and ureteropelvic junction obstruction. There were 60 cases in URL group and 53 cases in PCNL group. The stone burden, operative time, postoperative fever, perioperative hemoglobin decrease, hospital stay and stone free rate of the two groups were recorded.

Results: The stone size was $(1.0 \pm 0.3) \mathrm{cm}$ in the URL group and $(1.4 \pm 0.4) \mathrm{cm}$ in the PCNL group. The procedure was aborted in 1 case in the URL group due to stone moving up to the renal pelvis. The other 112 procedures were successful with stones completely removed. The operative time was $(54.0 \pm 24.4) \mathrm{min}$ in the URL group and $(59.4 \pm 37.0) \mathrm{min}$ in the PCNL group. The perioperative hemoglobin decreased by $(4.3 \pm 5.7) \mathrm{g} / \mathrm{L}$ in the PCNL group and by $(9.5 \pm 8.0) \mathrm{g} / \mathrm{L}$ in the URL group. Postoperative fever $\left(\mathrm{T} \geq 38^{\circ} \mathrm{C}\right)$ occurred in 4 patients in the URL group and in 6 patients in the PCNL group. The stone free rate was $98.3 \%(59 / 60)$ in the URL group and $100 \%(53 / 53)$ in the PCNL group. The hospital stay was $(6.4 \pm 2.1) \mathrm{d}$ in the URL group and $(8.5 \pm 3.4) \mathrm{d}$ in the PCNL group.

Conclusions: Both URL and PCNL are effective and safe in the treatment of upper ureteral calculi. PCNL is applicable to patients with large stones, bearing high stone free rate but longer hospital stays.

\section{MP28-04 Effect of percutaneous nephrolithotomy over the renal replacement therapy-free time on pa- tients with chronic kidney disease KDIGO IV and V}

KA Mendoza, A Ávila, E Cruz, M Basulto, A Esqueda, A Romero, J Flores, H Rendon, S Canul, T Diaz

Hospital Regional de Alta Especialidad de la Península de Yucatán

Introduction \& Objective: The end-stage chronic kidney disease (CKD) can be caused by kidney stones (KS), timely detected, can be well managed through medical and surgical procedures. Percutaneous nephrolithotomy (PNL), is a surgical approach to remove renal stones reaching better stone-free status and low retreatment rates, this initial strategy to decreased KSrelated complications, and postoperative effects on renal function lacking. Objective: Determine the effect of PNL over the renal replacement therapy-free time of patients with CKD KDIGO IV and V due to KS.

Methods: Data from patients who came to the KS Clinic and Transplant Unit at Hospital Regional de Alta Especialidad de la Península de Yucatán between 2015-2018 were prospectively collected. Descriptive statics was used for the clinical characteristics and medical procedures.

Results: PNL was performed in 55 patients, of which, 40 patients (Group A) did not require Renal Replacement Therapy (RRT), and 15 patients that after PNL required RRT (Group B). Group A HBP and DM2 was found in $27.5 \%$ and $7.5 \%$, respectively, mean BMI 27, with a pre-PNL mean $\mathrm{CrS}$ of 5.4, and current $\mathrm{CrS}$ of $4.74 \mathrm{ng} / \mathrm{dl}$, with a mean decrease of $\mathrm{CrS}$ of $0.66 \mathrm{ng} / \mathrm{dl}$. CT changes $17 \%$ without no parenchymal abnormalities, $15 \%$ with thinning/atrophy (PTA), 45\% with hydronephrosis, 5\% nephrocalcinosis, $7 \%$ with chronic inflammatory changes (CIC). Regarding the mean post-op CrS decrease, it was $2.9 \mathrm{ng} / \mathrm{dL}$ in $62.5 \%$ of patients and an increase of $2 \mathrm{ng} / \mathrm{dL}$ in $30 \%$ of them, without showing changes in $\mathrm{CrS}$ in $7.5 \%$ of patients. Group B, patient's mean age was 44.5 years, with a mean BMI of $29.5 \mathrm{~kg} / \mathrm{m}^{2}$, predominantly female $(80 \%)$. HBP was observed in $26 \%$ and there were no patients with DM2. The CT changes in 


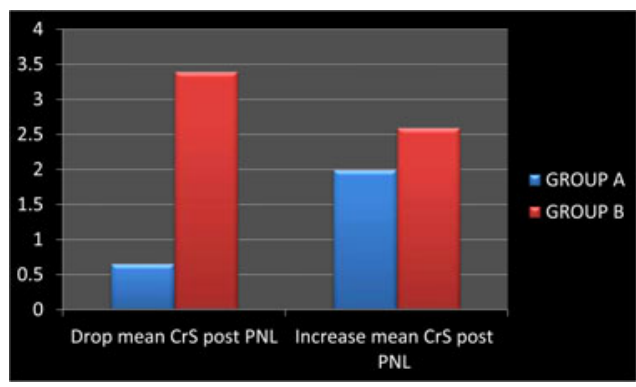

$86 \%, 53 \%$ with PTA, $26 \%$ with hydronephrosis and $6 \%$ with CIC. Changes in the $\mathrm{CrS}$ pre and post PNL in $26 \%$ with a drop mean of $\mathrm{CrS}$ of $3.4 \mathrm{ng} / \mathrm{dL}, 6 \%$ with no variation in $\mathrm{CrS}$ levels and an increase in $\mathrm{CrS}$ in $66 \%$ with a mean increase $\mathrm{CrS}$ of $2.6 \mathrm{ng} / \mathrm{dl}$. With a CKD-EPI qe found an improvement in GFR in $26 \%$ with a mean of $6.75 \mathrm{ml} / \mathrm{min}, 6 \%$ without change and impairment of GFR, $66 \%$ with an mean of $7.9 \mathrm{ml} / \mathrm{min}$. 9 patients required HD and $6 \mathrm{PD}$, with a delay time of RRT of at least 14 days minimum and maximum 1800 days (60 months) with a mean of 760.9 days ( 25 months). With 9 patients in transplant protocol program and 2 of them have already successfully transplanted.

Conclusions: The most relevant predispositions and conditioning factors of the patients that require RRT after PNL had the following characteristics female sex, being overweight, patients with HBP, tomographic changes such as atrophy or loss of renal parenchyma and CKD KDIGO V. These characteristics conditioning the patient in a mean of 60 months of surgery can to require RRT after PNL.

MP28-05 Ambulatory Percutaneous Nephrolithotomy Performed in a Free-standing Surgery Center: Outcomes of the First 550 Cases

MM Dunne, AI Kim, MV Silva, JE Abbott, JG Davalos

University of Maryland Medical Center

Introduction \& Objective: Percutaneous Nephrolithotomy (PNL) is a procedure that has traditionally been performed in an inpatient setting with at least an overnight stay. Many surgical procedures have evolved over time from an inpatient setting to an ambulatory surgery center (ASC) setting. Feasibility of Ambulatory PNL (aPNL) was shown in our initial pilot series of 25 cases. [1] This 550-case series is reviewed to further evaluate outcomes with a more robust data set.

Methods: We present our initial series of 550 patients who underwent PNL in an ASC from April 2015 to May 2019. Each aPNL was performed by one of two surgeons with the same operative team. All procedures were performed with the operative surgeon obtaining renal access and all procedures were performed tubeless (ureteral stent without a nephrostomy tube). All patients also had hemostatic plugs placed into the access tract with a local intercostal block performed to aid with pain control. [2] All cases were reviewed, and demographic data and case details were analyzed.

Results: Our initial 550 consecutive aPNL cases were reviewed, identifying 251 men and 299 women, 255 right side and 295 left, one bilateral, mean age 57.6 years (16-86), mean BMI 31.0 (1649), mean ASA of 2.3 (range 1-4) and mean stone burden $30.5 \mathrm{~mm}(5-140 \mathrm{~mm})$, mean fluoroscopy time $86 \mathrm{sec}(0-299)$. Mean OR time was 102 min (range 32-305). Mean PACU time was 96 min (range 37-247). A mini-PNL procedure was conducted in $114(21 \%)$ patients. Stone free rate was $86 \%$. Twentyone patients had complications ranging from Clavien I-IVa, of which 13 were hospital transfers.

Conclusions: This consecutive 550 case series further demonstrates the safety of aPNL. While twenty-one patients experienced complications, the site of service did not lead to an alteration in the outcomes of the adverse events. Each complication that occurred was managed in an appropriate fashion without notable treatment delay. Our complication rate in the ASC is lower than reported in large cohort studies and lower than our hospital-based patient population, however recognizing these patients are preselected. With an experienced surgeon, well trained operative team and with modifications to the procedure focusing on post-operative pain control, PNL can be safely and effectively performed in an ASC.

1. Davalos JG, Abbott JE. Ambulatory PCNL: Initial Case Series. J Urology. April 2016. Vol. 195 (4), Supplement 1: MP51-20, e688-e689.2

2. Abbott JE, Cicic, A, Jump III RW, Davalos JG. Hemostatic Plug: Novel Technique for Closure of Percutaneous Nephrostomy Tract. J Endourol. March 2015, 29(3): 263269.

\section{MP28-06 Bacteriology and Antibiotic Sensitivity Pattern of Isolates in Patients Who Underwent Per- cutaneous Nephrolithotripsy at the Philippine General Hospital}

J Flor, M Lapitan

University of the Philippines- Philippine General Hospital

Introduction \& Objective: Despite being a clean contaminated procedure, PCNL is associated with significant infectious morbidities such as pyrexia and postoperative bacteremia. We determined the prevalence of post-PCNL infectious complications and distribution, and antibiotic sensitivity of microorganisms isolated from patients admitted for PCNL. Risk factors associated with positive culture results and development of post-PCNL fever and bacteremia were also analyzed.

Methods: A cohort of 102 adult patients who underwent PCNL from January to December 2018 under the PCNL Urinary Tract Infection Surveillance Protocol at a tertiary government hospital was studied retrospectively. The susceptibility patterns of isolated bacteria from urine, stone and blood cultures were evaluated against the most common antibiotics in the hospital. Chi-square and Student's T-test were used to determine differences in the frequencies and means for different risk factors for those who developed fever and urosepsis and those who did not.

Results: Ten isolates were recovered from the preadmission urine culture study (CS), seven from intraoperative urine CS, forty-seven from stone CS, none from febrile urine CS and four from febrile blood CS. The most common organism isolated on the urine specimens was Escherichia coli, which showed high sensitivity to aminoglycosides. This organism is also among the most common isolate found in stone CS but significant number of Pseudomonas aeruginosa and Stenotrophomonas maltophilia were also cultured which showed higher sensitivities to fluoroquinolones. Twenty-five percent (26/102) and $3.9 \%$ (4/102) of PCNL-treated patients developed postoperative fever and urosepsis, respectively, despite receiving antibiotic prophylaxis. 
No significant association was found between the different risk factors studied and the development of postoperative fever and urosepsis.

Conclusions: The current antibiogram formulated showed higher sensitivity to aminoglycoside and fluoroquinolones. Despite nearly consistent resistance to ceftriaxone of the isolates in our cases, it does not warrant a change in the antibiotic prophylaxis utilized in the surveillance protocol, given the lower rates of the post-PCNL infectious complications compared to published literature. Further surveillance is required to justify a shift in prophylactic antibiotics and identify significant risk factors for the development of fever and urosepsis post-PCNL.

\section{MP28-07 Comparison of Cost-Effectiveness and Postoperative Outcomes following Integration of a Stiff Shaft Glidewire into Percutaneous Ne- phrolithotripsy}

C Valadon, CU Nottingham, T Large, A Krambeck

University of Louisville School of Medicine

Introduction \& Objective: Healthcare providers are experiencing increased pressure to attain a higher level of cost efficiency. The purpose of this study is to analyze the cost effectiveness of integrating a stiff shaft glidewire (SSGW) in percutaneous nephrolithotripsy (PCNL) relative to standard technique (ST).

Methods: ST for PCNL at our institution involves a hydrophilic glidewire during initial percutaneous access and then two new stiff shaft wires. The SSGW is a hydrophilic wire used for initial access and the remainder of the procedure. We collected operating room costs for all primary, unilateral PCNL cases over a two-month period during which ST for PCNL was used at a single institution with a single surgeon and compared to a twomonth period during which a SSGW was used. Mean costs for each two-month period were then compared along with stone free rates measured by $\mathrm{CT}$ and complications.

Results: We included 27 total cases in the ST group and 26 in the SSGW group. The average OR supply cost for the ST group was $\$ 1,966.11$ and $\$ 1,523.58$ in the SSGW group. The use of the SSGW resulted in a savings of $\$ 442.53$ per case or a projected $\$ 27,613.87$ per year at our institution. There was no difference in postoperative stone-free rates $(88 \%$ vs $91 \%, \mathrm{p}=1.0$, respectively) or complications ( $8 \%$ vs $14 \%, \mathrm{p}=0.659$, respectively) between ST and SSGW groups.

Conclusions: Transitioning to a SSGW has reduced OR supply cost by reducing the number of supplies required. The change in wire did not affect stone free rates or complications.

\section{MP28-08 Analysis of the clinical efficacy and safety of PCNL in patients with anatomical variations: A single center retrospective study}

DT Rubio, JA Abraham

National Kidney and Transplant Institute

Introduction \& Objective: Percutaneous nephrolithotomy (PCNL) is the standard of care for renal stones $>2 \mathrm{~cm}$. Kidneys with anatomical disparities resulting from fusion (horseshoe), malrotation, ectopic location (renal allografts) and bifid col-

\begin{tabular}{|c|c|c|c|c|c|}
\hline & \multirow[b]{2}{*}{ Total } & \multicolumn{4}{|c|}{ Anatomical variants } \\
\hline & & $\begin{array}{c}\text { Allograft } \\
\text { Kidney }\end{array}$ & Horshoe & $\begin{array}{l}\text { Malrotated } \\
\text { Kidney }\end{array}$ & $\begin{array}{l}\text { Bifid } \\
\text { pelvis }\end{array}$ \\
\hline Access: Upper & $37(88.1 \%)$ & $2(100 \%)$ & $18(100 \%)$ & $5(71.4 \%)$ & $12(80 \%)$ \\
\hline Mid & $4(9.5 \%)$ & $0(0 \%)$ & $0(0 \%)$ & $1(14.3 \%)$ & $3(20 \%)$ \\
\hline Inferior & $3(7.1 \%)$ & $0(0 \%)$ & $0(0 \%)$ & $1(14.3 \%)$ & $2(13.3 \%)$ \\
\hline Tract: Single & $36(85.7 \%)$ & $2(100 \%)$ & $18(100 \%)$ & $7(100 \%)$ & $9(60 \%)$ \\
\hline Multiple & $6(14.3 \%)$ & $0(0 \%)$ & $0(0 \%)$ & $0(0 \%)$ & $6(40 \%)$ \\
\hline
\end{tabular}

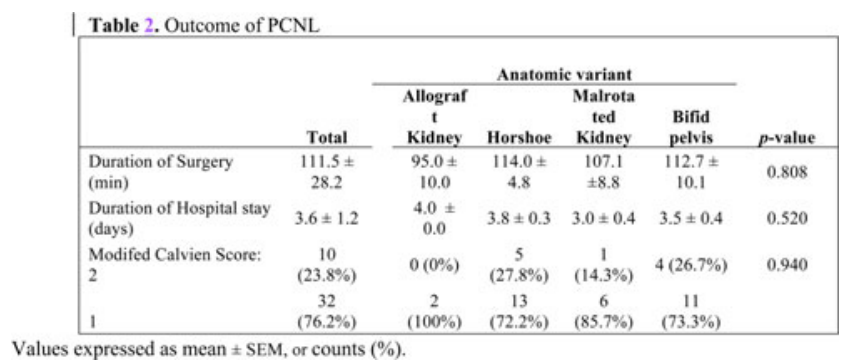

lecting systems present as a unique challenge because variations in vasculature, calyceal rotation and possible intervening viscera make percutaneous access treacherous. This study aims to evaluate the clinical safety and efficacy of PCNL for patients with anatomical variants and we report our experience with PCNL in these types of kidneys.

Methods: A chart review was done on all patients who underwent PCNL in our institution from 2012-2017. Those with anatomical variations were identified and analyzed. Patient demographics (age, gender, co-morbidity) and stone characteristics (Guy's stone score, laterality, stone size) were summarized. Intraoperative parameters such as location of puncture site (upper, mid, inferior calyces), number of tracts (single vs. multiple), operative time, and estimated blood loss (EBL), length of hospital stay (LOS) were analyzed. The primary endpoints were stone-free and complication rates according to the ClavienDindo classification.

Results: A total of 1,657 PCNLs were performed during the study period, of which 42 had anatomical variants. The mean age was $45.2 \pm 8.8(\mathrm{R}=28-65)$ with a male to female ratio of $3: 1$. There were 18 horseshoe (42.9\%), 15 bifid (35.7\%), 7 malrotated (16.7\%) and 2 renal allografts (4.8\%.); Laterality wise, $28(67 \%)$ were left sided, 12 (29\%) were right sided and $2(4 \%)$ had a right sided pelvic kidneys (allografts). The Guy stone scores were 3 and 4 in 13 patients $(30 \%)$ and $29(70 \%)$ patients, respectively. Mean stone diameter is $3.8 \pm 0.6 \mathrm{cms}(\mathrm{R}=2.5-5.5)$. Majority, $\mathrm{n}=37(88 \%)$ were treated with an upper pole access. Thirty-six patients $(86 \%)$ utilized a single tract and while six (14\%) required multiple tracts (all had bifid pelvis). The mean operative time was $111.5 \pm 28.1$ mins. $(\mathrm{R}=65-188)$, EBL was $461 \pm 278.4 \mathrm{cc}$. $(\mathrm{R}=200-1300)$. LOS was $3.6 \pm 0.94$ days $(\mathrm{R}=2-7)$. The postoperative stone-free rate was $95 \%$. Twenty-five $(59.5 \%)$ complications were documented. Fifteen $(35.7 \%)$ had fever: Grade I $\mathrm{CD}$, and $10(23.8 \%)$ had required transfusion: Grade II CD. There was no mortality.

Conclusions: PCNL still persists as the treatment of choice for nephrolithiasis in kidneys with variations in anatomy or position. A high stone clearance rate can be achieved while minimizing complications. 
MP28-09 Investigating the Roll of Smaller Tract Percutaneous Nephrolithotomy in the Fully Anticoagulated Patient

MM Dunne, JE Abbott, JG Davalos

University of Maryland Medical Center

Introduction \& Objective: Both AUA and EAU guidelines for stone disease include anticoagulation therapy as a contraindication to Percutaneous Nephrolithotomy (PNL). Smaller tract surgery, such as mini-PNL (mPNL) miniaturized to 14-22 Fr, has gained popularity in the United States over the past few years. Decreasing tract diameter is shown to minimize renal parenchymal trauma and likely leads to less bleeding, as demonstrated in studies revealing lower blood transfusion rates and stable postoperative hemoglobin/hematocrit with mPNL. Under this basis, the aim of this study is to analyze surgical outcomes and determine the feasibility of performing mPNL on patients who are fully anticoagulated at the time of procedure.

Methods: We present a pilot case series of 10 patients who underwent mPNL while on full anticoagulation therapy in the hospital setting at a single institution performed by two high volume surgeons from 5/2018 to 5/2019. Renal access was obtained by the operative surgeon in all cases. All procedures were performed tubeless with a ureteral stent placed. Patients were observed overnight with CT scan performed on post-operative day one and laboratory studies tracked. Anticoagulation therapies included patients taking apixaban (Eliquis), rivaroxaban (Xarelto), ticagrelor (Brilinta), and clopidogrel (Plavix).

Results: mPNL was performed on 10 patients that remained on full anticoagulation therapy indicated for cardiac or antithrombotic prophylaxis. Mean stone burden was $23 \mathrm{~mm}$. Mean surgery time was 97 minutes. Absolute stone free rate was $90 \%$. No patients underwent secondary procedures. Average estimated blood loss was $10 \mathrm{~mL}$. There were no intraoperative complications related to bleeding. Hematocrit on post-operative day 1 declined by a mean of $3.1 \%$ and no blood transfusions were performed.

Conclusions: With well-trained operating room staff and surgeon in a high-volume center, mPNL may be cautiously considered for patients on anticoagulation therapy. Furthermore, these patients may be at higher risk of surgical complication for a cardiovascular or thrombotic event should their anticoagulation be stopped to perform PCNL. Although ureteroscopic lithotripsy remains the standard of care for these patients that require continued anticoagulation therapy, operative time and stone free rates are still important considerations for these high-risk patients that need optimal treatment in a single surgical setting. Additional studies with a larger cohort are necessary to determine if the result of this pilot series is valid.

\section{MP28-10 Prospective Outcomes of Single Stage Tu-} beless mini-PCNL for Renal Stones $\geq 20 \mathrm{~mm}$

\section{S Khadgi, M Darrad, AR EL-Nahas, A Al-Terki \\ Urology Department, Al-Amiri Hospital, Kuwait}

Introduction \& Objective: To evaluate the outcomes of single stage tubeless mini-PCNL for patients with large renal stones $\geq 20 \mathrm{~mm}$. To identify variables that help to predict the likelihood of successful stone clearance.

Methods: In this prospective study, we identified consecutive patients who underwent tubeless mini-PCNL (16-20F) with noncontrast CT confirmed renal stone burden of $20 \mathrm{~mm}$ or greater in 2 referral centers between July 2015 - November 2018. Primary outcomes evaluated were immediate stone-free rates, 30-day procedure-related complications, length of postoperative hospital stay, and need for blood transfusion. $\mathrm{Chi}^{2}$ or Fisher's exact test were used for categorical variables and Student's t test or the Mann-Whitney U test for continuous variables. A p-value $<0.05$ was considered to indicate statistical significance.

Results: We identified 225 renal-units from 218 adult patients for the study. The mean average age was 42.9 years old and $75 \%$ of patients were male. The mean cumulative stone size was $30.2 \mathrm{~mm}$ per renal unit, of which 63 renal-units contained staghorn calculi. The overall single stage stone-free rate was $87.6 \%$ with overall 30-day procedure-related complication rates of $8.4 \%$ (Clavien-Dindo I-II of $7.6 \%$, III of $0.9 \%$, IV-V of $0 \%$ ). 1 patient required blood transfusion and the average length of postoperative hospital stay was 3.0 days. Cumulative stone size was the best predictor of the stone-free rate $(\mathrm{p}<0.001)$. Other significant variables affecting the stone-free rates was multicalyceal stone locations $(\mathrm{p}=0.001)$, multiple stones $(\mathrm{p}=0.014)$, Guy's stone score $(\mathrm{p}=0.018)$, and the presence of staghorn calculi $(\mathrm{p}=0.02)$. Patients under 40 were more likely to develop 30day procedure-related complications $(\mathrm{p}=0.023)$.

Conclusions: Tubeless mini-PCNL is an effective treatment option for patients with large renal stones of $20 \mathrm{~mm}$ or greater and should be considered as one of the primary treatment options for this subgroup.

\section{MP28-11 Evaluation of Efficacy and Safety Profile of Bilateral Simultaneous Endoscopic Stone Surgery in Kidney Stones}

\author{
Y Tanidir, T Sener, G Ozgur
}

Marmara University School of Medicine, Department of Urology

Introduction \& Objective: Advancements in technology and endourological armamentarium has led us to bilateral surgical interventions to avoid multiple operations. The present study evaluates the outcomes of simultaneous bilateral endoscopic surgery (SBES) in kidney stones as a combination of supine percutaneous nephrolithotomy (PCNL) and contralateral retrograde intrarenal surgery (RIRS).

Methods: All patients in which related intervention can be carried out according to the EAU guidelines were prospectively recorded (NCT03599297). Each patient had a non-contrast computed tomography (NCCT) scan and kidney-ureter-bladder (KUB) X-ray before the procedure. Stone burdens were calculated with the 3D-Doctor software. The safety and efficiency of SBES were evaluated with the operative findings, the stone-free status, and complications. The immediate stone-free status decision was done according to both the endoscopic view and the postoperative imaging with KUB X-ray. Stone-free was defined as having fragments less than $3 \mathrm{~mm}$.

Results: A total of 27 patients (male/female $=2.9$ ) with a mean age of $51.7( \pm 14.1)$ years were operated between August 2017 and May 2019. The median stone burden on PCNL and RIRS sides were found to be $3021 \mathrm{~mm}^{3}(\min 728, \max 11848)$ $554 \mathrm{~mm}^{3}$ (min 68.3, max 5026), respectively. The median operation duration and fluoroscopy dose were found to be $180 \mathrm{~min}$ ( $\min 100, \max 300)$ and $53 \mathrm{mGy}(\min 14, \max 223)$, respectively. The mean size of the residual fragments in the PCNL-treated kidneys $(\mathrm{n}=8)$ was $4.5 \mathrm{~mm}( \pm 2.8 \mathrm{~mm})$ and the median size of residual fragments in FURS-treated kidneys $(n=5)$ was $7 \mathrm{~mm}$ $( \pm 3.5 \mathrm{~mm})$. The stone-free rates on the PCNL and FURS sides were found to be as $81.5 \%(n=22)$ and $85.2 \quad(n=23)$, 
respectively. Following the operation, a median hemoglobin drop of $1.0 \mathrm{gr} / \mathrm{dL}$ was found $(\mathrm{p}<0.001 ; 0.4-9 \mathrm{gr} / \mathrm{dL})$. Fever $(\mathrm{n}=5 ; 18.5 \%)$ and transfusion $(\mathrm{n}=4 ; 14.8 \%)$ were the most common complications. Of all patients, $11(40.7 \%)$ were treated with antibiotics. Three patients were transferred to the intensive care unit; due to hypotension $(\mathrm{n}=2)$, urosepsis $(\mathrm{n}=1)$. Overall 18 patients $(66.8 \%)$ had no complications, 1 patient $(3.7 \%)$ had grade 1,7 patients $(25.9 \%)$ had grade 2 and 1 patient $(3.7 \%)$ had grade 4 complications according to the Modified Clavien Dindo Grading System. The median hospital stay of patients with and without complications was found to be 3 days $(\min 1, \max 7)$ and 7 days ( $\min 2, \max 14)$, respectively.

Conclusions: The complication and success rates of SBES in kidney stones seem to be similar to PCNL and RIRS. The major disadvantage of the procedure was the need for at least two devoted and experienced surgeons as well as a well-equipped institution, such as a tertiary referral hospital.

MP28-12 A control research between super-mini percutaneous nephrolithotomy (SMP) and minimally invasive percutaneous nephrolitripsy (MPCNL)

Z Guoxi, W Xiaoning, Z Xiaofeng, Y Yuanhu, X Rihai, W Gengqing

Introduction \& Objective: To compare the difference between super-mini percutaneous nephrolitripsy (SMP) and minimally invasive percutaneous nephrolitripsy (MPCNL) for upper urinary stones, and to evaluate SMP for urinary stones.

Methods: To collect the data of 49 patients with up urinary stones treated by SMP (SMP group), and the data of 49 patients with up urinary stones treated by MPCNL (MPCNL group) during the same period. The operative time, postoperative one-day instant stone free rate, postoperative hemoglobin, postoperative $24 \mathrm{~h}$ visual analog pain score (VAPS), the incidence of complications, hospital stay and postoperative 3- month stone free rate was compared.

Results: In the SMP group, 44 cases were successfully completed, but 5 cases were conversed to MPCNL (18F peer-away sheath) due to intraoperative hemorrhage. In MPCNL group, 48 cases were successfully completed, but 1 case failed due to failure of puncture, who was treated by flexible ureteroscope lithotripsy later. Compared with MPCNL group, the mean decreased hemoglobin level of SMP group was lower $(8.3 \mathrm{~g} / \mathrm{L}$ vs $9.8 \mathrm{~g} / \mathrm{L})$, the incidence of SIRS of SMP group was lower $(2.3 \%$ vs $4.2 \%)$, the rate of tubeless SMP was higher (93.2\% vs $37.5 \%)$, and the length of hospitalization of SMP group was shorter (3.5d vs $4.5 \mathrm{~d}$ ). But the mean operative time of SMP group was longer $(70.5 \mathrm{~min}$ vs $58.6 \mathrm{~min})(\mathrm{P}<0.05)$. There was no significant difference $(\mathrm{P}>0.05)$ in the postoperative complication rate $(13.6 \%$ vs $18.8 \%)$, the postoperative 24-hourvisual analogue pain score (VAPS) (3.6 vs 4.1), instant stone free rate rate(90.9\% vs $89.6 \%$ ) and postoperative 3 -month stone free rate $(95.5 \%$ vs $97.9 \%)$.

Conclusions: SMP with less blood loss, lower pressure in the renal pelvis, lower incidence of postoperative complications, faster recovery and shorter hospitalization time, provide a new option for the treatment of upper urinary calculus.

MP28-13 Feasibility of Percutaneous Nephrolithotomy (PCNL) under Spinal Anesthesia for Staghorn Kidney Stones: Safety and Efficacy

JA Kalathia, S Agarwal, C Saurabh

Fortune Urology Clinic
Introduction \& Objective: Percutaneous nephrolithotomy (PCNL) for specifically staghorn kidney stones performed under general anesthesia can induce diluted anemia, higher analgesia, hypothermia, higher blood loss as well as the possibility of fluid absorption and electrolyte imbalance. We prospectively evaluated the safety and efficacy of PCNL for staghorn kidney stones under spinal anesthesia.

Methods: 22 patients of the 106 patients who underwent PCNL for the partial and complete staghorn kidney stones from January 2017 to April 2017 were included. Patient's demographics, perioperative parameters, stones free rate (absence of residual stones under fluoroscopy), blood loss and post-operative analgesia requirements were prospectively evaluated.

Results: Mean patients age, stone burden and stone free rate was $50.22 \pm 8.38,53.18 \pm 6.72 \mathrm{~mm}, 86.2 \%$. The mean operating time was $80.44 \pm 28.34$ minutes. Length of hospital stay was $4.5 \pm 2.0$ days. Only $4.5 \%$ (1 patient) required blood transfusion. Postoperative analgesia requirement was very low. There were no significant postoperative complications.

Conclusions: PCNL for staghorn kidney stones under spinal anesthesia is safe and effective with its own advantages of shorter operative time, less analgesia requirements and early hospital discharge.

MP28-14 A Nomogram to Predict Significant Estimated Glomerular Filtration Rate Reduction After Percutaneous Nephrolithotomy.

U Falagario, A Mangiatordi, M Auciello, A Martini, P Treacy, JN Bamberger, M Gupta, G Carrieri, L Cormio

Introduction \& Objective: Percutaneous nephrolithotomy (PCNL) is a minimally invasive technique to treat large or otherwise complex renal or proximal ureteral stones. Though considered a safe procedure, it may cause acute kidney injury (AKI) due to puncturing and dilating the renal parenchyma as well as to release of bacteria from the stone. Moreover, stone themselves may cause obstructive uropathy. Indeed, the reported rate of AKI after PCNL ranges from $6.7 \%$ to $38.2 \%$ in different series and may to poor postoperative outcomes and a longer hospital or intensive care unit stay. In the present study we attempted to develop a nomogram to predict AKI following PCNL.

Methods: We extract data of 605 patients from our Internal Review Board approved PCNL database. Estimated GFR (eGFR) was calculated according to the Chronic Kidney Disease Epidemiology Collaboration formula. Patients with eGFR $<30$ were excluded from the analysis. AKI was defined as $>25 \%$ reduction in preoperative baseline eGFR or $>1.5$-fold increase in preoperative creatinine, both at discharge from hospital. A nomogram was built based on a binary logistic regression that ultimately included age, sex, baseline eGFR and Operative time. Internal validation was performed using the leave-one-out cross validation. Calibration was graphically investigated. The decision curve analysis was used to evaluate the net clinical benefit. Results: Descriptive characteristics are shown in Table 1. Median (IQR) age at surgery was $54.8(43.8,63.4)$ years; 320 $(52.9 \%)$ patients were female, while $285(47.1 \%)$ were male. Median (IQR) eGFR was $86.3(71.9,99.7) \mathrm{ml} / \mathrm{min} / 1.73 \mathrm{~m}^{2}$. Median (IQR) Operative time was 75.0 (60.0, 100.0) $\mathrm{min}$. Postoperative AKI occurred in $65(10.7 \%)$ patients. All variables fitted in the model significantly $(\mathrm{p}<0.01)$ predicted AKI and were therefore used to build a nomogram (Figure 1). After internal validation, the AUC was $82 \%$. The model demonstrated excellent calibration and improved clinical risk prediction at the decision curve analysis. 
Nomogram Predicting AKI after PCNL

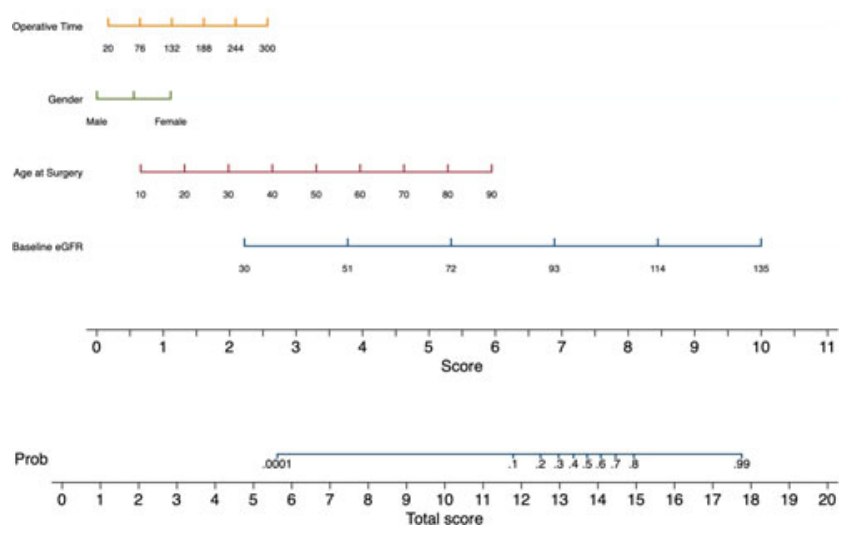

\begin{tabular}{|c|c|c|c|c|}
\hline \multirow[t]{2}{*}{ Variables } & \multirow{2}{*}{$\begin{array}{l}\text { Overall Population } \\
\mathrm{N}=605\end{array}$} & \multicolumn{2}{|c|}{ Multivariate Analysis } & \multirow[b]{2}{*}{$P>|z|$} \\
\hline & & Odds Ratio & 95\% C.I. & \\
\hline Age, years & $54.8(43.8,63.4)$ & 1.08 & $1.05,1.11$ & $<0.001$ \\
\hline \multicolumn{5}{|l|}{ Gender $M / F, n(\%)$} \\
\hline Male & $285(47.1 \%)$ & Ref. & & \\
\hline Female & $320(52.9 \%)$ & 3.55 & $1.92,6.53$ & $<0.001$ \\
\hline Operative time, $\min$ & $75.0(60.0,100.0)$ & 1.01 & $1.00,1.02$ & 0.009 \\
\hline Baseline eGFR & $86.3(71.9,99.7)$ & 1.09 & $1.06,1.12$ & $<0.001$ \\
\hline \multicolumn{5}{|l|}{ AKI, n (\%) } \\
\hline Absent & $540(89.3 \%)$ & & & \\
\hline Present & $65(10.7 \%)$ & & & \\
\hline
\end{tabular}

Conclusions: We developed a nomogram that accurately predicts AKI in patients undergoing PCNL. This model may serve in the preoperative setting to counsel patients regarding their risk of suffering AKI as well as in the immediate postoperative period to identify patients who would benefit from an early multidisciplinary evaluation.

MP28-15 Does skeletal muscle quality impact the outcomes of percutaneous nephrolithotomy?

\author{
Y Wu, Y Lu, Y Xun, H Hu, S Wang, Q Wang
}

Tongji Hospital

Introduction \& Objective: To evaluate whether skeletal muscle quality has an impact on the outcomes of percutaneous nephrolithotomy (PCNL).

Methods: We retrospectively reviewed patients who underwent PCNL at our institution from 2014 to 2018. Skeletal Muscle Index (SMI) was analyzed by computed tomography scans using the third lumbar vertebrae skeletal muscle index and standardized according to patient height $\left(\mathrm{cm}^{2} / \mathrm{m}^{2}\right)$. Sarcopenia was defined as $<40.8 \mathrm{~cm}^{2} / \mathrm{m}^{2}$ for men and $<34.9 \mathrm{~cm}^{2} / \mathrm{m}^{2}$ for women. Sarcopenic obesity was also assessed in patients with BMI $43.0 \mathrm{~kg} / \mathrm{m}^{2}$. Specifically, stone-free rates, complications, and hospital length of stay were assessed. Multivariable logistic regressions were used to evaluate the relationship between sarcopenia and variables.

Results: A total of 896 patients (592 men and 304 women) with a mean age of 49 years, BMI of $23.9 \mathrm{~kg} / \mathrm{m}^{2}$, and SMI of $49.7 \mathrm{~cm}^{2} /$ $\mathrm{m}^{2}$ were included. Furthermore, 312 patients $(35 \%)$ were sarcopenic, and 106 patients (12\%) had sarcopenic obesity. The overall stone-free and complication rates were $92.1 \%$ and $17.0 \%$, respectively. Univariate and multivariable analyses showed no association between skeletal muscle quality and stone-free or complication rates. However, patients with less skeletal muscle quality had significantly higher transfusion rates $(\mathrm{P}<0.05)$ and were significantly more likely to have a prolonged hospital length of stay $(\mathrm{P}<0.05)$.

Conclusions: Less skeletal muscle quality is associated with higher transfusion rates and longer hospital length of stay in patients with renal stones. The results of this study suggest that identifying patients with low muscularity contributes to better stratification in this population.

\section{MP28-16 Novel use of betadine and antibiotic irri- gation for renal pelvis sterilization during PCNL - a pilot study}

D Mikhail, E Lynch, A Rai, T Benjamin, M Mekhail, AD Smith, D Hoenig, Z Okeke

Introduction \& Objective: As complex stone incidence increases, rates of percutaneous nephrolithotomy (PCNL) for continue to rise. Efforts are made to prevent infectious complications, including pre- and postoperative antibiotic regimens and ensuring urine is sterile pre-operatively. Despite this, infectious complications, such as fevers and urosepsis, are the most common complications following PCNL. We developed a technique aimed at further sterilizing the collecting system during PCNL. We report early results on feasibility, safety and operative benefits of our technique.

Methods: From March to May 2019, we modified our PCNL technique for 32 consecutive patients. In traditional prone, cystoscopy and passing of a wire and ureteral catheter were performed as usual. After a renal pelvis urine sample was taken, we instilled $10 \mathrm{~mL}$ of betadine solution through the ureteral catheter. Contrast for retrograde pyelography was also mixed 50/50 with betadine. Gentamicin $80 \mathrm{mg}$ was injected into the first two $3 \mathrm{~L}$ bags of normal saline irrigation. Percutaneous access was obtained with fluoroscopy; stone treatment and drainage were decided intraoperatively based on stone burden and composition. A chest $\mathrm{x}$-ray and serum metabolic panel were obtained postoperatively for all patients.

Results: Our first 32 patients were analyzed. Patients were 53\% female and median age was $61 \pm 13$ years. About $30 \%$ of patients were diabetic and $47 \%$ were hypertensive. All patients had stone burden $>2 \mathrm{~cm}$. Six had positive urine cultures treated preoperatively, while 3 patients had contaminated cultures. All patients were treated with our antibiotic irrigation and betadine protocol regardless of pre-operative cultures or antibiotics. Chest $\mathrm{x}$-rays postoperatively were normal except 2 patients with small pleural effusions. Mean baseline creatinine was $0.87 \pm 0.35$, with a mean change of 0.24 post-op day 1 . No patients experienced significant changes in electrolytes. Three of 32 patients experienced fevers post-operative day 1 , although only 1 was found to have a positive culture as the cause. There were no adverse reactions to the betadine or antibiotic irrigation. No complications were attributed to our sterilization protocol.

Conclusions: We report a novel technique for renal pelvis sterilization using intrarenal instillation of betadine and antibiotic irrigation. Confirmation of access to the renal pelvis was easier to establish given the change in color from betadine. There were no adverse reactions or complications related directly to our protocol. Having established safety and easier access with our protocol - we are beginning a randomized control trial to determine whether this decreases infectious complications. 


\section{MP28-17 Withdrawn}

MP28-18 Comparative analysis of microbial contamination of stone and urine in recurrent nephrolithiasis

A Pushkarev, V Pavlov

Bashkir State Medical University

Introduction \& Objective: The main feature of the modern stage of development of surgical treatment of nephrolithiasis is the transition from traditional open interventions, the share of which in the world is $0.7-2 \%$, to minimally invasive high-tech methods. However, there is an increase in the incidence of urolithiasis disease. Chronic pyelonephritis plays a significant etiological and pathogenetic role in stone formation. In $92 \%$ of patient's urolithiasis occurs on the background of pyelonephritis. Chronic inflammation is based on highly organized bacterial communities - biofilms enclosed in an exopolymer matrix and adhered to inert or living surfaces.

Methods: Bacteriological examination of urine from the bladder, renal pelvis and calculus removed during PCNL was performed in 159 patients with nephrolithiasis. 74 patients had recurrent kidney stones. As methods of verification of microorganisms in urine and stones, we used mass spectrometry, standard culture techniques, and PCR diagnostics.

Results: According to our studies, which carried out PCNL microbial flora isolated from the bladder, in $38 \%$ of cases did not match the results of urine culture from the cavities of the affected kidney. The proportion of infected stones in recurrent nephrolithiasis was $71.8 \%$. Only in $16.7 \%$ of patients the study of urine and stone did not give results. Gram-positive flora prevailed in the microbial spectrum of microflora isolated from stones, and gram-negative microbial flora prevailed in the renal pelvis isolated from urine. In most patients, the types of microorganisms in the urine and stone differed and were represented mainly by uropathogens: Ent. faecalis, Staph. aureus, Staph. epidermidis, K. pneumoniae, P. aeruginosa, Strept. haemolyticus, E. coli. When microorganisms of the same species were detected in the urine and in the stone, different sensitivity to antibacterial drugs was determined.

Conclusions: With minimally invasive types of surgical treatment of nephrolithiasis, such as percutaneous nephrolithotripsy, concretions are crushed, and biofilms are destroyed in the cavities of the kidney. This causes the release of microorganisms and their toxins into the urinary tract. Antibacterial therapy conducted according to the results of urine culture will not be effective, will not lead to eradication of the actual microbial flora. Chronic inflammatory process contributes to the rapid formation of infectious stones. It is necessary to introduce microbiological examination of a removed stone into the standard of examination of patients with nephrolithiasis.

\section{MP28-19 Withdrawn}

\section{MP28-20 Analysis of Risk Factors for Pulmonary Complications in Patients undergoing Upper Pole Access Percutaneous Nephrolithotomy: A Single Center Ex- perience}

ME Amponin, JA Abraham

National Kidney and Transplant Institute /Philippine Urological Association
Introduction \& Objective: Upper pole access PCNL (UpPCNL) is our preferred approach because it is easy to perform and provides a panoramic view of the collecting system, leading to a high stone clearance rate. UpPCNL however, remains unpopular to many because of the perceived increased pulmonary complications. We summarized the pulmonary complications in our UpPCNL cases and analyzed the risk factors that contributed to them.

Methods: A chart review was done of all patients who underwent UpPCNL from January 2015 to December 2017. Percutaneous access was performed uniformly using a "bullseye" technique to the uppermost posterior calyx; while the depth was confirmed with an oblique view. Patient (age, gender, body mass index, co-morbidity) and stone demographics (Hounsfield unit, Guy's stone score, laterality, stone location), intraoperative parameters (operative time, number of tracts, estimated blood loss, and length of hospital stay) were summarized. Point Biserial correlation and Pearson Chi-Square for independent tests were used to identify the independent predictors of pulmonary complications.

Results: A total of 992 patients underwent UpPCNL during the study period. Fifty-two (5.2\%) had pulmonary complications. A total of sixty-seven pulmonary complications were tallied. The most common was pleural effusion: 35 (48\%), atelectasis: $16(30 \%)$, hospital-acquired pneumonia: $14(27 \%)$ and acute respiratory distress syndrome: $2(4 \%)$. Forty-one $(78.8 \%)$ and $11(21.2 \%)$ were managed medically or surgically, respectively. Preoperative creatinine ${ }^{3} 4.5 \mathrm{mg} / \mathrm{dl}$, stone size of $>5.0 \mathrm{~cm}$, a Guy's stone score of 3-4, and length of hospital stay of ${ }^{3} 8$ days were significant predictors for developing pleural effusion. Patients with higher preoperative creatinine and longer hospital stay were significantly associated with possible surgical intervention $(\mathrm{p}<0.05)$.

Conclusions: UpPCNL is generally safe and leads to low incidence of pulmonary complications. When risk factors such as those mentioned above are present, these patients need close monitoring for possible pulmonary complications, or alternatively, another calyceal entry may be considered to avoid these pulmonary complications from occurring.

\section{MP28-21 ECIRS in treatment of Large Upper Ure- teric Stones}

DK Mishra, MS Agrawal

Global Rainbow Healthcare, Agra, India

Introduction \& Objective: Endoscopic Combined Intra Renal Surgery (ECIRS) involves simultaneous use of Ureteroscopy and Percutaneous renal surgery in supine position, allowing access to all parts of the urinary tract at the same time. While doing ureteroscopy in cases of large upper ureteric stones, the problem lies in upward migration of stone fragments. On the other hand, antegrade percutaneous approach carries the risk of not being able to reach the stone in the upper ureter due to angulation. We have analyzed the outcomes of ECIRS in such cases of large upper ureteric stones.

Methods: Data was collected prospectively from January 2016 to December 2018 for all upper ureteric stones admitted at our center. All patients with large upper ureteric stones with or without co-existing renal stones were included in the study. Pediatric patients $(<18 \mathrm{yrs})$ were excluded. All procedures were done in general anesthesia. ECIRS was performed using semirigid ureteroscope (6/7.5 F, Richard Wolf) along with mini- 
PCNL (15 F sheath with 12 F Nephroscope, Karl Storz) in supine modified Valdivia position. Ultrasonography was used to make puncture in pcs followed by single-step dilation. Laser energy was used for stone fragmentation in all cases.

Results: we had a total of 32 cases with 20 males and 12 females. The mean age was $43.4 \pm 12.5$ yrs. laterality was equal with 16 cases each. Mean stone size was $16.1 \pm 4.6 \mathrm{~mm}$. There were 16 renal units with middle calyceal puncture and 12 with upper calyceal punctures. 4 renal units had double puncture. Most of the times the fragments were retrieved from renal tract. Operative time was $54.6 \pm 8.5$ minutes. All patients had a double-J stent put in and none required a nephrostomy. There were minimal intra operative complications with 4 cases having a failed access and ureteric perforation in 1 case. The average length of hospital stay was $28.5 \pm 4.6 \mathrm{hrs}$. The stone clearance rate was $96.8 \%$.

Conclusions: ECIRS using a semi-rigid ureteroscope and mini PCNL is a viable option for large upper ureteric stone with excellent outcomes.

MP28-22 Can CT-based measurements of renal parenchyma, perirenal fat and subcutaneous fat predict the complication rate of Percutaneous Nephrolithotomy? A single center retrospective study

J Torres, C Gonçalves, N Morais, S Anacleto, R Rodrigues, P Passos, P Mota, R Leão, E Lima

Introduction \& Objective: Percutaneous Nephrolithotomy (PCNL) is associated with various complications that are difficult to predict. In an attempt to identify which patients would be more susceptible to complications, we investigated if the renal parenchymal, perirenal fat and subcutaneous fat thicknesses are risk factors for PCNL complications.
Methods: From January 2011 to June 2018, 322 patients with nephrolithiasis were submitted to PNCL in a single center. Stonefree rate and the intra and postoperative complications (pain, fever, hematuria, nausea/vomiting, anemia, sepsis, hemoglobin drop and need for transfusion) were evaluated. The overall complication rate was classified as minor or major using the Clavien-Dindo score (0-2 vs. 3-5 respectively). Pre-surgical CT scan was used to measure the renal parenchymal thickness (from stone to renal capsule), perirenal fat thickness (from renal capsule to transversus abdomins muscle), subcutaneous fat thickness (from external oblique to skin), intra-abdominal distance (parenchymal and perirenal fat added) and total abdominal wall thicknesses (from stone to skin). These measurements were made in the lowest plane of the stone using the angle of the desired puncture.

Results: Regarding parenchymal thickness, we verified a significant association between thinner parenchyma and infection $(p=0.012)$. Additionally, there was a trend towards thinner parenchyma being associated with greater hemoglobin loss $(p=0.058)$ and more severe overall complications $(p=0.076)$. Perirenal fat thickness was not associated with more severe complications $(p=0.45)$. When analyzing subcutaneous fat thickness, there was a trend pointing to a relationship between shorter length and hemorrhagic and more severe complications ( $p=0,078$ and $p=0,089$, respectively). We found a significant association between shorter length of intra-abdominal thickness and infectious complications $(p=0.01)$. Finally, we did not find any significant associations between total abdominal wall thickness and the severity of complications $(p=0.525)$.

Conclusions: In this study, we show that thinner renal parenchyma and shorter distances between the stone and the skin appear to predispose to more severe complications after PCNL. Future studies are needed to confirm our results in order to establish preoperative risks and anticipate complications.

\section{MODERATED POSTER SESSION 29: CLINICAL STONES: URETEROSCOPY (II)}

\begin{abstract}
MP29-01 Impact of an Enhanced Recovery After Surgery Protocol on Patient Symptoms in the Immediate and Intermediate Post-operative Period After Ureteroscopy for Stones
\end{abstract}

BA Johnson, J Tse, A Akhtar, J Crivelli, RL Steinberg, J Sasaki, JA Antonelli, M Pearle

University of Texas Southwestern

Introduction \& Objective: Ureteroscopy (URS) for stone disease generates an unusually large number of unexpected patientinitiated encounters in the peri-operative period. An Enhanced Recovery After Surgery (ERAS) protocol for patients undergoing URS was developed. We sought to determine whether implementation of an ERAS protocol would reduce the need for patient-initiated encounters after URS.

Methods: We initiated an ERAS protocol, involving the use of intravenous ketorolac, and oral pregabalin, acetaminophen, and methocarbamol, for patients undergoing URS for stones. Prior to initiation of the protocol, URS patients were followed prospec- tively from October 2017 to January 2018. Following a 3-month wash-in period, we collected demographic and operative data on 100 consecutive patients scheduled to undergo URS between May and August 2018. Patients that did not undergo ureteroscopy were excluded. Post-anesthesia care unit (PACU) visual analog pain scale (VAS) scores and morphine milligram equivalent (MME) usage recorded. All 7-day post-operative clinic/oncall telephone encounters or secure internet messages ("calls") and 30-day emergency department (ED) visits, and readmissions were recorded.

Results: A total of 62 pre-ERAS (median age 57 years IQR $50-$ 66) and 92 post-ERAS (median age 57 years IQR 43 - 68) patients were included in analysis. Median operative time for the cohort was $78 \mathrm{~min}$ (IQR $52-114$ ) and $23 \%$ of procedures (36/ $154)$ were bilateral. The need for ED visits and post-operative readmissions were similar between the two groups. A total of 76 calls within 7 days of surgery from 154 patients was documented. The number of calls in the pre-ERAS group was more than twice that in the post-ERAS group ( 0.73 vs 0.34 calls per patient-week, respectively, $\mathrm{p}=0.011)$; likewise, calls specifically related to pain declined by more than half after ERAS (0.27 vs 0.12 calls 
Table. Post-procedure patient-initiated encounters

\begin{tabular}{lccc}
\hline & $\begin{array}{c}\text { Pre-ERAS } \\
(\mathrm{n}=62)\end{array}$ & $\begin{array}{c}\text { Post-ERAS } \\
(\mathrm{n}=92)\end{array}$ & p-value \\
\hline ED visits & $10(16 \%)$ & $11(12 \%)$ & 0.481 \\
$\quad$ Pain-related ED visits & $9(15 \%)$ & $9(10 \%)$ & 0.446 \\
$\begin{array}{l}\text { 30-day Re-admissions } \\
\quad \text { Pain-related re-admissions }\end{array}$ & $2(3 \%)$ & $5(5 \%)$ & 0.589 \\
$\begin{array}{l}\text { No. of Calls } \\
\quad \text { Calls per patient-week }\end{array}$ & 0.73 & $5(4 \%)$ & 0.800 \\
$\begin{array}{l}\text { No. of Pain-related calls } \\
\quad \text { Calls per patient-week }\end{array}$ & 17 & 31 & \\
$\begin{array}{l}\text { No. patients with made at } \\
\text { least one call }\end{array}$ & $\mathbf{0 . 2 7}$ & 0.34 & 0.011 \\
$\begin{array}{l}\text { Mean PACU VAS scale at } \\
\text { time of discharge }\end{array}$ & $29(46 \%)$ & 11 & \\
$\begin{array}{l}\text { MME requirements during } \\
\text { recovery }\end{array}$ & 3.6 & 0.12 & 0.008 \\
& 27.2 & $21(22 \%)$ & 0.002 \\
& & 8.5 & $<0.001$ \\
\end{tabular}

per patient-week, respectively, $\mathrm{p}=0.008)$. Twice as many patients in the pre-ERAS group had at least one call $(46 \%$ vs $22 \%$, respectively, $p=0.002$ ). Finally, ERAS patients had less pain and required significantly less narcotic in PACU (table).

Conclusions: Early analysis after implementation of an ERAS protocol shows reduced unplanned patient-initiated communication within 7 days of surgery and a lower narcotic requirement in PACU suggesting that the ERAS protocol can improve the patient experience after ureteroscopy.

MP29-02 FRAIL: Feasibility of Retrograde IntrArenal Surgery In eLderly - Preliminary Outcomes

S Biligere, C Heng, V Gauhar

$\mathrm{Ng}$ Teng Fong General Hospital

Introduction \& Objective: Ageing/ immobility and comorbidities can be a double threat by imposing physical and metabolic disability, which lead to higher rates urolithiasis \& related problems. Since Retrograde Intrarenal Surgery (RIRS) is an acceptable option even in renal stones up to $2 \mathrm{~cm}$, we assessed its safety, feasibility and outcomes in a predominantly elderly population, $>65 y$ rs of age.

Methods: In a single centre, patients $>65 y$ rs were subjected to RIRS by Modified ergonomic Lithotripsy (MEL) from July 15 May19. Demographics, co-morbidities, stone characteristics and

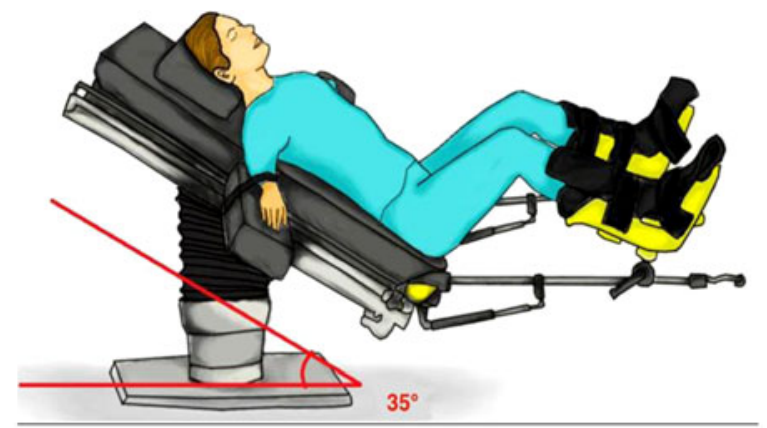

Figure 1. Placement of the patient into the MEL position

\begin{tabular}{|l|l|l|}
\hline STANDARD & DIFFERENCES & MEL \\
\hline FULLYFLAT & POSITION OF TABLE & 30 -35 degree HEAD UP \\
\hline LITHOTOMY & POSITION OF PATIENT & $\begin{array}{l}\text { LITHOTOMY WITH HEAD } \\
\text { UP }\end{array}$ \\
\hline STANDING & POSITION OF SURGEON & SITTING \\
\hline CLOSER TO GENITALIA & POSITION OF ACCESS \\
SHEATH & $\begin{array}{l}\text { SLIGHTLY AWAY FROM } \\
\text { GENITALIA }\end{array}$ \\
\hline
\end{tabular}

Table 1: Demographics and Stone characteristics $[>65 \mathrm{vrs}$ age]

\begin{tabular}{|l|l|l|}
\hline \multicolumn{2}{|l|}{ TOTALCASES $=60$} \\
\hline Stone size & $>1.5 \mathrm{~cm}$ & $33(55 \%)$ \\
\cline { 2 - 3 } & $<1.5 \mathrm{~cm}$ & $27(45 \%)$ \\
\hline \multirow{5}{*}{ Co-morbids } & $\begin{array}{l}\text { Hyertension(HTN)+/- } \\
\text { Diabetes (DM) }\end{array}$ & $56(93.3 \%)$ \\
\cline { 2 - 3 } & $\begin{array}{l}\text { Chronic Kidney } \\
\text { disease (CKD) }\end{array}$ & $10(16.67 \%)$ \\
\cline { 2 - 3 } & Anti-coagulation & $3(5 \%)$ \\
\hline \multirow{5}{*}{ Location } & PU//Pelvis & 5 \\
\cline { 2 - 3 } & Upper pole & 3 \\
\cline { 2 - 3 } & Midpole & 5 \\
\cline { 2 - 3 } & Lowerpole & 21 \\
\cline { 2 - 3 } & Multiple & 26 \\
\hline Pre-stented & Yes & $38(63.3 \%)$ \\
\cline { 2 - 3 } & No & $22(36.7 \%)$ \\
\hline Complex Anatomy & Yes & $4(6.7 \%)$ \\
& & \\
\hline
\end{tabular}

Table 2-Outcomes
\begin{tabular}{|l|c|c|c|}
\hline & $\leq 1.5 \mathrm{~cm}$ & $\geq 1.5 \mathrm{~cm}$ & Overall \\
\hline $\begin{array}{l}\text { SFR 1 } \\
\text { sitting }\end{array}$ & $25 / 27(92.6 \%)$ & $19 / 33(57.6 \%)$ & $44 / 60(73.33 \%)$ \\
\hline Sepsis & $0 \%$ & $4 / 12(12.1 \%)$ & $4 / 60(6.7 \%)$ \\
\hline
\end{tabular}

SFR 2nd sitting (overall) - $96.7 \%$

outcomes in terms of Stone Free rate (SFR) and sepsis were assessed. Fig. 1 - Description of MEL position.

Results: Table 1 and 2 showing Demographics, Stone characteristics and Outcomes.

Conclusions: RIRS can be performed in a safe and effective manner in elderly patients despite physical disability. These patients are at a higher risk of developing venous thromboembolism and hip fractures. Hence, adequate pre-op planning, positioning of patient and approach to surgery play a crucial role. The MEL technique along with use of High-power Laser, reduces operative time, improves ergonomics, hence, minimizing risk of complications, especially sepsis. Our study demonstrates comparable SFR and complication levels to current published data. RIRS by MEL may be a preferred treatment option for elderly patients with large stone volumes when compared to PCNL.

\section{MP29-03 Novel Biocompatible Adhesive to remove stone dust: Usability Trial in a Kidney Model}

TJ Hausmann, C Netsch, B Becker, A Gross, CM Rosenbaum

Asklepios Clinic Barmbek, Hamburg, Germany

Introduction \& Objective: Residual fragments after endoscopic intracorporal lithotripsy are still a challenging problem in modern endourology. To improve the stone-free rates, we tested the viability of a novel bioadhesive system for intrarenal embedding and retrieval of residual fragments after endoscopic lithotripsy in a kidney model.

Methods: All procedures were performed in a standardized setting including a kidney model (SAMED, Dresden) in a plastic basin filled with water. We used a Viper URF flexible endoscope (6.6/8 Fr., Richard Wolf, Knittlingen). A mean amount of $138 \mathrm{mg}$ (SD $\pm 11.7 \mathrm{mg}$ ) of sand grains (range, $0.2 \mathrm{~mm}-0.8 \mathrm{~mm}$ ) was inserted in different renal calyxes of the kidney model. We assessed the extraction efficacy of flexible ureterorenoscopy using the bioadhesive system with conventional retrieval baskets $\left(\right.$ NCircle $^{\circledR}$ Nitinol Tipless Stone Extractor (Cook Medical, Bloomington) and NGage ${ }^{\circledR}$ Nitinol Stone Extractor (Cook Medical, Bloomington)). In total 8 different surgeons performed one intervention, respectively. The endoscopic and macroscopic stone-free rates, the experience of the surgeons and the overall time of stone retrieval were assessed. 

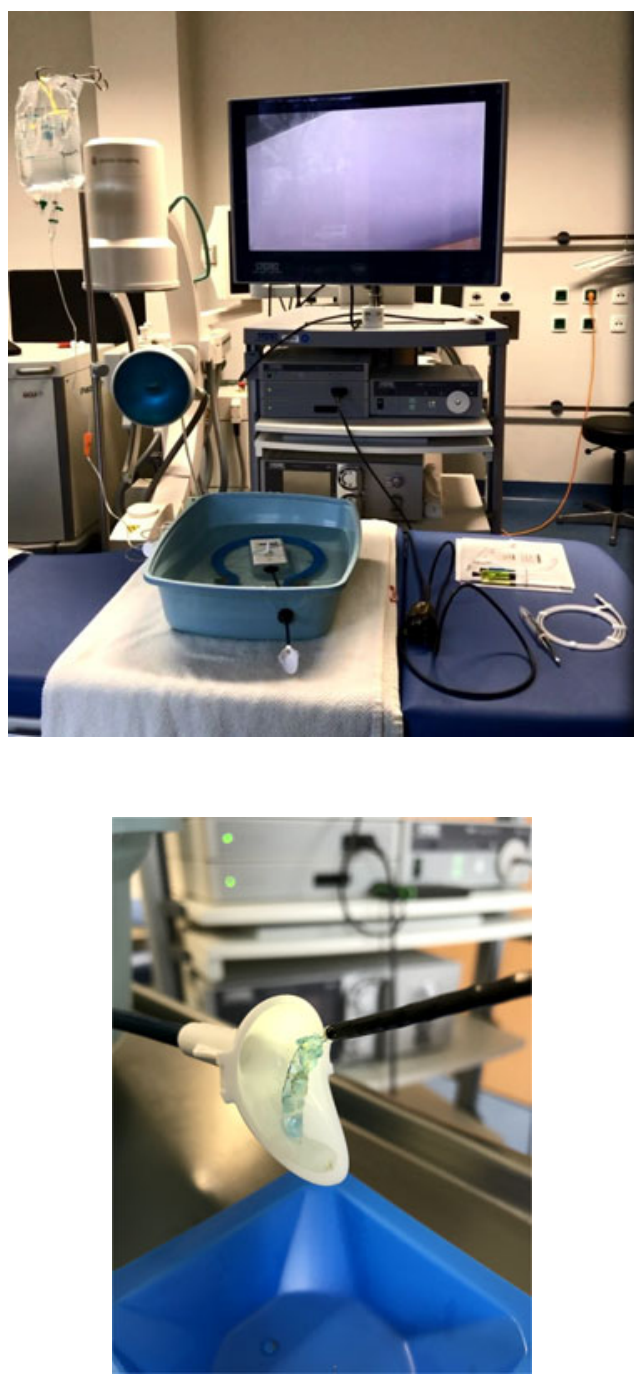

Results: The extraction of the sand grains was only possible while using the bioadhesive system, otherwise they were too small-sized to grab with a retrieval basket. The total stone-free rate was $84 \%$. In a postoperative survey all surgeons stated that it was a promising technique which they could imagine using on a daily basis. Conclusions: This novel technique to remove residual fragments from the kidney has enabled the surgeons to retrieve even fragments which could otherwise not be removed with a basket. Therefore, it significantly improves the stone-free rate with fragments $<1 \mathrm{~mm}(0 \%$ vs. $86.7 \%)$. This method technique might improve the stone free rate in difficult anatomic conditions, i.e. lower calyxes or anomalous kidneys. More tests, especially inpatient studies, are required to further evaluate the bioadhesive based extraction of stone fragments after lithotripsy.

\section{MP29-04 Efficacy and Safety of Semi-rigid Uretero- scopic Lithotripsy for Proximal Ureteral Stone Larger than $10 \mathrm{~mm}$}

\section{Bangash, SM Nazim, S Jamil, M Ghani, S Naeem}

The Aga Khan University Hospital, Karachi Pakistan

Introduction \& Objective: Large proximal ureteric calculi are more likely to get impacted and infected and usually dealt with flexible ureteroscope but due to high cost and maintenance of flexible ureteroscope, semirigid ureteroscope (URS) is still used in developing countries. We intended to evaluate the efficacy and safety of the use of semirigid ureteroscopy (URS) in the management of proximal ureteral calculi more than $10 \mathrm{~mm}$ in size. Methods: We reviewed a total of 103 patients (70 male and 33 female), who underwent URS for proximal ureteral calculus larger than $10 \mathrm{~mm}$ diameter from January 2007 to December 2017. All adult patients who had single unilateral radio-opaque proximal ureteral stone larger than $10 \mathrm{~mm}$ in size were included. Patients with solitary kidney, Uro-sepsis, pregnancy or preoperative drainage with nephrostomy or JJ stent were excluded. Stone free rate was defined as no evidence of radiopaque stone on X-Ray KUB done after 4 weeks of URS.

Results: During the study period, 2709 URS cases were performed out of which 404 were for proximal ureter stone. 103 cases had stone size greater than $10 \mathrm{~mm}$. All cases were performed as day care procedures. Stones had a mean size of $13.20 \pm 4.80 \mathrm{~mm}$. Our stone-free rate was $83.5 \%$. JJ stents were placed in $80.2 \%$. Mean operative time was $41.50 \pm 15.60 \mathrm{~min}-$ utes. Efficiency Quotient (EQ) was calculated using a standard formula, taking into considerations the retreatment rate and ancillary procedures performed and the calculated EQ was 0.72 . The minor complications included only self-limited postoperative fever $(5.82 \%)$ and hematuria $(15.5 \%)$. They were mostly Clavein Grade 1. Other complications were stone migration $(4.8 \%)$ and urosepsis (1.9\%). No major complications were encountered. 17 cases required ancillary procedures mainly ESWL $(13.5 \%)$ and secondary URS $(2.9 \%)$.

Conclusions: Semirigid URS is a safe and effective treatment option for large proximal ureteral calculi with limited access to flexible instruments.

\section{MP29-05 What is the Current Role of Single Use Ureteroscopes? A Snapshot World Survey}

M Ravindraanandan, W Mahmalji, Y Phan

Hereford County Hospital

Introduction \& Objective: Flexible ureteroscopy is a mainstay of kidney stone treatment, including fibre-optic and digital scopes. Differing opinions have been raised with scope durability, infection control, safety and cost-benefit. Single-use ureteroscopes have shown possible superiority within these fields. This multi-center world snapshot study demonstrates views on users' experiences with single-use ureteroscopes.

Methods: An online survey was created with respect to single use ureteroscopy. Questions focused on user experience, general opinion, advantages, disadvantages and cost. Invitations to participate were distributed via social media and email to urologists. Results: 62 responses were obtained. Clinical grade of responders is highlighted in Graph $1.75 .4 \%$ of responders were UK based, with $24.6 \%$ coming from the rest of the world. A total of $66.1 \%$ had used a single-use ureteroscope, $95.1 \%$ liked it. Positive comments included good vision and convenience. Negative comments were cost $46.7 \%$, inferiority to a traditional scope $20 \%$, environmental concerns $16.7 \%$, or all of the above $16.7 \%$. After currency conversion responders estimated mean cost from $£ 100-£ 6000$ (Mean $£ 991.36$ ).

Conclusions: The results suggest that once tried the single-use scope is popular for its light weight, lack of fear of damage, vision and maneuverability, however the main factor restricting widespread use appears to be costs (Graph 2). With such wide 


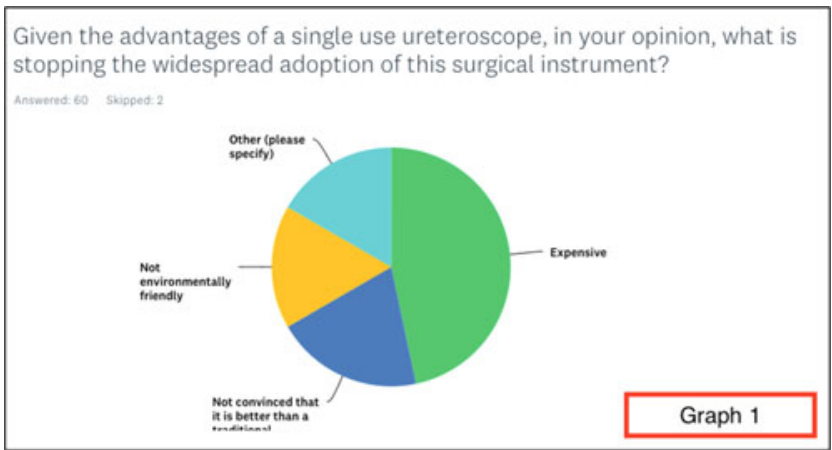

What is your designation in the hospital?

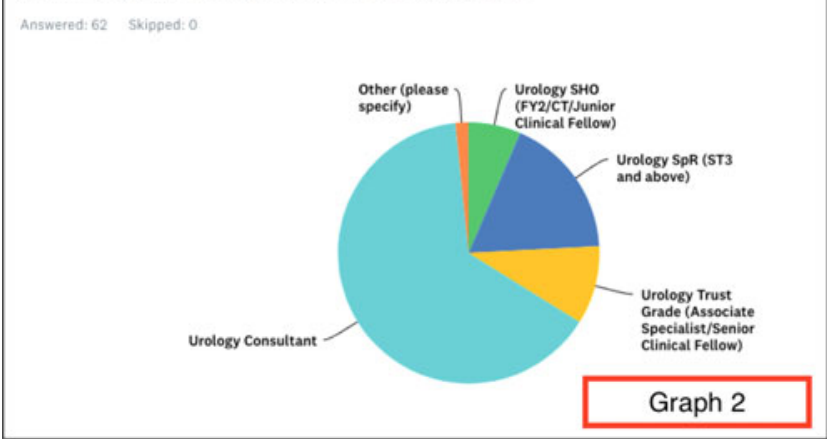

views on cost variations of $£ 100-£ 6000$ there may be a role of education from industry as to the recommended retail price which may alter these views. The cost of a single-use scope may vary from centers depending on user volume so there may be a role in a 'standardized' single price. Accurate costs of sterilizing, storing and repairing usable scopes have to be considered. The environmental impact of single-use instruments must also be considered. The modern stone-unit could adopt a combination of both reusable and single-use scopes on a case dependent basis.

\section{MP29-06 The Impact of Endourological Experience on Flexible Ureteroscopy (fURS) Outcomes. Retro- spective Multifactorial Analysis}

T Ozimek, MW Kramer, MC Hupe, JM Laturnus, JP Struck, M Hennig, AS Merseburger, J Cordes

Universitätsklinikum Schleswig-Holstein (Campus Lübeck)

Introduction \& Objective: Due to its minimal-invasiveness and acceptable level of difficulty fURS has been incorporated already in many departments as a part of residents' training. The aim of our study was to retrospectively analyze the influence of residents' participation in fURS procedures on intra- and postoperative outcomes.

Methods: Intra- and postoperative parameters were compared in retrospective monocentric setting between three groups: "Resident group" (47 cases) for surgeries performed by experienced residents (PGY4, PGY5) alone, "Consultant group" (245 cases) for surgeries performed by consultants alone, "Resident plus consultant group" (124 cases) for training surgeries between September 2013 and June 2017.

Results: Patients operated by residents alone were characterized by significantly smallest median kidney stone diameter $(5.0 \mathrm{~mm}$ vs. 7.0 for "Consultant group" vs. 6.0 for "Resident plus consultant group", $\mathrm{p}=0.011$ ) and statistically shortest postoperative hospital stay, although with median of 2 days for all groups $(p=0.013)$. "Resident group" had statically the shortest operating (median $47.0 \mathrm{~min}$ vs. 63.0 vs. $77.0, \mathrm{p}<0.001$ ) and fluoroscopy time (median $39.0 \mathrm{sec}$ vs. 69.5 vs. $89.0 \mathrm{p}<0.001$ ). Laser application rate was the smallest in "Residents group" compared to the both other groups $(5 / 47 ; 10.64 \%$ vs. $77 / 245 ; 31.43 \%$ vs. $37 / 124 ; 29.84 \%, \mathrm{p}=0.009)$. Univariate analysis revealed no relevant differences regarding fURS defect rate, postoperative stone-free rate (SFR) or Clavien Dindo $\geq$ Grade 2 complications between the groups $(\mathrm{p}>0.05)$. In multivariate logistic regression, performed for parameters significant in univariate analysis stone diameter, length of hospital stay, total laser energy, fluoroscopy and operation time - no independent parameters distinguishing cases operated by residents alone from cases with consultant participation were found $(\mathrm{p}>0.05)$.

Conclusions: A proper case selection of less complicated diagnostic or kidney stone cases, especially without laser application, could balance the natural experience deficit of the residents. fURS can be safely and effectively incorporated as a part of residents' training, without impact on fURS device defect rate or clinical outcomes.

MP29-07 The role of antegrade irrigation via percutaneous nephrostomy on surgical outcomes in semirigid ureteroscopy among patients with upper ureteral stones

W Jung, H Byun, D Lee

Keimyung University School of Medicine, Dongsan Medical Center

Introduction \& Objective: We aimed to investigate the role of antegrade irrigation via percutaneous nephrostomy on surgical outcomes in retrograde ureteroscopy in patients with upper ureter stones.

Methods: In this retrospective study, we analyzed 134 patients who underwent retrograde semi-rigid ureteroscopy for upper ureter stones between August 2012 and December 2017. Patients were divided into two groups: retrograde irrigation group (not using percutaneous nephrostomy) and antegrade irrigation group

Table 1. Main surgical outcomes

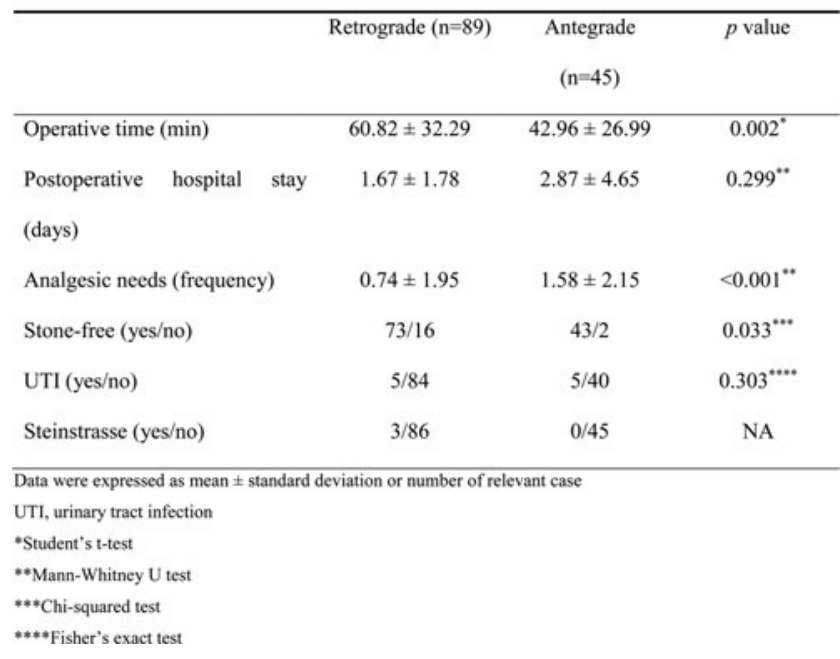


(using percutaneous nephrostomy). Operation time, postoperative hospital stay, analgesic use, complications, and stone-free rate were measured for each patient after ureteroscopy.

Results: The mean age in the retrograde irrigation and antegrade irrigation groups was 53.3 and 60.7 years, respectively $(\mathrm{p}=0.007)$. The operation time was $60.8 \mathrm{~min}$ vs. $43.0 \mathrm{~min}(\mathrm{p}=0.002)$, analgesic use was 0.74 vs. $1.58(\mathrm{p}<0.001)$, and stone-free rate was $82.0 \%$ vs. $95.5 \%(\mathrm{p}=0.033)$ (Table 1$)$. Stone size, laterality, the proportion of male patients, and urinary tract infection prevalence were comparable between the groups. In the subgroup analysis of stone size $>10 \mathrm{~mm}$, the antegrade irrigation group had a shorter operation time and a higher stone-free rate. For stone size of 5-10 $\mathrm{mm}$, operation time in the antegrade irrigation group was lower and the stone-free rate between the two groups was comparable.

Conclusions: Antegrade irrigation via percutaneous nephrostomy during ureteroscopy has a higher stone-free rate with a shorter operation time without an increased urinary tract infection risk. Therefore, if percutaneous nephrostomy is necessary before ureteroscopy, antegrade irrigation of external fluid via percutaneous nephrostomy is strongly recommended.

\section{MP29-08 Frequency and predictors of access failure in patients undergoing Ureterorenoscopy for upper urinary tract calculi}

A Rafi, H Ather, W Aziz

Aga Khan University Hospital

Introduction \& Objective: Failure to access during primary Ureterorenoscopy (URS) for renal or ureteric stones is a frustrating problem both for patient and surgeon. We aimed to determine the frequency of access failure during URS and factors correlating with it. Objective: To determine frequency of access

\begin{tabular}{|c|c|c|c|c|c|c|}
\hline \multirow[t]{3}{*}{ Table 1} & & & & & $\begin{array}{l}\text { Access } \\
\text { failure }\end{array}$ & \\
\hline & & Number & $\mathrm{N}=110$ & $n=95$ & $n=15$ & p-value \\
\hline & Age & $\begin{array}{r}\text { Mean } \\
\text { SD }\end{array}$ & $\begin{array}{c}44.65 \pm \\
14.56\end{array}$ & $\begin{array}{c}45.51 \pm \\
14.30\end{array}$ & $\begin{array}{c}39.27 \pm \\
15.57\end{array}$ & 0.16 \\
\hline \multirow{7}{*}{$\begin{array}{l}\text { Patient } \\
\text { factors }\end{array}$} & BMI & $\begin{array}{r}\text { Mean } \\
\text { SD } \\
\end{array}$ & $\begin{array}{c}27.26 \pm \\
5.17\end{array}$ & $\begin{array}{c}26.83 \pm \\
5.24\end{array}$ & $\begin{array}{c}29.11 \pm \\
5.32\end{array}$ & 0.17 \\
\hline & Gender & Male & 73 & 62 & 11 & 0.74 \\
\hline & & Female & 37 & 33 & 4 & 0.17 \\
\hline & Pre stented & Yes & 22 & 22 & None & 0.03 \\
\hline & \multirow{3}{*}{ History of } & Stone passage & 29 & 29 & None & 0.02 \\
\hline & & Prior JJ stenting & 23 & 21 & 2 & 0.52 \\
\hline & & Prior URS & 16 & 16 & None & 0.12 \\
\hline \multirow{12}{*}{$\begin{array}{l}\text { Stone } \\
\text { Factors }\end{array}$} & \multirow[t]{4}{*}{ Location } & Distal & 28 & 26 & 2 & 0.16 \\
\hline & & Mid & 14 & 14 & 0 & \\
\hline & & Proximal & 33 & 26 & 7 & \\
\hline & & Kidney & 35 & 29 & 6 & \\
\hline & Size & & $\begin{array}{c}10.08 \pm \\
4.77\end{array}$ & $\begin{array}{c}10.00 \pm \\
4.66\end{array}$ & $\begin{array}{c}10.61 \pm \\
5.62\end{array}$ & 0.7 \\
\hline & \multirow[t]{5}{*}{ Number } & 1 & 73 & 61 & 12 & 0.24 \\
\hline & & 2 & 17 & 16 & 1 & \\
\hline & & 3 & 8 & 8 & 0 & \\
\hline & & 4 & 6 & 4 & 2 & \\
\hline & & 5 or more & 6 & 6 & 0 & \\
\hline & \multirow[t]{2}{*}{ Laterality } & Right & 56 & 47 & 9 & 0.45 \\
\hline & & Left & 54 & 48 & 6 & \\
\hline
\end{tabular}

failure in patients undergoing Ureterorenoscopy for upper ureteric and renal stones and factors associated with it.

Methods: After ERC approval, this prospective study was conducted at urology department of a University hospital. All patients undergoing Ureterorenoscopy for ureteric or renal stones were included. URS was performed with semirigid 6.8 FR Ureterosocpe followed by $8 \mathrm{FR}$ semirigid ureteroscope or $9 \mathrm{Fr}$ flexible ureteroscope as appropriate. Whether stone could be reached with any of these ureteroscopes (accessed) or not (access failure) during procedure was noted. Data was collected for demographics, prior history of stone passage, previous ureterorenoscopy/JJ stenting and whether patient is pre-stented at time of URS. Level of access failure (proximal /mid / distal ureter) was also noted. Postoperative complications within one month and need of ancillary procedure were recorded.

Results: We included 110 participants undergoing Ureterorenoscopy for upper urinary tract calculi. Mean age was $44.72 \pm 14.56$. Mean stone size was 10.08 . A total of $15(13.63 \%)$ patients had access failure. None of the patients in access failure group were pre-stented or had previous history of stenting/stone passage. (table 1). Complications requiring intervention were noted in 3 patients.

Conclusions: Access failure is a fairly common problem during ureterorenoscopy. Patients who are presented and have a history of stone passage are less likely to fail access. Information from this study will help in counselling patients pre operatively and may guide urologists on selective prestenting in higher risk patients.

\section{MP29-09 Fluoroless Ureteroscopy for ureteric stones: feasibility, outcomes and assessment of factors neces- sitating fluoroscopy}

\section{R Gupta, G Garg}

Introduction \& Objective: Patients with urolithiasis receive significant radiation exposure during their workup and endoscopic procedure. Also, Urologists and operating room personals are also at risk of significant radiation exposure. We prospectively evaluated Fluoroless Ureteroscopy for patients with ureteric stones and evaluated outcomes and factors which necessitated use of fluoroscopy in these patients.

Methods: Patients undergoing fluoroless ureteroscopy were evaluated prospectively. Direct access to ureter was done with $6 / 7.5$ Fr Wolfe ureteroscope. Where the ureteric orifice was tight or there was difficulty in advancing the ureteroscope, 4.5 Fr ureteroscope was used to perform the procedure. Holmium laser was used to dust the stone. After clearance, guidewire was passed under vision and with tactile feedback. DJ stent was placed at the end of procedure under vision. X-ray KUB was done the next day to document stone clearance and stent position.

Results: A total of 30 patients (31 renal units) undergoing fluoroless ureteroscopy were assessed. Stone location was in lower ureter in 22, midureter in 8 and upper ureter in 1 patient. Stone size was $58.69 \pm 38.66 \mathrm{~mm}^{2}$. Lasing time was $7.33 \pm 5.28$ minutes. Fluoroless ureteroscopy was successful in 28/31(90\%) of the renal units. Fluoroscopy was required in 3/31 (10\%) renal units. Reasons for use of fluoroscopy were, 1. tight ureter despite using 4.5 Fr ureteroscope necessitating ureteral dilatation. 2. Ureteral tortuosity due to chronic stone impaction. There was no intraoperative complication. Postoperative X- ray KUB showed normal position of stent in all patients.

Conclusions: The study demonstrates that fluoroless Ureteroscopy is feasible and successful for stones at all levels in ureter. It 
significantly reduces the radiation exposure to the patient and to urologists who are doing the endourological stone surgeries.

MP29-10 Outcomes and complications of ureteroscopy and ureterorenoscopy for stones management using Holmium laser in a tertiary stone center for ureteric and renal stones

MA Hamdoon, MO Breish, M Smith, D Whiting, D Browne, H Marsh, F Anjum, S Sriprasad

Dartford and Gravesham NHS Trust

Introduction \& Objective: The aim of this study was to assess the ureteroroscopy and ureterorenoscopy (URS) outcomes and complications using holmium YAG (Yttrium-AluminumGarnet) lithotripsy in patients with ureteric and renal stones in a tertiary stone center.

Methods: The data was collected prospectively from 1003 patients between 2013 to 2018 for who underwent URS for stones in the ureter and kidney. The patient demographics, site, size, number of stones were noted. Stone type, location and stone free rates (SFR) were analyzed. Complications were recorded as per the Clavien-Dindo (CD) system.

Results: Sixty four percent of the patients were male with a mean age 55 years (17-94; SD 16). The location had left side preponderance $(52 \%)$ and $40 \%$ were ureteric stones. The stone locations are shown in Figure 1. Mean stone size was $7.98 \mathrm{~mm}$ $(1-30 \mathrm{~mm})$ and the median stone burden (cumulative stones size) was $10 \mathrm{~mm}$ for multiple stones. Eighty six percent were radiopaque and $14 \%$ radiolucent. The median stone burden was: $<10 \mathrm{~mm}(43.5 \%), 10-19 \mathrm{~mm}(43.7 \%)$ and $>20 \mathrm{~mm}(13 \%)$. The SFR following first URS was $76 \%$ and with second URS reached

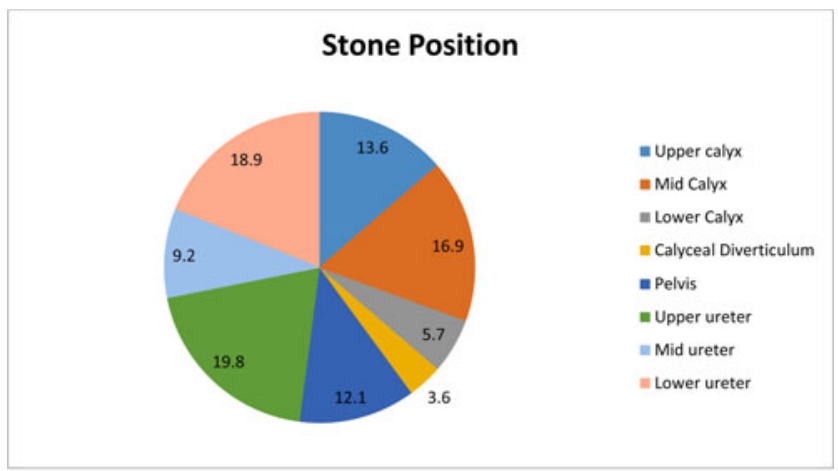

Figure 1: Stones position percentage within the kidney and ureter

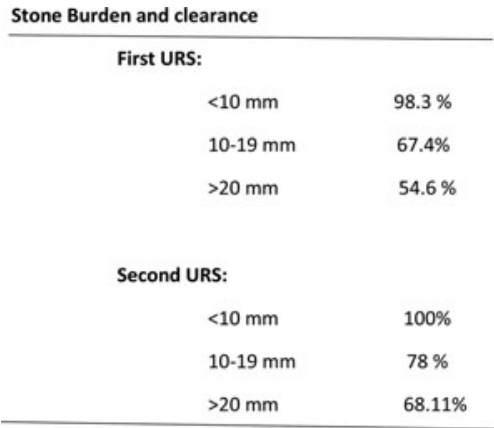

Table 1: Stone burden for multiple stones and clearance
$88 \%$. The stone burden and clearance is shown in table 1 . The overall complications rate was $7.3 \%$. Urosepsis (CD grade 2) happened in 23 patients (2.3\%); ureteric injury (Grade $1 \& 2$ ) was in 12 patients $(1.2 \%)$ and these patients were stented at surgery. One patient went into retention and 1 patient complained off erectile dysfunction. Two patients had laryngeal spasms (anesthetic complication), treated conservatively. One patient had a myocardial infarction (CD grade 4) and there was 1 death (CD grade 5). This patient had several comorbidities including large pressure sores, ischemic heart disease, diabetes and high body mass index. The patient died of urosepsis (CD 5).

Conclusions: This prospective date reiterates that URS with Holmium laser lithotripsy is safe with good stone clearance. The larger stones require more than one procedure and complications rate are acceptable.

MP29-11 The efficacy of the disposable flexible ureterorenoscopy $\left(\right.$ Lithovue $\left.^{\circledR}\right)$ for renal stone smaller than $2 \mathrm{~cm}$.

I Seo, J Lee, S Park, T Oh

Wonkwang University School of Medicine \& Hospital

Introduction \& Objective: To evaluate the efficacy of the disposable flexible ureterorenoscopy $\left(\right.$ Lithovue $^{\circledR}$ ) for renal stone smaller than $2 \mathrm{~cm}$.

Methods: From January 2018 to December 2018, patients who underwent retrograde intrarenal surgery (RIRS) used by Litho$v^{\circledR}{ }^{\circledR}$ after diagnosing renal stone smaller than $2 \mathrm{~cm}$ at our hospital were included in study. Patients who did not have follow-up, or who did not have any evidence of stones free were examined through general urinalysis, plain radiography (KUB, DIP), and computed tomography were excluded. Stone free was defined as the absence of any residual disease, or a remnant of less than $3 \mathrm{~mm}$ without any symptoms.

Results: A total of 66 patients were enrolled in study. The mean age of the patients was $56.29 \pm 13.7$ years and the male to female ratio was $46(69.7 \%)$ to $20(30.3 \%)$. The mean stone size was $17.8 \pm 2.1 \mathrm{~mm}$ and mean Hounsfield Unit (HU) was 823.1 \pm 358.0 . The stone composition was calcium oxalate monohydrate (41, $62.1 \%)$, uric acid $(16,24.2 \%)$, carbonite apatite $(3,4.5 \%)$. The 3month stone free rate was $77.3 \%(51 / 66)$ and the mean remnant stone size was $6.14 \mathrm{~mm}$. The mean operation and laser lithotripsy time was $46.3 \pm 20.5$ and $8.9 \pm 10.7 \mathrm{~min}$. The mean duration of hospitalization was 2.1 days. Three patients experienced postoperative pyelonephritis.

Conclusions: Our results show that Lithovue ${ }^{\circledR}$ is effective treatment for renal stone smaller than $2 \mathrm{~cm}$ as like the results of reusable flexible ureterorenoscopy. Although our cases is small, if we experience more cases and overcome learning curve of Lithovue $^{\circledR}$, we will have a higher success rate for renal stone smaller than $2 \mathrm{~cm}$.

MP29-12 Developing a free three-dimensional tool for kidney stones' surgical planification: "calculator"

F Panthier, S Doizi, L Berthe, O Traxer

Service d' Urologie, Hospital Tenon, APHP

Introduction \& Objective: Flexible ureteroscopy is a common surgical procedure in urology. Its duration may vary from a few 
minutes to one and a half hour. Beyond, practitioners are told to interrupt the procedure and plan a second intervention. We aimed to develop a three-dimensional (3D) tool for kidney stones' surgical planification.

Methods: 3DSlicer is a free open-source software platform for medical image computing (DICOM) and 3D visualization. Both MATLAB and Python scripting are available to develop new modules, using the "Extension Developing tool". Major developing criteria were: suited for CT-scan DICOM series, definition of a region of interest (ROI), stone single- or multiple-detection based on Hounsfield Units, 3D visualization. Minor criteria were available expected duration of lithotripsy beyond laser source (Holmium:YAG or Superpulse Thulium Laser Fiber), core-diameter laser fibers and laser settings. Module should succeed to measure a known volume and a clinical situation.

Results: Calculator is a Python scripted module, available on the "Extension Manager" in 3DSlicer. Before using it, definition of a ROI is required with "Crop Volume" module. ROI is adjustable upon axial, sagittal and coronal views of a nonenhanced CT-scan DICOM series. From ROIs, Calculator defines a adjustable segmentation based on thresholds to detect stones. «Split islands into segments » option is useful in case of multiple stones but requires a minimal size criteria (voxel). Various laser pre-settings are available to calculate lithotripsy duration (Figure 1). 3D view is optional in order to maximize computing time. Final view contains axial, sagittal and coronal views, with segment's volume and lithotripsy duration among chosen laser settings. Figure 1 shows an in vitro example of a $10 \mathrm{~mm}$ begostone cube measurement and a clinical example of a single kidney stone measurement and expected lithotripsy duration.

Conclusions: Calculator is a free 3DSlicer module that provides stones volumes and expected lithotripsy duration among laser

Figure 1a: Example from a non-enhanced CT-scan of a begostone volumetric measure. Stone phantom is a $10 \mathrm{~mm}$-cube.

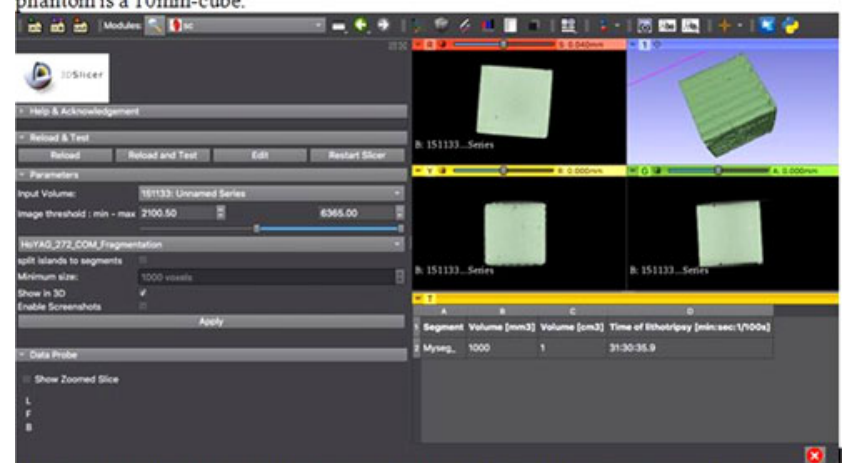

Figure 1b: Example from a non-enhanced CT-scan of a single stone volumetric measure in the left renal pelvis et treated according to dusting settings $(0,5 \mathrm{~J}-30 \mathrm{~Hz})$ with Holmium : YAG the left 1 .
laser.

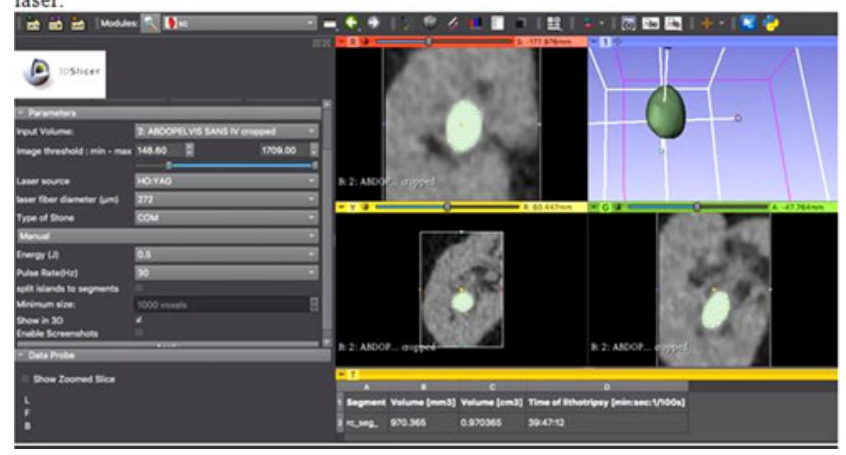

settings, based on segmentation principle with CT-scan DICOM series. Surgical planification should be useful in kidney stone procedures. Further clinical studies are needed to assess volumetric evaluation as the gold-standard.

MP29-13 Durability of fiber optic reusable flexible Ureterorenoscopes :Retrospective analysis after 313 consecutive cases of Retrograde Intra Renal Surgery ; the lesson learned

\section{Y KK, S Prabhakar}

Renai Medicity Multi Super Specialty Hospital, Kochi, Kerala, India

Introduction \& Objective: To evaluate the durability of fiber optic reusable flexible Ureterorenoscopes (fURSs) and the factors affecting it and to suggest possible preventive measures to increase the scope life.

Methods: All patients who were treated for renal or upper ureteric calculi using fURS were retrospectively studied. Study period was from Jan 2016 to Sep 2018 in a single center. Data on patient characteristics, stone size, density and location, presence of a prior DJ stent, total endoscopy time, the number of fURS passages through the ureteric access sheath (UAS), the number of passages of accessory devices through the working channel (WC), need for lower pole access and lasing and type and severity of scope damage were collected. The primary endpoint for each scope was its irreparable damage. Scope durability was measured as the total number of cases per scope and total ureteroscopy time before damage.

Results: Retrograde Intra Renal Surgery (RIRS) was performed in 266 consecutive patients (total of 313 renal stone units including 47 bilateral cases) using four different fURSs (one URFP6, one Flex x2 and two Flex x2S). Lithotripsy was done with $200 \mu \mathrm{m}$ fiber using Holmium Laser $(100 \mathrm{~W})$. A 9.5 or $12 \mathrm{~F}$ UAS was routinely used. Active stone extraction of all the fragments with a $2.2 \mathrm{~F}$ basket were done to achieve maximum stone free rate. The method of sterilization was complete immersion in Peracetic acid. Mean age of the patient was 50.4 years (07-86). Mean stone size was $16.97 \mathrm{~mm}(02-55 \mathrm{~mm})$. The average number of procedures per scope was 78.25 (37-126). Most durable scope in our series was Flex-X2 (122hrs). Most common cause of irreparable scope damage was deflection unit failure followed by fiberoptic bundle breaks. Total flexible ureteroscopy time (mean 60.29 minutes), the number of fURS passages through UAS (mean 38.69) and the number of passages of accessory devices through the WC (mean 5.05) were found to be statistically significant $(p<0.001)$. Limitations of this study were single center analysis, retrospective design, three different fURS models and surgeons.

Conclusions: The durability of fURSs in our series is was up to 78 cases per scope. The deflection unit was the most fragile part of the fURS. Decreasing total scope time and passage of scope through UAS and limiting passage of accessories through WC will maximize the overall durability of a fURS.

MP29-14 Donor and post-transplant ureteroscopy for stone disease in patients with renal transplant - Evidence from a systematic review

T Reeves, A Pietropaolo, BK Somani, H Ho 
Introduction \& Objective: Renal transplant remains the gold standard treatment for end stage kidney disease. Urolithiasis was traditionally considered a contraindication to transplant and a cause of rare but significant morbidity in the post-transplant setting. With advancements in technology ureteroscopy (URS) is increasingly performed to treat urolithiasis associated with renal transplant. Here we review the challenges and complications of pre and post-transplant URS.

Methods: A Cochrane style review was performed in accordance with the Preferred Reporting Items for Systematic Reviews and Meta-analyses guidelines to evaluate the outcomes of donor and post-transplant URS for stone disease, including all English language articles between January 1996 and December 2018.

Results: Eighteen articles (167 patients), seven ex-vivo or donor URS and 11 post-transplant URS met inclusion criteria and were included in the review. A pre-transplant URS showed a stonefree rate (SFR) of $100 \%$ with an overall complication rate of $7.5 \%$ (four Clavien I and one Clavien $\geq 3$ ), where as a posttransplant URS showed SFR of $100 \%$ in five studies and 60-91\% in four studies with an overall complication rate of $12.9 \%$ (10 Clavien I and three Clavien $\geq 3$ ).

Conclusions: Advancements in endourological technique have made URS for donor and post-transplant urolithiasis a safe and effective procedure. Although data were based on small retrospective caser series, it shows that in experienced centers it can be performed with low morbidity and a high SFR.

\section{MP29-15 Reporting outcomes of a successful treat- ment in Ureteroscopy and Retrograde intrarenal surgery for stones: The STONE-FECTA.-A Single center experience.}

\author{
N Iqbal, A Hasan, M Mughal, S Akhter, J Rahim \\ Pakistan Kidney and Liver Institute and Research Center
}

Introduction \& Objective: To adopt a new method for reporting outcomes during URS/RIRS for stones, the SToNE-FECTA (Successful Treatment in Endourology) concept at our center. Its said in recent literature that endourological procedures outcomes should be seen as composite in terms of stone free status and the absence or presence of minor/major complications. It's helpful in providing patients with counselling regarding the composite outcomes.

Methods: From April 2018 till September 2018, total of 210 consecutive patients underwent URS/RIRS by 2 well experienced endourologists at hospital. These patients were prospectively analyzed. Those Patients who achieved simultaneously a stone free rate in single procedure, absence of urinary infections and having no urological complications were considered to have achieved the SToNE-fecta. Continuous and categorical variables were showed as mean, standard deviation (SD) and frequencies, percentages $(\%)$ respectively. For the statistical univariate analysis Kruskal-Wallis, chi-squared and Fisher exact tests, were used to compare continuous and categorical variables as needed. A multivariable logistic regression model was prepared to assess independent factors responsible for achieving SToNE-fecta. A p value $<0.05$ was considered statistically significant. All statistical analyses were performed using SPSS v.16.0 (SPSS Inc, Chicago, IL, USA).

Results: Single-procedure stone free rate was seen in $186 / 210$ (89\%), absence of UTI in 206/210 (98.10\%), and no procedural urological complications rates in 198/210 (94.3\%) at a median follow-up of 2 months. SToNE-fecta rate at 2 months was $87.4 \%$.
On multivariable logistic regression analysis, Stone length and width, prior urinary tract infection and impacted nature of stones were independently related with the SToNE-fecta.

Conclusions: We concluded that adopting SToNE-fecta outcomes helps manage patients' and surgeon expectations post URS/RIRS procedures. This new predictive model may be useful tool for a composite counseling of patients and can be considered for the improvement of standardized quality assessment during the residents training as well.

\section{MP29-16 Intramuscular Ketorolac Administered at Time of Stent Removal Decreases Pain-related Return Visits in a Randomized Control Trial}

BA Johnson, I Sorokin, RL Steinberg, JA Antonelli, M Pearle

University of Texas Southwestern

Introduction \& Objective: Ureteroscopy (URS) for stones has been associated with significant post-operative morbidity, thought to be largely due to symptoms associated with stent placement. Furthermore, in some patients stent removal precipitates debilitating renal colic that may last for hours following stent removal. We sought to determine whether intramuscular (IM) ketorolac given at the time of stent removal reduces the occurrence of post-stent removal renal colic.

Methods: With institutional review board approval, we performed a prospective, randomized, double-blind, placebocontrolled trial assessing the effects of IM ketorolac administered at the time of office stent removal. We included patients with and without stent tethers after undergoing URS for stones. Patients were randomized via random sequence generator to receive an $\mathrm{IM}$ injection of $30 \mathrm{mg}$ in $1 \mathrm{~mL}$ of ketorolac or $1 \mathrm{~mL}$ of $0.9 \%$ saline immediately prior to office stent removal. Injections were drawn up by a research nurse, and the surgeon, nurse administering the injection, and patient were blinded. Demographic

\begin{tabular}{|c|c|c|c|c|}
\hline & Group & $\begin{array}{l}\text { Control } \\
\text { Group } \\
(n=62)\end{array}$ & $\begin{array}{c}\text { Treatment } \\
\text { Group } \\
(n=62)\end{array}$ & p-value \\
\hline \multirow{10}{*}{ 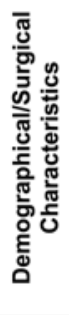 } & \multirow{6}{*}{$\begin{array}{l}\text { Age } \\
\text { Female } \\
\text { Mean BMI } \\
\text { Mean ASA } \\
\text { Mean stone size } \\
\text { Location }\end{array}$} & 56 & 54 & NS \\
\hline & & $45 \%$ & $50 \%$ & NS \\
\hline & & 28.9 & 30.1 & NS \\
\hline & & 2.2 & 2.3 & NS \\
\hline & & 9.1 & 8.9 & NS \\
\hline & & & & NS \\
\hline & Renal & $52 \%$ & $46 \%$ & \\
\hline & Ureteral & $19 \%$ & $26 \%$ & \\
\hline & Both & $29 \%$ & $28 \%$ & \\
\hline & Mean stent duration & 10.2 & 9.0 & NS \\
\hline \multirow{7}{*}{ 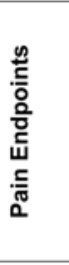 } & & 2.7 & 2.4 & NS \\
\hline & $\begin{array}{l}\% \text { pts with VAS } \geq 7 \text { at } 24 \\
\text { hours }\end{array}$ & $14 \%$ & $17 \%$ & NS \\
\hline & \multirow{2}{*}{$\begin{array}{l}\text { Subjective renal colic } \\
\text { Narcotic use at } 24 \text { hours }\end{array}$} & $16 \%$ & $10 \%$ & NS \\
\hline & & $27 \%$ & $26 \%$ & NS \\
\hline & Mean Vas 7 Days & 0.5 & 0.9 & NS \\
\hline & \multirow{2}{*}{$\begin{array}{l}\text { Mean days missed work (if } \\
\text { working) }\end{array}$} & $2 \%$ & $3 \%$ & NS \\
\hline & & 3.2 & 3.8 & NS \\
\hline \multirow{3}{*}{ 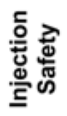 } & \multirow{3}{*}{$\begin{array}{l}\text { Injection site reaction } \\
\text { Injection site pain } \\
\text { Injection complication }\end{array}$} & $0 \%$ & $0 \%$ & NS \\
\hline & & $2 \%$ & $2 \%$ & NS \\
\hline & & $0 \%$ & $0 \%$ & NS \\
\hline$\stackrel{v}{\underline{L}}$ & Return to clinic or ED & $\begin{array}{c}8 / 62 \\
(13 \%)\end{array}$ & $\begin{array}{l}1 / 62 \\
(2 \%)\end{array}$ & 0.032 \\
\hline
\end{tabular}


and operative data were collected prospectively. Patients were contacted 24 hours and 7 days following stent removal to assess level of pain using the visual analog pain scale (VAS); the occurrence of renal colic symptoms; the use of narcotics; Emergency Department (ED) or urgent clinic visits; or the need for surgical/medical interventions related to renal colic.

Results: A total of 124 patients (62 patients in each group) were included. Demographics, stone size/location, and stent duration were similar between groups. No difference between the groups was detected in VAS pain scores or number of patients with severe $(\geq 7)$ pain. Need for narcotic pain medication within 24 hours of stent removal was similar between groups. There were no injection site reactions or complications. However, ketorolac resulted in significantly fewer renal colic-related ED/clinic visits in the treatment group (2\%) versus the control group (13\%) $(\mathrm{p}=0.032)$ (table).

Conclusions: Although administration of ketorolac prior to stent removal does not reduce overall post-stent removal pain, it does reduce the likelihood of severe renal colic requiring ED or office visits. Patients may benefit from routine use of ketorolac at the time of stent removal. Further evaluation of risk stratification may identify patients most likely to benefit from ketorolac.

MP29-17 Intrarenal arteriovenous malformation following flexible ureterorenoscopy and holmium laser stone fragmentation: report of a case

A Bashar, F Hammad

Salford Royal NHS Foundation Trust

Introduction \& Objective: Flexible ureterorenoscopy (FURS) and holmium laser lithotripsy is currently considered as one of the treatment options for large renal calculi. It has been shown to be safer than percutaneous nephrolithotomy. The latter can sometimes be complicated by the formation of intrarenal arteriovenous malformation (AVM). AVM is extremely rare following FURS and laser lithotripsy. Indeed, only one case has been reported on reviewing the literature up to June 2018. We report on the second case illustrating the possibility of developing this major complication following this procedure.

Methods: A 79 years old diabetic and hypertensive male with stage-4 chronic kidney disease who previously had left extracorporeal shockwave lithotripsy and FURS with Holmium laser lithotripsy, presented with bilateral large renal calculi. He un-

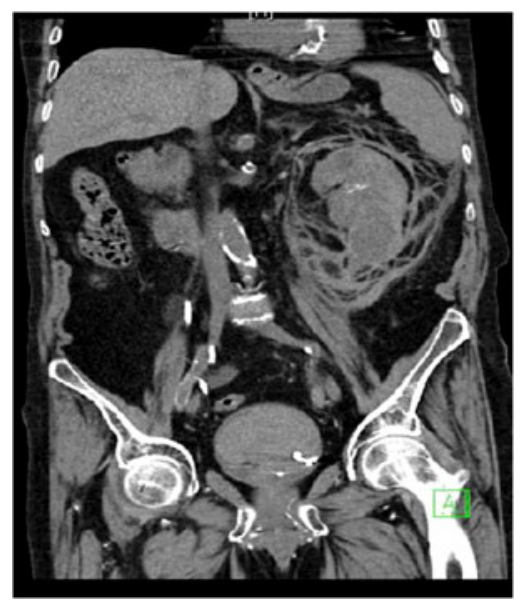

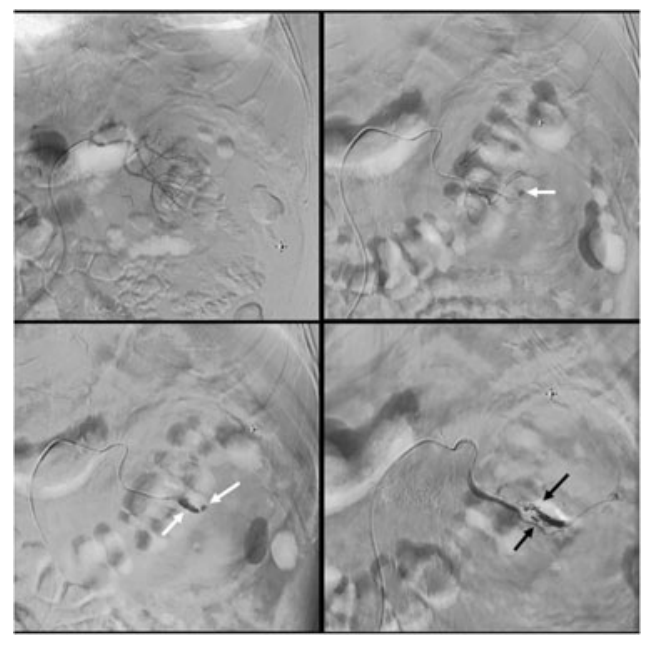

derwent simultaneous bilateral FURS and Holmium laser lithotripsy and was discharged home the following day with almost clear urine. Four days post-discharge, he presented with gross hematuria for which he required hospitalization and blood transfusion. CT scan demonstrated left subcapsular, perinephric and retroperitoneal hematoma. Angiography showed contrast extravasation from pseudoaneurysms in two small branches of left renal artery. Both were selectively embolized with microcoils and this led to the cessation of the hematuria.

Results: Despite the relative safety of FURS and Holmium laser lithotripsy, it can be associated with major complications like intrarenal AVM. This can probably be prevented by careful and judicious use of laser energy in patients with large stone burden and premorbid conditions.

Conclusions: Despite the relative safety of FURS and Holmium laser lithotripsy, it can be associated with major complications like intrarenal AVM. This can probably be prevented by careful and judicious use of laser energy in patients with large stone burden and premorbid conditions.

MP29-18 S.T.O.N.E nephrolithometry scoring system vs. Guy's scores for predicting stone-free status post sheathless retrograde intrarenal surgery: Which is better?

SN Mahmood, AA Saeed, HM Toffeq, SS Fakhraldin

University of Sulaimania, College of Medicine, Department of Surgery

Introduction \& Objective: Introduction: In the past few years, various nomograms and scoring systems have been used to estimate the SFR after RIRS, However, neither of these scoring systems can precisely and easily anticipate the outcomes of the procedure Objective: The objective of the present study was to evaluate and compare the predictive efficiency of S.T.O.N.E and Guy's scores in stone-free rates post sheathless retrograde intrarenal surgery (RIRS).

Methods: Between May 2016 and August 2018, 187 patients with renal stones $<2 \mathrm{~cm}$ who were treated with sheathless retrograde intrarenal surgery were included in the study. The S.T.O.N.E score and Guy's Stone Score (GSS) grades were based on preoperative non-contrast computed axial tomography (NCCT). RIRS procedures were done using a standard sheathless technique. 
Table 1: S.T.O.N.E score

\begin{tabular}{|l|c|c|c|}
\hline \multicolumn{1}{|c|}{ Features } & 1 Point & 2 Points & 3 Points \\
\hline (S)ize & $<10 \mathrm{~mm}$ & $10-15 \mathrm{~mm}$ & $>15 \mathrm{~mm}$ \\
\hline (T)opography & pelvis & $\begin{array}{c}\text { Upper pole or mid } \\
\text { pole }\end{array}$ & Lower pole or multiple calices \\
& & The preoperative stent or \\
no Hydronephrosis & Mild to moderate & Moderate to severe \\
& 1 Ho)bstruction & 2 stonephes & Hydronephrosis \\
\hline (N)umber & $<750 \mathrm{HU}$ & $750-1000 \mathrm{HU}$ & $>1000 \mathrm{HU}$ \\
\hline (E)valuation of HU & & & \\
\hline
\end{tabular}

Table 2: Patients demographic and perioperative characteristics

\begin{tabular}{|l|l|}
\hline Variables & Mean \pm SD $(\%)$ \\
\hline Patient Age (Means \pm SD) (ranges) & $41+\_13.86(3-70)$ years \\
\hline $\begin{array}{l}\text { Gender \% } \\
\text { Male } \\
\text { Female }\end{array}$ & $115(61.5)$ \\
\hline $\begin{array}{l}\text { Stone side \% } \\
\text { Left side } \\
\text { Right side } \\
\text { Bilateral }\end{array}$ & $72(38.5)$ \\
\hline Stone size (mm $\left.{ }^{2}\right)$ & $104(55.6)$ \\
\hline HU & $82(43.9)$ \\
$0-750$ & $1(0.5)$ \\
$750-1000$ & $12.44 \pm 3.48 \mathrm{~mm}$ \\
$>1000$ & $79(42.4 \%)$ \\
\hline Operative time (min) & $59(31.5 \%)$ \\
\hline Lasing time (min) & $49(26.2 \%)$ \\
\hline $\begin{array}{l}\text { Stone free \% } \\
\text { Complete free }\end{array}$ & $34.9 \pm 14.05$ \\
\hline Residual & $22.68 \pm 10.66$ \\
\hline Post-operative hospital stays (hours) & $160(85.5)$ \\
\hline HU: Hounsfield units, SD; Standard deviation & $27(14.5)$ \\
\hline
\end{tabular}

Table 3: Patient distribution and stone-free status according to guy's and STONE nephrolithometry scoring systems

\begin{tabular}{|l|c|c|c|}
\hline \multicolumn{1}{|c|}{ Nephrolithometry scoring } & Number of patients & Number of stone-free patients (\%) & $p$ \\
& $\%$ & & \\
& & & \\
\hline S.T.O.N.E scoring system & 26 & $26(100 \%)$ & \\
Grade 1 (5-6) & 67 & $64(95 \%)$ & 0.0198 \\
Grade 2 (7-8) & 94 & $82(87 \%)$ & \\
Grade 3 (9-13) & & & \\
& 112 & $108(96 \%)$ & 0.040333 \\
\hline The Guy's scoring system & 69 & $59(85 \%)$ & \\
Grade 1 & 6 & $5(83 \%)$ & \\
Grade 2 & 0 & 0 & \\
Grade 3 & & & \\
Grade 4 & & & \\
\end{tabular}

Table 4: comparison between S.T.O.N.E. score and Guys stone score system in predicting stone free rate.

\begin{tabular}{|c|c|c|c|}
\hline variable & Stone free & Residual calculi & $P$ \\
\hline S.T.O.N.E. score (mean \pm SD) & $8.52 \pm 1.77$ & $9.64+\_1.54$ & 0.023 \\
\hline Guys stone score(mean \pm SD) & $1.40 \pm 0.54$ & $1.69+\_0.48$ & 0.064 \\
\hline
\end{tabular}

Results: The present study population was comprised of 187 patients with a mean age of $41 \pm 13.86$ (range 3-70) years; the mean stone size was $12.44 \pm 3.48 \mathrm{~mm}$. The mean operative time was $34.9 \pm 14.05$ minutes, and the mean lasing time was $2.68 \pm 10.66$ minutes. The complete final stone-free rate was achieved in $173(85.5 \%)$ of patients, while in $27(14.5 \%)$ patients, residual stones $>4 \mathrm{~mm}$ in diameter were detected. Both
Table 5: The STONE scoring system variables and stone-free rates

\begin{tabular}{|l|c|l|l|}
\hline Variables & Number of patients & Number of stones-free patients (\%) & $p$ \\
\hline No. of stone & 121 & $114(94 \%)$ & \\
Single & 49 & $45(91 \%)$ & 0.215 \\
2 stone & 17 & $14(82 \%)$ & \\
3 stones and more & 39 & $38(97 \%)$ & \\
\hline Degree of hydronephrosis & 74 & $69(93 \%)$ & 0.390 \\
No & 58 & $52(89 \%)$ & \\
Mild & 16 & $12(75 \%)$ & \\
Moderate & 79 & $74(93 \%)$ & 0.618 \\
Severe & 59 & $52(88 \%)$ & \\
\hline Hounsfield unit & 49 & $40(81 \%)$ & 0.000 \\
$<750$ & 70 & $69(98 \%)$ & \\
$750-1000$ & 66 & $66(100)$ & \\
$>1000$ & 51 & $39(76 \%)$ & \\
\hline Stone location & & $44(100 \%)$ & \\
Pelvis & 120 & $114(95 \%)$ & \\
Mid or upper pole & 23 & $15(65 \%)$ & \\
Lower pole & & & \\
\hline Stone size & & \\
$<10 \mathrm{~mm}$ & & & \\
$10 \mathrm{~mm}-15 \mathrm{~mm}$ & & & \\
$>15 \mathrm{~mm}$ & & & \\
\hline
\end{tabular}

scores were significantly correlated with the stone-free rate of the procedure. When comparing both scoring systems, the accuracy of the S.T.O.N.E scores was higher than the Guy stone scores in predicting a stone-free result $(p=0.023$ vs. $p=0.064)$. Among several STONE variables, stone size and lower pole stones were significant predictors of the stone-free rate (SFR).

Conclusions: GSS and S.T.O.N.E scores are effective in predicting the stone-free rate, with the ST.O.N.E. score being more valid in predicting the success of RIRS. The S.T.O.N.E score is an ideal nomogram - simple, not time consuming and easily applied in daily practice.

MP29-19 Does the working channel position have a role on the efficacy of Ho:YAG laser lithotripsy? Results from a laboratory test

L Villa, E Ventimiglia, S Doizi, S PROIETTI, G Giusti, A Briganti, A Salonia, F Montorsi, O Traxer

Division of Experimental Oncology/Unit of Urology; URI; IRCCS Ospedale San Raffaele, Milan, Italy

Introduction \& Objective: To evaluate whether the position of the working channel affects the effectiveness of flexible ureteroscopy.

Methods: We compared Flex-X2S and Flex-XC (working channel at 9 and 3 o'clock position, respectively) in eight cavities of the K-Box simulating the distribution of right and left intrarenal calyces. In the first and second settings each cavity contained $1 \mathrm{~cm}$ stone lying on the bottom and fixed on the anterior sheet covering the box, respectively. In the third setting the posterior and lateral surface of each cavity was draped with graph paper. Once the f-URS entered each cavity, we measured and compared a) the proportion of stone surface targeted by the laser (STL) and b) the proportion of graph paper burned by the laser (PBL) obtained with the two instruments.

Results: 3 o'clock position obtained higher STLs and PBLs than 9 o'clock position in the right posterior and left anterior cavities (mean STL $\pm S D: 0.87 \pm 0.15$ vs. $0.46 \pm 0.38$ and $0.78 \pm 0.35$ vs. $0.43 \pm 0.24$, respectively, $\mathrm{p}<0.05$, Fig. 1 e Fig. 2 ), and on the right posterior surfaces of the middle-lower/lower cavities $(\mathrm{PBL}=$ 0.72 vs. $0.31 / 0.77$ vs. $0.35, \mathrm{p}<0.01$, Fig. 3 ) and on the left lateral surface of the lower cavities $(\mathrm{PBL}=0.45$ vs. $0.25, \mathrm{p}=0048$, Fig. 4), respectively. Conversely, 9 o'clock position provided 
higher STLs and PBLs than 3 o'clock position in the left posterior and right anterior cavities (mean STL \pm SD: $0.84 \pm 0.20$ vs. $0.65 \pm 0.28$ and $0.79 \pm 0.30$ vs. $0.44 \pm 0.35$, respectively, $p \leq 0.02$, Fig.1 and Fig.2), and on the left posterior and right lateral surfaces of the lower cavities (PBL $=0.59$ vs. 0.34 and 0.50 vs. 0.21 , $\mathrm{p} \leq 0.04$, Fig.3 and Fig.4), respectively.
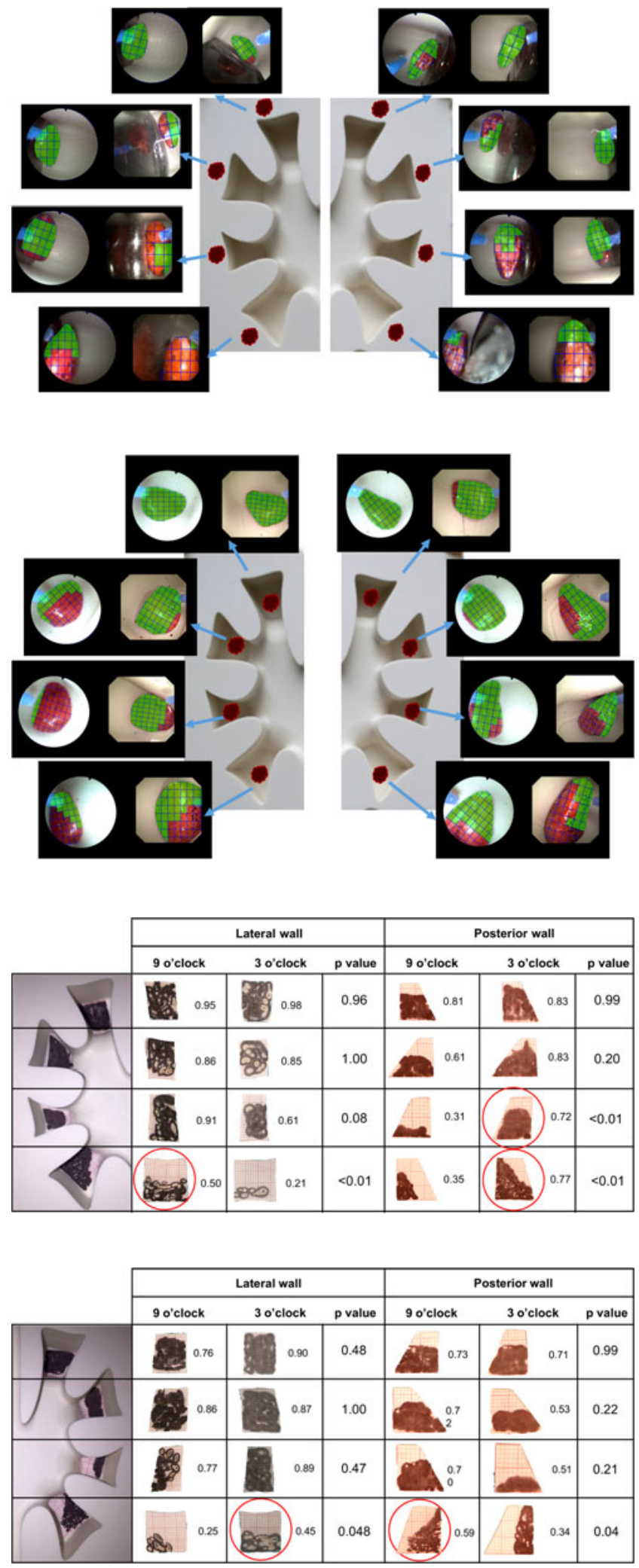

Conclusions: Kidney side and location of the calyx should be considered when choosing the instrument to be used for f-URS. The 9 o'clock position might provide better effectiveness than the 3 o'clock position in the right anterior and left posterior cavities. Conversely, the 3 o'clock position might perform better than the 9 o'clock position when accessing left anterior and right posterior cavities. These differences might be more evident in the lower pole.

MP29-20 S.O.L.V.E. nephrolithometry: a novel scoring system for predicting the stone-free rate of flexible ureteroscopy

S Luo, D Hu, W Tang

Department of Urology, The First Affiliated Hospital of Chongqing Medical University

Introduction \& Objective: To propose S.O.L.V.E. nephrolithometry scoring system, and to evaluate the predictive value of the novel scoring system for the stone-free rate (SFR) of flexible ureteroscopy (FURS).

Methods: A literature review was performed to identify the variables which could affect the SFR of FURS. Five reproducible variables were included in S.O.L.V.E. scoring system: stone surface area (S), obstruction(O), length of calyces funnel (L), visible number of calyces $(\mathrm{V})$ and essence of stone $(\mathrm{E})$. Variables were measured based on preoperative non-contrast computed tomography of urography. Clinical data of 439 patients who underwent FURS with holmium laser for upper urinary tract stones in our department were retrospectively analyzed from January 2017 to June 2018. The correlation of S.O.L.V.E. scoring system and SFR, postoperative hospital stay, surgical complications and operation time were analyzed. Receiver operating characteristic (ROC) curves were drawn to detect sensitivity and specificity of S.O.L.V.E. scoring system in predicting SFR of FURS.

Results: All cases FURS were performed successfully and the SFR was $75.6 \%$. The mean score was 6.3 (range 4-11). The patients were divided into low score (4-5) group, moderate score (6-8) group and high score (9-11) group due to S.O.L.V.E. scoring system. The SFR was $94.2 \%, 72.5 \%$ and $22.5 \%$, respectively $(\mathrm{p}<0.001)$. The area under receiver operating characteristic curve of S.O.L.V.E. score was 0.798 , higher than that of each variable in S.O.L.V.E. scoring system. Additionally, the score was correlated with the operation time $(\mathrm{p}<0.001)$, but not with postoperative hospital stay $(p=0.401)$, intraoperative bleeding $(\mathrm{p}=0.536)$ and postoperative infectious fever $(\mathrm{p}=0.763)$.

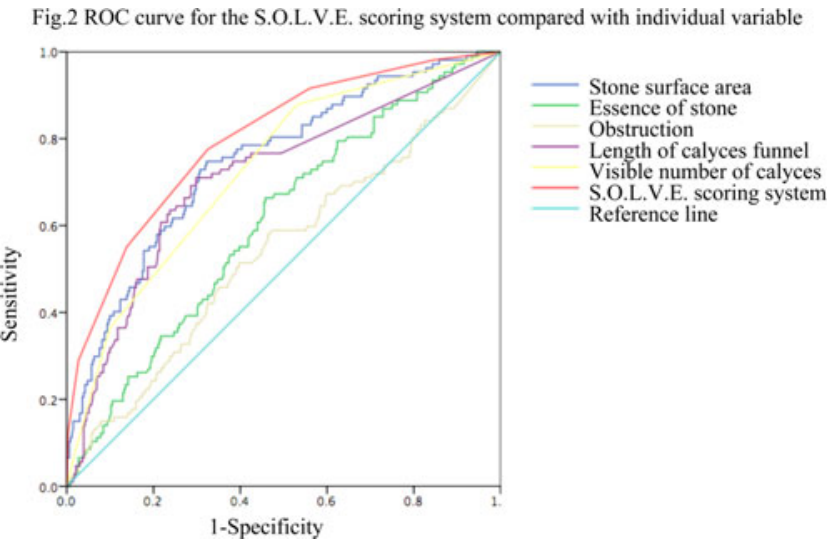




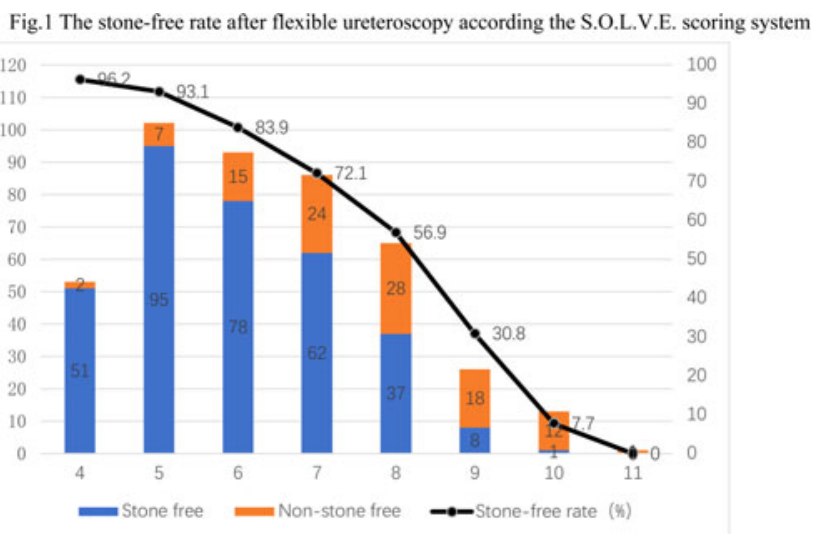

Conclusions: The S.O.L.V.E. nephrolithometry scoring system can predict stone free status after FURS. This novel scoring system can provide assistance of preoperative communication and making clinical decisions. Further prospective randomized studies with large samples are needed to validate and revise this system.

MP29-21 Treatment of isolated small renal stone leads to resolution of symptoms and should be routinely offered to patients: Outcomes (of stone $\leq 7 \mathrm{~mm}$ and $8-10 \mathrm{~mm}$ ) from a University Hospital

\section{A Pietropaolo, R Geraghty, BK Somani}

Introduction \& Objective: While treatment of large renal stone is recommended, treatment of smaller renal stone and the symptomatic value it offers to patients is often debated. We wanted to analyze prospective surgical outcomes for treatment of small renal stone and whether it leads to symptom resolution.

Methods: Over a period of 7-years (March 2012 to June 2018), a total of 860 patients underwent ureteroscopy and stone treatment. Of these $100(11.6 \%)$ had a single renal stone $\leq 10 \mathrm{~mm}$ (48 with stones $\leq 7 \mathrm{~mm}$ and 52 with stones $8-10 \mathrm{~mm}$ ). Data was collected and collated for patient and stone demographics, surgical outcomes and pre and post-operative symptoms.

Results: A total of 100 patients with small renal stone $\leq 10 \mathrm{~mm}$ underwent URS and stone treatment during the study period with a male: female ratio of 2:1 and a mean age of 50.8 years (range: 2-91 years). While patients with single renal stones were included, multiple stones or ureteric stones or patients with a prior stent in situ were excluded. Of the patients who had pain, UTI and hematuria, the symptoms resolved in majority of patients for both stones $\leq 7 \mathrm{~mm}$ and $8-10 \mathrm{~mm}$. The operative time and length of stay

\begin{tabular}{|c|c|c|c|}
\hline \multirow{2}{*}{$\mathrm{N}=100$} & \multicolumn{2}{|c|}{ Size, $\mathrm{mm}$} & \multirow{2}{*}{$P$ (test) } \\
\hline & $7, \mathrm{n}=48$ & $8-10, n=52$ & \\
\hline Stone Free, n (\%) & $47(98 \%)$ & $49(94.0 \%)$ & 0.196 \\
\hline Complications, n (\%) & $\begin{array}{c}2(4 \%) \\
2 \text { stent pain readmission- } \\
\text { Clavien I }\end{array}$ & $\begin{array}{c}3(5.7 \%) \\
1 \text { urosepsis - Clavien II } \\
1 \text { bladder washout - Clavien } \\
\text { IIla } \\
1 \text { respiratory sepsis - } \\
\text { Clavien IVa }\end{array}$ & 0.679 \\
\hline $\begin{array}{l}\text { Mean Operative time, mins } \\
\pm \text { SD }\end{array}$ & $30.34 \pm 13.44$ & $43.91 \pm 20.71$ & $<0.001$ \\
\hline Day-case, $\mathrm{n}(\%)$ & $37(77.0 \%)$ & \begin{tabular}{|l}
$30(58.6 \%)$ \\
\end{tabular} & 0.048 \\
\hline Symptom & Resolutio & of symptom & $\begin{array}{l}\text { Overall symptom } \\
\text { resolution }\end{array}$ \\
\hline Asymptomatic $(\mathrm{n}=10)$ & $\begin{array}{l}n=6 / 6(100 \%) \\
n=19 / 21(90 \%)\end{array}$ & $\begin{array}{r}\mathrm{n}=4 / 4(100 \%) \\
\mathrm{n}=33 / 35(94 \%)\end{array}$ & $\begin{array}{l}100 \% \\
94.6 \%\end{array}$ \\
\hline $\begin{array}{l}\text { Pain }(\mathrm{n}=56) \\
\text { UTI }(\mathrm{n}=18)\end{array}$ & $\begin{array}{c}\mathrm{n}=19 / 21(90 \%) \\
\mathrm{n}=7 / 9(77 \%)\end{array}$ & $\begin{array}{c}\mathrm{n}=33 / 35(94 \%) \\
\mathrm{n}=8 / 9(89 \%)\end{array}$ & $\begin{array}{l}94.6 \% \\
83.3 \%\end{array}$ \\
\hline Pain + UTI (n=6) & $\mathrm{n}=1 / 1(100 \%)$ & $n=5 / 5(100 \%)$ & $100 \%$ \\
\hline Pain + Haematuria $(n=10)$ & $\mathrm{n}=6 / 6(100 \%)$ & $n=4 / 4(100 \%)$ & $100 \%$ \\
\hline
\end{tabular}

was both longer for larger renal stones $(p<0.001$ and $p=0.048$ respectively). Conversely, the stone free rate and complication rates were not different between the different stone sizes.

Conclusions: Treatment of small renal stones $(\leq 10 \mathrm{~mm})$ is safe and leads to complete resolution of preoperative symptoms in majority of patients. The patients should therefore be counselled, and an informed decision making should be done for treatment of these stones.

MP29-22 Retrograde ureteroscopy versus antegrade mini percutaneous flexible ureteroscopy for treating impacted proximal ureteric stones $\geq 10 \mathrm{~mm}$

O Elgebaly, H Abdeldaiem, F Idris, A Zahran

Urology Department, Alexandria University

Introduction \& Objective: To prospectively compare between the safety and efficacy of retrograde ureteroscopy and antegrade mini percutaneous ureteroscopy in treating impacted proximal ureteric stones $\geq 10 \mathrm{~mm}$.

Methods: The study was conducted on 60 patients admitted to the Urology Department in Alexandria Main University Hospital, presenting with impacted proximal ureteric stone $\geq 10 \mathrm{~mm}$. Patients were randomly divided into two groups, Group A were treated with retrograde ureteroscopy using semirigid or flexible ureteroscope going up to the stone, while Group B were treated by antegrade miniperc ureteroscopy, were a $14 \mathrm{~F}$ renal tract was obtained to pass a ureteric access sheath, then flexible ureteroscope was used going from upside downwards to the stone. Holmium laser was used for stone fragmentation. DJ was inserted in all cases. Follow up with non-contrast CT was performed after one month.

Results: Mean stone size was comparable in both groups. Stone free rate was significantly higher in group B (73.3\%) than group A $(60 \%)$. Mean operative time was significantly shorter in group A $62.8 \pm 20.1 \mathrm{~min}$ versus group B $111 \pm 16.6 \mathrm{~min}$, while mean lithotripsy time was comparable in both groups. The mean radiation exposure time was significantly less in group A $37 \pm 11.9 \mathrm{sec}$ compared to group B $195 \pm 45.1 \mathrm{sec}$. Both groups were comparable concerning minor complications with no major complications.

Conclusions: Antegrade miniperc flexible ureteroscopy is more effective than retrograde ureteroscopy in treating large impacted proximal ureteric stones.

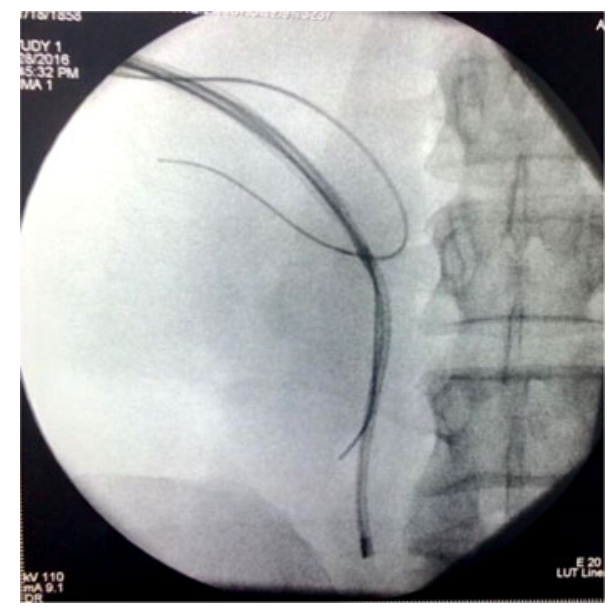




\section{MODERATED POSTER SESSION 30: CLINICAL STONES: URETEROSCOPY (III)}

MP30-01 Does Previous History with Urosepsis Predict the Likelihood of Urosepsis Post-Ureteroscopy

OA Ramadan, J Dorrell, SS Kommu, J Hale, P Keirandish, R Krishnan, N Shrotri

Kent \& Canterbury Hospital. East Kent Hospitals NHS Foundation Trust

Introduction \& Objective: There is a paucity of information to predict the likelihood of urosepsis after definite ureteroscopy in the cohort of patients who initially present with urosepsis secondary to obstructive calculi and managed with urgent stent/nephrostomy. This also applies to those who had positive urine culture and treated successfully with antibiotics prior definitive ureteroscopy. The aim of this study was to predict the likelihood of urosepsis after definitive treatment in this cohort of patients. Herein, we present our findings.

Methods: This retrospective study included 100 patients identified from our hospital database who underwent ureteroscopy for stone fragmentation during in the period of January 2016 to January 2017. Inclusion criteria was (1) patients who had previous history of urosepsis due to obstructive stones, (2) documented positive urine cultures and (3) patients who had been treated successfully with antibiotics before the elective definitive ureteroscopy. A matched-pair analysis was performed using three parameters (age, gender and race,). Fifty patients satisfied the inclusion criteria. This was compared to a matched cohort of patients who had neither previous history of urosepsis nor isolated positive urine cultures prior to the elective ureteroscopy.

Results: The study included 100 patients, 31 females (31\%) and 69 males (69\%) with median age 67.5 (range 21-89). Patients with previous history of sepsis had similar patient characteristics and stone free rates ( $80 \%$ vs $77 \%$ ) compared to patients without previous history of sepsis $(\mathrm{p}>0.05)$. Patients with previous history of sepsis had higher rates of post URS sepsis (14\% vs 4\%) compared to those who had not $(\mathrm{p}<0.05)$. [Table 1].

\begin{tabular}{|c|c|c|}
\hline Patients who developed Sepsis post URS: & $\begin{array}{l}\text { History of sepsis and UTI } \\
7 \text { patients (14\%) }\end{array}$ & $\begin{array}{l}\text { No history of sepsis nor UTI } \\
2 \text { patients }(4 \%)\end{array}$ \\
\hline $\begin{array}{l}\text { Sepsis grade: } \\
\text { - SIRS: confirmed by (>38 } \\
\text { Temp, WBC >12000, HR } \\
>90) \\
\text { - Sepsis: the above + } \\
\text { confirmed sepsis on } \\
\text { Blood and urine culture } \\
\text { - Severe sepsis: } \\
\text { confirmed by ( SBP< } \\
90 \mathrm{mmHg}, \mathrm{LDH}>4 \mathrm{mmol})\end{array}$ & $\begin{array}{l}4(57.14 \%) \\
2(28.57 \%) \\
1(14.3 \%)\end{array}$ & $\begin{array}{c}2(100 \%) \\
- \\
-\end{array}$ \\
\hline $\begin{array}{l}\text { Urinary tract decompression } \\
\text { before elective URS: } \\
\text { - Ureteric stent } \\
\text { - Nephrostomy } \\
\text { - Ante grade stenting }\end{array}$ & $\begin{array}{c}5(71.43 \%) \\
1(14.3 \%) \\
1(14.3 \%)\end{array}$ & $\begin{array}{c}2(100 \%) \\
- \\
-\end{array}$ \\
\hline Mean Operative time: & $67.42 \mathrm{~min}$ & $48.5 \mathrm{~min}$ \\
\hline
\end{tabular}

Conclusions: Previous history of urosepsis predicts the likelihood of further urosepsis after definitive treatment with ureteroscopy. Patients who initially present with urosepsis who then proceed to definitive management are at higher risk of further episodes of urosepsis as a result of definitive ureteroscopy. These patients should be managed with close liaison with the microbiologist and appropriate mechanisms place in preparation. Other factors that seemed to predict the risk are operative times. Further large scale multi-centered studies are warranted to develop risk stratification models.

MP30-02 Efficacy and outcomes of ureteroscopy and Holmium laser on calculus anuria by ureteric stones: A single center experience

JA Kalathia, C Saurabh, S Agarwal, R Agarwal

Fortune Urology Clinic

Introduction \& Objective: To present safety, efficacy and outcomes of ureteroscopic lithotripsy (URSL) via Holmium laser on recovery of renal function in adult patients with calculus anuria due to ureteric calculi.

Methods: Between April-2013 to March-2015, 37 patients of calculus nephropathy were treated, out of which $17(45.94 \%)$ (10 males and 7 females) had calculus anuria with mean age of 46.35 years. Fourteen $(82.35 \%)$ patients had bilateral ureteric stones and $3(17.64 \%)$ had unilateral calculi with solitary functioning kidney. Serum creatinine was $5.8-21.6 \mathrm{mg} \%$ (mean $12.5 \mathrm{mg} \%$ ) and hyperkalemia (ten patients) was between 5.5 $7.1 \mathrm{mmol} / \mathrm{L}$. Preliminary percutaneous nephrostomy, Double-J stenting and hemodialysis was performed in 7 (41.17\%) and 6 $(35.29 \%)$ and $4(23.52 \%)$ patients respectively. Twenty-nine URSL with holmium laser performed using $230 \mathrm{~mm} / 365 \mathrm{~mm}$ fibre with energy setting $1.2 \mathrm{~J}$ and frequency $8-10 \mathrm{~Hz}$. Outcomes in terms of stone free rate and recovery in renal function were evaluated.

Results: Mean follow-up time was 6 months. 16 patients (29 procedure) successfully managed with URSL laser lithotripsy and one patient was converted to open ureterolithotomy due to severe kinking of ureter. Mean operative-time was 52 min (28-80) with stone free rate $94.8 \%$. Post-operative complications of mild hematuria and fever occurred in $10(58.82 \%)$ and $3(17.64 \%)$ respectively. Complete recovery, improvement in renal function and no recovery occurred in $14(82.35 \%), 2(11.76 \%)$ and 1 (5.88) patients respectively. Conclusions: URSL with Holmium laser is safe, effective, minimally invasive treatment in compromised renal function with high success rates and minimal morbidity.

MP30-03 Is the use of a ureteral access sheath necessary for maintaining safe irrigation temperatures during flexible ureteroscopy when a high power Holmium:YAG laser is used? Clues from an in-vivo experimental study

C Adamou, E Farsari, P Ntasiotis, A Vagionis, A Gerakaris, K Gkialas, GA Callas, K Pagonis, E Liatsikos, P Kallidonis

University of Patras 
Introduction \& Objective: To assess the effect of high power Holmium:YAG laser in the temperature of the irrigation fluid under different ureteral access sheath (UAS) configurations during flexible ureteroscopy in a porcine model.

Methods: 22Fr percutaneous tracts were established in the upper calyces of the kidneys of a female pig. A custom-made balloon nephrostomy catheter containing a K-type thermocouple was inserted in the pelvicalyceal system. The thermocouple was connected to a data logger (TC08, Pico Technologies, UK) for real time temperature measurements. A Flex-X2 Ureteroscope (Karl Storz GmbH, Germany) was used with a continuous irrigation pump (Endoflow II, Rocamed SAM, Monaco) set at $100 \mathrm{mmHg}$ pressure. Ureteroscopy was performed without ureteral access sheath (UAS) and with a $10 / 12 \mathrm{Fr}$ or $12 / 14 \mathrm{Fr}$. A holmium:YAG laser (Moses Pulse 120H, Lumenis Ltd. Israel) was activated at powers of 20, 40 and $60 \mathrm{~W}$ for $60 \mathrm{sec}$, (when the
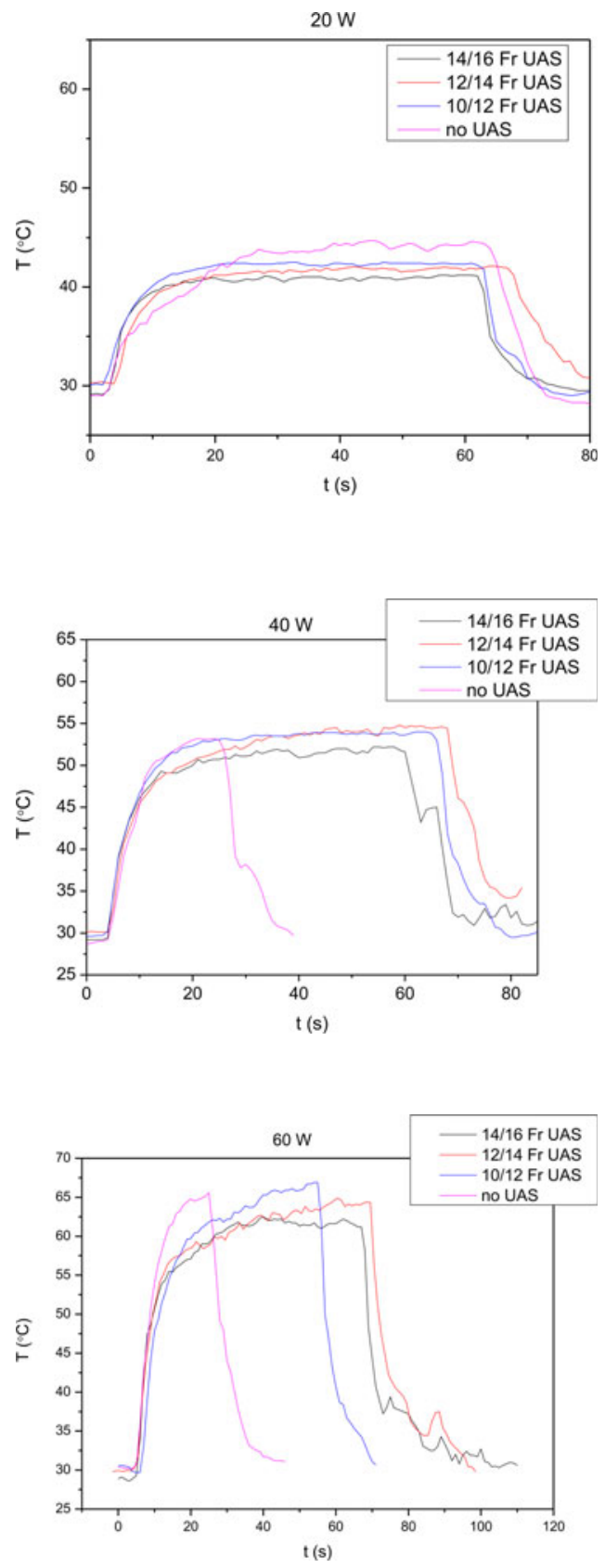

temperature exceeded $65^{\circ} \mathrm{C}$ the experiment was intentionally interrupted). Temperature measurements were obtained.

Results: When UAS was not used the respective highest temperatures developed in the irrigation fluid for 20,40 and $60 \mathrm{~W}$ were $45^{\circ} \mathrm{C}, 53.5^{\circ} \mathrm{C}$ and $65.5^{\circ} \mathrm{C}$ respectively. The respective figures for $10 / 12 \mathrm{Fr}$ were $42.5^{\circ} \mathrm{C}, 54^{\circ} \mathrm{C}$ and $67^{\circ} \mathrm{C}$. When a $12 / 14 \mathrm{Fr}$ UAS was used the highest temperatures developed were $41^{\circ} \mathrm{C}$, $55^{\circ} \mathrm{C}$ and $65^{\circ} \mathrm{C}$. When a $14 / 16 \mathrm{Fr}$ UAS was used the highest temperatures developed were $41^{\circ} \mathrm{C}, 52^{\circ} \mathrm{C}$ and $61.5^{\circ} \mathrm{C}$.

Conclusions: The invitro experiments showed that without the use of a UAS the temperature of the irrigation fluid rapidly increases under conditions of continuous flow with continuous pressure within the acceptable limits for renal intraparenchymal backflow.

\section{MP30-04 Pulse Modulation with Moses Technology Improves Popcorn Laser Lithotripsy}

K Black, A Aldoukhi, J Teichman, WW Roberts, T Hall, K Ghani

Department of Urology, Univeristy of Michigan

Introduction \& Objective: With the introduction of pulse modulation and the Moses technology, especially Moses Distance (MD) which is designed for lithotripsy at 1-2 mm distance, it is unclear if this affects fragmentation for popcorn laser lithotripsy. The aim of this in vitro study was to compare MD, with short pulse (SP) and long pulse (LP) modes, for popcorning based on fragment size distribution and imaging analysis of the fragmentation strike rate.

Methods: 10 BegoStones were fragmented in $127 \mathrm{~mm}^{3}$ spherical test-tubes using a 120W Ho:YAG laser (P120, Lumenis). A $230 \mu \mathrm{m}$ core fiber (Moses, Lumenis) was introduced through a ureteroscope (Lithovue, Boston Scientific) using a 3D positioning system and kept fixed $2 \mathrm{~mm}$ away from the stones. 20W settings ( $1 \mathrm{Jx} 20 \mathrm{~Hz}, 0.5 \mathrm{Jx} 40 \mathrm{~Hz}$ ) were assessed using SP, LP and

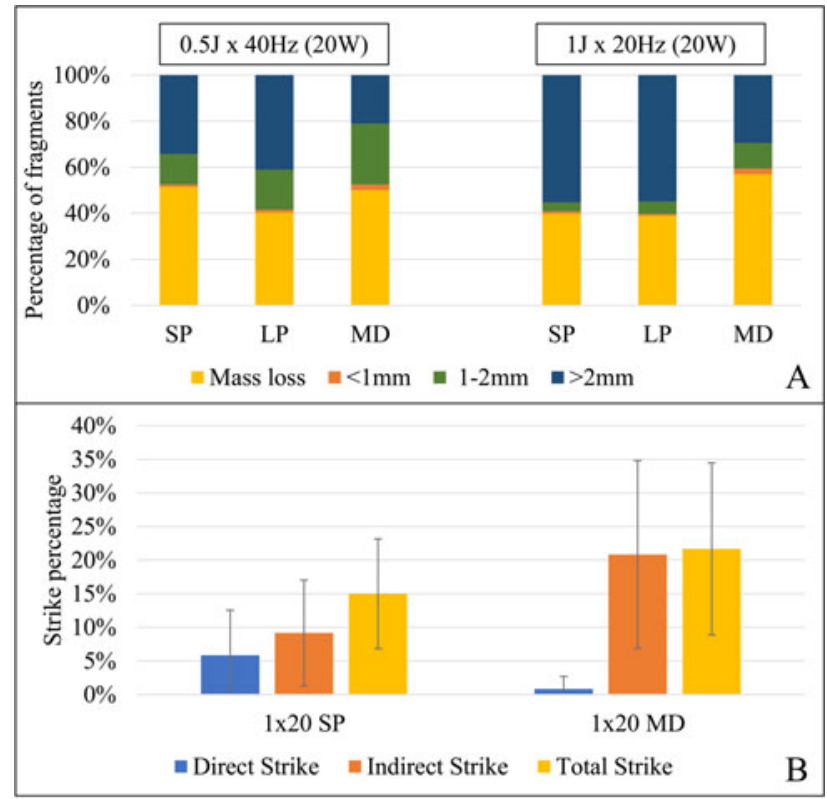

Figure 1: (A) Fragment distribution for 20W settings during popcorn laser lithotripsy in an in-vitro calyceal model. SP — Short Pulse; LP-Long Pulse, MD - Moses Distance. (B) Laser fragmentation strike percentage for $20 \mathrm{~W}$ settings using SP and MD mode. 
MD modes. Total energy delivered was constant at $4.8 \mathrm{KJ}$. Five trials were done per cohort. Study outcome was fragment size distribution including mass loss, which was calculated by subtracting the final dry mass of all collected fragments from the initial mass. High speed video analysis was performed for $1 \mathrm{~J} \times 20 \mathrm{~Hz}$ on SP and MD modes at $10,000 \mathrm{fps}$ to calculate laser fragmentation strike percentage. Strikes were categorized as: Direct - a visual plume of dust ejected from stone while in contact with the fiber-tip; or Indirect - a visual plume of dust ejected from stone with distance between stone and fiber-tip. Strike rate was calculated as the number of strikes divided by the total number of laser fires in one second duration.

Results: For all experiments, the overall mean mass loss was $46 \%$ (Figure 1A). Compared to SP and LP, MD mode at $1 \mathrm{~J} \times 20 \mathrm{~Hz}$ resulted in the greatest mass loss $(57 \%)$ and the lowest distribution of fragments $>2 \mathrm{~mm}(29 \%) \quad(\mathrm{p}<0.05)$. For $0.5 \mathrm{~J} \times 40 \mathrm{~Hz}$, MD mode resulted in lower $\%$ of fragments $>2 \mathrm{~mm}$ when compared to SP $(\mathrm{p}<0.05)$ and LP modes $(\mathrm{p}=0.08)$. Video analysis demonstrated that when using MD mode, $96 \%$ of total fragmentation strikes were indirect (Figure 1B).

Conclusions: MD mode was superior to SP and LP modes for popcorn laser lithotripsy. Video analysis suggests that MD achieves fragmentation through non-contact laser lithotripsy.

\section{MP30-05 RIRS with SuperPulse Thulium fiber laser for kidney stones larger than $2 \mathrm{~cm}$}

A Dymov, D Tsarichenko, G Akopyan, D Chinenov, S Ali, R Klimov, V Lekarev, D Korolev, L Rapoport

Institute for Urology and Reproductive Health, Sechenov University

Introduction \& Objective: According to current EAU Guidelines PNL is a first-line treatment of larger kidney stones $>2 \mathrm{~cm}$ and In case PNL is not an option flexible ureteroscopy may be used with a higher risk of a follow-up procedure. This is mostly due to the longer operative time needed to stone disintegration during RIRS and increased risk of infectious complications. Recently, we have evaluated in vitro performance of SuperPulse Thulium (Tm) fiber laser (IRE Polus, Russia) with wavelength of $1.94 \mu \mathrm{m}$ and found its efficacy to be higher than the leading Ho:YAG laser for fragmentation $(\times 1.7$ times $)$ and dusting $(\times 2.3$ to 4 times) for equal energy and repetition rate settings. Our clinical experience confirms the preclinical performance of this laser. This study aimed to assess the efficacy of RIRS with Tm fiber laser lithotripsy for larger stones.

Methods: The SuperPulse Tm fiber laser device has been cleared for clinical use by the Ministry of Health of Russian Federation and the protocol has been approved by the institutional Ethical Review committee. We performed 18 for larger stones $>2 \mathrm{~cm}$ with SuperPulse Tm fiber laser. Access sheath was used in all cases. Stone size, its density, lithotripsy time (from the first to last pedal press) and "lasering" (laser emission) time were measured. Based on surgeon's feedback, retropulsion and intraoperative visibility were assessed. Postoperative parameters were recorded.

Results: The average stone size was $24.7 \mathrm{~mm}(20.5-36 \mathrm{~mm})$. The average stone density was $848 \mathrm{HU}(350-1600 \mathrm{HU})$. The average lithotripsy time was $43.7 \mathrm{~min}(23-70 \mathrm{~min})$. The average "lasering" time was $11.5 \mathrm{~min}(5.9-26.5 \mathrm{~min})$. The retropulsion was insignificant in all cases with energy level less than $0.5 \mathrm{~J}$. Visibility was estimated as optimal by surgeons in most cases. No major complications infectious included were observed in this cohort. In one case with stone, up to $36 \mathrm{~mm}$ a second procedure was required. The overall stone-free rate was $89 \%$.

Conclusions: The SuperPulse Thulium Fiber laser can be successfully utilized for efficient lithotripsy of larger stones during RIRS with acceptable operative time without compromising the safety of the procedure. It might be a possible reason to reconsider the current guidelines on stone management, apparently, further studies and data are necessary.

\section{MP30-06 Evaluation of Risk Factors for Urinary Tract Infection After Flexible Uretero-Renoscopy: A Retrospective Study Including 604 Interventions}

R Boissier, M Baboudjian, B Gondran-Tellier, G Karsenty, E Lechevallier

Aix-Marseille University, Marseille, France; Department of Urology and Renal Transplantation, APHM, Conception University Hospital, Marseille, France

Introduction \& Objective: Urinary tract infection (UTI) is a common complication after flexible ureteroscopy (fURS) despite technical precautions to avoid infectious complications. The aim was to investigate incidence and predictive risk factors of UTI following fURS procedure.

Methods: We conducted a retrospective study including consecutive fURS performed in our center from January2015 to March2019. The indications were: nephrolithiasis management and diagnosis and conservative treatment of upper urinary tract urothelial carcinomas (UTUC). Since 2015, we have technical precautions to avoid postoperative infectious complications: centralized collection of preoperative urine cultures whose are examined daily by an urologist and a service provider, systematic use of ureteral access sheath and application of standardized antibiotic prophylaxis measures. The primary endpoint was occurrence of UTI within 15 days following fURS.

Results: Six hundred and four fURS were included for nephrolithiasis $(n=462)$ and UTUC management $(n=142)$. The median (IQR) age in the study cohort was 61(48-68) years, 268 female patients were included $(44.4 \%)$, the median (IQR) charlson score was 2(1-4) and single-use fURS were used in 186cases $(30.8 \%)$. Postoperative UTI occurred in 41 cases $(6.7 \%)$. On multivariate analysis, female gender (OR 2.30[1.05-5.36], $\mathrm{p}=0.04$ ), UTI within last 6months (OR 2.44[1.14-5.42], $\mathrm{p}=0.02$ ), preoperative polymicrobial urine culture (OR 4.72[2.04-11.29], $\mathrm{p}<0.001)$ and increased operative time (OR 1.01[1.002-1.032], $\mathrm{p}=0.02$ ) remain associated with postoperative UTI.

Conclusions: In a large cohort study, female gender, prior UTI, increased operative time and preoperative polymicrobial urine culture were associated with the occurrence of postoperative UTI. Limiting operative time and improving our knowledge of polymicrobial urine cultures could reduce the infectious risk.

\section{MP30-07 Negative Ureteroscopies For Ureteric Stones}

M Moghul, O Elhalawaty, L Da Silva, A Almushatat, P Patki, Z Ali

Barts Health NHS Trust

Introduction \& Objective: Ureteroscopic surgery for stone disease has seen major advancements with the advent of modern ureteroscopes, which offer better imaging quality and less 


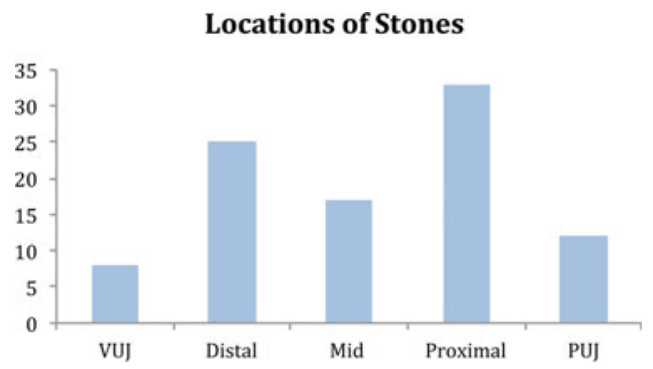

trauma. Ureteroscopic surgery is accepted to be safe but crucially it is not complication free. Ureteric perforation or avulsion is the most serious complications, notwithstanding anesthetic risks. There is no consensus regarding repeating a CTKUB to confirm stone passage prior to ureteroscopy. The balance of patient risks between performing repeated CT scans and offering surgery varies between individual patients. There is also a cost-benefit analysis to be considered with the average cost of a ureteroscopy around $£ 2000$ (\$2500) compared with around $£ 200$ (\$250) for a non-contrast CT scan.

Methods: In our institution we audited our ureteroscopies for ureteric stones over a 6-month period (January 2018-June 2018). Negative ureteroscopies were defined as those where the stone had already passed.

Results: During the 6-month period 158 ureteroscopies were performed in our department for stone disease, 95 for ureteric stones. $13(13.7 \%)$ were negative. Figure 1 shows the CT-based locations of ureteric stones prior to surgery. The mean stone size was $9.51 \mathrm{~mm}( \pm 4.65 \mathrm{~mm})$, ranging from $2-11 \mathrm{~mm}$. 43 ureteroscopies were performed for stones $7 \mathrm{~mm}$ and smaller, of which 12 were negative $(27.9 \%)$. Therefore $12 / 13$ negative ureteroscopies were for ureteric stones $\leq 7 \mathrm{~mm}$. Of the 13 negative ureteroscopies, 3 patients had a repeat CT prior to surgery. 11 of the patients had no JJ stent prior to surgery, whilst 2 patients did (neither of these had a repeat CT scan).

Conclusions: The debate between repeating CT scan/imaging prior to ureteroscopy for ureteric stones will be on-going with multiple patient and logistical factors needed to be taken into consideration. At our institution, following on from this audit, we plan to repeat a CTKUB on the morning of surgery for all patients planned for primary ureteroscopy for stones $\leq 7 \mathrm{~mm}$. We shall continue to list patients directly for ureteroscopy for stones $>7 \mathrm{~mm}$ and remove them from the list if there is patient reported stone passage. These patients will be seen in a stone clinic for follow up. We envisage this will reduce the rate of negative ureteroscopy whilst also facilitating a streamlined and quicker time to surgery for our patients.

\section{MP30-08 Worldwide survey of Flexible Ureteroscopy (FURSL) Practice: A survey from EAU sections of Young Academic Urologists (YAU) and Uro- Technology (ESUT)}

A Pietropaolo, A Skolarikos, E Liatsikos, N Rukin, E Niewad, T Sener, P Kallidonis, M Oezsoy, E Emiliani, MA Maestro, T Tailly, M talso, OM Aboumarzouk, S Proietti, G Giusti, BK Somani

Fundacion Puigvert. Universidad Autonoma de Barcelona, Spain

Introduction \& Objective: Ureteroscopy is an accepted method for treatment of stone disease. We wanted to assess the world-

\begin{tabular}{|c|c|c|c|c|c|}
\hline \multicolumn{6}{|c|}{ What is your subspecialty of interest? } \\
\hline \multicolumn{6}{|c|}{ Endourology $-75 \%$, General Urology $-25 \%$} \\
\hline \multicolumn{6}{|c|}{ How many renal/ureteric stone cases does your unit deal with annually? } \\
\hline$<25$ & $25-50$ & $50-100$ & $100-150$ & $150-200$ & $>200$ \\
\hline $2 \%$ & $2 \%$ & $6 \%$ & $14 \%$ & $19 \%$ & $56 \%$ \\
\hline \multicolumn{6}{|c|}{ How many flexible ureteroscopes do you have in your unit? } \\
\hline 1 & 2 & 3 & 4 & $>5$ & $>10$ \\
\hline $5 \%$ & $27 \%$ & $22 \%$ & $13 \%$ & $23 \%$ & $9 \%$ \\
\hline \multicolumn{6}{|c|}{ How many of these flexible scopes are 'digital' scopes? } \\
\hline 1 & 2 & 3 & 4 & $>5$ & $>10$ \\
\hline $30 \%$ & $30 \%$ & $10 \%$ & $9 \%$ & $8 \%$ & $1 \%$ \\
\hline \multicolumn{6}{|c|}{$\begin{array}{l}\text { What is the estimated number of flexible ureteroscopy performed in your unit } \\
\text { annually? }\end{array}$} \\
\hline$<25$ & $25-50$ & $51-100$ & $101-200$ & $>201$ & not sure \\
\hline $11 \%$ & $14 \%$ & $28 \%$ & $23 \%$ & $23 \%$ & $1 \%$ \\
\hline \multicolumn{6}{|c|}{$\begin{array}{l}\text { Over the last year how many flexible ureteroscopes have been } \\
\text { damaged? }\end{array}$} \\
\hline 1 & 2 & 3 & $>5$ & $>10$ & \\
\hline $37 \%$ & $29 \%$ & $10 \%$ & $16 \%$ & $8 \%$ & \\
\hline \multicolumn{6}{|c|}{ Which is the most frequent damage to your scope? } \\
\hline $\begin{array}{l}\text { Laser } \\
\text { damage }\end{array}$ & $\begin{array}{l}\text { Loss of } \\
\text { deflection }\end{array}$ & $\begin{array}{l}\text { Optical } \\
\text { damage }\end{array}$ & Don't know & & \\
\hline $24 \%$ & $25 \%$ & $31 \%$ & $20 \%$ & & \\
\hline
\end{tabular}

wide pattern of availability in equipment and technology, with the technique used by urologists while treating stone disease.

Methods: The survey consisted of 42 questions distributed between November 2017 and December 2018 during the bi-annual EAU section of uro-technology (ESUT) meeting and via survey monkey to Endourologists across Europe.

Results: A total of 114 responses were obtained of which 100 $(88 \%)$ had successfully completed it. The average time taken for it was 5.4 minutes. Of the responders, $75 \%$ were endourologists and $25 \%$ were general urologists. The case volume and the number of available flexible ureteroscopes are as stated in the table. Disposable scopes were either not used at all $(60 \%)$ or used in up to $20 \%$ patients by a third of respondents. A single perioperative prophylactic antibiotic dose was given by $67 \%$. The

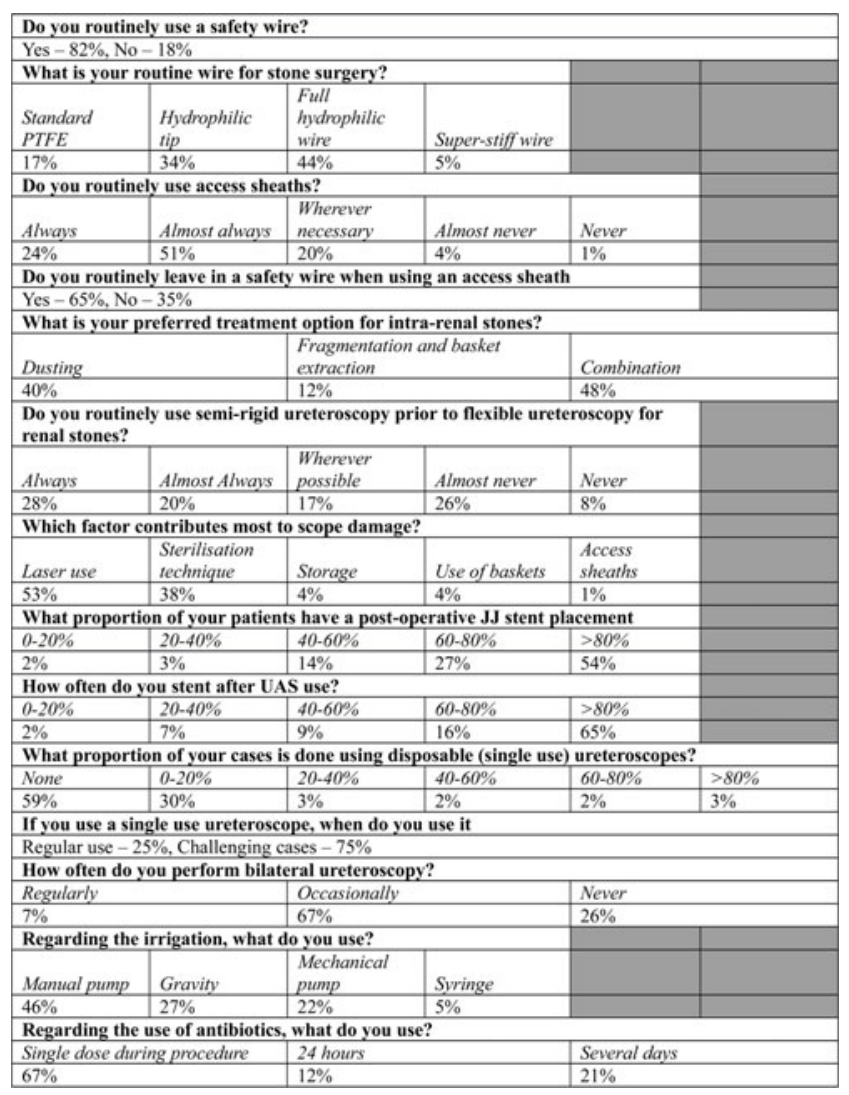


type of irrigation used was manual $(46 \%)$, mechanical $(22 \%)$ or gravity $(27 \%)$. An access sheath was always or almost always used by $75 \%$ responders. The mode of intra-renal stone treatment was chosen as dusting (40\%), fragmentation with basketing (12\%) or a combination of both $(48 \%)$. Post-operative stent was used by $54 \%$ respondents in $>80 \%$ of their patients and $28 \%$ in $60-80 \%$ of their patients.

Conclusions: There seems to be a wide variation in the availability of technology and technique used by urologists. While most respondents work in a medium or high-volume centre and have access to digital scopes, disposable scopes are still not used widely. Among endourologists, the use of single dose peri-operative antibiotics, access sheath, stone dusting and post-operative stent seems to be the most common treatment pathway.

\section{MP30-09 Fluoroscopy-free ureterorenoscopy for kidney stones treatment: technique description by an endourology expert.}

G Verzotti, G Capon, F Bladou, J Bernhard, H Bensadoun, G Robert, J Ferriere, V Estrade

Hospital Pellegrin

Introduction \& Objective: In the last few decades, semi-rigid and flexible ureterorenoscopy (URS) have emerged as the first choice treatment for urinary stones $<20 \mathrm{~mm}$. The localization of the stone, the placement of the safety wire, and the insertion of ureteral access sheath and ureteral stent are traditionally performed under fluoroscopic (FS) control. Is FS necessary during all these steps to treat stones? We describe a FS-free URS technique and analyze its feasibility.

Methods: Prospective cohort study of 40 patients operated for urinary stones in two hospitals by a single experienced endourologist. The following key steps are performed to achieve FS- URS. The guidewire is placed under visual control. The guidewire reaches the upper calyx when it cannot ascend any further and the ureteric orifice moves backwards. If needed, the surgeon may decide to perform ureteral dilation. A ureteral access sheath may be used according to clinical situations and surgeon judgment. Afterwards, the endoscope is passed up to the kidney and the upper calyx placement of the guidewire is confirmed by visual control. Then exploration of the cavities is performed, stones are identified, relocated and treated with a Holmium YAG laser. All these steps are carried out under visual control only. At the end of the procedure, the surgeon checks the upper calyx position of the guidewire and for any ureteral injury. Finally, he may decide to put a ureteral stent. This last step is also performed under visual control without FS.

Results: 40 patients were operated from URS between March and May 2019. Collected data were: patient age, sex, weight and height, stone location, size, Hounsfield Units density, multiplicity and bilaterally, presence/absence of double J stent, use of a ureteral access sheath, history of past URS, endoscope type, difficulty of the procedure (scale 1 to 5), X-ray dose and duration of FS, use of a JJ stent at end of procedure, operating time, complications. 37 patients were operated without FS. FS was needed for 3 patients (duplicated ureter/ ureteropelvic junction stenosis/ stone identification). Dosimetry: 38/99/134 cGycm2. We observed no intraoperative complications.

Conclusions: URS performed without FS by an expert is feasible without major issues. It is a modern evolution in endourology for urinary stones management. A study protocol has been submitted to the ethics committee of our center to consider the continuation of this prospective study.
MP30-10 Predictors and results of negative ureteroscopy for treatment of consecutive ureteric stones done as a primary procedure: Prospective outcomes from a university hospital

\author{
S Prattley, P Rice, A Pietropaulo, H Ching Ho, BK Somani
}

Introduction \& Objective: Negative ureteroscopy (URS) can be considered an unnecessary procedure with medico-legal consequences. However, it avoids radiation from repeat CT scan and can reassure patients with ongoing symptoms. We wanted to analyze our predictors and results of negative ureteroscopy for treatment of ureteric stones.

Methods: Between 2012 and 2018, data on consecutive patients with ureteric stones undergoing primary URS (without a preoperative stent) was prospectively collected. Comparison was completed for patients with diagnostic and therapeutic primary URS for ureteric stones, renal stones were excluded.

Results: A total of 270 patients underwent URS of which 35 $(13 \%)$ had a negative procedure. Patients undergoing negative URS were younger $(\mathrm{p}=0.001)$, had smaller stones $(\mathrm{p}<0.001)$ and more stones located distally $(\mathrm{p}=0.034)$. No patients who had a negative URS were stented or had any complications.

Conclusions: Although negative URS levels should be as low as possible, it is a safe procedure, and reassures symptomatic patients. Based on our data, patients with small distal stones should undergo repeat imaging to avoid an unnecessary procedure. Informed consent, patient counselling and appropriate up-to-date imaging is key to minimizing negative ureteroscopies. We have therefore introduced a re-imaging algorithm to attempt to reduce our negative URS rates.

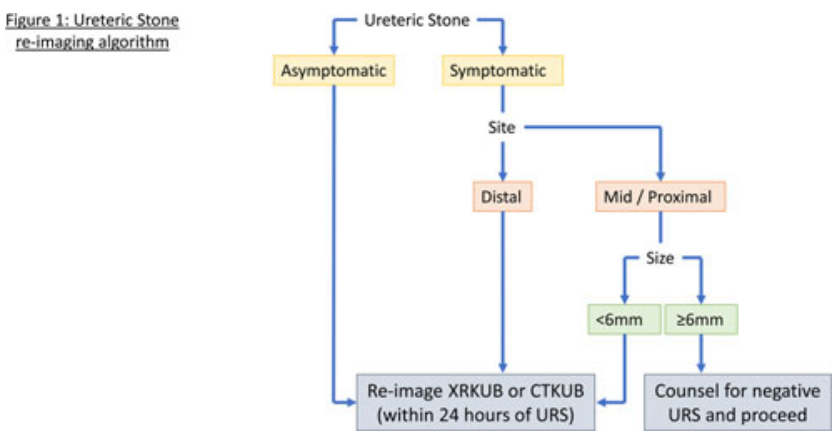

\section{MP30-11 Factors Influencing the Cost of Ureteroscopy}

M Alsyouf, P Stokes, D Hur, S Kown, A Amasyali, M Hajiha, M Shah, R Belay, J Maldonado, D Baldwin

Introduction \& Objective: Cost containment during ureteroscopy has been advocated recently, in the setting of reduced reimbursement rates. Academic centers must balance outcomes, efficiency and resident training, with little evidence regarding the effect of these factors upon cost. The purpose of this study was to evaluate factors during ureteroscopy that can potentially impact hospital cost.

Methods: A retrospective review of 129 consecutive patients undergoing elective ureteroscopy at a single academic medical center was performed. Average direct cost data was obtained and was defined as hospital cost incurred due to operating room expenses only including operating room time, staffing, equipment, and supplies. Data regarding patient, procedural, and operating room staffing characteristics were compared between the most expensive and least expensive cases. Univariate and logistic 
regression analysis were performed to identify factors predictive of higher costs.

Results: The mean direct cost of ureteroscopy was \$3298.0. Ureteroscopy cases in the highest 50th cost percentile had larger mean stone size ( 170.1 vs. $146 \mathrm{~mm} 2 ; \mathrm{p}=0.03$ ), higher percentage of cases performed for non-stone indications (21.4 vs. $7.2 \%$; $\mathrm{p}=0.03$ ), longer average operative times (95.3 vs. $49.9 \mathrm{~min}$; $\mathrm{p}<0.01)$, were more likely to have a resident present $(65.5$ vs. $43.6 \% ; \mathrm{p}=0.02)$, and less likely to have a dedicated urology scrub technician (38.2 vs. $61.8 \% ; \mathrm{p}=0.01)$ when compared to the lowest 50th percentile. The presence of a resident in a case, larger stone burden $(>100 \mathrm{~mm} 2)$, absence of a dedicated scrub tech, and longer operative time ( $>1$ hour) were associated with an average increase in case cost of $\$ 516, \$ 700, \$ 1122$, and $\$ 1401$; respectively. On multiple logistic regression analysis, operating room time was the only factor to remain significantly associated with higher case cost (OR 12.8, 95\% CI 2.0-84.0).

Conclusions: Operating room time is the primary determinant of case cost during ureteroscopy. Indirect factors that can impact operating room time include large stone burden, resident training, and availability of dedicated operating room staff. All efforts should be made to decrease operative time in order to decrease cost while balancing patient safety and maintaining a quality training environment.

\section{MP30-12 Removing Stones from the Lower Pole during Retrograde Intrarenal Surgery (RIRS)- Improved Stone Free Rates or a Waste of Time? A Prospective Randomized Study}

B Gallante, JN Bamberger, RA Chandhoke, W Atallah, M Gupta, AM Zampini

\section{Mount Sinai}

Introduction \& Objective: Renal stones located in the lower pole (LP) are more difficult to access, more difficult to fragment, more likely to cause ureteroscope damage, and more likely to result in residual fragments (RF). To improve the stone free rate and cause less damage to the ureteroscope, some urologists will move a LP stone to a more accessible part of the kidney. While many urologists use this technique of displacement as a matter of preference, its usefulness has not been clearly determined. We sought to compare the effect of stone fragmentation in situ versus fragmentation after displacing the stone on stone clearance in patients with LP intrarenal stones. Our primary outcome was stone free rates.

Methods: In this IRB approved prospective, randomized study, patients were included if they had stones only in the LP, total stone burden was $7-15 \mathrm{~mm}$, and did not have anatomic kidney abnormalities or pre-existing ureteral stents. Patients were randomized into the in situ or displacement group prior to the procedure then underwent URS. Demographics, comorbidities, and operative parameters were documented. We determined stone free rates using renal ultrasound and $\mathrm{x}$-ray (KUB) at 1-month follow-up. ANOVA, Chi-Square, and $t$-tests were conducted to assess differences between the groups.

Results: Our data includes 60 patients. 28 in situ patients and 32 displaced patients have completed the study with imaging. Displaced stone patients had higher stone free rates when compared to in situ patients (97\% vs. 85\%). In addition, total surgery time was higher in the displaced group $(\mathrm{p}=.048)$. There was no difference between groups in any other parameters, and there were no major complications in either group. Table 1 shows our results.

\begin{tabular}{|c|c|c|c|}
\hline & In situ Stone & Displaced Stone & $p$-value \\
\hline & $\mathrm{N}=28$ & $\mathrm{~N}=32$ & \\
\hline \multicolumn{4}{|l|}{ DEMOGRAPHICS } \\
\hline $\begin{array}{l}\text { Mean Age (Standard Deviation), } \\
\text { years }\end{array}$ & 54.54 (18.3) & $59.06(11.7)$ & $0.253^{\wedge}$ \\
\hline Male, $\%$ & 28.60 & 53.10 & $0.054^{\mathrm{B}}$ \\
\hline White, \% & 67.90 & 78.10 & $0.370^{\mathrm{B}}$ \\
\hline Mean Height, m & 1.65 & 1.68 & $0.206^{\mathrm{B}}$ \\
\hline Mean Weight, kg & 77.70 & 84.87 & $0.164^{\mathrm{B}}$ \\
\hline Mean Body Mass Index, $\mathrm{kg} / \mathrm{m}^{\wedge} 2$ & 28.70 & 29.25 & $0.729^{\mathrm{B}}$ \\
\hline ASA & & & $0.172^{\mathrm{B}}$ \\
\hline 1 & 16.70 & 3.10 & - \\
\hline 2 & 70.80 & 75.00 & - \\
\hline 3 & 12.50 & 21.90 & - \\
\hline \multicolumn{4}{|l|}{ CO-MORBIDITIES } \\
\hline Diabetes Mellitus, \% & $19.20 \%$ & $25.00 \%$ & $0.600^{\mathrm{B}}$ \\
\hline Hypertension, \% & $40.70 \%$ & $37.50 \%$ & $0.799^{\mathrm{B}}$ \\
\hline Hypercholesterolemia, \% & $11.10 \%$ & $20.00 \%$ & $0.358^{\mathrm{B}}$ \\
\hline Hyperlipidemia, \% & $7.70 \%$ & $21.90 \%$ & $0.138^{\mathrm{B}}$ \\
\hline Chronic Pain, \% & $23.10 \%$ & $29.00 \%$ & $0.611^{\mathrm{n}}$ \\
\hline Metabolic Syndrome, \% & $8.00 \%$ & $12.50 \%$ & $0.583^{\mathrm{B}}$ \\
\hline \multicolumn{4}{|c|}{ PRE, INTRA- \& POST-OPERATIVE PARAMETERS } \\
\hline Pre-Operative Hydronephrosis & & & $0.221^{\mathrm{B}}$ \\
\hline None & $46.40 \%$ & $56.30 \%$ & $\cdot$ \\
\hline Mild & $50.00 \%$ & $31.30 \%$ & - \\
\hline Moderate & $3.60 \%$ & $12.40 \%$ & $\cdot$ \\
\hline Severe & $0.00 \%$ & $0.00 \%$ & - \\
\hline Access Sheath Used, \% & $22.20 \%$ & $40.60 \%$ & $0.132^{\mathrm{B}}$ \\
\hline Intra-Operative Stent Placed, \% & $57.10 \%$ & $75.00 \%$ & $0.143^{\mathrm{B}}$ \\
\hline $\begin{array}{l}\text { Previous Ureteroscopy Performed, } \\
\%\end{array}$ & $34.80 \%$ & $33.30 \%$ & $0.914^{\mathrm{n}}$ \\
\hline Mean Estimated Blood Loss, mL & 7.74 & 7.77 & $0.991^{\mathrm{C}}$ \\
\hline Mean Irrigation Used, mL & 986.36 & 1160.42 & $0.425^{\mathrm{C}}$ \\
\hline Mean Laser Energy Used, kJ & 2.43 & 6.18 & $0.051^{\mathrm{c}}$ \\
\hline Mean Total Surgery Time, min & 63.04 & 90.96 & $0.048^{\mathrm{C}}$ \\
\hline Mean Pain Score 1-hour Post-Op & 2.43 & 3.72 & $0.094^{\mathrm{C}}$ \\
\hline Mean Pain Score 24-hour Post-Op & 0.18 & 0.79 & $0.156^{\mathrm{C}}$ \\
\hline $\begin{array}{l}\text { Mean Total Morphine Equivalents, } \\
\text { mg }\end{array}$ & 3.57 & 7.02 & $0.08^{c}$ \\
\hline Total stone burden, mm & 14.61 & 14.20 & $0.879^{c}$ \\
\hline $\begin{array}{l}\text { Stone Free at 1-Month Follow-Up, } \\
\%\end{array}$ & $85.20 \%$ & $96.90 \%$ & $0.108^{n}$ \\
\hline
\end{tabular}

Conclusions: Displacing LP stones may require more OR time but does improve stone free rates when compared to patients who are treated in situ. Increased OR time may be due to the need for increased time to completely remove stone fragments.

\section{MP30-13 Incidence of Hydronephrosis and Ureteric Stricture with the Use of Ureteral Access Sheaths in the Treatment of Nephrolithiasis}

LF Reynolds, D Ghiculete, AM Althunayan, JY Lee, J Honey, M Ordon, KT Pace

\section{St. Michael's Hospital}

Introduction \& Objective: The ureteral access sheath (UAS) is an effective adjunct for ureteroscopic management of stone disease. While there are many benefits, there is a risk of ureteric injury with UAS use. A clear causative link between UAS use and ureteral stricture disease has not been demonstrated but is possible. Our objective was to assess the incidence of hydronephrosis or ureteric strictures following ureteroscopy (URS) for urolithiasis with and without the use of a UAS.

Methods: Consecutive patients undergoing URS for urolithiasis with and without ureteral access sheaths were compared. A control group of patients undergoing semi-rigid and flexible URS without a UAS was used to compare outcomes of patients undergoing flexible URS with either a 9.5/11 Fr or 12/14 Fr UAS 
for ureteric and renal stones. The primary outcome was the development of a new ureteric stricture post-operatively. Secondary outcomes were the persistence or development of hydronephrosis at 3 months post operatively and long-term hydronephrosis ( $>3$ months post-URS).

Results: A total of 236 patients were included in this retrospective series. No patients in the UAS group developed a stricture with a mean follow-up of 21.7 months while one patient in the no-UAS group developed a stricture at the site of an impacted ureteric stone. Post-operative hydronephrosis was the same or worse 3 months after URS in 4 patients in each group (3.3\% of patients in the UAS group vs. $3.5 \%$ in the no-UAS group, $\mathrm{p}=\mathrm{NS}$ ). All other cases of hydronephrosis at 3 months and at long term follow-up were caused by non-obstructing unresolved hydronephrosis or residual stone fragments.

Conclusions: We did not identify any patients who developed a de novo ureteric stricture as a consequence of UAS use. Judicious use of UAS provides surgeons with the ability to perform URS safely without added risk of post-operative hydronephrosis or strictures.

\section{MP30-14 Pain Intensity and Interference after Ur- eteroscopy for Nephrolithiasis}

G Lin, A Fombona, B Friel, H Stambakio, GE Tasian, JB Ziemba

Perelman School of Medicine at the University of Pennsylvania

Introduction \& Objective: Pain is one of the most common complaints following ureteroscopy. How this pain is characterized and its duration following surgery remains poorly described. Therefore, we captured patient-reported pain intensity and interference in a prospective cohort of patients undergoing ureteroscopy for nephrolithiasis.

Methods: A pragmatic, longitudinal cohort study began enrollment in 11/2018. Data for this analysis included the initial 6months (end 5/2019). Any adult patient who underwent ureteroscopy for a renal or ureteral stone and who was accessible for further electronic follow up was eligible for inclusion. Exclusion criteria included vulnerable populations, inability to speak English, or inability to receive email. Enrolled subjects completed PROMIS-Pain Intensity and PROMIS-Pain Interference instruments on the day of surgery (pre-op), POD \#1, and POD \#7. All patients were discharged with a ureteral stent (all removed POD \#4) and a standing non-narcotic pain regimen. All scores are reported as T-scores (normalized to US population) with a change of 3.5-5.5 considered a clinically significant difference.

Results: A total of 67 subjects completed enrollment with the pre-op questionnaire. Of these, $36(54 \%)$ and 23 (34\%) subjects completed the questionnaire on POD \#1 and \#7, respectively. The range, median, and interquartile range for each instrument at each time point are depicted in Figure 1. As compared to the US population mean (50), intensity (mean diff. -5.4 ) and interference (mean diff. +5.5$)$ were significantly different at pre-op (1sample T-test; $\mathrm{p}<0.001)$, but only interference continued to be significantly different at POD \#1 (mean diff. +12.8) and POD \#7 (mean diff. +11.4$)(1$-sample T-test; $\mathrm{p}<0.001)$. From pre-op to POD \#1, both intensity (mean diff. +7.5) and interference (mean diff. +7.5) were significantly different (paired sample $T$-test; $\mathrm{p}<0.001$ ). No difference was observed from POD \#1 to POD \#7 or pre-op to POD \#7 for either measure (all p >0.05).

Conclusions: Pain intensity and interference are elevated immediately after surgery, but intensity returns to baseline by 1 -

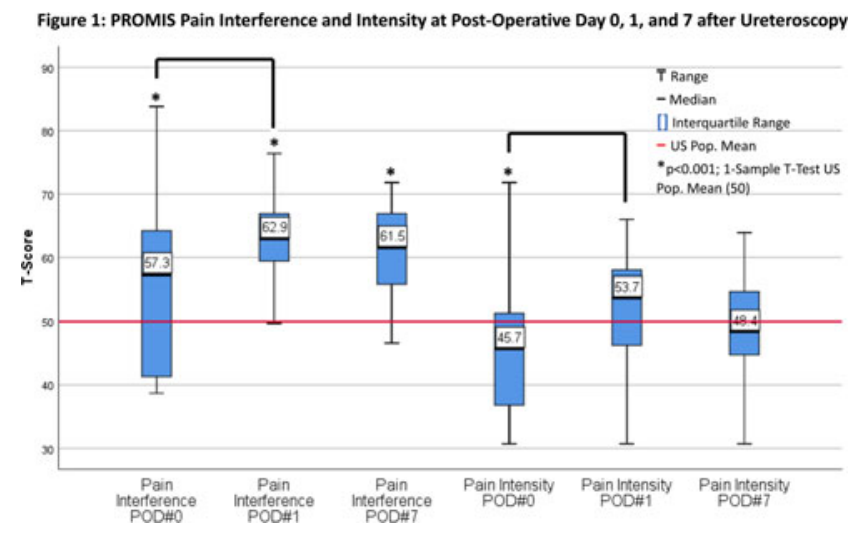

week, while interference is persistently elevated. Pain interference rather than intensity appears to be a more sensitive measure for capturing discomfort after ureteroscopy.

\section{MP30-15 Use of a Coagulum during Ureteroscopic Stone Removal Adds No Additional Case Time or Complications}

C Valadon, CU Nottingham, T Large, A Krambeck

University of Louisville School of Medicine

Introduction \& Objective: Ureteroscopic basketing of small stone fragments can be time-consuming and challenging, especially if the patient maintains a large overall burden of stone material. The purpose of this study was to evaluate if use of a blood coagulum provides any benefit or burden to ureteroscopic stone basket extraction.

Methods: We enrolled adult patients undergoing ureteroscopic stone removal with laser lithotripsy for renal and ureteral stones under $20 \mathrm{~mm}$ in total size with IRB approval. Patients with a coagulative disorder or on anticoagulant medication were excluded. Following laser lithotripsy, a peripheral blood sample or "coagulum" administered through the ureteroscope into the areas of the kidney containing stone fragments. After five minutes of coagulation time, ureteroscopic stone basket extraction was initiated. We then matched these patients 1:1 to standard patients who had previously undergone ureteroscopic stone removal without coagulum by age, stone composition, and preoperative stone size.

\begin{tabular}{|c|c|c|c|}
\hline $\begin{array}{l}\text { Patient Characteristics and } \\
\text { Outcomes }\end{array}$ & Coagulum ( $n=25)$ & Matched Standard $(n=25)$ & p-value \\
\hline Median Age in Years (IQR) & $51(37-62)$ & $39(33-60)$ & 0.090 \\
\hline $\begin{array}{l}\text { Preoperative Median Stone Size } \\
\text { (IQR) in Millimeters }\end{array}$ & $8(4-10)$ & $7(4-10)$ & 1.000 \\
\hline $\begin{array}{l}\text { Median Milliliters of Coagulum } \\
\text { Used (IQR) }\end{array}$ & $7(5-10)$ & $\cdot$ & - \\
\hline Predominant Stone Type, $n(\%)$ & & & 0.960 \\
\hline Ammonium Hydrogen Urate & 0 & $1(4.0)$ & \\
\hline Brushite & $1(4.0)$ & $1(4.0)$ & \\
\hline Calcium Phosphate & $12(48.0)$ & $12(48.0)$ & \\
\hline Calcium Oxalate Dihydrate & $1(4.0)$ & $1(4.0)$ & \\
\hline Calcium Oxalate Monohydrate & $7(28.0)$ & $7(28.0)$ & \\
\hline $\begin{array}{l}\text { Magnesium Ammonium } \\
\text { Phosphate }\end{array}$ & $1(4.0)$ & 0 & \\
\hline Struvite & $1(4.0)$ & $1(4.0)$ & \\
\hline Uric Acid & $2(8.0)$ & $2(8.0)$ & \\
\hline $\begin{array}{l}\text { Median Total Operating Room Time } \\
\text { (IQR) in Minutes }\end{array}$ & $110(97-132)$ & $83(58-152)$ & 0.258 \\
\hline $\begin{array}{l}\text { Median Procedure Time (IQR) in } \\
\text { Minutes }\end{array}$ & $80(67-99)$ & $54(29-124)$ & 0.258 \\
\hline Stone-Free, $n(\%)$ & $21(84.0)$ & $19(76.0)$ & 0.480 \\
\hline Complication & 0 & $4(16.0)$ & 0.110 \\
\hline
\end{tabular}


Results: We included 25 patients in each group for a total of 50 patients, with all results summarized in table 1 . A median of 7 milliliters of coagulum was used. We observed no difference in total operating room ( 110 vs 83 minutes; $\mathrm{p}=0.258)$, procedure time ( 80 vs 54 minutes; $p=0.258)$, stone-free rate $(84 \%$ vs $76 \%$; $\mathrm{p}=0.480)$, or complication rate $(0$ vs $16 \% ; \mathrm{p}=0.110)$ between coagulum and matched standard patients. All complications were Clavien-Dindo grade II.

Conclusions: Coagulum is a useful tool for ureteroscopic stone basket extraction that adds no additional procedural time or morbidity and does not alter the stone-free rate.

\section{MP30-16 Fire the Laser: Lithotripsy activation pat- terns for ureteroscopic stone dusting}

A Aldoukhi, K Black, T Hall, JM Hollingsworth, SN Ambani, CA Dauw, WW Roberts, K Ghani

Department of Urology, University of Michigan

Introduction \& Objective: Advances in laser technology have increased the popularity of dusting for ureteroscopy (URS). The technique consists of two phases, contact and non-contact lithotripsy. However, it is unclear how much time is spent on each phase, how much energy is utilized, and overall patterns of performance. We studied laser activation patterns during dusting lithotripsy for renal stones.

Methods: Digital laser logs (pulse energy, pulse frequency, total energy, and timing of pedal activation) from de-identified URS procedures by 5 endourologists at an ambulatory center using a high-power holmium laser (P120; Lumenis, CA) were analyzed. Inclusion criteria were cases with total lasing time $>3$ minutes and where non-contact laser lithotripsy (i.e. pop-dusting) was performed using moderate pulse energy $(0.5-0.8 \mathrm{~J})$ and high frequency $(70-80 \mathrm{~Hz})$. We analyzed (1) total lithotripsy time, defined as time from the first laser activation to end of last laser activation including $\mathrm{ON}$ and OFF times, (2) contact and noncontact lithotripsy time, (3) lasing time, which is the cumulative

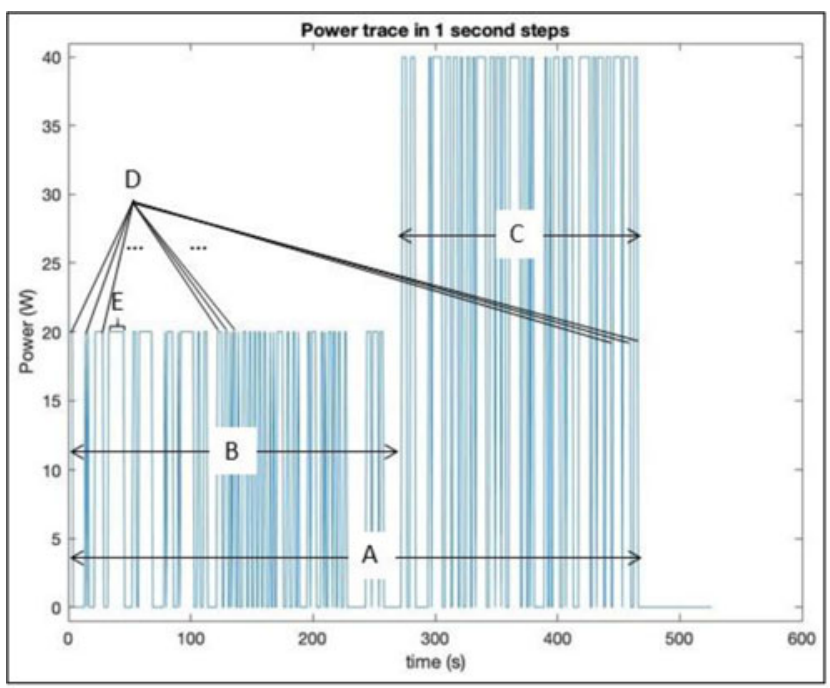

Figure 1: Laser activation patterns when dusting renal stones using a highpower holmium laser. (A) Total lithotripsy time, (B) Contact lithotripsy time, (C) Non-contact lithotripsy time, (D) Lasing time, the cumulative time the laser was active (total $\mathrm{ON}$ time), (E) Longest pedal activation time.

\begin{tabular}{|c|c|c|}
\hline & Time (mm:ss) & Range (mm:ss) \\
\hline Total lithotripsy time & 14:12 & $(05: 59-26: 27)$ \\
\hline Contact time & $08: 42$ & $(03: 27-23: 14)$ \\
\hline Non-contact time & $05: 30$ & $(01: 44-14: 24)$ \\
\hline Lasing time & $05: 43$ & $(03: 27-14: 44)$ \\
\hline $\begin{array}{c}\text { Longest pedal activation time } \\
\text { during contact phase }\end{array}$ & $00: 27$ & (00:05-01:31) \\
\hline Longest pedal activation time & $00: 20$ & $(00: 05-01: 49)$ \\
\hline
\end{tabular}

time the laser was active (total ON time), (4) longest pedal activation time, and (5) total energy used.

Results: A total of 18 cases were analyzed. A representative case is shown in Figure 1. Mean total lithotripsy time was $14.1 \mathrm{~min}-$ utes (Table 1). Mean total energy used was $8.2 \mathrm{~kJ}$ (range 3.320.3). Total power used during contact and non-contact phases ranged between $8-25 \mathrm{~W}$ and $40-56 \mathrm{~W}$, respectively. Contact and non-contact phases represented $61.2 \%$ and $38.8 \%$ of total lithotripsy time, respectively.

Conclusions: When using a high-power holmium laser for dusting renal stones, approximately $40 \%$ of the time is spent on non-contact lithotripsy. On average, lasing time accounts for $1 / 3$ of total lithotripsy time. Future work should explore strategies to improve laser lithotripsy efficiency by decreasing the lasing to total lithotripsy time ratio.

\section{MP30-17 Predictors of urosepsis after ureteroscopy} (URS): Prospective results from 900 consecutive patients

\section{S Chugh, A Pietropaolo, BK Somani}

\section{Portsmouth Hospitals NHS Trust}

Introduction \& Objective: Post-ureteroscopy urosepsis is a cause for morbidity and potential mortality. We wanted to look at the rates of urosepsis in our cohort of patients who underwent ureteroscopy (URS) for stone disease.

Methods: Between March 2012 and September 2018, consecutive cases of URS performed or supervised by a single surgeon were recorded in a prospective database. Patients underwent protocol-based antibiotics prior to surgery based on microbiology guidelines. Data on patient demographics, stone parameters, pre-operative assessment, operative details, length of stay, stonefree rate and complication rates were collected and analyzed.

Results: Nine-hundred patients underwent URS in the study period of which $12(1.3 \%)$ developed urosepsis (8 managed on the ward and 4 had a brief intensive care unit (ICU) stay. The

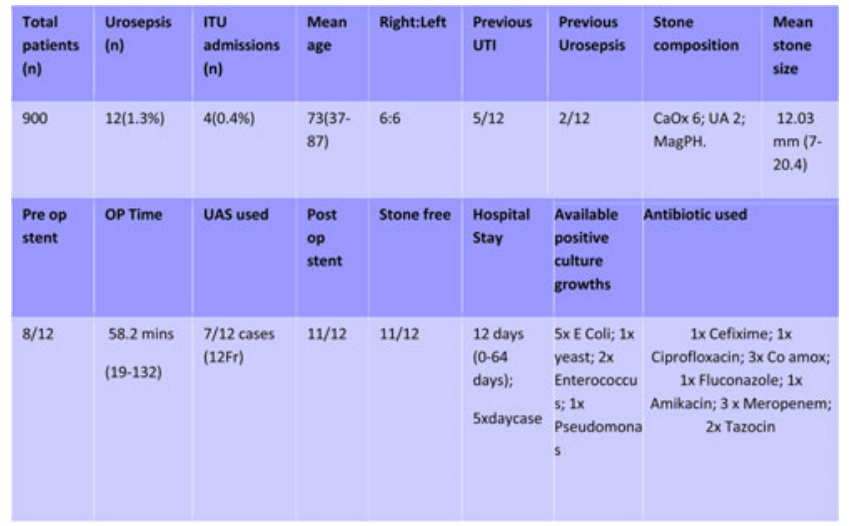


mean age was 73 years (range: $37-87$ years), with a history of previous recurrent UTIs $(n=5)$ and urosepsis $(n=2)$. Five $(41 \%)$ had ${ }^{3} 2$ comorbidities and $8(67 \%)$ had a history of prior endoscopic procedure. The mean stone size was $12 \mathrm{~mm}$ (range: $7-$ $20.5 \mathrm{~mm}$ ) with a pre-operative stent in $8(67 \%)$ patients and a mean operative time of 58 minutes (range: 19-132 minutes). The stone location was in the ureter $(n=3)$, kidney $(n=5)$ and both locations $(n=4)$. A ureteric access sheath was used in $7(58 \%)$ patients with a post-operative stent in $11(92 \%)$ patients. Eleven patients were stone free after the procedure and there was no mortality in our series.

Conclusions: The risk of post-URS urosepsis remain despite preoperative optimization and meticulous protocol-based antibiotics. Patients with a prior history of recurrent UTIs, urosepsis and indwelling stent seem most at risk of this dreaded complication.

\section{MP30-18 RIRS for renal pelvic stones 1.5 to $2.0 \mathrm{~cm}$ using semi-rigid uretero-renoscopy}

H Qureshi, G Sultan, M Hussain, T Gazdar, A Hashmi, A Rizvi

Sindh Institute of Urology and Transplantation

Introduction \& Objective: RIRS has nowadays become widely used treatment modality for stones located in upper urinary tract. ESWL and RIRS are options for stones less than $2 \mathrm{~cm}$ within renal pelvis, upper and middle calyx. Stones with housefield units more than 1000 have low clearance (30 to 50\%) with ESWL.

Methods: Prospective descriptive study was done from June 2014 to Dec 2016 at Sindh institute of urology and transplantation. 82 consecutive patients with stone $1.5-2.0 \mathrm{~cm}$ located in renal pelvis who underwent retrograde intrarenal surgery as Primary procedure were included.

Results: The stone free rate at six weeks after the first procedure was $79.2 \%$ (65 patients), which further improved to 84.1 (69 patients) after auxiliary procedures mostly SWL. The mean operating time was 32.03 mins (SD 12.9) and 60 (73.1\%) patients required double $\mathrm{J}$ stenting after the procedure. Complications including fever and hematuria were reported in 13 patients.

Conclusions: Retrograde intrarenal surgery using Rigid Ureterorenoscopy is safe and effective for renal stones 1.5 to $2.0 \mathrm{~cm}$ with HU more than 1000. The procedure can be carried out as day care and has low morbidity. It is especially useful in low resource centers were costly flexible scopes are not available.

\section{MP30-19 Infectious pathogens in urine, blood and ureteral stents in ureterolithotripsy}

TM Brasil, MQ Fortes, BN Lana, GB Araújo, RF Ferraz, CM Celjar, DC Perdigão, AT Dias, JP Pedrosa, HS Almeida, RM Medeiros, AA Carvalho, B Marroig

\section{Hospital Quinta D’Or}

Introduction \& Objective: Drainage of upper urinary tract with ureteral stents (US) is frequent after ureterolithotripsy and is associated with an increased risk of infection due to US colonization by diverse uropathogens. The aim of this study was to analyze and correlate the incidence and bacteriological profile of positive cases in urinary culture (UC), stent culture (SC) after ureteroscopy and blood culture (BC) from patients that presented with post-operative urinary sepsis.

Methods: Patients underwent US insertion after urgent ureterolithotripsy due to obstructive ureterolithiasis. UC and hemogram were collected before the procedure and all patients received a 7day course of antibiotics. After the removal of the US, the distal end was sent for microbiological assessment. The indwelling time of US was recorded, and BC was collected, as part of the standard care pathway, in patients who developed urinary sepsis. Continuous variables were expressed in mean \pm standard deviation (SD) and categorical variables in number and percentage. Chisquared test and Pearson correlation were used to analyze data.

Results: From May 2018 to May 2019, 124 patients (92 (74.2\%) male and $32(25.8 \%)$ female) were analyzed, 74 of whom had UC. All of them had SC at the time of US removal. The indwelling time was 32.5 (19.25-43.75) days. Mean age was $46.1 \pm 11.38$ years old. We observed a total of $9(12.16 \%)$ positive UC and $20(16.12 \%)$ positive SC. Positive UC was more frequent in older patients $(\mathrm{p}=0.045, \mathrm{R}=0.181)$ and in females $(\mathrm{p}=0.0003)$. The average indwelling time was $34 \pm 19$ days. Longer stenting periods positively correlated with positive UC $(\mathrm{p}=0.056, \mathrm{R}=0.172)$. Among 4 patients admitted in the intensive care unit due to sepsis, $3(75 \%)$ had positive BC with a different pathogen from previously isolated in SC. However, we observed a $100 \%$ concordance of UC and BC in the presence of sepsis. Both sterile UC and SC were found in $58(46.77 \%)$ cases. Nine patients with sterile UC had positive SC. In all patients with positive UC, 3 had negative SC, and 4 patients presented sepsis. The most commonly isolated bacteria in SC was E. faecalis, and E. coli and $K$. pneumoniae were often associated with UC.

Conclusions: Our results indicated that female sex, older age and longer indwelling time correlates with positive UC. Bacteria isolated in SC usually differ from the isolated in UC. In sepsis, the same bacteria were isolated in pre-operative $\mathrm{UC}$ and $\mathrm{BC}$. Therefore, the UC results appear to be effective in guiding the treatment. Since bacteria isolated in SC did not correlate with causative agents of severe infection, this work indicates that, SC might not need to be performed routinely.

\section{MP30-20 Effect of ureteric stenting on female sexual function- A prospective cohort study}

\section{Z Kazmi, D Umer, H Ather}

The Aga Khan University Hospital

Introduction \& Objective: Introduction and

Objectives: Female sexuality is an important aspect of a woman's personality and is plays a vital role in quality of life. Female sexual dysfunction (FSD) is a rarely talked about clinical entity, especially in our part of the world. Much work has been done to elucidate the adverse effects of DJ stent placement; however, the effect of ureteric stents on female sexual function remains largely unexplored. We aimed to find the impact of ureteric stenting on female sexual function.

Methods: Following approval from the Ethical Review Committee, all women presenting to the section of Urology at a University hospital (2018 to 2019) requiring ureterorenoscopy (URS or fURS) for ureteric stone disease and fulfilling the inclusion criteria were enrolled in the study. Female patients requiring double $\mathbf{J}$ ( $\mathrm{JJ}$ ) stent placement (Group A) were compared with sexually active females who did not require $\mathrm{JJ}$ stent insertion (Group B) following URS. The outcome variable was to assess the impact on sexual function after JJ stent placement. Patients were requested to fill the FSFI form (Female Sexual Function Index) at initial visit, 04 weeks, and at 12 weeks.

Results: A total of 106 sexually active women were included in the study. In total, 37 women refused to participate; most 
common reason for refusal was hesitation to discuss female sexuality. The final study population included 69 women. Women in group A, the mean FSFI score at initial presentation was $31.706 \pm 4.246$. FSFI score at 4 weeks was lower compared to the baseline score $14.242 \pm 5.272$. (p-value $<0.0001$ ). At 12 weeks the FSFI score returned to baseline $32.053 \pm 5.35$ ). There was no significant difference in FSFI score at 12 weeks as compared to baseline 31.706 vs ...32.053 (p-value 0.76). In comparison to women in group B, the mean FSFI score at 4 weeks was significantly lower in Group A (p-value $<0.0001)$.

Conclusions: Sexual activity is an important aspect of a woman's overall wellbeing. Endourologic interventions (specifically DJ stent placement) result in transient post-operative sexual dysfunction in women, which resolves spontaneously within a span of 12 weeks.

\section{MP30-21 Ureteric stenting versus non-stenting fol- lowing uncomplicated ureteroscopic lithotripsy: A Prospective randomized trial}

S Al Demour, A Alrabadi, A AlSharif, M Ababneh, M Melhem, H Mansi, S Aljamal, M Abufaraj

The University of Jordan/ Jordan University Hospital/ Urology Department

Introduction \& Objective: There is no consensus about whether a double-J ureteric stent (DJ-US) should be placed following uncomplicated ureteroscopy for stone retrieval. This study aimed to compare three groups of patients who underwent uncompli- cated ureteroscopic lithotripsy (URSL) and to evaluate whether stents could be eliminated after the procedure.

Methods: A total of 105 patients underwent uncomplicated URSL for ureteric stones were prospectively randomized into three groups: group 1 (34 patients) with DJ-US, group 2 (35 patients) with DJ-US on extraction string, and group 3 (36 patients) with no DJ-US after the procedure. In group 1, DJ-US were cystoscopically removed after 2 weeks, whereas patients in group 2 had their DJ-US removed in the floor after 48 hours. The outcomes measured were; postoperative Visual Analog Score (VAS) for flank pain and dysuria score, urgency, frequency, suprapubic pain, hematuria and analgesia requirement at 5 hours, 24 hours, 48 hours, 1 week, and 1 month. In addition, patient and stone characteristics, operative time, re-hospitalization, duration of hospitalization, and return to normal physical activity.

Results: There were no statistically significant differences between the three groups regarding patient or stone characteristics. Mean operative time was significantly longer in groups 1 and 2 compared to group 3 [mean time $\pm \mathrm{SD}, 22.2 \pm 9.1 \mathrm{~min}$, $20.2 \pm 6 \mathrm{~min}, 15.1 \pm 7.1 \mathrm{~min}$ respectively, $\mathrm{p}<0.0001]$. The results of the VAS for flank pain and dysuria showed a significant difference at all time points of follow-up, with significantly higher mean VAS in groups 1 and 2 compared to group 3. Urgency, frequency, hematuria, and suprapubic pain were frequent complaints for patients in groups 1 and 2 compared to patients in group 3 with statistically significant differences. Further analysis showed that flank pain and dysuria scores, urgency, frequency, hematuria, suprapubic pain, and analgesia need for groups 1 and 2 were similar, except that group 2 experienced less flank pain, dysuria, urgency, frequency, hematuria, suprapubic pain, and analgesia need at 1 week and at 1 month compared to group 1 .

Table 3: The presence of urinary tract symptoms and the need for analgesia

\begin{tabular}{|c|c|c|c|c|c|}
\hline & Time & $\begin{array}{c}\text { Group } 1 \\
n(\%)\end{array}$ & $\begin{array}{c}\text { Group } 2 \\
n(\%)\end{array}$ & $\begin{array}{c}\text { Group } 3 \\
n(\%)\end{array}$ & P-value \\
\hline \multirow{5}{*}{ Urgency } & $5 \mathrm{~h}$ & 32 (94.1) & $35(100)$ & $21(58.3)$ & $<0.0001$ \\
\hline & $24 \mathrm{~h}$ & $30(88.2)$ & 34 (97.1) & $7(19.4)$ & $<0.0001$ \\
\hline & $48 \mathrm{~h}$ & $30(88.2)$ & $31(88.6)$ & $3(8.3)$ & $<0.0001$ \\
\hline & $7 d$ & $29(85.3)$ & $9(25.7)$ & $1(2.8)$ & $<0.0001$ \\
\hline & $1 \mathrm{~m}$ & $9(29.5)$ & $2(5.7)$ & $1(2.8)$ & $<0.004$ \\
\hline \multirow{5}{*}{ Frequency } & $5 \mathrm{~h}$ & $32(94.1)$ & $35(100)$ & $21(58.3)$ & $<0.0001$ \\
\hline & $24 \mathrm{~h}$ & $30(88.2)$ & $33(94.3)$ & $7(19.4)$ & $<0.0001$ \\
\hline & $48 \mathrm{~h}$ & $30(88.2)$ & $30(85.7)$ & $2(5.5)$ & $<0.0001$ \\
\hline & $7 d$ & $29(85.3)$ & $8(22.9)$ & $1(2.8)$ & $<0.0001$ \\
\hline & $1 \mathrm{~m}$ & $9(26.5)$ & 0 & 0 & 0.0001 \\
\hline \multirow{5}{*}{ Suprapubic Pain } & $5 \mathrm{~h}$ & $32(94.1)$ & $33(94.3)$ & $15(41.7)$ & $<0.0001$ \\
\hline & $24 \mathrm{~h}$ & $29(85.3)$ & $32(91.4)$ & $4(11.1)$ & $<0.0001$ \\
\hline & $48 \mathrm{~h}$ & $26(76.5)$ & $22(62.9)$ & $2(5.5)$ & $<0.0001$ \\
\hline & $7 \mathrm{~d}$ & $27(79.4)$ & $6(17.1)$ & $1(2.8)$ & $<0.0001$ \\
\hline & $1 \mathrm{~m}$ & $5(14.7)$ & $1(2.9)$ & 0 & 0.0141 \\
\hline \multirow{5}{*}{ Hematuria } & $5 \mathrm{~h}$ & $29(85.3)$ & 34 (97.1) & $26(72.2)$ & 0.0082 \\
\hline & $24 \mathrm{~h}$ & $25(73.5)$ & $21(60)$ & $10(27.9)$ & 0.0004 \\
\hline & $48 \mathrm{~h}$ & $22(64.7)$ & $3(8.6)$ & $1(2.8)$ & $<0.0001$ \\
\hline & $7 d$ & $12(52.7)$ & $3(12.5)$ & $1(4)$ & $<0.0001$ \\
\hline & $1 \mathrm{~m}$ & $1(2.94)$ & 0 & 0 & - \\
\hline \multirow{4}{*}{ Analgesia } & $5 \mathrm{~h}$ & $31(91.2)$ & $34(97.1)$ & $21(58.3)$ & $<0.0001$ \\
\hline & $24 \mathrm{~h}$ & $28(82,4)$ & $29(82.9)$ & $8(22.2)$ & $<0.0001$ \\
\hline & $48 \mathrm{~h}$ & $24(70.6)$ & $17(48.6)$ & $3(8.3)$ & $<0.0001$ \\
\hline & $7 d$ & $26(76.5)$ & $8(22.9)$ & $1(2.8)$ & $<0.0001$ \\
\hline
\end{tabular}


Conclusions: DJ-US placement appear to be unnecessary in procedures considered uncomplicated by operating urologists at the time of surgery. The advantages of DJ-US with extraction string over DJ-US only include earlier and easy removal with earlier relief of symptoms, and less analgesia requirements.

\section{MP30-22 Peri-Calculus Ureteral Thickness (P-CUT) on CT Predicts Stone Impaction at Time of Surgery: A Prospective Analysis}

RA Chandhoke, B Gallante, JN Bamberger, W Atallah, M Gupta, AM Zampini

Mount Sinai

Introduction \& Objective: Impacted ureteral stones are associated with edema and hypertrophy of the ureteral mucosa, which often makes endoscopic ureteral stone surgery more challenging. If certain preoperative CT scan parameters could predict significant ureteral stone impaction, a urologist would be better prepared and could better counsel a patient regarding associated risks and outcomes of surgery.

Methods: After IRB approval, we prospectively collected intraoperative data on 34 patients who had a preoperative CT scan and underwent ureteroscopy (URS) for ureteral stones. Surgeons were blinded to preoperative CT parameters except for stone size, location, and degree of hydronephrosis. Stones were considered impacted at surgery if contrast had difficulty passing proximal to the stone, or if a guidewire could not pass, and the stone was visually impacted $>5$ on a Likert scale. We correlated intra-operative findings to preoperative CT measurements of ureteral thickness on a coronal series directly above, around (ie P-CUT), and below the calculus. Other variables measured included hydronephrosis, stone volume, longest dimension, and stone location (Table 1). Chi-square, ANOVA, and logistic regression analyses were conducted.

Results: Of the 34 patients, 13 were found to have an impacted ureteral stone at the time of surgery. Patients with an intra-

\begin{tabular}{|c|c|c|c|}
\hline & Impacted & $\begin{array}{c}\text { Non- } \\
\text { Impacted }\end{array}$ & P-value \\
\hline $\mathrm{N}$ & 16 & 26 & \\
\hline Male : Female & $8: 8$ & $16: 10$ & $0.576^{\mathrm{B}}$ \\
\hline Mean Age (SD) & $56.71(13.3)$ & $61.65(10.7)$ & $0.184^{\mathrm{A}}$ \\
\hline Mean Height (m) & 1.67 & 1.69 & $0.594^{\mathrm{A}}$ \\
\hline Mean Weight (kg) & 82.1 & 79.7 & $0.681^{A}$ \\
\hline Mean BMI & 29.4 & 27.9 & $0.342^{\mathrm{A}}$ \\
\hline ASA & & & $0.796^{8}$ \\
\hline 1 & 1 & 3 & \\
\hline 2 & 11 & 18 & \\
\hline 3 & 4 & 5 & \\
\hline Stone Location & & & $0.285^{8}$ \\
\hline Proximal Ureter & 8 & 6 & \\
\hline Middle Ureter & 2 & 3 & \\
\hline Distal Ureter & 6 & 14 & \\
\hline Mean Stone Size (mm) & 7.35 & 6.45 & $0.626^{A}$ \\
\hline Mean Stone Volume $\left(\mathrm{mm}^{\wedge} 3\right)$ & 221.8 & 99.2 & $0.086^{A}$ \\
\hline PreOp Degree of Hydronephrosis & & & $0.144^{8}$ \\
\hline None & 3 & 1 & \\
\hline Mild & 4 & 14 & \\
\hline Moderate & 7 & 10 & \\
\hline Severe & 2 & 1 & \\
\hline \multicolumn{4}{|l|}{ Ureteral Thickness Parameters } \\
\hline Ureteral Thickness Above Calculus & 11.51 & 7.33 & $0.001^{* \mathrm{~A}}$ \\
\hline Peri-Calculus Ureteral Thickness (P-CUT) & 5.29 & 1.71 & $\begin{array}{l}0.001 * A \\
0.048^{* C}\end{array}$ \\
\hline Ureteral Thickness Below Calculus & 8.53 & 4.46 & $0.001^{* A}$ \\
\hline
\end{tabular}

operative finding of an impacted stone had significantly higher ureteral thickness above, around (P-CUT), and below the calculus (all three p-values <0.001) compared to non-impacted ureteral stones. Multivariate analysis revealed that only P-CUT was independently predictive of impaction at the time of surgery. A mean P-CUT of $5.3 \mathrm{~mm}$ and $1.7 \mathrm{~mm}$ was noted for the impacted and non-impacted stone groups, respectively.

Conclusions: Increased ureteral thickness as measured on CT coronal imaging above, around (P-CUT), and below the calculus is predictive of impacted stones at the time of surgery. This information is valuable for surgical planning and patient counseling of risks and outcomes expectations pre-operatively.

\section{ABSTRACT VIDEO SESSION}

\section{ABSTRACT VIDEO SESSION 01: BPH \& FEMALE UROLOGY}

V01-01 Step by step ThuLEP (Thulium laser enucleation of prostate): an Indian multicentric experience

J Jagtap, S Veer, G Agrawal, K Jadhav, P Rao, S Mistry

Introduction \& Objective: To share our clinical experience of thulium laser enucleation of the prostate (ThuLEP) for management of being prostatic hyperplasia and to describe the technique in a stepwise manner for easy reproducibility.
Methods: Patients with an International Prostate Symptom Score (IPSS) of $>15$ and a quality-of-life (QoL) score of $>3$ not responding to medical management, with a significant post-void residual urine volume (PVR; $>100 \mathrm{~mL}$ ) on ultrasound, with or without recurrent urinary tract infection and/or acute urinary retention were included in the study. Exclusion criteria were patients with urethral strictures, bladder stones, neurogenic bladder and previously failed transurethral procedures.

Results: A total of 42 men were included. The average age was 64.7 years. The mean preoperative IPSS and QoL scores were 
20.8 and 4.1 respectively. These improved to a mean value of 4.2 and 1.6 postoperatively. The average flow rate increased from 10.6 to $22.6 \mathrm{~mL} / \mathrm{s}$ and there was a decrease in PVR from a mean of $142 \mathrm{~mL}$ to $35 \mathrm{~mL}$. Mean operative time was $78 \mathrm{~min}$ with no major intraoperative complications. No patient required a blood transfusion. The average duration of catheterization and hospital stay were 2.2 days and 2.8 days respectively. Three patients (7.1\%) developed post-operative complications in the form of reintervention for bleeding control in one and formation of urethral and bladder neck strictures in the other two.

Conclusions: ThuLEP is an efficacious and safe modality of treatment for symptomatic BPH. Advantages are manifold in the form of shorter duration of catheterization and hospitalization, higher improvement of symptoms and low complication rates.

\section{V01-02 Endoscopic management of bladder mesh erosion following previous incontinence surgery}

\section{A Pietropaolo, BK Somani, M Ramachandra}

Introduction \& Objective: Intravesical mesh erosion following surgery for stress urinary incontinence (SUI) is a recognized but rare complication. The removal entails either open surgical removal or endoscopic laser excision.

Methods: A 57-year female with a history of Transvaginal tape (TVT) surgery for SUI presented with recurrent urinary tract infections (UTIs) 3 years after her initial surgery. A flexible cystoscopy revealed an encrusted mesh eroding the bladder. An endoscopic laser excision of the eroded mesh was electively performed.

Results: Under a general anesthesia a rigid cystoscopy was performed and Ho:YAG laser $(272 \mu$ m laser fiber; $1 \mathrm{~J}, 8-15 \mathrm{~Hz})$ was used to incise the mesh to detach it from the anterior bladder wall. Once this was achieved, the encrustations were lasered and the mesh was gradually incised and divided piecemeal. A grasper was used for retraction and to position the mesh intermittently. Once the mesh was detached from the right lateral wall, a deeper incision of bladder mucosa was done to ensure adequate removal of the intravesical mesh. A post-operative urethral catheter was left on free drainage for 3 weeks to allow the urothelium to heal over the bladder wall defect. A flexible cystoscopy under a local anesthesia was done at 6 weeks. This showed a complete resolution with no mesh seen in the bladder with urothelium covering the bladder defect. The patient remained free of UTI at 12 months follow-up.

Conclusions: Endoscopic laser excision of eroded bladder mesh is a minimally invasive procedure which would otherwise require an open surgical retrieval.

\section{V01-03 New Advancements of Moses for HoLEP: Transforming Enucleation into a Day Case}

\section{CU Nottingham, T Large, C Stephens, A Krambeck}

Introduction \& Objective: Bleeding complications during Holmium Laser Enucleation (HoLEP) are fortunately an infrequent complication, with reported rates of blood transfusion up to $2 \%$. However, bleeding control during the operation makes the case challenging and adds time especially in cases with very large glands, patients on anticoagulation, and patients with documented coagulopathy. The purpose of this study was to evaluate the initial experience with a novel modification of Moses technology in HoLEP optimized for HoLEP.

Methods: We performed one HoLEP with the novel technology using a Moses laser fiber. Adenoma enucleation was performed using a setting of 2 joules and 20 to 40 hertz for dissection, and 1 joule and 20 hertz for coagulation.

Results: We performed HoLEP on one patient with a prostate measuring approximately $200 \mathrm{~mL}$ volume by transrectal ultrasound. The patient was taking aspirin $81 \mathrm{mg}$ due to coronary artery disease that could not be stopped for the procedure. A total of 160 grams of adenoma was enucleated over 95 minutes. The patient was discharged home after 2 hours in the recovery area, and his urethral catheter was removed on postoperative day 1 in the office. He experienced no complications including bleeding, and he was able to void without difficulty following catheter removal.

Conclusions: The novel holmium technology allows for excellent hemostasis, visibility, and fiber stability, thereby improving surgeon experience and surgical outcome. At our institution, we are incorporating this technology into our outpatient HoLEP program.

\section{V01-04 Aquablation of the prostate: a guide through a novel robotic procedure for $\mathrm{BPH}$}

\section{A Hajj, J El-Asmar, N Abdallah, G Bustros, M Bulbul}

Introduction \& Objective: Aquablation is a novel minimally invasive procedure for patients with benign prostatic hyperplasia $(\mathrm{BPH})$. A robotic assisted high-velocity water jet is used for prostatic parenchymal tissue ablation under dynamic ultrasound guidance. Hemostasis is achieved by an innovative four-way double-balloon 24 Fr Foley catheter. Compared to a transurethral prostate resection, it has a shorter resection time and a lower rate of anejaculation.

Methods: A 60-year-old gentleman with a known history of $\mathrm{BPH}$ refractory to dual medical therapy presented to our clinic with urinary retention and a prostate size of 45 grams. Upon that, a robotic-assisted aquablation was performed. With the patient in dorsal lithotomy position, a bi-planar transrectal ultrasound device was secured, and a 24-Fr hand-piece accommodating scope was used for bladder access. Both devices were stabilized by articulating arms. Thereafter, the procept biorobotics software was activated and the mapping of depth, angle, and boundaries of resection was determined. A high-velocity water jet was applied by a foot-pedal pump.

Results: Total resection time was 4 minutes. Estimated blood loss was minimal, and the catheter was removed on Day 1. At three months follow-up, the patient had a post-void residue of 50 cc, an IPSS score of 5, and a quality of life score of 1. His Qmax velocity was recorded at $17.5 \mathrm{~mL} / \mathrm{sec}$. In addition, patient denied any post-operative ejaculatory variation.

Conclusions: Aquablation is a novel robotic platform allowing standardization of BPH surgery. It offers the advantage of ultrasound treatment planning and guidance, coupled with fast resection with a heat free waterjet energy.

\section{V01-05 Moses Laser Enucleation of the Prostate: Early Experience}

MW Pickhardt, T Large, CU Nottingham, A Krambeck

Introduction \& Objective: Moses Enucleation of the Prostate uses modulated, pulsed, laser energy to improve energy delivery to the targeted biologic material. The initial application of Moses technology was toward the treatment of kidney stones but has subsequently been applied to Holmium Laser Enucleation of the Prostate (HoLEP) for lower urinary tract symptoms secondary to benign prostate hyperplasia (LUTS/BPH). After an initial experience, we felt Moses was more hemostatic and therefore we sought to compare it to standard HoLEP. 
Methods: This video demonstrates examples of procedures done on patients enrolled in our study. This was a retrospective review of 152 men with LUTS/BPH who underwent HoLEP between August 2018 and January 2019. Patients were assigned to group 1 (50 patients), group 2 (50 patients) and group 3 (52 patients) based on their HoLEP being completed with a Slimline $550 \mu \mathrm{m}$, Slimline $1000 \mu \mathrm{m}$, or MOSES ${ }^{\circledR} 550 \mu \mathrm{m}$ laser.

Results: There were no differences between the groups in age, comorbidities, American Society of Anesthesia (ASA) classification, BPH medication or antiplatelet/anticoagulation (AP/AT) status between the groups. Prostate enucleation times differed significantly $(47.1 \pm 17.9,41.4 \pm 14.6,39.9 \pm 15.1 \mathrm{~min} \mathrm{p}=0.01)$ between the groups. Time to achieve hemostasis ( $\mathrm{min}$ ) was statistically different between the different groups $(10.6+6.1,7.7+5.2,6.3+4.8 \mathrm{~min}$ $\mathrm{p}<0.001)$. The change in hemoglobin was significantly less between the groups $(1.5+1.2,1.5+1.6,1.0+1.1 \mathrm{p}=0.02)$.

Conclusions: Our findings suggest that modulated pulsed laser energy, such as MOSES, can improve hemostasis during the enucleation phase of a HoLEP resulting in shorter procedure times and less blood loss.

\section{V01-06 Staying Out of Incontinence Zone: Our} technique of Ultra Low Power Precision HoLEP

S Prabhakar, Y KK

Introduction \& Objective: HoLEP is touted to be new gold standard surgery for Benign Prostatic Hypertrophy. High rates of temporary incontinence is reported for this procedure in literature. Hereby we present our technique of HoLEP and technical modifications to decrease incontinence.

Methods: We present edited videos of selected cases to demonstrate our technique. Core modifications we made in our technique include (1) Using only Ultra low power settings $(30 \mathrm{~W})$, (2) Curtain raiser mucosal incision over lower lateral aspect of lateral lobe (3) Avoiding mechanical push on lobes instead using precise lasing (4) Preserving anterior mucosa (5) Mucosal strip incision for lateral lobe enucleation (6) Roof top detachment of lateral lobes by precise lasing.

Results: Out of 453 patients, Temporary incontinence was observed in 4 patients $(0.9 \%)$. None had permanent incontinence. Conclusions: Ultra Low Power enucleation with this technique is an effective way to bring down incontinence incidence.

\section{V01-07 Thulium Laser Enucleation of Prostate In A} Patient Who Has 170 cc Prostate Volume

HC Aybal, TB Aydogan, E Gazel, E Kaya, S Yalcin, S Yilmaz, L Tunc

Introduction \& Objective: Thulium laser enucleation of prostate (ThuLEP) is a popular technique among surgical urology. It is now gradually becoming a contender for surgical treatments, trans- urethral resection of the prostate (TURP) and open prostatectomy (OP). Endoscopic prostate surgery has some difficulties in patients with big prostate volume. The most important problem is the bleeding of mucosa occur in the prostate tissue due to big prostate volume. In this case we present a ThuLEP surgery applied to a patient who has $170 \mathrm{cc}$ prostate volume.

Methods: A 68-year-old male patient was applied to our clinic with lower urinary tract symptoms. In his medical history it is determined that median lobe resection was performed in TURP operation 5 years ago. He has used alpha blocker and 5-alpha reductase inhibitor for two years. In his uroflowmetry test Qmax revealed $8.3 \mathrm{~mL} / \mathrm{sec}$ and average flow rate revealed $4.0 \mathrm{~mL} / \mathrm{sec}$.
In his urinary system ultrasound prostate size was determined as $170 \mathrm{cc}$. Three-lobe technique ThuLEP surgery was planned to patient and performed successfully.

Results: ThuLEP surgery allows removal of the entire adenomas of the prostate in the presence of the right anatomical line. In patients who have big prostate volume, the main difficulty is bleeding of mucosa. ThuLEP is a surgery with a long learning curve because of the need for high anatomical and endoscopic instrument experience. These experience gains much more importance in patients who have big prostate volume. Since our center has sufficient experience in this regard, we have successfully performed the surgery.

Conclusions: If ThuLEP surgery is performed in a patient who has big prostate volume, surgeons who have sufficient experience about ThuLEP must perform this surgery. Such cases can result with serious complications for surgeons at the learning stage.

\section{V01-08 Different Approaches For Robotic Simple Prostatectomy For Benign Prostatic Hyperplasia}

BD Dayal, K Meilika, A Beksac, KE Okhawere, AA Elbakry, KK Badani

Introduction \& Objective: Robotic simple prostatectomy is gaining popularity as an alternative management for large size adenoma due to benign prostatic hyperplasia. In this video we demonstrate two different approaches for the procedure.

Methods: The first case is a demonstration of the retropubic approach. We start by dropping the bladder to access the space of retzius. Prostatic capsule is dissected from the surrounding fat and an incision is made at the bladder neck. The median lobe is retracted upward, and an incision is made just proximal to the median lobe to develop the plane between the adenoma and the capsule of the prostate. Blunt dissection and electrocutarization are used to completely dissect the adenoma all around. After removal of the adenoma, we anastomose the posterior aspect of the bladder neck to the distal urethral stump. The anterior opening at the bladder neck is closed. The second case is a demonstration of trans-vesical approach. We start by performing a vertical incision at the dome of the bladder, and 4 extracorporeal sutures are applied through the abdominal wall for retraction. An incision is made just proximal to the median lobe to develop the plane between the adenoma and the capsule of the prostate. Adenoma is dissected as discussed prior. After removal of the adenoma, we perform a complete circumferential bladder neck reconstruction to the distal urethral stump. The cystotomy is then closed.

Results: Both approaches are feasible and safely completed robotically. In our experience, there was no difference between the two approaches regarding estimated blood loss, hospital stay or complication rate. However, the transvesical approach catheter time was 6 days (range 5-7), whereas the retropubic approach had a dwell time of 10 days (range 8-14).

Conclusions: We demonstrated a stepwise technique for both transvesical and retropubic robotic simple prostatectomy for benign prostatic hyperplasia disease. Both approaches had similar operative outcome, and the transvesical approach led to a shorter catheter time.

\section{V01-09 ProTouch Laser Enucleation of the Prostate:} A retrospective analysis of the first 77 cases

M Emmerling, S Wason, W Pate

Boston Medical Center Department of Urology 
Introduction \& Objective: The ProTouch (1470nm) laser is a novel diode laser for use in endoscopic enucleation of the prostate. ProTouch laser enucleation of the prostate (ProLEP) may provide similar outcomes to traditional HoLEP with the additional benefit of improved hemostasis and tissue vaporization.

Methods: This is an IRB approved single surgeon chart review of 77 patients (average age 69.9 \pm 9.9 years) who underwent ProLEP (10/16-3/19). Pre-operative, intra-operative, and postoperative variables were collected and analyzed.

Results: Average prostate gland size was $92.4 \mathrm{~g}$ (range: $27-$ $290 \mathrm{~g}$ ) by pelvic ultrasound and average pre-operative International Prostate Symptom Score (IPSS) (on medical therapy) was $22.3 \pm 6.9$. Post-operative IPSS was significantly reduced at 1,3 , 6 , and 12 months [12.9 $\pm 9.3,9.5 \pm 8.1,7.3 \pm 5.0,9.2 \pm 6.0$ (pvalue $<0.0001)]$. Quality of life (IPSS bother scale) was significantly improved from baseline (4.7 \pm 1.2$)$ at $1,3,6$ and 12 months $[2.5 \pm 1.9,1.7 \pm 1.6,1.8 \pm 1.5,2.5 \pm 1.9$ (p-value $<0.0001)]$. Average pre-operative $\mathrm{Q}_{\max }$ was $9.1 \pm 4.5 \mathrm{cc} / \mathrm{sec}$ with significant post-operative improvement at 1,3 and 6-months [27.2 \pm 11.3 $\mathrm{cc} / \mathrm{sec}, 15.1 \pm 11.1 \mathrm{cc} / \mathrm{sec}, 20.8 \pm 6.6 \mathrm{cc} / \mathrm{sec}$ (p-value <0.05)]. 25 patients $(32.5 \%)$ were catheter dependent prior to surgery, 19 of whom had indwelling catheters while the remaining 6 performed daily clean intermittent catheterization. All patients were voiding spontaneously at 1-month post-procedure. Average operative time was 130.7 minutes and average EBL was $51.8 \mathrm{~mL}$. An average of $30.4 \mathrm{~g}$ of prostatic tissue was resected during the ProLEP procedure, with an average of $34.2 \%$ of total prostatic tissue mass resected. Thirty-eight patients $(49.4 \%)$ were discharged from the recovery room, with 72 patients $(93.5 \%)$ being discharged on or before POD\#1. 53 patients $(68.8 \%)$ had their catheters removed on or before POD\#1. 14 patients (18.2\%) experienced intraoperative or post-operative complications. 8 patients required recatheterization for post-operative acute urinary retention, urethral false passage occurred in 1 patient, 2 patients required antibiotics for a UTI, and 1 patient underwent cystoscopy for persistent hematuria. Two patients returned to the operating room for clot evacuation and fulguration. No patients received a blood transfusion. All of the complications were Clavien-Dindo IIIb classification or less (Clavien I-64.3\%, Clavien II-14.3\%, Clavien IIIa-7.1\%, Clavien IIIb-14.3\%). No patients required repeat surgery.

Conclusions: To our knowledge, this study is the first to investigate ProLEP for the treatment of LUTS in men with BPH. Our data suggest that ProLEP is a safe and effective alternative to TURP.

\section{V01-10 HoLEP with Moses technology: MoLEP}

\section{Y Hussein M.I., S Corti, F Ceresoli, R Milesi, I Vavassori}

Introduction \& Objective: The endoscopic technique of enucleation of the prostatic adenoma with Holmium laser (HoLEP) was introduced in 1998 by Peter Gilling. The enucleation technique and holmium laser production technology has remained almost unchanged for 20 years. After years of research, Lumenis has developed a system capable of modify the production of the Holmium laser pulse with the Moses technology in order to optimize the laser to lithotripsy and enucleation of the prostate. In the video we show how Moses technology can improve HoLEP execution.

Methods: The Moses technology allows to modify the shape of the laser pulse and therefore of the plasma bubble produced by the interaction between Holmium laser and water. The laser energy in HoLEP is used in two different ways, one is vapor- ization of prostate tissue making the incisions prior to the enucleation; the second mode exploits the mechanical effect called "photoacoustic effect", due to the expansion of the plasma bubble, resulting in a pressure wave that propagates to promote cleavage of tissues in course of detachment of the adenoma from the capsule during enucleation.

Results: As shown the application of Moses technology during HoLEP allows a better interaction between laser and prostatic tissue; during incisions the MoSES is able to produce a vaporization of greater depth, therefore more effective, during anterograde incision; the oblong plasma bubble allows the tissue flaps to be kept out of the way, while proceeding retrograde, allowing the capsular plane already exposed to be kept under visual control, optimizing the course and duration of the incisions. The photoacoustic effect produced with Moses technology allows obtaining a longer detachment of the tissues with respect to the conventionally produced impulse, resulting in a greater efficiency in enucleation due to the separation of longer tissue segments and for better exposure of the plan.

Conclusions: The Moses technology makes the HoLEP more effective when performed by an expert operator due to reduction of incision and enucleation times and is able to simplify the learning curve for beginners given due to the better development and exposure of enucleation plans.

V01-11 4D UroLift ${ }^{\circledR}$ approach to Anatomically chal-
lenging Benign Prostatic Hyperplasia with middle lobe

\section{KL Ng, S Giona, NJ Barber}

Introduction \& Objective: Urolift prostatic surgery is an effective minimally invasive surgical technique for the treatment of lower urinary tract symptoms (LUTS) secondary to benign prostatic hyperplasia (BPH). However, anatomically challenging $\mathrm{BPH}$ with obstructive middle lobe in a trilobar prostatomegaly present difficulties in the standard delivery technique for urolift in order to provide an effective disobstruction. The new 4D technique of urolift placements ( $P$. Chin, Australia) have managed to treat the middle lobe to achieve good urine outflow outcomes (similar to MEDLIFT study) with no serious adverse events or sexual dysfunction.

Methods: During flexible cystoscopy, 17 patients with symptomatic LUTS were diagnosed to have enlarged lateral and middle lobes. Informed consents for 4D urolift procedure were obtained. Four urolift implants were placed adjacent to bladder neck region in 4 different directions to maximally widen the bladder neck. All patients were discharged on same day, 12 patients without catheter while 5 patients discharged with catheter with a trial without catheter (TWOC) on post op day 3. International Prostate Symptoms Score (IPSS) and urine flow rates results were collected and analyzed.

Results: $65 \%$ reduction (14.6 points) in mean IPSS was noted (preop mean 22.4; postop 7.8). QoL scores reduced from 4.3(preop) to 1.7(post op). Uroflowmetry showed an improvement of mean Qmax from 12mls/sec (preop) to $26.3 \mathrm{mls} / \mathrm{sec}$ (postop) and reduction of post void residual volume of $52.7 \mathrm{mls}$ (mean). No erectile or ejaculatory dysfunction were reported. All patients had successful TWOC at the planned schedule post op. One patient returned with urinary retention within a week but with subsequent successful TWOC. There were no significant adverse events noted.

Conclusions: Obstructive middle lobes of BPH can be effectively treated with this novel technique of 4D prostatic urethral lift 
surgery. Our early encouraging results will provide for future larger trials assessing the effectiveness this 4D urolift technique in managing anatomically challenging obstructing middle lobes in $\mathrm{BPH}$.

\section{V01-12 Colposacropexy laparoscopic: Technique and functional Results}

C Conde Redondo, F Castroviejo Royo, A Alonso Villalba, S Martin Martin, J Torrecilla, J Diez, J Cortiñas González

Introduction \& Objective: A recent Cochrane review stated that abdominal sacrocolpopexy is the more effective procedure and is considered by many authors to be the gold standard in the treatment of vaginal vault prolapse. In this video we show the surgical technique step by step, and our functional results.

Methods: 55 women with symptomatic prolapse of the central compartment (Pelvic Organ Prolapse Quantitative [POP-Q] stage 2) underwent laparoscopic sacrocervicopexy. Surgical technique: 1. Fixation of sigma and uterus. 2. Opening of the peritoneum on the sacral promontory, and laterally to de sigma. 3. Liberation of the right pararectal space, up to the levator ani 4.
Liberation of the left pararectal space, up to the levator ani. 5. Fixing the mesh bilaterally. 6. Fixation of the mesh to the uterosacral ligaments and to the posterior vaginal aspect. 7. Opening of the broad ligament of the uterus. 8. Dissection of the anterior vaginal face. 9. Fixing the mesh to the anterior vaginal face. 10 . Fixation of the mesh to the sacral promontory. 11. closure of the peritoneum.

Results: The mean follow-up period was 53 months. The success rate was $90 \%$. The present study showed :8,5/10 satisfaction rate, a high amelioration on incontinence satisfaction, ICIQ-SF 8 vs 2,4 (range 0-21). Sexual results showed no changes (IFSF 22,75 vs 22,79 no SD). $60 \%$ showed incontinence previously, and $20 \%$ after surgery (SD). Incontinence cure rate was $77,7 \%$, incontinence maintained $11,1 \%$, de novo SUI $2.86 \%$. No changes in dyspareunia has been shown, $50 \%$ has bowel dysfunction, $5,7 \%$ mesh erosion, $2,8 \%$ bowel injury during the surgery and $2,8 \%$ iliac injury.

Conclusions: Laparoscopic sacrocervicopexy is an effective option for sexually active women with pelvic organ prolapse. No incontinence surgery should be done at the same time, under risk of overtreatment.

\section{ABSTRACT VIDEO SESSION 02: MISCELLANEOUS}

\begin{abstract}
V02-01 Indocyanine green (ICG) assisted video endoscopic inguinal lymphadenectomy (VEIL) for penile carcinoma
\end{abstract}

C Gurbani, K Chen, A Sim

Introduction \& Objective: Inguinal lymph node dissection has shown significant survival benefit in penile carcinoma and remains the gold standard for nodal staging and disease control in intermediate to high risk disease. Recent years have seen the emergence of the minimally invasive video-endoscopic inguinal lymphadenectomy (VEIL), thereby encouraging a shift away from traditional open nodal dissection and its associated high morbidity. We report the first case of bilateral VEIL in Singapore, aided also by indocyanine green (ICG) for deeper tissue penetration and visualization of nodal and vascular structures in a foreign operative field.

Methods: A 63 year-old with pT1aN3M0 squamous cell carcinoma of the penis underwent bilateral VEIL following partial glansectomy and 4 cycles of neoadjuvant chemotherapy. Port placement comprised the camera port 4 centimeters distal to the apex of the femoral triangle, with 2 working ports sited 6 centimeters laterally on either side. A plane was developed deep to Scarpa's fascia via a combination of digital dissection and introduction of the endoscope, and operative pneumoperitoneum was established at $8 \mathrm{mmHg}$. ICG-assisted nodal dissection was then performed abiding by the boundaries of the femoral triangle as the operative limits of dissection.

Results: Staged VEIL was performed. Operative time for right VEIL was 255 minutes, with the patient discharged on postoperative day 3 , and a brief readmission on day 25 for a right thigh seroma treated initially with oral antibiotics. Left VEIL and concurrent ultrasound guided aspiration of the right thigh collection were then performed on day 35 with a total operative time of 170 minutes. The patient was discharged 5 days thereafter, with bilateral drain removal on day 17 in clinic and no significant complications following. Nodal yield was 11 and 8 nodes respectively for right and left VEIL, with a total of 5 metastatic nodes overall.

Conclusions: ICG proved an invaluable aide in the identification of vital structures such as the femoral vessels, thereby facilitating more precise dissection. Whilst increased exposure is required to mitigate the learning curve for this operation, ICG-assisted VEIL is a promising safe alternative to open nodal dissection in this novel local experience.

\section{V02-02 Conservative treatment of a large UTUC in solitary kidney}

L Defidio, M De Dominicis, M Antonucci, N Russo

Introduction \& Objective: A conservative treatment for a large UTUC in solitary kidney 61 years old patient 2005 Right radical nephrectomy 2018 Left TCC : pielic $3 \mathrm{~cm}+$ lower calyx $2 \mathrm{~cm}$, negative for lynphonode involvements or metastasis 2018/01 RIRS + double JJ positioning : thullium ablation of a large TCC pielic tumor, urine cytology negative 2018/04 RIRS + double JJ positioning : partial ablation of lower calyx TCC, urine cytology negative 2018/10 PCN monopolar resection of large lower calyx tumor.

Methods: Puncture needle $12 \mathrm{~cm} 18$ Gauge Amplatz one shot dilator 30 Fr Amplatz sheath 30 Fr Storz monopolar resectoscope 26 Fr Storz flexible cystoscope 16 FR Nephrostomy tube $20 \mathrm{Fr}$ Double JJ 6Fr, $24 \mathrm{~cm}$ Storz Flexible ureteroscope Flex XC Ureteral access sheath $12 / 14 \mathrm{Fr}, 35 \mathrm{~cm}$.

Results: 3 months follow up RIRS, negative for tumor, urine cytology negative.

Conclusions: Percutaneous monopolar resection of a UTUC in solitary kidney is a conservative treatment for selected and motivated patient. 


\section{V02-03 Ultrasound Visibility of Instruments in Zero- exposure Retrograde Intrarenal Surgery}

D Takahashi, Y Kawanishi, K Izumi, M Yamanama, T Tsujioka, K Seto, Y Atagi, K Hirota, H Bando

Introduction \& Objective: Ultrasound (US) guidance techniques have increased precision and are not invasive. Therefore, they are being used in more and more medical procedures in recent years. However, fluoroscopy is still conventionally used for retrograde intrarenal surgery (RIRS). All surgical instruments for RIRS on the market are designed to be used under fluoroscopy guidance, without regard for their visibility under US guidance. The aim of our research is to evaluate the visibility of several currently available instruments under US guidance.

Methods: One hundred and seventeen consecutive patients underwent zero-exposure RIRS at our institution. The group consisted of 85 males $(72.6 \%)$ and 32 females $(27.4 \%)$. Mean age was $63 \pm 14.6$ years, mean body mass index was $24.5 \pm 4.4 \mathrm{~kg} / \mathrm{m}^{2}$, and mean stone size was $14.3 \pm 15.6 \mathrm{~mm}$. Forty-two $(35.9 \%)$ stones were renal, $44(37.6 \%)$ were in the proximal ureter, and 24 $(20.5 \%)$ were in the middle or distal ureter. Seven patients had multiple stones $(6.0 \%)$. The visibility of 2 types of guidewires, 5 types of ureteral access sheaths, 3 types of ureteral stents, and 4 types of laser fibers was evaluated subjectively by 7 surgeons and 4 ultrasonography technicians. Convex 5-MHz and linear 9-MHz ultrasound probes were used.

Results: Both guidewires were visualized satisfactorily. The most visible ureteral access sheath had a flat metallic coil intended to prevent kinking. However, sheath tips could not be seen for any of the 5 sheath types. All ureteral stents were visible, with no difference between those made of polyurethane and those made of silicone, but visibility was poor by any standard. No laser fibers could be seen under US guidance.

Discussion: Guidewires and ureteral sheaths containing metallic materials had good visibility. However, one issue was that sheath tips were unable to be seen for any of the 5 sheath types. The development of new instruments whose tips are visible under US guidance is desirable. Laser fibers were made of glass and were expected to be visible under US guidance. However, with a width of 200 micrometers, they were in fact too thin to be seen. In the case of fibers, their visibility under US guidance is much less of an issue as their use is ureteroscope-guided.

Conclusions: While it is true that zero-exposure RIRS can be sufficiently performed using currently available instruments, the development of instruments with improved visibility under US guidance is indeed desired. The availability of such instruments will contribute to advancements in the sophistication of RIRS.

\section{V02-04 Endoscopic Management of Giant Ureteral Fibroepithelial Polyp With Laser Ablation}

B Onal, Y Ilki, E Bulbul, B Ercili, S Citgez

Introduction \& Objective: Around 300 cases of fibroepithelial polyps (FEP) have been reported in the medical literature. Onefifth of the tumors in the ureter are benign, while the majority of these are FEPs originating from the mesoderm. In a series of 22 patients, ureteral stones were found in the etiology of the disease. Cases have been found in the renal pelvis, bladder and urethra, but mostly in the ureter. In most cases, FEP is benign. However, some reports indicate it may accompany malign transformation and urothelial carcinomas.
Methods: A 50-year-old woman was admitted to another hospital with macroscopic hematuria, suprapubic pain, dizury and urge incontinence. The patient, who had experienced the symptoms for approximately two months, suffered from no known diseases, while no pathology was detected in the physical examination. The MR urography showed protruding lesions in the distal ureter. Examinations revealed that the lesions were not hydrenophosis, but they could not determine whether they were inflammation or malignancy. An external ureteroscopy revealed a giant stem mass of $8 \mathrm{~cm}$ in the middle ureter, while the biopsy pathology was reported as reactive urothelial hyperplasia. The patient was referred to our hospital. When a ureteroscopy was performed with a ureterorenoscopy in semirigid, a polypoid $8 \mathrm{~cm}$ smooth mucosal surface lesion with a small stalk was seen. The $365 \mathrm{~nm}$ holmium laser probe was set to $1.2 \mathrm{~J}$ and $15 \mathrm{~Hz}$. The polypoid lesion ureter stalks was separated from the ureter with a laser ablate. The polypoid lesion was removed with a zero tip nitinol retrievel basket, while a 4.8 fr double $\mathrm{J}$ catheter was inserted into the right renal pelvicalyceal system.

Results: The total operation time lasted 40 minutes. The patient was discharged home one day later. One week after the examination, the patient's urinary incontinence regressed markedly. The pathology was reported as a urotelyal fibroepitial polyp. A double $\mathbf{J}$ catheter was removed one month later. Within a month, the patient reported no more complaints regarding the issue.

Conclusions: A holmium laser is a feasible and safe method of treating giant FEP. A ureteral double $\mathrm{J}$ stent can be inserted due to postoperative ureteral mucosal edema. Although FEP is rare, it should be considered in differential diagnoses in such giant urethral masses

\section{V02-05 Laser Endopyelotomy: where are we in 2019?}

\section{G Celentano, H Abboudi, F Schoofs, S Choong}

Introduction \& Objective: Pelviureteric junction obstruction (PUJO) is defined as a functional or anatomic obstruction to outflow of urine from the pelvis to the ureter, which if left untreated may result in a deterioration of kidney's function. PUJO is treated with three primary treatments: open pyeloplasty, minimally invasive pyeloplasty, and endopyelotomy. The evidence regarding the comparative effectiveness of these three treatments is limited. Over the last 20 years, the surgical management of PUJO has been revolutionized by the development of endourological instrumentation and several minimally invasive procedures.

Methods: A systematic search of the published literature was performed to assess the success rate and perioperative complications of laser endopyelotomy.

Results: The long success rate of the pooled data for the endopyelotomy was up to $89 \%$ with an average overall success of $75 \%$. The success rate for open pyeloplasty and minimally invasive pyeloplasty was up to $96 \%$ and $97 \%$ respectively. A recent study, which analysed 1125 minimally invasive pyeloplasties, 775 open pyeloplasties and 1315 endopyelotomies, shows that the failure rates were $7 \%, 9 \%$, and $15 \%$ for minimally invasive pyeloplasty, open pyeloplasty, and endopyelotomy, respectively. Conclusions: Laser endopyelotomy has a good success rate. Patients often choose this method as it is the least invasive with no abdominal incisions and has the shortest recovery. It can be performed as a day case with the least major complication rate. It is also the salvage technique of choice for failed open/ laparoscopic/ robotic pyeloplasty. 


\section{V02-06 Ureteroscopic management of parapelvical renal cysts.}

A Martov, N Baykov, D Ergakov

Introduction \& Objective: Renal cysts are the most common lesions of the kidney, which occur in about $50 \%$ of the population aged older than 50 years. Symptomatic parapelvical cysts could either obstruct urinary tract or make challenging the diagnosis of renal tumor. Open, laparoscopic and robotic management could be difficult due to the central location of pathology. Retrograde intrarenal laser incision has been proposed as an effective and minimally invasive treatment for such cysts. In this study, we report 2 cases with ureteroscopic laser management of parapelvical cyst.

Methods: 64 y.o. male was admitted with the revealed intrarenal lesion in the left kidney. He complained on the recurrent painless hematuria during last several months CT scan showed a round tumor with the diameter $36 \mathrm{~mm}$ and a density $7,2 \mathrm{HU}$. Our second patient was 35 y.o. female with MRI suspicion on $35 \mathrm{~mm}$ sinus mass in left kidney. Both patients were managed ureteroscopically. After having visualized the relationship between the cyst and the renal pelvis by retrograde pyelography, Ho:YAG laser incision of renal pelvis wall was carried out. A small hole was then made using a $400 \mu$ holmium laser fiber (Auriga ${ }^{\circledR}$, Boston Scientific) set at $0.8 \mathrm{~J}$ power with a $16 \mathrm{~Hz}$ frequency. Once sure of being into the renal cyst, the hole was enlarged as much as possible (usually $7-10 \mathrm{~mm}$ ) in order to obtain a wide communication between the 2 compartments and to easily place inside the cyst a 6 Fr double-J ureteral stent. An indwelling urethral catheter was finally left in the bladder.

Results: The postoperative period proceeded without complications. No signs of relapse were observed at follow-up examinations (ultrasound, CT/MRI, ureteroscopy) 3, 6 and 12 months after the operation.

Conclusions: Our small data showed the ureteroscopic management proved to be a safe and effective treatment for symptomatic renal parapelvical cysts. Further results are needed.

\section{V02-07 Transperineal prostate biopsy under local anesthetic in the outpatient setting with a reusable needle guide}

\section{A Patel, T Poloskey, C Rogers}

Introduction \& Objective: Transrectal ultrasound-guided biopsy of the prostate remains the current mainstay of diagnosis of prostate cancer in the US, however is associated with risk of infection $(\sim 10 \%)$ and severe sepsis $(\sim 0.6 \%$ in the state of Michigan). This is leading to gradual shift to performing prostate biopsy via a transperineal route (TPbx) with recent studies suggesting TPbx associated with a $0 \%$ risk of severe sepsis. Several different techniques for TPbx have been reported thus far; in this video we demonstrate a TPbx under local anesthetic (LA) in the outpatient clinic (OPC) using a reusable needle guide.

Methods: A 61-year-old, biopsy naïve, gentleman with a PSA of $5 \mathrm{~g} / \mathrm{dl}$ underwent a TPbx under LA in the OPC. The patient is supine with legs in stirrups exposing the perineum. The perineum is shaved, scrotum elevated with taping to groin and prepped with chlorhexadine. $10 \mathrm{mls}$ of $1 \%$ lidocaine is infiltrated via a $25 \mathrm{~g}$ needle at the skin at the proposed needle puncture site bilaterally, followed by deeper LA using $20 \mathrm{~g}$ spinal needle, using further $10 \mathrm{mls}$ of $1 \%$ lidocaine bilaterally focusing on infiltration to space between prostate capsule and pelvic floor muscles. Using a biplanar probe, prostate measurements are taken and 12 sector
TPbx are taken following a modified barzell map with avoidance of peri-urethral transition zone. Biopsy needle access to perineum is achieved with a disposable $17 \mathrm{G} 7.8 \mathrm{~cm}$ coaxial needle fitted to the reusable needle guide. To reach the anterior zone the needle guide is move to a higher position, for better access.

Results: The patient experienced moderate pain during LA infiltration, however during prostate biopsy the procedure was well tolerated. Only 2 puncture sites required in the perineum. No complications observed in follow-up at 2 weeks. TPbx results revealed Gleason grade group 2 disease in 3/12 cores max tumor length $6 \mathrm{~mm}$.

Conclusions: This video demonstrates a safe and feasible freehand TPbx technique using a reusable needle guide. The reusable nature of the needle guide provides a cost-effective approach to TPbx.

\section{V02-08 Percutaneous Ablation of Upper Renal Ur- othelial Carcinoma with Thulium laser}

A Breda, L Mosquera Seoane, D Vanacore, A Territo, F Regis, F Sanguedolce, O Mayordomo Ferrer, A Gallioli, A Mercadé, R Boissier, J Palou

Introduction \& Objective: In low-risk (LR) upper tract urothelial carcinoma (UTUC) conservative treatment has been considered a treatment valuable option to preserve renal function without compromising oncological outcomes. Retrograde ureteroscopy is the usual approach, even though percutaneous approach can be considered in selected cases. Laser endoscopic ablation has been traditionally undertaken with Holmium YAG; more recently, Thulium laser has been proposed as an alternative with improved spatial beam quality, more precise tissue incision, and operation in continuous-wave/pulsed modes. We describe a case of incidental right UTUC treated with a percutaneous approach by using Thulium laser.

Methods: A 62 yo male referred to his GP because of chronic lumbar pain. A CT scan of the abdomen was performed showing a $38 \mathrm{~mm}$ lesion of the pancreatic tail associated with a thrombus invading the splenic vein. Further findings included the incidental detection of a $17 \mathrm{~mm}$ right renal pelvis lesion, enhancing with contrast, suggestive for a UTUC. A distal pancreatectomy with splenectomy and lymphadenectomy was firstly undertaken; histology reported a poorly differentiated pancreatic adenocarcinoma, pT2N1, for which adjuvant chemotherapy was indicated. Before that, a retrograde intrarenal surgery was attempted to diagnose and treat the right renal pelvis lesion. Because of a tight uretero-pelvic junction the procedure was abandoned; a right $\mathrm{J} \mathrm{J}$ stent and a nephrostomy tube were inserted. The procedure was rescheduled after 4 cycles of chemotherapy via a percutaneous approach. Through a nephrostomy tube a guidewire was advanced and a 10/12 Fr ureteral access sheath was inserted. The renal pelvis and collecting system were explored with a digital flexible ureteroscope (Flex-Xc, Storz): a sessile tumor of $20 \mathrm{~mm}$ in size was found in the renal pelvis. The lesion was sampled with a $3 \mathrm{fr}$ piranha forceps and a $6 \mathrm{fr}$ BIGopsy forceps cup. The lesion was first contoured all around with Thulium laser and then completely ablated.

Results: Neither intra- nor post-operative complications were recorded. Histology reported a low grade UTUC so that the patient was deemed suitable for conservative management of his UTUC. At 3-month follow-up an antegrade pyelography did not show filling defects and selective cytology was negative.

Conclusions: Percutaneous ablation is a valuable option in patient suitable for conservative treatment of LR UTUC in the 
kidney, especially in those patients where the retrograde approach is not feasible or not indicated. The aid of new technologies such as the thulium laser seem to increase efficacy reducing risks of complications.

\section{V02-09 Robot-assisted Retroperitoneal Lymph Node Dissection in Post-chemotherapy Residual Mass Ad- herent to Left Kidney Vessels}

\author{
O Aytac, A GARAYEV, F Atuğ
}

Introduction \& Objective: To report robot-assisted retroperitoneal lymph node dissection (RA-PLND) in 27-year-old man with mixed testicular germ cell carcinoma.

Methods: A 27-year-old man with left testicular mass presented to our hospital. Scrotal ultrasonography revealed a $3 \mathrm{~cm}$ solid mass in the testicular parenchyma. Tumor markers were elevated $(\mathrm{AFP}=85 \mathrm{mg} / \mathrm{dl}, \mathrm{b}-\mathrm{HCG}>10.000 \mathrm{mIU} / \mathrm{ml})$. After first evaluation patient underwent radical orchiectomy. The pathology report showed mixed germ cell tumor with teratoma (\%10), choriocarcinoma (\%85) and yolc sac (\%5) component. Due to paraaortic and lung metastasis he received 4 course BEP chemotherapy. After completion of medical treatment computed tomography revealed residual mass in left paraaortic region near to the kidney vessels. Robot-assisted laparoscopic RPLND was planned.

Results: After placing the patient in the right flank position robot docked from the right side of the patient. A total of 5 ports were used. Due to improved visualization of the robotic technology, mass was gently dissected from adherent aorta, VCI and ureter. Robot provided also slight and careful retraction and dissection of renal vein and artery. This allowed to complete operation without any vessel injury. Total operation time and surgical console time were 250 and 190 minutes. Blood loss was $100 \mathrm{ml}$. Patient discharged after 3 days without any complication. The final pathology showed necrotic cells without any viable tumor. Conclusions: In this case we showed safety and feasibility of robotic approach for surgical treatment of RPLND. Further studies will demonstrate advantages of da Vinci System for large retroperitonal masses.

\section{V02-10 MRI-based 3D printing of prostate for clinical-radiological-pathological correlation}

\section{T Chan, C Kan, K Lai, L Lam, T Chung, Y Stone, N Chia,} $\mathrm{W} \mathrm{Au}$

Introduction \& Objective: Surgical margin is always an important factor in predicting cancer specific survival or tumor recurrence. However, direct comparison of pathological slides with radiological images is often difficult due to their different alignment of cuts, therefore limiting our knowledge on disease anatomy and affecting patient's management. With the current knowledge on 3D printing technology, we would like to introduce its application in our daily surgical practice.

Methods: A gentleman with positive surgical margin after a robotic assisted laparoscopic radical prostatectomy was recruited. His preoperative multi-parametric MRI was obtained and was used for 3D reconstruction. The association between the Prostate Imaging Reporting and Data System (PI-RADS) lesion and pathology slides was analyzed.

Results: The pre-operative MRI images was used to outline the region of interest and applied on segmentation. The reconstructed model was sent for pathological sectioning and put together with pathological slides for direct comparison. After adjustment of printing materials to mimic a comparable patho- logical slicing, several models were made to test on its color contrast as well as stability during the slicing. The location of tumor breach was identified on the model, which is compared with intraoperative findings and reflected on the pathological slides. The location of positive surgical margin was found to be indeed beneath a pre-prostatic fat, which was taken out and sent separately during the surgery. The pre-prostatic fat had no evidence of malignancy, and therefore patient was subsequently spared from adjuvant treatment as he does not have a genuine positive surgical margin.

Conclusions: We introduced the index case of 3D printing using MRI images, instead of the usual CT films. It allows the direct visualization of region involved in positive margin, provides a good and reliable histological correlation, and thus opens up another potential application of 3D printing. We also touched on the 3D model creation process, technical difficulties encountered during model printing, and its future application in clinicalradiological-pathological correlation.

\section{V02-11 Office-Based, Ultrasound-Guided Renal Mass Biopsy: Technique and Results}

FA Jefferson, Z Okhunov, J Sung, C Cottone, RM Patel, J Landman

Introduction \& Objective: Imaging advances have led to an increase in the incidental detection of small renal masses (SRM) (i.e. $\leq 4 \mathrm{~cm}$ ) and, perforce, of renal cell carcinoma (RCC). Notably, $20 \%$ of SRM are benign, and some indolent malignant tumors can be safely managed with active surveillance; yet, preexcision biopsy is practiced by a minority of urologists. Renal mass biopsy (RMB) could alter the management of SRM by reducing the number of benign masses excised. Historically, RMB required patient sedation, CT-guidance, and was a radiologist-performed, hospital-based procedure. Advances in ultrasound (US) technology now enable the urologist to perform office-based, US-guided RMB. This video presentation details our office-based, US-guided RMB technique in an 80-year-old male with a renal mass and provides further details on our experience with this approach in over 50 patients.

Methods: A few hours prior to the scheduled biopsy, the patient applies anesthetic cream (lidocaine $2.5 \%$ /prilocaine $2.5 \%$ ) to the skin overlying the previously outlined biopsy entry point. The patient positions himself/herself in the prone position. Once the target lesion and renal anatomy are identified on US, $1 \%$ lidocaine is injected into the skin and along the entire biopsy needle track up to a few mm off the surface of the tumor. A standard 18G biopsy gun is used to insert a biopsy needle under direct USguided vision using a facilitated US targeting system; 4-6 cores are obtained. After the procedure, the patient's vital signs are monitored; one hour after the biopsy, a Doppler US is performed in the office to assess for complications. The patient is evaluated for pain (scale 0-10) before and after the biopsy, and a follow-up appointment is made in order to discuss the biopsy results.

Results: Our prospectively maintained RMB database $(\mathrm{N}=52)$ revealed a diagnostic rate of $73 \%$ and a concordance with final pathology in 100\% (16/16) for primary histology, 93\% (14/15) for histologic subtype, and 90\% (9/10) for low (I-II) vs. high (III-IV) Fuhrman grade. RMB revealed benign pathology in $21 \%(11 / 52)$ of patients. No patient with a benign lesion underwent surgery. Of note, 39\% (20/52) of our patients have opted for active surveillance. There were no complications. Patients reported a median pain score of $1 / 10$ (range $0-5$ ) and $0 / 10$ (range 0-5.5) immediately after RMB and at one hour after RMB, respectively. Fewer than $10 \%(4 / 52)$ of patients required narcotics for pain. 
Conclusions: Office-based RMB can safely be performed by a urologist skilled in renal ultrasonography. RMB guides patient management and markedly reduces the rate of surgery for benign renal masses.

\section{V02-12 Robotized Laparoscopy demonstration in various Urological scenarios}

K Ramaswami, P Bhirud, R Raveendran, H Pothiyedath

Introduction \& Objective: Today laparoscopy has become the essential standard care for most of the urology related diseases like stone disease, uro-oncology, reconstructive urology, pediatric urology and renal transplantation. 2 Dimension laparoscopy was upgraded to 3 Dimension laparoscopy which made the laparoscopy much easier with short learning curve. Robotic surgery gave the maximum advancement and comfort to the operating surgeon with good result and its main drawback is the high cost of investment and maintenance. Robotized laparoscopy with the $3 \mathrm{D}$ vision is a new alternative which is providing the benefits of robotic surgery at a lower cost and good result. We share the techniques, outcomes, feasibility and experience of major reconstructive urology cases of Partial nephrectomy, radical prostatectomy, Pyeloplasty, ureteric reimplantation done with robotized laparoscopic instruments.

Methods: All the cases for robotized laparoscopy is given a bowel preparation prior night with poly ethylene glycol and done under general anesthesia. Initial camera port was $(10 \mathrm{~mm})$ placed under vision by open method and working ports were placed under camera guidance. All the cases were approached with transperitoneally and abdomen pressure was maintained at $12-15 \mathrm{~mm} \mathrm{Hg}$. 3-Dimensional laparoscopy monitor was used in all cases. The robotized scissors and needle holder were used for reconstructive steps. Harmonic energy source was used in all cases.

Results: The operating time and blood loss was similar to standard laparoscopic procedures but with more comfort for the surgeon of our own series. No technical malfunction of robotized equipment noted during the procedure. There was no conversion to open surgery in the series. No blood transfusion was required in the above cases.

Conclusions: Robotic assistance is an adjunct to 3 Dimension laparoscopy, being far superior ergonomically. The main advantage of this technology is enhanced dexterity, precision, ergonomics, less surgeon fatigue, tremor filter, haptic feedback, seven degrees of movement, 360-degree rotation and cost effectiveness. The learning curve is very short.
V02-13 Application of ureteroscopy combined with laparoscopy in the treatment of ureteral septal injury after cervical cancer operation

J Huang, X Zhan, G Hu, H Zhang, Y Xu

Introduction \& Objective: To explore the surgery and surgical techniques of laparoscopy combined with ureteroscopy in the treatment of ureteral injury after cervical cancer.

Methods: Patient information: Female 45 years old, admitted to 'pathologically diagnosed cervical cancer for 1 week'. During the process of radical surgery of cervical cancer, the thermal injury of the ultrasonic knife was not recognized in time. Urine leakage occurred 1 week later. Urinary tract angiography suggested right ureteral pelvic injury. Ureteroscopy considered urteral disconnection. The patient took the bladder lithotomy position, the gynecologist placed the laparoscopic kit in parallel with the original tunnel of laparoscopic operation to find the proximal end of the ureter. Meanwhile, the urologist operated the F8/9.8 WOLF ureteroscope through the bladder ureter opening, finding the distal end of the ureter. The operation was completed by two physicians at the same time. During the operation, the proximal ureter and stump were seen from the front of the psoas muscle. In order to maintain the stability of the proximal ureter, the ureter is not excessively free, and the stump ureter is properly cut and trimmed. The ureteroscope is placed from the ureteral opening and then to the ureteral stump. Under the monitor of laparoscopic visual and auxiliary operation, the zebra guide wire is placed along the stump opening through the proximal ureter. Next, the ureteroscope enters the proximal ureter, confirming that the guide wire is placed in place, then guiding the F6 ureteral stent tube through the guide wire, and then performing the distal end and the proximal ureter end-to-end anastomosis through the laparoscope.

Results: Ureteroscopy combined with laparoscopic surgery for ureteral septal injury after cervical cancer operation, the time of operation is 65 minutes, the process is smooth and satisfied, KUB prompts the double $\mathrm{J}$ tube is in place, the urine leakage disappears, and the hydronephrosis is obviously relieved. The patient was discharged one week after the surgery.

Conclusions: Ureteroscopy combined with laparoscopic surgery for ureteral septal injury after cervical cancer surgery, the operation is easy to proceed, the patient expects smaller damage, shorter decubation and the short-term curative effect is verified. However, the long-term efficacy is still waiting for further exploration.

\section{ABSTRACT VIDEO SESSION 03: RADICAL PROSTATECTOMY}

V03-01 Intrafascial radical prostatectomy by robotic laparoscopic assisted surgery with complete Neurovascular bundle and Urethra preservation

\author{
F Adawi, M Alhamad, A Alkhalili, A Demey, X Taccoen, \\ P Loock, F Ozone
}

Introduction \& Objective: Objective: erectile dysfunction and Postoperative urinary incontinence are two complications to be feared after the radical prostatectomy, several surgical techniques were proposed to conserve erectile function and urinary continence we present in this video the technique of Intrafascial radical Prostatectomy with neurovascular bundles and urethra preservation

Material and Methods: 63-year-old Patient under treatment with Alpha blocker for voiding disorders, prostatic biopsies performed for PSA elevation at 7.3 diagnosed with prostate adenocarcinoma G $6(3+3)$ Briganti Score 0.001, rectal digital exam finds prostate $70 \mathrm{~g}$ non-suspect, MRI T2a right lobe. Patient very desired to keep his sexual activities. Robot Da Vinci 4 arms Intervention begins by dissection of Retzius space then dissection of bladder necks up to the plane of the seminal vesicles 
Section of vas deferens thereafter we performed Intrafacial prostatic discussion with preservation of neurovascular bundles by remaining in contact with the prostate. discussion of the prostatic apex with the preservation of the urethra is performed without section of pubo-prostatic ligament the anastomosis Between the urethra and the bladder neck was performed by 2 continues suture using a $\mathrm{V}$ lock $3 / 0$.

Results: The intervention duration was 105 minutes Bleeding 30 cc no drainage, the patient left the service at day one next operative The histopathological exam confirms the presence of a prostatic Adenocarcinoma PT2b R0 G6 (3+3), The bladder catheter was removed at day 76 months later the patient has spontaneous erection and mainland with only rare leakage to excessive exertion. Conclusions: intrafascial radical prostatectomy by laparoscopic assisted surgery May be performed in selected patients with a priori best results on the continence and the preservation of the erection.

\section{V03-02 Robotic-Assisted Radical Prostatectomy with minimal apical dissection and lateral prostatic fascia preservation: an evolution of our conventional technique.}

M Covas Moschovas, O Fikret, S Bhat, T Rogers, V Patel

Introduction \& Objective: Radical prostatectomy is the gold standard surgical treatment for prostate adenocarcinoma. The robotic-assisted radical prostatectomy (RARP) advantages such as lower hospital stay and blood loss are established in the literature. After more than ten thousand robotic prostatectomies, we notice that patients presented a faster potency and continence recovery after the modification of our conventional technique. Our objective is to illustrate and describe the robotic-assisted radical prostatectomy with minimal apical dissection and lateral prostatic fascia preservation (MAD/LPFP), an evolution of our technique, and compare to our conventional RARP (cRARP).

Methods: In this compilation, we have organized our video in four surgical steps to present the differences between MAD/LPFP and cRARP procedures. The surgical technique described for both cases presented six transperitoneal ports placed at the traditional RARP sites (four robotic and two assistant trocars). The same surgeon performed both techniques illustrated in this video with the DaVinci Xi console.

Results: The MAD/LPFPdiffers from the cRARP by the following steps:

1. Endopelvic fascia dissection (EFD): In the cRARP, the first step was the endopelvic fascia opening to dissect the prostate until the apex. In the MAD/LPFP technique, the lateral prostatic fascia is preserved, and the EFD step is performed after the posterior prostate dissection.

2. Santorini complex stitch: This step used to be performed after the endopelvic fascia opening and apical dissection. The cRARP described one single stitch at Santorini complex before the suspension stitch step. In the current technique, the Santorini bleeding control is performed with a running suture after the apical dissection and before the urethral division.

3. Suspension stitch: The stitch used to be applied to replace the puboprostatic ligaments divided during the apical dissection. However, this step is not performed in the MAD/LPFPtechnique due to the anatomy preservation after the apical dissection.

4. Apical dissection (AD): In the cRARP, both puboprostatic ligaments are divided, while the MAD/LPFP approach spares the ligaments and the lateral prostatic fascia.
Conclusions: Robotic radical prostatectomy with minimal apical dissection and lateral prostatic fascia preservation is a safe procedure and should be considered for radical prostatectomies. The data regarding the modification of our conventional technique after ten thousand cases suggest that the apical anatomy preservation and the anterior neuronal plexus sparing are crucial factors related to the earlier potency and continence recovery.

\section{V03-03 Less is More: Modified Robotic Prosta- tectomy Techniques to Decrease Operative Time and Improve Outcomes}

NH Patel, M Iorga, M Stern, M Zhang, R Yau, M Esposito, G Lovallo, M Ahmed

Introduction \& Objective: Robotic radical prostatectomy is a common minimally invasive technique used to treat prostate cancer in appropriately selected patients. Herein we sought to describe our modified operative technique and present the effects on surgical outcomes of robotic-assisted laparoscopic prostatectomy (RALP). This modified technique helps eliminates additional steps and combines them with knowledge of the anatomy.

Methods: As compared to the standard robotic prostatectomy technique we omit division of the endopelvic fascia and suturing or stapling of the dorsal venous complex for our modified approach. Alternatively, this technique incorporates division of the endopelvic fascia with the nerve sparing, creates wide develop of the posterior/lateral plane allowing for single clip placement on the pedicle, and sutures the dorsal venous complex with the vesicourethral anastomosis for hemostasis as well as urethral support. Furthermore, we retrospectively examined a total of 106 patients in two group: group $1(\mathrm{n}=53)$ under robotic radical prostatectomy using the standard approach and group $2(n=53)$ underwent the modified approach. The demographics, perioperative and postoperative data and results were compared retrospectively. We examined continence data for all patients and erectile function data for patients with a SHIM score above 16 , no history of previous radiation, and no history of diabetes mellitus for greater than five years.

Results: When we examined demographic and operative factors such as age, PSA, gleason score, body mass index (BMI), estimated blood loss, and positive surgical margins no statistically significant difference was seen between the two groups. The operative time was lower in the modified group $(125.679 \pm 23.001$ minutes vs $112.981 \pm 17.059$ minutes, $p=0.0017)$. When continence was quantified as a categorical variable, the 3-month and 6month continence rates were significantly greater in group 2 (41.5\% vs $69.9 \%, p=0.0059 ; 85.0 \%$ vs. $98.1 \%, p=0.0313$, respectively). No difference in erectile function post-operatively was seen in both groups.

Conclusions: Our modified technique for control of the DVC and delayed entry into the endopelvic fascia resulted in a faster operative time and a quicker recovery to continence. This technique is easily reproducible and can combine multiple related steps simultaneously for better outcomes.

\section{V03-04 An analysis of the trans-umbilical approach of reduced port surgery in laparoscopic radical pros- tatectomy}

K Araki, K Ohtsuka, K Hou, T Suyama, S Kojima, Y Naya, H Masuda, K Mikami

Introduction \& Objective: Radical prostatectomy is an established treatment procedure for localized prostate cancer. 
Currently, the procedure is mainly performed using robotassisted surgery, which offers the advantage of good degree of motion range; however, the operation wound in robot-assisted surgery is slightly larger than that in conventional laparoscopic radical prostatectomy (LRP). Thus, we attempted the transumbilical approach of reduced port surgery in laparoscopic radical prostatectomy (reduced port LRP) to reduce the size of wound, which is an advantage of LRP.

Methods: We included 15 patients who underwent reduced port LRP from November 2017 to March 2019 at Teikyo University Chiba Medical Center. The surgery was performed using a 4-port trans-umbilical extra-peritoneal approach with a head-down angle of $20^{\circ}$. A U-shape incision was placed on the caudal side of the umbilicus. The extra-pelvic pre-vesical space was maintained using a balloon dilator, and the multi-channel port (E.Z AC$\mathrm{CESS}^{\circledR}$, E.Z trocar $5 \mathrm{~mm}^{\circledR}$, Lap protector $\left.{ }^{\circledR}\right)$ was placed in the umbilical wound. Three $5 \mathrm{~mm}$ ports were positioned in the caudal side of umbilicus. Despite the elevated risk, reduced port LRP was indicated, because there was adequate space from the umbilical port to perform extended lymph node dissection (LND). Radical prostatectomy was performed same modality as conventional LRP, LND was performed if necessary, and all specimens were removed from the umbilical wound.

Results: The patients' age, preoperative PSA level, operating time, and estimated blood loss were $69 \pm 3.81$ (63-72) years, $9.57 \pm 6.66$ (4.73-30.75) ng/ml, $328 \pm 50.6$ (245-423) minutes, and $419 \pm 316(100-1443) \mathrm{ml}$, respectively. The specimens could be removed from umbilical wound in all except two cases, in whom the prostate volume was more than $50 \mathrm{ml}$. Pathological diagnoses of T1c, 2a, 2b, and 2c were established in 9, 2, 1, and 3 cases respectively. The Gleason scores were $3+3=6,3+4=7$, $4+3=7$, and $4+4=8$ in $8,3,3$, and 1 cases respectively. The number of dissected lymph nodes was $9.5 \pm 6.13$, and no lymph node metastasis was observed. Complications greater than Clavien- Dindo grade 1 were not observed, except in 1 patient in whom ureteral stenosis developed as a post-operative complication, and internal urethrotomy was performed.

Conclusions: Reduced port LRP demonstrated a cosmetic advantage over robot-assisted surgery and LRP. The relatively long operating time was owing to the reconstruction of the peritoneal injury in the initial period.

\section{V03-05 Modified Dissection Technique of the Urethra provided Improved Early Urinary Continence after Robot-Assisted Radical Prostatectomy}

\section{R Munver, M Degen, JF Tsui, B Desroches}

Introduction \& Objective: Urinary continence after robotassisted radical prostatectomy (RARP) is affected by surgical technique, patient adherence to postop Kegel exercises, and membranous urethral length. While there are a variety of surgical techniques described to improve urinary continence and patient adherence to Kegel exercises can be improved with pelvic floor therapy, membranous urethral length has been a nonmodifiable factor for urinary continence in patients undergoing RARP. Herein, we describe a urethral dissection technique for obtaining greater urethral length when performing RARP in patients at higher risk for postop urinary incontinence.

Methods: The modified dissection technique (MDT) for obtaining additional urethral length begins with transection of the dorsal venous complex. Excellent hemostasis is needed to allow for adequate visualization and dissection of tissue planes. Next, the tissues surrounding the urethra laterally are dissected off the urethra to expose the apical boundaries of the prostate. With the prostate on traction, the apical edges of the prostate are circumferentially dissected proximally to expose additional urethra at the prostato-urethral junction. At the posterior aspect of the urethra, a plane is identified that allows for the surgeon to dissect this portion of the apical prostate from the urethra as well. Once the apical prostate has been adequately dissected proximally, the urethra is transected as proximally as possible. If there is any concern for a positive surgical margin in this area, the proximal margin of the urethra can be sent for frozen section pathologic analysis.

Results: Eight patients, with Gleason Score $3+4$ or $4+3$ prostate cancer, underwent RARP (5 nerve sparing, 3 non-nerve sparing) using the MDT. Mean BMI was 33 (range 21-46), mean membranous urethral length was $14 \mathrm{~mm}$ (range 9-20), and mean catheter duration was 10 days (range 6-16). EBL was $<100 \mathrm{cc}$ in all patients. All patients had an uncomplicated postop course and were discharged home on postop day \#1. One patient had prostatic apical disease on biopsy and intra-op frozen section of the urethra was negative. All patients had negative margins. All patients performed preop and postop Kegel exercises. At 1-month follow-up, 1 patient with BMI $>35$ and membranous urethra $<1 \mathrm{~cm}$ on MRI was continent and required no pads, 4 patients required 1 pad per day, and 3 patients required 2 pads per day with no pads being soaked.

Conclusions: In patients with a short membranous urethra or elevated BMI that are at higher risk for post-RARP urinary incontinence, the MDT allows for compensation of a shorter urethral length such that patients may be less likely to have significant early post-RARP incontinence.

\section{V03-06 Robotic assisted radical prostatectomy in a patient with complete ureteral duplicity with ectopic prostate drainage}

\section{LM Calazans, NJ Barretto, N Barretto, T Mourão, R Oliveira}

Introduction \& Objective: Robotic Assisted Radical Prostatectomy (RARP) is one of the treatment modalities for prostate cancer. The increasing use of this technique is based on the reduction of hospital readmission rates and blood loss, with potential oncological and functional outcomes when compared with open or laparoscopic. Ureteral duplicity is the most frequent anomaly in the urinary tract, and when it is complete, upper renal pole is usually drained by an ectopic ureter. The aim of this video is to show the challenges and the surgical strategy used in a RARP in a patient with complete duplicity and ectopic drainage for the prostate.

Methods: The video was recorded in high definition and edited evidencing the main steps of RARP that demonstrated the strategies and difficulties due to this anomaly.

Results: A 60-year-old male patient with PSA $5.4 \mathrm{ng} / \mathrm{dl}$ with a Prostate Multiparametric MRI showing a $2 \mathrm{~cm}$ PIRADS-5 lesion with suspicion to invade the prostate capsule. A biopsy was performed with ISUP 3 prostate adenocarcinoma in 4/16 cores. Patient had previous diagnosis of complete ureteral duplicity, with ectopic drainage for prostate and functional exclusion of the upper renal pole. RARP, with preservation of the endopelvic fascia, no dorsal vein section and bilateral neurovascular bundles sparing was performed. Before manipulation of the prostate, dilated ureter was dissected, with its section and closure of the proximal segment. Distally was introduced a guide wire to facilitate location of the ectopic ureter implanting at the prostate. There were no complications during de surgery and the anatomopathological reported an ISUP 5, T3a lesion with 10 negative lymph nodes and negative surgical margins. The 45-days PSA 
was $0.04 \mathrm{ng} / \mathrm{dl}$ and the patient had early continence. Three months after surgery, the patient had a reminiscent upper pole infection. At the CT there was a hidronephrotic upper pole, with $14 \mathrm{~cm}$. As the patient was septic it was performed a nephoureterectomy with a complete resolution of the infection.

Conclusions: Although prostate cancer and ureteral duplicity are relatively common, the association between them, added to ectopic prostate drainage, is a rare event. This is the first report of a RARP performed in this context and can contribute to the definition of the best strategies for future cases.

\section{V03-07 Salvage robot-assisted laparoscopic prosta- tectomy: tips and tricks for challenges due to different primary treatments.}

\section{O Fikret, M Covas Moschovas, S Bhat, T Rogers, V Patel}

Introduction \& Objective: Salvage robot-assisted laparoscopic prostatectomy (sRARP) is a technically demanding procedure due to significant alterations in anatomical landmarks and loss of tissue planes. In this video, we present the challenges associated with different primary treatments and describe key points in their management.

Methods: Between 2008 and 2018, 126 patients underwent sRALP by a single experienced surgeon. Ninety-four patients had received external or internal radiation (EBRT: 39, IMRT: 15, proton beam: 3, brachytherapy: 23, EBRT+brachy: 14) and 32 had received focal therapy (cryotherapy: 20, HIFU: 9, microwave/electroporation: 3) as primary treatment. Difficulties caused by different primary therapies at each surgical step were identified and key points in their management were presented.

Results: 1. Endopelvic fascia dissection (EFD): Opening the endopelvic fascia at the prostate base where the space between the prostate and the levators is least vascular and dissection away from the prostate capsule towards the apex facilitated EFD. Apical dissection around the sphincter was typically difficult in brachytherapy patients due to inflammation caused by misplaced seeds. 2. Bladder neck dissection (BND): In cases with HIFU, the prostate was atrophic and fused anteriorly behind the pubic tubercle. In such cases, opening the anterior BN provided better identification of the anatomy followed by antegrade prostatectomy.

3. Posterior dissection (PD): IMRT and proton beam were noted to cause more extensive fibrosis in the pelvis. In such cases, identification of the correct plane for posterior dissection was especially difficult due to lack of prerectal fat and 'tenting' of the rectum. We have used the instant toggling feature of DaVinci Xi robot (180 degrees upward rotation of the 30-degree camera) to facilitate visualization of the posterior plane and careful cold dissection.

4. Vesicourethral anastomosis (VUA): Loss of tissue vascularization after EBRT and proton beam radiation adversely affects VUA vitality and leads to anastomotic dehiscence or leaks. In our experience, use of an acellular and resorbable scaffold graft resulted in decreased leak rates and catheterization times (Ogaya-Pinies et al, EurUrol 2018).

Conclusions: sRALP should be performed by experienced surgeons due to lack of tissue planes and anatomical landmarks. The surgeon should be familiar with challenges specific to different primary therapies. Opening endopelvic fascia away from prostate capsule, antegrade prostatectomy when prostate and $\mathrm{BN}$ are fused to the pubis, $30^{\circ}$ Toggling during posterior dissection, and Acell graft to reinforce VUA are keys in management of complex cases.

\section{V03-08 Transvesical Robot Assisted Radical Prosta- tectomy}

\section{G Wang, X Zhou, B Fu, C Zhang}

Introduction \& Objective: To introduce the technique and report our initial experience of transvesical robot assisted radical prostatectomy (TvRARP).

Methods: 10 TvRARP for prostate cancer performed by a single surgical team between 2018 April and 2018 October were retrospectively reviewed. Preoperative data of patients [patient age $(58.6 \pm 9.4)$ years, BMI $(26.5 \pm 3.1) \mathrm{kg} / \mathrm{m}^{2}$, tPSA $(19.5 \pm 4.1)$ $\mathrm{ng} / \mathrm{ml}$, biopsy Gleason score $6(6 \sim 7)$, prostate volume $(33.4 \pm$ 15.8) $\mathrm{ml}$ and IIEF-5 score $12(10 \sim 16)]$ were collected. Preoperative study revealed 8 cases of $\mathrm{cT}_{1 \mathrm{c}}, 1$ case of $\mathrm{cT}_{2 \mathrm{a}}$ and 1 case of $\mathrm{cT}_{2 \mathrm{~b}}$. All patients were continent preoperatively.

Results: All 10 cases were successfully performed robotically without conversion, transfusion or other major intraoperative and postoperative complications. Postoperative pathology confirmed 6 pT2a cases, 3 pT2b cases and 1 pT2c case [Gleason score $6(6 \sim 7)] .1$ case reported positive surgical margin $(10 \%)$. Operative time was $(140.5 \pm 35.5) \mathrm{min}$. Estimated blood loss was $(65.5 \pm 35.5) \mathrm{ml}$. Urethral catheter was removed at postoperative 7-day. 9 patients achieved urinary continence ( 0 pads) immediately after the removal of urinary catheter, while 1 patient returned to full continence in 2 weeks postoperatively. During a
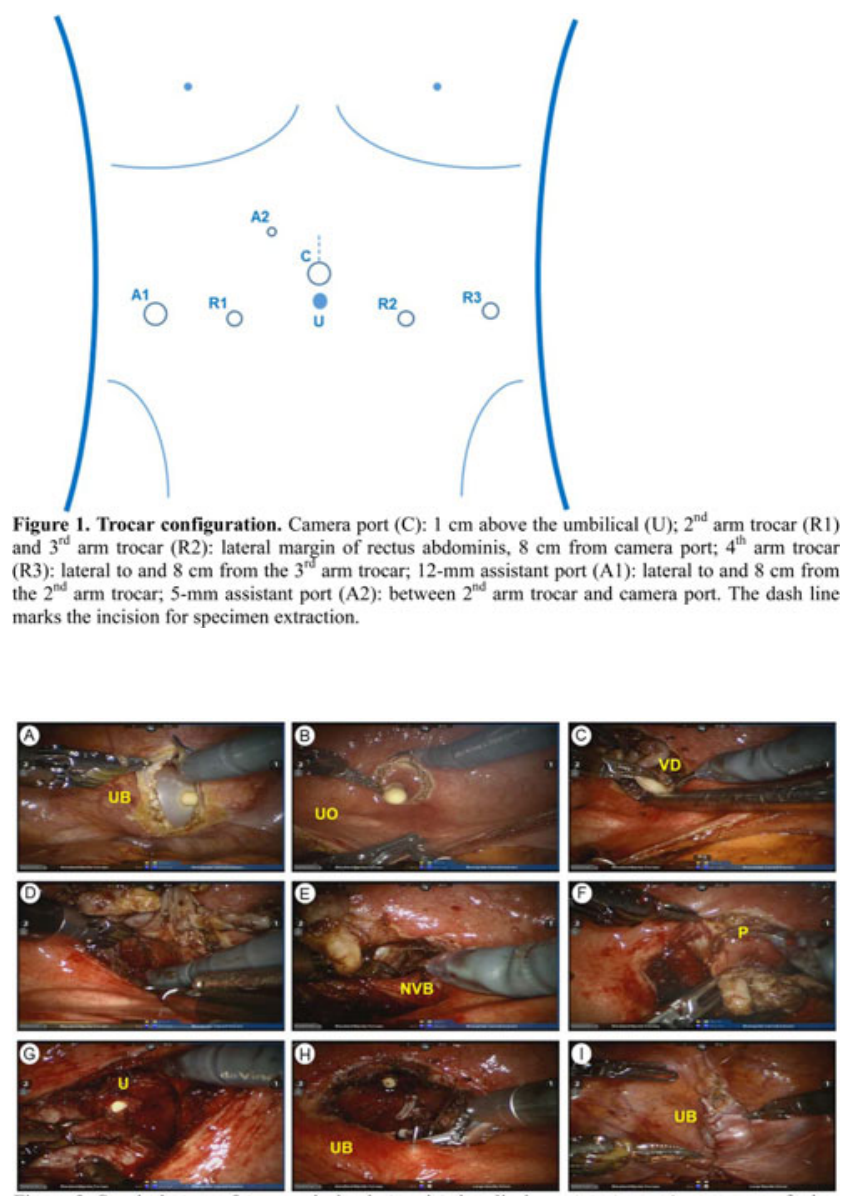

Figure 2. Surgical steps of transvesical robot assisted radical prostatectomy. A: cystotomy, further orifice through the mucosa and muscular layer. $\mathrm{C}$ : initial dissection of the vas deferens and semi vesicles is carried out through the lower half of the circumferential incision around the internal urethral orifice. D posterior dissection towards the apex of the prostate. E: dissection of bilateral NVB. F: anterior dissectio towards the apex of the prostate. G: dissection and transection of the urethra. H: urethrovesical anastomosis. I: 2-layer closure of bladder. Abbreviations: UB (bladder), UO (ureteral orifice), VD (vas deferens), NVB (neurovascular bundle), $\mathrm{P}$ (prostate), $\mathrm{U}$ (urethra). 


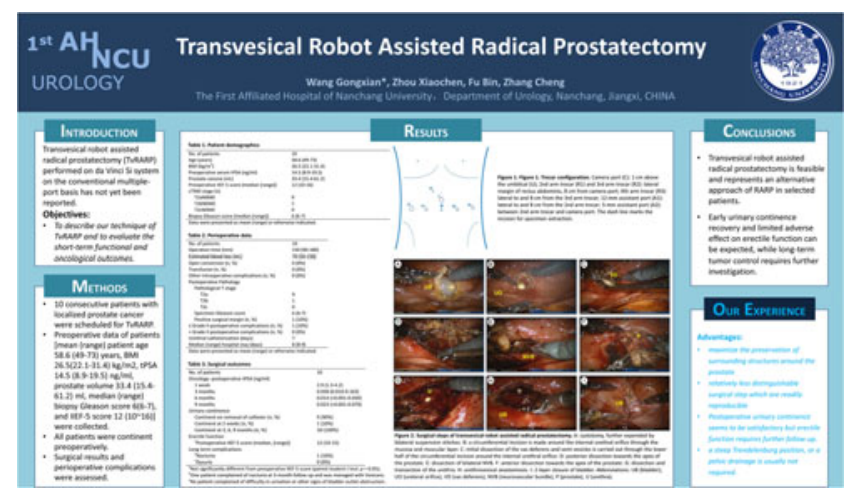

mean follow up of 3 months ( $2 \sim 4$ months), no biochemical recurrence was noted (tPSA $<0.2 \mathrm{ng} / \mathrm{ml}$ ). At 2 -month postoperatively, IIEF-5 score was $11(8 \sim 13)$ and not statistically different from preoperative status $(\mathrm{p}>0.05)$.

Conclusions: Transvesical approach is a valid alternative of performing RARP in patients bearing localized low-risk prostate cancer. Tumor control and preservation of erectile function remains to be determined in long term follow up.

V03-09 Surgical Factors Improving Continence in Robotic Assisted Laparoscopic Radical Prostatectomy, Real Surgical Experience In Ramathibodi Hospital, A Case Report.

W Ratanapornsompong, P Sungkum, S Pacharatakul, C Leenanupan, W Kongcharoensombat

Introduction \& Objective: Continence is an important outcome of pentafecta after robotic-assisted laparoscopic radical prostatectomy (RARP). Incontinence after prostatectomy is mostly due to intrinsic sphincter deficiency. The factors affecting continence are age, continence status before surgery, length of remaining urethra and striated sphincter, etc. There are multiple procedures that can enhance continent recovery. we share the real surgical aspects of continent-enhancing procedures in RARP in a specific case study at Ramathibodi Hospital.

Methods: A 71-year-old Thai male patient had $13 \mathrm{ng} / \mathrm{ml}$ prostate-specific antigen (PSA). Transrectal ultrasound-guided prostatic biopsy showed a prostatic adenocarcinoma Gleason score of $4+4=8$ for the left lobe of the prostate gland. The patient underwent RARP, and we performed continenceenhancing procedures. First, we preserved the bladder neck using a posterolateral approach when separating the bladder and prostate. The puboprostatic ligament has been deemed a continence complex, and we performed puboprostatic ligament preservation before controlling the dorsal vein complex. Anterior retropubic suspension was performed using a V-Loc ${ }^{\mathrm{TM}}$ no. 0 to hold up the urethra with the pubic bone. Then, we preserved the right cavernous nerve using the interfascial technique. Afterward, the urethra length was preserved as much as possible using the collar technique. After removal of the prostate specimen, we performed a modified Rocco stitch to repair the Denonvilliers' fascia. A urethrovesical anastomosis was created with the running mucosa-to-mucosa technique using a 3-0 V-Loc ${ }^{\mathrm{TM}}$ and was stented with a 20 Fr Foley catheter.

Results: The surgical procedure was finished in 110 minutes, and the estimated blood loss was $240 \mathrm{ml}$. The patient was doing well after the procedure and was discharged home in 3 days. The
Foley catheter was removed at post-operative day 7 , and the patient had only mild symptoms of stress urinary incontinence. Conclusions: The continence-enhancing procedures were technically feasible and helped the patient gain early continence. The technique needs to be adapted individually, and a randomized control trial is needed to statistically prove the results.

V03-10 Athermal and Clip-free Nerve-Sparing Technique in Robotic Radical Prostatectomy: Feasibility Study of Autologous Fibrin Glue as Hemostatic Agent

O Aytac, A Garayev, F Atuğ

Introduction \& Objective: Nerve-sparing techniques with less traction, athermal and clip-free bleeding control comprises critical step for improved postoperative erectile functions. Several methods still continue to develop to maintain neurovascular bundles and minimize mechanic and thermal trauma in robotic radical prostatectomy. In this study we describe the safety and feasibility of autologous fibrin glue application for NVB preservation avoiding any thermal energy, clips or sutures.

Methods: The present technique was used in 45 men undergoing RARP due to prostate cancer with unilateral or bilateral nerve sparing. The decision for NVB preservation was based on preoperative Gleason score, digital rectal examination (DRE) or MRI findings. Operation technique All cases were operated with standard transperitoneal approach. After incising of posterior peritoneal layer, the seminal vesicles were isolated using cold dissections or judicious cautery use. Athermal dissection was performed along the lateral side of seminal vesicles due to its proximity to the NVBs. The NVBs were gently retracted and released in retrograde manner along the prostate capsule toward the base. Hem-o-lok clips were placed to the prostatic vascular pedicle at some distance from posterolateral edge according to MRI and DRE findings. The vascular pedicles were transected using cold scissor. After complete mobilizing NVB from prostatic capsule, the prostate apex completely mobilized and dissected from NVBs. After removing the prostate, the NVBs observed for bleeding in low abdominal pressure. Following complete release of NVBs, a 2-3cc of Vivostat (Vivostat A/S, Alleroed, Denmark) autologous fibrin glue were applied over the bundles and prostatic fossa. In case of bleeding after aFG application, fibrin layer elevated and 4/0 absorbable suture was tautly placed for hemostasis. All patients underwent water-tight urethrovesical anastomosis technique with two barbed V-loc ${ }^{\mathrm{TM}}$ sutures.

Results: Control of small bleeding areas on the NVBs are achieved using fibrin glue without electrocautery. Postoperative blood loss, delayed bleeding or prolonged drainage were not observed.

Conclusions: In order to control bothersome bleeding from neurovascular bundles we successfully applied fibrin glue to maintain hemostasis It is reliable method for sparing NVBs to avoid of electrocautery and surgical sutures to gain rapid return of sexual functions. Further studies with randomized setting are needed to confirm our results.

V03-11 Design and Validation of a full-immersion robot assisted radical prostatectomy simulation using three-dimensional printing and hydrogel molding technology

A Ghazi, M Witthaus, R Melnyk, G Wu, TP Frye, H Rashid, JV Joseph

Introduction \& Objective: Simulation has emerged as a key training tool designed to facilitate training outside of the 
operating room. Virtual reality systems are the primary platform for robotic skills training but lack physical interaction with tissues necessary for surgical education. Animal \& Cadaver models require objective forms of assessment in order to evaluate current expertise and track progression. In our study, we validate a highfidelity, inanimate robot assisted radical prostatectomy (RARP) model within a full-immersion simulation environment.

Methods: Anatomically accurate models of the human pelvis, bladder, prostate, seminal vesicles, urethra, neurovascular bundle (NVB) and relevant adjacent structures were created from a patient's MRI using polyvinyl alcohol (PVA) hydrogels and threedimensional-printed injection molds. Pertinent steps of the nervesparing RARP were simulated: bladder neck dissection, seminal vesicle mobilization, nerve-sparing prostatectomy and urethrovesical anastomosis (UVA). Five experts ( $>250$ caseload) and 10 novices $(<50$ caseload $)$ completed the simulation. Face and content validity were calculated using expert model ratings for realism, similarity of the model to cadaveric models and usefulness as a training tool. Differences in performance among groups with various levels of experience using the clinically relevant performance metrics were used to calculate construct validity.

Results: $80 \%$ of the experts found the bladder neck dissection, nerve sparing, vesicourethral anastomosis and apical dissection o be the most realistic. $>80 \%$ of the experts found the model to be superior to cadavers in its bleeding capability, ability to replicate all steps of the procedure and anatomical resemblance to live surgery. $60 \%$ of expert found the texture of tissue to be superior to cadavers and $40 \%$ found it similar. All experts agreed that this model is suitable as a training tool and a mandatory prerequisite in assessing their trainees prior to live surgical training. There were significant differences between novices and expert operative metrics including operative time, margin status and UVA leak rates. Experts were superior to novices in each category with the exception of estimated blood loss, which did not reach significance.

Conclusions: This inanimate, full-task hydrogel model was highly rated for realism and suitable as a training tool for RARP. Construct validity between experts and novices was demonstrated using clinically relevant performance metrics. This full-immersion simulation platform provides a comprehensive tool for surgical skill development and evaluation before hands-on exposure.

\section{V03-12 Maximum conservation in laparoscopic prostatectomy step by step}

C Conde Redondo, F Castroviejo Royo, A Alonso Villalba, J Torrecilla, S Martín Martín, F Lara Pérez,

R Cortiñas Gonzalez

Introduction \& Objective: Radical prostatectomy is the most common cause of stress urinary incontinence in men. Surgical techniques to improve postoperative continence rates are based on the concept of maintaining normal anatomy and the function of pelvic structures as much as possible: Preservation, Reconstruction or Reinforcement.

Methods: A 61-year-old patient with a diagnosis of prostate cancer, with PSA $6.54 \mathrm{ng} / \mathrm{ml}$, DRE: T1c and Gleason $6(3+3) 1 /$ 12 fragments. Surgical points: Increases postoperative continence Rates: Preservation of the Endopelvic fascia $\bullet$ Preservation of the bladder neck: To maintain most of the circular muscle fibers. - The Musculofascial preservation (formed by the Puboprostatics ligaments, the Tendineous Arch of the pelvis and the puboperineal muscle) is performed by the careful separation of the apex from the Puboprostaticcomplex. Preservation of the urethral length to reach the maximum urethral length. Accurate recognition of the joint between the membranous urethra and the prostatic apex is the most crucial point for maintaining the maximum urethral length without increasing the surgical edges Increases the rate of erectile function: - Preservation of the seminal vesicle • Preservacción of Neurovascular Bandeletas is important for the preservation of erectile function and helps to improve postoperative urinary incontinence.

Results: The total operating time was 150 minutes. The estimated blood loss was $300 \mathrm{ml}$. The drainage was removed on the first postoperative day and the patient left high on the second day. The Foley catheter was removed after 10 days and the patient remained completely dry. Pathological anatomy was adenocarcinoma Gleason 6, negative margins. PSA after 30 days was 0.02 .

Conclusions: Post-prostatectomy incontinence can be influenced by many factors, including the patient's aspects, the surgeon's experience, and the operative technique. Anatomical restoration suggested to improve early continence rates.

\section{ABSTRACT VIDEO SESSION 04: URETEROSCOPY}

\section{V04-01 Ultrasound-guided retrograde intrarenal surgery is safe and useful}

T Tsujioka, Y Kawanishi, D Takahashi, K Hirota, Y Atagi, K Seto, K Izumi, M Yamanama

Introduction \& Objective: Conventional retrograde intrarenal surgery (RIRS) is usually underwent with fluoroscopy. Fluoroscopy exposes patients and medical staff to the health hazard of radiation. We considered that ultrasound-guided RIRS without fluoroscopy would be safe for all types of patients because of advancements in ultrasonography and ultrasonographer technic. This study aimed to determine whether ultrasound-guided RIRS without fluoroscopy is safe and useful for treating urinary stones. Methods: The study included all patients $(\mathrm{n}=117)$ who underwent RIRS at our hospital between April 2018 and April 2019.
We evaluated surgical duration as well as rates of zero exposure, insertion of ureteral access sheaths, and stone-free outcomes. Perioperative complications were evaluated in patients after assessment by ultrasound-guided RIRS with zero exposure. U1trasonography was used to place guidewires, insert ureteral access sheaths into the ureteral orifice, appropriately position sheaths in the ureter, measure the sizes of stones during lithotripsy, identify residual stone fragments, and put ureteral stents. Results: We assessed 113 (96.6\%) patients using ultrasoundguided RIRS with zero exposure. The mean operative duration was $95.5 \pm 42.9 \mathrm{~min}$, and the stone-free rate was $82.1 \%$. Complications were developed in eight $(6.8 \%)$ patients comprised urinary tract infection $(n=4)$, ureteral mucosal injury $(n=2)$, ureteral perforation $(n=1)$, and inability to position a ureteral stent $(n=1)$. Conclusions: Ultrasound-guided RIRS without fluoroscopy offers several advantages. For example, the proximal ureter can be 
confirmed in patients with impacted stones, and X-ray negative stones can be recognized. Ultrasound-guided RIRS is straightforward, safe, and effective for treating urinary stones.

\section{V04-02 Challenging case of RIRS in Horse Shoe Kidney}

A Puri

Introduction \& Objective: Horse shoe kidney (HSK) is the most common renal fusion anomaly- with a prevalence $0.25 \%$. The normal ascent of kidneys arrested by fusion of lower poles with malrotation with anterior displacement of collecting system. The insertion of ureter onto the renal pelvis is superiorly and laterally displaced. Urolithiasis is the most common complication with an incidence of $21 \%$ to $60 \%$. Various modalities to manage HSK stones have refined over years from open surgery to shock wave lithotripsy to PCNL to RIRS. There are various complexities and challenges in managing renal stones in HSK in each modality. This video illustrates the difficulties faced with RIRS in HSK, especially with stones lying in inferomedial calyces and isthmus region.

Methods: This is a case of a 34 year old gentleman with complaints of left flank pain for last 3 years. He had undergone left sided PCNL in 2016, which failed to retrieve the renal calculi. The patient had no co-morbidities. His general and systemic examination were normal. Contrast CT Whole Abd with Urography had revealed horseshoe kidneys with multiple calculi in inferiomedial left renal calyces with normal excretion of contrast on both sides. The patient was planned for RIRS. Flexible ureteroscope URF-P6 with 9.5/11.5 Fr, $28 \mathrm{~cm}$ access sheath was used for the procedure along with Holmium laser ( $200 \mu$ fibre). Pre-stenting was not done.

Results: The following were the results of the RIRS surgery:

- Total stone burden- $3.8 \mathrm{~cm}$

- Total surgical time- $90 \mathrm{~min}$

- Blood loss- minimal

- Post-operative stay- 1 day

- Post-operative period- uneventful

- Stone analysis- Calcium oxalate dihydrate

- Post-operative Xray KUB and USG KUB at 3 weekscomplete stone clearance

Conclusions: RIRS is a safe and minimally invasive treatment option for managing HSK stones. In this case, because of entire stone load in the inferiomedial calyces and isthmus region, it was the best option amongst other options like laparoscopy assisted PCNL, open surgery and ESWL. Yet there are difficulties faced with this modality due to peculiar anatomy of HSK. The pelvis of HSK is flatter than normal kidney with narrower intra-renal space. There is increased difficulty of deflecting and steering ureteroscope inside kidney. The high insertion of the ureters and acute infundibulopelvic angle, negatively affect stone-free rate $\&$ increase the risk of second intervention.

V04-03 Moving away from Percutaneous Nephrolithotomy; Retrograde intrarenal surgery in complex stone scenarios

\section{Y KK, S Prabhakar}

Introduction \& Objective: To assess the feasibility of Retrograde Intrarenal surgery (RIRS) using fiber optic reusable flexible Ureterorenoscope as an alternate to Percutaneous Nephrolithotomy (PCNL) in treating patients with complex renal stone burden.
Methods: We present edited videos of our RIRS technique for renal stones in two different scenarios ie large stone bulk $>2 \mathrm{~cm}$ and a partial staghorn calculus.

Results: A total of 62 renal stone units with large stone bulk of more than $2 \mathrm{~cm}$ were cleared. Ureteral Access Sheath size 9.5 or $12 \mathrm{~F}$ were placed in all cases. Stone retrieval was done with $2.2 \mathrm{~F}$ NGage stone extractor. We use a combination of dusting and fragmentation followed by fragment extraction which will results in maximum stone free rate (SFR). All patients were discharged within or immediately after 24 hours of the procedure, except those who had febrile urinary tract infection in the postoperative period.

Conclusions: RIRS is a feasible option in large and complex stone disease. Overall SFR is excellent with low complication rates.

\section{V04-04 Single-use digital flexible ureteroscopy: Pelvic kidney with pyelic lithiasis}

O Angerri, L Sabiote, J Balañà, E Emiliani, A Kanashiro, F Sánchez-Martín, F Millán Rodriguez

Introduction \& Objective: Flexible ureteroscopy with intrarenal surgery is a valid alternative to extracorporeal lithotripsy and percutaneous surgery. When there is a complex anatomy of the urinary tract the ureteroscope has a high risk of rupture, so it is advisable to use a single-use ureteroscope.

Methods: Here we present a 52-year-old male with hematuria and recurrent abdominal pain who was diagnosed with left pelvic kidney with a pyelic lithiasis of $25 * 13 \mathrm{~mm}$ and 550 Hounsfield Units treated with endoscopic intrarenal surgery with single-use flexible ureteroscope.

Results: In a lithotomy position a retrograde pielography was performed and subsequently a semi-rigid ureteroscopy was made showing a complex anatomy of the urinary tract. An intrarenal endoscopic surgery with single-use flexible ureteroscope was made (Lithovue, Boston Sci.) with intracorporeal lithotripsy with High-power Holmium laser (Lumenis 120H) pulverizing the pyelic lithiasis without fragmentation.

Conclusions: The disposable ureteroscope allows access to all parts of the kidney, even in anatomical abnormalities, without risk of endoscopic instrument rupture.

\section{V04-05 Endoscopic Removal of Foreign Body within the Ureter}

\section{S Setia, L Deane}

Introduction \& Objective: Migration of surgical clips is a wellknown phenomenon that is not uncommon. Migration of clips into the ureter is a rarely described event with only few case reports described in the literature. This is a case of a 60-year-old female with a history of multiple abdominal surgeries who presented for vague abdominal discomfort. She was found to have an encrusted surgical clip embedded within the wall of the $\mathrm{mid} /$ distal ureter.

Methods: Ureteroscopy and holmium laser lithotripsy with basket extraction was performed for elimination of encrustation. The surgical clip was embedded within the wall of the ureter and was unable to be removed in its entirety with basket extraction. A small endoureterotomy was performed and fragmentation of the base of the surgical clip was done using the holmium laser. The clip pieces were subsequently extracted, and a stent was placed.

Results: A small portion of the surgical clip remained embedded in the side wall and was unable to be extracted. The goal was for 
the ureteral wall to heal with the remaining fragments outside the lumen. Unfortunately, the patient was lost to follow after the first post-operative visit.

Conclusions: Erosion of a surgical clip into the ureter is rarely described and can be complicated by encrustation leading to obstruction. Endoscopic removal of the entire surgical clip has been reported in the literature, but it may not always be possible. Careful partial removal of embedded clips can be performed safely but long-term outcomes are unknown.

\section{V04-06 Withdrawn}

\section{V04-07 Blue spritz technique - a simple way to identify the elusive ostium of a calyceal diverticulum}

W Low, N Mohd Zam

Introduction \& Objective: Calyceal diverticuli often contain stones and can lead to recurrent infections. Endoscopic access to the diverticuli can be challenging. This video demonstrates the blue spritz technique to identify the ostium of a calyceal diverticulum during retrograde intrarenal surgery.

Methods: The patient is a 53-year-old male with flank pain and multiple small calculi within an upper pole calyceal diverticulum. Retrograde intrarenal surgery is performed. After identifying the ostium, laser diverticulotomy is done and the calculi within are basketed out.

Results: There were no complications. KUB xray 2 weeks postop showed no residual calculi.

Conclusions: The blue spritz technique is an effective way to identify the elusive ostium of the calyceal diverticulum during retrograde intrarenal surgery, saving the need for percutaneous access. Diverticulotomy can then be performed and any stone can be removed.

\section{V04-08 The Cost of Ureteroscopy: Can Single-Use Flexible Ureteroscopes Advance Past Reusable?}

C Tabib, V Vasudevan, D Hoenig, Z Okeke, AD Smith

Introduction \& Objective: Flexible ureteroscopy was introduced in 1971 and has experienced significant improvements over the years. With an ever increasingly competitive market, manufacturers have developed scopes that are lighter in weight, have improved maneuverability as well as optics, with the ability to use smaller caliber instruments to aid during surgery. Flexible ureteroscopy has become a mainstay in the treatment of renal calculi, ureteral strictures, as well as the diagnosis and treatment of upper tact TCC. However, durability remains a major concern. Repeated as well as inappropriate use increases the risk a scope will be damaged. This ultimately increases costs incurred in the form of repairs. In this video, we aimed to describe the types of damage that occurs to reusable ureteroscopes, the frequency of damage, as well as the costs required to repair the damages. We then identify the role of single-use ureteroscopes and their advantages.

Methods: Videos clips were taken from the operating room and includes other clips of both disposable and reusable ureteroscopes. A Pubmed search was completed in order to obtain comparative information regarding both disposable and reusable ureteroscopes.
Results: Damages to reusable scopes decrease overall cost effectiveness with wide variations in number of uses until repair (615 uses per scope). In head to head comparisons, disposable scopes show equivalent effectiveness without the concern for durability.

Conclusions: Single use flexible ureteroscopes provides advantages in deflection and irrigation with comparable optics and stone free rates while eliminating needs for maintenance or repairs. It is feasible to say that a transition to single use scopes provides a cost effective and clinically sound option for patients requiring endoscopic treatment.

\section{V04-09 Impacted stone or Basket}

AZ Osman, AZ Osman

NMC Royal Hospital, Dubai, UAE

Introduction \& Objective: Retrograde Intra renal surgery becomes a well-established main modality of treating renal stones. Daily development of accessory instruments allows treating more and more difficult stones. Introducing laser fibre and retrieval basket in the same working/irrigation channel of flexible scope becomes feasible owing to the minute size of both. It is a point of technical skill which each endourologist should practice, not only a tip or trick to treat impacted basket but also in different situations of stone lithotripsy procedure. Some of such indications, relocation of stone from lower to an upper calyx, large stone burden specially of calyceal parts, a highly moving stone of considerable size that need to be fragmented, and as in our case, an impacted stone in a narrow and flat neck calyx with evidence of recurrent infections which led to sticking of the stone to calyceal wall.

Methods: Introducing laser fibre 200 micron and N-gage basket (COOK) through same working/irrigation channel of disposable (PUSEN) scope and dusting/fragmenting impacted stone precisely and with minimal invasive maneuvers of moving laser fibre (to and from) and of the basket (in rotatory movement). Lasing was done with $0.5-0.8$ Joules, $10 \mathrm{~Hz}$ of frequency and 5-8 watts. using short pulse pattern for fragmenting with in basket premises or long pulse pattern for projecting parts of stone beyond that.

Results: Most of the stones treated with this maneuver were fragmented/dusted successfully and retrieved easily without any complications or injuries of renal unit. Few baskets were broken with the laser beam; however, most were in early cases treated due to impaction of large fragments and basket without any injuries of urinary tract. The overall surgery time increased by 8 to 30 minutes in comparison to routine RIRS for straight forward procedures for almost similar stones sizes.

Conclusions: Introducing laser fibre beside a basket in the same working channel of flexible scope is a good and easy to master point of technique to facilitate and successfully treat intra renal or ureteric stones without any complications or extra costs of such procedures.

\section{V04-10 Piecemeal retrograde removal of encrusted and encased stuck ureteral stent: Video tips and tricks}

A Pietropaolo, BK Somani

Introduction \& Objective: Severe encrustation leading to complete encasement of the stent is relatively rare. Retrograde ureteroscopic removal is challenging and in extreme cases the only option is either a piecemeal removal using laser transection of the stent or open surgical approach. Background: Severe encrustation leading to complete encasement of the stent is 
relatively rare. Retrograde ureteroscopic removal is challenging and in extreme cases the only option is either a piecemeal removal using laser transection of the stent or open surgical approach.

Methods: A 67 Year male with a stent in situ for 4 weeks came for his stent removal. Aim of this video is show tip and trick in removal of an encased heavily encrusted ureteric stent through ureteroscopic retrograde approach.

Results: In our patient the bladder curl was not heavily encrusted, and we were able to get the stent to the urethral meatus using the grasper. However, it was impossible to remove the rest of the stent due to its encasement from heavy encrustation. A cystoscopic guided safety wire was inserted to the kidney. A storz semi-rigid ureteroscope was then inserted parallel to the stent. Due to stent encasement and lack of space to navigate the scope proximally, the distal end of the stent was cut by a laser. Using the scope, the encrustations around the stent were then laser fragmented using the Ho:YAG laser to gradually release the stent. The stent was then divided using the cutting setting $(10 \mathrm{~Hz}-$ 1.0J). This allowed to create space and proximal progression of the ureteroscope to release the next segment of the stent. The cut end of the stent was removed using Ngage basket. The process was repeated to allow complete removal of the stent retrogradely. We had to divide the stent several times to finally release and remove it.

Conclusions: Piecemeal stent removal is necessary in extreme cases of stent encrustation where it is encasing the stent completely. This approach allows successful retrieval of stents which otherwise may need an open surgical approach.

\section{V04-11 Establishment of internal circulation system in rigid ureteroscopy}

X Zhan, g Hu, J Huang, H Zhang, Y Xu

Introduction \& Objective: To explore the surgery and new surgical techniques of rigid ureteroscopy in obstructive ureteral calculi.

Methods: Patient information: Male 68 years old, complained of right lumbar colic for one week. Physical examination reveals a percussion pain in the right kidney region and tenderness in the right ureter walking area. CT scan suggests right kidney stone, right upper ureter multiple stones, resulting in upper ureter, pyelic effusion dilation. We perform ureteroscopic lithotripsy to deal with ureteral calculi (URSL) first and schedule percutaneous nephrolithotomy (PCNL) for secondary treatment. During the URSL, we find complete upper ureteral obstruction, the flow can't circulate causing the field of vision of the operation blurred. So we insert an 5F ureter catheter through the guide wire under surveillance of the ureteroscopy in advance. The rigid ureteroscopy is inserted in parallel with the catheter and adjusts the position of the catheter in the visual field of surgery so that it slightly exceeds the ureteroscope while does not reach the stone. Results: By pre-inserting the ureter catheter we create an extra way for the water drainage, the internal circulation system is established. The water flow is smooth and the small fragments is discharged with the current which results in a clearer vision and shorter operation time than expected. Post-operation vital signs are stable, urine color is light red. The patient is discharged 1 day after the operation without discomfort complaint.

Conclusions: Establishing an internal circulation system is an effective way in dealing with obstructive ureteral calculi. It provides clearer surgical field of vision, the patient expects smaller damage, less pain and better recovery.

\section{V04-12 The Complete Endoscopic Laser Manage- ment Of A Ureterocoele With An Associated Ob- structive Calculus}

\author{
M Ravindraanandan, W Mahmalji, Y Phan
}

Hereford County Hospital

Introduction \& Objective: A ureterocoele is defined as a cystic segment of the distal ureter. It is commonly associated with defects in the bladder wall, neck and posterior urethra, as well as ureteric stenosis and duplex systems. It has an approximate incidence of 1 in 4000 people with a variety of clinical presentations and a vast number being discovered incidentally on imaging. Not uncommonly, ureterocoeles may be complicated by a calculus with the literature quoting rates between 4 and $39 \%$. Methods: Formal methods of investigation would include imaging such as ultrasound, radioisotope imaging, MRI, voiding cystourethrograms and cystoscopy. Ureterocoeles can be challenging to treat due to their variance in anatomy and presentation. They are predominantly managed conservatively, or with endoscopic incision. Traditionally in cases that are complicated by a calculus, a two-step process of diathermy incision of the ureterocoele is performed, with the stone subsequently treated with variable modalities.

Results: We present a video of an incidental ureterocoele complicated by a calculus found on MRI imaging of the prostate, managed by a single, safe and effective holmium laser endoscopic treatment of the ureterocoele and calculus. Follow up imaging shows no stone or reoccurrence of the ureterocoele.

Conclusions: We have demonstrated that single step endoscopic treatment of a ureterocoele and calculus with a holmium laser has given a safe and effective outcome for our patient, with no stone burden or recurrence. Early treatment for obstructing ureterocoeles should be considered to reduce the risk of renal tract injury and potentially invasive surgery.

\section{V04-13 Tips and tricks: Limiting radiation exposure during retrograde intrarenal surgery}

\section{T Chi, T Chi}

Introduction \& Objective: Radiation exposure may be linked to increased risk of malignancy and can cause tissue damage at high doses. Patients are requesting treatment modalities that limit or eliminate radiation exposure. Retrograde intrarenal surgery (RIRS) typically utilizes fluoroscopy to aid in surgical guidance to help optimize stone free status. Our objective was to demonstrate tips and tricks to help reduce radiation exposure during RIRS.

Methods: We recorded fluoroscopy reduction techniques during RIRS. These included novel methods of ureteroscope handling, optimization of fluoroscopy settings, strategies for limiting duration of fluoroscopy, and utilization of ultrasound guidance. Footage was obtained from the endoscopic camera view, surgical hand maneuvers, fluoroscopy C-arm controls, and ultrasound screen recordings.

Results: We demonstrate a compilation of effective strategies for radiation free flexible ureteroscopy with direct intubation of the ureteral orifice. This was performed using both a free-hand approach and with utilization of guidewire assistance. Significant reductions in radiation exposure can be achieved by utilization of pulsed fluoroscopy exposure settings of 2 to 4 frames per second, low dose radiation, and $\mathrm{C}$-arm collimation during placement of a ureteral access sheath. Ultrasound guidance during flexible 
ureteroscopy with laser lithotripsy and stent placement can also be achieved without need for fluoroscopy.

Conclusions: RIRS can be performed safely and effectively with significant reductions in radiation exposure using judicious fluoroscopy, alternative ureteroscope handling techniques, and ultrasound guidance. These techniques are achievable and should be considered by the surgeon to limit radiation utilization during RIRS.

\section{ABSTRACT VIDEO SESSION 05: ADRENAL \& LAPAROSCOPY: UPPER TRACT- BENIGN}

\begin{abstract}
V05-01 Laparoscopy assisted PCNL for Staghorn calculus in Pelvic kidney using Stone Morcellator - A Novel Technique
\end{abstract}

PK Pattnaik, SK Pattnaik, DP Pattnaik

Introduction \& Objective: Here we present a Novel technique of Laparoscopy assisted PCNL for a Staghorn calculus in a pelvic kidney using "stone morcellator" - Olympus shockpulse.

Methods: This patient presented with lower abdominal colicky pain and a palpable mass in lower abdomen. On detailed clinical and radiological investigations, it was revealed that the patient had a staghorn calculus in the pelvic kidney. Here we demonstrate the single sitting successful, complete clearance of staghorn calculus in pelvic kidney without any complications using Laparoscopy assisted PCNL via. two port technique under Fluoroscopic guidance and real time vision using 2 camera ports and olympus shockpulse. lithotripter (simultaneous stone fragmentation and suction without any impact on opposite calyceal/ pelvic wall and prevention of fragment migration to different calyces).

Results: The operating time was 67 minutes. There was negligible blood loss. Patient was completely stone free in one sitting. Conclusions: We recommend use of Olympus Shockpulse (stone morcellator) for patients with calculi in anomalous kidneys for morbidity free complete stone removal.

V05-02 Laparoscopic ureterocalicostomy for complicated upper urinary tract obstruction without hydronephrosis after treatment of staghorn calculi: A case report.

K Mikami, K Ohtsuka, K Hou, T Suyama, H Masuda, S Kojima, Y Naya, K Araki

Introduction \& Objective: Laparoscopic ureterocalicostomy has been reported in few studies till date. The surgery is performed for complicated upper urinary tract obstruction with hydronephrosis. We report a case of laparoscopic ureterocalicostomy for upper urinary tract obstruction without hydronephrosis.

Methods: A 64-year-old man was referred to our institute with right complicated ureteropelvic junction and renal pelvis stricture after undergoing treatment twice for staghorn calculi. He had unsuccessfully undergone endoscopic dilation twice for treatment of urinary tract stenosis. A right ureteral stent and a nephrostomy tube were successfully placed and there was no evidence of hydronephrosis. As stenosis of the upper urinary tract was complex, conventional pyeloplasty was not attempted in this case; therefore, an ureterocalicostomy was performed to reconstruct the kidney. On the patient's request, the surgery was performed laparoscopically. The surgery was performed via a
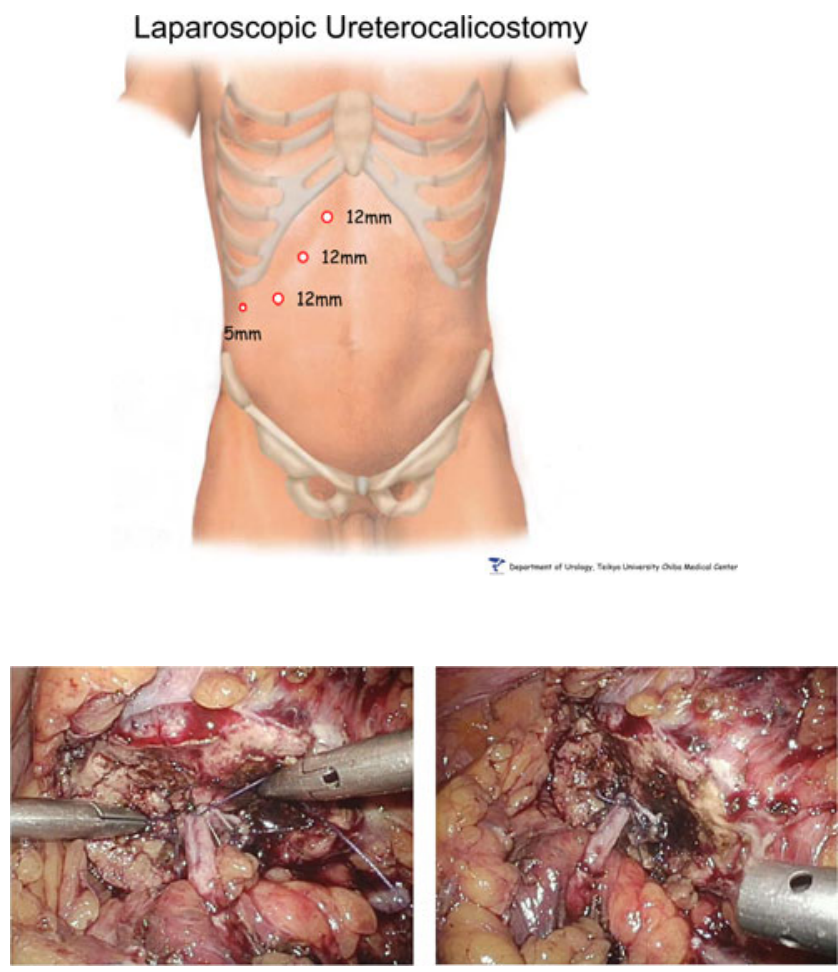

transperitoneal, 4-port approach. Partial nephrectomy of lower pole of the kidney was done to open the lower calyx and clamp the renal artery. The lower calyx was identified by injecting indigo-blue natural saline into the renal pelvis through the nephrostomy. The kidney was dissected from the renal bed and moved to the pelvic region sufficiently to gain access to the ureteral end and the renal opening calyx. The ureter was cut close to the stenosis, and an ureterocalicostomy was performed.

Results: The operating time was 412 minutes and estimated blood loss was $310 \mathrm{ml}$. A renogram 4 months after the surgery showed kidney function to be the same as before the surgery.

Conclusions: Laparoscopic ureterocalicostomy is feasible for complicated upper urinary tract obstruction without hydronephrosis.

V05-03 Is this man pregnant?-An unusual presentation of a renal cyst

O Andura, D Marghawal, C Wülfing

Introduction \& Objective: Renal cysts are a common imaging finding, and usually remain untreated. Giant renal cyst measuring 
more than $15 \mathrm{~cm}$ in diameter and containing more than $2000 \mathrm{cc}$ of serous fluid are rarely seen. We report a case of a 58-year-old man with a giant left renal cyst.

Methods: A 58-year-old man presented with a five years history of suprapubic pain, abdominal distension. He had no urological symptoms. Physical examination revealed a distended abdomen with shifting dullness. Routine hematology, biochemistry, and serum tumor markers were within normal limits. The diagnosis of giant renal cyst was finally made by MRI, this showed a $30 \mathrm{~cm}$ of diameter (giant) left cortical renal cyst. The Patient underwent Tc99m-MAG3 renal scintigraphy, which showed impaired function of the left kidney (about 15\%) combined with an obstruction. We performed a retroperitoneoscopic laparoscopy; the resection of the cyst wall and evacuation of $11000 \mathrm{cc}$ were achieved.

Results: The mean operative time and hospitalization time were 45 minutes and 2 days, respectively. Minor or major complications were not observed.

Conclusions: Giant renal cysts are uncommon; the retroperitoneoscopic laparoscopic approach of symptomatic renal cysts is feasible, effective, and safe, even if the cyst is very large.

V05-04 A prospective evaluation of outcomes of transperitoneal 3-D laparoscopic adrenalectomy in adrenal masses

A Kumar, S Yadav, S Kumar, K Saurav, Y Prashant, V Tyagi, V Prasad

Introduction \& Objective: Laparoscopic transperitoneal adrenalectomy (LA) is the Gold standard for adrenal masses. However, LA is technically challenging and confined to centers with advanced laparoscopic expertise. The 3- D laparoscopy has advantages over 2-D laparoscopy in terms of better vision and depth perception. We prospectively evaluated the outcomes of 3D laparoscopic transperitoneal adrenalectomy (LA) in adrenal masses.

Methods: All consecutive patients undergoing 3-D LA for adrenal masses by a single surgeon between June 2015 and May 2019 at our institution were included. The various clinical data were recorded and analyzed. We are presenting video of one such case.

Results: A total of 57 patients were included in the study. The mean age was 47.4 years with male /female distribution of $26 / 31$. The mean tumor size was $6.3 \mathrm{~cm}$. The indications for surgery included: Incidentaloma (Non functioning, more than $6 \mathrm{~cm}$ )43.8\%, Adrenal carcinoma - 7\%, Adrenal Cushing syndrome $12.2 \%$, Pheochromocytoma $-28.3 \%$, Conn syndrome- $8.7 \%$ The mean operating time and estimated blood loss were $119.1 \mathrm{~min}$ and $143.1 \mathrm{ml}$ respectively. Three (5.2\%) patients were converted to open. The mean hospital stay was 2.3 days. The introperative and postoperative complications were $5.2 \%$ and $7.1 \%$ respectively and mainly Clavien 1 and 2 only. In histopathology adrenal cortical carcinoma, adenoma, cyst, pheochromocytoma and angiomyolipoma were seen in 7\%, 47.3\%, 14\%, $28.3 \%$ and $3.5 \%$ respectively. At mean follow up of 32.3 months, adrenal insufficiency was found in $4(7 \%)$ patients. The hypertension was improved or resolved in Pheochromocytoma(43.7\%), Cushing syndrome $(57.1 \%)$ and Conn syndrome $(60 \%)$.

Conclusions: 3-D Laparoscopic transperitoneal adrenalectomy for adrenal masses is feasible, effective with acceptable complications and good perioperative outcomes. There is Improvement in Hypertension in substantial number of patients after LA in Pheochromocytoma, Cushing and Conn syndrome. However, it is a technically challenging procedure and should be done by surgeons of significant laparoscopic expertise.

\section{V05-05 Sling Technique for Ureteropelvic Junction Repair - An Autologous Gonadal Vein Sling For Transposing the Crossing Vessel}

\section{J Goodman, T Chi, M Stoller, T Chi}

Introduction \& Objective: The gold standard for ureteropelvic junction (UPJ) obstruction correction is dismembered pyeloplasty. There are times when a dismembered approach is not technically feasible. The Hellström technique is an alternative approach resulting in the transposition of the crossing vessel using peri-renal tissue, but appropriate tension on the vessels can be challenging to achieve. Our objective is to describe a novel technique for management of UPJ obstruction caused by a crossing vessel using an autologous sling created from the left gonadal vein.

Methods: In this case a 58-year-old female with flank pain and a history of left UPJ obstruction from a crossing vessel was consented for laparoscopic repair. Due to patient anatomy a dismembered approach was not feasible. To achieve increased tension for vessel transposition, a novel sling was created using the gonadal vein fastened to Gerota's fascia to suspend the crossing vessel away from the UPJ. The vein was transected distally above clips and spatulated longitudinally. The transected vein was then looped under the crossing vessel and tacked to Gerota's fascia on the anterior superior surface of the kidney. This created a sling to lift the crossing vessel away from the UPJ. Results: A sling was created to mobilize the crossing vessel away from UPJ relieving the obstruction. At three months following surgery, the patient's flank pain was resolved, and MRI visualized the sling in place lifting the crossing vessel away from the UPJ.

Conclusions: This novel technique demonstrates that a gonadal vein sling can be effectively used to transpose crossing vessels away from the UPJ. This may provide an advantage to the traditional Hellström technique by creating increased tension away from the UPJ. Further studies comparing outcomes should be performed to confirm benefits.

\section{V05-06 Technical Tips in Ureterocalicostomy in Failed Pyeloplasty}

\section{S Nachimuthu, R Manickam, S Kallappan}

Introduction \& Objective: Laparoscopic management of failed pyeloplasty is a challenging surgery. Here we illustrate the technical tips of redo laparoscopic pyeloplasty in a failed open pyeloplasty case with dense adhesion and more of a calyceal dilatation and minimal dilatation of pelvis.

Methods: A 28 years old male who had undergone open pyeloplasty two years ago elsewhere presented with left loin pain of 3 months duration. Clinically unremarkable. UTI was treated. CECT showed left pelviureteric junction obstruction with secondary calculi. By transperitoneal approach, left colon was mobilized. Upper ureter, UPJ and pelvis were densely adherent to posterior wall as the pelvis was thin and started tearing in few areas, it was decided to go ahead with nondismembered pyeloplasty and if necessary to extend to ureterocalicostomy. Accordingly, dissection was continued to mobilize lower calyx off the parenchyma as much as possible. As still it looked like it will end up with tense anastomosis, renal descendens was also done. Even then it looked like it apical suture with lateral end of calicotomy may cut through, we started the ureterocalicostomy from midway by interrupted 4-0 vicryl sutures and continued to 
complete the lateral layer as uretro calicostomy. Antegrade stenting was done. As further manipulation to remove secondary calculi appeared to be unfruitful we decided to tackle calculi by PCNL later. Subsequently anterior layer of ureterocalicostomy was done. Torn pelvis was closed with vicryl sutures. Tube drain was placed and ports were closed.

Results: Post operatively patient had about $150 \mathrm{ml}$ of drain over 3 days and settled. He made a smooth recovery.

Conclusions: Ureterocalicostomy is probably the best option in a failed pyeloplasty with thinned out small pelvis having a dilated calyx.

\section{V05-07 Laparoscopic pyeloplasty and pyelolithotomy in a pelvic kidney}

MA Hamdoon, D Whiting, MO Breish, S Madaan, S Sriprasad

Introduction \& Objective: Management of stones in a pelvic kidney is challenging. It's even more complicated if there is associated Pelvi-ureteric junction (PUJ) obstruction. In this video we present the management of PUJ obstruction with laparoscopic pyeloplasty with concomitant laparoscopic pyelolithotomy.

Methods: Fit and healthy 40 years male patient, presented with a history of right iliac fossa pain following consumption of fluids. This is been ongoing for 3-4 years. Clinical examination was unremarkable. Investigations revealed that he had a right PUJ obstruction in the pelvic kidney with associated large multiple right renal stones. The stones were considered to be partial staghorn although it was found out after operation it was a cluster of stones giving that appearance. MAG 3 (Meracpto Acetyl triglycine) renogram confirmed the PUJ obstruction with $36.52 \%$ split function on the right kidney.

Results: He had a right retrograde study and 4.8 French ureteric stent was placed. He was then placed in the left lateral position, ports replaced and pneumoperitonum created. The right colon mobilized, the redundant pelvis with crossing vessels was dissected, pyelolithotomy was done and with laparscopic nephroscopy the stones were extracted. Dismembered pyeloplasty was performed. Drain was placed. Our video shows all these steps in a comprehensive fashion.

Conclusions: The patient recovered well without complications and he is stone and stent free.

\section{V05-08 Combined Antegrade Intrarenal Surgery, Laparoscopy and Retrograde Intrarenal Surgery for absolute clearance of large volume renal stone - A versatile approach}

\section{JM Ramanathan, A Konay}

Introduction \& Objective: Desire for complete stone clearance is always there in the minds of both the patient and the surgeon. However, as the stone volume increases it becomes difficult. We wish to demonstrate a new versatile technique which could achieve this aim in certain scenarios.

Methods: A 60-year-old diabetic gentleman with a $5 \mathrm{~cm}$ right sided partial staghorn calculus and multiple secondary calculi (ranging from $5 \mathrm{~mm}$ to $1.3 \mathrm{~cm}$ ) presented with recurrent flank pain. His creatinine was $2 \mathrm{mg} / \mathrm{dl}$ due to diabetic nephropathy. Patient opted for parenchyma preserving procedure. On table we first stented the ureter. The staghorn calculus was removed by laparoscopic pyelolithotomy. Then through the right-hand working port a 12-14 Fr access sheath was introduced. Through which flexible fibre optic ureteroscope was passed into the kidney through the pyelotomy. Secondary calculi were basketed out and collected into a retrieval bag and the pyelotomy closed. At the time of DJ stent removal flexible ureteroscopy was done to check for any residual stones. Only two small (3 to $4 \mathrm{~mm}$ ) stones remained and were basketed out without re stenting.

Results: The patient underwent only two procedures - the first one where the majority of the stone bulk was cleared using this unique technique and the second one was basketing out the remaining two tiny fragments at the time of DJ stent removal. Thus, patient got $100 \%$ stone free rate without injuring renal parenchyma.

Conclusions: In scenarios where patient have large staghorn calculus with multiple secondary stones and extrarenal pelvis, this combined procedure could significantly reduce the number of sittings needed for $100 \%$ stone free rate and do so without injuring the renal parenchyma. Comparing flexible ureteroscope over flexible nephroscope it has the advantage of ability to pass through narrow infundibulum and even into ureter for any dislodged stones.

\section{V05-09 Laparoscopic Ileal Ureter In Failed Laparo- scopic Ureterocalicostomy}

\section{R Manickam, S Nachimuthu, S Kallappan}

Introduction \& Objective: Laparoscopic Ileal ureter is complex procedure reserved for unrepairable stricture ureter or failed pyeloplasty especially with multiple secondary stones. We illustrate the technique of total laparoscopic ileal ureter in a case of failed ureterocalicostomy with multiple secondary stones.

Methods: 45 yrs old female who had undergone laparoscopic ureterocalicostomy (following multiple URSL) for stricture UPJ with intra renal pelvis and secondary stones in 2014. Now she presented to us with left loin pain of 3 months duration. Clinical examination was unremarkable. Urine culture was positive and was treated accordingly. CECT revealed stricture at ureterocalicostomy site; intra renal pelvis with grossly dilated calyces and multiple secondary calculi of left kidney. With patient in torque position using 6 ports left colon was mobilized; adhesions were released, and lower calyx was mobilized. Using endoscopic staplers about $20 \mathrm{~cm}$ of distal ileum isolated and Ileo-ileal continuity was restored. Staple lines were excised and 6fr double pigtail stent placed. Calicotomy of lower calyx done for about $2 \mathrm{~cm}$, multiple stones were removed and ileocaliceal anastomosis was done using 3-0 vicryl continuous running fashion. Distal end of isolated ileum was anastomosed to $2 \mathrm{~cm}$ long cystotomy made in the left dome and lateral aspect of bladder using 3-0 barbed suture. Tube drain placed and ports were closed.

Results: Postoperatively patient made uneventful recovery. Tube drain was removed on the 3rd POD and Foley catheter was removed on 10th POD.

Conclusions: Total laparoscopic ileal ureter for failed ureterocalicostomy is technically demanding but a viable option.

V05-10 Two cases of laparoscopic surgery for the local recurrence of pheochromocytoma in patients with multiple endocrine neoplasia

K Tomita, T Yoshida, S Kageyama, K Johnin, M Narita, A Kawauchi

Introduction \& Objective: Surgery for pheochromocytoma is often difficult because of the tumor's endocrine activity. We report the two multiple endocrine neoplasia (MEN) cases for whom laparoscopic surgery was performed for a recurrence of pheochromocytoma.

Methods: Case 1 was a 52-year-old woman who had undergone a bilateral cortical-sparing adrenalectomy 28 years previously. 
The patient presented with palpitations. Magnetic resonance imaging (MRI) revealed two tumors at medial and dorsal to the inferior vena cava (IVC). Metaiodobenzylguanidine (MIBG) scintigraphy indicated abnormal uptake at the same lesion. Blood sampling revealed an elevated norepinephrine level. Case 2 was a 30-year-old man who had undergone a previous bilateral adrenalectomy. Follow-up computed tomography (CT) detected the local recurrence of pheochromocytoma. Local recurrence was confirmed by MRI and MIBG scintigraphy.

Results: In case 1, We selected laparoscopic surgery using five trocars. Initially, we detected a medial tumor by exposing tissue ventral to the IVC, cutting the peritoneum under the liver, and sealing and cutting the short hepatic veins. After separating the adhesions of surrounding tissue, the tumor was resected. We then removed the tumor found dorsal to the IVC being careful not to damage the IVC. Although the patients' blood pressure was elevated, intaraoperative care by an anesthetist enabled the operation be performed safely. Total surgery time was 4 hour and 37 min. with a blood loss of $200 \mathrm{~mL}$. The pathological diagnosis was pheochromocytoma. In case 2, after sufficiently mobilization of the descending colon, intestine and spleen, we detected the tumor at superior and medial to the left kidney. The tumor was carefully separated from surrounding tissue using a sealing device. Total surgery time was 4 hour and 21 min with only a small amount of blood loss. The pathological diagnosis was pheochromocytoma.

Conclusions: Given that for patients with MEN, the risk of malignancy is low whereas the risk of bilateral disease is significant, partial cortical-sparing adrenalectomy has been advocated. However, the risk of recurrence is 3.4-fold higher in patients with familial disease than in those with sporadic tumors. Furthermore, complete and safe dissection of a dissection of a local recurrence is often difficult. From our experience of these two cases, the decision to perform a partial cortical-sparing adrenalectomy in patients with MEN should be carefully considered.

V05-11 Appendiceal interposition for $3 \mathrm{~cm}$ midureteric stricture

\section{A Alaqqad, W Hassen}

Introduction \& Objective: Mid ureteral strictures are challenging to manage. Appendiceal interposition has long been noted to be a feasible option as a ureteral substitute in children. Few case reports have explored the use of appendix in the adult management of mid ureteral strictures. We describe our technique for the minimally invasive use of the appendix as a substitute in the management of a long right sided mid ureteral stricture and the ureteroscopic appearance of the repair postoperatively.

Methods: A 36-year-old male presented with a $3 \mathrm{~cm}$ high mid ureteral stricture secondary to previous ureteroscopy for a complex ureteral calculus. The ureter was mobilized a dense $3 \mathrm{~cm}$ mid ureteral stricture was encountered. The appendix was mobilized on its pedicle and used as an interposition without tension. Flexible ureteroscopy via a port was used to delineate the extent of the stricture. The ends of the appendix were anastomosed with 5-0 PDS suture in tension free manner. Operative time was approximately 2.5 hours and hospital stay were one day.

Results: Ureteroscopic evaluation of the repair six weeks postoperatively revealed a widely patient interposition graft. A nuclear scan afterwards confirmed free drainage.

Conclusions: Appendiceal interposition is a useful tool in the management of difficult right ureteral strictures. It obviates the need of bowel or bladder manipulation and is not associated with urinary reabsorption. This may be accomplished with minimally invasive techniques.

V05-12 Successful laparoscopic resection of a paraganglioma immediately behind the inferior vena cava and bilateral renal veins.

\section{T Hakariya, Y Shida, Y Miyata, H Sakai}

Introduction \& Objective: Paragangliomas have a rich blood flow and are located around large vessels; thus, resection is often difficult. We herein report a case of a paraganglioma that was located immediately behind the inferior vena cava and bilateral renal veins and successfully resected by laparoscopic surgery.

Methods: A 72-year-old man was incidentally diagnosed with a $7-\mathrm{cm}$ retroperitoneal mass immediately behind the inferior vena cava and bilateral renal veins by computed tomography. The mass was diagnosed as a retroperitoneal paraganglioma. The patient underwent laparoscopic surgery in the left lateral decubitus position. The tumor was dissected completely with no complications. Results: In the present case, the most difficult and dangerous step in the surgical procedure seemed to be dissection of the tumor from the inferior vena cava. Therefore, to safely release the tumor from the back of the inferior vena cava, we dissected the tissue around the tumor and obtained mobility of the tumor before manipulation of the inferior vena cava. To avoid damage during the course of these operations, we secured the main blood vessels with vascular tape during the procedure. Additionally, the CT and magnetic resonance imaging findings were carefully evaluated before surgery, and we discussed the surgical strategy in a preoperative conference. Through careful preoperative planning, surgical safety was secured, and complications were avoided. Conclusions: Resection of retroperitoneal paragangliomas is often a surgical challenge. The feasibility of the laparoscopic approach to such paragangliomas was demonstrated in the present case.

\section{Figure.1 Schema and CT scan}

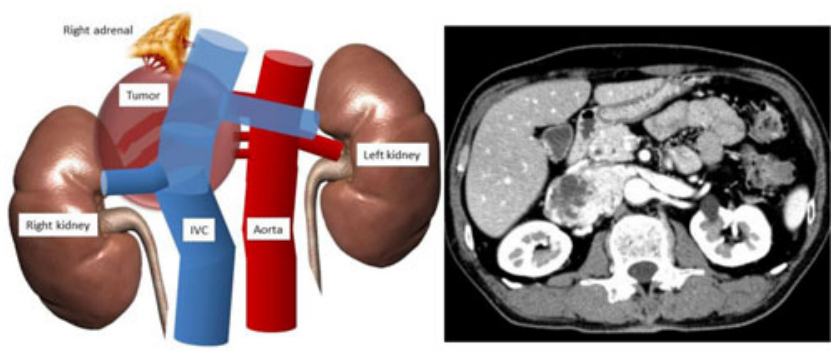

Figure.2 Intraoperative finding

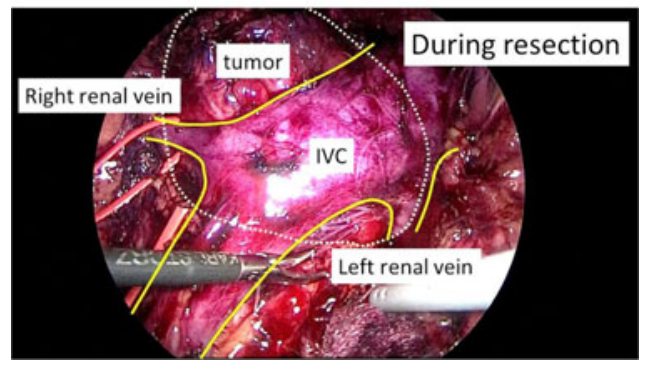




\section{ABSTRACT VIDEO SESSION 06: PEDIATRICS \& TRANSPLANTATION}

V06-01 Robotic Assisted Laparoscopic Lymphatic Sparing Varicocelectomy In An Adolescent Male

J Jensen, A Rensing, D Frimberger, B Meenakshi-Sundaram

Introduction \& Objective: The gold standard technique for management of pediatric varicoceles is controversial. Previously, the laparoscopic approach has been associated with an increased risk of a postoperative hydrocele formation secondary to disruption of the lymphatics draining the testis and scrotum. The use of isosulfan blue dye to identify and preserve the lymphatics during varicocele repair was recently described. We present a case in which isosulfan blue was used to identify the lymphatics for a robotic varicocelectomy.

Methods: A 13-year-old boy presented with a left grade III varicocele as well as a decrease in size of the affected testicle. Ultrasound demonstrated a $20 \%$ difference in volume between the right and left testicles. The decision was made for a varicocele repair utilizing the robotic approach. Isosulfan blue was injected preoperatively $(1.5 \mathrm{~mL}$ in Dartos and $1 \mathrm{~mL}$ intratesticularly). The patient was place in the supine position and pneumoperitoneum was established. A camera port was placed in the umbilicus and two additional $8 \mathrm{~mm}$ ports were placed triangulating towards the groin. The DaVinci XI robot was docked and targeted towards the gonadal vessels. The lymphatics were seen coursing over the gonadal vessels. They were dissected free of the vessels and spared. The gonadal artery and vein were separately clipped and divided. The port sites were closed with absorbable suture.

Results: Total operative time was 60 minutes and estimated blood loss was less than $5 \mathrm{~mL}$. The patient tolerated the surgery well and was discharged home two hours postoperatively. There were no postoperative complications.

Conclusions: Our video demonstrates a novel technique in which the robotic approach for varicocele repair is combined with isosulfan blue dye injection for identification and sparing of lymphatics to reduce the postoperative hydrocele rate. It is an efficient and safe method for varicocele repair in pediatric patients and reduces operative time compared to the microscopic subinguinal approach.

\section{V06-02 Safe Laparoscopic Technique Port Placement In Pediatric Patient}

B Onal, D Abdullahoglu, Y Ilki, E Bulbul, B Ercili, S Citgez

Introduction \& Objective: Laparoscopic port incertion requires special care in children. Complications such as solid organ andvascular injury during port incertion is higher in infants and children than in adults. Children have smaller abdominal cavity (in relation to surface area). Abdominal wall and specially the peritoneum is very elastic in infants and children what increases the difficulty of safe port incertion.

Methods: In our technique first port is inserted by open Hasson's technique, which is safe technique to create pneumoperitonium. Working ports are placed under direct vision safely, between 2 full- thickness abdominal wall sling sutures (including the fascia and peritoneum from the abdominal skin). In this video, 5-year- old boy patient. In right kidney, solitary, $4 \mathrm{~cm}$, pure cystic lesion have been followed with ultrasounds during 2 years. Patient is referred to our hospital because cyst has grown to $6 \mathrm{~cm}$. MRI shows normal right kidney size. Cortical cystic lesion containing thin septa of $51 * 34 * 64 \mathrm{~mm}$ in upper pole. Wide hypoactive area in the upper pelvicalyceal system in right kidney in DMSA. Renal function left $52 \%$ and right $48 \%$. Periodic right flank pain and recurrent urinary tract infections are detected during follow-up. Laparoscopic cyst excision performed without any complication. Results: We've discharged this case the next day. No peroperative vascular or solid organ injury occurred in our cases. We applied this port insertion technique with two sling suture in 20 children.

Conclusions: We didn't experience pressure drop through sling sutures. Without any serious complication and experienced that this technique is safe, effective and useful. Safe techniques should be developed in pediatric patients because of the high risk.

V06-03 Patient-Specific Surgical Simulation Using 3D Printed Hydrogel Models for Living Donor \& Recipient Kidney Transplant Surgery

A Ghazi, R Melnyk, E Belfast, T Campbell, G Wu, R Kashyap, JV Joseph

Introduction \& Objective: Robotic-assisted kidney transplantation (RAKT) offers key benefits for patients that have been demonstrated in several studies. A barrier to the wider uptake of RAKT is surgical skill acquisition. Simulation is a well-established method of developing surgical skill in a safe and controlled environment away from the patient. We have developed a patientspecific realistic, perfused hydrogel simulation model using 3D printing and hydrogel molding technologies.

Methods: We present our technique combining 3D printing and hydrogel injection molding to develop patient-specific kidney phantoms suitable for use as a simulation platform for a preoperative rehearsal of donor and recipient RAKT. The model replicates all steps of the kidney transplant operation including both donor and recipient potions. It is anatomically accurate, based on the $\mathrm{CT}$ scans of patients and it incorporates perfused vascular tissue. The first model is an abdominal simulation platform for donor nephrectomy based on the donors CT scan. The second model is a pelvic model based on the recipients CT scan where the donor kidney model is used. Crucially, it was developed to be used in the robotic operating theatre with the operating robot to enhance its fidelity.

Results: After completing a comprehensive simulation curriculum that included virtual reality and these patient specific hydrogel models. A robotic naïve surgeon was able to complete 3 donor nephrectomy cases with average estimated blood loss of $<150 \mathrm{cc}$, no complications and ischemia time of $<30$ minutes. The surgeon was also able to complete the recipient robotic kidney transplant operation successfully without any vascular or major complications, an average hospital stay of 2 days and ischemia time of 19 minutes. The surgeon practiced on each model an average of 2.5 times prior to feeling confident to perform the live procedure.

Conclusions: Software that incorporates patient imaging into 3D printing applications, together with hydrogel molding techniques 
is capable of creating human kidney phantoms with authentic anatomical, physical, and functional properties. Application of this versatile, inexpensive and reproducible method for creating kidney phantoms has demonstrated a realistic platform for surgeons to rehearse their procedures prior to live surgery resulting in direct transfer of the skills acquired in the simulation to the operating theater.

\section{V06-04 Solo-Surgeon Pure Laparoscopic Living Do- nor Nephrectomy Using Camera Holder}

D An, D You, Y Kim, J Aum, K Kim, J Han

Introduction \& Objective: This video demonstrates a pure laparoscopic living donor nephrectomy(left) in a female patient performed by solo-surgeon using camera holder.

Methods: The patient was 59-year-old female and had one left renal artery and one left renal vein. Body weight and height of the patient was $45.6 \mathrm{~kg}$ and $149.8 \mathrm{~cm}$. BMI was $20.92 \mathrm{~kg} / \mathrm{cm}^{2}$. The Gelpoint (Applied Medical, Rancho Santa Margarita, California) was placed at the area of peri-umbilicus by open method for camera introduction and kidney extraction, and two 12-mm working ports were placed at the left side of abdomen. After scope holder system was manually fixed on the right side of the operating table, solosurgery was started. Only the roles of a first assistant were substituted by the passive camera holder (Karl Storz, Tuttlingen, Germany). Scope holder was modulated whenever surgeon needs to change the field of vision. The numbers of camera modulation were 4 times during this surgery. Renal artery and vein was clamped by the Endopath(Ethicon, Somerville, US).

Results: Total time of operation was 168 mins. Solo-surgery time (camera docking to bleeding control) was 74 mins. Estimated blood loss was less than $100 \mathrm{cc}$. JP drain was not inserted and Foley catheter was removed at post-operative day (POD)\#1. The patient was discharged from hospital at POD\#6 without immediate post-operative complication.

Conclusions: Solo-surgeon laparoscopic donor nephrectomy is technically feasible and safe.

\section{V06-05 Robot-assisted Bilateral Orchidopexy: Per- sistent Mullerian Syndrome}

\section{Al Khalaf, AM Alenizi, TA Al Saad}

Introduction \& Objective: Persistent Mullerian Syndrome is a rare entity of sexual development disorders. More than 200 case reports have been published. Our video presents the least common type among the 3 known types, which involves a transverse testicular ectopia with both testes in the same hernia sac along with Mullerian structures.

Methods: We analyzed the data of a 4-year old 46 XY child who had an empty scrotum, impalpable testes bilaterally with welldeveloped scrotum and normal phallus. Ultrasound pelvis revealed a right inguinal gonad while the left gonad was not visualized. Anti-Mullerian was within normal limits and beta hCG stimulation test was showing an acceptable response. Diagnostic laparoscopy showed a crossed ectopic left gonad that is connected to the right gonad intraabdominally with an underdeveloped Mullerian remnant (uterus). Through a transperitoneal approach, the patient underwent robot-assisted bilateral orchidopexy and gonadal biopsies. Adhesions around both gonads were released, and gonadal vessels were dissected to maximize length. The mullerian remnant was divided in the midline, which revealed an underdeveloped blind-ending uterus. Bilateral orchidopexy was then performed. We believe this video is of great value demonstrating the ana-

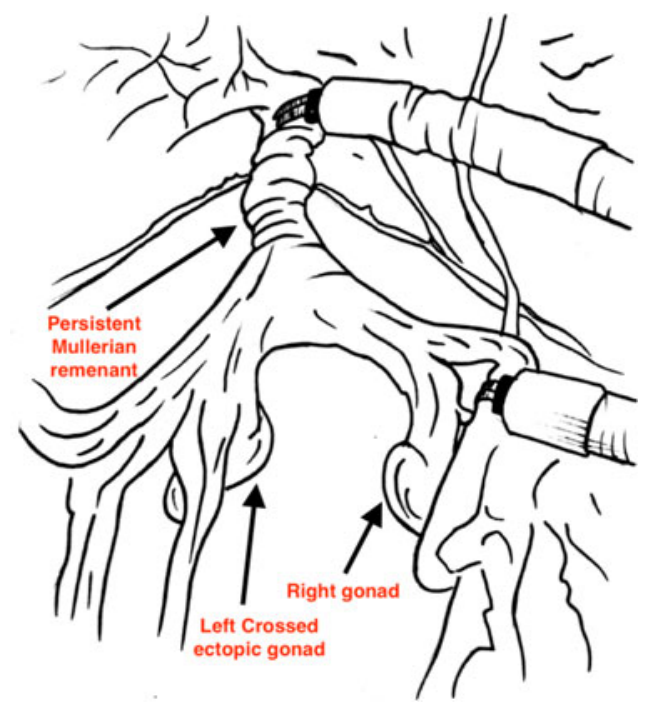

tomical findings and emphasizing the role of surgical robots in this rare urological condition.

Results: Operative time and blood loss were $200 \mathrm{~min}$ and $100 \mathrm{cc}$ respectively. Gonadal biopsies showed seminiferous tubules. Patient was discharged on postoperative day 3 with uneventful recovery.

Conclusions: Although rare, Urologists have to consider robotassisted surgery for definitive management of Persistent Mullerian Syndrome.

\section{V06-06 Four Renal Artery Kidney Donor and Transplant technique}

V Agarwal

Introduction \& Objective: Kidney donor normal anatomy include single renal artery and renal vein.in $10 \%$ cases there are variation in vasculature. In this case patient donor has left side four renal artery and right side three renal artery with bilateral single renal vein. Patient was taken for left side laparoscopic donor nephrectomy.

Methods: Standard three ports was placed after pneumoperitoneum, colon was reflected down. ureterogonadal packet was lifted and hilum was dissected. pre marked pfanenesteil incision was prepared for delivery of kidney graft. fore artery was doubly clamped and cut.renal vein was clipped and cut. Graft removed. complete homeostasis checked. and drain placed. On bench table four vessel was perfused, Two renal artery joined to make one stump and other two was joined.two separate anastomosis done with EIA. graft became pink after clamp release and urine came promptly.

Results: total duration of graft nephrectomy was 80 minutes. blood loss about $350 \mathrm{ml}$. There was no acute tubular necrosis. Urine came immediately. Patient discharged on 4th post op day. Conclusions: Multiple vessel kidney transplant are challenging case but with due care and expertise, donor pool can be increased with these multiple vessel donor with good graft outcome.

\section{V06-07 Pneumovesicoscopic Bilateral periureteric diverticulum excision with Ureteric Reimplantation in 6years old child}

\section{A Aljneibi}



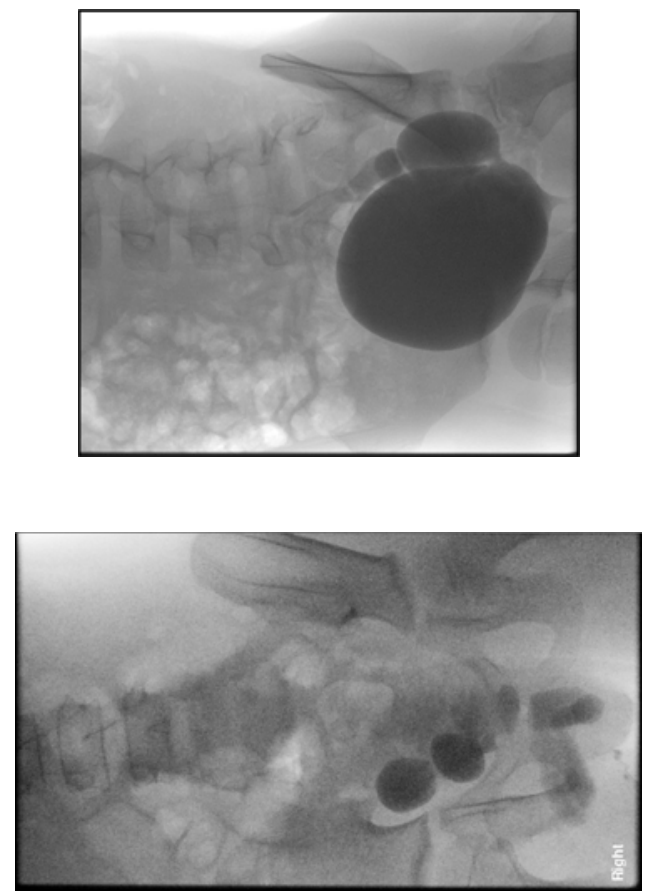

Introduction \& Objective: Feasibility of Minimal Invasive Surgery approaching Symptomatic Bladder diverticulum in 6 years child, had history of recurrent attacks of afebrile urinary tract infection with day time frequency urination and nocturnal Enuresis.

Methods: Single case presentation with symptomatic bladder diverticulum.

Results: complete resolving the clinical symptoms and duration of the follow up 3 years.

Conclusions: Pneumovesicoscopic approach is safe approach in children treating the symptomatic bladder diverticulum and Reimplantation the ureters

\section{V06-08 Laparoscopic retroperitoneal living donor nephrectomy with simultaneous cholecystectomy}

\section{Perlin, I Alexandrov, V Zipunnikov, I Dymkov, M Kretov}

Introduction \& Objective: The shortage of donor organs leads to a gradual extension of the selection criteria for living donors including age and presence of comorbidities. It was noted, that chronic cholecystitis is most often surgical comorbidity in living donors Performing simultaneous procedures with donor nephrectomy would increase the number of living donors and increase the attractiveness of operations. It was previously shown that retroperitoneal donor nephrectomy access has improved safety of the procedure and enhanced recovery of donor. Now we present our experience of simultaneous cholecystectomy with laparoscopic retroperitoneal live donor nephrectomy via the same approach.

Methods: Patient was placed on the operating table at the contralateral side with over extension the space was done through the incision is $1-1.5 \mathrm{~cm}$ on posterior axillary line $1 \mathrm{~cm}$ below the 12th rib. After forming the workspace by balloon and $\mathrm{CO} 2$ insufflation another two ports on the mid-axillary and anterioraxillary line was placed also retroperitoneally under laparoscope control. Renal artery, vein, ureter and kidney dissection were done I usual manner. The retroperitoneal tunnel was created to the pubis. After clipping and dividing of the ureter, renal artery and vein, kidney was removed through previously made suprapubic incisions.

Results: Then parietal peritoneum was transected for approximately $15 \mathrm{~cm}$ along Told's line. Through the incision cystic duct and artery was dissected, clipped and transacted, using the same ports. Gale bladder was dissected and removed. We have used this technique in 5 patients. No perioperative complications were seen in donors and recipients.

Conclusions: This procedure has three major advantages: no contact with the abdominal cavity when performing the access, the easiest access to the renal arteries, and the absence of a systematic increase in intraperitoneal pressure. Such access may be particular important for the patients who have had previous interventions on the abdominal organs.

\section{V06-09 Combined antegrade and retrograde endo- scopic approach for management of a forgotten stent in the pediatric patient}

\section{JT Barnard, AJ Hajiran, CE Morley, O AL-Omar}

Introduction \& Objective: Forgotten stents with significant incrustation remain a unique surgical challenge. Adult studies suggest a mean of 1.5 operative interventions to achieve stone free status and remove the incrusted stent. The degree of incrustation has been correlated with stent dwell time, need for multiple operations, operative times, and length of hospital stay. A combined antegrade and retrograde approach has been described in the literature to decrease operative times and avoid multiple operations. Our objective is to describe a technique where modified Barts flank free supine position is utilized in the pediatric patient to allow two teams of surgeons to work simultaneously in both antegrade and retrograde fashion to remove a forgotten stent in a pediatric patient with over $10 \mathrm{~cm}$ of total calcification.

Methods: The patient was an 11-year-old male referred for forgotten, heavily incrusted stent status post pyeloplasty over 2 years prior for congenital UPJ obstruction. Preoperative CT imaging showed a distal coil calcification of $3 \mathrm{~cm}$, numerous calcifications of the mid-portion of the stent (largest $1.2 \times$ $4.2 \mathrm{~cm}$ ), and $3.7 \mathrm{~cm}$ proximal coil calcification. The patient underwent simultaneous percutaneous cystolitholapaxy and percutaneous nephrolithotripsy followed by antegrade and retrograde ureteroscopy with laser lithotripsy. A Barts flank free modified supine position was utilized.

Results: Total operative time was 333 minutes which is above average for the average forgotten stent case in the adult literature; however, it must be noted that this patient is an outlier due to the extremely large stone burden (over $10 \mathrm{~cm}$ of stone). The distal coil was successfully freed of stone using a 550-micron holmium laser fiber through an $18 \mathrm{Fr}$ suprapubic access sheath. The proximal coil was similarly addressed using an ultrasonic lithotripter through a $30 \mathrm{Fr}$ access sheath. A 200-micron holmium laser fiber was used from both the antegrade and retrograde approach to free the calcifications of the midportion of the stent and successfully remove it. Once all clinically significant stone burden was removed an 8.5 Fr nephroureteral stent and a foley catheter were placed. The patient experienced no complications intraoperatively and was radiographically free of stone at the conclusion.

Conclusions: A combined antegrade and retrograde approach to the heavily incrusted, forgotten stent allows for shorter operative times and higher opportunity for stone free status in a single operation. The Barts flank free supine position is technically feasible for the pediatric population and allows simultaneous antegrade and retrograde access to the kidney, ureter and bladder. 
V06-10 25 Year Old Male with Voiding Dysfunction in the Setting of Prostatic Utricle Cyst

\section{K Parikh, J Moore, R Pak}

Introduction \& Objective: A 25-year-old male presented to clinic with severe voiding dysfunction and an AUA score of 24. $\mathrm{He}$ also described pain on ejaculation. He had previously undergone a cystoscopy with no discernable abnormalities. Pelvic MRI clearly delineated a 2 -centimeter midline prostatic cyst. The patient had previously undergone two transrectal drainage procedures of the cyst, but the cyst recurred after both procedures. He was offered re-peat transrectal drainage, IR sclerosing agent, or robotic surgery. The patient elected to proceed with robotic assisted laparoscopic excision of the prostatic utricle cyst.

Methods: This video demonstrates a robotic assisted laparoscopic excision of a prostatic utricle cyst.

Results: Patient was discharged home as outpatient surgery. At his six weeks follow-up appointment, patient's voiding and ejaculatory symptoms had resolved. He maintained antegrade ejaculation.

Conclusions: Robotic assisted laparoscopic excision of a prostatic utricle cyst can be a safe and effective means of managing prostatic utricle cysts.

V06-11 Robot-assisted Simple Nephrectomy for Management of Persistent Urinary Incontinence Secondary to an Ectopic and Atrophic Right Kidney and Ureter

M Lee, Z Lee, AM Asghar, MG Packer, DD Eun
Introduction \& Objective: The presence of an ectopic ureter in the setting of an ectopic kidney is rare. Patients, especially girls, with ectopic ureters may present with continuous urinary incontinence. We describe a patient with an ectopic right kidney and ureter who was managed with a robot-assisted simple nephrectomy.

Methods: A 16-year-old girl was referred for evaluation and management of continuous urinary incontinence. The patient had constant urinary leakage requiring 2 pads per day and her incontinence was refractory to behavioral modifications. Based on prior ultrasounds, she was presumed to have a congenitally absent right kidney with no other urologic abnormalities. We obtained a magnetic resonance urography that demonstrated an ectopic and atrophic right kidney near the aortic bifurcation associated with a single-system ectopic right ureter that inserted into the patient's vagina. Cystoscopy confirmed the absence of a right ureteral orifice and vaginoscopy demonstrated that the right ureteral orifice inserted into the anterior vaginal wall. Given the patient's constant incontinence and atrophic right kidney, we performed a robotic simple right nephrectomy.

Results: For this case, total operative time was 70 minutes and the estimated blood loss was $<25$ milliliters. The patient had no postoperative complications and was discharged home on postoperative day 0 . Following the procedure, the patient had immediate resolution of urinary incontinence. At 6 weeks followup, the patient continues to be symptom free.

Conclusions: Robotic simple nephrectomy may be performed for management of patients with an ectopic ureter in association with an ectopic kidney who have continuous urinary incontinence and negligible renal function in the affected kidney.

\section{ABSTRACT VIDEO SESSION 07: BPH}

\section{V07-01 Rezum Steam Ablation of the Prostate: A New Wave of Day Case, Effective Prostate Surgery with no Sexual Dysfunction}

M Ravindraanandan, W Mahmalji, Y Phan

Hereford County Hospital

Introduction \& Objective: Symptoms associated with benign prostatic hypertrophy (BPH) include lower urinary tract symptoms (LUTS) and sexual dysfunction; common findings within the aging male population. Conventional surgery for $\mathrm{BPH}$ is frequently associated with adverse risks such as an impact on sexual function. There are new minimally invasive procedures to treat $\mathrm{BPH}$ that have the advantage of reducing adverse complications. We look at the efficacy and advantages of Rezum compared to well established procedures.

Methods: The principles of Rezum rely on convection of heat that exploits the thermodynamic properties of water. A radiofrequency current is applied through a coil producing energy in the form of water vapor. The water vapor is transmitted through a needle that makes contact with the prostatic tissue. The energy is transferred into the tissue, promoting instant cell necrosis. The lateral lobes are ablated first, followed by the median lobe.
Results: We present a video of an enlarged prostate treated by Rezum surgery. Post-operative imaging shows a widened channel and an improvement in flow rate studies.

Conclusions: We have demonstrated that Rezum treatment of $\mathrm{BPH}$ has given a safe and effective outcome for our patient, with improvements in channel width and flow rates. Furthermore, no sexual dysfunction was reported after surgery. Treatment for BPH using Rezum should be considered in those willing to preserve their sexual function.

\section{V07-02 The New 4D and Standard Prostatic Urethral Lifting (UroLift) Techniques: Day Case, Effective, No Sexual Dysfunction}

M Ravindraanandan, W Mahmalji, Y Phan

Hereford County Hospital

Introduction \& Objective: Benign prostatic hypertrophy (BPH) associated with lower urinary tract symptoms (LUTS) and sexual dysfunction is common amongst the aging male population. Surgery for LUTS is one of the most common in the UK, with a rise in prevalence increasing financial pressure and burden on the 
Table 1 - primary outcomes from UroLift

\begin{tabular}{|c|c|c|c|c|c|}
\hline Characteristics & $\begin{array}{c}\text { Baseline } \\
\text { Mean }\end{array}$ & $\begin{array}{c}4 \text { months } \\
\text { post-op } \\
\text { mean }\end{array}$ & $\begin{array}{c}\text { Mean } \\
\text { Difference }\end{array}$ & P-value & $\mathbf{n}$ \\
\hline $\begin{array}{l}\text { International } \\
\text { Prostate Symptom } \\
\text { Score (IPSS) }\end{array}$ & 21.8 & 13.8 & 8 & 0.02 & 37 \\
\hline Quality of life & 4.6 & 28 & 1.8 & 0.04 & 37 \\
\hline $\begin{array}{l}\text { Post-void residual } \\
\text { volume (mI) }\end{array}$ & 229 & 138 & 91 & 0.04 & 37 \\
\hline QMAX (m/s) & 7.7 & 12.1 & 4.4 & 0.04 & 37 \\
\hline
\end{tabular}

healthcare system. UroLift is a minimally invasive surgery used to treat $\mathrm{BPH}$ with advantages compared to conventional techniques.

Methods: The UroLift procedure was performed on a moderately sized cohort within a single institution. Parameters measured included IPSS score, post-void residuals, QMAX, sexual dysfunction, theatre time and length of stay. Data was analyzed using a two-sample equal-variance $t$-test.

Results: We performed UroLift on 37 patients, average age 69 years (range 53-90). Table 1 summarizes primary outcomes achieved following the UroLift procedure, with significant improvements in IPSS $(\mathrm{p}<0.02)$, post-void residuals $(\mathrm{p}<0.04)$ and QMAX $(\mathrm{p}<0.04)$. No patients suffered any sexual dysfunction side effects with quality of life scores improving by 1.8 $(\mathrm{p}<0.04)$. Hospital stay and theatre time were significantly reduced. Average length of stay was just 10.6 (6-18) hours and average theatre time just 18.7 (12-30) minutes.

Conclusions: UroLift is substantially shorter than other surgery for LUTS. Significant benefits are felt for both the patients, who are discharged home much sooner, and also the hospital, who can perform a higher number of procedures on patients in a similar time period. Patients also do not require an inpatient bed so should not be cancelled on the day of theatre.

\section{V07-03 En Bloc MoLEP (Moses HoLEP) With Early Apical Release}

F Gómez Sancha, ME Rodriguez Socarras, G Saitta, J Reinoso Elbers, J Fernandez Del Alamo, L Llanes Gonzalez, J Gomez Rivas

Introduction \& Objective: Significant early temporary stress urinary incontinence (SUI) rates have been published after HoLEP. Despite its resolution on the majority of patients, it is bothersome both for patients and surgeons. The new Moses technology for HoLEP seems to provide better hemostasis and cutting properties, due to the long reach of the energy pulse through the saline medium used for irrigation. We believe the sphincter's mucosa is ripped off the sphincter during the classic procedure and this could be one of the factors involved in the development of SUI. We present a new En-bloc technique that aims to protect the sphincter's mucosal lining, to provide better chances of early continence.

Methods: A new technique was developed starting at the prostatic apex, scoring the mucosa circumferentially at the junction of the external sphincter with the prostatic apex, to ensure the protection of its mucosal lining. The attachments of the apex to the sphincter are carefully cut posteriorly from 3 to 9 o'clock. The posterior plane is then developed to generate some working space. Attention is then drawn the anterior attachments, from 9 to 3 , cutting $5 \mathrm{~mm}$ in depth into the prostatic apex, to ensure proper sphincter's protection. Then the anterior anatomic plane is followed to liberate the apex completely from the sphincter. Once the apex is free, the plane is followed circumferentially until the bladder neck is reached and cut. The adenoma is then pushed En- bloc into the bladder. Hemostasis and morcellation follow. A video was recorded, and drawings were developed to show the tips and tricks of this technique.

Results: Results of this technique have been previously published by this group (World J Urol 2019 Feb 8). The rate of SUI was $5.8,1.5$ and $0.7 \%$ at 1,3 , and 6 months post-op, respectively. This compares favorably with previously published literature, supporting that this concept is valuable. This is the first video of the technique submitted to a urology congress or published elsewhere.

Conclusions: En-bloc MoLEP (En-Bloc HoLEP carried out with Moses technology) with early apical liberation is a new surgical technique designed to protect the external sphincter's mucosa and to prevent its traction and distention. This procedure tries to reduce the incidence of early postoperative SUI. The new Moses technology facilitates the early dissection of the sphincter during the procedure.

\section{V07-04 Top-Down Green Light Laser enucleation of the prostate (GreenLEP): Technical Aspects}

H Elmansy, A Hodhod, FA Oquendo Aponte, W Shahrour

Introduction \& Objective: Laser prostatectomy is currently considered one of the best modalities for the surgical management of benign prostatic hyperplasia (BPH). Recently, the TopDown technique was introduced as a modification to traditional holmium laser enucleation (HoLEP). Herein, we describe the Top-Down technique using green light laser technology and its feasibility.

Methods: Between 2018 and 2019, we prospectively collected the data of 10 patients who underwent green light enucleation of the prostate using the Top-Down technique (Top-Down GreenLEP). A greenlight XPS machine and a 2090 laser fiber were utilized for these procedures. Using $80 \mathrm{~W}$ for vaporization and 35 $\mathrm{w}$ for coagulation, one posterior groove was created at either 5 or 7 o'clock up to the verumontanum allowing simultaneous enucleation of the median lobe with the attached lateral lobe. The anterior commissure mucosa was then incised starting from the bladder neck at the 12 o'clock position. Once the plane between the adenoma and surgical capsule was created, a top-down lateral lobe dissection was performed and extended anteroposteriorly, towards the apical adenoma at the 6 o'clock position. As the apical dissection was performed from top-down, the mucosal strip was easily visualized. The mucosal strip appears as a bandshaped structure attached to the sphincter on the medial side of

\begin{tabular}{|c|c|c|}
\hline \multicolumn{2}{|c|}{ Data } & Finding \\
\hline \multicolumn{2}{|l|}{ Age median (range) } & 64.5 years $(56.7-88.3)$ \\
\hline \multicolumn{2}{|c|}{ Size of prostatic adenoma median (range) } & $96 \mathrm{cc}(82-150)$ \\
\hline \multicolumn{2}{|c|}{ Preoperative PSA median (range) } & $4.8 \mathrm{ng} / \mathrm{dl}(2.1-12.6)$ \\
\hline \multicolumn{2}{|c|}{ Enucleation time median (range) } & $82(35-170)$ \\
\hline \multicolumn{2}{|c|}{ Morcellation time median (range) } & $13.8(5-26)$ \\
\hline \multicolumn{2}{|c|}{ Median resected weight $(\mathrm{g})$ median (range) } & $88(52-130)$ \\
\hline \multicolumn{2}{|c|}{ Laser energy median } & $156 \mathrm{~J}(141-169)$ \\
\hline \multicolumn{2}{|l|}{ Catheter stay } & $<24$ hours \\
\hline \multicolumn{2}{|c|}{ Stress Urinary incontinence $n(\%)$} & $1(10 \%)$ \\
\hline \multicolumn{2}{|c|}{ Urge Urinary incontinence $n(\%)$} & $1(10 \%)$ \\
\hline \multirow{2}{*}{ IPSS median (range) } & Preoperative & $21(7-30)$ \\
\hline & Postoperative & $6(0-10)$ \\
\hline \multirow{2}{*}{ QoL median (range) } & Preoperative & $5(2-6)$ \\
\hline & Postoperative & $1(0-5)$ \\
\hline \multirow{2}{*}{ Qmax median (range) } & Preoperative & $8.8(5.2-14)$ \\
\hline & Postoperative & $23.1 \mathrm{ml} / \mathrm{s}(18-36.1)$ \\
\hline \multirow{2}{*}{ PVR median (range) } & Preoperative & $198 \mathrm{ml}(87-380)$ \\
\hline & Postoperative & $48 \mathrm{ml}(0-78)$ \\
\hline
\end{tabular}

Table 1: Preoperative and postoperative data 
the scope. By incising the band-shaped mucosa close to the adenoma, the apex of the adenoma was completely released without damaging the ring formed by the sphincter muscle. We recorded patient demographics and collected intraoperative and postoperative data. The preoperative and postoperative IPSS, QoL, Qmax, and PVR were evaluated.

Results: The median prostatic volume was $96 \mathrm{cc}$ (82-150), and the median age at surgery was 64.5 years. There were no intraoperative complications or blood transfusions. All patients had their catheters removed the next day postoperatively. Only 1 patient (10\%) had failed a trial of voiding (TOV) initially but succeeded after 3 days. The one-month postoperative data are presented in Table 1.

Conclusions: Top-Down GreenLEP is a feasible procedure. Early outcomes are satisfactory and promising. To better evaluate this technique, further studies with larger populations and longer follow up are required.

\section{V07-05 Ablation in Holmium Laser Enucleation of the Prostate: A Novel Method to Avoid Termination of Difficult Morcellation}

BB Whiles, K Glavin, KL Thurmon

Introduction \& Objective: An important aspect of holmium laser enucleation of the prostate (HoLEP) is effective tissue morcellation. Although morcellation can be difficult for various reason, inability to endoscopically evacuate the tissue results in the need for increased intraoperative time or additional procedures. This can include an open cystotomy or a staged morcellation procedure, both of which subject the patient to increased morbidity. Much to our frustration in our own clinical practice, we sought to develop an alternative method for prostatic tissue clearance in HoLEP.

Methods: This is a descriptive case report on a method for holmium laser ablation of prostatic adenoma in HoLEP which is commonly encountered during the morcellation segment of the HoLEP procedure. The specific steps and materials for completing this procedure were described.

Results: The enucleation procedure proceeds in a routine fashion. After all obstructing prostatic tissue has been successfully enucleated and displaced into the lumen of the bladder, a morcellator is introduced. Dense, fibrotic tissue is encountered. The morcellator is unable to effectively break up the tissue, increasing the patient's risk for a prolonged morcellation time or the need for an additional procedure. The morcellator is removed and a $550 \mu \mathrm{m}$ side fire Xpeeda fiber is introduced. The adenomatous tissue is ablated with ease. This decreases the total amount of tissue for morcellation but also created textured, rough edges. Both of these result in improved and successful morcellation thereafter.

Conclusions: Ablation of difficult to morcellate tissue during HoLEP is a viable alternative to open cystotomy or staged morcellation and likely has decreased patient morbidity. Urologists should consider this method when morcellation difficulty occurs.

\section{v07-06 Top Down technique of Holmium laser enu- cleation of prostate}

K Ramaswami, P Bhirud, R Raveendran, H Pothiyedath

Introduction \& Objective: Traditionally, the gold standard for treatment of BPH has been the electrocautery- based Trans Urethral Resection of the Prostate (TURP). However, the number of laser techniques being performed is rapidly increasing. Potential advantages of laser therapy over traditional TURP include decreased morbidity and shorter hospital stay. There are several techniques for laser prostatectomy that continue to evolve. We present here a Top Down approach of performing HOLEP.

Methods: We demonstrate our version of this technique and present early operative outcomes. After cystoscopy and urethral calibration, laser incision is made in the floor of prostate and deepened up to capsule. The edges of the laterallobes on either side of the veru are incised. The scope is rotated to visualize at $12^{\prime} \mathrm{O}$ clock position and laser incision is given at 12' O clock. Laterallobe dissection is then dissected from the top down allowing faster dissection time than the traditional technique.

Results: In our series of Holep between December 2014 and April 2019, we have performed 20 cases using Top Down technique at our center. Median patient age was 65 years. Mean time required for laser enucleation was 58 minute and mean morcellation time was 14.3 minutes. No major complications were observed during this technique.

Conclusions: Holep with Top Down techniques a safe method of performing the procedure and can be executed without much technical difficulty.

\section{V07-07 Holmium Laser Enucleation of the Prostate after Failed Urolift: Surgical Considerations for Morcellation}

\section{K Parikh, C Dora}

Introduction \& Objective: Objective: To demonstrate the surgical considerations for managing retained UroLift implants when performing HOLEP. Prostatic Urethral Lift via the UroLift System has become a common treatment modality to manage symptoms associated with benign prostatic hyperplasia. The UroLift procedure uses non-absorbable implants to retract obstructing prostate lobes. Retreatment rates following UroLift have been reported at $13.6 \%$ over 5 years. ${ }^{1}$ We anticipate an increasing number of men seeking definitive surgical management after failed Urolift. There have been reports in the literature of UroLift implants causing morcellator device jams when attempting holmium laser enucleation of the prostate (HOLEP).

Methods: From August 2018 to April 2019, we reviewed 118 consecutive patients who underwent HOLEP by a single surgeon. Three men were identified who had previously undergone Urolift. Video footage was obtained. As demonstrated in the video, during enucleation the metallic clip of the UroLift implants were incorporated in the adenoma specimen. For morcellation, we use the Piranha morcellator (Richard Wolf, Knittlingen, Germany). Morcellation was carried out in a slow and controlled manner. When the metal clip comes into contact with the morcellator, a catch and release is performed by releasing the morcellator pedal and withdrawing the morcellator into the nephroscope to release the adenoma. Remnant clips and sutures can be retrieved with a grasper. We perform a 3-month follow-up cystoscopy in the office to exclude any remnant implant material in the prostatic urethra or bladder.

Results: Procedures were completed uneventfully. In developing this technique, we experienced jamming of the morcellator blades in 2 cases requiring replacement of the disposable blades. Follow up in-office cystoscopy did not reveal any remnant implant material that needed to be removed.

Conclusions: HOLEP can be performed safely in the UroLift failure patient population. Careful morcellation techniques can decrease the risk of costly morcellator blade replacement. 
V07-08 Thulium Laser enucleation of the prostate: Top or down technique adopting during the early learning curve?

M Omar, M El Shazly

Introduction \& Objective: Since2010, Herrmann and colleagues introduced Thulium laser enucleation of the prostate (ThuLEP) as a versatile minimally invasive enucleation procedure; followed by several studies that have confirmed its feasibility and efficacy such as HoLEP. Our aim was to present the feasibility of adopting both top or down techniques during a selftaught, ThuLEP, learning curve for a single surgeon with no previous HoLEP experience.

Methods: Between February 2018 and March 2019, a welltrained endourologist; who didn't perform laser enucleation of the prostate before, started reviewing the available literature attended more than 15 procedures at another hospital and participated in 3 laser enucleation of prostate workshops. 29 patients with a prostate size between 80 and 120 grams underwent ThuLEP without tutoring or mentor supervision. We used Revolix DUO ${ }^{\text {TM }}$ machine with a 40-watt setting for both cutting and coagulation with a 550-mic fiber for all cases.

Results: In the 1 st 4 cases, the surgeon was unable to completely enucleate the $1^{\text {st }}$ lobe and ended with open prostatectomy. In the following 4 cases, one lobe was completely enucleated and morcellated while the other lobe was finished by bipolar resection. The following 10 cases the down technique was the primary strategy for a successful enucleation. Then the last 11 cases were successfully completed by a combination of down and top technique. All patients had a satisfactory voiding outcome with a mean Qmax of 24.5 after surgery.

Conclusions: ThuLEP extraordinary hemostatic effect and the limited penetration depth may allow it to be the standard step for beginner surgeon, while adopting both top and down technique may be the easiest way for accelerating the learning curve for surgeons performing ThuLEP.

\section{V07-09 En-bloc Laser Enucleation of Prostate with Multi-pulse laser}

\section{T Chang, J Tseng}

Introduction \& Objective: Laser enucleation of prostate is nowadays recognized as an alternative surgical treatment for benign prostatic hyperplasia besides from traditional Mono-polar or Bipolar instruments. Different variants of laser surgical method had been developed. The classical "3-lobes laser technique" method and some variants had been proposed to be a sufficient way for enucleation. However, various modifications of this surgical technique had been developed, including the socalled "En-bloc enucleation" - that is to enucleate the prostate in one piece. Here we presented a step-by-step approach to en-bloc Multipulse laser enucleation of the prostate for benign prostatic hyperplasia surgical treatment. In our experience, this procedure is a fast, efficient way to treat obstructive prostates and offers comparable surgical outcomes.

Methods: We used Multipulse laser for En-bloc enucleation of the prostate. The initial incision was made over 5 o'clock to the capsular layer. The adenoma was enucleated from the capsule carefully like peeling an orange. The plane is followed ascending towards the bladder neck from 5 to 1 o'clock, separating the left lobe from the prostatic capsule. The median and right lobes were then enucleated together following the same plane clockwise from 5 to 7 to 11 o'clock. Finally, the residual attachments of mucosa over 11 to 1 o'clock were cut with laser. The whole prostatic adenoma was removed in one piece. Morcellation was used to remove the adenoma from bladder. Hemostasis were secured solely by laser.

Results: Total operational time was 35 minutes and morcellation time was 4 minutes. The resected adenoma weighted 20 grams out of the original 36-gram prostate.

Conclusions: En-bloc laser enucleation of prostate provides an efficient approach for endoscopic enucleation of prostatic adenoma. In our experience, we believe that this technique provides a better surgical outcome compared with traditional resection of $\mathrm{BPH}$ by reducing surgical time and reducing the amount of tissue energy exposure.

\section{V07-10 Non-anatomical thulium laser enucleation of prostate for elderly BPH patients with oversize pros- tate volum}

Y Jing, Y Zhu, Y Ruan, F Zhao, B Han, S Xia

Introduction \& Objective: The treatment of elderly benign prostatic hyperplasia (BPH) patients with oversize prostate volum is a challenge for urologist. Here we introduce a nonanatomical thulium laser enucleation of prostate (ThuLEP) for these cases.

Methods: A total of $63 \mathrm{BPH}$ patients more than 80 years old with oversize prostate volum $(>150 \mathrm{ml})$ were accepted ThuLEP from May 2017 to February 2019. The ages of the patients are from $80 \mathrm{y}$ to $91 \mathrm{y}$. The volum of the prostate is from $150 \mathrm{ml}$ to $251 \mathrm{ml}$. At $12 \mathrm{o}$ 'clock, the parietal prostate tissue was cut from the inside of the external urethral sphincter and reached the bladder neck, and then at the prostate apex, the tissue was cut to surgical capsule at 5 o'clock beside the verumontanum. The integrity of the external urethral sphincter was reconfirmed before the urethra mucosa was cut to form a marker line close to the inside of the sphincter. Then, the prostate tissue beside the proximal end of the marker line was cut to the surgical capsule. And the cutting points were merged counterclockwise along this plane from 5 o'clock to 12 o'clock. The prostate tissue was cut in an arc shape repeatedly alongside the surgical capsule, and the whole left lobe was cut off and pushed into the bladder. The right lobe and the middle lobe (if there were a middle lobe) were cut into bladder in the same way. The residual prostate tissue in bladder was removed by the morcellator.

Results: The enucleation time was $45 \sim 85 \mathrm{~min}$, and the morcellating time was $12 \sim 18 \mathrm{~min}$. No patient needed blood transfusion. The indwelling catheter time was $36 \sim 48 \mathrm{~h}$. The IPSS, QoL, Qmax and PVR were improved greatly when evaluated after 1moth and 3mothe separately. 2/63(3.17\%) patients were suffered from urgent urinary incontinence after removing the catheter and recovered in 2days and 8days separately.

Conclusions: The non-anatomical ThuLEP is an effective and save method for the treatment of elderly BPH patients with oversize prostate volum.

\section{V07-11 Various techniques of Holmium Laser En- ucleation of Prostate}

K Ramaswami, P Bhirud, H Pothiyedath

Introduction \& Objective: Holmium laser enucleation of prostate (HoLEP) has emerged as an effective transurethral treatment option for patients suffering from symptomatic BPH of any size. The efficiency of HoLEP lies in its excellent tissue debulking capabilities. We present different techniques of doing Holmium laser enucleation of prostate in this study. 


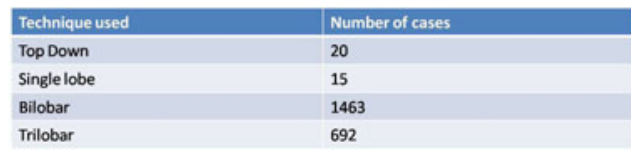

\begin{tabular}{|l|l|}
\hline Baseline Patient Characteristics & \\
\hline Number of patients & 2190 \\
\hline Age(yrs) & $66.5 \pm 8.1$ \\
\hline Q Max(m//s) & $8 \pm 3.1$ \\
\hline IPSS & $24 \pm 5.6$ \\
\hline PVR (ml) & $115 \pm 35$ \\
\hline Prostate volume (cc) & $62 \pm 34$ \\
\hline Total Serum PSA (ng/ml) & $4.6 \pm 3.5$ \\
\hline Urinary retention & 122 \\
\hline Bladder Calculi & 47 \\
\hline Bladder Diverticuli & 43 \\
\hline
\end{tabular}

\begin{tabular}{|l|l|}
\hline Intra- and perioperative data & \\
\hline Total operative time $(\mathrm{min})$ & $80.8 \pm 39.7$ \\
\hline Laser enucleation time $(\mathrm{min})$ & $54 \pm 20.9$ \\
\hline Morcellation time $(\mathrm{min})$ & $18.3 \pm 9$ \\
\hline Morcellation efficiency (g/ $\mathrm{min})$ & $2.89 \pm 1.5$ \\
\hline Resected weight (g) & $40 \pm 27.5$ \\
\hline Hb drop ( $/$ / dl) & $1.1 \pm 0.9$ \\
\hline Na drop (meq/ I) & $2 \pm 1.4$ \\
\hline Catheter time (hrs) & $36 \pm 14.7$ \\
\hline Hospital stay (hrs) & $48 \pm 26$ \\
\hline
\end{tabular}

Methods: Procedure was carried out using a Pulsed 100W Holmium:YAG Laser with a 550 micron end fire laser fibre. Initially $26 \mathrm{~F}$ continuous flow resectoscope with a laser bridge-later $24 \mathrm{~F}$ continuous flow resectoscope and morcellation with the Lumenis VERSACUT tissue morcellator system. All patients were preoperatively assessed with ultrasound prostate volume estimation, maximum urine flow rate (Q MAX), post void residual urine (PVR) assessment and International prostate symptom score (IPSS). We demonstrate various techniques of HoLEP such as bilobar, trilobar, single lobe and Top down techniques.

Results: In our series of HoLEP between December 2014 and April 2019, we have performed 2190 cases: 20 cases using top down technique, 15 cases single lobe technique and remaining carried out using bilobar and trilobar techniques. Median patient age was $66.5 \mathrm{yrs}$ with a mean operative time of 54 minutes.

Conclusions: Bilobar and trilobar techniques are easy to perform especially for beginners. Efficiency and better enucleation possible with single lobe and top down technique. The evolving techniques may reduce operating time of the procedure

\section{V07-12 Improving the safety profile of morcellation technique during Hoelp}

H Pothiyedath, K Ramaswami, P Bhirud

Introduction \& Objective: Holmium laser enucleation of the prostate (HoLEP) is an effective treatment option for bladder outflow obstruction secondary to prostatic enlargement. How-

\begin{tabular}{|l|l|}
\hline Baseline Patient Characteristics & \\
\hline Number of patients & 2190 \\
\hline Age(yrs) & $66.5 \pm 8.1$ \\
\hline Q Max(m//s) & $8 \pm 3.1$ \\
\hline IPSS & $24 \pm 5.6$ \\
\hline PVR (ml) & $115 \pm 35$ \\
\hline Prostate volume (cc) & $62 \pm 34$ \\
\hline Total Serum PSA (ng/ml) & $4.6 \pm 3.5$ \\
\hline Urinary retention & 122 \\
\hline Bladder Calculi & 47 \\
\hline Bladder Diverticuli & 43 \\
\hline
\end{tabular}

ever, complications such as bladder mucosal injuries during morcellation of the prostatic adenoma after enucleation slow the learning curve and prevent some urologists from learning the procedure. We present some tips and tricks to perform a safe and quick morcellation based upon experience in Holep over last 4 years.

Methods: A retrospective review of a prospective database of all HoLEP procedures performed during the period of Dec 2014 to April 2019(2190 cases) at our center was undertaken. Procedure was carried out using a pulsed $100 \mathrm{~W}$ Holmium:YAG laser with a 550 micron end fire laser fiber, Initially a $26 \mathrm{Fr}$ continuous flow resectoscope with a laser bridge - later $24 \mathrm{Fr}$ Continuous flow resectoscope and morcellation with the Lumenis VERSACUT ${ }^{\mathrm{TM}}$ tissue morcellator system. All patients were preoperatively assessed with ultrasound prostate volume estimation, maximum urine flow rate (Q Max), post void residual urine volume (PVR) assessment and International Prostate Symptom Score (IPSS). Data were recorded for operative duration, morcellation time and all case notes were reviewed for morcellation injury.

Results: Between December 2014 and April 2019, 2190 cases of HoLEP were performed at our center. Median patient age was 66.5 years. Mean time required for laser enucleation was 54 minutes and mean morcellation time was $18.3 \mathrm{~min}$. Bladder mucosal injury(superficial) occurred in 44 patients during morcellation. A bladder irrigation system was placed only when evidence of significant post-op hematuria (3\%) was present and was placed for no longer than $12 \mathrm{hrs}$. No other major complication was observed during the series.

Conclusions: Holep with morcellation is an effective and safe surgical intervention for benign prostatic hyperplasia of all sizes. By taking certain precautions, morcellation can be performed quickly and safely without injuring urinary bladder wall.

\begin{tabular}{|l|l|}
\hline Intra-and perioperative data & \\
\hline Total operative time ( $\mathrm{min}$ ) & $80.8 \pm 39.7$ \\
\hline Laser enucleation time ( $\mathrm{min})$ & $54 \pm 20.9$ \\
\hline Morcellation time $(\mathrm{min})$ & $18.3 \pm 9$ \\
\hline Morcellation efficiency (g/ $\mathrm{min})$ & $2.89 \pm 1.5$ \\
\hline Resected weight (g) & $40 \pm 27.5$ \\
\hline Hb drop ( g/ dl) & $1.1 \pm 0.9$ \\
\hline Na drop (meq/ l) & $2 \pm 1.4$ \\
\hline Catheter time (hrs) & $36 \pm 14.7$ \\
\hline Hospital stay (hrs) & $48 \pm 26$ \\
\hline
\end{tabular}




\section{ABSTRACT VIDEO SESSION 08: ROBOTIC CYSTECTOMY}

\begin{abstract}
V08-01 Simultaneous Robot-assisted Radical Cystectomy and Nephroureterectomy for Concomitant Bladder and Upper Urinary Tract Urothelial Carcinoma in Patient with Solitary Kidney
\end{abstract}

A Garayev, O Aytac, F Atuğ

Introduction \& Objective: Patients with multifocal unresectable bladder and concomitant upper urinary tract tumors are the candidates of the simultaneous cystectomy and nephroureterectomy. In this study we report our result of robotassisted simultaneous cystectomy and nephoureterectomy.

Methods: A-62-year-old man with previous history of left laparoscopic nephroureterectomy (LNU) due to the renal pelvis urothelial carcinoma referred to our clinic with macroscopic hematuria. After LNU he recieved intravesical BCG treatment in sign of metastasis. Laparoscopic cystectomy was planned. After induction of anesthesia, pneumoperitoneum was achieved. One $10 \mathrm{~mm}$ and $25 \mathrm{~mm}$ trochars were placed. After dissection of the peritoneum, perivesical tissue was dissected. After ligation of the dorsal venous complex,prostatic urethra was dissected. Ligation of the bladder vessels was performed. Bilateral ureters were dissected and cut from the most distal segment. Specimen was placed into an organ retrieval bag. $10 \mathrm{~mm}$ port was removed and appropriate ileal segment was pulled out. After separation of the $15 \mathrm{~cm}$ ileal segment and ileoileal anastomosis, ureteroileal anastomosis was performed. Cutaneous placement of the distal portion of the ileal conduit was performed. Results: Patient was ambulated and oral intake was initiated by liquids on first post-operative day. Drain was removed on 3rd post-operative day. Patient was discharged from the hospital on 5th post-operative day. On first month follow-up, no significant pathology was observed.

Conclusions: Cystectomy operation has high morbidity and mortality rates. Laparoscopic technique has advantages in terms of peri-operative and post-operative complications, when compared with open surgery. Oncological results are also comparable with open surgery.

V08-02 Robot-Assisted Radical Cystectomy with Intracorporeal Ileal Conduit in a Patient with Situs Inversus Totalis

\section{F Munshi, C Polotti, SE Elsamra}

Introduction \& Objective: Situs invesus totalis (SIT) is a rare congenital anomaly characterized by the mirror-image transposition of abdominal and thoracic organs. While feasible, operating on patients with situs inversus offers unique technical challenges to the surgeon due to its rarity and the contralateral disposition of the viscera. Urologists in particular need to be aware of the genitourinary abnormalities associated with situs inversus when planning to operate. We report the case of a 67year-old male with invasive bladder cancer in the presence of situs inversus totalis and associated bilateral duplicated ureters. This is only the second case of bladder cancer in the context of situs inversus reported in the literature and the first one managed with robot-assisted radical cystectomy and urinary diversion with an intracorporeal ileal conduit.

Methods: From a urologic perspective, several renal cell and urothelial carcinomas have been reported in patients with SIT, but only one case prior to ours has been reported of a patient with invasive bladder cancer and SIT till date. SIT may pose several technical difficulties during laparoscopic operative procedures, due to the mirror image of the laparoscopic view, which requires a constant reorientation of anatomy from surgeons, even when carrying out typical maneuvers. We discuss our approach when operating on 1 patient with invasive bladder cancer and SIT as well as necessary considerations when operating on urologic patients with SIT.

Results: We successfully and safely performed a robot-assisted laparoscopic radical cystoprostatectomy with intracorporeal ileal conduit urinary diversion in a patient with SIT and bilateral ureteral duplication.

Conclusions: Although the incidence of intra-abdominal malignancies in a patient with SIT is very rare, the surgeon must anticipate the complexity of the surgical procedure in cancer patients with SIT. Advanced surgical skill is required to perform a precise radical cystectomy with lymphadenectomy and urinary diversion via ileal conduit in a patient with SIT by visualizing the exact mirror image of the anatomy during the operation. Preoperative recognition of the anatomic variations might be needed when operating on a patient with SIT, and three-dimensional CT angiography reconstruction and training with a surgical simulator prior to the operation can augment the surgeon's preparedness for operating. In conclusion, robot-assisted radical cystectomy with intracorporeal ileal conduit in a patient with invasive bladder cancer and SIT can be safely performed when adequate consideration is given to the patient's unique anatomy.

\section{V08-03 Evolution in technique of robotic intracorporeal continent catheterizable pouch after cystectomy}

\section{R Matulewicz, G Chesnut, A Goh}

Introduction \& Objective: Robotic assisted radical cystectomy (RARC) with intracorporeal urinary diversion (ICUD) has become an increasingly utilized approach to treating various bladder pathologies requiring extirpation. As surgeons have become more experienced with ICUD, especially with lesser used diversions such as continent cutaneous reservoirs, the technique has evolved.

Methods: We describe several advances in the technical aspects of robotic ICUD with a continent catheterizable pouch (CCP) built upon the open principles of the previously described "Indiana Pouch," which uses the distal ileum and right colon. Modifications in anatomic mobilization and exposure, bowel segmentation using novel robotic bowel staplers, and catheterizable channel creation are highlighted. Use of indocyanine green to assess vascularity in bowel and ureter intraoperatively is demonstrated. Also described are short-term outcomes of our initial experience.

Results: Eleven consecutives patients ( 4 female) who have undergone ICUD with CCP were included. Of the eleven, five had malignant indications (bladder or urethral cancer) while 4 were for benign causes (2 radiation cystitis, 2 interstitial cystitis). Mean blood loss was 235cc (range 100-500cc) and mean operative time was 8.5 hours (range $7.5-13$ ). There were no conversions to an open procedure and no patient required a blood transfusion. Hospital stay was 7.2 days on average (range 4-18) with no 30-day post discharge readmissions. One patient had a 
Clavien grade 3 or greater complication at 30 days. At three months follow up, all patients were continent and satisfied with their diversion.

Conclusions: RARC with CCP ICUD is a safe and effective approach to bladder removal and reconstruction. Patients should be offered CCP ICUD as a reconstructive option following RARC.

V08-04 Minimally Invasive management of RobotAssisted Radical Cystectomy Related complications: 15 Years of Experience

NA Aldhaam, AS Elsayed, M Durrani, Z Jing, AA Hussein, KA Guru

Introduction \& Objective: There is a lack of data regarding the robot-assisted management of complications after robot-assisted radical cystectomy (RARC). We reviewed our data for specific complications after RARC during our 15-year experience to assess the feasibility and safety of robot-assisted approach in managing such complications.

Methods: We retrospectively reviewed the records of all patients who underwent surgical interventions for robot-assisted radical cystectomy specific complications (ureteroileal stricture, urinary fistula, and parastomal hernia) between 2004 and 2019.

Results: Out of 614 consecutive patients who underwent RARC, 153 patients $(25 \%)$ experienced surgical intervention for complications. Mean follow-up was 30 months. Average time to any surgical intervention after cystectomy was 16 months. Sixtyseven patients $(44 \%)$ managed by robot-assisted surgical approach. $88 \%$ of surgical interventions were performed after 90 days of RARC. Interventions for ureteroileal stricture were the most common (43 cases) followed by interventions for urinary fistulas (17 cases) and parastomal hernia (7 cases).

Conclusions: Robot-assisted management of robot-assisted radical cystectomy complications appears safe and feasible. The decision to proceed with robot-assisted repair was largely driven by the surgeon experience.

\section{V08-05 A Novel Technique of Intracorperal Ileal Neobladder After Robot Assisted Radical Cystectomy: Modified Anatolian Pouch}

\author{
B Onal, C Demirdag, B Selcuk, B Ercili, S Citgez, A Erözenci \\ Istanbul University- Cerrahpaşa
}

Introduction \& Objective: We performed a robot asissted laparoscopic radical cystoprostatectomy and intra-corporeal modified Anatolian Pouch ileal neobladder on a 49-year-old male with non-metastatic stage T1 high grade with glandular differentiation. Preoperative staging was negative. His body mass index was 21.2. His ASA (American Society of Anesthesiologists) score was 1.

Methods: We used the 6-port transperitoneal technique. The camera port position is measured at up to $2 \mathrm{~cm}$ cranial to the umbilicus. A 12-mm left-sided port was needed for the Endo GIA stapler. Three $8-\mathrm{mm}$ robotic trocars and a $10-\mathrm{mm}$ assistant port were used. A $45 \mathrm{~cm}$ ileal segment was separated, starting at an appropriate point 15 to $20 \mathrm{~cm}$ from the ileocecal junction. The left ureter is transposed. The silk stay suture on the Hem-o-lok clip is delivered to the assistant's forcep, to pull the ureter under the mesocolon and 2 ureters were anastomosed with each other by Wallace type anastomosis technique prior to un-docking. The ileal segment is disconnected, and the entero- enterostomy performed, using 4 reloads of the Endo GIA linear stapler $60 \mathrm{~mm}$. The whole separated ileal segment was cut at the antimesenteric border for detubularization. Proximal and distal sides of the ileal loop were anastomosed side-to-side and a bagel-shaped detubularized ileal segment was formed. Three identical points, starting from the medial border of the anastomosis segment, were identified and united at the center. Then, the medial edges of the ileal loop were joined by a running through-and-through suture of 3-0 V-lock continuously, resulting in a goosefoot image in the centrum. The urethroileal anastomosis was performed over a transurethral 18-French catheter with two $30 \mathrm{~cm} \mathrm{3-0} \mathrm{V-lock} \mathrm{su-}$ tures. The afferent limb and ureters are lined up on the right side. The Wallace ureteral-ileal (neobladder afferent limb) anastomosis is performed using 4.0 vicryl. A small opening remains at the anterior wall just below the afferent limb, which is where the ureteric stents will pass through the neobladder and into the ureters. The two 6 Fr ureteral stents are inserted via a urethral catheter. The small gap in the anterior neobladder wall, is closed. Results: The docking time was 9 minutes. The robotic radical cystoprostatectomy with extended pelvic lymphadenectomy took 1 hours, 45 minutes; the neobladder took 2 hours, 55 minutes, for a total operative time of 5 hours, 15 minutes. The estimated blood loss was $400 \mathrm{~mL}$.

Conclusions: Our study demonstrated the feasibility of novel technique (Anatolian neobladder) in the treatment of bladder cancer after robot assisted laparoscopic radical cystectomy. It can be an alternative to other ileal neobladder techniques.

\section{V08-06 Treatment of Multipl Diseases in One Session by Robot Assisted Laparoscopic Method: A Case Report}

\section{B Onal, C Demirdag, B Selcuk, B Ercili, U Aferin, M Ertem}

Introduction \& Objective: Robotic surgery is being used more and more nowadays and is a surgical method that offers important advantages and comfort to both patients and surgeons. Sometimes, surgical treatment of more than one disease can be done in a single session. In this video, we aimed to present a case who had a robotic right adrenalectomy, left partial nephrectomy and cholecystectomy in the same session.

Methods: Abdominal ultrasonography, magnetic resonance imaging and positron emission tomography were performed in a 49-year-old woman who presented with palpitations, back pain and tremors in her hands. A $21 \times 19 \mathrm{~mm}$ lesion in the right adrenal gland and $33 \times 21 \mathrm{~mm}$ contrast holding lesion in the anterior of the left kidney was detected. As a result of hormonal tests performed by endocrinology, the patient was diagnosed with pheochromocytoma. In addition, cholecystectomy was decided to be performed in the patient who had stones in the gallbladder and was symptomatic. The patient was first placed in the left lateral decubitus position, $8 \mathrm{~mm}$ camera port was placed from the left lateral side of the rectus muscle at umbilicus level. After the formation of pneumoperitoneum, one $8 \mathrm{~mm}$ camera port, three $8 \mathrm{~mm}$ working ports and a $10 \mathrm{~mm}$ assistant port were placed. After the docking, retroperitoneal area was reached. The $4 \mathrm{~cm}$ mass on the anterior of the left kidney was revealed by opening the gerota fascia. 2 bulldog clamps were placed in the renal artery and warm ischemia was achieved. The mass was excised with scissors. The defect in the renal parenchyma was repaired with $2 / 0$ v-lock sutures. Clamps were opened. The warm ischemia time was calculated as 14 minutes. Then the robot was undocked 
and the patient was placed in the right lateral decubitus position. Three $8 \mathrm{~mm}$ working ports and a $10 \mathrm{~mm}$ assistant port were placed. Right below the liver gerota fascia was opened. The right adrenal gland was removed together with the mass by dissecting from the surrounding tissues. Then cholecystectomy was performed. The operation was terminated by placing two silicone drainage tubes separately in the both operation sites.

Results: The operation time was 230 minutes and the bleeding amount was 350 milliliters. The patient who was taken drainage tube at the post-operative 48th hour was discharged without any complications in the post-operative 72th hour.

Conclusions: Robotic surgery is used more and more frequently in the treatment of oncologic masses. The use of robotic surgery in the presence of more than one mass requiring surgery provides advantages such as fewer incisions, less bleeding and less hospital stay. Robotic surgery can be performed safely and effectively in the surgical treatment of different masses in a single session.

\section{V08-07 Robot-assisted radical cystectomy with in- tracorporeal urinary diversion}

V Pavlov, R Safiullin, M Urmantsev, A Deneyko

Introduction \& Objective: Laparoscopic radical cystectomy (LRC) and robot-assisted radical cystectomy (RARC) are increasingly popular, but high-level evidence for these techniques remains lacking. Objective: To compare the outcomes of patients undergoing open radical cystectomy (ORC), RARC, and LRC. Methods: From January 2018 to May 2019, 40 patients received robot-assisted radical cystectomy for muscle-invasive bladder cancer or at high risk of non-muscular-invasive bladder cancer. Primary end points were 30 - and 90-d complication rates. Secondary end points were perioperative clinical, pathologic, and oncologic outcomes, and quality of life (QoL).

Results: The 30-d complication rates (classified by the ClavienDindo system) varied significantly between the three arms. ORC complication rates were significantly higher than LRC and RARC. The 90-d complication rates did not differ significantly between the three arms. Mean operative time was significantly longer in LRC compared with ORC or RARC. ORC resulted in a slower return to oral solids than RARC or LRC. There were no significant differences in QoL measures.

Conclusions: The 30-d complication rates varied by type of surgery and were significantly higher in the ORC arm than the LRC arm. There was no significant difference in 90-d Claviengraded complication rates between the three arms. Patient summary: We compared patients having open, robotic, or laparoscopic bladder removal surgery for bladder cancer and found no difference in Clavien-graded complication rates at 90-d.

\section{V08-08 Robotic-assisted anterior pelvic exenteration with intracorporeal urinary diversion}

V Pavlov, R Safiullin, M Urmantsev, A Deneyko, $\mathrm{R}$ Abdrahimov

Introduction \& Objective: To report the outcomes of 5 patients whom we performed robot-assisted anterior pelvic exentration with intracorporeal urinary diversion (ileal conduit) for bladder cancer.

Methods: Between February 2018 and May 2019, we performed robot-assisted anterior pelvic exentration with intracorporeal urinary diversion (ileal conduit). Patient demographics, operative and postoperative variables, pathological variables, complications (according to modified Clavien system) and functional outcomes were evaluated.
Results: The mean (sd, range) operative duration, intraoperative estimated blood loss and mean lymph node (LN) yield were 5.1 (4.3-6.4) h, 450 (100-1200) $\mathrm{mL}$ and 24.8 (8-46), respectively. The mean (sd, range) hospital stay was 10.5 (7-18) days, lodge drains were removed at a mean of 9.3 (5-15) days and surgical margins were negative in all but one patient who had pT4b disease. The postoperative pathological stages were: pT2b (one), pT3a (one), pT3b (one), pT4a (one) and pT4b (one). Positive LNs were detected in 3 patients, respectively and at a mean follow-up of 8.3 (2-14) months, one patient died from metastatic disease. According to the modified Clavien system, there were 2 minor (Grade 1 and 2) and 1 major (Grade 3-5) complications in the perioperative (0-30 days) period; 1 minor and 1 major complications in the postoperative (31-90 days) period.

Conclusions: Robot-assisted anterior pelvic exentration with intracorporeal urinary diversion are complex procedures with acceptable morbidity, excellent short-term surgical and pathological outcomes and satisfactory functional results. Studies with more patients and longer follow-ups are required to evaluate the feasibility of these RA totally intracorporeal complex procedures.

\section{V08-09 Robotic intracorporeal Studer pouch recon- struction with internal $\mathbf{J J}$ stenting following robotic radical cystectomy: Balbay's technique}

\section{Balbay, AE Canda, MC Kiremit, E Koseoglu}

Introduction \& Objective: We present the surgical technique of robotic intracorporeal Studer pouch reconstruction with internal JJ stenting following robotic radical cystectomy for bladder cancer by applying Balbay's technique.

Methods: Overall,5 ports were used including a $12 \mathrm{~mm}$ sized assistant port on the left side. $4^{\text {th }}$-arm was controlled with the left hand. Starting from the ileoceacal junction, distal $20 \mathrm{~cm}$ of terminal ileum was spared and a total of $55 \mathrm{~cm}$ ileal segment length was used for pouch construction. Laparoscopic staplers for ileum were used to spare the segregated ileum to perform side-to-side ileo-ileal anastomosis. Stay sutures on lateral sides of the ileum were placed at every $10^{\text {th }} \mathrm{cm}$. All measurements were made with the use of a pre-measured vascular tape. An anastomosis between urethra and antimesenteric side of the ileum at $10^{\text {th }} \mathrm{cm}$ from the distal cut point was made over a Foley catheter. Using the proximal $15 \mathrm{~cm}$ segment of the ileum as the chimney, remaining $40 \mathrm{~cm}$ of the ileal segment was incised and opened on its antimesenteric border. Ileal segment was folded from its mid-point at the $20^{\text {th }} \mathrm{cm}$ and posterior plate was constructed horizontally between adjacent intestinal edges. Corresponding $10^{\text {th }}$ and $30^{\text {th }} \mathrm{cm}$, and $0^{\text {th }}$ and $40^{\text {th }}$ $\mathrm{cm}$ points were brought together with stay sutures, and posterior plate was closed using additional stay and absorbable or barbed continuous sutures. Foley catheter was replaced by an N/G tube and, anterior plate was constructed vertically by joining the lateral serosal edges at $20^{\text {th }}$ and $40^{\text {th }} \mathrm{cm}$, with additional stay and continuous sutures. Wallace type uretero-ileal anastomosis was made. Bilateral double-J stents were introduced through the external urethral meatal end of the N/G tube in and up to renal pelvis which directs patients own urine into the newly formed pouch.

Results: Pouch is distended with $150 \mathrm{ml}$ sterile saline to confirm watertight state. In case of a leakage, additional barbed sutures are applied. Postoperatively, drain creatinine is measured in order to confirm no urine leakage.

Conclusions: Application of Balbay's technique replicates open Studer pouch construction when creating robotic intracorporeal neobladder formation, assuring double folding which is a sine qua non, if low pressure and high capacity pouch is to be created. 
Key points include exact use of measured $55 \mathrm{~cm}$ ileum, urethroileal anastomosis at the $10 \mathrm{~cm}$ from the distal end and double folding of the segregated ileal segment. Internal double-J stenting additionally provides a safe, low pressure continuous irrigation of the pouch with patients own sterile urine.

\section{V08-10 Robotic Nerve Sparing Radical Cystectomy: Maintaining Oncologic Efficacy while Improving Quality of Life}

\section{Billah, S Ahsanuddin, G Lovallo, M Stern, M Ahmed}

Introduction \& Objective: Radical cystoprostatectomy remains the gold standard treatment for muscle invasive bladder cancer though it has significant effects on patient's quality of life particularly regarding continence and sexual function. The introduction of robotic assistance has reduced complications, improved recovery, and shortened hospitalizations. Robotic nerve sparing approaches are commonly discussed in the setting of prostatectomy but are lightly addressed in the literature with respect to robotic cystoprostatectomy. Nerve sparing leads to improved outcomes of continence and sexual function while maintaining oncological efficacy. Our aim is to apply and demonstrate the benefits of both robotics and nerve sparing in a patient undergoing radical cystoprostatectomy.

Methods: This video presents a 70-year-old male with muscle invasive bladder cancer on the right lateral wall undergoing radical nerve sparing cystoprostatectomy with extended bilateral pelvic lymph node dissection and orthotopic neobladder. The patient is continent, has good erectile function and is sexually active. Patient wishes to maintain erectile function after surgery. Results: Nerve sparing is accomplished by careful dissection of the inferior vesical pedicles. Minimal electrocautery is used near the inferior vesical pedicles. Clips are used instead of end block staplers. Nerve sparing is performed in both antegrade and retrograde fashion. After the ureters are ligated and pelvic lymphadenectomy is performed, the inferior vesicle pedicles are dissected. During initial dissection, nerve sparing is performed antegrade from the bladder towards the prostate using blunt dissection to push the nerves laterally. After the nerves are carefully dissected from the bladder, nerve sparing is performed retrograde from the prostate towards the bladder. The neurovascular bundle is bluntly dissected from the prostatic fascia in a retrograde fashion from the prostate towards the bladder. After the neurovascular bundle is spared, clips are used to obtain hemostasis. Cystoprostatectomy continues with ligation of the dorsal venous complex, ligation of the urethra and anastomosis of the neobladder to the urethra and ureters.

Conclusions: Combined use of robotic surgery and nerve sparing aims to provide improved patient outcomes via reduced complications, shorter hospitalizations, improved sexual function and urinary continence. Nerve sparing can provide oncologic efficacy while improving patient outcomes and quality of life.

V08-11 Sex-sparing vs standard Robot Assisted Radical Cystectomy with Intracorporeal Padua Ileal Neobladder in female: step-by-step surgical technique, perioperative, oncologic and functional outcomes

G Tuderti, R Mastroianni, R Flammia, S Guaglianone, F Minisola, U Anceschi, L Misuraca, A Brassetti, M Ferriero, M Gallucci, G Simone
Introduction \& Objective: Despite the widespread use of Robot assisted radical cystectomy (RARC), there is paucity of data concerning outcomes of Sex-sparing RARC with intracorporeal ileal neobladder (iN) performed in female patients. In this video we demonstrate surgical steps of standard vs sex-sparing RARC, and we compare perioperative and functional outcomes of both approaches.

Methods: Our single center IRB approved bladder cancer database was queried for Female, RARC, iN and Sex-sparing, including patients treated between January 2013 and June 2018. Key surgical steps are: isolation of the ureters, preparation of umbilical, uterine and superior vesical arteries. In Sex-sparing approach development of vesicouterine plane is performed, and uterine feeding arteries are preserved, while in standard procedure uterine and vesical pedicle are transected close to their origins from iliac internal artery. Notably, sex-sparing RARC allows to preserve utero-vaginal component of hypogastric plexus that is almost sacrificed during standard RARC. Baseline demographic, clinical, perioperative, pathologic and functional data were collected and reported. Kaplan-Meier method was performed to compare daytime continence recovery probabilities between Sex-sparing and standard RARC cohorts.

Results: Out of 47 patients included, 11 underwent sex-sparing RARC $(23.4 \%)$. The two cohorts were homogeneous for all baseline, clinical and pathologic features (all $\mathrm{p} \geq 0.14$ ), except for age, being sex-sparing patients significantly younger (47.1 vs $61.7 \mathrm{yrs}, \mathrm{p}<0.001)$. Perioperative complications and hospital stay were comparable between groups $(\mathrm{p}=0.25$ and $\mathrm{p}=0.67$, respectively). With regard to functional outcomes, no significant differences were observed for last estimated glomerular filtration rate $(p=0.64)$, neobladder stone formation rate $(p=0.93)$ and $1-$ $y r$ incidence of ureteroileal strictures $(p=0.56)$. Day-time continence recovery probability was significantly higher in Sexsparing cohort $(1-y r$ rate $90.9 \%$ vs $74 \%, \log$-rank $\mathrm{p}=0.02)$. A trend towards a significantly higher adoption of intermittent self-catheterization was observed in Sex-sparing cohort $(\mathrm{p}=0.07)$. At a median follow-up of 19.9 months, no patients of Sex-sparing cohort developed recurrences.

Conclusions: In select cohort of patients, Sex-sparing RARC-iN seems an oncologically sound procedure, associated with favorable perioperative and functional outcomes. Due to small sample size and intrinsic selection biases, oncologic and functional outcomes of sex-sparing RARC-iN should be assessed in larger cohorts, while outcomes comparison with conventional RARC-iN technique requires prospective randomized trials.

\section{V08-12 Tips For Optimizing the Outcome of Robotic Segmental Ureterectomy for Distal Ureteral Ur- othelial Carcinoma}

KE Okhawere, BD Dayal, AA Elbakry, K Meilika, A Beksac, KK Badani

Introduction \& Objective: Segmental ureterectomy is an alternative for nephroureterectomy as a management for upper tract urothelial carcinoma confined to the urethral, especially in patients with chronic kidney disease or patients with a solitary kidney. Robotic approaches are increasingly being utilized for this procedure.

Methods: In this video, we are presenting our technique of distal ureterectomy and Boari flap uretero-vesical reimplantation. Importantly, we demonstrate our surgical tips to optimize the outcome by utilizing indocyanine green (ICG) and florescence imaging for direct tumor identification, intraoperative ultrasonography and 
flexible ureteroscopy. The patient is a 70-year-old male, with stage III chronic kidney disease and was diagnosed with right ureteral urothelial carcinoma involving the distal and mid ureter.

Results: The tumor was clearly identified using florescence imaging after injection of ICG through the nephrostomy tube. Ultrasonography and flexible ureteroscopy were used in confirming the tumor location and absence of disease in the renal pelvis or calyces. Ureterectomy and boari flap were created. The patient was discharged on postoperative day 1 . The catheter and the nephrostomy tube were removed on post-operative day 6 and 10, respectively. The pathology report confirmed high grade carcinoma with negative surgical margins.

Conclusions: Injection of ICG through a preplaced nephrostomy tube can be used for intraoperative identification of ureteral urothelial carcinoma. This novel observation should be studied on a large cohort of patients to confirm our findings.

\section{ABSTRACT VIDEO SESSION 09: SINGLE PORT ROBOTIC SURGERY \& UPPER TRACT ROBOTIC SURGERY}

\begin{abstract}
V09-01 Robotic assisted, single-docking nephroureterctomy and bladder cuff excision with regional lymph node dissection
\end{abstract}

W Li, L Tsai, G Chen, C Huang, C Chang, C Yang, H Wu

Introduction \& Objective: The prevalence of upper urinary tract urothelial carcinoma (UTUC) was increasing and Taiwan has one of the world's highest incidence rates. The gold-standard management of UTUC was radical nephroureterctomy with bladder cuff excision (BCE). Lymph node dissection (LND) in conjunction with RNU was not routinely performed due to controversial therapeutic benefits. However, LND during RNU allows more accurate staging and prediction of survival. In this demonstration, we introduced robotic-assisted, single-docking radical nephroureterctomy(RANU) with BCE and LND.

Methods: We performed the surgery with the da Vinci Si surgical system. The patient was placed in a modified decubitus position with the ipsilateral arm slightly extended. During the operation, the instruments were switched once to manage distal ureter and bladder cuff without patient repositioning or robot redocking. The template of LND was individualized.

Results: Twenty-one patients had undergone RANU with BCE and LND using the modified method. Mean operative time was 210 minutes and mean blood loss was $75 \mathrm{~mL}$. The mean examined number of dissected lymph nodes was 13 (range, 1-32 nodes), and positive lymph nodes were reported in two cases (one and two nodes separately). The average follow-up time was 11.8 months (range, 2-23 months). During the follow-up periods, one case encountered tumor recurrence in bladder and another case developed distal metastasis disease.

Conclusions: The procedure of robotic-assisted, single-docking RANU with BCE and LND provided shorter operative time and avoided patient repositioning or robot re-docking. Moreover, concurrent LND should be performed in selected cases not only for better tumor staging but also for prognosis improvement.

V09-02 Single stage transperitoneal robotic nephroureterectomy in patient with ventriculoperitoneal shunt

A Veccia, A Tracey, R Autorino

Introduction \& Objective: Robotic nephroureterectomy is being adopted for the treatment of upper-tract urothelial cell carcinoma. Ventriculoperitoneal shunt (VPS) is a device placed to reduce intraventricular pressure for a variety of indications. $\mathrm{Pa}-$ tients with VPS may be candidates for minimally invasive (laparoscopic or robotic) transperitoneal surgery. However, there might be concerns related to presence of the VPS in the abdomen. Herein we describe a case of single stage robot-assisted nephroureterectomy in a patient with VPS and to highlight technical nuances of the procedure.

Methods: A 74 years old woman presented with history of gross hematuria. During her workup, a CT scan revealed an atrophic left kidney and a $5 \mathrm{~cm}$ mass of the left collecting system. An additional $5 \mathrm{~cm}$ length enhancing mass was detected within the middle tract of the left ureter. The patient was candidate to single stage left robotic nephroureterectomy. The procedure was performed with the robotic device DaVinci ${ }^{\circledR} \mathrm{Xi}$ (Intuitive Surgical ${ }^{\circledR}$, Sunnyvale, CA, USA). The patient was placed in $60^{\circ}$ modified flank position with a $15^{\circ}$ Trendelenburg. The camera and robotic ports were placed all on the same oblique straight line along the lateral rectus abdominis. The AirSeal $^{\mathrm{TM}}$ (Conmed, Utica, NY, USA) device was used and pneumoperitoneum pressure was constantly kept at $10 \mathrm{mmHg}$. The VPS was immediately identified and kept open, but it was retracted laterally by passing two sutures through the anterior abdominal wall. Once the colon was medialized, the psoas muscle and left ureter were identified, and this was traced distally, clipped with a Hem-oLok, but not transected. Attention was then given to the renal hilum, which was dissected to identify renal artery and vein. A $45 \mathrm{~mm}$ laparoscopic stapler was used for the resection of the vessels. Then, the left ureter and its intramural tract were dissected. The ureter and bladder cuff were excised and a single layer cystorraphy was performed by using a V-lok barbed suture. Hydraulic test was negative, and a Foley catheter was left in place. Careful hemostasis was performed with the use of hemostatics agents. No drain was placed. Results: Estimated blood loss was $75 \mathrm{~mL}$. Console time 210 minutes. No intraoperative or post-operative complications or neurological sequelae occurred, and patient was discharged on postoperative day 2. Surgical pathology report described a high grade papillary invasive urothelial carcinoma (pT3N0). 6-month follow-up is negative for recurrence.

Conclusions: A single stage $\mathrm{Xi}$ transperitoneal robotic nephroureterectomy can be safely performed in patients with VPS by adopting few precautions during the procedure.

\section{V09-03 Robotic Uretero-Vesical Reimplantation For Ureteral Stricture After Kidney Transplant}

A Beksac, AA Elbakry, KE Okhawere, BD Dayal, K Meilika, KK Badani

Introduction \& Objective: Robotic approach is gaining more popularity in performing abdominal urological reconstructive 
cases. We are presenting a complex case of Robotic ureterovesical reimplantation for a case of ureteral stricture at the site of uretero-vesical anastomosis after kidney transplant with an indwelling nephrostomy tube.

Methods: A 61-year-old male patient with history of hydronephrosis of transplanted kidney that was managed with nephrostomy tube placement. Nephrostogram showed stricture at the site of ureterovesical anastomosis. Procedure was started by identifying and dissecting the transplanted ureter at the plane between the transplanted kidney and the bladder. Indocyanine green was injected through the nephrostomy tube and immunofluorescence imaging was employed to guide the dissection. After the ureter was identified and completely freed from the surrounding, the bladder was dissected to decrease the tension on the new anastomosis. The stricture point was identified, and the ureter was resected and spatulated and the cystostomy was done. The new anastomosis was carried out and the bladder was hitched to the pelvic sidewall and the perinephric fat. A double-J stent was fixed.

Results: The procedure was successfully done robotically. Operative time was 155 minutes and estimated blood loss was minimal. The patient was discharged the next morning. Follow-up serum creatinine showed improvement to the normal level.

Conclusions: Robotic approach for complex urological reconstructive procedures is feasible in the hand of an experienced robotic urologist. The role of robotic surgery in such complex cases should be considered when appropriate to provide the patients with the advantages of minimally invasive approach.

\section{V09-04 Robot assisted supine extra peritoneal (RASE) approach for RPLND: Our experience}

\section{GK Pooleri, A LAddha}

Introduction \& Objective: Robot-assisted lymph node dissection (RA- RPLND) for post-chemotherapy residual mass is a challenging surgery and is usually done through transperitoneal route in deep Trendelenburg position. In this video, we demonstrate robot-assisted supine extraperitoneal (RASE) RPLND where the patient was positioned in flat supine position and approach is extraperitoneal.

Methods: From Jun 2017 to May 2019, five cases of robot-assisted retroperitoneal lymph node dissection were attempted through extraperitoneal route out of which four cases were completed successfully. Two cases were converted (One to transperitoneal approach due to loss of space and one to open surgery due to tumor adhesion. Here we are presenting a video of RASE RPLND done for a 31-year-old gentleman with post-chemotherapy residual mass who had an IVC thrombus preoperatively. Because of the IVC thrombus and severe bleomycin toxicity, the anesthetist was not comfortable for a steep Trendelenburg position. The procedure was done using the da Vinci Xi system.

Results: We could perform RASE RPLND successfully in three patients out of 5 patients The operation duration of 250 minutes (220-270 min) and blood loss was $150 \mathrm{ml}$. (100-250 cc).

Conclusions: RASE RPLND minimally invasive technique and is a novel approach for retroperitoneal lymph node dissection. RASE RPLND has an advantage that bowel handling can be avoided and that the patients can be positioned flat which is helpful in case a conversion / resuscitation is required as an emergency. Anesthesia risk may be reduced in post-chemotherapy settings as ventilation is safer in the flat supine position.
V09-05 Retroperitoneal Approach Single-Site RobotAssisted Partial Nephrectomy in High Complexity Renal Mass: Step-by-step technique using da-Vinci Xi system

Y Yoon, J Na, D Kim, K Rha, W Han, H Lee

Introduction \& Objective: To demonstrate a step-by-step technique of retroperitoneal single site robot-assisted partial nephrectomy using the da Vinci Xi surgical system.

Methods: A 66 years old woman had diagnosed with $4.5 \mathrm{~cm}$ lateral renal mass located on the mid pole kidney. The da-Vinci Xi surgical system (Intuitive Surgical, Sunnyvale, CA, USA) was used to perform retroperitoneal single site robot-assisted partial nephrectomy. The patient was placed in the full flank position and the surgical table was fully flexed to increase the space between the 12 th rib and iliac crest. The port access was on $3 \mathrm{~cm}$ above the iliac crest just lateral to the triangle of Petit with $3 \mathrm{~cm}$ incision. A PDB balloon dilator (Tyco, Princeton, NJ, USA) was placed into the retroperitoneal space to get enough space for surgery. Lapsingle Vision (Sejong med, Paju, Korea) advanced access platform was used as single site assess port. 8.5-mm cannula was connected to the robotic camera system, and the two 5-mm curved cannulas were inserted. The flexible robotic instruments were inserted Fig 1.
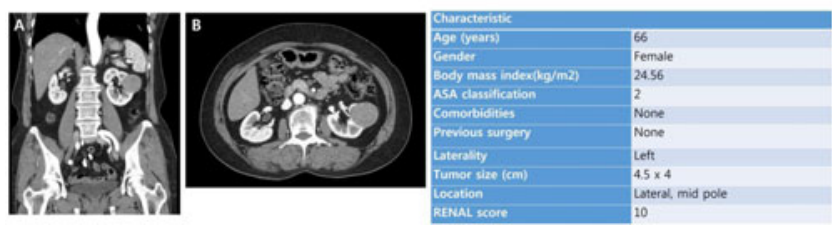

Fig 2.

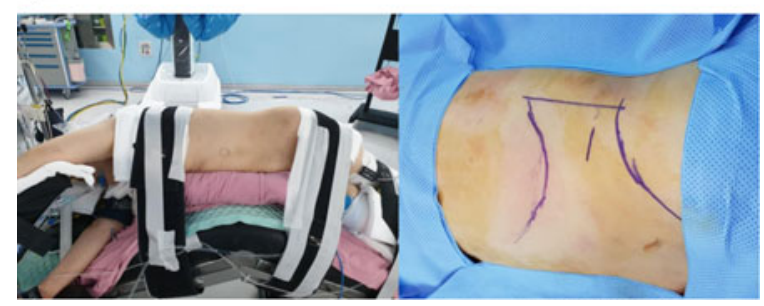

Fig 3.

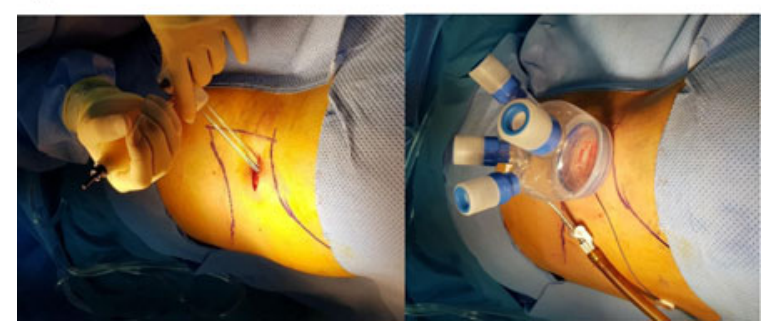

Fig 4.

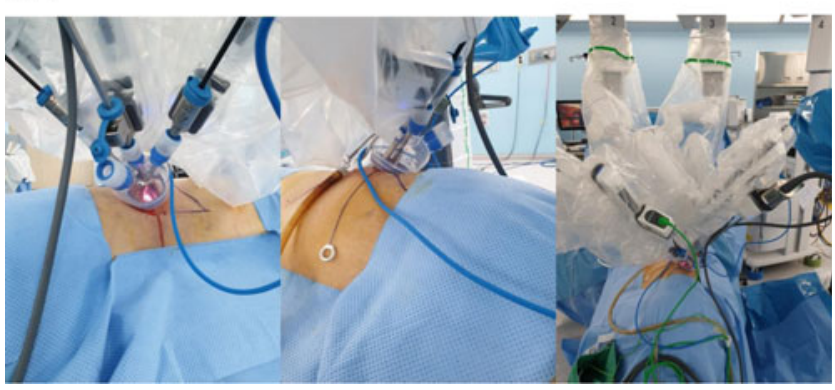


Fig 5 .

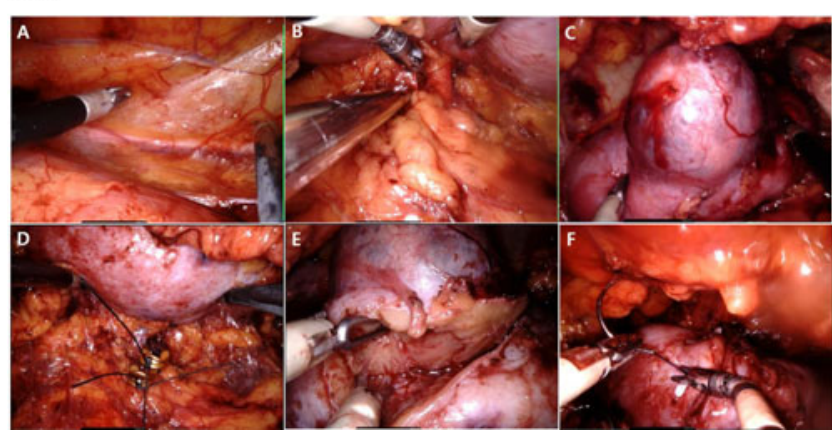

Fig 6.

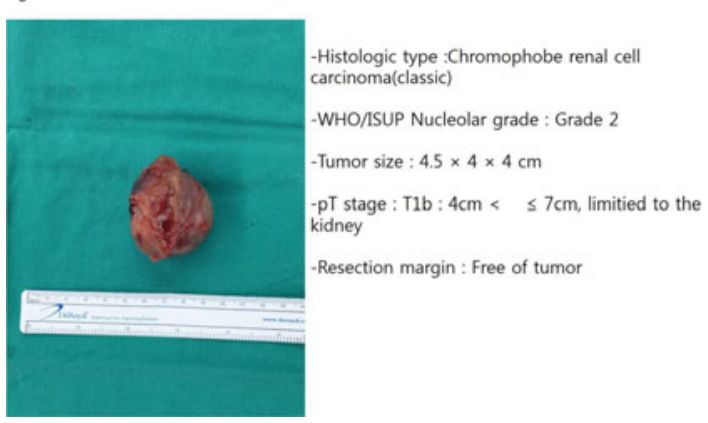

Fig 7.

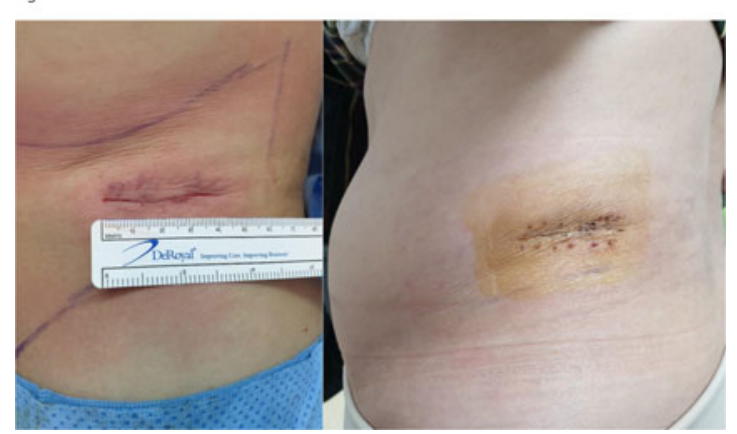

through the curved cannulas. A suction device could be inserted through Lap-single Vision accessory port if needed.

Results: The surgery was successfully completed without additional port. The operation time was 100 minutes (docking time was 9 minutes), and warm ischemia time was 23 minutes. Estimated blood loss was $30 \mathrm{~mL}$. The pathology results revealed chromophobe renal cell carcinoma with negative surgical margin.

Conclusions: Retroperitoneal single site robot-assisted partial nephrectomy is feasible treatment modality for treatment of posterior or lateral renal mass including high complexity mass. Further cases are needed to confirm the feasibility and safety of this technique.

V09-06 Embolizing left renal artery before Robotic assisted radical left nephrectomy and renal vein thrombectomy to treat left renal cancer with renal vein thrombosis

\section{J Wu, M Chen, L Zhang}

Introduction \& Objective: We present a new technique to treat left renal cell carcinoma with renal vein thrombosis.

Methods: From October 2018,10 patients suffered from left renal cell carcinoma with renal vein thrombosis were operated in our department. The characteristics of the patients were reviewed, and the surgical technique was discussed. When the radical nephrectomy was scheduled the patient received left renal artery angiogram and the left renal artery was embolized, then the patient was sent for robotic assisted surgery.

Results: All the 10 patients received radical nephrectomy and left renal vein thrombectomy with this technique. No major complication was encountered, and the patients were discharged on postoperative day 5-7 days. Pathological diagnosis showed negative surgical margin. All the patients were alive on the last follow up. Conclusions: Embolizing left renal artery before Robotic assisted radical left nephrectomy and renal vein thrombectomy is safe and feasible to treat left renal cancer with renal vein thrombosis.

\section{V09-07 Single-site Robotic-Assisted Pyeloplasty Using the Da Vinci SP Robotic System}

RL Steinberg, A Garbens, BA Johnson, JA Cadeddu

Introduction \& Objective: Robotic assisted pyeloplasty has been adopted as the standard of care in reconstruction for ureteropelvic junction obstruction in adults. Single site robotic pyeloplasty has been reported using the da Vinci Si and Xi systems but requires novel, curved instruments and is performed in a cross-arm fashion. This leads to increased difficulty and has led to poor adoption of the technique. The recent release of the daVinci SP robotic system (Intuitive, Sunnyvale, CA, USA) now provides a purpose built single-site surgical robot to the surgical market. We aim to evaluate the safety and efficacy of single site robotic assisted pyeloplasty using the Da Vinci SP robotic platform.

Methods: Six patients underwent a single-site robotic pyeloplasty using the da Vinci SP platform at a single institution from November 2018 to May 2019. After placement into the modified flank position, a peri-umbilical incision was made and the abdomen entered using the Hasson technique. A GelPoint (Applied Medical, Rancho Santa Margarita, CA, USA) was placed through which the robotic cannula was placed, along with $2,8 \mathrm{~mm}$ Gel Point ports. A ROSI suction device (Vascular Technology, Nashua, NH, USA) was placed through one port and the other used to pass sutures. At the completion of the procedure a drain was placed through a separate stab incision. Demographics, pre-operative disease characteristics, intraoperative, and outcomes data were all collected. Descriptive statistics were generated.

Results: All cases were successfully completed without conversion to a standard robotic system, placement of addition assistant ports or use of an assistant suction device. No intraoperative or immediate post-operative complications were noted. Table 1 demonstrates the pre- and post-operative characteristics of this cohort. Mean operative time was $155 \pm 9$ minutes and mean blood loss was $17 \pm 5 \mathrm{~mL}$. All patients were discharged to home on post-operative day 2. All patients had relief of their pre-operative pain after stent removal.

Conclusions: Single-site robotic pyeloplasty using the daVinci SP robot is safe and effective. Use of the ROSI irrigation system obviates the need for assistant suction and allows the surgeon to be completely autonomous except for suture passage.

V09-08 Perineal Single-Port Approach for Robotic Radical Prostatectomy Using The New SP ${ }^{\circledR}$ da Vinci Surgical System: An option for selected patients

GV Sawczyn, RJ Valero Carrion, J GaristoRisco, RJ Yau, J Kaouk 
Introduction and Objective: to describe and present our technique of Perineal single-port robotic radical prostatectomy using the new $\mathrm{Sp}^{\circledR}$ da Vinci surgical system (Intuitive Surgical, Sunnyvale, CA).

Methods: From October 2018 to May 2019 a total of fifteen patients underwent to perineal single pot robotic radical prostatectomy using this technology. The patient is in the lithotomy position with stirrups, a $3 \mathrm{~cm}$ omega shape incision is made using ischial tuberosities as an anatomical landmark, dissection of the perirectal space is done carefully, after that, a GelPOINT Mini advanced access platform (Applied Medical, Rancho Santa Margarita, CA), is inserted and fixed to the skin with interrupted sutures. A $25 \mathrm{~mm}$ multichannel single-port robot cannula and a 12$\mathrm{mm}$ accessory laparoscopic port are placed through the GelSeal cap and after plugged to the Alexis ${ }^{\circledR}$ wound retractor, followed by the docking of the da Vinci $\mathrm{SP}^{\circledR}$ robot. Pneumoperineum is achieved, the prostate is accessed and the procedure is done following these steps: creation of perirectal space, identification of the prostate, dissection of the neurovascular bundles, dissection and division of the urethra, dissection of the prostatic apex, dissection of the bladder neck, identification of vas deferens and seminal vesicles, opening bladder neck, lymph node dissection, urethrovesical anastomosis.

Results: Mean age and BMI were 61.5 years old and $30.5 \mathrm{~kg} / \mathrm{m}^{2}$ respectively. Mean operative total time was 266.7 minutes, mean estimated blood loss was $168.3 \mathrm{ml}$. One intraoperative complication was recorded -a ureteral injury that was repaired intraoperatively with anastomosis and double $\mathrm{J}$ stent. No drain, conversion or transfusion was needed. Mean length of stay was 32.5 hours. Median days with a catheter was 11 days. Positive margins were present in $33 \%$ of patients and three patient presented anastomotic leak — all managed with extended foley period, and one with acute kidney injury managed with fluids.

Conclusions: Perineal robotic radical prostatectomy using this technology is an option regarding minimally invasive techniques for treating prostate cancer in a subpopulation of patients multiples abdominal surgeries, ileostomies, J pouch, etc. Our initial experience revealed that this procedure is feasible and safe, and an adaptation process and learning curve is involved regarding the approach and identification of anatomical landmarks.

\section{V09-09 Bladder Cuff Excision: An Integral Compo- nent of Radical Nephroureterectomy}

\section{R Pathak, A Hemal}

Introduction \& Objective: The gold standard for high-risk upper tract urothelial carcinoma is Radical Nephroureterectomy with Bladder Cuff Excision (RNUBCE). The primary objective was to showcase various techniques of bladder cuff excision and report our perioperative and long-term oncologic outcomes (overall survival, cancer-specific survival, and recurrence-free survival) for patients undergoing robot-assisted RNUBCE.

Methods: 90 patients underwent robot-assisted RNUBCE from 2006 to 2017. The video presented demonstrates different techniques in performing cuff excision. We evaluated overall survival (OS), cancer-specific survival, local recurrence- (LR), distant recurrence- (DR), and bladder recurrence-free survival (BRFS) were calculated using Kaplan-Meier curves with estimated survival rates at 12, 36, and 60 months. Follow-up consisted of cystoscopy and abdominopelvic and chest imaging at regular intervals.

Results: A total of 90 patients with a mean age of 70.9 and BMI 27.4 underwent robot-assisted RNUBCE. Bladder cuff was excised utilizing monopolar scissors in a majority of cases $(>90 \%)$, with ultrasonic device and vessel sealer used sparingly. Average operative time was 215.3 minutes with a mean estimated blood loss of 139.8 . $36.7 \%$ of patients were diagnosed with pT3 or greater disease with a $\mathrm{pN}+$ rate of $16.6 \%$. Median follow-up was 31.5 months with a range of 6 to 114.2 months. 5-year overall and cancer-specific survival rate was 41.5 and $57.9 \%$, respectively. Conclusions: RNUBCE with robot-assistance achieves all components of UTUC surgery safely, effectively, efficiently and ergonomically in a minimally-invasive fashion. In this video, we showcase various techniques of bladder cuff excision.

\section{V09-10 Single-port Robotic Partial Nephrectomy Using The New SP ${ }^{\circledR}$ da Vinci Surgical System}

RJ Valero, GV Sawczyn, J GaristoRisco, RJ Yau, J Kaouk

Introduction \& Objective: to describe and present our technique of single-port partial nephrectomy using the new $\mathrm{Sp}^{\circledR}$ da Vinci surgical system (Intuitive Surgical, Sunnyvale, CA).

Methods: Three consecutive patients underwent to robotic partial nephrectomy between October and December 2018. Using a transperitoneal approach, patient is lateral supine position with a 45-degree angle, a $3 \mathrm{~cm}$ supra-umbilical incision is made two finger breath above the umbilicus, a GelPOINT standard access platform (Applied Medical, Rancho Santa Margarita, CA), is inserted directly into the incision. A $25 \mathrm{~mm}$ multichannel singleport robot cannula and an additional $12 \mathrm{~mm}$ laparoscopic port are placed through the GelSeal cap and after plugged to the Alexis ${ }^{\circledR}$ wound retractor, followed by the docking of the da Vinci SP ${ }^{\circledR}$ robot. Pneumoperitoneum is achieved and the procedure is performed replicating the technique previously describe for the multiarm robotic platform.

Results: Total operative time range from 150 to $210 \mathrm{~min}$ with a mean of 186.6 \pm 32.1 minutes, mean estimated blood loss was $183.3 \pm 104 \mathrm{ml}$. No intraoperative complications were reported, and no surgeries were converted to open approach. There was no blood transfusion during or following the procedures.

Conclusions: Single-port robotic partial nephrectomy using this technology is an option regarding minimally invasive techniques for treating small renal tumors. Our initial experience revealed that this procedure is feasible and safe. Further studies must be done to validate the results and establish the indications for the use of the technique.

\section{V09-11 Robotic Single Port Partial Nephrectomy: First Experience Using a Magnetic Retraction Device.}

\section{J Fulla, E Kaplan-Marans, M Palese}

Introduction \& Objective: Efforts to limit the footprint of laparoscopic and robotic surgery have focused on reducing the size and number of ports. Recently, the Food and Drug Administration approved both the da Vinci SP and the Levita ${ }^{\mathrm{TM}}$ Magnetic Surgical System. Our objective is to demonstrate the use, safety and feasibility of the da Vinci SP single port robotic system and the Levita ${ }^{\mathrm{TM}}$ Magnetic Surgical System.

Methods: We present a 71-year-old female with a $1.8 \mathrm{~cm}$ left renal mass found incidentally noted on MRI for evaluation of liver cysts. The single port system requires a $2.5-\mathrm{cm}$ skin incision which was made paramedian to the umbilicus. An additional $12 \mathrm{~mm}$ assistant port was placed parallel to the single port cannula. The Levita ${ }^{\text {TM }}$ Magnetic Surgical System includes a deployable, one-time use grasper and a reusable external magnet. A bedside assistant manipulated the deployable grasper by moving the extracorporeal magnet. 
Results: Operative time was 95 minutes with an ischemia time of 11 minutes. Estimated blood loss was $150 \mathrm{~mL}$. The patient required minimal pain medications and was discharged on postoperative day 2 . There were no intraoperative or perioperative complications. The magnetic system was especially useful for the renal hilar and tumor dissection.

Conclusions: This is the first case of a Single-Port da Vinci SP robotic system using the Levita ${ }^{\mathrm{TM}}$ Magnetic System. The use of the magnetic-assisted retractor is safe and feasible. Advantages may include use of fewer ports, improved cosmesis, and lower costs.

V09-12 Bilateral Pyeloplasty performed with the da Vinci Single Port Robotic System: A new approach to a classic case.

M Zhang, NH Patel, G Lovallo, M Ahmed

Introduction \& Objective: The introduction of the da Vinci Single Port Robotic System (SP) represents the next generation of robotic technology. With changes in technologies, new ap- proaches classic surgeries are made possible. In this case, we demonstrate the ease and feasibility of performing bilateral retroperitoneal surgery through a small midline incision.

Methods: In this video, we demonstrate our set up and technique in performing a bilateral Anderson-Hynes pyeloplasty using the SP in a young adult with bilateral UPJ obstructions. The technique is demonstrated by a high-volume robotic surgeon, including 40+ SP cases to date.

Results: Our patient tolerated the procedure well. Her postoperative course was uncomplicated. Her JP was removed, and patient was discharged on post-operative day one. Ureteral stents were removed after four weeks with resolution of pain and hydronephrosis. Operative time was 118 minutes with minimal EBL.

Conclusions: We show the ease, feasibility and excellent cosmetic outcome in performing bilateral retroperitoneal surgery using the da Vinci Single Port Robotic System. Although we show the use of an assistant port in this video, our more recent cases are true single port cases by switching to the remotely operated suction irrigation system (ROSI). As our experience and case volume increases, we will be able to better show cosmetic and clinical outcomes.

\section{ABSTRACT VIDEO SESSION 10: LAPAROSCOPY: UPPER TRACT}

\section{V10-01 3D Retroperitoneoscopic nephrectomy. A pa- tient friendly procedure.}

A Soomro, M Azad, S Saulat, S Qadri, A Ayub, M Ejaz

Introduction \& Objective: To evaluate safety and efficacy of 3D retroperitoneoscopic nephrectomy in patients with non functioning kidneys.

Methods: Clinical data of 77 patients who underwent retroperitoneoscopic nephrectomy was evaluated. All cases were diagnosed as non functioning kidneys by ultrasound and mercapto acetyl tri glycine (MAG-3) scans or di-mercapto succinic acid (DMSA) scans. All patient underwent 3D retroperitoneoscopic nephrectomy using one camera port and 2 working ports. Demographic data (gender, age, laterality), perioperative indices including operative time, blood transfusion. Complication like wound infection, fever and injuries to bowel and other organs were recorded. Numerical demographic and perioperative data were analyzed by using Student's $t$-test. Categorical data were compared using Pearson's Chi-square test.

Results: Mean age of patients was 35.326 (min 3 years \& max 75years) with 46 male and 27 female patients. Right renal units were $35(45.50 \%)$ and left were $42(54.50 \%)$. Mean operative time 130.84 mins \pm 25.4 mins. Only $4(5.2 \%)$ patient required conversion to open surgery. $5(6.5 \%)$ patients required blood transfusion. Complication like wound infection 01 (1.3\%), fever 01 (1.3\%) were recorded. Pleural, bowel and other organs were safe in all cases. Conclusions: 3D retroperitoneoscopic nephrectomy is safe and effective. Because of the retroperitoneal approach, the anatomy is familiar to urologist, access to renal hilum and its vascular control is easy and peritoneal organs are safe from in iatrogenic injury. Due to three-dimensional optics tissue identification and depth perception improves remarkably and hence it reduces rate of complication.

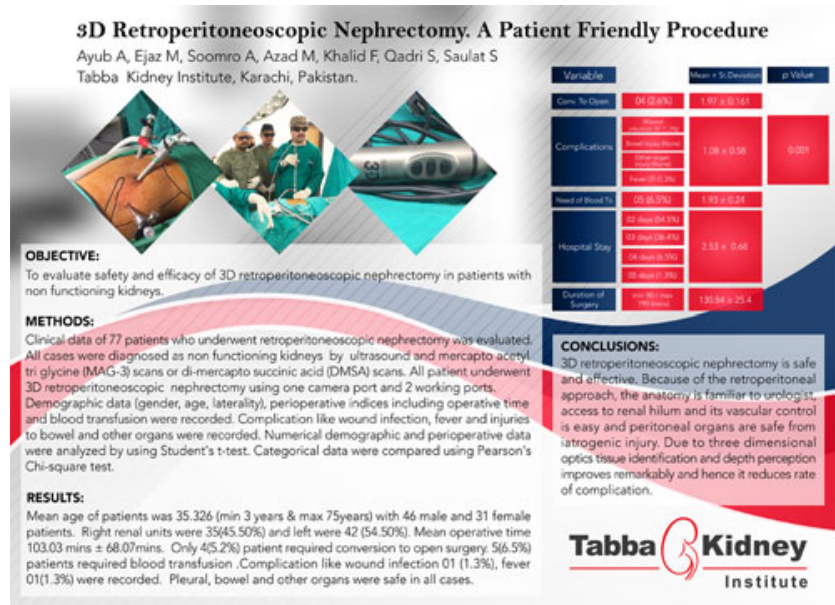

V10-02 Retroperitoneoscopic Partial Nephrectomy The technique step by step

C Wülfing, D Marghawal, O Andura

Introduction \& Objective: International guidelines recommend nephron sparing surgery over radical nephrectomy whenever technically feasible. The retroperitoneoscopic approach in minimally-invasive partial nephrectomy is the preferred standard at our institution. In this video we present the standardized technique after more than 800 procedures.

Methods: In this video we present the key steps of retroperitoneoscopic partial nephrectomy in a step-by-step manner to facilitate this minimally-invasive approach for standard partial nephrectomy. Several scenes from different cases with various 
tumor sites and the use of different suturing techniques are shown.

Results: In more than 800 cases, our technique has evolved as a standard procedure and allows to quickly and safely accomplish a high trifecta rate.

Conclusions: The retroperitoneoscopic approach is an excellent variant in minimally-invasive partial nephrectomy and allows excellent results.

\section{V10-03 “Unlock" accidents of polymeric Hem-O-Lok clips during laparoscopic kidney surgeries}

T Abe, A Sazawa, D Iwami, T Osawa, R Matsumoto, H Kikuchi, S Murai, N Shinohara

Introduction \& Objective: Polymeric Hem-O-Lok clips are widely utilized for renal vascular control during laparoscopic procedures. Although the lock-engagement feature offers security, no surgical device is free from accidents or failures. We have encountered several "unlock" accidents of Hem-O-Lok clips during laparoscopic kidney surgeries. In the current movie, we show our experiences and discuss preventive measures in order to avoid those accidents.

Methods: We presented three video-clips showing "unlock" accidents of Hem-O-Lok clips during laparoscopic kidney surgeries.

Results: Clip 1: A case of left radical nephroureterectomy with a retro-peritoneal approach. One Hem-O-Lok clip unlocked without any significant manipulation during control of the left renal artery, which fortunately did not result in severe bleeding. The scrub nurse did not know how to apply a clip into its applicator, and its incorrect application to the applicator squashed the tip of the clip, which caused this incident. Clip 2: A case of right radical nephrectomy with a trans-peritoneal approach. Two Hem-O-Lok clips unexpectedly unlocked without any significant manipulation during control of the right renal artery, which fortunately did not result in bleeding. The manufacturer's investigation revealed a defect of the applicator, which caused half-lock of the clip. Clip 3: A case of right radical nephrectomy with a retroperitoneal approach. One Hem-O-Lok clip unexpectedly unlocked without any significant manipulation during dividing the right renal artery, which fortunately did not result in bleeding. A video review did not reveal the precise reason for this incident, although the surgeon conducted the surgery with staff who were unfamiliar with laparoscopic surgery at another hospital.

Conclusions: In our experience, a scrub nurse's insufficient knowledge, device malfunction, and an uncoordinated surgical team were the underlying causes of "unlock" accidents involving polymeric Hem-O-Lok clips during laparoscopic kidney surgeries. We believe that sharing the backgrounds among surgical team members is the first step to prevent this type of incident.

\section{V10-04 Laparoscopic resection of retrohepatic su- prarenal mass}

\section{Tan, A Sim Soon Phang}

Introduction $\boldsymbol{\&}$ Objective: In the era of minimally-invasive techniques, laparoscopic resection of suprarenal masses such as adrenal and adrenal masses has been a topic for debate. Current consensus has agreed that minimally-invasive methods are the treatment of choice for benign lesions with a diameter of less than $6 \mathrm{~cm}$. However, retrohepatic tumors are known to be challenging to resect because of their proximity or adhesions to the infrahepatic portion of the inferior vena cava, therefore open surgery with extensive mobilization of the liver might be done instead. We will be demonstrating our method of laparoscopic resection of a retrohepatic suprarenal mass.

Methods: We present a case of laparoscopic resection of suprarenal mass measuring $5.6 \times 3.1 \times 3.9 \mathrm{~cm}$ in the right suprarenal space, it is retrohepatic and abutting the inferior vena cava. This method involves the use of stay sutures to assist in mobilization and providing tension for dissection.

Results: Total operative time was 160 minutes and the patient's intra-operative blood pressure was stable The patient recovered well after surgery and was discharged on post-operative day 3 and histology of the suprarenal mass was a cellular schwannoma. Conclusions: Laparoscopic resection is a feasible method for resection of retrohepatic suprarenal masses. The method described allows limited mobilization of the liver and allows patient to benefit from advantages of minimally invasive surgery such as early mobilization and shorter hospitalization.

\section{V10-05 Robotized Laparoscopy In Stone Disease}

\section{R Raveendran, K Ramaswami, P Bhirud, H Pothiyedath}

Introduction \& Objective: Ever since its inception, stone surgery has historically been pivotal in the specialty of Urology. Large upper tract stones can be tackled through either of open, percutaneous or laparoscopic approaches. Here we are presenting the usefulness of Robotized laparoscopy in the treatment of large stone burden cases like Staghorn calculi and large ureteric calculi.

Methods: Data was collected in a retrospective manner between May 2017 and April 2019. All patients admitted for laparoscopic management of stone disease were included in the study. The robotic arms used consisted of dissecting scissors and needle holder. Basic demographical data, intra and post-operative parameters were recorded.

Results: Out of 121 robotized laparoscopy procedures performed, there were 16 Ureterolithotomies, 10 Pyelothotomies and 3 Anatrophic Nephrolithotomies. The operating time and blood loss, hospital stay was similar to standard laparoscopic procedures but with more comfort for the surgeon of our own series. No major complications or conversion to open surgery were observed in this series. Robotized laparoscopy systems are hand held systems which are very close to full-fledged robotic console systems in terms of functionality at a fraction of the cost of latter. The learning curve is simple and helps the surgeon in difficult dissection and reconstruction scenarios.

Conclusions: Robotized laparoscopy is a safe and reproducible adjunct to conventional laparoscopy in the treatment of high stone burden.

\section{V10-06 Laparoscopic Ureterolithotomy for large distal ureteric stone}

JM López, R Martos, A Sierra, M d́anna, P Luque, A Alcaraz

Introduction \& Objective: Technology has allowed increasingly less invasive surgeries to be developed. Currently we have endourological and percutaneous techniques with minimal approaches for the treatment of renal and ureteral stones. In selected cases, European guidelines consider ureterolithotomy in large volume lithiasis as the most effective surgery. The laparoscopic approach provides less morbidity to the technique, considering the choice of abodaje in these cases. 
Methods: We present the case of a 76-year-old male patient, with a history of liver cirrhosis due to HCV, diagnosed of two ureteral stones due to right back pain. The CT reports a $35 \mathrm{~mm}$ asymptomatic left distal stone and a small right distal ureteral lithiasis. Renal scintigraphy reported that the left kidney contributed $30 \%$ of the total function. After the placement of a left nephrostomy catheter and right ureteroscopy, it was decided to perform laparoscopic ureterolithotomy as the most effective option due to the stone burden. Four 5-mm trocars and one 12$\mathrm{mm}$ optical trocar were used. Surgery started by making a window in the peritoneum and identifying the left distal ureter. After localization of the stone, a longitudinal ureterolithotomy of about $3 \mathrm{~cm}$ is performed to extract the complete stone. Subsequently, double $\mathbf{J}$ catheter placement is performed endoscopically. The surgery was carried out without incident in 2 hours. The patient was discharged on the third postoperative day, and the ureteral catheter removed at 3 weeks. Postoperative CT showed residual ureterohydronephrosis with a non-obstructive renographic curve. Results: The surgery was carried out without incident in 2 hours. The patient was discharged on the third postoperative day, and the ureteral catheter removed at 3 weeks. Stone composition was: calcio oxalate monohydrate $55 \%$, uric acid $35 \%$, protein $10 \%$. Postoperative CT showed residual ureterohydronephrosis with a non-obstructive renographic curve.

Conclusions: 1) According with European guidelines, laparoscopic approach for ureteral stones is indicated when ESWL or endoscopic treatment are unlikely to be successful. 2) Laparoscopic ureterolithotomy is a safe treatment for large ureteral stones, achieving stone-free status in a single treatment.

\section{V10-07 Laparoscopic Management of Vascular In- juries}

\section{Vaddi, S PM, R Paidakula, P Gopi, M B, S Ganesan}

Introduction \& Objective: Iatrogenic major vascular injury occurs in $0.11-2 \%$ of laparoscopic surgeries. Major vascular injuries may require conversion to open laparotomy, which can be avoided by insertion of extra ports and meticulous repair. We present four cases of major vascular injuries during laparoscopic surgeries and their management.

Methods: Case 1: A 40-year female underwent left nephrectomy for nonfunctioning kidney. During dissection of hilum, left renal artery got injured partially and blood spilled on to the camera. Immediately with the left-hand instrument renal artery was compressed, camera cleaned, additional ports were placed and the artery was clipped with hem-o-lok clips just below the lefthand instrument. Renal vein was also clipped and procedure was completed. Case2: A 25-year male posted for left pyeloplasty. Pelvis was incised anteriorly and while incising the posterior lip with a hook cautery, there was a small perforation on the left renal vein. It was controlled with the left-hand instrument and a rescue stitch with 5-0 prolene was taken over the rent. Later bleeding got controlled and pyeloplasty completed. Case3: A 25year male presented with complaints of lower abdominal pain with fever and chills for 2 weeks. History of left pyeloplasty in childhood. On evaluation, he was found to have a nonfunctioning left pelvic ectopic kidney and normally functioning and positioned right kidney. After evaluation he was taken up for lap left nephrectomy. After standard port placements, grossly enlarged left kidney noted in the pelvis with dense adhesions to the pelvic side wall. During the dissection external iliac vein was lacerated, immediately pneumoperitoneum was raised to $20 \mathrm{~mm} \mathrm{hg}$ and the lacerated part identified and grasped. Then with the 5-0 prolene rescue stich venous repair done. Due to dense adhesions, subcapsular nephrectomy was done. Case 4: A 30- year female posted for left ureteric reimplantation for lower ureteric stricture. During mobilization of the distal ureter there was bleeding from the pelvis. With the help of suction apparatus. the site of bleeding was identified to be an internal iliac vein tributary. It was clipped with a metal clip and procedure was completed.

Results: All these patients had uneventful post-operative period. All of them are on follow up with no complaints.

Conclusions: Major vascular injuries can be managed laparoscopically in experienced hands and smart quick-witted action. One must be vigilant and be prepared for the emergent tackling of iatrogenic injuries.

\section{V10-08 Ureteral Obstruction Due To Crossing Vessel At The Level Of The Pelvis}

HV Patel, BJ Lichtbroun, SE Elsamra

Rutgers Robert Wood Johnson Medical School

Introduction \& Objective: Ureteral obstruction has many potential etiologies, largely categorized into either intrinsic or extrinsic causes. Intrinsic causes arise from within the ureter as is seen in the case of calculi or intrinsic ureteral fibrosis. Extrinsic causes are caused by a force external to the ureter that presses on the ureter to cause an obstruction as seen in the case of retroperitoneal fibrosis or malignant ureteral obstruction. Ureteropelvic junction obstruction (UPJO) is a well-studied cause of ureteral obstruction, which is thought to be caused by both an extrinsic and/or intrinsic component. One common cause of UPJO is a crossing vessel at the level of the lower pole that mechanically compresses the ureter. While crossing vessels leading to obstruction are relatively common at the level of the ureteropelvic junction (UPJ), it is a rare phenomenon at other locations, notably at the level of the pelvis. Herein, we describe the case of a 59-yearold male with a history of nephrolithiasis who was found to have severe unilateral hydroureteronephrosis. Intraoperatively, he was found to have an aberrant crossing vessel at the level of the pelvis, mechanically compressing the ureter.

Methods: A literature review was performed to put this phenomenon in context of other vascular anomalies leading to ureteral obstruction outside of the UPJ that have been reported in the literature. Results: Ovarian vein and testicular vein syndrome are wellstudied causes of obstructive uropathy discussed in the literature. Other congenital causes are transcaval and retrocaval ureter. Aneurysmal has also been discussed as a potential cause of obstruction. Conclusions: Obstruction secondary to a crossing vessel is a common and well-studied cause of ureteral obstruction at the level of the UPJ. Outside of the UPJ crossing vessel obstruction is exceedingly rare. Our case of a 59-year-old male with a crossing vessel at the level of the pelvis contributed to recurrent nephrolithiasis and severe hydroureteronephrosis. It was repaired via robotic excision of redundant ureter and translocation of ureter via primary anastomosis.

\section{V10-09 Laparoscopic Transposition of Cecal Appen- dix in Ureteral Injury Repair}

CA Gonzalez-Santana, D Soriano-Morillo, MA FernandezCamilo, JP Mejía-Rosario, I Peralta-Bernal, M Joa-Cabrera, G Castillo, M Pichardo-Garcia

Introduction \& Objective: Ureteral lesion is quite infrequent and mostly iatrogenic and a surgical challenge. These lesions can 
occur during open or laparoscopic abdominal procedures, vaginal and endourologic procedures as well. When there is loss of a long ureteral segment, complex procedures such as psoas hitch, Boari flap, transuretero-ureteral anastomosis, autotransplant or intestinal loop interposition can be done.

In 1912, Melnikoff, reported the first cecal appendix ureteral substitution. Since that there have been some other case reports. In this case report we present the surgical technique of ureteral repair in a male subject with late diagnosis of ureteral injury.

Methods: We present the case of a male subject 48 years older, with medical history of psoriasis and surgical background of cholecystectomy in 2017 with a histopathologic report of gallbladder adenocarcinoma with local recurrence after one year. He had a hepatic segmentectomy and retroperitoneal lymph node dissection. A month after he presented to the clinic with the complaint of tenderness in surgical area. Abdominal computed tomography scan performed which reported a perirrenal collection with renal contrast extravasation, and no ureteral visualization. Retrograde urethrogram showed right ureteral disruption. Was scheduled for surgical repair. We started with an endoscopic placement of an open-end catheter. In lateral decubitus standard position, a transperitoneal laparoscopic approach was then performed and 2,500 cc of subhepatic and retroperitoneal urinoma collections were drained. After ureterolysis a $9 \mathrm{~cm}$ injury was found in the proximal and medium ureter. We confirmed that a termino-terminal tension free anastomosis could not be performed. After evaluating the available options, we decided to substitute the ureteral segment with transposition of the cecal appendix. We ligated the appendix base, maintaining the vascular structures of mesoappendix, the tip of the appendix was sectioned and anastomosed to the proximal ureter and the base to the medium ureter. A double $\mathrm{J}$ ureteral catheter was placed, and a closed-suction drain was left in place.

Results: There was drainage of $1,950 \mathrm{ml}$ during the first 24 hours. Patient was discharged after 72 hours. Drainage was removed on day 7. A pericatheter retrograde pyelogram was performed at 8 weeks before double $\mathbf{J}$ stent removal.

Conclusions: Laparoscopic cecal appendix interposition is an alternative for minimally invasive repair. An advantage of ap- pendix could be the antegrade peristalsis, good blood flow, no urine and electrolites absortion, similar appendix-ureteral diameter. We should be aware of this option for the repair of proximal and midureteral injuries.

\section{V10-10 Withdrawn}

\section{V10-11 Total Laparoscopic Retroperitoneal tumor excision: challenging but possible.}

J Jagtap, S Veer, G Agrawal, P Rao, S Mistry

Introduction \& Objective: Retroperitoneal tumors pose a challenge to minimally invasive excision techniques especially pure laparoscopy due to the limited range of mobility when compared to robotic assistance. The difficulty lies in the close proximity of major vessels and renal vasculature to the tumors and the risk of damaging them during dissection.

Methods: We have successfully performed three such total laparoscopic excisions and are describing one of them in the video. A 70-year-old female presented with occasional hypertensive spells and headache. Her CT showed a $4 \mathrm{~cm}$ solid enhancing mass medial to uncinate process of pancreas in the aortocaval region. Utilizing a four port technique (three $10 \mathrm{~mm}$ and one $5 \mathrm{~mm}$ port) the mass was completely mobilized free and excised en mass after clipping the supplying vessels.

Results: There were no major intraoperative or postoperative complications. No blood transfusion was required. The total operative time was $128 \mathrm{~min}$. The patient was started on oral diet in a day and was discharged on the 2 nd postoperative day.

Conclusions: Total laparoscopic excision of retroperitoneal masses is both safe and feasible though it requires and advanced level of proficiency in performing pure laparoscopy. If conducted in an appropriately selected patient it affords a smaller cosmetic scar along with faster convalescence.

\section{ABSTRACT VIDEO SESSION 11: ROBOTIC SURGERY LOWER TRACT}

\section{V11-01 Bladder Neck Sparing Robot-assisted Simple Prostatectomy}

M Shahait, DI Lee

Introduction \& Objective: To report a step-by-step technique for bladder neck sparing robot-assisted simple prostatectomy. Methods: The key elements of the procedure include bladder mobilization; removal of peri-prostatic fat pad; then limited incision of the endopelvic fascia; suture ligation of the dorsal vein runoff, bladder neck dissection using modified graded bladder sparing technique; adenoma enucleation; followed by watertight anastomosis and approximation of the anterior fat overlying the prostate-vesical junction to the anterior bladder. Voiding trial 4 to 6 days in the office.
Results: Between March 2015 and December 2018, a total of 30 consecutive non-randomized patients with BPH underwent bladder neck sparing RASP. The mean (SD) operative time was 107.5 (22.2) minutes, and the mean (SD) estimated blood loss was 132.4(35.4) ml. All cases were completed robotically, no intra-operative complications were encountered, continuous bladder irrigation was not needed in any patient; all the patients were discharged with 24 hours of the procedure. All the patients were continent ( 0 pad), and able to void after catheter removal except one patient with underlying neurogenic bladder who resumed clean intermittent catheterization. Of the 19 patients who had antegrade ejaculation before surgery, 8 patients (42\%) reported of enjoying antegrade ejaculation after surgery. 
Conclusions: 1) Bladder neck sparing RASP is a reproducible procedure with a short learning curve. 2) Bladder neck sparing may play a major role in preserving antegrade ejaculation. 3) Complete circumferential anastomosis decreases postoperative hematuria and obviates the need for continuous bladder irrigation.

\section{V11-02 Robotic-Assisted Excision of Retroperitoneal Schwannoma}

\section{A AlMulhim, A AlGosaibi, AM Alenizi}

Introduction \& Objective: Schwannomas are slow-growing usually benign tumors that arise from nerve sheaths. Patients tend to present with lesions in the head, neck, and flexor surfaces of the upper and lower limbs. The retroperitoneal region is a rare location for these tumors to develop, approximately $0.3-3.2 \%$ of all benign schwannomas. Patients usually present with vague symptoms: poorly localized dull pain or discomfort. Total surgical excision is the standard modality of treatment in retroperitoneal schwannomas. Robotic assisted laparoscopy is promising method and quickly becoming preferred due to it having the advantage three-dimensional visualization and superior mobility.

Methods: The patient is a 74-year-old female. She is a known case of diabetes mellitus type 2, hypertension and hypothyroidism. She presented to the emergency department as a case of abdominal pain and vomiting for three weeks. On physical examination, the patient was found to have abdominal distention. Computed tomography was done for her to rule out any functional cause for her presentation. The CT scan revealed a large mass displacing the kidney and ureter inferiolateraly measuring $70 \times 55 \times 50 \mathrm{~mm}$ with evidence of central necrosis. After adequate preparation the patient underwent robotic excision of the mass. The mass was successfully resected with good surgical margins. The resected mass was 88.8 grams in weight and measuring $70 \times 55 \times 50 \mathrm{~mm}$.

Results: Upon pathological examination, the specimen showed a fibrous slightly nodular ulcer surface. Serial sectioning of the mass showed nodular fibro myxoid surface with one cyst showing a central myxoid degeneration. The cyst measured $40 \mathrm{~mm}$ in greatest dimension and was $2 \mathrm{~mm}$ from the surgical margin. The cell neoplasm was positive for immunstain 100 , protein $\mathrm{B}$ and vimentin; it was negative for pancytokeratin, smooth muscle actin, CD117, CD34 and desmin. Post-operatively, the patient suffered no complications from the surgery.

Conclusions: Robotic assisted surgery is a modality gaining traction as being superior for excision of abdominal masses. The combination of increased vision as well as planes of manipulation of surgical tools not possible with open or laparoscopic techniques are one of the main reasons robotic surgery was chosen to remove this mass. Given the possibility of retroperitoneal schwannomas to become malignant, recur, or metastasize, it is of paramount importance that the mass be visualized as clearly as possible and resected along a surgical plane which allows full removal with wide margins.

\section{V11-03 Testicular vein syndrome: First described case of robotic assisted treatment}

JB de Oliveira, L Welter, VR Jr, CP dos Santos, HO Santos, GF Canuto, TB Santana, LM Calazans, NJ Barretto

Introduction \& Objective: The testicular vein syndrome (TVS) is a rare cause of ureteral obstruction, resulting in ureterohydronephrosis. The cause of obstruction is an extrinsic compression of the ureter in the intersection of the ipsilateral gonadal vein. The first case found in literature was reported in 1975. This is the $9^{\text {th }}$ case described and the first one treated by robotic assisted surgery. The objective of this paper is to describe a rare pathology with video presentation of the surgery technique used, robot assisted surgery, and literature review.

Methods: Video recordings from the surgery were edited. Comments on robotic surgical technique and literature review were added for better understanding. Literature research in English language publications until May 2019 was performed, using the terms "testicular vein syndrome", "testicular vein compression on ureter"; "spermatic vein compression on ureter" and "hydronephrosis caused by testicular vein/spermatic vein" on PubMed database.

Results: 58-year-old male presented with a 5 years history of colicky lumbar pain, with progressive worsening in the last months and a single episode of gross hematuria. He had well controlled hypertension and no previous surgery. He never had history of urinary calculi. First exam performed was an abdominal contrast tomography (CT) scan, that demonstrated hydronephrosis and focal thickening of the right ureter. Posteriorly, magnetic resonance imaging (MRI) of the urinary tract was performed, with the founding of ureteral stricture of $2,0 \mathrm{~cm}$ just posterior to the testicular vein intersection. This stricture was $5,5 \mathrm{~cm}$ distal from ureteropelvic junction and was associated with moderate collecting system dilatation. Due to the suspicion of TVS, ureteroscopy and pyelogram were performed, with the founding of a luminal stenosis of approximately $90 \%$. A ureteral stent was placed. Opted for transperitoneal robotic assisted laparoscopic approach with identification of gonadal vein and artery that transited above the ureter. Vessel ligation was performed as well as resection of the stenotic segment of the ureter. An uretero-ureteral anastomosis was performed with separated 5-0 Polydioxanone sutures. The patient was discharged after 2 days of surgery, with no urinary catheter or drain.

Conclusions: TVS is a rare and challenging diagnosis. This case demonstrates that the robotic approach facilitates ureter anastomosis and permits faster recuperation.

\section{V11-04 Robotic-Assisted Laparoscopic Excision Of Calyceal Diverticulum And Removal Of Calyceal Calculi}

\section{Y Phan, M Ismail, O Karim}

Introduction \& Objective: A 39-year-old lady was referred from a local hospital with symptomatic calyceal calculi in her left kidney which was not amenable to be cleared endoscopically. After discussing the risks and benefits of the procedure, she underwent a robotic-assisted laparoscopic excision of calyceal diverticulum and removal of calyceal calculi in our institution. During the procedure, pus was drained from her calyceal.

Methods: An ultrasound scanner was used to confirm the calyx with calculi. The calyx was then incised through the thin cortex and calculi removed. Copious amounts of pus were drained and aspirated. The calyceal wall was then enucleated with minimal normal renal tissue.

Conclusions: She made an uneventful post-operative recovery and her follow-up CT showed clearance of the calyceal stones. More importantly, her symptoms have resolved completely when she was seen in the follow-up clinic.

\section{V11-05 Simplified transvescical robot-assisted simple prostatectomy: technical nuances}

A Veccia, A Balthazar, L Hampton, R Autorino 
Introduction \& Objective: Open prostatectomy and laser enucleation represent the most common surgical procedures for large prostate adenomas $(\geq 80-100 \mathrm{gr})$. Robotic simple prostatectomy is being adopted in several centers worldwide, and various surgical techniques have been described. Aim of this video is to report a step-by-step technique for robot-assisted transvesical simple prostatectomy.

Methods: A 71 years old man with long history of LUTS/BPH and recurrent episodes of urinary retention presented to our clinic. The ultrasound reported an enlarged prostate with a volume of $122 \mathrm{~mL}$. The uroflow showed a Qmax $<10 \mathrm{ml} / \mathrm{sec}$, and a post-void residual of $300 \mathrm{~mL}$. Different treatment options were discussed, and the patient was candidate to a robotic simple prostatectomy. The procedure was performed with the DaVinci ${ }^{\circledR}$ $\mathrm{Si}$ (Intuitive Surgical ${ }^{\circledR}$, Sunnyvale, CA, USA). Ports were placed in a ' $\mathrm{W}$ ' configuration, similar to radical prostatectomy, but with only one assistant trocar (12 mm Airseal ${ }^{\mathrm{TM}}$-Conmed, Utica, NY, USA). Surgery started with transversal incision and opening of the bladder, which was left in place in its natural position. Ureteral orifices were identified and a circumferential incision of the bladder mucosa at the level of the adenoma was performed. Exposure and traction of the adenoma during the resection was aided by using the Endowrist ${ }^{\circledR T}$ Tenaculum forceps. Anterograde dissection of the adenoma was performed till the achievement of the urethra which was sectioned using cold scissors. Hemostasis of the enucleation bed was performed with the use of hemostatic agents and bipolar electrocauterization. V-Loc 3/0 sutures were used to obtain a vesicourethral anastomosis. Bladder closure was performed in double layer, again using V-Loc 3/0 sutures. A water-tightness test was performed. A 3-way 20 Fr Foley catheter was left. No drain was placed, and the specimen was retrieved via the midline port.

Results: Estimated blood loss was $295 \mathrm{~mL}$. Console time 98 minutes. No perioperative complications were recorded. Continuous bladder irrigation was used for 12 hours, and patient was discharged the following morning. Surgical pathology report described an $8.0 \times 6.1 \times 5.5 \mathrm{~cm}$ prostate adenoma of $125 \mathrm{gr}$. $\mathrm{Pa}$ tient passed a successful voiding trial on postoperative day 10 .

Conclusions: Robot-assisted simple prostatectomy is a safe, effective, and reproducible minimally invasive surgical procedure for the treatment of large prostate adenomas.

\section{V11-06 Robotic Fascial Sparing Rectus Abdominis Harvest}

LC Zhao, ML Schulster, ML Schulster, GW Dy, NM Vranis, JP Levine, M Jun

Introduction \& Objective: The rectus abdominis muscle is a useful flap for multiple surgical repairs including complex posterior urethral fistula repair. Traditional open harvest with anterior sheath violation is associated with significant donor site morbidity including hernia, bulge, and wound infections. We describe a robotic assisted fascial sparing technique for rectus abdominis flap harvest to mitigate donor site morbidity.

Methods: In 2018, four robotic rectus muscle harvests were performed at our institution by the senior authors, a urologist and a plastic surgeon, for robotic vesical or urethral fistula repair. The fistula repair was initially approached via a robotic transabdominal approach. After repair of the fistula, the abdomen is desufflated, and the robot is undocked. Next, a $3 \mathrm{~cm}$ skin and anterior fascial incision was made, and a GelPoint was placed into this opening in the fascia. The space between the anterior and posterior rectus sheath was insufflated, and the robot (da Vinci XI or SP) was docked into the GelPoint. The rectus muscle was freed robotically up to its costal and subxiphoid margin. The abdominal cavity is then re-insufflated, and the flap was passed into the abdominal cavity for pelvic soft tissue repair. Demographic data, peri-operative variables and outcomes were recorded.

Results: All patients were male with a mean age was 65.8 (range 58-74) and mean BMI 28 (range 22.4-32.7). Mean EBL for the rectus harvest portion of the procedure alone was 10cc. Two harvests were for rectourethral fistula repair, one vesicocutaneous fistula and one vesicopubic fistula repair. All harvests were completed robotically without the need for open conversion. All flaps were deemed viable intra-operatively with the use of Doppler. No patients experienced a hernia, bulge or infection at the harvest site with a mean of 5.6 months follow up (range 212.2). One patient experienced a harvest site seroma necessitating drainage.

Conclusions: Robotic, fascial sparing rectus abdominis harvest is safe and feasible. Preservation of the anterior and posterior rectus sheath allows for decreased morbidity.

\section{V11-07 Robotic Bladder Neck Reconstruction Tech- niques for Retropubic and Trans-Vesical Approaches}

A Beksac, AA Elbakry, KE Okhawere, BD Dayal, K Meilika, R Purohit, KK Badani

Introduction \& Objective: In this video, we present a case of a bladder neck reconstruction of a 46-year-old male. The patient has a history of prostate cancer treated with primary radiotherapy. 14 years after radiation, patient received treatment for benign prostatic hyperplasia. He developed stress urinary incontinence, nocturia and recurrent urinary tract infections following treatment. 2 years after bph treatment, patient developed acute urinary retention. He was catheterized suprapubically. Flexible cystoscopy revealed completely obliterated bladder neck. Patient had no evidence of recurrent prostate cancer.

Methods: The patient was positioned in the low lithotomy position. We started the case with mobilization of the bladder. Following mobilization, bladder was dissected towards the endopelvic fascia. Bladder was incised from the midline, and the obliterated urethrovesical junction was observed. A flexible cystoscope was inserted into the urethra and immunofluoresence guidance was used to create the passage. Urethral foley catheter was inserted using glidewire assistance. The fibrotic tissue in the bladder neck was excised. Bilateral bladder flaps were used from the proximal side of the bladder. Healthy urethral tissue was advanced toward the bladder neck and posterior plate was formed. Healthy proximal bladder tissue was used to reconstruct the lateral and anterior bladder surface. Running barbed sutures were used for anastomosis. Finally, the cystotomy was closed with running suture.

Results: Surgery was completed in 2:17 hours, with an estimated blood loss of $50 \mathrm{~mL}$. Drain was removed at day 1 and patient was discharged on POD 1 without complications. Foley catheter was removed at postoperative 10 days. Patient was able to urinate without intervention.

Conclusions: This video demonstrates a stepwise technique for the challenging scenario of radiation induced bladder neck stricture. Immunofluorescence can a useful tool in identification of the urethra in patients with an obliterated bladder neck.

\section{V11-08 Robot-assisted transvesical simple prostatec- tomy with concurrent bladder stone removal.}

O Aytac, A Garayev, F Atuğ 
Introduction \& Objective: Robot-assisted simple prostatectomy (RASP) continues to be preferred surgical treatment option for patients with large benign prostate hyperplasia. In this video we aimed to report transabdominal, transvesical RASP with bladder stone with trigonization.

Methods: The male patient included in present report had preoperative bothersome lower urinary tract symptoms (LUTS) unresponsive to several medications. Preoperative performed prostate MRI revealed enlarged prostate with $100 \mathrm{~cm}^{3}$ and concurrent $2 \mathrm{~cm}$ bladder stone. Robot-assisted simple prostatectomy and bladder stone removal was planned.

Results: The patient was 65 -year-old with prostate specific antigen (PSA) level of $2.1 \mathrm{ng} / \mathrm{ml}$. Preoperative International Prostate Symptom Score was 24 . He had severe LUTS despite of several alpha-blockers. Preoperative prostate MRI showed PIRADS I and II lesions with benign nature of prostate tissue. The operation was planned with standard 6-port transabdominal transvesical approach. Estimated blood loss was $150 \mathrm{ml}$ and console time was 135 minutes. The patient had a simultaneous bladder stone removal. The adenoma tissue removed with enbloc. There were no acute intraoperative or perioperative complications. Hospital stay was 2 days and adenoma weight was 95 grams.

Conclusions: Robotic-assisted retropubic simple prostatectomy is gaining popularity with safe and feasible options alternative to an open and endoscopic techniques.

\section{V11-09 Robotic assisted Ureteric Mitrofanoff conduit with Augmentation Cystoplasty and Malone Ante- grade Continence Enema procedure} S Harinatha, M Keshavamurthy, S Tabrez, P Kumar,
B Neelagar

Introduction \& Objective: Neurogenic bladders requiring selfcatheterizations have primarily been treated using appendix as the preferred mitrofanoff channel. But in some cases appendix may be absent or maybe required for other procedures as in MACE (Malone Antegrade Continence Enema) for chronic constipation. Here we demonstrate the feasibility of Ureter as the Mitrofanoff channel combined with bladder augmentation and MACE procedure done robotically.

Methods: The patient is a 14-year-old male with neurogenic bladder due to spinal dysraphism with inability to do per urethral self-intermittent catheterization due to sensitivity and recurrent urinary tract infection. He also had chronic constipation requiring digital evacuation frequently. Evaluation with urodynamics and cystogram showed poor compliance with small capacity bladder with no reflux. He was planned for Robotic Augmentation Cystoplasty and Ureteric Mitrofanoff conduit with MACE procedure. The patient was placed in trendelenburg position and standard 4 port docking of Da Vinci XI robot was done with additional assistant port. Ileal segment of $20 \mathrm{~cm}$ was marked with sutures and delivered out of the abdomen through a small suprapubic incision and was partially detubularizsed and refashioned into an $U$ shaped patch with chimney and was returned to the abdominal cavity. Augmentation of bladder was done with single layer barbed sutures. Right ureter was divided at the lower one third level. Proximal ureter was anastomosed to the ileal chimney and distal ureter was brought out at the right iliac fossa as cutaneous stoma. Appendix was brought out at a separate site for cutaneous stoma for antegrade continence enema.

Results: Total operative time was approximately 4 hours. Estimated blood loss was about $200 \mathrm{ml}$. Patient was discharged on the 4 th postoperative day. 4 weeks after the procedure, ureteric stent was removed and patient initiated on self-catheterization and antegrade enemas, which he was able to do comfortably.

Conclusions: Robotic assisted ureteric mitrofanoff procedure with augmentation cystoplasty and MACE procedure is technically feasible with good outcome in selected patients with combined neurogenic bladder and bowel dysfunction.

\section{V11-10 Robot-Assisted Radical Prostatectomy of a 500g Gland}

AM Asghar, Z Lee, DD Eun

Introduction \& Objective: Prostate cancer (PCa) is the most common solid organ malignancy in men and the second cause of cancer-related death in the US. Surgical extirpation or radiation therapy are the standard of care for patients with intermediaterisk prostate cancer. Robot-Assisted Radical Prostatectomy (RARP) is now the most common approach surgical management of PCa. We present a case of a patient with intermediaterisk $\mathrm{PCa}$ and 500-gram prostate who desires surgical treatment. Methods: A 72-year-old male was referred to our tertiary referral center for management of urinary retention, significant lower urinary tract symptoms (LUTS), and reported $400 \mathrm{~g}$ prostate on retroperitoneal ultrasound. The patient had avoided prostate cancer screening for $>10$ years and was hesitant to obtain labs and imaging. After a comprehensive discussion, PSA was obtained which was $32.7 \mathrm{ng} / \mathrm{mL}$, prompting a prostate biopsy. He was diagnosed with Gleason $3+4$, Group Grade $2 \mathrm{PCa}$ in 1/12 cores up to $8 \%$. An MRI was also obtained which showed a $580 \mathrm{~g}$ gland, bilateral pelvic lymphadenopathy, and possible extracapsular extension and seminal vesicle invasion. Given his significant LUTS and urinary retention, he opted for surgical extirpation. Our surgical approach was similar to our routine RARP with bilateral extended pelvic lymph node dissection with a few alterations. A Vessel Sealer Extend was used instead of a Maryland forceps, avoiding the use of clips in the small working space. The complex anatomy also made the bladder neck location unclear, so the bladder neck dissection was begun with two incisions at 10 and 2 o'clock before connecting them. Lastly, the large bladder neck was reconstructed prior to anastomosis.

Results: Our console time was 327 minutes with 400cc estimated blood loss. The patient was discharged post-operative day 1 with no immediate post-operative complications. His final pathology was pT2N0M0 Gleason $3+4 \mathrm{PCa}$ with negative margins and a total weight of $500 \mathrm{~g}$. He is currently awaiting his outpatient follow up visit.

Conclusions: Prostate cancer in exceedingly large glands, such as this $500 \mathrm{~g}$ gland, may be managed with RARP using standard steps while staying true to surgical principles. Some alterations such as the use of a vessel sealer, modified bladder neck dissection, and bladder neck reconstruction will allow for complete oncologic control and watertight anastomosis.

\section{V11-11 Combined robot assisted laparoscopic ne- phrectomy and ureterocystoplasty}

\section{Wetterauer, A Feicke, S Moehr, T Horn, H Seifert}

Introduction \& Objective: We present the case of a 21-year-old patient with severe neurogenic bladder dysfunction secondary to a menigomyelocele with Arnold-Chiari-malformation typ II. Serial urodynamic studies revealed Sphincter-DetrusorDyssynergia together with increasing detrusor muscle overactivity and poor bladder compliance The patient performed clean 
intermittent self-catheterization (CIC). Bladder capacity was limited to $300 \mathrm{ml}$. Anticholinergic therapy and Botolinum Toxin injections were unsuccessful in reducing intravesicular pressure. Cystography revealed left-sided grade $\mathrm{V}$ vesicoureteral reflux and nuclear scintigraphy showed a non-functioning left kidney. Methods: Supine positioning with a 30-degree lateral tilt to elevate the left flank. The camera trocar was inserted supraumbilically in line with Hasson'stechnique. The remaining trocars were inserted as shown. We initially performed a left nephrectomy utilizing cephalic side-docking of the Robot from the left side. The proximal ureter was separated following placement of dividing clips. The ureter was mobilized and spatulated down to the point where it crosses the iliac artery. The kidney specimen was retrieved via the supraumbilical port. The robot was re-docked in a more caudal direction. The distal part of the ureter was spared to a length of $5 \mathrm{~cm}$, the ureter was spatulated and looped to the shape of the letter $\mathrm{U}$. The medial sides were sutured together using 3-0 Monocyl with a running stitch creating a ureteral patch. After opening the retropubic space, the bladder was mobilized, and the dome was widely incised transversely. We performed a cystoplasty by suturing the ureteral patch to the open bladder wall and achieved significant augmentation of the bladder. A small easy-flow drainage was inserted. Finally, the peritoneal line of the bladder was fixed to the abdominal wall with a running barb suture.

Results: Urodynamic studies after 3-month demonstrated a drastic decrease in bladder pressure and improvement in bladder compliance. After one year the patient was still performing CIC. Bladder capacity had improved to $650 \mathrm{ml}$.

Conclusions: Combined robot assisted laparoscopic nephrectomy and ureterocystoplasty is a feasible treatment option and led to a highly satisfied patient.

\section{V11-12 Robotic assisted repair of traumatic intra- peritoneal bladder rupture}

M Stanowski, MM Quraishi, E Latif, SS Kommu, BA Eddy
Introduction \& Objective: Traumatic bladder rupture is a relatively uncommon presentation, and typically occurs during blunt lower abdominal trauma in the presence of a distended bladder. It can be in relation to pelvic fractures or be an isolated injury. Iatrogenic bladder trauma is well documented in urology, gynecology and colorectal surgery. Often the patient has difficulty voiding after the trauma and hematuria is very common. A cystogram, either retrograde or CT based will typically confirm the diagnosis. Intraperitoneal injuries will not usually heal without surgical intervention. Traditionally, open repair is the suggested method for management, however minimally invasive options can be used also. We present the first reported case of robotically assisted traumatic bladder rupture repair. This case we present was the result of blunt abdominal trauma and left the patient with a large "blow out" defect at the dome of their bladder. This was the only significant injury that this patient endured during their trauma and were otherwise clinically stable. This enabled time for safe transfer of the patient from another facility and planning of this novel method of repair.

Methods: The repair was performed under general anesthesia and steep trendelenberg. A 3 robot arm setup and single Air Seal assistant port was used. A satisfactory 2-layer continuous repair was performed and passed a leak test of $250 \mathrm{mls}$. A $20 \mathrm{Fr}$ urethral catheter and a pelvic drain was left insitu and the patient was discharged the following day after removal of the drain.

Results: A fluoroscopic cystogram was performed at day 14 post operatively which was normal. The urethral catheter was removed. At review 3 months post-operative the patient is well and voiding normally.

Conclusions: This robotic assisted repair of intraperitoneal traumatic bladder rupture is unique case where the patient was stable with an isolated injury which enabled the safe use of a robotic technique for repair. It avoided the need for a more morbid laparotomy and enabled a more precise and secure repair than standard laparoscopy. This demonstrates that in carefully selected novel cases, robotic surgery is feasible and can deliver excellent outcomes.

\section{ABSTRACT VIDEO SESSION 12: LAPAROSCOPY: LOWER TRACT}

V12-01 Laparoscopic pyelolithotomy in a cross-fused renal ectopia guided by 3 Dimensions imagine reconstruction.

\section{Ye, J Wu, Y Wei}

Introduction \& Objective: Stone disease in cross-fused renal ectopia is rare, because of the abnormal anatomy, treatment options in such cases may be challenging. In this study, we present a case of right to left cross-fused renal ectopia with a $22 \mathrm{~mm}$ stone in the crossed kidney managed by transperitoneal Laparoscopic pyelolithotomy.

Methods: A 61-years-old woman presented with a dull left lower abdomen pain and gross hematuria, CTU confirmed this was a right to left cross-fused renal ectopia with multiple stones, the largest one was $22 \mathrm{~mm}$ in the ectopic renal pelvis with suspicious ureteropelvic junction obstruction. 3D imagine reconstruction was performed which provided anatomic details of crossed right kidney. Pros and cons of each treatment options including observation, ESWL, flexible ureteroscopic lithotripsy, PCNL, laparoscopic pyelolithotomy and open surgery were discussed, she was leaning towards a laparoscopic approach. Under general anesthesia, the patient was initially placed in a lithotomy position for ilateral ureteric catheters insertion, then she was replaced in a lateral position, transperitoneal and transmesocolic approach was used to give direct access to the renal pelvis, which was opened and calculi were removed from kidney, there was no obvious UPJ stricture, a double $\mathrm{J}$ stent was inserted antegrade, the pelvis was closed with continuous 4-0 absorbable sutures.

Results: The procedure was completed in 120 minutes without any intraoperative complications, estimated blood loss was less than $50 \mathrm{~mL}$. The patient had an uneventful postoperative recovery, she passed flatus and had diet on postoperative day (POD) 1, urethral catheter was removed on POD 4, drain tube 
was removed on POD5 and she was discharged on the sixth postoperative day. Post-operative KUB revealed no residual stone in the ectopic kidney and she was scheduled to remove the double $\mathrm{J}$ stent 6 weeks postoperatively. The patient would be followed up regularly for early detection of stone recurrence and surveillance of left kidney stones.

Conclusions: Laparoscopic pyelolithotomy is a safe and feasible option in crossed fused renal ectopia that offers excellent outcomes with low morbidity. Preoperative 3D imagine reconstruction is very helpful for surgical planning. Intraoperative ureteral cannulation on both sides should be considered to avoid iatrogenic injury to the contralateral ureter.

V12-02 3-D Transperitoneal Lap anterior exenteration in females with muscle invasive bladder carcinoma :A prospective evaluation

A Kumar, S Yadav, S Kumar, Y Prashant, K Saurav, V Tyagi, V Prasad

Introduction \& Objective: Laparoscopic transperitoneal anterior exenteration (LAE) is the Gold standard in females with muscle invasive bladder carcinoma. However, LAE is technically challenging and confined to centers with advanced laparoscopic expertise. The 3- D laparoscopy has advantages over 2D laparoscopy in terms of better vision and depth perception. We prospectively evaluated the outcomes of 3-D laparoscopic transperitoneal anterior exenteration (LAE) in females with muscle invasive bladder carcinoma.

Methods: All consecutive female patients undergoing 3-D LAE for muscle invasive bladder carcinoma by a single surgeon between July 2015 and April 2019 at our institution were included. The various clinical data were recorded and analyzed. We are presenting video of one such case.

Results: A total of 121 patients were included in the study. The mean age was 61.3 years. The prior abdominal surgery was found in $35(28.9 \%)$ patients. The neo-adjuvant chemotherapy was given in $21(17.3 \%)$ patients. The clinical stage was T2 in $85(70.2 \%)$ and ${ }^{3} \mathrm{~T} 3$ in $36(29.8 \%)$ patients. The extracorporeal diversion was ileal conduit in $96(79.3 \%)$ and ileal neo-bladder in $25(20.7 \%)$ patients. The mean operating time and estimated blood loss were $143.3 \mathrm{~min}$ and $183.1 \mathrm{ml}$ respectively. There was no conversion to open. The mean hospital stay was 4.1 days. The introperative and postoperative complications were $4.9 \%$ and $5.7 \%$ respectively and mainly Clavien 1 and 2 only. In histopathology pT2, pT3, pT4, pN0 and pN1-2 were found in $69.4 \%, 25.6 \%, 4.9 \%, 75.2 \%$ and $24.8 \%$ respectively. The mean number of lymph nodes removed were 19. At mean follow up of 34.7 months, local recurrence and distant recurrence were found in $2.4 \%$ and $1.6 \%$ patients respectively. The estimated 5 year disease free and cancer specific survival were $88.4 \%$ and $90.9 \%$ respectively.

Conclusions: 3-D Laparoscopic transperitoneal anterior exenteration (LAE) in females with muscle invasive bladder carcinoma is feasible, safe with acceptable perioperative morbidity and excellent oncological outcomes. However, it is a technically challenging procedure and should be done by surgeons of significant laparoscopic expertise.

V12-03 Modified Ileal Conduit Intracorporeally Accomplished following Laparoscopic Radical Cystectomy

Y Niu, H Wei
Introduction \& Objective: To introduce a modified ileal conduit (MIC) intracorporeally performed following laparoscopic radical cystectomy (LRC) and dissection of lymph nodes for bladder cancer (BC).

Methods: Using linear anastomosis stapler (LAS) $15 \mathrm{~cm}$ terminal ileum was isolated with mesentery transilluminated to preserve the blood supply. The continuity of the ileum was restored by end-to-end ileoileal anastomosis using LAS. The isolated loop were flushed with normal saline containing gentamycin, two single-J stents were pulled through the lumen of the loop. At stoma site a circular incision was made and an extra-peritoneal tunnel was bluntly created with index finger. Through the tunnel the loop and stents were pulled out of the incision. The left ureter was brought over the great vessels to the right side. The posterior wall of spatulated ureters were sutured to the loop in running suture, the anterior wall was closed after stents were inserted into ureters respectively. Finally, the conduit and the ureterointestinal anastomosis were totally re-peritonealized by closing the rent of retroperitoneum.

Results: Twenty-six patients of BC were included. Male/female 20/ 6 , age $63.57 \pm 9.96$ years old, BMI $23.91 \pm 2.957 \mathrm{~kg} / \mathrm{m}^{2}$, operation time $329.08 \pm 96.91 \mathrm{mins}$, estimated blood loss $232 \pm 222.62 \mathrm{~mL}$, ambulation 2 (1-4) days, flatus 3 (1-5) days, hospital stay 11 (6-25) days. Peri-operative complications (Clavien-Dindo) within 90 days include minor (I-II) 11 (44\%) cases and major (III-V) 1 (4\%) cases. Hydronephrosis was found in 3 cases (mild, 1; moderate, 1; severe, 1) and compromised renal function in 9 cases (mild, 7; moderate, 1; severe 1).

Conclusions: The MIC was intracorporeally accomplished with maximally preserved blood supply of involved intestine, end-toend reflux ureterointestinal anastomosis with conduit anchored in the extra-peritoneal tunnel, and the ureterointestinal anastomosis were totally retroperitonealized, thus obviating any chance of herniating small bowel lateral to the conduit, reducing the incidence of urinary intestinal leak and secondary infection, and facilitating to handle subsequent complications. MIC is feasible and safe although technique challenging, which preserves satisfying renal function while not increasing perioperative complications.

\section{V12-04 Laparoscopic Excision Of Infected Urachal Cyst Following Laparoscopy Assisted Vaginal Hys- terectomy}

C Vaddi, S PM, R Paidakula, P Gopi, MB, S Ganesan

Introduction \& Objective: In the era of minimally invasive surgery, port placement has seen various complications even in experienced hands. One such rarely reported complication which often goes unrecognized is, injury to hitherto undetected urachal cyst during umbilical port placement. Only 3 such cases have been reported in the literature and hereby we present the fourth case, which was managed by laparoscopic excision.

Methods: A 43yr old female presented with infra umbilical pain and fever. She had undergone laparoscopic assisted vaginal hysterectomy 3 weeks before. On examination she was found to have an abscess at infra umbilical port site. MRI abdomen revealed a subcutaneous abscess communicating with a tract suggestive of urachal cyst. After adequate drainage and treatment of abscess, laparoscopic excision of the urachal cyst was done 3 weeks later. 3 ports were placed in the upper half of the abdomen, omental adhesions to the anterior abdominal wall released, the entire tract leading to the bladder was visualized. Adhesions between the tract and abdominal wall were released meticulously and the adherent 
part of posterior rectus sheath was excised. The fluid filled cavity reaching the dome of the bladder was excised in toto along with part of the dome to avoid recurrence. Bladder was closed in 2 layers. Specimen was retrieved through an infraumbilical incision, encircling the previous scar and the defect in the rectus sheath was closed with far- near, near- far technique.

Results: Patient had an uneventful post-operative course. Drain was removed on the $2^{\text {nd }}$ post-operative day (POD). Foley catheter was removed on the $7^{\text {th }}$ POD. Histopathology confirmed the diagnosis of urachal cyst.

Conclusions: Urachal cysts usually present after they get infected. One of the reasons being port placement injury. Preoperative imaging has to be carefully analyzed and placement of ports has to be planned accordingly." The eye does not see what the mind does not know".

V12-05 Radical cystectomy, urethrectomy and ileal substitution in woman

C Conde Redondo, F Castroviejo Royo, F Lara Pérez, J Torrecilla, S Martín Martín, A Alonso Villalba, R Cortiñas Gonzalez

Introduction \& Objective: Treatment of infiltrating bladder tumor with bladder neck involvement is radical cystectomy with urethrectomy. In women is a pathology much rarer than in the male. In this video we present the laparoscopic surgery technique step by step.

Methods: Placement of the patient in Trendelemburg forced position with legs in half-opened leg for urethral access. Positioning of the trocars in fan, placing the camera two fingers above the navel to facilitate access to the iliac common in the lymphadenectomy. 2 Trocars of 10 and 3 of 5 are placed. We start the surgery by fixing and suspending the uterus. We release the infundibulum-pelvic ligaments. Then before starting the Linfadectomy we release and section the ureter to avoid accidents. We performed a bilateral extended linfadenecomy, extracting the ganglia in separate pouches. We pass the ureter above the retrosigma, after performing presacral lalinfadenectomy if necessary. We open in the bottom of the sack of Douglas, we identified the vagina and open it posteriorly and anteriorly, section the bladder Pediculae until reaching the urethra. This is released longitudinally, until its insertion into the urethral meatus, which is released from external genitalia. Finally, we introduce a bag of extraction by vagina. We perform the vaginal closure with continuous suture and suspension of the vaginal dome to the Uterosacros. After passing the ureters through the Retrosigma, we chose a segment of ileum that is individualized. We performed a side to side ileum anastomosis. We do an anastomosis to the bricker individually and we use Uni $\mathrm{J}$ catheters. Finally, we use the port of 10 to perform the Briker.

Results: Surgical time: 240 minutes. Bleeding: $200 \mathrm{ml}$. Transfusion No.

Conclusions: Laparoscopic radical cystectomy with urethrectomy in women is not very common, it can be performed entirely by laparoscopic means, with extraction of the piece by vaginal way preserving the integrity of the wall. Posterior reconstruction can be performed intracorporeally when there is laparoscopic D or Robot, which facilitates the pure laparoscopic technique without firing the surgical times.

V12-06 Suprapubic cystoscopy guided Heygrove dilator placement in anastomotic urethroplasty for PFUI

S Nachimuthu, R Manickam, S Kallappan
Introduction \& Objective: During anastomotic urethroplasty in PFUI, usually one needs to place a Heygrove dilator blindly to guide location of the membranous urethra. Occasionally this can end up with a false passage. To obviate this we have improvised this technique of supra pubic guided Heygrove insertion alongside scope.

Methods: 16-year-old male patient of PFUI on SPC, was planned for anastomotic urethroplasty. In lithotomy position, midline perineal incision, bulbocavernous muscle was divided, urethra mobilized. Crural separation done, urethra divided at the level of stricture. Instead of blind Heygrove dilator placement we dilated the SPC tract with Amplatz dialators upto 30 Fr. 10 Fr pediatric cystoscope was introduced first and Hegrove dilator was inserted alongside under vision making sure that there is no undue force (avoiding contusion and bleeding of membranous urethra). With this, optimal placement of Heygrove, membranous urethra was identified and incised. Proximal and distal urethra was spatulated and end-to-end urethroplasty was done over $14 \mathrm{~F}$ silicon foley catheter using 4-0 interrupted vicryl sutures. Post-operative period was uneventful.

Results: Patient voided with good stream after three weeks and did not have any recurrence of stricture in a follow up of 2 years.

Conclusions: We found that supra pubic cystoscopy guided urethral dilator placement is better than blind placement in PFUI.

\section{V12-07 Laparoscopic treatment of uterovesical fistula after caesarian delivery}

V Agarwal

Introduction \& Objective: Uterovesical fistula is rare complication after caesarian delivery, but multiple and emergency cesarian leads to uterovesical fistula. 38-year-old lady presented with whitish and dirty urine and continous leakage from vagina after 2 week of caesarian. After investigation with ct urography and cystoscopy with vaginosocpy diagnosis was confirmed. She was taken for laparoscopic management of fistula with consent for hysterectomy.

Methods: After detailed preoperative work up, she was taken for laparoscopy management. Initialy with cystoscopy and vaginoscopy, fistula tract was cannulated with 6fr ureteric catheter. Both side ureteric catheter was placed. Three port was placed. Uterus was separated from bowel and omentum. Uterus was bulky and soft. Pouch of douglas was dissected. Fistula tract identified. Hysterectomy was done. Uterus was removed from vagina in two pieces. Margins of bladder opening was cut and sent for hpe. Bladder closed with viryl 2-0.vagina opening closed with vicryl 2-0 Separately. Omentum was placed in between, drian placed and closed.

Results: Patient tolerated procedure well. Total surgical time was 160 minutes. Blood loss was $200 \mathrm{ml}$ mailnly during hysterectomy. Patient started accepting feed from next days and discharged with foleys catheter on 4th postoperative day. Cather was removed after cystogram on 14th day. She was on follow up till 9 months and doing well

Conclusions: Uterovesical fistula can be managed with laparoscopy and hysterectomy is required to treat it well. Ideally 6-8 week waiting period is required postpartum for uterus to involute but in selected cases time is no bar.

\section{V12-08 Laparoscopic urachal remnant excision}

DG Sucupira, HS Andrade, MA Arap, V Srougi, G Eufrasio da Silva, A Mitre, RJ Duarte, M Srougi 
Introduction \& Objective: Urachal remnants are originated from failure in its segmental or total obliteration, resulting in the presence of cystic structures at any site throughout its course. They are classified as patent urachus, umbilicalurachus sinus, urachal cyst and vesicourachal diverticulum. Evidences suggesting that urachal cysts can present a malignant transformation are unclear and treatment should be limited to symptomatic patients. Our objective is to demonstrate a step-by-step laparoscopic technique to urachal remnants excision.

Methods: a 34-year-old woman presented to our service with pelvic pain and intermittent fever. She was previously treated with various courses of antibiotics and anti-inflammatory therapy without symptoms resolution. Pelvic CT-scan and MRI showed a $2.8 \mathrm{~cm}$ median infraumbilical cystic formation in the preperitoneal fat topography, suggestive of an infected urachal cyst. A three port configuration was utilized: one $11 \mathrm{~mm}$ midline supraumbilical camera port, and two $5 \mathrm{~mm}$ ports in each abdominal side. The table was set in a $30^{\circ}$ trendelenburg position. The preperitoneal fat containing the urachus and its remnant was excised from the umbilicus to the bladder dome and a partial cystectomy was performed. The bladder defect was closed with a 3-0 polyglactin running suture. The histopathological analysis showed a benign smooth muscle tumor - injury of uncertain potential of malignancy. The symptoms were solved during the early followup period.

Results: since 2011, six patients were surgically treated. The mean age was 33,2 (18-48), mean surgical time was 140 (30 $330 \mathrm{~min}$ ), there were no complications. Discharge occurred within 100h of hospital staying (48-192h) and indwelling catheter was removed 3 days after surgery (1-5d). 4 histopathological analyses demonstrated urachal cysts, one urachal diverticulum and one smooth muscle tumor - injury of uncertain potential of malignancy.

Conclusions: laparoscopic excision is an effective and minimally invasive definitive treatment for urachal remnants.

\section{V12-09 Recurrent Epididymo-orchitis: Uncommon Presentation of Zinner Syndrome}

\section{F ALHAJERI, S Salem, A Harraz, S Alkhudir, K Alali}

Introduction \& Objective: Several case reports have been published in the literature regarding uncommon presentation of Zinner syndrome. We present a case of an unusual presentation including recurrent epididymo-orchitis and lower abdominal pain.

Methods: A 46 years old male, who is married with 5 children, presented with long history of recurrent left testicular and suprapubic pain mainly with full bladder along with one episode of acute urinary retention. Extensive investigations have been carried out including abdominopelvic ultrasonography, CT scan and MRI, and a diagnosis of Zinner syndrome has been established. He underwent trans-rectal aspiration with major symptomatic relieve. Patient lost follow-up and presented with recurrence of the previous symptoms intermittently along with occasional perineal discomfort and dysuria. Physical examination showed tender left epididymis otherwise unremarkable. All laboratory investigations were normal. Scrotal ultrasound confirmed to have left epididymitis. Abdominopelvic MRI showed non-visualized left kidney, large left cystic lesion arising from the left seminal vesicle indenting the inferior wall of urinary bladder without infiltration and retained secretions with high proteinacious contents with no post contrast en- hancement in the ejaculatory duct. The patient underwent laparoscopic left seminal vesiculectomy with an uneventful periand post-operative recovery.

Results: Zinner syndrome is a congenital anomaly of the mesonephric duct development early in the embryogenesis. It mainly refers to the triad of unilateral renal agenesis, ipsilateral seminal vesicle cysts, and ipsilateral ejaculatory duct obstruction. Presentation ranges from being asymptomatic with incidental discovery or symptomatic. Our case was uncommon presentation and clinical suspicion should be raised in patients with lower urinary tract symptoms and unilateral kidney agenesis.

Conclusions: The concept of having unusual presentation of Zinner syndrome is known in the literature, however, high index of suspicion should be raised in patients with recurrent urological symptoms with congenital renal agenesis.

\section{V12-10 Technical Tips In Laparoscopic Excision Of Giant Bladder Diverticulum}

S Nachimuthu, R Manickam, S Kallappan

Introduction \& Objective: This video illustrates the technical tips of laparoscopic excision of giant bladder diverticulum.

Methods: 78 years old male with a history of having had TURP in 2013 was presently admitted with acute urinary retention. Ultrasound showed a large bladder diverticulum. CT scan showed a large diverticulum in relation to the left lateral wall of bladder. Cystoscopy was done and guide wire was coiled in the diverticulum. Laparoscopic diverticuluctomy was done after placing a left ureteric stent. Coiling of the guidewire helped in dissection of the diverticulum from the bladder wall. Presence of stent on the ipsilateral side helped to prevent injury to the ureter. Diverticulum was excised after reaching close to its neck. Bladder wall closed with 3-0 barbed suture and drain placed. Patient had smooth recovery and drain removed on POD 3. Catheter was removed on 10th day.

Results: Cystogram done on 14th day showed normal bladder outline. Subsequently he underwent TURP after three months for prostatomegaly. He voided with a flow of $23 \mathrm{ml} / \mathrm{sec}$.

Conclusions: Laparoscopic excision of large bladder diverticulum has less morbidity. Guide wire coiled in the diverticulum helps in identification of the diverticulum. Stent prevents to the injury to the ureter.

\section{V12-11 A new transurethral approach to repair a Vesicovaginal Fistula}

E Hernandez-Mendez, G Morales-Montor, C Martinez-Arroyo, P Cortes-Raygoza, I Calvo-Vazquez, M Cantellano-Orozco, G Fernandez-Noyola, C Pacheco-Gahbler

Introduction \& Objective: Vesicovaginal fistulas (VVF) are the most commonly acquired fistulas of the urinary tract, but we lack a standardized algorithm for their management. Surgery is the most commonly preferred approach to treat women with primary gynecologic or urological surgery. All fistula surgeries should be conducted under the basic principle that the fistula and scar will be completely excised. The success rate of treatment of a simple VVF is reported to be $75 \%$ to $97 \%$. Most surgeries are performed with an open approach. We describe a transurethral repair of a 
vesicovaginal fistula using a nephroscope and mini-laparoscopic surgery equipment.

Methods: This is the case of a 58 yo female with past medical history of total hysterectomy, 3 cesarean sections and a pneumatic cystolithotripsy due to a bladder calculus. She developed urine leaking from the vagina that began 3 months after cystolithotripsy. This leakage was continuous and related to activities that increased intra-abdominal pressure. Her quality of life was affected using 3 pads per day. During speculoscopy we identified an orifice in the anterior wall of the vagina near the scar of the previous gynecologic surgery that continuously leaked urine. We completed the study protocol and diagnosed a retro-trigonal VVF after performing a cystography and cystoscopy.

Results: Our surgical repair consisted in a transurethral approach using a 24 Fr nephroscope. First, we used Amplatz dilatators in the urethra and introduced a 26 Fr renal access sheath to the bladder. After that, we worked with the nephroscope and a Collins loop to resect the fistula and we closed the defect with mini-laparoscopic surgery equipment (needle driver and scissors). Bladder catheter was removed 3 weeks after surgery, with a postoperative cystography that showed no evidence of urine leakage. In the 2 nd month follow-up, the patient reported complete absence of urinary symptoms.

Conclusions: VVF can be repaired with this new approach safety and with good results. We need to reproduce this technique and if postoperative results are similar, this could be a new standard of minimally invasion surgery for these patients.

\section{V12-12 Laparoscopic VVF Repair Using Barbed Su- ture And 3D Camera}

\section{R Manickam, S Nachimuthu, S Kallappan}

Introduction \& Objective: Gynecological causes for vesicovaginal fistulae are common nowadays. Early repair was done in most of our cases. Here we illustrate laparoscopic repair with a video wherein we used barbed suture and 3D camera.

Methods: 22 women developed vesicovaginal fistulae following hysterectomy. Early repair was planned after confirming the absence of urinary infection. Cystoscopy was done to examine the fistula to confirm the absence of slough. Bilateral ureteral catheters or stents were placed. Laparoscopy was done using 5 ports. Lessons learnt to make the procedure successful are as follows. Foley balloon with about $40 \mathrm{ml}$ in the balloon was used to prevent gas leak from vagina. As it was an early repair the adhesions were flimsy. The wall of the bladder was not fibrosed unduely and could be dissected sharply. Bladder was bivalved and the fistula was excised with a $5 \mathrm{~mm}$ margin. The vaginal wall was separated adequately from the bladder wall. Vaginal edges were sutured using 3-0 barbed suture maintaining watertight closure. Omental flap was tacked to anterior vaginal wall. Urethral foley was left in. Bladder rent was also closed with 3-0 barbed, single layer closure. Tube drain was placed at flank trocar site. With the use of barbed suture, the suturing time and overall operative time has reduced by 30 minutes. Also, 3D camera has improved the precise movements and surgeon's comfort level.
Results: The time taken for the surgery was between 150 to 180 minutes. Patients were in the hospital for 5 days. Drain was removed before discharge. Catheter was removed on the 14th day. None of the patients had a recurrence of the fistula.

Conclusions: Laparoscopic repair of gynecological vesicovaginal fistula is more efficient when barbed suture is used.

V12-13 Rare cause of persistent hematuria: Diverticulum formation after conservative management of extraperitoneal bladder injury associated with pelvic bone fracture

\section{Gr Choudhary, P Gupta, L Pallagani}

Introduction \& Objective: Urogenital injuries are commonly associated with pelvic bone fracture, the extent of injuries and organ involvement depends upon the mode of injury and the type of fracture. 83-95\% cases of bladder trauma are associated with a pelvic bone fracture. Extra peritoneal bladder injuries are usually managed conservatively with an indwelling per-urethral catheter for prolonged time. However, when associated with bony spicules projecting into the bladder or other concomitant injuries requiring exploration, such extraperitoneal bladder injuries should be repaired surgically. Here we report the management of a rare case of persistent hematuria after conservative management of extraperitoneal bladder injury associated with pelvic fracture.

Methods: A 41 years old gentleman suffered a road side accident 9 months ago, that time he sustained multiple site pelvic bone fractures and extraperitoneal bladder injury. The pelvic bone fracture managed with traction and bladder injury managed with continuous bladder drainage for 21 days. After removal of perurethral catheter patient voided well. However, he continued to have persistent hematuria and occasional dysuria, which aggravated on walking and physical exertion. on evaluation, he was found to have a bladder diverticulum anteriorly just proximal to bladder neck. Trans-peritoneal laparoscopic bladder repair was successfully performed and lead to rapid resolution of symptoms. Results: The urinary bladder is well protected by the bony cage of the pelvis. However high impact injuries may cause pelvic fracture and resultant bladder injury due to shear forces or bony spicules. Hematuria is a cardinal sign and is seen in almost all cases of bladder injury. If associated with blood at the meatus, concomitant urethral distraction injury should be suspected. Displacement of bone fragments leads to tear urinary bladder at its fascial attachments and in the index case because of comminuted fracture and persistent displacement of pubic symphysis bladder laceration did not heal. During surgery the defect was seen like diverticulum and that was the cause of non-remitting hematuria on movement of distracted bony fragments. We could repair this bladder injury laparoscopically and patient went home on day 2 of surgery.

Conclusions: Persisting bladder injury with pelvic bone fracture management may be challenging specially near to bladder neck, however laparoscopic repair is good option with minimal morbidity. 


\section{ABSTRACT VIDEO SESSION 13: SINGLE PORT ROBOTIC SURGERY}

\section{V13-01 Magnetic-assisted Robotic Radical Prosta- tectomy Using the Da Vinci SP Robotic System}

RL Steinberg, A Garbens, BA Johnson, JA Cadeddu

Introduction \& Objective: Robotic assisted radical prostatectomy has been rapidly adopted and is the most common surgical approach utilized in the United States. Retraction is typically achieved using robotic or laparoscopic instruments. Magnetic retraction using in place of $\mathrm{a} 4^{\text {th }}$ robotic arm has been reported using the da Vinci Si and Xi systems. The recent release of the daVinci SP robotic system (Intuitive, Sunnyvale, CA, USA) continues to minimize the surgical footprint on patients. We aim to evaluate the safety and efficacy of magnetic assisted robotic radical prostatectomy (MA-RRP) using the da Vinci SP robotic platform.

Methods: Eighteen patients underwent MA-RRP using the da Vinci SP platform and a LevitaÒ Magnetic Surgical System (Levita Magnetics, San Mateo, CA, USA) at a single institution from November 2018 to May 2019. The robotic cannula was placed $3 \mathrm{~cm}$ above the umbilicus in the midline. Abdominal access was obtained using the Hasson technique. A separate $12 \mathrm{~mm}$ assistant port was placed $10 \mathrm{~cm}$ from the robotic cannula in the left lateral abdomen to facilitate suction and suture passage. A suprapubic tube was placed at the end of the procedure which is standard for the surgeon. No drains were placed. Demographics, pre-operative disease characteristics, intraoperative, and outcomes data were all collected. Descriptive statistics were generated.

Results: All cases were successfully completed without conversion to a standard robotic system, placement of addition assistant ports or use of an assistant suction device. No intraoperative or immediate post-operative complications were noted. Mean operative time was $244 \pm 33$ minutes and mean blood loss was $262 \pm 114 \mathrm{~mL}$. All patients were discharged to home by postoperative day 2.

Conclusions: Magnetic-assisted robotic radical prostatectomy using the da Vinci SP robotic platform appears to be both safe and effective. Magnetic retraction continues to make the surgeon more autonomous and obviates the need for assistant-based retraction. Further analysis in larger cohorts and cost analysis is warranted.

\section{V13-02 Robotic, Single Port, Trans-vesical Bladder Diverticulectomy}

K Fareed, J GaristoRisco, J Kaouk

Introduction \& Objective: Bladder diverticulae complicate chronic bladder outlet obstruction \& can become retentive. Urine stagnation inside the diverticulae can account for persistent urinary retention despite relieving the infra-vesical obstruction, chronic urinary infections \& stone formation. Resecting large, retentive diverticulae is often indicated at the time of bladder outlet procedure. Smaller diverticulae may not need to be resected if the opening is wide (or been incised) as long as the bladder outlet obstruction has been relieved. We present Trans-vesical resection of bladder diverticulum using the DaVinci-SP ${ }^{\circledR}$ platform.

Methods: 77 years old patient with very large prostatic adenoma underwent simultaneous robotic Simple Prostatectomy \& Resection of a large, retentive bladder diverticulum. Single port access was obtained through mid-line supra-umbilical incision. DaVinci-SP ${ }^{\circledR}$ robotic platform was used. Extra-vesical dissection was attempted; however, trans-vesical dissection was found to be faster and safer in this case. A smaller diverticulum was managed by removing the calculi retained inside \& incising the diverticular neck. Ureteral stents were used to protect and help identify the ureters. intramural incision of the right ureter was managed by transverse closure \& stent for 6 weeks.

Results: Operative time including the prostatectomy was nearly 3 hours. Blood loss was $200 \mathrm{~mL}$. patient was discharged the following day and passed voiding trial 1 week later. No interval urinary infections or need for catheterization at 3 months follow up. Conclusions: Single port Robotic diverticulectomy is feasible and could be done simultaneously at the time of managing the prostatic adenoma. It provides a minimally invasive alternative to open surgical approach to such complex urological condition.

\section{V13-03 Circumferential Periumbilical Incision for Single Port Robot-Assisted Surgery}

JR Slawin, GW Dy, R Bluebond-Langner, LC Zhao

NYU Langone Health

Introduction \& Objective: We describe a novel skin incision used when performing surgery with the da Vinci Single Port Robotic Surgical System.

Methods: A $2.7 \mathrm{~cm}$ diameter circumferential skin incision is made around the umbilicus and carried down through the subcutaneous tissue while leaving the umbilical stalk intact. The fascia adjacent to the umbilicus is exposed by retracting the skin incision in a direction determined by the operative field. The fascia and peritoneum are entered, and the Single Port robotic trocar is inserted in the standard fashion. This technique allows for a cosmetic incision partially hidden within the umbilicus and places less tension on the skin than a standard linear incision.

Results: This technique was utilized in approximately 70 patients at a single institution between October 2018 and May 2019. This method of skin incision has been used for a wide variety of robotic reconstructive urologic procedures including penile inversion vaginoplasty, vaginectomy, pyeloplasty, ureteral reimplant, Boari flap, cystectomy and intracorporeal ileal conduit, bladder neck reconstruction, and posterior urethroplasty. No patients have yet to experience complications related to the incision.

Conclusions: A circumferential periumbilical skin incision is a versatile skin incision that can be used for a wide array of cases using the Single Port Robotic Surgical System. Moreover, it may have benefits compared to a standard linear incision.

\section{V13-04 Single Port Robotic Y-V Plasty of Bladder Neck Contracture}

K Fareed, J GaristoRisco, H Wood, J Kaouk

Introduction \& Objective: Management of Recurrent Bladder neck contracture is particularly challenging. When first line 
endoscopic management fails, major reconstructive procedures are the next option. At our institution we've performed Y-V Plasty of recurrent/recalcitrant BNC using conventional multiport Robotic approach. In this abstract we aimed at even less invasive approach utilizing the DaVinci ${ }^{\circledR}$ Single Port Robotic Platform.

Methods: Patient with recurrent, intractable BNC following multiple procedures for $\mathrm{BPH}$, who failed multiple attempts at endoscopic incisions, resections, dilations, underwent Robotic Single Port Y-V Plasty of the bladder neck after endoscopic confirmation of a stenotic ring proximal to the external sphincter $\&$ negative urine culture. In supine position, 3-cm open intraperitoneal access was obtained via midline supra-umbilical incision. The Alexis ${ }^{\circledR}$ wound protector/retractor ring was placed followed by the GelPoint-Mini ${ }^{\circledR}$ through which the DaVinici Single port trocar \& instrument cannula were placed. $12 \mathrm{~mm}$ AirSeal ${ }^{\circledR}$ assistant port was placed next to the main trocar. The DaVinici-SP Robot was dock'ed from the patient's side (no need for stir-ups/ lithotomy position). using mono-polar scissors, fenestrated grasper \& Prograsp forceps, the bladder was dropped from the anterior abdominal wall \& the space of Retzius was developed. The anterior Bladder wall was incised in Y-shaped fashion and the distal extent of the incision was extended through the stenotic ring of the bladder neck at 12 o'clock position with simultaneous cystoscopic guidance to ensure sphincter integrity. The cystotomy was then closed in V-shaped fashion, advancing an apex of healthy detrusor onto the the split bladder neck ring. 2 layers water-tight closure was achieved using 3-0, V-Locking (uni-directional, barbed) suture on $\mathrm{SH}$ needle. Foley was left for 3 weeks.

Results: Operative time was nearly 3 hours, with $20 \mathrm{~mL}$ estimated blood loss. pt was observed \& discharged home 15 hours later with Foley for 3 weeks. The Patient underwent successful voiding trial at 3 weeks. AUA-SS was 13 at 4 months post operatively compared to 26 pre-operative. QoL was down from 5 to 2. Flow Rate was $22 \mathrm{ml} / \mathrm{s}$ at 3 weeks \& 2 months, and 16 at 4 months.

Conclusions: Single port Robotic platform is a technically feasible approach to bladder neck reconstruction. It may offer a less invasive approach to the traditional open reconstruction or the standard Robotic, multi-port approach.

\section{V13-05 Robotic Assisted Laparoscopic Posterior Ur- ethroplasty Using the SP Robot}

M Jun, W Liu, GW Dy, X Meng, LC Zhao

NYU Langone Health

Introduction \& Objective: To describe our technique and outcomes for the treatment of posterior urethral stenosis with the da Vinci Single Port (SP) ${ }^{\circledR}$ platform.

Methods: We retrospectively reviewed 5 patients who underwent SP robot-assisted laparoscopic posterior urethroplasty (SPRALPU) by a single surgeon from October 2018-January 2019. Compared to multi-port robotics, the SP robot allows for improved exposure and less instrument clashing in the deep pelvis. We evaluated patient demographics, diagnosis, etiology, prior interventions, intraoperative variables, and outcomes. Success was defined as passage of a 17 Fr. flexible cystoscope or absence of urinary symptoms. The operative technique involves SP port placement at a periumbilical location for transabdominal mobilization of the bladder neck and urethra. Cystoscopy is used to identify the level of the urethral stenosis. The stenotic segment is excised, and the anastomosis completed using either excision and primary anastomosis or Y-V plasty. Combined abdominoperineal approach may be used for distal urethral mobilization to reduce tension.

Results: The mean age was 64.8 years. Posterior urethroplasty was performed for vesicourethral anastomotic strictures \pm rectourethral fistula or bladder neck contracture \pm urethral stricture, caused by prostate cancer treatments (brachytherapy, radiation therapy, prostatectomy), or prostatic urethra false passage due to traumatic intermittent catheterization in a patient with neurogenic bladder. Prior interventions included endoscopic balloon dilation, urethral incision under direct vision, and Foley catheter placement. Four patients underwent SPRALPU without open conversion. Mean operative time was 417 minutes, estimated blood loss $220 \mathrm{~mL}$, and length of stay 5.2 days. There were no intraoperative complications. One case required open conversion due to SP robot failure; however, surgery was completed robotically using the $\mathrm{Xi}$ robot. Post-operative complications included ileus $(n=2)$, small bowel obstruction $(n=1)$, deep venous thrombosis $(n=1)$, urinary tract infection $(n=3)$, and hematuria $(n=1)$. Two patients required cystectomies for osteomyelitis with urethrocutaneous fistula $(n=1)$ and recurrent necrotic debris $(n=1)$. Catheters were removed at a median of 44.2 days. All cases had patent urethral anastomoses with a median followup of 4.4 months. No patients experienced de novo urinary incontinence.

Conclusions: SPRALPU is a feasible approach to an otherwise difficult reconstructive procedure due to challenges in exposure

\section{V13-06 Pure single-site robot-assisted pyeloplasty with the da Vinci SP ${ }^{\circledR}$ system: Initial experience}

J Heo, S Kang, D Koh, J Na, Y Lee, W Han, Y Choi, W Jang

Introduction \& Objective: Laparoendoscopic single-site surgery (LESS) reduces the limited invasiveness of conventional laparoscopy, while providing superior cosmetic results. However, LESS remains a challenging surgical technique even in robotic surgery primarily due to the lack of triangulation and limited instrument movement. The da Vinci $\mathrm{SP}^{\circledR}$ system was recently introduced to overcome these limitations. We describe our initial experience with pure single-site robot-assisted pyeloplasty (RAP) for ureteropelvic junction obstruction (UPJO) using the SP system.

Methods: Three consecutive patients who were diagnosed with UPJO underwent RAP with the SP system from December 2018

Figure 1. Perioperative figures. (A) Umbilical incision; (B) Placement of GelPORT; (C) Docking; (D) Cosmetic result.

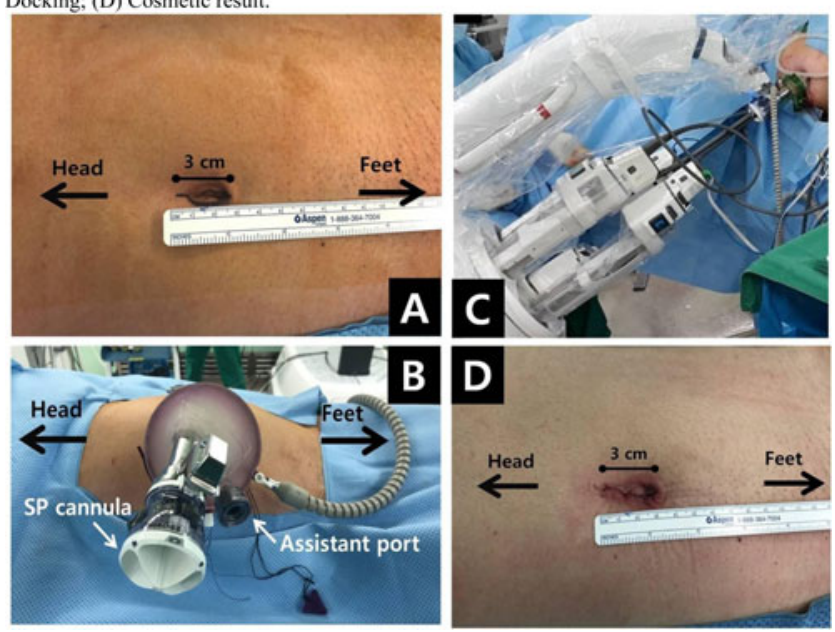


Table 1. Patients' characteristics and perioperative data

\begin{tabular}{llll}
\hline & Case No.1 & Case No.2 & Case No.3 \\
\hline Age & 42 & 30 & 71 \\
Sex & Male & Male & Female \\
Affected side & Left & Right & Left \\
BMI $\left(\mathrm{kg} / \mathrm{m}^{2}\right)$ & 22.49 & 27.08 & 18.82 \\
Preoperative eGFR $\left(\mathrm{ml} / \mathrm{min}^{2} \mathrm{~m}^{2}\right)$ & 91 & 108 & 43 \\
Grade of UPJO & High & Low & High \\
Operative time $(\mathrm{min})$ & 139 & 180 & 213 \\
Docking time $(\mathrm{min})$ & 5 & 10 & 10 \\
Console time $(\mathrm{min})$ & 118 & 145 & 185 \\
Suture time $(\mathrm{min})$ & 29 & 47 & 53 \\
Intraoperative complications & No & No & No \\
Conversion to other surgery & No & No & No \\
Estimated blood loss (ml) & Minimal & Minimal & 50 \\
Discharge & POD 4 & POD 3 & POD 3 \\
Postoperative eGFR $\left(\mathrm{ml} / \mathrm{min} / \mathrm{m}^{2}\right)$ & 105 & 113 & 81 \\
Postoperative complications & No & No & No \\
Pain at discharge, NPIS score & 2 & 2 & 3 \\
\hline
\end{tabular}

to February 2019 at our institution. The surgical technique involved reproducing the steps of multi-port RAP. A 30-mm umbilical incision was made, and the GelPOINT was inserted. The multichannel robotic port and the assistant's port were placed through the GelSeal cap. In all patients, Anderson-Hynes dismembered pyeloplasty was performed. The ureteral double-J stent was inserted antegrade, and the drain was not placed.

Results: The procedures were successfully completed using a pure single-site approach. There was no need for additional port placement or conversion to laparoscopic or open surgery. The total operative time was 139,180 , and 213 minutes for the three patients. No intraoperative complications occurred, and blood loss was minimal. The postoperative course of all patients was uneventful with no complications greater than Clavien-Dindo grade I surgical complications.

Conclusions: Pure single-site RAP using the da Vinci SP $^{\circledR}$ system is feasible and safe. Further studies involving more patients and long-term outcomes are needed to confirm our results.

\section{V13-07 Pure Transvesical Single-Port Robotic Simple Prostatectomy Using The New SP ${ }^{\circledR}$ da Vinci Surgical System}

GV Sawczyn, RJ Valero Carrion, J GaristoRisco, RJ Yau, J Kaouk

Introduction and Objective: to describe and present our technique of pure transvesical single-port robotic simple prostatectomy (pTSP-RSP) using the new $\mathrm{Sp}^{\circledR}$ da Vinci surgical system (Intuitive Surgical, Sunnyvale, CA).

Methods: Eight consecutive patients underwent to pTSP-RSP between February and May 2019. Using a transvesical approach, the patient is in a supine position, a $3 \mathrm{~cm}$ infra-umbilical incision is made after percutaneous needle identification of the bladder dome, a GelPOINT Mini advanced access platform (Applied Medical, Rancho Santa Margarita, CA), is inserted directly into the bladder. A $25 \mathrm{~mm}$ multichannel single-port robot cannula and a 12-mm accessory laparoscopic port are placed through the GelSeal cap and after plugged to the Alexis ${ }^{\circledR}$ wound retractor, followed by the docking of the da Vinci SP ${ }^{\circledR}$ robot. Pneumovesicum is achieved, the prostate is accessed, and the adenoma is enucleated. Finally, a mucosal advancement flap is sutured to the urethra to cover the resected area.

Results: Mean operative total time was 182.2 minutes, mean estimated blood loss was $350 \mathrm{ml}$ and the mean postoperative hemoglobin deficit was $2.5 \mathrm{mg} / \mathrm{dL}$. The mean length of stay was 37 hours and 26 minutes. Fifty percent of the patients went out of surgery with a 2-way foley catheter and no bladder irrigation. No drain or additional port was needed. No intraoperative complications were reported, and no surgeries were converted to open approach. There was no blood transfusion during or following the procedures.

Conclusions: Transvesical robotic simple prostatectomy using this technology is an option regarding minimally invasive techniques for treating benign prostatic hyperplasia with substantially enlarged glands. Our initial experience revealed that this procedure led to minimal bleeding, no need for additional ports, the possibility of skipping bladder irrigation, minimal usage of postoperative opioids, and thus favoring a shorter hospital stay.

\section{V13-08 Initial Report of da Vinci Single Port Robot- Assisted Orchiectomy for an Undescended Inguinal Testis}

\author{
R Munver, JF Tsui, K Basralian, B Desroches
}

Introduction \& Objective: Laparoscopic orchiectomy has been previously performed for undescended testes due to increased risk of testicular cancer in the affected testicle. As the robotic platform has continued to evolve with the introduction of the da Vinci SP (single port) platform (Intuitive Surgical, Inc.), the boundaries of robotic surgery continue to be advanced allowing for surgery to be performed through a single incision. Herein, we present a 40 y.o. male with a history of an undescended, nonintraabdominal, non-palpable left testis located in the inguinal canal proximal to the inguinal ring, who was referred for orchiectomy. We report on this patient who underwent the first single port robot-assisted laparoscopic left orchiectomy with the da Vinci SP ${ }^{\mathrm{TM}}$ Surgical System.

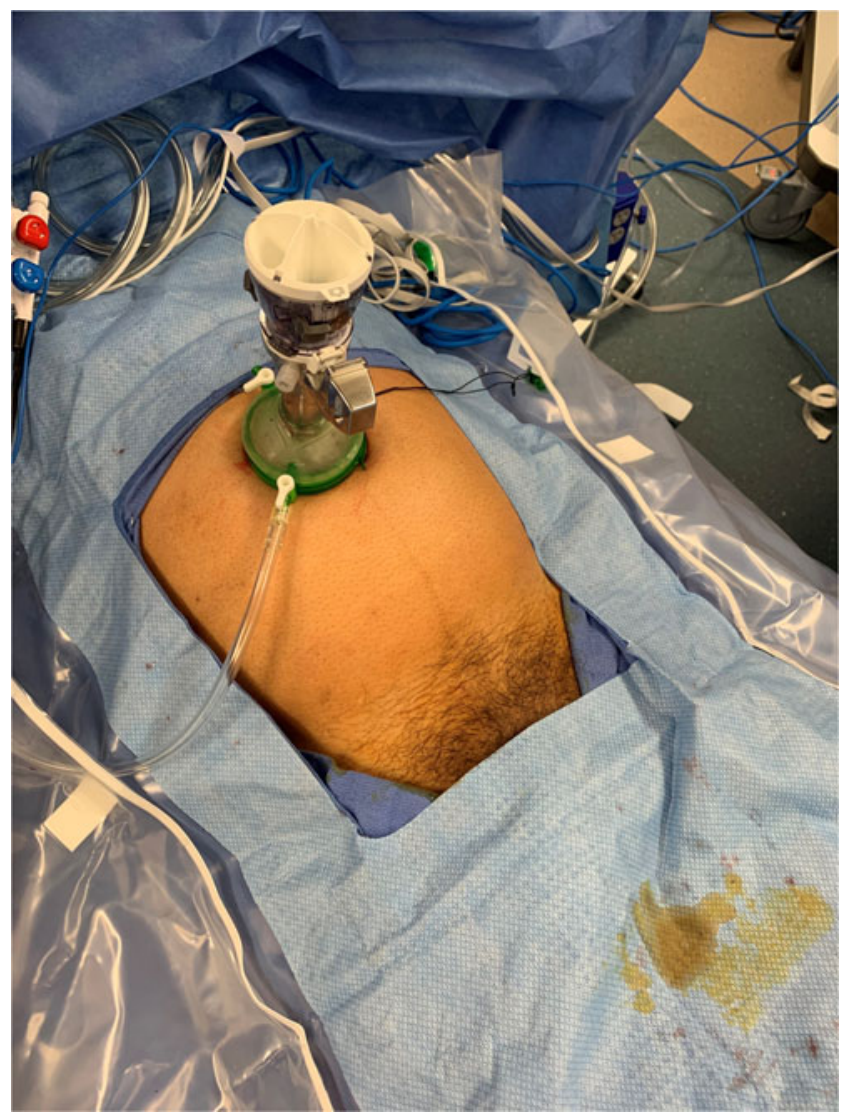


Methods: Abdominal access was obtained through a transverse supraumbilical incision carried through the fascia and into the abdomen where a GelPOINT (Applied Medical, Inc.) device was placed. The SP trocar was placed through the GelPOINT and the abdomen was insufflated (Figure 1). Due to the presence of adhesions in the vicinity of left vas deferens, the right vas deferens was initially identified to establish an appropriate landmark. Lysis of adhesions was performed on the left and then dissection was carried out carefully at the site mirroring the location of right vas. The left vas was identified, and dissection was carried distally until the cephalad portion of the left testis was identified. Traction was applied to the vas and left testicle was mobilized using a combination of blunt dissection and electrocautery. Once the testis was dissected free of its gubernacular attachment, the specimen was placed in a specimen bag and extracted through the periumbilical incision. The fascia was closed using interrupted monofilament sutures and the skin was closed with absorbable subcuticular suture.

Results: The patient had an uncomplicated course with a total operative time of 2 hours and 19 minutes. Estimated blood loss was minimal. The patient was observed overnight and was discharged on the first postoperative day. Pathology revealed a benign atrophic testis measuring $3.5 \times 2.0 \times 1.7 \mathrm{~cm}$ with attached spermatic cord showing focal fibrosis and chronic inflammation. Conclusions: We present the initial surgical application of the da Vinci SP ${ }^{\mathrm{TM}}$ Surgical System in performing robot-assisted laparoscopic orchiectomy.

\section{V13-09 Single Port Robotic Assisted Laparoscopic Radical Prostatectomy}

\section{Stern, M Billah, S Ahsanuddin, G Lovallo, M Ahmed}

Introduction \& Objective: Single Port system represents a new advance in surgical robotic technology. We set out to demonstrate the feasibility of single port prostatectomy even in setting of difficult anatomy.

Methods: Completion of robotic assisted laparoscopic prostatectomy with single port system. Assistant port and SP trocar were used via Alexis wound retractor and gel poart. Da Vinci SP system was used with bipolar forceps, Cadiere forceps, monopolar scissors and a single needle driver. Suction was controlled by robotic surgeon with use of Remotely Operated Suction Irrigation (ROSI) system.

Results: Prostatectomy was concluded in 151 minutes.

Conclusions: Single port robotic system can be used to safely and efficiently treat prostate cancer, even in setting of difficult anatomy.

\section{V13-10 Single Port Robotic Pyeloplasty: Similar Outcomes, Excellent Cosmesis}

M Billah, D Kim, PM Youssef, PD Kothari, JC Deolankar, SS Komaravolu, MD Stifelman

Introduction \& Objective: Single Port Robotic Assisted Laparoscopic Surgery is a new technology released by Intuitive in 2018. This technology further advances minimally invasive surgery. It enables multiple instruments with fully wristed motions through one $25 \mathrm{~mm}$ port. As with all new technologies, there is an associated learning curve and this video aims to demonstrate appropriate use of this technology for pyeloplasty.

Methods: 33-year-old female with no significant past medical history presented with right flank pain. Workup demonstrated right ureteropelvic junction obstruction.

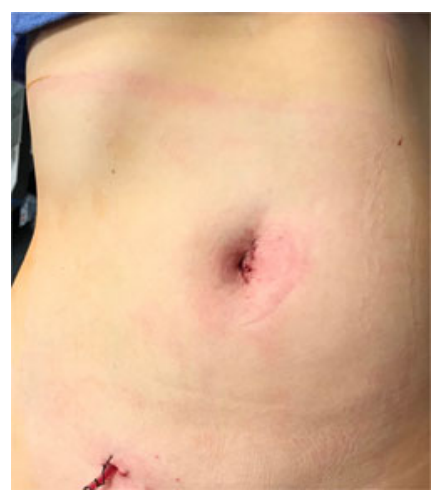

Results: Patient underwent single port robotic assisted laparoscopic right pyeloplasty. A single $25 \mathrm{~mm}$ incision was placed in the umbilicus for the robotic instruments. A $5 \mathrm{~mm}$ assistant port was placed in the ipsilateral lower quadrant within the pant line for the assistant port. The procedure mostly mimicked the multiport approach. Adjustments were made to instrument placement and traction to accommodate the different mobility of the single port system.

Conclusions: The single port enables excellent mobility and control. It is similarly safe and effective as the multiport. In our unpublished data we have found no significant difference between our multiport and single port outcomes.

\section{V13-11 Robotic, Single Port, Trans-peritoneal Simple Prostatectomy}

\section{K Fareed, J GaristoRisco, J Kaouk}

Introduction \& Objective: Robotic Assisted Simple Prostatectomy (RASP) is an alternative surgical option for patients with bladder outlet obstruction secondary to larger glands. Direct Intra-vesical insufflation and access may avoid consequences of trans-abdominal, intra-peritoneal surgery, however with large intra-vesical component of the adenoma, there may not be adequate intra-vesical space for surgical instrument manipulation and dissection.

Methods: Patients with bothersome, symptomatic and/or complicated Benign Prostatic Obstruction (BPO) with adenomas $>200 \mathrm{cc}$ (measured by TRUS, MRI or CT scan). Cystoscopic evaluation of the bladder \& Urodynamic proven BPO were confirmed in addition to adenoma measurements. With patients in supine position, a $3 \mathrm{~cm}$ supra-umbilical, midline open intraperitoneal access was obtained through which the DaVinci-SP was inserted. Multi-Instrument Cannula was introduced through the trocar. $12 \mathrm{~mm}$ AirSeal Assistant port was placed in right lower quadrant position. DaVinci-SP was docked. The bladder was dropped from anterior abdominal wall, then anterior cystotomy was performed. The adenoma was dissected from the bladder neck, prostatic fossa \& the apical attachments. The Prostatic fossa was oversewn with running hemostatic suture. Continuous bladder irrigation was performed for 1 day and voiding trial was performed 1 week postoperatively.

Results: We have performed the procedure on 3 patients with chronic retention (indwelling Foley or suprapubic catheters). Adenoma volumes ranged from 240-433 cc. Mean operative time was 3 hours, blood loss ranged from $400-800 \mathrm{ml}$. \& hospital stay ranged from 1-7days. All patients passed voiding trials with mild to moderate Stress incontinence (2-5 pads per day). 
Conclusions: Single Port Robotic Simple Prostatectomy offers less invasive alternative to patients with very large prostatic adenomas. Using standard trans-peritoneal approach (as opposed to direct trans-vesical access) is more suitable for patients with large intravesical adenoma component.

V13-12 Same Day Discharge Extra-peritoneal SinglePort Robotic Radical Prostatectomy: New Device, New Approach, New Paradigm

RJ Valero Carrion, GV Sawczyn, J GaristoRisco, RJ Yau, J Kaouk

Introduction \& Objective: To describe and present our technique and protocol of extraperitoneal single-port robotic radical prostatectomy (extraP-SPRRP) using the new $\mathrm{Sp}^{\circledR}$ da Vinci surgical system (Intuitive Surgical, Sunnyvale, CA).

Methods: From February to May 2019 we have performed 34 cases of extraP-SPRRP. Data was prospectively collected regarding demographics and perioperative outcomes including age, BMI, PSA, NCCN cancer risk classification, operative time, estimated blood loss, complications, length of stay, days with a foley catheter and surgical pathology report. Surgical technique: a $3 \mathrm{~cm}$ infraumbilical incision is done, extra-peritoneal space is created using a kidney shape space-maker balloon (Covidien, Dublin, Ireland). After that, a GelPOINT Mini advanced access platform (Applied Medical, Rancho Santa Margarita, CA) a dedicated 25-mm multichannel port and a 12-mm accessory laparoscopic port are placed through the gel-seal cap and plugged with into the same incision and the robot is docked. Robotics instruments are introduced, and the procedure is done replicating the technique described for transperitoneal single port prostatectomy. Intraoperative protocol: patient in a supine position, no stirrups, minimal use of opioids and $30 \mathrm{mg}$ of ketorolac divided into two doses - one after finishing the procedure and one before discharge from PACU.

Results: Thirty-four cases with a median operative time $203 \pm 37.9$ minutes, estimated blood loss $175 \pm 334.9$, no intraoperative complications were recorded, no drain, conversion or transfusion were needed. Ninety percent of patients had a mean length of stay less than 24 hours with a mean of 5 hours. Sixty percent of patients have no need for pain medication in the postoperative time. Median days with a catheter was 7 days. Positive margins were present in $9(27 \%)$ patients and one patient presented an abscess that required percutaneous drainage.

Conclusions: Our technique and protocol of ExtraP-SPRRP using this new technology is safe, feasible and suggest the possibility of outpatient management in most of the cases with a significant reduction in postoperative pain, use of analgesic medication and length of stay.

\section{ABSTRACT VIDEO SESSION 14: ROBOTIC PARTIAL NEPHRECTOMY}

\section{V14-01 Microwave Assisted Clampless Laparoscopic and Robotic Partial Nephrectomy}

B Pham, A Parker, R Venkatesh

Introduction \& Objective: Robotic assisted laparoscopic partial nephrectomy, in the majority of cases, is performed with renal hilar vessel(s) clamping and parenchymal renorrhaphy. Clampless laparoscopic partial nephrectomy is associated with bleeding and poor visualization of resection margin. The microwave needle ablation system has been successfully used for percutaneous ablation of liver, lung and renal masses. We present the feasibility of clampless renal tumor resection without parenchymal renorrhaphy using microwave assistance.

Methods: We recruited selected patients with a solitary small renal mass who were candidates for partial nephrectomy. Choice of laparoscopic or robotic partial nephrectomy was performed based on tumor location and surgeon preference. We utilized the Neuwave ${ }^{\mathrm{TM}}$ microwave system, Ethicon, WI, at 95 watts for 30-45 seconds pulse for renal tissue coagulation and hemostasis. A $17 \mathrm{G}$ probe was utilized to create a $1 \mathrm{~cm}$. diameter coagulation zone extending proximally from the tip of the needle. The needle was inserted into normal renal tissue along an ultrasound guided pre-marked line of resection around the tumor. Tumor resection was performed through coagulated renal tissue around the tumor. We avoided use of microwave when close to the collecting system. Collecting system openings were closed with a deep running suture. No parenchymal renorrhaphy or hilar vessel(s) clamping was performed.

Results: Three patients underwent laparoscopic assisted partial nephrectomy. Two patients underwent robotic assisted laparo- scopic transperitoneal partial nephrectomy and one patient underwent laparoscopic retroperitoneal partial nephrectomy. All three patients were discharged on postoperative day 1 in good condition with an average decrease in hemoglobin of $0.67 \mathrm{~g} / \mathrm{dL}$ and an average elevation of creatinine at $0.24 \mathrm{mg} / \mathrm{dL}$. The average estimated blood loss was $60 \mathrm{~mL}$ with an average operative time of 168.33 minutes. Size of mass ranged from $1.3 \mathrm{~cm}-3.4 \mathrm{~cm}$ on pathology and all patients had negative tumor margins. At a median follow-up of 12 weeks, all patients were doing well without complications.

Conclusions: It was feasible to perform clampless laparoscopic/robotic partial nephrectomy utilizing needle microwave assistance without parenchymal renorrhapy in selected patients. Needle microwave assisted tissue coagulation, except near the collecting system, provided effective hemostasis without any urine leak/fistula. Use of intraoperative doppler ultrasound may facilitate more precise coagulation of peritumoral blood vessels.

\section{V14-02 Robotic assisted partial nephrectomy for en- dophytic renal tumor}

T Lin, C Ou, Y Lin

Introduction \& Objective: Partial nephrectomy for an endophytic renal tumor can be difficult. Even more challenging in robotic assisted surgery. Here we introduce the surgical procedure of robotic assisted partial nephrectomy to treat a case with left endophytic renal tumor. 
Methods: This 50-year-old male suffered from epigastric pain. Then computed tomography and MRI were done and left renal nodule, suspected renal cell carcinoma, was incidentally found. He received left robotic assisted partial nephrectomy. During the operation, we performed cystoscopy and left R-P tube placement first. Robotic system we used was Da Vinci Si Surgical system. The patient was placed in a modified right lateral decubitus position at 60 degrees with a gel roll supporting the lower back. The camera port was set at the left lateral border of the rectus muscle three to four centimeters above the umbilicus. The two $8-\mathrm{mm}$ robotic instrument ports were placed $8-10 \mathrm{~cm}$ from the camera port and more laterally. An assistant $12-\mathrm{mm}$ port was placed below the umbilicus in the midline and for left-sided procedures an additional 5-mm port was placed. After ports setting, we performed partial nephrectomy using transperitoneal approach. The followed surgical steps were colon mobilization, hilar dissection, exposure of renal surface, intra-OP ultrasound and surface marking, renal pedicle clamp, remove disc of renal cortex, 2nd intra-OP ultrasound to identify tumor, excision of endophytic tumor, identify and repair renal pelvis defect, and rhinorrhaphy.

Results: After operation, the patient recovered smoothly. The pathology report showed renal cell carcinoma, clear cell type and the surgical margin was free from tumor involvement.

Conclusions: We share our successful experience in dealing with renal endophytic tumors using robotic assisted partial nephrectomy.

\section{V14-03 Management of Major Complication After Robot Assisted Partial Nephrectomy}

\section{B Onal, M Simsekoglu, U Aferin, B Ercili, A Erözenci}

Introduction \& Objective: Partial nephrectomy is a gold standard curative option in patients with T1 stage renal cancers. As a result of technological developments, nowadays robot assisted partial nephrectomy became more beneficial and frequently preferred technique. Thus, that it's important to optimize perioperative and postoperative management of complications in robotic surgery. In this video, we present our initial experience with managing a major complication in a patient with a history of hemorrhage after robot assisted partial nephrectomy.

Methods: 62-year-old man presented with an incidental left renal mass. Ultrasonography demonstrated a cystic lesion. Magnetic Resonance imaging confirmed a $3.3 \times 3.1 \times 3 \times 8 \mathrm{~cm}$ solid, contrast enhanced renal mass that localized at lower pole of left kidney (RCC). PADUA score was 7. We performed robot assisted left partial nephrectomy. Perioperative hemorrhage was $100 \mathrm{cc}$. Warm ischemia time was 26minute. Operation time was 3 hours. There was no other unexpected event during the operation. Postoperative early hgb/hct was $10.1 / 30.5$. Postoperative first day in early morning, patient had syncope, hypotension and tachycardia. Patient had sensitivity in the abdominal examination. Urgent ultrasonography demonstrated a $7 \times 6 \mathrm{~cm}$ collection area in retroperitoneum (hematoma). Patient quickly referred to department of interventional radiology. Endovascular coiling had performed to selective artery of kidney with two coils. The patient's post angioembolization course was uneventful without any other postoperative complications. Patient discharged after 5 days follow up. Pathology report showed clear cell type of renal cell cancer. Pathological size was $3.9 \mathrm{~cm}$. Surgical margin was negative (T1N0M0).

Results: Angioembolization is an ideal option that prevent patients from diagnostic laparoscopy or laparotomy in case of hemorrhage after robot assisted partial nephrectomy.
Conclusions: Robot assisted partial nephrectomy is technically feasible in patients with T1 stage renal cancer. However, ideal technique and timing is an important part of treatment for the management of complications developing after robot assisted partial nephrectomy.

\section{V14-04 Sutureless technique in robot-assisted partial nephrectomy}

M Ishida, Y Miyazaki, Y Konnai, K Shojo, H kawasaki, S Watanabe, H Kobayashi, M Kosugi

Introduction \& Objective: Bleeding is one of the most unfavorable post-operative complications of partial nephrectomy. It is generally accepted that renorrhaphy is necessary for hemostasis because conventional coagulation is uncertain for hemostasis of renal tumor bed. In soft coagulation mode, the output voltage is regulated below $190 \mathrm{~V}$ and generates Joule heat without producing sparks which cause tissue carbonization. Carbonized tissue sometimes drops off and it leads bleeding, however protein degeneration caused by Joule heat is durable. We introduce our minimally invasive sutureless technique using soft coagulation in robot-assisted partial nephrectomy (RAPN).

Methods: We performed RAPN for 76 consecutive patients with cT1 renal tumor from December 2014 to March 2019. We utilize VIO 300D (ERBE, Germany) for hemostasis of renal tumor bed. The device was set in a "soft coagulation" mode with a power setting of effect 7 and $80 \mathrm{~W}$. In each case, the renal artery was clamped without cooling. During tumor excision, the assistant used a suction/irrigator and a monopolar electrocautery coagulating renal tissue concurrently with the resection of tumor. The renal artery was unclamped after the bleeding was controlled using soft coagulation (early unclamping). After unclamping, additional coagulation was done until complete hemostasis was gained. We didn't suture the renal parenchyma when sufficient hemostasis was achieved. The renal artery was re-clamping if there was an arterial bleeding and additional coagulation was done.

Results: Twenty-four out of 76 cases underwent RAPN by complete sutureless technique which carried out with neither suture nor renorrhaphy. There was no case with above 10 points of RENAL score, 6 cases were with between 7 to 9 points and 18 cases are with below 6 points of the score. The median age was 65 (39-85) and the median BMI was $24.6 \mathrm{~kg} / \mathrm{m}^{2}(19.3$ - 31.8). The median console time was $106 \mathrm{~min}$ (71-169) and the median estimated blood loss was $21 \mathrm{ml}(0-250 \mathrm{ml})$. The median tumor size was $2.3 \mathrm{~cm}(1.1-4.0 \mathrm{~cm})$ and the median warm ischemic time was $9.0 \mathrm{~min}(6.0-29.1 \mathrm{~min})$. All cases had negative surgical margins. The median rate of change in estimated glomerular filtration rate (eGFR) was $-3.9 \%(-22.7-24.4 \%) 1$ month postoperatively. Clavien-Dindo Grade IIIa urinoma was occurred in 2 patients and postoperative bleeding was not observed in all cases.

Conclusions: Soft coagulation is an effective technology for tissue hemostasis. Sutureless hemostasis together with early unclamping is safe and feasible minimally invasive technique for RAPN.

\section{V14-05 Robot-assisted Partial Nephrectomy in Pa-} tient with solitary Kidney and multiple renal masses

\section{O Aytac, A Garayev, F Atug}

Introduction \& Objective: In present report we aimed to share our experience of robot-assisted partial nephrectomy in patient with solitary kidney with 2 renal masses. 
Methods: A-39-year-old male patient with left robotic radical nephrectomy operation history presented with newly developed right kidney mass. A computed tomography (CT) scan revealed a $2-\mathrm{cm}$ solid mass near to renal hilum as well as $4 \mathrm{~cm}$ mass in the posterior mid-pole with close relation to renal calyces. Before proceeding to robotic partial nephrectomy step, cystoscopy was performed to place ureteral catheter to fulfill of the collecting system for identification of calyces. After positioning in flank position two $8-\mathrm{mm}$ robotic ports, $12-\mathrm{mm}$ periumbilical camera port, airseal port and 5-mm assistance ports were placed. After incising of Toldt white line the bowel and after that duodenum completely mobilized medially. After identification of ureter and gonadal vein dissection continued toward the renal hilum. According to CT arterial images hilar microdissection was performed to identify specific posterior renal artery branches supplying the tumor. A small tumor $(2 \mathrm{~cm})$ was resected without arterial clamping. Posterior renal artery branch occlusion was used to devascularize the tumor. Pelvi-calyceal entry was repaired with absorbable suture in a watertight manner. Sliding-clip renorrhaphy was performed for renal parenchymal hemostasis.

Results: There were not any peri- and postoperative complication. Estimated blood loss was $200 \mathrm{ml}$. Operation time was 145 mins. Warm ischemia time was 25 mins. Preoperative and postoperative $2^{\text {nd }}$ day blood creatinine levels was $0,9 \mathrm{mg} / \mathrm{dl}$ and $1 \mathrm{mg} / \mathrm{dl}$. Foley catheter and drain catheter were removed at $2^{\text {nd }}$ and $3^{\text {rd }}$ days, respectively. Final pathology reported clear cell renal cell carcinoma with negative surgical margins.

Conclusions: Robot-assisted partial nephrectomy for multiple renal tumors in solitary kidney offers feasible and safe treatment option. Delicate hilar dissection may provide selective arterial clamping with minimally renal function loss.

\section{V14-06 Off-clamp Robot-assisted Partial Ne- phrectomy (RPN) for cT2 renal cancers}

G Simone, U Anceschi, A Brassetti, M Costantini, M Ferriero, R Flammia, S Guaglianone, R Mastroianni, F Minisola, G Tuderti, M Gallucci

Introduction \& Objective: To present our series of cT2 renal tumors treated with off-clamp Robot-assisted Partial $\mathrm{Ne}$ phrectomy (RPN).

Methods: From July 2010 to September 2017, a consecutive series of patients undergoing RPN for large (cT2) renal tumors at our center were prospectively enrolled. A contrast CT scan was always performed at baseline and the R.E.N.A.L. score calculated. An off-clamp partial nephrectomy was always attempted in these cases: only patients reporting gross hematuria were not offered a nephron-sparing approach. In the video we present the case of a 40 years old woman, with no comorbidities, with an incidental diagnosis of cT2aN0M0 tumor, mainly endophytic, posteriorly located, at the mid third of her solitary functioning right kidney. With the patient laying on the left flank, 5-trocars (three for the DaVinci system and two for the assistant) are placed as per standard robotic kidney surgery. With monopolar cautery, the line of Toldt is incised and the ascending colon is medialized. The Gerota fascia is incised and the posterior aspect of the kidney is directly accessed. The hilum is neither identified nor prepared. Following the surface of the kidney, the tumor is identified and its peripheral limit with the healthy parenchyma is marked with monopolar coagulation. The enucleation is started at the level of the coagulated tissue and the almost avascular plane of the pseudo capsule is followed. The table-side assistant uses the suction device to compress small bleeding vessels eventually encountered, thus contributing to maintain a clear surgical field. These are further secured with pin-point coagulation. Once the enucleation is completed, the parenchymal breach is sutured with a "sliding clips" technique.

Results: Overall, 48 patients were treated, $75 \%$ were male, median age was 61 yrs (IQR: 52.5-68.7) and BMI 26.4 (24.929.4). Median tumor size was $84 \mathrm{~mm}(79.5-100)$ and most of cases $(84 \%)$ had a RENAL score $\geq 10$. Median operative time and length of hospital stay were $70 \mathrm{~min}$ (70-80) and 5 days (4-5), respectively. Median estimated glomerular filtration rate reduction was - $12 \%(-37 /+4)$. Two (4\%) Clavien Dindo grade $\geq 3$ occurred. The trifecta was achieved in two third of the cases.

Conclusions: Nephron sparing surgery is a safe and feasible option, also in patients with cT2 renal masses. In experienced hands, purely off-clamp RPN is a reasonable approach, providing negligible impact on renal function.

\section{V14-07 The Utility of 3D Virtual Reality Models In Preoperative Planning For Robotic Partial Ne- phrectomy}

BD Dayal, AA Elbakry, KE Okhawere, K Meilika, A Beksac, KK Badani

Introduction \& Objective: The review of pre-operative CT scan or MRI are usually useful when planning for partial nephrectomy of small renal tumors. However, axial imaging modalities provide two-dimensional images and intraoperative anatomic findings may be different. A novel 3D modeling software (Ceevra Inc, San Francisco, CA) can be used to create 3D models based on the preoperative CT scan or MRI. This technology is currently under investigation in a multi-center trial to determine utility in surgical planning.

Methods: We are participating in a multi-institutional prospective randomized controlled trial to evaluate the impact of using 3D Virtual reality models in planning for robotic partial nephrectomy. In this video, we present a case of partial nephrectomy demonstrating the utility of a virtual reality $3 \mathrm{D}$ model. The model can demonstrate separate arterial supply, venous drainage, collecting system, and tumor location. In addition to the model, we also utilized other available technologies including ultrasound and immunofluorescence vision.

Results: Based on the model, there was a distinct arterial and venous supply to the tumor and kidney. We planned on dividing the tumor hilum and clamping only the lower artery/vein for ischemia to the normal kidney. Warm ischemia time was 11 minutes, and the remnant kidney was well perfused after this approach. The estimated blood loss was $100 \mathrm{~mL}$. The patient was discharged on postoperative day 1 . The pathology report showed chromophobe renal cell carcinoma, confined to the kidney with negative surgical margins.

Conclusions: We were able to successfully plan a complex partial nephrectomy procedure using a 3D model created by preoperative imaging. After completion of the ongoing study, we will be able to assess the benefit of using 3D models in clinical practice.

\section{V14-08 Robotic Partial Nephrectomy after Previous Open Ipsilateral Partial and Contralateral Robotic Partial Nephrectomy}

\section{S Aly, JF Tsui, R Munver, B Desroches}

Introduction \& Objective: The approach to performing a partial nephrectomy $(\mathrm{PN})$ for a metachronous renal cell carcinoma in a patient with a prior history of an ipsilateral open PN can be 
extremely challenging. Repeat PN in the ipsilateral kidney is considered a highly complex procedure due to the presence of dense adhesions that may form as a result of prior mobilization of the kidney and renal hilar dissection. Oftentimes, radical nephrectomy or open PN are the preferred forms of surgical management. We have extensive experience in approaching these tumors with minimally invasive PN. Herein, we present a 66 y.o. female with a history of right lower pole open PN and left robotassisted PN (RPN) in whom we performed a right lower pole RPN for a new, nephrometry score 8a, right lower pole renal mass.

Methods: Abdominal access was obtained using a Veress needle and trocars were placed in the as shown in Image 1. Once the liver was mobilized, the lower pole of the kidney was meticulously freed due to the presence of extensive perinephric adhesions. The colon was mobilized and investing tissues covering the vena cava were incised to further exposure. The gonadal vein and ureter were encased in fibrotic tissue and were dissected carefully. Adipose tissue lateral to the ureter and below the lower pole of the kidney outside of Gerota's fascia was dissected off the psoas muscle and divided with extensive effort due to the presence of severely fibrotic tissue. In progressing from the lower pole towards the mid kidney, the renal artery was identified posterior to the renal vein and was meticulously dissected free from surrounding tissues. Renal ultrasonography was performed. The renal mass was excised following arterial clamping with cold scissors, ensuring an adequate surgical margin. The base of the excision defect was oversewn with running 3-0 Vicryl suture. Renorrhaphy was then performed with interrupted 0 Vicryl sutures using Hem-o-lok and Lapra-ty clips, and the defect was closed in a clam style fashion over a bed of Floseal.

Results: The patient had an uncomplicated procedure with an operative time of 3 hours and 37 minutes and estimated blood loss of $270 \mathrm{~mL}$. Warm ischemia time was 35 minutes. The patient had an uncomplicated postoperative course and was discharged on the second postoperative day. Pathology revealed a $3.4 \mathrm{~cm}$ clear cell renal cell carcinoma with nuclear grade 4 , confined to the kidney with negative margins of resection. Renal function was preserved with postoperative creatinine of 0.83 (eGFR 68.78).

Conclusions: Despite increased difficulty, robot-assisted partial nephrectomy after previous ipsilateral open partial nephrectomy can be performed safely with excellent outcomes at experienced centers.

V14-09 Renorrhaphy during robotic partial nephrectomy: technique evolution

A Patel, T Poloskey, C Rogers

Introduction \& Objective: Robotic partial nephrectomy (RPN) is well established in the management of the small renal mass. Renorrhaphy is a key step in achieving hemostasis and closure of collecting system whilst maximizing preservation vascularized renal parenchyma. Techniques, suture material and clip applicators have evolved over time, allowing for a multitude of approaches; with this video we demonstrate our current strategies for rennorrhaphy during RPN.

Methods: 3 cases were selected of dual (inner and outer) layer renorrhaphy from our institution's video library collected over the last 10 years. Cases were chosen to demonstrate, key features in renorrhaphy and it evolution with time.

Results: Case 1 demonstrates, inner layer renorrhaphy (ILR) with a 3.0 monocryl suture with attention paid at closure of venous sinuses, collecting systems and exclusions of clips. Outer layer renorrhaphy (OLR) is completed with interrupted 2.0 vicryl. Sutures are secured with Lapra-TY Case 2 demonstrates use or 3.0 monocryl-barbed suture, for ILR and OLR with interrupted 2.0 moncocryl with omission of lapra-TY. Case 3 shows continuous 2.0 monocryl-barbed suture with sliding clip renorrhaphy with multifire WECK clip applicator.

Conclusions: In this video we have demonstrated evolution of the dual layer renorrhaphy technique with the adoption of barbed suture for both inner and outer layers, allowing for the omission of lapra-TY clips. Continuous OLR using barbed suture with multi-fire weck clip applicator permits for more efficient closure and improvement in ischemia time.

V14-10 Robotic-assisted laparoscopy Partial nephrectomy for intra parenchymal renal tumor assisted by endoscopic ultrasound in a patient known case of multi cystic kidney disease.

\section{A Alkhalili, F Adawi, M Alhamad}

Introduction \& Objective: Small renal tumors is a common finding associated with multi cystic kidney disease in our practice. In this video we describe our technique for performing partial nephrectomy by robotic-assisted laparoscopy partial nephrectomy combined with the use of endoscopic ultrasound probe intraoperatively to locate and find the tumor and cystic limitations.

Methods: A 53-year-old patient presents with a $3 \mathrm{~cm}$ renal tumor incidentally found by ultrasound performed for an abdominal pain. The assessment and investigations completed by an MRI and a CT scan confirms an intra-parenchymal Medio-renal tumor. In addition, there are 2 large simple cysts, located at the upper and lower renal adjacent to the tumor. We used Robot Da Vinci Xi 4 arms, and endoscopic ultrasound probe to locate and delineate the tumor and cysts. We performed a resection of the upper pole cyst and then we resected the tumor, macroscopic examination of the resected tumor shows resection in the negative margin. We Closed the renal parenchyma with 2 layer, superficial and deep layer by continues suture using a $3 / 0 \mathrm{~V}$ lock thread, blocked with multiple hem-o'lok clips, Bleeding $20 \mathrm{CC}$, No drainage.

Results: The duration of procedure was 129 minutes, arterial clamping time 18 minutes, the patient left the hospital on the next postoperative day, the histopathological examination confirms a renal cell tumor classify PT1a, R0. The CT scan at 3 months was normal.

Conclusions: The treatment of small intra parenchymal renal tumor associated with renal cysts may be achieved by roboticassisted laparoscopy the use of intraoperative endoscopic ultrasound provides more safety and facilitates operation with more precision.

V14-11 Robotic-assisted laparoscopic partial nephrectomy for renal cell carcinoma in horseshoe kidney: a hybrid technique with conventional laparoscopic surgery

Y Muto, K Numakura, T Nara, S Kanda, M Huang, M Saito, S Narita, T Inoue, S Satoh, T Habuchi

Introduction \& Objective: Robotic-assisted partial nephrectomies (RAPN) have come up to standard treatment for small renal 
tumors, with a growing indication to accomplish this procedure. Although a horseshoe kidney is one of the most common congenital renal fusion anomalies, surgical planning for tumors is considered difficult because of poor mobility and abnormal vascular supply.

Methods: We showed our experience of RAPN in combination with conventional laparoscopic kidney mobilization and dissection in a patient with renal cell carcinoma in a horseshoe kidney. Results: A 66-year-old male was referred to our hospital with right incidental renal mass. A CT scan revealed $26 \mathrm{~mm}$ renal mass (cT1aN0M0) in the posterior of the right lower pole of the horseshoe kidney and two right renal arteries. Port placement was the same as the standard RAPN with one camera port, two robotic arm ports, and three assistants' ports. By conventional laparoscopic procedure without robotic assistance, the two right renal arteries were exposed and secured by vascular tapes. The right ureter and an unexpected artery were identified and secured by vascular tapes. After the right lobe of the horseshoe kidney was almost completely mobilized and exposed, the robot was docked, and the tumor was exposed. After the excision margin was marked by coagulation, the three arteries were clamped individually, and the tumor was successfully removed with a $0.5 \mathrm{~cm}$ rim of normal parenchyma. The inner medullary hemostatic sutures were performed, followed by early unclamping and additional hemostatic sutures. Total operative time was 290 minutes and warm ischemic time was 12 minutes. The postoperative course was uneventful. Pathological diagnosis was T1a Grade 2 clear cell renal carcinoma with a negative surgical margin.

Conclusions: Renal cell carcinoma in a horseshoe kidney is a rare entity and minimally invasive surgery for that can be challenging because of its poor mobility and abnormal vasculatures.
RAPN in combination with conventional laparoscopic technique, which endows sufficient mobilization and identification of unexpected vasculatures, is a feasible and may be the best option in the treatment of a small tumor in the horseshoe kidney.

V14-12 Robotic zero ischemic partial nephrectomy with quantitative three dimensional reconstruction and triple-screen real-time display (Tilepro ${ }^{\circledR}$ )

W Xiong, P Guo

Introduction \& Objective: High definition virtual threedimensional reconstruction and triple-screen real-time display (Tilepro ${ }^{\circledR}$ ) imaging method would facilitate robotic partial nephrectomy. Herein, we display our techniques in the robotic zero ischemic partial nephrectomy.

Methods: A 72 years old female was admitted for the detected right renal mass with $8 \mathrm{~cm}$ diameter by CT scan. She had hypertension for $10+$ years. The NSS-complexity evaluation indicated that R.E.N.A.L. score 10xh pts, PADUA score 11 pts and $\mathrm{ABC}$ score $3 \mathrm{H}$. The nephron sparing surgery was performed by robotic.

Results: The robotic zero ischemia partial nephrectomy was performed successfully, and the patient recovered evenly with unchanged eGFR before and after operation.

Conclusions: Quantitative three-dimensional virtual reconstruction and triple-screen real-time display could improve the surgeon's intuition and risk management in precise partial nephrectomy.

\section{ABSTRACT VIDEO SESSION 15: ROBOTIC SURGERY: UPPER TRACT}

\begin{abstract}
V15-01 Robot-assisted ureteral stricture resection and primary anastomosis in a solitary kidney: illustration of an optimized technique utilizing indocyanine green and reconstructive surgical principles
\end{abstract}

PM Gurung, G Wu

Introduction \& Objective: Ureteral strictures causing obstructive renal impairment in solitary kidneys are challenging entities to manage. Definitive surgical correction of such ureteral strictures, particularly in patients with prior complex abdominal surgery, needs to be carefully planned with the surgeon prepared to utilize a wide range of techniques in his/her armamentarium. Robot-assisted reconstructive procedures, which are increasingly reported in the literature, attempt to recapitulate open surgical techniques in a minimally-invasive platform. Herein, our objective was to demonstrate, in our video, our technique of safely and effectively treating such challenging ureteral strictures.

Methods: A 68-year-old female patient was referred by the Vascular surgeons after being found to have an atrophic Left kidney and an obstructed Right kidney 4 months following an Open Aorto-Bifemoral Bypass surgery. The right kidney was nephrostomised and nephrostograms suggested a tight $2 \mathrm{~cm}$ stricture in the mid-ureter. Cystoscopic retrograde studies and ureteroscopy confirmed this. A ureteral stent was possible, but the patient preferred a definitive surgical correction. Therefore, robot-assisted "laparotomy" was performed to release intraabdominal adhesions and to release the Right ureter. The strictured segment of mid-ureter was identified by mobilizing the ureter and by deploying indocyanin green (ICG). Use of ICG allowed resection until healthy ureteral margins remained. Release of the ipsilateral medial umbilical ligament and bladder allowed further mobilization of the distal ureter. A ureteroureteral anastomosis was performed using semi-continuous 4:0 dissolving (Stratafix) suture over a 6Fr ureteral stent. Tacking sutures were placed to relieve any tension on the anastomosis. Posterior peritoneal tissue flaps were mobilized and placed behind the anastomosis to further secure the anastomosis.

Results: On post-operative day 1, the patient was pain-free after overnight clamping of the nephrostomy tube. The pelvic drain output was $110 \mathrm{ml}$ and was proven to be biochemically serous. Renal function was stable. As such, the patient was discharged after removal of nephrostomy, pelvic drain and urethral catheter. The ureteral stent was removed in the office at 6 weeks.

Conclusions: Robot-assisted resection of relatively short segments of strictured ureter with primary uretero-ureteral anastomosis can be safe and feasible, even in a solitary kidney within a hostile abdomen with complex prior surgery, when deploying 
traditional reconstructive techniques and more recent applications such as ICG on the robotic platform.

V15-02 Left sided Robot assisted radical Nephrectomy for RCC with thrombus in the renal vein reaching up to IVC

\section{S Schakaki}

Introduction \& Objective: Left-sided radical nephrectomy for RCC with large thrombus in the renal vein is one of the most challenging surgical procedures. The technique of robot assisted left nephrectomy for a thrombus reaching up to the edge of the IVC is still uncertain. Our objective is to demonstrate the feasibility and key steps of the robotic surgical treatment in left kidney cancer with venous involvement.

Methods: A 56-year-old women with an $8 \mathrm{~cm}$ tumor of the left kidney with a tumor thrombus in the renal vein which was reaching up to the edge of the IVC. The day before surgery, we performed angiography, a direct inferior vena cavography to evaluate the extension of the thrombus, its fixation, and the left renal artery embolization. The operation was done in both flank positions. We started on the left side to secure and cut the renal vein, then on the right side to do the nephrectomy.

Results: Nephrectomy time was $140 \mathrm{~min}$ without significant blood loos. Patient was discharged 5 days after surgery. The pathology report described a clear renal cell carcinoma, Fuhrman II with level I tumor thrombus.

Conclusions: Radical left nephrectomy for RCC with large thrombus in the renal vein can be done by minimally invasive intervention. It is a challenging but safe and feasible option in a carefully selected patient.

\section{V15-03 Robotic arm assisted ureteroureterostomy}

\section{$\mathrm{K}$ Lin, C Ou, K Lin}

Introduction \& Objective: Introduce robotic arm assisted ureteroureterostomy on an idiopathic upper third ureter stricture of the right kidney.

Methods: Surgical procedure. Initially, we put the patient as lithotomy position performing ureteroscopy to identify the ureter stricture location. Insert protective ureter catheter as a stent. Then, infuse Methyl blue dye into the right ureter via protective ureter catheter in order to indicate obstruction level of the right ureter. Change the patient to a supine position with the right flank elevated. Insert Veress needle to create pneumoperitonium. Insert one $12 \mathrm{Fr}$. trochar for the robotic camera and three $8 \mathrm{Fr}$. trochars for robotic arms. Dissect layer by layers to Identify right ureter and ureter stricture site. Perform right ureter segmental resection. Spatulation of both ureter end. insert a double-J stent and perform ureteroureterostomy with 4-0 Monocryl. Place a 10 French Jackson-Pratt drain to the ureter anastomosis site. Close wound layer by layer.

Results: Right ureteroureterostomy was performed effectively and precisely by robotic arm assistance.

Conclusions: Robotic arm assisted ureteroureterostomy was accurate surgical technique toward ureter stricture.

V15-04 Robotic Assisted Laparoscopic Bilateral Ureterolysis: A Novel Approach

M Billah, SS Komaravolu, JC Deolankar, D Kim, PD Kothari, PM Youssef, MD Stifelman
Introduction \& Objective: Retroperitoneal fibrosis is a rare cause of hydronephrosis secondary to inflammatory response. Traditionally managed with open surgery, ureterolysis is now performed laparoscopically and robotically. When performed robotically, the trocars are typically placed midline in order to access both sides. Here we present a robotic approach with the trocars placed along a Pfannenstiel incision. The objective of this video is to demonstrate the robotic approach to bilateral ureterolysis with retroperitoneal biopsy and omental wrapping with trocars in a Pfannenstiel incision.

Methods: 83-year-old female with past medical history of diabetes mellitus and hypothyroidism who developed bilateral hydronephrosis with associated flank pain. Workup revealed retroperitoneal fibrosis.

Results: Robotic bilateral ureterolysis was performed with retroperitoneal biopsy and omental wrapping. Incisions were placed along a Pfannenstiel incision and bowel was mobilized superiorly. This provided excellent access to bilateral ureters. Landmarks, stents and ultrasound were used to identify ureters. Retroperitoneal biopsies were performed which were consistent with fibrosis. Omental wrapped was used bilaterally and fluorescence imaging using indocyanine green dye confirmed adequate blood supply.

Conclusions: Robotic bilateral ureterolysis with a Pfannenstiel incision is feasible and is an effective alternative to a midline approach. This approach provides excellent exposure and robotic mobility.

\section{V15-05 Robotic pyeloplasty in anomalous kidney: point of technique}

\section{A Singh, S Agrawal, A Ganpule, R Sabnis, M DESAI}

Introduction \& Objective: Pyeloplasty is now commonly performed using the robotic platform but, performing pyeloplasty in kidney with anomalies of lie and position is rare using robotic platform.

Methods: In this video, we present three clinical cases with pelviureteric junction obstruction (PUJO), two in ectopic pelvic kidney and one case of horseshoe kidney. All the three cases were managed using robotic approach and different operative method. First case was a left ectopic kidney with PUJO (Obstructed on DTPA renogram) and a dismembered pyeloplasty was done. Second case was and right ectopic kidney with PUJO and a flap based pyeloplasty was done using robotic platform. The third case was a right sided PUJO in a horseshoe kidney, a dismembered pyeloplasty was done for the same.

Results: Console time for the three cases was $63 \mathrm{~min}, 75 \mathrm{~min}$ and $58 \mathrm{~min}$ respectively. Estimated blood loss was $50 \mathrm{cc}, 80 \mathrm{cc}$ and 60 cc respectively. Postoperative course was uneventful, and the patients were discharged on postoperative day 2 . All the patients were asymptomatic at 6 months with minimal hydronephrosis on ultrasound.

Conclusions: Robotic pyeloplasty is anomalous kidney is safe and feasible. Technical modifications make the procedure more effective. Direction of spatulation of ureter is the key and is different than in normally located kidney. Incision on the pelvis and the ureter depend on the lie of the kidney. Flap pyeloplasty can be effectively done in pelvic kidneys using robotic platform.

V15-06 Combined robotic-assisted laparoscopic and endoscopic surgery for a ureteropelvic junction obstruction caused by aberrant vessel with removal of kidney stones

F Adawi, M Alhamad, A Alkhalili 
Introduction \& Objective: ureteropelvic junction obstruction (UPJO) find regularly in the adults and sometimes complicated with nephrolithiasis, and recurrent fibrile UTI. The cause of this anomaly may be un aberrant vessel crossing anterior to the UPJ for the lower renal pole in this video, we present our technique for combined treatment by simultaneous robotic and endoscopic surgery of both the UPJO and the kidney stones.

Methods: A 55-yr-old patient presenting with complicated pyelonephritis the, investigations revealed the presence of the ureteropelvic junction obstruction with hydronephrosis (pelvis measure $70 \mathrm{~mm}$ ), and renal $14 \mathrm{~mm}$ calculus in median calyx. Further assessment and investigations confirmed the presence of an aberrant vessel crossing the UPJ towards the lower renal pole. After treatment of the infection and ureteric stent, the Pt. posted for surgical repair of the UPJO, and removal kidney stone at the same time. After pelvis dissection, we easily found the inferior polar artery. we reduced the size of pelvis, mais we could not find stone. We hold up the pelvis open by using robotic forceps in static position, and we used a flexible bladder fiberscope with the use of 2 nd column video for find the stone. We performed the anastomosis of the ureter anterior to the aberrant vessel.

Results: The total duration of the procedure was 115 minutes the patient discharged at next day post op., and the double J stent was removed 4 weeks later. Post OP follow up imaging with CT scan showed normal appearance of the kidney.

Conclusions: The treatment of ureteropelvic junction obstruction with kidney stone and a crossing aberrant vessel can be performed by a combined robotic and endoscopic surgery. It is a feasible, simple, reproducible, minimal invasive surgery.

\section{V15-07 Robot-assisted Synchronous Bilateral Ne- phrectomy for Autosomal Dominant Polycystic Kid- ney Disease}

\section{PM Gurung, TP Frye, H Rashid, JV Joseph, G Wu}

Introduction \& Objective: Autosomal dominant polycystic kidney disease (ADPKD) is a major cause of end stage renal disease (ESRD) necessitating bilateral nephrectomy in a significant proportion of patients. Laparoscopic, hand-assisted laparoscopic, and robot-assisted bilateral nephrectomies have gained popularity in recent years, in the quest to lessen the invasiveness of open surgical approaches. Herein, we describe our technique of robot-assisted synchronous bilateral nephrectomy for ADPKD.

Methods: Given prior abdominal surgery/transplant in most patients, we prefer an open cut-down access to place a $15 \mathrm{~mm}$ port $10 \mathrm{~cm}$ below the umbilicus in the midline. Four $(8 \mathrm{~mm})$ robotic trocars are placed under vision in a fan distribution along the umbilical level. The operating table is placed in reverse Trendelenburg (15 degrees) and tilted opposite to the targeted side. Provided there are no concerns for malignancy, some cysts encountered in large kidneys $(>2.5 \mathrm{~L})$ may require puncture, to facilitate access and mobilization. The resected kidney is placed in a large bag and tucked in the pelvis. A similar procedure is carried out on the contralateral side after redocking the robot and tilting the table in the opposite direction. The specimen bags are extracted by elongating the lower midline $15 \mathrm{~mm}$ port site.

Results: Of 15 synchronous robotic bilateral nephrectomies (2009 to 2018) in our prospective database, there were 7 cases performed for ADPKD (December 2015 to December 2018). Median [Interquartile range, IQR] values for patient demographics were: Age $=59 y$ yrs [47-63], Body Mass Index $=29$ [2632], Charlson Comorbidity Index $=5$ [3-7] and American Society of Anaesthesiology grade $=3$. Three (3) patients had prior Deceased Donor Transplant and four (4) had prior Living Donor
Transplant. Of these, 2 had prior Peritoneal Dialysis and 3 had Hemodialysis. Indication for nephrectomy were: Pain (5), Hemorrhage into cysts (3), and Renal masses (2). Perioperative outcomes were: Operating Room time $=388 \mathrm{mins}$ [324-453], Estimated Blood Loss $=200 \mathrm{ml}[75-300]$, Hematocrit change $=5$ [3.5-7.5], Hemoglobin change $=1.3[0.9-2.5]$, Transfusion $=0$, Length of hospital stay =3days [1.5-3.5], Grade I Clavien-Dindo complications $=2$ cases. All patients were alive at a median follow-up of 3.8yrs.

Conclusions: The accompanying video and outcomes data illustrate that robot-assisted synchronous bilateral nephrectomy is safe and effective in ADPKD, even in the context of prior renal transplant patients with attendant comorbidities.

\section{V15-08 Robotic Stone Removal in the Challenging Patient}

C Crigger, MW Salkini, A Pearce

Introduction \& Objective: Robotic surgery is an emerging option for challenging patients with nephrolithiasis. In some patients the body habitus, anatomy, concurrent pathology or past surgical history may preclude removal of large stone burdens by more conventional methods such as PCNL or ureteroscopy. Our objective is to review our experience with robotic stone removal and reinforce this technology as an option in urologists' arsenal. Methods: We retrospectively reviewed our series of patients undergoing robotic removal of their ureteral or renal stones since 2016. We reviewed demographics and relevant history, stone volume, BMI, prior surgeries, need for concurrent robotic surgery, clinical outcomes and follow-up. 23 patient records were found based on CPT codes with 21 patients included in the final analysis as 1 patient's case was aborted due to anesthesia concerns and the other patient elected to undergo staged ureteroscopies.

Results: 21 patients (mean age 52.9 years, equal sex distribution) underwent robotic removal of their ureteral and/or renal stones since 2016, including one patient who underwent staged bilateral removal. Average BMI in all patients was $37.4 \mathrm{~kg} / \mathrm{m}^{2}$ (range $21-$ $64 \mathrm{~kg} / \mathrm{m}^{2}$ ), average ASA classification 2.65, and all had prior abdominal surgeries (mean 2.4, range 1-5). Reasons for a robotic approach included severe obesity $\left(\mathrm{n}=3\right.$, mean BMI $\left.56.4 \mathrm{~kg} / \mathrm{m}^{2}\right)$, need for concurrent renal surgery such as pyeloplasty $(n=3)$, severe contractures limiting positioning for retrograde endoscopic or percutaneous nephrolithotripsy $(n=4)$, symptomatic calyceal diverticular stone with failed endoscopic approach $(n=1)$ and patient preference over percutaneous nephrolithotripsy $(n=1)$. Patients had a mean of 2.3 stones and total stone volume of $19 \mathrm{~cm}^{3}$ (range $0.7-75 \mathrm{~cm}^{3}$ ) per kidney. Average blood loss was $72 \mathrm{~mL}$ and mean operative time was 198 minutes. Mean hospital stay was 2 days. Mean follow-up was 90 days and the stone free rate for our cohort was $96 \%$.

Conclusions: Robotic stone removal is an emerging and efficacious option in patients with poor body habitus such as severe obesity, failed endoscopic or percutaneous management, or those undergoing concurrent renal surgery.

V15-09 Diagonal renorrhaphy technique in robotassisted partial nephrectomy for endophytic mid-pole renal tumor of size $>4 \mathrm{~cm}$

G Wang, X Liu, X Zhou, B Fu, L Chen, Y Li 
Introduction \& Objective: The safety and efficacy of diagonal renorrhaphy technique during robot-assisted partial nephrectomy (RAPN) for management of endophytic middle pole renal tumor of size $>4 \mathrm{~cm}$ was retrospectively evaluated.

Methods: For retrospective clinical study: data of patients with endophytic middle pole renal tumor of size $>4 \mathrm{~cm}$ who underwent RAPN from January 2016 to January 2019 were collected. All surgeries used diagonal renorrhaphy technique, following the same procedure: the renal artery was dissected and clamped, the tumor was enucleated from the kidney, defects of the vascular and collecting system were repaired using 3-0 absorbable barbed suture, then the edge of the renal parenchyma was sutured diagonally along the border using 2-0 absorbable barbed suture followed by unclamping. We retrospectively analyzed warm renal ischemic time, time required for renorrhaphy, blood loss and postoperative complications.

Results: For clinical study: among the 21 patients, 15 were male (median age 62 years, range 39-73 years). Mean tumor diameter was $6.4 \pm 1.3 \mathrm{~cm}$ and median R.E.N.A.L. nephrometry score was 9 (range $8-10$ ). The mean warm ischemic time was $20.1 \pm 5.6 \mathrm{~min}$ and mean time required for renorrhaphy was $12.4 \pm 3.3 \mathrm{~min}$. Median blood loss during surgery was $130 \mathrm{ml}$ (range $50-360 \mathrm{ml}$ ). No active bleeding was observed after renorrhaphy in all cases. Postoperative eGFR was $(70.7 \pm 13.2)$ $\mathrm{ml} / \mathrm{min} / 1.73 \mathrm{~m}^{2}$ at three-month follow-up. The imaging evaluation of 3 months after operation show that the operation site of kidney recovered well.

Conclusions: The diagonal renorrhaphy technique seems to be safe and effective for the management of endophytic mid-pole renal tumor of size $>4 \mathrm{~cm}$ during RAPN in selected patients.

\section{V15-10 Magnetic-assisted laparoscopic and robotic renal surgery: Applications of a new technology}

\section{J Fulla, E Kaplan-Marans, M Palese}

Introduction \& Objective: The Levita ${ }^{\mathrm{TM}}$ Magnetic Surgical System is a new magnetic retraction technology that reduces the number of ports used during laparoscopic and robotic surgery. It is FDA approved for laparoscopic cholecystectomy, bariatric surgery, and robotic radical prostatectomy. The objective of this video is to demonstrate the uses, safety, and feasibility of the Levita $^{\mathrm{TM}}$ Magnetic Surgical System during laparoscopic and robotic renal surgery.

Methods: A prospective, single center, single-arm, open-label study was performed to assess the safety and performance of a Levita $^{\mathrm{TM}}$ Magnetic Surgical System. The system includes a deployable, one-time use grasper and a reusable external magnet. The magnetic grasper is opened when the deployment handle is spread and closed with squeezing. The handle can be squeezed further which releases the tissue grasper from the handle. Bedside assistant moves the external magnet over the corresponding site until the magnets are coupled. For robotic cases, the magnetic retractor was used instead of a fourth robotic arm.

Results: A total of five surgeries were performed using the magnetic device: laparoscopic donor nephrectomy (1), laparo-

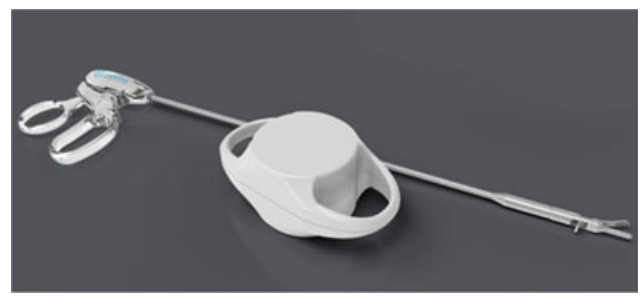

scopic radical nephrectomy (1), robotic partial nephrectomy (2), single port robotic partial nephrectomy (1). No robotic cases required a 4th arm or additional assistant port. No intraoperative or perioperative complications were noted.

Conclusions: Magnetic-assisted laparoscopic and robotic renal surgery is safe and feasible. Routine use of this system may avoid an extra incision and port as well as the need for a $4^{\text {th }}$ robotic arm. Advantages may include lower complication rates, improved cosmesis, and possibly lower cost.

\section{V15-11 ICG-Near Infrared guided robot-assisted pyeloplasty in patient with retrocaval ureter}

\section{A Veccia, A Tracey, R Autorino}

Introduction \& Objective: Retrocaval ureter is a rare congenital anomaly. The use of ICG-Near Infrared Fluorescence technology might be beneficial during robotic surgical repair of retrocaval ureter. Aim of this video is to report step-by-step ICGNear Infrared Fluorescence guided robot-assisted pyeloplasty in patient with retrocaval ureter.

Methods: A 26 years old woman presented to our clinic with recurrent episodes of dysuria, right flank pain and UTIs. CT scan and $3 \mathrm{D}$ rendering showed a right hydronephrosis and dilation of the proximal right ureter due to retrocaval ureter. Renal scan confirmed the right obstruction with a functional delay of about 27 minutes. The patient was candidate to right robotic pyeloplasty. The procedure was performed with the DaVinci ${ }^{\circledR} \mathrm{Xi}$ (Intuitive Surgical $^{\circledR}$, Sunnyvale, CA, USA). A 4-arm configuration was used as for other kidney surgery procedures. One 12mm Airseal ${ }^{\mathrm{TM}}$ assistant port (Conmed, Utica, NY, USA) was also used. The colon was medialized by incising the peritoneum, and the inferior vena cava was identified. Following the course of the inferior vena cava, the right renal pelvis was exposed, whereas no ureter could be seen in its regular anatomical position. The ICGgreen solution was injected through the percutaneous tube the patient had, and this allowed enhancement and identification of the right retrocaval ureter medial to the inferior vena cava. The proximal portion of the right ureter was identified, and it was confirmed to have a retrocaval course. Once the retrocaval ureter was completely identified, its narrow portion at level of the UPJ was excised and the renal pelvis was widely opened for dismembered pyeloplasty. The distal end of the ureter was spatulated using robotic scissors, and a $6 \mathrm{Ch}$ ureteral double-j ureteral stent was advanced into the spatulated ureter. The pyeloplasty was completed by using an Anderson-Haynes technique with two hemi-continuous 4-0 Vicryl sutures. No drain was placed.

Results: Estimated blood loss was $25 \mathrm{~mL}$. Console time 82 minutes. No perioperative complications were recorded, and patient was discharged on postoperative day 1 . Stent was removed after 4 weeks.

Conclusions: Robotic pyeloplasty is feasible and safe in patients with retrocaval ureter. The use of ICG-green could facilitate the procedure enhancing the course of the ureter.

\section{V15-12 Robotic Inferior Vena Cava Thrombectomy Using A Novel Intracaval Balloon Occlusion Techni- que}

A Alahmari, K Algarni, T Alamoudi, A Abdelsalam, T Kaid, A Kaki, E Younes, R Jamjoom, R Azhar

Introduction \& Objective: Robotic inferior vena cava (IVC) thrombectomy is a complex and challenging surgical operation. Optimal control of the IVC is crucial to avoid major 
intraoperative complications such as bleeding and embolism. Two approaches have been described for proximal control of the IVC in robotic surgery: 1. extraluminal approach by cross clamping using Rummel tourniquets or with one or more bulldog clamps or a Satinsky clamp replacing one or more tourniquets and 2. endoluminal approach by intracaval balloon occlusion using a Fogarty balloon catheter inserted through a cavotomy. Herein, we describe another endoluminal method using a Reliant ${ }^{\circledR}$ stent graft balloon catheter (SGBC) inserted through endovascular access through the right internal jugular vein (IJV) under fluoroscopy and placed above the tumor thrombus.

Methods: The case is a 60-year-old male patient who presented with a $10-\mathrm{cm}$ left renal cell carcinoma (RCC) with a level II thrombus. Left renal artery embolization with gel foam was performed the night before the procedure. Two right renal veins were found, and vessel loops and Rummel tourniquets were applied. Then, IVC mobilization above and below the IVC thrombus was obtained. The IVC was vessel looped below the thrombus, and Rummel tourniquets were applied between the 2 right renal veins and above the thrombus. Next, the Reliant ${ }^{\circledR}$ SGBC was inserted through the IJV under fluoroscopy and in- traoperative ultrasound guidance and placed above the thrombus in the suprarenal IVC. Then, vessel loops on the first right renal vein and infra-thrombus IVC were cinched, followed by balloon inflation above the thrombus. A vascular stapler was applied across the left renal vein at the junction with the IVC. Next, a cavotomy was made and the thrombus and stapled IVC wall were removed in a retrieval bag. The IVC was repaired using 4-0 Gortex ${ }^{\circledR}$ sutures followed by removal of all the vessel loops.

Results: The procedure was successfully performed in a minimally invasive fashion. The estimated blood loss was $250 \mathrm{cc}$, the operative time was 6 hours and 43 minutes for both IVC thrombectomy and radical nephrectomy. The IVC clamp time was 41 minutes. The postoperative period was uneventful, and the patient was discharged home on day three post-operatively. Histopathology report showed clear cell RCC. pT3c N0 M1. Negative margins with metastasis to the adrenal gland.

Conclusions: The application of advanced techniques and instrumentation has allowed renal tumors involving the IVC to be managed in a purely minimally invasive fashion. Our technique has demonstrated the feasibility of obtaining proximal control of the IVC using a Reliant ${ }^{\circledR}$ SGBC.

\section{ABSTRACT VIDEO SESSION 16: LAPAROSCOPY: UPPER TRACT- MALIGNANT}

\begin{abstract}
V16-01 Complete retroperitoneal Laparoscopic radical nephroureterectomy for the treatment of upper urinary tract urothelial carcinoma
\end{abstract}

J Wu, B Xu, M Chen, L Zhang

Introduction \& Objective: To present a new technique to accomplish retroperitoneal laparoscopic nephroureterectomy without changing the position of the patient in the procedure.

Methods: From February 2016 to December 2018, 12 patients suffered from upper urinary tract carcinoma were operated in our department, laparoscopic surgery was executed, and modified technique was used to avoid changing the position of the patients. Results: All the surgery were successful. No one need open conversion. Blood loss was minimal, all patients started oral intake without bloating 6 hours after surgery. Cystoscopic examination was scheduled one month after surgery, no recurrence was found in the bladder and the ureteral orifice of the affected side was fully resected.

Conclusions: Retroperitoneal laparoscopic nephroureterectomy without changing the position of the patient in the procedure is safe and feasible

\section{V16-02 Pure intraperitoneal laparoscopic ne- phroureterectomy with bladder cuff resection for upper urinary tract urothelial carcinoma}

G Kaneko, S Hattori, S Shirotake, K Kanao, S Hara, M Oyama

Introduction \& Objective: Laparoscopic nephroureterectomy (LNU) is an established procedure for low stage upper urinary tract urothelial carcinoma (UTUC). Combination laparoscopic procedures for removing a kidney and upper ureter with lapa- rotomy for removing lower ureter and bladder cuff (BC) is widely used [conventional LNU (cLNU)]. To reduce invasiveness, pure LNU (pLNU) has been reported, however, BC resection (BCR) is performed using "pluck" technique, stapler or clip in most cases. Therefore, there are several problems, such as possibility of postoperative urine leakage or incomplete BCR. The aim of the study was to describe our operative procedures of intraperitoneal (intra) pLNU and to assess surgical and oncological outcomes.

Methods: Surgical and oncological outcomes of intra pLNU for UTUC from 2015 to 2019 were reviewed. After transection of renal hilum and dissection of kidney from around tissue as like laparoscopic nephrectomy, an additional port was inserted if necessary, to retract colon or small intestine in lower abdomen without patient reposition, and ureter was caudally dissected until a ureterovesical junction could be identified. A stay suture was placed on a ureterovesical junction. And a ureteral orifice was circumferentially cut by cold scissor, and kidney, ureter and $\mathrm{BC}$ were removed en block. The opened bladder wall was closed with running stitches. Oncological outcomes of intra pLNU were compared with intra cLNU.

Results: Thirty-three intra pLNUs were performed (renal pelvic cancer in 20 cases, upper ureteral cancer in 5 cases, and lower ureteral cancer in 7 cases.). No intraoperative complications or open conversions were found. Median total operative time, pneumoperitoneal time and estimated blood loss were 180 minutes, 145 minutes, and $5 \mathrm{ml}$, respectively. Median time for dissection of lower ureter, BCR, and suturing the opened bladder was 74 minutes. Template lymph node dissection was performed in 8 cases, and median numbers of removed lymph nodes were 14. Tumor locations, tumor size, histology, pathological stage, grade, lymphovascular invasion, and positive surgical margin rate of pLNU were not significantly different from cLNU. No significant difference of recurrence free, intravesical recurrence 
free and cancer specific survival between pLNU and cLNU was found.

Conclusions: Intra pLNU was able to safely perform and shortterm oncological outcomes were comparable with intra cLNU.

V16-03 A prospective evaluation of impact of adherent perirenal fat on perioperative outcomes of 3-D laparoscopic transperitoneal partial nephrectomy for clinical $\mathbf{T} 1$ renal tumors

A Kumar, S Yadav, S Kumar, K Saurav, Y Prashant, V Tyagi, V Prasad

Introduction \& Objective: The adherent perirenal fat (APF) is an inflammatory fat sticking to renal parenchyma, making dissection difficult during LPN. We prospectively evaluated the impact of APF on perioperative outcomes of 3-D transperitoneal LPN for clinical T1 renal tumors.

Methods: All consecutive patients undergoing 3-D LPN for a clinical T1 renal tumors by a single surgeon between June 2013 and March 2019 at our institution were included. The patients were divided in 2 groups: Group A (with APF) and Group $\mathrm{B}(\mathrm{No} \mathrm{APF})$. The various clinical data were recorded and analyzed for these 2 groups. We are presenting video of one such case with APF.

Results: A total of 110 patients were included in the study. Group A and Group B had 39 and 71 patients respectively. The demographic data including mean age, BMI, mean tumor size and mean preoperative GFR were comparable in 2 groups. ( $>0.05)$. Similarly, the tumor location (Upper pole/Mesorenal/Lower pole) and tumor growth pattern (Cortical /Corticomedullary)were comparable in 2 groups. ( $p>0.05)$. The mean operating time and estimated blood loss were significantly higher in Group A (APF) in comparison to Group B, $(\mathrm{p}=0.001)$. Similarly, the blood transfusion rate and open conversion rate were significantly higher in Group A (APF) in comparison to Group B, $(p=0.001)$. However, the mean warm ischemia time, mean hospital stay and mean convalescence were comparable in 2 groups. The intra-operative bleeding and complication rate was significantly higher in Group A (APF) in comparison to Group B, $(\mathrm{p}=0.001)$. However, postoperative complications were comparable in 2 groups $(p=0.27)$. In histopathology, renal cell carcinoma was the predominant histology in both groups. The positive surgical margins were also comparable in 2 groups. At mean follow up of 42.1 months in Group A and 46.9 months in Group B, there was no local or distal recurrence in either group. The mean estimated GFR at 4 years was comparable in 2 groups and also not significantly lesser than preoperative value in both groups $(\mathrm{p}=0.57)$.

Conclusions: The presence of APF during 3-D transperitoneal LPN for clinical T1 renal tumors results in increased intraoperative bleeding, intraoperative complications, longer operating time and increased risk of conversion to open surgery. There are no adverse effect on preservation of renal function and oncological outcomes.

V16-04 Solitary kidney and thirteen tumors (13): laparoscopic radical nephroureterectomy, bench exvivo nephron-sparing surgery and auto transplantation

LA Zanettini, LA Zanettini, AC Zanettini, AA Paulmichl, OV Eye, MV Rizzon, IE Litvin, RA Guelfii, O Santos,

F Ribeiro, LA Zanettini
Introduction: This is a complex case of a 44-year-old man with a solitary left kidney, and at least eleven solid tumors diagnosed by Magnetic resonance imaging (MRI). He was in good general condition, no symptoms and normal serum creatinine. Four months before, he had undergone right radical open, nephrectomy by a large tumor of $20.2 \times 11.6 \mathrm{~cm}$ with diagnosis of multifocal papillary renal carcinoma stage pT2NOMx.

Objective: After multidisciplinary planning and patient's consent, we opted for surgery to remove all tumors and preserve the kidney.

Methods: Left laparoscopic radical nephroureterectomy, renal cooled solution perfusion and ex-vivo nephron-sparing resection of the tumors were performed. We used ultrasonography to identify deep tumors. After meticulous reconstruction, auto transplantation was performed in the right iliac spot.

Results: Dialysis was performed only in the first week. The patient was reoperated on the 9th postoperative day (PO) to drain a retroperitoneal urinoma. He was discharged on the 19th PO with a fistula that spontaneously closed at the 40th PO. Serum creatinine at the 60th PO was $1.74 \mathrm{mg} / \mathrm{dl}$. She had no other complications. Thirteen (13) tumors were finally resected with a diagnosis of multifocal papillary renal carcinoma type 1 and 2 . MRI control was performed at the 4th, 8th, and 12th PO and no relapses or metastasis were detected. At the time of publication of this text, in the 13th month PO, it has normal life and serum creatinine $1.69 \mathrm{mg} / \mathrm{dl}$.

Conclusions: Laparoscopic radical Nephroureterectomy, bench ex-vivo nephron-sparing surgery for multiple renal tumors and auto transplantation is a viable option in special situations. The laparoscopic approach should be used whenever possible to reduce the morbidity. This approach offers several advantages over hemodialysis therapy and should be considered after discussion with the patient.

\section{V16-05 Retroperitoneal Laparoscopic Radical Ne- phrectomy Technique for Advanced Left T3a Renal Cancer}

\section{Yogeswaran, J Roberts, S Hugo, K Ekwueme}

Introduction \& Objective: Locally advanced renal cancer comprises up to $40 \%$ of patients presenting with renal cell carcinoma. The optimum surgical technique (open vs laparoscopic) remains subject of intense debate. Laparoscopic retroperitoneal approach is technically challenging due to limited working space and the need to ensure oncological clearance of tumor thrombus from the renal vein (RV). Therefore, techniques to describe renal vein dissection using the retroperitoneal approach in locally advanced renal cancer is lacking. In this video, we demonstrate step-by-step dissection of the left RV in a T3a renal mass with tumor thrombus extending to the mid segment.

Methods: The index case is a 76-year-old male who presented with an incidental $10 \mathrm{~cm}$ tumor with left RV invasion. Following recommendation of radical surgery at multi-disciplinary meeting, the patient was prepared for retroperitoneal laparoscopic radical nephrectomy (RPLN). The patient was positioned in right lateral decubitus position with left side uppermost on surgical table flexed to 45 degrees under general anesthesia. The surgeon (KE) performed RPLN using a 4-port technique $(12 \mathrm{~mm}$ camera $+3 \times 10 \mathrm{~mm}$ ports). Balloon dilatation of the retroperitoneal space was performed. The anterior Gerota's fascia was opened and the ureter was identified and followed to the renal hilum. The renal artery was dissected and divided between three Hem-o-lok-

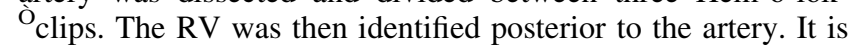
mandatory to isolate the renal vein in entirety along its length. 
Hence, the gonadal, lumbar and adrenal veins must be identified, isolated, clipped and divided. With the RV now free of any tributary, it is bluntly dissected beyond the tumor thrombus and stapled with ECHELON FLEX ${ }^{\mathrm{TM}} 35 \mathrm{~mm}$ Powered Vascular Stapler, ensuring a clear margin. This is achieved by a 360degree dissection of the distal thrombus free portion of the RV. The now avascular kidney is mobilized after division of the ureter.

Results: The patient's recovery was uneventful, and he was discharged on post-operative Day 2 with no complications. The final histology revealed a Fuhrmann Grade 4 clear cell carcinoma with involvement of the left renal vein pT3a. The resections margins were clear.

Conclusions: Retroperitoneal laparoscopic radical nephrectomy for advanced T3a RCC is feasible with quick recovery and good oncological outcomes in experienced hands.

\section{V16-06 Treatment of complex renal tumors, laparo- scopic is still available?}

C Conde Redondo, F Castroviejo Royo, S Martín Martín, J Torrecilla, F Lara Pérez, A Alonso Villalba,

R Cortiñas Gonzalez

Introduction \& Objective: There is no doubt that Da Vinci technology facilitates the learning curve of laparoscopy and allows facing increasingly risky challenges due to the neatness of the movements, and the precision that allows the technique. Now, it requires a time of installation and removal of the robot that can take from 60 to 10 minutes depending on the degree of training of the team. In surgeries where it is possible to foresee a serious vascular accident, laparoscopy is a useful alternative since it allows a conversion time higher than robotics. In this video we present two difficult cases of renal tumor that displaces and compresses the cava and a hiliar renal tumor.

Methods: CASE 1: We present the case of a 61-year-old male patient who presented a large mass that depends on the right kidney of 12 centimeters. The epicenter of the tumor starts from the lower pole with extrarenal extension, compressing and displacing the vena cava. There is no evidence of thrombosis of the cava or renal vein. There is a second synchronous tumor of $4.2 \mathrm{~cm}$ in the posteromedial renal area CASE 2: A 66-year-old patient with a history of laparoscopic left nephrectomy due to clear cell tumor in 2008. After 9 years of follow-up, he presented a heterogeneous mass of $5 \mathrm{~cm}$ in the lower pole of the right kidney with cranial growth to the hilar region. The biopsy of the mass was a renal adenocarcinoma. The right renal artery bifurcates in its middle third, originating a lower branch on which the vascularization of the tumor of the lower pole depends. The upper branch originates a branch that is directed to the anterior face of the lower pole, contacting it peripherally with it.

Results: CASE 1: Surgical time $180 \mathrm{ml}$, bleeding $100 \mathrm{ml}$, time of admission 5 days Piece of nephrectomy of 1242 grams and measures: $17 \times 8 \times 3 \mathrm{~cm}$. Necrotic tumor of $10 \times 9.5 \mathrm{~cm}$ in the lower pole. Pathological Anatomy: chromophobe type renal carcinoma that infiltrates the renal pelvis, capsule and perirenal adipose tissue. Clear cell carcinoma limited to the kidney of $3 \mathrm{~cm}$ of major axis. Negative edges pT3a, NOM0 CASE 2: Surgical time 280 minutes. Bleeding $75 \mathrm{ml}$, transfusion no. Cr post iq 1,8 , hospitalization time 4 days' Time ischemia 40 minutes. AP of the kidney cell adenocarcinoma piece, free edges. Need for dialysis: no.

Conclusions: Pure 3D laparoscopy allows great technical precision, leaving us a time and optimal reconversion in case of serious vascular injury.

\section{V16-07 Off-Clamp Laparoscopic Standard Partial Nephrectomy using PlasmaJet Surgical System}

R Papalia, a Civitella, E Luperto, P Tuzzolo, F Prata, R Scarpa

Introduction \& Objective: Standard Partial Nephrectomy (SPN) is routinely performed using clamping techniques to minimize blood loss. In this video we evaluated the feasibility and safety of an entirely no-clamping SPN Using Plasma Jet System.

Methods: A 65 years old men was admitted to our department for a $51 \mathrm{~mm}$ right renal tumor. Padua Nephrometry Score was 8 . Asa score was 2. Preoperative sCratinine was $1.09 \mathrm{mg} / \mathrm{dl}$ and eGFR 70,9ml/min, respectively. The patient underwent offclamp laparoscopic SPN using PlasmaJet Surgical device. The PlasmaJet Surgery Device is an advanced energy system delivering pure plasma to the tissues. Plasma is a highly energized phase of gas which is short-lived and quickly dissipates at the targeted site of application. A transperitoneal approach was used. The right kidney was isolated and flipped in order to allow circumferential exposure of the tumor. A cuff of fatty tissue was preserved to facilitate exposure of the mass during resection. Renal parenchyma was incised using PlasmaJet and a SPN carried out without clamping the main renal artery. During dissection specific vascular branches supplying the tumor were identified and treated using PlasmaJet surgical device without any need of clip ligation or suture. Hemostasis was completed by closing renal capsule on the PN bed.

Results: The procedure was successfully completed. Operative time was 110 minutes. Estimated blood loss was $150 \mathrm{cc}$. No intraoperative and postoperative transfusions were necessary. The patient was discharged 3 days after surgery. sCreatinine and eGFR at discharge were comparable to preoperative levels: $1.09 \mathrm{mg} / \mathrm{dl}$ and $70.9 \mathrm{ml} / \mathrm{min}$, respectively.

Conclusions: Off-clamp SPN using PlasmaJet Surgical System was feasible and safe. Further and more complex cases are necessary to evaluate the easy reproducibility of this procedure.

\section{V16-08 Renal cell carcinoma in left kidney with tu- mor caval thrombus: full laparoscopic management}

D Perlin, I Alexandrov, I Dymkov, A Shmanev, V Zipunnikov

Introduction \& Objective: Background: Treatment of level IIIII inferior vena cava (IVC) tumor thrombus for left renal cell carcinoma is among the most challenging open urologic oncologic surgeries with $38 \%$ complications and 4-10\% mortality rate. The procedure includes IVC thrombectomy, left radical nephrectomy and lymph nodes dissection. There are increasing numbers of centers using a right side laparoscopic radical nephrectomy with thrombectomy at last years but only few reports about laparoscopic management of IVC thrombus from left kidney.

Methods: We describe the method and present the initial series of full laparoscopic level II-III IVC thrombectomy in three patients with tumor in left kidney. One patient had been diagnosed distant metastases before operation, the another - pancreas involvement in the tumor process. The follow up time consist 4-26 months after surgery. All procedures completed successfully without conversion to open surgery. Tumor sizes were $5-16 \mathrm{~cm}$, length of thrombus in IVC $-2.4-7 \mathrm{~cm}$. Volume of blood loss ranged from 300 to $2500 \mathrm{ml}$. One patient received blood transfusion after surgery. One patient has died because of distant metastases 5 months after surgery. Two others were alive at 4and 26-months follow-up without signs of progression. 
Results: All procedures completed successfully without conversion to open surgery. Tumor sizes were $5-16 \mathrm{~cm}$, length of thrombus in IVC $-2.4-7 \mathrm{~cm}$. Volume of blood loss ranged from 300 to $2500 \mathrm{ml}$. One patient received blood transfusion after surgery. One patient has died because of distant metastases 5 months after surgery. Two others were alive at 4- and 26-months follow-up without signs of progression.

Conclusions: Laparoscopic IVC tumor thrombectomy for level II-III thrombi in cases of left kidney cancer is feasible, safe and allow use all main principles of oncology and vascular surgery. This kind of procedure doesn't lead to serious perioperative complications or major blood loss and can gain a quicker recovery after surgery. It needs more operations and longer followup for evaluation oncological efficiency.

\section{V16-09 Pure laparoscopic nephroureterectomy with bladder cuff excision and lymph node dissection}

\section{J Fang, L Tsai, G Chen}

Introduction \& Objective: Nephroureterectomy with bladder cuff excision (NUBCE) is the standard management of localized upper urinary tract urothelial carcinoma (UTUC). The prevalence of UTUC in Taiwan is relatively higher than in western countries. Lymph node dissection at the time of NUBCE is controversial due to lack of evidence of randomized control study and no precise parameter for preoperative staging. We shared our experience of laparoscopic NUBCE with lymph node dissection at the tertiary medical center.

Methods: We retrospectively reviewed patients with UTUC who received NUBCE between May 2015 and May 2018 in China medical university hospital. There were 229 patients. Of them, 153 patients $(66.8 \%)$ was treated with laparoscopy. Robotic method was used in 60 patients $(26.2 \%)$ and 16 patients $(7 \%)$ underwent open surgery. Surgeon mainly decided to perform lymph node dissection due to the locally advanced primary tumor or lymphadenopathy noted at preoperative images. The template of lymph node dissection was according to Kondo's study.

Results: Among the laparoscopic NUBCE patients, 81 patients $(52.6 \%)$ had lymph node dissection. 14 patients $(17.3 \%)$ had lymph node metastasis. The median number of retrieved lymph nodes was 9 (IQR 4-15). The mean operation time was 280 minutes and the mean blood loss was $110 \mathrm{ml}$. The average length of hospital stay was 8.7 days. None of the patients had intraoperative complication. All of the postoperative adverse events were below Clavein Dindo grade 3 (fever: 3 patients; ileus: 2 patients; chylous ascites: 2 patients). Analyzing the subgroup of patients with pathological stage T2 to T4 renal pelvis tumor, there was no significant difference of cancer-specific survival rate, progression-free survival rate and overall survival rate between the groups of lymph node dissection or not. However, the case with UTUC, pT4N2M0, presented in this video, seemed to have the benefit of lymph node dissection. She had no tumor recurrence during 28-month follow-up even without adjuvant chemotherapy.

Conclusions: It's a challenge to perform complete lymph node dissection template with laparoscopy due to high risk of large vessels injury. However, as time goes by, our techniques of pure laparoscopic NUBCE with lymph node dissection evolved and became mature nowadays. We have more ability to complete the template and dissect more lymph nodes. It's a safe and effective procedure in our experience. Although the therapeutic role of lymph node dissection is unclear in this case series, it may be caused by the incomplete template of lymph node dissection in the early period, we found the survival benefit in patients with enough range of lymph node dissection.

\section{V16-10 Spill Free Technique of Laparoscopic Ne- phroureterectomy In Multiple Lower Ureteral TCC}

S Nachimuthu, R Manickam, S Kallappan

Introduction \& Objective: The major concern in laparoscopic nephroureterectomy for multiple ureteric TCC is the spill of tumor tissue around the hiatus. Here we illustrate the spill free technique and inclusion of bladder cuff.

Methods: A 53-year-old female presented with hematuria associated with clots for two weeks duration. On evaluation Contrast CT Abdomen showed multiple right lower ureteric tumors. Cystoscopy revealed no bladder tumor. Laparoscopy in our spill free technique, we mobilized the distal ureter up to hiatus and further exposed the peri ureteric orifice mucosa by dividing the detrusor adequately. Then ureter was ligated at the orifice level. Before dividing the ureter, stay was secured in the bladder mucosa one $\mathrm{cm}$ distal to the orifice. Bladder closed in two layers.

Results: Patient made an uneventful recovery. Drain was removed on post-operative day 4 and discharged on next day with catheter in situ. Bladder cuff margin was tumor free in histopathology.

Conclusions: Our technique to prevent spill in nephroureterectomy is novel and easy to adopt.

V16-11 Partial nephrectomy by robotic-assisted laparoscopy for intra parenchymal renal tumor assisted by endoscopic ultrasound in a patient presents a multi-cystic kidney

\section{F Adawi, X Taccoen, A Demey, F Ozone, P Loock}

Introduction \& Objective: Regularly we discover small renal tumor associated with multiple kidney cysts. In this video we describe our technique for performing partial nephrectomy by robotic-assisted laparoscopy surgery combined with the use in perioperative an endoscopic ultrasound probe to locate and find the tumor and cystic limitations.

Methods: 53-year-old patient presents a $3 \mathrm{~cm}$ renal tumor discovered fortuitous by ultrasound performed for abdominal pain. The assessment and investigations completed by an MRI and a CT scan confirms an intra-parenchymal medio-renal tumor. In addition, there are 2 large simple cysts. polar superior and inferior in contact of the tumor. We used Robot Da Vinci Xi 4 arms, and endoscopic ultrasound probe to locate and delineate the tumor and cysts, We performed a resection of the upper pole cyst and then we resected the tumor, Macroscopic examination of the resected tumor shows resection in the negative margin. We Closed the renal parenchyma with 2 layer, superficial and deep layer by continues suture using a $3 / 0 \mathrm{~V}$ lock thread, blocked with multiple hem o'lok clips, Bleeding $20 \mathrm{CC}$, No drainage.

Results: The duration of procedure was 129 minutes, arterial clamping time 18 minutes, the patient left the hospital on the next postoperative day, the histopathological examination confirms a renal cell tumor classify PT1a, R0. the CT scan at 3 months was normal.

Conclusions: The treatment of small intra parenchymal renal tumor associated with renal cysts may be achieved by roboticassisted laparoscopy the use of perioperative endoscopic 
ultrasound provides more safety and facilitates operation with more precision

V16-12 Laparoscopic Partial Nephrectomy and Adrenalectomy for Incidentally Detected Left Renal Hilar Mass with Left Adrenal Adenoma.

R T.P., D Sarma, SK Pratihar

Introduction \& Objective: Renal cell carcinoma (RCC) is the most common type of kidney cancer in adults, approximately 90 $95 \%$ of cases. The days of "hematuria, an abdominal mass, and weight loss" suggesting the diagnosis of RCC are a distant memory. More than half of cases of RCC are detected incidentally. The main diagnostic tools for detecting renal cell carcinoma are ultrasonography, computed tomography, magnetic resonance imaging. With the progression of minimally invasive surgery laparoscopic partial nephrectomy has become standard of care for small renal mass. Likewise, laparoscopic surgery is also advocated for adrenal mass. The objective of this video is to show that laparoscopic partial nephrectomy with adrenalectomy for incidentally detected small renal mass and adrenal mass can be performed safely and efficiently in experienced hands with reduced patient morbidity and early recovery.

Methods: It is a videographic presentation of a single patient. Surgery was done on May 2019 at Gauhati Medical College Hospital. Our patient was a 43 years old diabetic lady, presented with incidentally detected left renal hilar mass. On contrast enhanced computed tomography evaluation left renal hilar enhancing mass $3.5 \mathrm{~cm} \times 2.9 \mathrm{~cm}$ and left adrenal adenoma $3 \mathrm{~cm} \times$ $2 \mathrm{~cm}$ was detected. The adrenal adenoma was non functional on metabolic evaluation. After proper patient counseling laparoscopic partial nephrectomy with adrenalectomy planned. Patient underwent the surgery on May 2019.

Results: Operative time was 96 minutes with blood loss of approximately $200 \mathrm{ml}$. Post-operative ultrasound Doppler showed normal renal perfusion with resistive index (RI) of 0.46. Patient had uneventful post-operative recovery and discharged on $2^{\text {nd }}$ post-operative day.

Conclusions: Laparoscopic partial nephrectomy for small renal mass is very safe in experienced hands with early patient recovery. Adrenal mass if detected along with renal mass should also be resected.

\section{ABSTRACT VIDEO SESSION 17: TRANSURETHRAL ENDOSCOPY}

\section{V17-01 Holmium Laser En-Bloc Resection of Bladder Tumors: The Real Deal}

M Nobbs, C Chemalsé

Introduction \& Objective: En-Bloc Resection of Bladder Tumors (EBRT) has several stated advantages over conventional resection (cTURBT) including higher rates of detrusor involvement, improved histological assessment and potential reduced recurrence rates. Laser energy has been previously used in EnBloc resections but there are limited cases reported in the literature. The current study intends to demonstrate our experience with Holmium Laser EBRT, describe the technique and potential complications and limitations. This study is primarily expressed in a video medium.

Methods: This is a single center observational prospective cohort study conducted from March 2016 to May 2019. Assessment was made of demographics, histological parameters, complications and outcomes of patients undergoing Holmium Laser EBRT.

Results: A total of 25 patients were included with a total of 27 bladder tumors resected. The median age was 74 (range $50-88$ ) with $80 \%$ of participants being male. The mean diameter of tumor removed was $17.1 \mathrm{~mm}( \pm 7.0 \mathrm{~mm})$ with $52 \%$ low grade tumors, $44 \%$ high grade and a single papillary urothelial lesion of low malignant potential. $15 \%$ of tumors had associated CIS. The majority of tumors $(81 \%)$ were $\mathrm{Ta}, 19 \%$ were $\mathrm{T} 1$ and no $\mathrm{T} 2 / 3$ tumors were resected. All areas of the bladder had tumor resected with the exception of the anterior wall and dome. Overall pathology reports suggested complete resection in $96 \%$ of cases with a single case given an equivocal report. $78 \%$ of specimens had detrusor included. 6 patients $(22 \%)$ have subsequently had a recurrence noted following EBRT with a median follow-up time of 14 months. A single Clavien-Dindo Grade III complication is reported.

Conclusions: Our findings confirm the safety and efficacy of Holmium Laser EBRT with encouraging rates of detrusor inclusion and complete resection. We have also demonstrated safety of use at the bladder neck and a short, manageable learning curve. Care needs to be taken with laser resection with tumor involving the ureteric orifice with consideration given to concurrent stenting or conversion to conventional monopolar resection.

V17-02 A case of bladder cancer involving ureteral orifice, which was completely resected by photodynamic diagnosis assisted transurethral resection using oral but not intravesical administered 5aminolevulinic acid.

K Watanabe, S Shirotake, Y Umezawa, T Takahashi, T Yamanaka, N Santo, T Okabe, G Kaneko, K Sano, M Yasuda, K Kanao, M Oyama, K Nishimoto

Introduction \& Objective: In recent years, transurethral bladder tumor resection (TUR-BT) has been widely used in combination with photodynamic diagnosis (PDD-TUR-BT) to treat non-muscle invasive bladder cancer (NMIBC). The PDD-TURBT procedure performed in Japan involves the oral administration of 5 -ALA $(20 \mathrm{mg} / \mathrm{kg}$, oral-PDD-TUR-BT). It is unclear whether the oral or intravesical administration of 5-ALA is better for NMIBC; however, the oral administration of 5-ALA may be superior to the intravesical administration of 5-ALA for tumors 


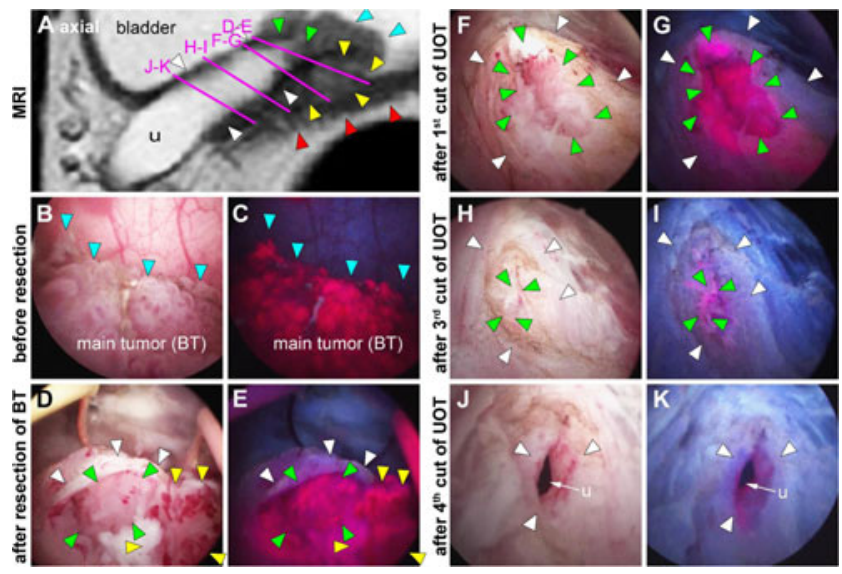

in the ureteral orifice when considering their intracorporeal delivery. Here, we experienced an intriguing case of bladder cancer, which invaded the ureteral orifice and was completely resected via oral-PDD-TUR-BT.

Methods: We describe intraoperative findings of this case with figure1 and supplemental video.

Results: This case involved a 71-year-old Japanese male who was diagnosed with bladder carcinoma. The main tumor was infiltrated into the right ureter. (Figure 1A) (cT1N0M0). The patient underwent oral-PDD-TUR-BT $(20 \mathrm{mg} / \mathrm{kg}$ of 5-ALA was administered 2 hours before the surgery) using the D-LIGHT system. The main tumor had invaded the bladder neck, the prostatic urethra, and the right posterior wall of the bladder. In addition, three daughter tumors were found on the posterior wall of the bladder. We deeply resected the largest tumor (Figures 1B-C). The right ureteral orifice was exposed, and it was found to have been filled with papillary tumors (Figures 1D-E). We resected the tumors in the ureteral orifice for 4 times (Figure 1F-K). After the $4^{\text {th }}$ resection, the hydroureter-affected lumen was exposed, the mucosa of which was smooth and did not emit fluorescence (Figures 1J-K). Low-grade cancer cells were detected in the ureteral mucosa after the $3^{\text {rd }}$ resection where an irregular and fluorescent mucosa was identified (Figure 1H-I). On the other hand, no cancer cells were found in the smooth and non-fluorescent mucosa after the $4^{\text {th }}$ resection. Thus, we succeeded in completely resecting the tumor in the ureteral orifice, which was accomplished via PDD using orally administered 5-ALA.Supplemental video shows detailed findings of PDD-TUR-BT.

Conclusions: Our experience indicates that oral-PDD-TUR-BT has the potential to treat ureteral tumor progressed from bladder tumors.

\section{V17-03 Memokath 051 migrated to bladder: a solu- tion}

J Torrecilla García-Ripoll, F Lara Pérez, S Martín Martín, M Bedate Núñez, J Soto Rodríguez, A Ruano Mayo, A Zamora Horcajada, F Natal Alvarez, M Pina Pallín, J Díaz Romero, J Cortiñas González

Introduction \& Objective: The Memokath051 stents are used in selected cases of ureteral stenosis, usually in a permanent way. However, we have observed that these can migrate in up to $35.48 \%$ of the cases in our series of 31 implanted stents. Our objective was to re-colocate a Memokath stent partially migrated to the bladder, in a simple and effective way.
Methods: We present a 77-year-old woman with a history of stenosis in the distal right ureter secondary to post-radiotherapy fibrosis, treated by placement of a $10 \mathrm{~cm}$ long Memokath stent. In the postoperative reviews, a distal migration of the stent was seen, with its distal end into the bladder, which caused urinary urgency, hematuria and recurrent urinary tract infections. We decided to perform a section of the stent with holmium laser.

Results: The patient evolved favorably, disappearing the urgency and hematuria, reducing the number of infections and maintaining the permeability of the ureteral stent.

Conclusions: The section of the memokath ureteral stent using holmium laser is a simple and effective technique in those cases of migration to the bladder where the stent remains permeable and in good condition.

\section{V17-04 Current status of thulium: fiber laser in the bladder cancer management.}

\section{A Martov, D Ergakov, N Baykov}

Introduction \& Objective: transurethral removal of bladder tumors in one piece becomes current "gold-standard" technique in the endoscopic management of non-muscle invasive bladder cancer (NMIBC). The rationale for it are oncological safety and excellent quality of pathology with the presence of the muscle layer. The proper detection of the tumors margins is essential during the procedure. It allows to obtain the negative surgical margin. For those purposes PDD, $1 \mathrm{~S}^{\circledR}$ and $\mathrm{NBI}^{\circledR}$ diagnosis are used. Thulium: fiber laser vaporization reserved for the patients with flat lesions, who unfit either for conventional TURBT or cystectomy. The aim of this study is to outline the thulium: fiber laser en-bloc bladder resection and vaporization.

Methods: since December 2017 till March 201858 pts (68-88 years) with NMIBC were treated with thulium:fiber laser en-bloc resection or vaporization under PDD, $1 \mathrm{~S}$ and NBI control. Tumors size were $1-3 \mathrm{~cm}$. The location of the tumors: bottom -12 , right lateral wall -23 , left lateral wall -19 , anterior wall -4.36 pts had primary tumors and 22 - recurrent. Time to recurrence were 3-24 mo. Thulium:fiber laser $\left(1940 \mathrm{~nm}\right.$, Fiberlase $\left.{ }^{\circledR}\right)$ with $600 \mathrm{mkm}$ fiber is used. The treatment mode was total energy $10 \mathrm{~W}$, frequency $-10 \mathrm{~Hz}$ and impuls energy $-1 \mathrm{~J}$. Vaporization mode was total energy $15 \mathrm{~W}$, frequency $-15 \mathrm{~Hz}$ and impulse energy - 1J. PDD were used in 12 cases, NBI - 28 and 18 pts had 1S technology.

Results: operation time is varied from 22 to $38 \mathrm{~min}$. There were no troubles during the removal of the detached tumor from the bladder. Differences at the detection of the tumors margin were revealed in 4/12 PDD-assisted operations, 5/28 NBI-assisted surgery and at 3/18 1S-controlled en-bloc resection. Totally, the application of modern optic and computer technologies allows to improve the results in 12 from 58 cases (21\%). Vaporization is used in 18 cases with a history of recent stoke (6pts) or AMI (9pts). The penetration depth of the laser is only $0,2 \mathrm{~cm}$ so there were no bladder perforations and blood transfusions. The catheter was removed on 1 day after the operation. Detrusor was presented in all cases of en-bloc removal, there were no positive surgical margins. Conclusions: thulium: fiber laser en-bloc bladder resection is current treatment of choice in the endourological treatment of the exofitic bladder tumors which are not exceed $3 \mathrm{~cm}$. This treatment is justified in cases of the patients, who couldn't stop anticoagulants, and those with severe comorbidities, which preclude use of muscle relaxants. PDD, $1 \mathrm{~S}$ and NBB permit to improve the operation results in $21 \%$ cases. Thulium: fiber laser opens additional possibilities in the office management of bladder cancer. 


\section{V17-05 MPM imaging, a new intraoperative tool for TURB diagnosis}

P Treacy, M Durand, I Pavlova, J Sfakianos, R Mehrazin, U Falagario, E Wajswol, F Barthe, Y Ahallal, N Tzavaras, D Chevallier, A Tewari, P Wiklund

Introduction \& Objective: Multiphoton microscopy is a nonlinear optical imaging technology, allowing imaging of fresh tissue, without any processing, up to a depth of 100 micrometers, with no photobleaching of the tissue. Signal is composed of autofluorescence (green channel) and second harmonic generation signal, helping to see non centrosymetric structures such as collagen (blue channel). Trans-urethral resection of bladder tumor (TURB) is fundamental in the diagnostic and staging tool, and the gold standard treatment for non-muscle invasive bladder cancer, and the absence of bladder muscle in the resection brings a significant increased risk of residual disease, disease recurrence and tumor understaging. The objective of the study was to evaluate the detection rate of bladder muscle and residual tumor cells on a cold cup biopsy of a resected bladder wall, after TURB, using the Multiphoton microscopy as a real-time imaging of the fresh bladder tissue, without processing, in order to have an intraoperative tool for real time diagnosis of bladder resection, and to avoid second look TURB.

Methods: Cold cup biopsies of the resected bladder wall were taken in the OR after TURB. Tissue was stored in PBS solution for conservation during transport to MPM room. Imaging of the tissue was done, with z stack images of 100 micrometer depth, and tiling of the 0.5 by $0.5 \mathrm{~mm}$ single images. After imaging, tissue was fixed in formalin, then embedded in paraffin, cut and stained with Haematoxylin and Eosin for histopathology analysis comparison. A correlation analysis was done with two pathologists.

Results: 4 patients were analyzed. Multiphoton microscopy allowed to show nuclear atypia of the carcinoma cells, elastin fibers (autofluorescence, green channel) and collagen fibers (second harmonic generation, blue channel) of the lamina propria, and the large bundles of regularly oriented muscle fibers, later confirmed by $\mathrm{H}$ and $\mathrm{E}$ staining histopathology. There was a $100 \%$ accuracy on resection depth, with 2 patients having a resection not including bladder muscle after histology results. Presence of tumor cells on Multiphoton images were confirmed on the $\mathrm{R} 1$ resection histopathology on 1 patient.

Conclusions: Multiphoton microscopy could be an interesting intra operative tool in order to evaluate both depth and margins of a TURB. Study is undergoing and more patients will be included in this study in order to have a bigger correlation analysis.

\section{V17-06 Holmium Laser Enucleation of a Bladder Tumor: Early Experience}

T Large, J Chavali, A Krambeck

Introduction \& Objective: We report our technique of transurethral laser enucleation of bladder tumor using the Holmium laser technique.

Methods: A 62-year old chronic smoker presents with gross hematuria. After initial CT evaluation, he was scheduled for cystoscopy, bilateral retrograde pyelogram and enucleation of bladder tumor. On cystoscopy, large fungating $>5 \mathrm{~cm}$ mass with calcifications identified on posterior bladder wall without ureteral orifices involvement. Bilateral pyelograms showed no signs of upper tract lesions. 1000-micron holmium laser was used for tumor enucleation using setting of 0.4 joules and $40 \mathrm{~Hz}$ for the entirety. Minimal bleeding was noted, and edges of tumor defect

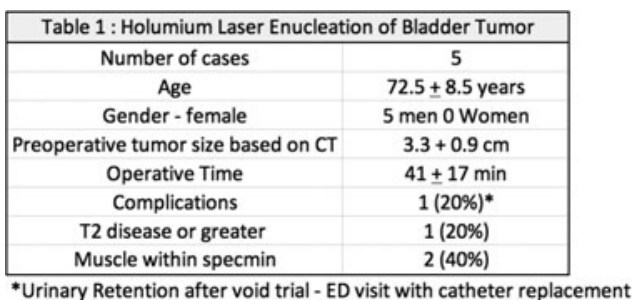

were ablated at 1 joule and $20 \mathrm{~Hz}$ with widened pulse width. Tumor base biopsies were obtained at the end and procedure was concluded after brief cystoscopy.

Results: We have completed 5 cases with only one instance of postoperative urinary retention. Table 1 outlines the cases. All patients have been men. Only one patient had muscle invasive disease. All cases were completed in a reasonable time $41+17 \mathrm{~min}$.

Conclusions: We demonstrate our technique for holmium laser enucleation of bladder tumor. It is associated with minimal bleeding post-operatively and preservation of tissue for pathological analysis.

\section{V17-07 Aggressive Bladder Tumor- Can intravesical morcellation help?}

A Puri

Introduction \& Objective: Bladder cancer is the sixth most prevalent malignancy in the United States. The most common clinical presentation is painless hematuria, which mandates evaluation with cystoscopy, renal function testing, and upper urinary tract imaging in adults 35 years and older. Transurethral resection of the bladder tumor allows for definitive diagnosis, staging, and primary treatment. This video presentation illustrates transurethral management of a highly aggressive bladder tumor, occupying the whole of the bladder lumen and initially suspected to be a mass of adherent organized bladder clot.

Methods: This is a case of a 89 year old gentleman who presented with gross painless hematuria with difficulty in urination along with other lower urinary tract symptoms. Patient gave a recent history of Optical internal urethrotomy with Bladder neck incision and cystolitholapaxy done elsewhere, in December 2018, two months prior to presenting to us. There was no mention of any bladder mass in the discharge slip from previous surgery. On evaluation, his performance status was 2 by ECOG. There was a firm palpable non tender bladder lump. Post-operative ultrasound was suggestive of bladder intraluminal mass (? Clots/? Neoplastic). Whole body PET CT revealed FDG avid (SUV max-25) heterogenously enhancing necrotic mass lesion with air foci within, $6.9 \mathrm{~cm} * 6.7 \mathrm{~cm} * 8.3 \mathrm{~cm}$ arising from anterior wall of urinary bladder with suspicious perivesical extension. Lesion reaching up to base of bladder inferiorly. Few non FDG avid subcentimeteric bilateral ext. iliac lymph nodes noted. Patient was planned for cysto-pan-endoscopy and proceed.

Results: Transurethral morcellation of the bladder mass followed by conventional transurethral resection of mass (TURBT) was done as surgery proceeded towards the bladder tumor base. Histopathological examination revealed high grade muscle invasive urothelial carcinoma. 1) Hospital stay- 2 days. 2) Need for blood transfusion- Nil. 3) Course in hospital- uneventful. 4) Catheter removal after 1 week. 5) Further management in conjunction with Oncologists (Medical and Radiation). 
Conclusions: The take home messages from this case are as follows: A complete and thorough cysto-pan-endoscopy is a must during every endoscopic evaluation. One must not only concentrate on the suspected pathology as per preoperative history and investigations but keep their eyes open for the unknown. Secondly, in case of a large blood clot or intraluminal bladder mass, careful initial intravesical morcellation by an experienced surgeon can be an option, although it is neither published nor approved for the same. Conventional TURBT remains the gold standard for managing a bladder mass initially.

\section{V17-08 En-bloc TURB: all over the bladder}

R Giulianelli, B Gentile, G Mirabile, P Tariciotti, L Albanesi, L Mavilla, G Tema, P Aloisi, A Lopes Mendes, G Rizzo, R Lombardo

Introduction \& Objective: Recently the EAU guidelines suggest patients with non muscle invasive bladder cancer undergoing en-bloc transurethral resection of the bladder (TURB) have a decreased risk of recurrence and progression. We present multiple cases of en-bloc TURB in different areas of the bladder.

Methods: All patients underwent en-bloc TURB using collins knife. A circular incision was made around the tumor, followed by level incisions beneath it with subsequent tumor retrieval. The circular incision connected marks made about $10 \mathrm{~mm}$. away from the tumor edge and continued until the superficial muscle layer was visualized.

Results: Al patients in our institution are treated with en-bloc TURB. Our recurrence rate at one year is $18 \%$ while no patients have progressed to pT2.

Conclusions: En-bloc TURB is feasible in all the regions of the bladder and we believe should be the standard technique in the management of non-muscle invasive bladder cancer.

\section{V17-09 Transurethral resection of a large bladder hemangioma: a case report.}

A Martov, D Ergakov, N Baykov

Introduction \& Objective: Hemangioma is a rare benign vascular tumor of the bladder, which occurs mainly in children. It has no specific clinical symptoms; this extremely rare tumor should be differentiated with muscle-invasive bladder cancer.

Methods: A 35 y.o. male patient has been admitted to D.D. Pletnev's clinical hospital in 2017 with complains on LUTS and hematuria. He noted 3 years ago sports injury of lower abdomen. Bladder tumor was suspected after office visit to urologist and the patient was referred to MRI, which showed large bladder tumor with thickening of the bladder wall. During videoendoscopic transurethral resection of the bladder using computer chromoendoscopy $\left(\right.$ SPIES $^{\circledR}$, resectoscope $26 \mathrm{~F}$ ), a solid $3 \mathrm{~cm}$ in diameter tumor was found on the right lateral wall of the bladder at a distance of $3 \mathrm{~cm}$ from the right ureteral orifice. The tumor had a wide base and ulcerative defects covered with blood clots. The dilated vessels of different caliber were observed in submucosal layer in computer-enhanced contrast mode in conditions of more uniform illumination ( $«$ Clara-Chroma» ${ }^{\circledR}$, Karl Storz, Germany). Pathological vascularization common for urothelial carcinomas was not found in the tumor, its base or bladder mucosa by computer modulation of a light beam (SPECTRA B ${ }^{\circledR}$, Karl Storz, Germany). A bladder transurethral resection of exophytic part of the tumor was performed. Moreover, there were visual signs of tumor pseudocapsule. Further, a separate removal of the tumor base located inside the bladder wall was performed. The final stage of the operation was hemostasis. The pathology showed a mixed hemangioma.

Results: The postoperative period proceeded without complications. No signs of relapse were observed at follow-up examinations (ultrasound, MRI, cystoscopy) 3, 6 and 12 months after the operation.

Conclusions: In current clinical observation, a 35-year-old patient had a large solid tumor of the bladder spreading into muscular layer. In histological and immunohistochemical analyses, verified hemangioma consisted of capillary, cavernous and arterio-venous components. The patient underwent transurethral resection of the bladder using computer chromoendoscopy. The diagnosis of bladder hemangioma has to be taken into consideration then manage the patients with bladder tumors.

\section{V17-10 Endoscopic Bladder Suture after ureteral and bladder cuff excision during laparoscopic $\mathrm{Ne}$ - phroureterectomy}

CA Oliveira, AA Barros, R Reis, E Dias, J Correia-Pinto, E Lima

Introduction \& Objective: Upper urinary tract urothelial carcinomas are usually managed by radical nephroureterectomy (RNU), often followed by intravesical chemotherapy to
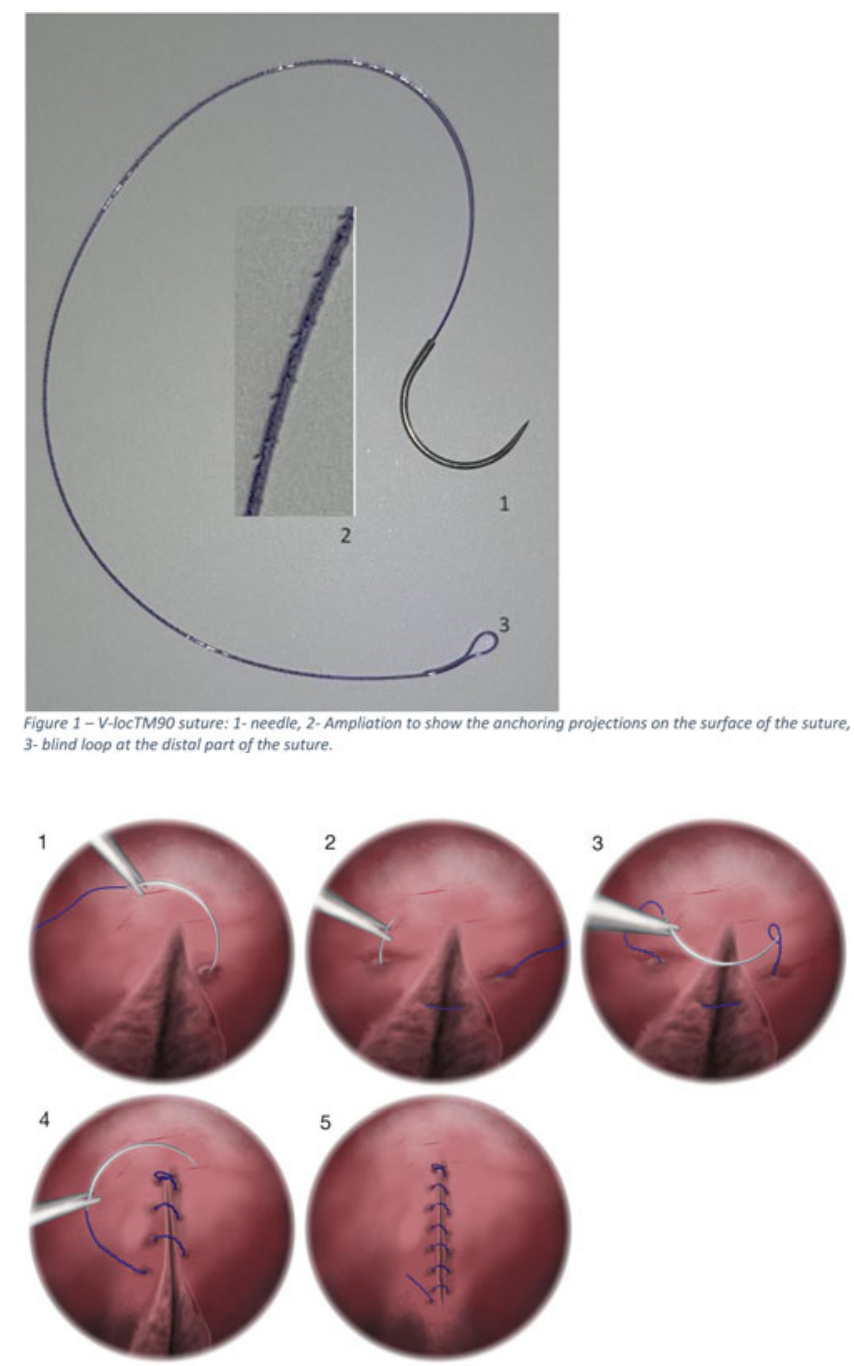

Figure 2 - Illustration of the steps (1-5) to close bladder incision using barbed sutures 
minimize recurrence. Open surgery is the gold standard procedure for RNU, but it associates with high morbidity, and it has been increasingly replaced by minimally invasive strategies, such as laparoscopy and endoscopy. Although effective, endoscopic ureteral excision leaves the bladder unsutured, precluding the immediate administration of intravesical chemotherapy. The objective of this video is to describe a new method to close the bladder wall after ureteral excision, using barbed sutures via the endoscopic access.

Methods: Seven patients with upper urinary tract urothelial carcinoma were submitted to endoscopic bladder wall closure in addition to standard laparoscopic nephroureterectomy. Ureteral disinsertion was performed using a bipolar resectoscope. The bladder wall defect was closed endoscopically using a $2 / 0$ barbed suture (Figures 1 e 2). The patients underwent standard laparoscopic nephroureterectomy and were submitted to intravesical instillation of $40 \mathrm{mg}$ mitomycin $\mathrm{C}$ in the $12 \mathrm{~h}$ pos-surgery.

Results: The procedure was performed in a simple fast and fluid manner. The total time of the endoscopic procedure was under 15 minutes. The bladder defect was completely closed. There were no complications to register. Patients underwent mitomycin bladder instillation without any adverse events to register.

Conclusions: We describe a simple, fast and safe technique of endoscopic bladder wall closure. With this method it is possible to maintain a minimal invasive approach and simultaneously administer early intravesical chemotherapy

\section{ABSTRACT VIDEO SESSION 18: PCNL \& URETEROSCOPY}

\author{
V18-01 Efficient Use of Low Watt Lasers: Good \\ Things Come in Small Packages - Pt. 2. Pulse Length
}

BF Schwartz, DT Lybbert

Introduction \& Objective: With technology improving laser lithotripsy, urologists are given greater freedom to adjust the settings to better suit their needs depending on the location of the stone, the type of stone, and how they are trying to break up the stone. This video will address how to adjust pulse length to make the most efficient use of the laser.

Methods: Video of cystoscopy from a live patient is presented to demonstrate how adjustments to pulse length to change how the stone is broken up or prevent retropulsion of the stone.

Results: The stone in this case was effectively obliterated in an efficient manner by adjusting pulse length depending on where we were at in the ureter and if we were intending to dust or fragment the stone.

Conclusions: Understanding the difference between long and short pulse length settings is essential in order to efficiently manage stone disease during ureteroscopy. By adjusting these setting throughout the case, urologists can decrease time spent in the operating room and have more patients that are stone free.

V18-02 Surgical treatment of matrix stone. Soft
stone, hard surgery JM López, A Sierra, P Luque, T Ajami, A Alcaraz

Introduction \& Objective: Matrix stone is a subtype of radiolucent lithiasis composed of a mucoprotein matrix with gummy and amorphous consistency. Correspond to type "VI a" in Daudon's classification. It is an unusual and challenging diagnosis due to the absence of pathognomonic radiological characteristics. Due to its amorphous consistency, PCNL seems to be a mandatory surgery to use suction systems to remove all protein matrix.

Methods: We present the case of 89-year-old man with CKD $(32 \mathrm{ml} / \mathrm{min} / 1.73 \mathrm{~m} 2 \mathrm{FG})$, past history of calcium phosphate stone with normal metabolic study and no documented recurrent UTI. As a result of recurrent macroscopic hematuria, with no findings in cystoscopy and negative cytology, a CT showed right hydronephrosis occupied by non-calcified material without contrast enhancement (20 UH). After the placement of the ure- teral stent, the patient was referred to our institution for surgical treatment.

Results: Supine Galdakao-modified Valdivia PCNL was performed. Retrograde pyelography showed a renal collection system completely occupied by a radiolucent matrix stone. $0-90^{\circ}$ $\mathrm{X}$-ray controlled puncture of lower calix papilla puncture is performed and 2-step dilatation with mini-percutaneous surgery equipment (MIP M, Karl-Storz) to accommodate mini-PCNL sheath of 17.5Fr. Extraction attempt with 16Fr mini-nephroscope and grasper is inefficient. Switch to $24 \mathrm{Fr}$ sheath with Amplatz dilator was performed in order to suction with US LithoClast master probe. Matrix of renal pelvis, upper and lower calix was removed. Second pyelography reveals filling defect in one upper calix and middle calyceal group. Flexible cystoscope though Amplatz sheath to access the upper occupied calix and extraction of matrix performed with $2.4 \mathrm{~F}$ Nitinol basket. Subsequently access to GCM was achieved using guidewire and rotating upside down the cystoscope due the posterior location of the entrance to the infundibulum. Finally, colleting system washed with physiological saline and gentamicin. 18Fr nephrostomy was placed for 24 hours without ureteral catheter. The patient was discharged at 72 hours with antibiotic and acidification of the urine with methionine. Surgical time of 90 minutes. CT reveal the absence of matrix material in the collecting system one month after the surgery.

Conclusions: Matrix stone is a rare entity with challenging diagnosis. Due to the protein nature and mucinous consistency, laser fragmentation is inefficient. Percutaneous surgery with suction system seems to be mandatory to eliminate the main volume of the matrix. The use of flexible scope helps to achieve stone-free status as it allows eliminate the residual material in smaller or narrow calix without accessory tracts.

\section{V18-03 Traditional Prone for PCNL: Fundamental Versatility}

D Mikhail, C Tabib, AD Smith, D Hoenig, Z Okeke

Introduction \& Objective: Percutaneous Nephrolithotomy (PCNL) for complex renal stones was originally described in the traditional simple prone position by Arthur Smith. Since then, dozens of modifications and other approaches have been attempted for PCNL. 
Methods: With each group pioneering modifications for supposed benefit, we continue to use a traditional prone approach at the Arthur Smith Institute for Urology in New York. As modified positioning has become the norm in the world of Endourology, we hope to review why we continue to use a simple prone approach - superior outcomes and true versatility.

Results: The primary debate is often in regard to Supine vs. Prone positioning. We touch on this debate in our video to confirm that prone continues to be the best approach and continues to be used by $80 \%$ of endourologists performing PCNL. However, prone positioning encompasses many modifications, the most common now being the split-leg prone approach. The urologic community believes that a split let position (whether supine or prone) is necessary for retrograde cystoscopy and ureteral access. We are the first to show in a video that we have similar access with a simple prone approach.

Conclusions: The benefits of simple prone compared to other approaches, including split-leg prone, are the superior outcomes of the prone position, with minimal extra equipment or personnel that can increase costs when a split-leg approach is taken. We show our success with prone cystoscopy, prone ureteroscopy, ECIRS (endoscopic combined intrarenal surgery), endoscopic guided access and using an access sheath - all in the traditional prone position.

V18-04 Percutaneous Nephrolithotomy for Erosion of Calcified Embolization Coils into the Renal Collecting System

\section{J Alamiri, J Joseph, C Fleming, ME Rivera}

Introduction \& Objective: Erosion of embolization coils into the renal collecting system is a rarely reported complication. Optimal management strategy is not well described. We presented a case of eroding calcified embolization coils managed with percutaneous nephrolithotomy (PCNL).

Methods: A 42-year-old female with remote history of coil embolization of a right renal arteriovenous malformation (AVM), presented with intermittent right flank pain and gross hematuria. Cross-sectional imaging and ureteroscopy demonstrated encrusted coils eroded into the right upper pole collecting system, with $2 \mathrm{~cm}$ of overlying stone. Patient elected to proceed with PCNL. Following fluoroscopic guided percutaneous access of the right upper pole calyx in the prone position by Interventional Radiology (IR), the patient was repositioned in a modified supine position facilitating prompt groin access for angiography in the event of major hemorrhage. The tract was established using a 30 Fr. balloon and sheath placement. A combination of ultrasonic and holmium laser lithotripsy was utilized to break the stone and expose the coils. The coils were retrieved using a grasper. After adequate stone and coil clearance, a nephrostomy tube was placed.

Results: Post-operative day (POD) 1 CT showed satisfactory stone clearance. Nephrostomy tube was subsequently removed, and the patient was discharged on POD 1. At 6 weeks follow up, ultrasound kidney showed no hydronephrosis and the patient reported resolution of flank pain and hematuria.

Conclusions: Percutaneous removal of an eroding calcified embolization coils utilizing a combination of ultrasonic and laser lithotripsy is safe and effective. However, collaboration with IR is warranted in managing any bleeding events.

V18-05 Efficient Use of Low Watt Lasers: Good Things Come in Small Packages - Pt. 1. Energy and Frequency

BF Schwartz, DT Lybbert
Introduction \& Objective: With technology improving laser lithotripsy, urologists are given greater freedom to adjust the settings to better suit their needs depending on the location of the stone, the type of stone, and how they are trying to break up the stone. This video will address how to adjust frequency and energy settings to make the most efficient use of the laser.

Methods: Video of cystoscopy from a live patient is presented to demonstrate how adjustments to frequency and energy change how the stone is broken up.

Results: The stone in this case was effectively obliterated in an efficient manner by adjusting energy and frequency depending on whether we were dusting the stone, fragmenting, or using the "popcorn" technique.

Conclusions: Understanding the relationship between energy and frequency are essential in order to efficiently manage stone disease during ureteroscopy. By adjusting these setting throughout the case, urologists can decrease time spent in the operating room and have more patients that are stone free.

\section{V18-06 Innovative technique Bullseye fluoroscopic renal access in supine PCNL}

\section{K Bhatti}

Introduction \& Objective: Percutaneous nephrolithotomy is still the first-choice treatment for renal stone $>2 \mathrm{~cm}$. Supine PCNL is gaining more acceptance and more urologist all over the world are adapting it recently. Many studies showed that there are no significant differences in stone free and complication rate between prone and supine PCNL. Renal access in supine PCNL is achieved either fluoroscopically with triangulation technique or with ultrasound guidance. In this study we are introducing an innovative way to perform Bullseye fluoroscopic renal access in supine PCNL. This approach is meant to facilitate renal access in challenging triangulation approach cases and when ultrasound not available, we also assume that it reduces the $\mathrm{x}$-ray exposure time during gaining initial access.

Methods: Approach description: The patient is placed in flank free complete supine position; a 6 Fr ureteric catheter is placed using flexible cystoscopy. The X-ray C-ram is introduced in 45 degrees to the operating table cephalically instead to the conventional 90 degrees approach (figure 1). In AP position a contrast study is done to localize the favored puncture calyx. The $\mathrm{C}$-arm is then rotated 45 degrees laterally towards the operating side. The wanted calyx is identified in this position too. The puncture needle tip is placed oved that calyx and then the needle is positioned coaxially with the $x$-ray tube until we get Bullseye configuration (figure 2). The needle in pushed further under fluoroscopy in this position, by shifting the $\mathrm{C}$-arm to AP position the depth of the needle and distance to the target calyx can be assisted (figure 3 ). Results: Pilot study, still 20 cases done.

Conclusions: Innovative technique Bullseye fluoroscopic renal access in supine PCNL really a new addition in pcnl techniques.

\section{V18-07 Mini Mini- Endoscopic Combined Intrarenal Surgery with LithoVue disposable ureteroscope for severely encrusted double-j ureteral stent}

A Mangiatordi, U Falagario, M Auciello, G Carrieri, L Cormio

Introduction \& Objective: Severely encrusted double-J stents are challenging clinical conditions usually requiring combined antegrade and retrograde endoscopic approach which can pose at high risk of damage flexible ureteroscope. Single-Use Digital ureteroscope has been developed as an alternative to reusable scopes 
showing safety and excellent performance, as they always perform as a new digital scope. Cost analysis revealed higher initial investments but lower costs per procedure in long term use for reusable scopes, however, the main factor for cost analysis of flexible ureterorenoscopes were referred to the instruments' longevity. The aim of this Video was to describe our technique for treatment of severely encrusted double-J stent using Mini- Endoscopic Combined Intrarenal Surgery and to report the optimal performance of LithoVue, a flexible disposable ureteroscope.

Methods: A 73-year-old man presented with a severely encrusted double-J stent placed six months before, due to obstructive urosepsis. Scan revealed multiple encrustations along the entire length of the stent associated with two stones of $4 \mathrm{~mm}$ in diameter in the lower calyx of the right kidney. The procedure was carried out under spinal anesthesia in galdakao-modified supine position. The lower pole calyx of the right kidney was punctured under fluoroscopic guidance. The percutaneous tract was dilated using metallic dilators up to $17.5 \mathrm{~F}$ and holmium laser was used to clear the proximal part of the encrusted stent. The LithoVue flexible disposable ureteroscope was then used to perform anterograde ureteroscopy to visualize first the stent in the proximal ureter and then to visualize the proximal pigtail of the stent in the upper calyx. Laser lithotripsy was used to clear the distal pigtail and to cut it at the level of the ureteral orifice. The proximal part of the stent was extracted through the Amplatz sheath. Flexible ureteroscopy with the LithoVue was carried out to examine the entire ureter and to check for further fragments into the kidney.

Results: Operative time was 75 minutes. The single-J ureteral catheter and the Foley urethral catheter were removed after 24 hours. Post-operative course was uneventful, and the patient was discharged on the 2 nd postoperative day. US and X-ray obtained 4 weeks after the procedure showed no residual fragments.

Conclusions: Severely encrusted double-J stents are challenging clinical conditions usually requiring combined antegrade and retrograde endoscopic approach. The Lithovue disposable flexible ureteroscope showed optimal antegrade and retrograde performance and should be recommended for such cases at high risk for flexible ureteroscope damage.

\section{V18-08 Supine Percutaneous Nephrolithotomy (PCNL) in Horseshoe Kidney}

\section{MO Breish, D Whiting, O Abdalla, S Sriprasad, F Anjum}

Introduction \& Objective: Horse-shoe kidney (HSK) is the most common renal fusion anomaly, with reported incidence of $1: 400$ mainly in males ( $M: F=2: 1$ ). Nephrolithiasis is frequently occurred in HSK patients with an estimated range of $21 \%-60 \%$ due to ureteropelvic junction obstruction, urinary stasis, recurrent infections, and metabolic abnormalities. Percutaneous nephrolithotomy (PCNL) is a procedure of choice for a high stone burden in a HSK with overall stone free rate of $72 \%$ to $93.1 \%$, and usually performed while patient in a prone position. In this video we present a case of a supine PCNL performed in our institution to treat stones in HSK.

Methods: 59 years old gentleman known to have HSK presented with left sided abdominal pain and a past medical history of renal stones. Previously, he was treated for renal calculi using shock wave lithotripsy and ureteroscopy. Blood investigations showed normal kidney function, however non contrast CT KUB demonstrated multiple calculi within the left moiety of HSK. He was counselled to undergo supine PCNL to treat the stone burden. Intraoperatively, the patient was prepared and positioned in the lithotomy position in order to perform retrograde studies. An Occlusion balloon catheter was placed within the ureter. An upper calyx puncture was performed to access the left moiety of HSK. Both hydrophilic and extra stiff guidewires were passed into the lower calyx. the puncture needle was removed leaving the guidewires in place. Balloon dilatation was performed followed by placement 30F Amplatz sheath. Flexible nephroscopy was performed in order to identify and basket the stones out of the kidney. $16 \mathrm{~F}$ Malecot nephrostomy was placed and secured.

Results: 1-2 days post operatively; the patient developed fever and was treated with Intravenous antibiotics. Post- operative CT KUB showed a patent nephrostomy in situ and confirmed stone free kidney. The nephrostomy was removed on day 7 and the patient was discharged 8 days post- operatively. The patient made a good recovery without major complications and was deemed stone-free following supine PCNL.

Conclusions: Supine PCNL is therefore feasible and safe option to treat stones in patients with HSK.

\section{V18-09 RIRS in Cross Cohen's ureteric re-impla- ntation : A difficult situation but safe and feasible.}

\section{KP Parikh, R Soni, AK Parikh}

Introduction \& Objective: Flexible ureteroscopy in a reimplanted ureter is always a challenging scenario and having its own set of difficulties. Purpose of this case is to demonstrate that flexible ureteroscopy for stone management in such cases is not only feasible but safe also.

Methods: Twenty-five years old young male presented with right sided dull aching, intermittent, nonradiating flank pain with previous history of some urologic intervention in the childhood for which no documentation available. On evaluation CT Urography suggestive of $1.1 \mathrm{~cm}$ right inferior calyceal calculus (HU1200) with bilateral cross cohen's ureteric re-implantation. Hydrophilic (Terumo) guidewire and ureteric catheter $(6 \mathrm{Fr})$ used to cannulate the ureteric orifices (UO). 8.5 Fr Flexible ureteroscope (Flex Xc) was introduced after putting the guide wire in right PCS without ureteral access sheath (UAS), calculus was placed in upper calyx with the help of basket then completely dusted with 200-micron laser lithotripsy with uneventful intraoperative and postoperative course. Results: Calculus was completely fragmented and dusted with laser lithotripsy with lasing time of 27 minutes with no intraoperative complication. Postoperative period was also uneventful.

Conclusions: Flexible ureteroscopy (RIRS) in ureteric reimplantation is a tricky situation, biggest challenge is to identify UO. Retrograde contrast study is very useful for guidewire placement, use of angled tip ureteric catheter makes job easier. UAS can be avoided; balloon dilatation of UO helps to introduce flexible scope smoothly, so with all these maneuvers RIRS is feasible and safe in such cases with no added complications.

\section{V18-10 SIMULTANEOUS BILATERAL ENDO- SCOPIC SURGERY (SBES): a new technique for the treatment of bilateral renal stones}

\section{S Proietti, ME Rodriguez Socarras, B Eisner, G Saitta, P Bellinzoni, F Gaboardi, G Giusti}

Introduction \& Objective: The incidence of bilateral and multiple renal stones is increasing. To date, some sparse data on simultaneous bilateral stone surgery are available in literature showing good outcomes in terms of both effectiveness and safety. The aim of this video is to report the outcomes of our series of patients with bilateral renal stones who underwent simultaneous bilateral endoscopic surgery (SBES) and to show step by step the details of the technique. 
Table 1- SBES outcomes

\begin{tabular}{|c|c|}
\hline \multicolumn{2}{|c|}{ SBES Demographic and stone characteristics of patients $(n=48)$} \\
\hline Gender, $n(\%)$ & \\
\hline Male & $31(64.6)$ \\
\hline Female & $17(35.4)$ \\
\hline Age $(y)$, mean $\pm S D$ & $48.5 \pm 12.4$ \\
\hline BMI $\left(\mathrm{kg} / \mathrm{m}^{2}\right)$, mean $\pm \mathrm{SD}$ & $24.1 \pm 3.3$ \\
\hline \multicolumn{2}{|l|}{ Stone size $(\mathrm{mm})$, mean $\pm \mathrm{SD}$} \\
\hline PCNL side & $30.1 \pm 9.2$ \\
\hline FURS side & $11.1 \pm 4$ \\
\hline R side & $20.6 \pm 10.8$ \\
\hline L side & $21 \pm 11.5$ \\
\hline \multicolumn{2}{|c|}{ Intraoperative and postoperative outcomes } \\
\hline Total OR time (min), mean \pm SD & $72.5 \pm 23$ \\
\hline Length of hospital stay (days), mean $\pm \mathrm{SD}$ & $2.75 \pm 1.5$ \\
\hline Primary SFR (both sides), n (\%) & $40(83)$ \\
\hline eGFR variation (preop, 1-day postop, 1-month post) & $P>0.05$ \\
\hline Complications by Clavien-Dindo grade & \\
\hline Grade I & $2 / 48(4.1)$ \\
\hline Grade II & $5 / 48(10.4)$ \\
\hline Grade III/TV/V & $0(0)$ \\
\hline
\end{tabular}

Methods: A prospective analysis of 48 consecutive patients who underwent simultaneous flexible ureteroscopy (fURS) in one side and percutaneous nephrolithotomy (PCNL) in the other side contemporaneously for bilateral renal stones at our institution between June 2017 and February 2019, was performed. The study was approved by the local ethics committee and patients provided informed consent. Routine preoperative and 1-month postoperative work-up included history, physical examination, urinalysis, urine culture, blood test including the evaluation of estimated glomerular filtration rate (eGFR) by using the Cockcroft-Gault equation. Abdominal non-contrast computerized tomography (NCCT) scan was performed in all cases preoperatively and 1-month postoperatively. Patients were reported to be stone free if there were no stones on post-operative NCCT or if there were residual fragments $<2 \mathrm{~mm}$ in diameter. Peri/postoperative complications were reported according Clavien-Dindo classification system. The primary endpoint of the study was stone free rate (SFR) and the secondary endpoints were Clavien-Dindo complications grade 1 or higher.

Results: The outcomes are reported in the Table 1.

Conclusions: SBES is a safe and effective procedure for the treatment of bilateral renal stones. Further randomized studies with larger population are needed to confirm these initial favorable outcomes of SBES as an alternative to staged surgeries in patients with bilateral renal stones.

V18-11 Endoscopic cystolithotripsy and Mini-PCNL with ballistic and ultrasonic energy for bladder and transplanted kidney stones

JM López, A Sierra, T Ajami, P Luque, L Peri, M Musquera, A Alcaraz
Introduction \& Objective: The incidence of kidney stones in patients after kidney transplantation is $1.0 \%$. Calcium based stones are the most common kidney stones after transplantation although struvite stones are not uncommon in kidney transplant recipients. The management of high burden stone $(>2 \mathrm{~cm})$ could be challenging in order to achieve high free stone rate maintaining renal function and avoiding septic complications. Percutaneous nephrolithotomy could be the therapy of choice for these cases.

Methods: We present the case of 54 years old female with history of chronic kidney disease. She has received 3 kidney grafts, the last one in left iliac fossa. She presented recurrent UTI in the last 6 months, with urine culture positive to Klebsiella and Proteus. A CT scan was performed, showing a 27 and $24 \mathrm{~mm}$ bladder stones $(750 \mathrm{HU})$ and a $22 \mathrm{~mm}$ pelvic stone $(800 \mathrm{HU})$. The patient was referred to lithiasis unit. She received 10-day ceftriaxone 1 gr SID (iv) administration prior surgery.

Results: The patient was placed in lithotomy position. An endoscopic cystolithotomy was done first using 22 Fr nephroscope (12Fr working channel). Ultrasonic and ballistic energy is applied using a 10.2 Fr probe. Total fragmentation time was 35 minutes. All fragments were removed neither Ellik evacuator nor grasper. An US and X-ray guided puncture was done through the superior calyx and dilatation with high $18 \mathrm{Fr}$ high balloon for mini-PCNL. The stone was localized in the renal pelvis using a $15 \mathrm{Fr}$ nephroscope (7.5 Fr working channel). $5.5 \mathrm{Fr}$ ballistic and ultrasonic probe was used for lithotripsy. Only one fragment was removed with basket for analysis. Mini PCNL OR time 40min. 8 Fr nephrostomy tube was placed for 72 hours without ureteral stent. Preoperative creatinine $1.28 \mathrm{mg} / \mathrm{dL}$, Postoperative creatinine $1.18 \mathrm{mg} / \mathrm{dL}$. No septic or hemorrhagic complications were reported. The spectroscopy analysis revealed a mainly carbonate apatite stone composition

Conclusions: Ultrasonic and ballistic energy may reduce endoscopic OR time for bladder and kidney stones. The increase of vacuum cleaner effect with active suction can make feasible treating $>2 \mathrm{~cm}$ bladder stones and reduction of amplatz size for kidney stones. For transplanted kidney stones, mini-PCL with ultrasonic and ballistic energy provides an efficient and safe treatment, and it can reduce the risk of bleeding and loss of function.

\section{V18-12 ECIRS in Kidney with Complex Anatomy}

F Pauchard, M Escobar

Introduction \& Objective: Endoscopic combined intrarenal surgery was first described by Dr. Ibarluzea at the beginnings of XXI century and internationalized by Dr. Scoffone in 2007. Its advantages are to provide versatility in the treatment of lithiasis, with the aim of reducing complications and number of procedures, increasing the success of surgery.

Methods: A 69-year-old woman with a history of hypertension and left lumbotomy due to renal lithiasis operated more than 20 years ago in other center. She was sent to our clinic due to recurrent urinary tract infection. Study with CT Urography reveals a left double pyelocaliceal system with a filiform stenosis that communicates the inferior with the superior ureters and determines hydronephrosis of the inferior system. In addition, a kink of the lower ureter with the lower pyelocaliceal cavity. Multiple stones in the lower ureter proximal to the stenosis and in the pyelocaliciary cavity. The first flexible endoscopic surgery was decided to evaluate the anatomy and eventual lithiasis extraction. We achieve to advance flexible ureteroscope through the stenosis and the kink with difficulty. Pigtail catheter was installed. Operating time 90 minutes, discharged at $24 \mathrm{hrs}$. Second surgery was scheduled, this time combined, planning mobilization of 
urinary lithiasis from the ureter to the lower pyelocaliceal cavity with flexible ureteroscope and extraction of stones by percutaneous tract. In supine position we dilated a $24 \mathrm{Fr}$ tract and flexible nephroscope was used, simultaneous work with a flexible ureteroscope without access sheath retrogradely. Previously narrow stenosis zone is displayed of good caliber. All stones were extracted. Surgery was completed as planned without incidents in $135 \mathrm{~min}$, nephrostomy was left for $48 \mathrm{hrs}$. and patient discharged. Results: Non contrast CT scan one month after surgery shows patient free of lithiasis and disappearance of obstructive factor. Conclusions: ECIRS is a synergy between PCNL and flexible ureteroscopy, becoming a useful, simple and safe alternative that should be considered for the personalized management of complex stones both by volume and by anatomy. We show a challenging clinical case that studied, planned and approached in an adequate way allowed a satisfactory result in a minimally invasive way, both from the point of view of lithiasis and stenosis.

\section{V18-13 Miniaturized PCNL for renal stone larger than $3 \mathrm{~cm}$ in Galdakao-modified supine Valdivia position}

\section{Liu, T Lin, H Huang}

Introduction \& Objective: Most percutaneous nephrolithotomy (PCNL) are performed by prone position. However, the disadvantages of prone position are prolonged operation time and difficulty dealing with cardiopulmonary emergent events. Galdakao-modified supine Valdivia position was first introduced in 2008. We demonstrate miniaturized PCNL for renal stone larger than $3 \mathrm{~cm}$ in Galdakao-modified supine Valdivia position. Methods: In this movie, we demonstrated mini-PCNL on a 68 y/o female with right renal stone, $36.93 \times 29.16 \mathrm{~mm} 2$. In our medical center, a total of 23 patients were undertaken with miniPCNL in Galdakao-modified supine Valdivia position.

Results: After general anesthesia, the patient was placed in Galdakao-modified supine Valdivia position. 11th, 12th ribs, and posterior axillary line were marked before disinfection. Antiseptic draping was done on both flank and perineal area. First, we performed cystoscopy and occlusion catheter placement. We then instilled contrast into renal pelvis via catheter. Second, renal puncture was achieved by combined fluoroscopic and ultrasonographic assists. Third, safety guidewire was placed into renal pelvis. Percutaneous nephrostomy tract was created by balloon dilator to 18 Fr. Mini-nephoscopy inserted with Holmium laser assisted lithotripsy. After completely stone removal, cystoscopyassisted double $\mathbf{J}$ was placed retrogradely. We checked double $\mathbf{J}$ position via PCN tract. Finally, we used 14 Fr. Foley as nephrostomy. One month after operation, abdominal plain film revealed minimal residual stones. To review the experience in $\mathrm{NCKH}$, a total 23 renal stone patients with mean age 57.61-yearold. Mean maximal stone length was $34.87 \mathrm{~cm}$. Stone free rate one month after mini-PCNL was $78.3 \%$. Only one patient needed blood transfusion.

Conclusions: Mini-PCNL in Galdakao-modified supine Valdivia position is time-saving and safe. Even for renal stones larger than $3 \mathrm{~cm}$, mini-PCNL can still be an effective therapeutic alternative. 